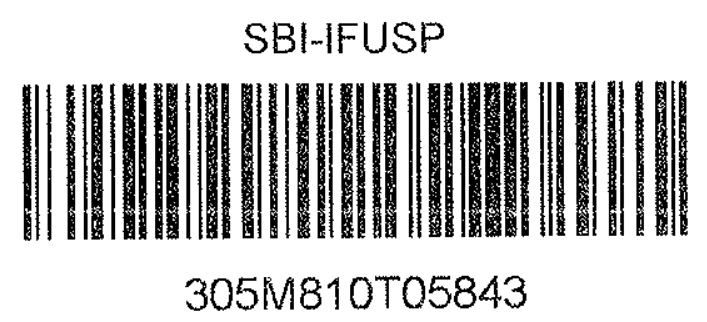

ESTUDO DE CRTSTAL THOUTDO HOTROPTCO NEMATTCO (TTPO IT) A PHMPERATURA AMBTENTE $\mathrm{B}$ DA FASE A BATXA TEMPERATURA

Tese de Livrembocencia apresentada ao motitu to de rusica da Univer sidade de säo paulo. 


$$
\text { - os }
$$

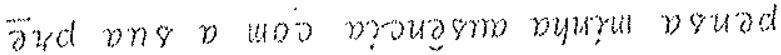

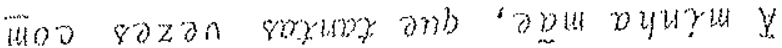
- opry9os byougsno bur sazan sor

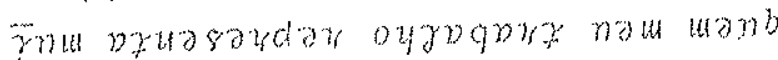

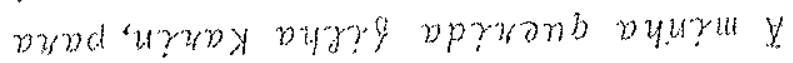




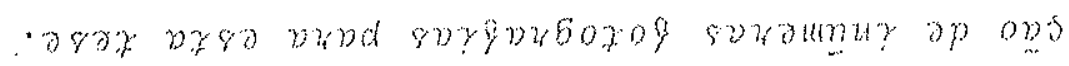

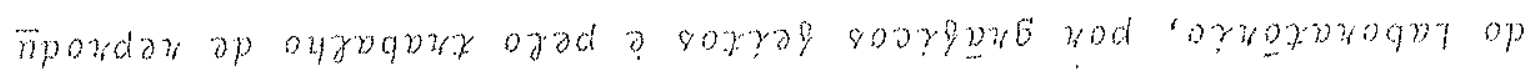

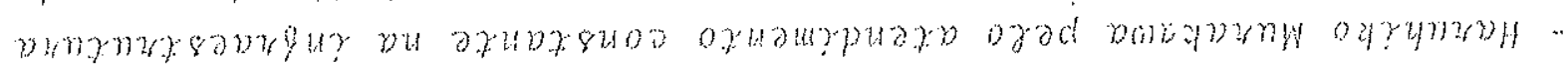

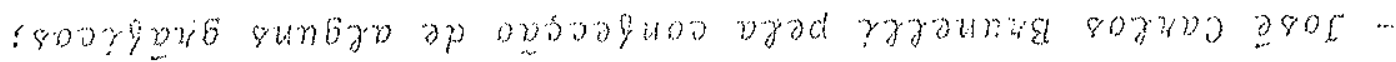
:

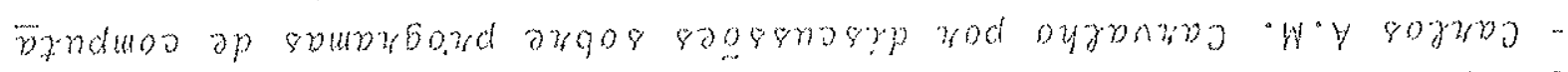

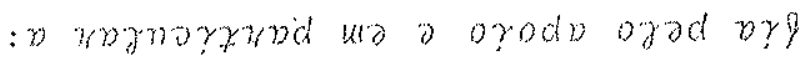

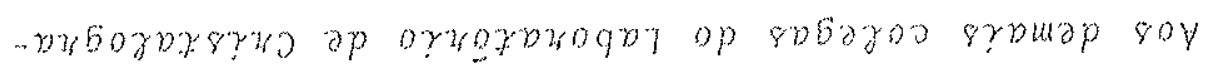

$$
\text { 9op) }
$$

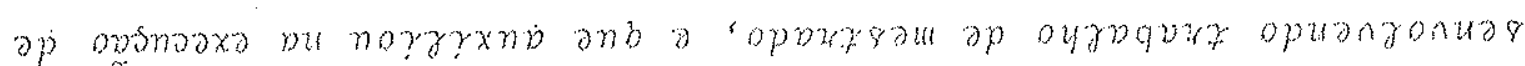

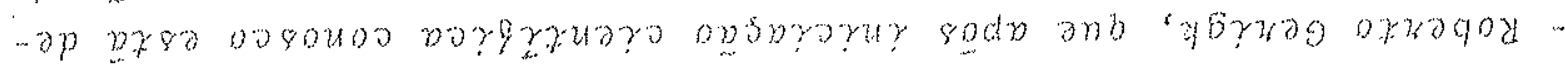
bygog

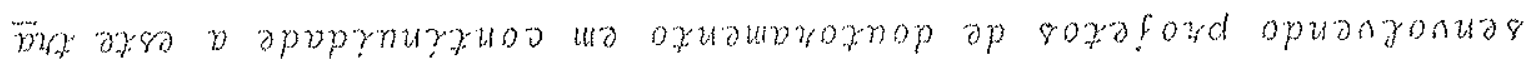

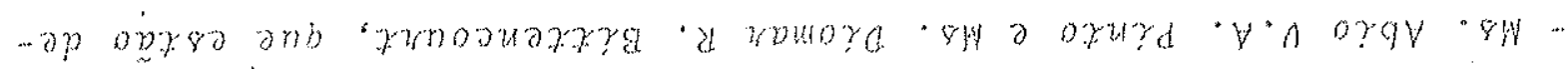
squpoxugrado ynbo woyu

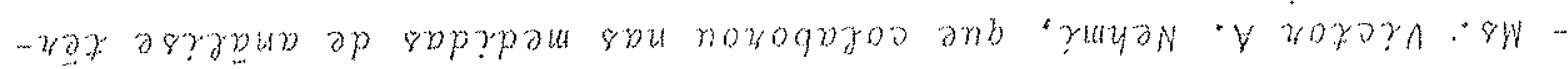
syogyos

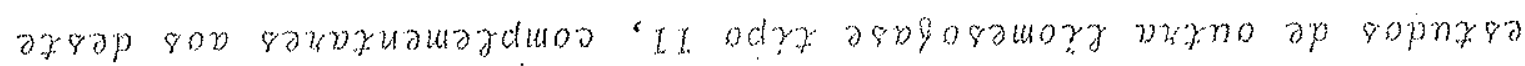

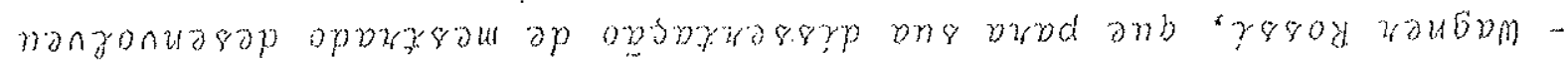
6yoxprogot op spurga

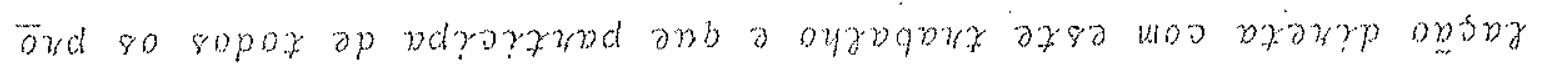

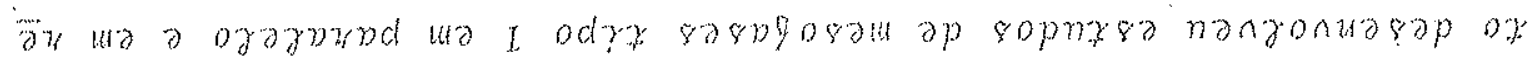

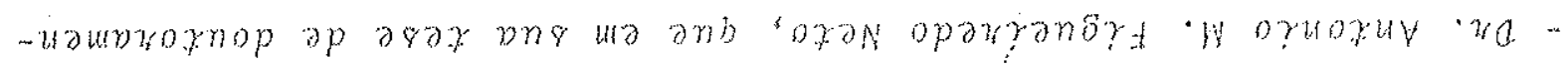
s opppratryoo

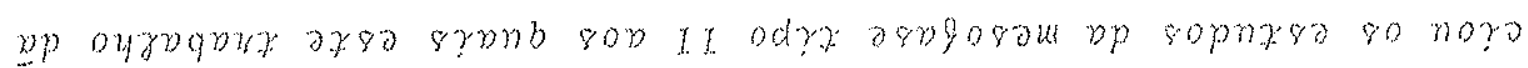

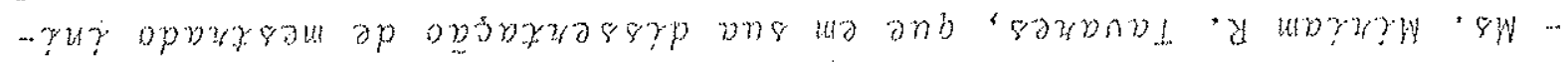

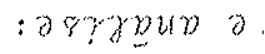

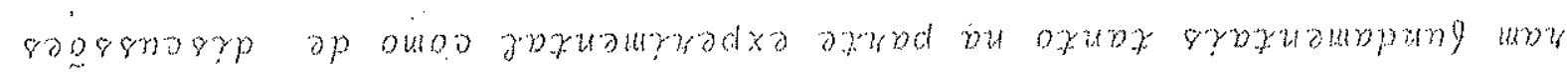

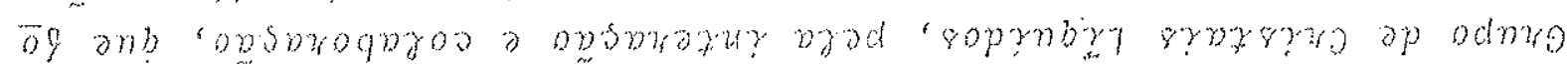

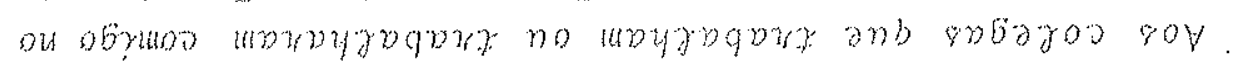

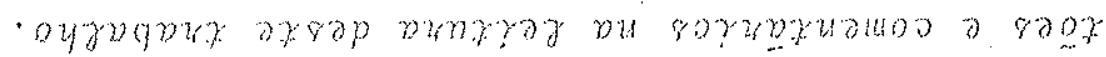

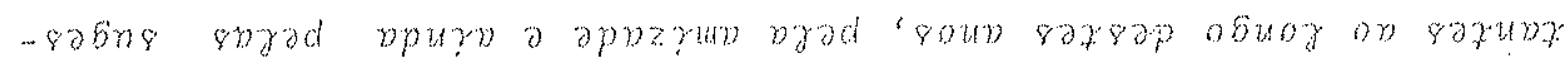

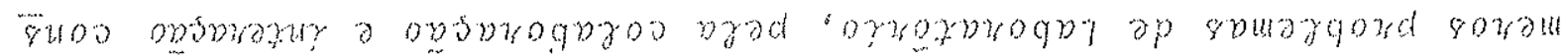

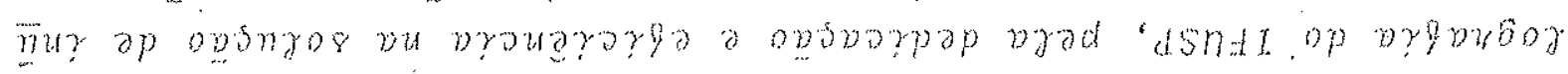

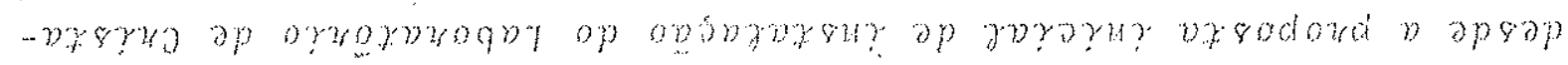

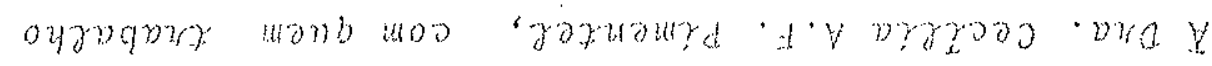




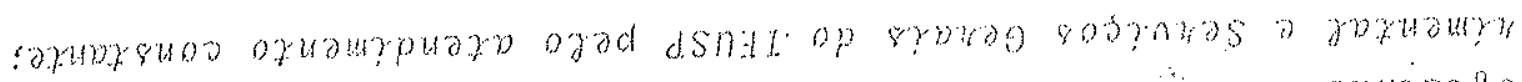

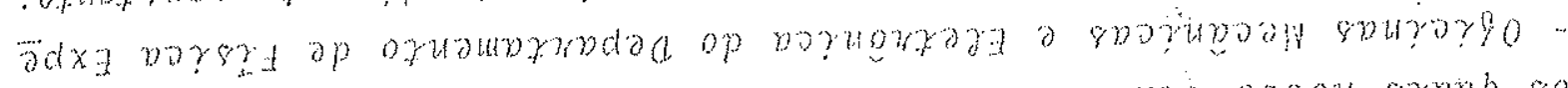

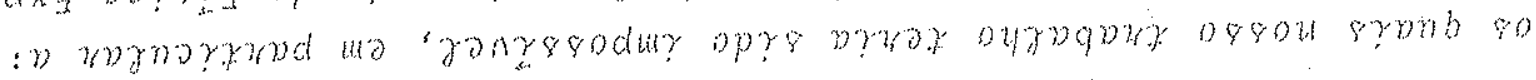

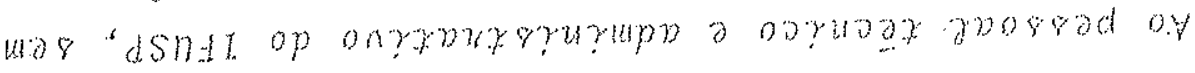

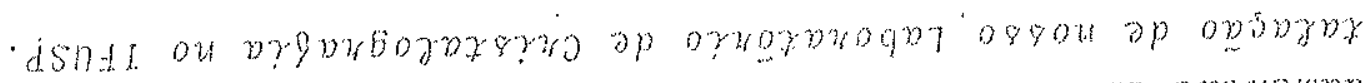

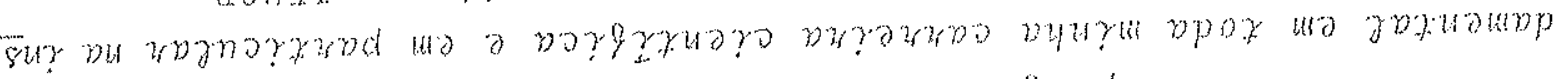

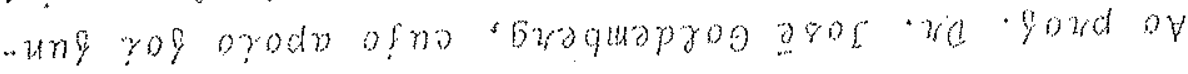

1\%9\%

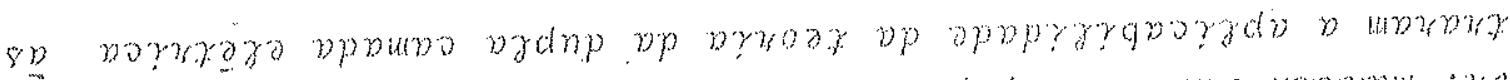

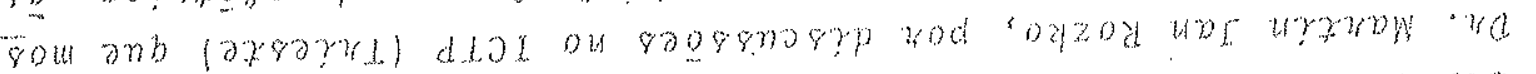
sdsnor op aryangyerapono

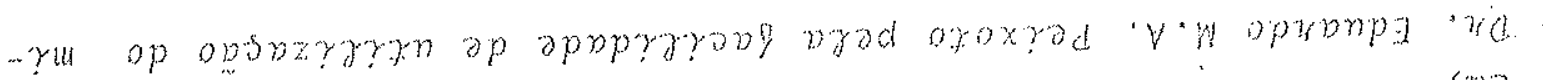
(D)

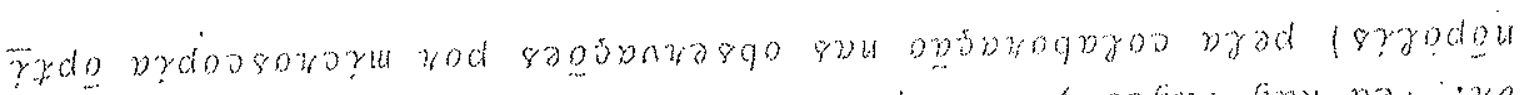

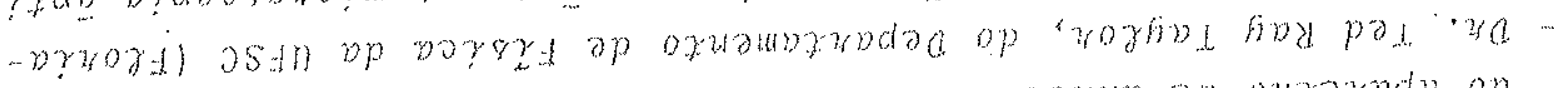

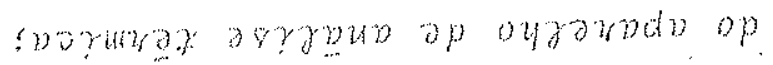

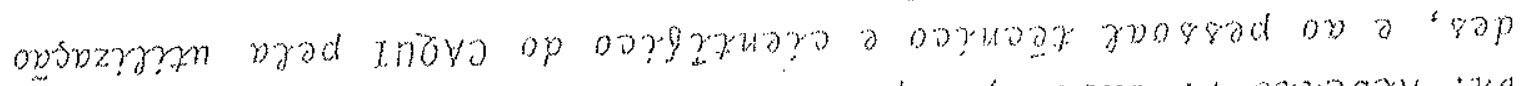

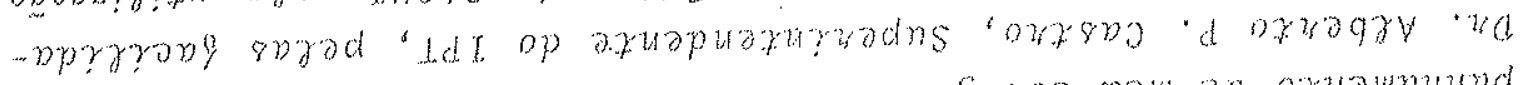

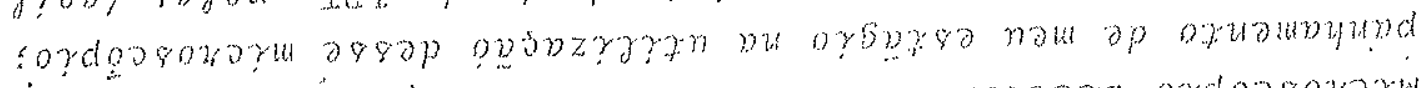

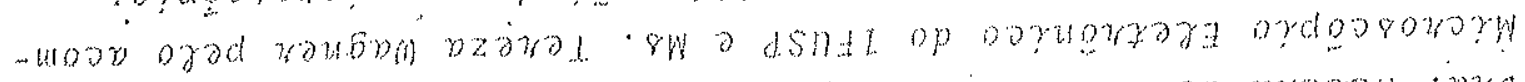

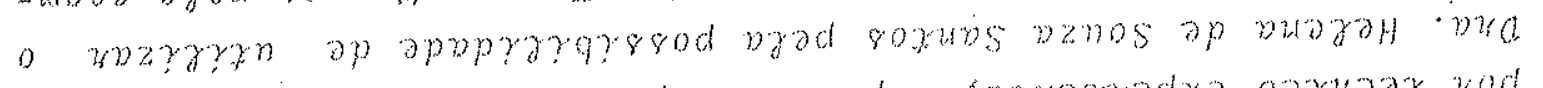

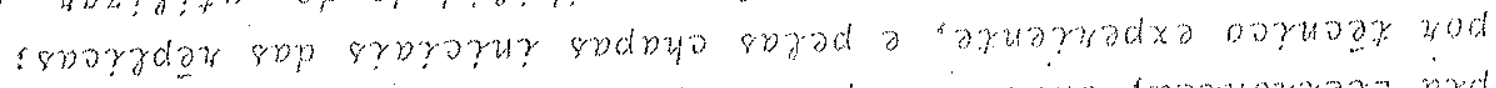

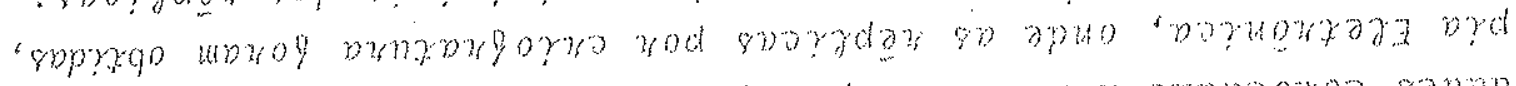

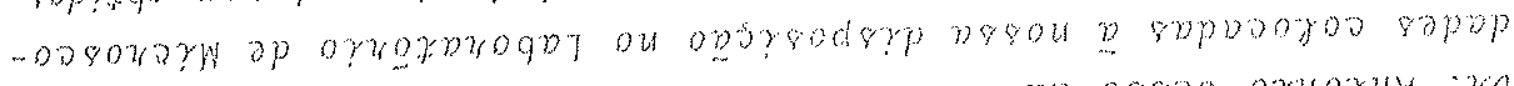

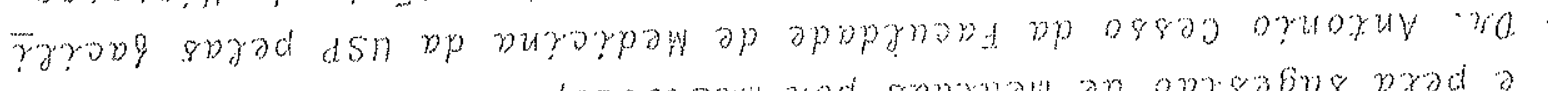

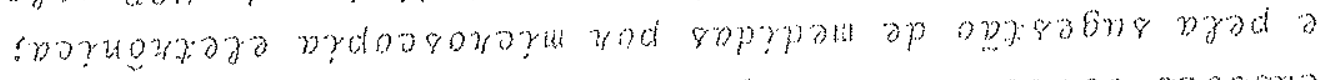

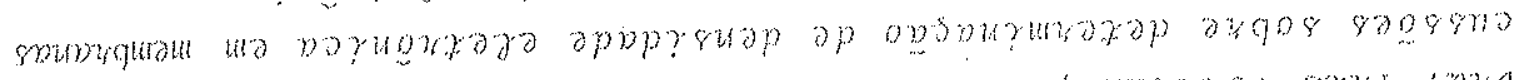

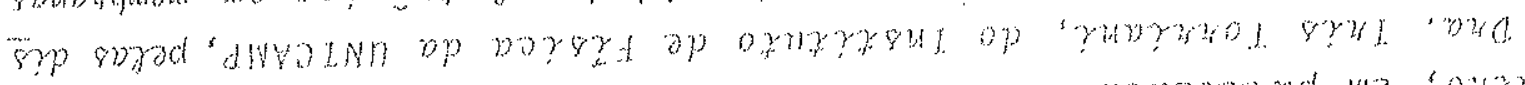

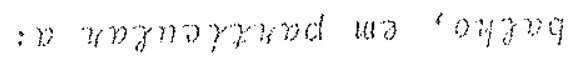

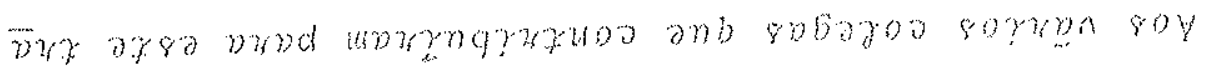

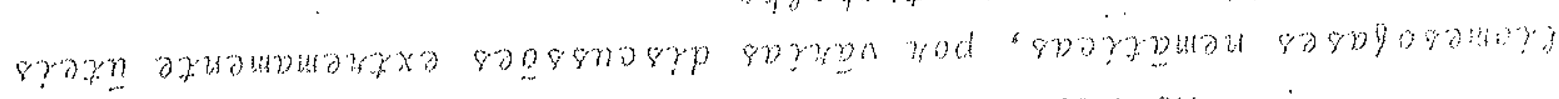

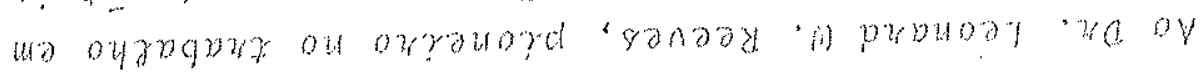

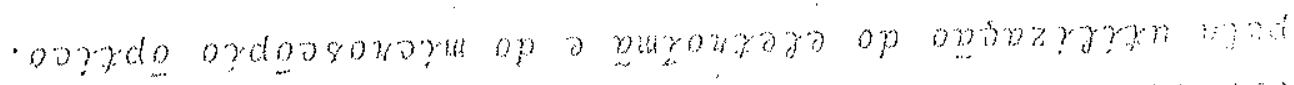

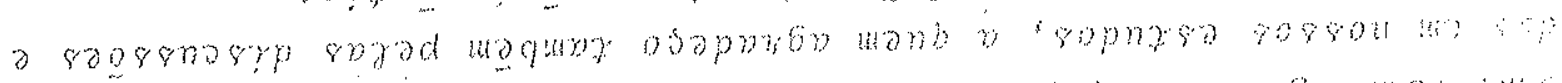

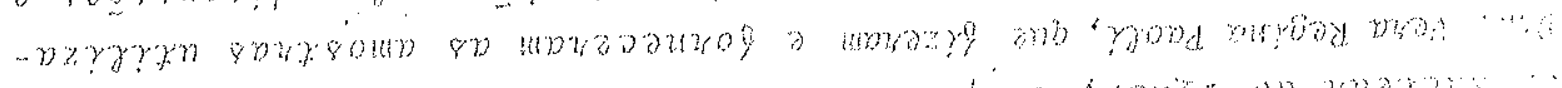

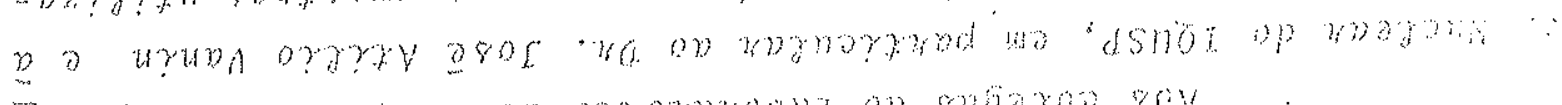

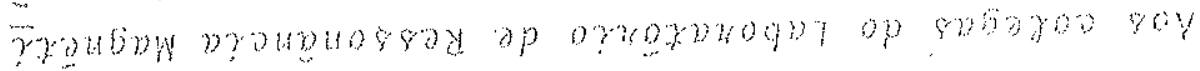




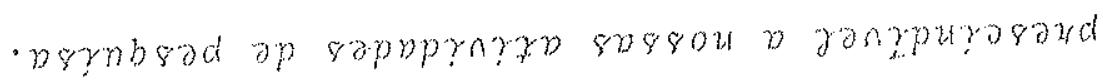

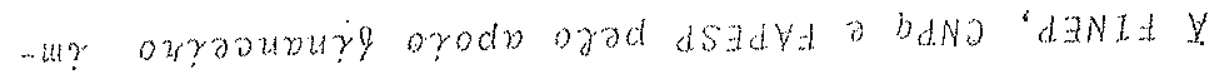

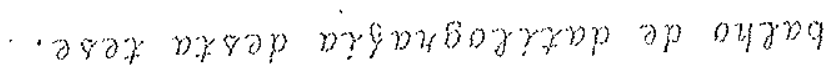

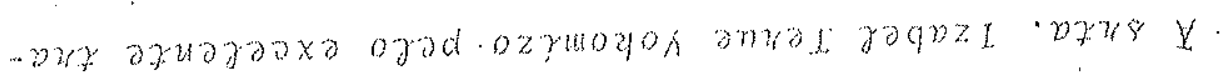

$$
\text { - moyomago }
$$

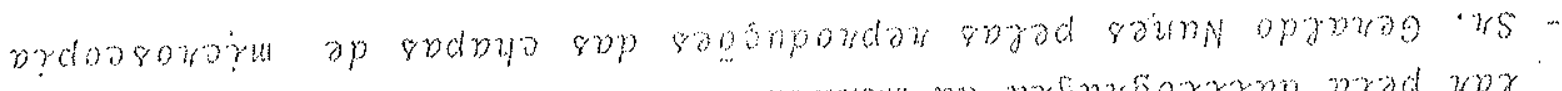

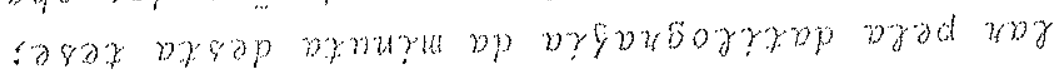

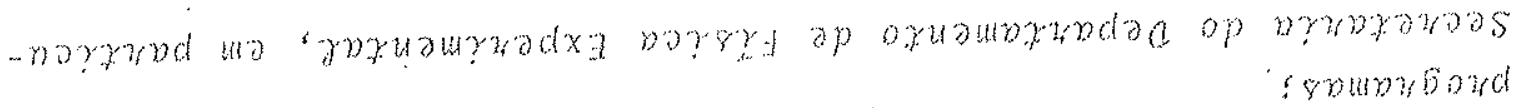

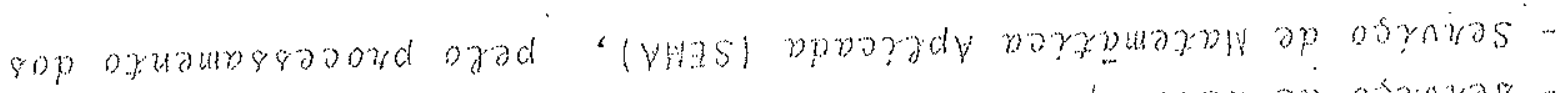

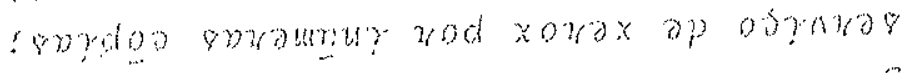

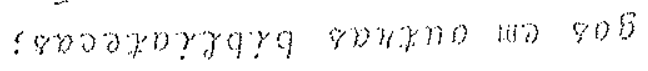

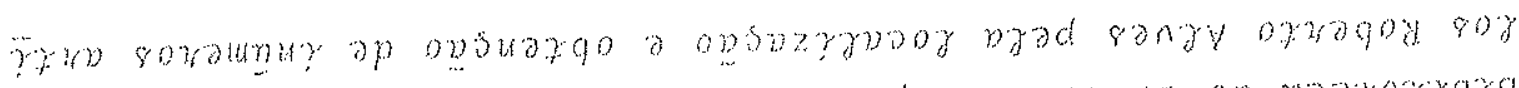

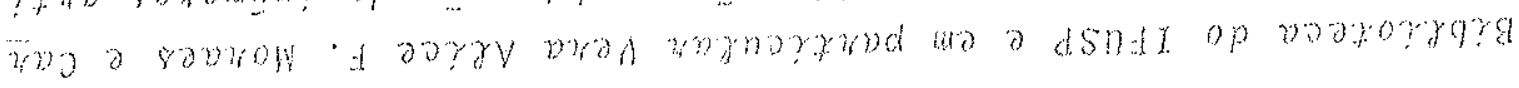




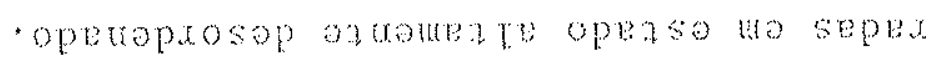

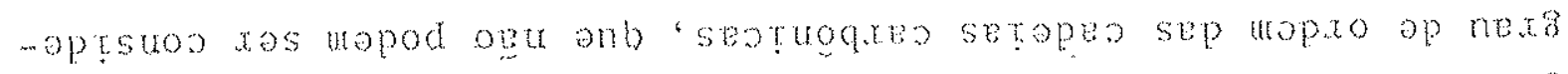

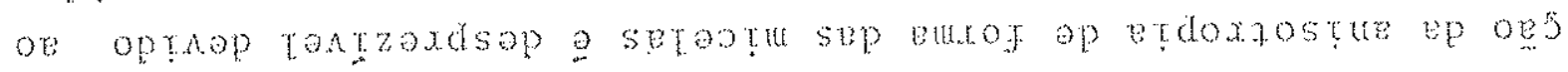

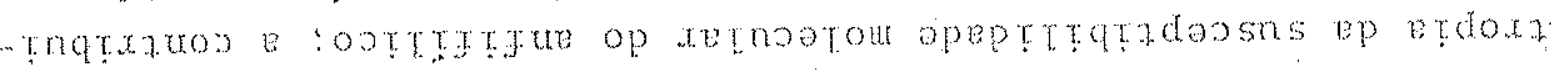

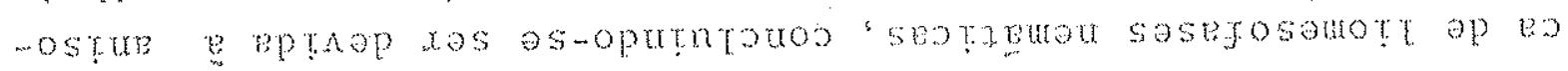

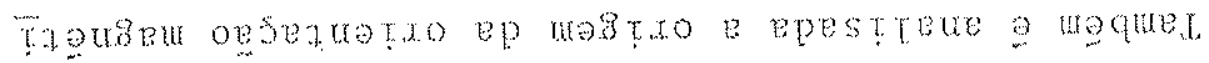

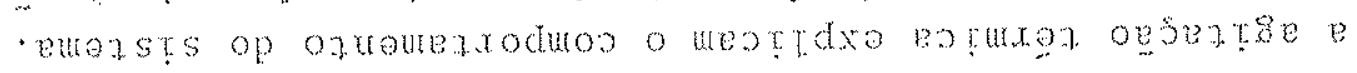

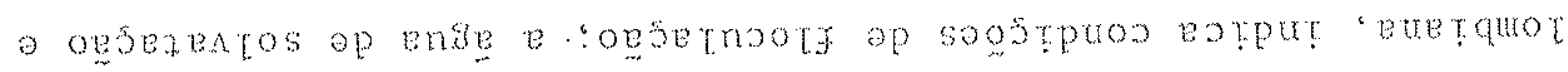

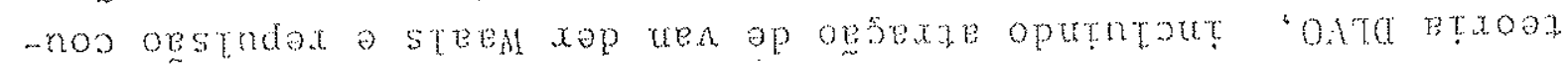

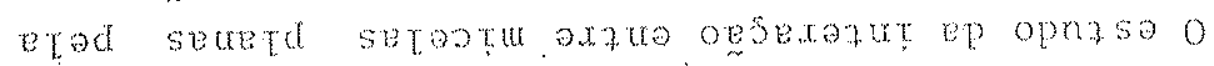

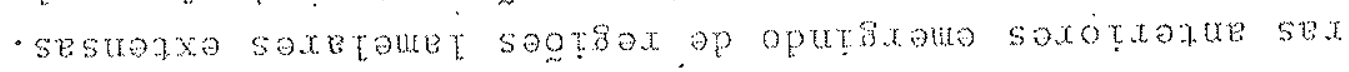

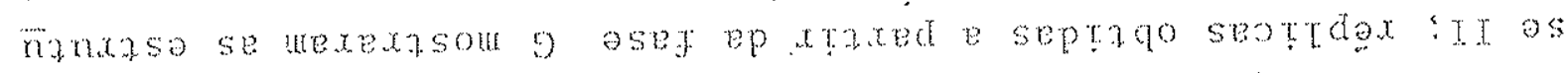

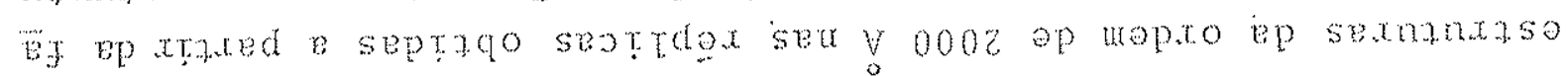

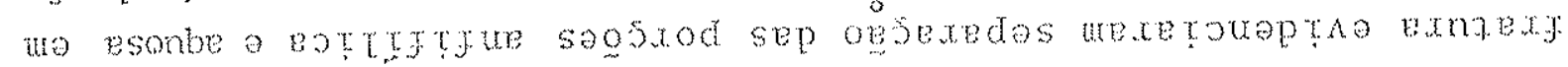

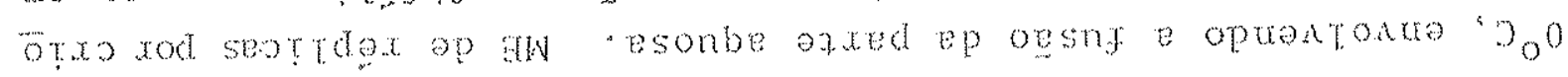
* gozr "o asej

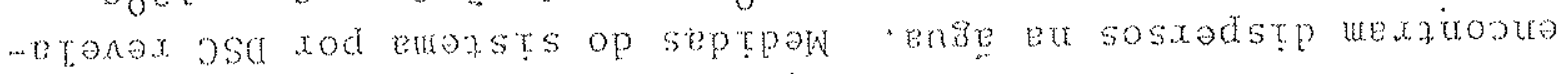

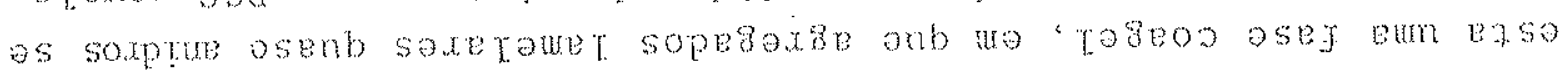

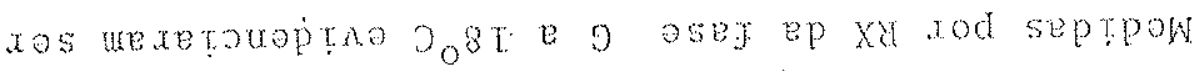

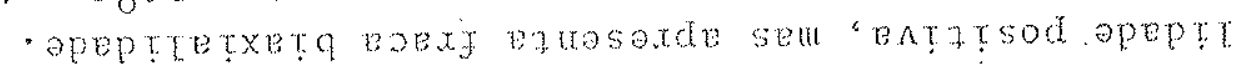
...

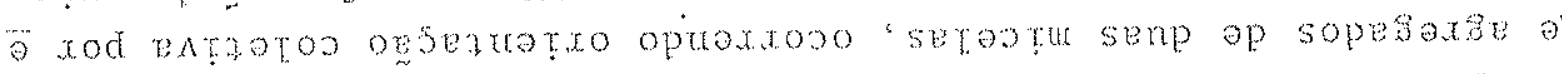

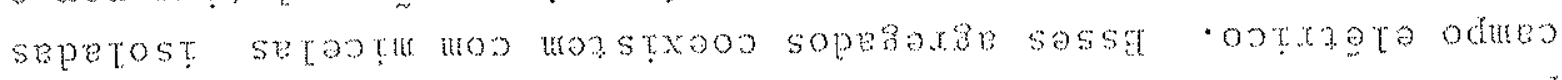

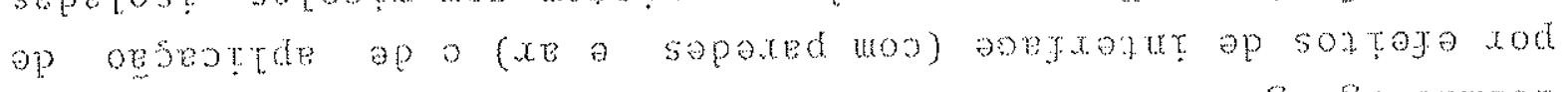

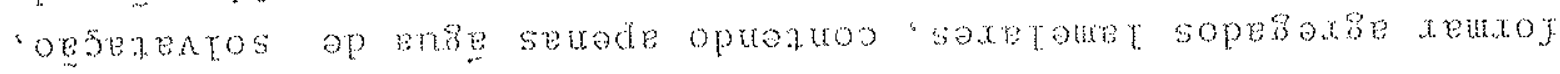

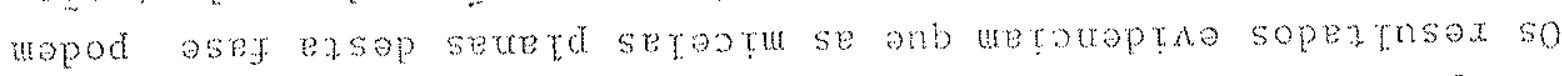

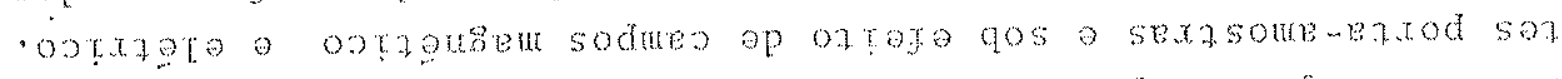

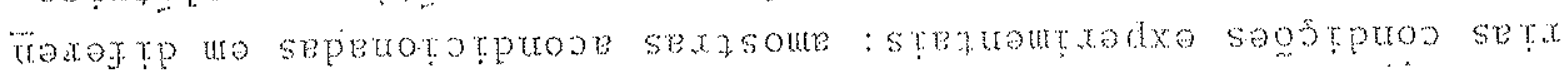

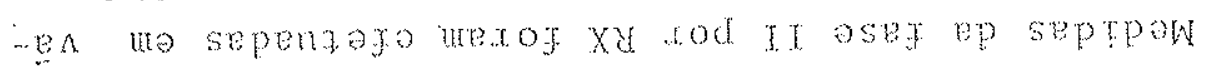

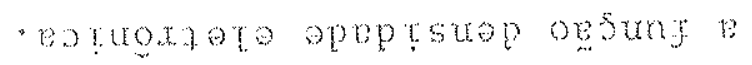

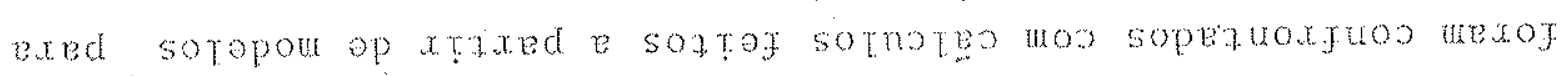

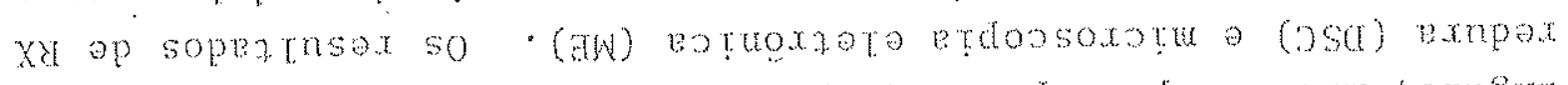

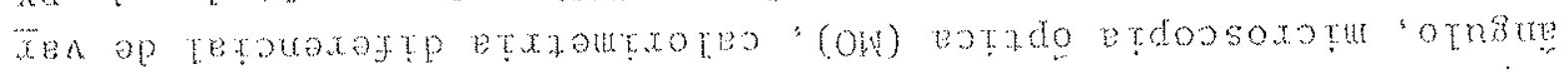

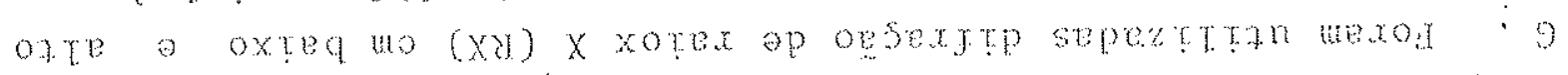

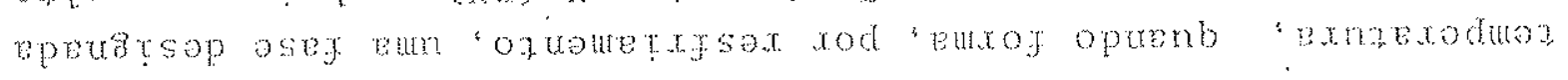

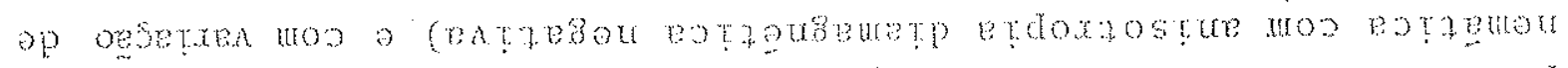

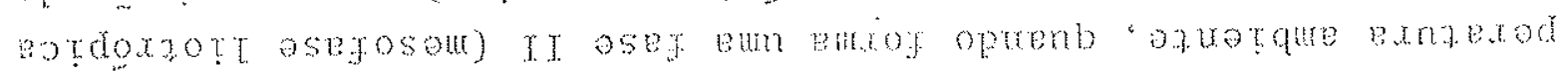

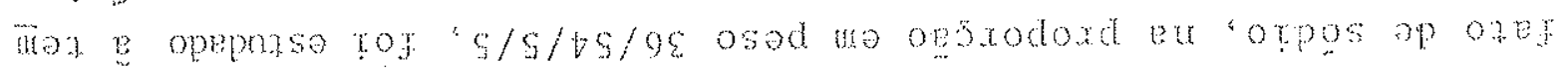

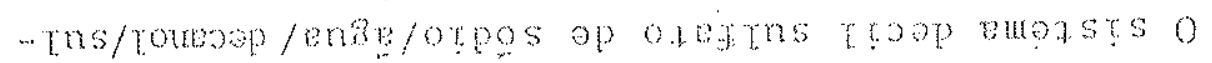




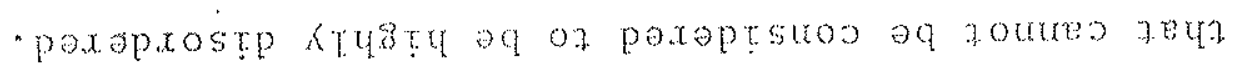

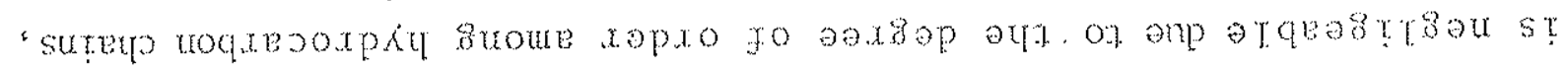

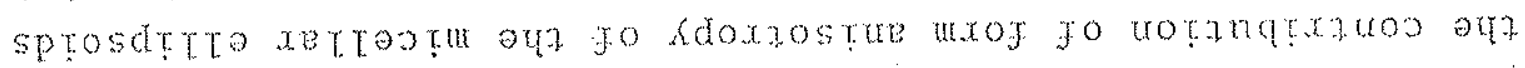

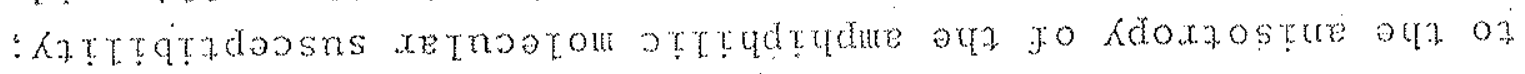

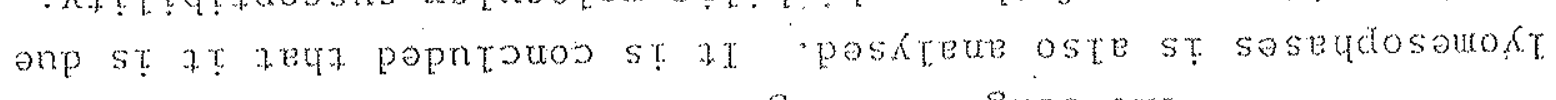

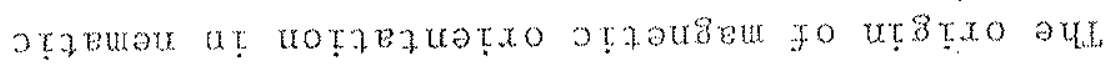

- Uazsk orr xo

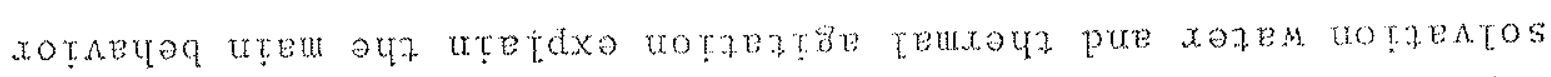

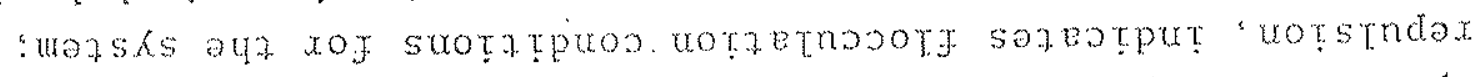

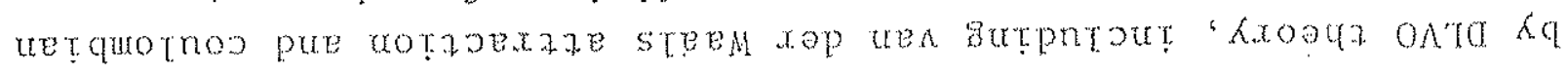

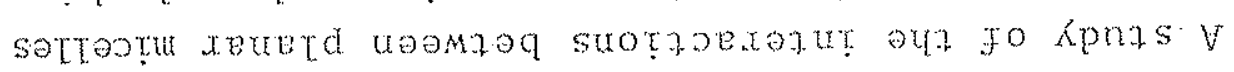

- strotsox xtromet astogxe mox

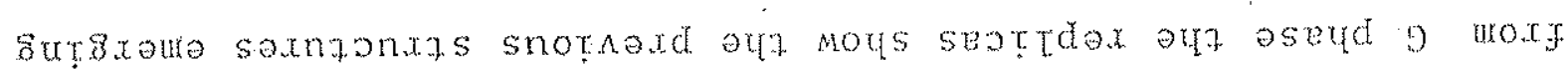

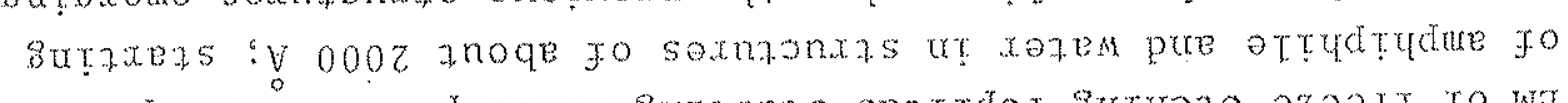

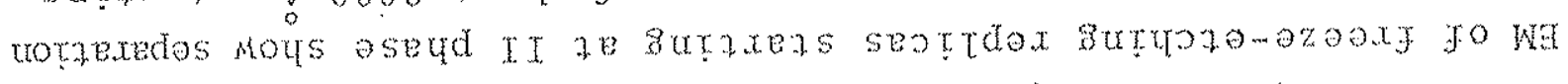

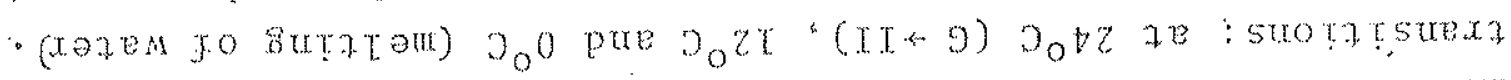

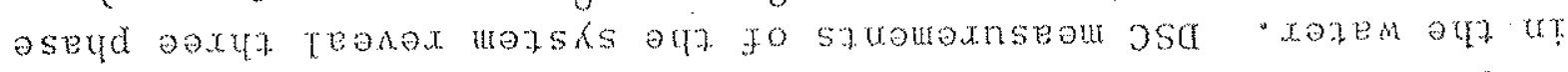

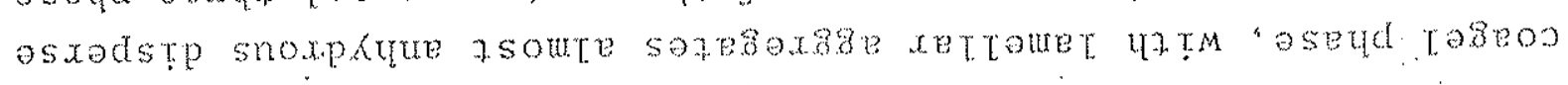

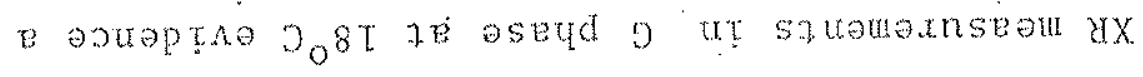

$\therefore$ itereta

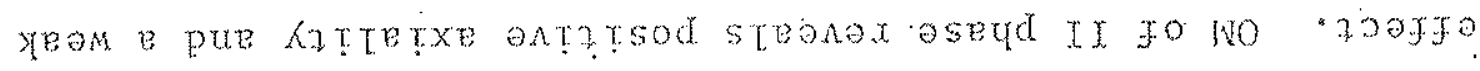

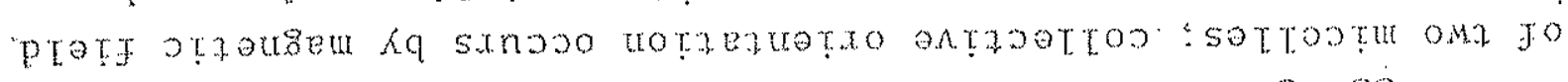

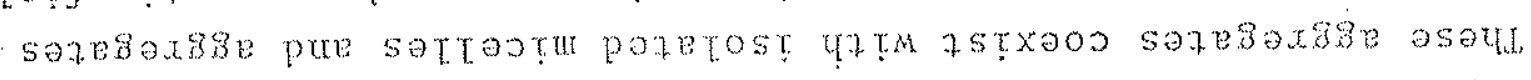

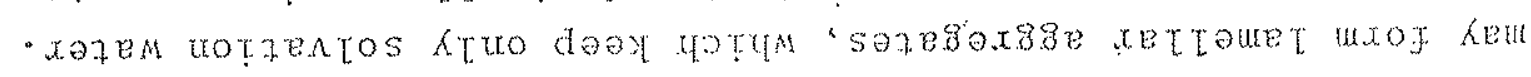

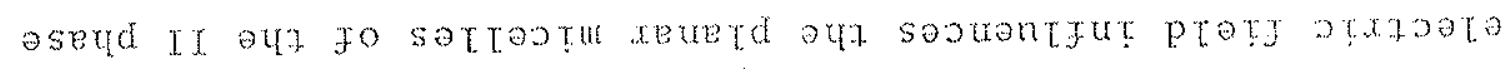

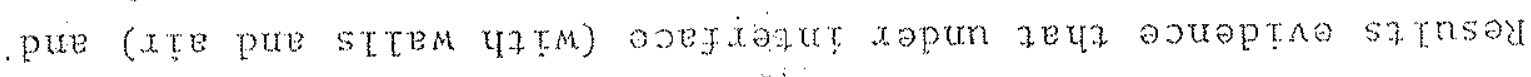

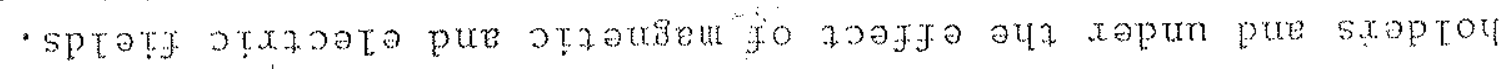

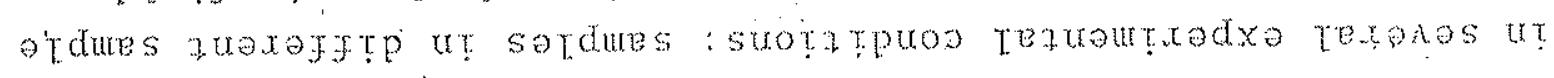

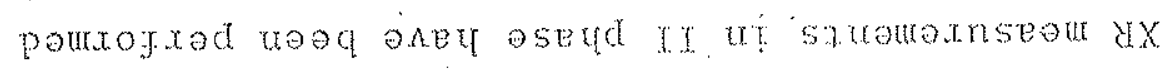

- votama katswop roxasoto

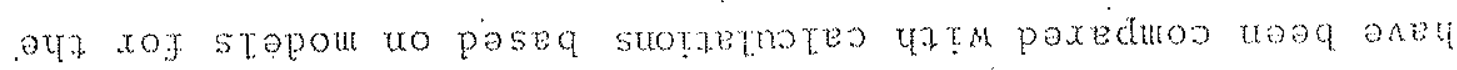

satnsox dx pakordme wooq anen (wa) Kdossoxoru roxasote puz

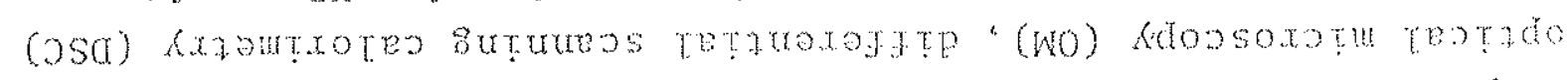

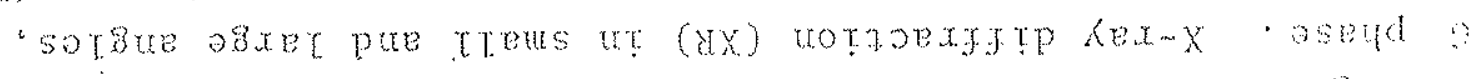

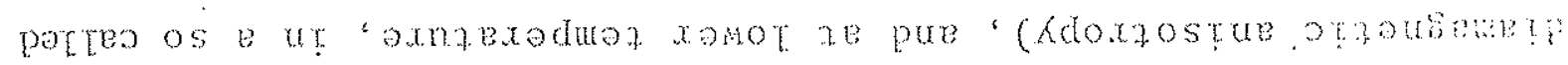

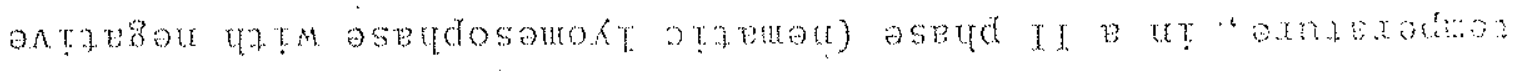

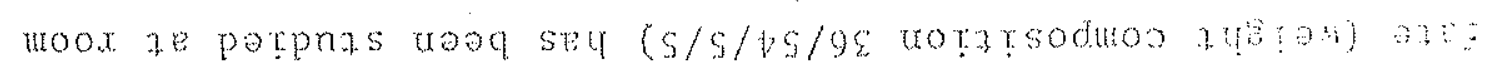

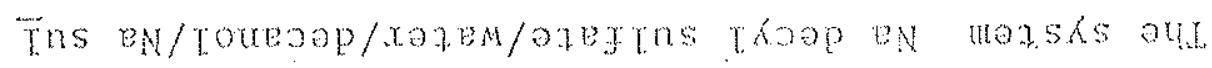




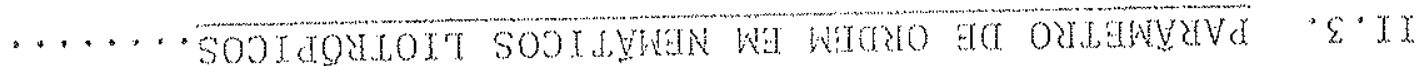

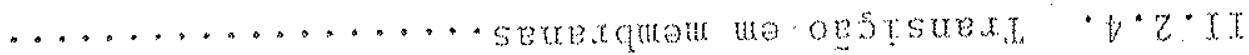

soserosenot the setopeo spp hopro op tTyod - $\mathrm{C} \cdot \mathrm{Z} \cdot \mathrm{T}$

bv

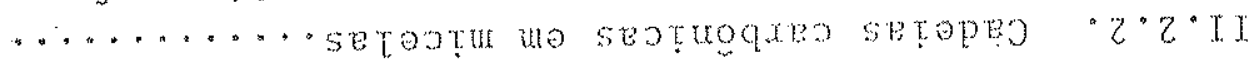

T.

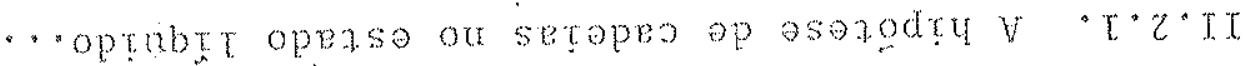

To

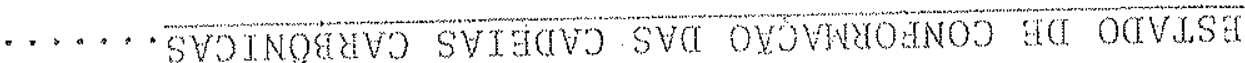

98

$z \&$

62

92

$\varepsilon ?$

$\varepsilon z$

IZ

it.

T. T

T. T

6

4

\&

S

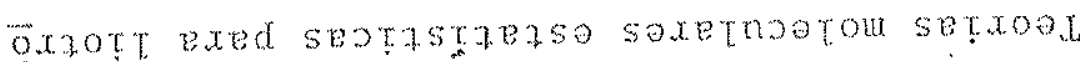

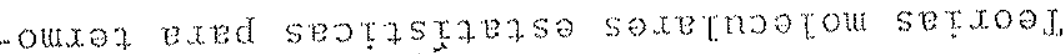

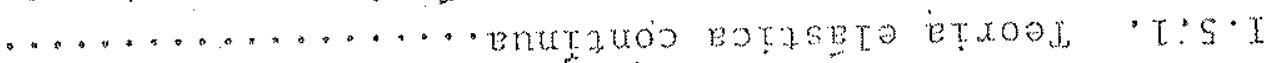

\section{SVDTUWWN SESVROSTWOIT}

$\cdot L \cdot I$

$\cdot 9 \cdot 1$

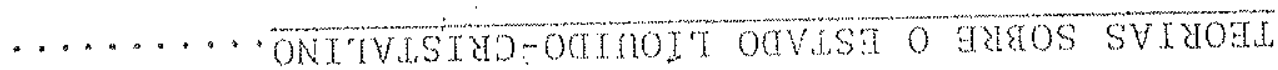

SVOTHOUDTT SY SVHNTZTA SASVAOSAW $\bullet \cdot$

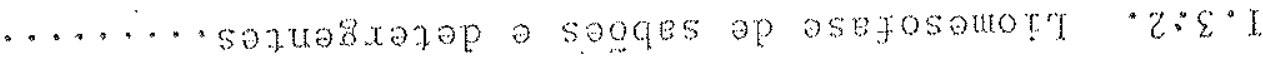

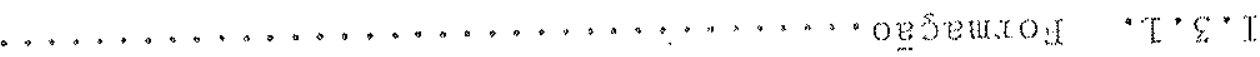

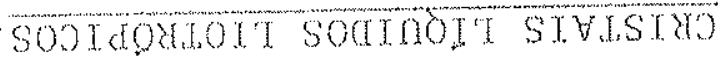

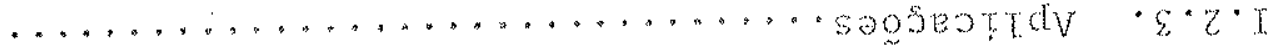

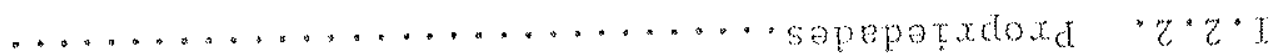

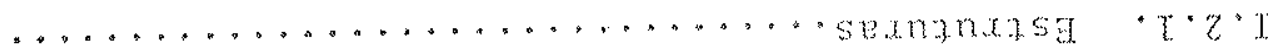

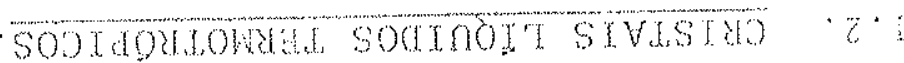
STSWOSAR $\cdot \mathrm{T}$

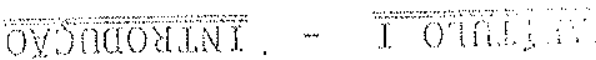

purged 


501
66
68
48

88
18
18

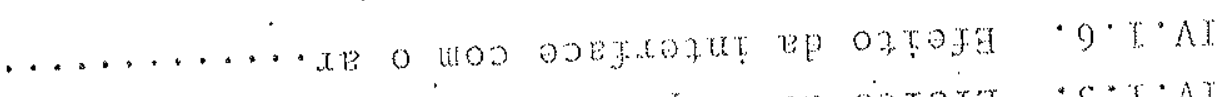

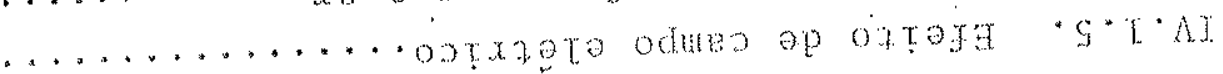

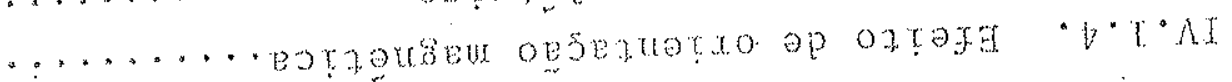

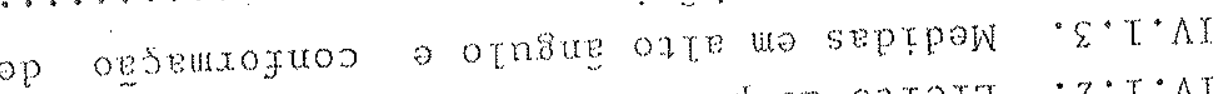

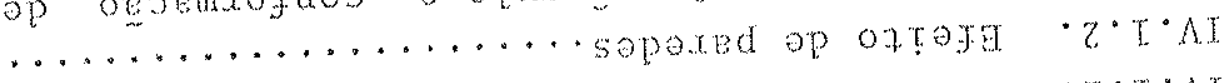
- I.T. $\mathrm{J}$

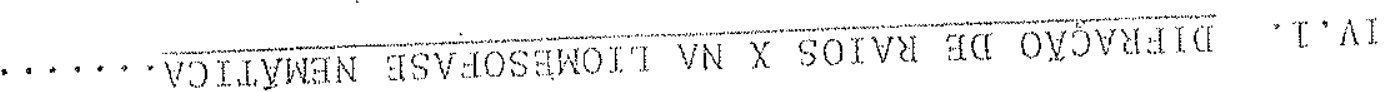

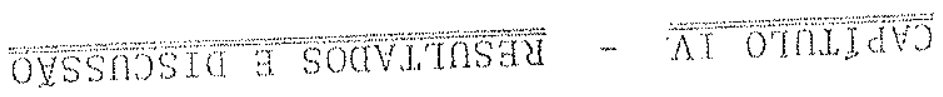
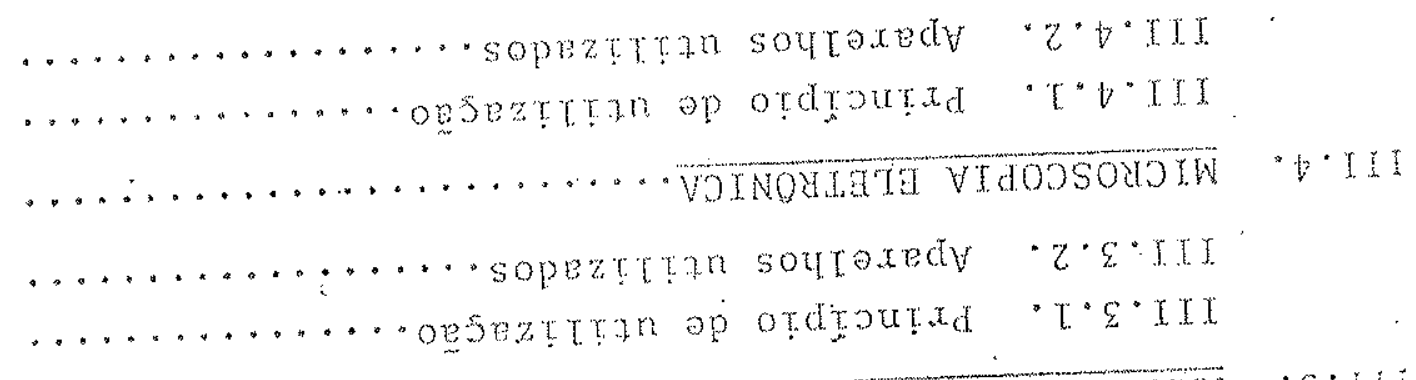

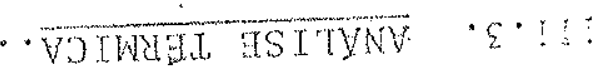

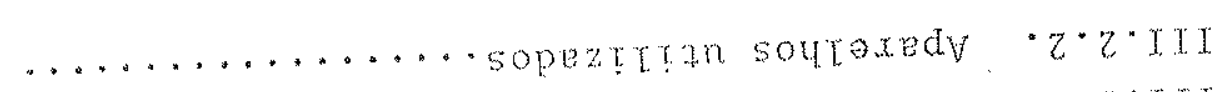

..........ogseztran op otdgoted $\cdot \tau \cdot z \cdot \operatorname{tr}$

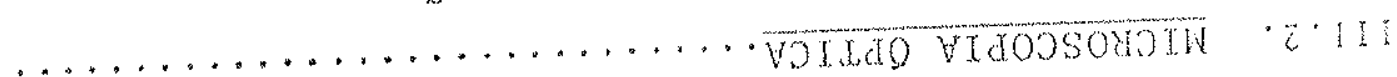
(9)

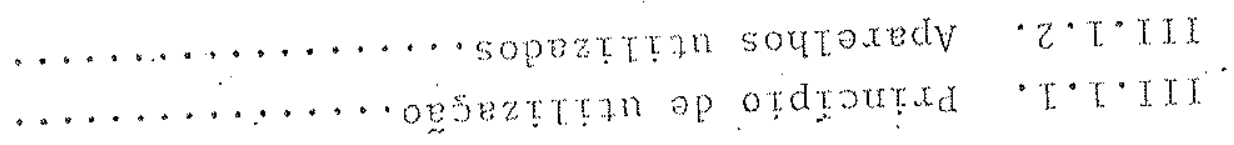

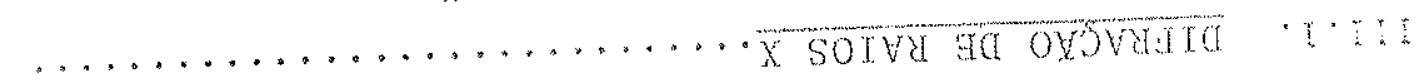

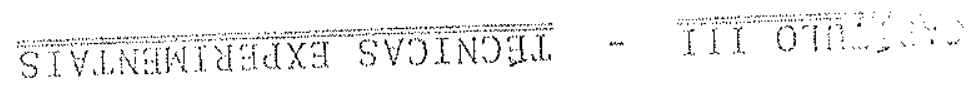

89

T.

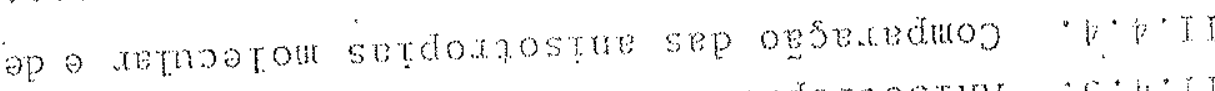

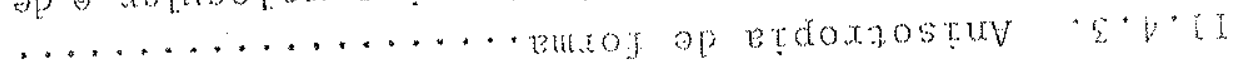

89

95

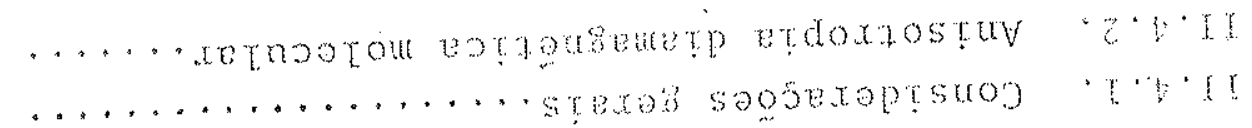

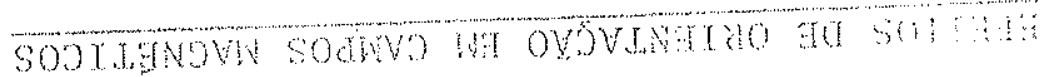

extsed 


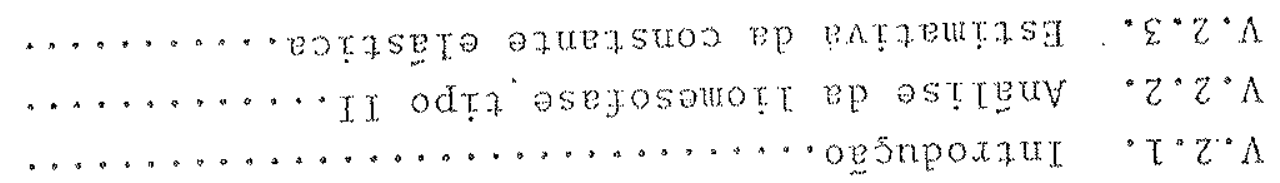

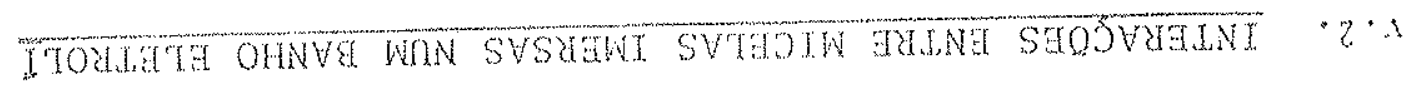

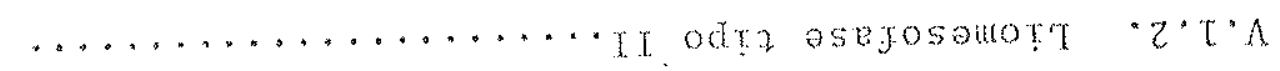




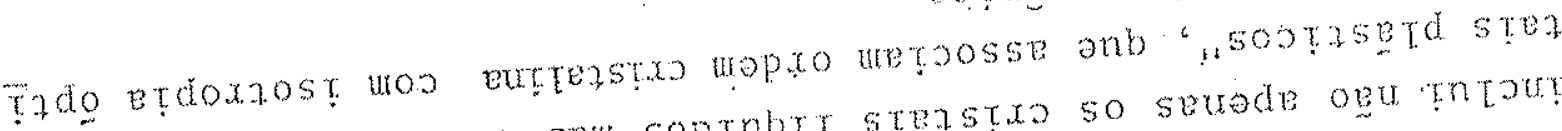

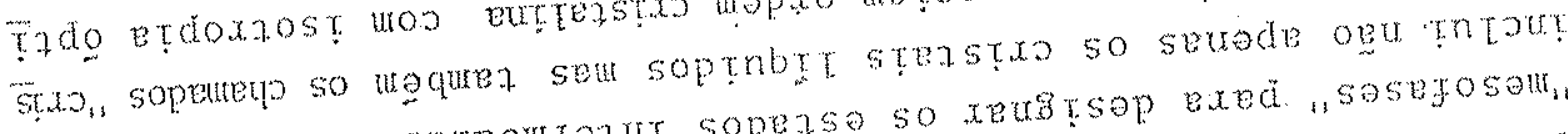

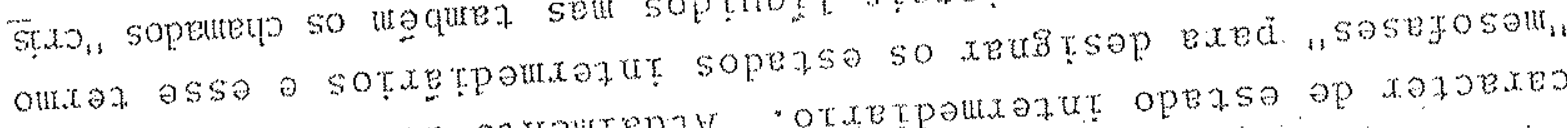

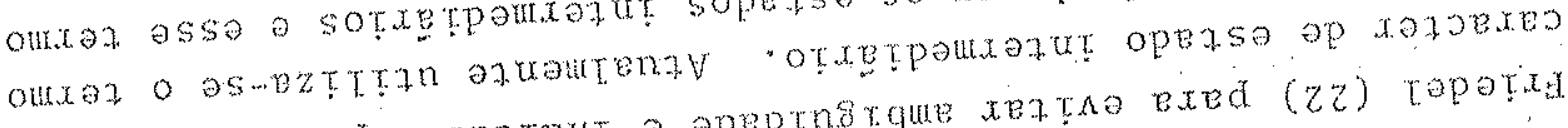

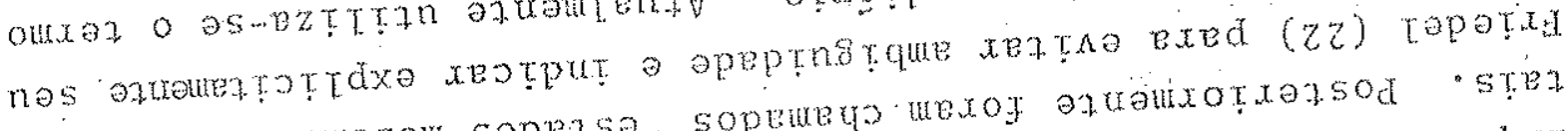

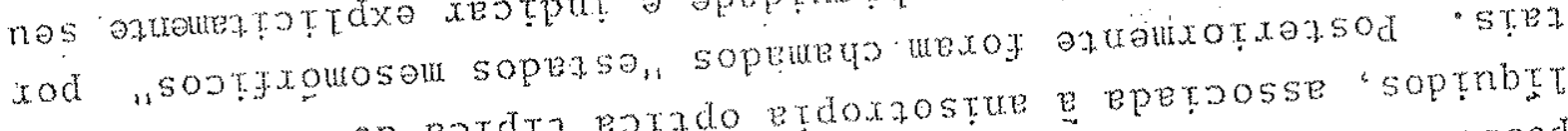
- stxo sojxo op eotdtz estado etdoxastue e epegosse soptrbt

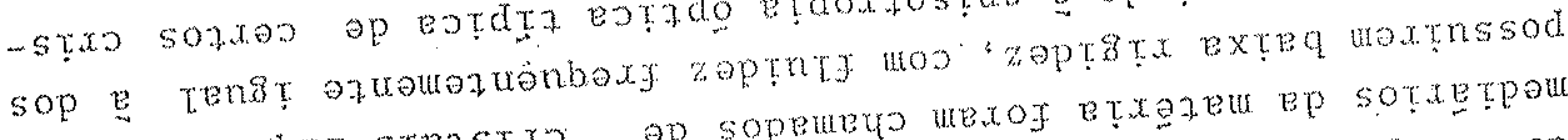

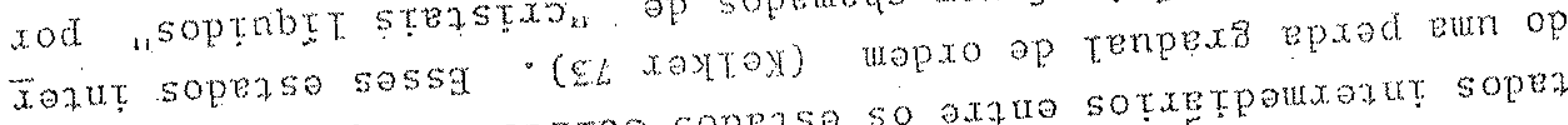

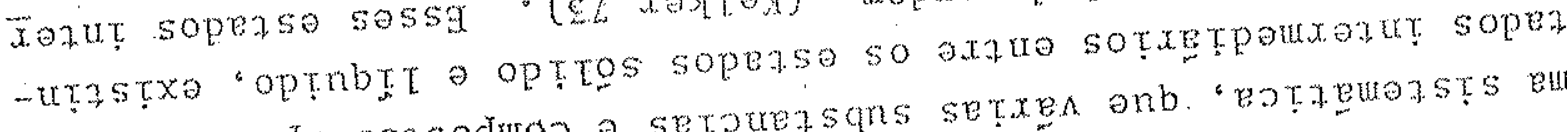

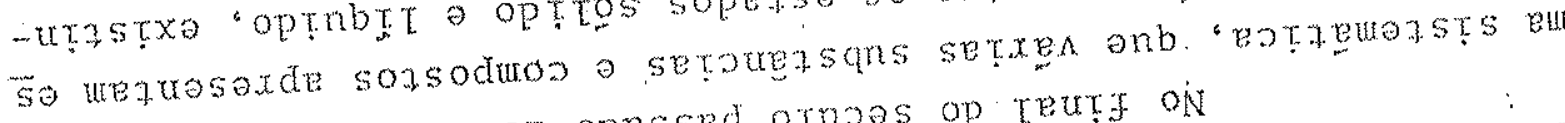

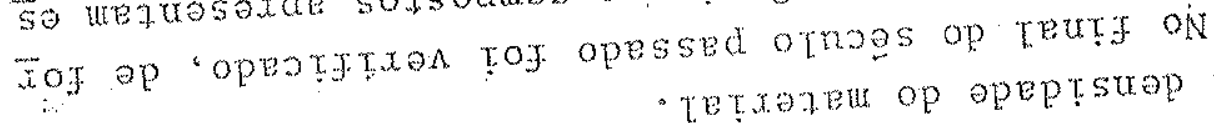

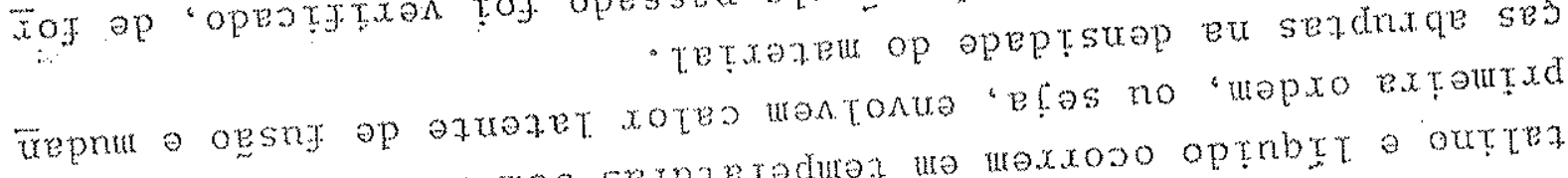

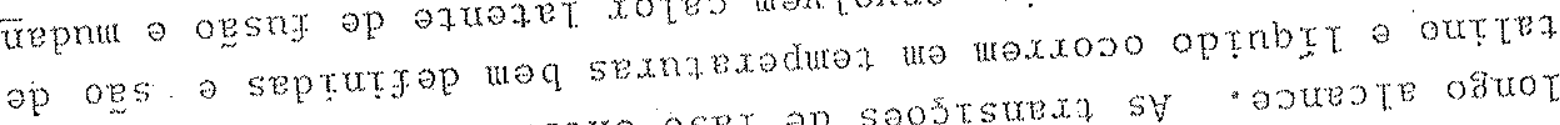

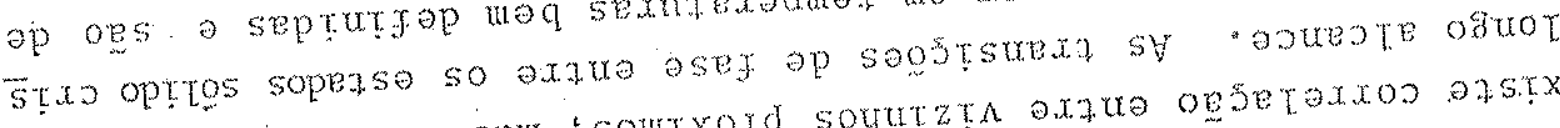

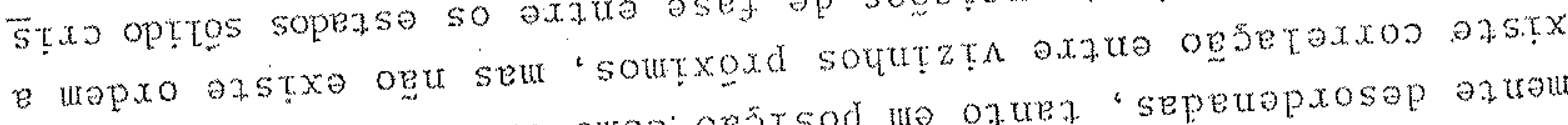

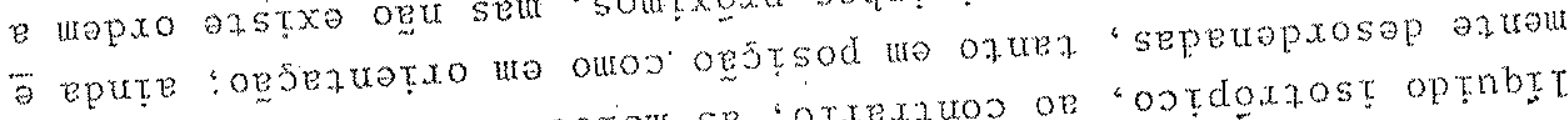

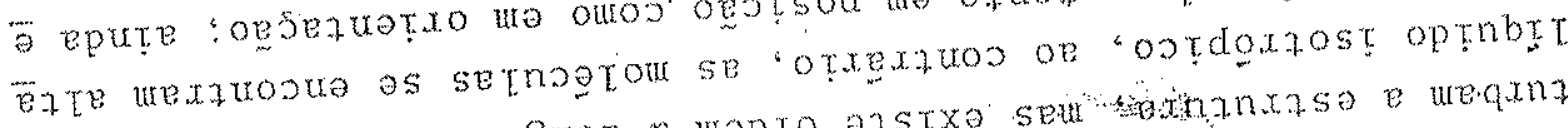

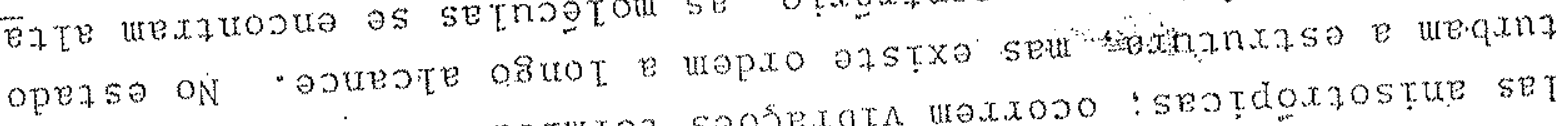

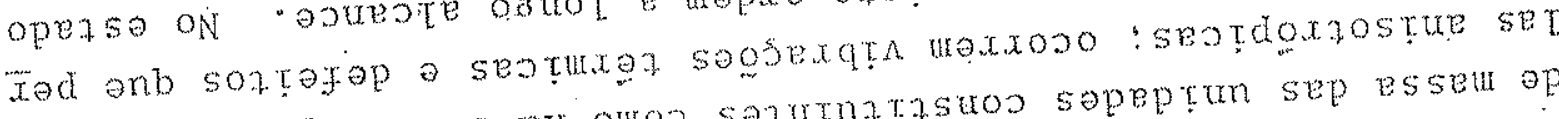

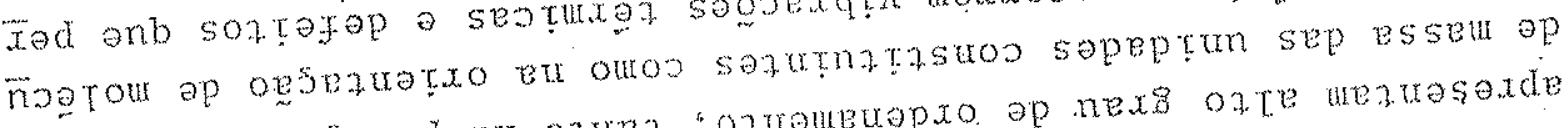

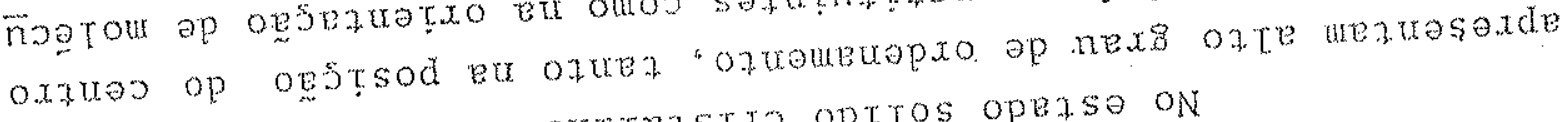
sernagtout somoze so orteterstre optros opease oN 


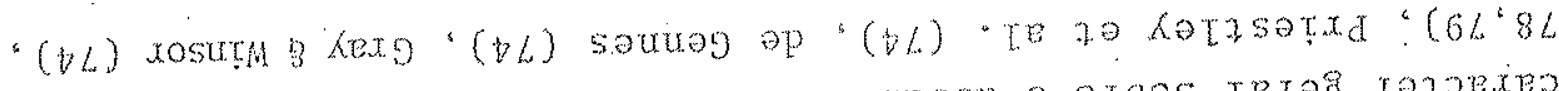

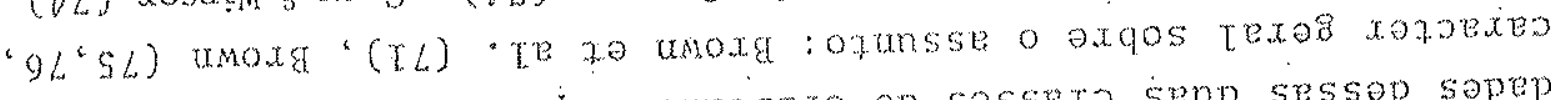

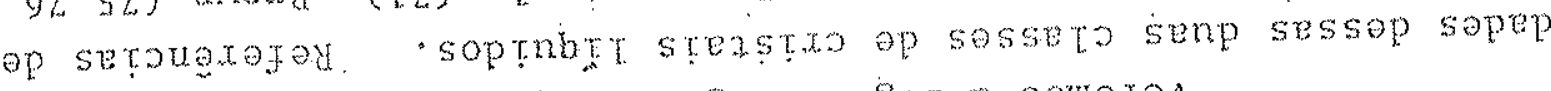

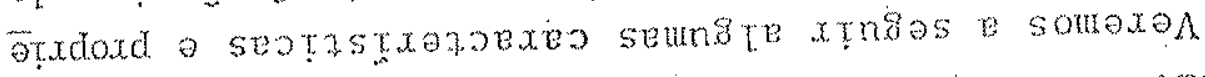

- enge wo sooprdt

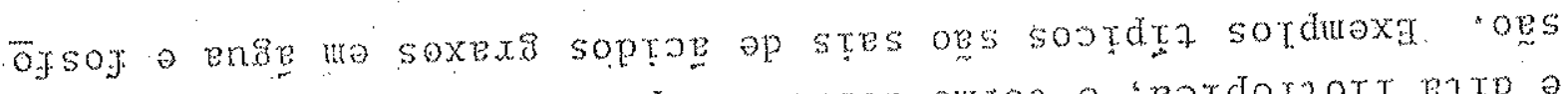

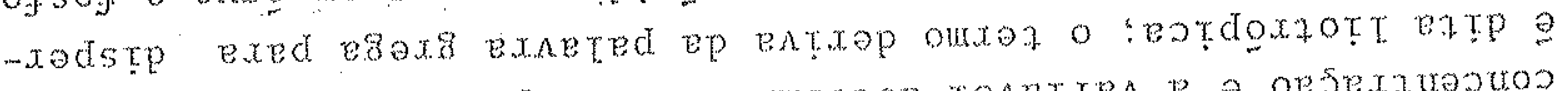

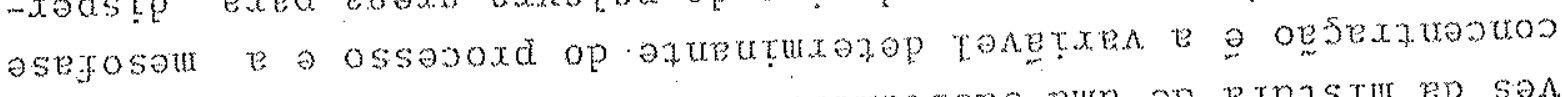

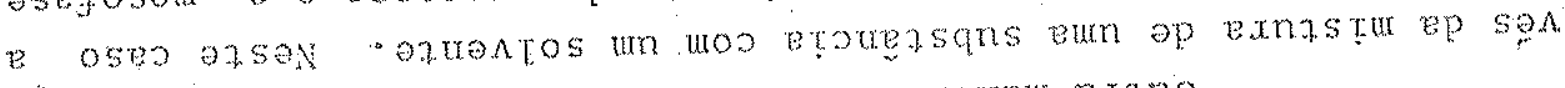

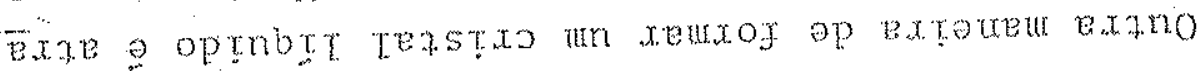

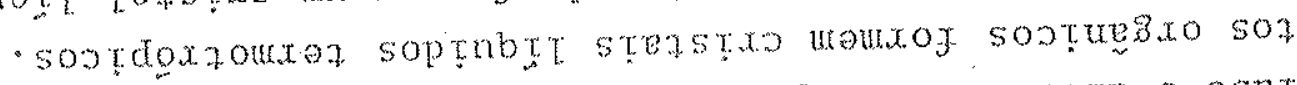

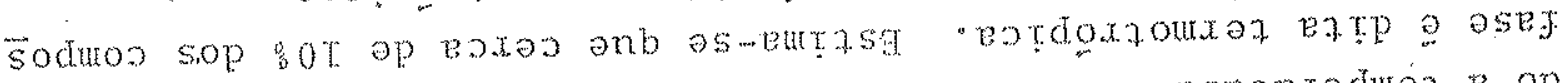

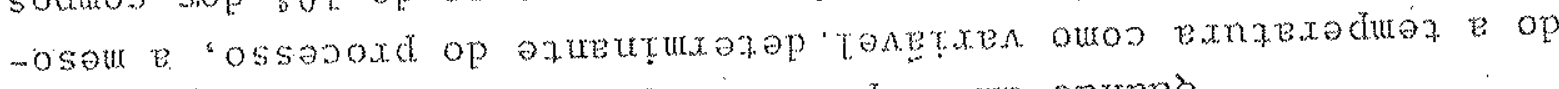

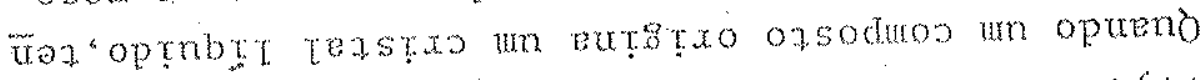

- (LL xeyxasexputo

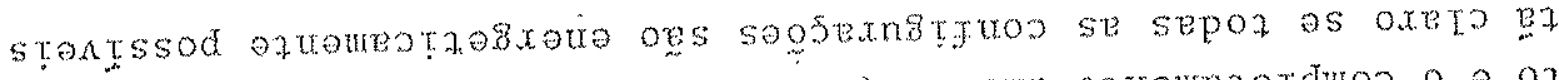

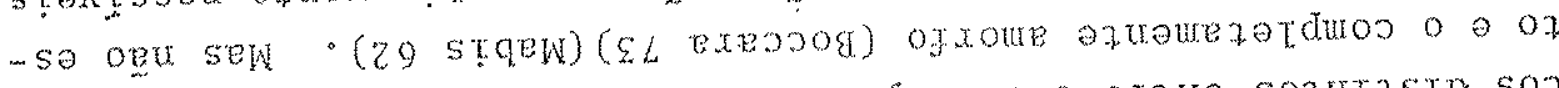

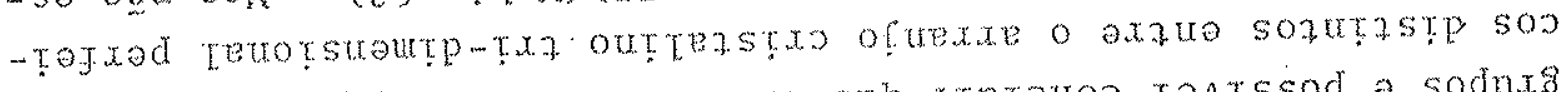

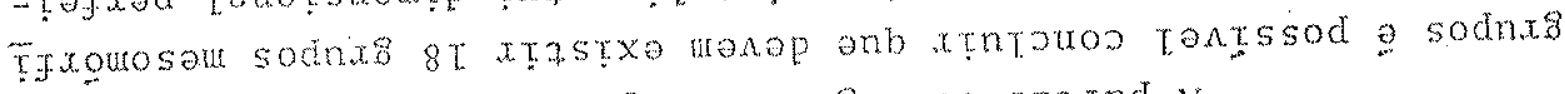

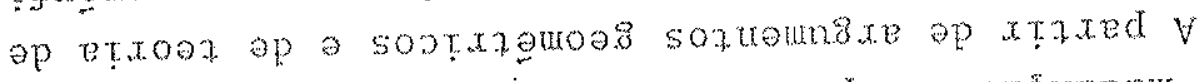

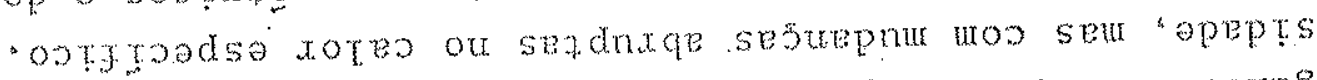

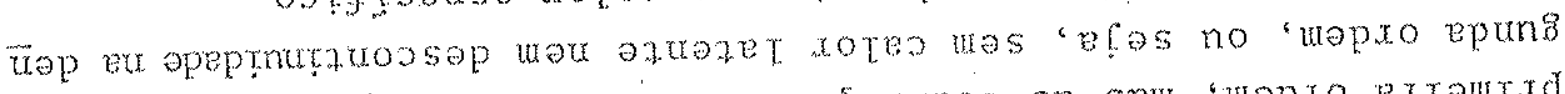

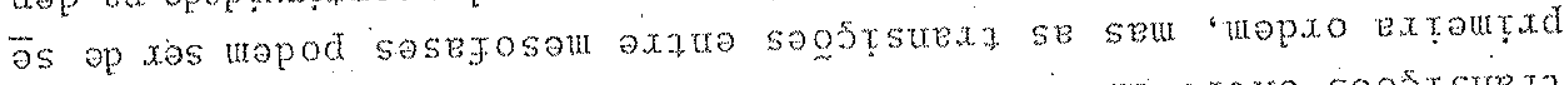

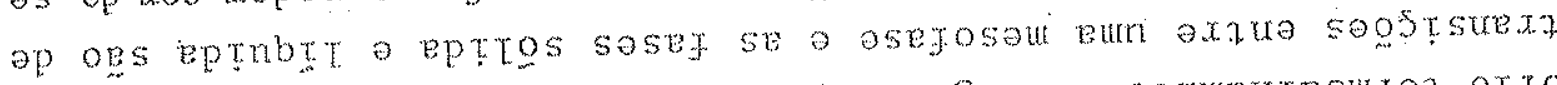

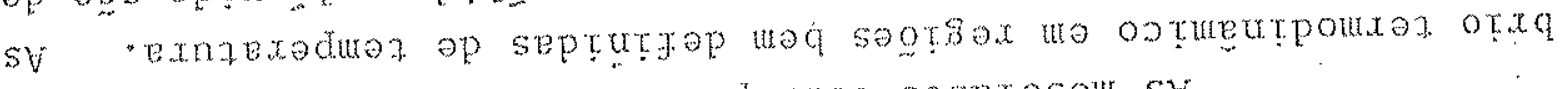

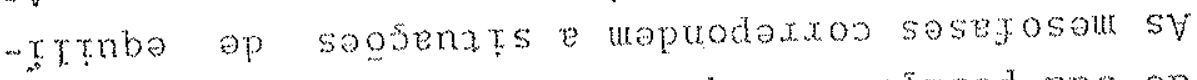

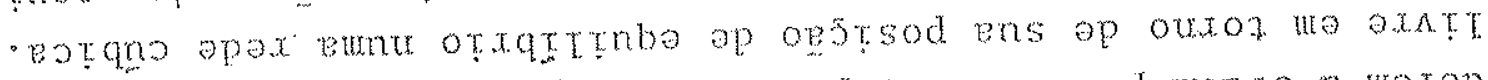

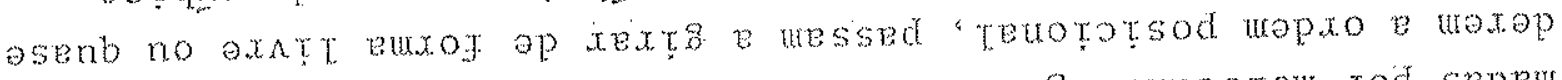

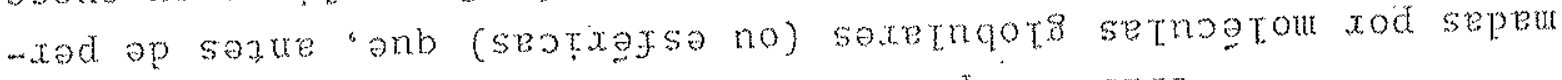

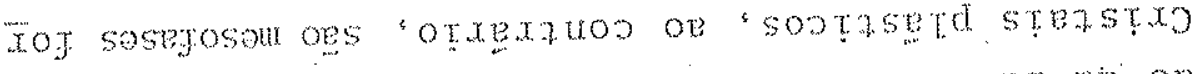

zant ep oesesedoxd ap oes

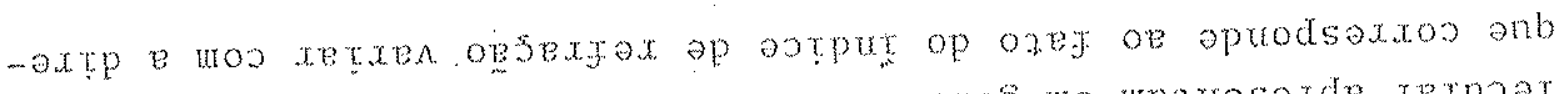

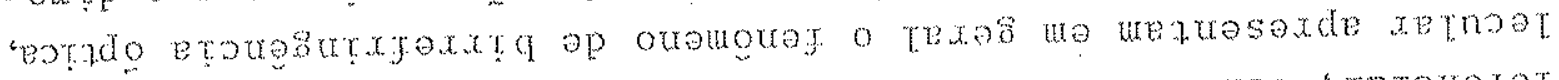

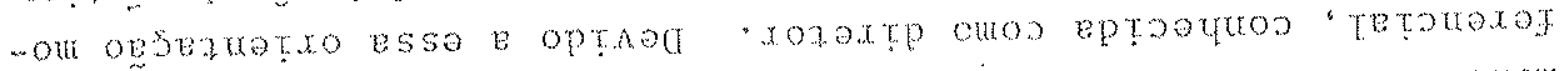

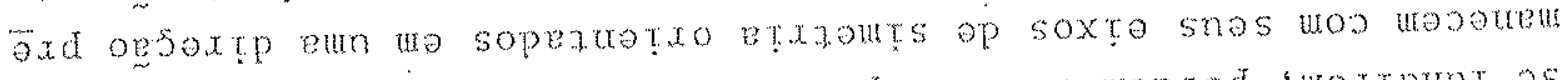

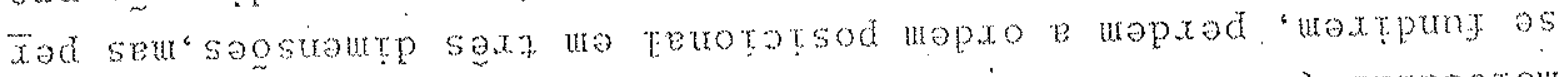

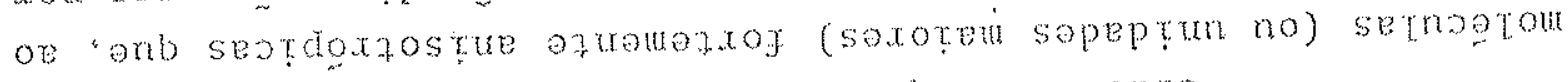

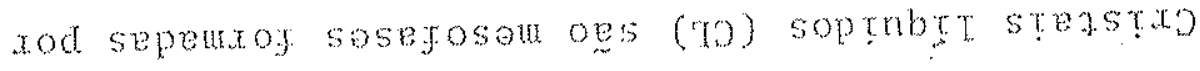




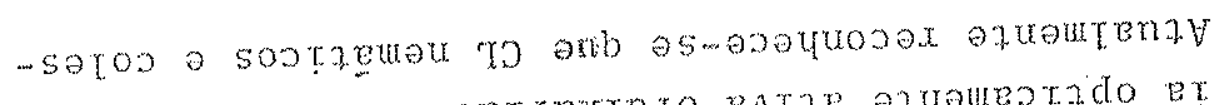

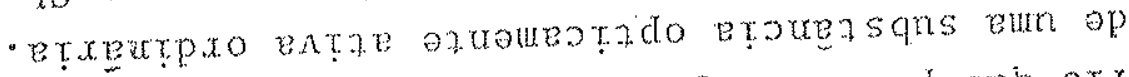

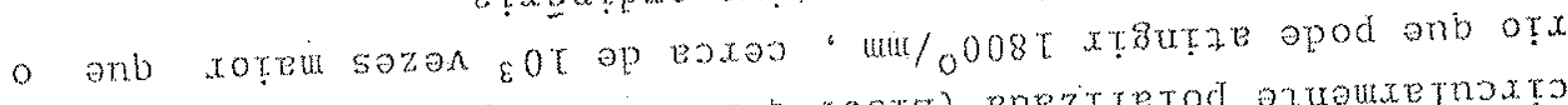

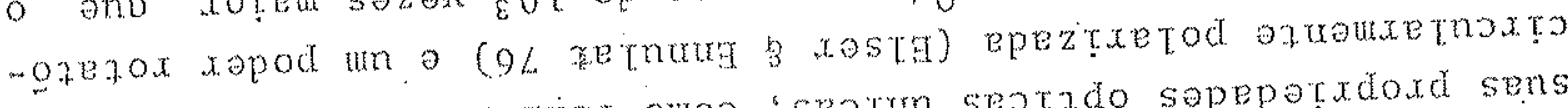

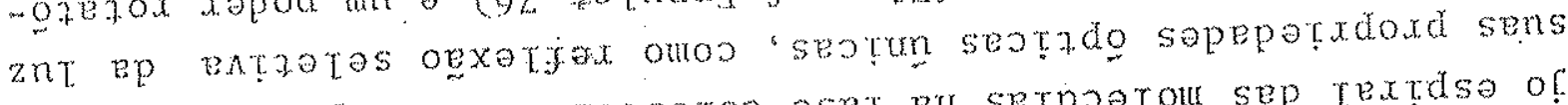

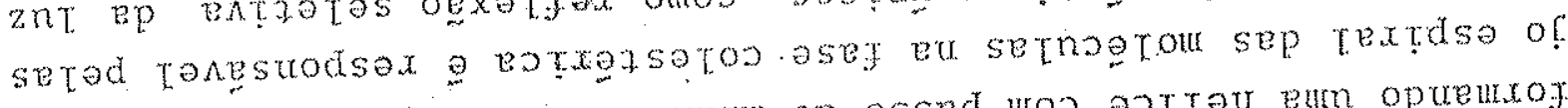

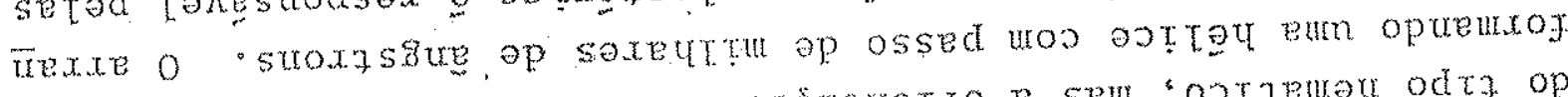

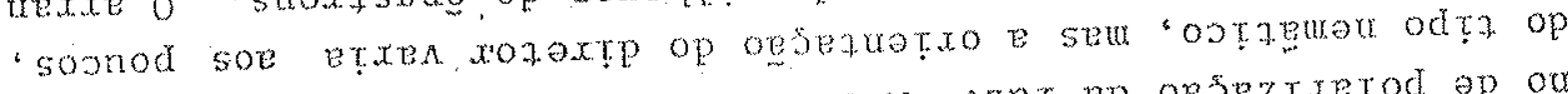

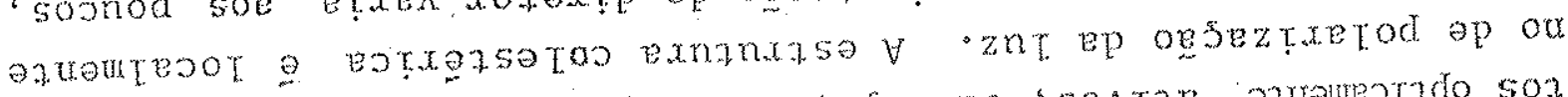

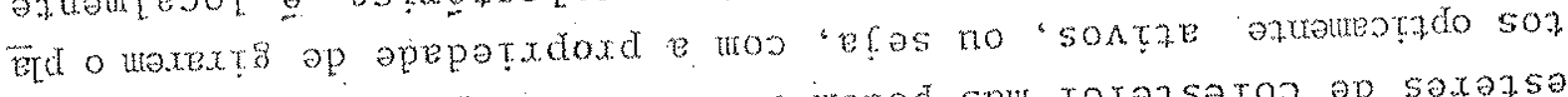
-soduos sotxen xod sopemoz xas mapod seu toxozsotoo op saxase

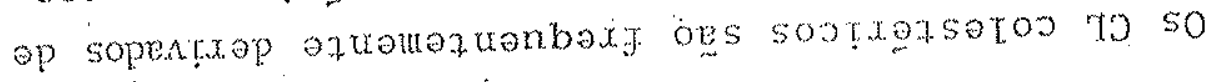

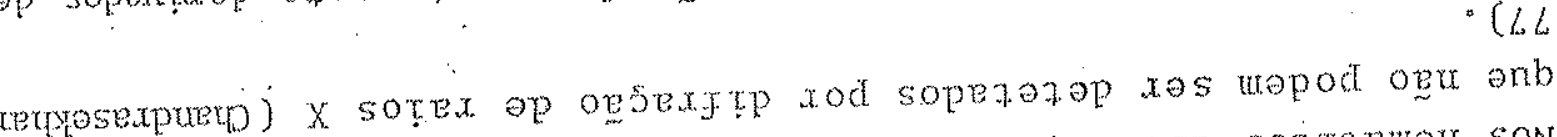

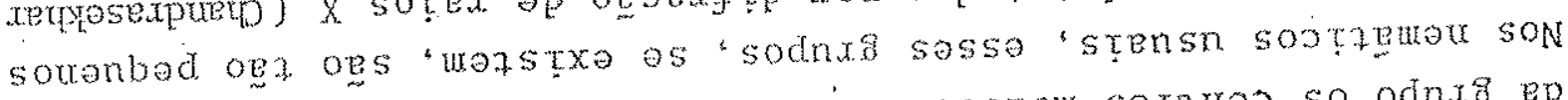

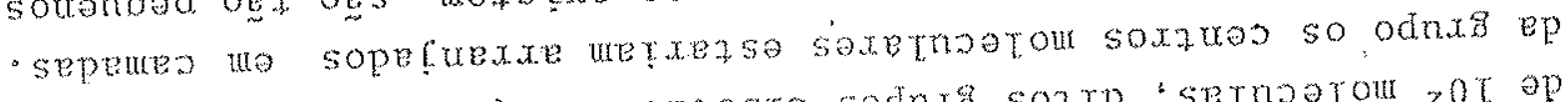

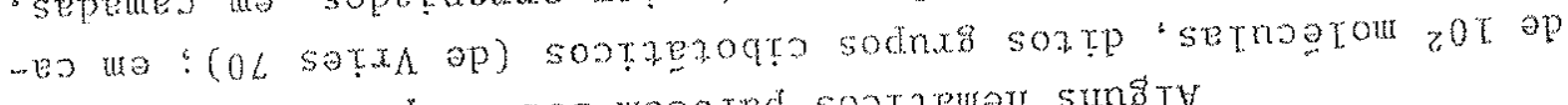
sodnx op sojsoduroo xos uevoxed soptaguex sungt

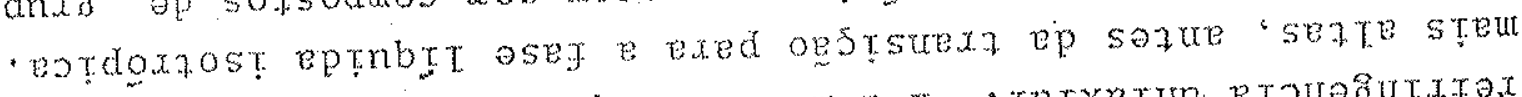

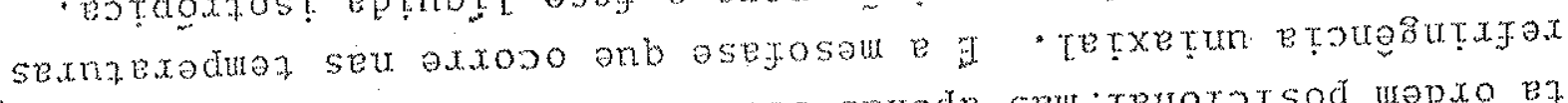

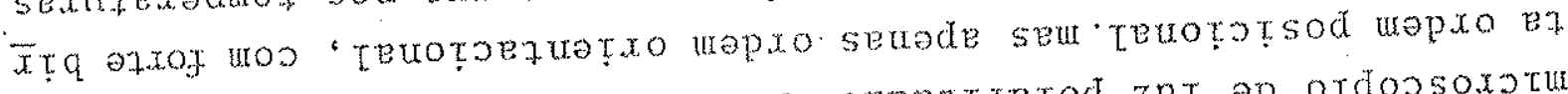

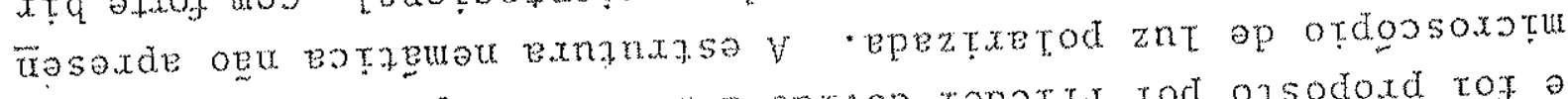

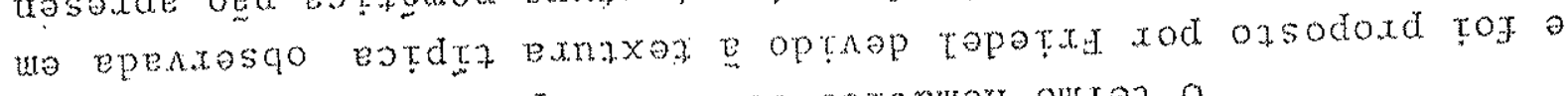

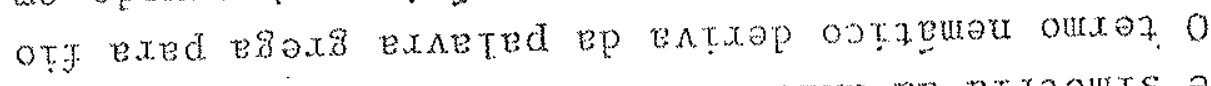

- xetnogrou opeptum ep exzauts ap oxto op etpell

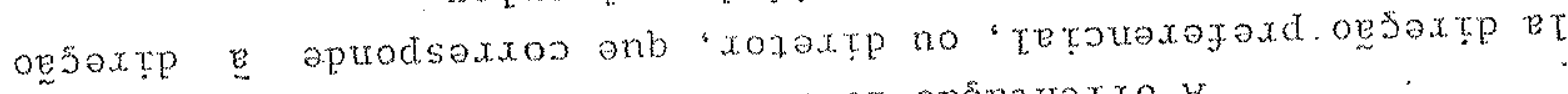

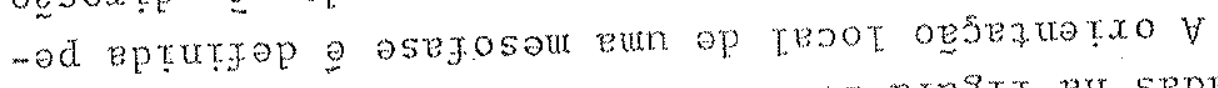

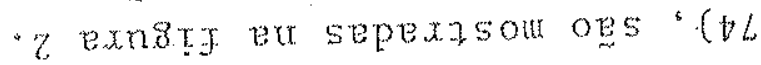

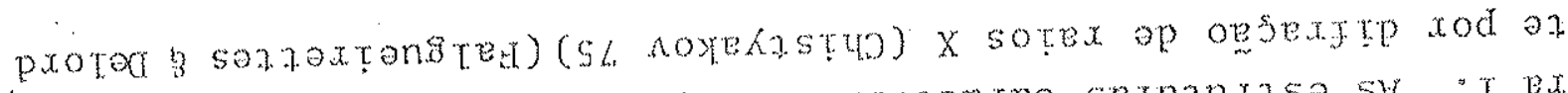

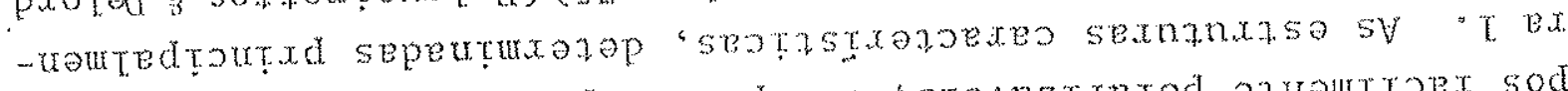

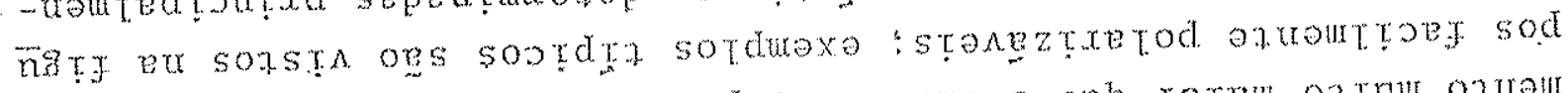

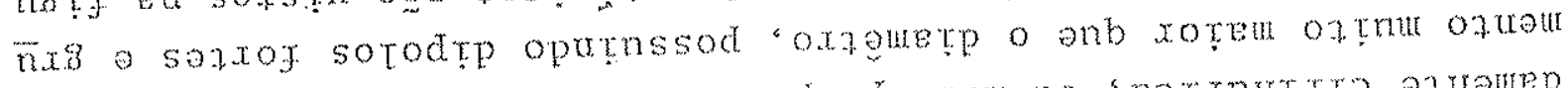

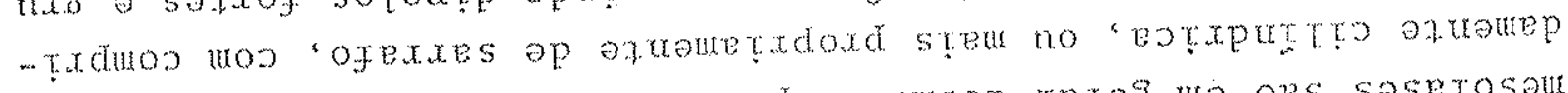

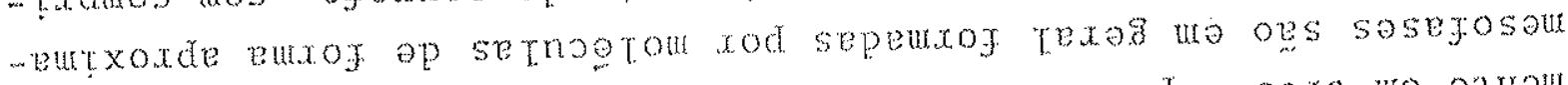

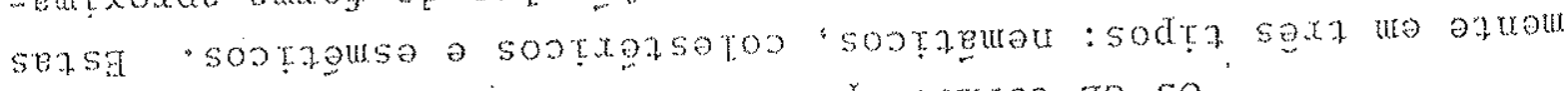

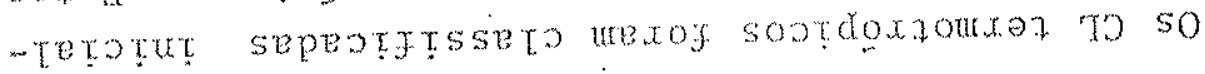

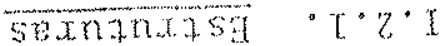

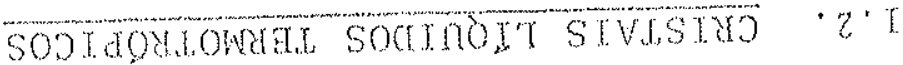




$$
\begin{aligned}
& \text { ) a } y \text { estouse (o) } \\
& \text { อकाxoาsoto (q) } \\
& \text { вวтrexpu (e) }
\end{aligned}
$$

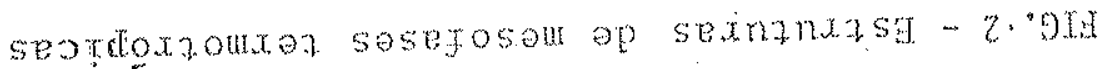

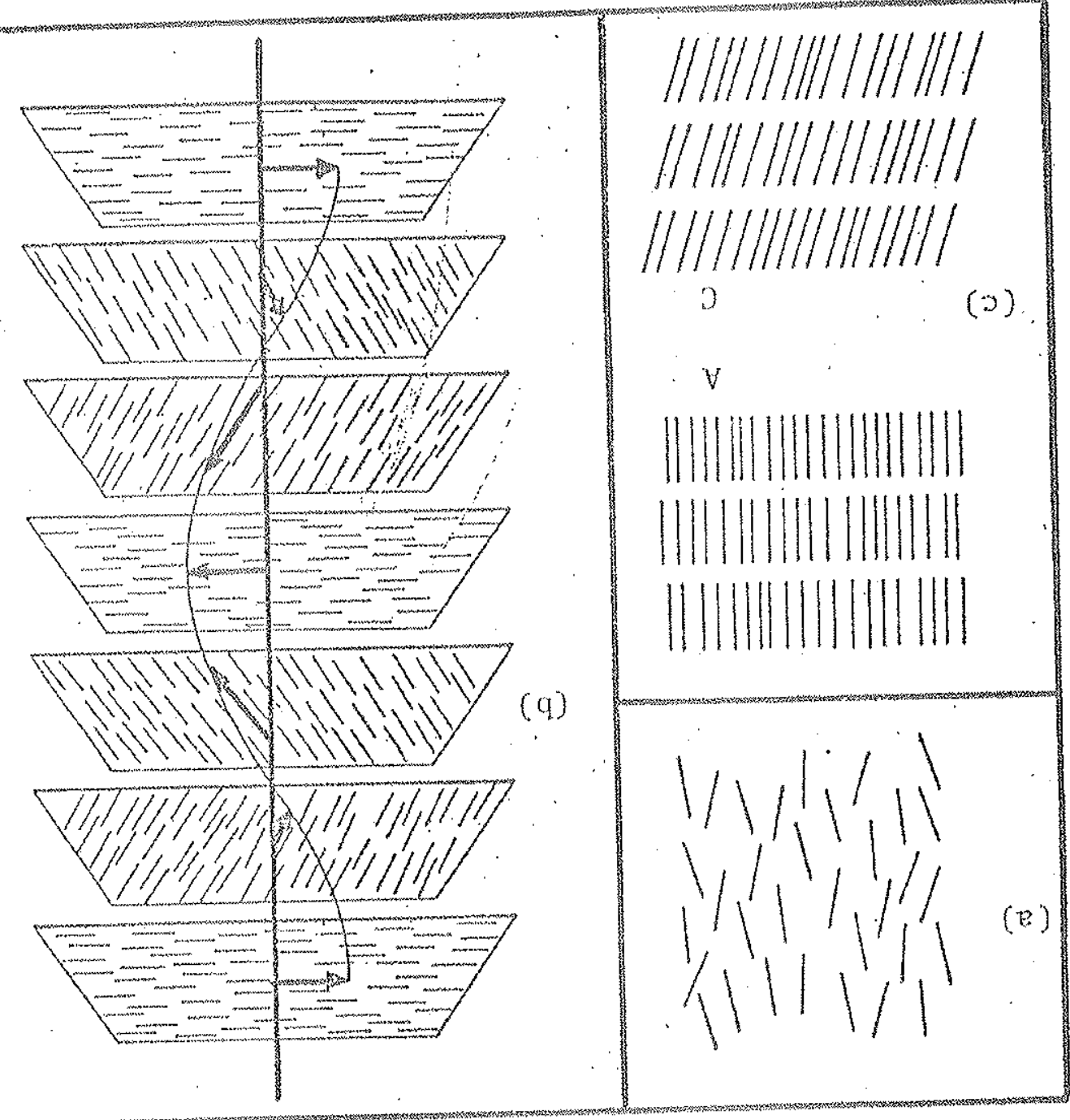

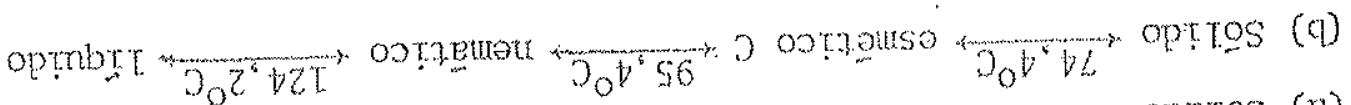

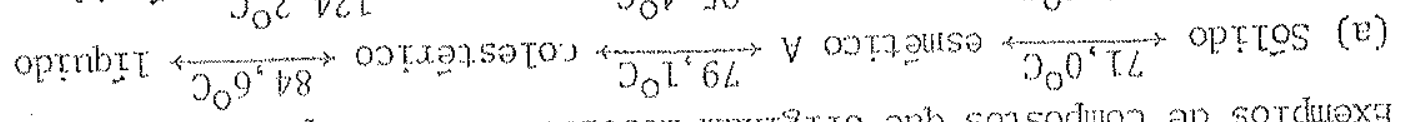

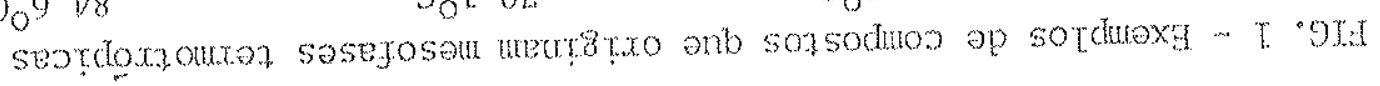

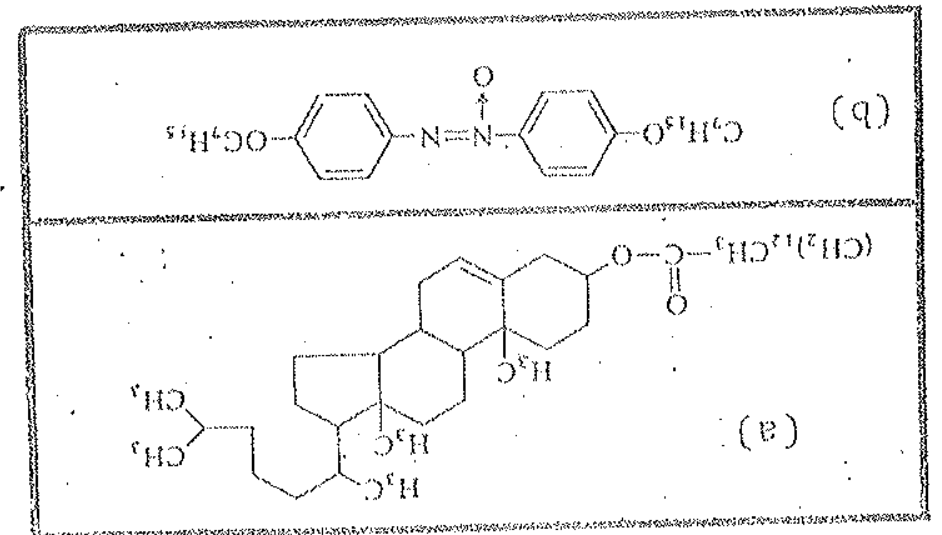


- eotdoxast eptnbt e

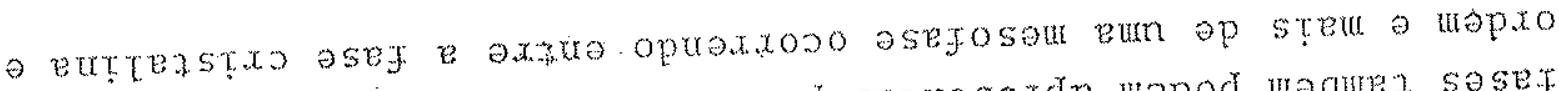
op tenpex epred tros ans?

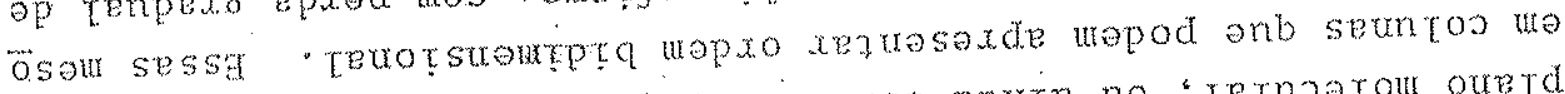

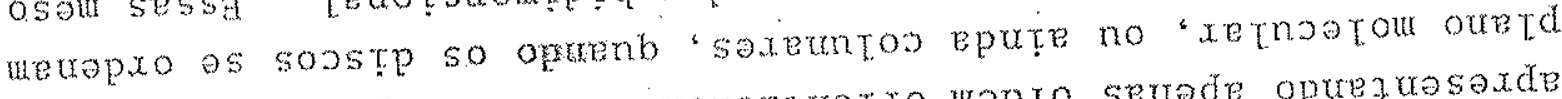

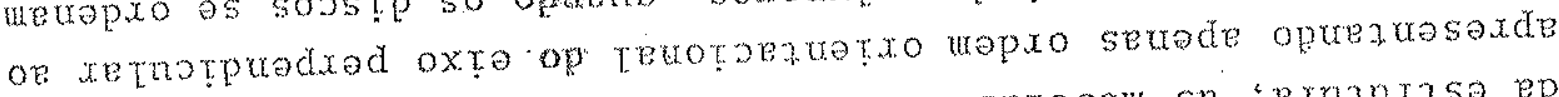
'ospzuar odta op xas mapod seotroostp sasejosem se 'exmanaso ep

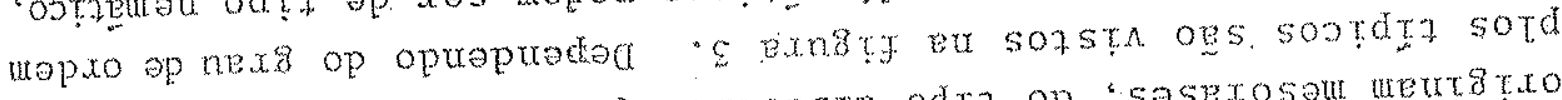

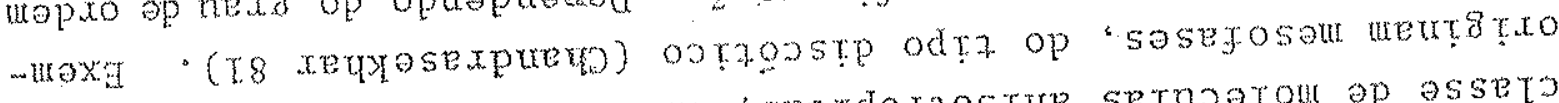

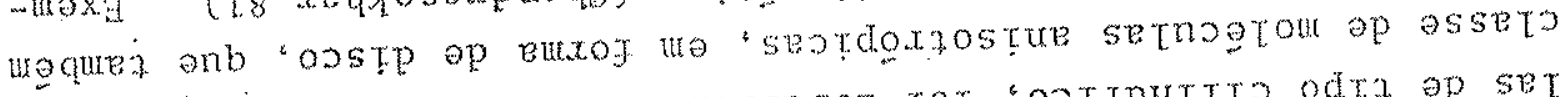

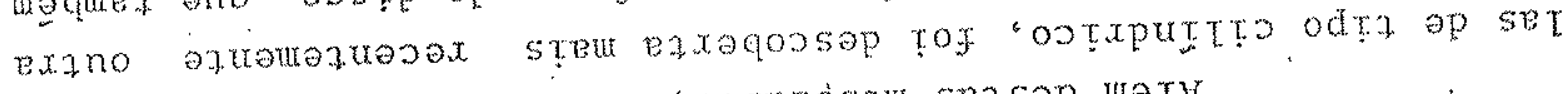
ñotou mos opuaxtojo sepoz"sesexosen seasep meTV

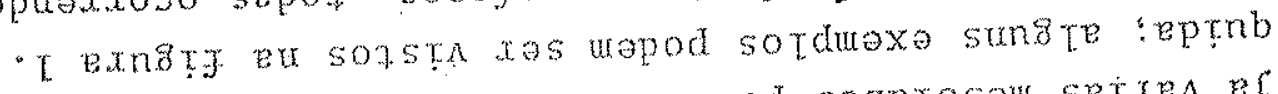

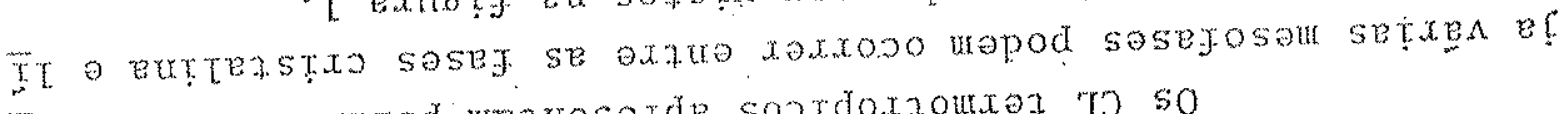

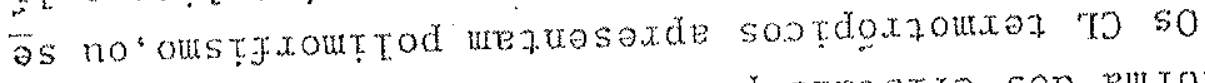

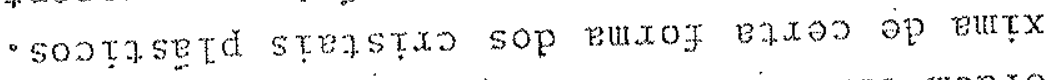

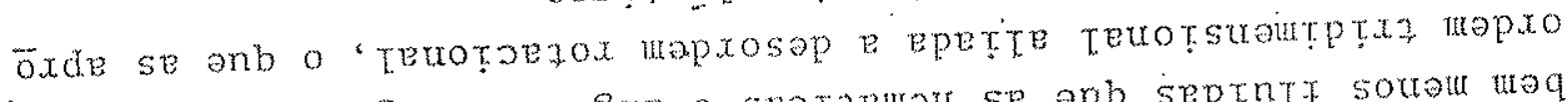

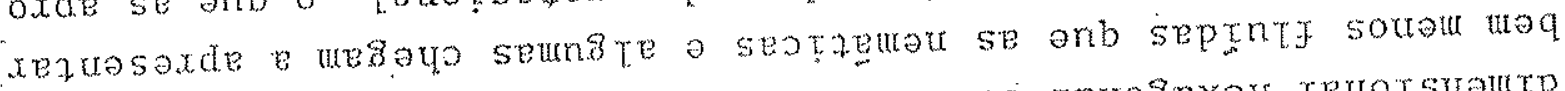

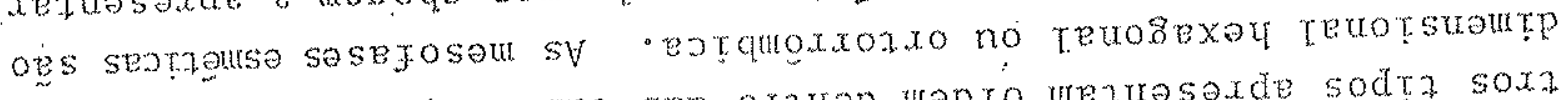

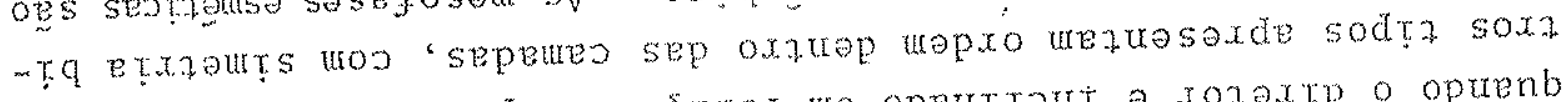

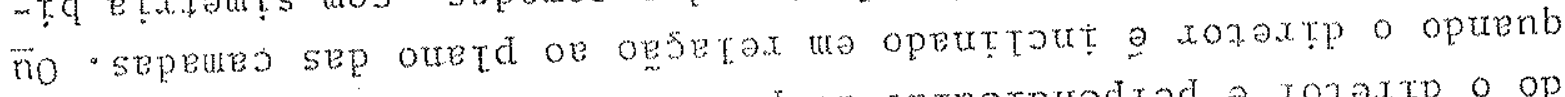

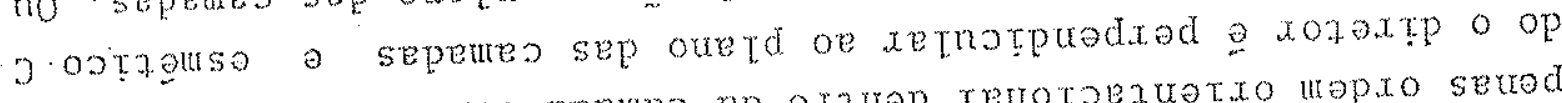

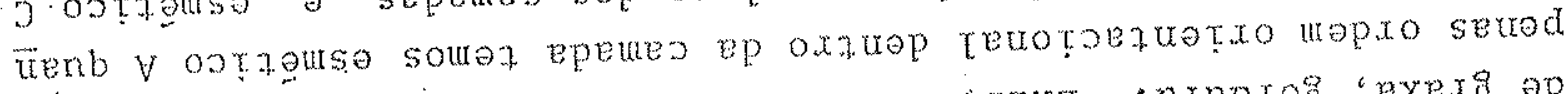

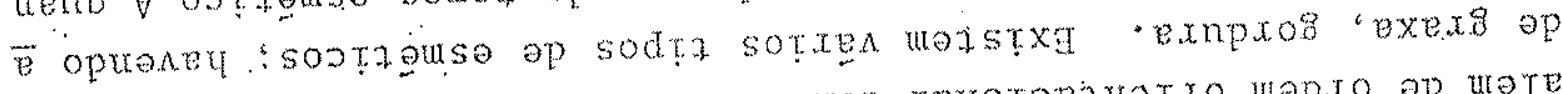

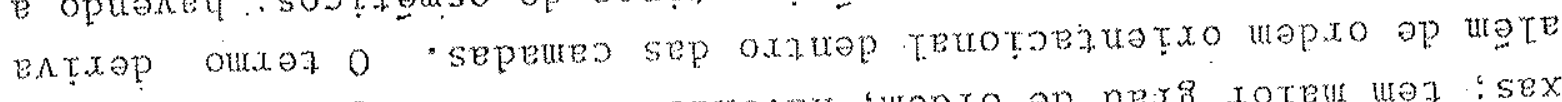

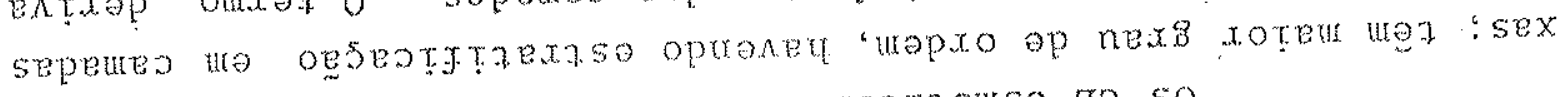

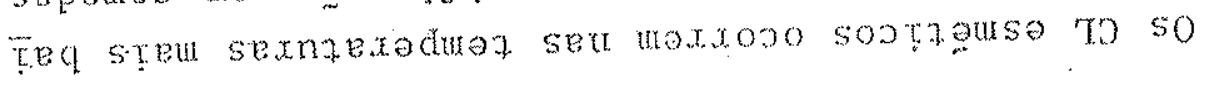

- $0250 z$

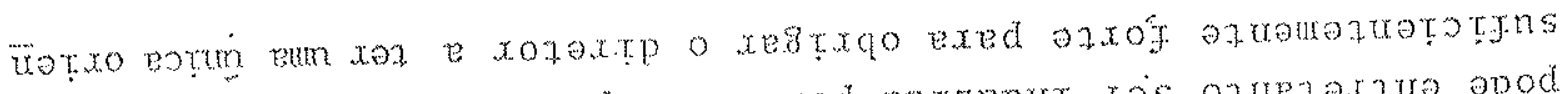

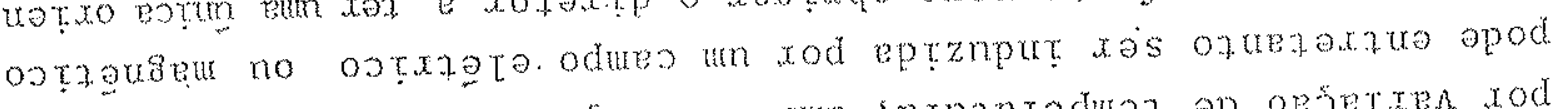

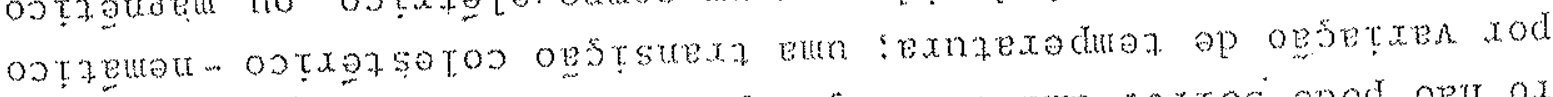

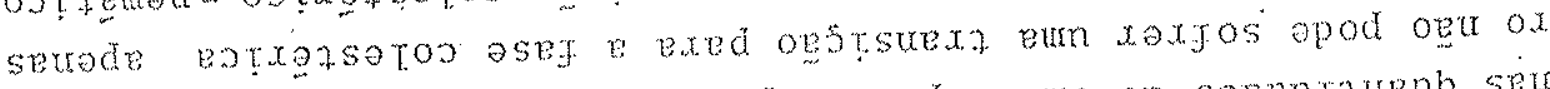

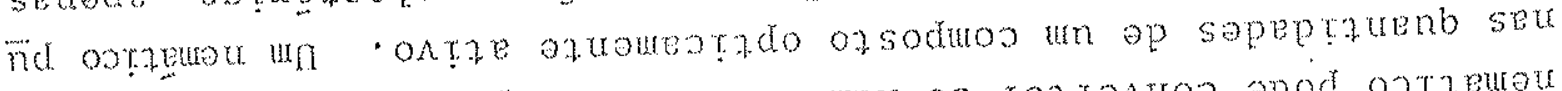

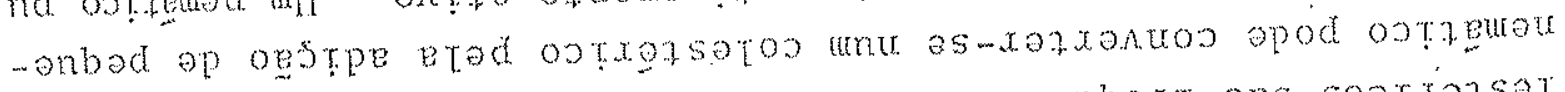

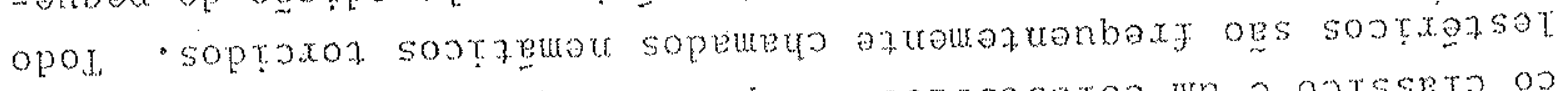

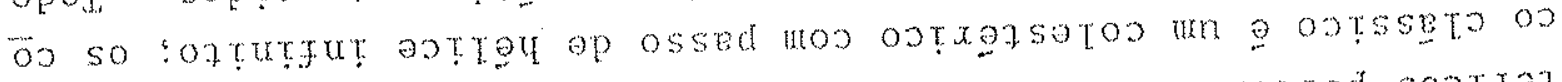

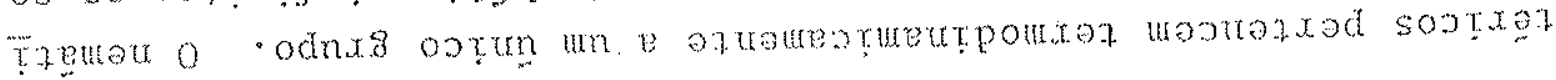



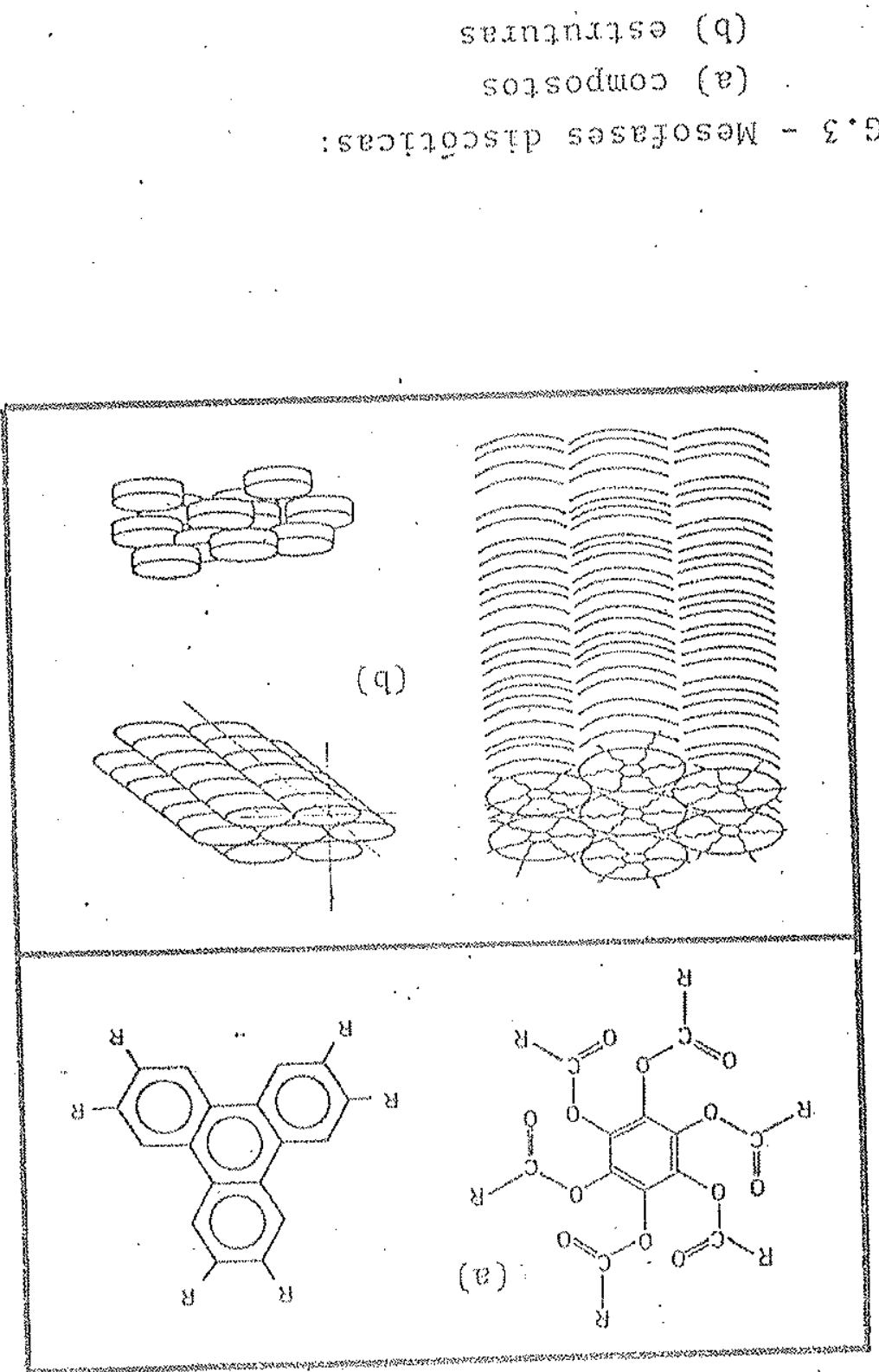


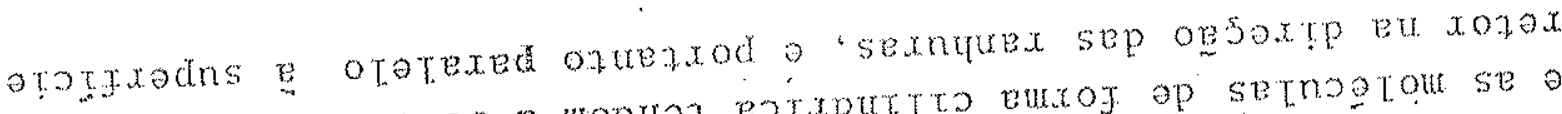

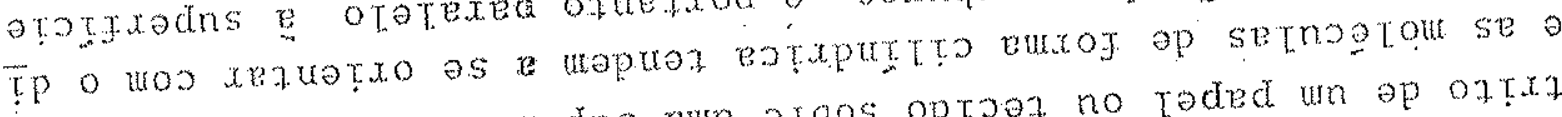

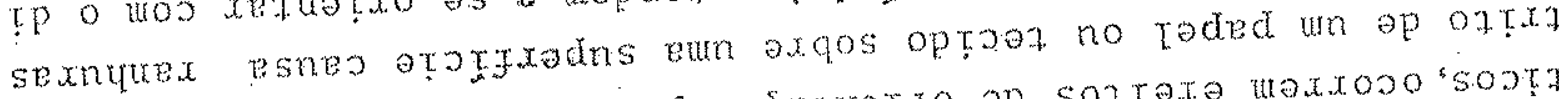

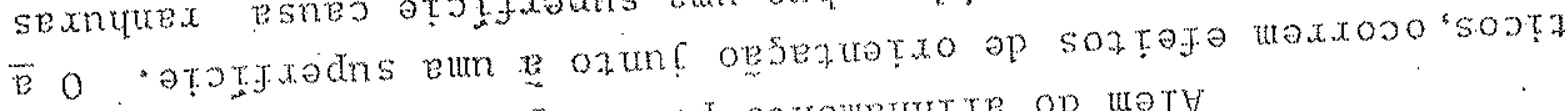

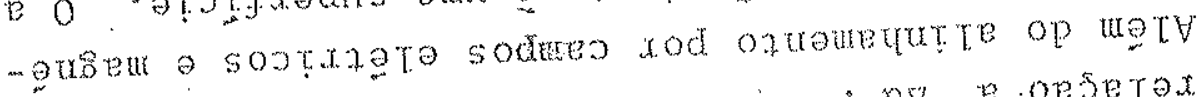

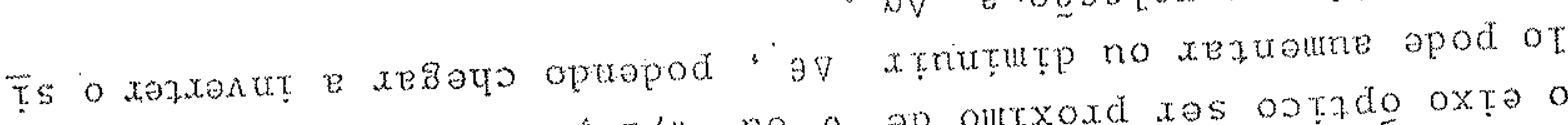

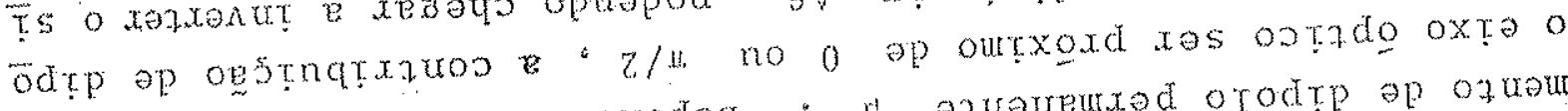

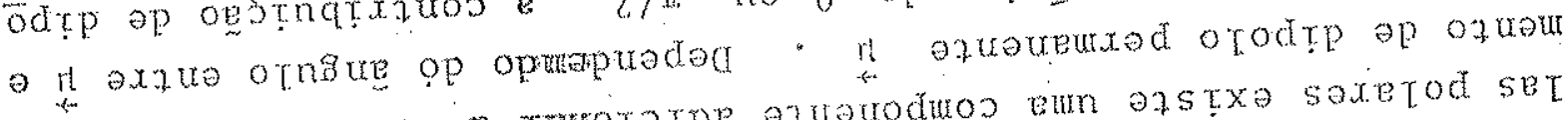

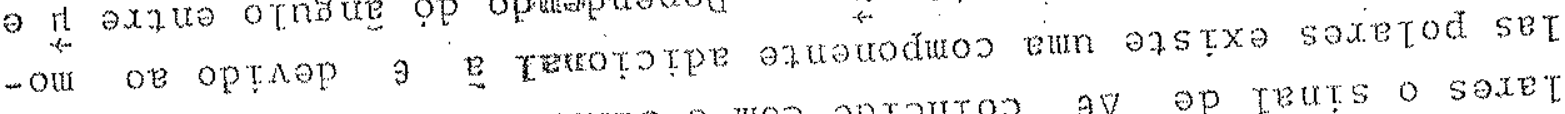

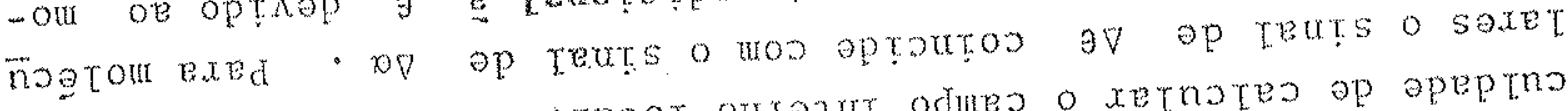

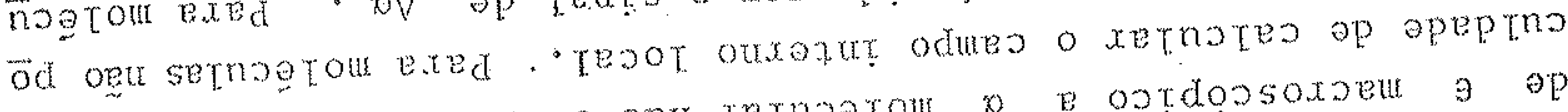

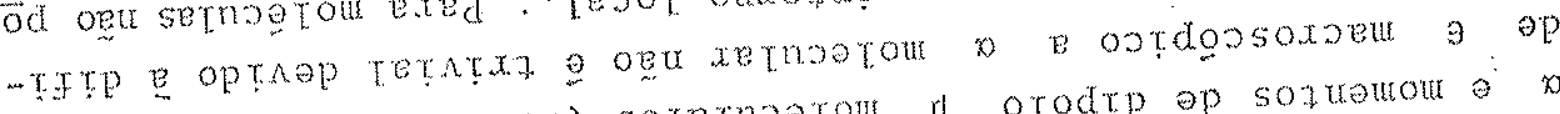
masessed e (8L nof op) saxetnogrou $\mathrm{r}$ orodtp ep solueur $a$

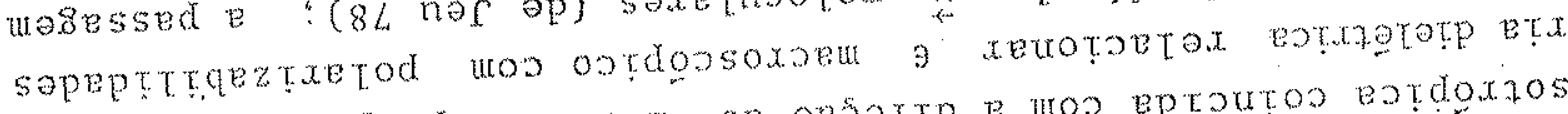

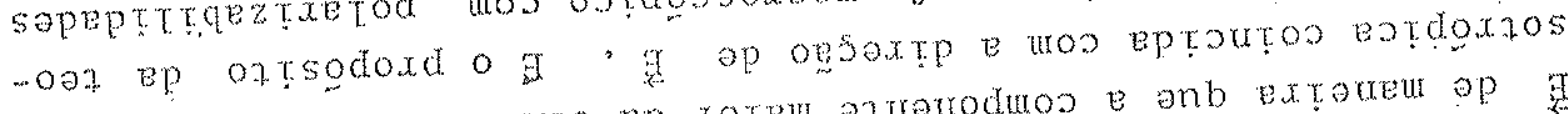

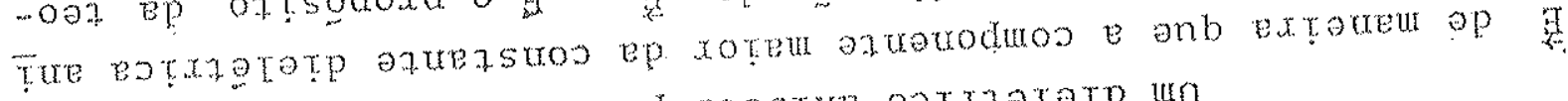

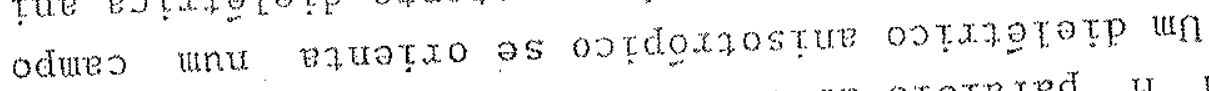

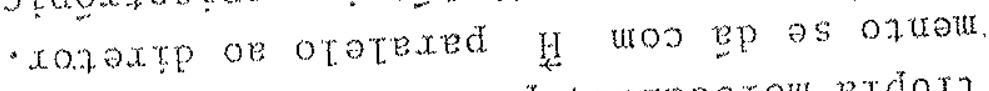

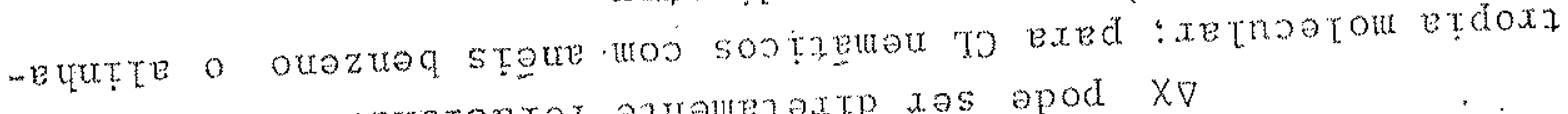

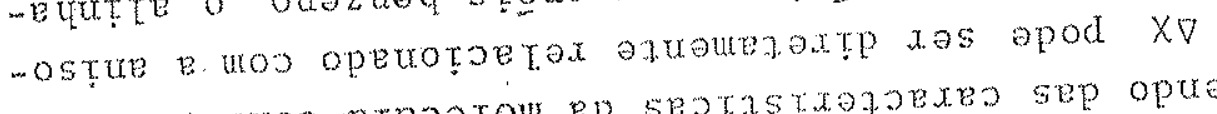

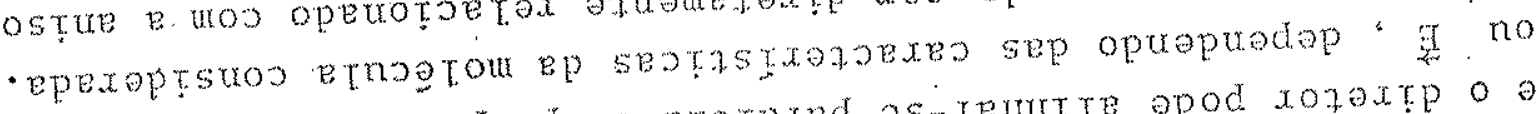
H 1 azuaternoppadxad no eratexed as-xequte opod xozoxpe o (Best"0 m) epter entrexadoon basodsax eun aztuxed sernoerou se axa

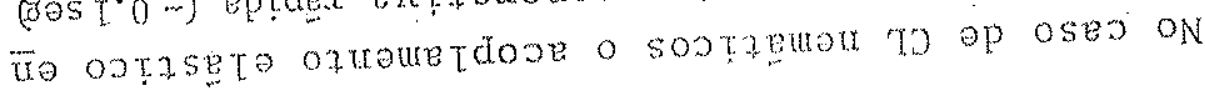

$$
\begin{aligned}
& \mu b / 29 \quad 0 \nabla \cdots=9 \\
& 2 \mathrm{H} \times \nabla \frac{2}{\mathrm{~T}} \cdots=\mathrm{m}_{B}
\end{aligned}
$$

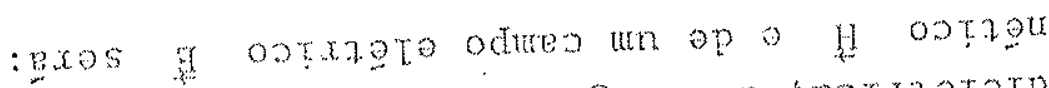

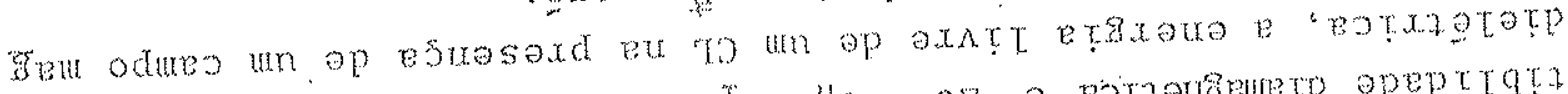

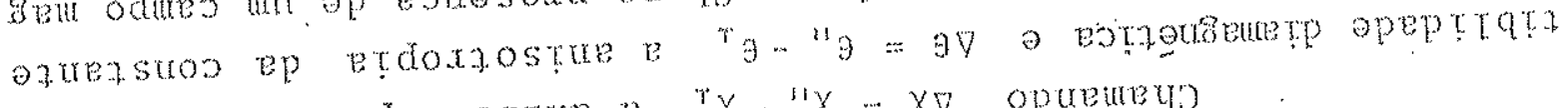

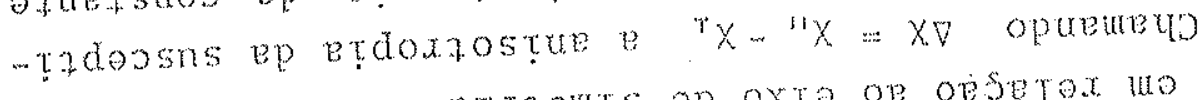

-etxams op oxto op oesetax uo saruatate soos

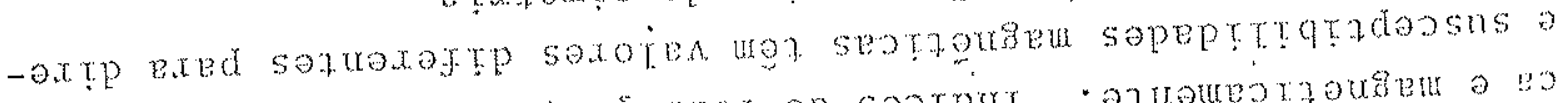

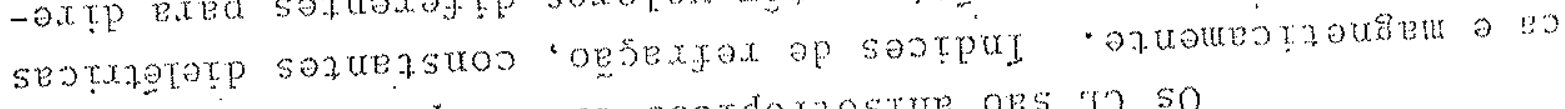

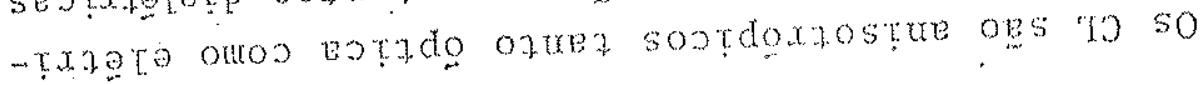




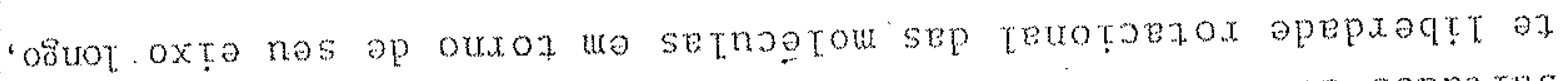

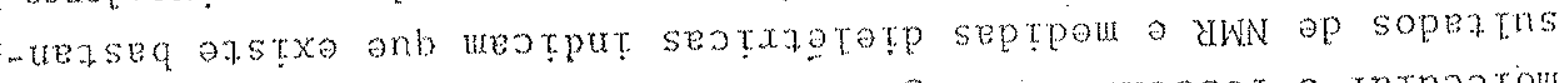
ad (

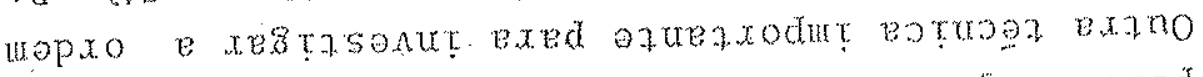

- otaur op oeseztretod ap sortejo soe

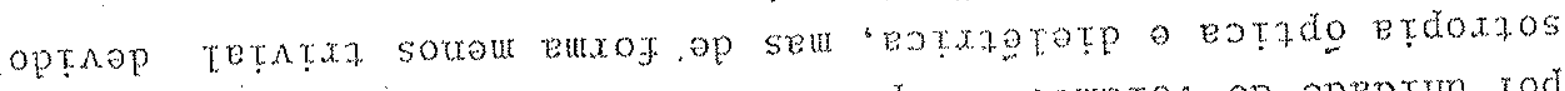

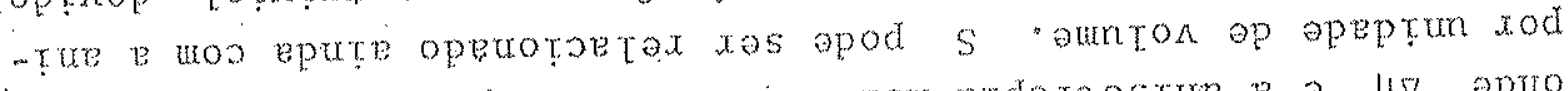

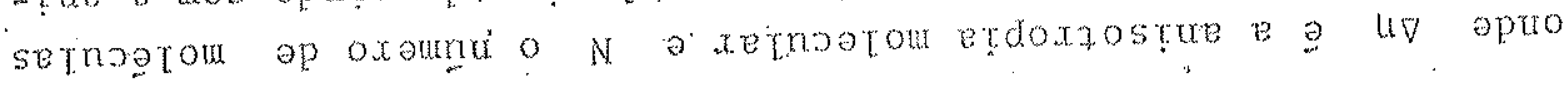

$$
U V S N=X V
$$

stod opeztretro optrb

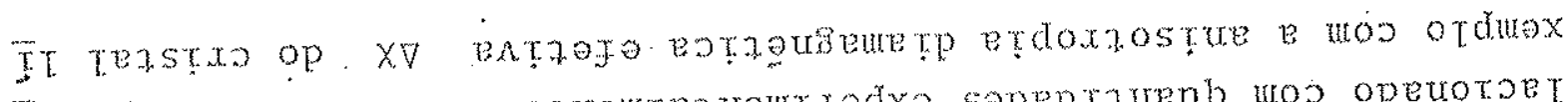

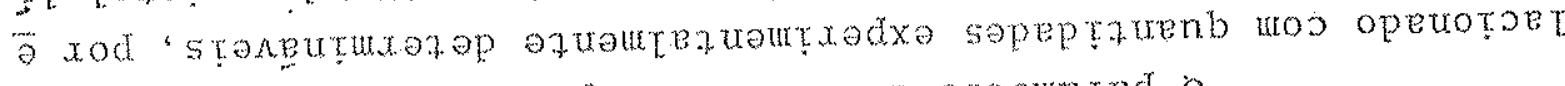
- ax azuouezaxte xes apod $s$ mopxo op oxamexed 0

- vsejosem ep x xojoxtp o a et

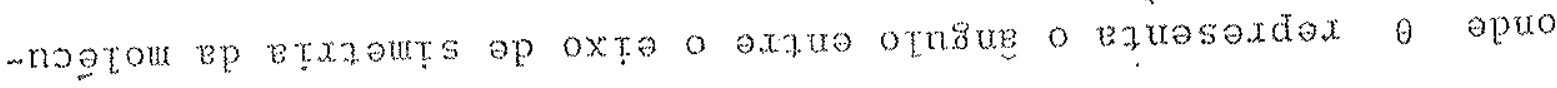
$<\quad<-\theta z \operatorname{sos} \&>\frac{Z}{T}=S$

mopto op oxzourexed un op sourtox ma ossaxd

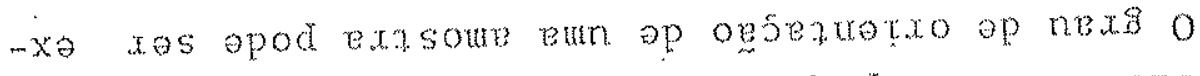

- soumbed equateatatex sodues ap oes

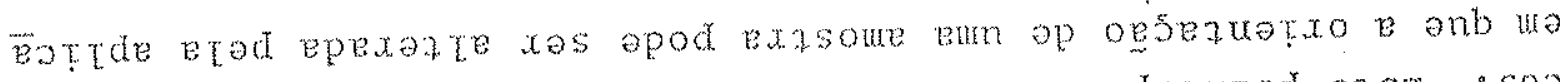

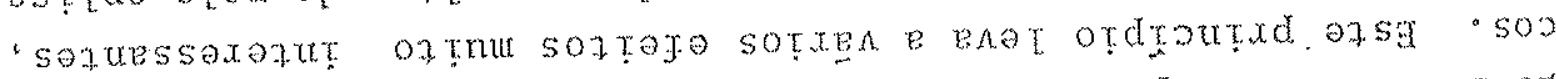

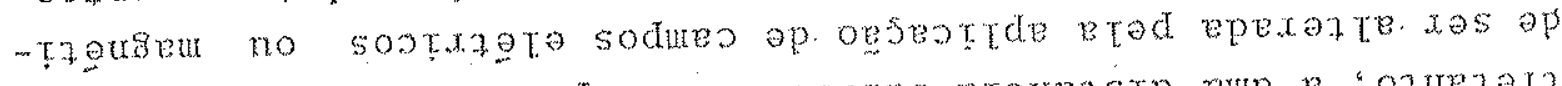

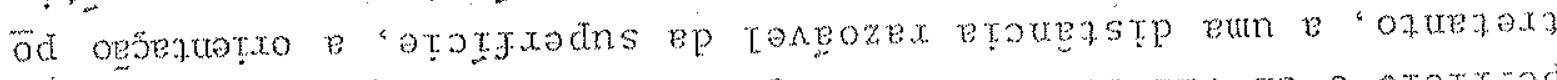

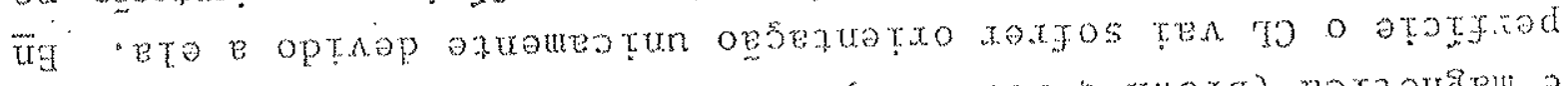

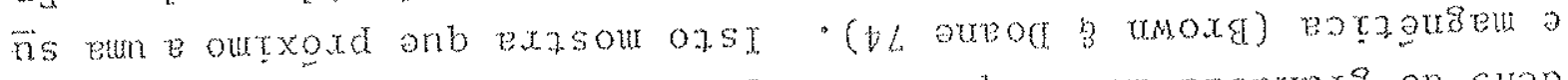

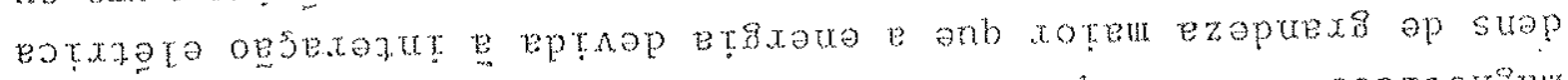

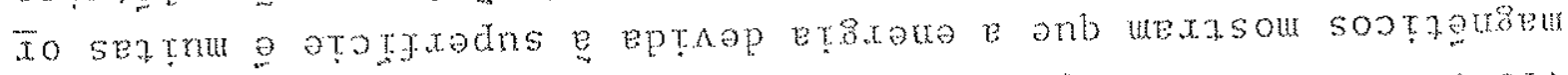

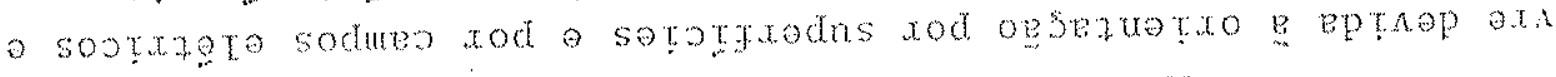
TT etrowe e exed sopenzofo sotnoteo op sopertnsoy

- stetrotraxaraxd sogsas

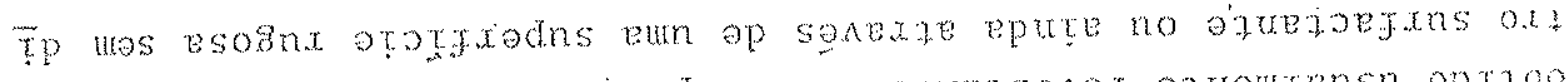

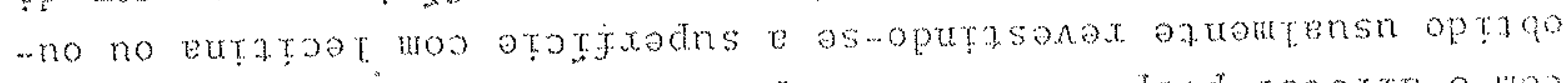

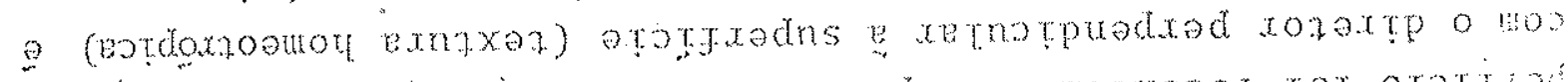

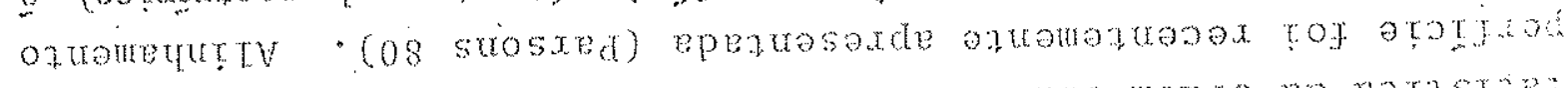

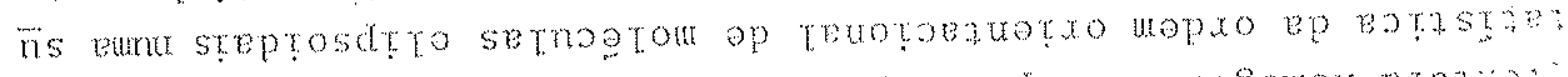

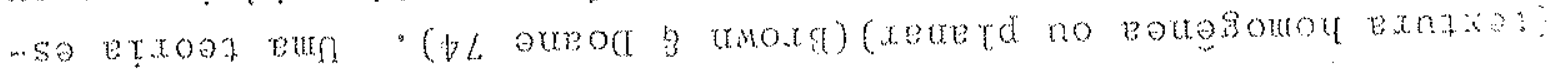




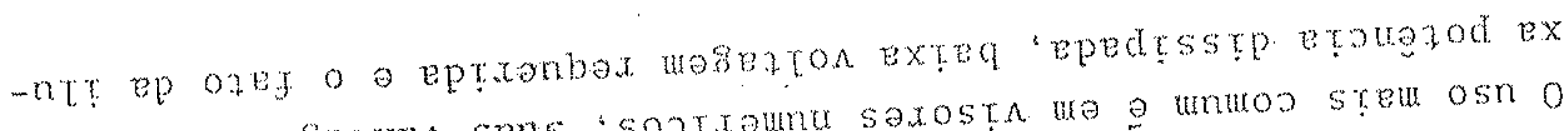

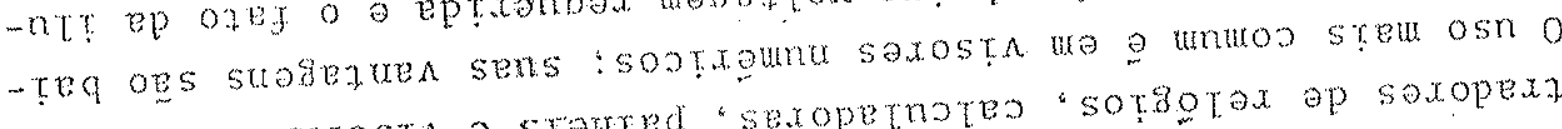

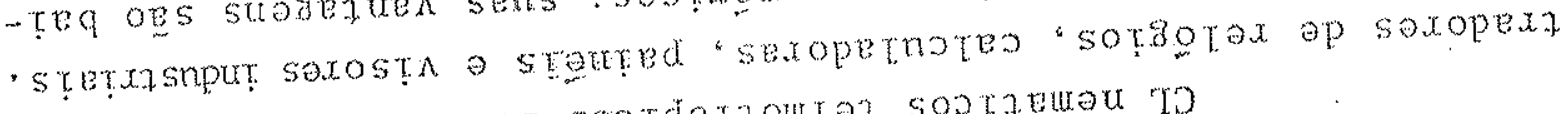

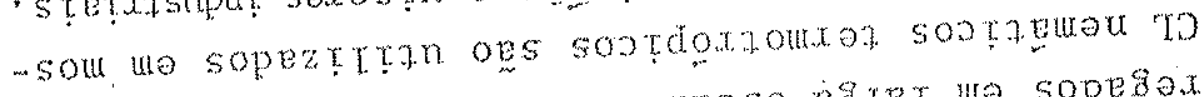

- eteose esxet แə sopesoxdur xas e urexes - omos woxod epesep entarn eason. opxozexoqer op opeptsorano eum

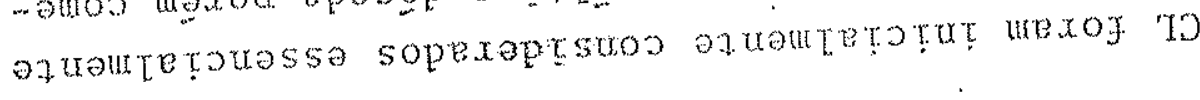

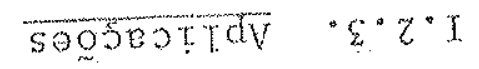

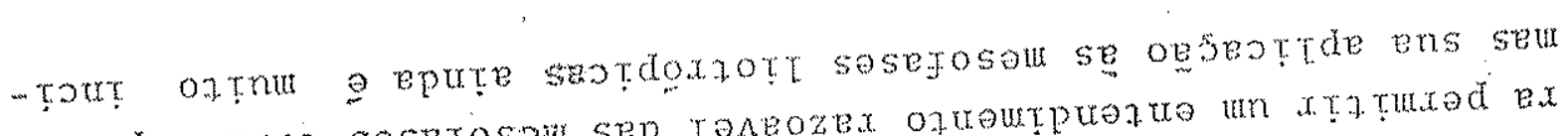

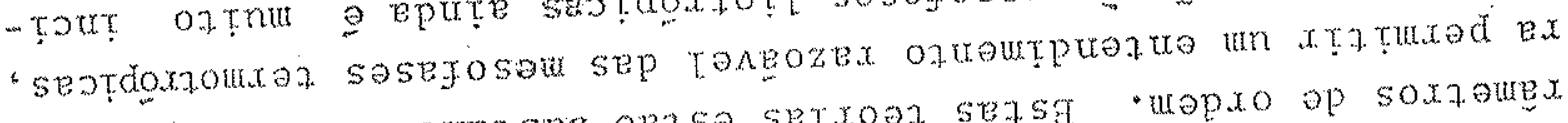

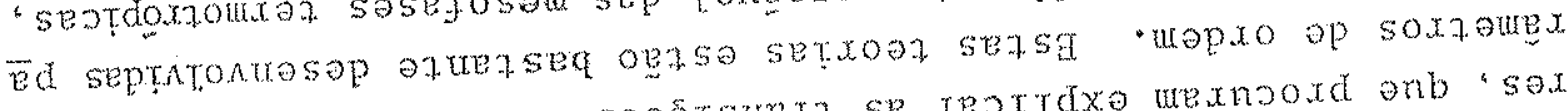

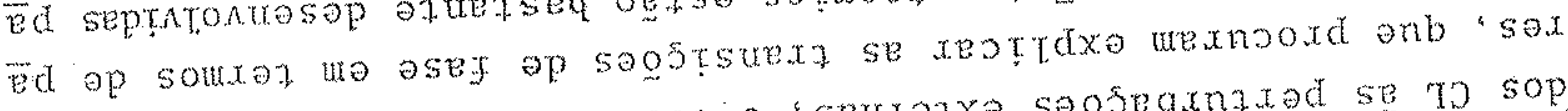

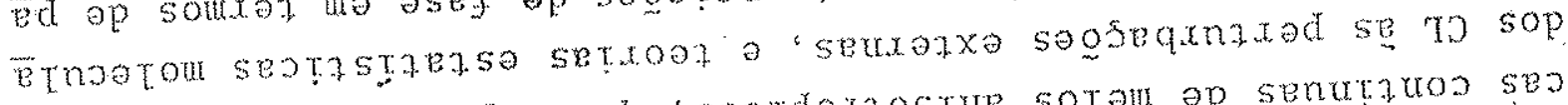

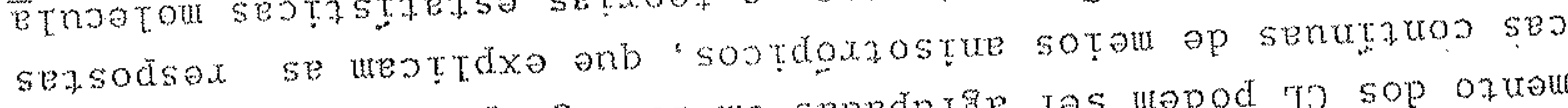

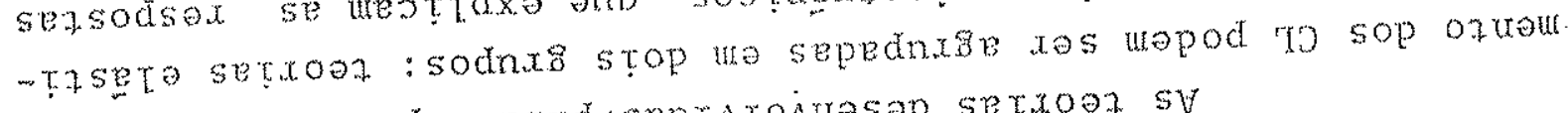

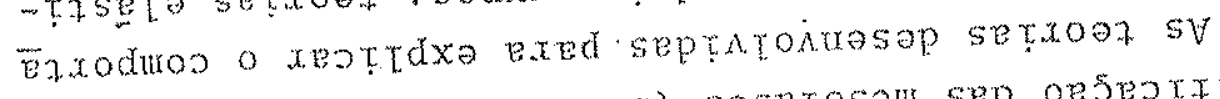

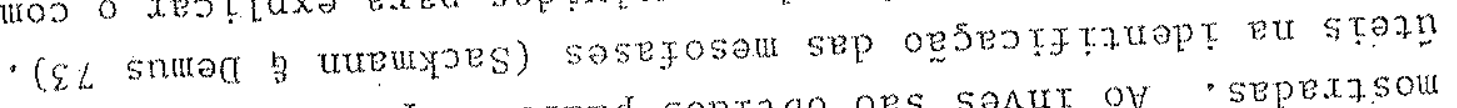
sem 'soxerduos sojtzde sooxped soptzqo oes seart ov sepextsou

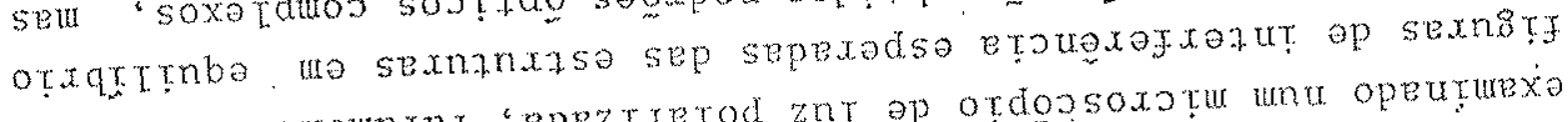

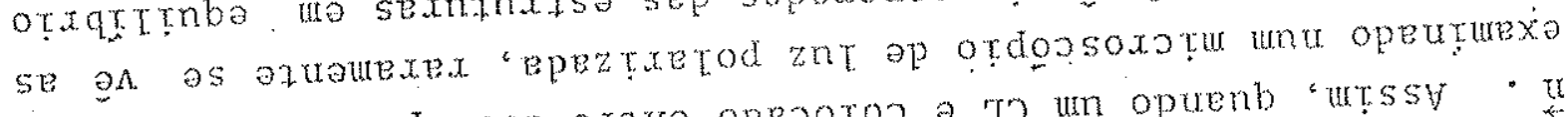

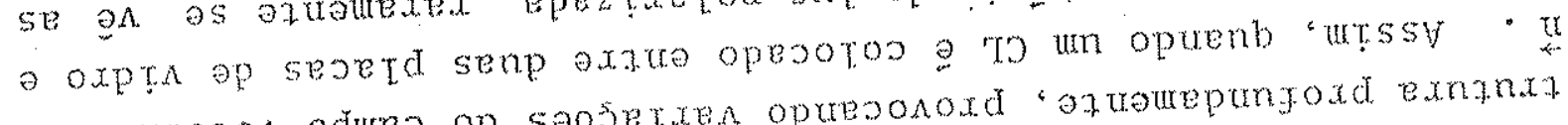

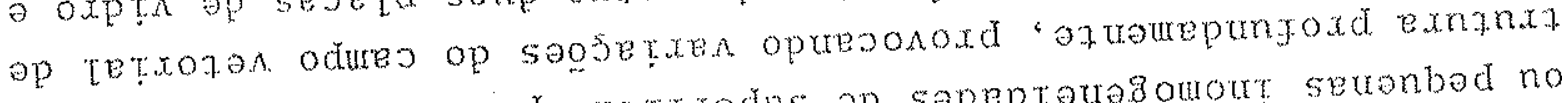

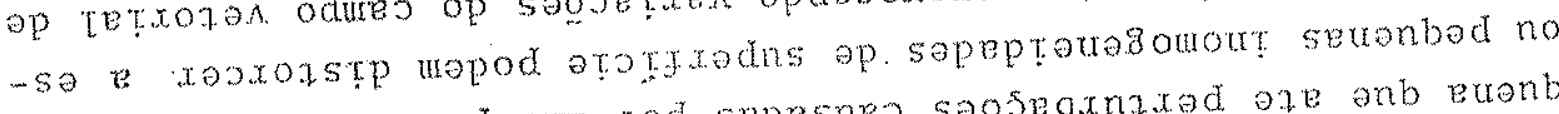

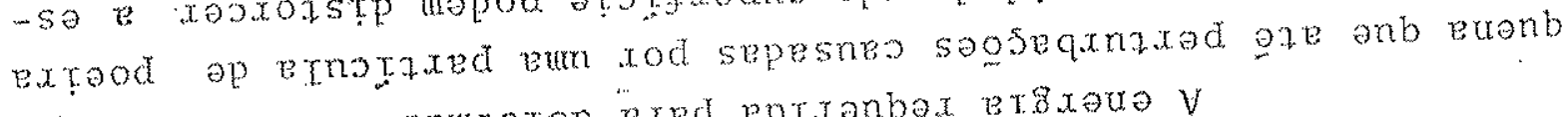

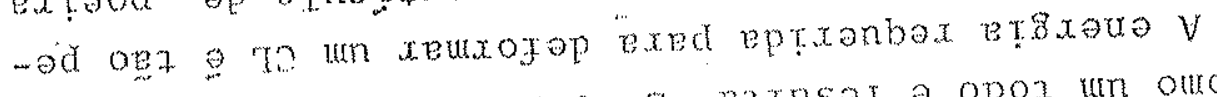

- $0=5$ patrsex a opoz un ouros pxasoure e ex

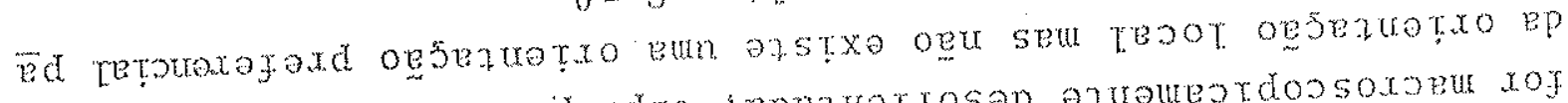

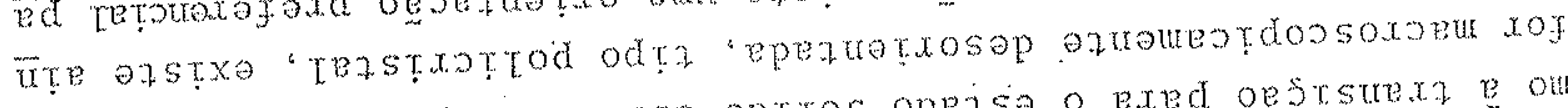

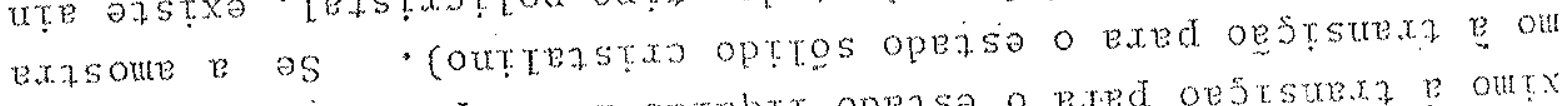

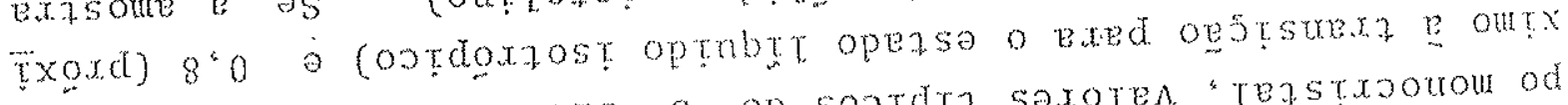

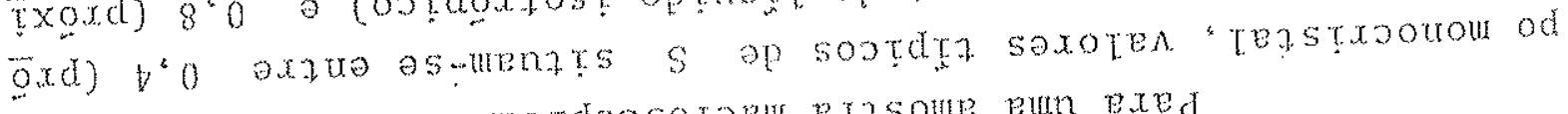

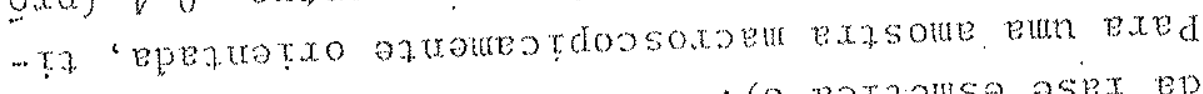

- (o eotrouse osez ep ospo) stetxeta

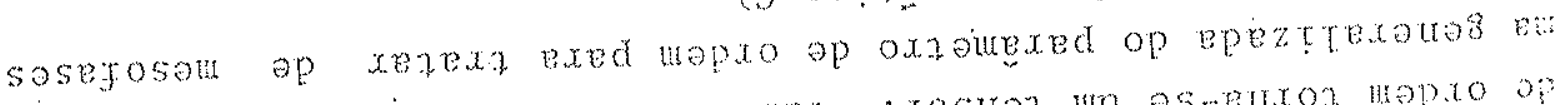

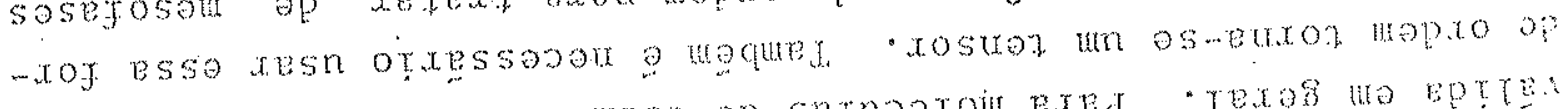

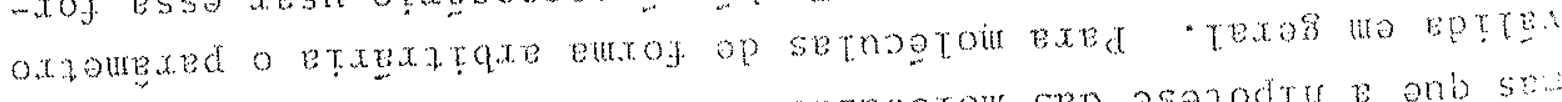

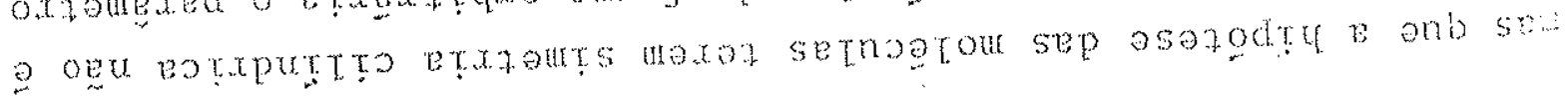


- $($ e-bl kexp) exajsomz eu soz

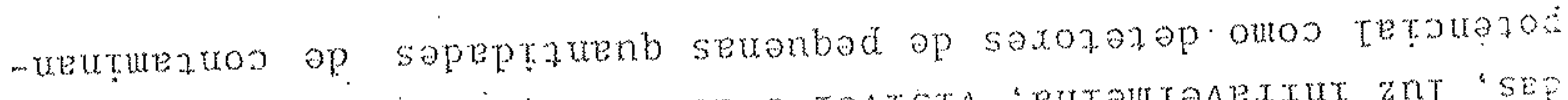

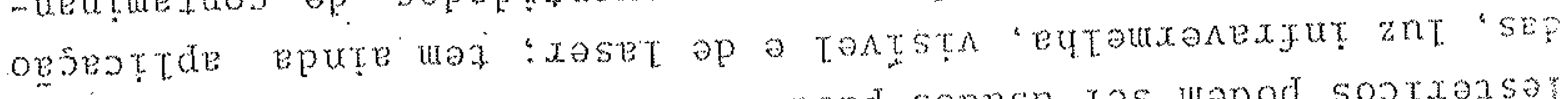

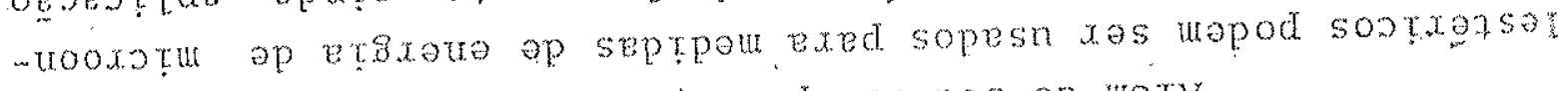

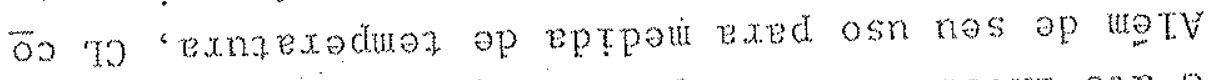

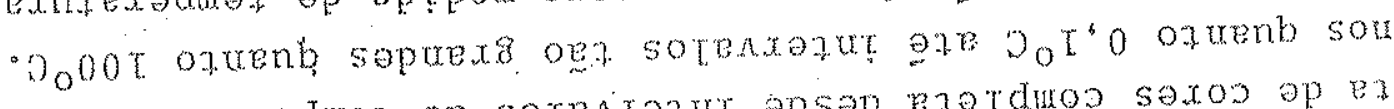

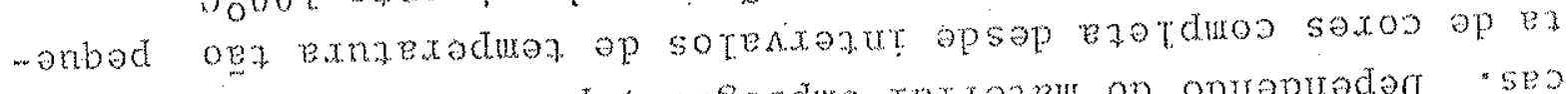
sodsax eum xorqo osmopod "opesoxdur rexazeu op opuepuodod "ses

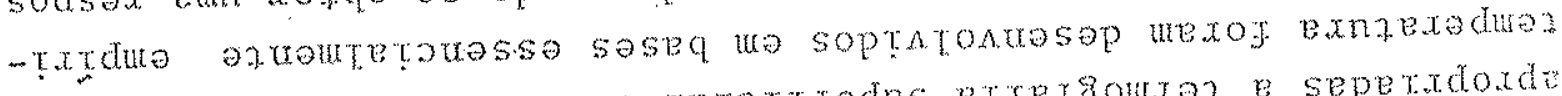

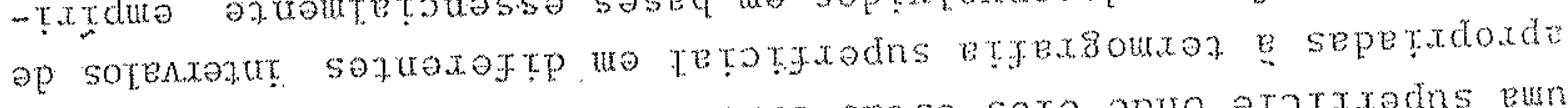

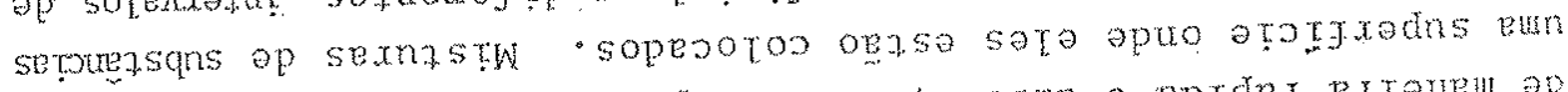

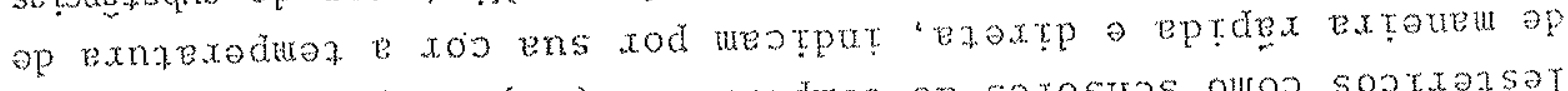

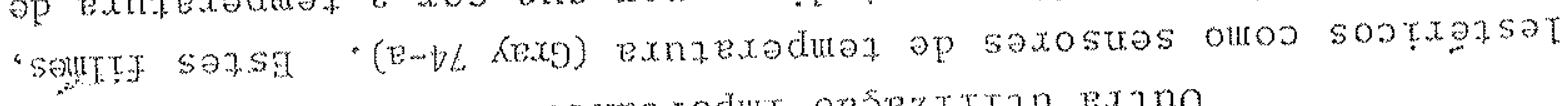

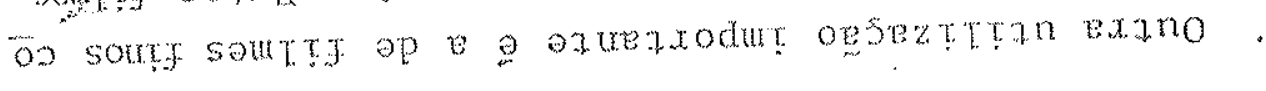

saxop

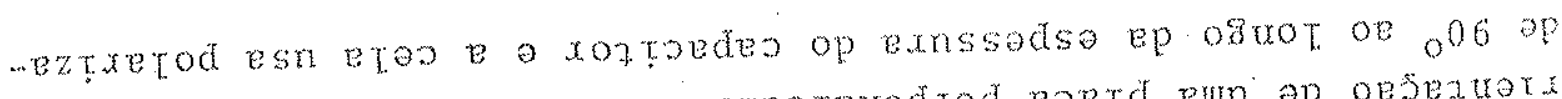

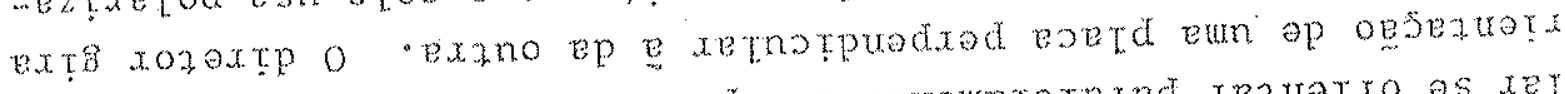

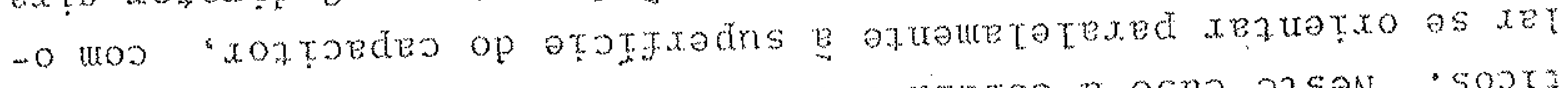

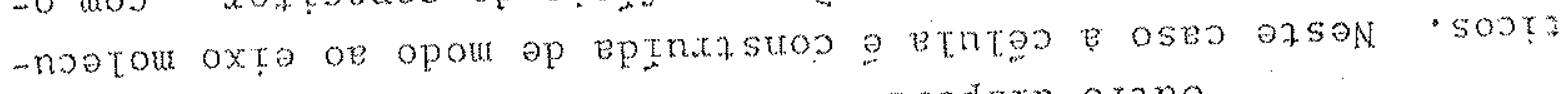
ËHox sop opsxoz op ortgye o esn oxtatsodstp oxjno

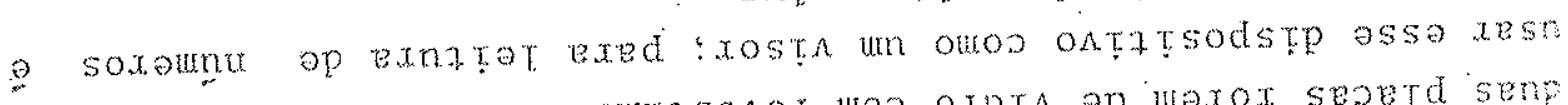

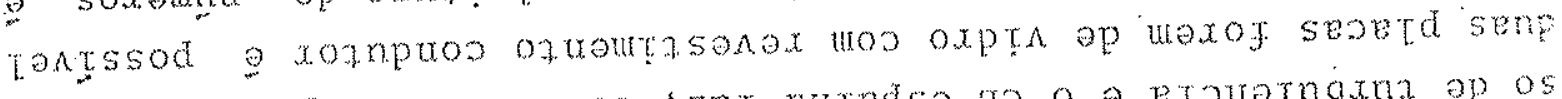

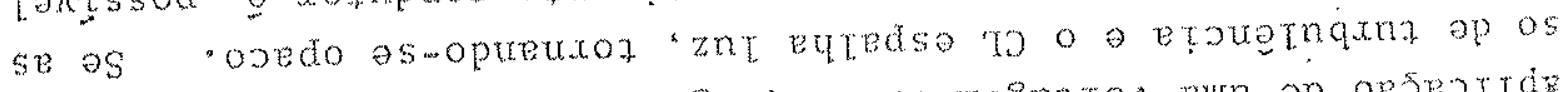

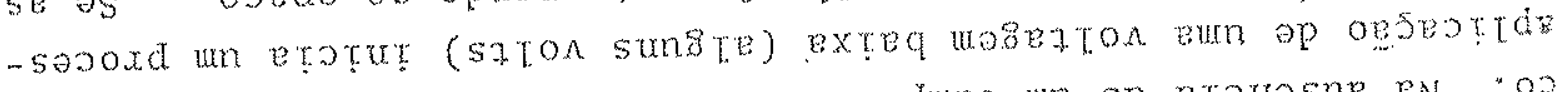

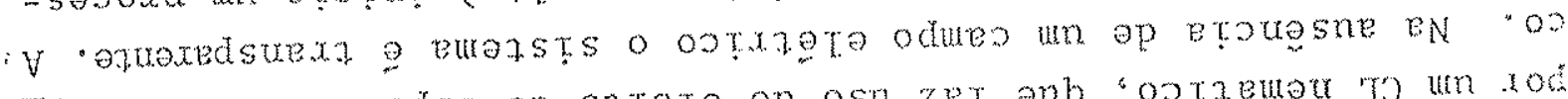

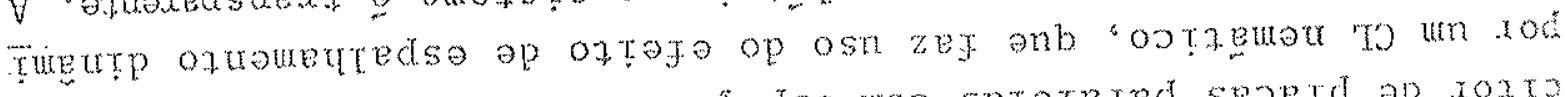

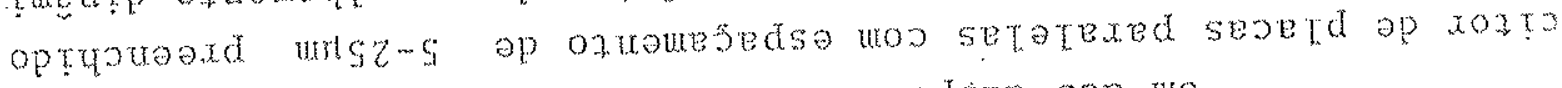

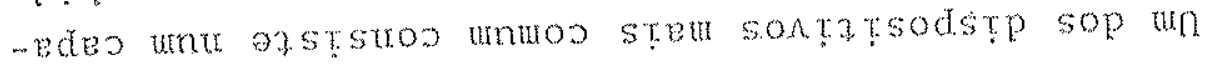

- wepuo ep opjanxazar p eptrop ppeytedse znt esuan?

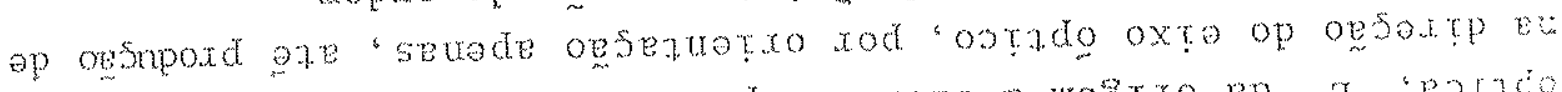

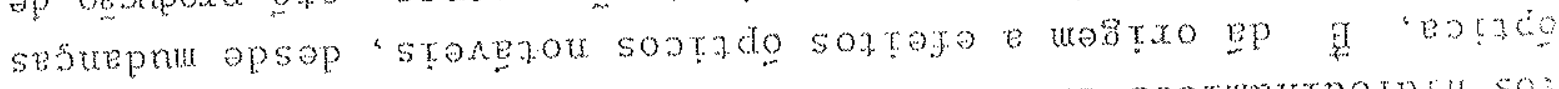

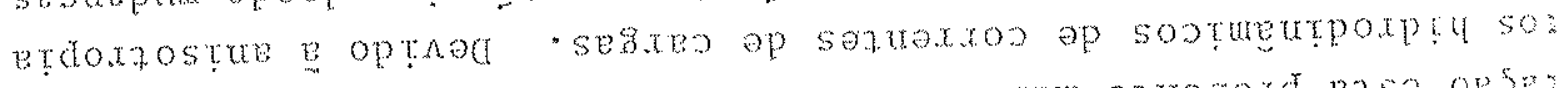

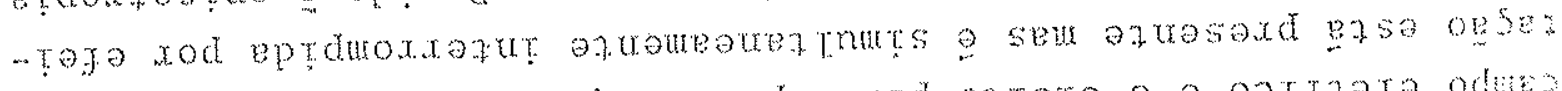

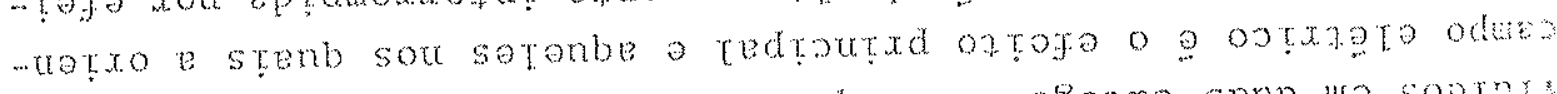

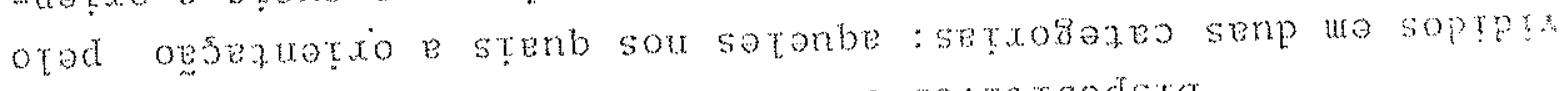

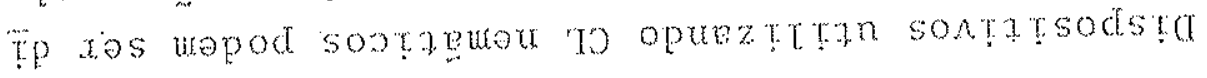

- (bl womsens

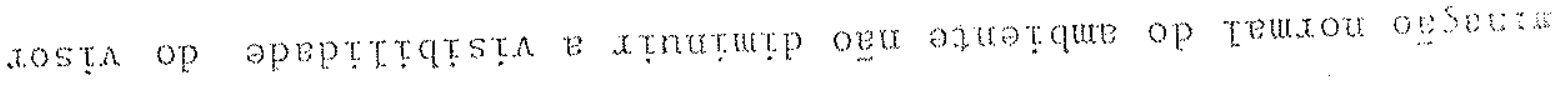


- ogsetyosse op saptotoo sozte ogs

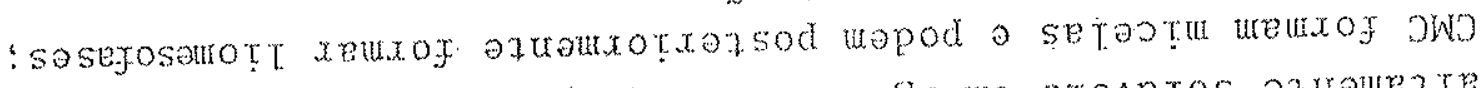
eurn op xtared e onb satonbe ogs a ense wo stantos onxautere (e : sod

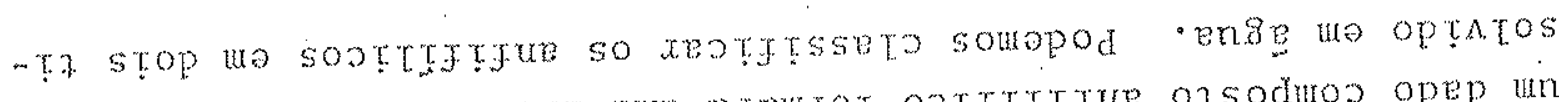

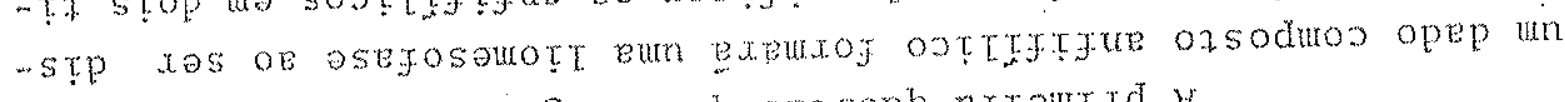

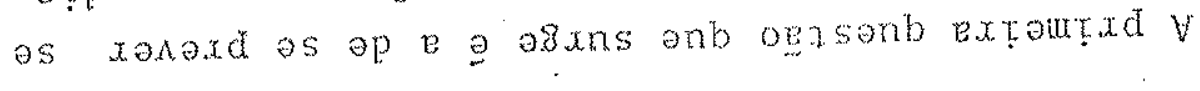

- ppejexpty extejsta oses eur epestres

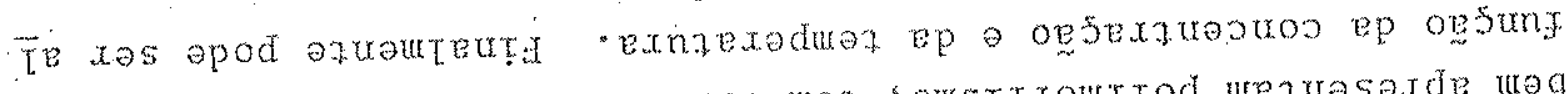

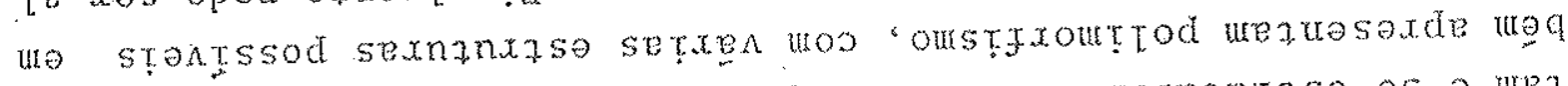

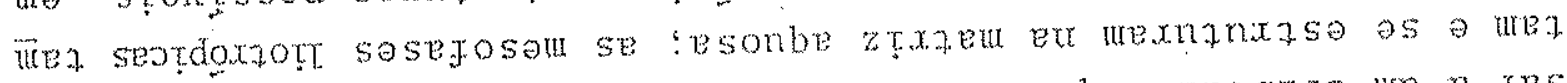

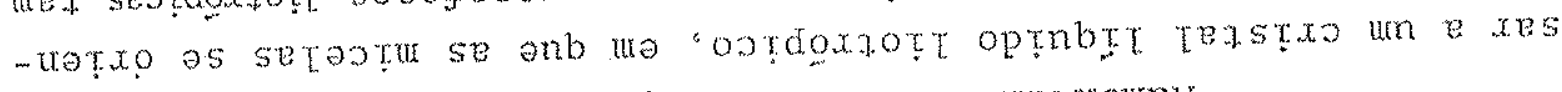

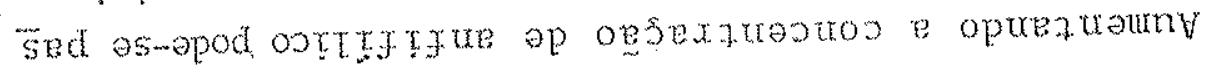

"toneorex opeptrqtaxets ep sout

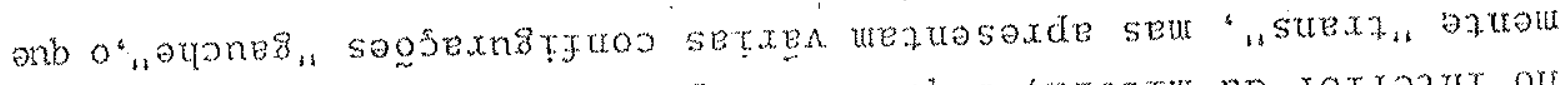

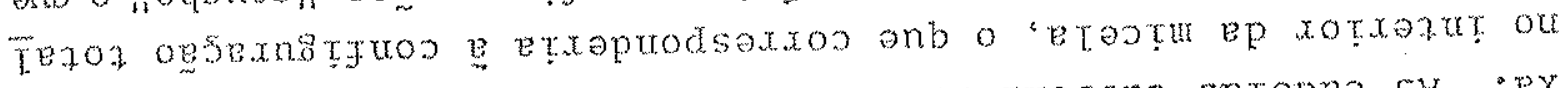

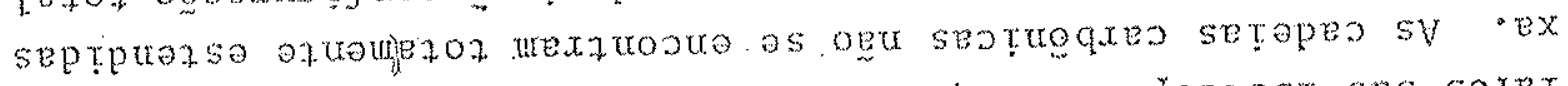

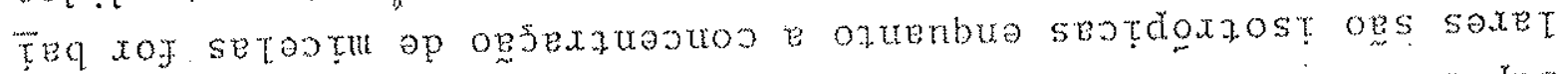

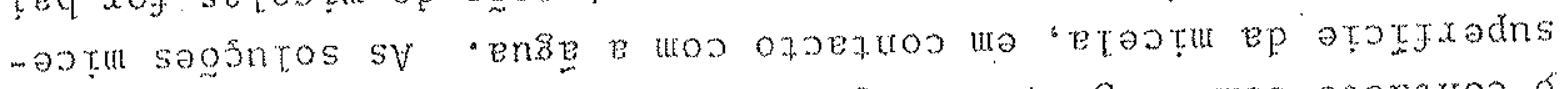

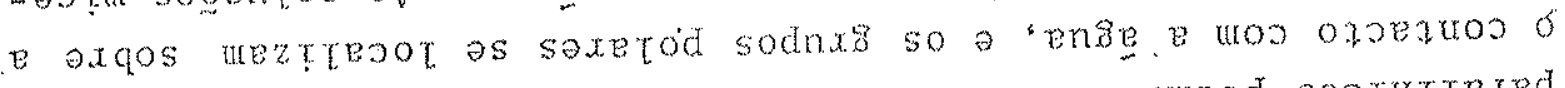

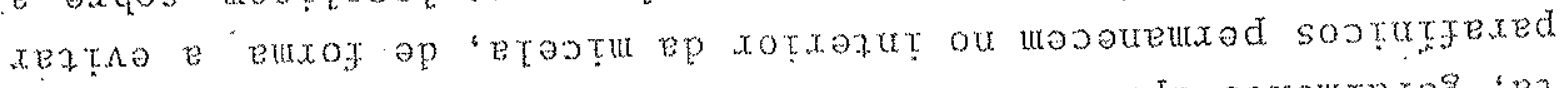

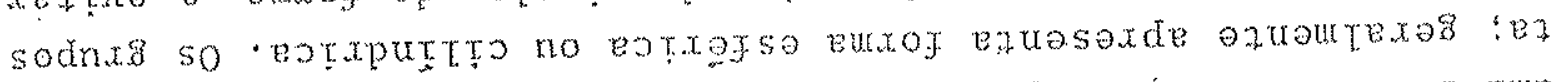

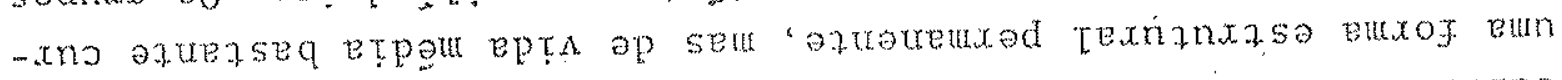

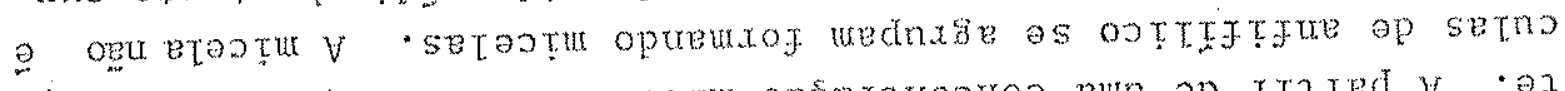

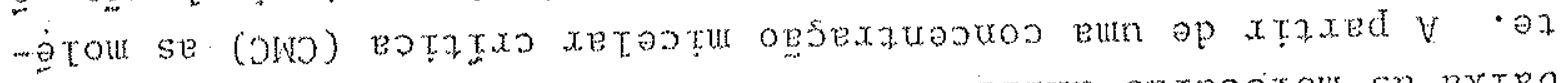

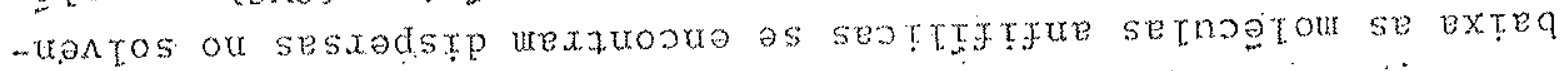

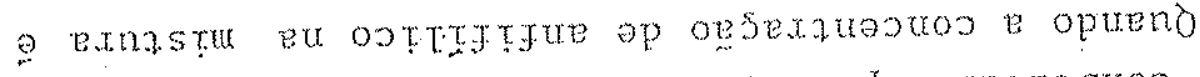

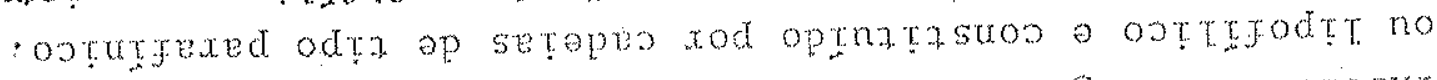

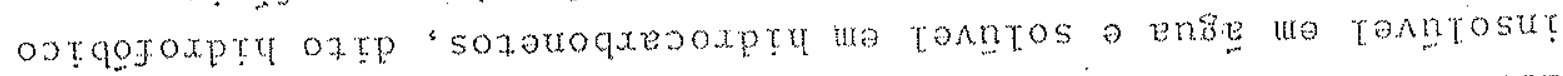

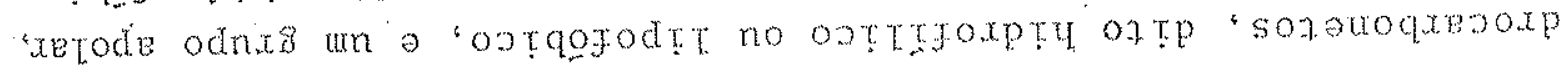

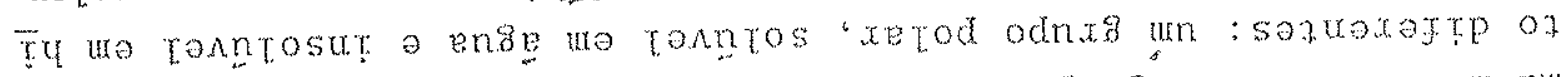

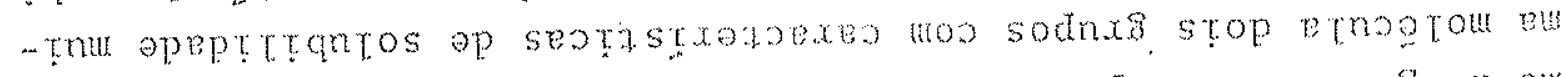

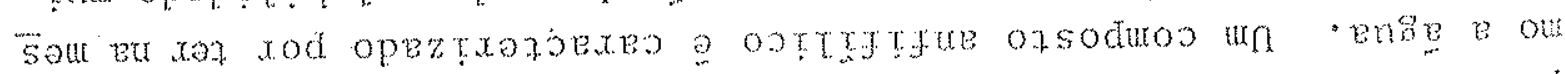

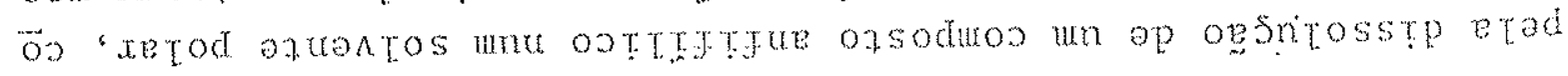

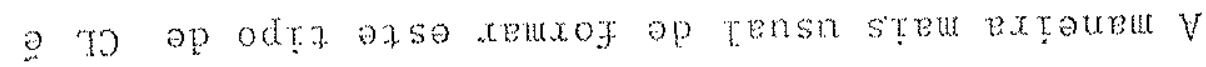

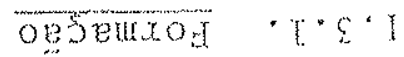




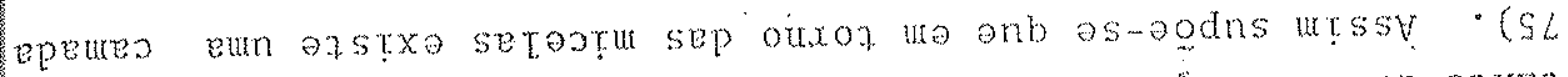

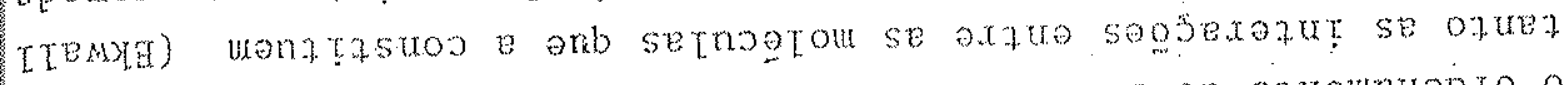

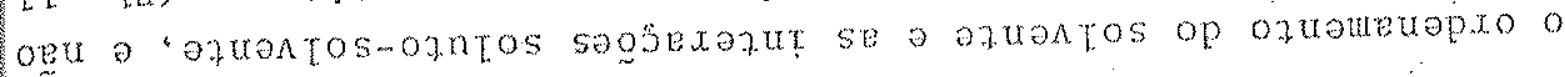

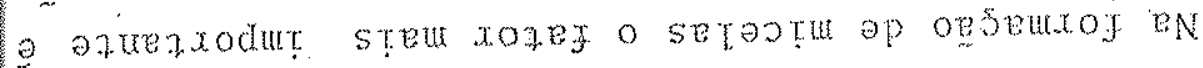

- enge pp exnanxase e gaxauestxatr

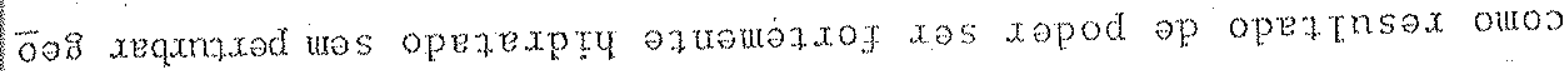

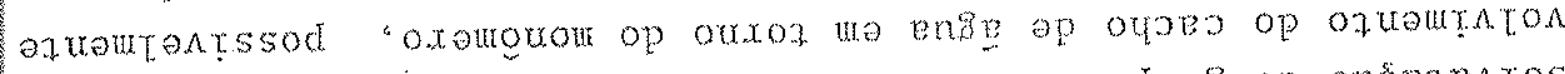

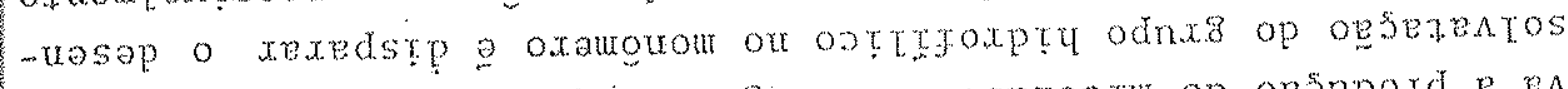

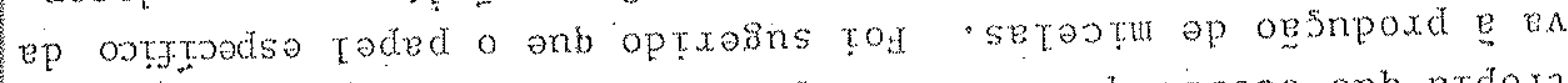

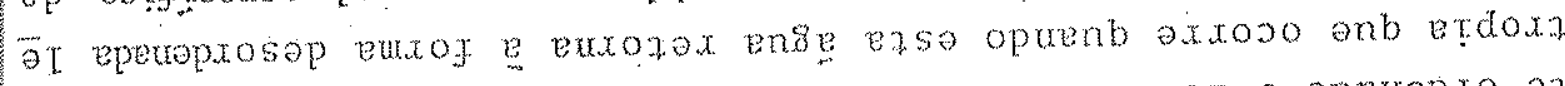

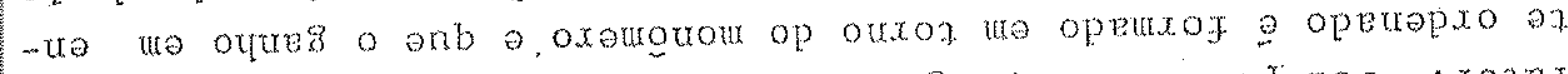

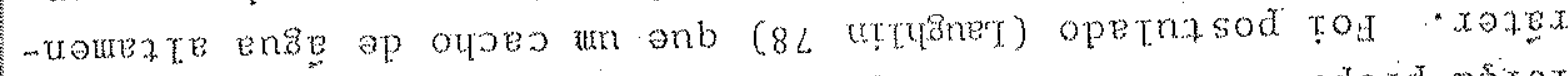

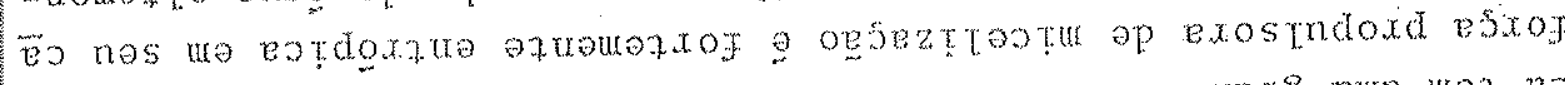

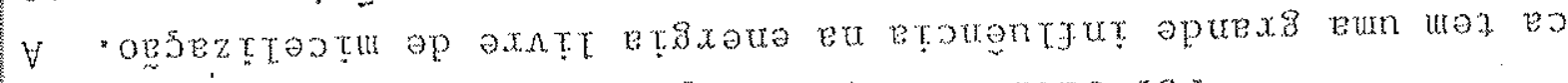

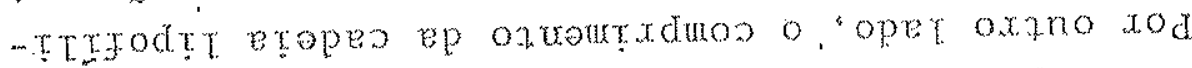

- xeztooe ap zedes a xatod odnas o H

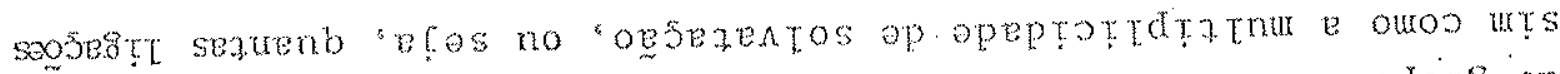

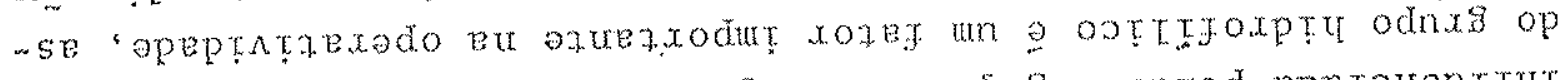

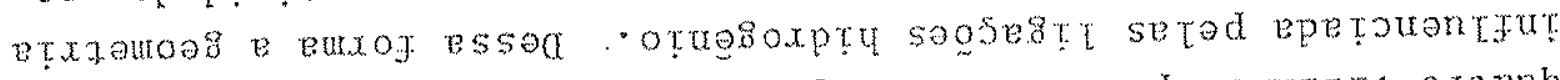

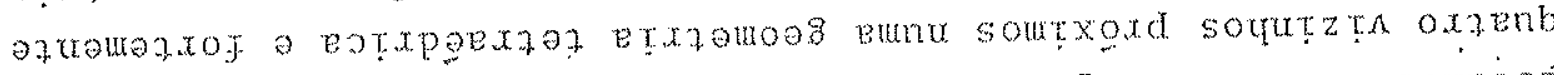

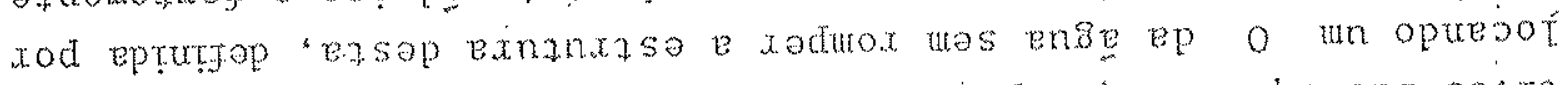

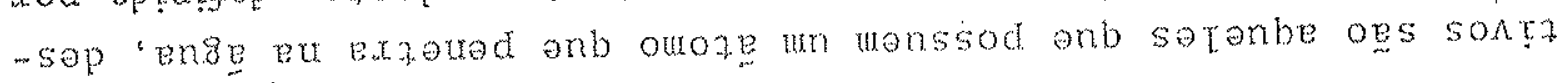

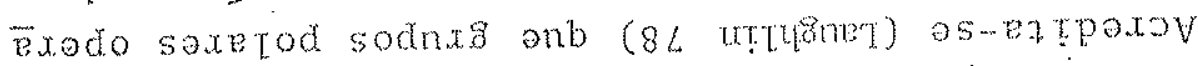

- otrosoxpty soosest ap

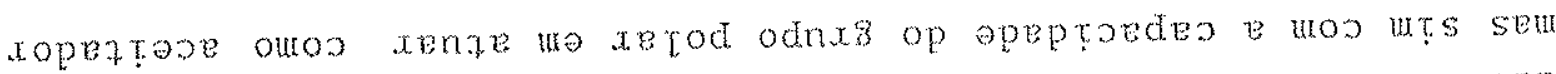

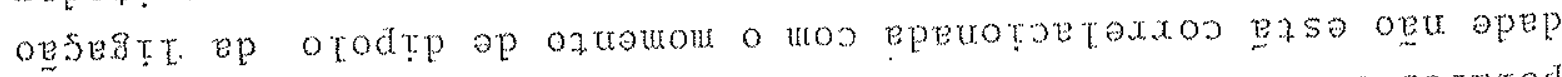

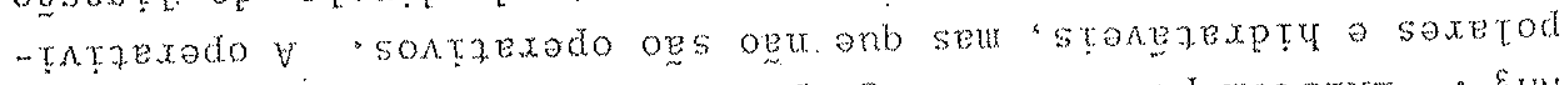

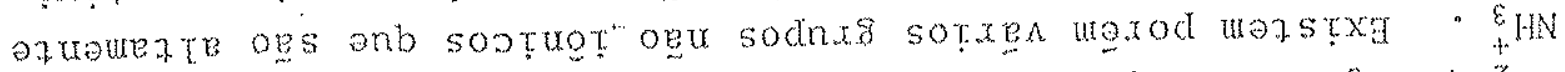

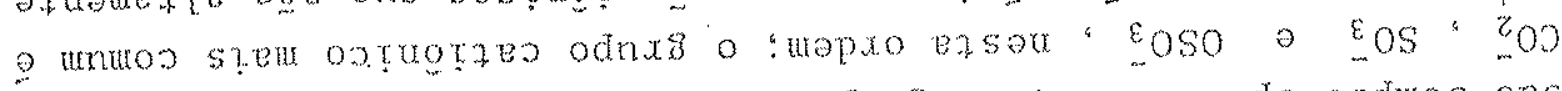
ots sontrexado stem soptrotu sodnx so sontaxedo andus ogs

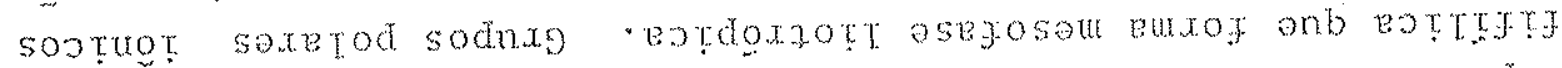

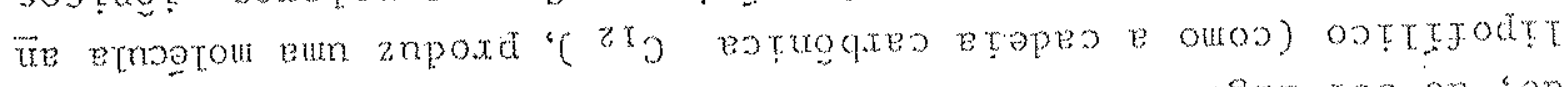

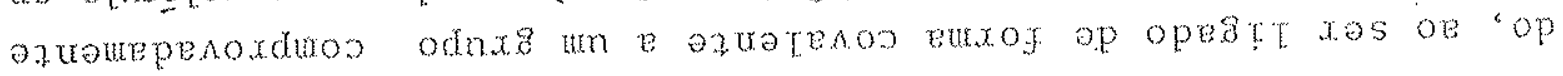

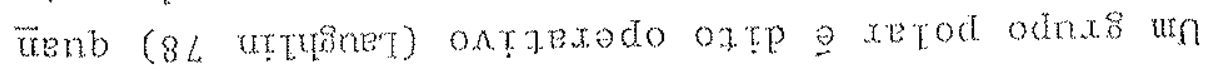

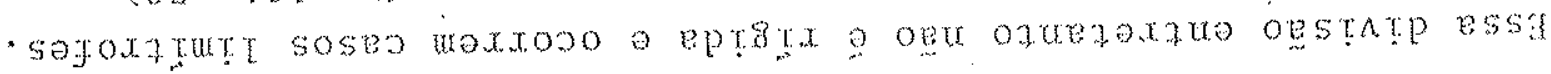
- sopezet

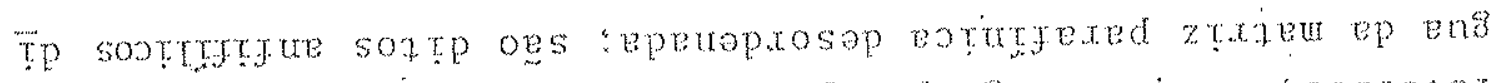

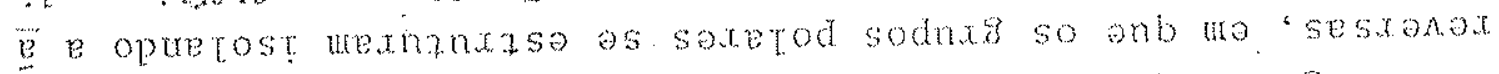

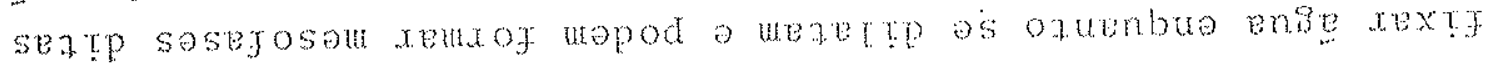

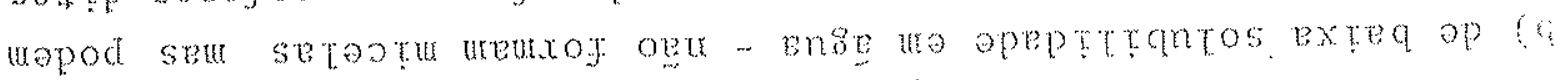




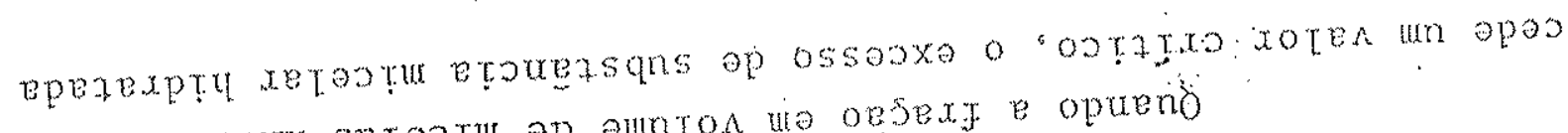

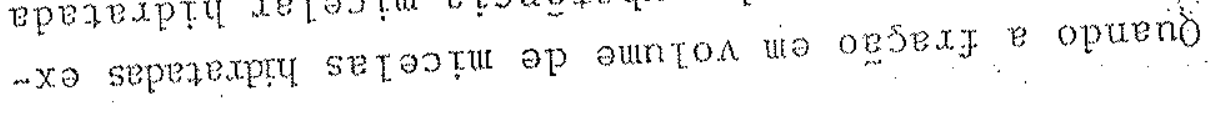

- optpuraso

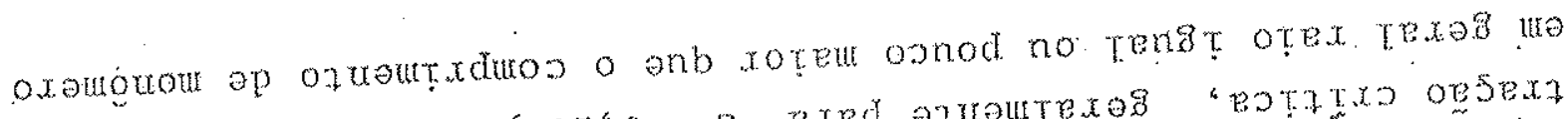

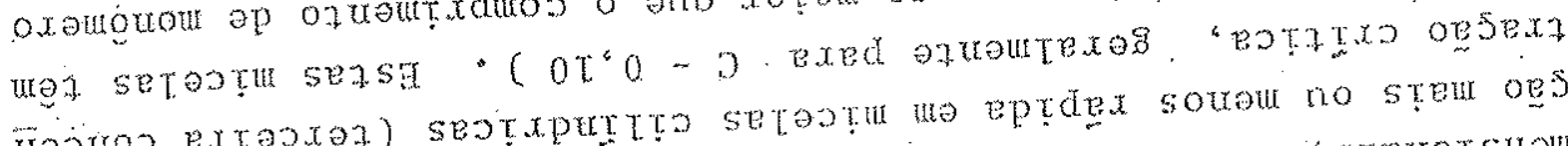

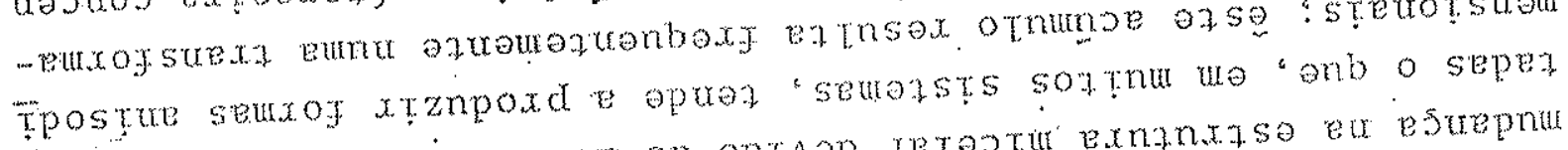

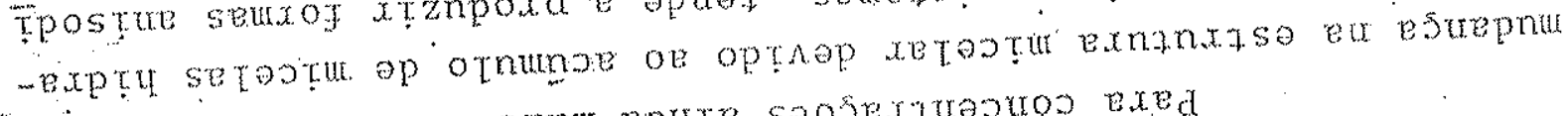

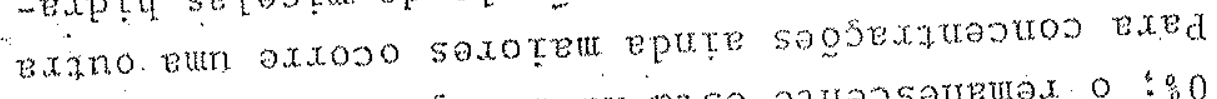

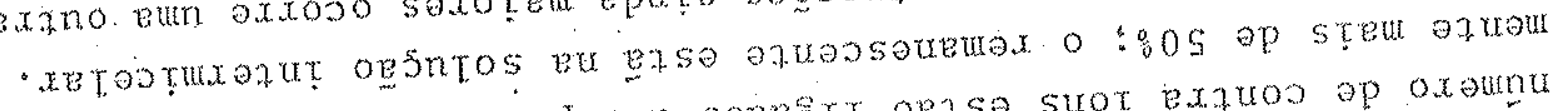

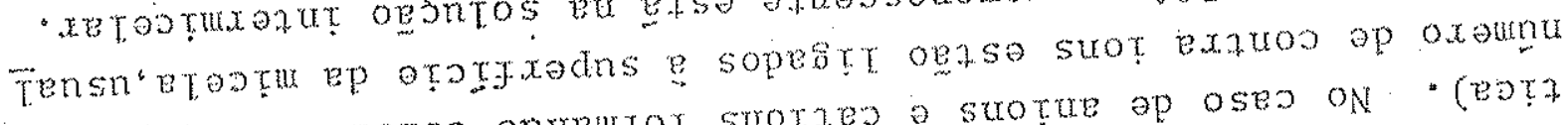

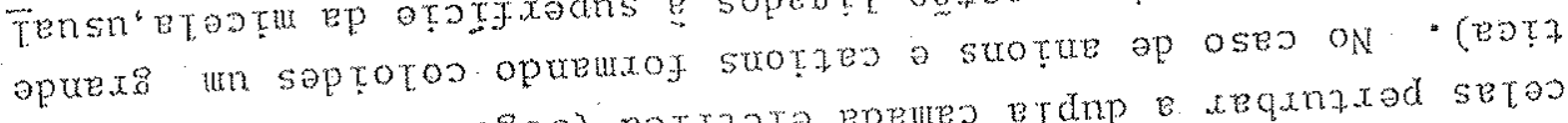

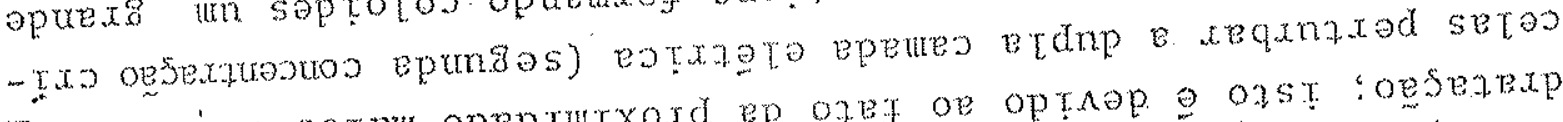

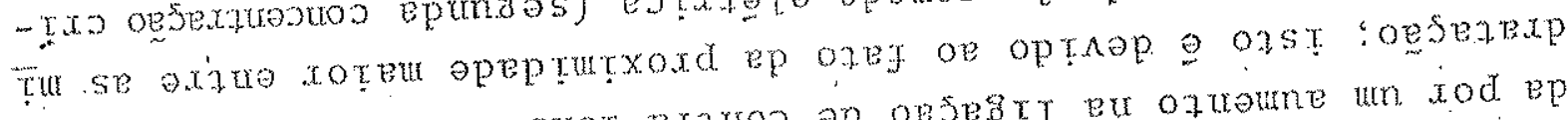
- Ty op anse ens woo suot exaroo op opsest ex ofueume un jod ep

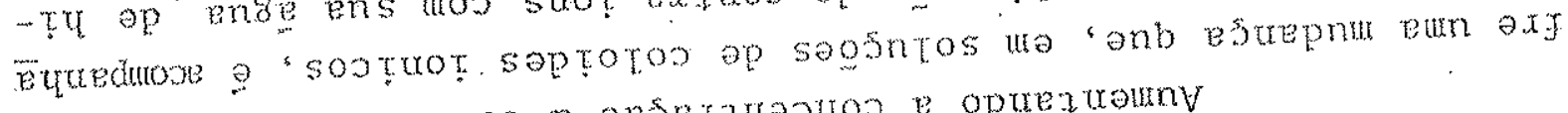

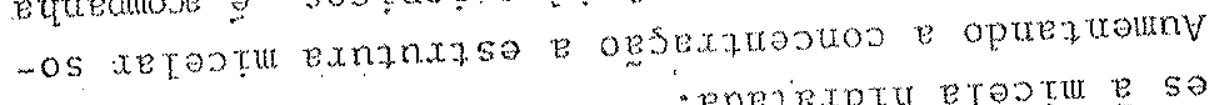

- eperaxptr ergotu e sazrasraxad owos sojsta xos wonep onb "etogtu e sopest enge op setnogtou o strot

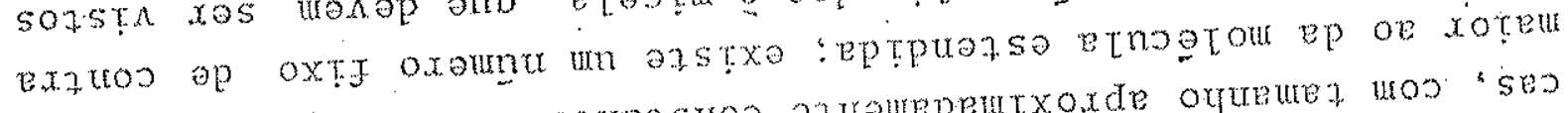

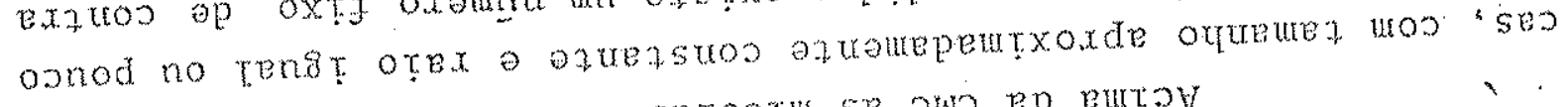

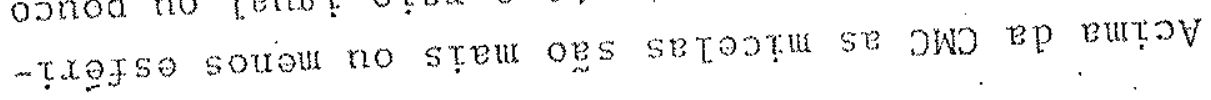

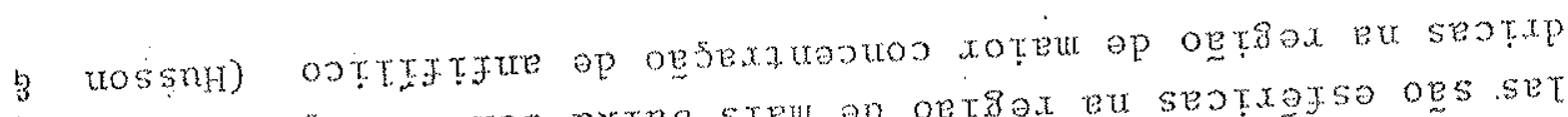

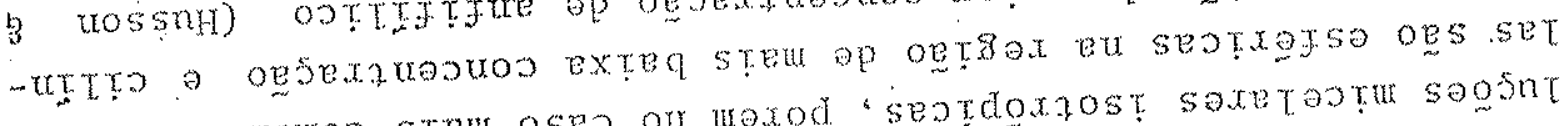
-astu se murod steu oseo or moxod "seotdoxast saxetooru soosnt

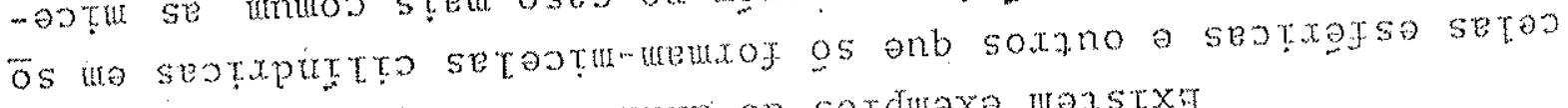

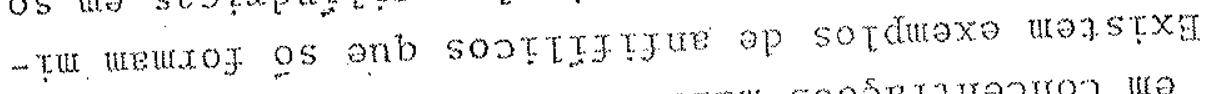

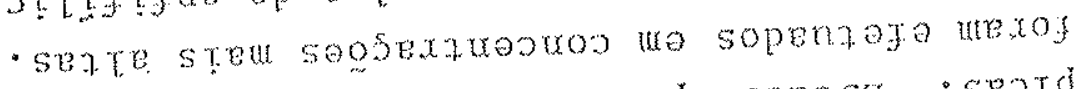

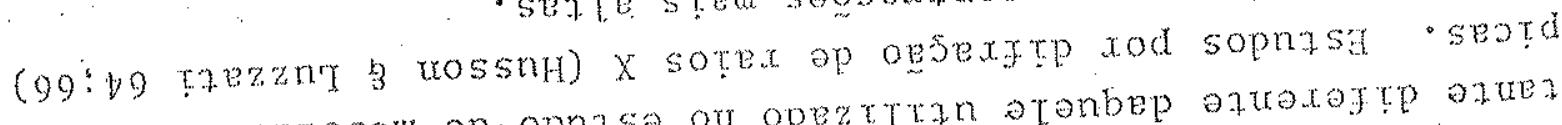

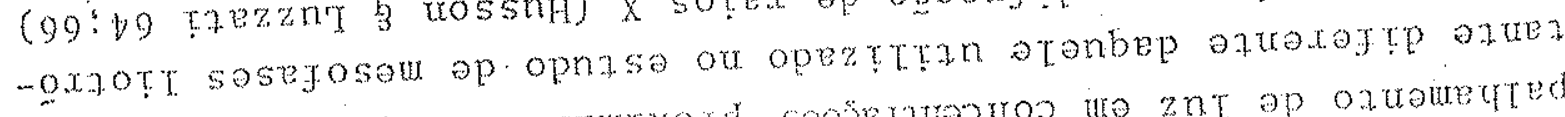

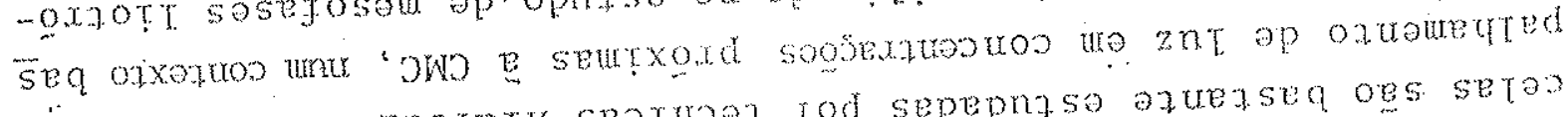

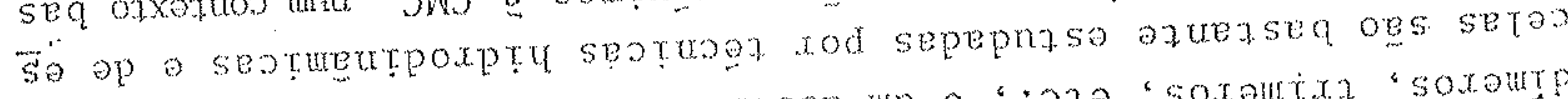

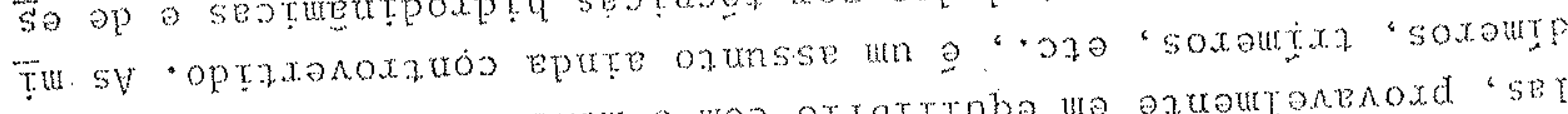

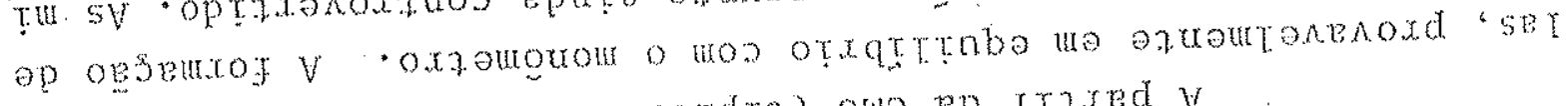

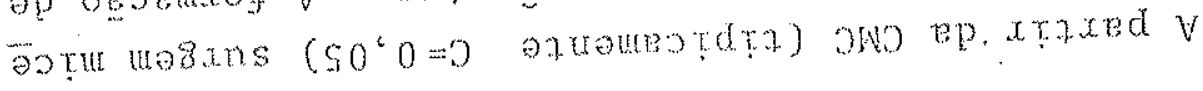




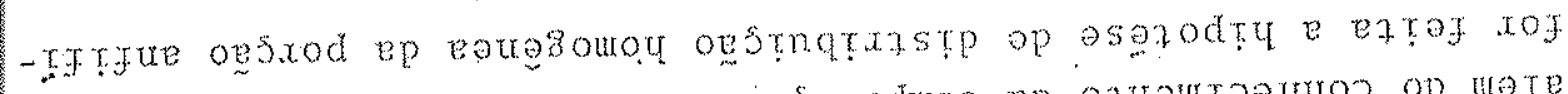

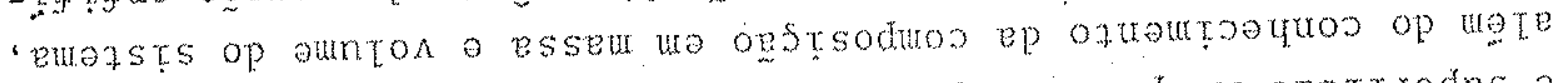

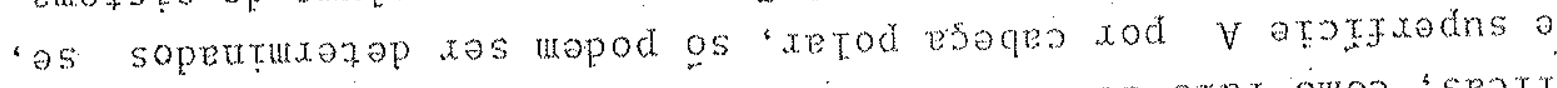

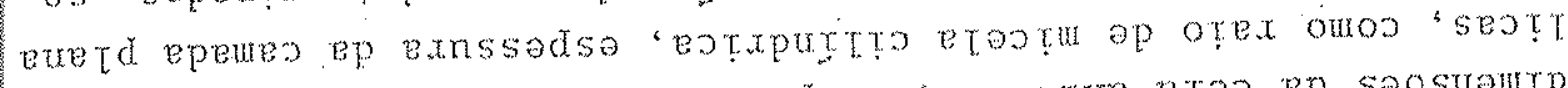

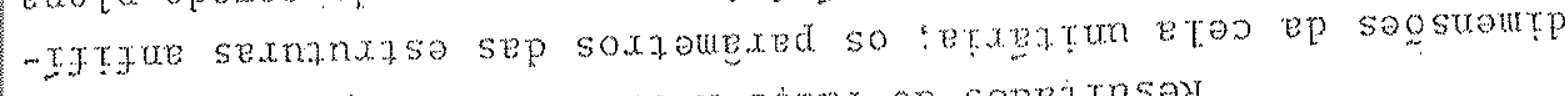

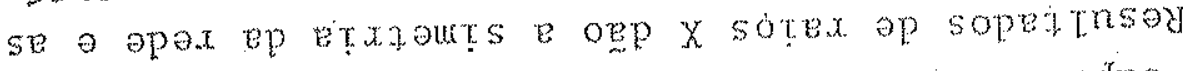

- ir ornateo ou sequexp sox

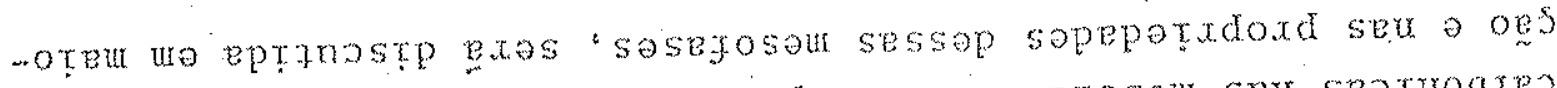

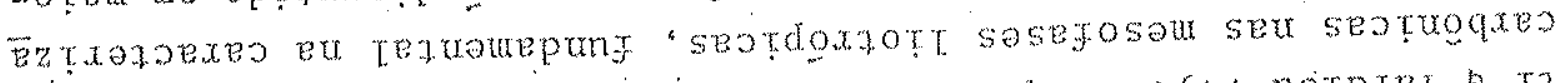

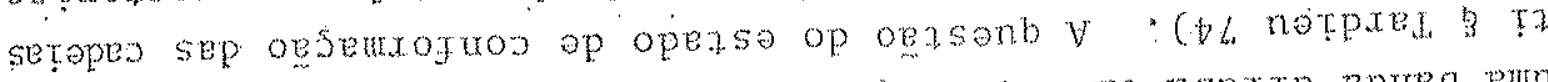

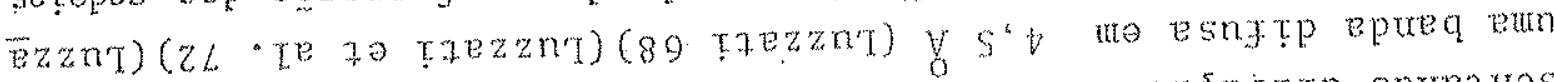

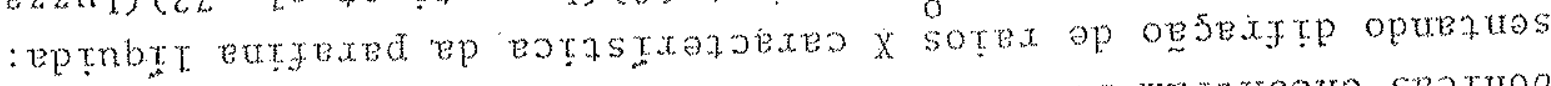

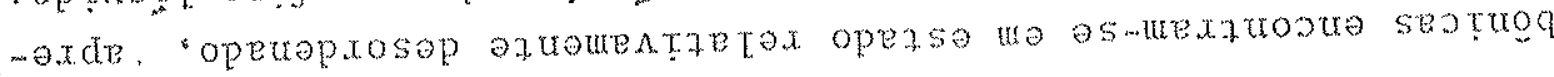
- xeo setapeo se seotidorzot sosejosen se sepol ug

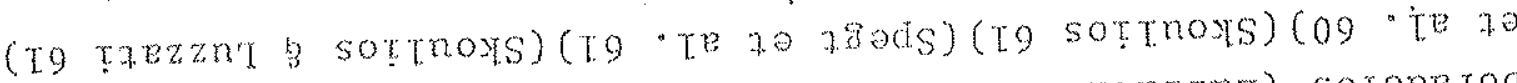

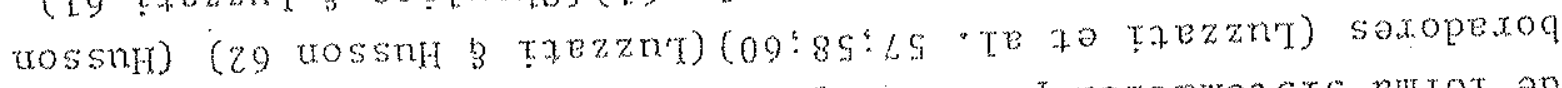

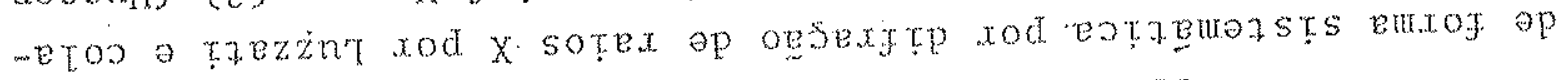
sopepraso xas e wexesauro ense/oeges semexsts so

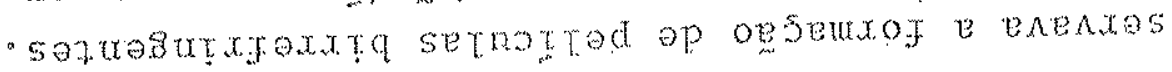
qo os opto "sooges op seotadex sep uan seotdoxat sosejoson axq

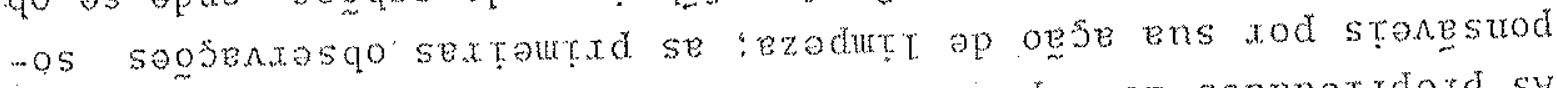
-sax ors sotsoduo sassap apeptrantos pxdmp ap sapepardoxd sy

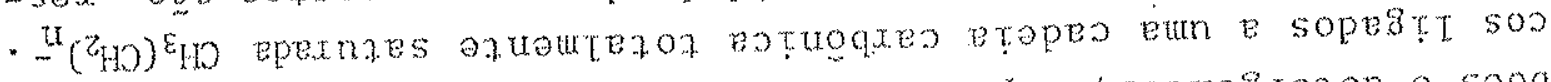

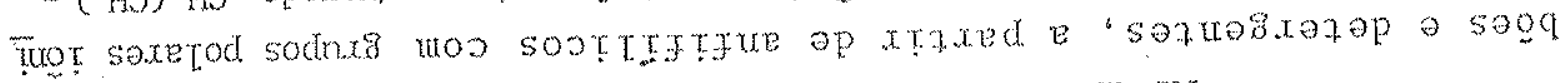
Es uon septaqo se oes sepepnase steu sosejosent sV

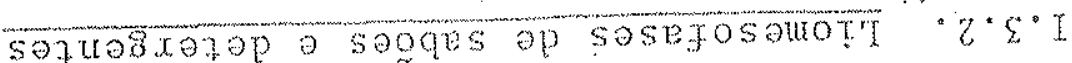

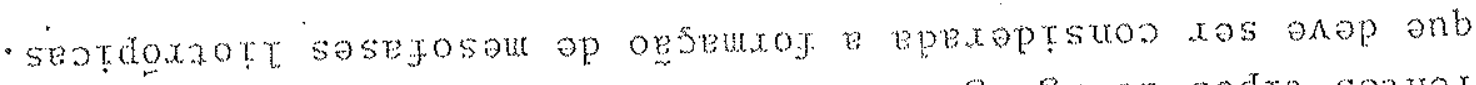

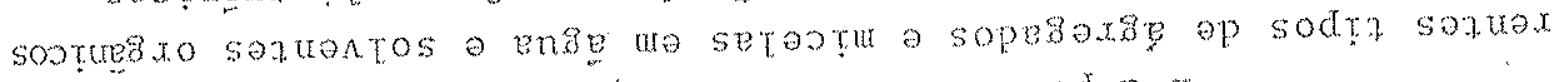

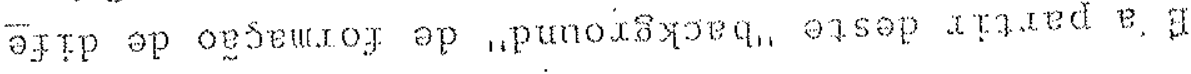

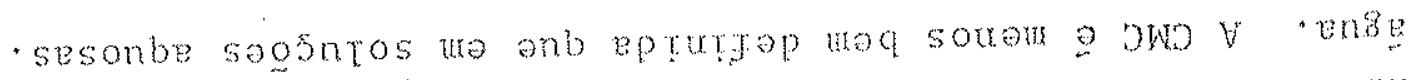

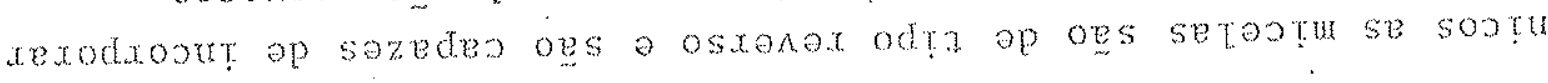

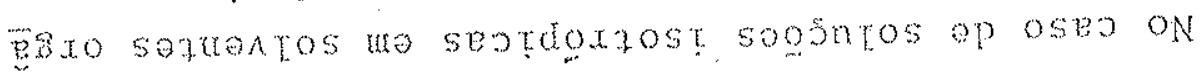

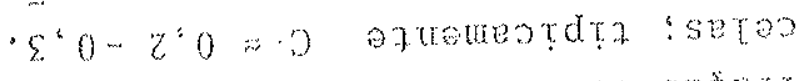

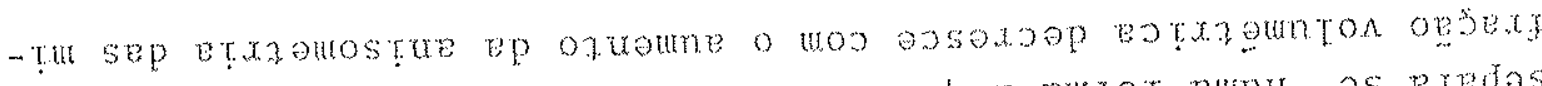

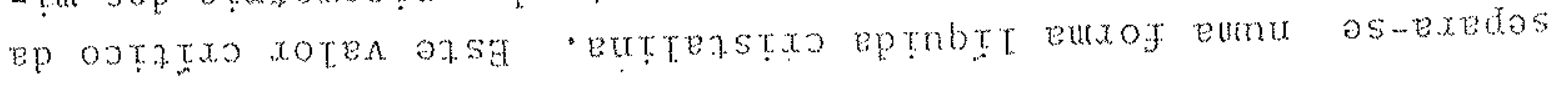




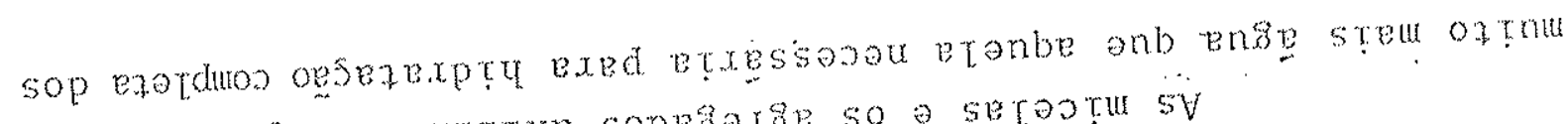

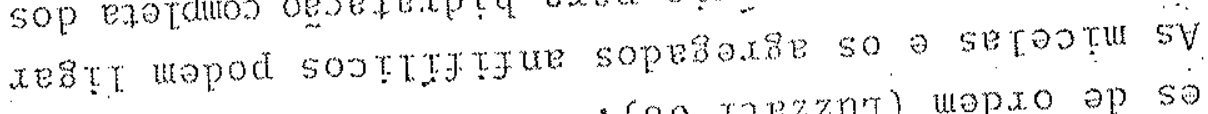

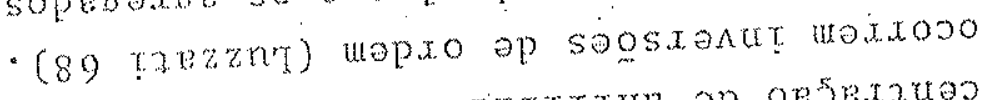

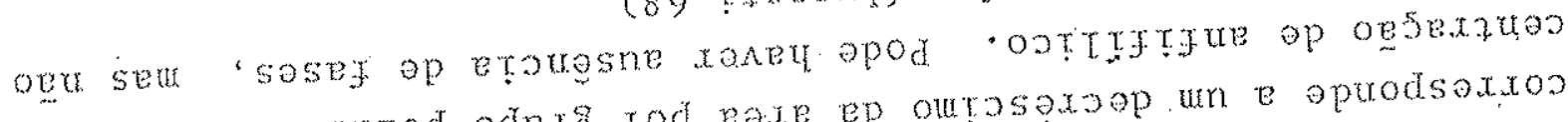

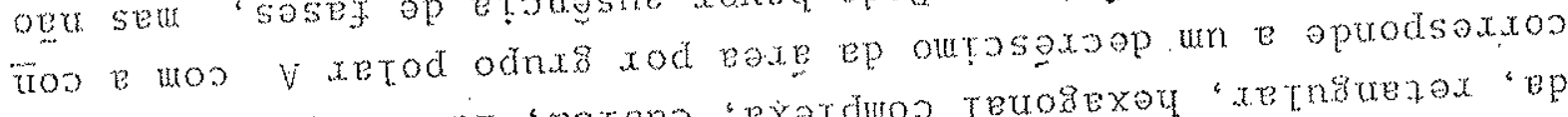

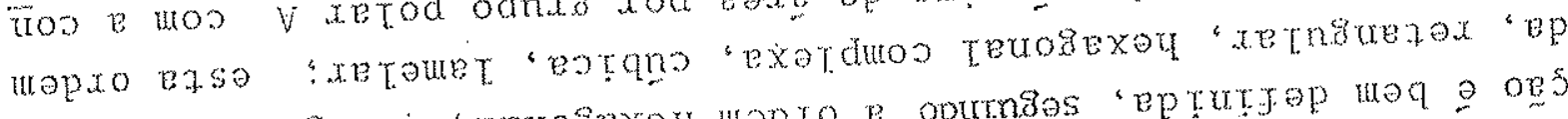

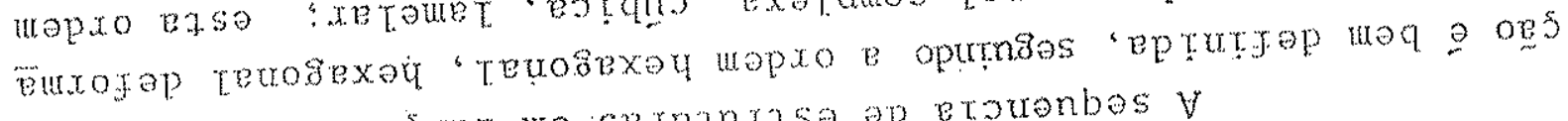

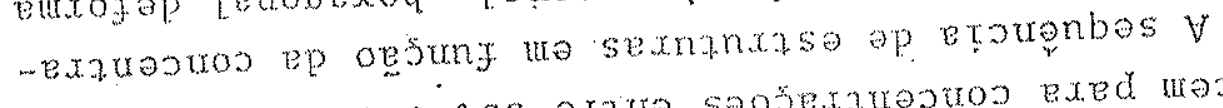

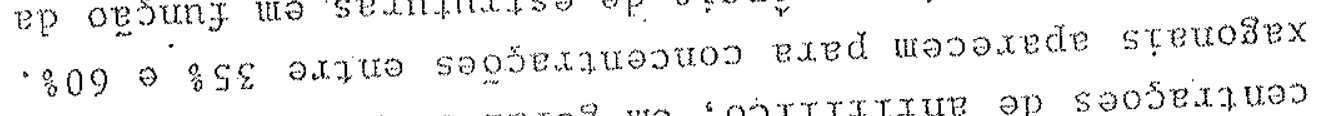

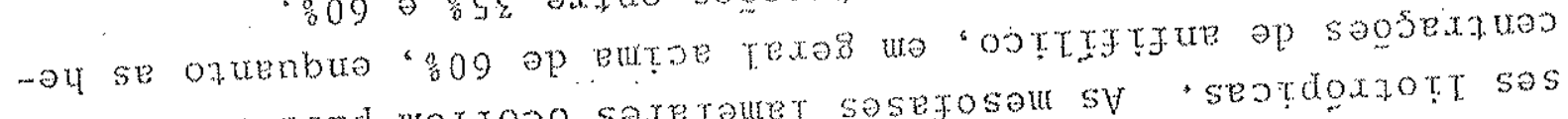

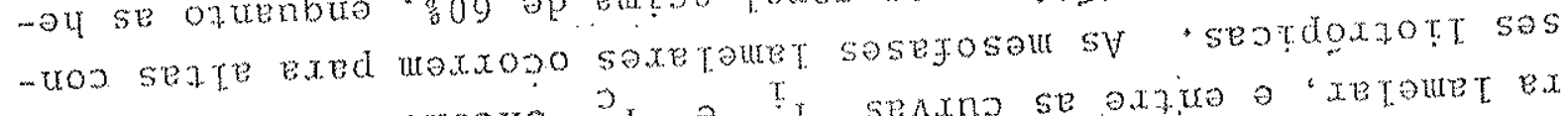

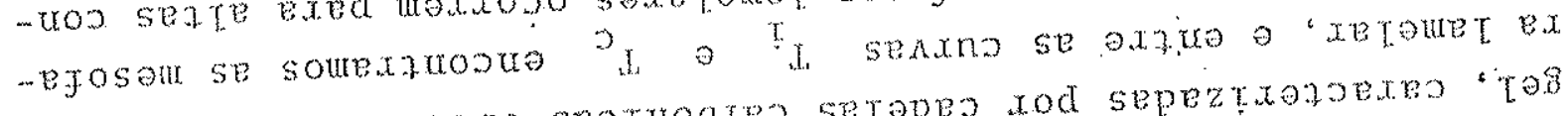

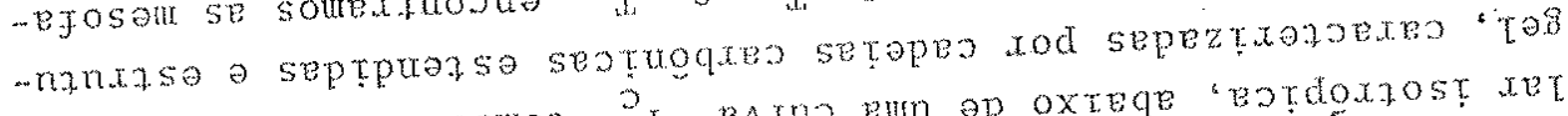

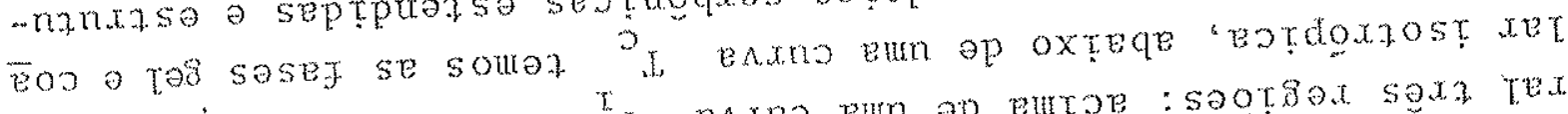

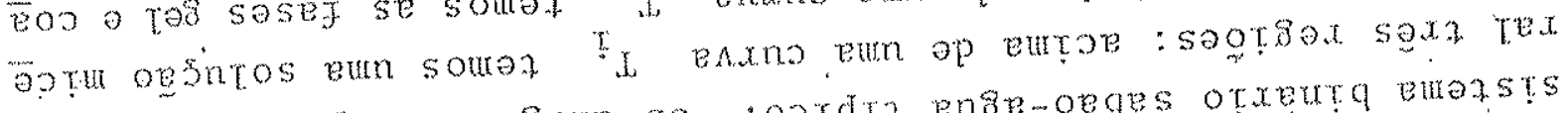

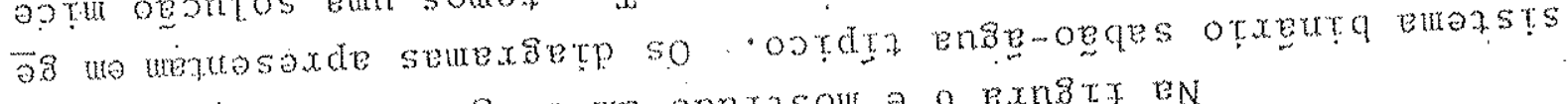

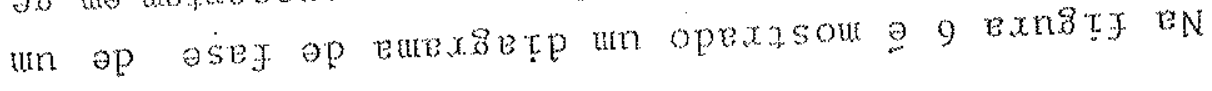

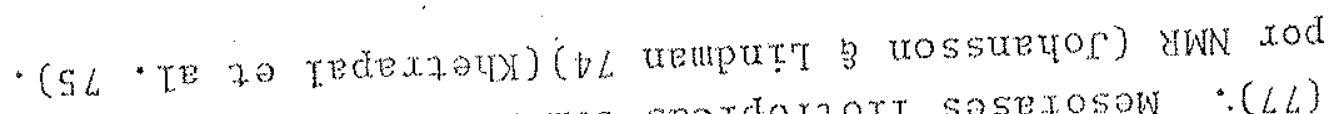

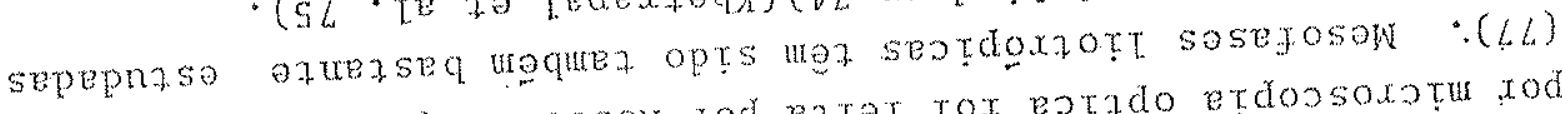

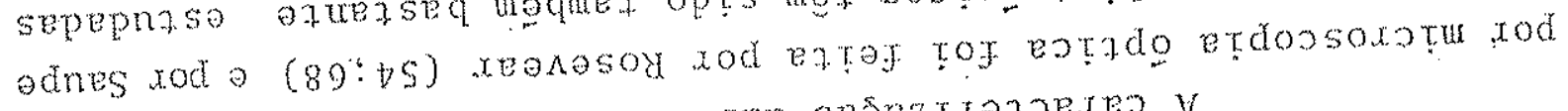

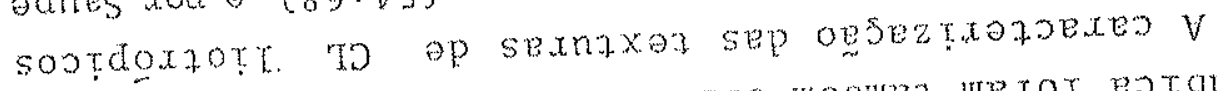

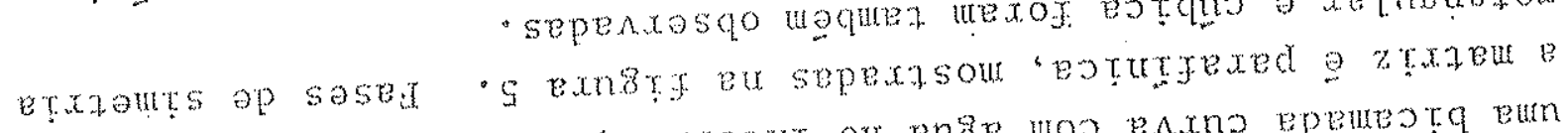

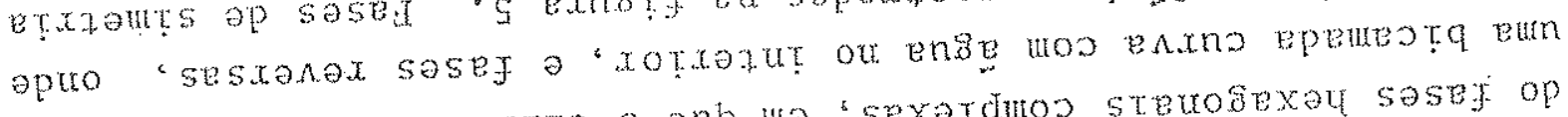

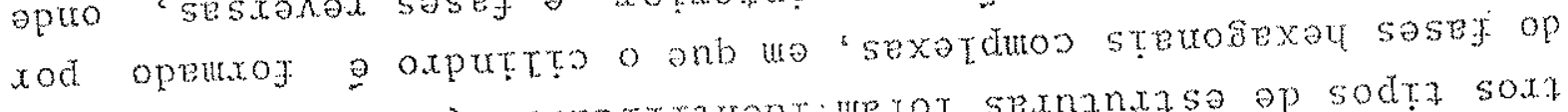

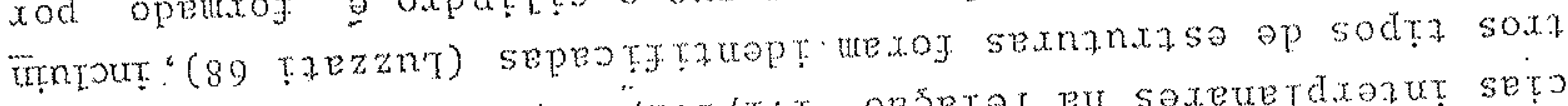

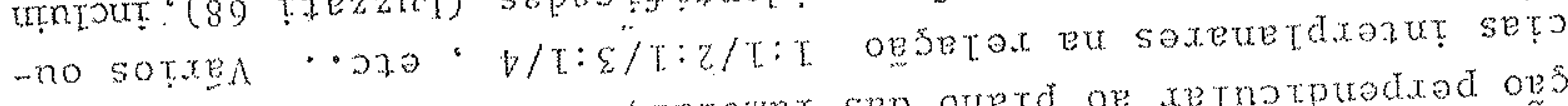

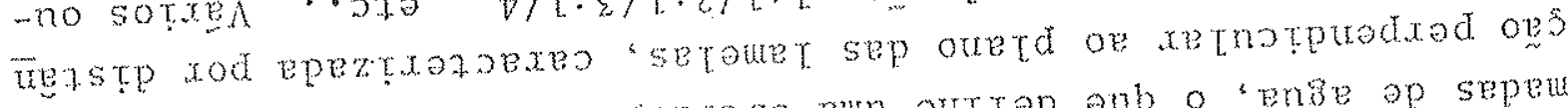

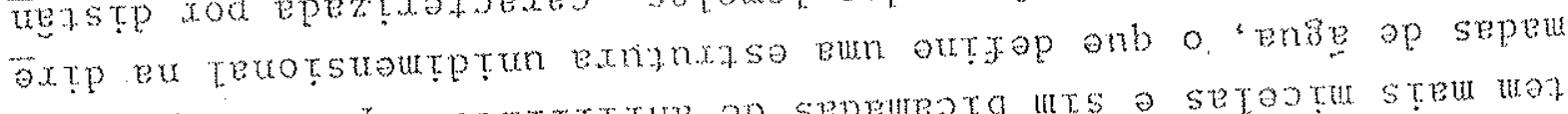

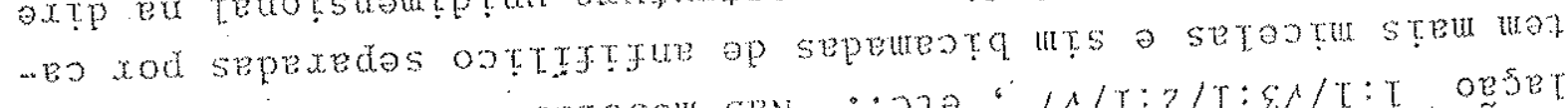

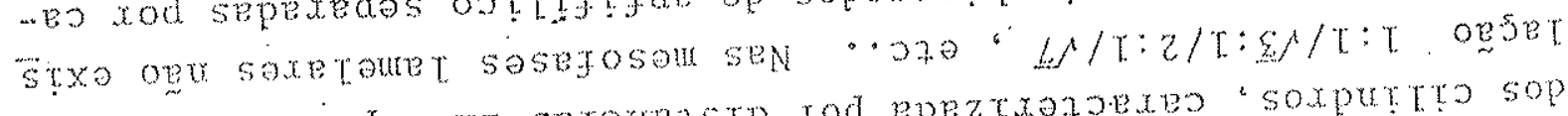

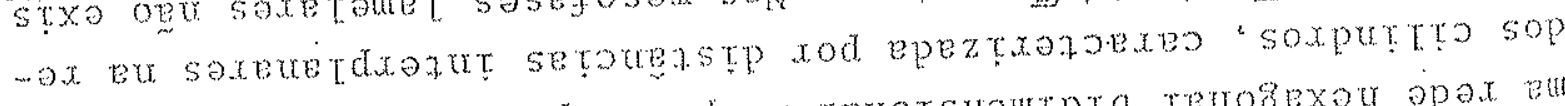

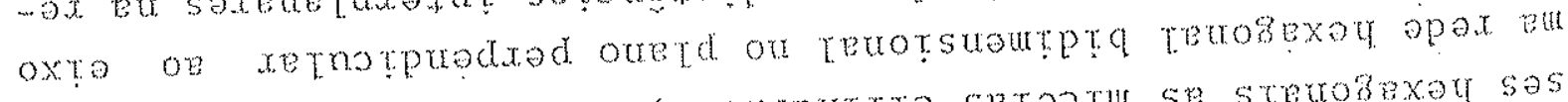

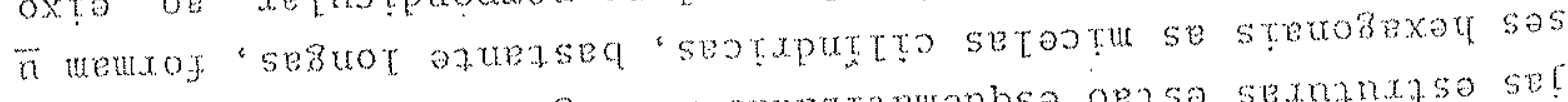

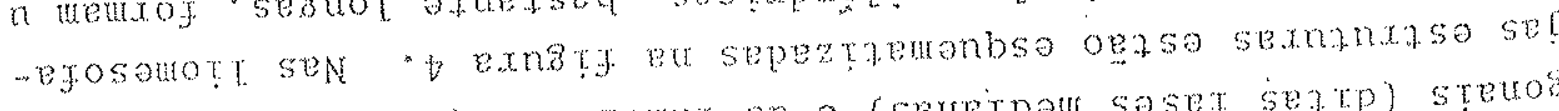

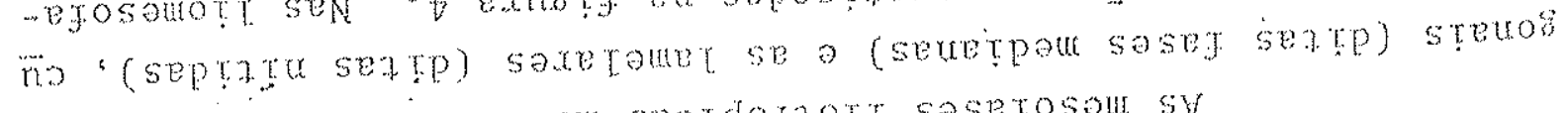

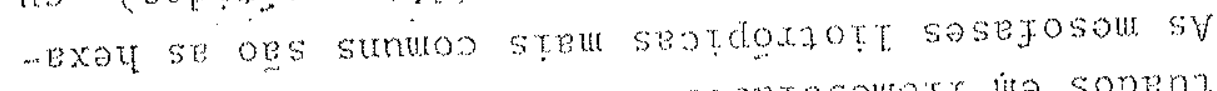

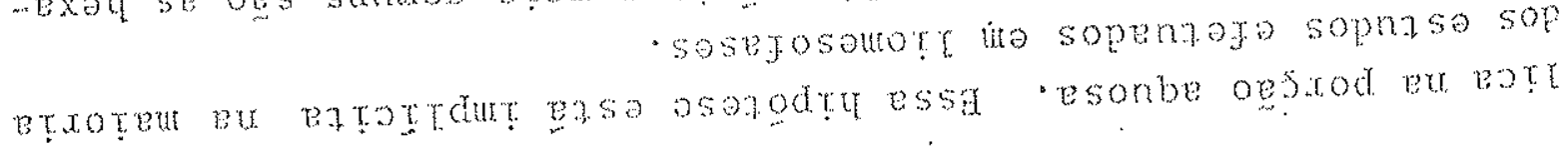




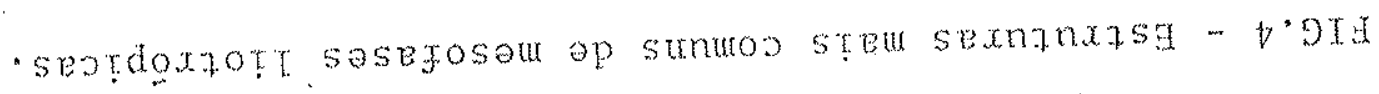

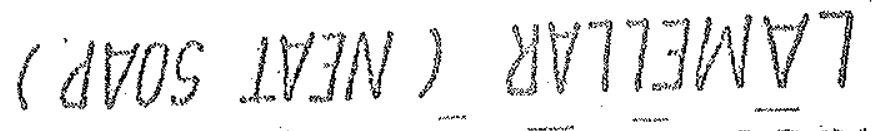

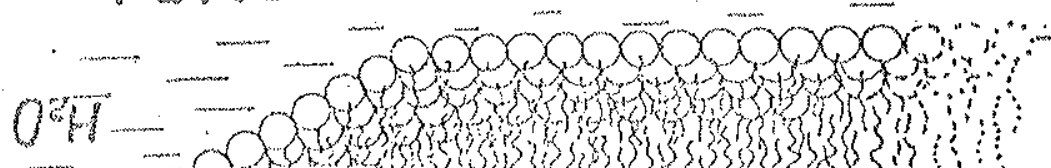

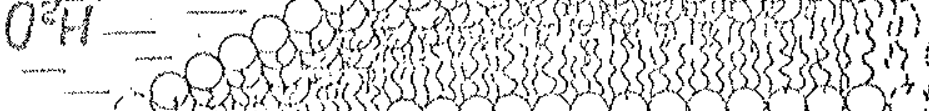

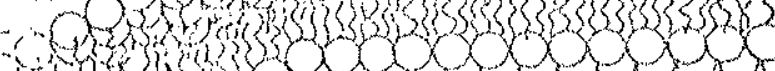

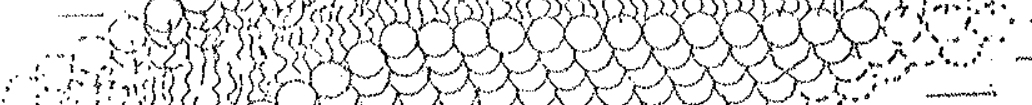

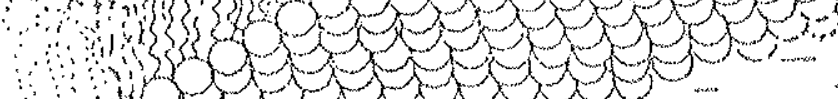

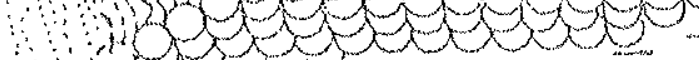

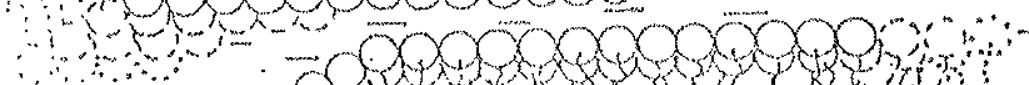

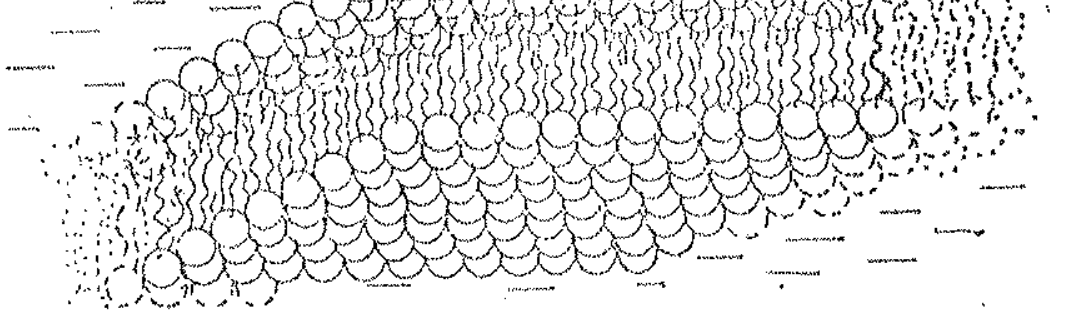

- $\cdots$

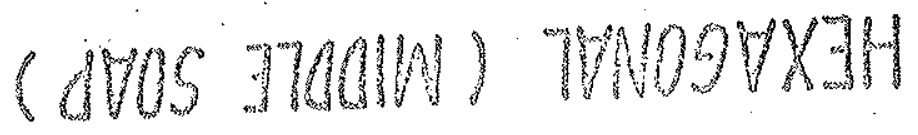
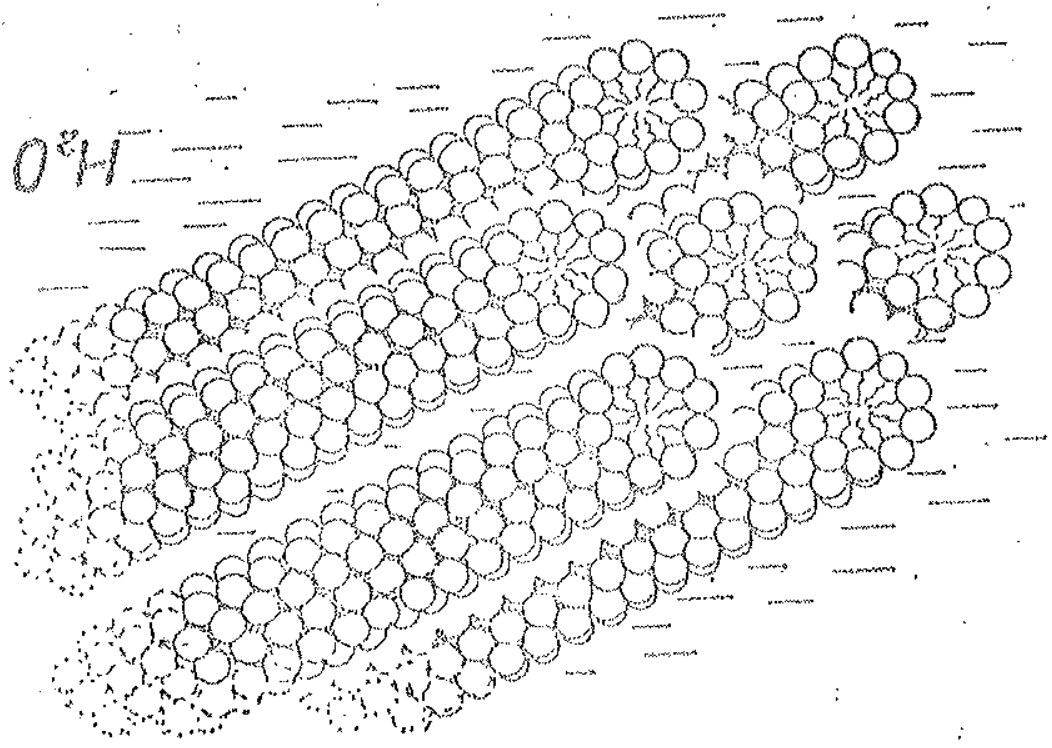


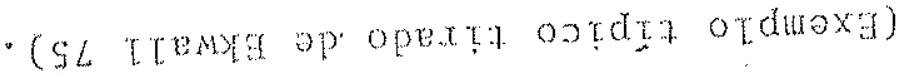

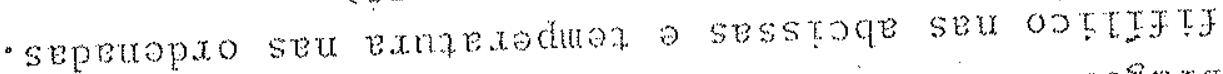

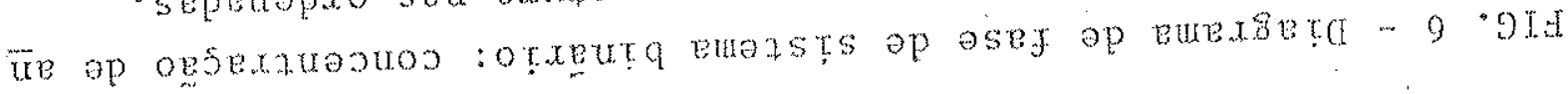

I00200 o to8 sases - 5

xetaut aseif - I

terosexol eses - H

estioxast og 5 ntos -1

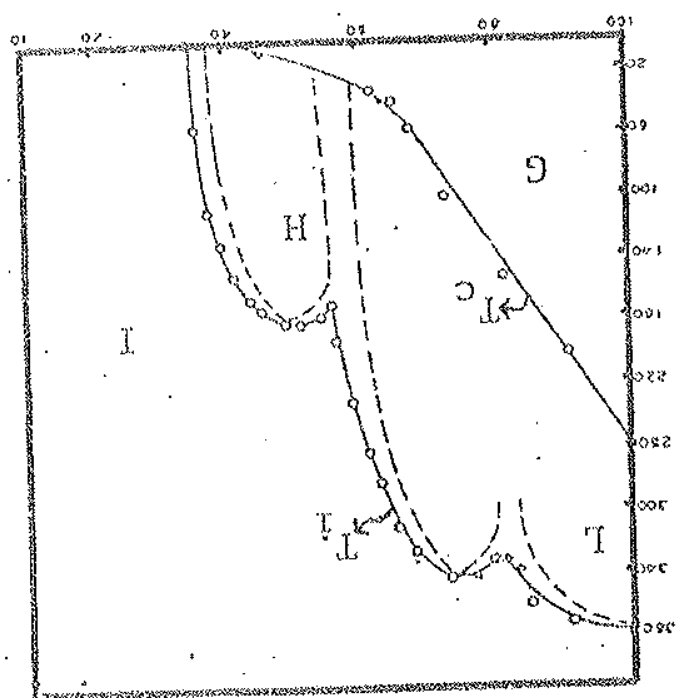

- sestdoxa

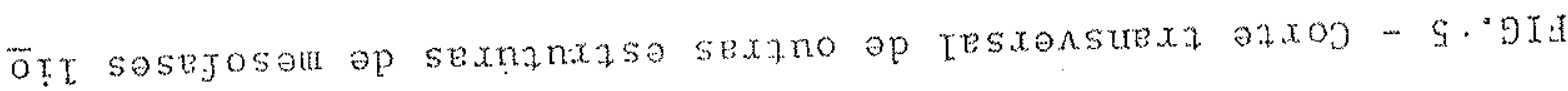

gxoratuos recrosexat (a) esxonor terosexou

(B)

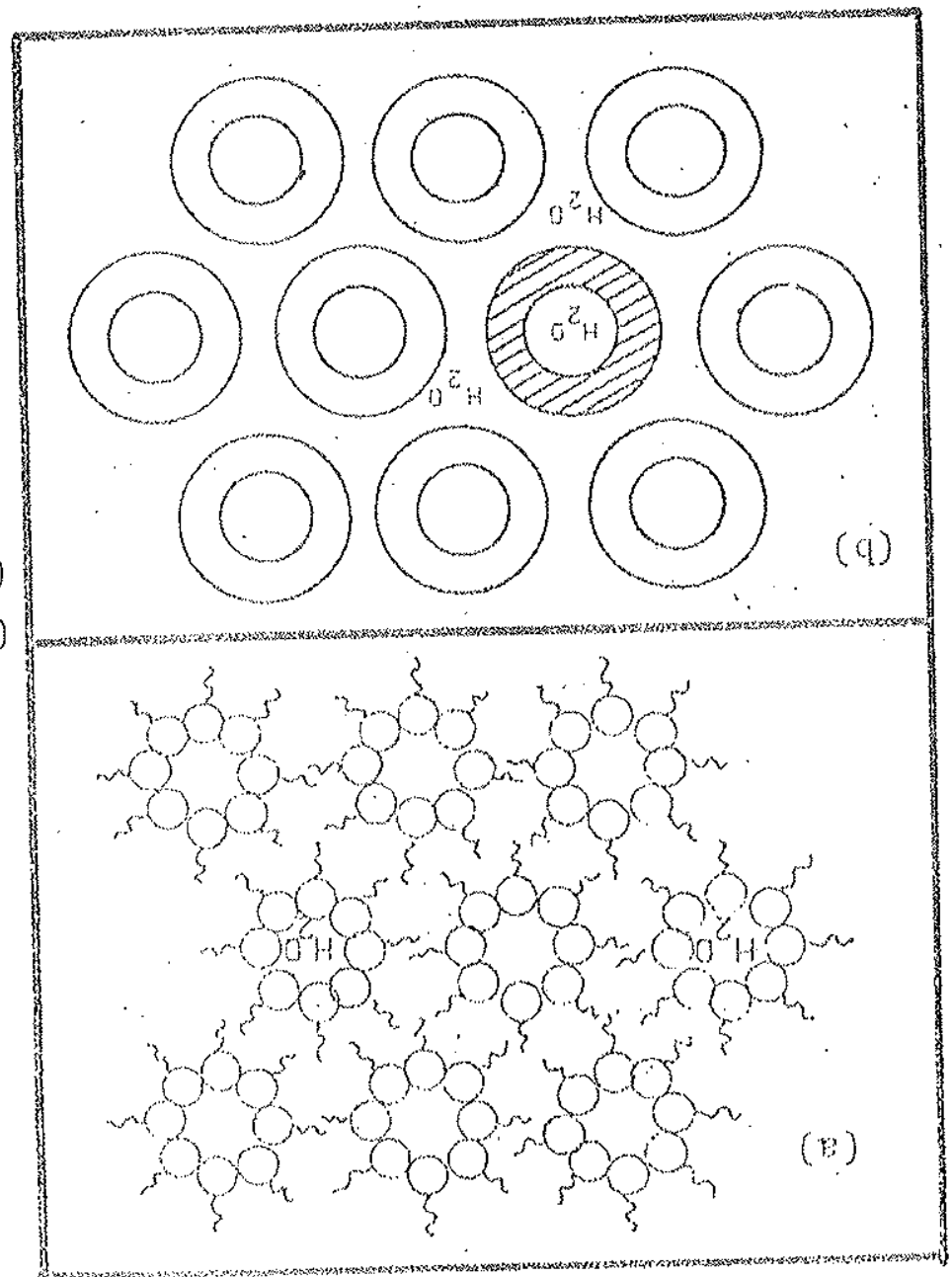




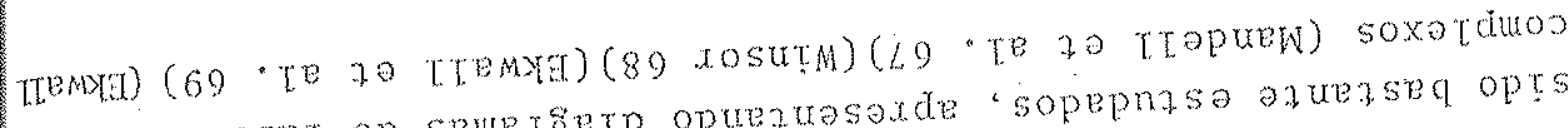

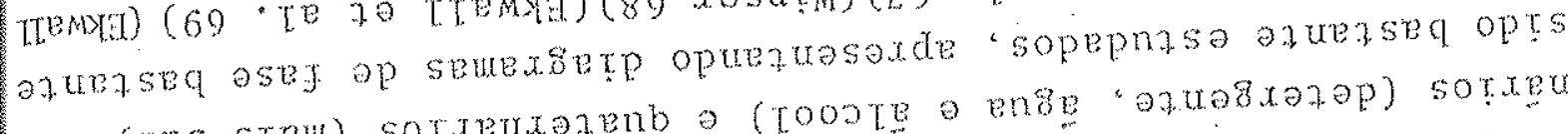

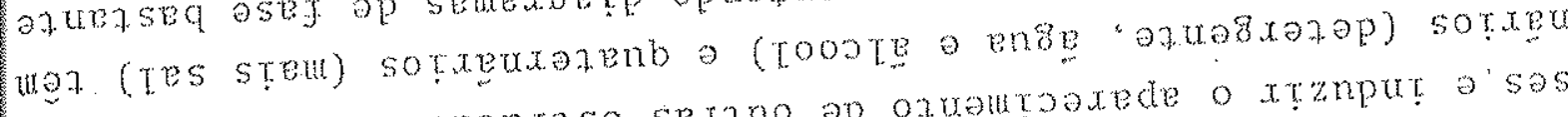

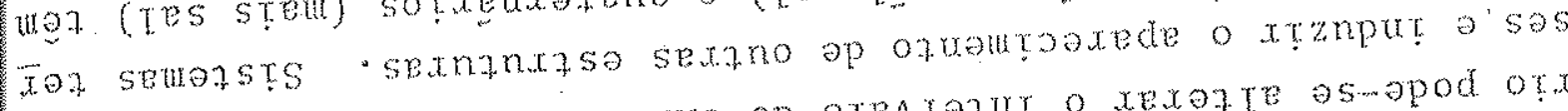

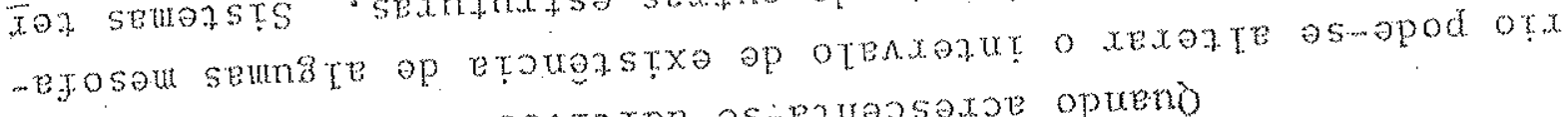

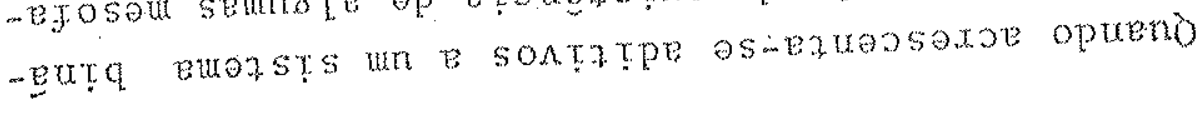

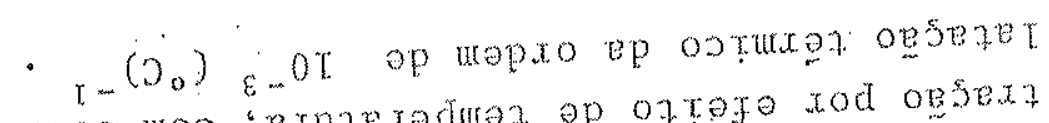

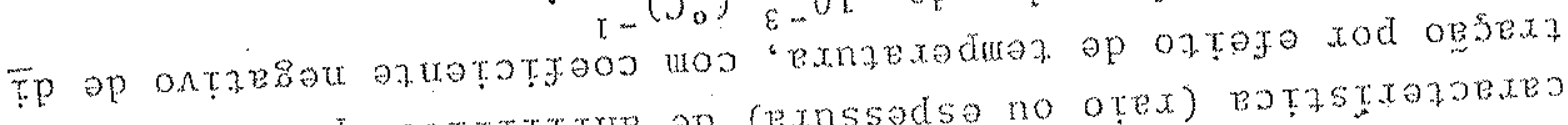

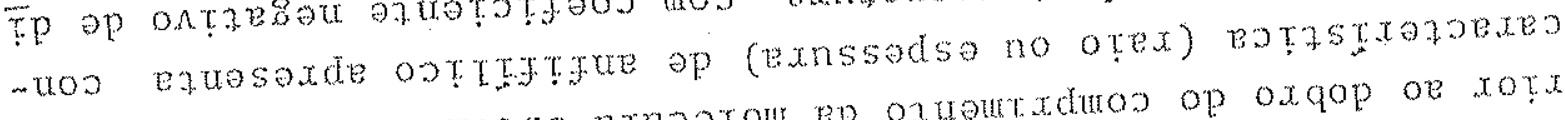

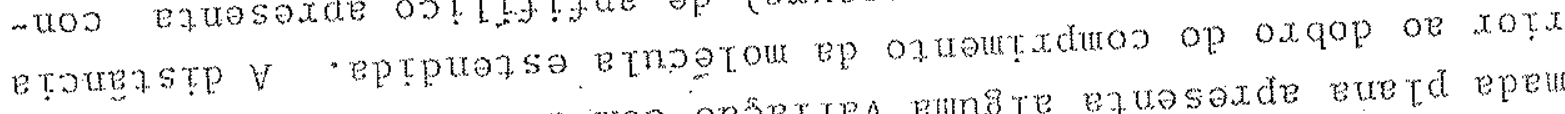

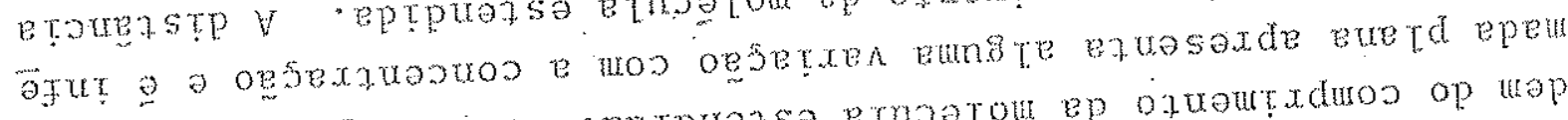

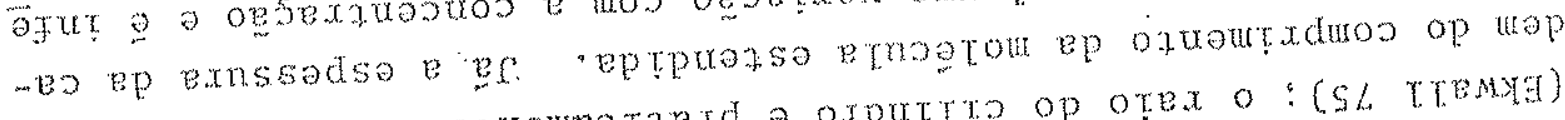

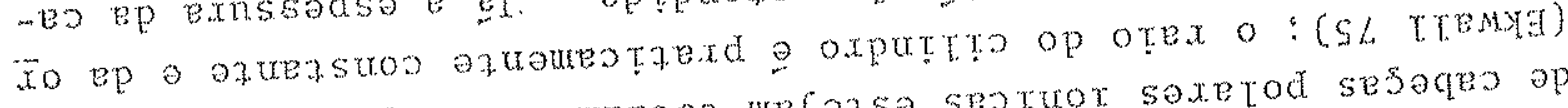

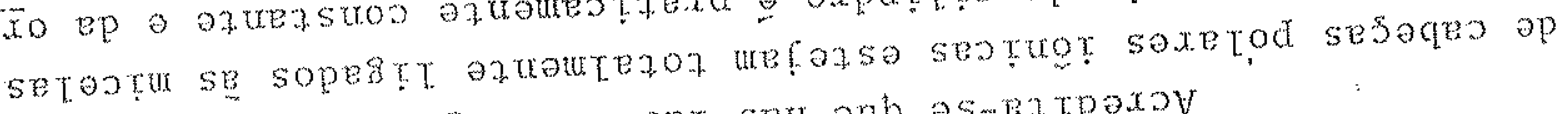

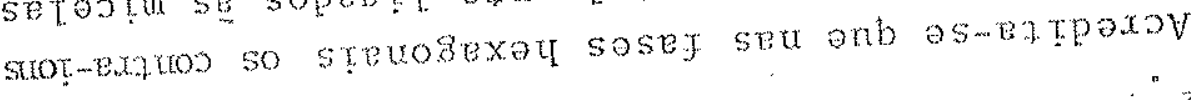

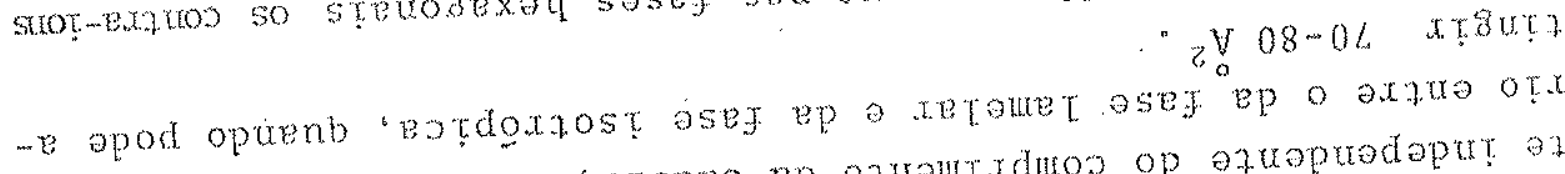

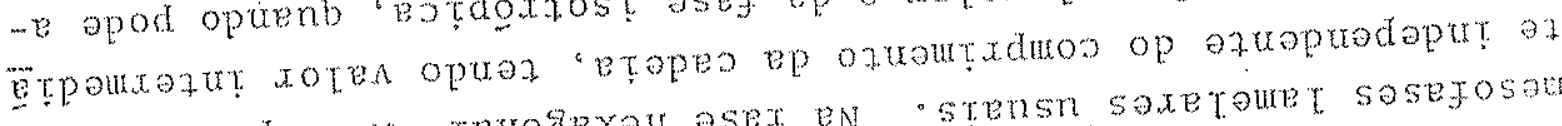

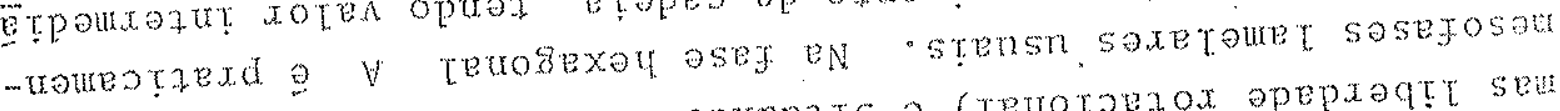

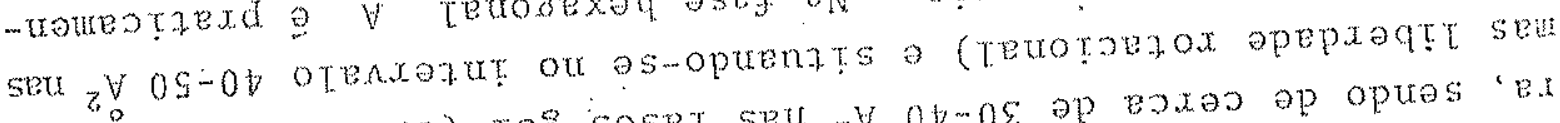

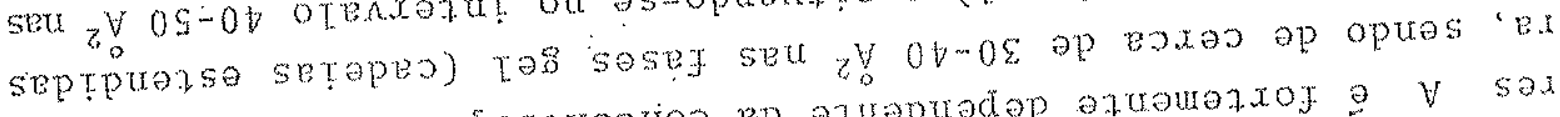

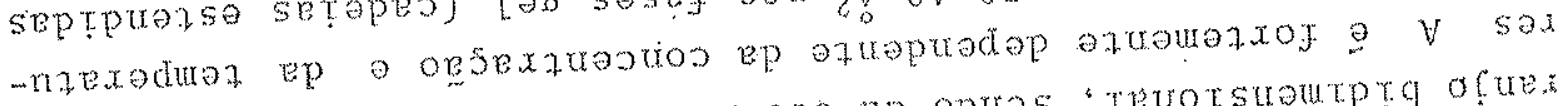

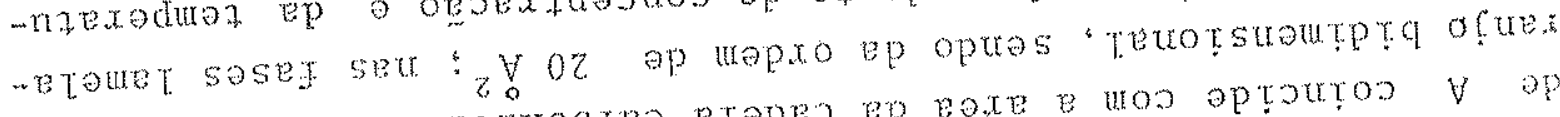

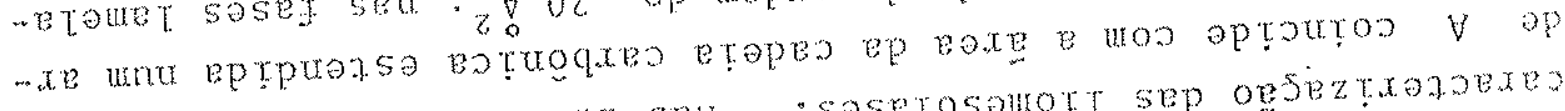

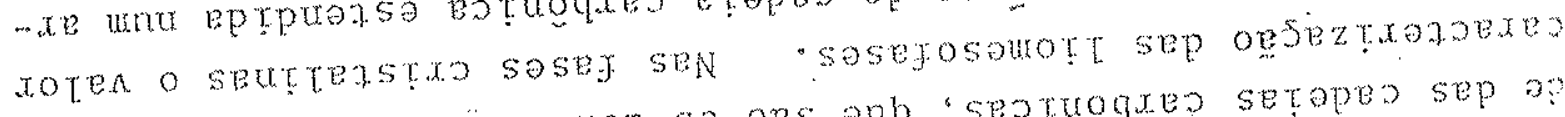

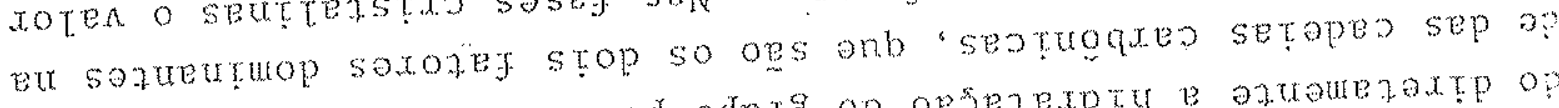

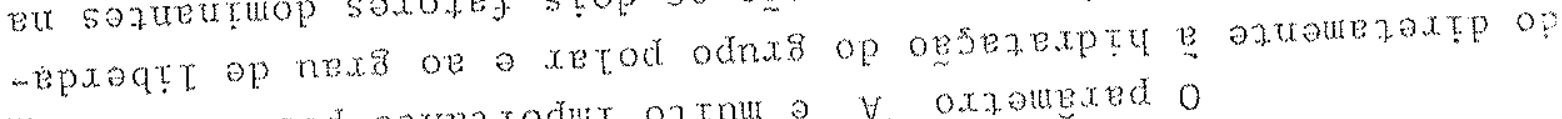

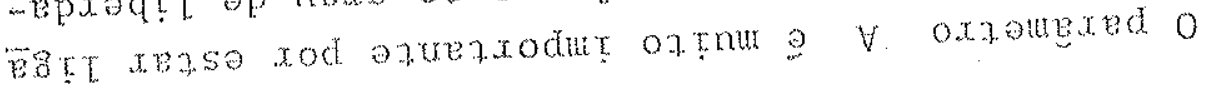

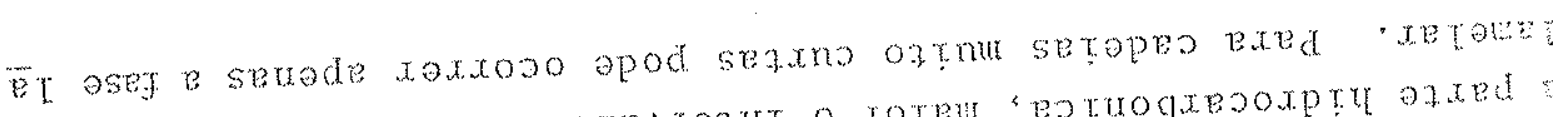

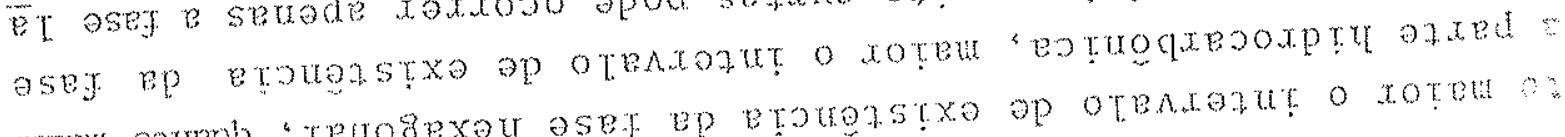

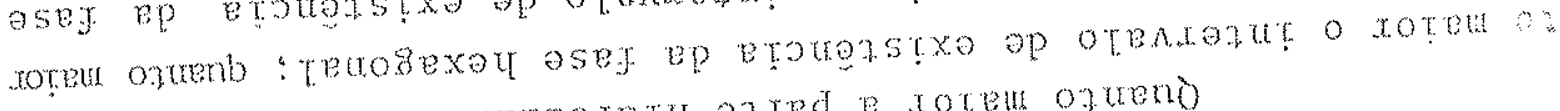

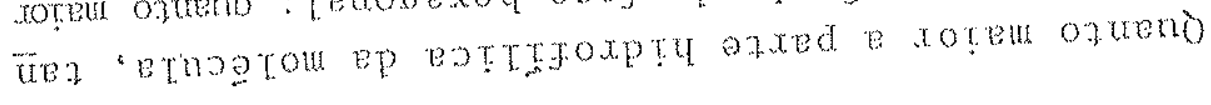

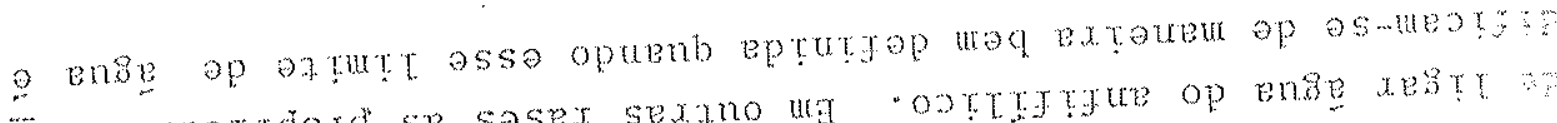

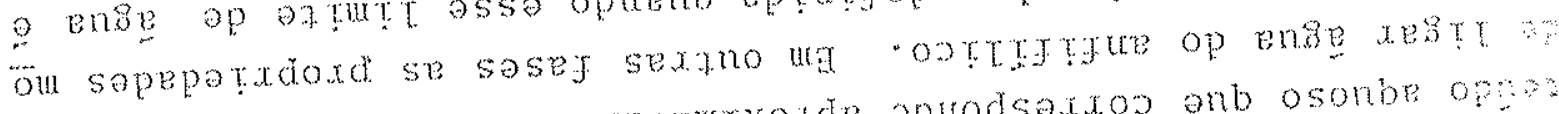

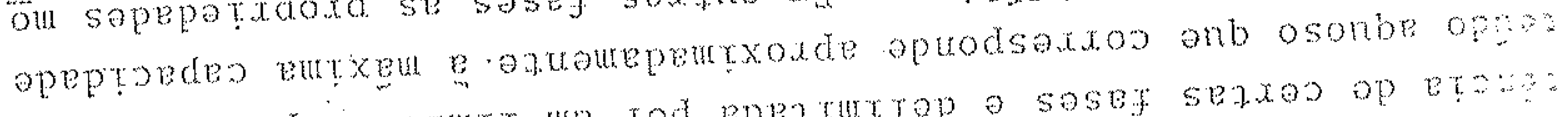

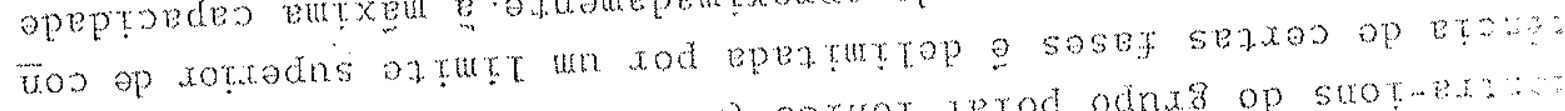

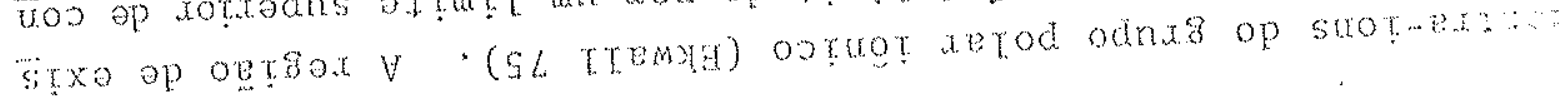


- (Ts rexeury 5

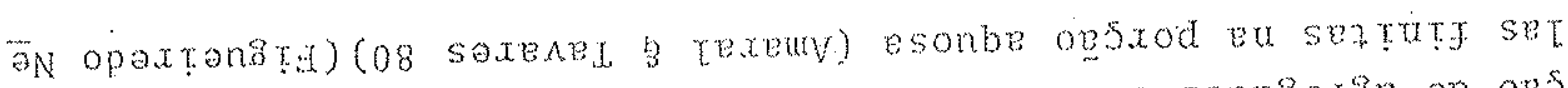

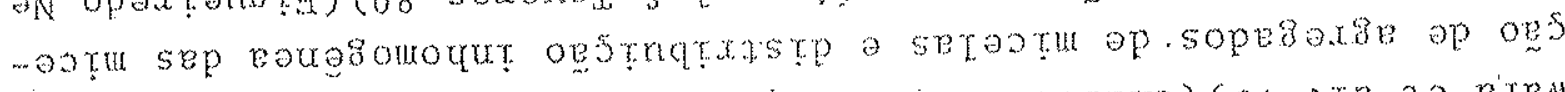

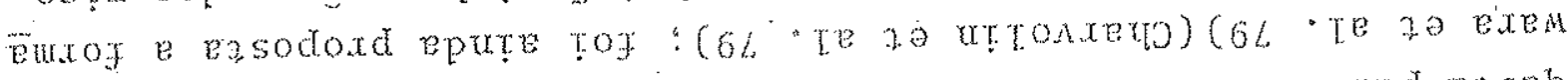

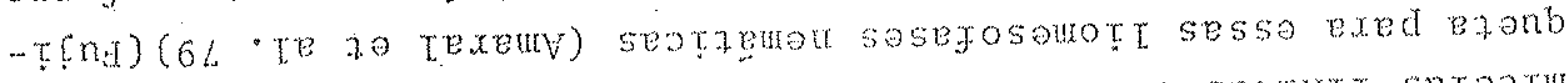

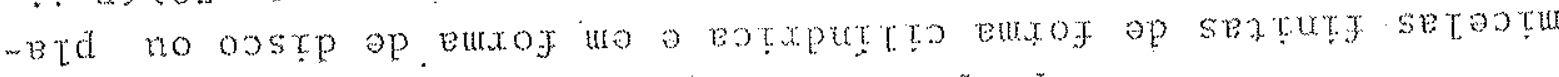
ap euxos ax semanxase ap sotopou soasodoxt wexor

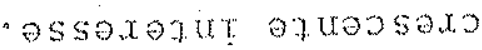

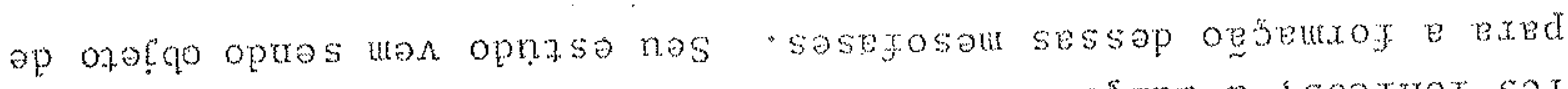

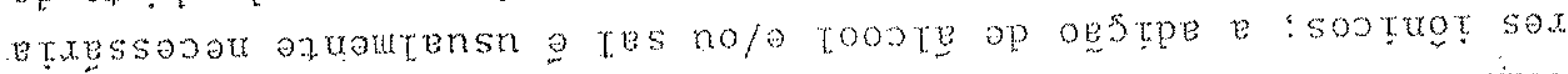

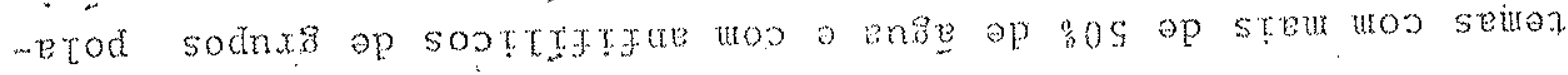

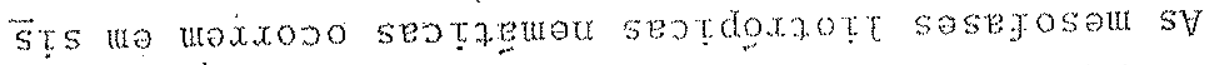

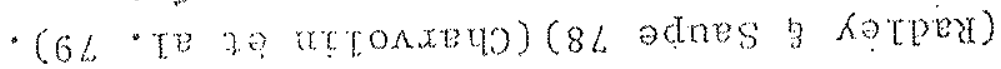

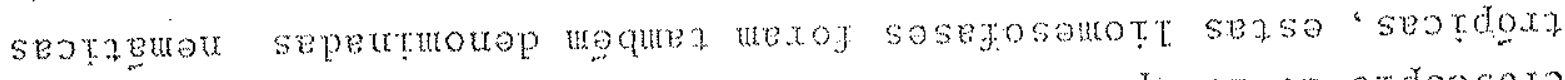

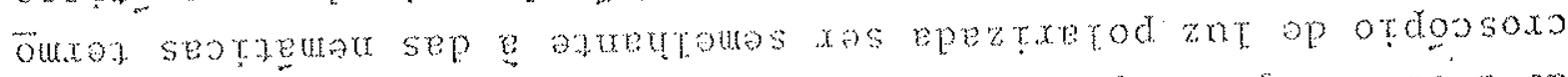

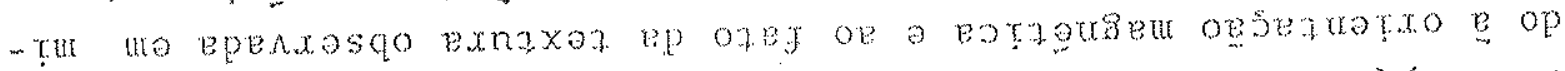

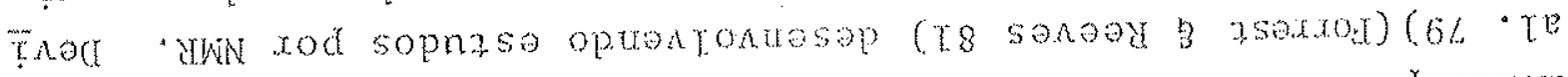

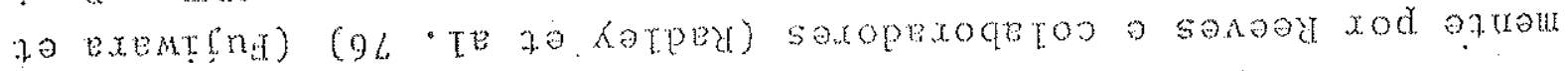

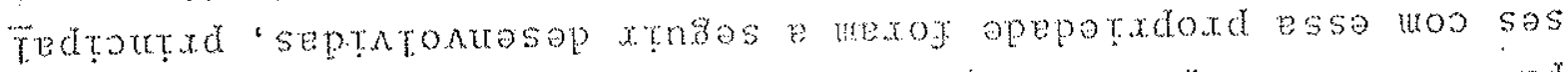

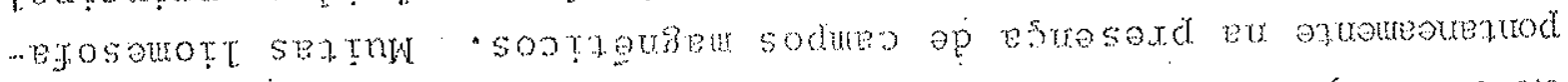

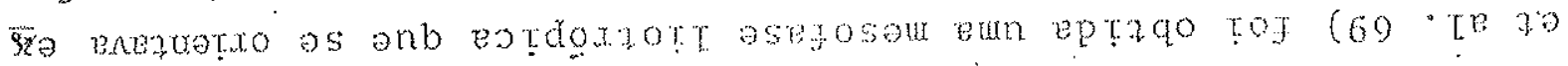

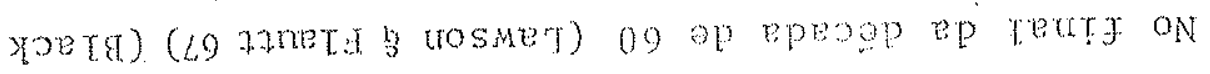

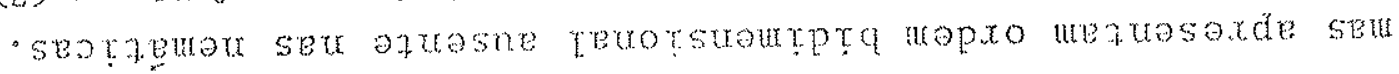

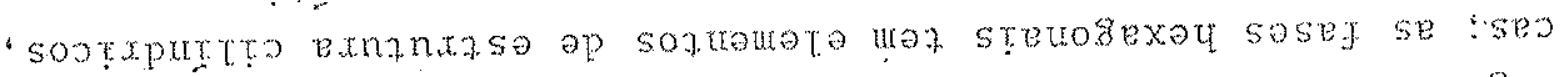

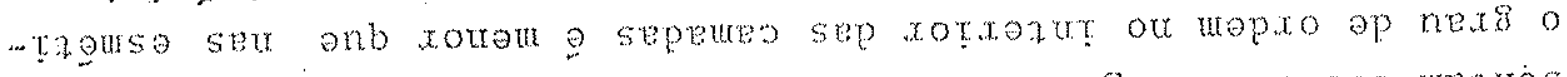

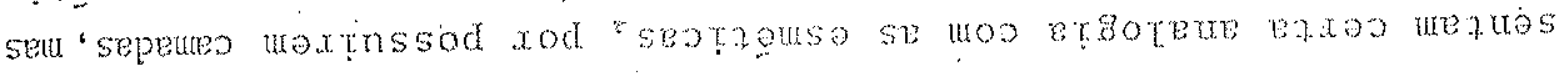

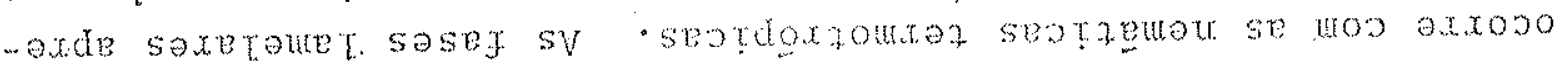

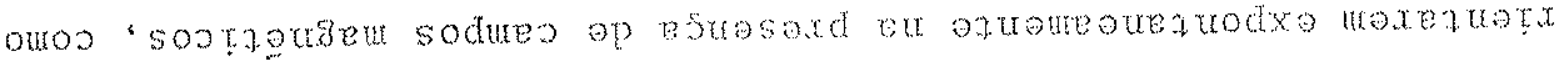

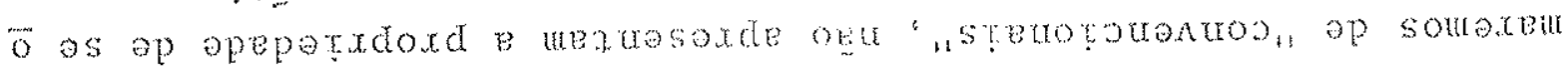

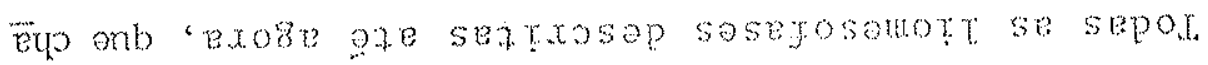

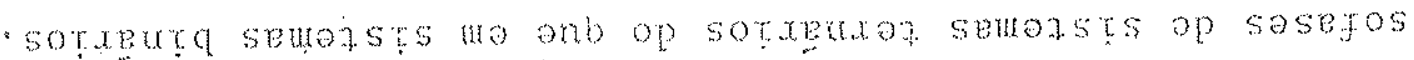

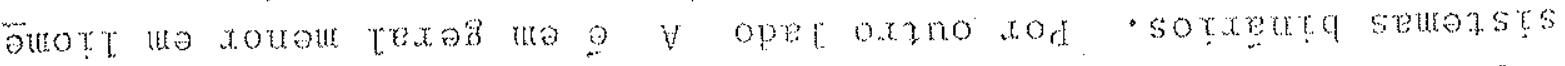

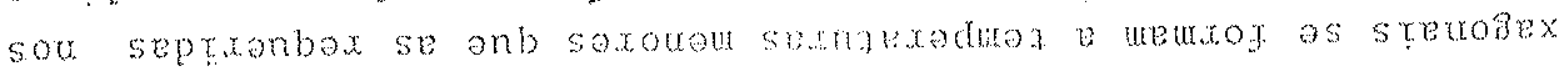

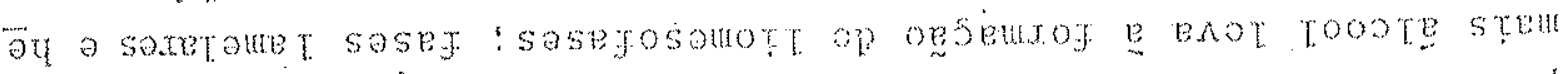

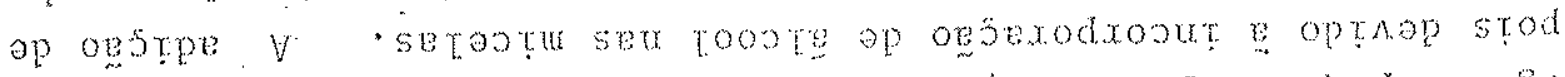

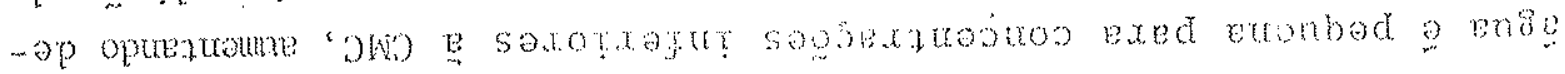

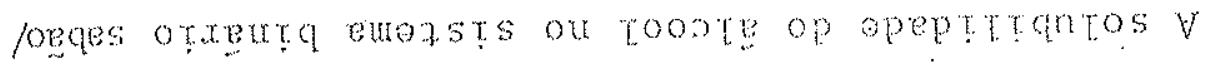




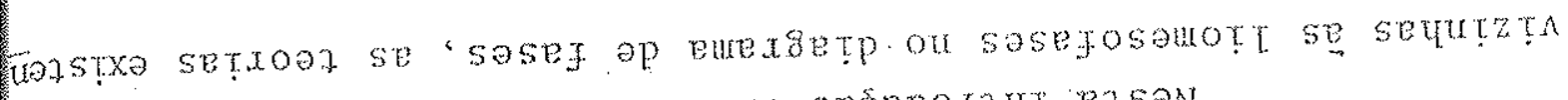
sosefosou se epute somoxtanostp opsnporame bason

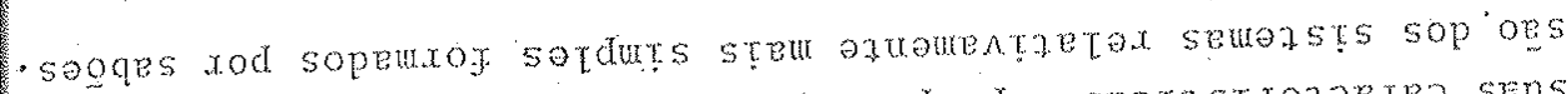

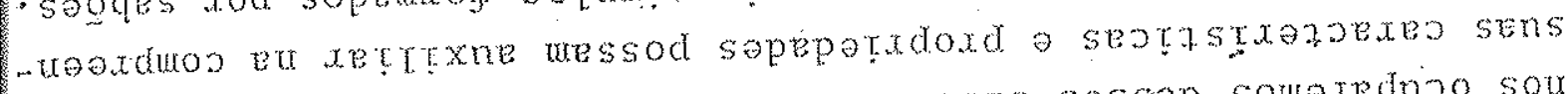

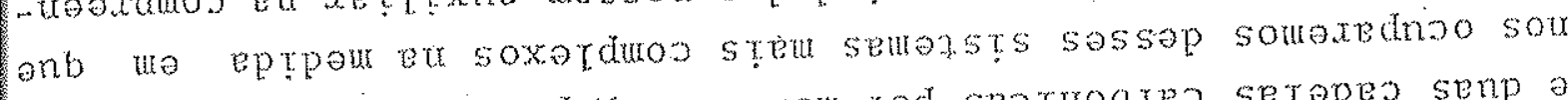

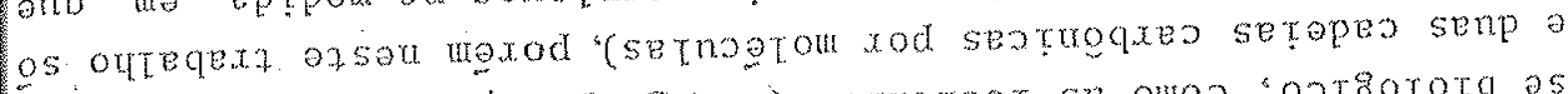

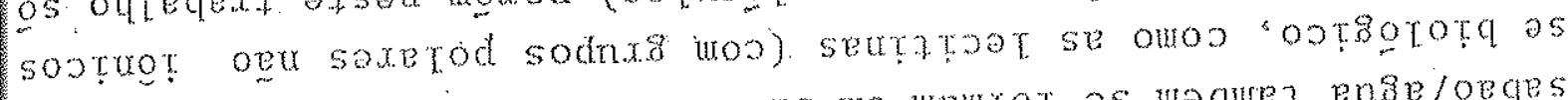

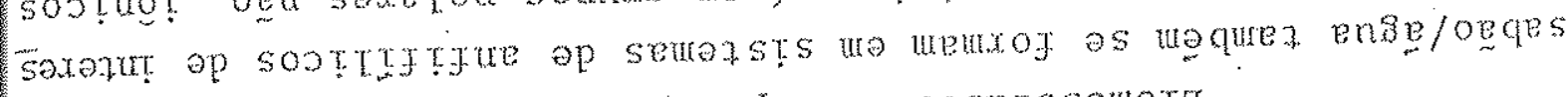

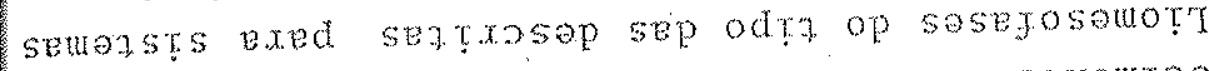

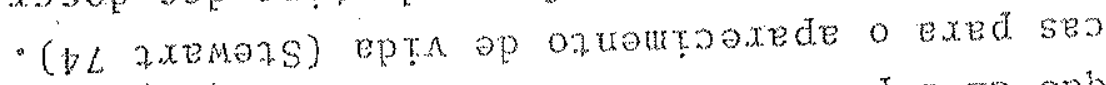

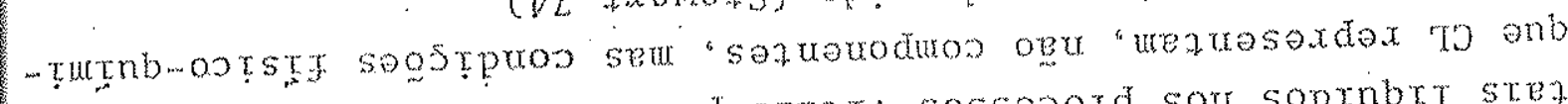
as - opraztp optunsax xos apod steas sossoboxd sou soptrbt stey stxo sop roded o enb optootoquaso ura azuntoneozex g a sonta sou

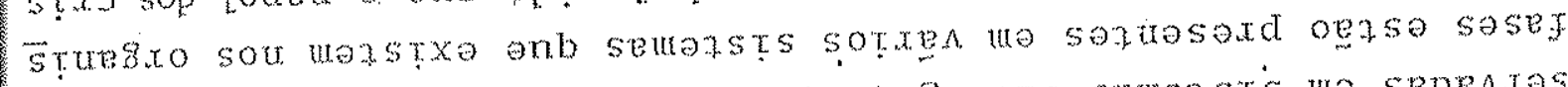

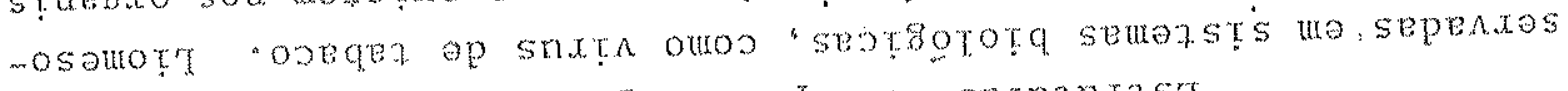
-qo mequea opts uga reuosexey odra op semanxas

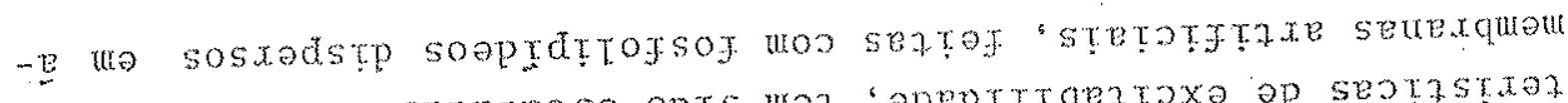

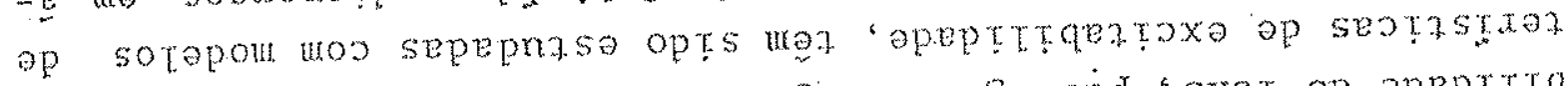

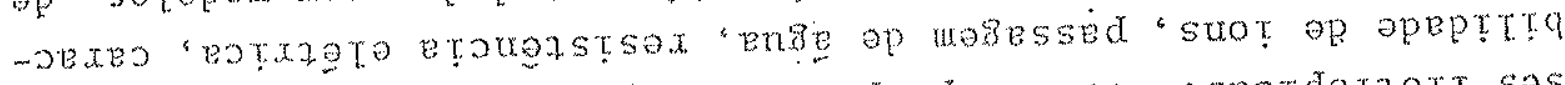

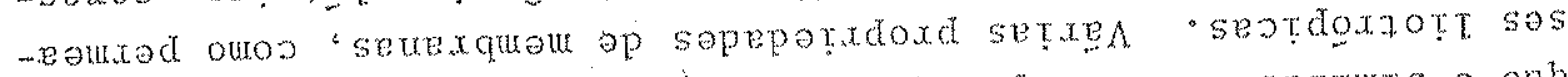

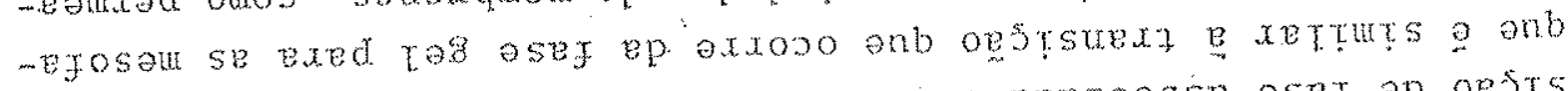

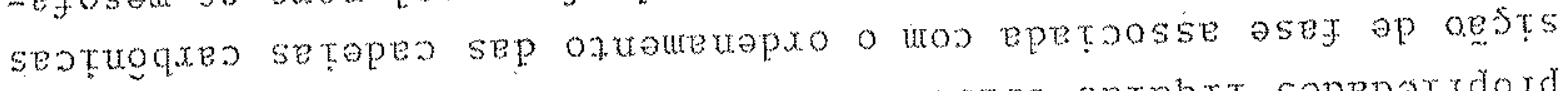

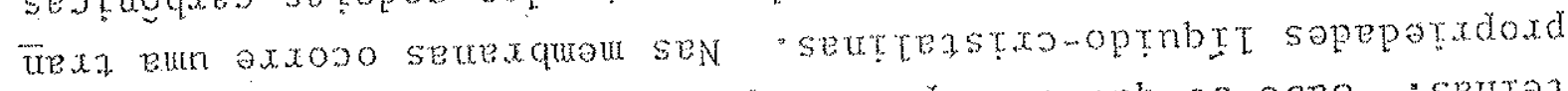

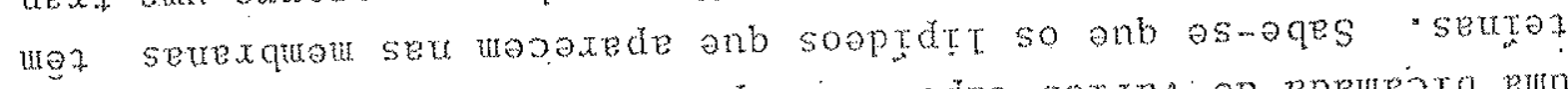

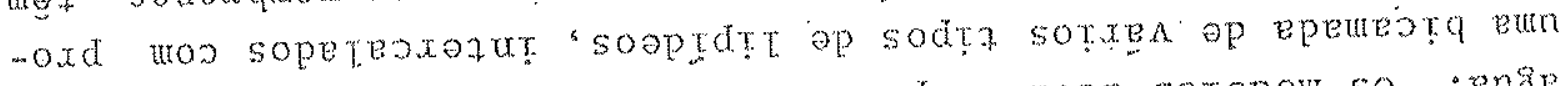

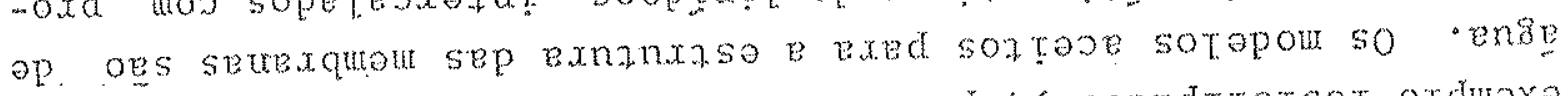

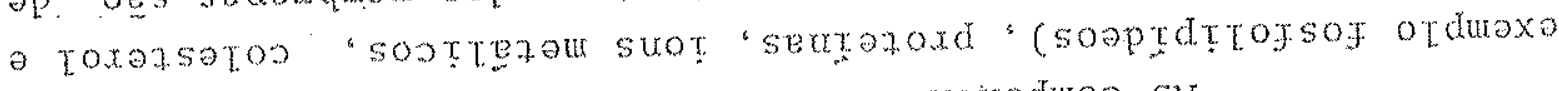
rod) sopxdrt wantout seurex quat sep saguotroduos $\mathrm{sV}$

- (VL zotmon rom) (6L weytom 3 tumote)

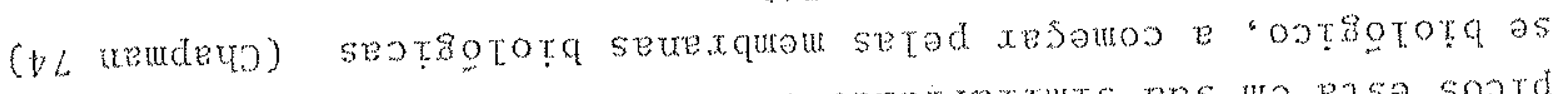

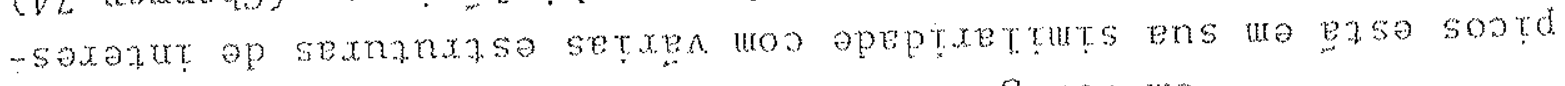

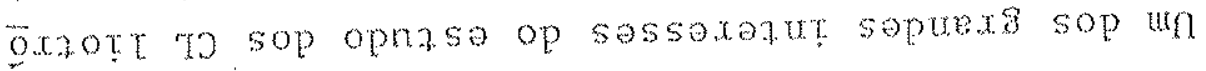

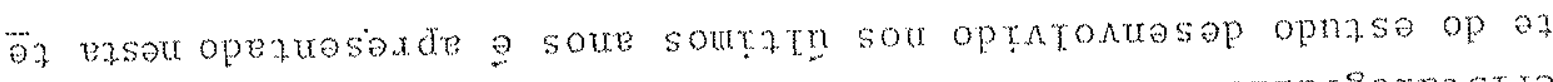

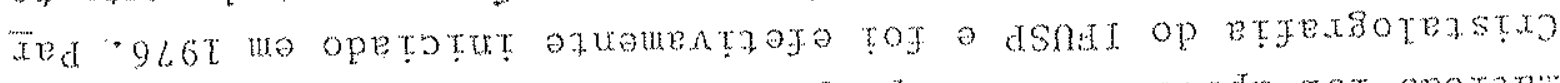

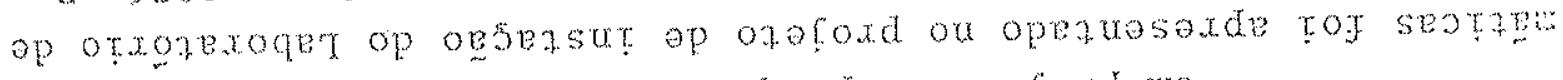

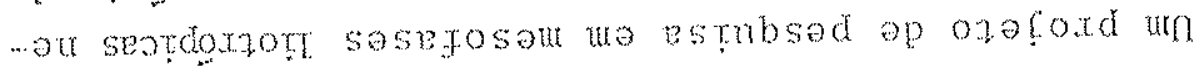




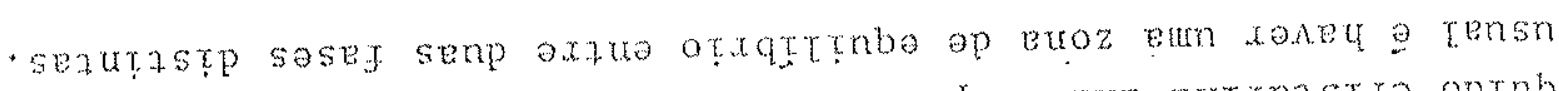

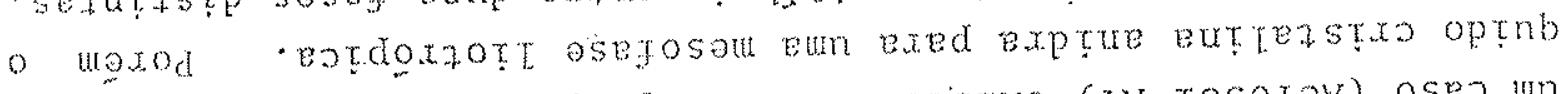

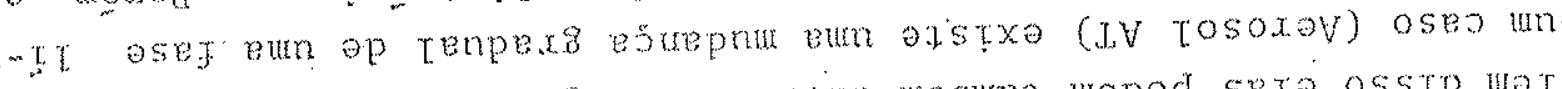
sorm otod ma. ense bunste xantosste mequez urapod seto osste wet

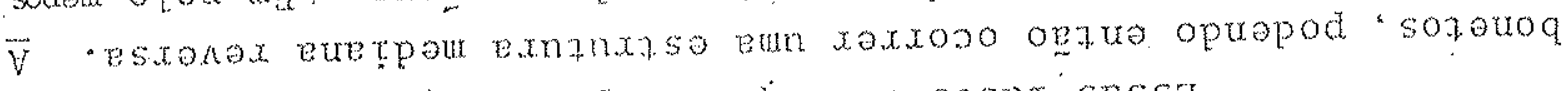
axooxpty antosstp upod sesonbe ogu sesey sess

- operopxosep opensa ue opzsa

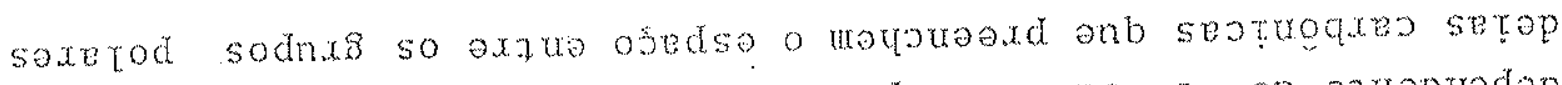

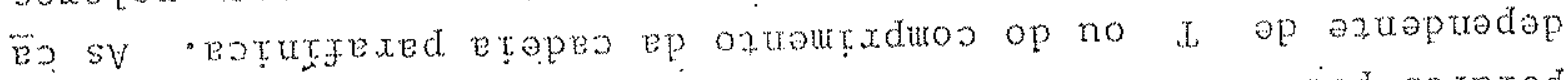

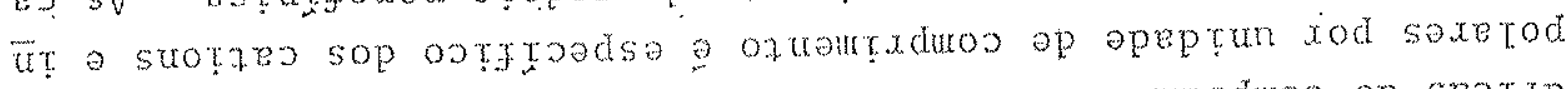

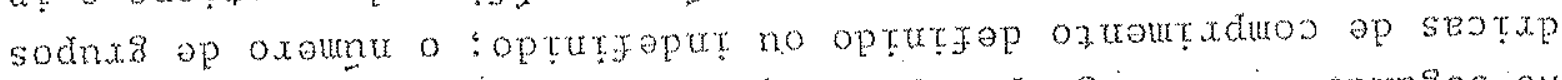

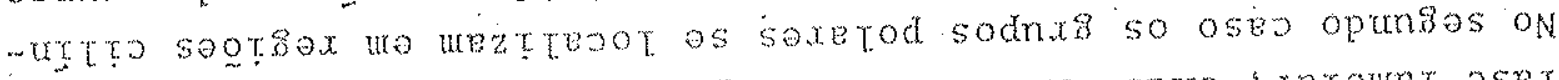

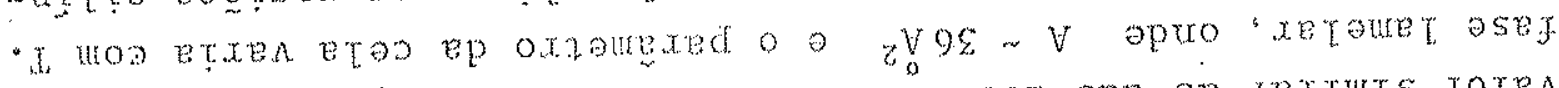

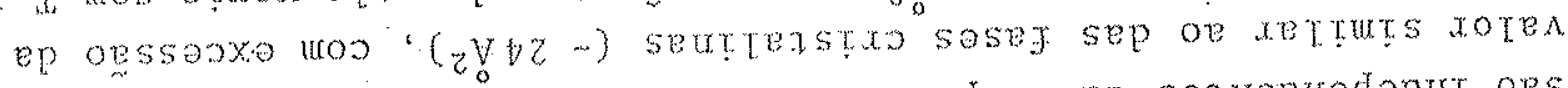

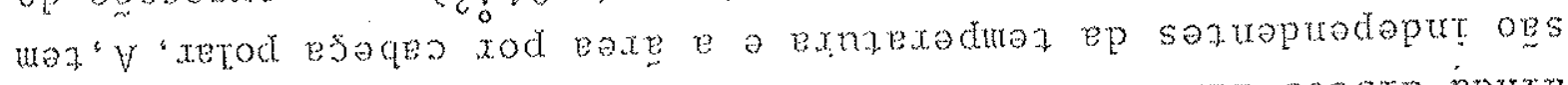

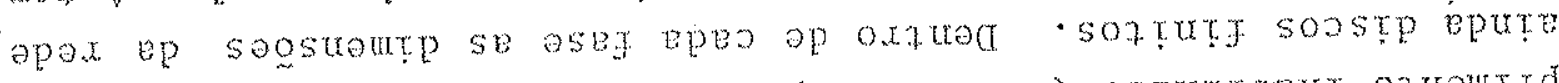

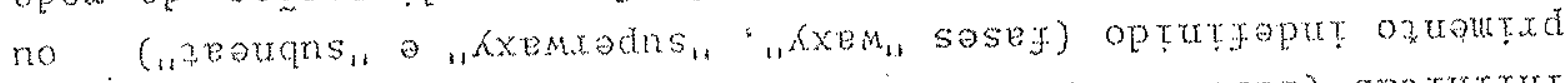

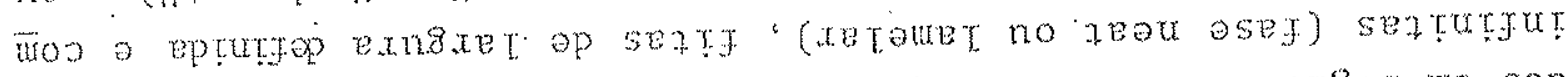

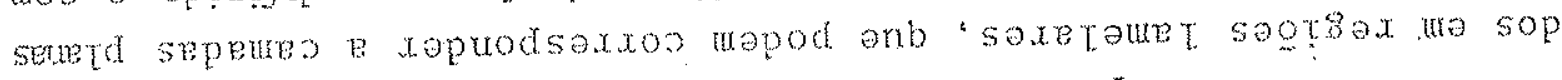

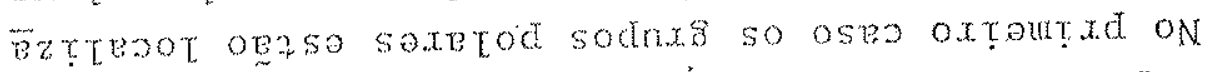

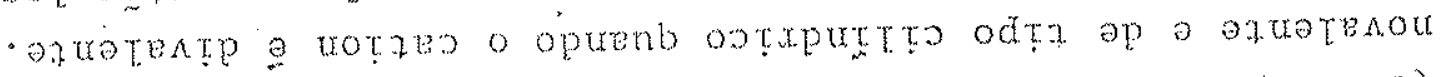
ou g trotzes o opuenb xetouret odrt op ogs (to razzn 3 sotrnoxs)

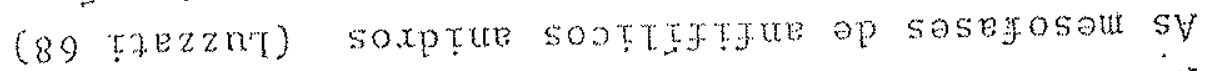

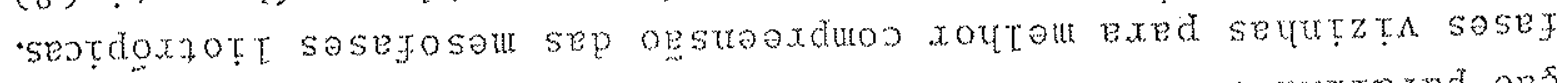

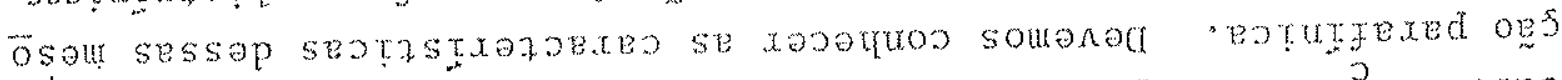

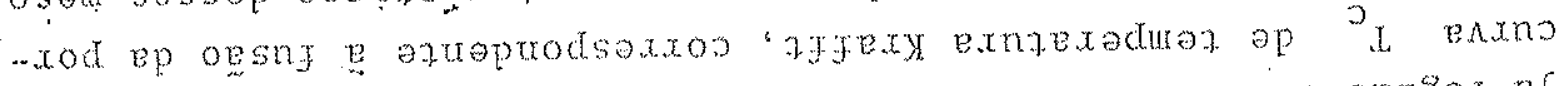

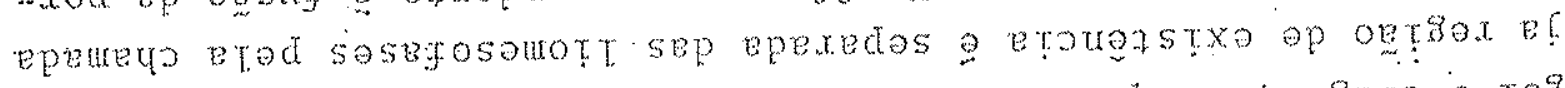

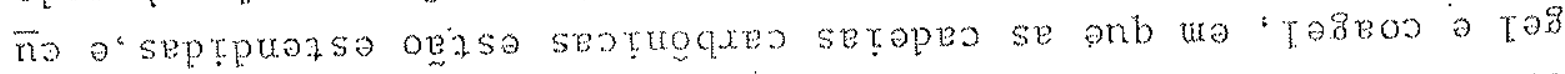

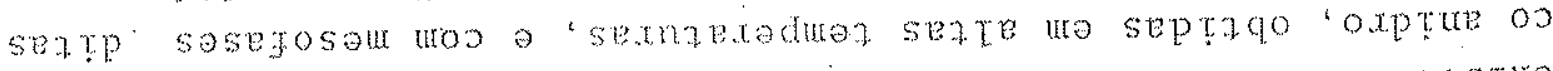

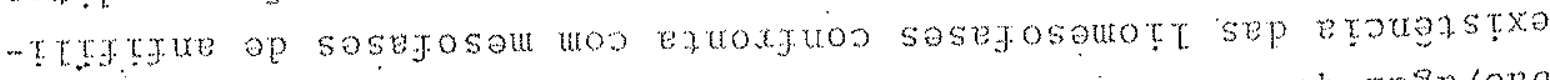

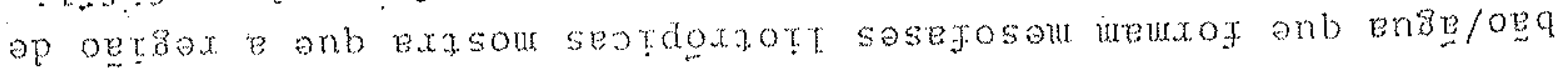

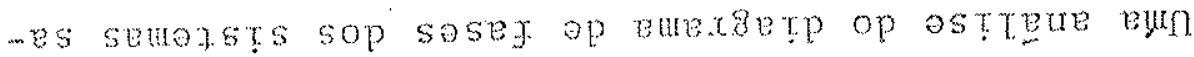

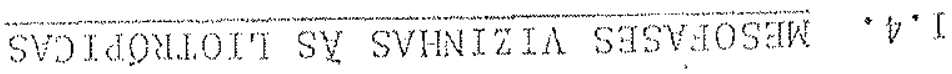

- oqfegera onsep sontrofqo so xenats sou

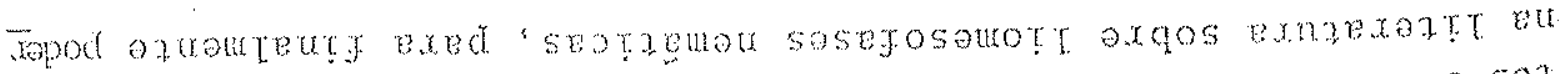

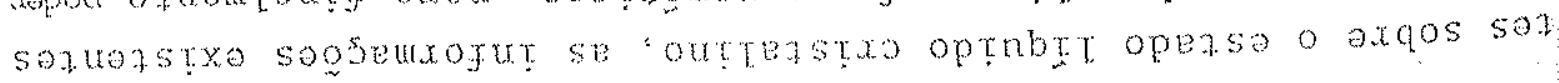




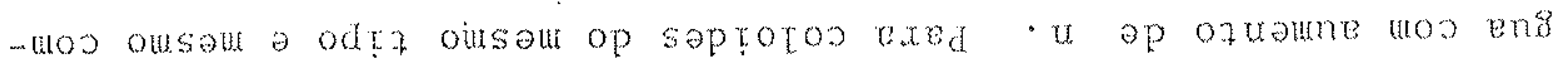

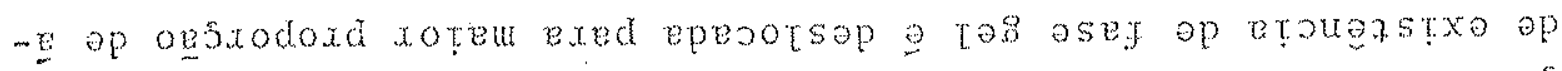

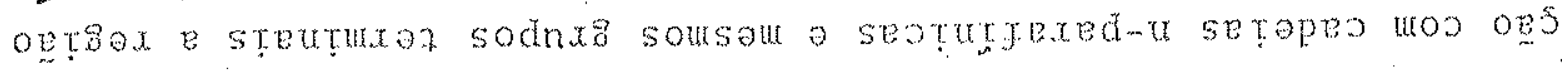

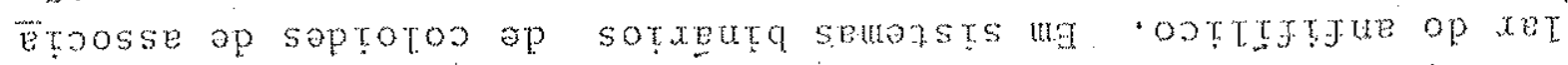

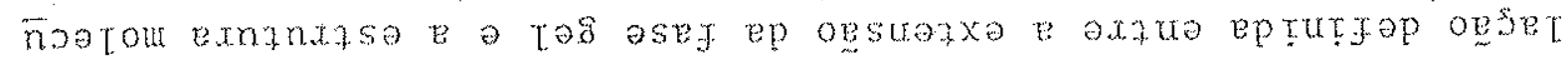

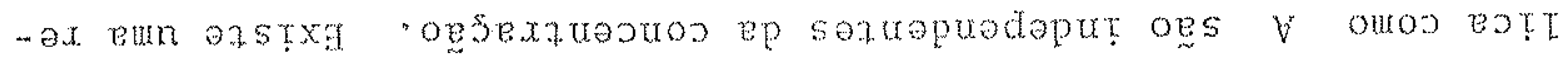

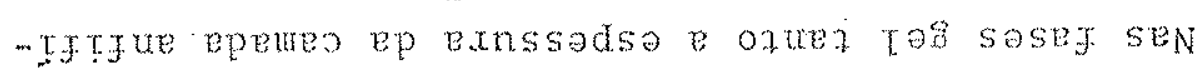

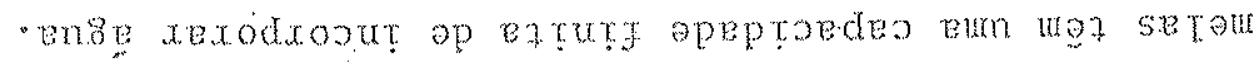

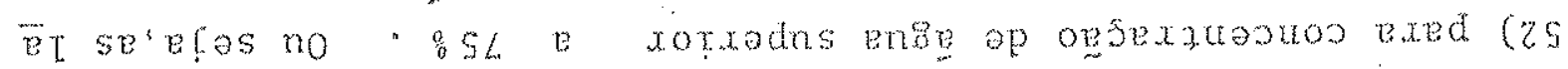

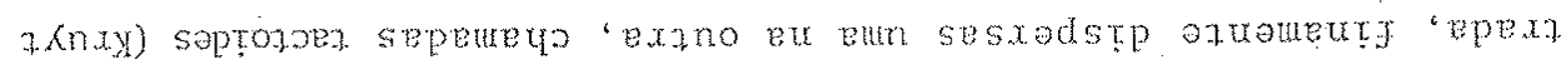

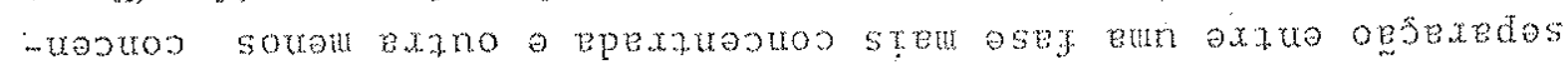

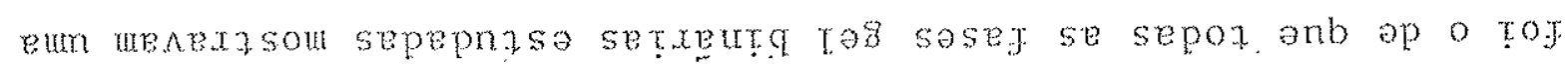

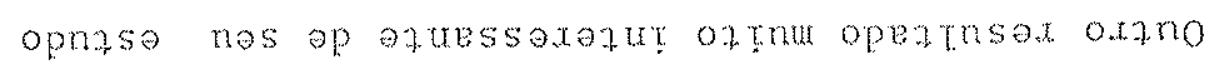

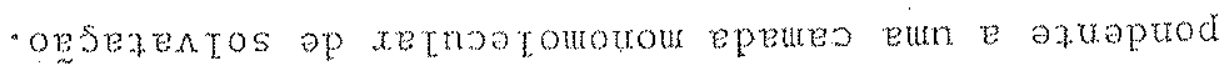

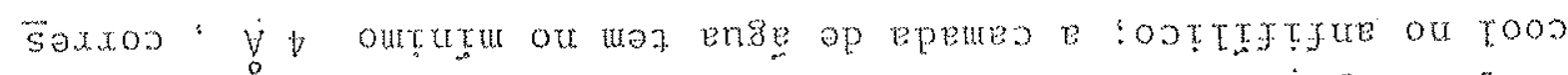
- Te op og Sntosstp e xanowoxd exed ense op as soubu otad op pjros

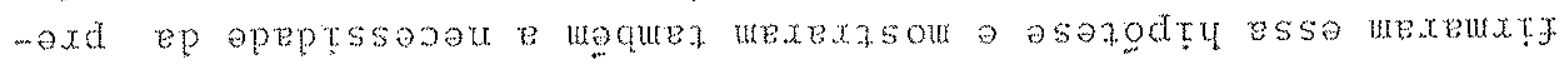

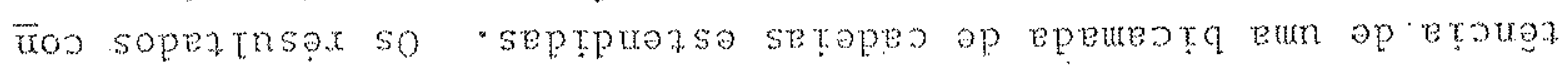

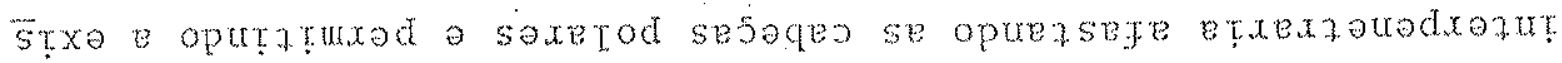

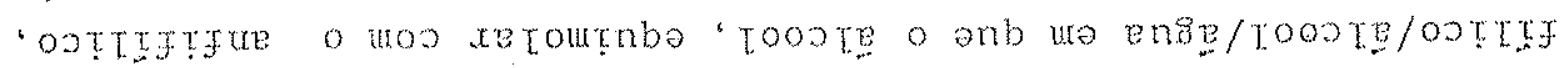

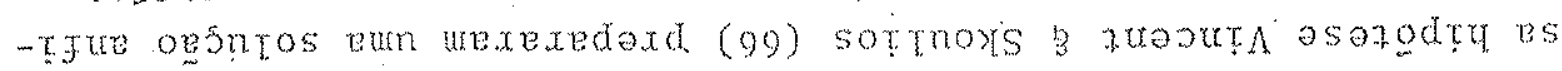

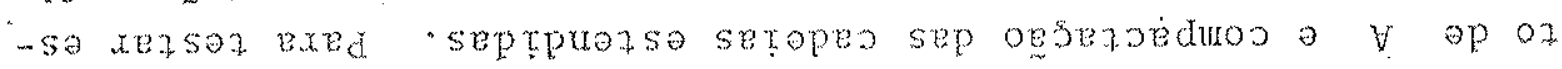

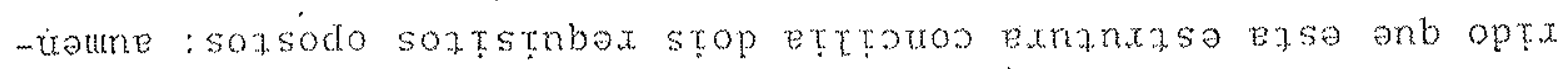

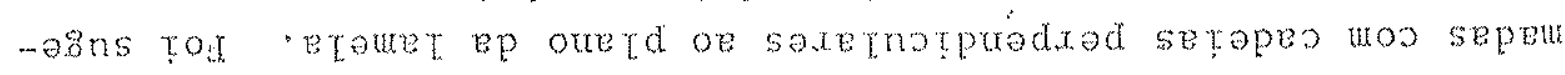

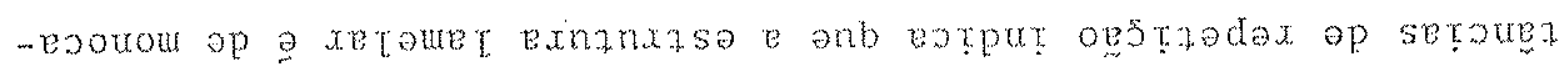

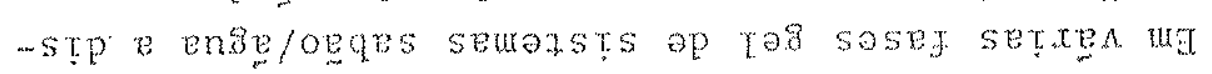

- 108000

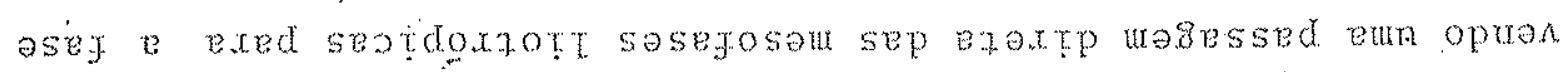
Eq 'Tos oseg axxoso oen eN a th ap sooqes op oses on "ertete

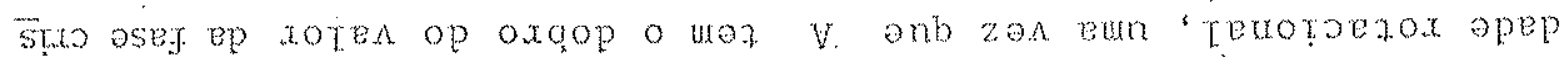

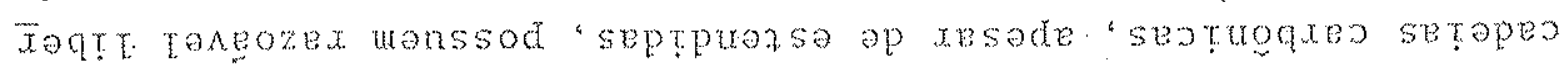

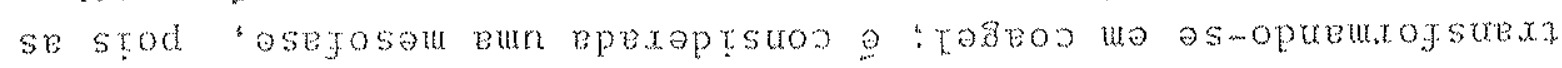

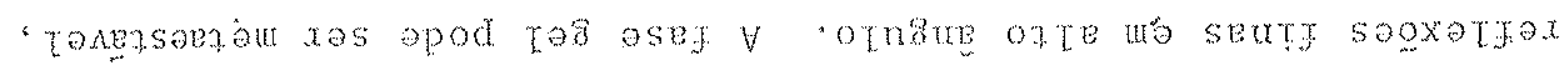

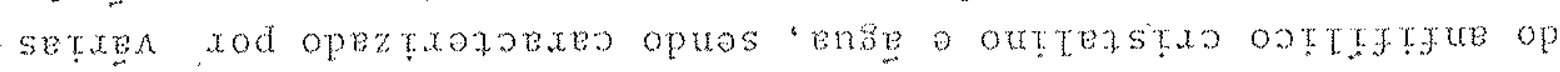

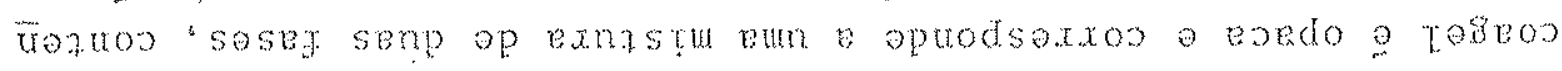

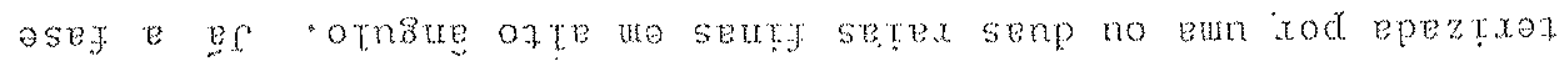

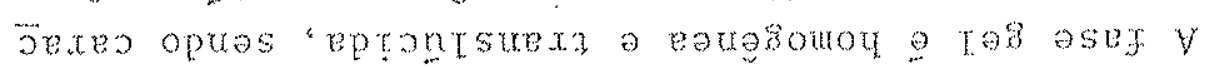

$$
\text { - x sotex ap opsexte xod (99) sommoxs }
$$

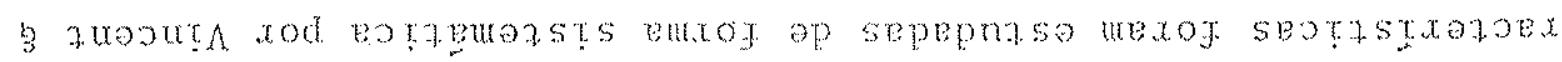

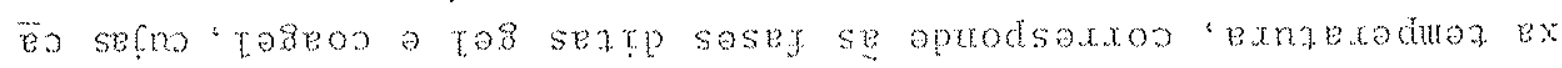

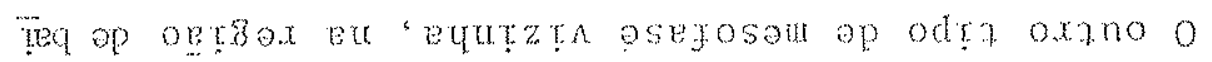


"sogtoxiostue sotou exed

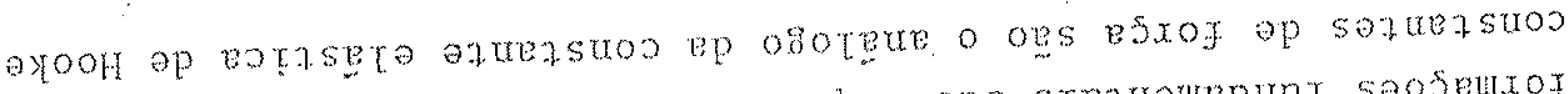

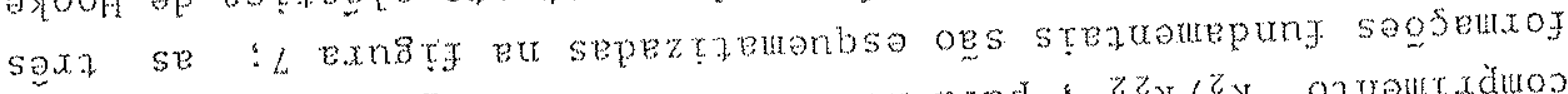

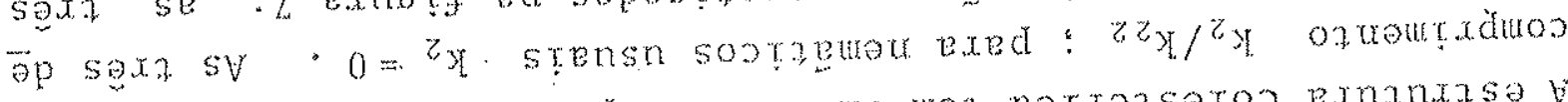

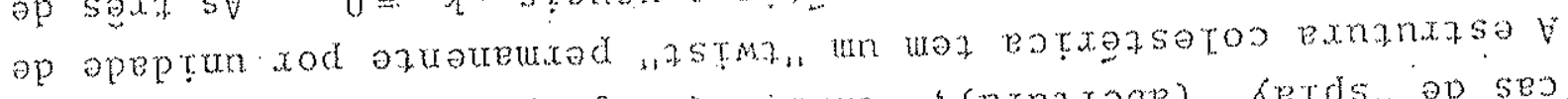

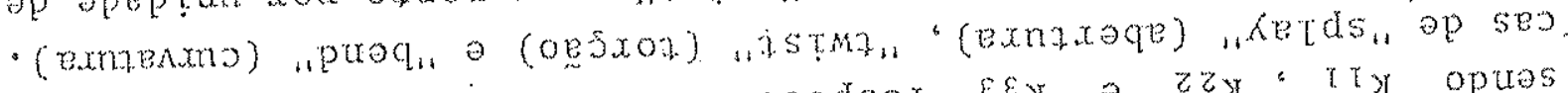

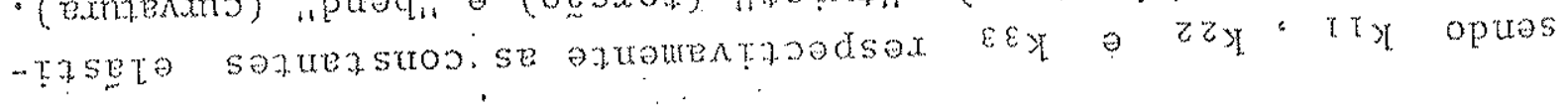

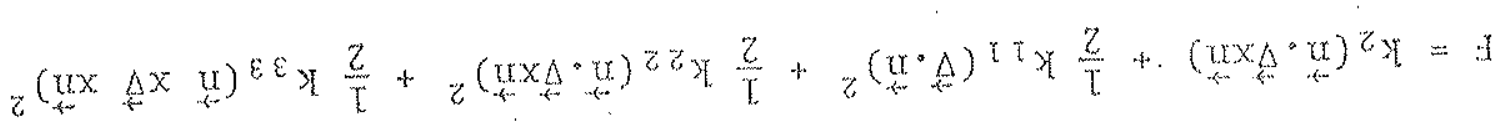

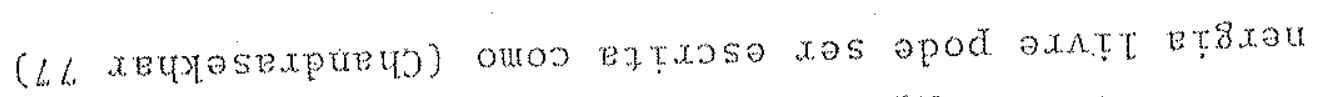

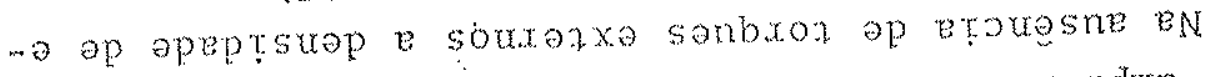

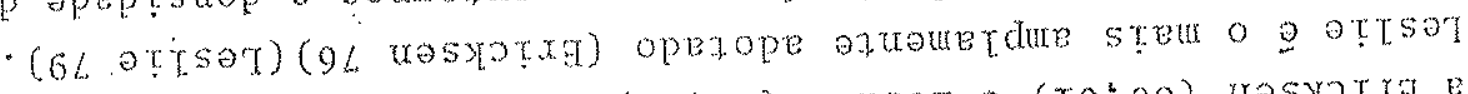

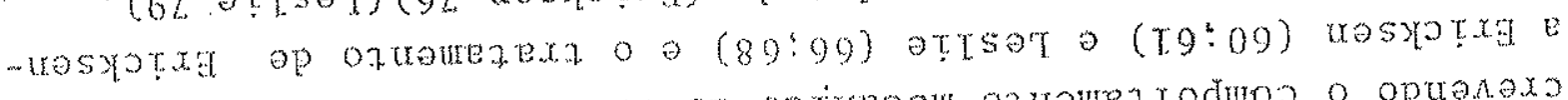

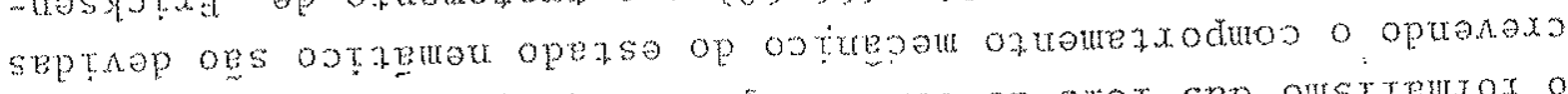

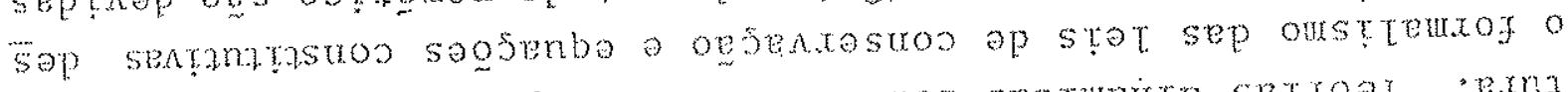

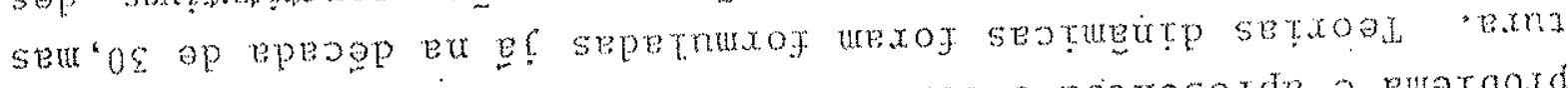

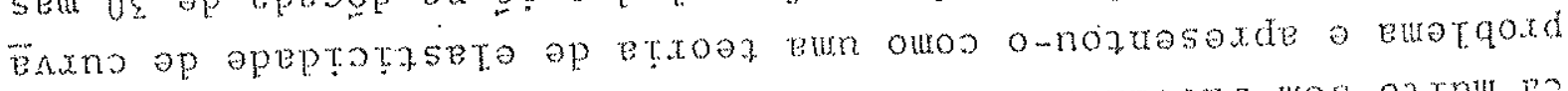

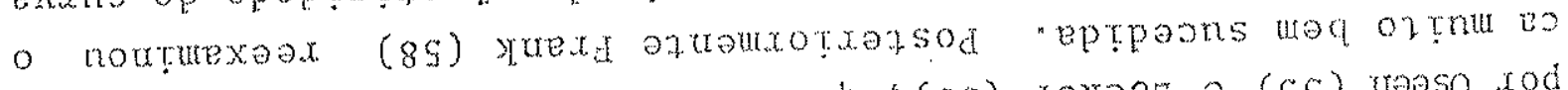

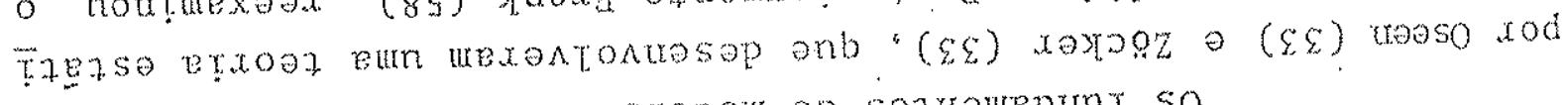

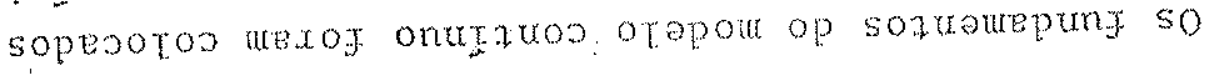

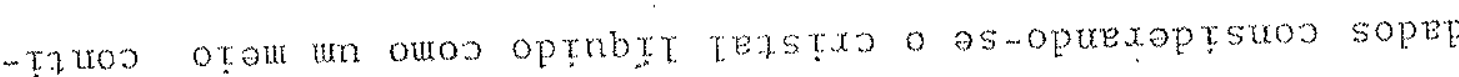

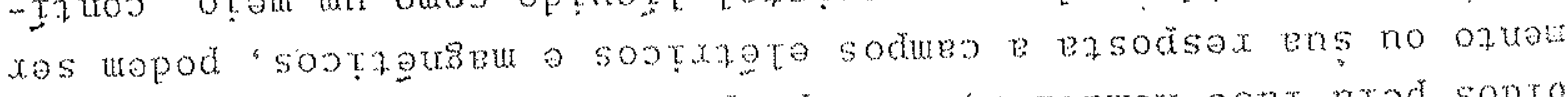

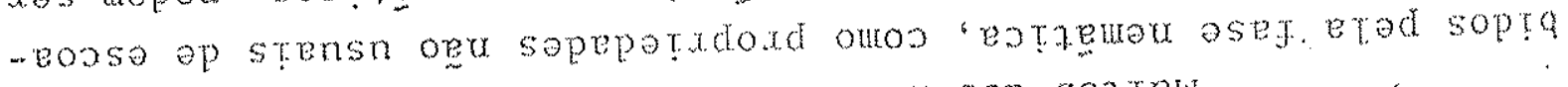

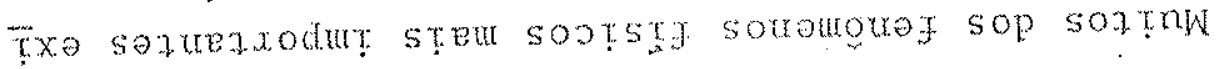

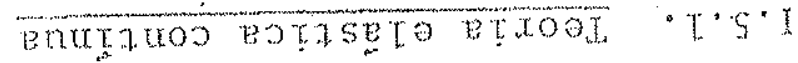

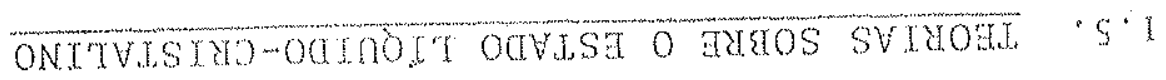

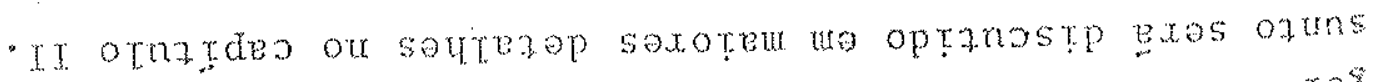

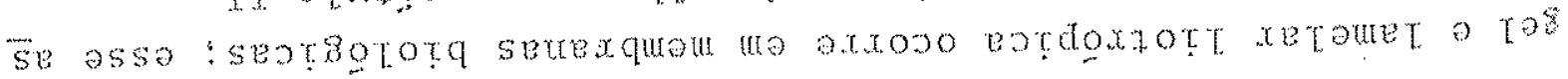

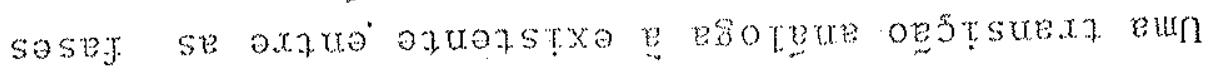

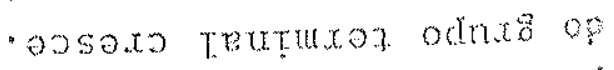

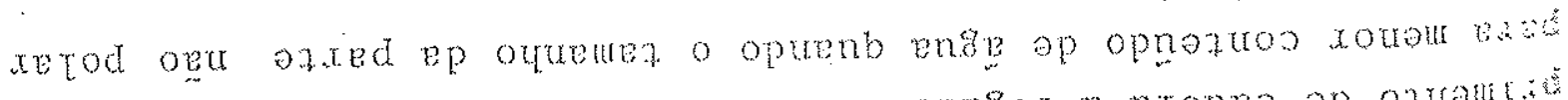

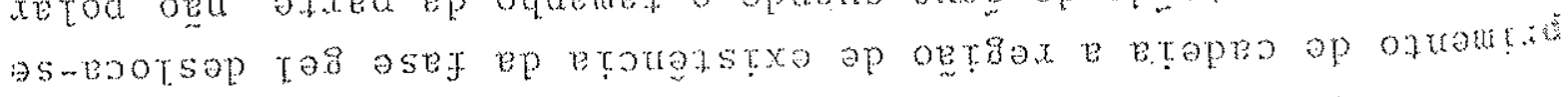


- H odues orad a sopaxd rod opsezrotxo op soz?ta sop opstsodxedns (q) sapared xod oesemeno (e)

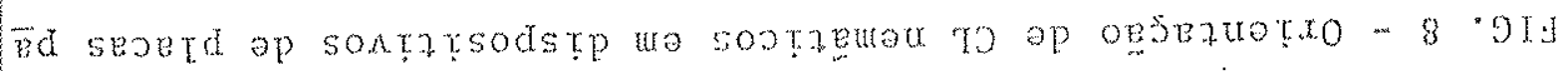

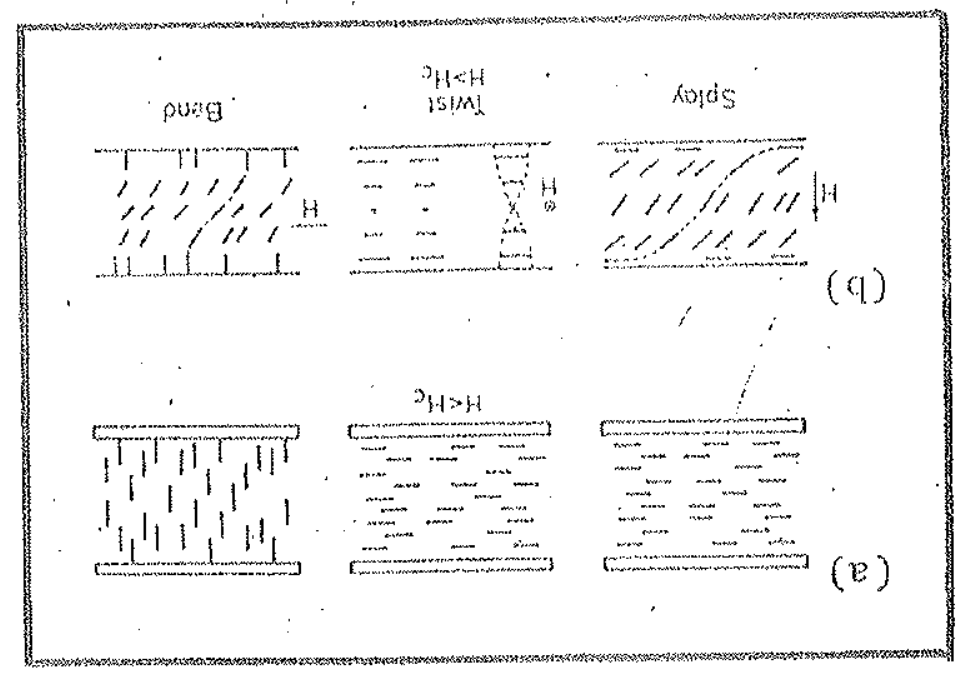

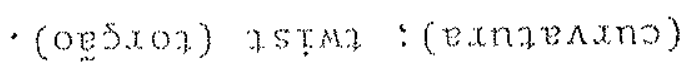

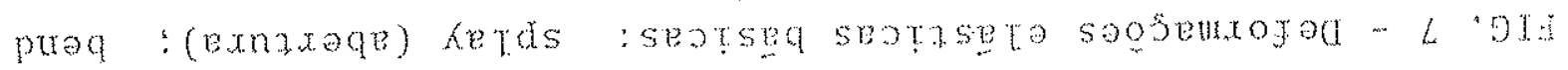

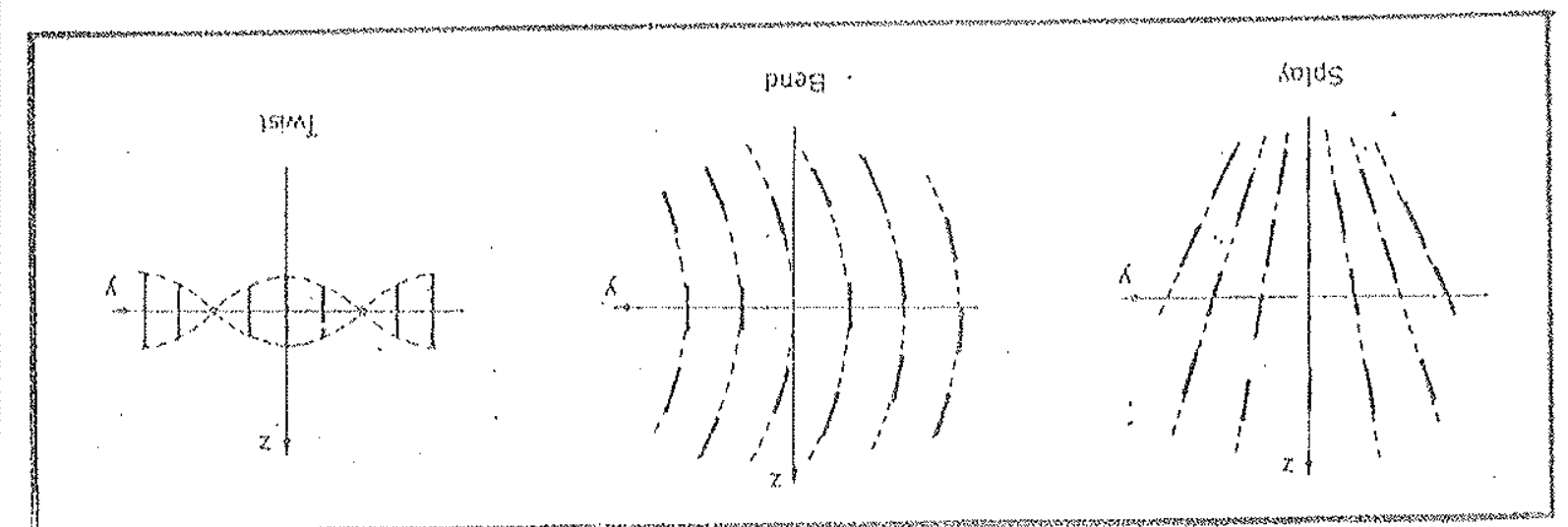




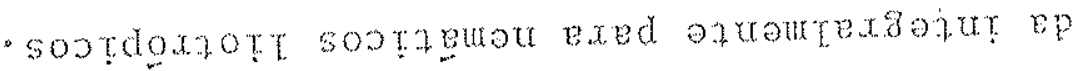

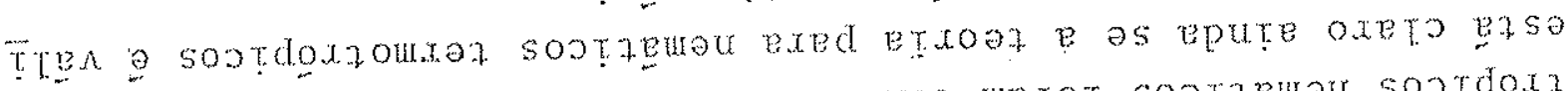

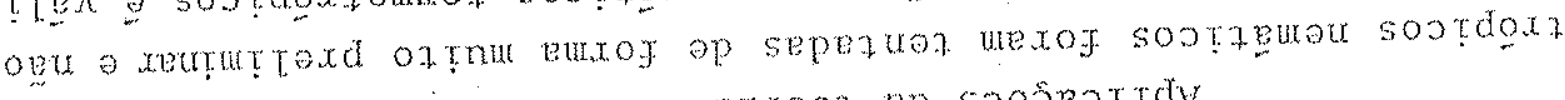

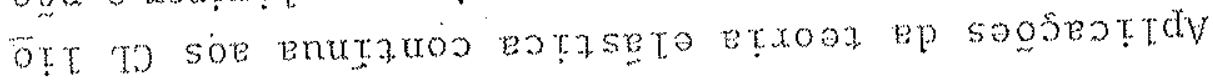

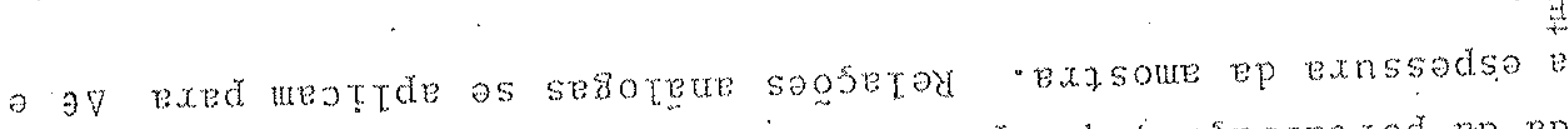

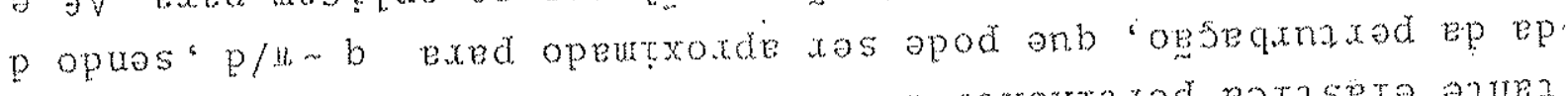

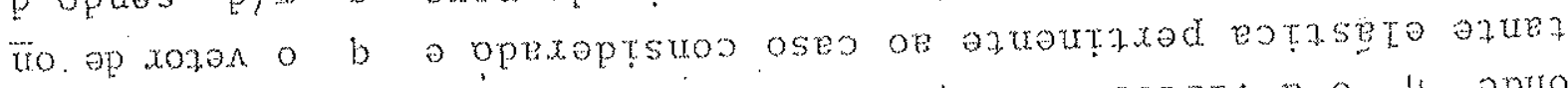

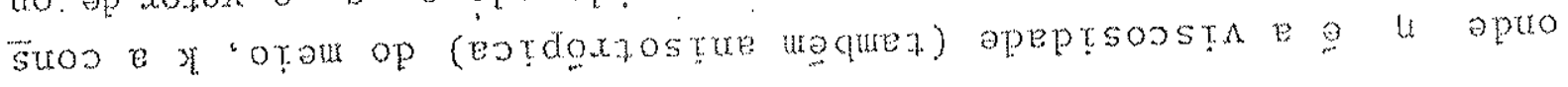

$$
\begin{aligned}
& \text { (r op etorgsine eu) } \\
& \frac{z^{b y}}{u}=\operatorname{epcosep} x
\end{aligned}
$$$$
(y) \text { estosend ex) }
$$$$
\frac{z^{b x-z^{X V}}}{u}=\operatorname{aprgns}
$$

$$
:(b L+\mathrm{TE}
$$

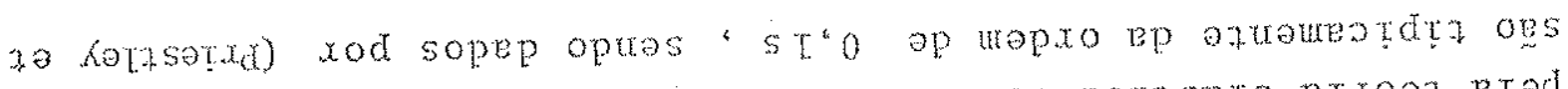

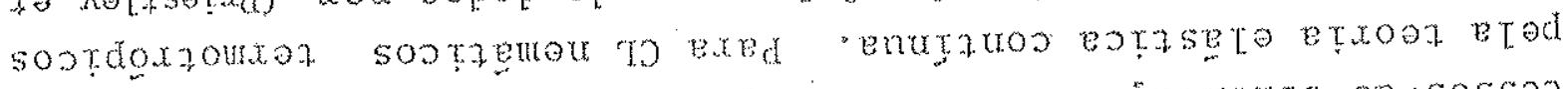

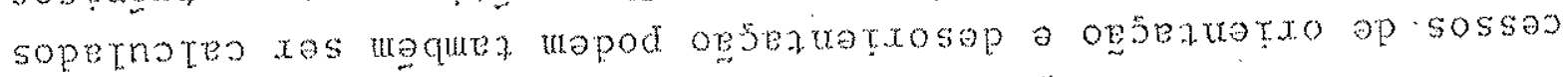

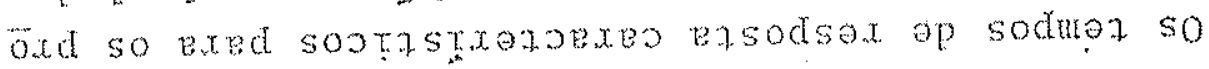

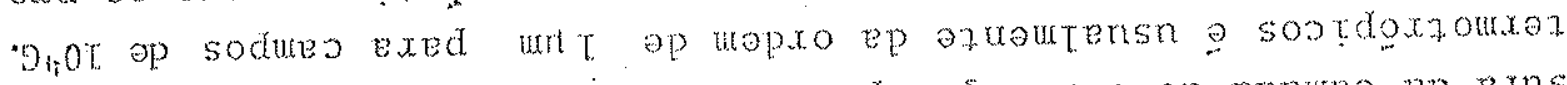

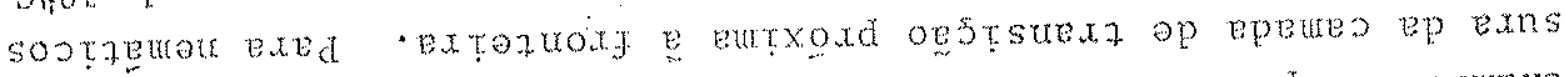

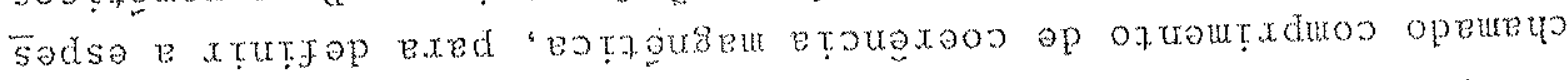

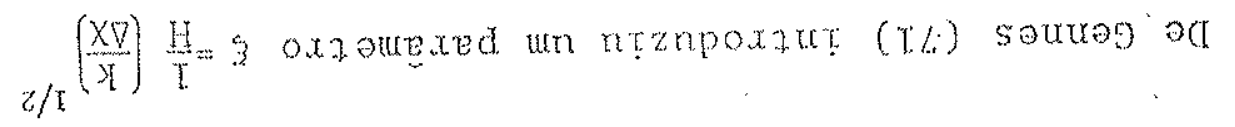

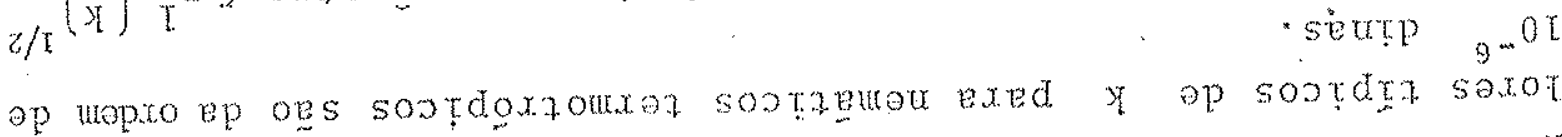

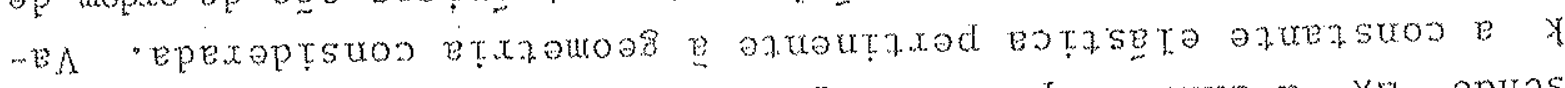

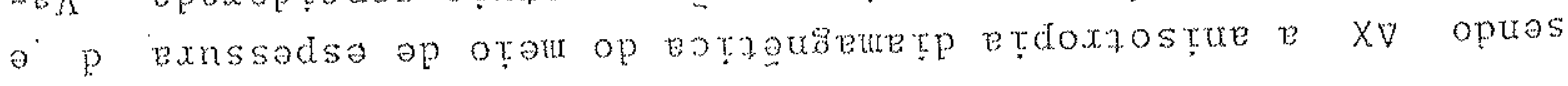

$$
\left(\frac{x \nabla}{x}\right) \frac{P}{H}=2 \mathrm{H}
$$

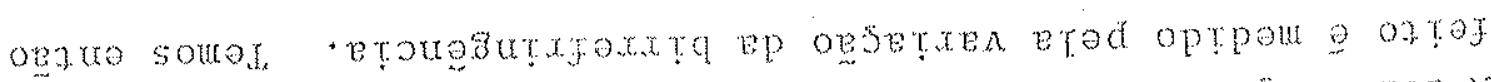

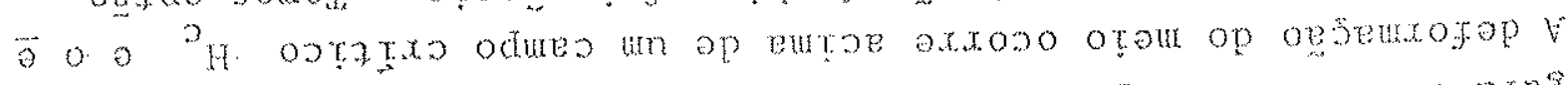

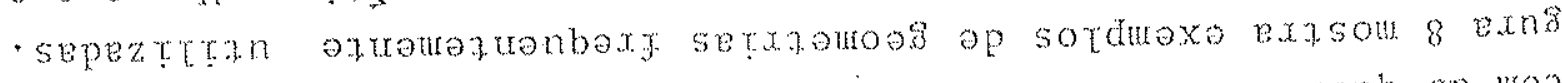

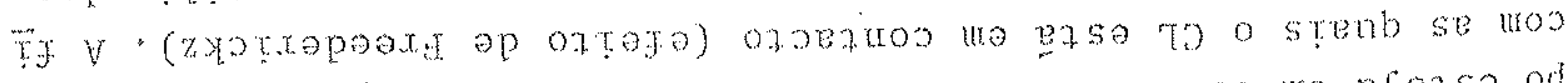

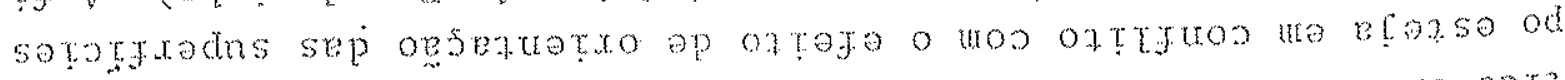

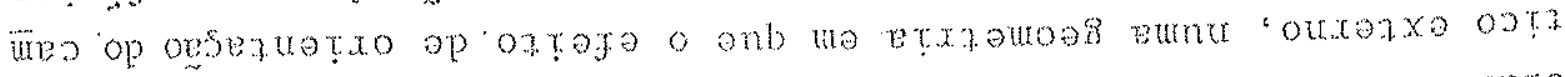

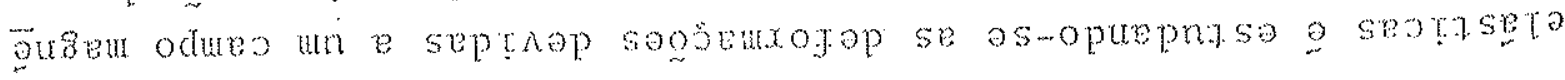

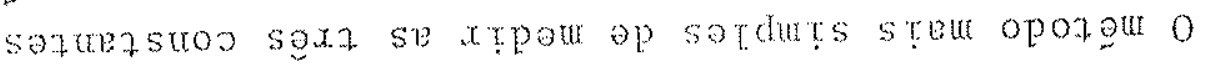




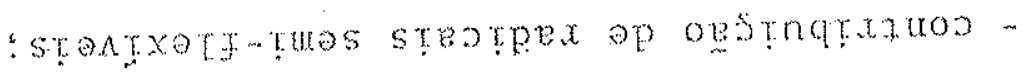

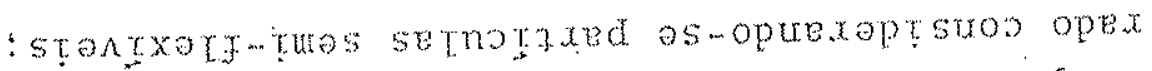

очтон

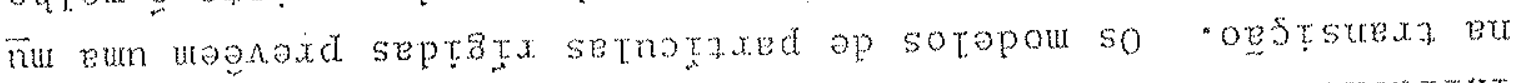

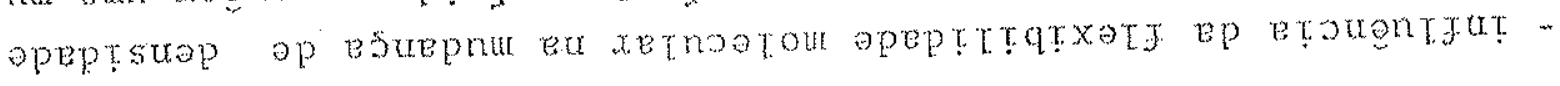

: seotaos

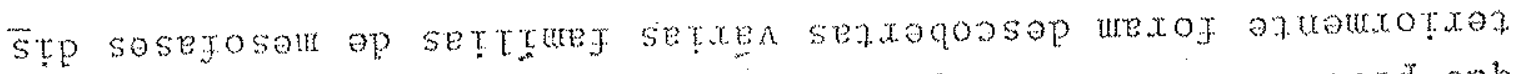

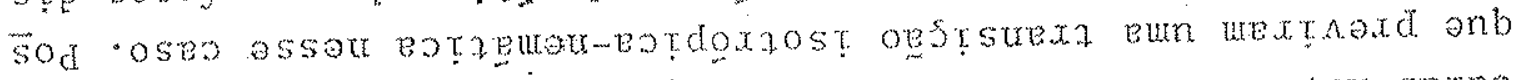

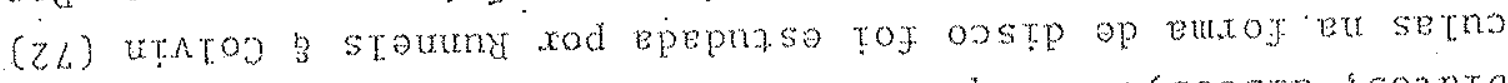

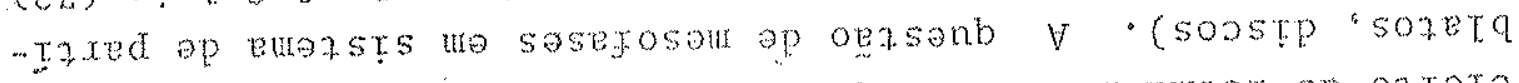

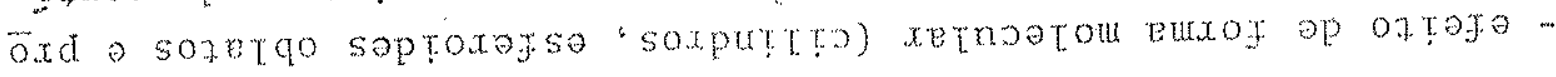
: setratgoxd somano epute xeprisa

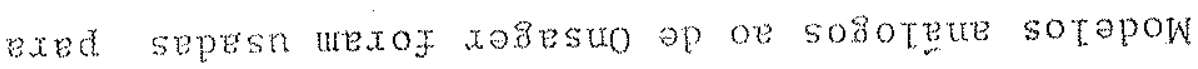

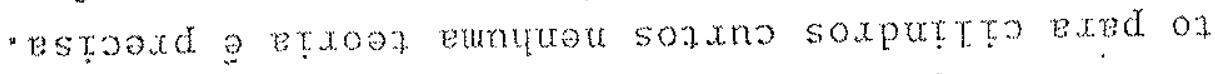

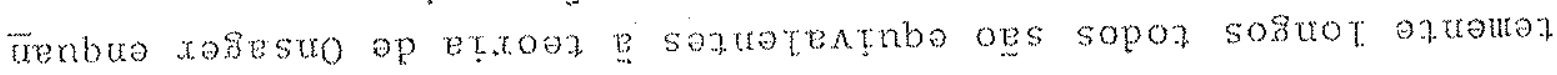

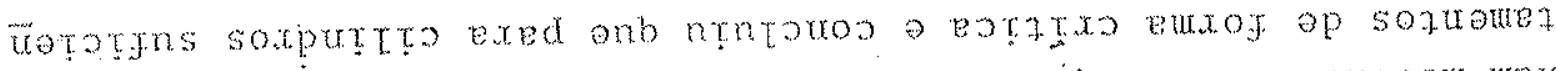

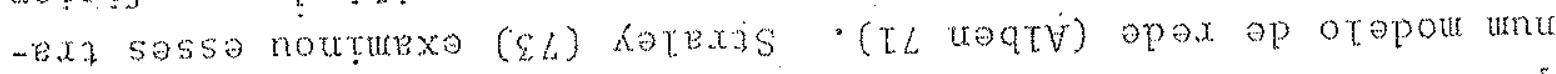

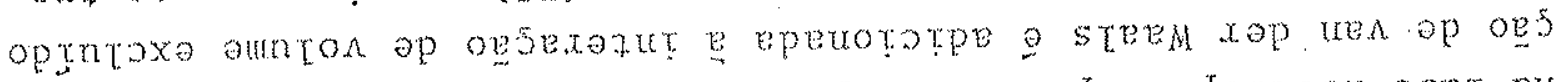

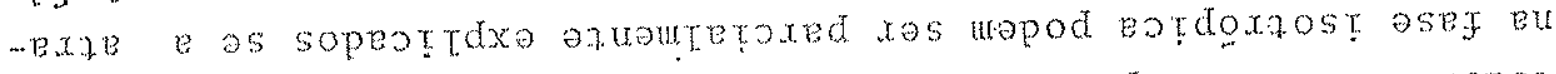

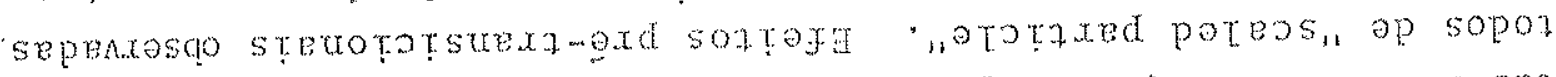

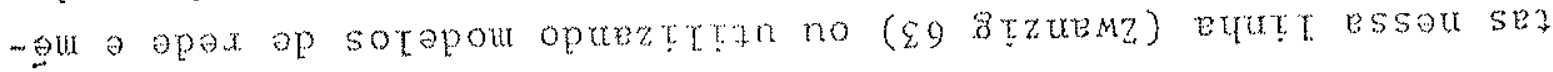

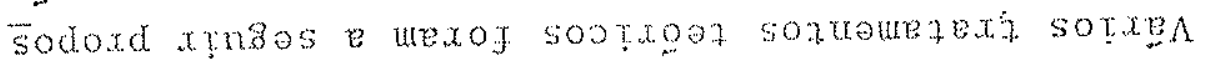

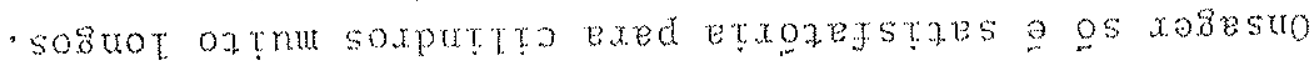

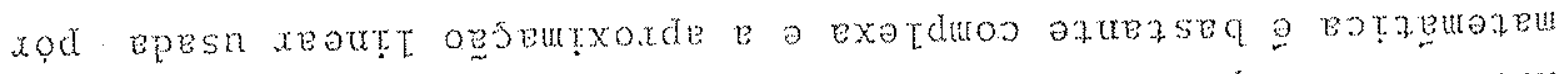

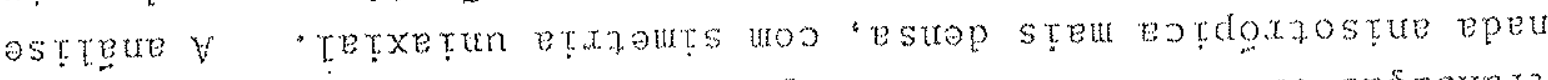

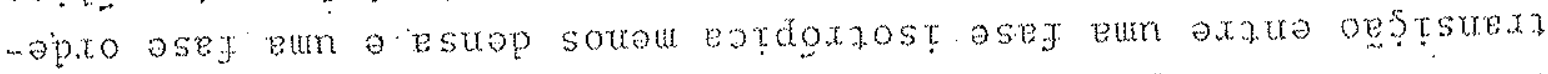

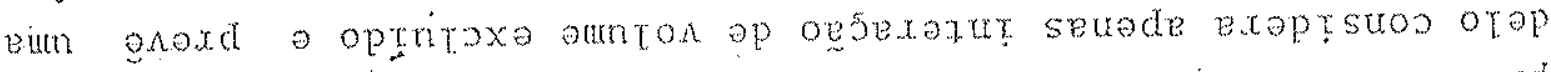

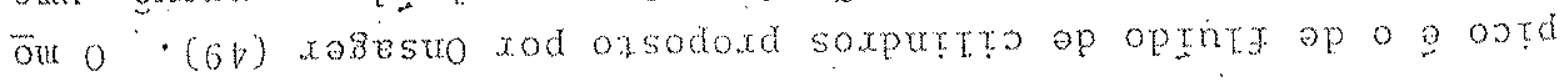

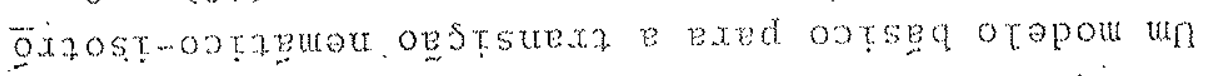

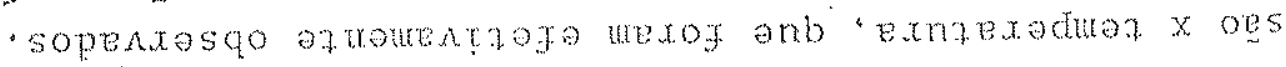

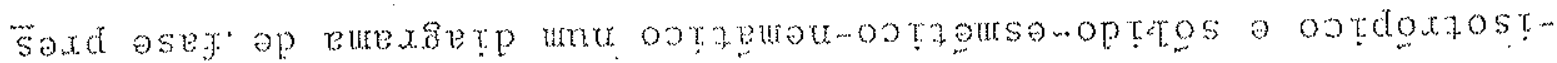

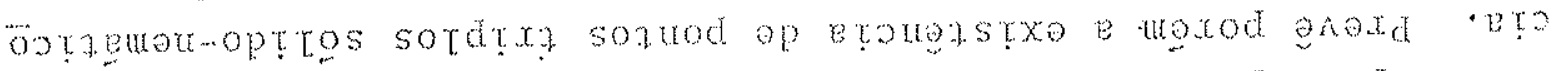

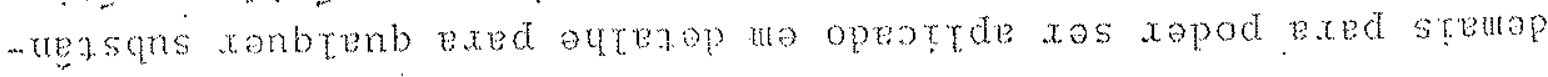

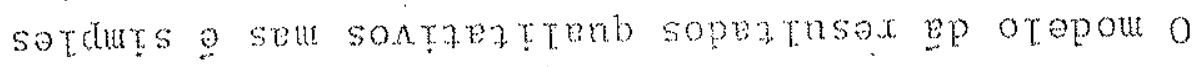

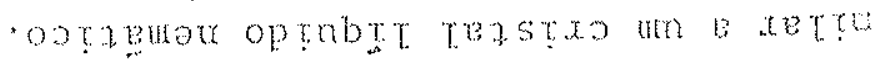

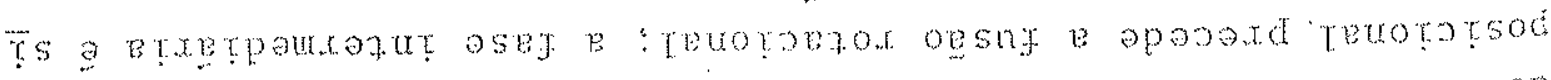

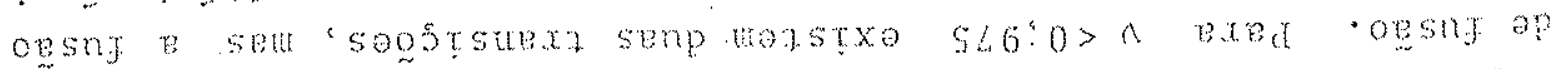

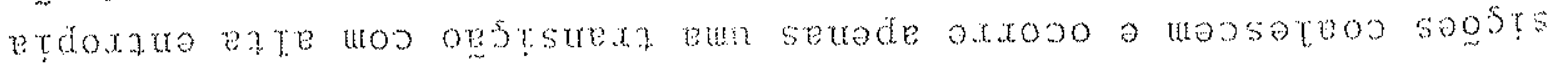

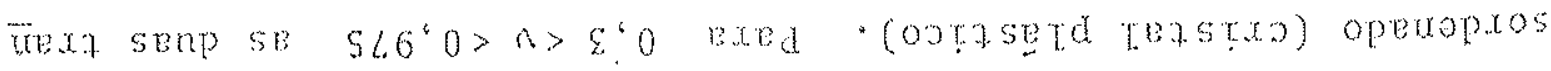




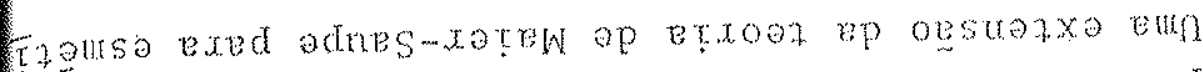

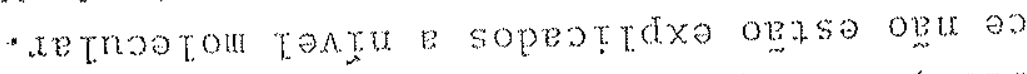

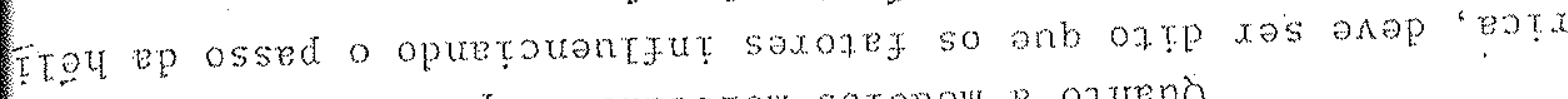

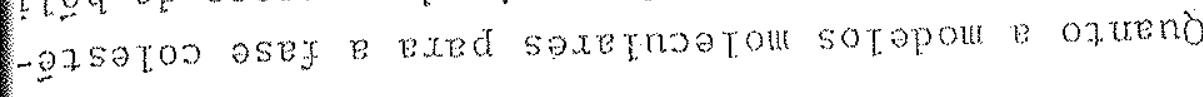

- ontagatamb

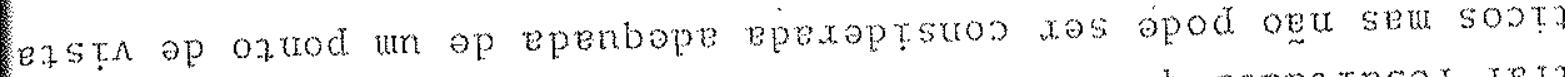

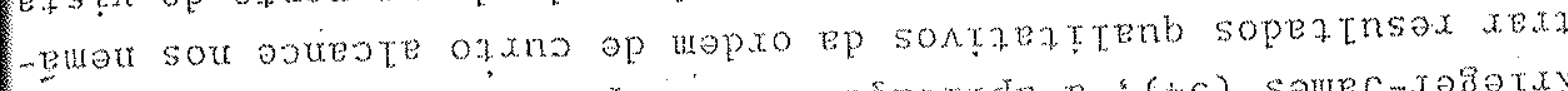

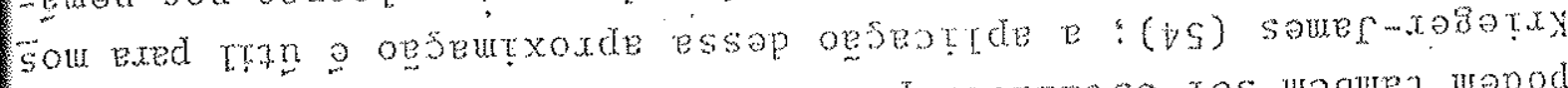

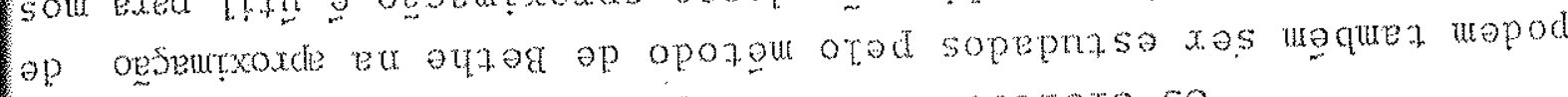

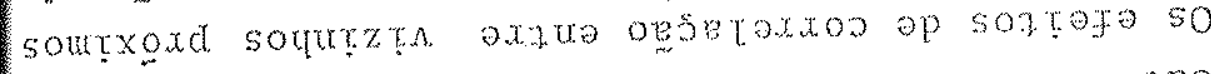

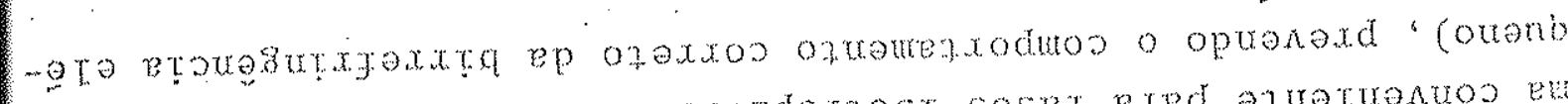

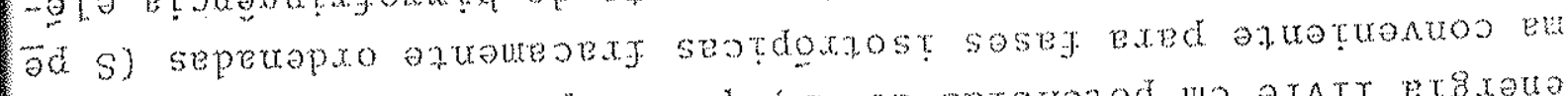

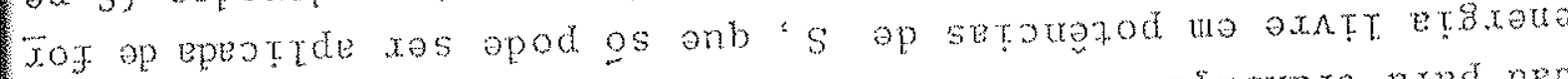

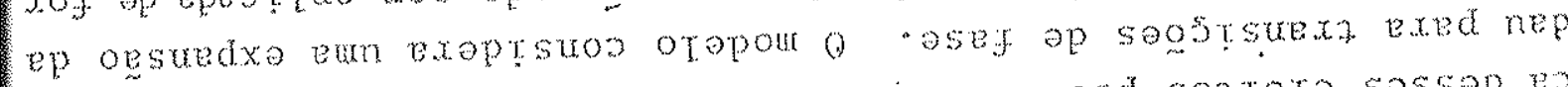

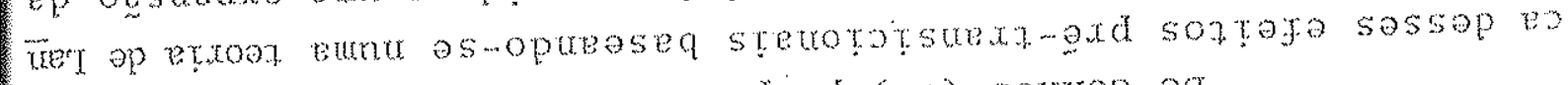

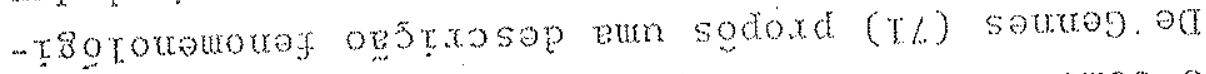

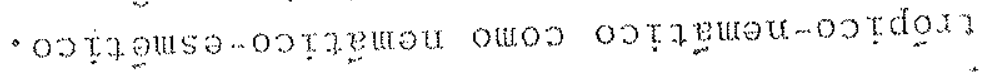

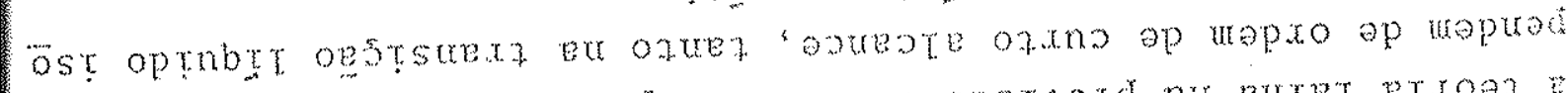

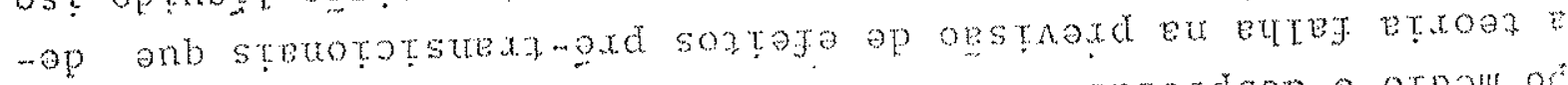

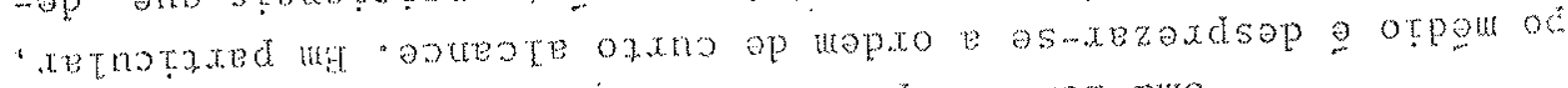

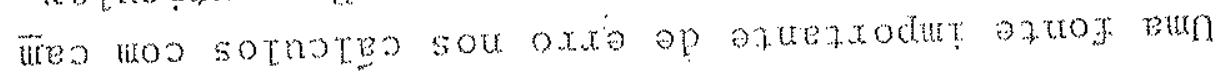

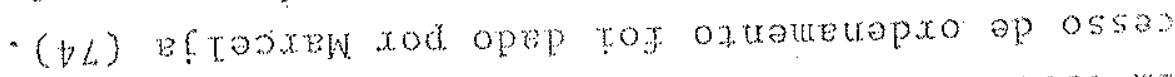

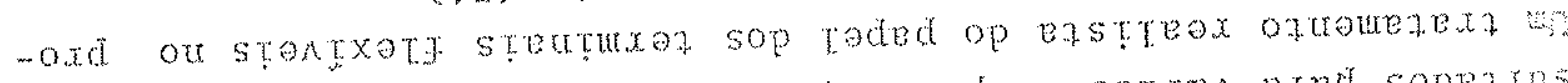

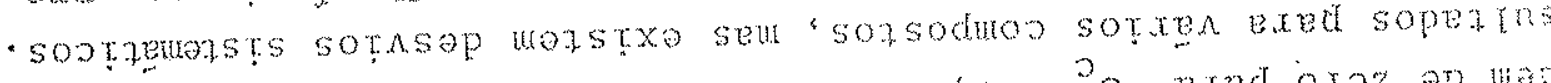

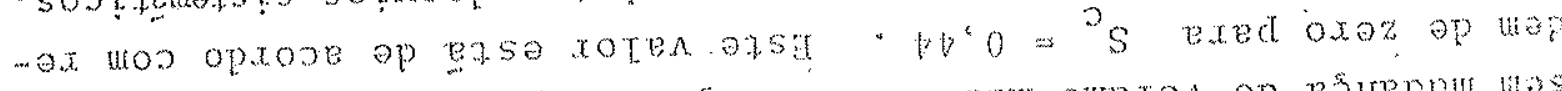

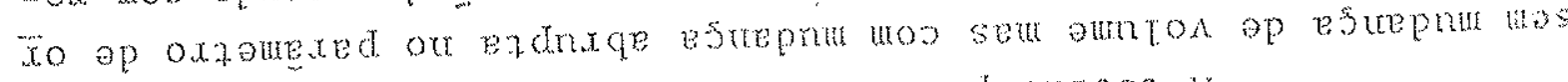

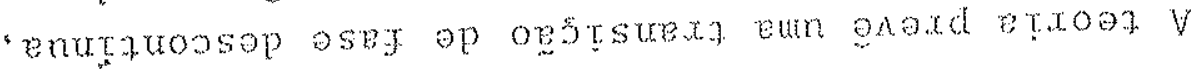

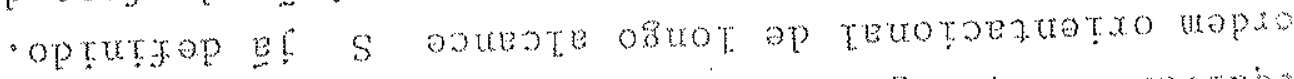

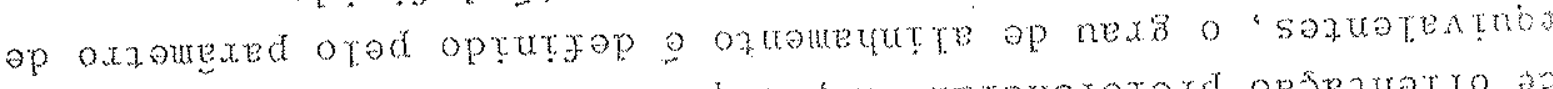

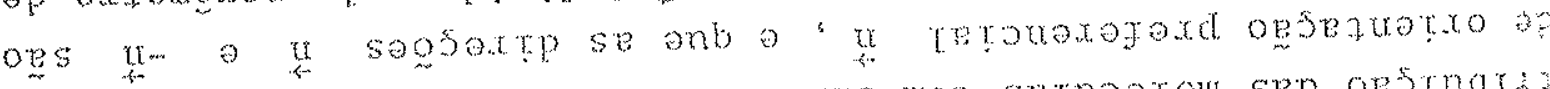

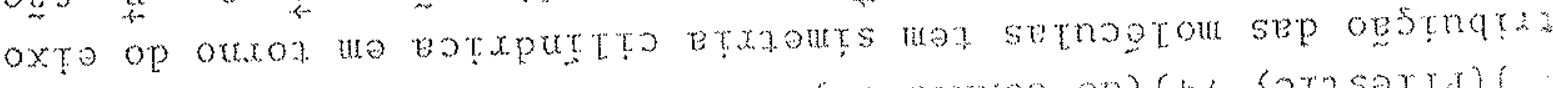

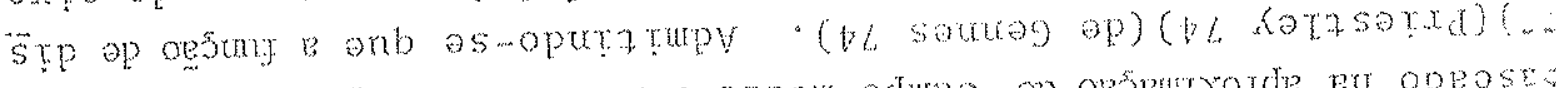

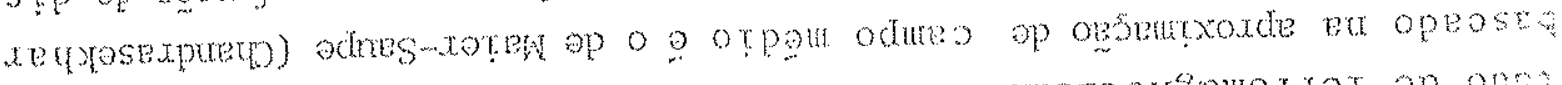

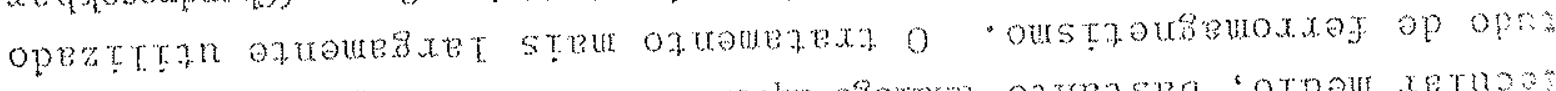

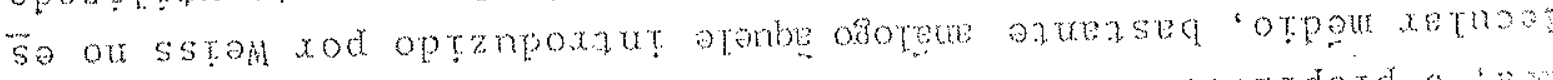

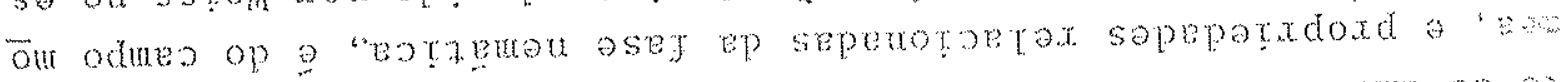

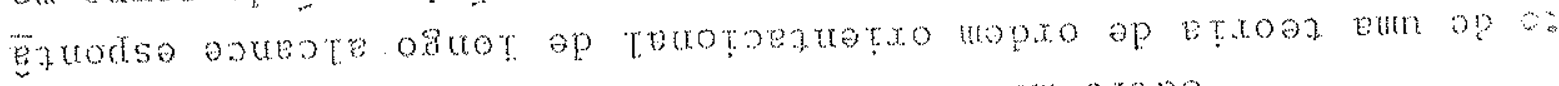

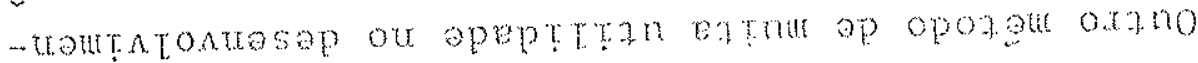

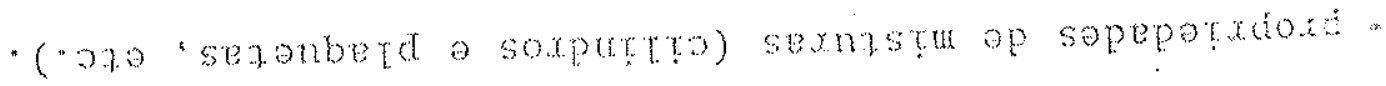




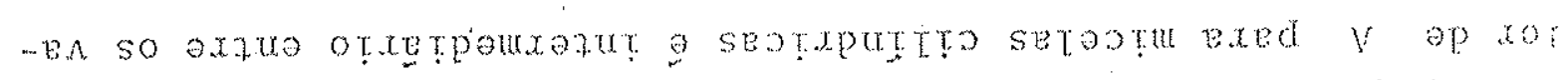

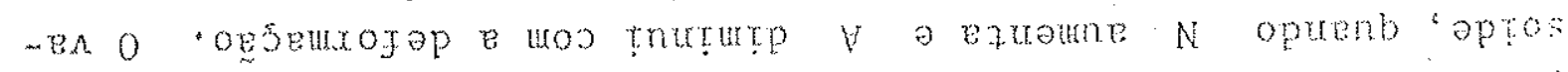

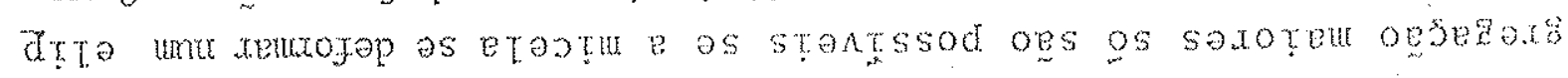

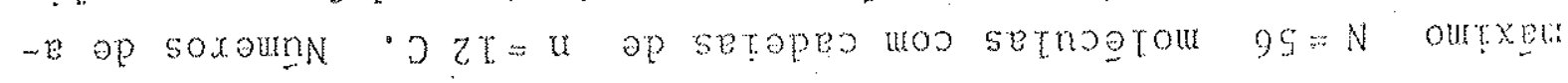

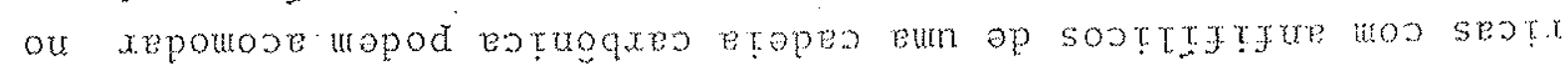

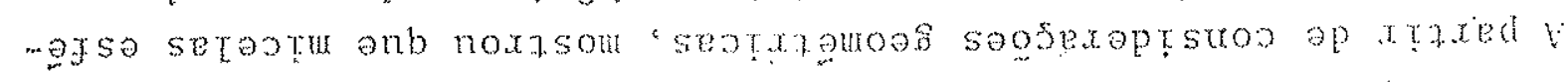

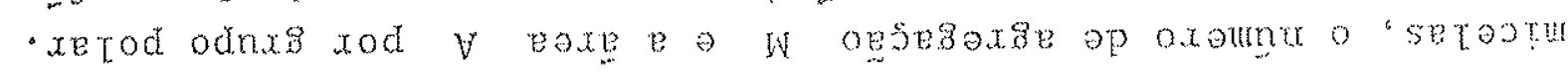

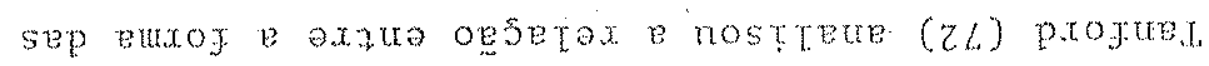

- soserosenot sos

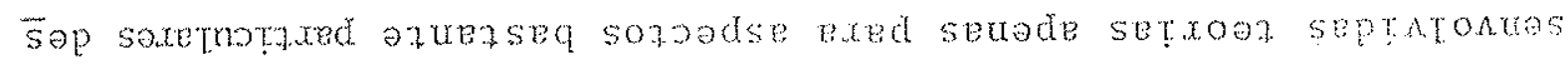

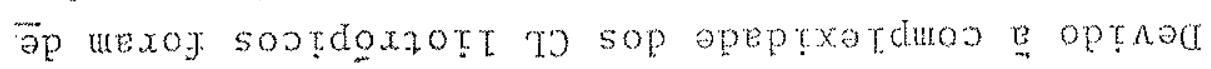

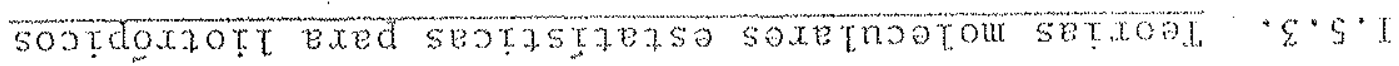

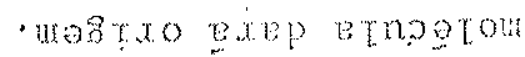

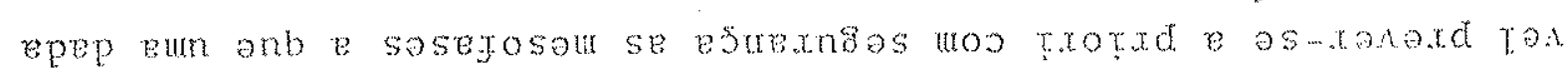

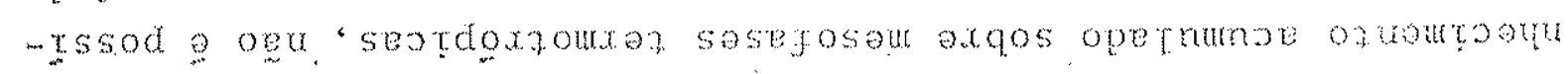
o. o opor ap xesede ónb a reatessex o ozud oxano

(b) dxe azromoduros but

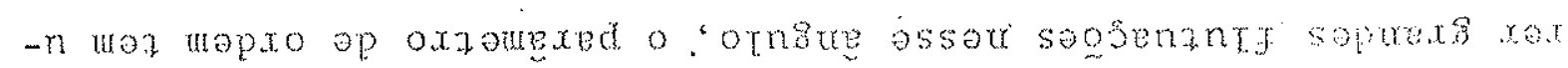

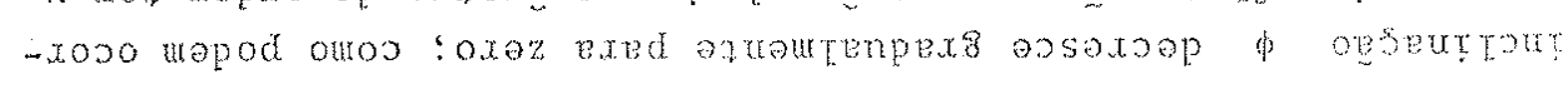

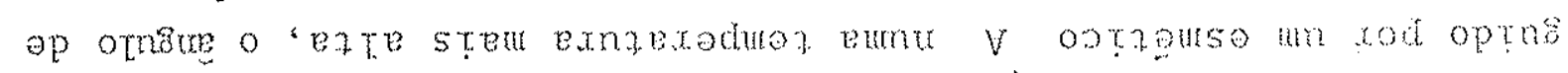

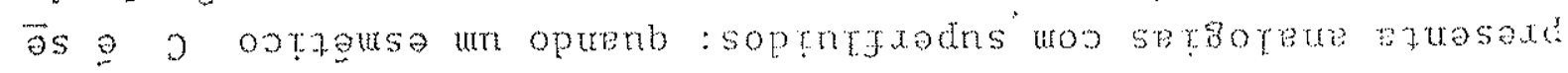

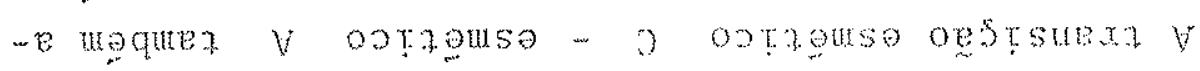

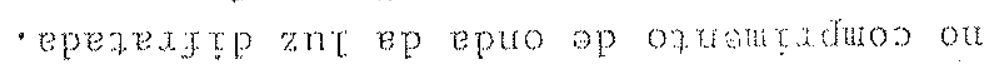

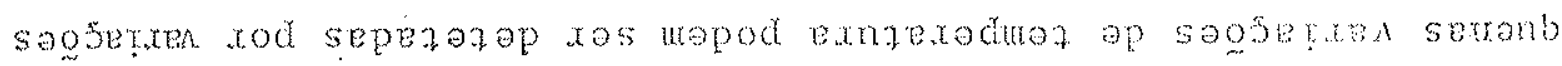

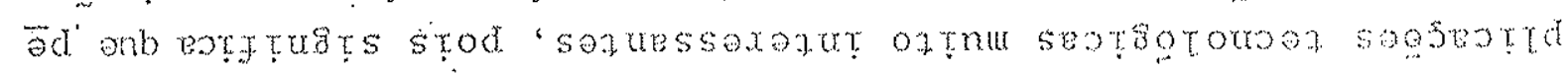

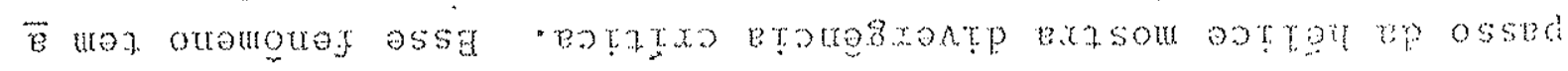

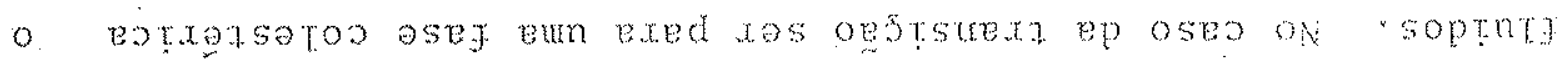

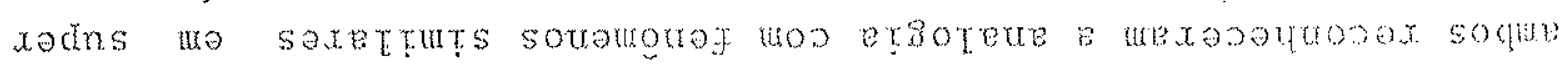

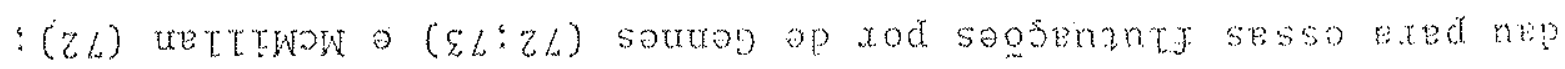

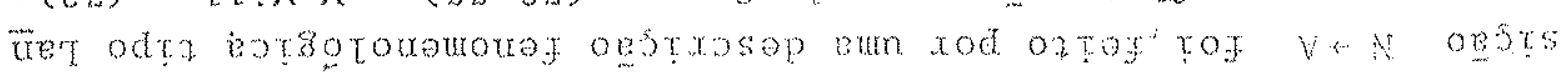

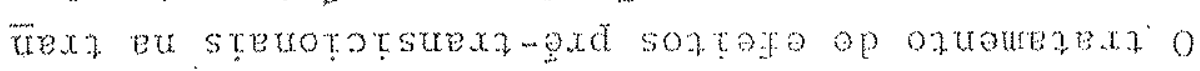

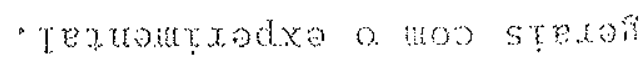

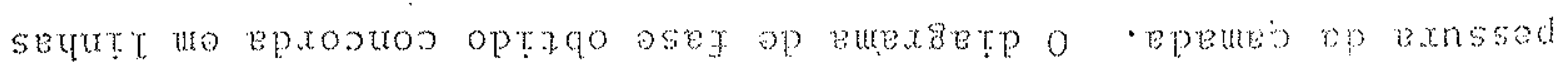

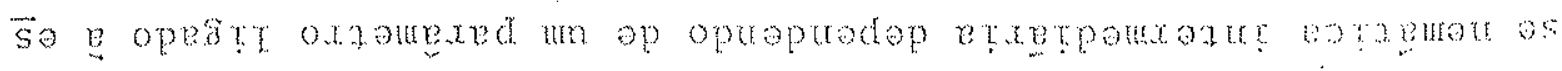

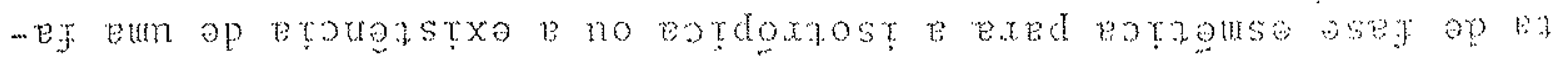

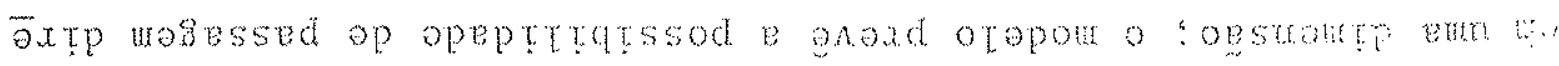

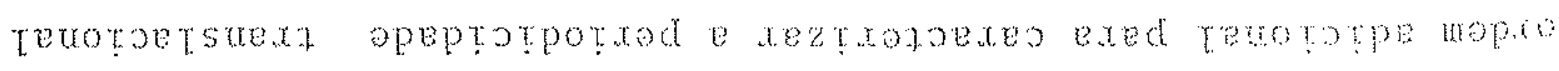

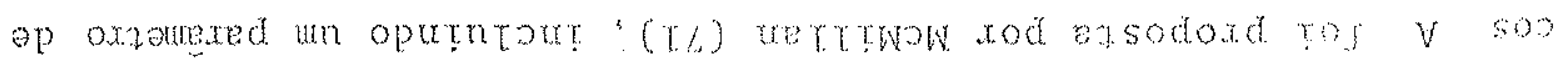




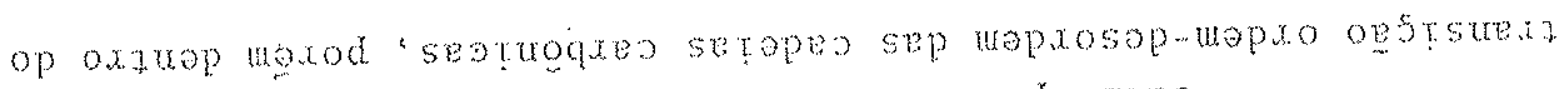

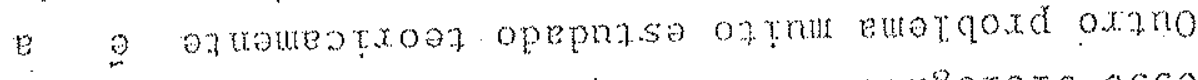

- ospotota assaxazut optex op

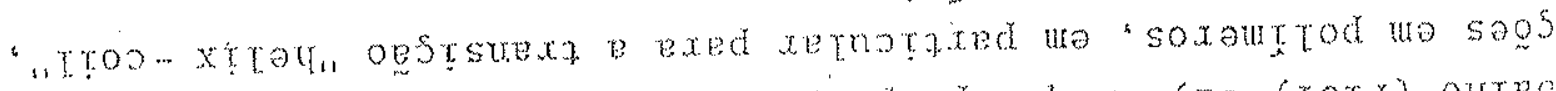

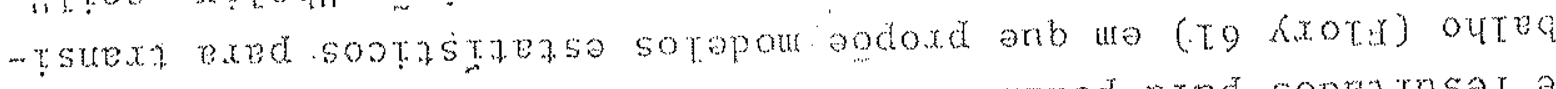

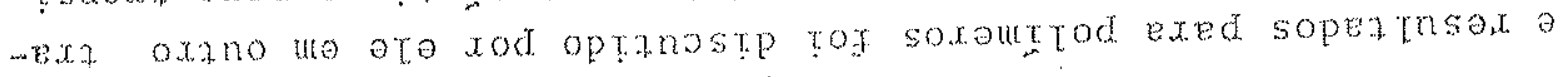

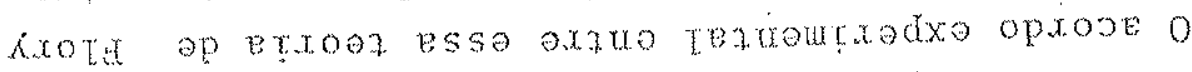

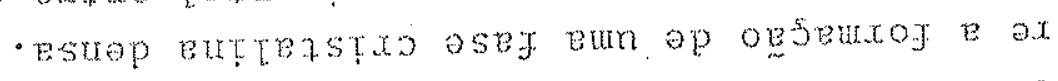

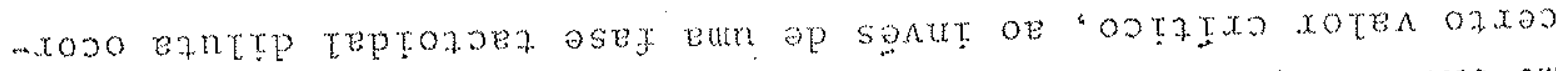

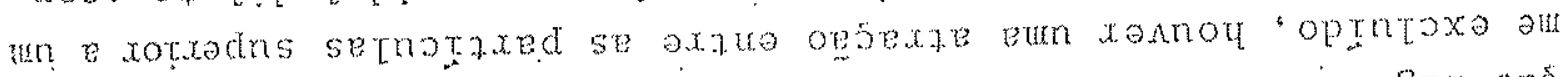

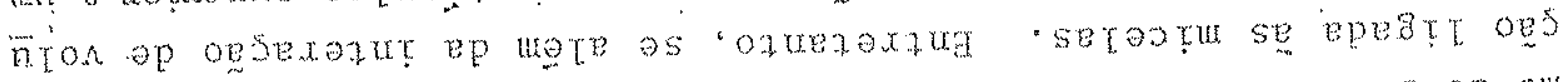

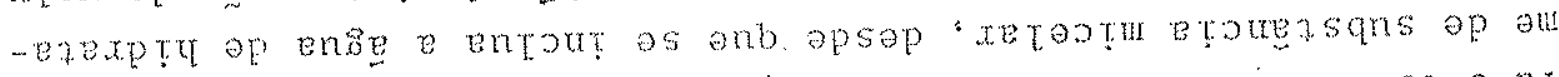

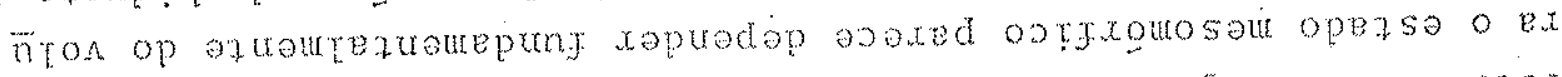

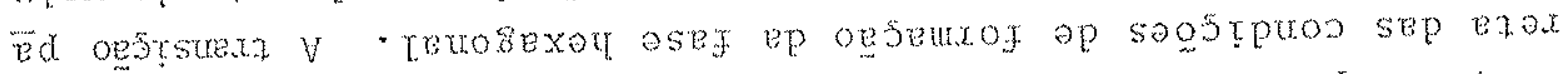

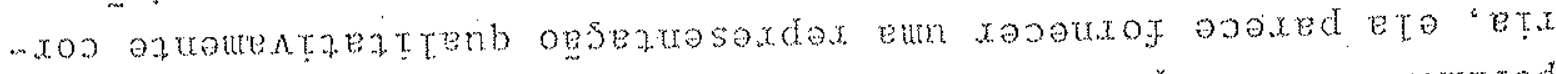

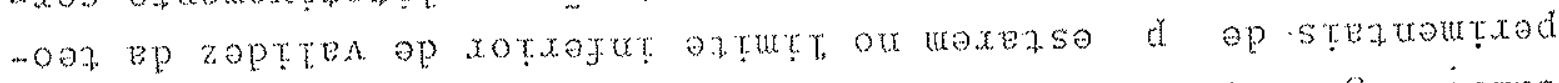

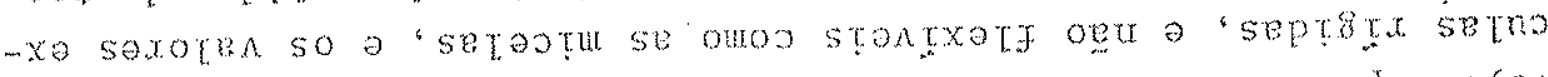

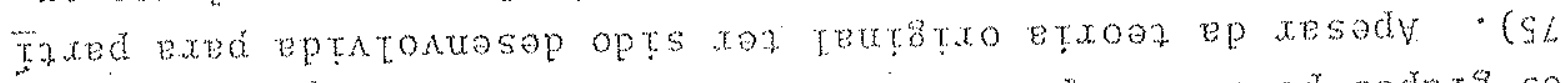

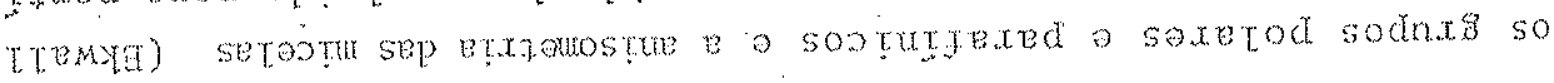

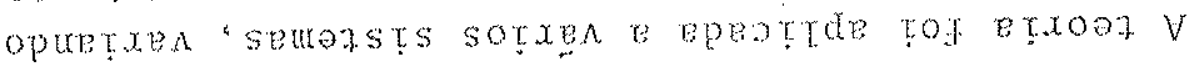

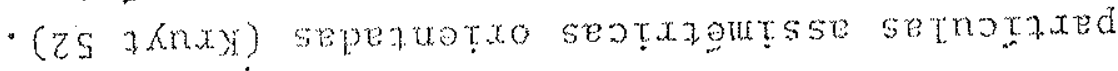

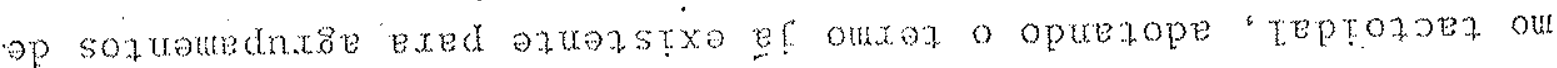

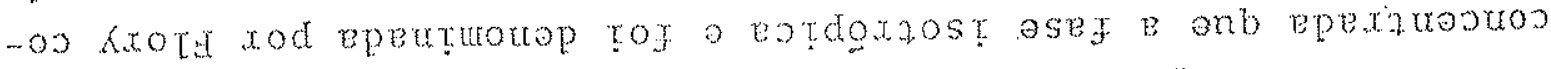

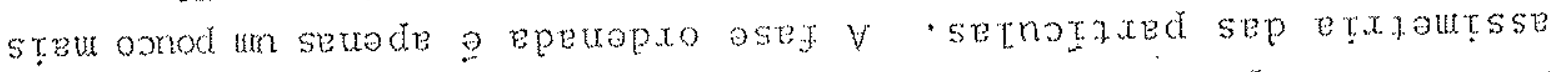

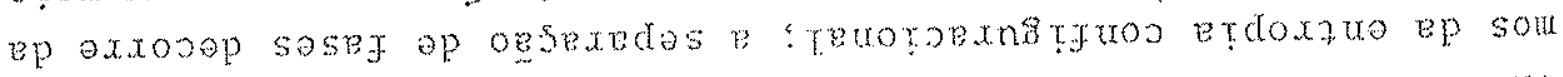

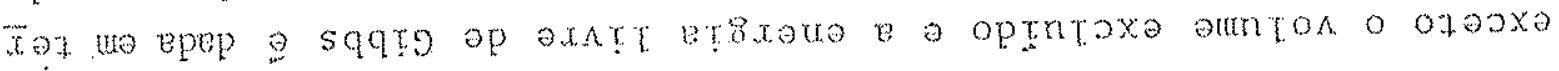

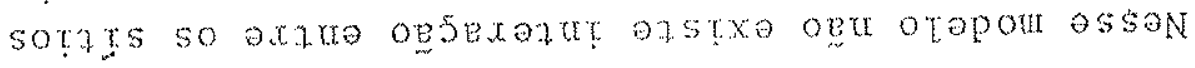

$$
\left[\begin{array}{ccc}
\frac{d}{3} & \cdots & \mathrm{T}
\end{array}\right] \frac{\mathrm{d}}{8}=x^{\phi}
$$

rod epep' soxputre sop d

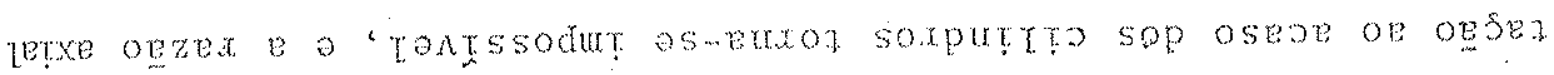

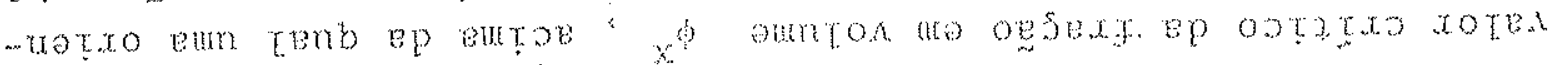

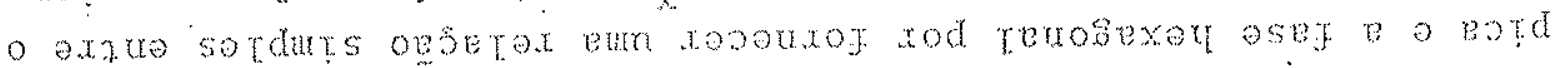

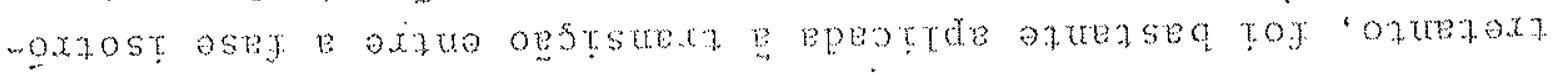

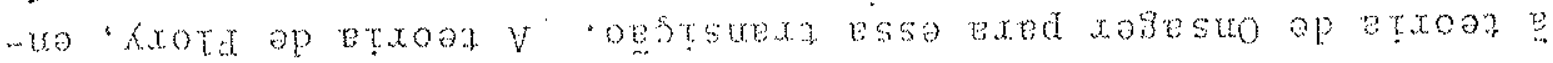

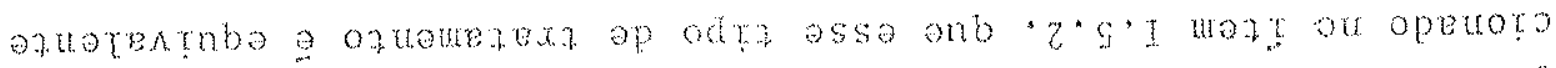

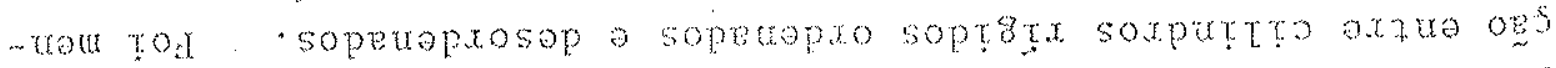

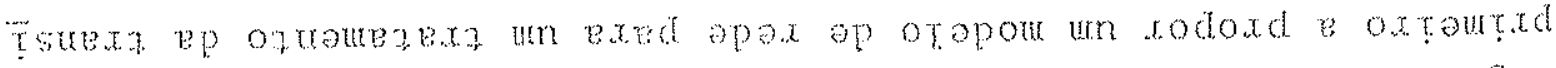

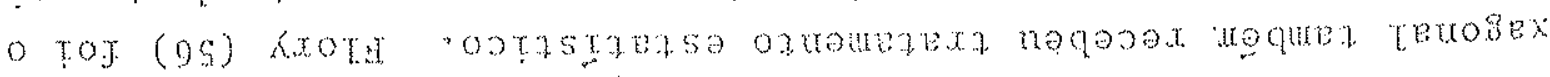




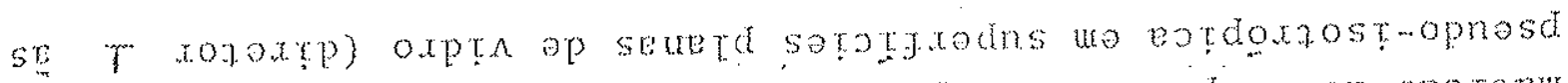

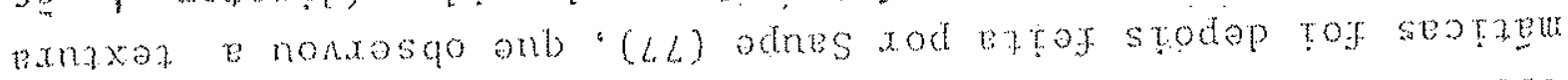

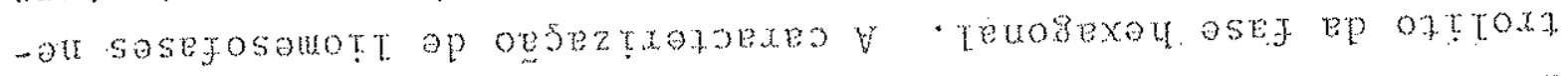

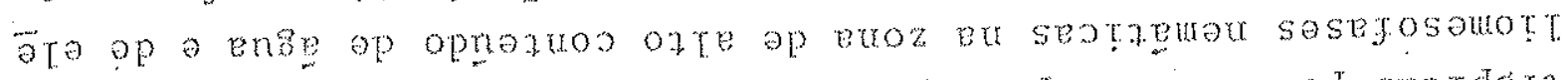

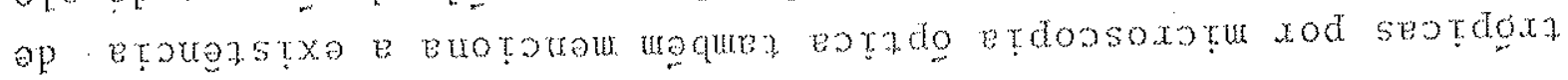
-ote seserosou oxqos opnase nes live (89) xeanesoy

"NWU

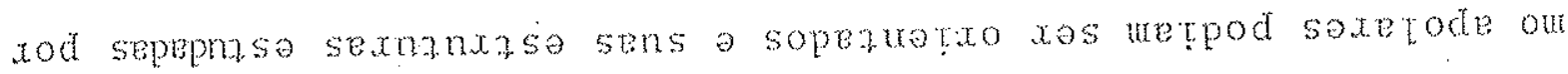

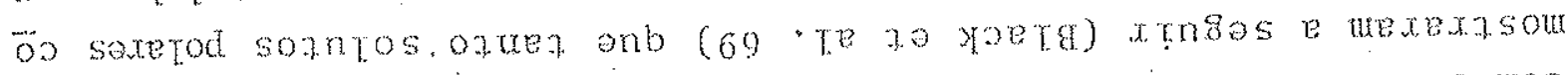

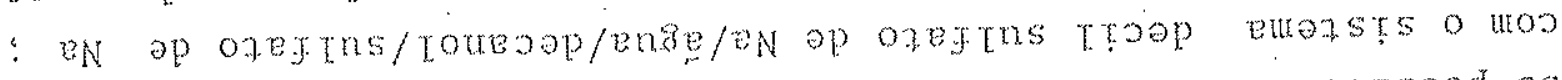

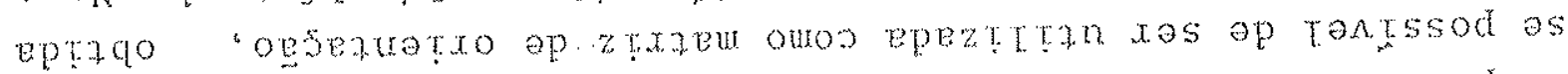

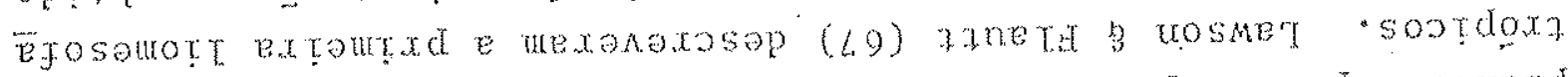

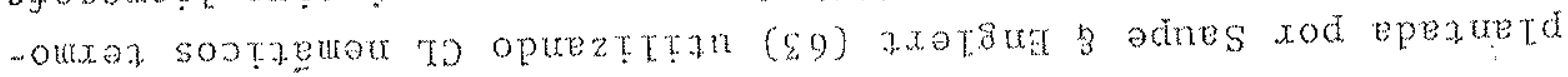

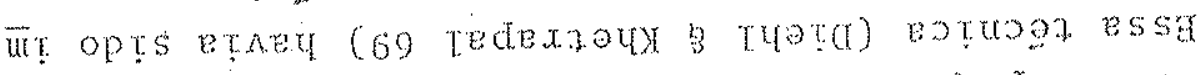

- sopeptruenb seuanbed ue seperototpe oantos

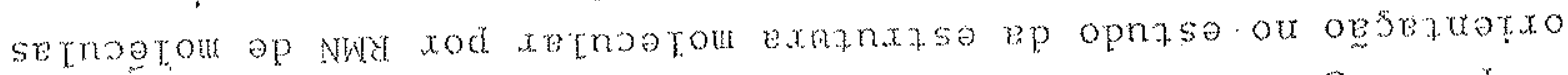

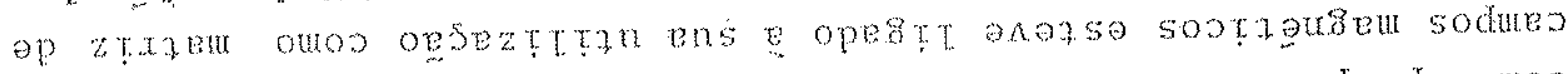

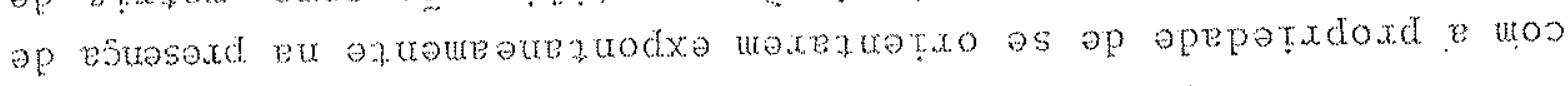

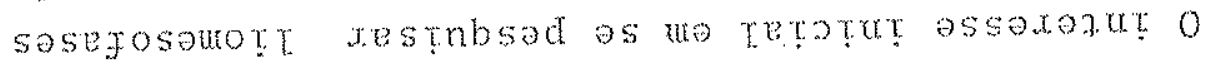

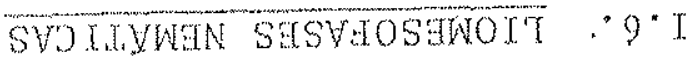

- seroogur op sopesaxse op ot

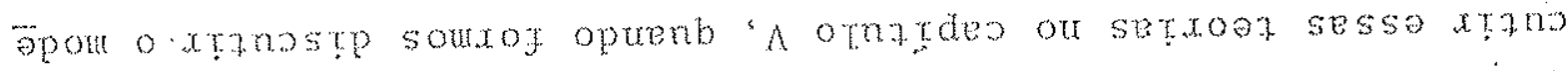

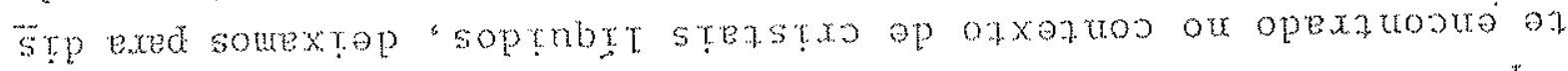

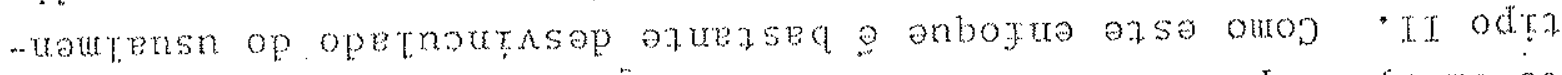

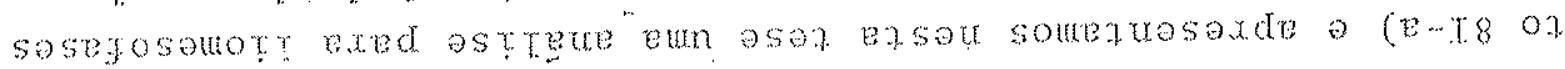

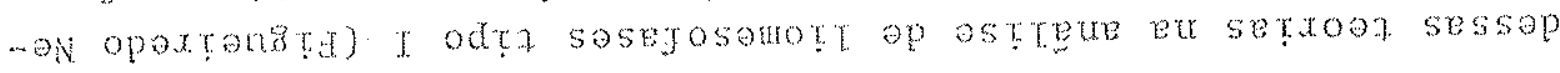

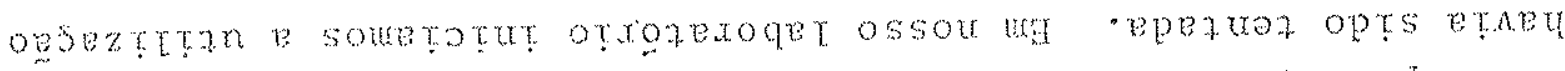

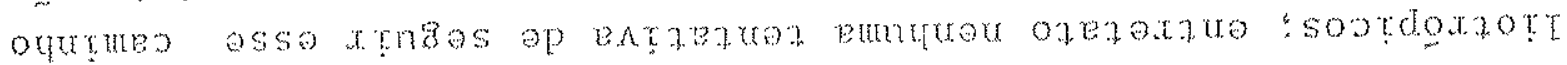

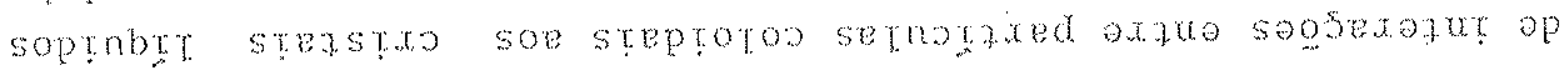

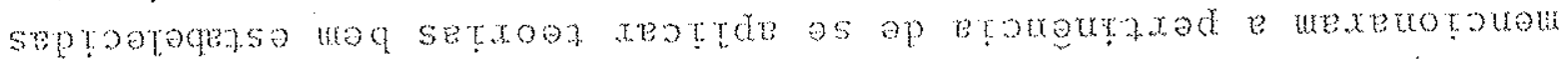

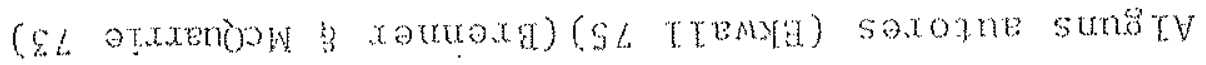

- tr otratapo ou seatroque stropeo

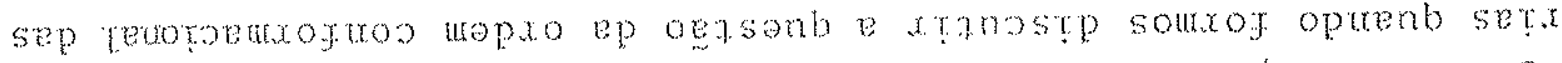

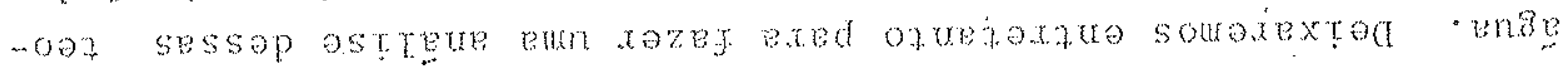

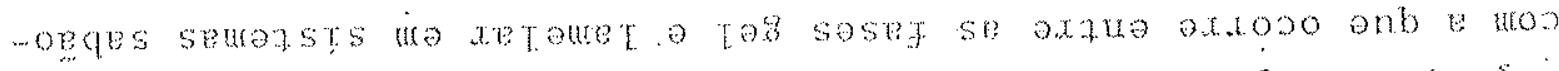

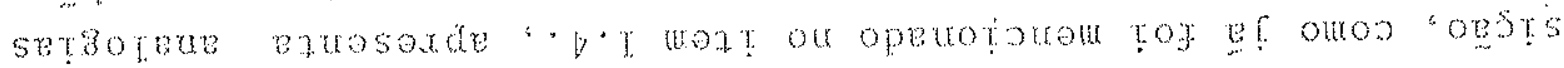

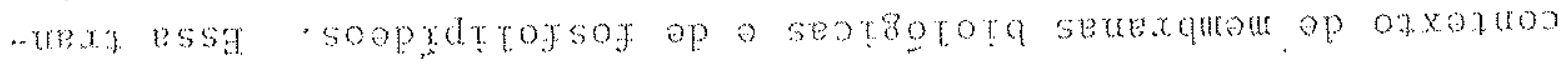




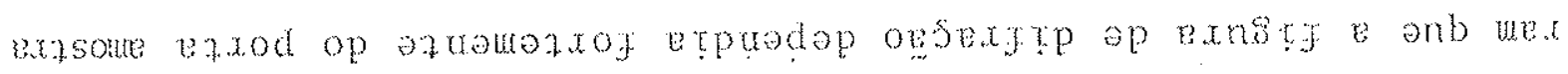

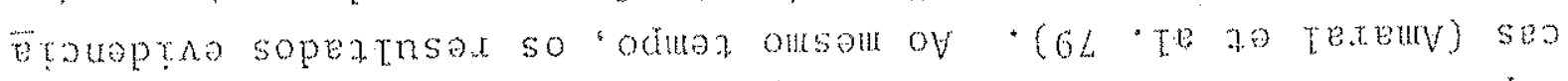

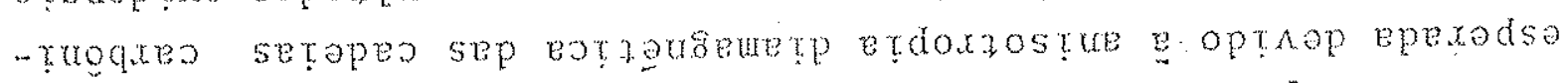

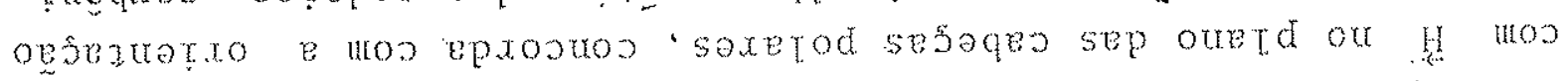

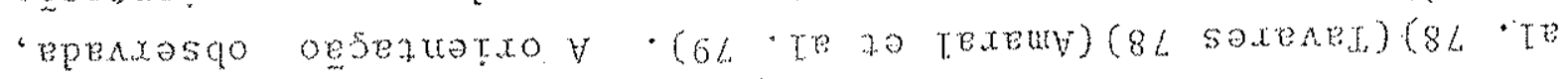

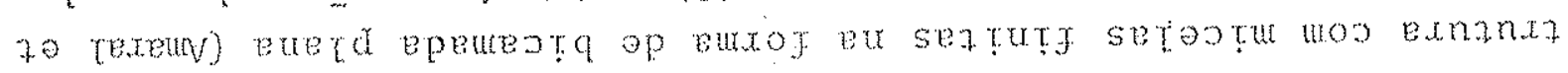

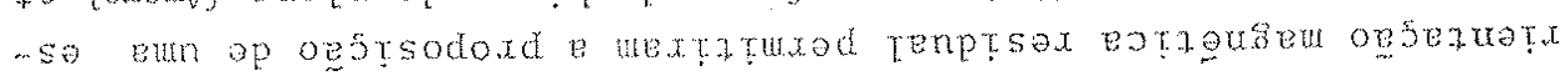
O moo sexasome no soptaqo soperansod -(eN op ozextrs trop woo)

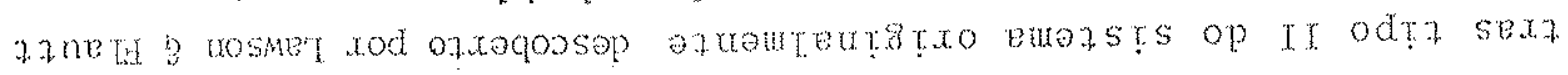

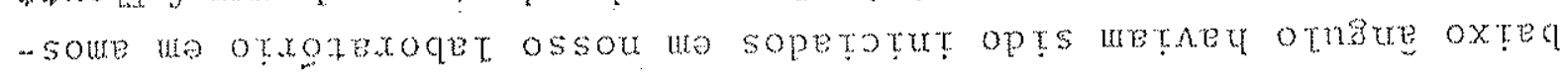
we $x$ sotex op opsbxtp op sopnaso "ofuourtetexed

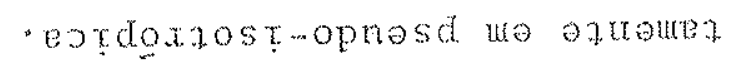

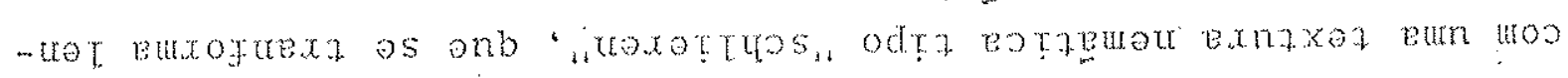

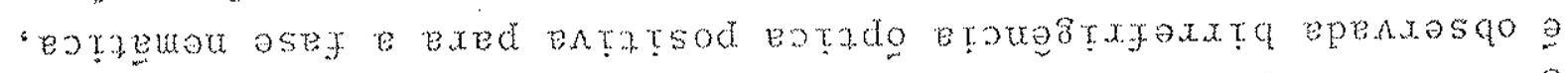

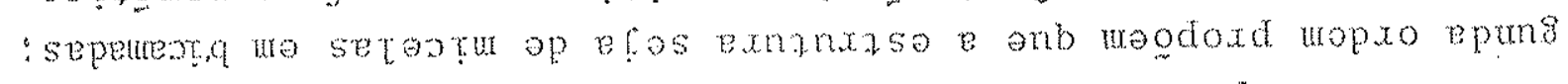

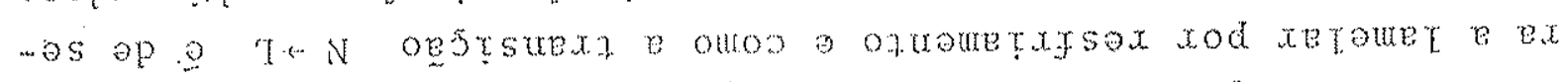

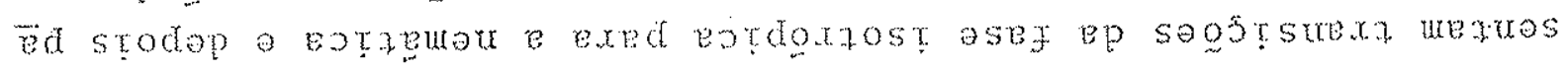
-axde onb sumetsts urexpmaso ( 32 ) adnes 3 kotpey

$$
(96 \cdot T \mathrm{~T} 20
$$

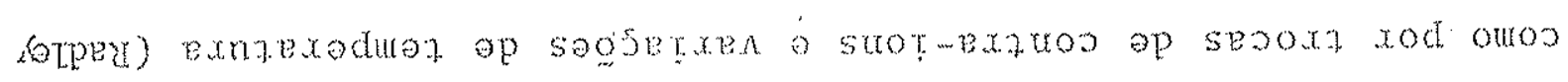

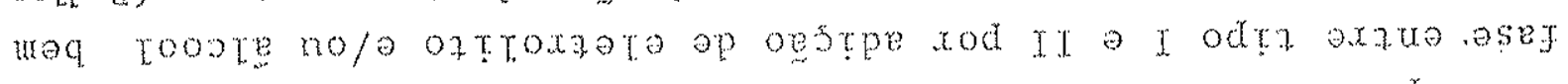

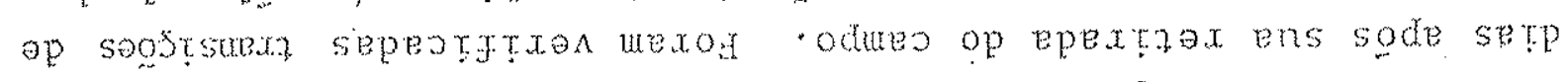

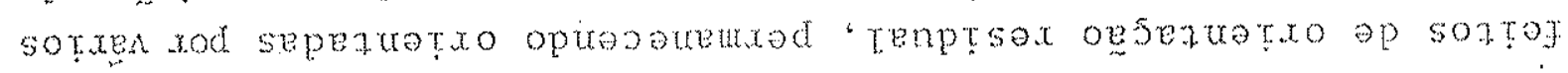

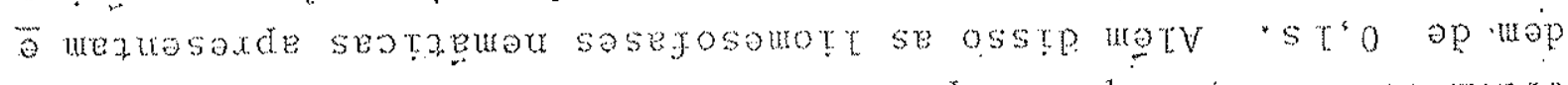

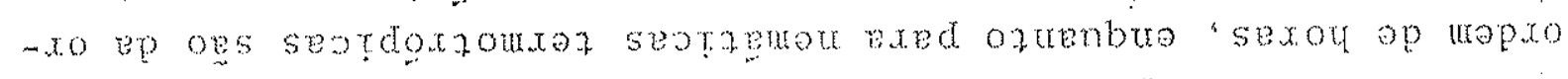

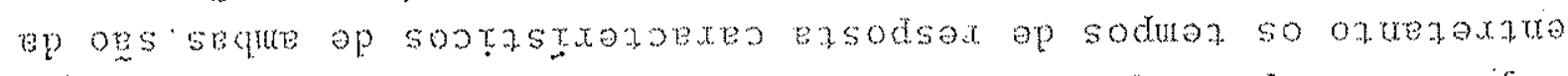

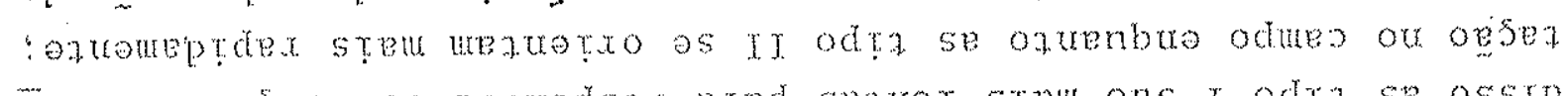

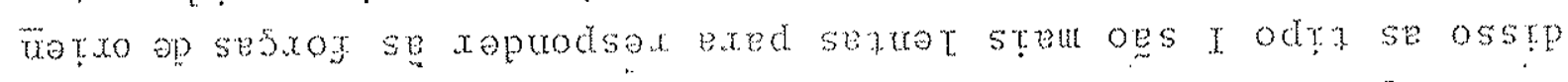

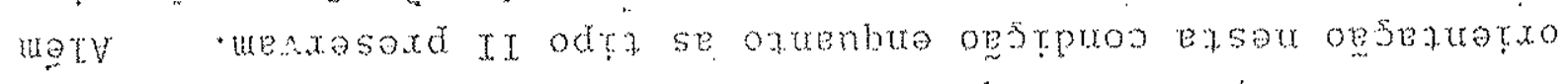

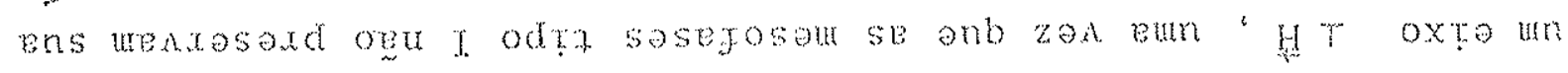

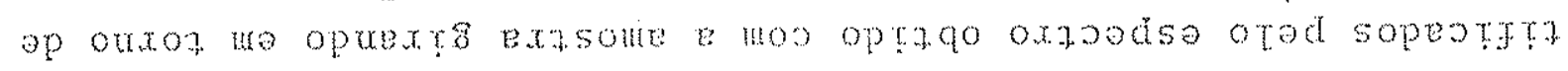

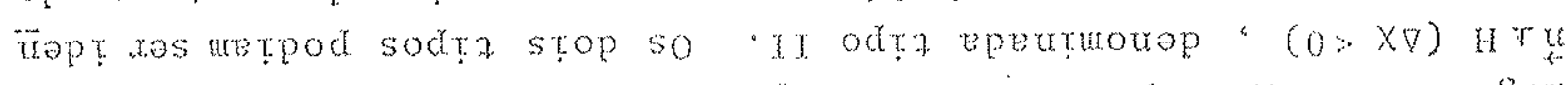

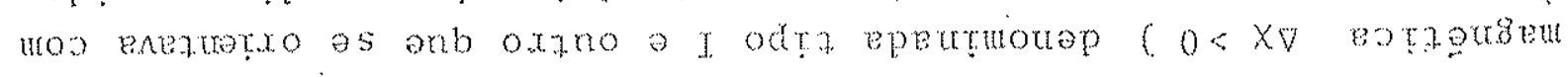

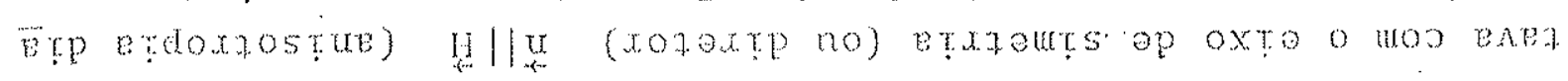

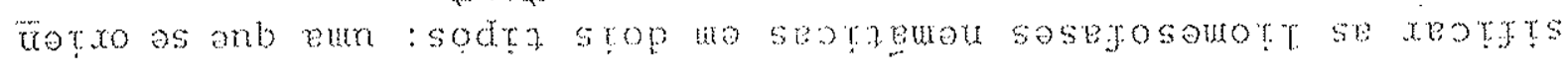

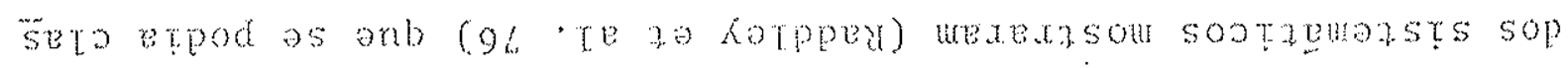

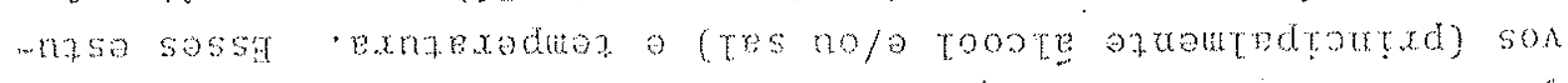

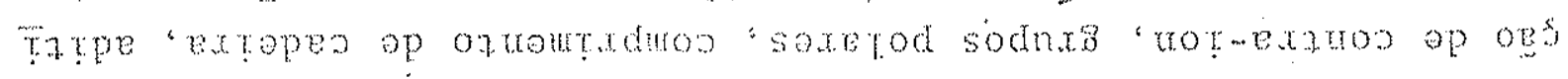

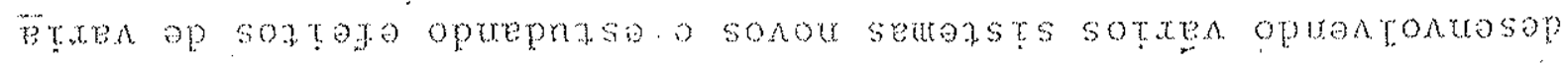

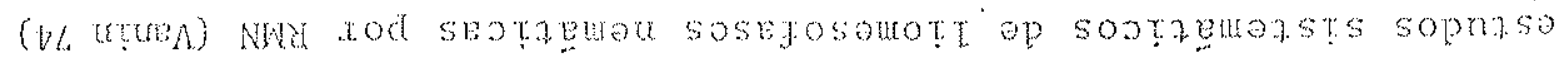

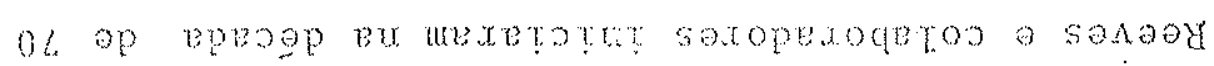




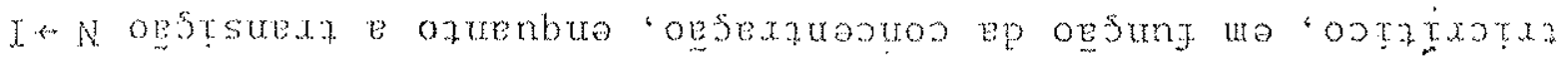

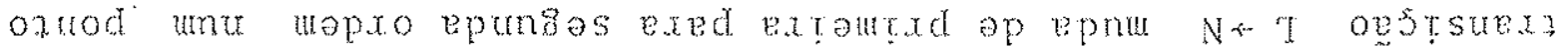
e anb bxasou a "osstp ap euxos bu setestu ap otepou un tros opxos

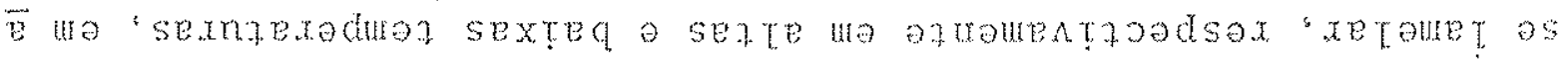

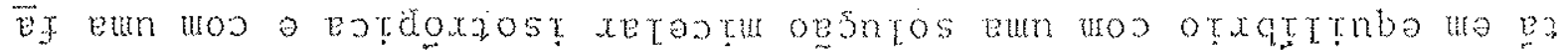

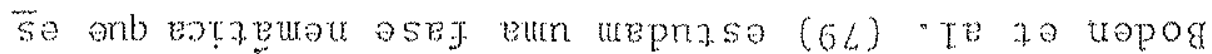

\section{- eztaj roj oed ol}

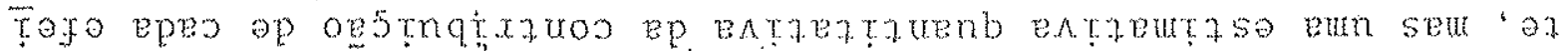

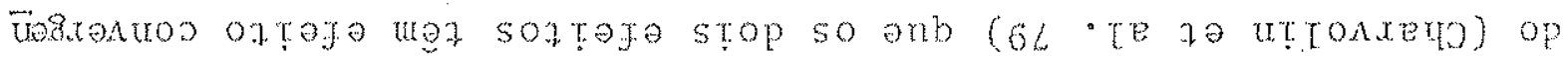

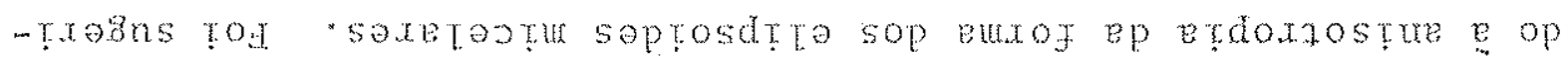

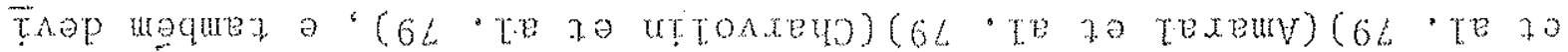

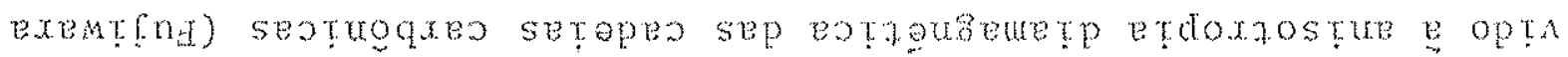

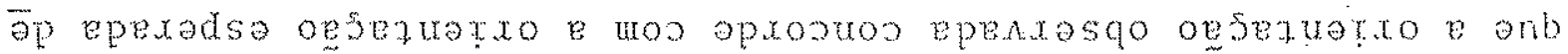
wros zey $\left(0>X_{\nabla}\right.$ uros sezanberd a $0<x \nabla$ wos soxpurtra) eorzan

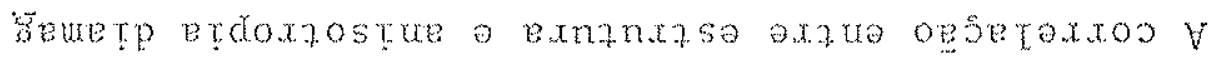

- asad basar a thejop lia sepr?

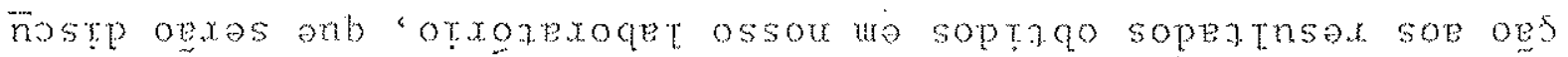

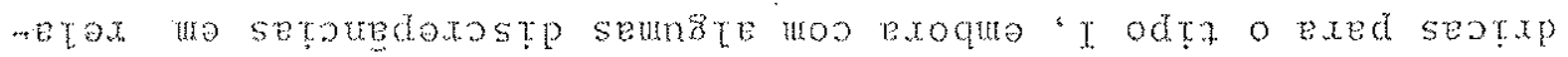

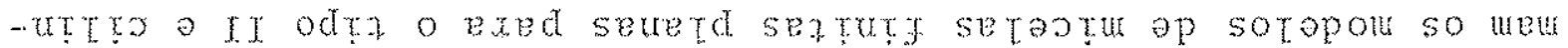

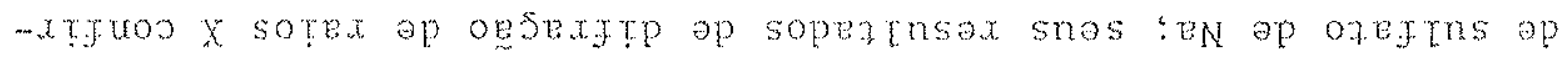

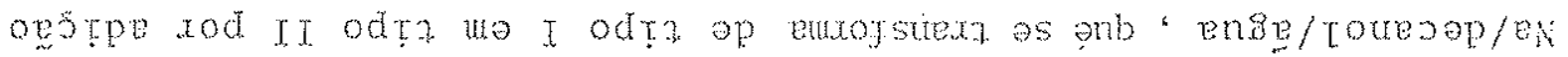

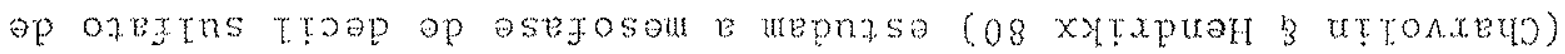

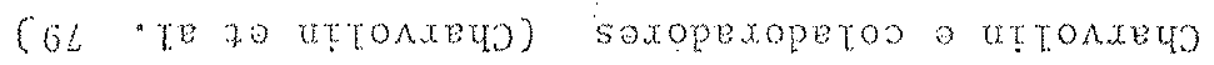

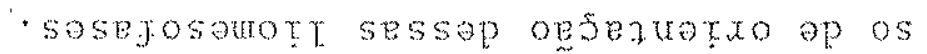

soooxd orod stangsuodsox seasodns "spotroqua setapea sep estrox

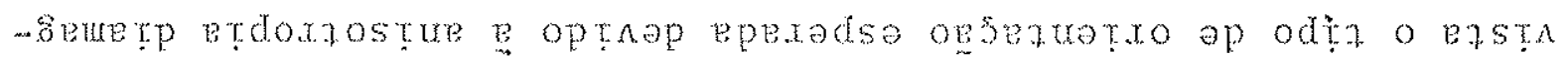

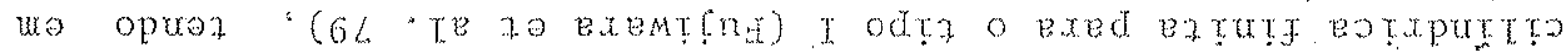

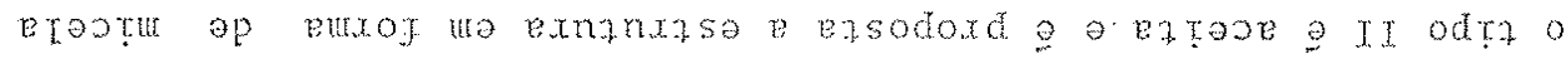

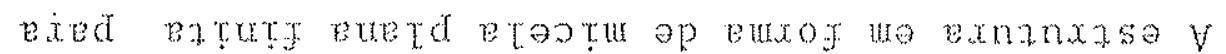

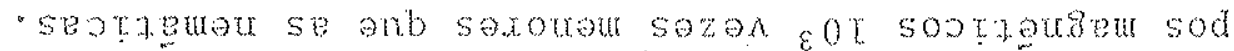

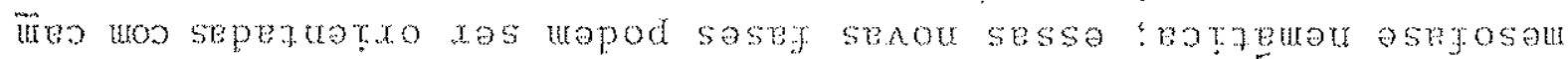

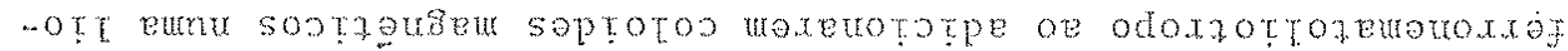

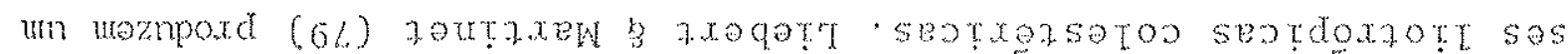

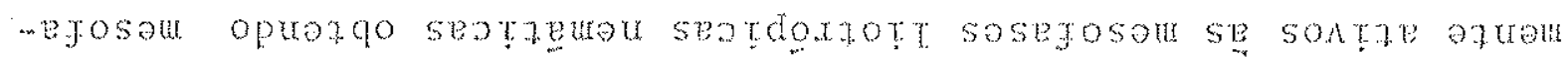

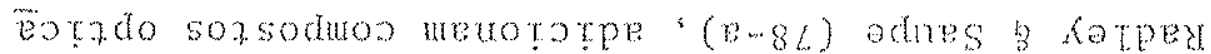

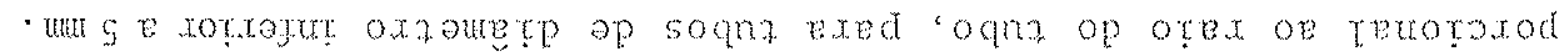

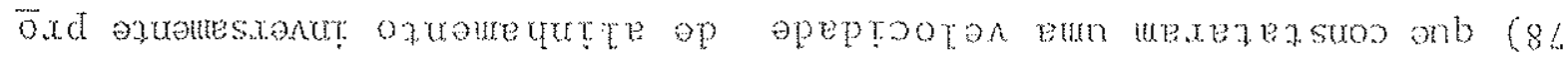

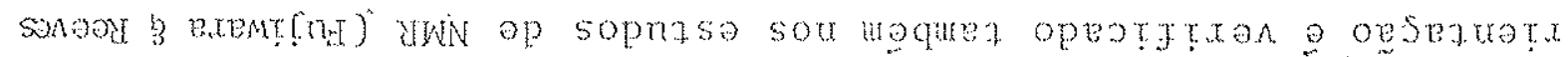

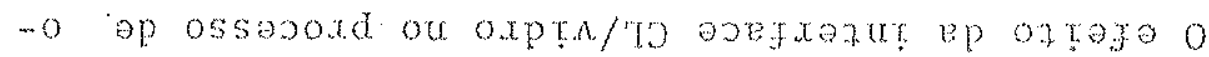

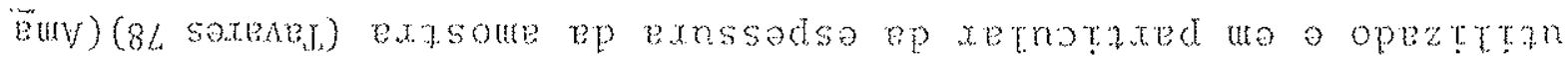


- oquo setopes sep se sez sodo seotagusemetp sepepotadoxd uos sos

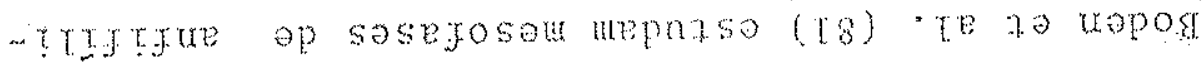

- (e-tr oxon opodtanitid) (os

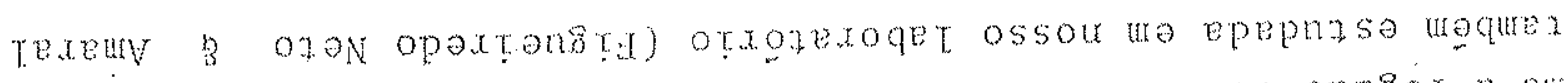

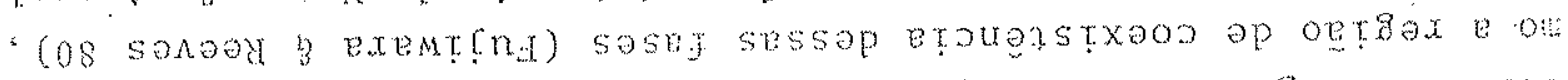

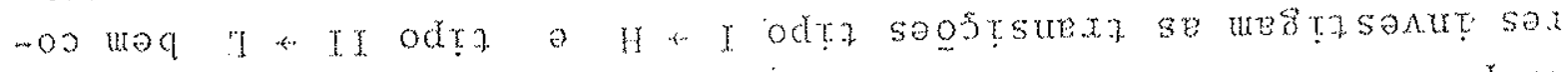

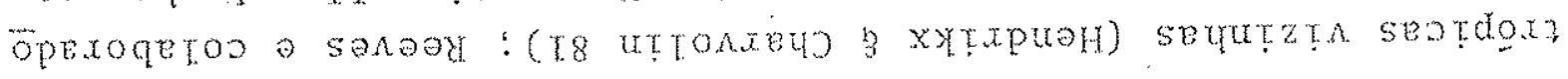

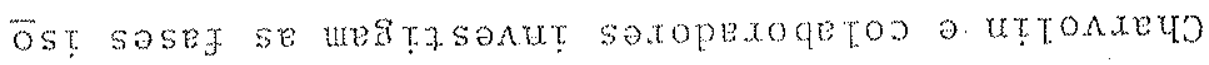

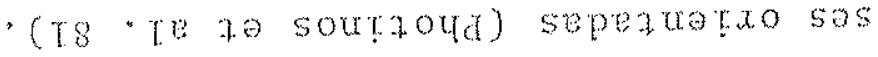

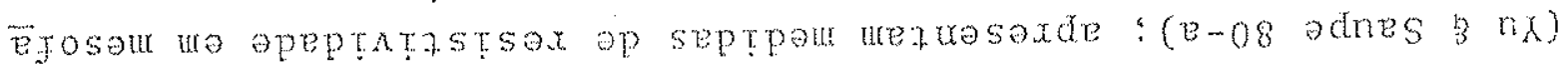

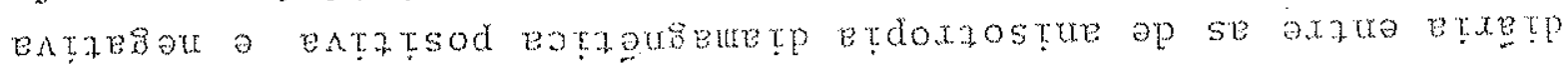

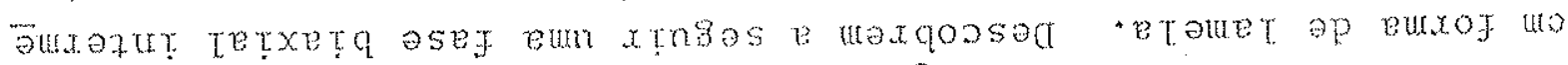

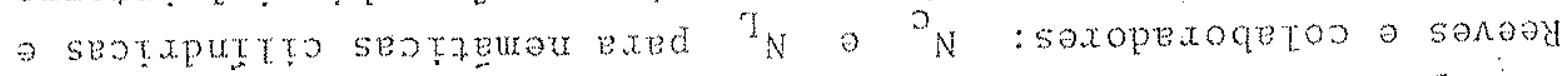

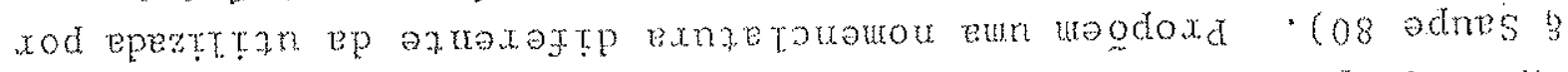

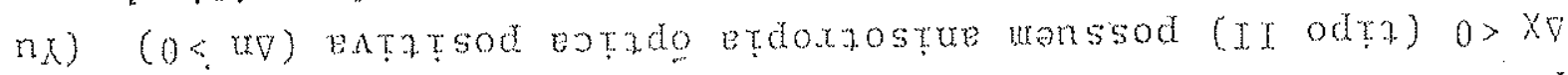

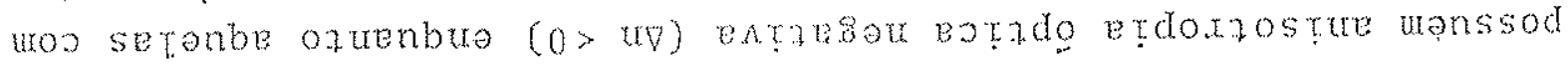
(r odra) $0<x y$ woo seseg se anb opreotgtan "sosegosourt sessop opnase o mensossoxd woque? soropexogeros a adnes

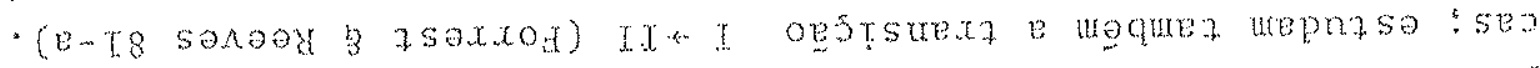

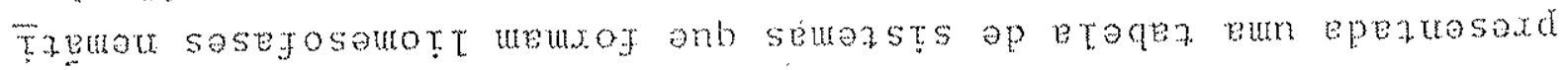

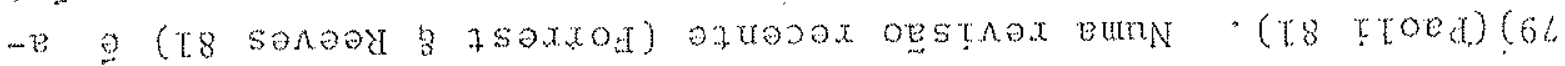

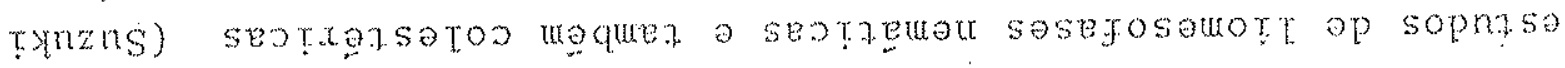
Honsossoxd saxopexoqetoo a sonood 'onturetotered

- C.8:08 TQP

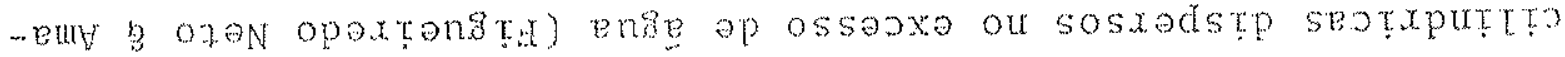

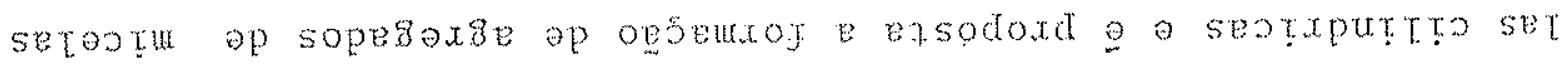

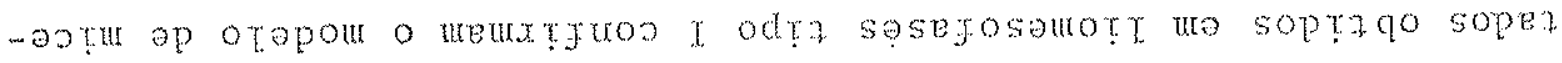

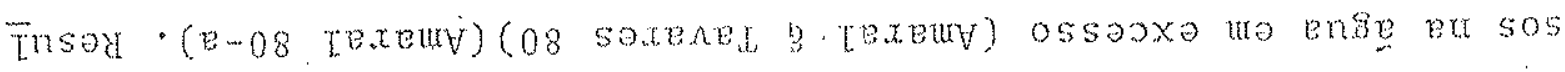

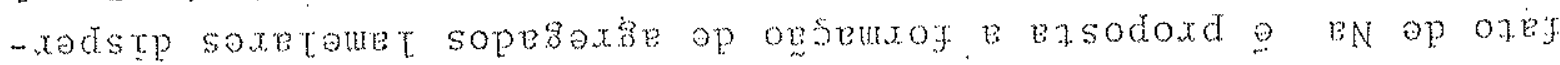

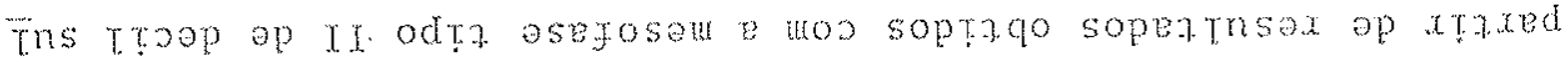
$V$ ofxomexoqu ossou we soptaqo $x$ sorex op ogsextrp ap soperths -ax sop xtaxd e eperotasonb e sasefosourot seu ense ex seratu

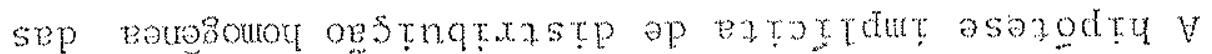

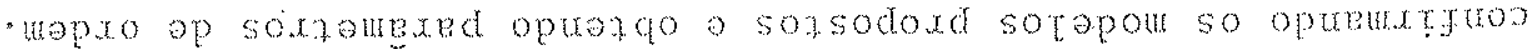

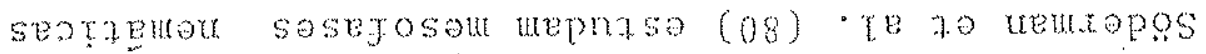

- aspasou up xozatip op

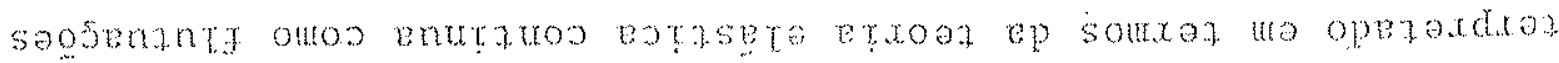

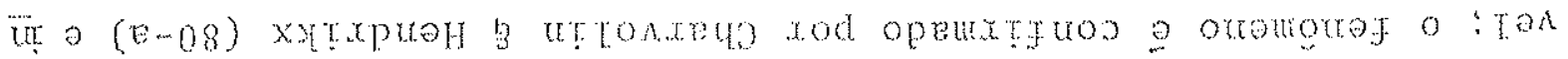

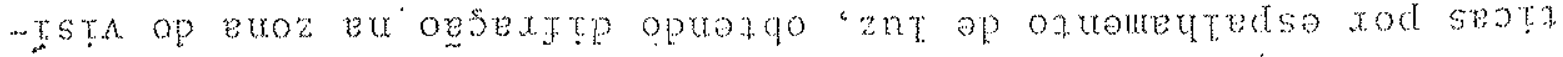

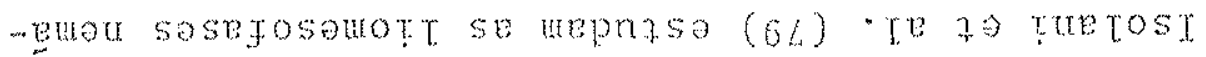

- mopxo patoutard ap axduso 


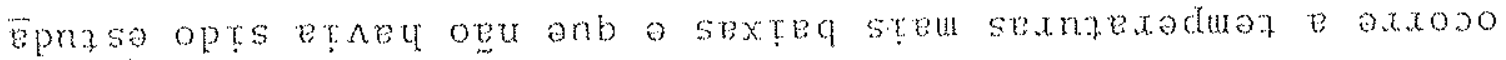

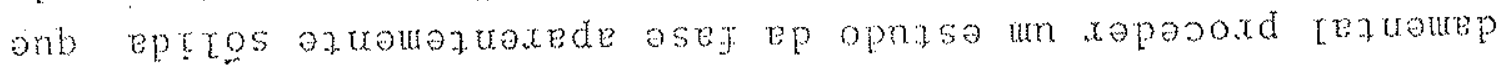

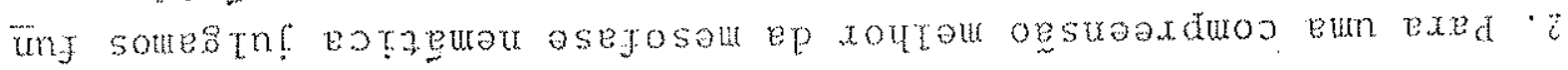

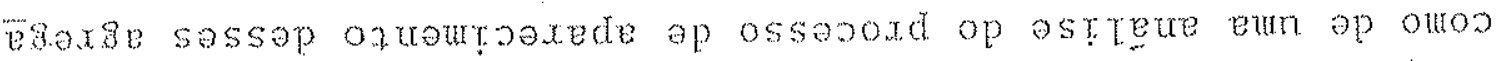

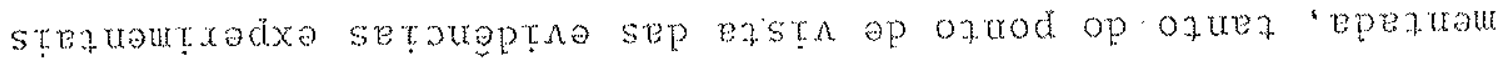
-epunt xoutan xas enestoxd "ense op ossoxxa ou sostadste sax.

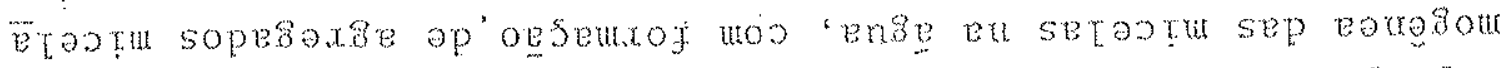

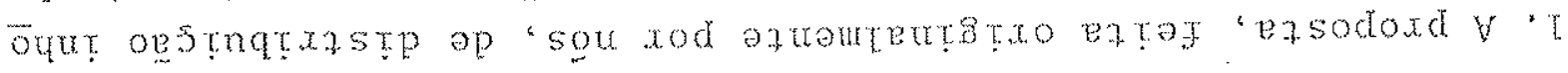
: ofuatrou ou sezuenetox steu se uexopoxed sou anb saozsonb op xtaxed a asmoxtontosep outequx onsa

$\therefore \cos 52 x e A$

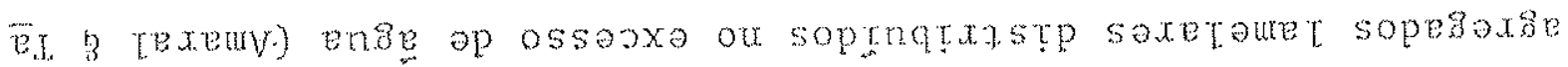

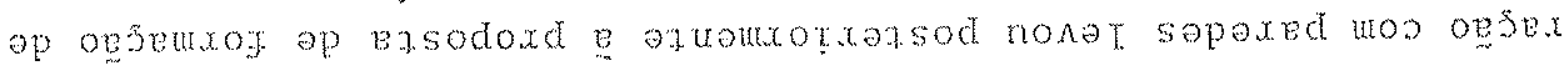

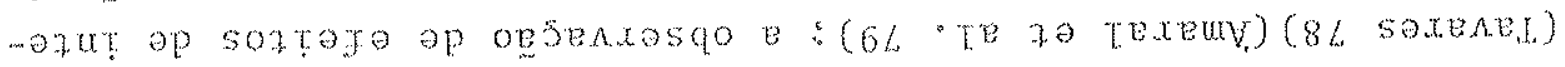

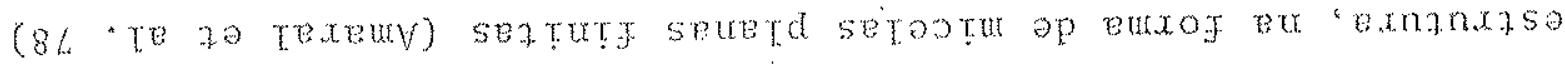

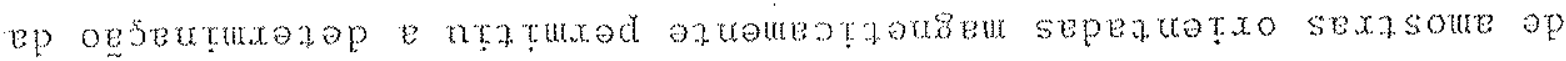
oproxe oxpeq we $x$ sotex op ogsexte rod opnase

$\cdot(8 L) \quad s a x$

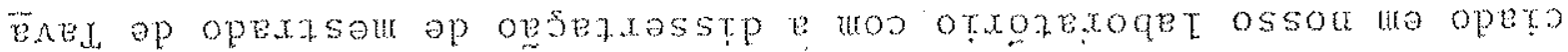

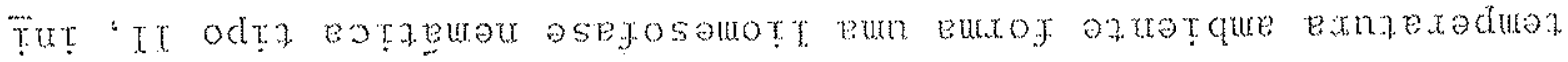

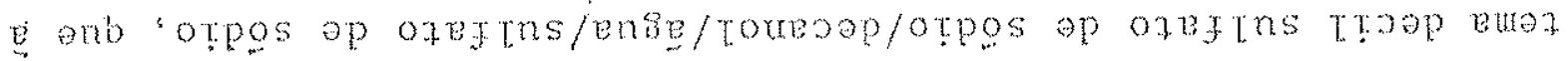

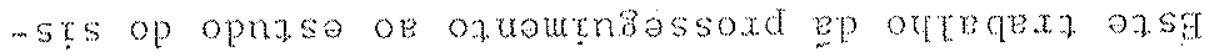

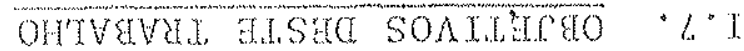

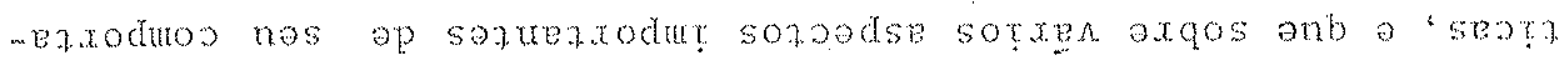

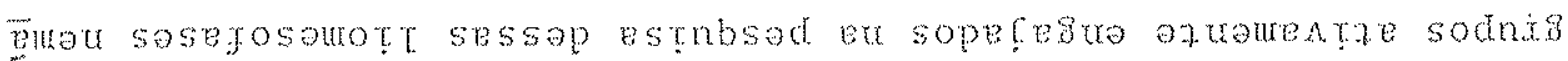

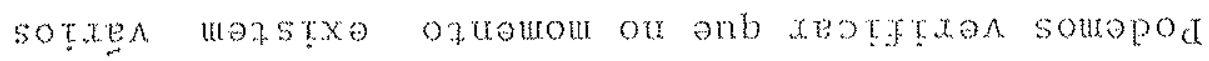

stepodse soottrtes

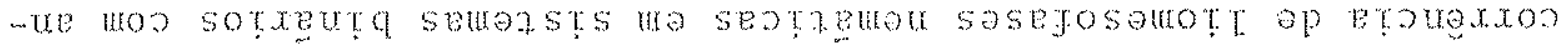

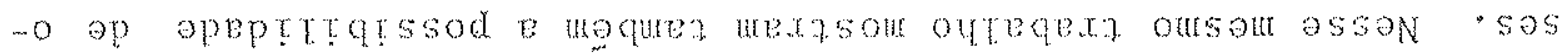

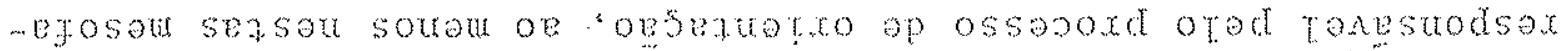

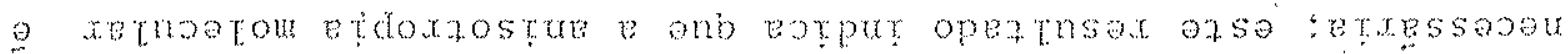

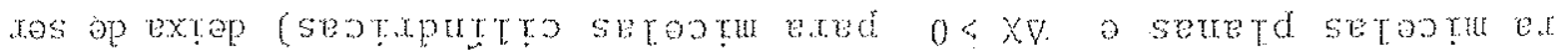

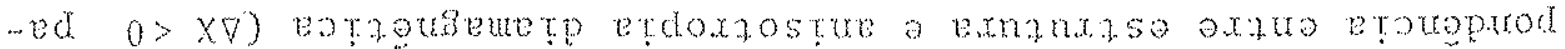

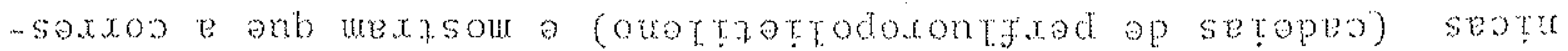




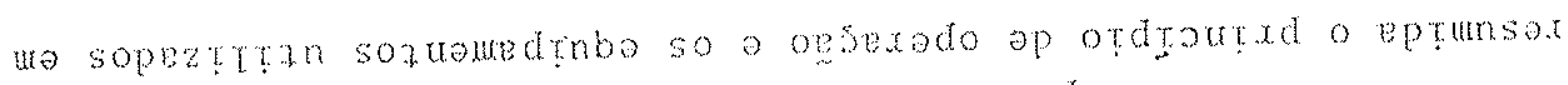

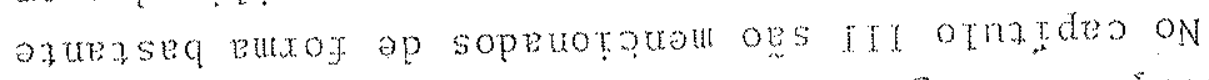

- eotrangemetp og 5 anduro op soustu

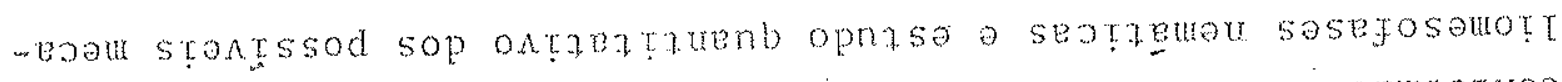

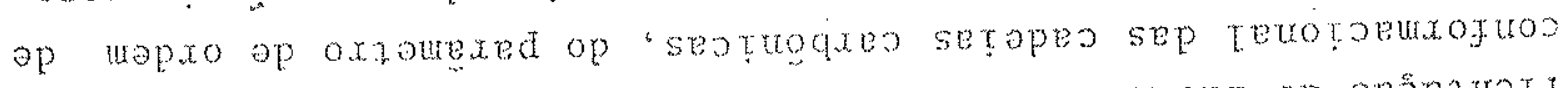

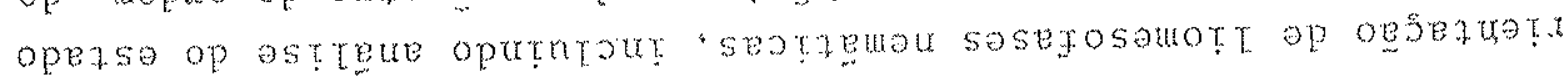

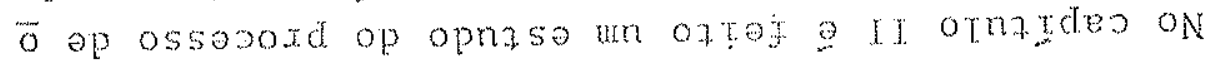

: epexnanase ursse pase asez easa

- ozsodond sopes

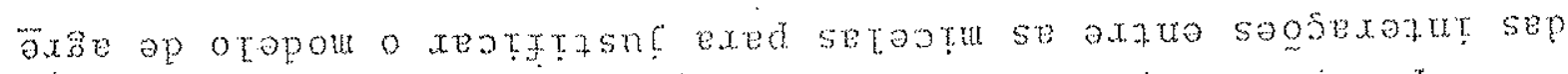

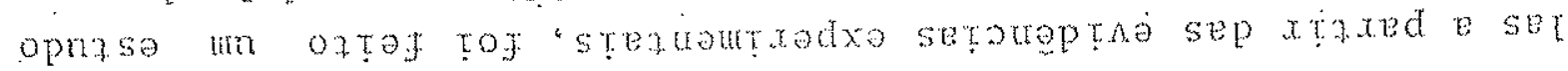

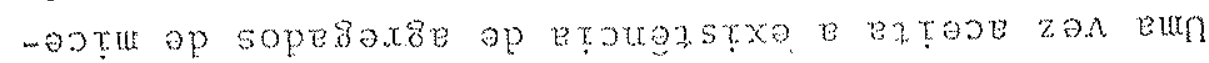

- estugata apeptiap ap sotapou asmopuezt

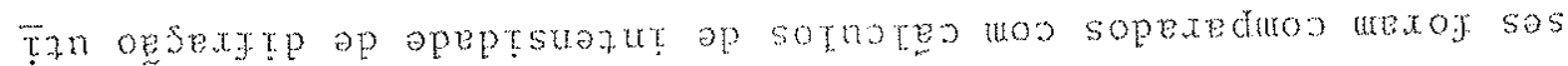
-ey senp sex $x$ sotex op opsexte op sopertusax so

- apepter ens xez

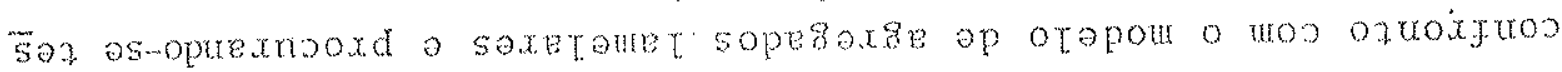
wa sopestefere wexoy soptrgo sopeatrsax so sopot

- exnegorto xod sertox op

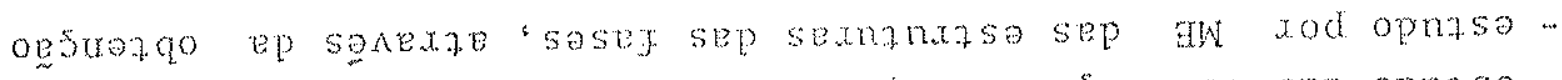

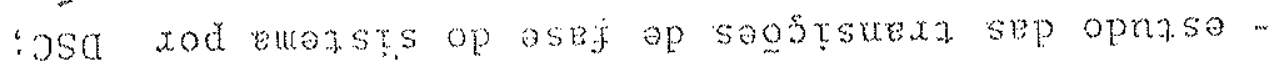

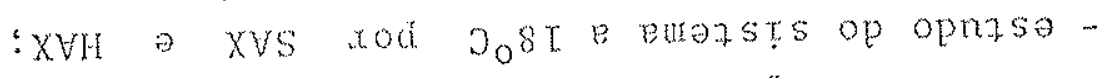

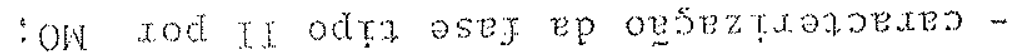

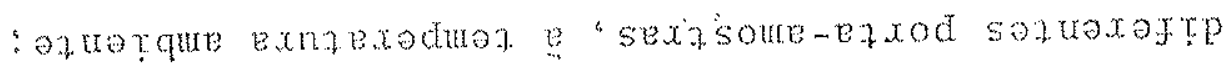

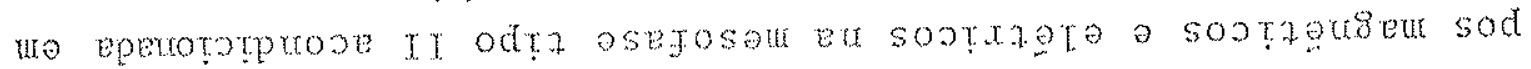

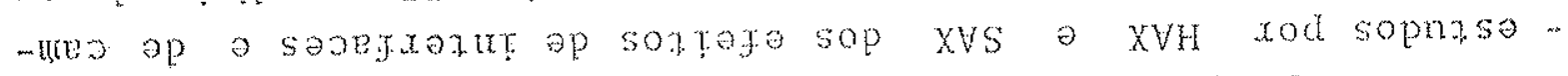
atreur onb septpou op eursoxd un opraton

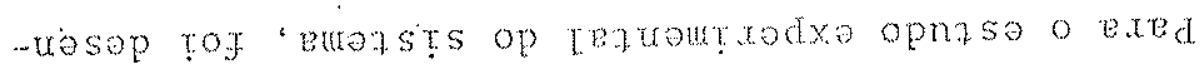

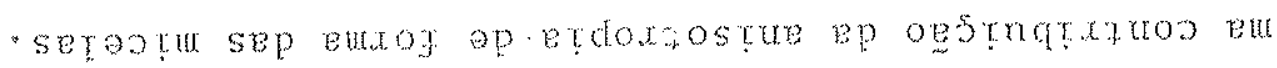

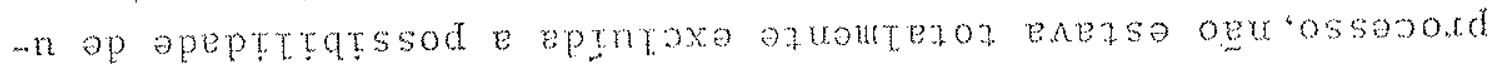

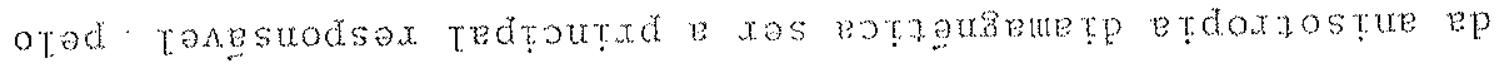

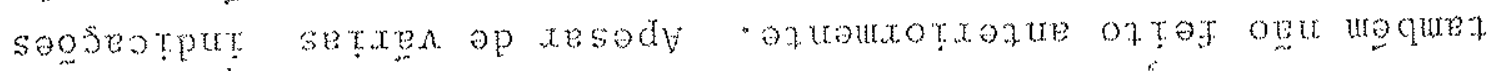

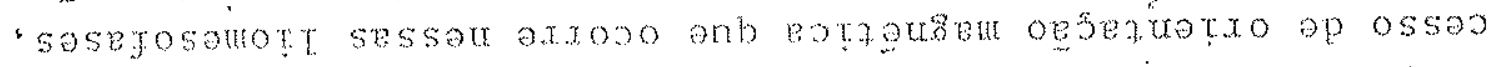

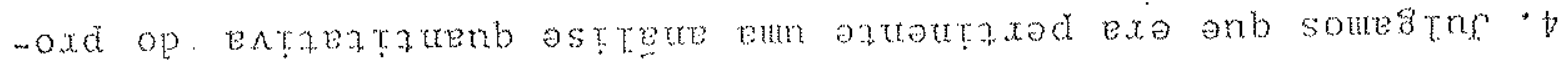

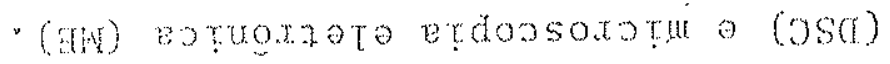

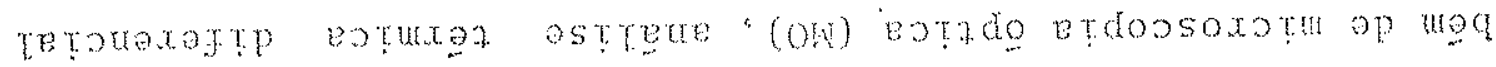

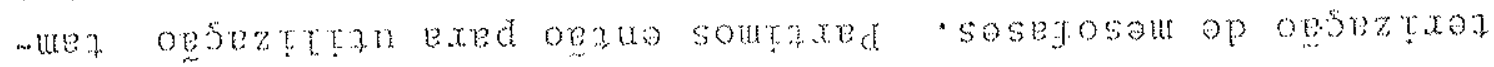

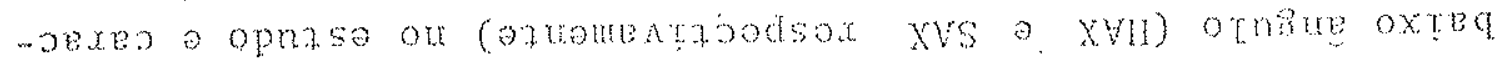

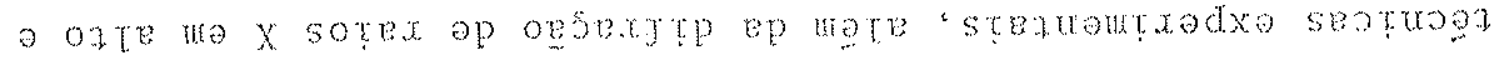

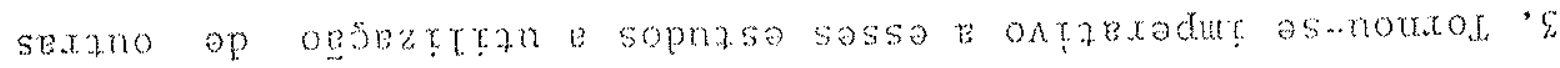


Spuozsts sossap opepts

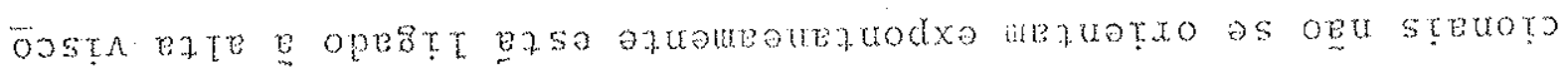

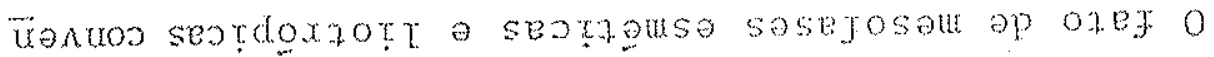

"sexoy tz souem otad rod ogs

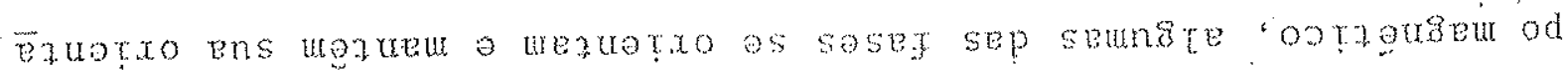

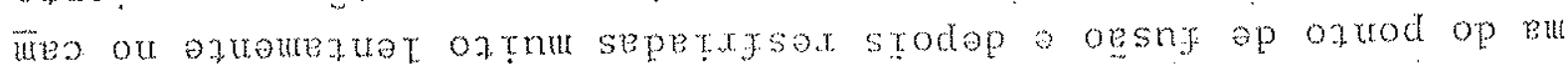

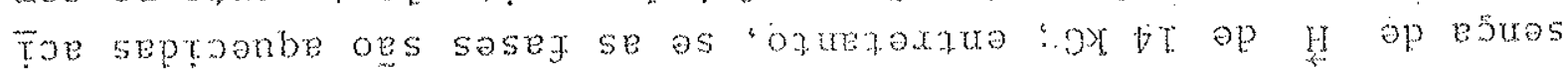

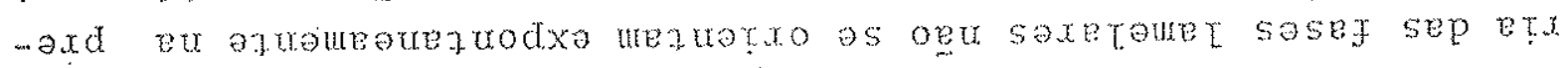

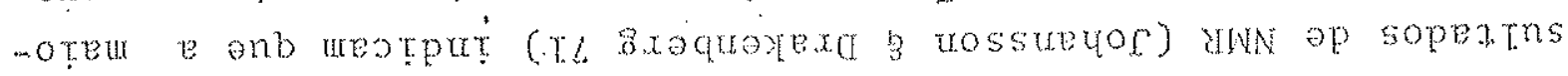

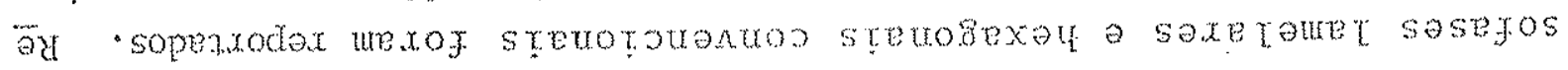

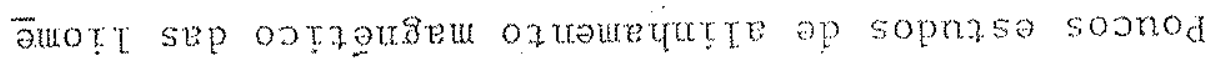

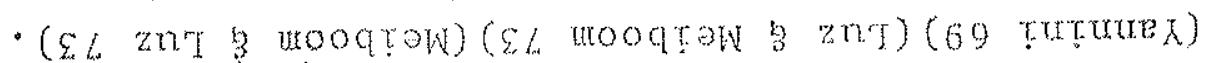

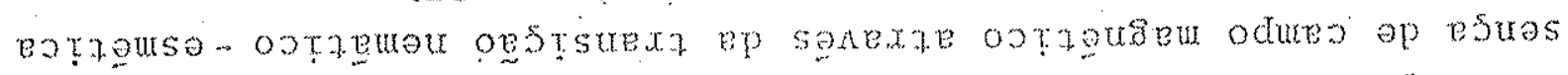

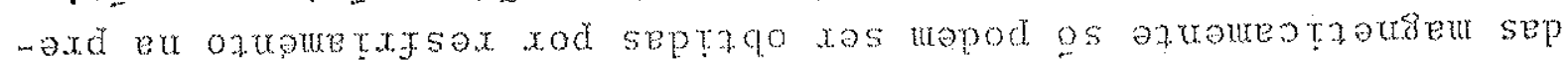

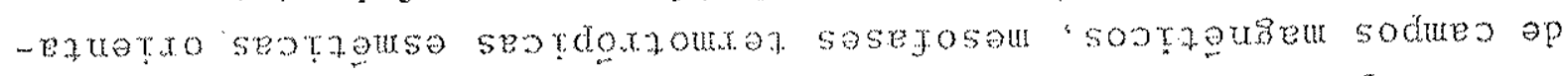

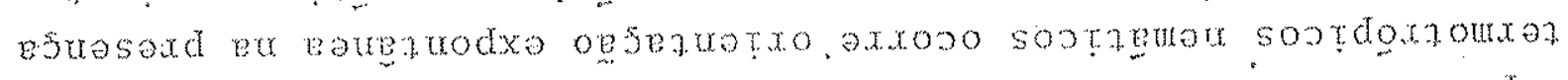

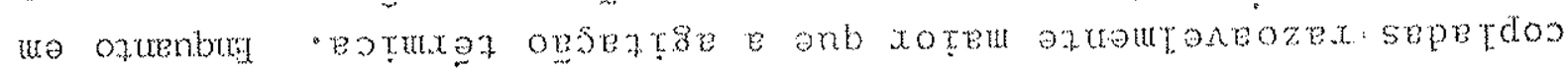

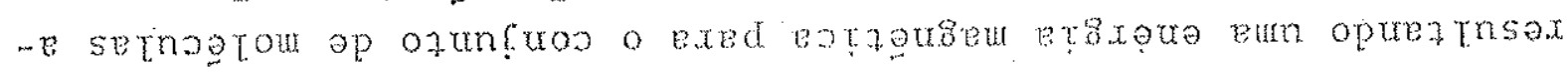

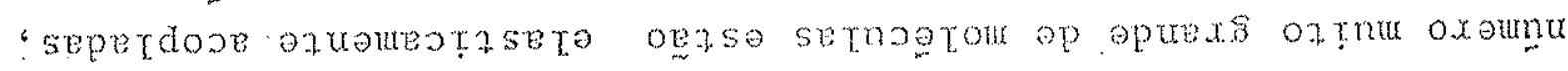

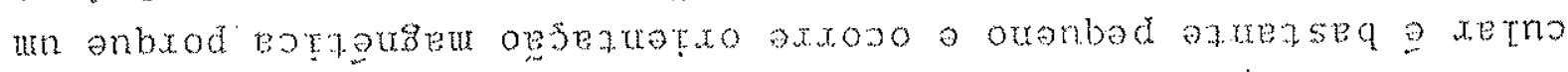

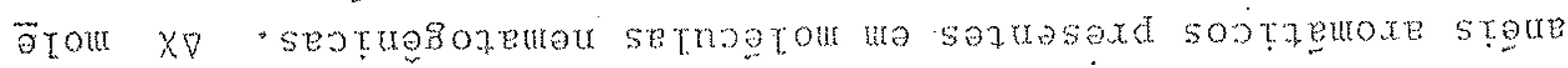

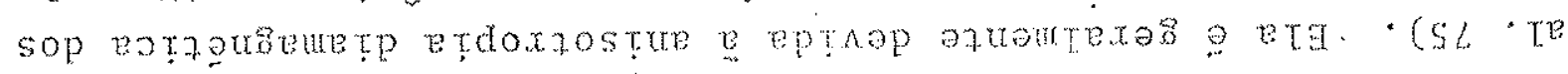

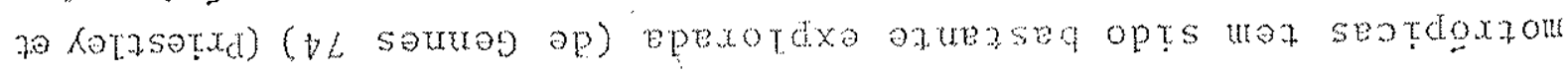

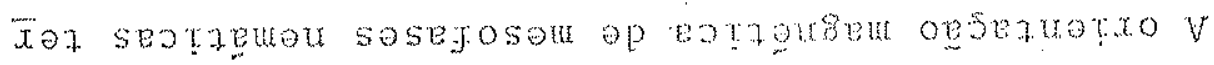

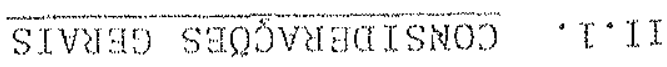

SVDINOgWV SUTHCO SVO

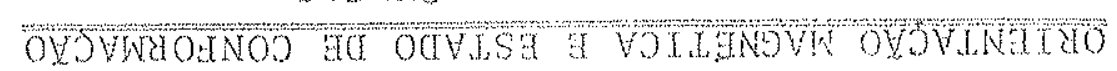

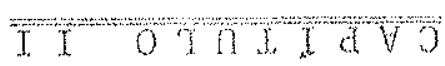




\section{tongraxdsop ecos}

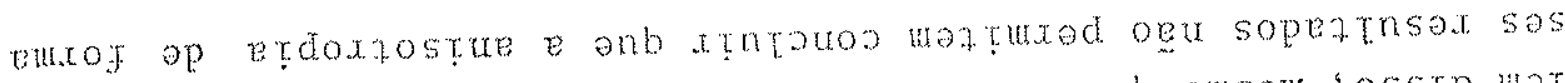

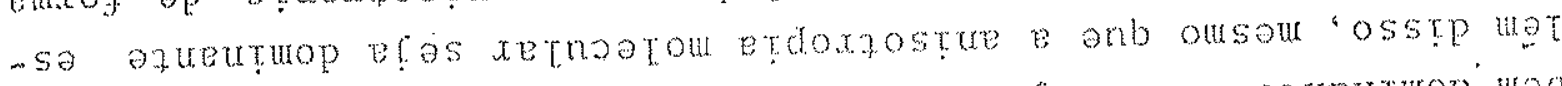

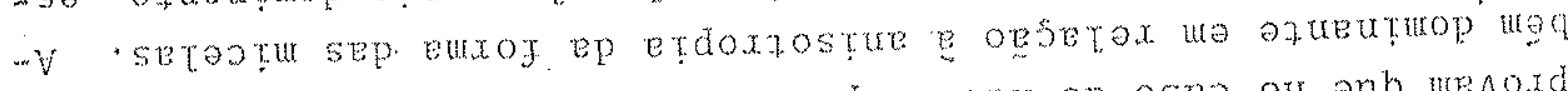

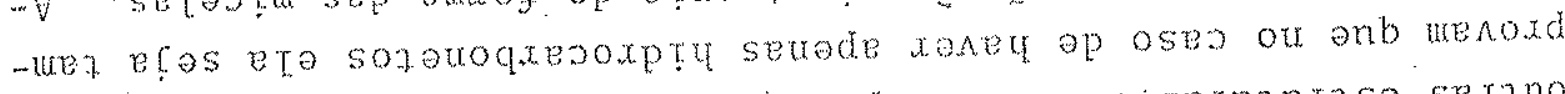

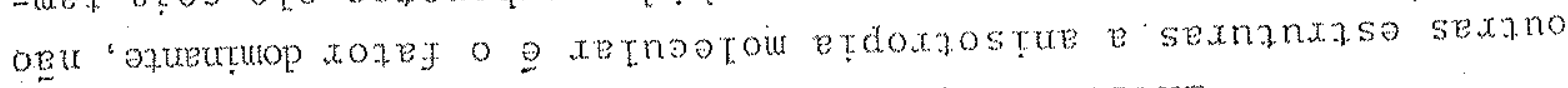

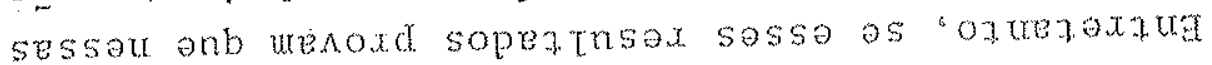

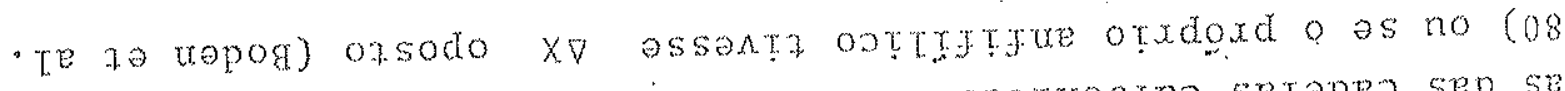
sonady) setoptu se spetrototpe messof seotroqxe setoped sep so

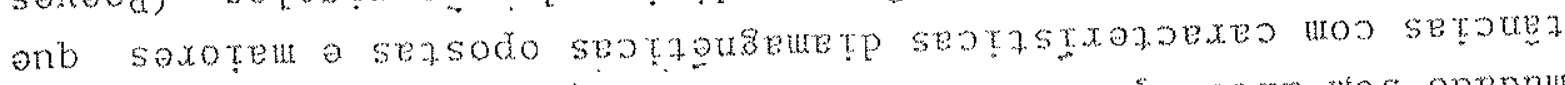

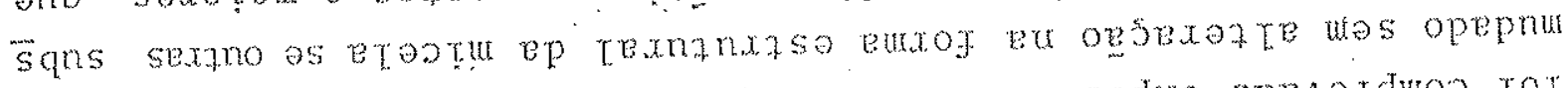

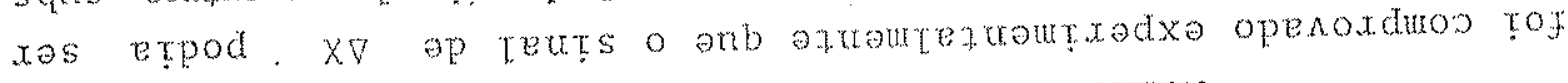

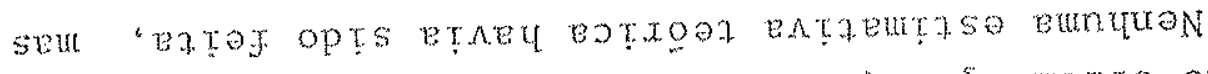

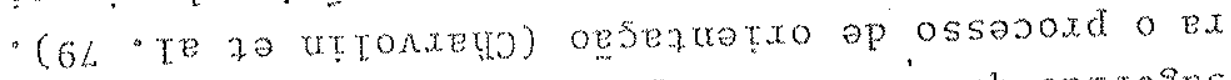

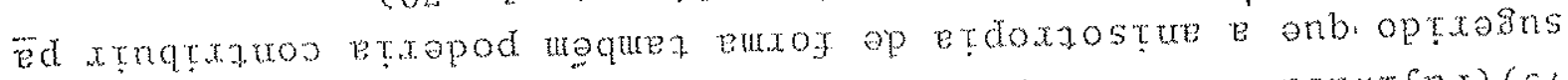

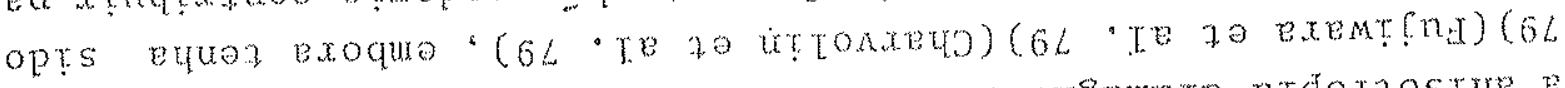

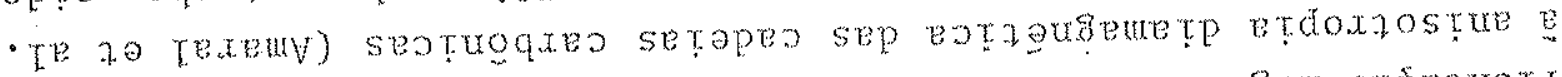

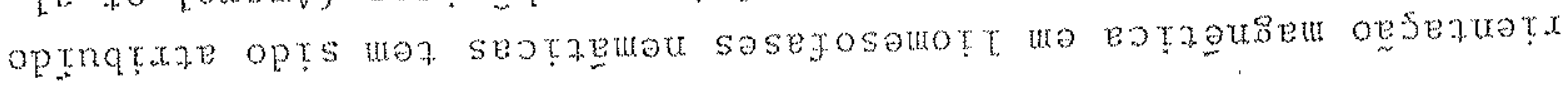

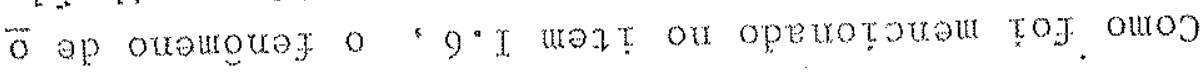

- seordoxzouxed sasejosou sep sedgoxzos

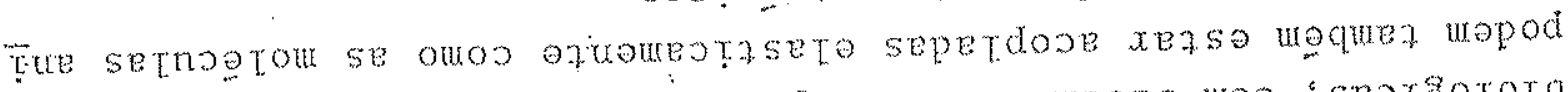
anb seu 'sbaxno no seuerd sooptrt op sepeutesta uod sporbototq

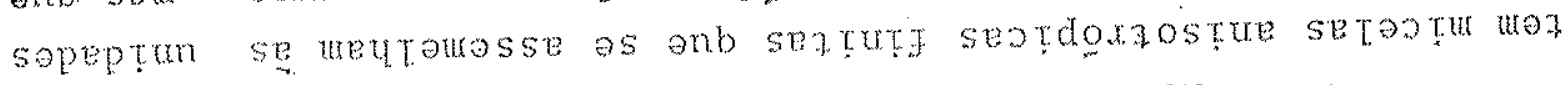
-stxe seotreurat seotdoxatr sasposall ap oseo on

- sestroques

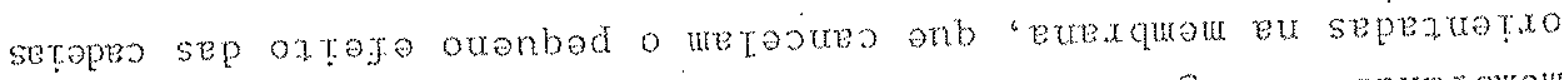

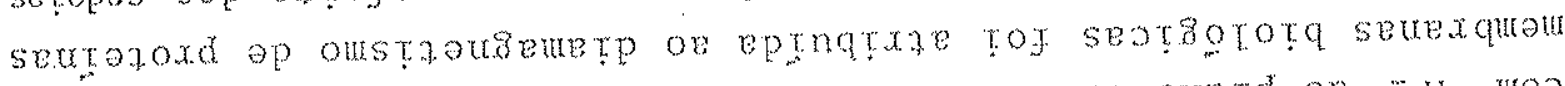

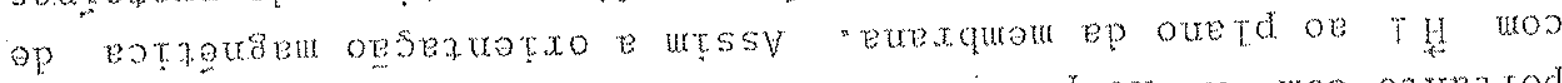

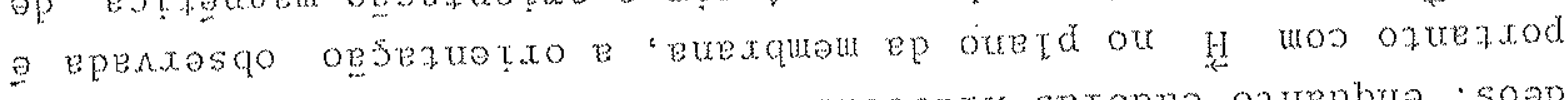

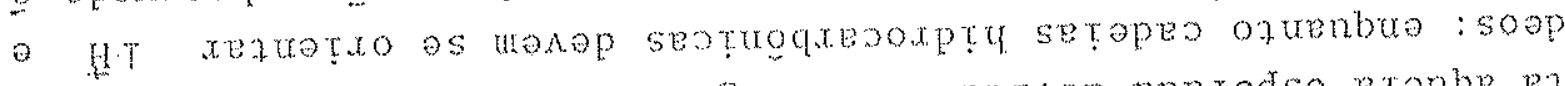

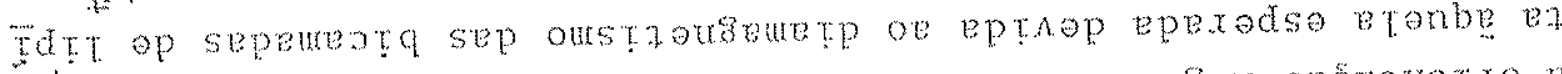

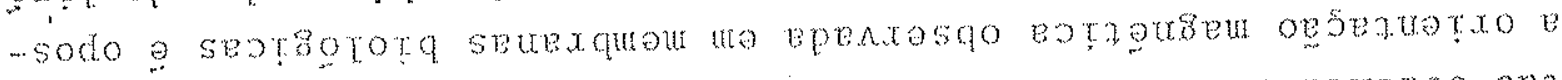

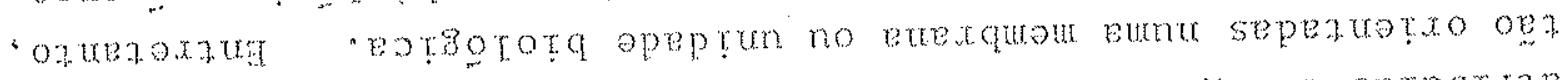

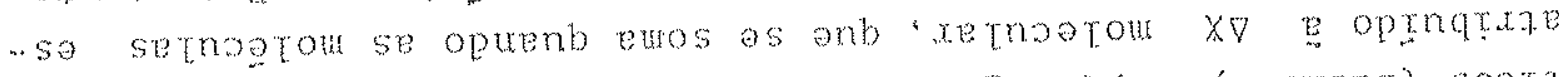

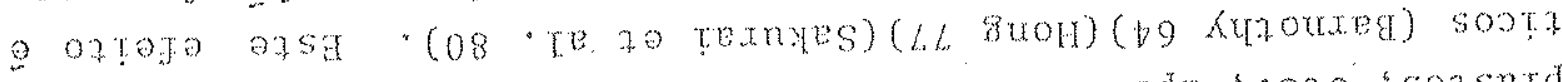

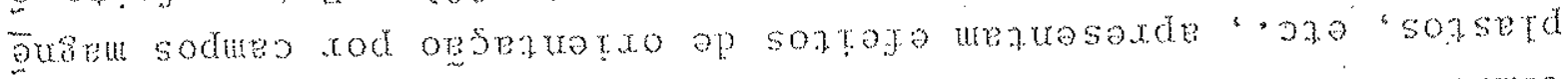

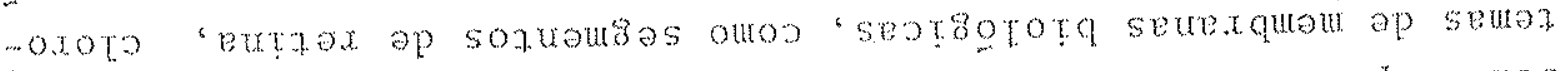

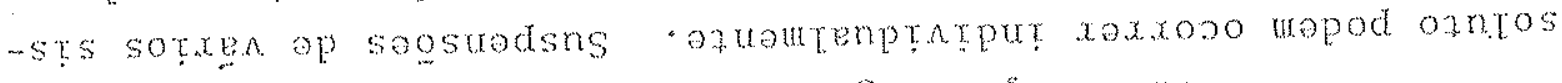

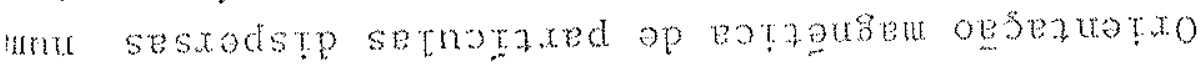




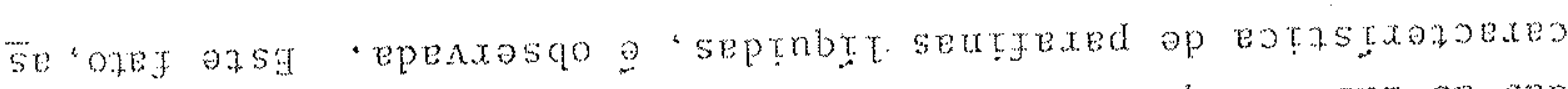

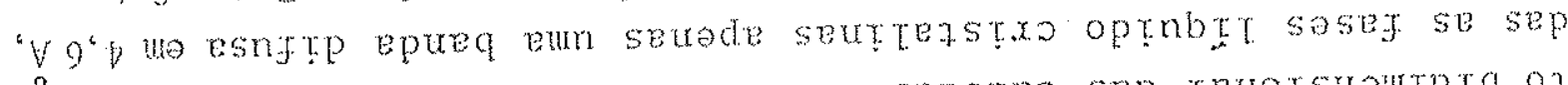

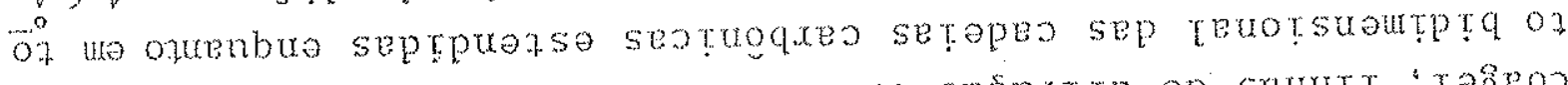

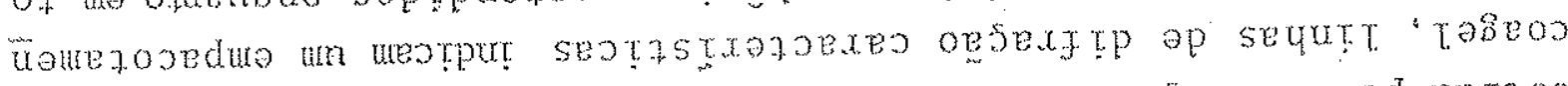

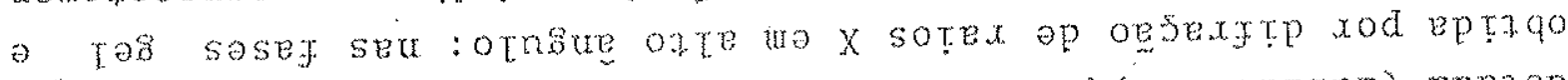

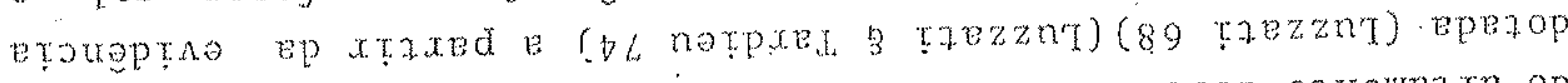

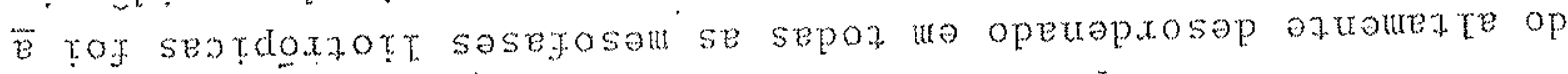

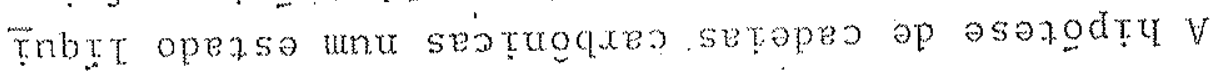

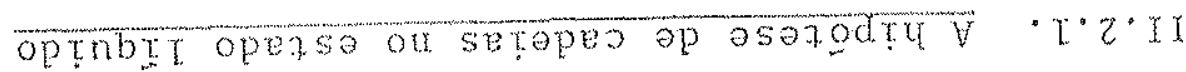

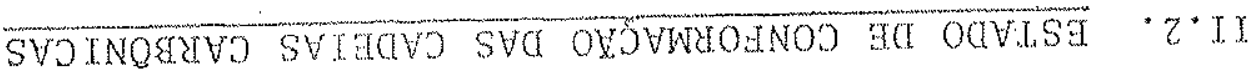

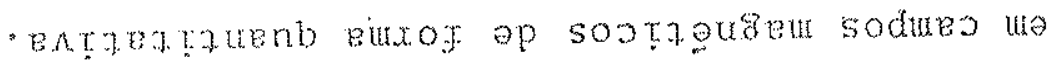
ogsezurxo ep sozrofe so sopestreue oes axuarteuta

- exptrdxa eurog

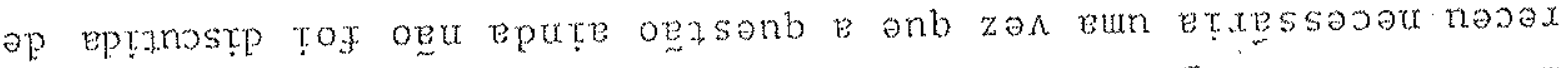

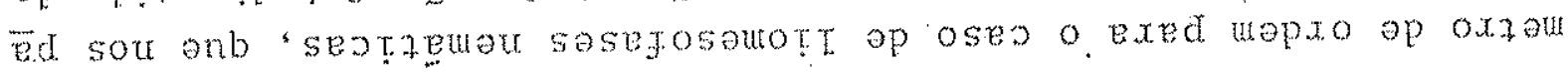

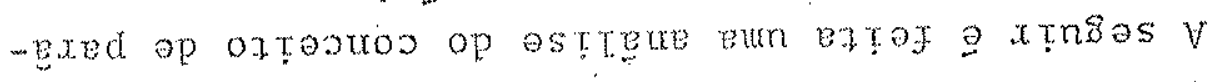

- oytequar ajsou sopezexz maxos e 507

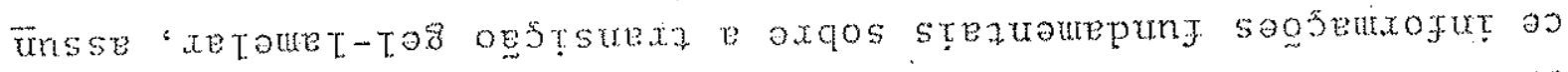

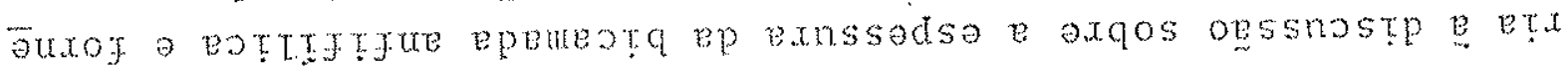

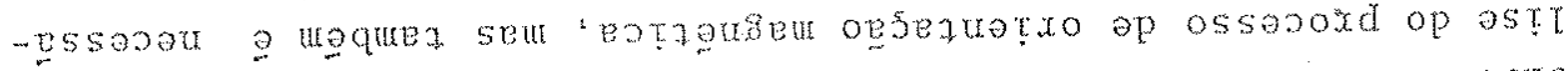

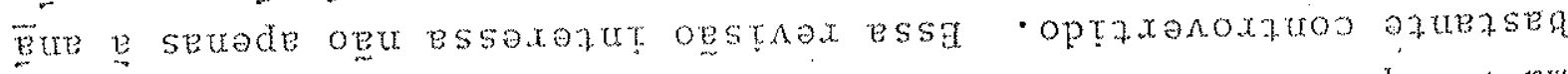

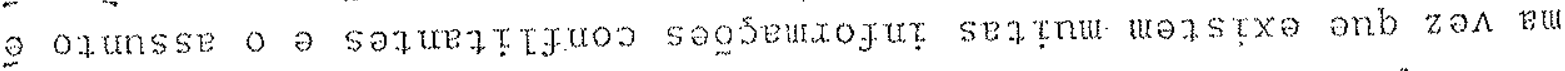

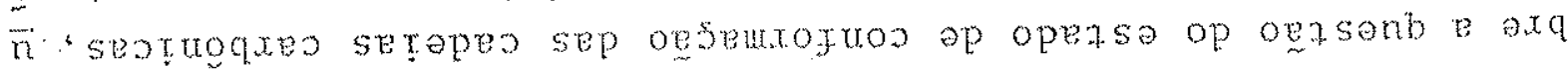

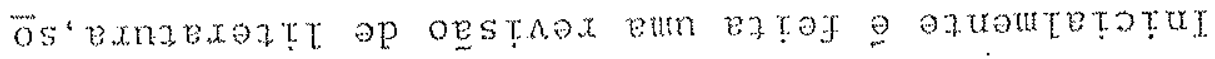

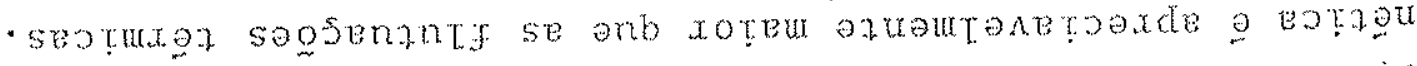

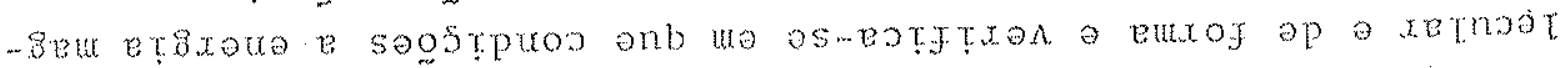

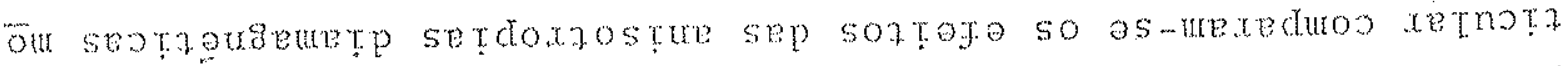

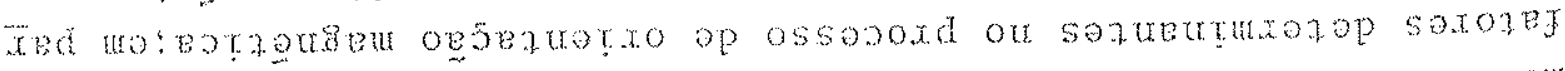

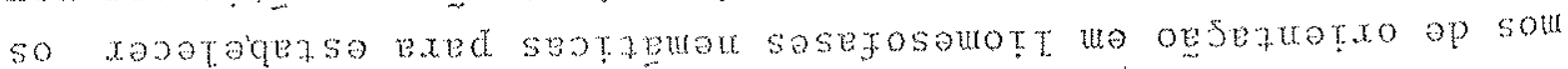
stmou stanssod so sopestreu oes ormades azson.

- entratos basodsox bur xtzrpoxd ex

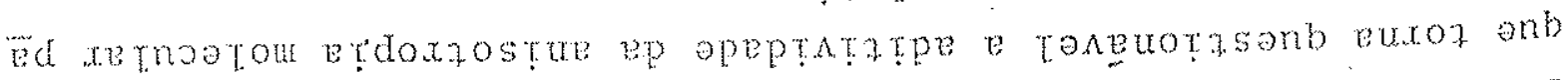

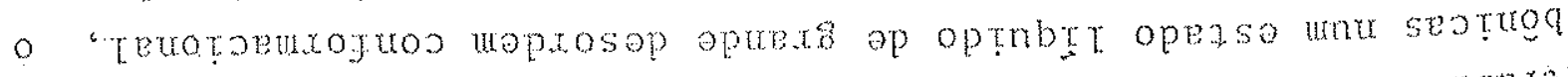

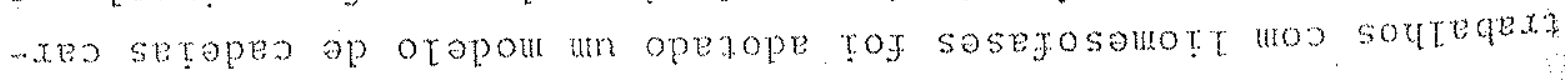
sorroutrd so epsep onbrod arwenotox e ogsanb $V$ 


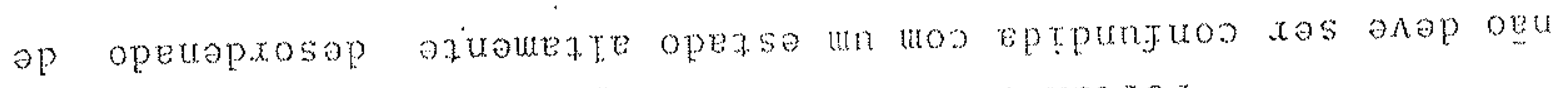

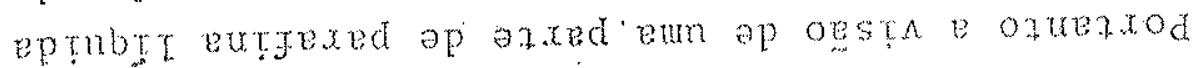

- seuanbod setouezstp exed seterexed aztrotep

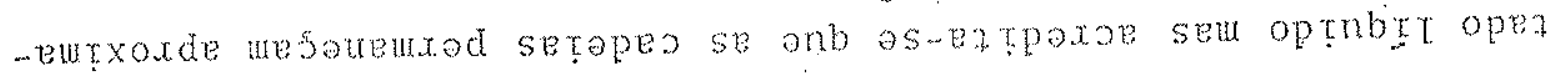

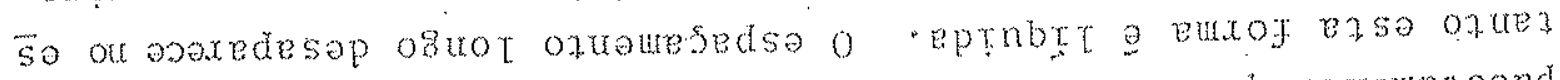

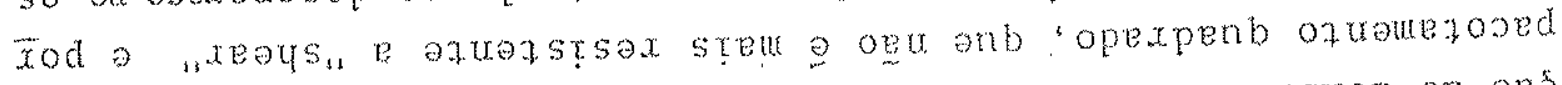

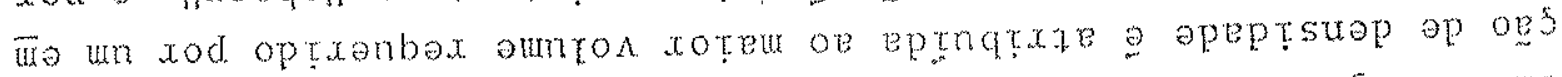

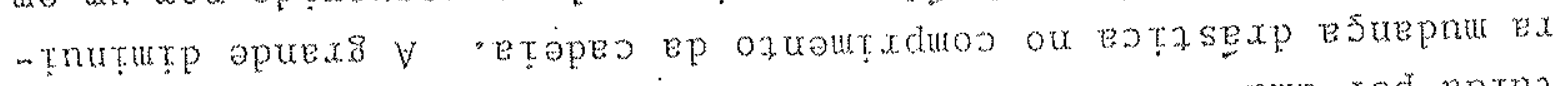

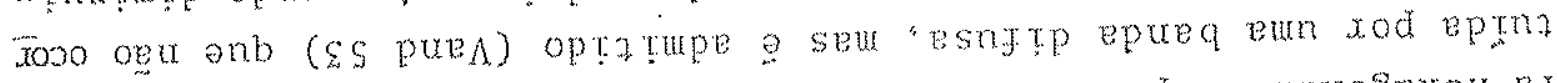

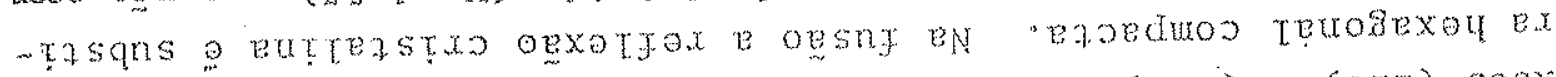

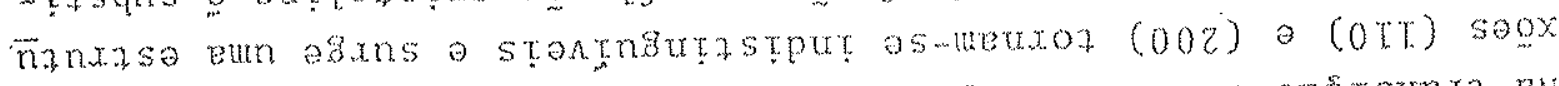

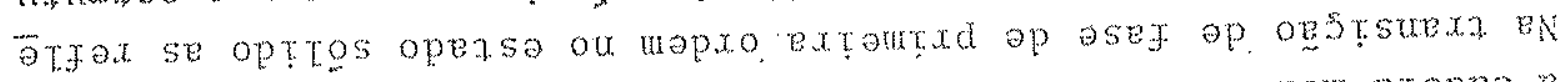

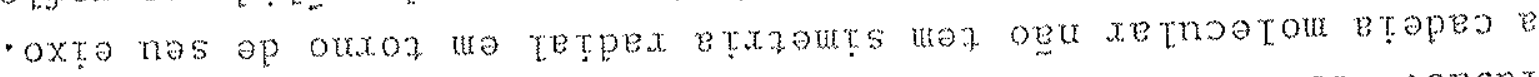
- $s^{\prime} \dot{c}$ op ozsuedxo ap oeberax em uar a o e soxta so oesny

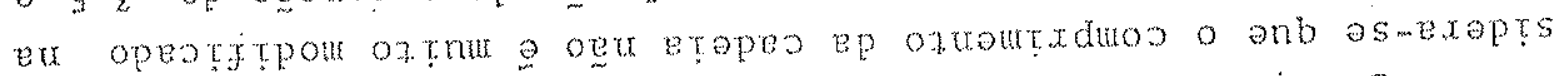

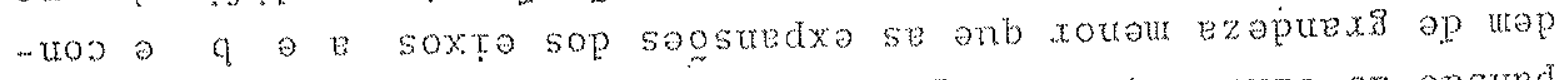

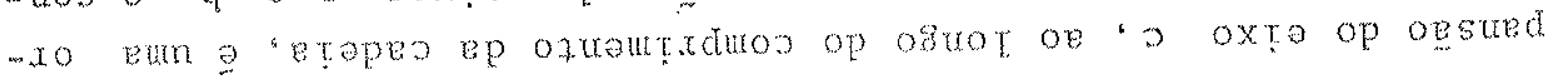

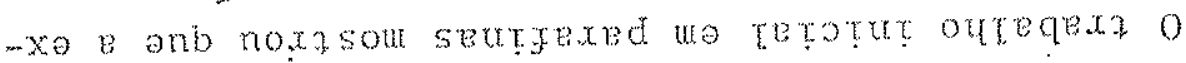

- epepnzsa azueasea opts uaz a oxto nos ap

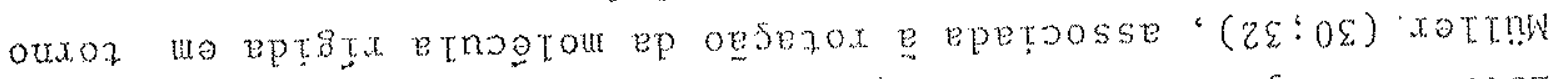

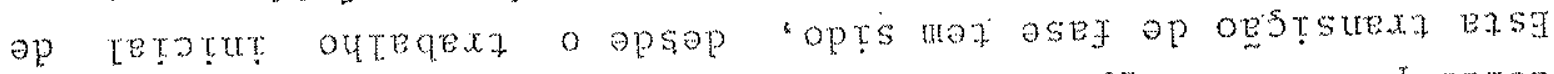

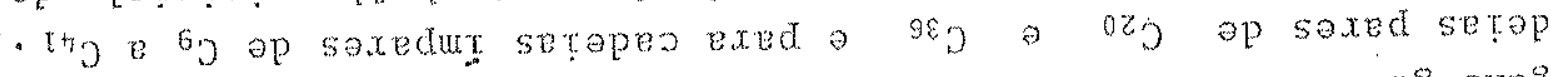

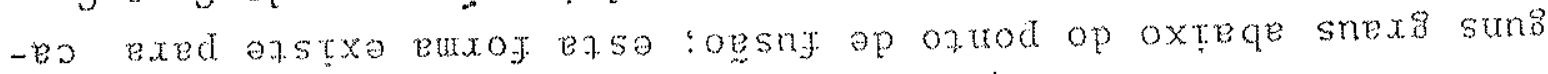
-

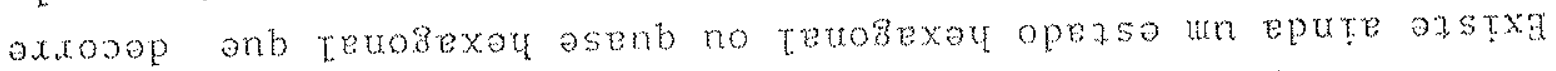

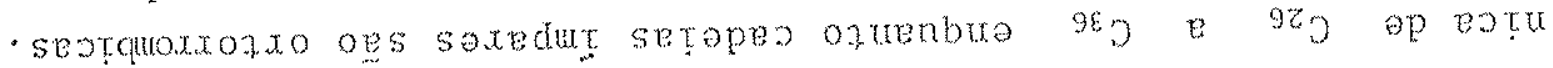

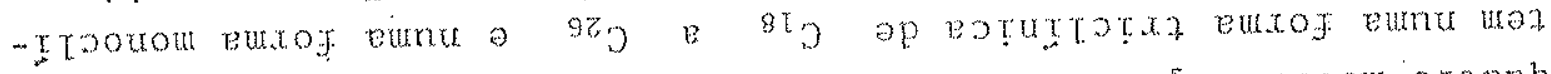

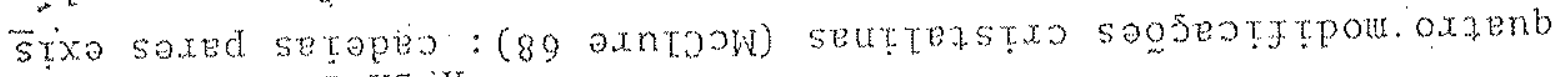

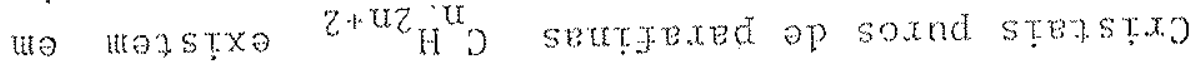

- etopes ep oxte oe xernotpuedxed ouetd or rexotsuam

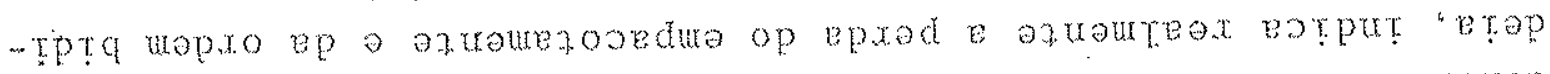

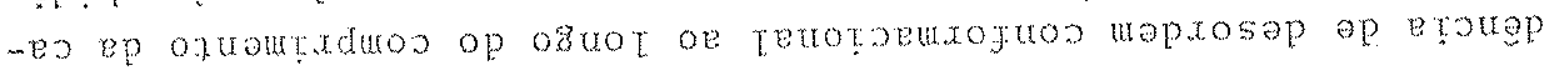

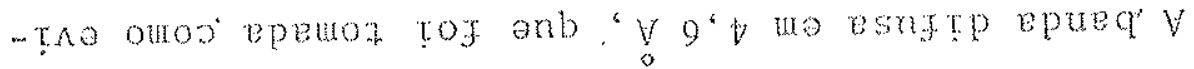

vepequa ster as

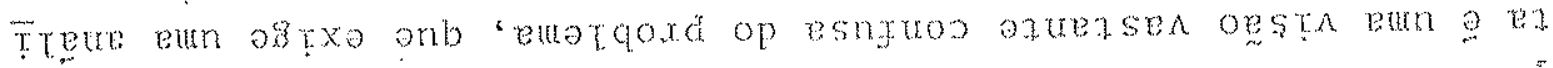

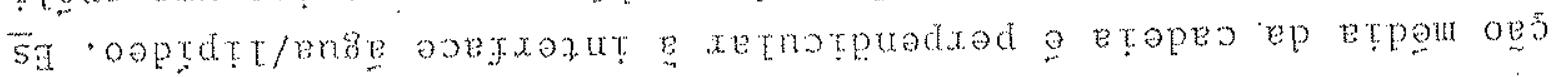

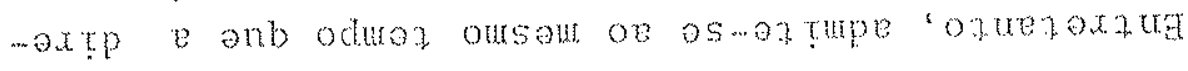

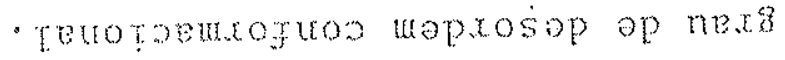

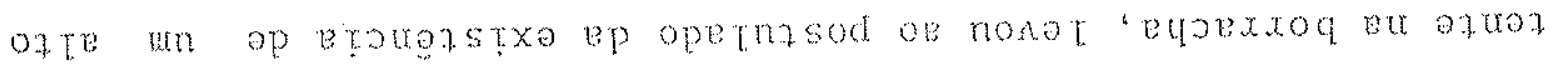

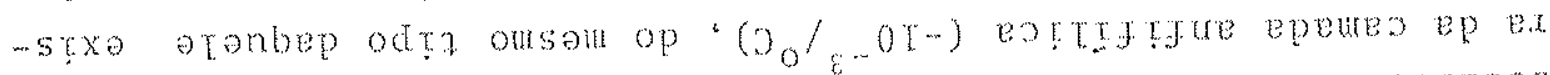

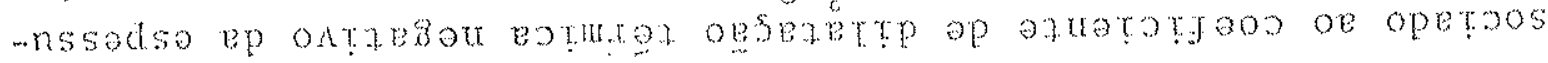




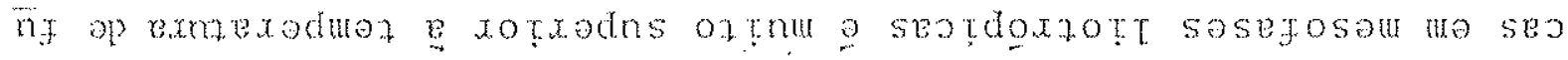
Tuoque seropeo sep oesng op astexy exnaexoduoz $\mathrm{V}$

soptoaxoner ofueneatrasxatro

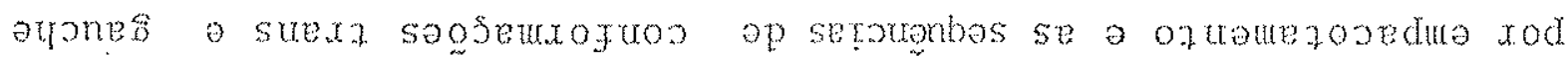

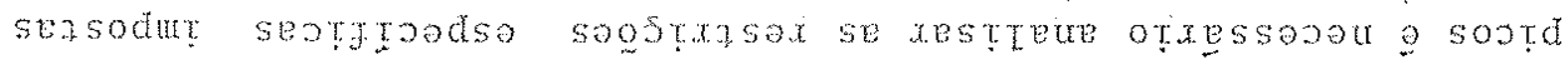

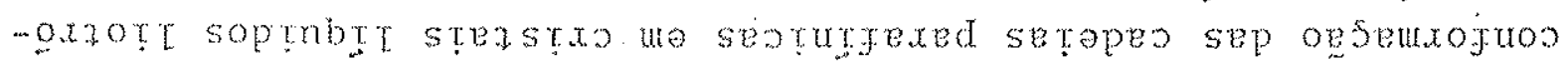
ap opessa op teax oesta eum xaz as exed ozuezod

optrbrt opezse ou setopes sep oregexed ozun

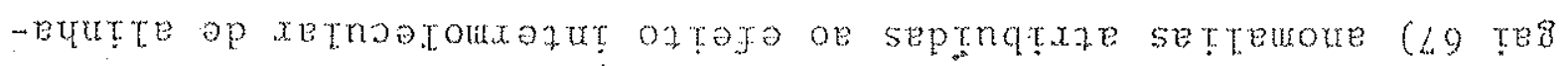

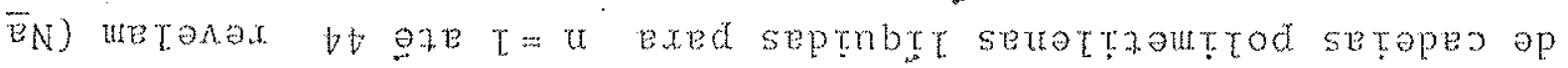

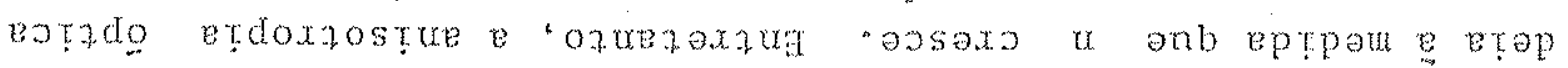

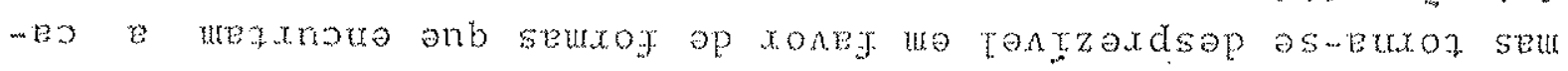

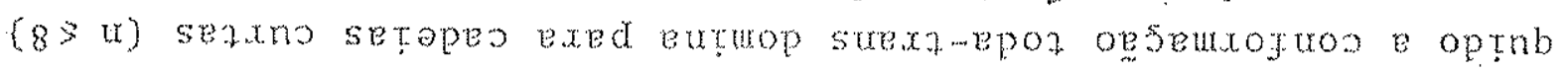

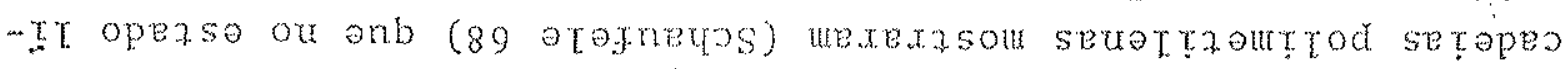

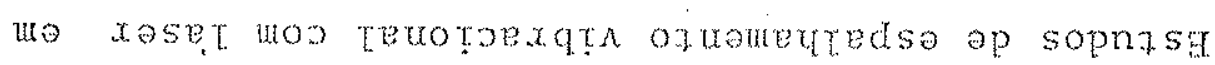

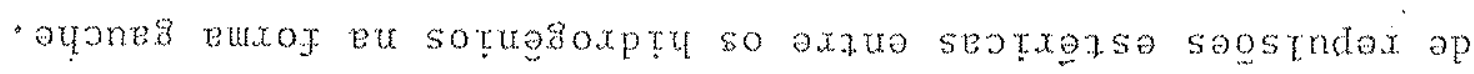

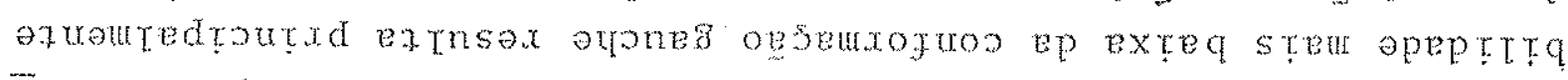

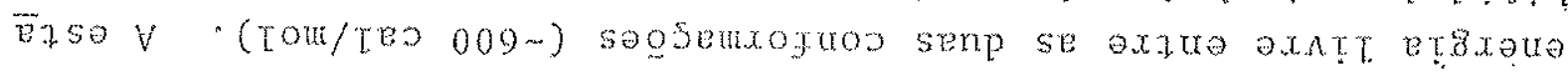

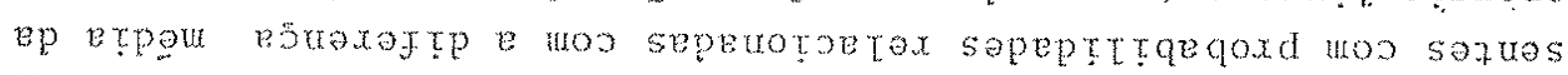

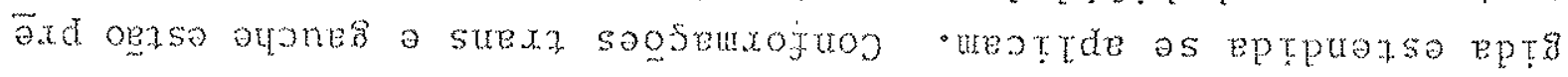

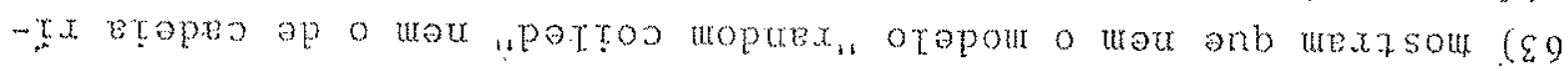
rqox a reateg) ososes opezso or ( әp botrouros? opsezox a exmaxse exed sopearnsod

- orteresta oetr opeaso ou

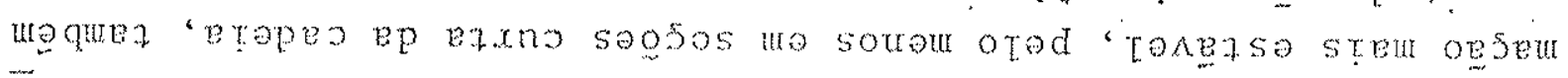

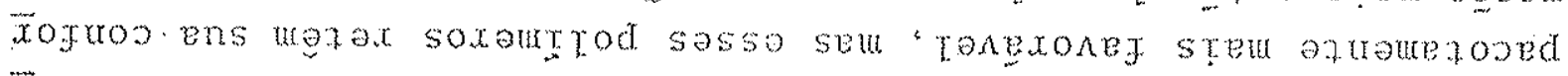

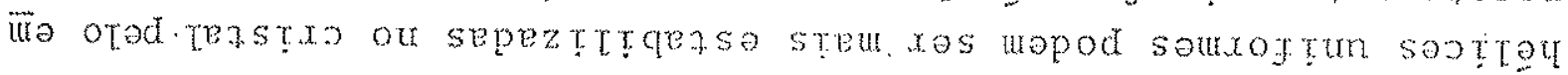

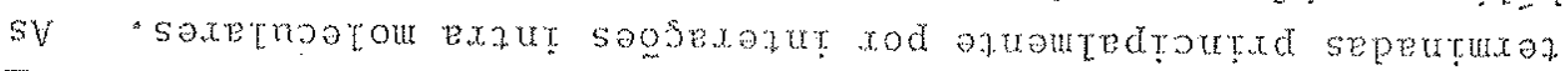

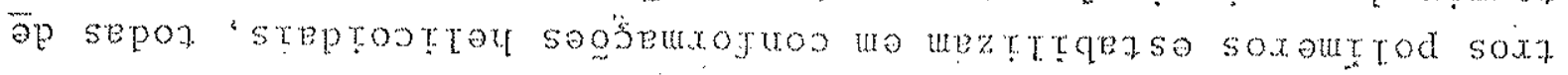

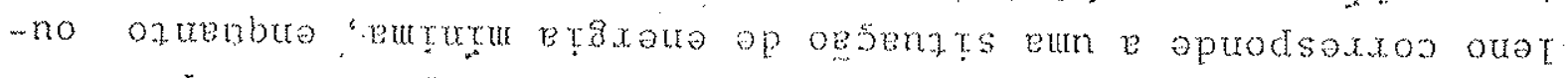

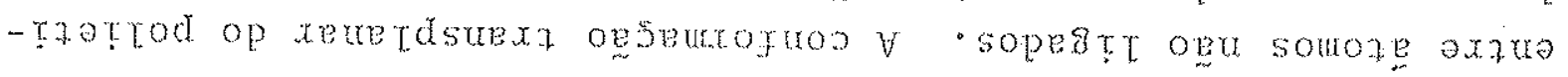

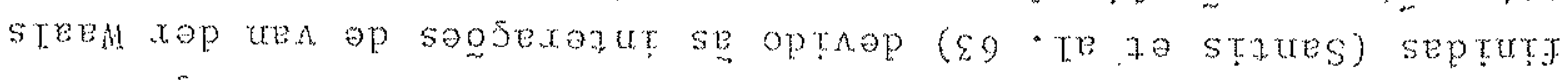

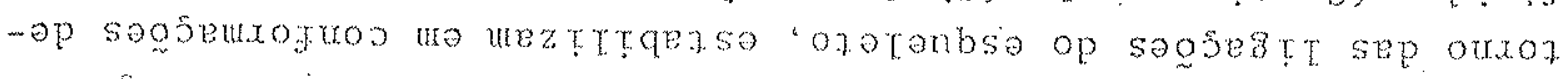

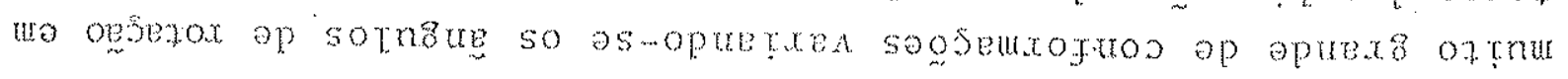

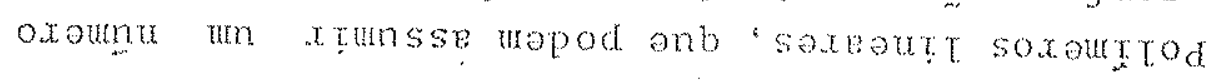

- sasejosamot ue saxet

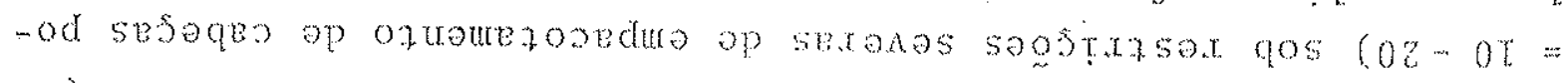

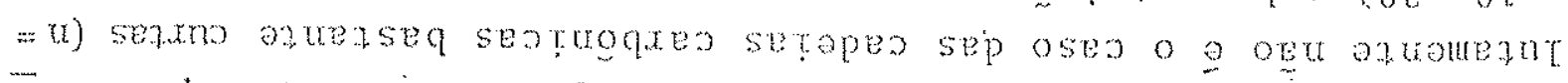

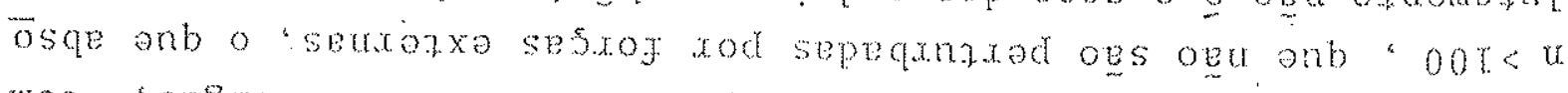

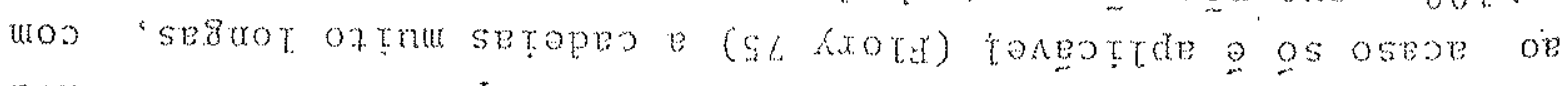

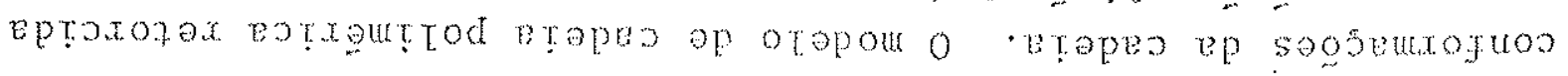




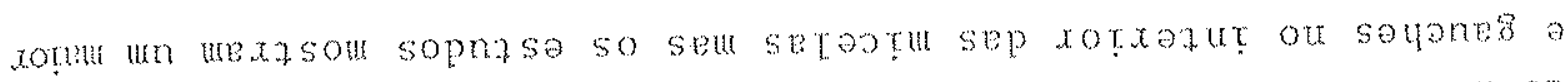

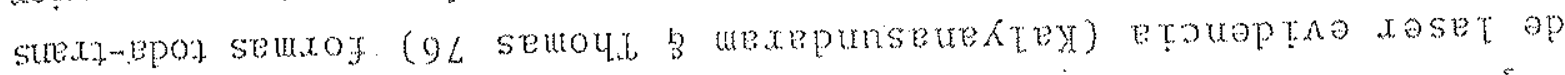

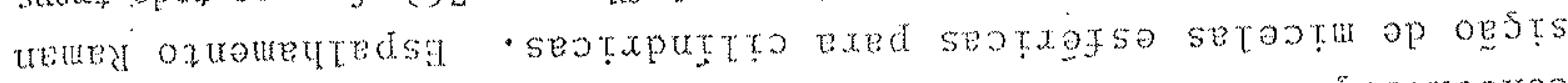

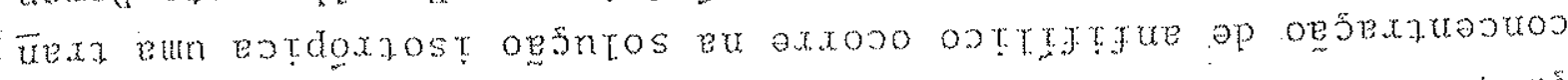

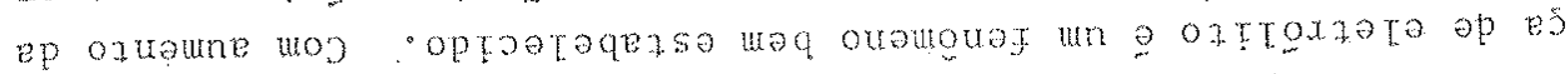

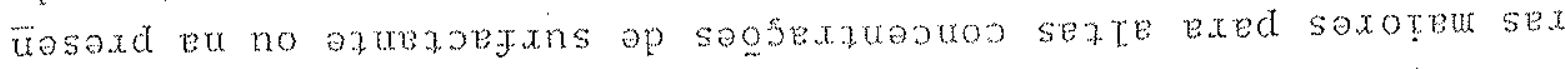

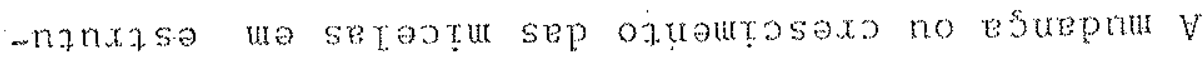

- xetnoetourotou oestadstp ep opezso ou onb op setostu seu tonezso steu g suextepot euxof

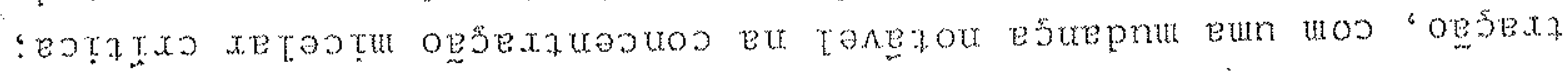

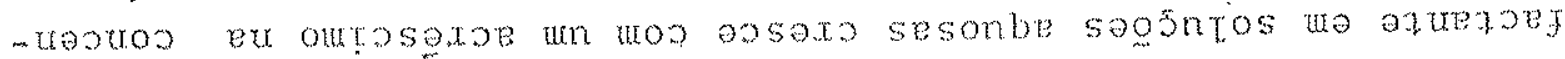

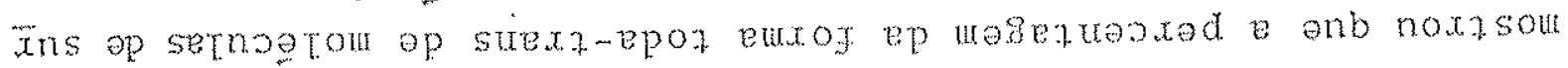
(SL te to rusekeqeyo) xaset op reured onumertedse

$\cdot 005$

ntos ex setios setnogtou onb op sonou seut optos opease ou setep -eo se onb op anones sooseuxoyno steu wonssod serostu seu serop

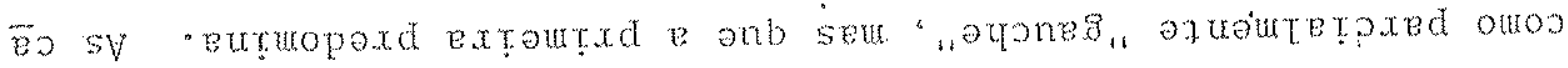

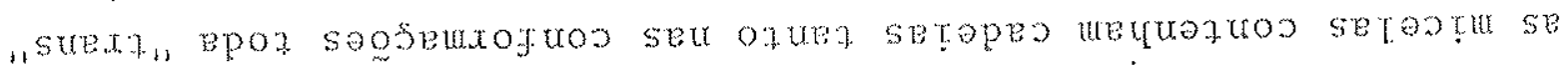

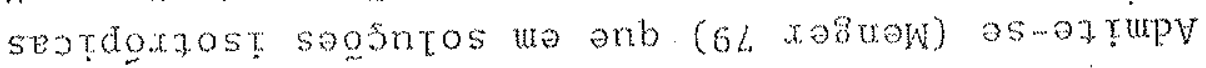

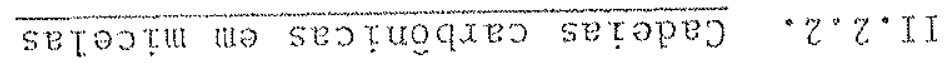

- seu

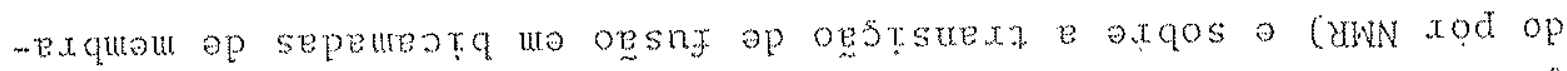

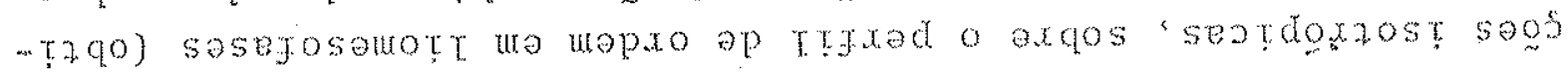

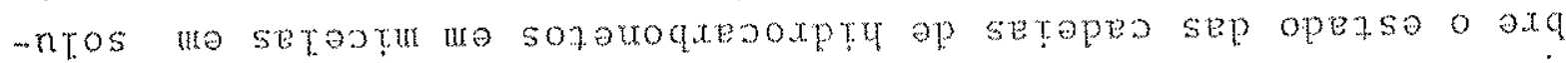

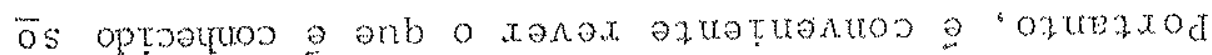

- enge-opqes semazts op eoruxer as Treme rod sopnaso sopentage mexoy opN ootgoxast optrbit ou our

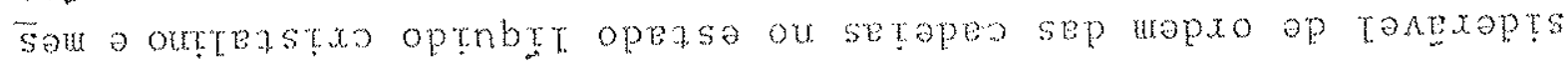

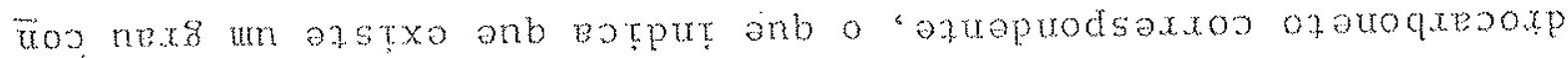

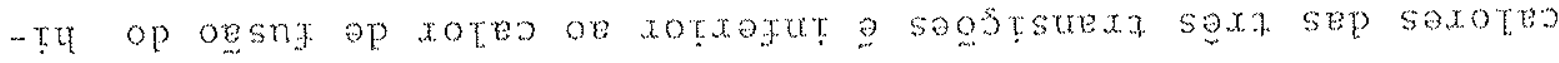

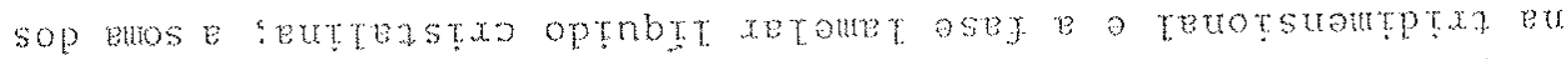

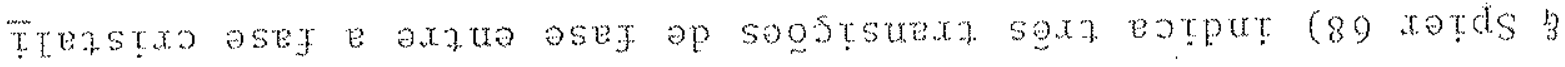

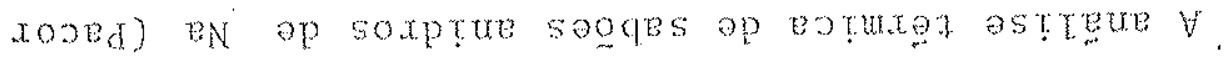

$\cdot 085$

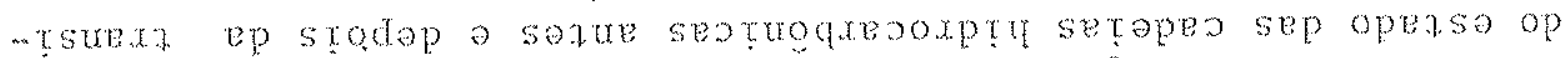

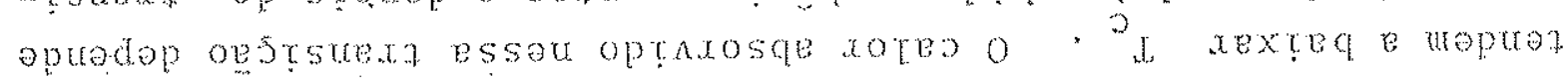

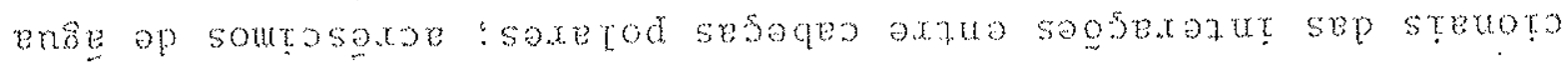

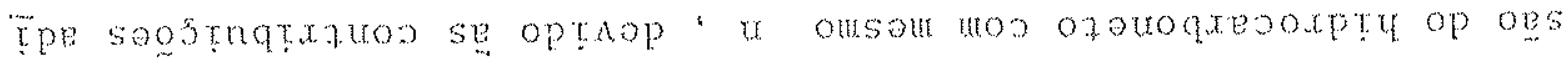




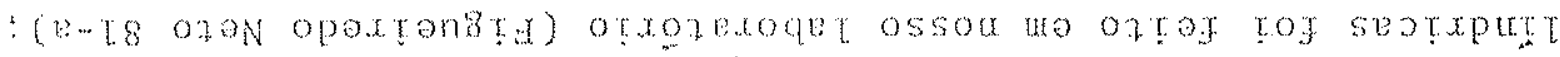
To seraotu exed oropou ap odrz assap oesuaze eur

- erootu te xotrazut

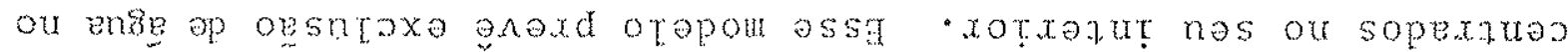

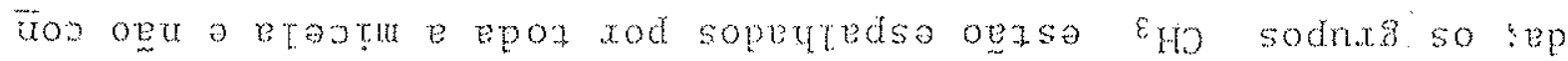

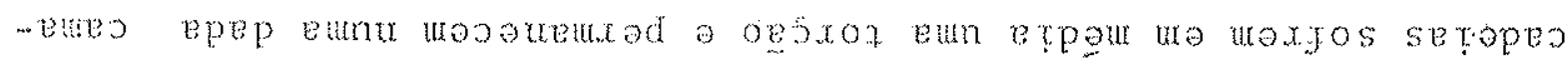

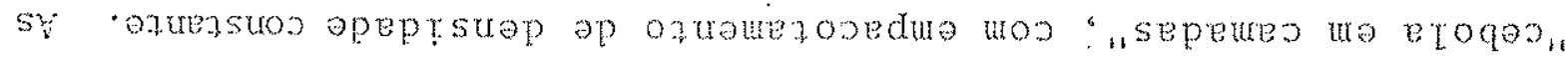
odtz op e sopertasex so wos opxose woq ep anb otepou o seprpuez

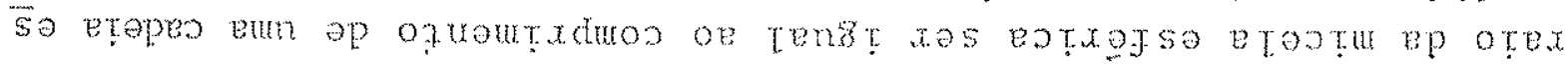

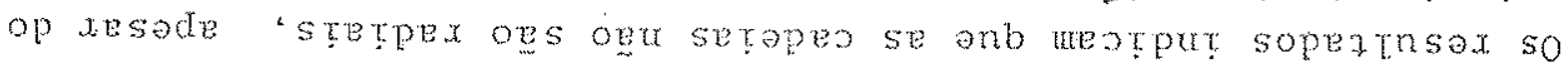
- setaper sep ozweurzosedue op sodrt sorxen exed soropou uos op

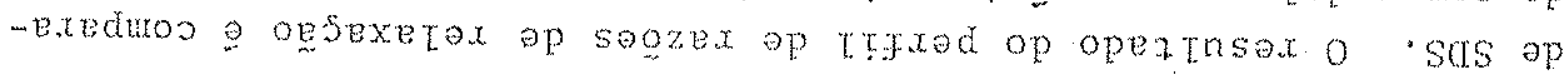

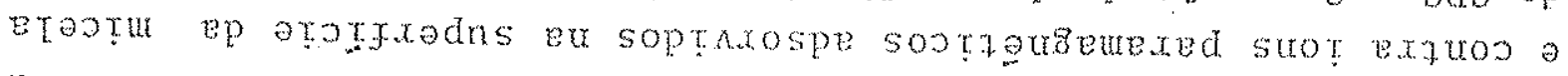

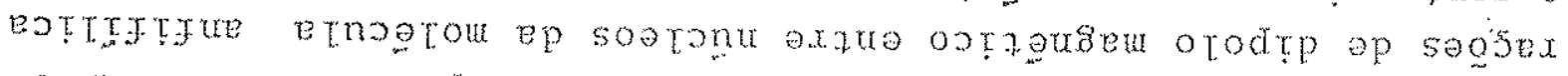

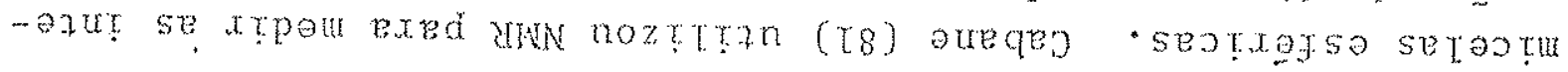

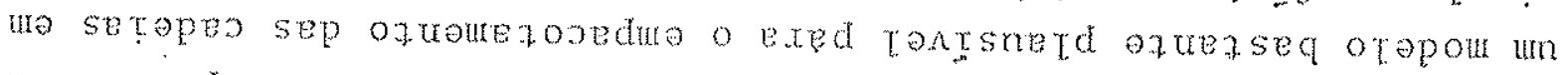

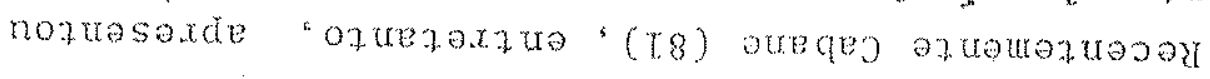

- xeurgeut assod as arb sor

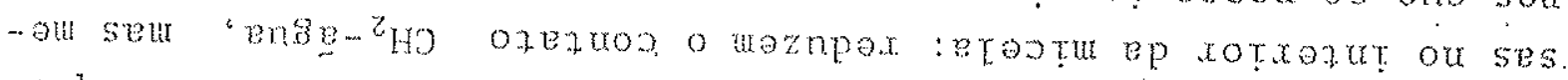

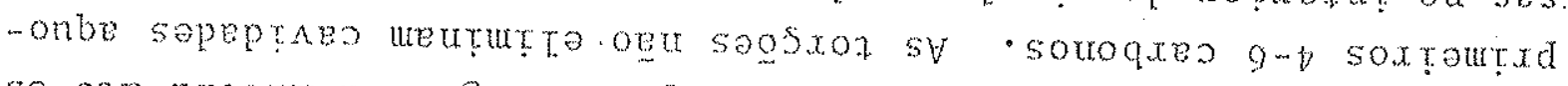
so axe erostu eu ense ap opsexamod xoney asop onb a (6L) xasuow

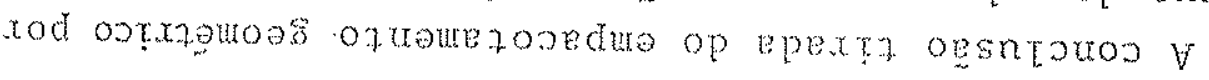

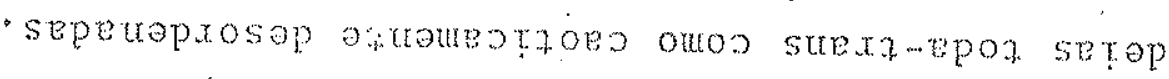

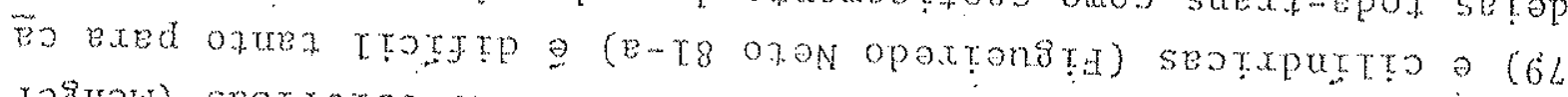

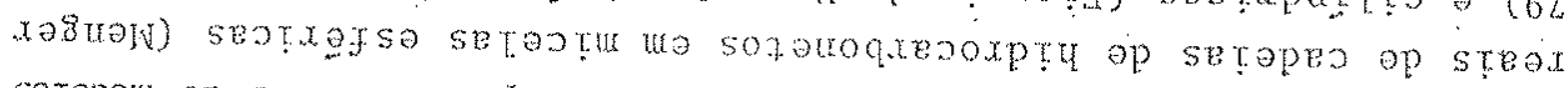

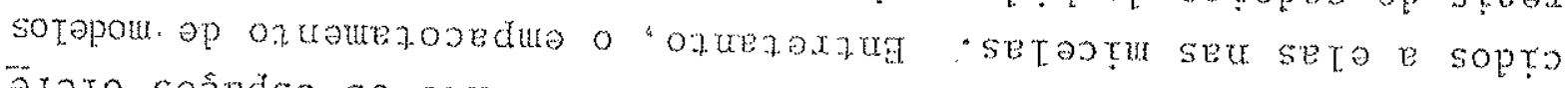

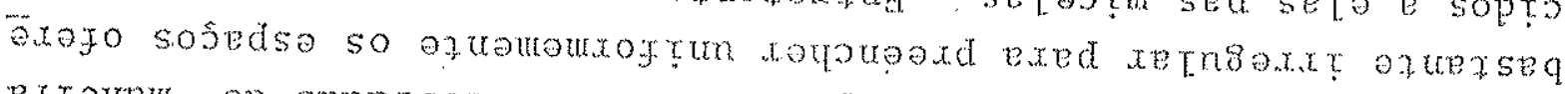

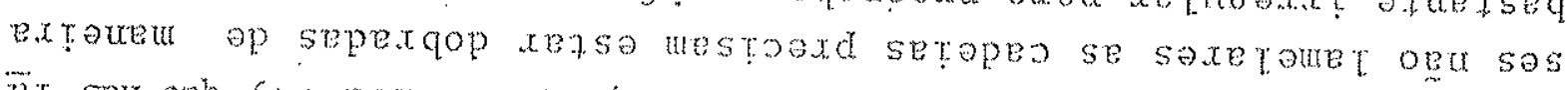

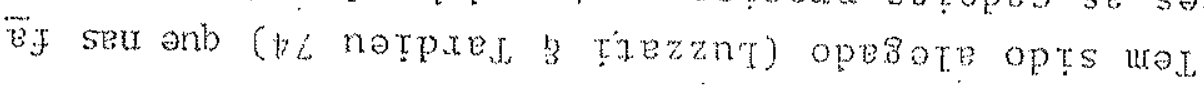

- opeprasux op nexis oxte uros.

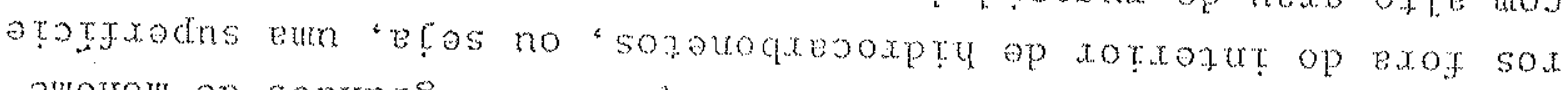

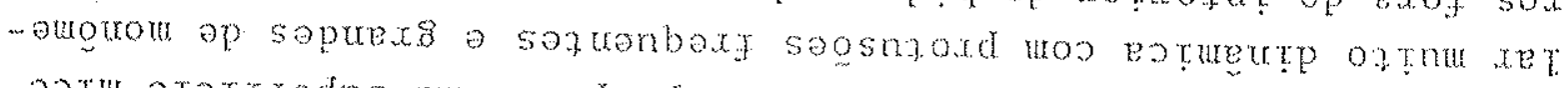

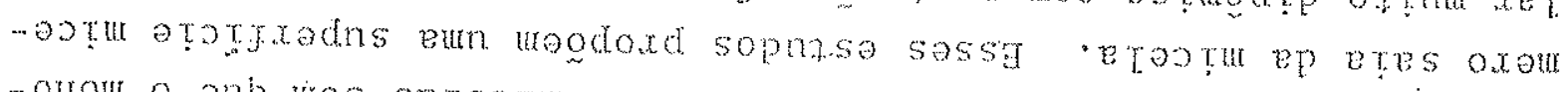

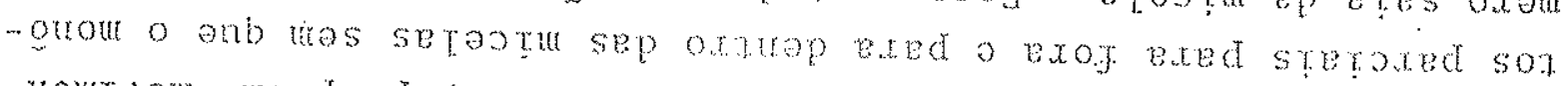

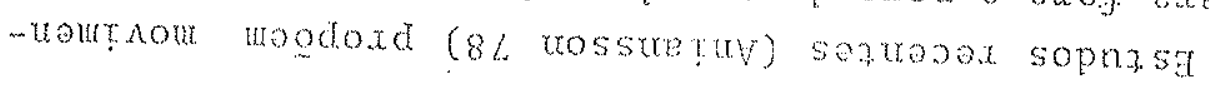

setepeo sep urg op opsexte ex 0

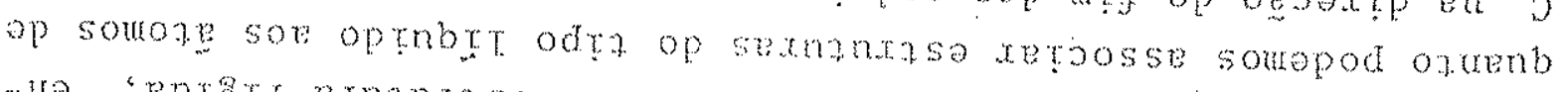

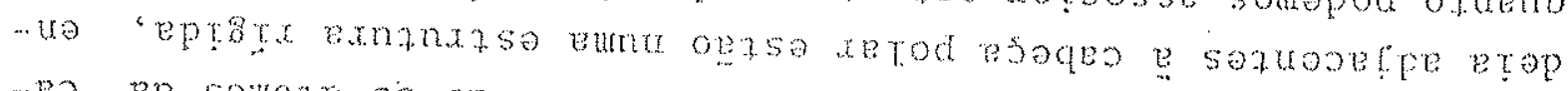
-

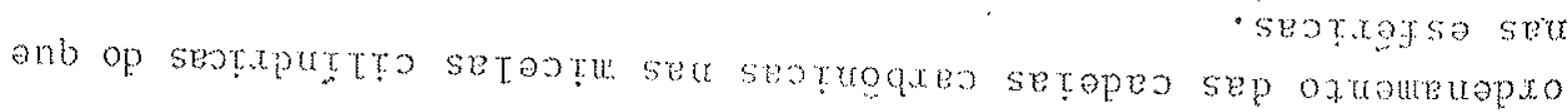




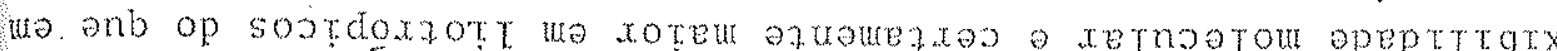

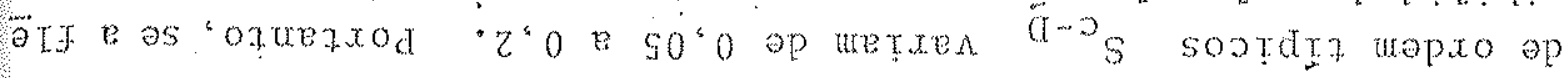

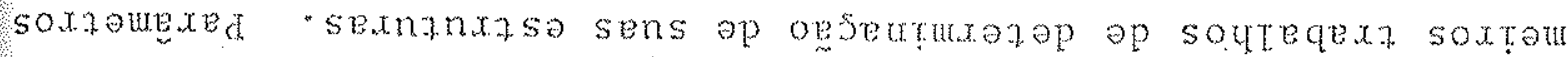

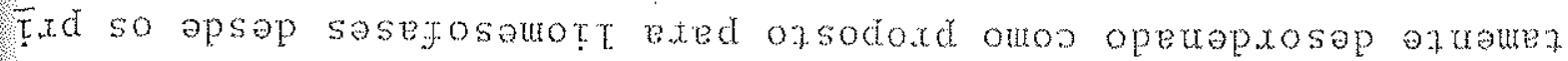

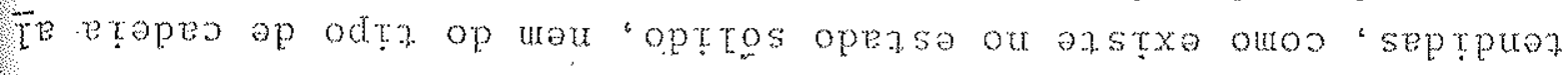

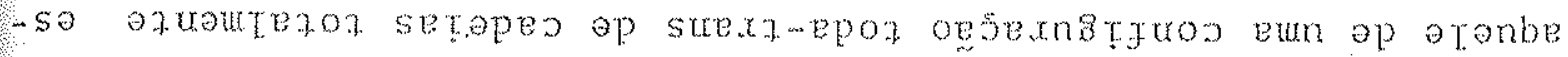

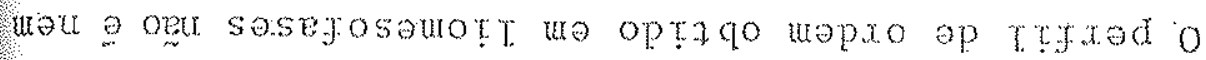

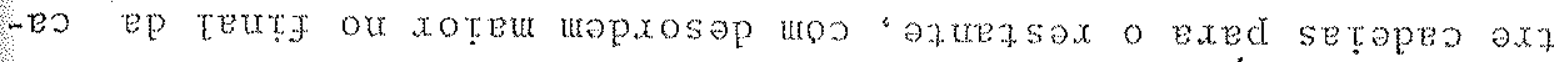

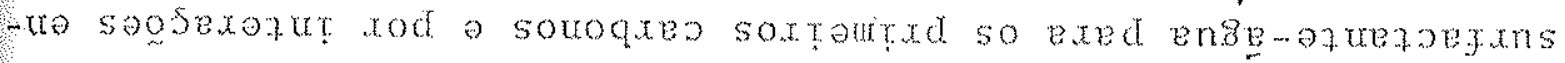

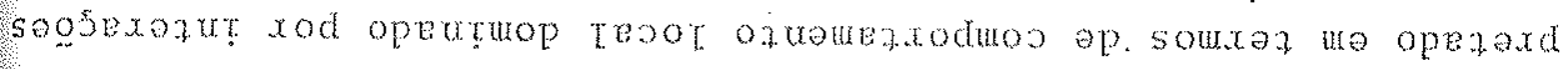

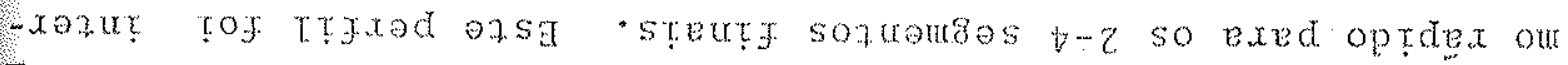

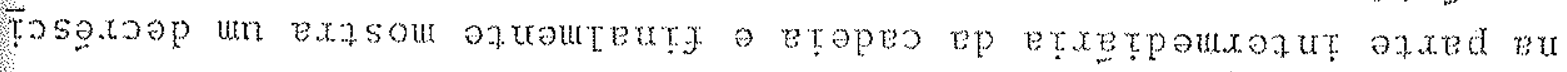

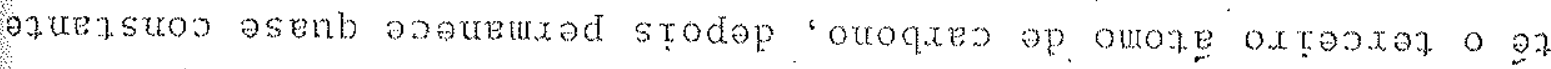

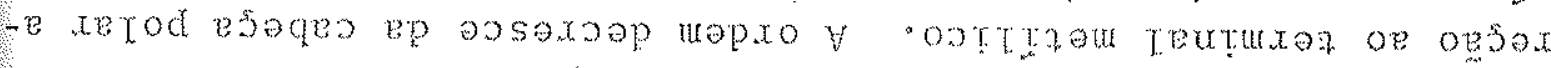

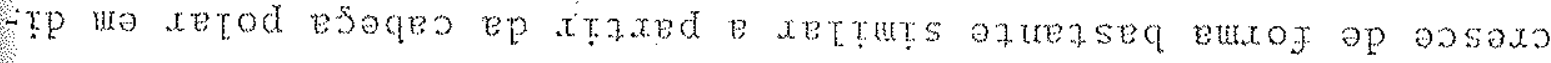

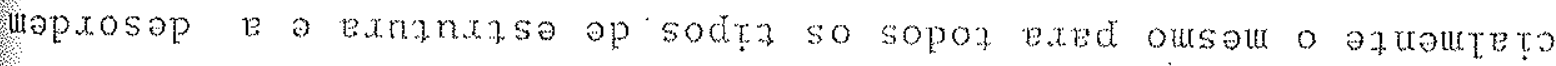

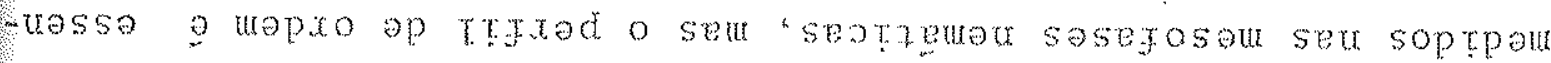

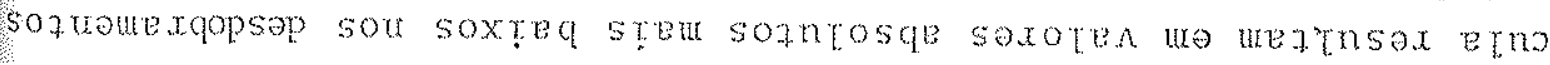

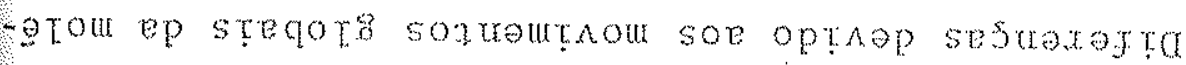

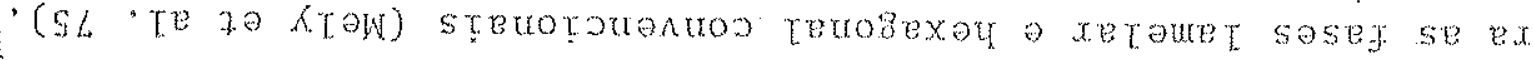

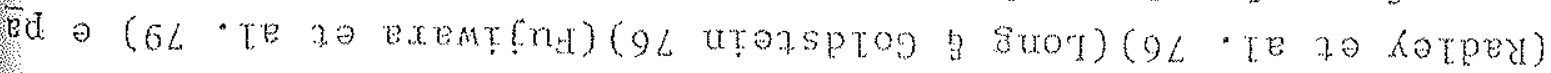

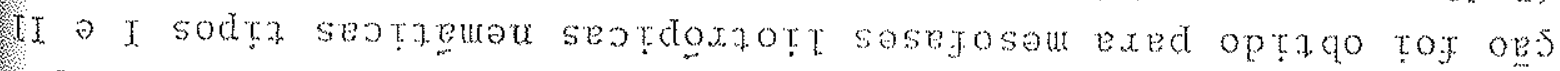

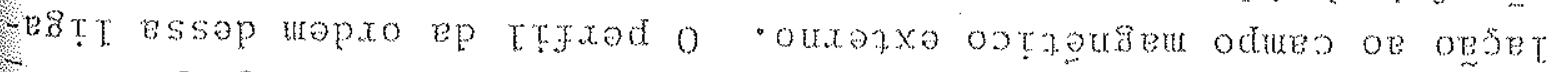

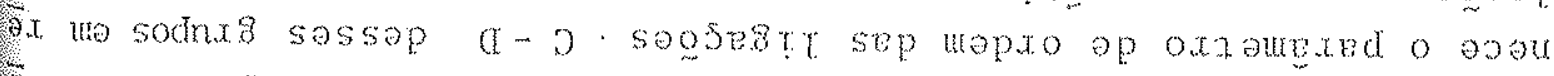

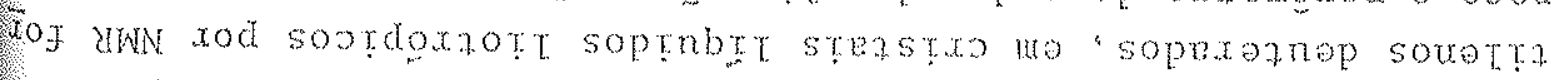

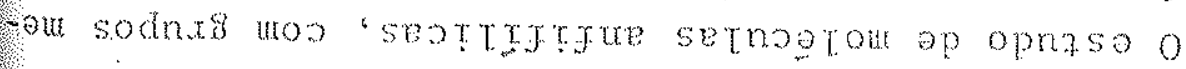

sasposourt

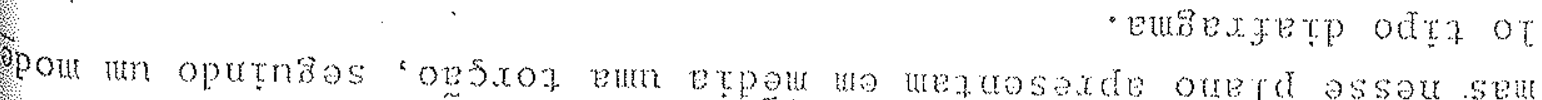

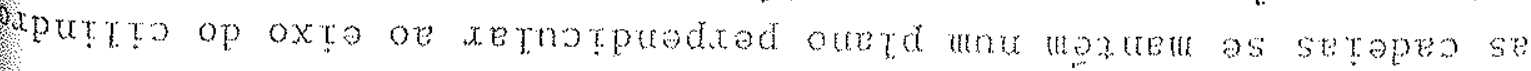




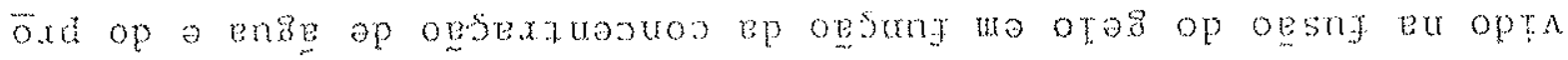

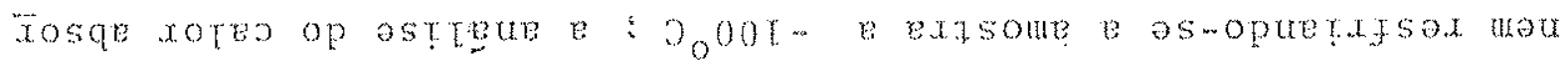

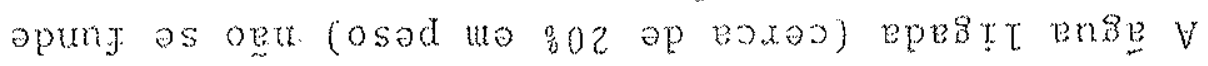

- steneaso setou

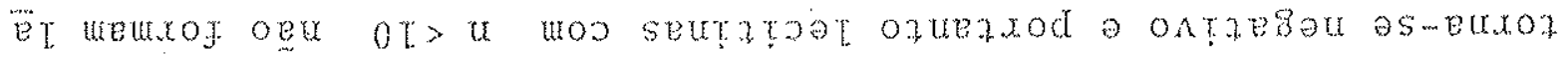

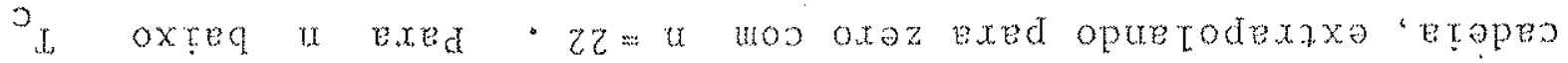

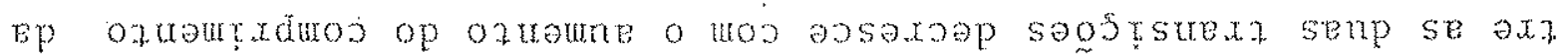

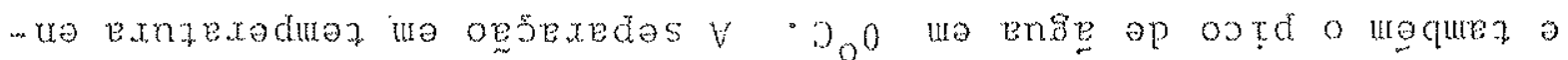

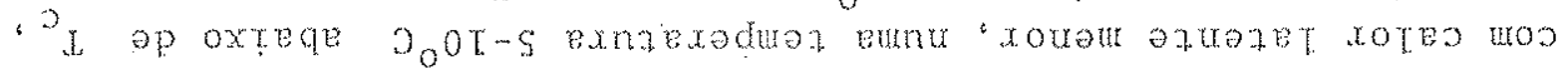

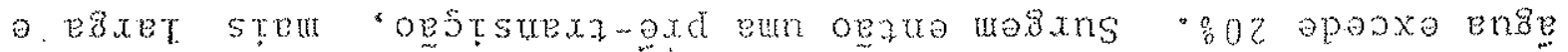

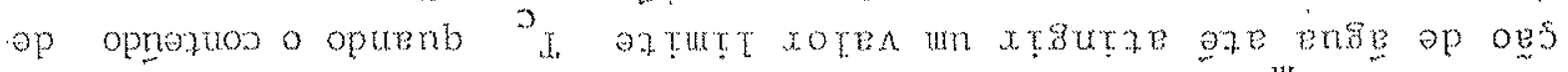

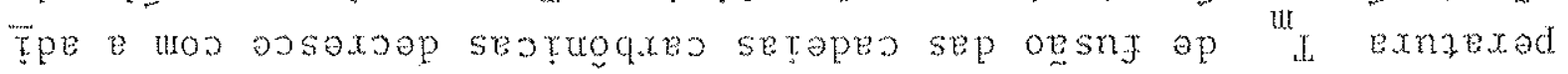

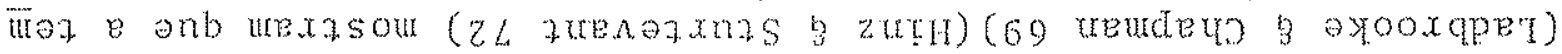

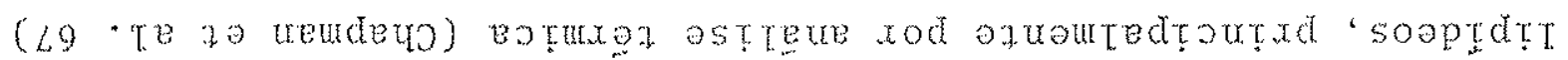

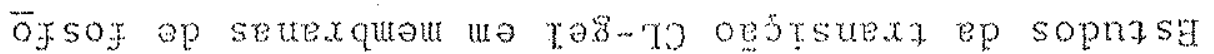

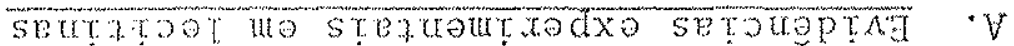

* otireqexa

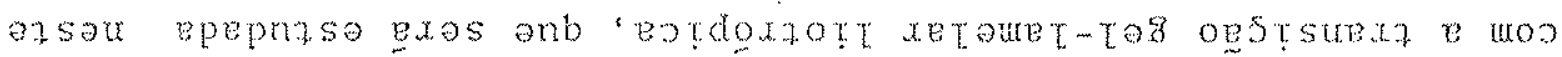

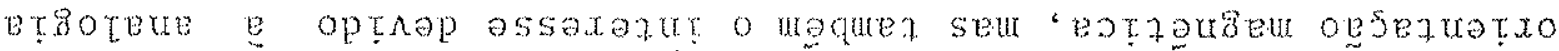
op ofxaztoo ou seotroqxeo setopes sep oxjeuxoguos ep oezsonb et

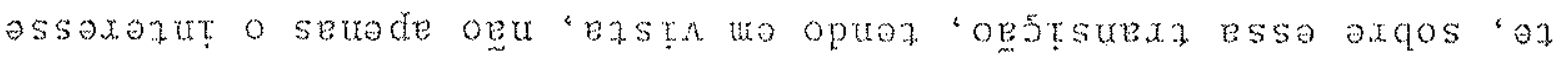

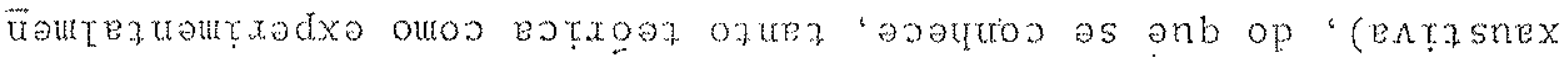
-o oet anb epute) oestax eun souax mox azsan

- opstarext ezse xenoxosop ex

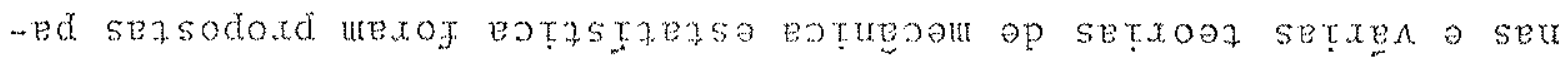

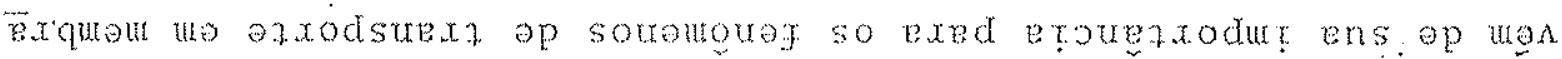

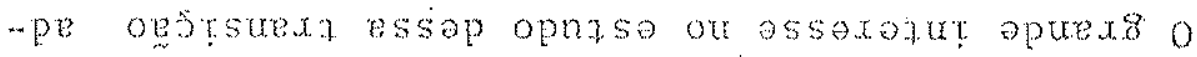

- sasegosemot exed oprago op odta omsem op e operopxosap op

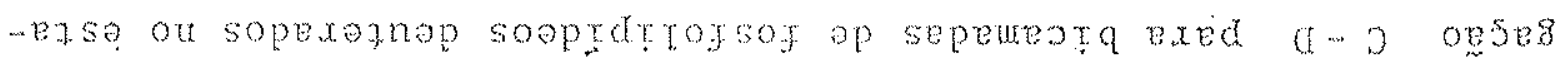

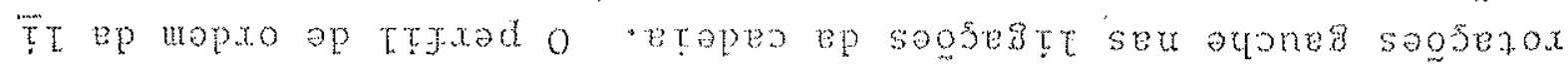

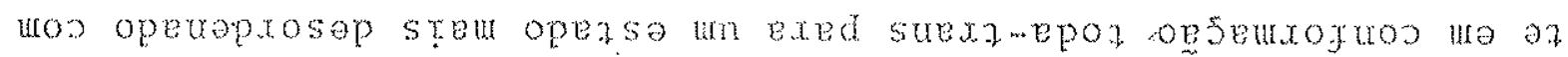

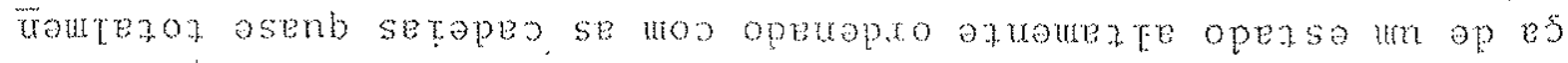

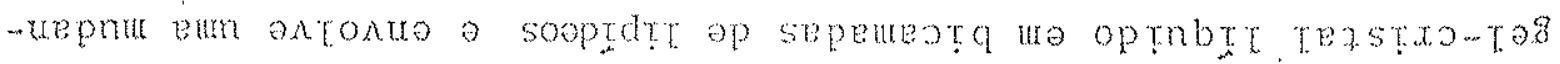

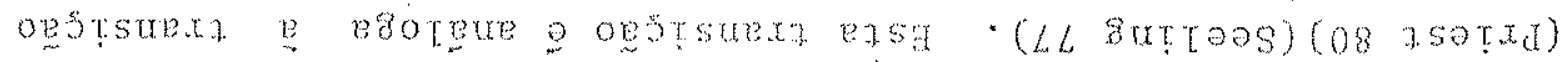

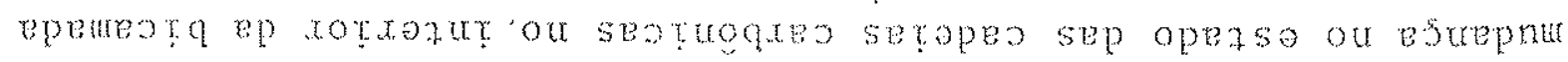

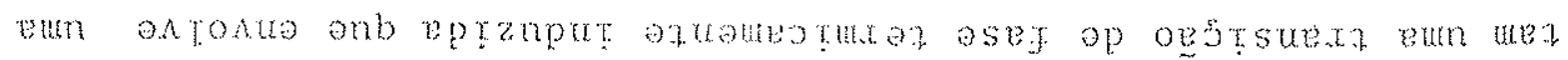

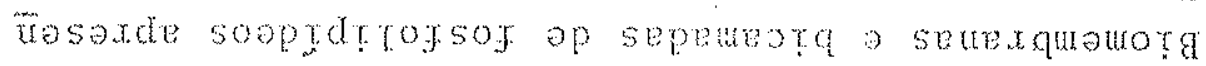




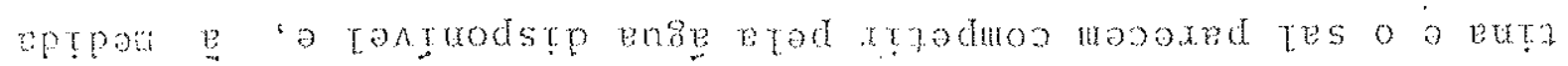

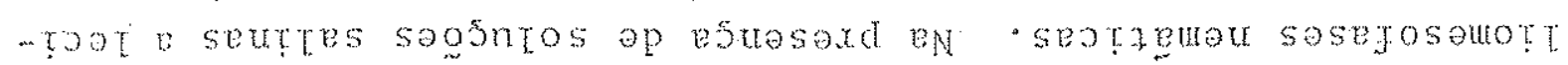

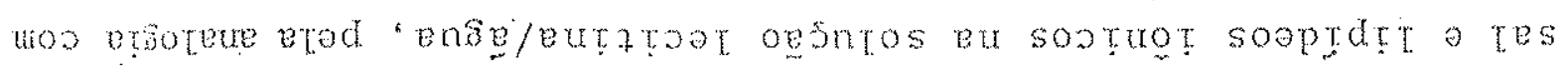

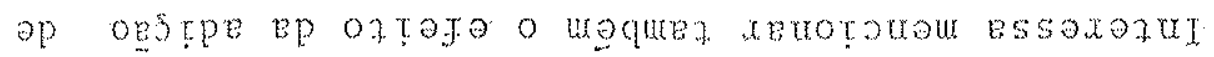

- SeTep

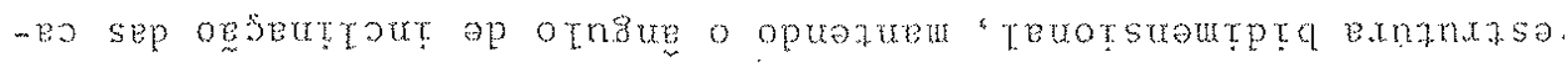

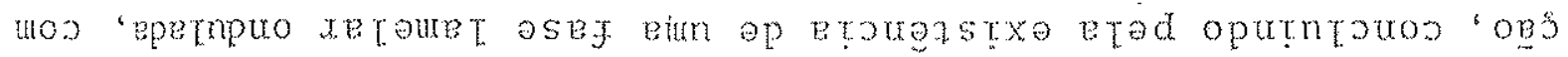

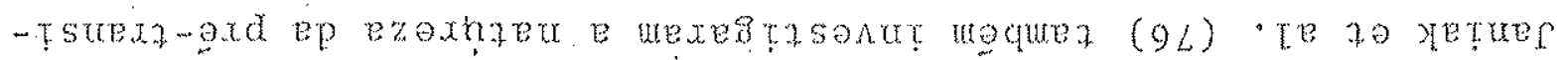

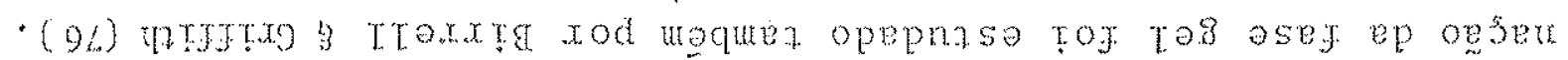

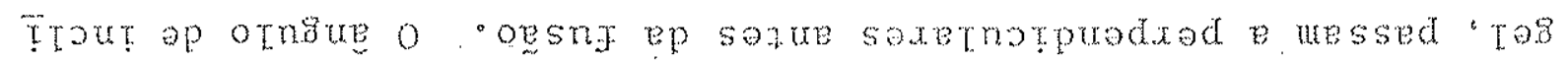

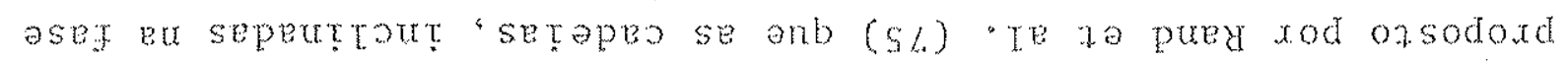

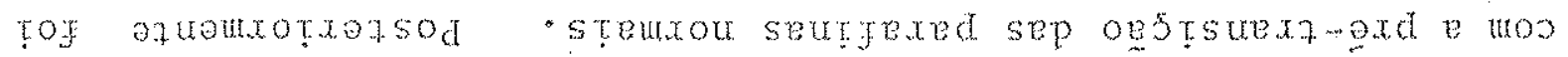

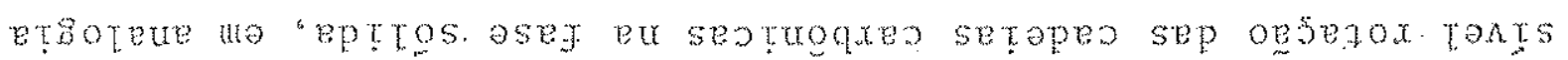
sod bun e sexerod seseqes sep exnanxase eu sesuepnu p arrourent

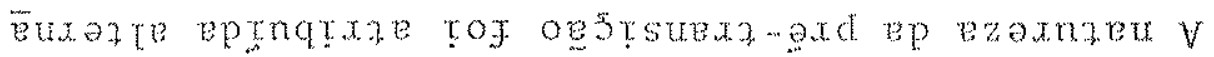

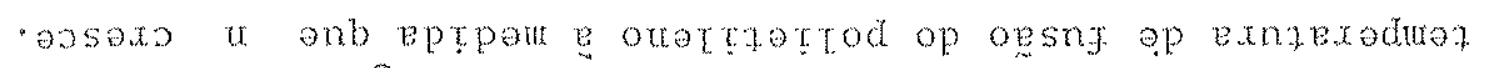

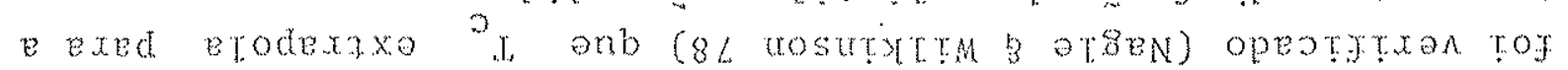

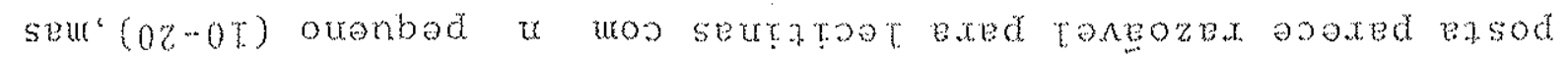

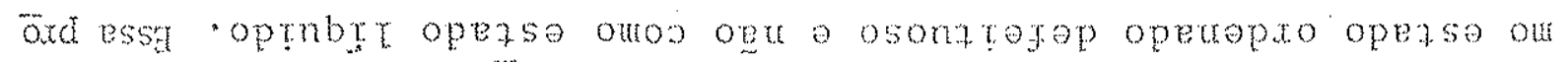

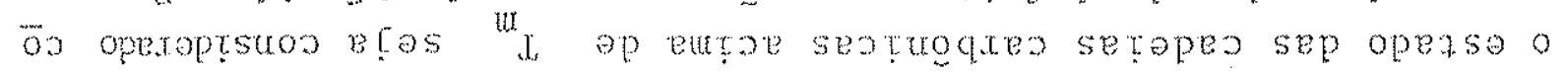

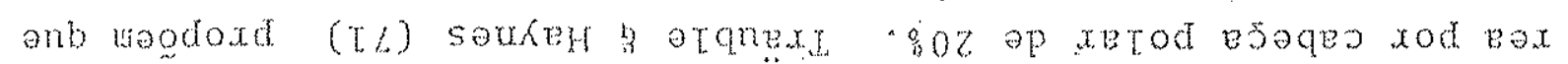

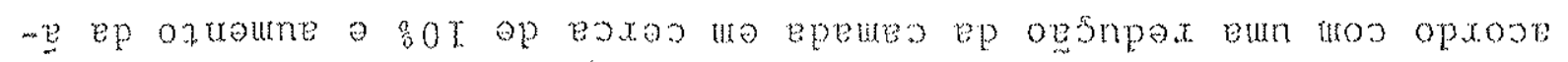

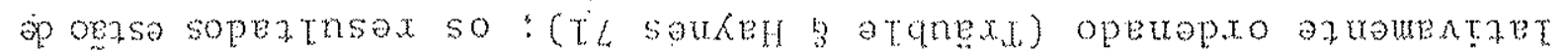

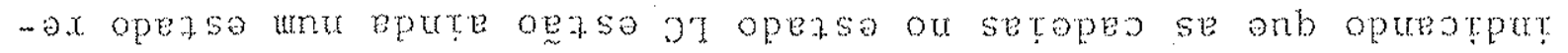

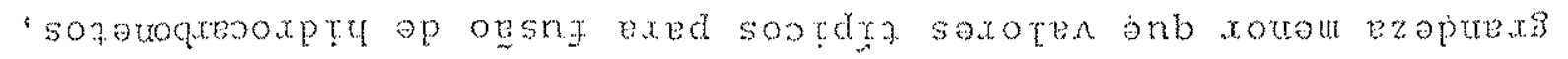

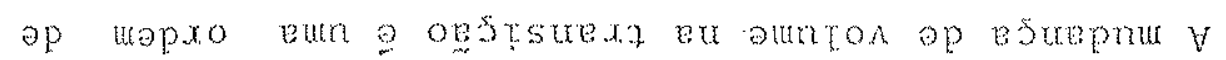

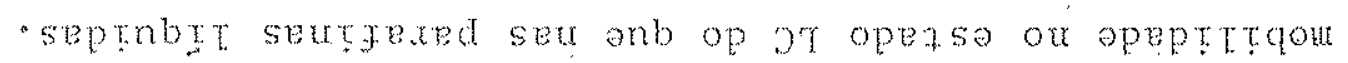

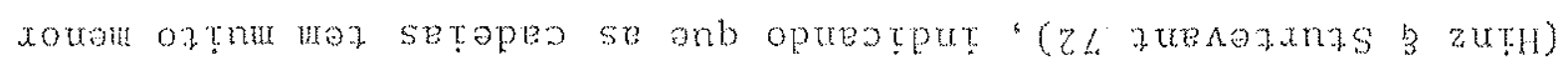

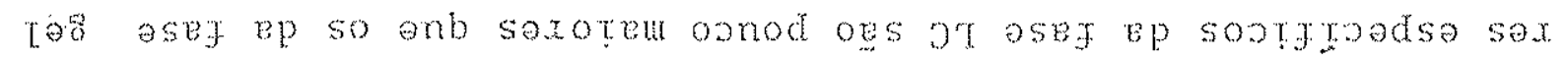

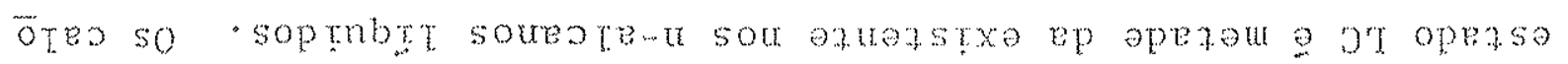

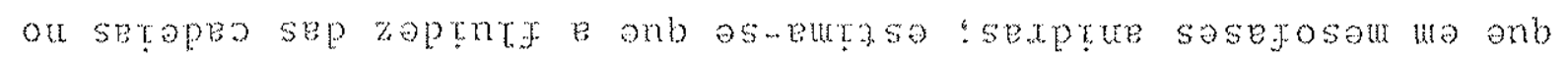

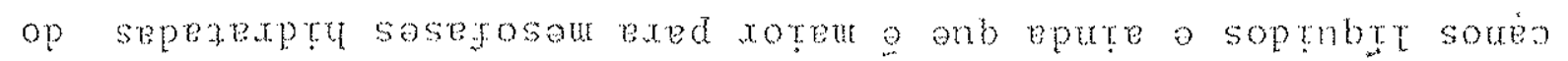

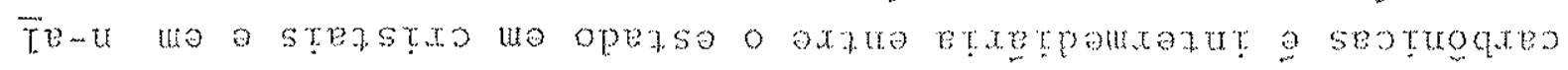

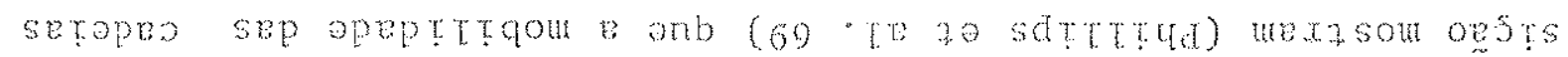

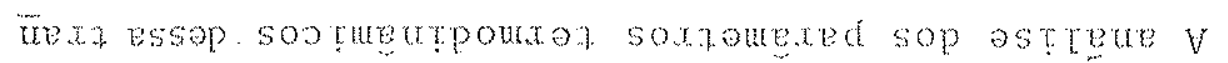

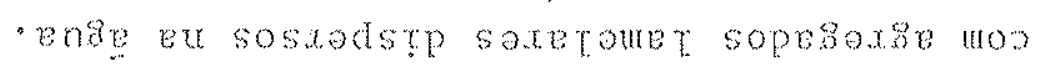

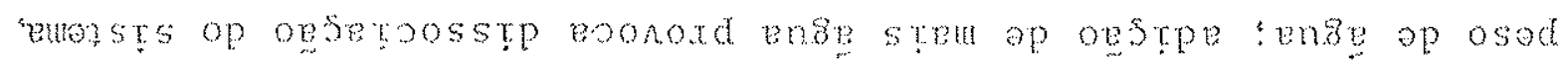

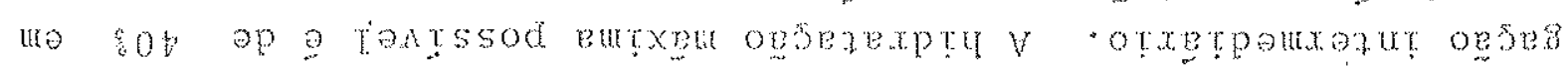

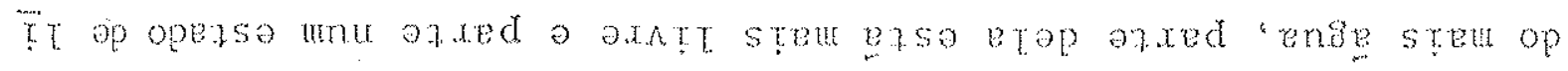

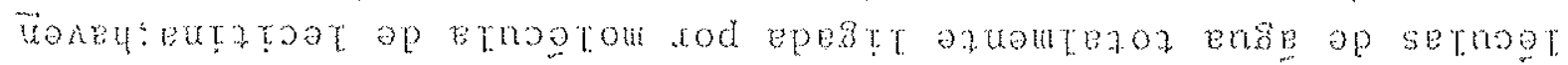

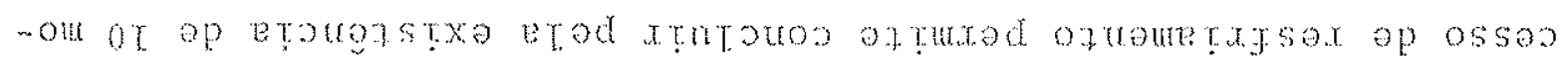




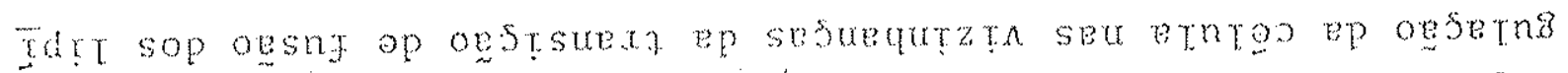

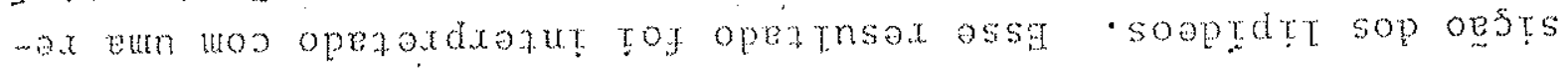

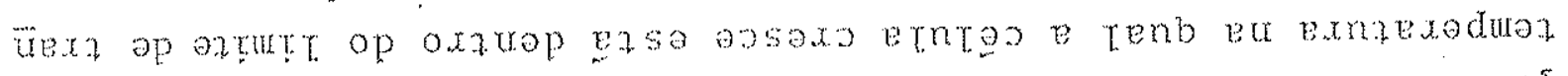

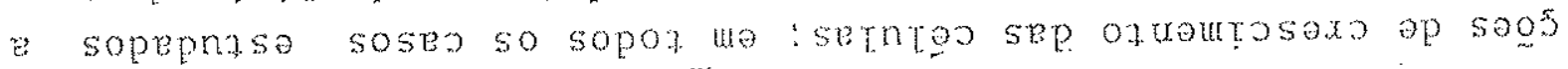

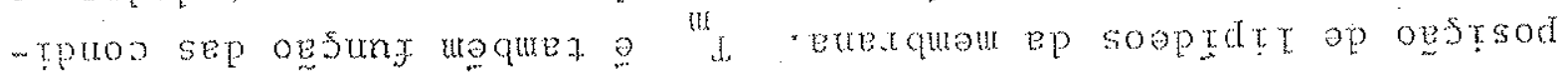

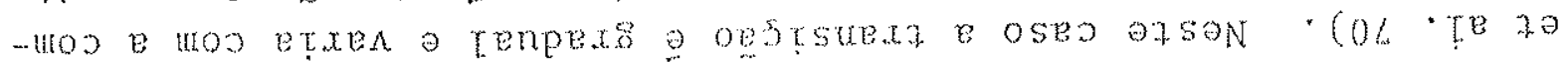

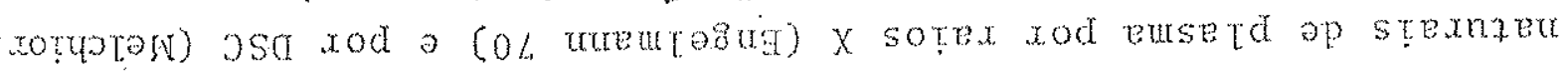

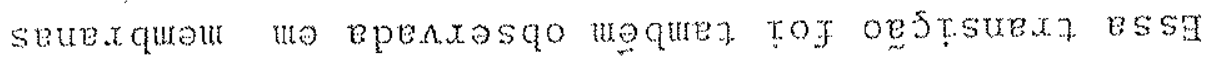

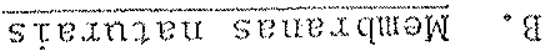

d ep soosetx

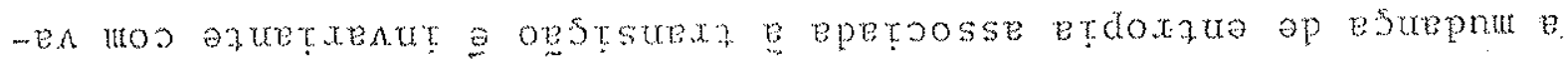

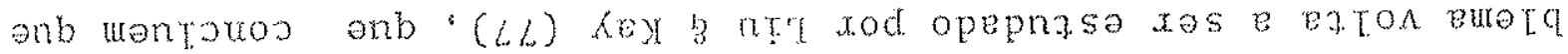

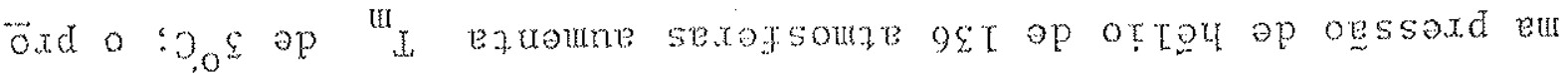

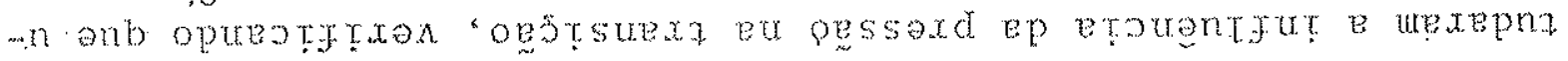

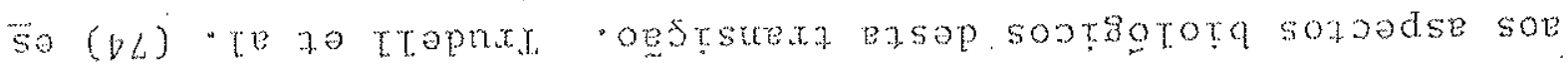

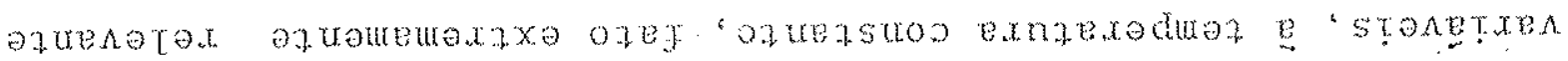

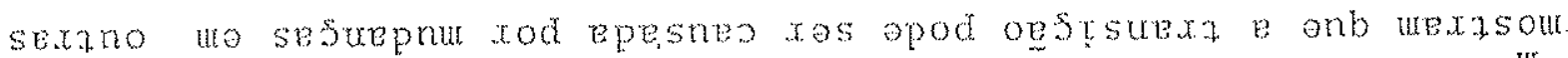

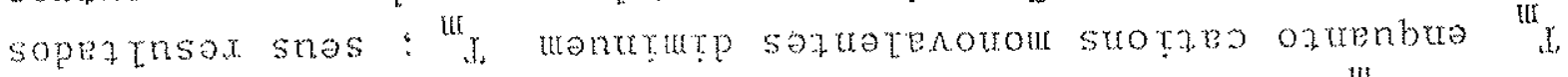

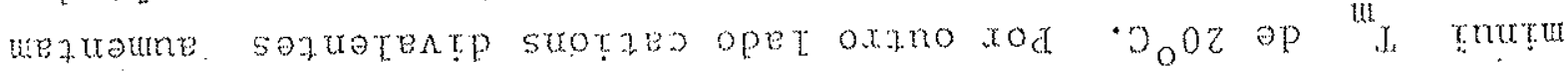

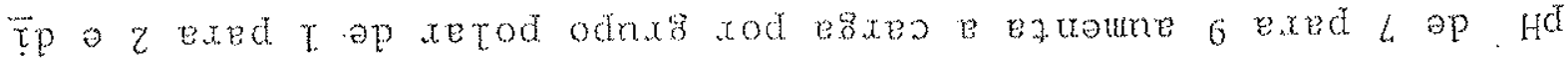

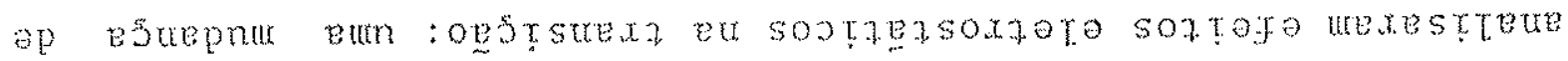

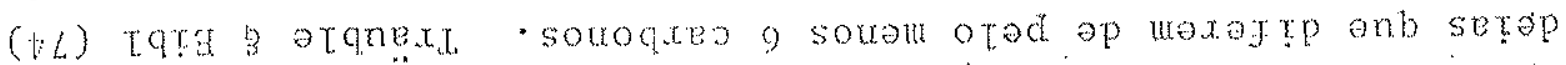

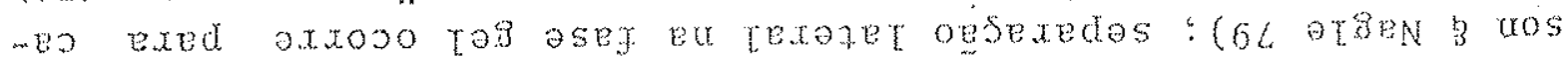

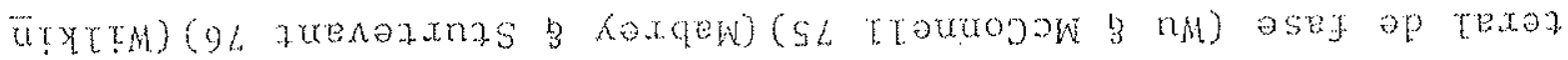

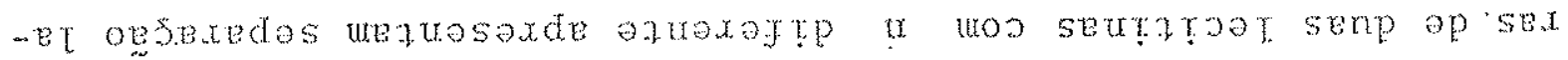

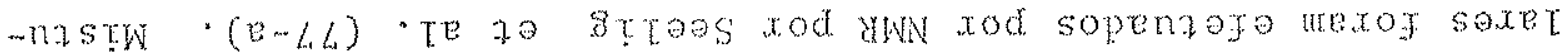

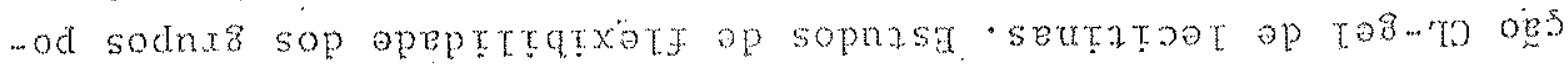

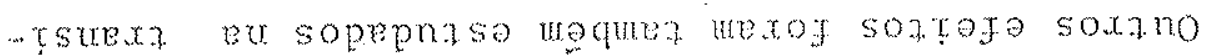

. osxet ster asmedroz ost o o

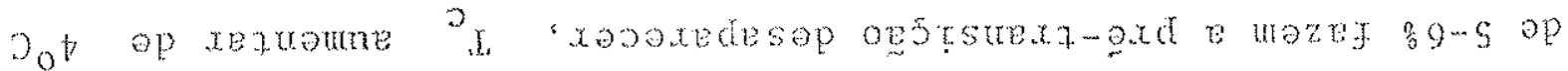

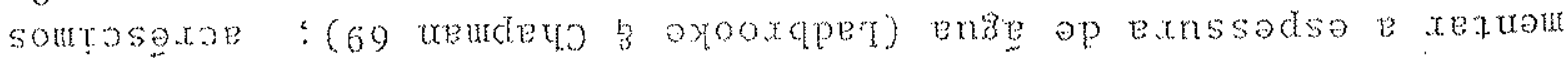

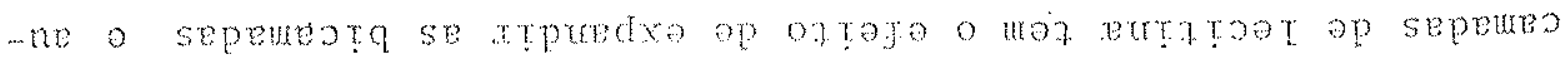

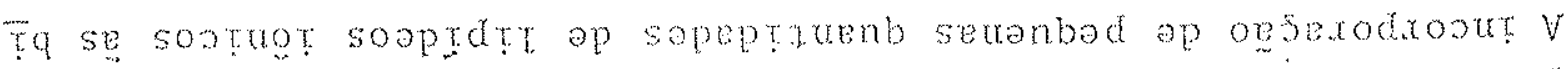

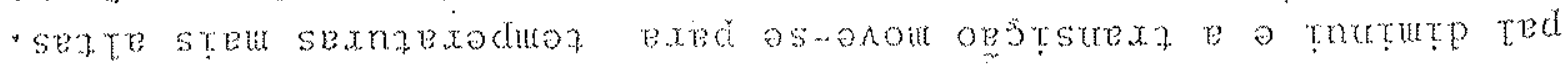

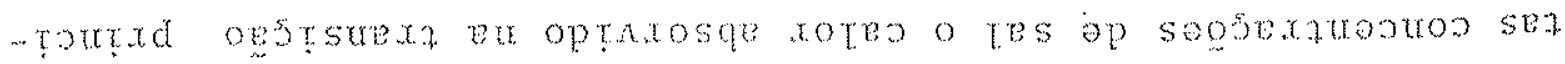

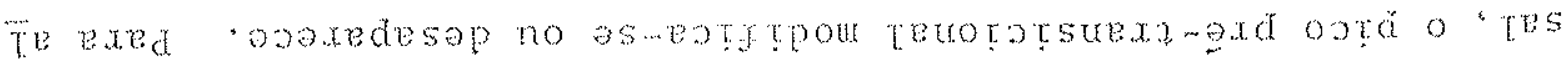

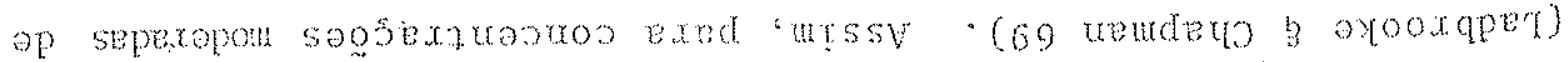

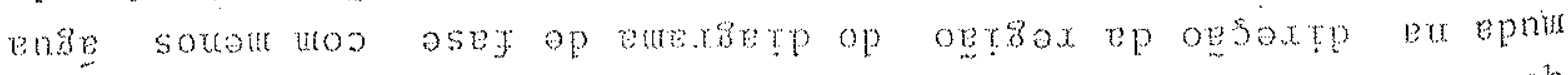

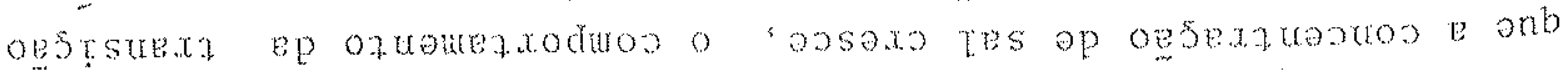




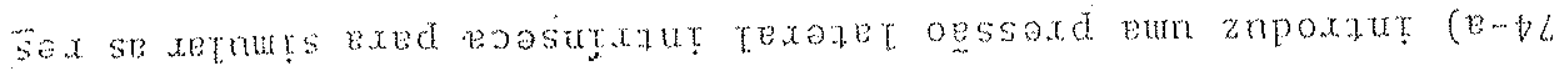

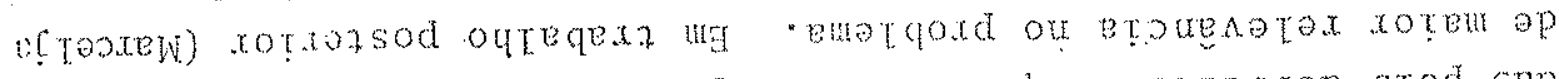

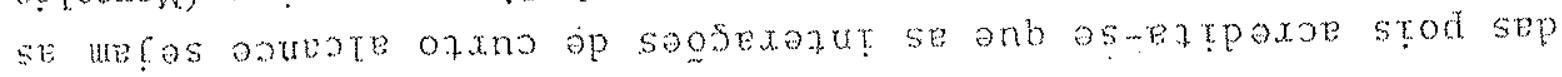

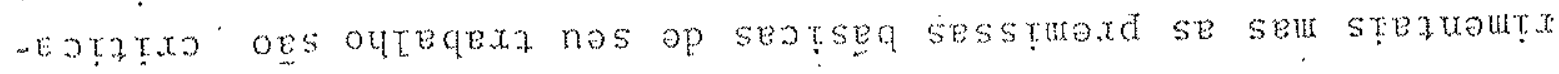

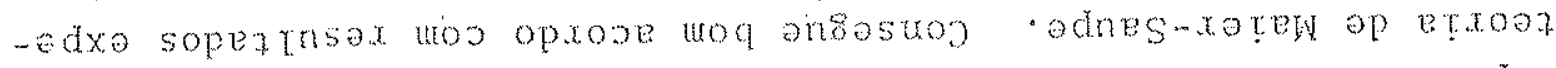

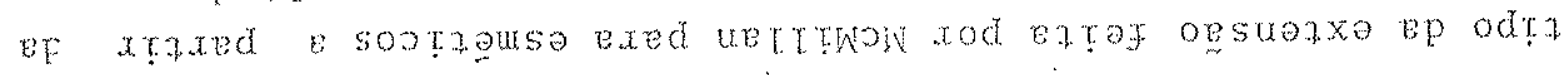
of "orpeu odues op ofopou un aodoxd (sL) ef [osx.eW

STPa

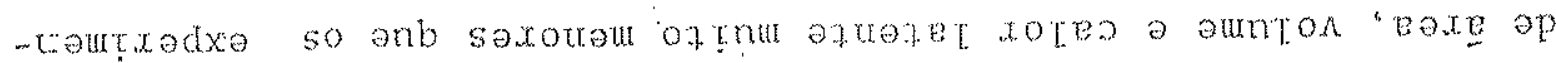

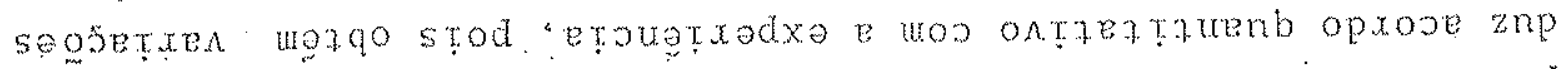

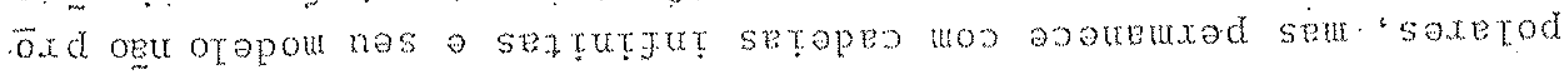

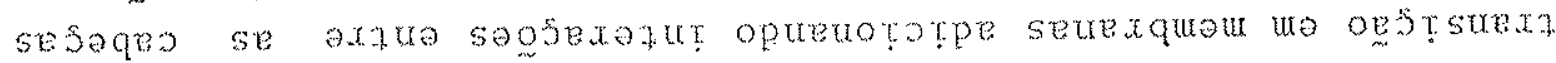

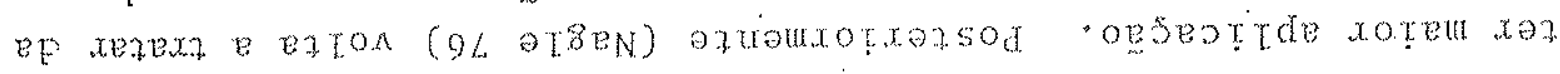

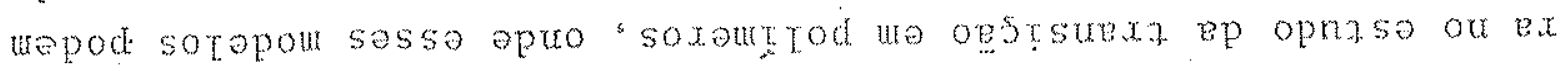

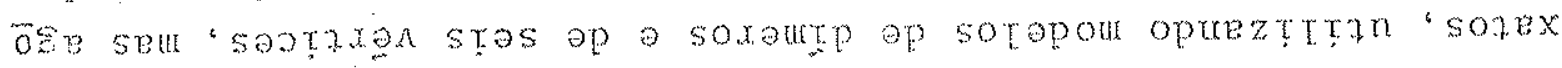

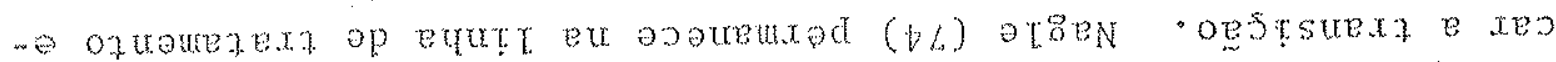

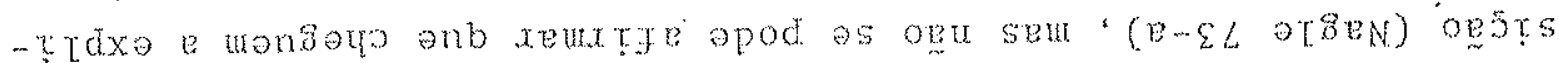

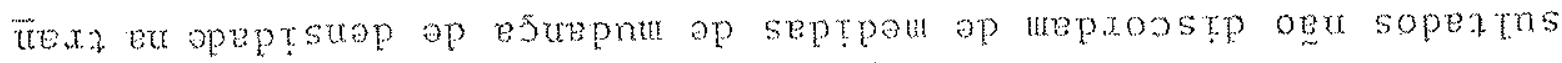

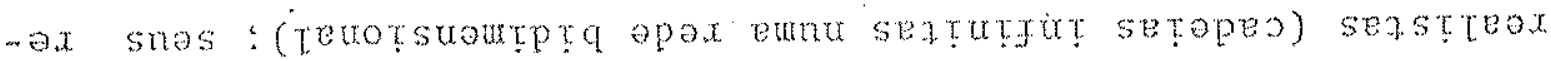

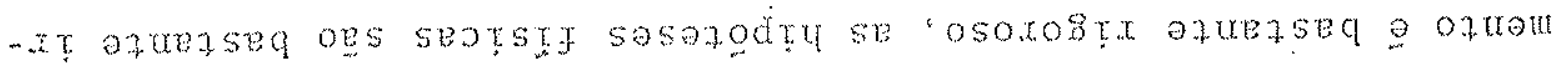

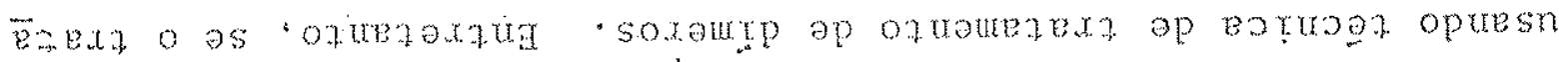

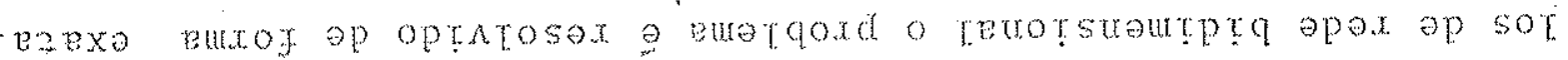

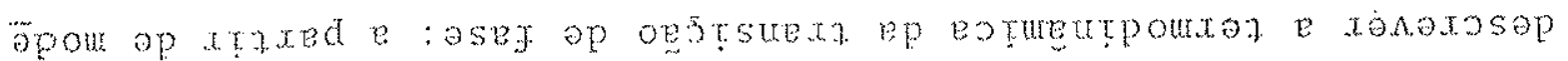

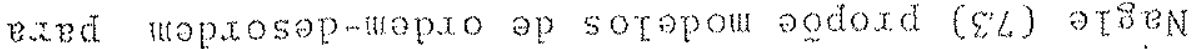

escdoxzost eptrbt asej eu

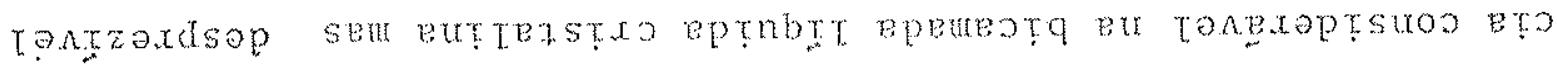

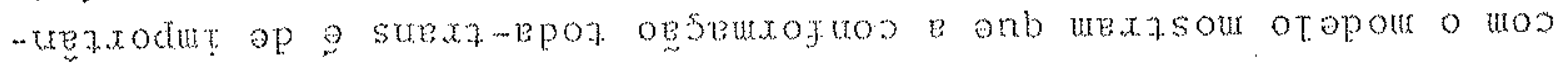

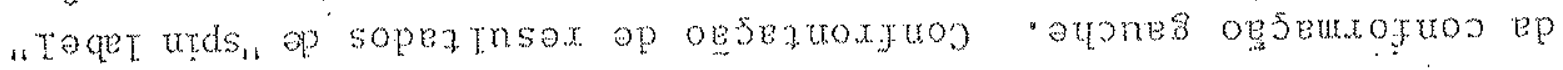

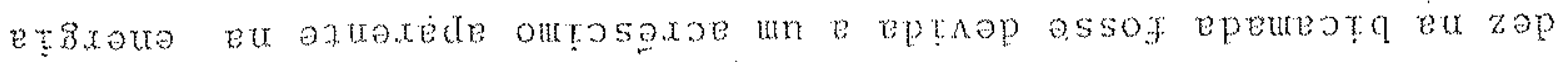

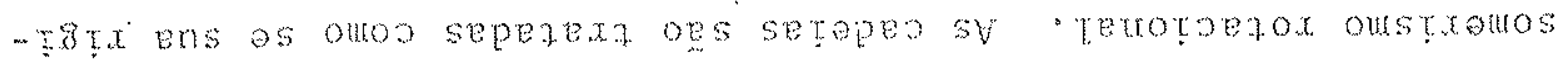

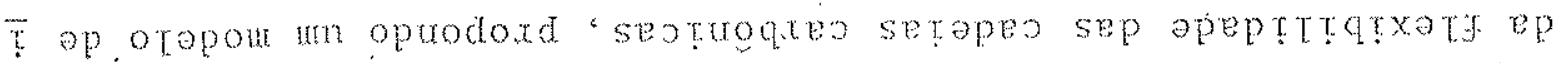

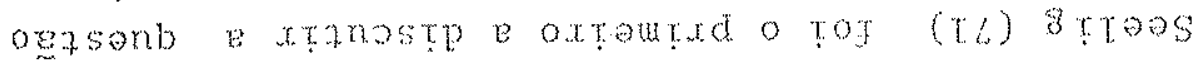

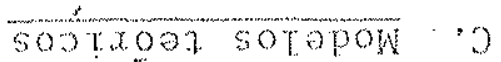

- exexquen ep eats

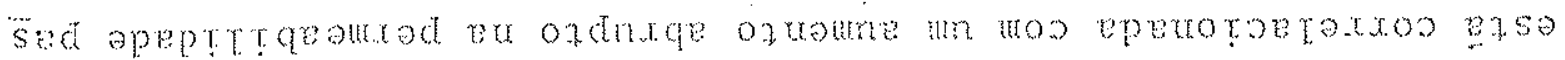

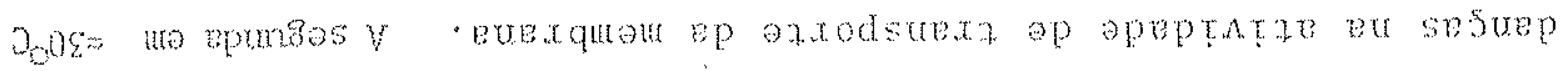

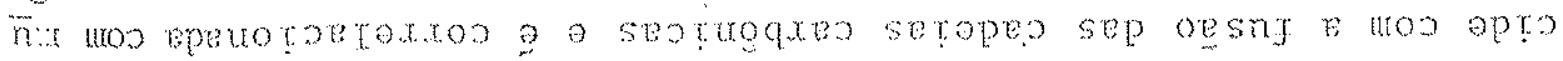

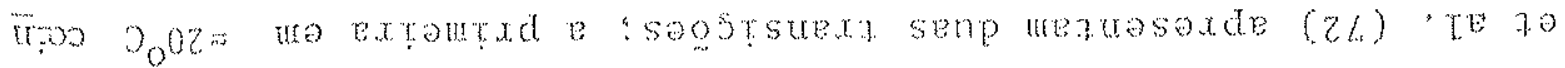

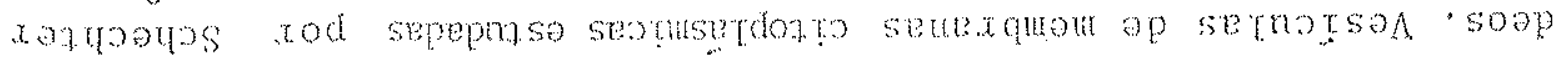




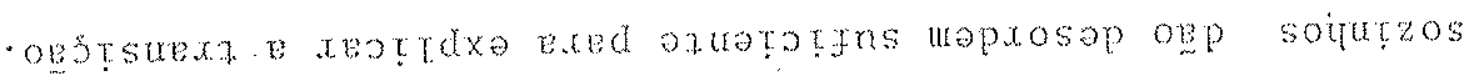

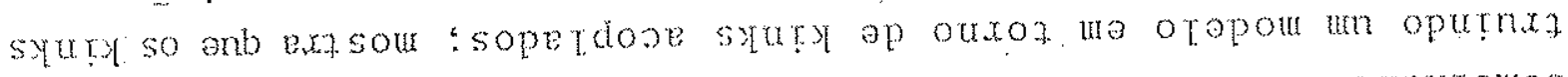

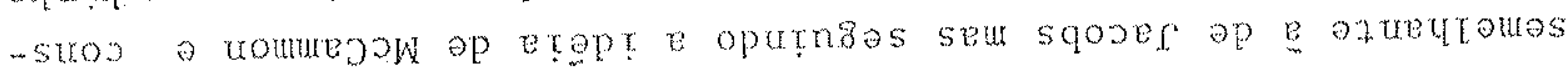

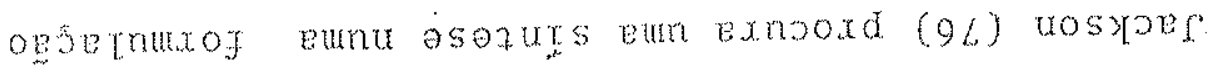

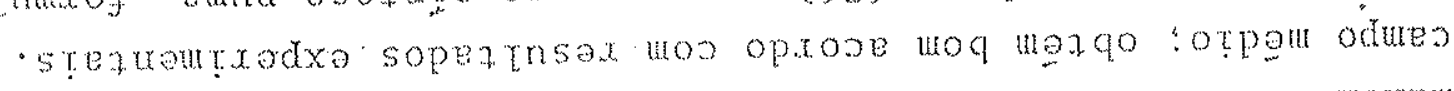

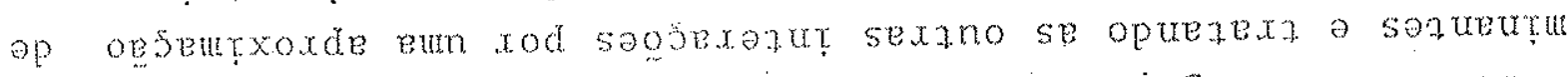

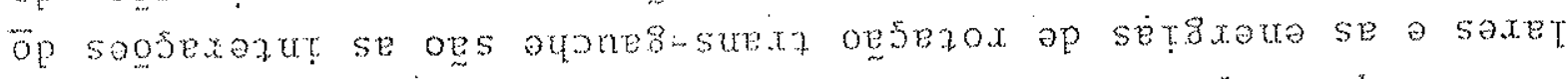

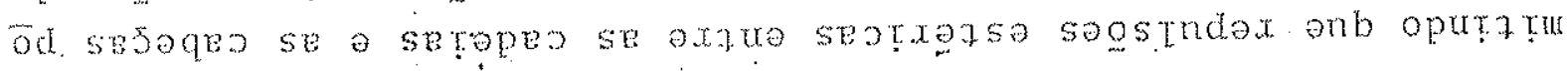

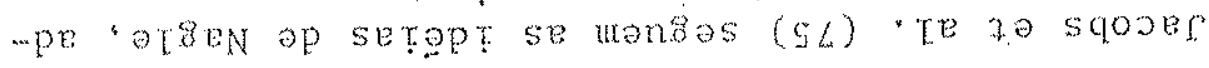

- serdurts

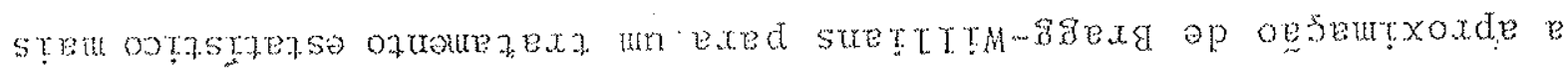

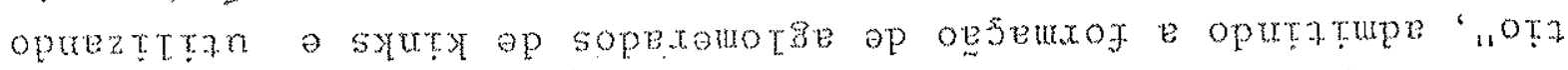

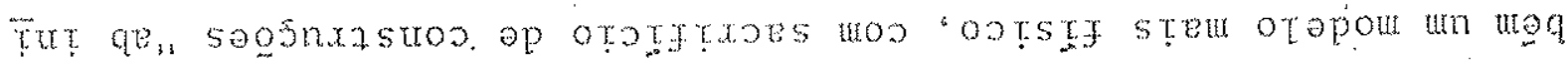

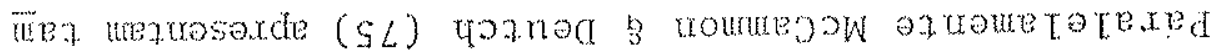

- eperareo

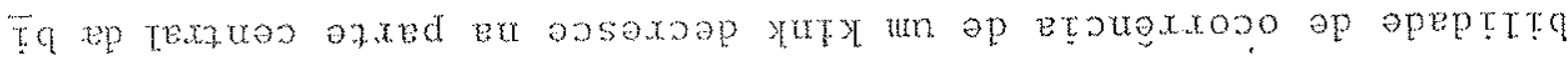

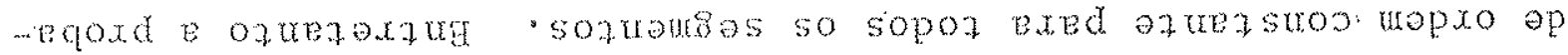

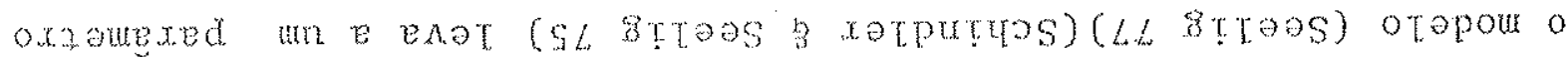

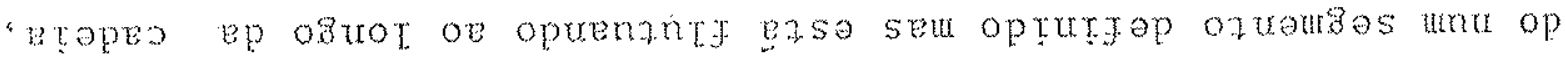

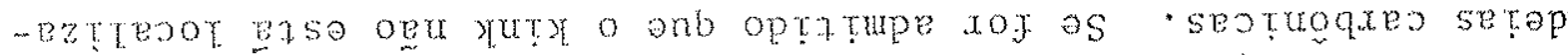

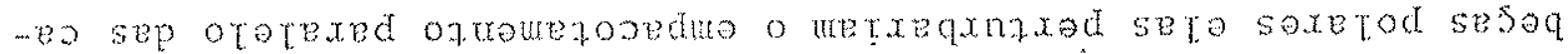

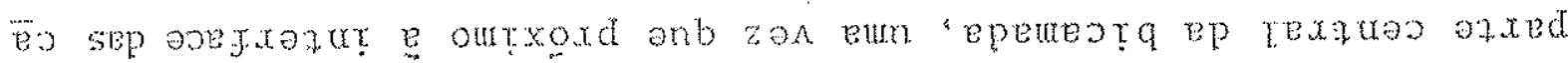

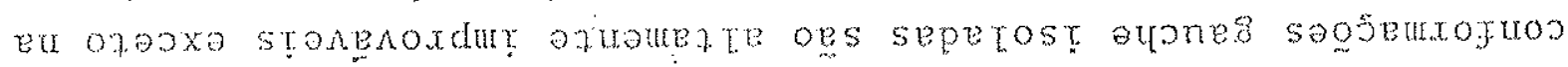

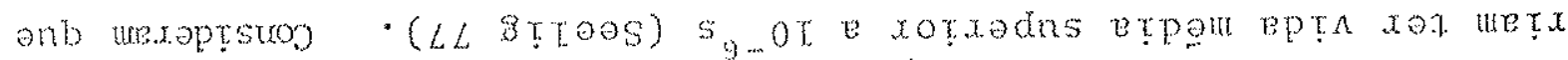

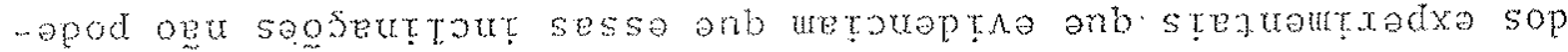

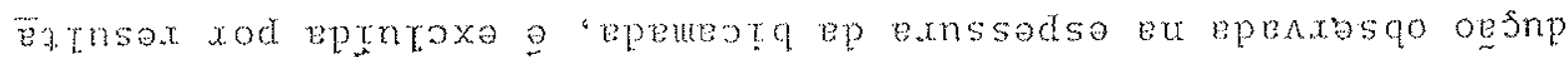

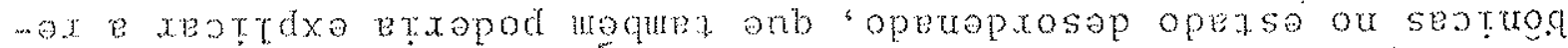

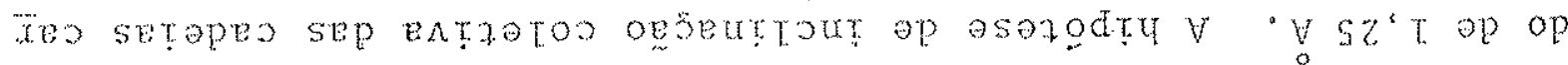

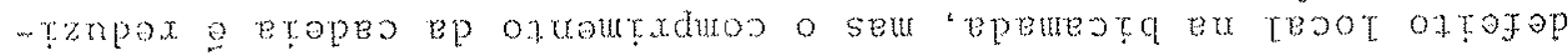

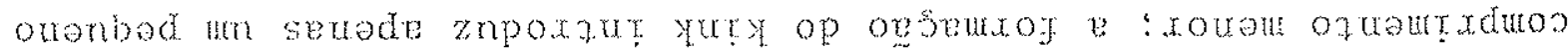

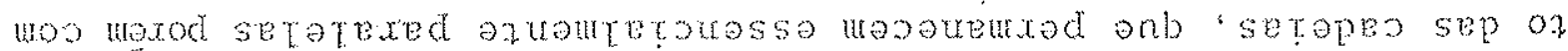

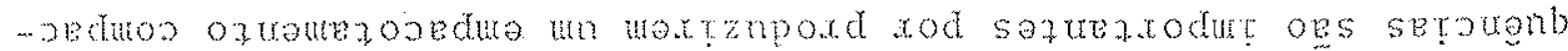

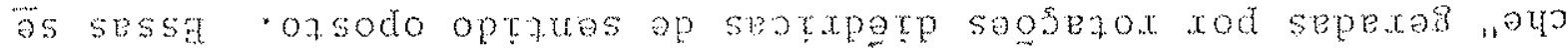

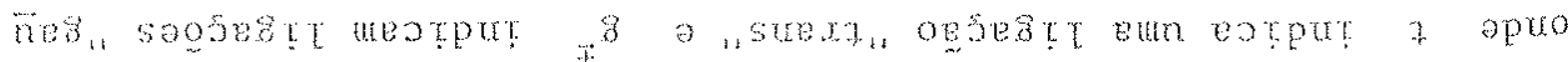

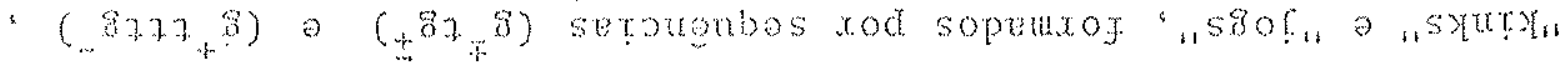

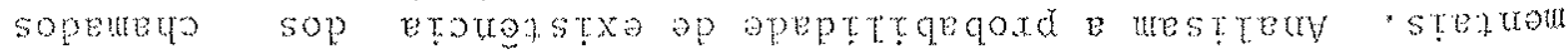

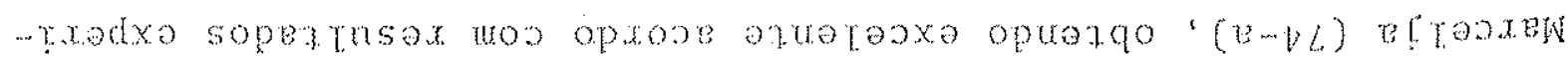

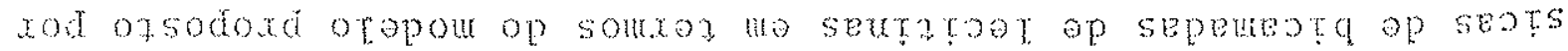

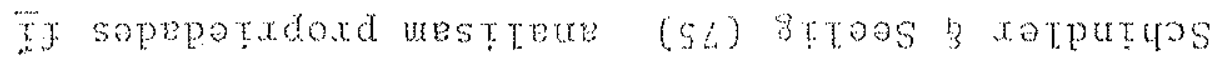

- setratrot axed sopertinsox uroo op

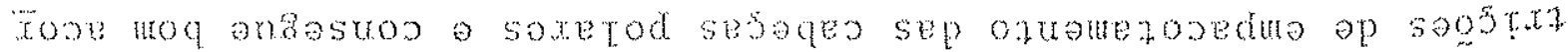




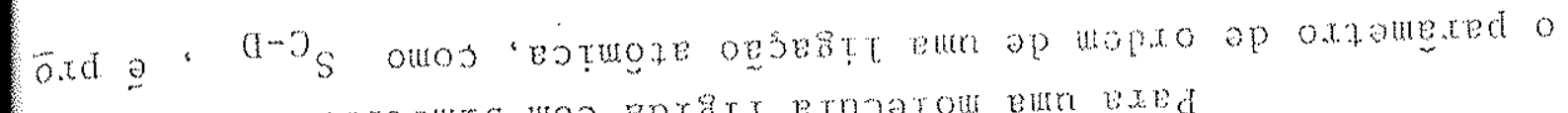

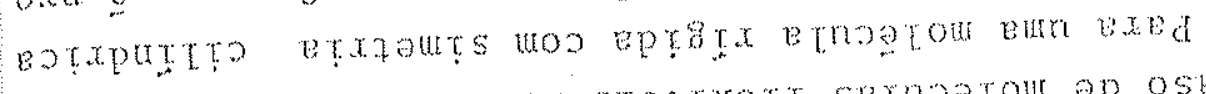

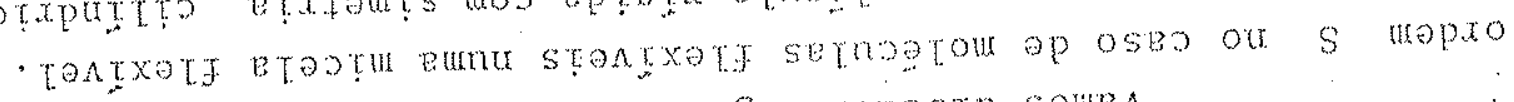

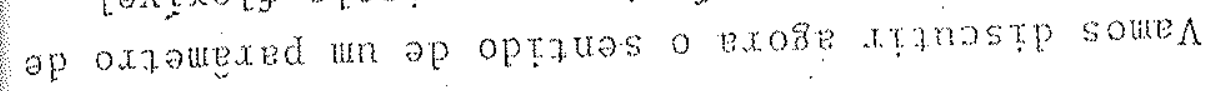

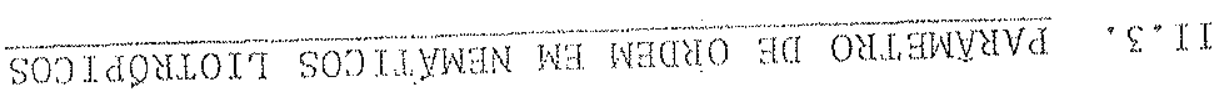

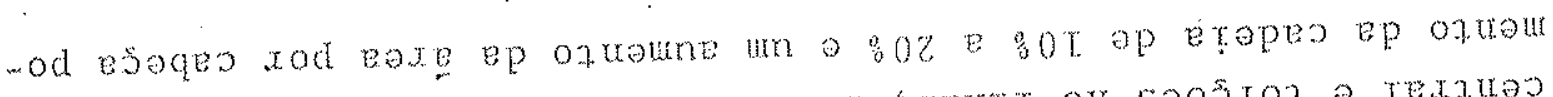

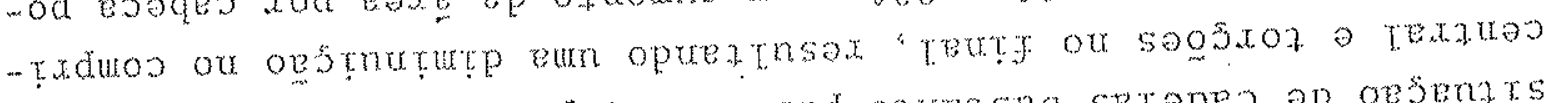

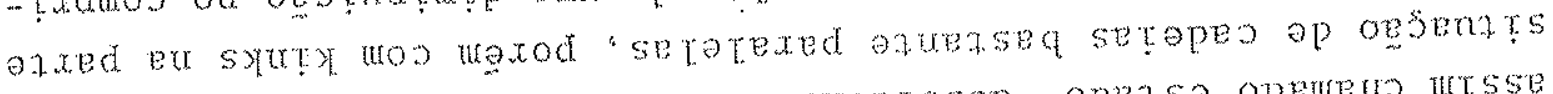

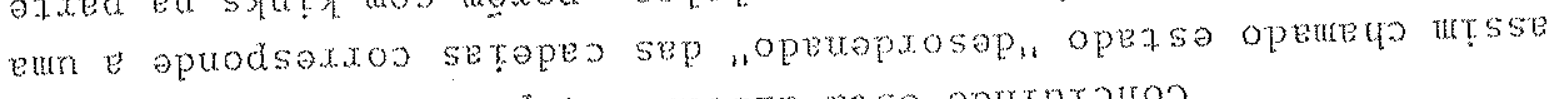

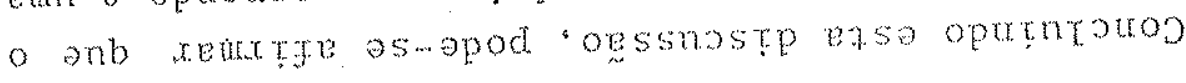

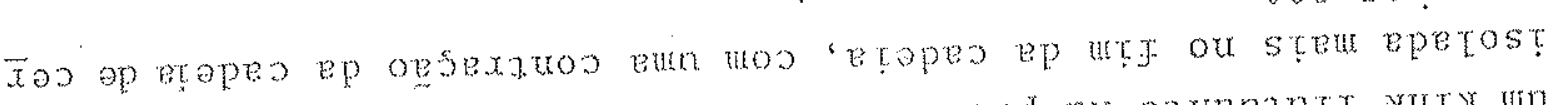

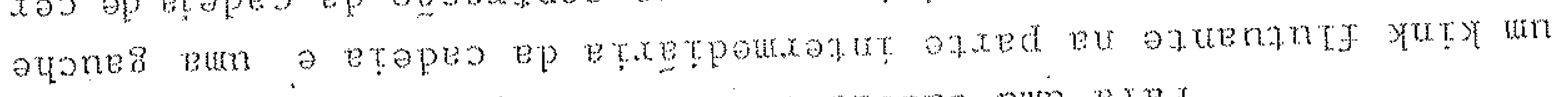

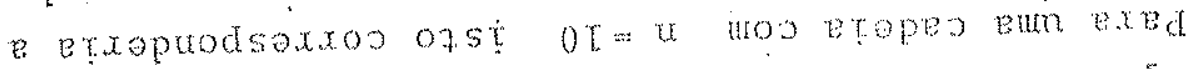

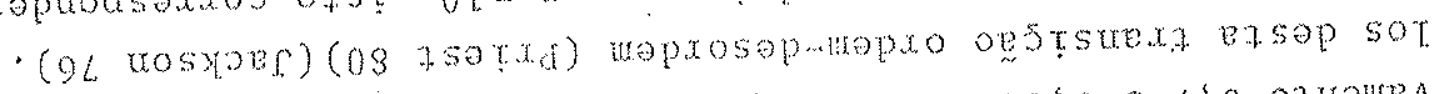

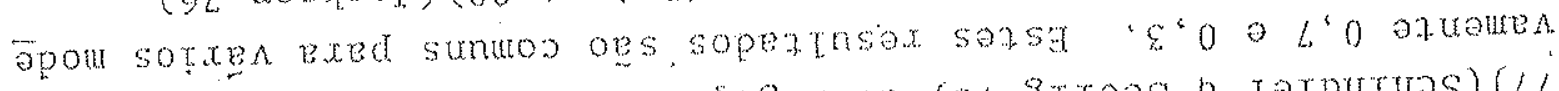

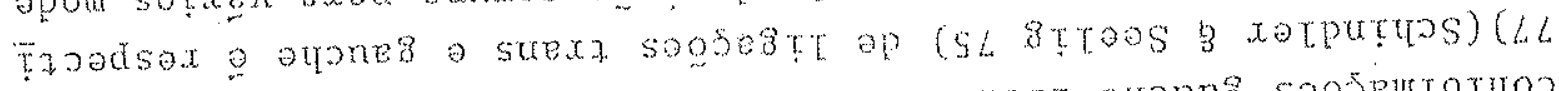

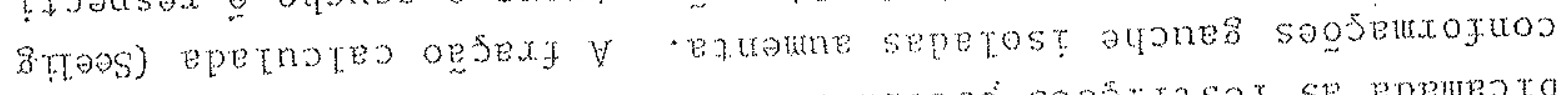

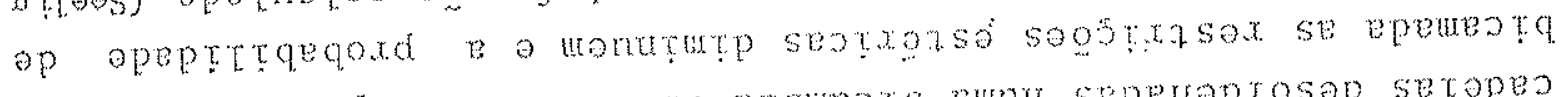

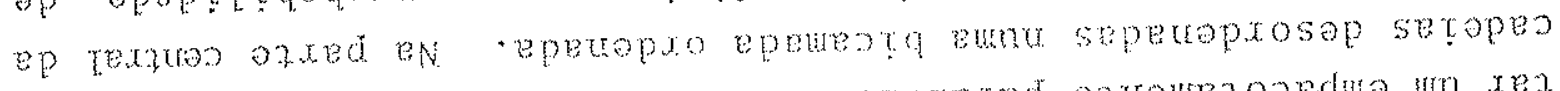

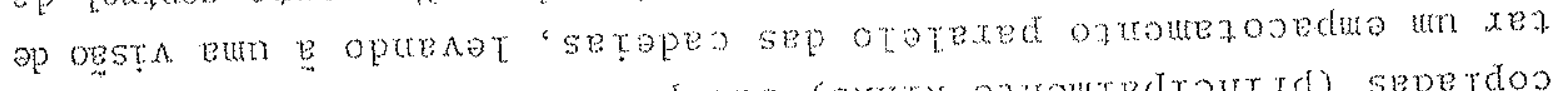

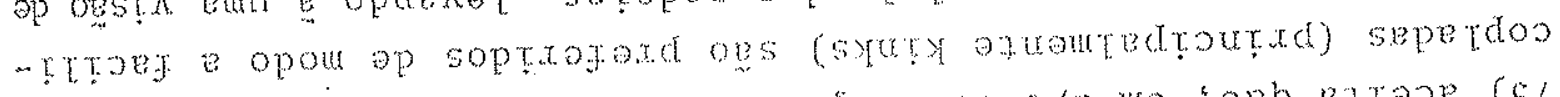

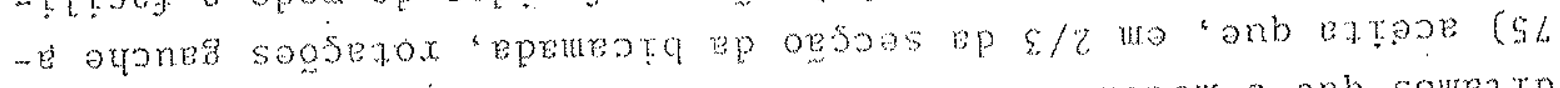

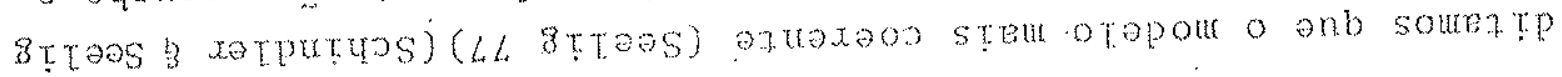
-axoe soptrandrasop sotapom sotxen so opuestretry

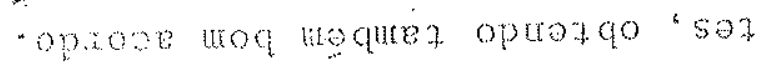

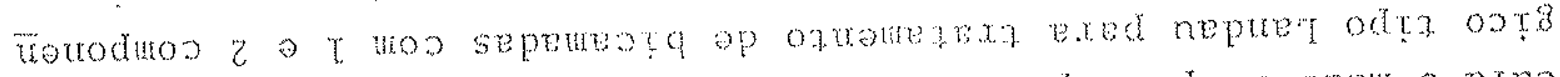

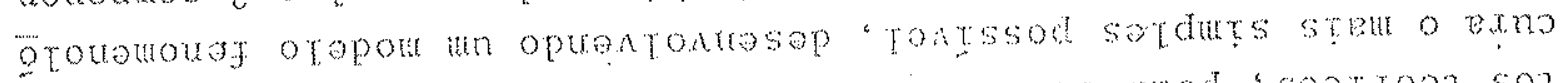

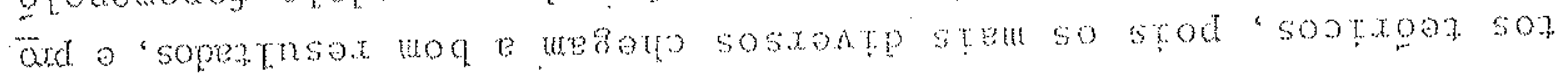

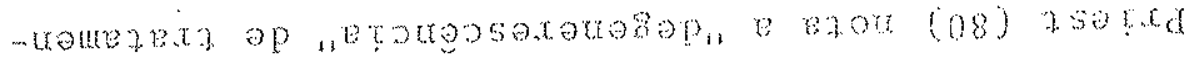

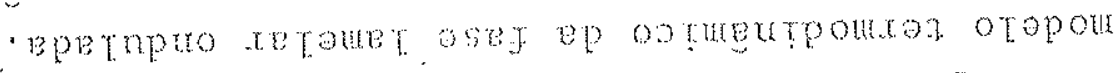

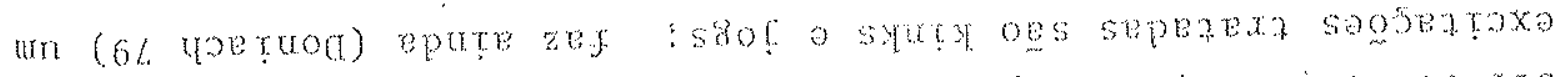

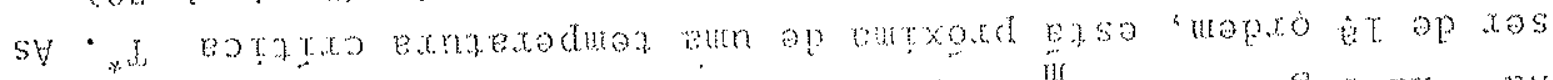

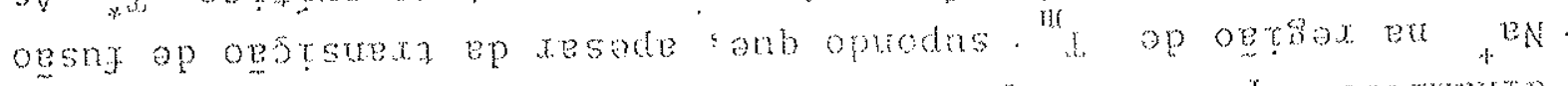

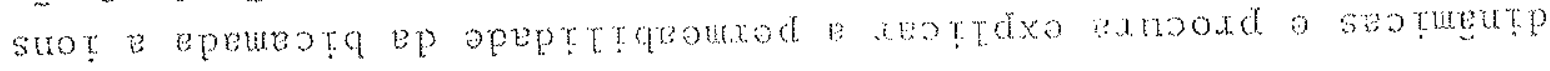

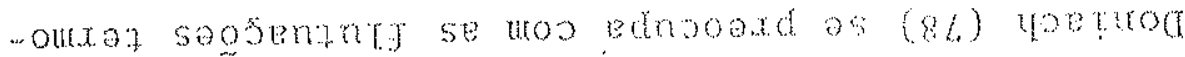




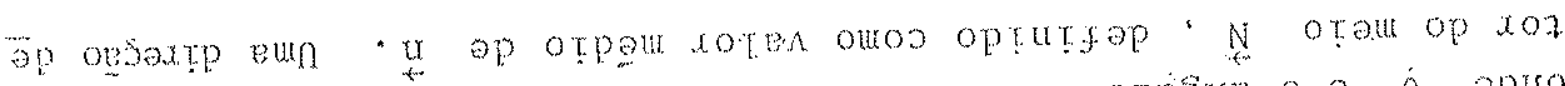

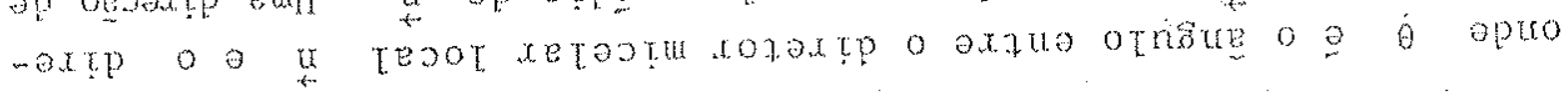

$$
<1-0,500 \xi>\frac{2}{T}=7 \times 9
$$

ouros oxtos

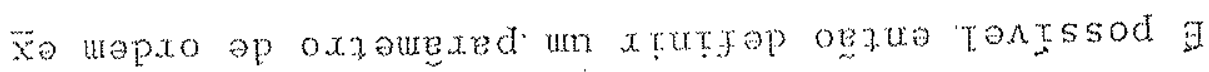

- seueta se tootur ap os

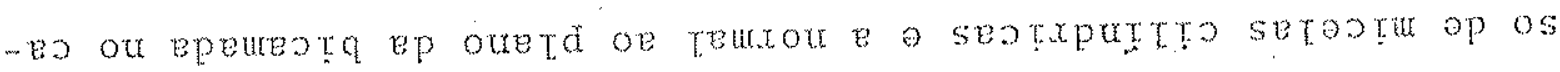

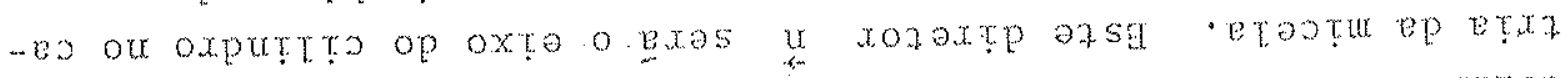

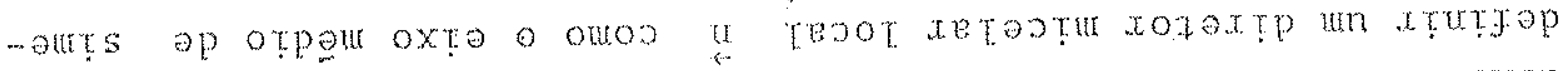

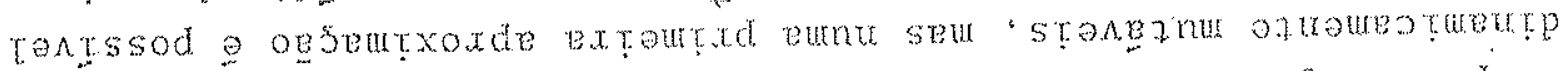

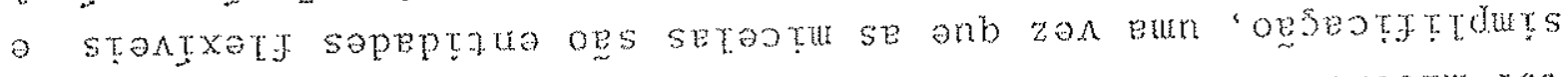

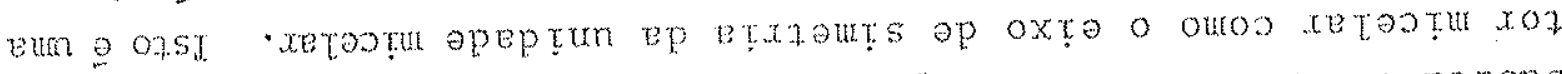

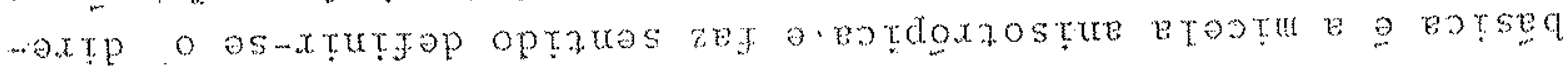
opeptun d sosegosamot sease axed opet oxano xod

- opoz un omos xetopru epeptun e

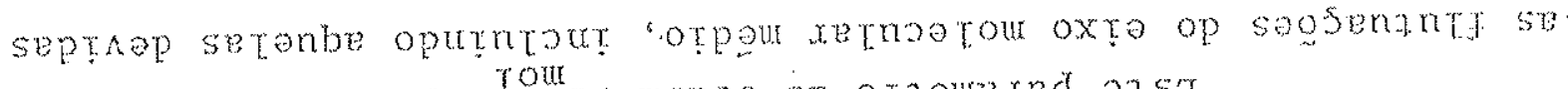

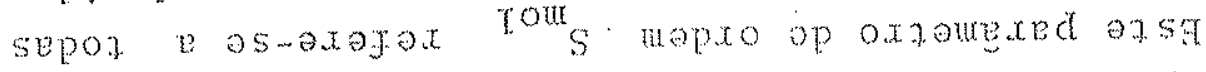

- eotrojsoxom bexsone

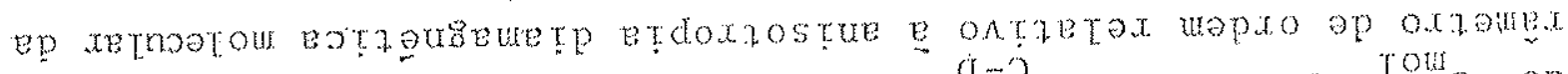

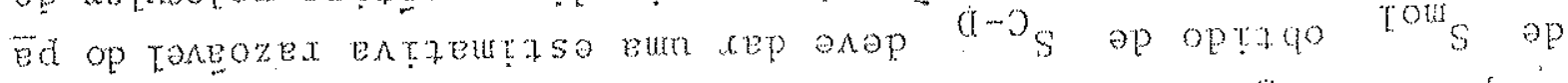

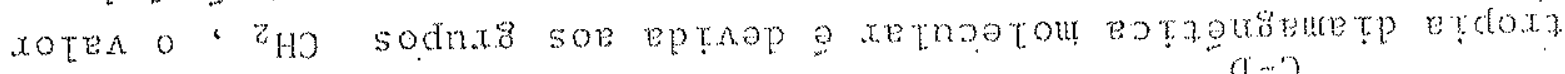

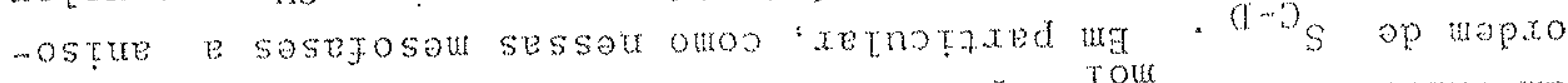

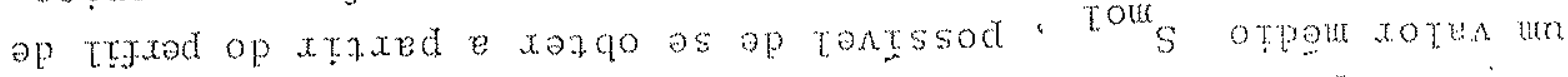

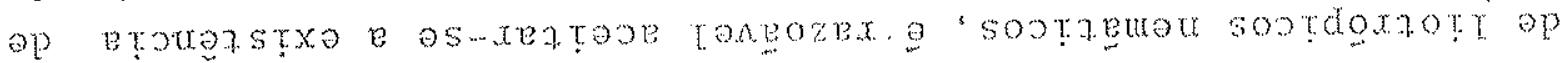

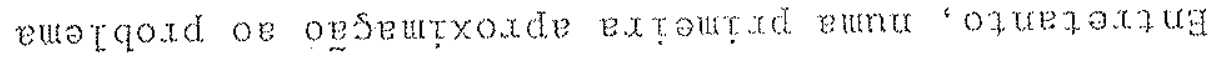

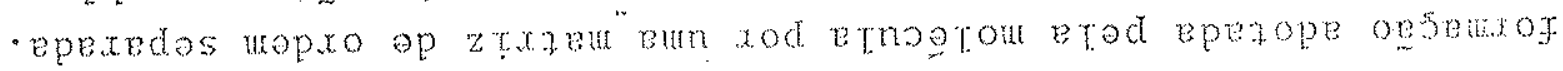

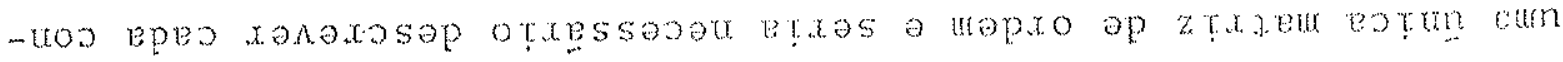

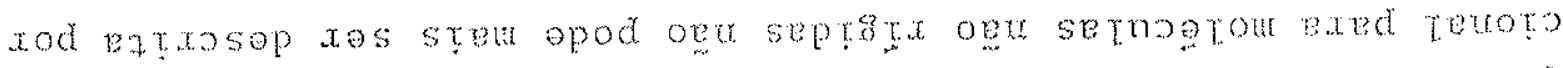

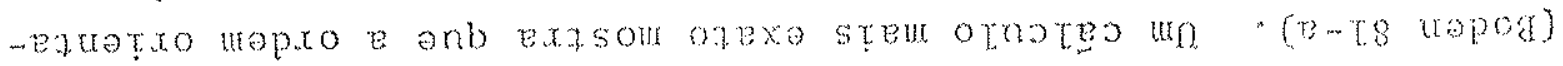

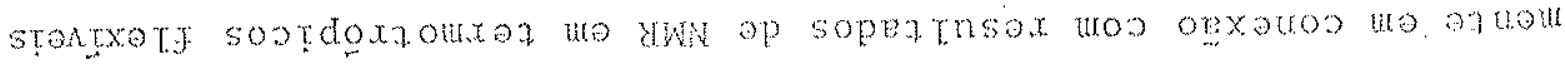

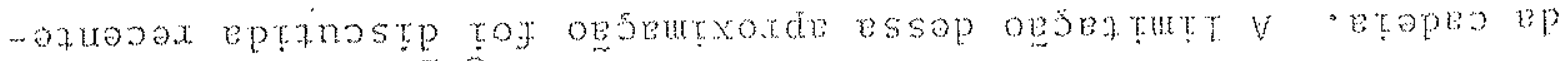
ostrot or etxen a-9 oprenb aten ogu xernotaxed he o stat ated

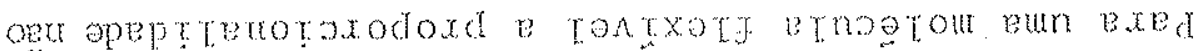

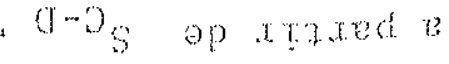

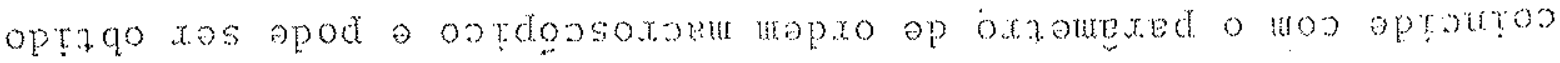

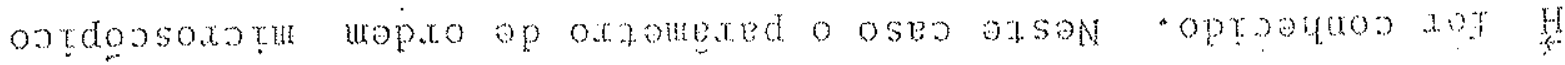

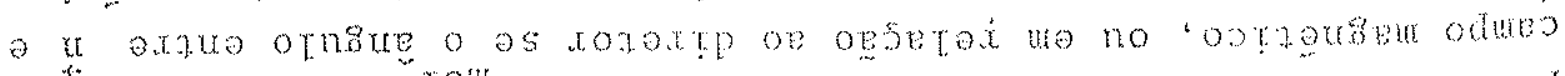

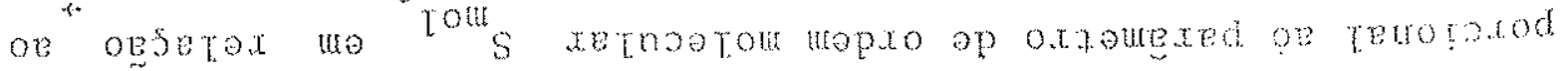




$$
\text { - Toms e stontsues opros 'xetrootom }
$$

Tontr on seosexalut ap uopuatop anb sepepotxdoxd uroo seperopot

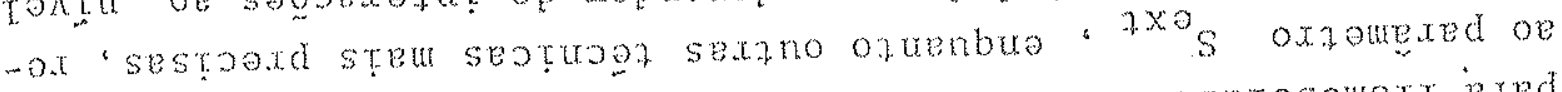

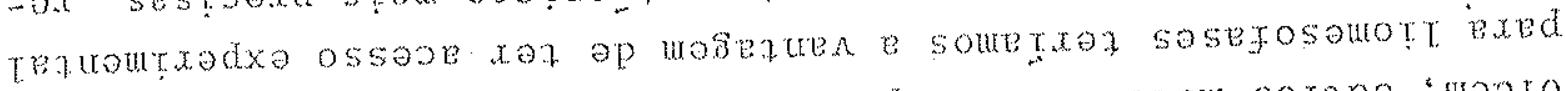

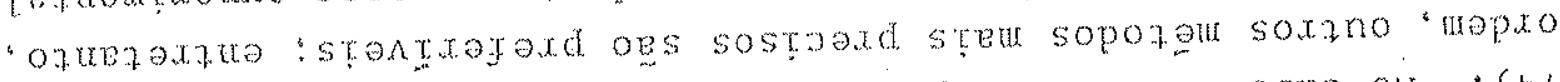

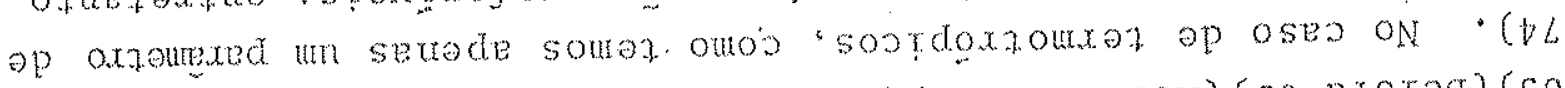

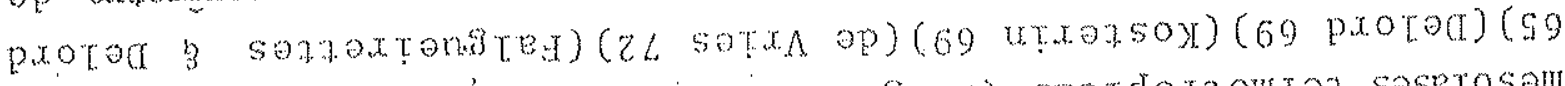

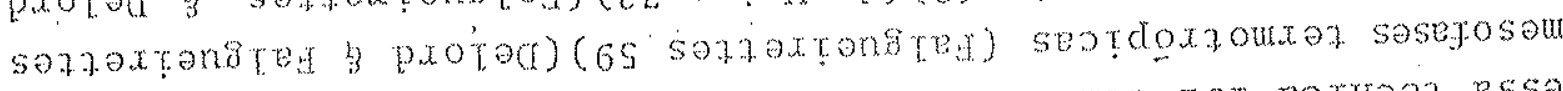

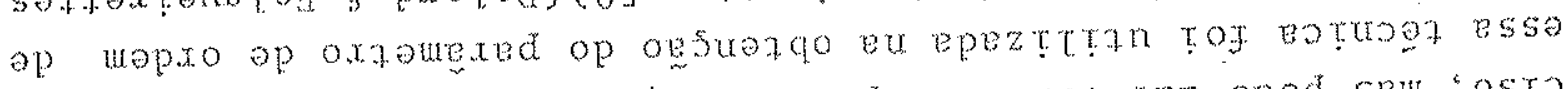

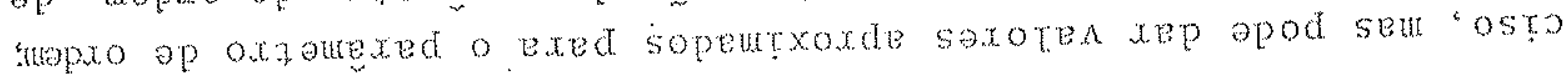
axd ofrnu opertrsax wn ep oed $x$ sorex ap opozen 0

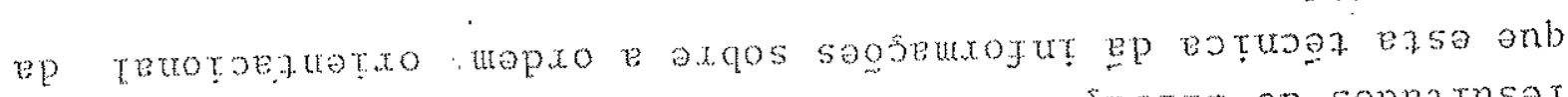

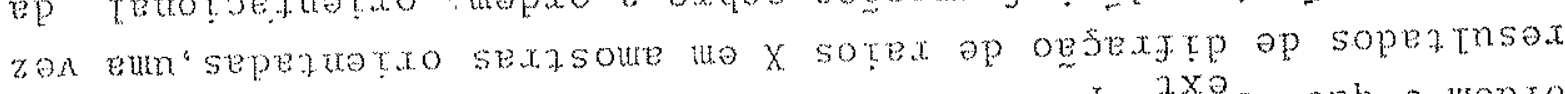

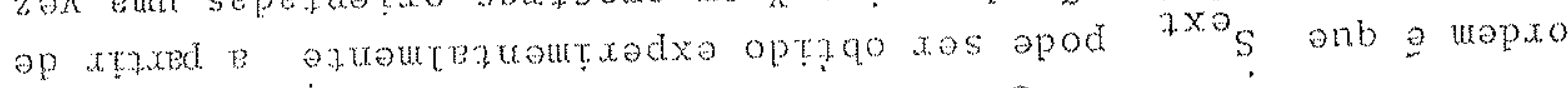

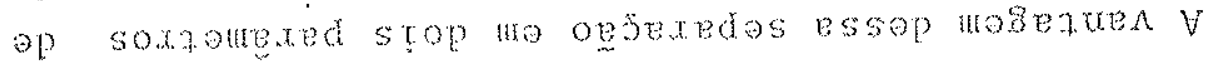

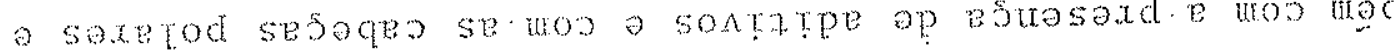

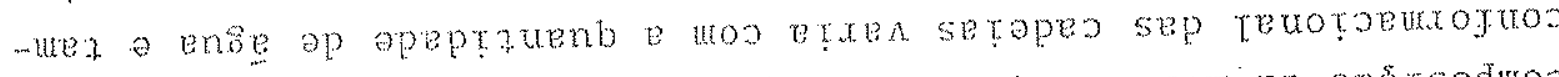

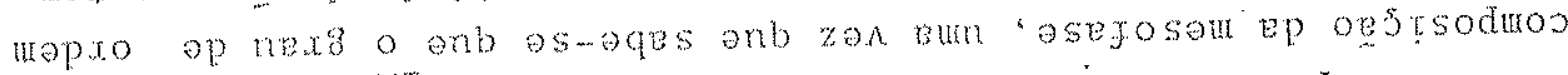

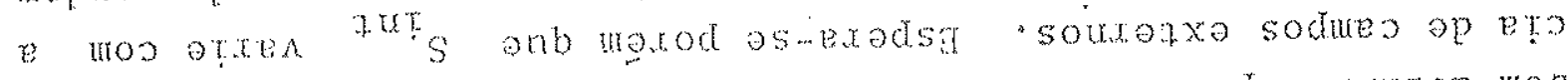

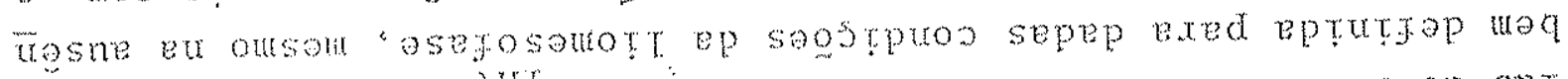

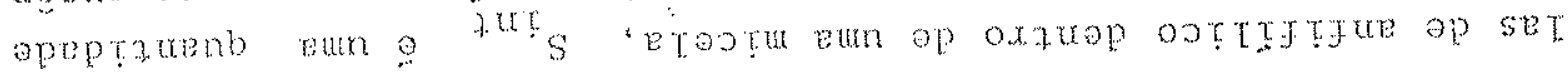

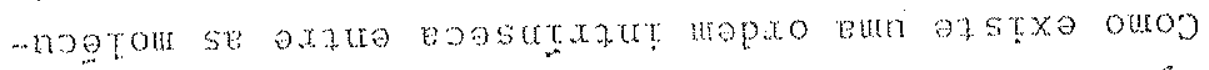

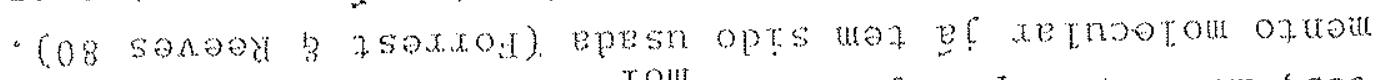

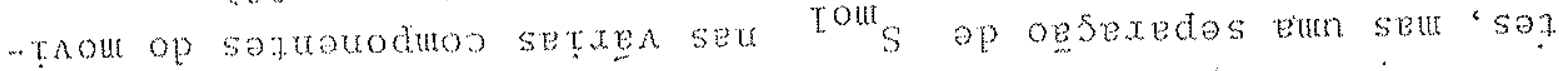

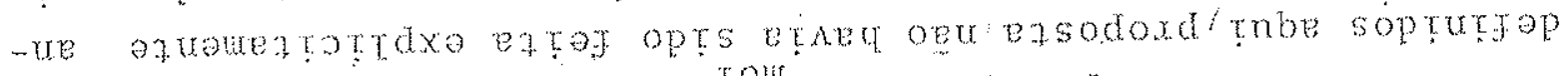

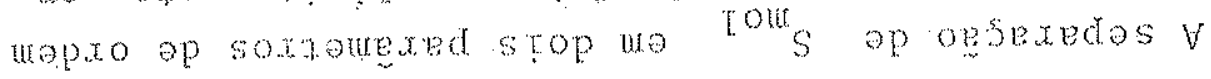

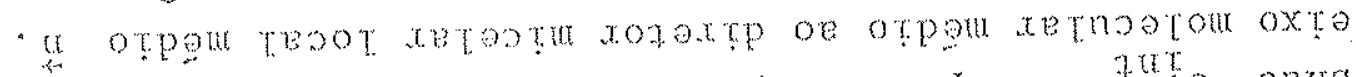

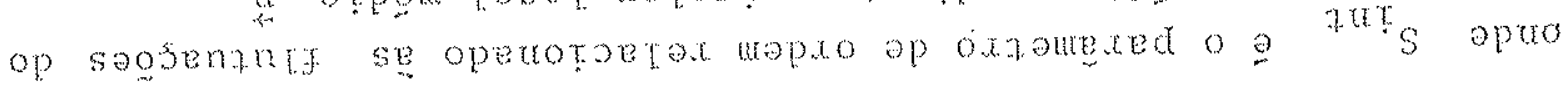

$$
2 \times 0 \operatorname{lng}_{S}=\text { tom }
$$

op sentare soperotoptox

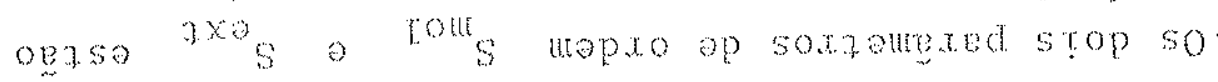

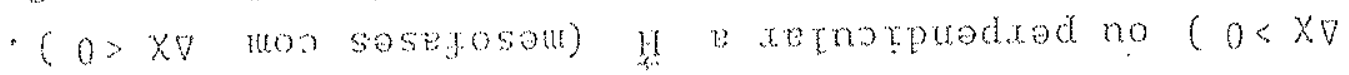

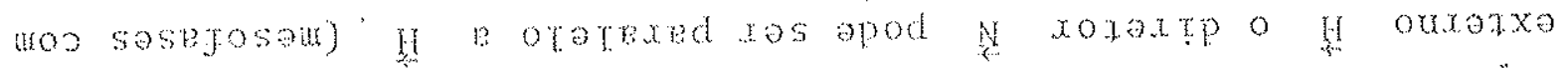

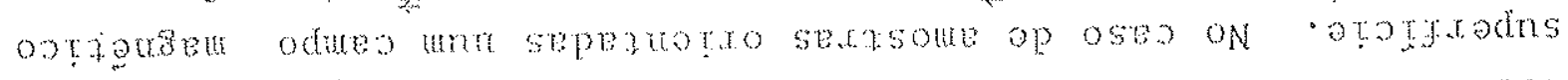

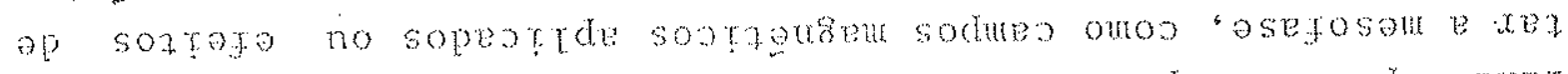

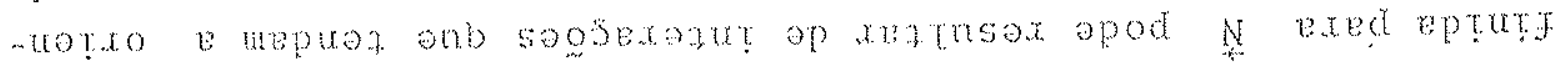


- odra assop suppom ar

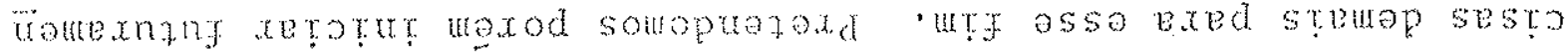

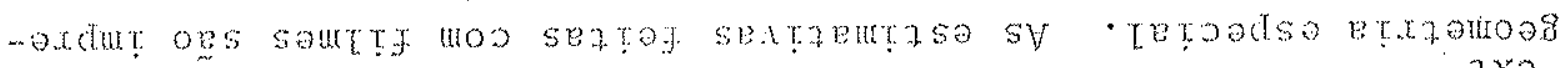

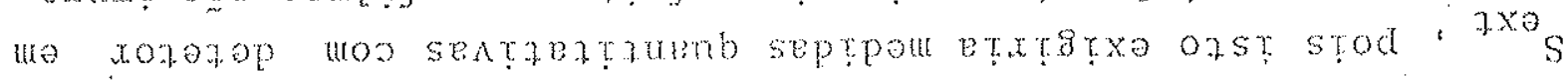

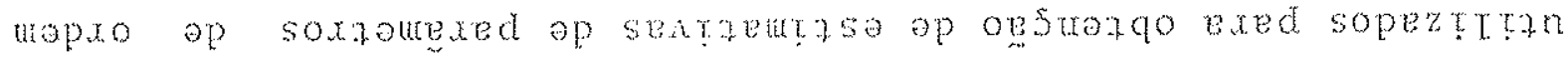

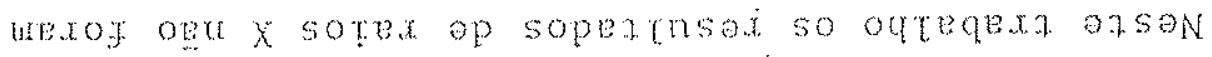

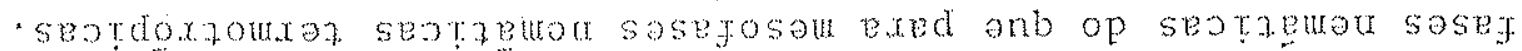

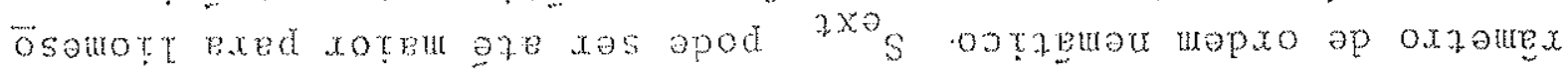

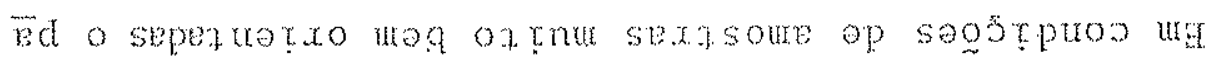

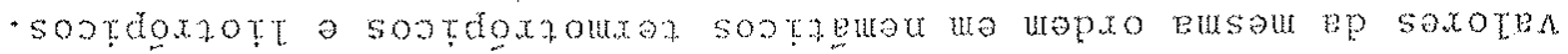

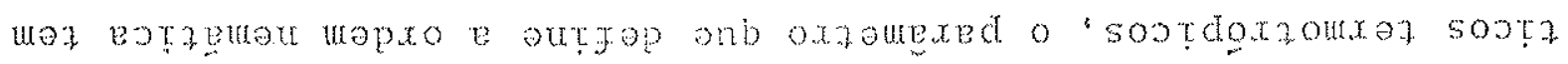

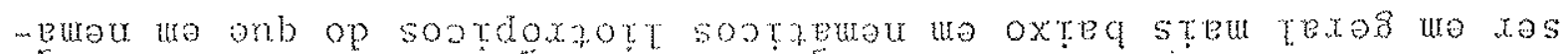

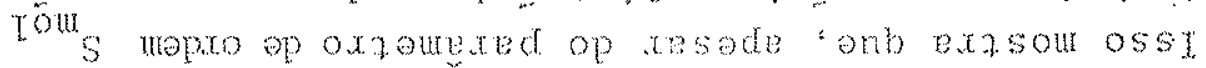

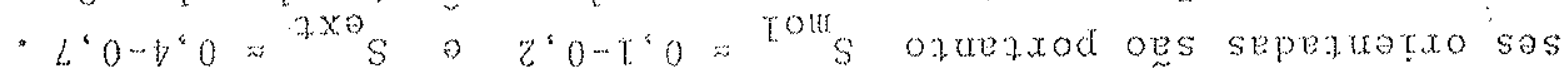

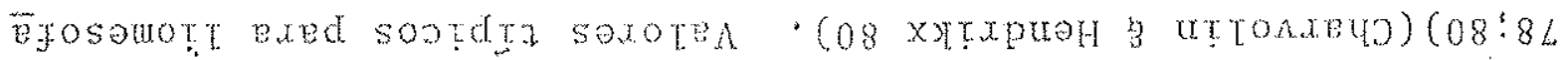

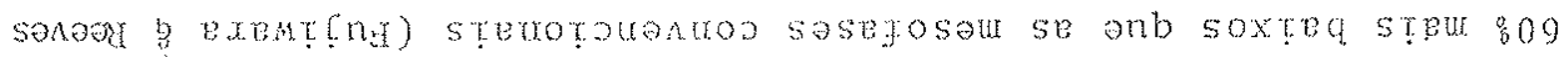

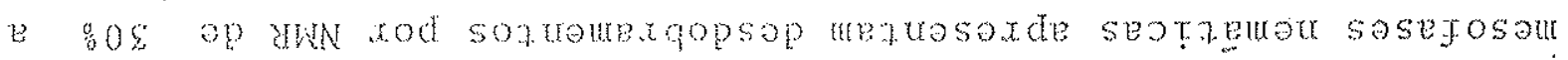

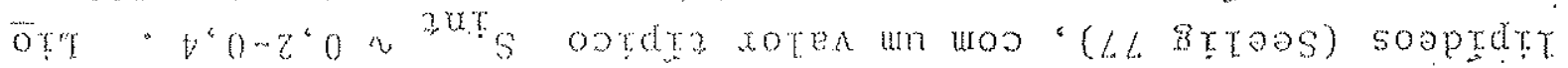
-oysor ap sepeneota axed soptago sotenbep uopxo euseu ep oes (h.

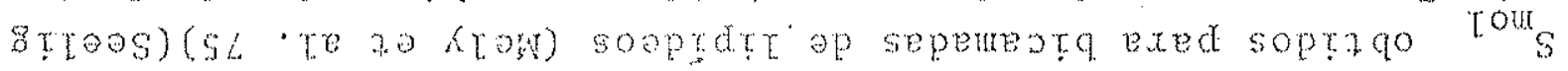

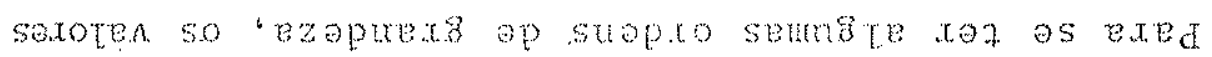

- tetaraz ogu op op

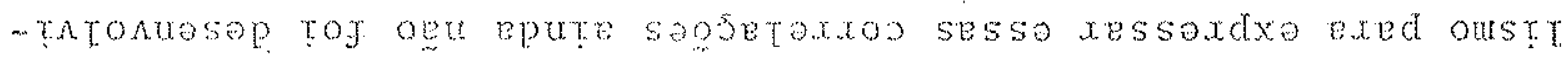

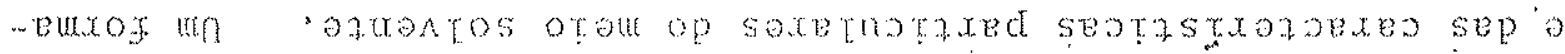

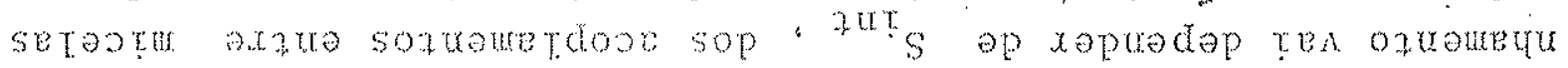

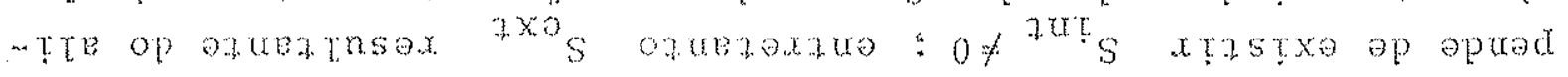

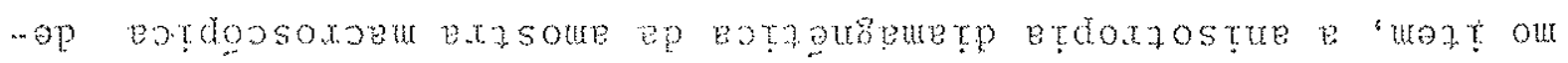

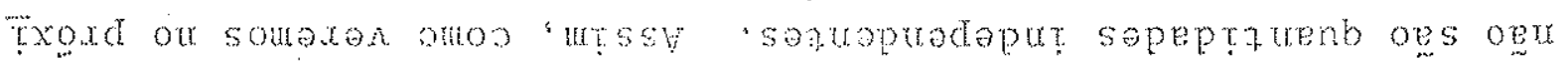

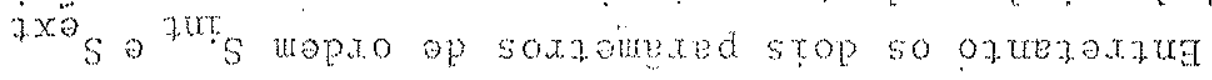

$\cdot(8.08 \div 08$

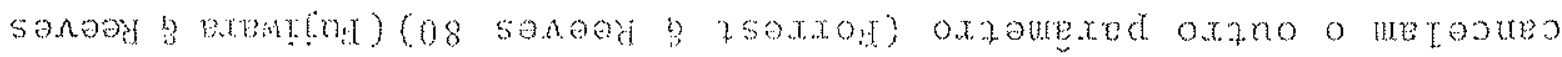

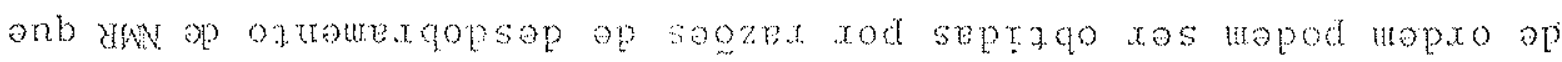

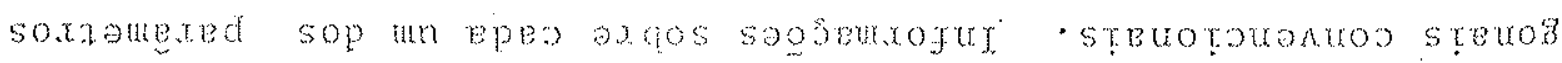

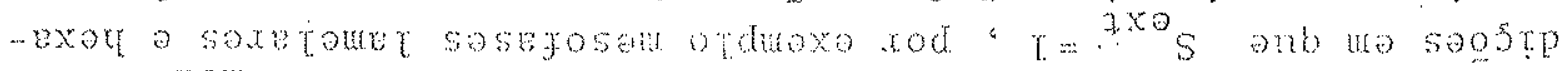

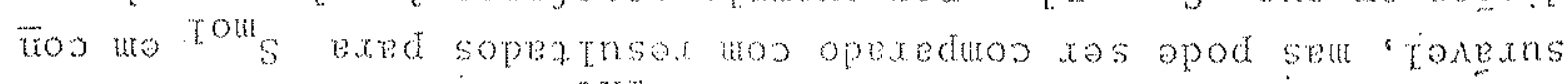

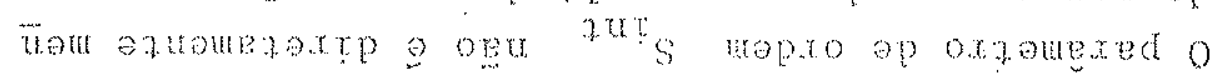

- tenpes

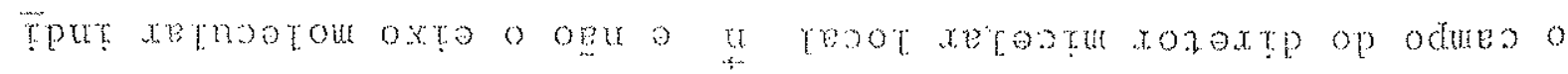

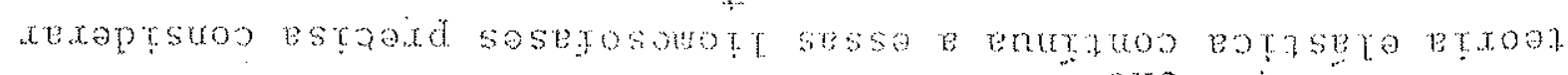

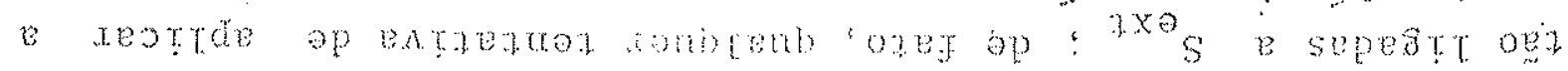

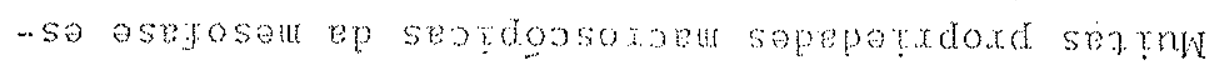




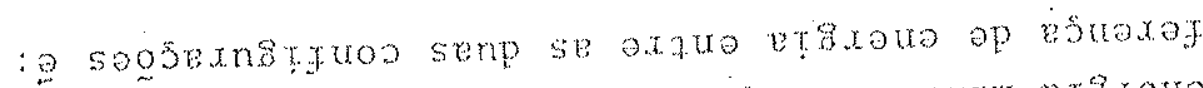

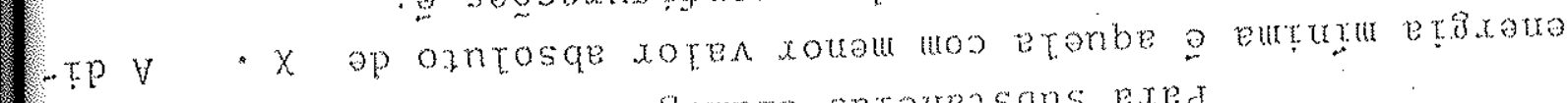
$2 p$

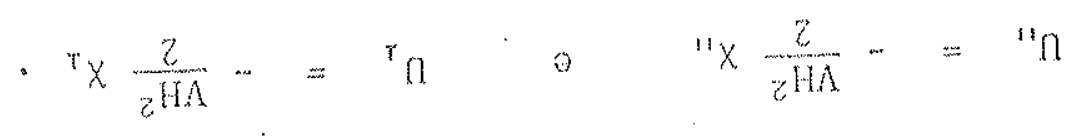

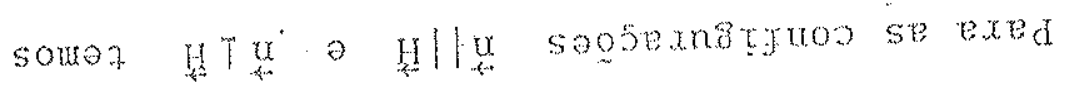

$$
\left[z^{(H \cdot u)} \times \nabla+{ }^{2} H^{\top} X\right] \frac{z}{\Lambda}=0
$$

$\because G L$

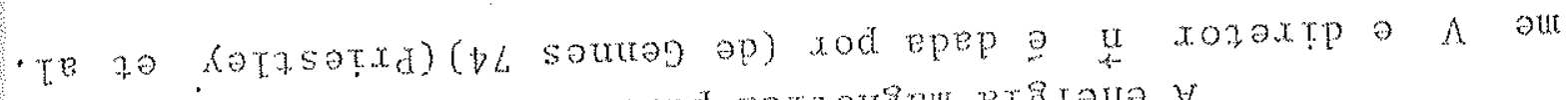

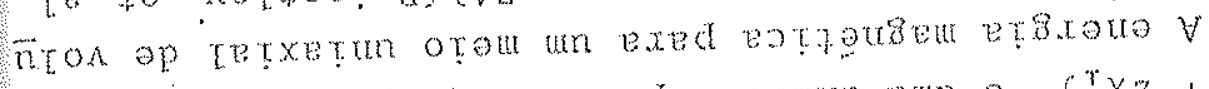

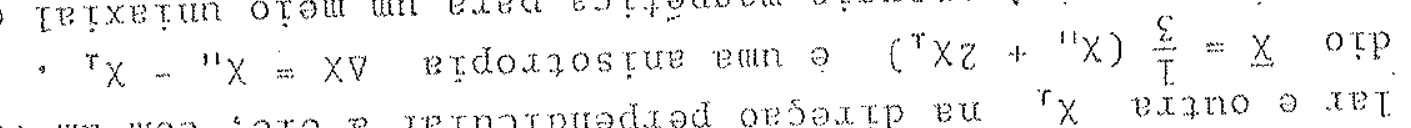

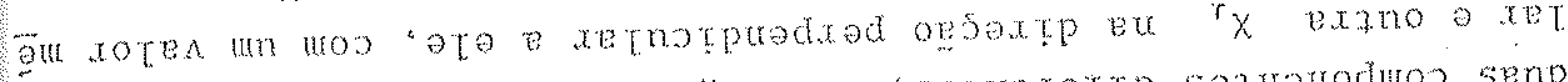

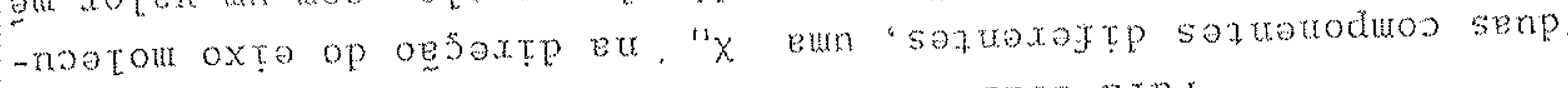

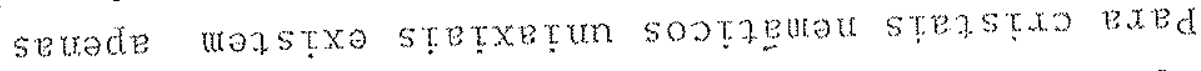

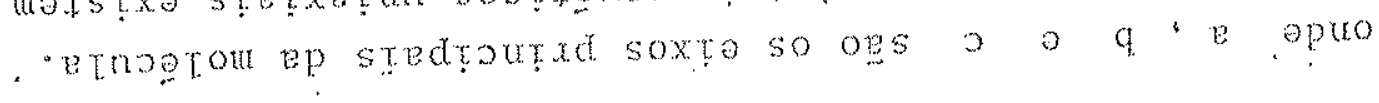

$$
\left.c^{2} x+9 x+e^{2} x\right) \cdot \frac{s}{T}=\underline{x}
$$

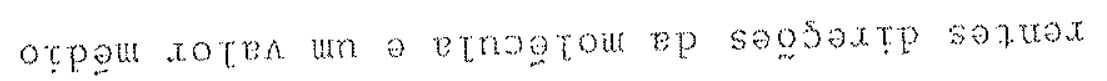

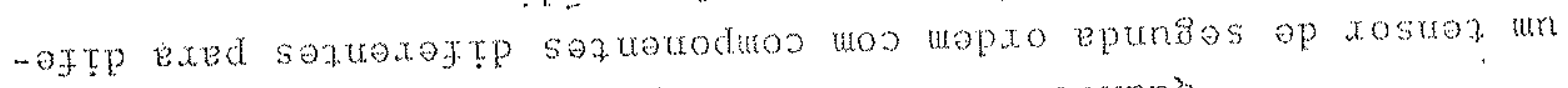
as-etrot $x$ xetnogrou etdoxzostre azstix opueno

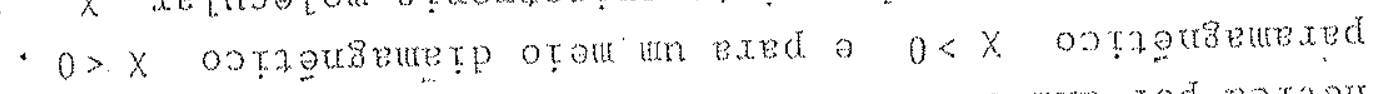

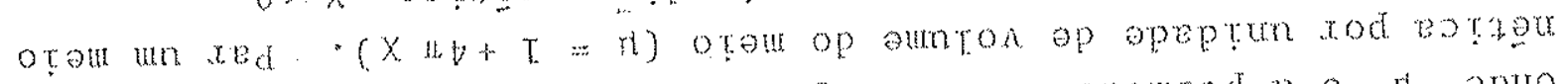

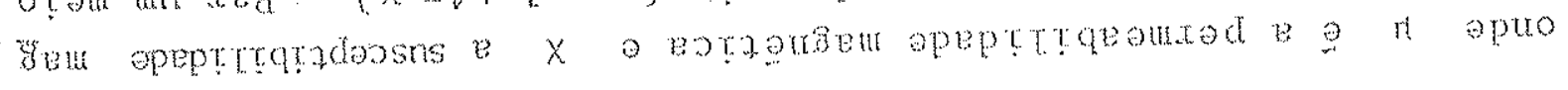

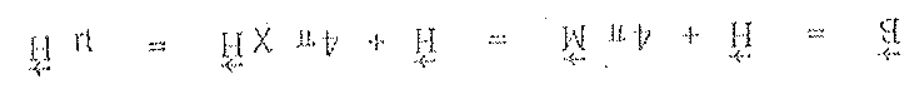

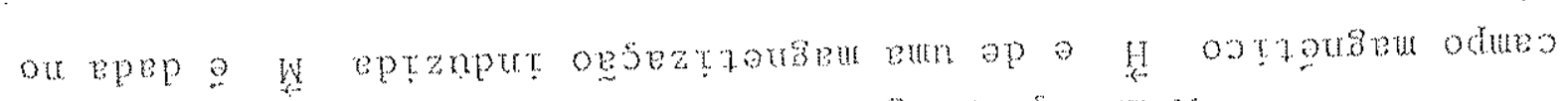

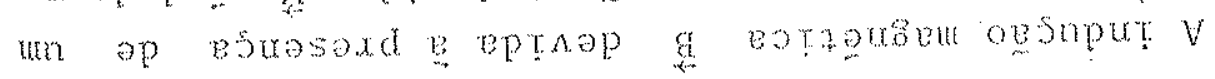
$5 T 0.00 \% 50050 x 00 \sin \cdot 1 \cdot 0^{\circ} \mathrm{TH}$ 


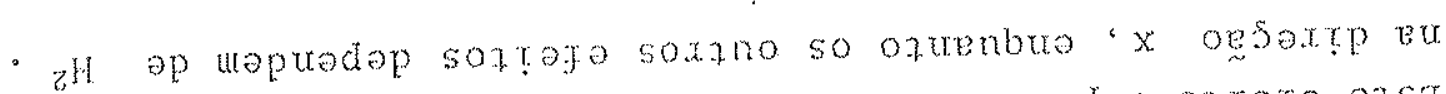

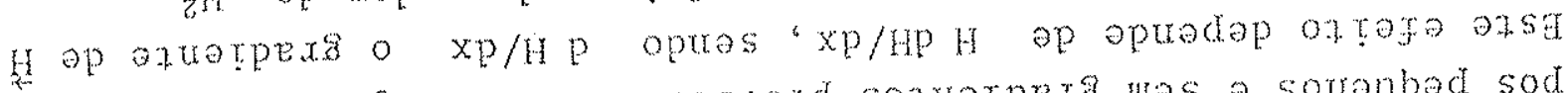

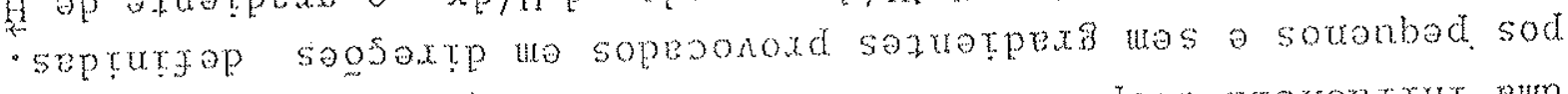

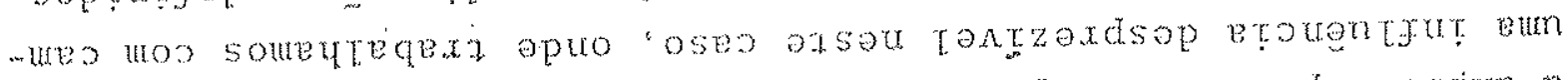

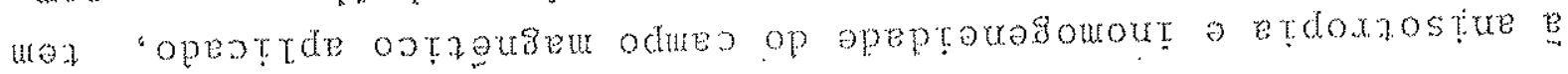

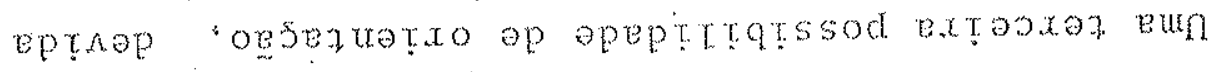

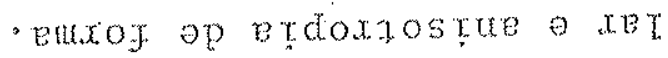

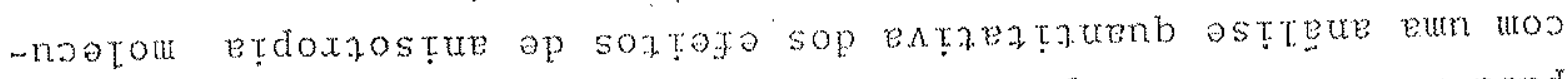

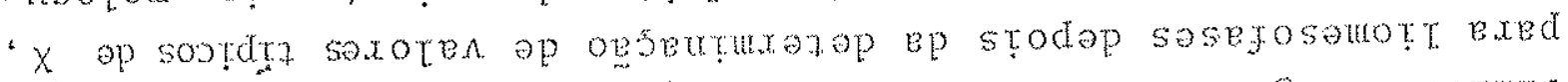

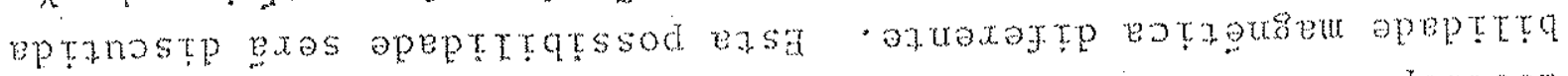

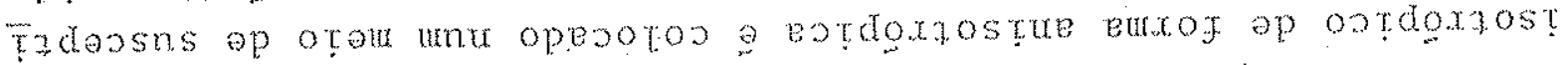

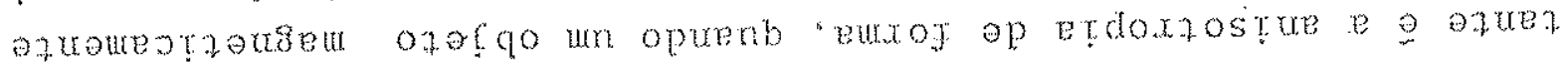

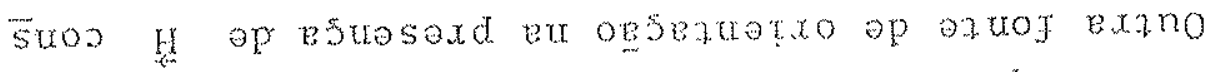

- setnogrou zzol: $\mathrm{N}$ anb wo sexasoure moo sontz

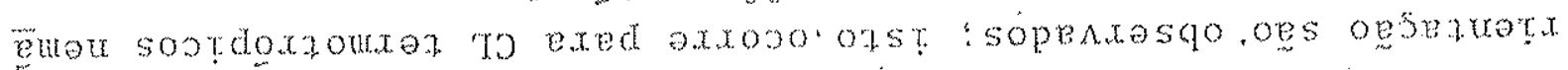

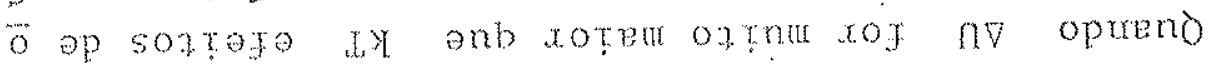

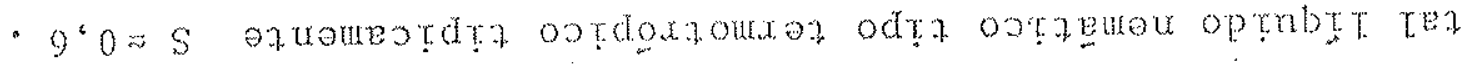

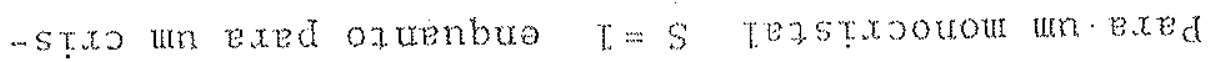

- auntor ep apeptun nod earzojo botrotgentep etdoxa

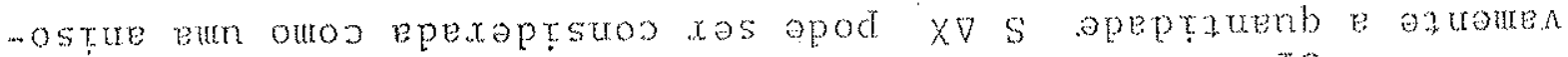

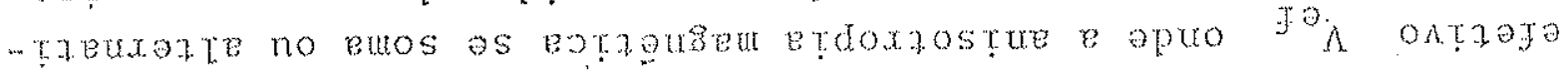
jumton un epexoptsuos xos opod sNA opeptumenb $V$

$$
x \vee \operatorname{SN} \frac{z}{z H} \cdots=\Omega \nabla
$$

e ersisous ap

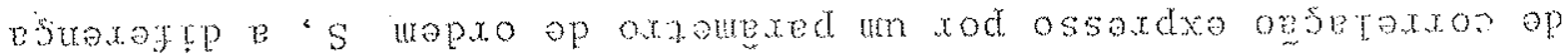

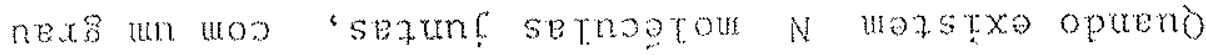

- stenptatert $5 a x$

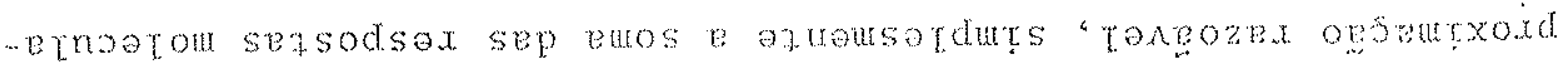

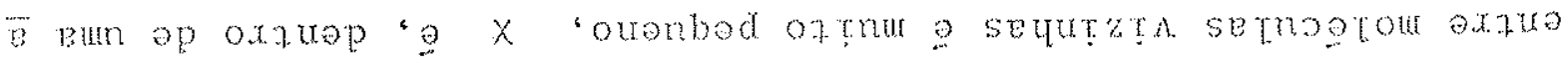

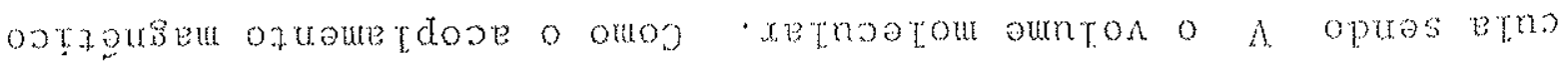
- otour run op $x$ bxed ueotrde os soossaxdxa sesse

- optrgensul odures op oes

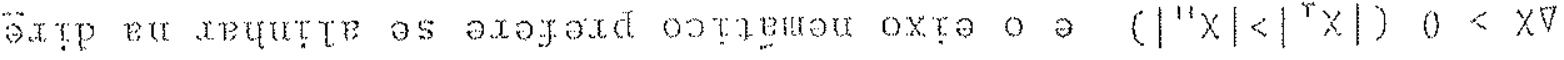

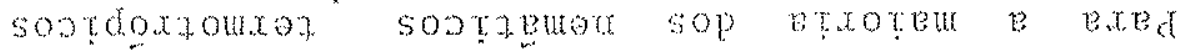

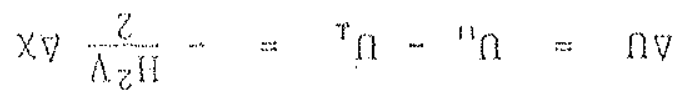




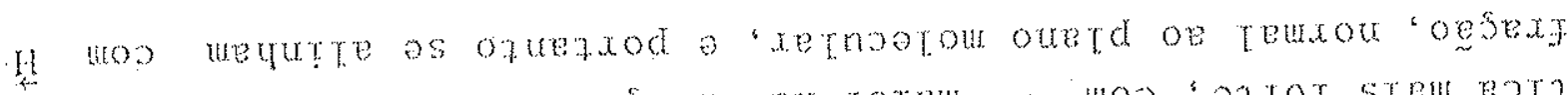

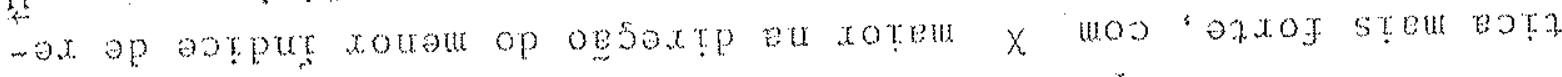

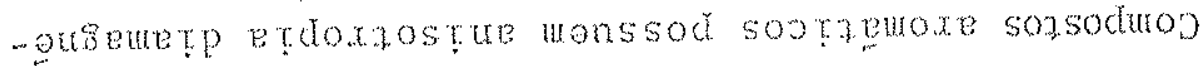

- soxetus

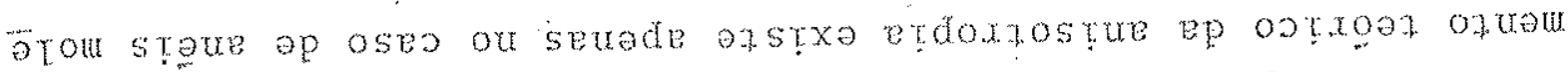

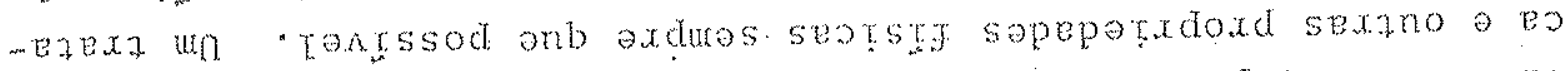

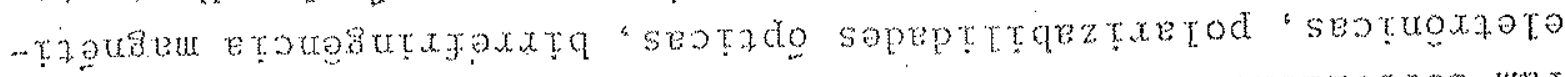

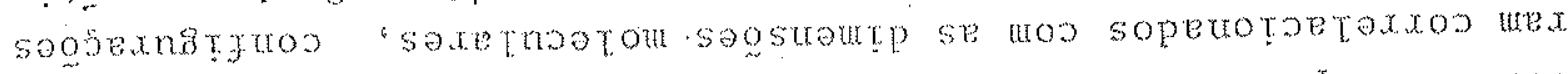

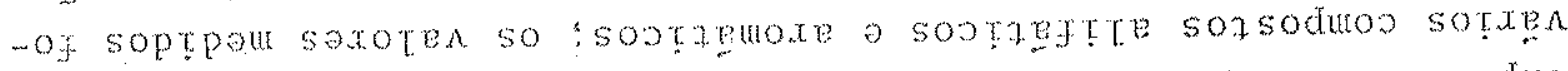

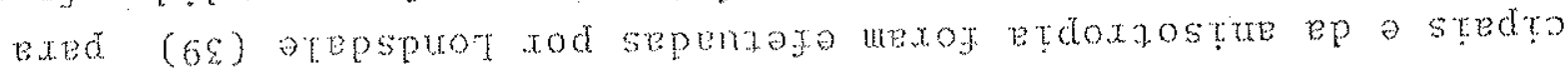
mad seotremourete sopeptrqtadoosns sep septpow

- sootif roodso steo

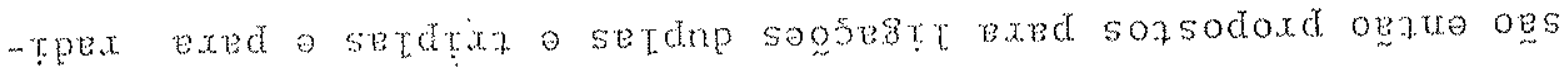

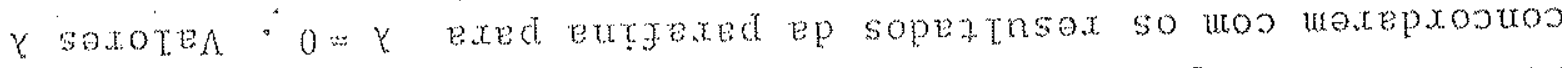

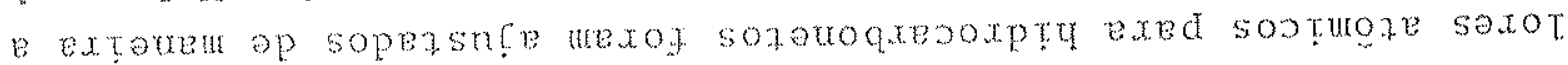

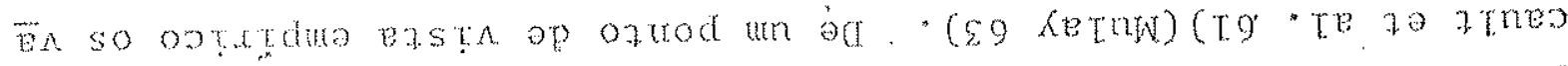

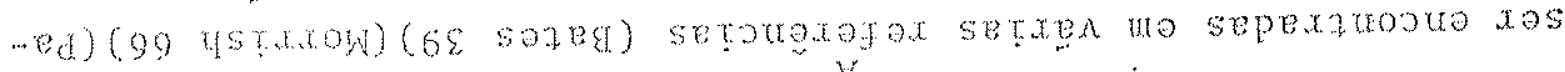
uapod sotnotes sossa red $x$ a $V_{x}$ Hoo setoqu.

$$
, \quad \times+V_{X},=\mathbb{N}_{X}
$$

Woo oproos ap' Ceposed

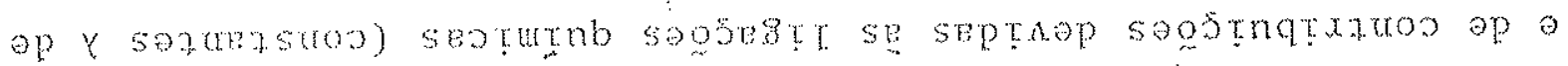

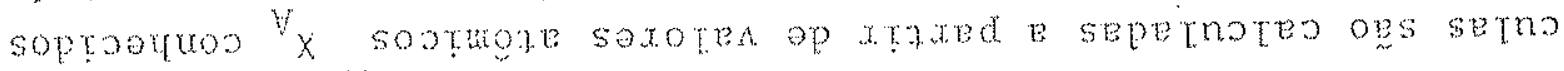

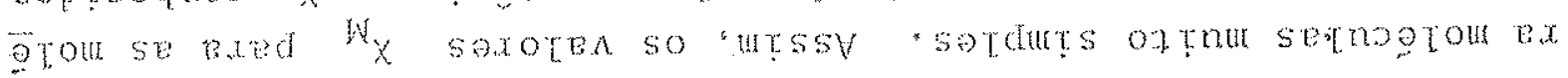

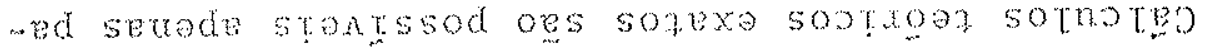

- stivd set.

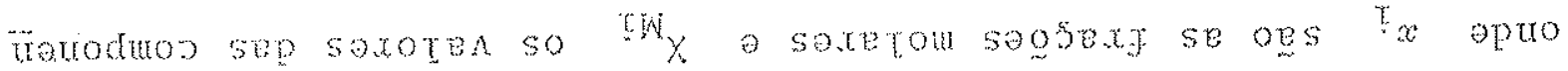

$$
\text { WX } T=W x
$$

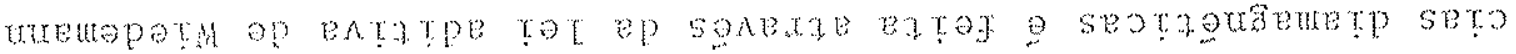

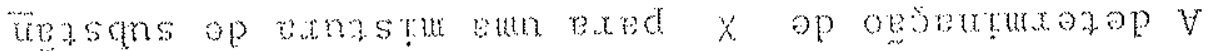

$$
\text { - armogrom no optuge osodo }
$$

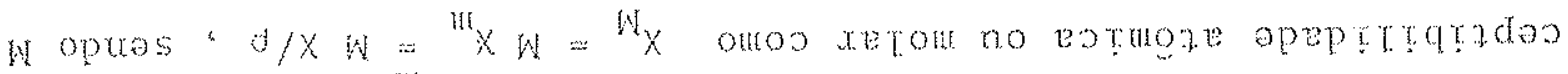

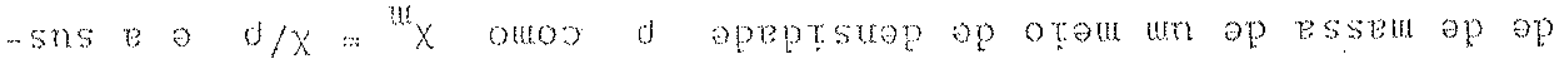

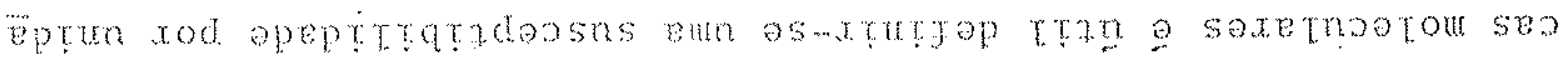

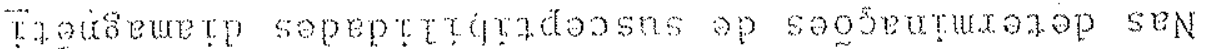

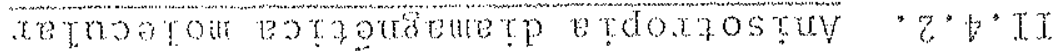




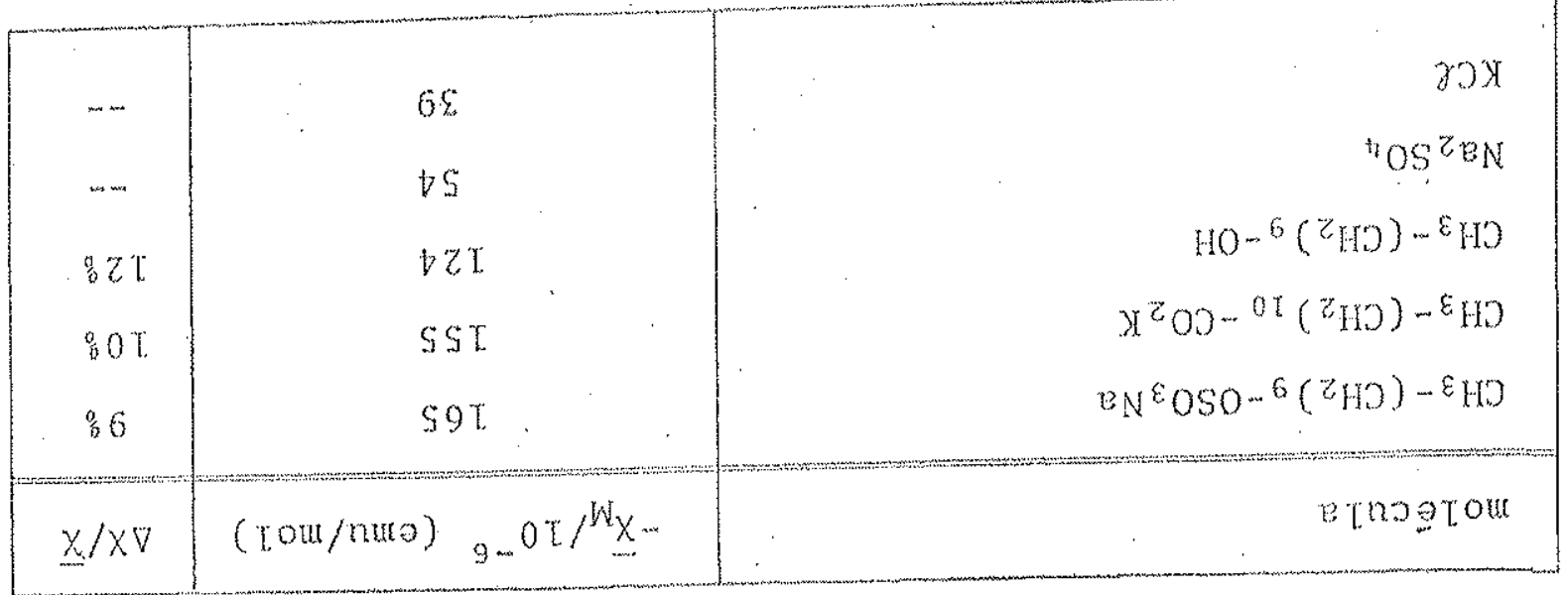

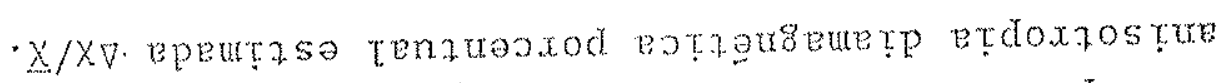

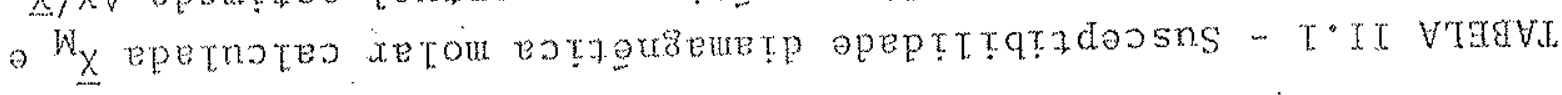

- (68 atepstror)

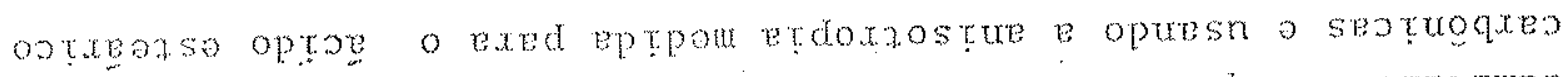

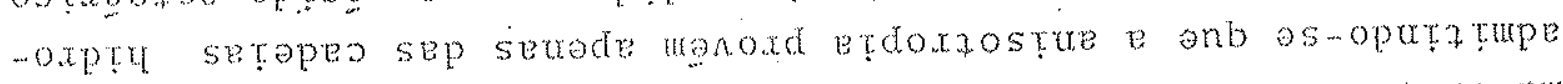

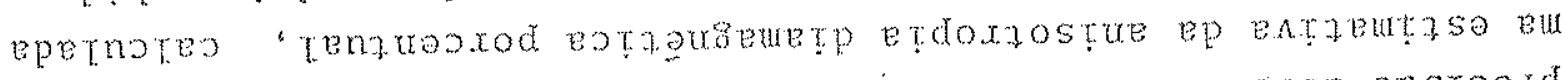
-n maquen ep taqez $\mathrm{V}$.

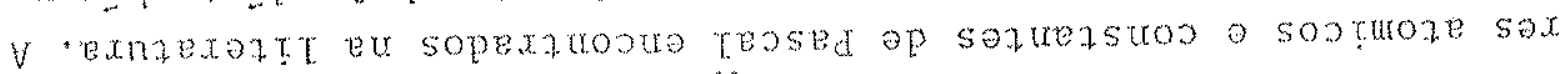

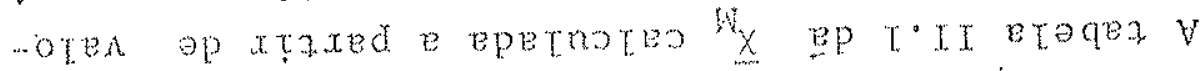

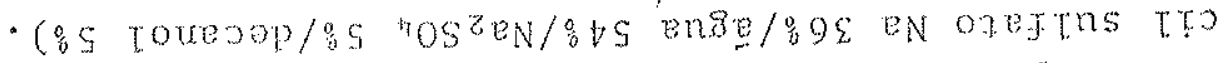

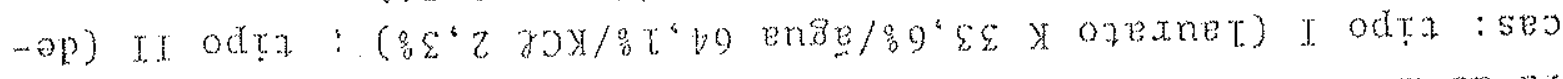

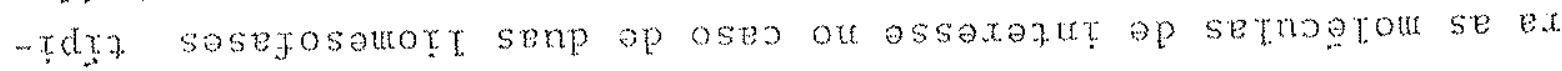
med sopertasarde exoge oys $x \nabla$ a $x$ ep sotnoted

- opeaxesqo e. onb ogs

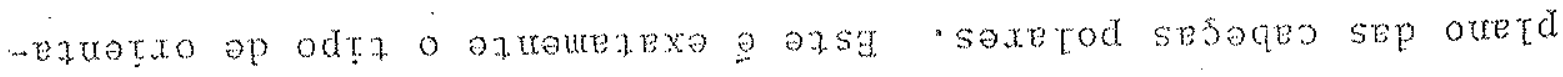

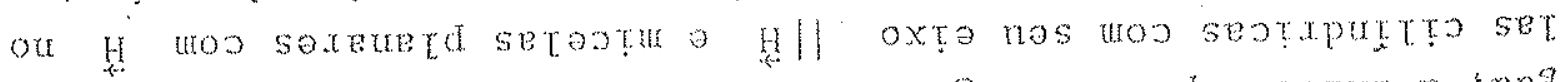

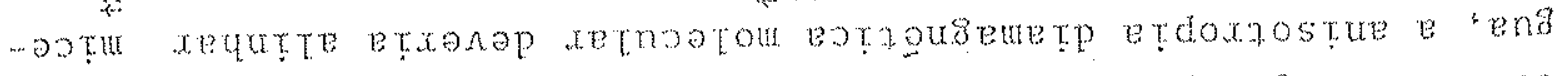

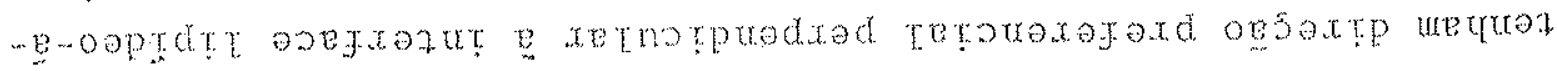

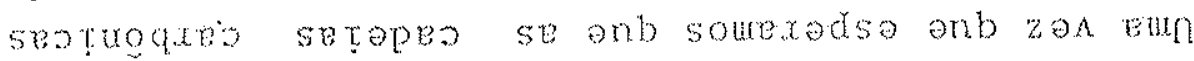

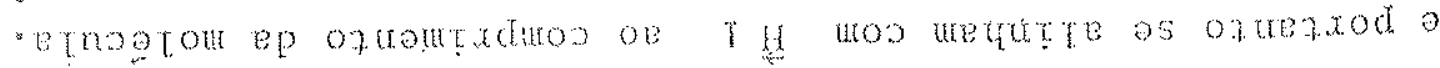

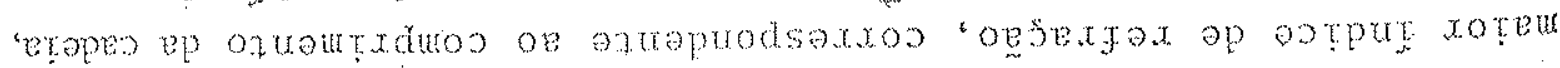

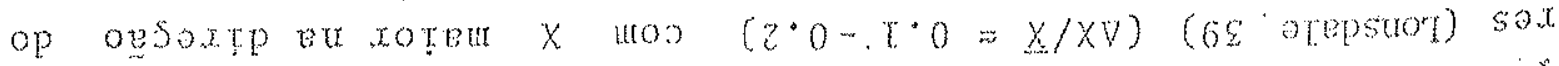

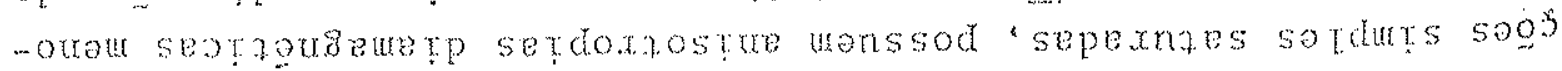

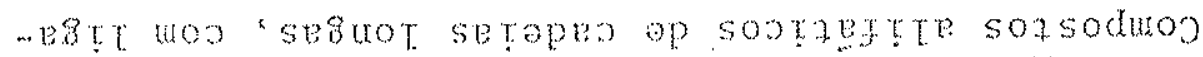

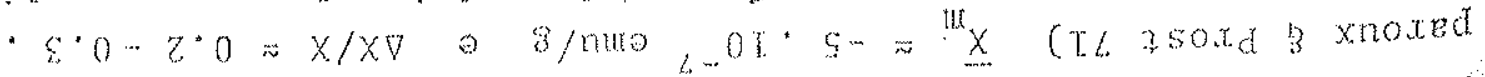

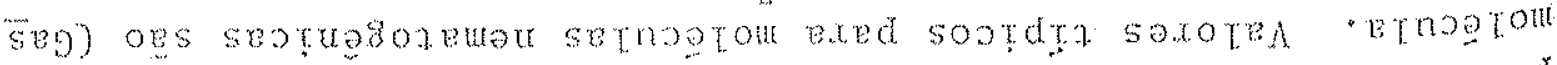

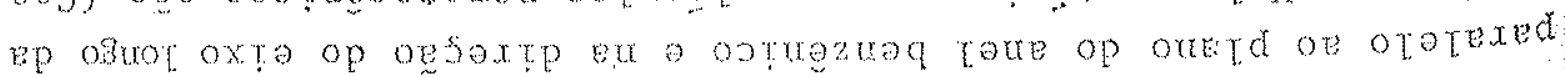




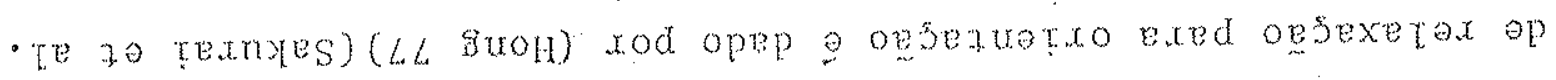

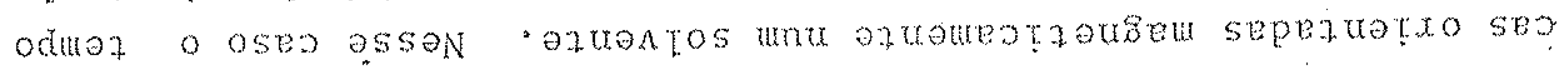

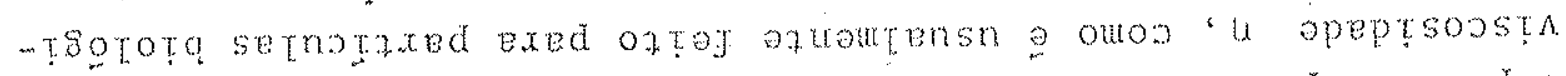

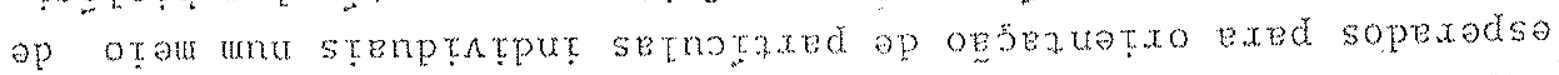
oesexerax op sodur sop otnotes otod ge tenptatput xetastu ogsta

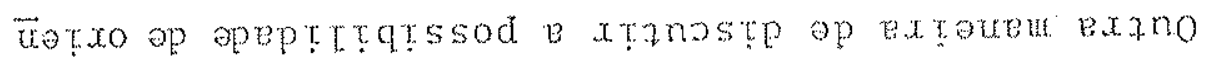

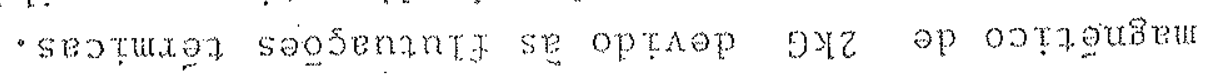

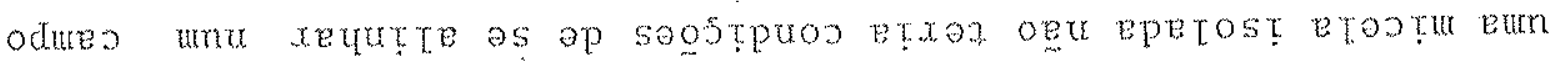
anb oreto a eurof xanbenb ad oses opunses o exed not cot wo

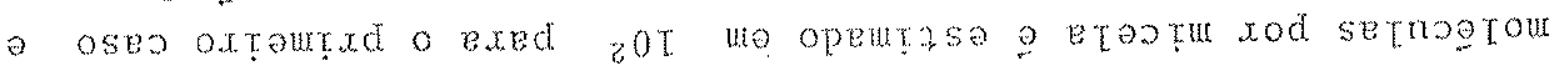

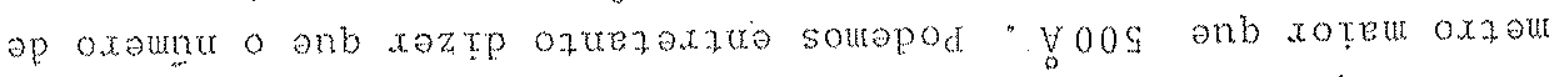

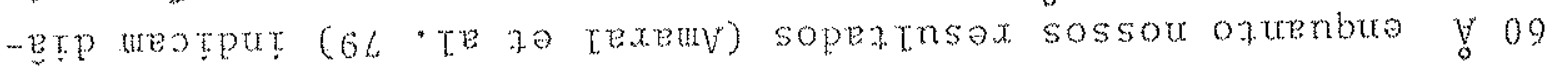

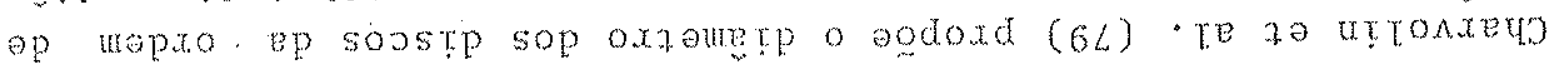

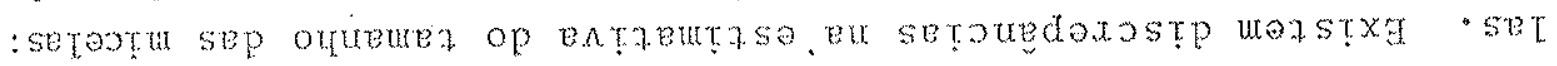

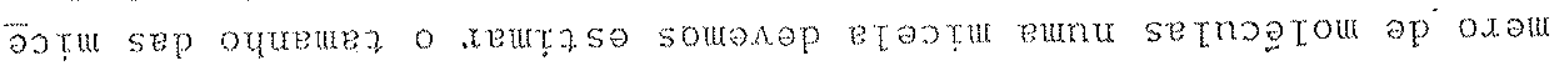

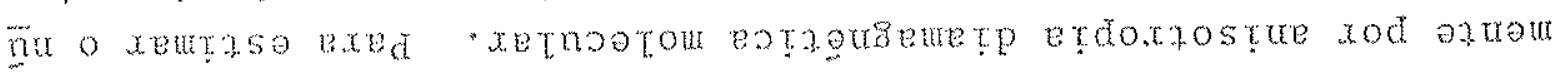

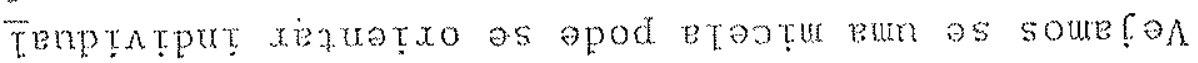

- opsezretido xtzopoxd paed seperdooz serno

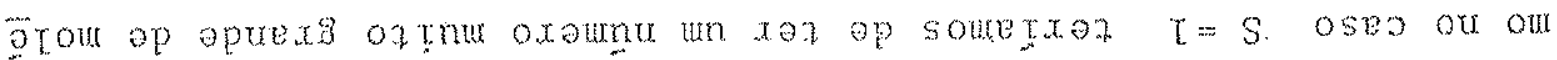

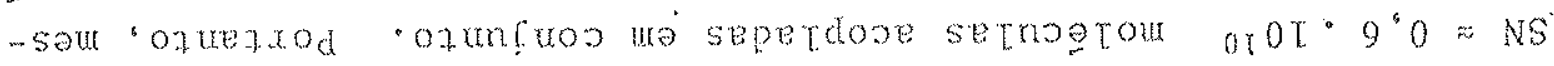

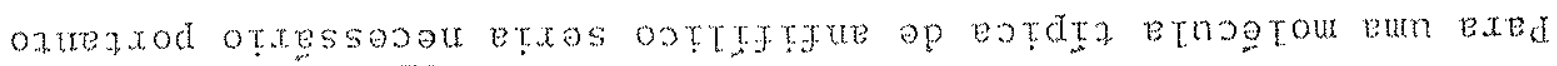

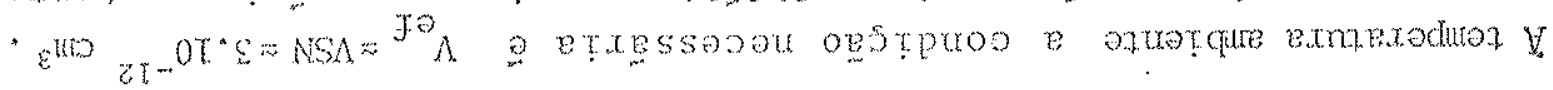

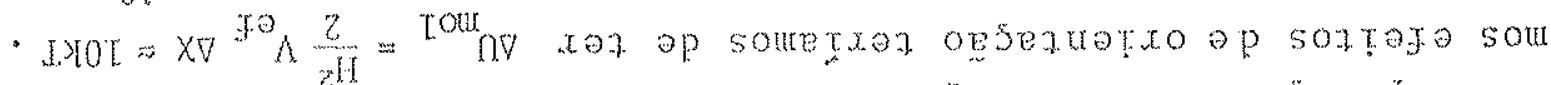

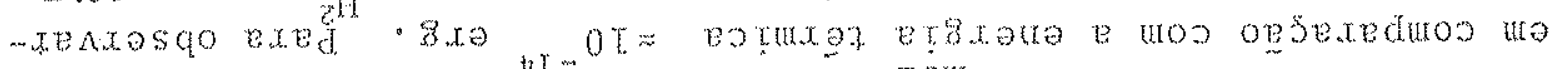

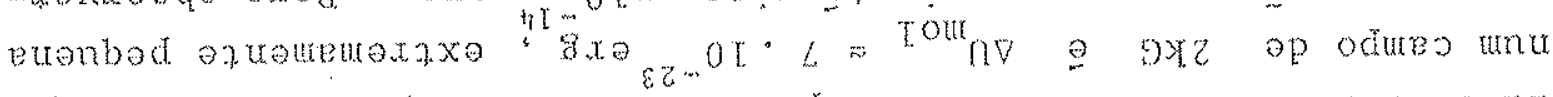

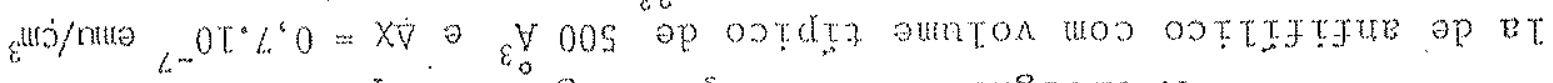

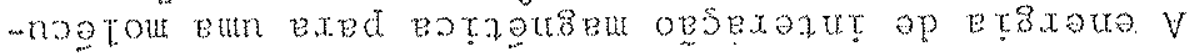

- xenzore as

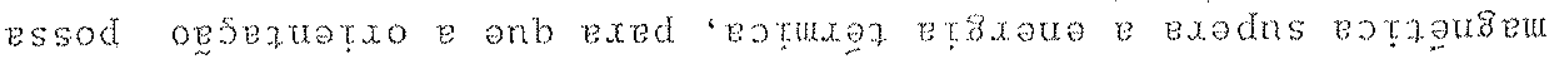

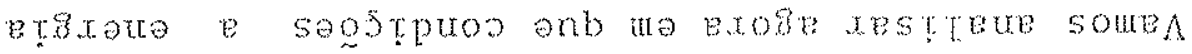

- atrontos ogoll o bued

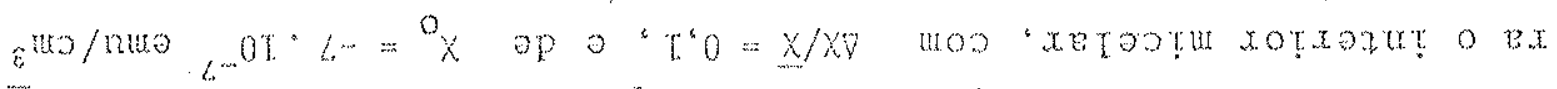
Ed

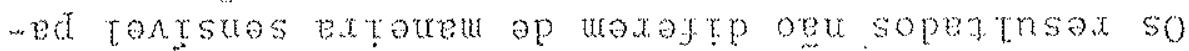

- ense pu

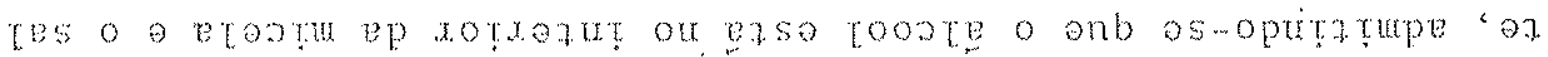

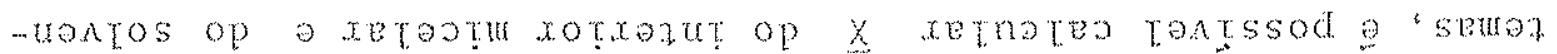

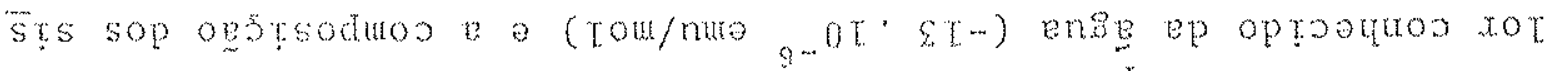
- an o optesn e sopetnoteo soroten sassop xitad $V$ 


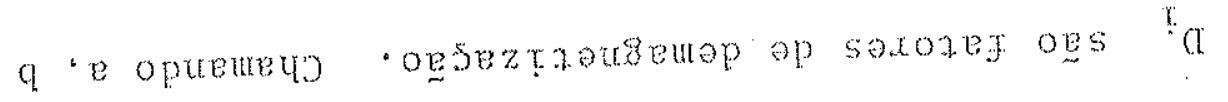

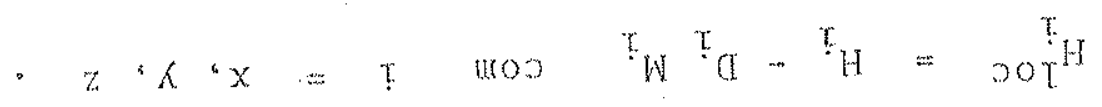

rod opep "oprosdtro op oxzup tesot odures o a sor opuo

$$
\text { . } \mathrm{H}^{\circ} \operatorname{sot} \mathrm{H}(\mathrm{H}-\mathrm{z}+\mathrm{H}) \frac{118}{\Lambda}=0
$$

3 องก

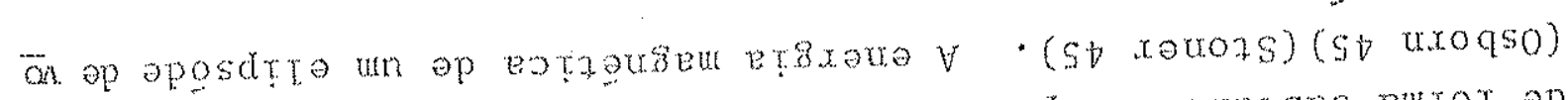

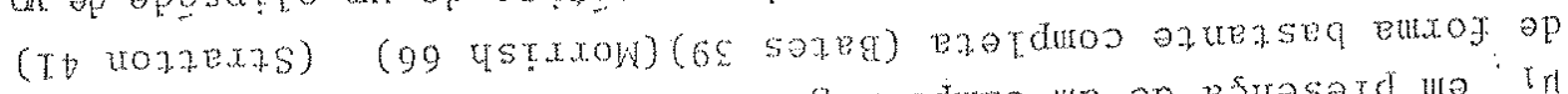

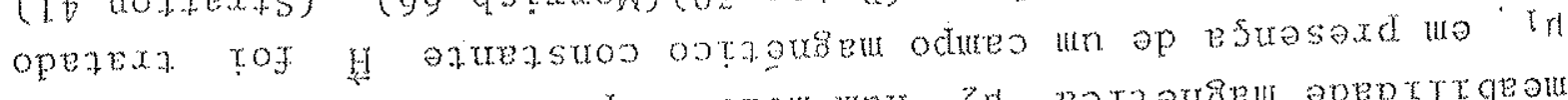

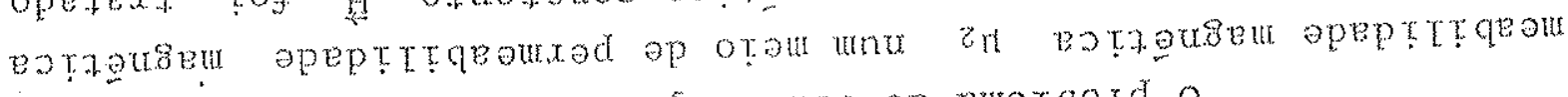

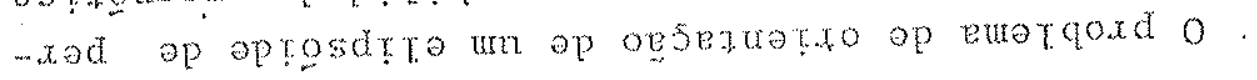

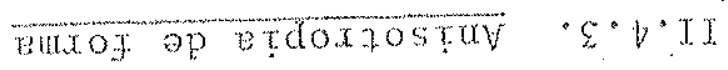

setoogu sep antzotoo oesezuatroot weo

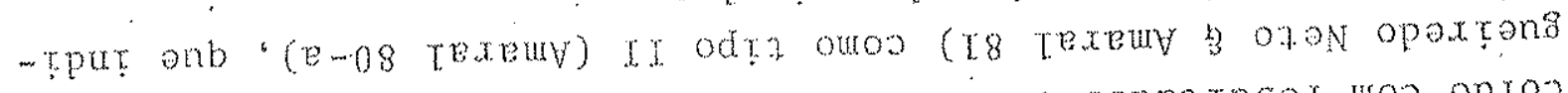

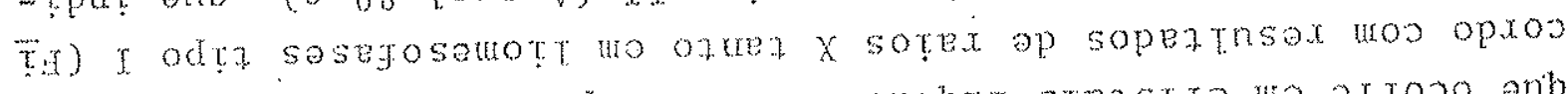

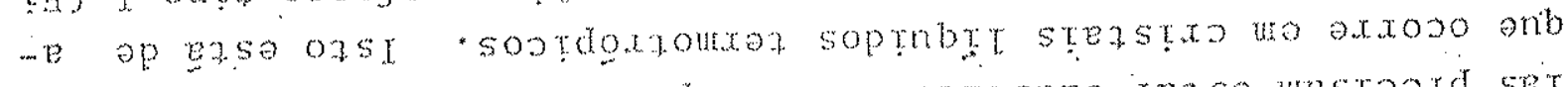

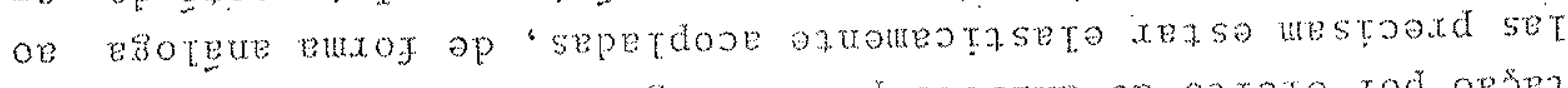

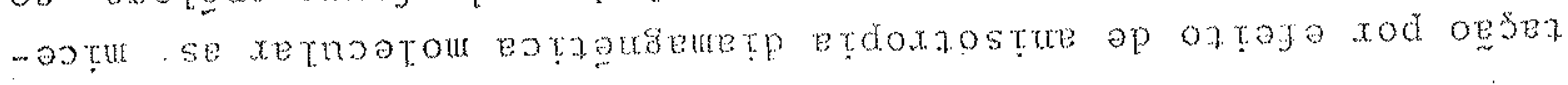
- netro xaxxoso exad anb ozwaxod trntotoo somanad

- Fa exed ourmertroter

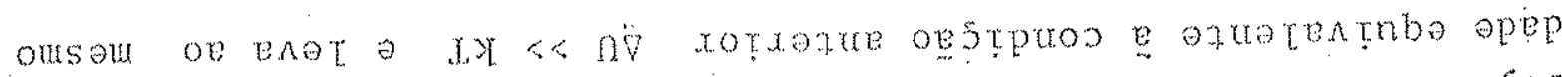

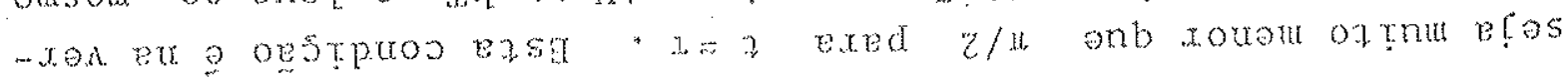

$$
2 \frac{x}{3 . x y}=208 /
$$

omermorg ofwempom on op

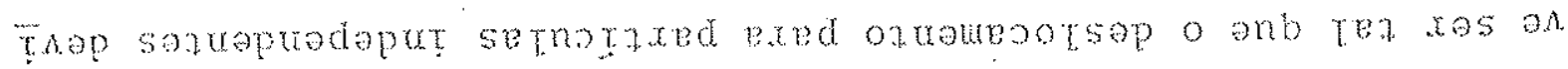

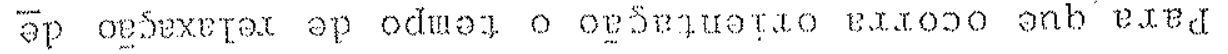

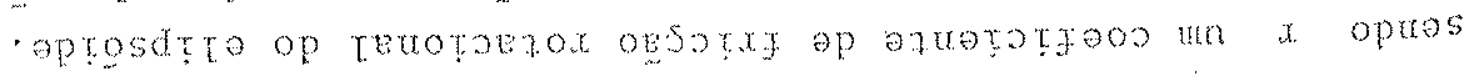

$$
\frac{2 \mathrm{HA} \times \mathrm{P}}{x}=3
$$




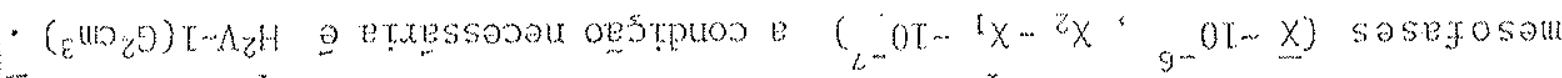

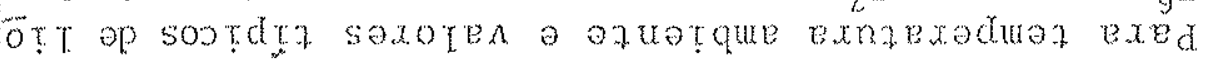

$$
\text { L. OT }=\Lambda_{z} \mathrm{H}^{2} \times(x-z x) \amalg z
$$

: botrdur of

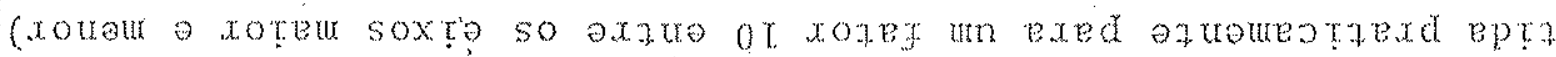

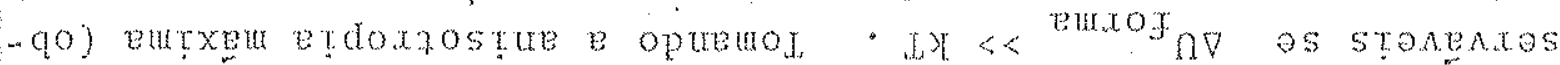

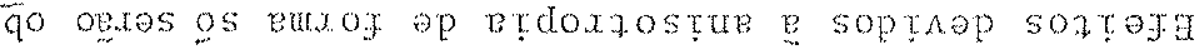

- oueta ou $y$ wos seamberd a $\mathrm{H}$

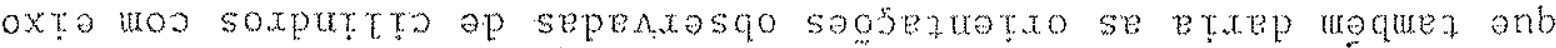
o'H op opsoxtp ex ozuraxo os optosdtro op xoteu oxta o onb as

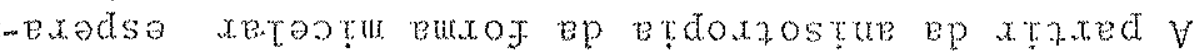

$$
\text { - sonerond septosdtra exed ur }=0 \text { a sozerqo sap }
$$

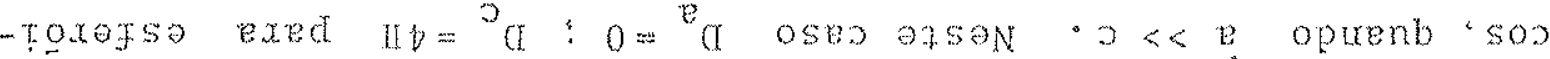

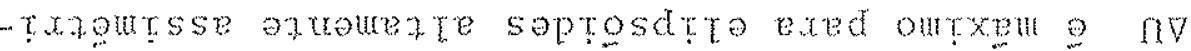

$$
2 x\left(a-2(n)\left({ }^{2} x-2 x\right) z H \frac{z}{\Lambda}={ }^{r} n-{ }^{2} n=n \nabla\right.
$$

arod epep o soosexnstroo senp se ax wa etsisue op estraxagt o $t>x$ anb oprexquet

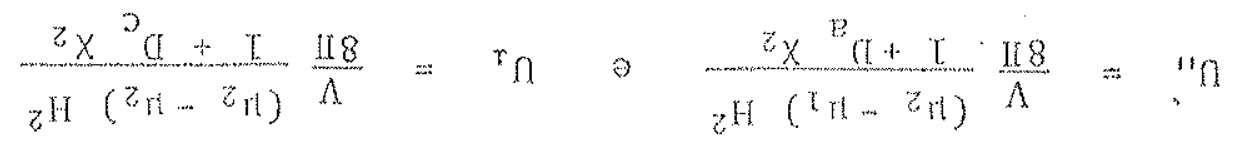

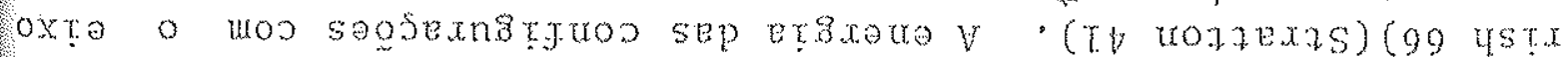

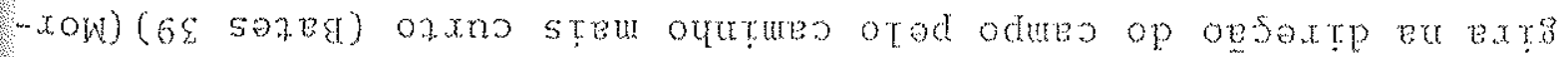

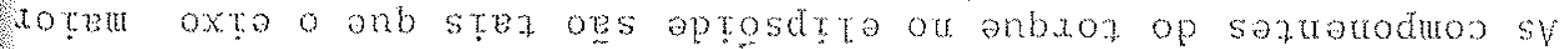

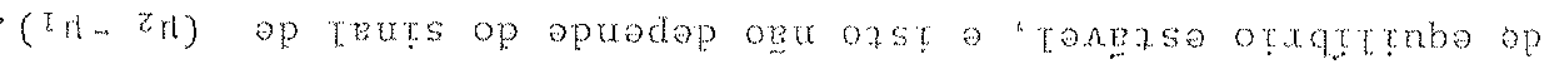

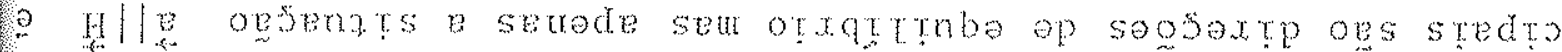

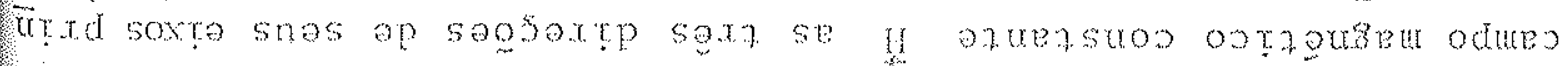

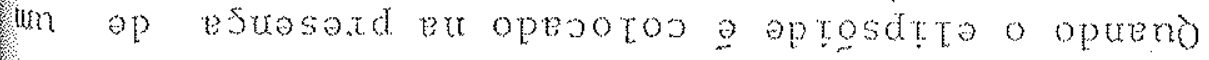

$$
\text { - } \theta=4 \text { optenb soavgo o } 2=9
$$

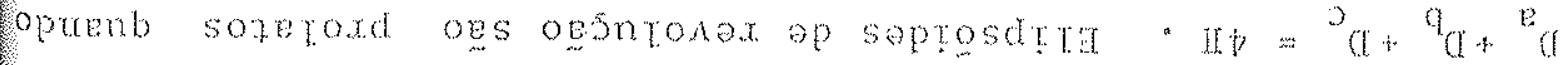

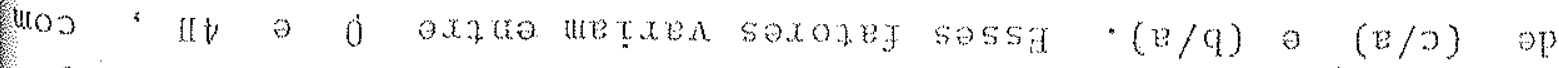

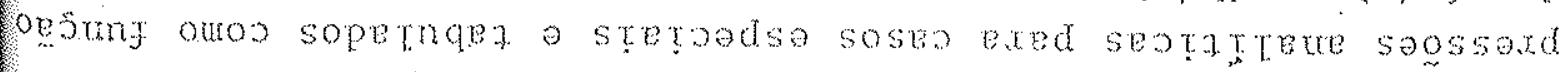

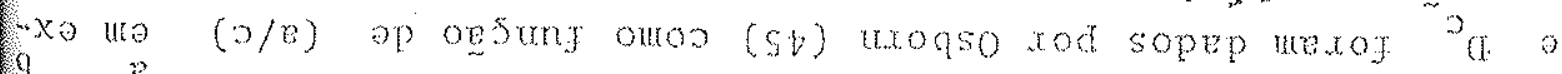

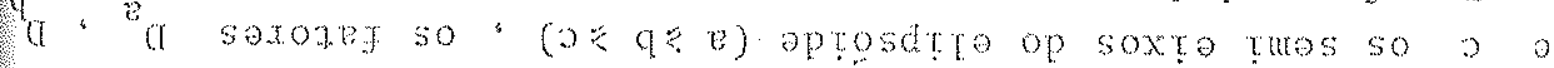




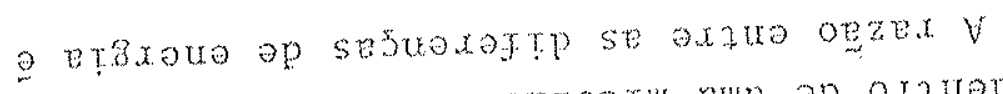

- toptu enn op oxame setnotom ax

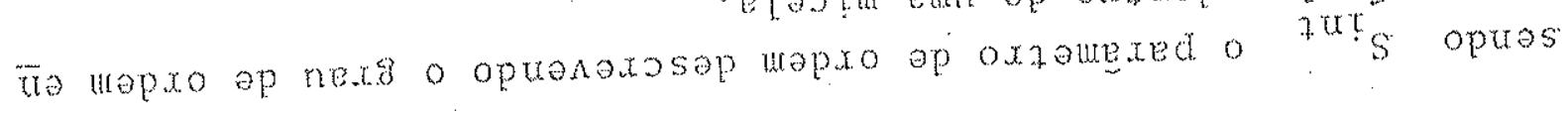

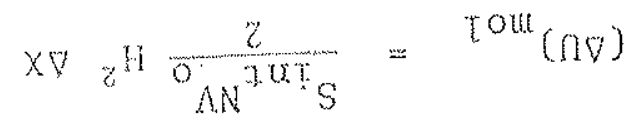

$$
\begin{aligned}
& \text { xilt }\left(x^{0} x-x\right) z^{H} \frac{z}{{ }_{M N}}=\operatorname{euxox}(n v)
\end{aligned}
$$

(xo

$-9$

q10

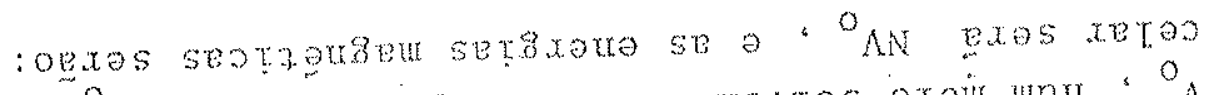

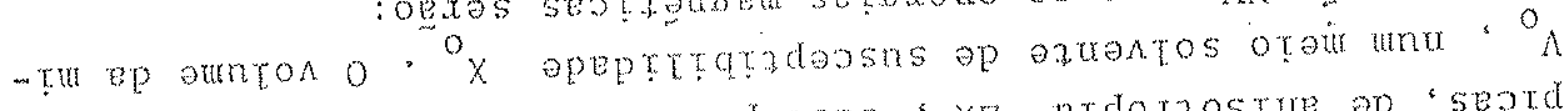

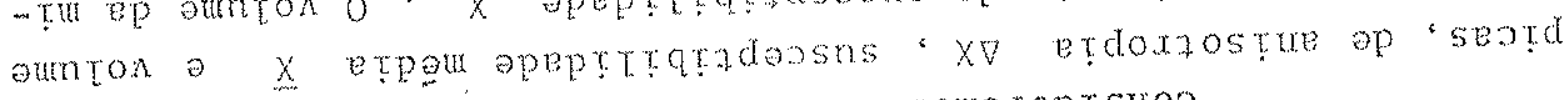

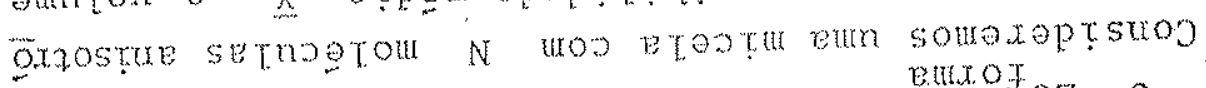
- veriogar a roun op steax

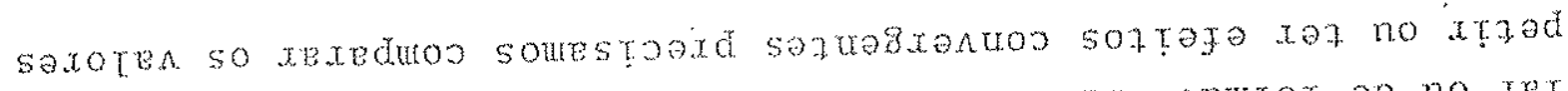

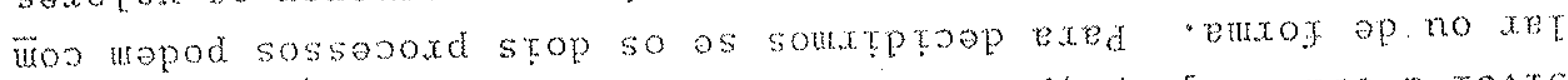

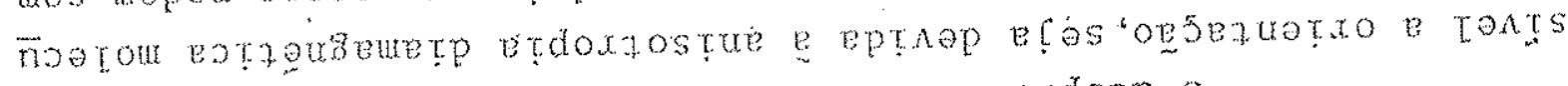

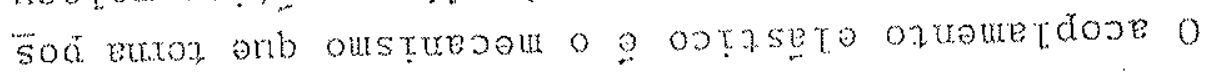

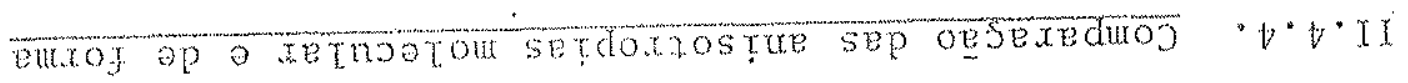

- sepestasonut sexzsoure setad extorstas xos bta

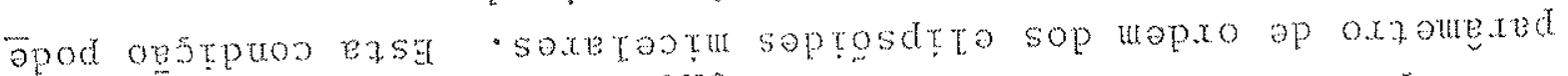

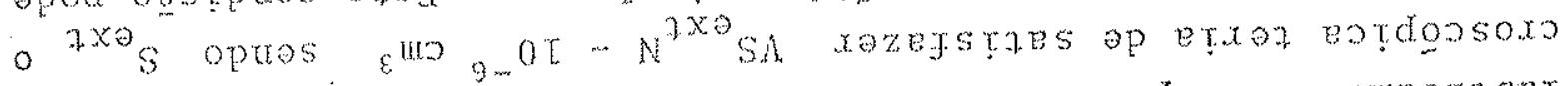

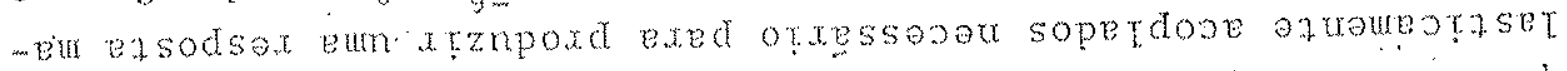

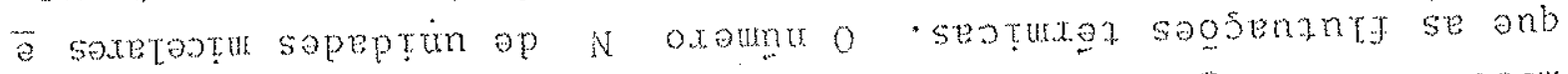

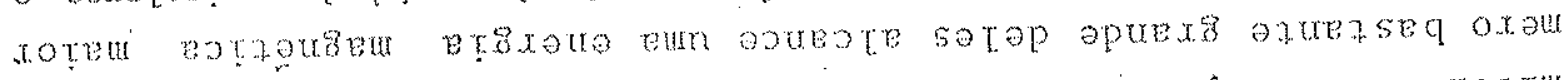

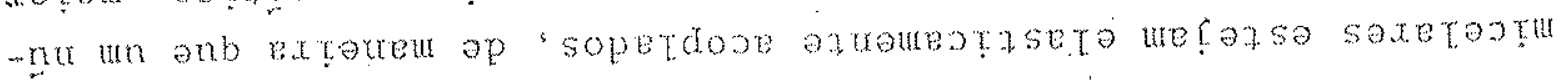
soptosdrte so anb op opeptrgtssod e eprete oistxa

- eurcos

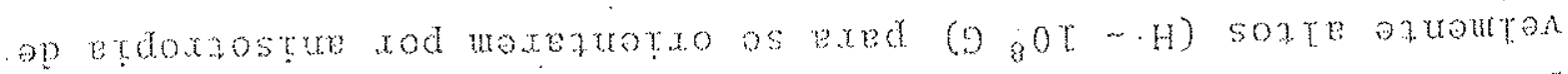

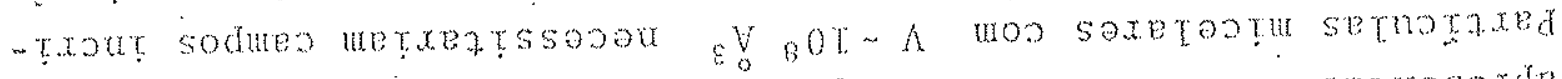

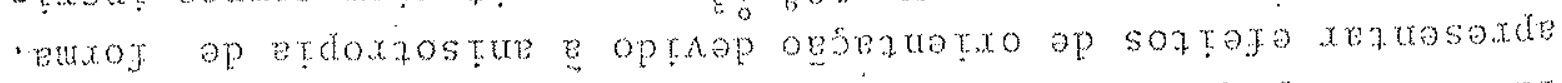

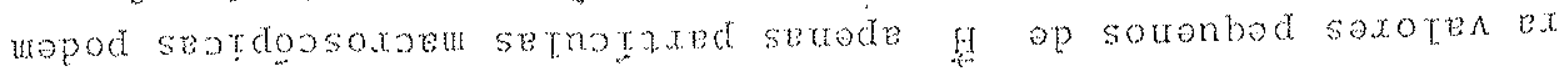

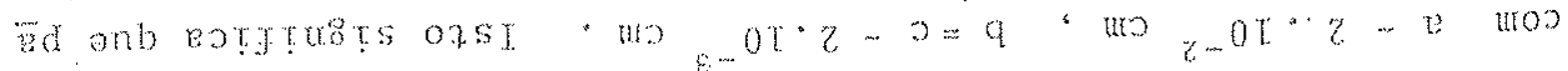

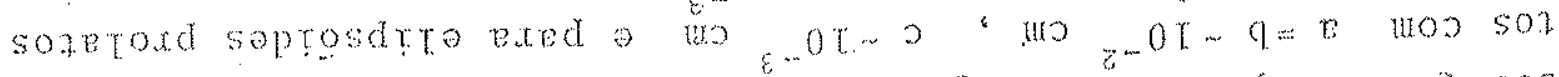

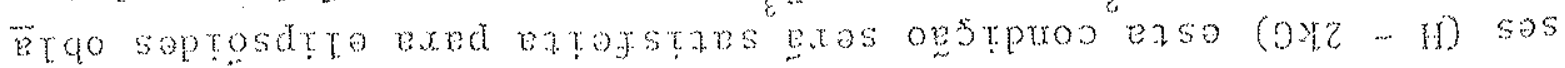

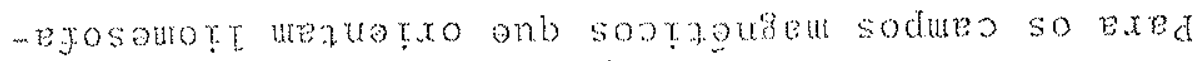


- aronbep oxad tangaxdsop a zu

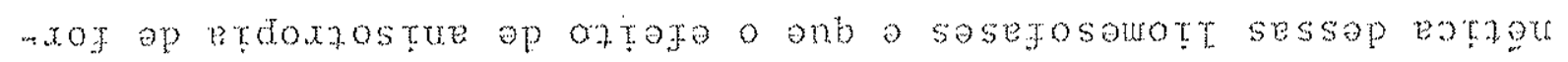

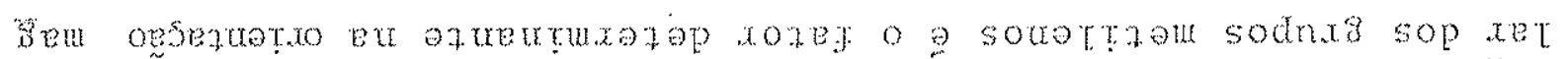

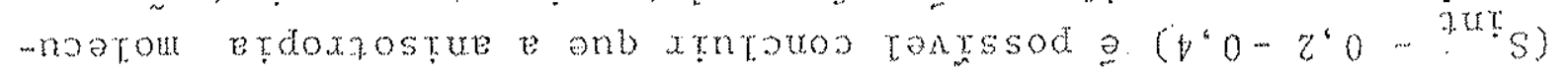

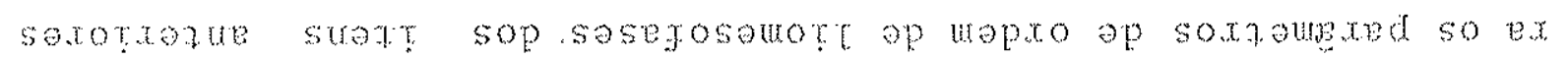

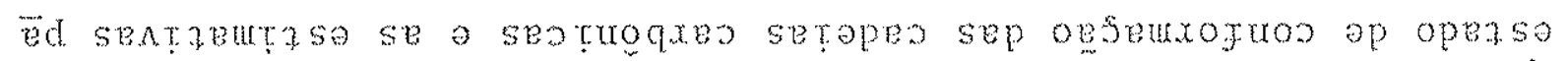

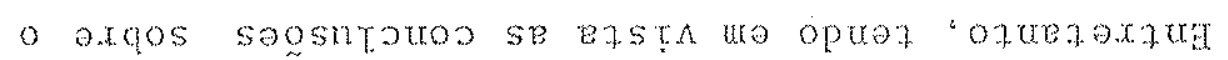

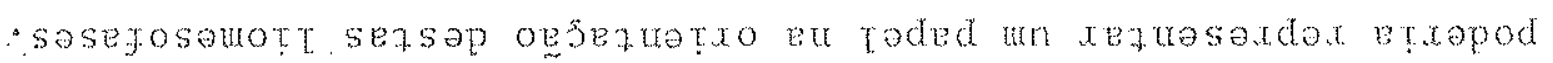

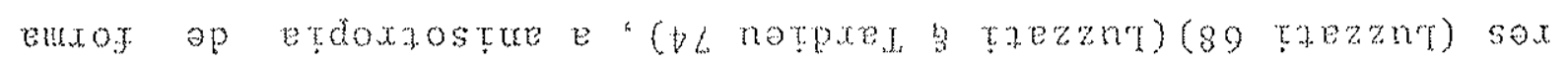

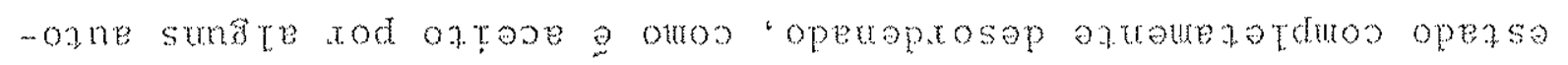
urn wessontaso seotuogres setopes se as 'ozuezod

\section{- optorazst optrot oper.}

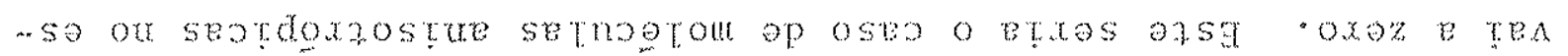

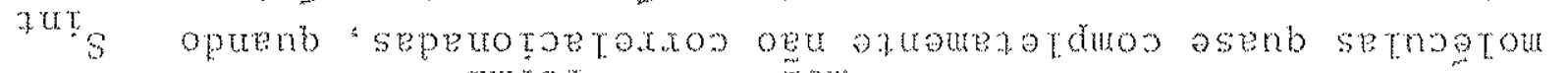

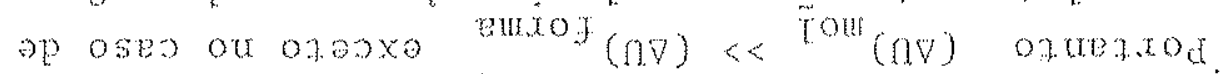

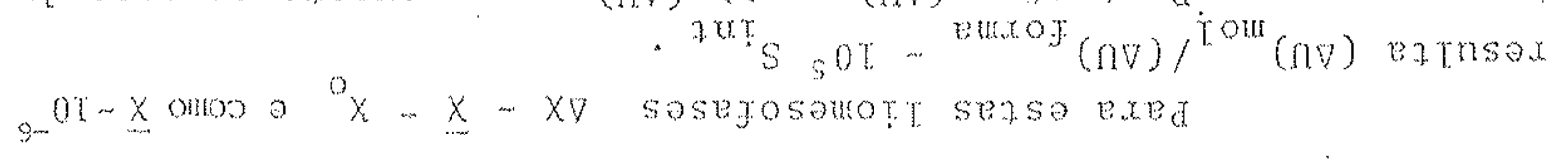

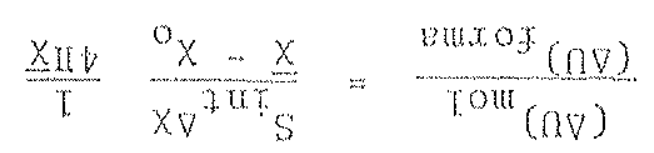




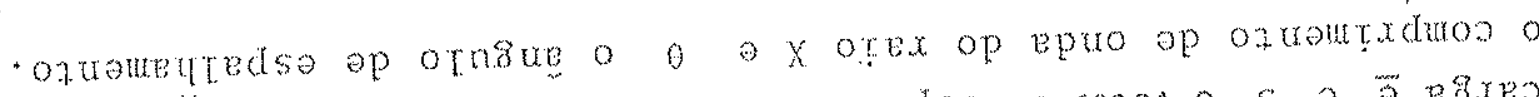

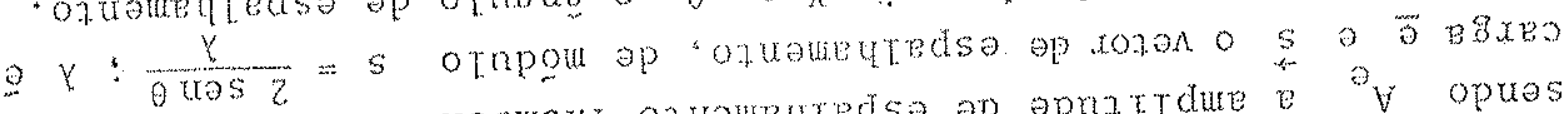
$a p$

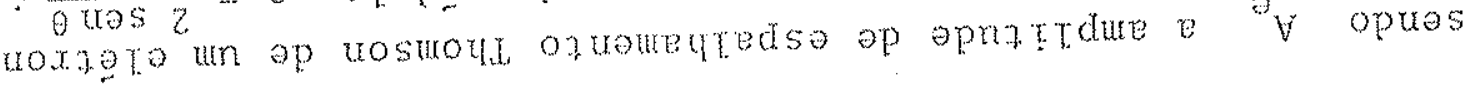

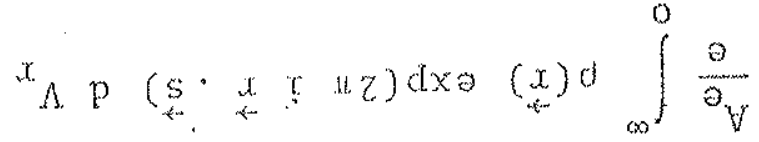

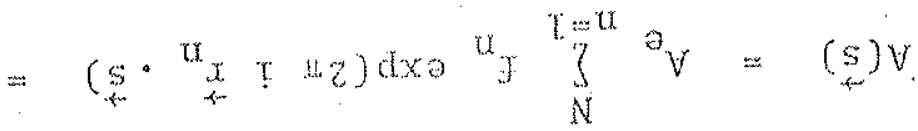

out

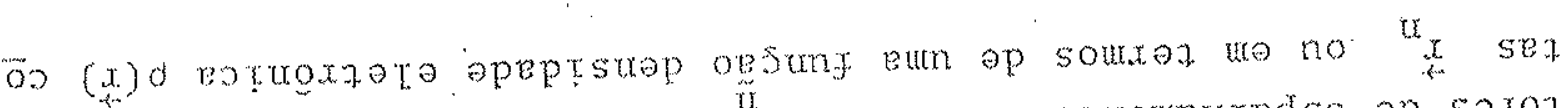

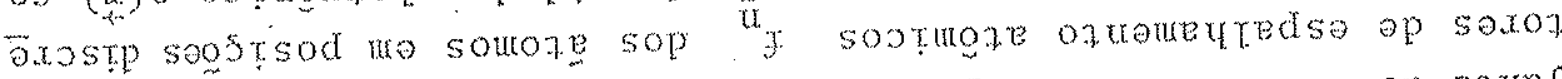

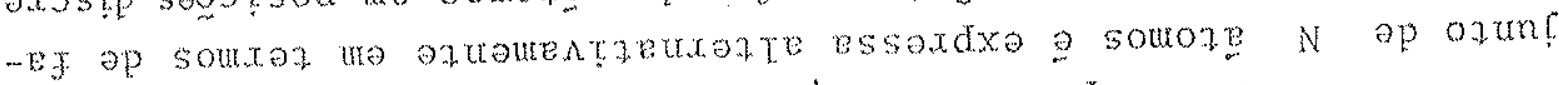

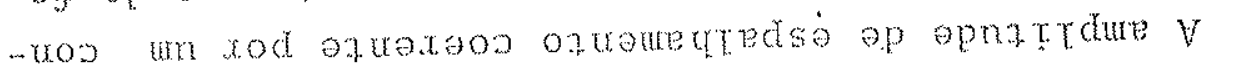

- (ss xatrung) ornsue oxteq ap borrogr e epeztr

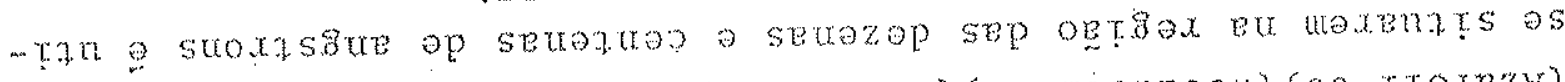

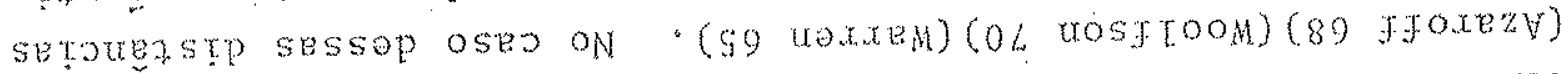

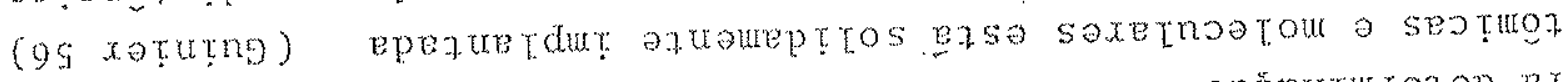

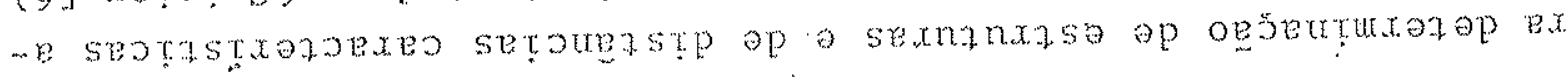
ed $x$ sotex ap oesexte ap atrog ep opseztran $V$

opseztran op ondrated T.T.TI

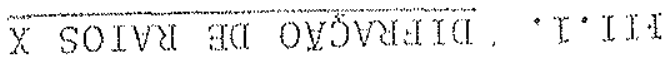

STVWNGNUGAX SVOWOHA 


$$
\cdot \frac{\theta 9000}{\gamma}=\nabla
$$

xaxays ap eperrxoxde opssoxdxe ep son

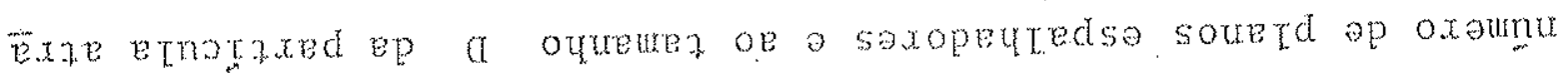

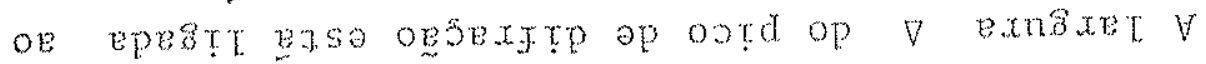

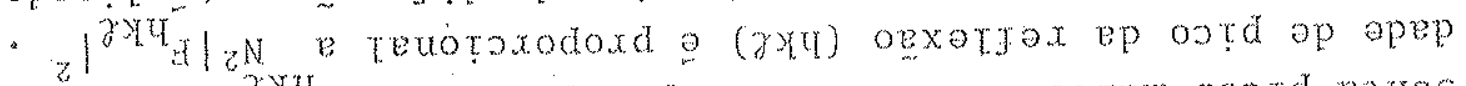

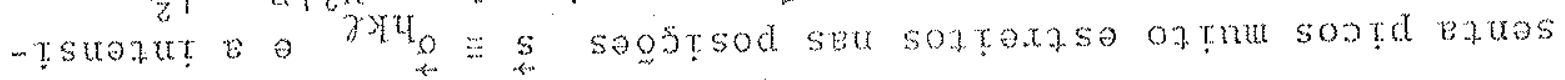

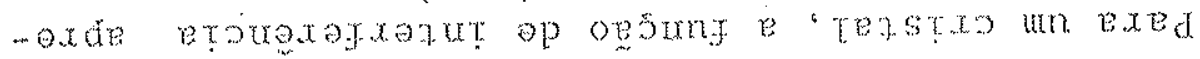

- secozextp sepeptun $N$ se oxzue extanta

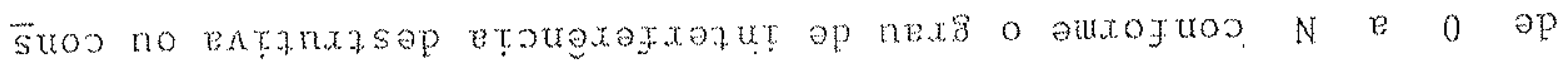

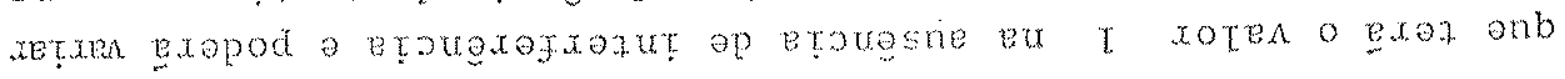

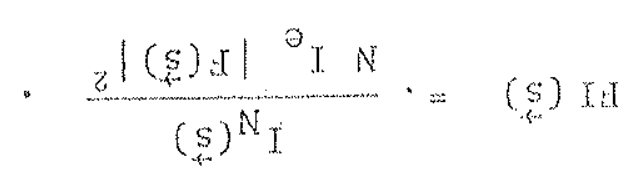

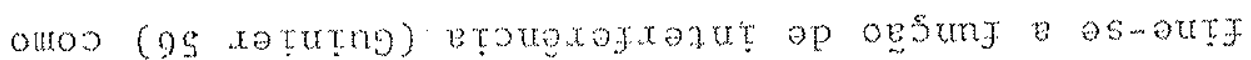

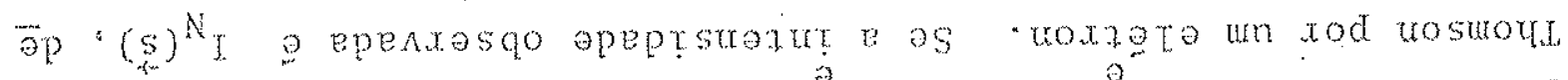

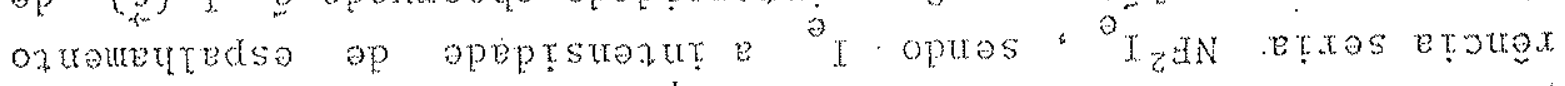

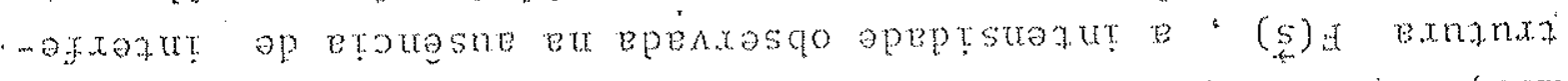

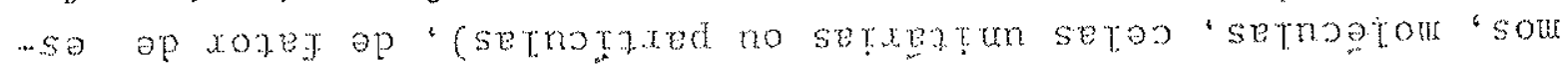
o.p) seatruept serozextp sepeptum $\mathrm{N}$ ap oseo on

$$
\begin{aligned}
& \text { - }\left(u_{z} \cdot u^{\prime} \cdot u_{x}\right)=u_{x} \text { optes }
\end{aligned}
$$

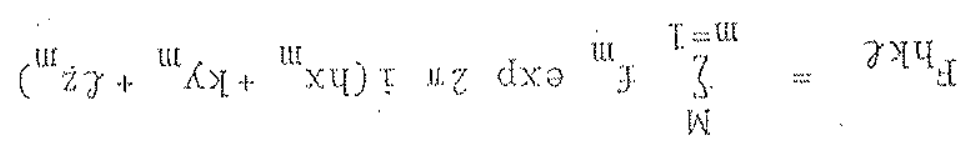

optuo etos somote

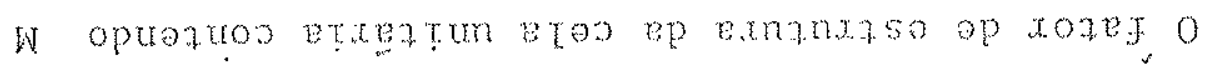

$$
\theta 4057 \mathrm{xp} z=\mathrm{r}
$$

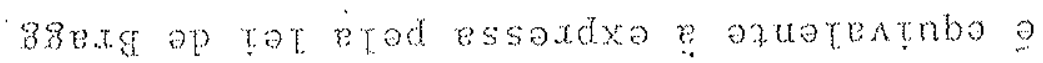

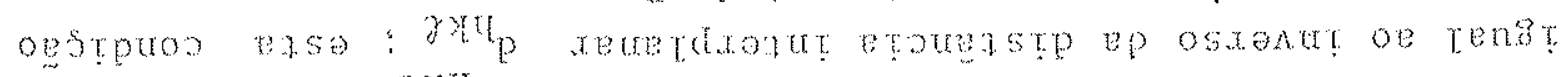

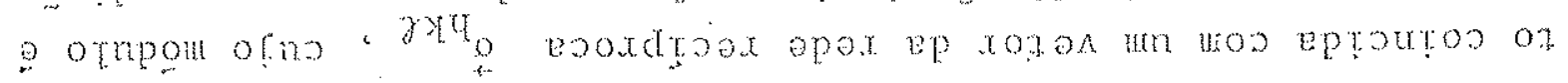

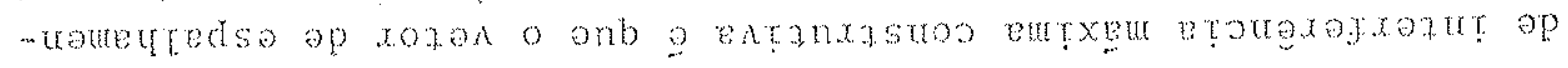

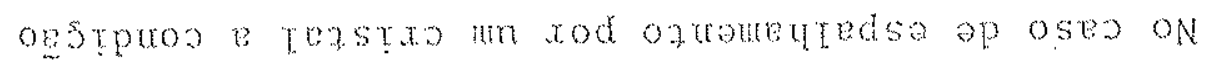




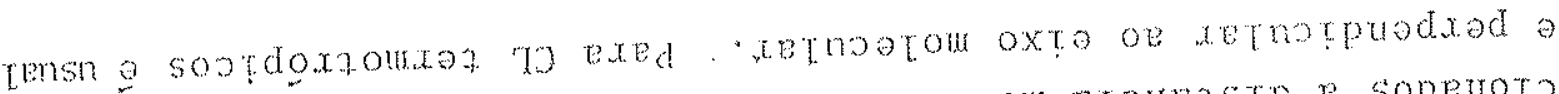

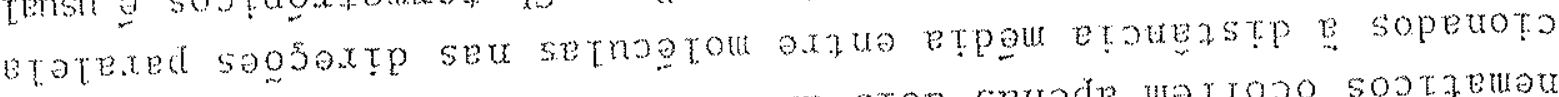

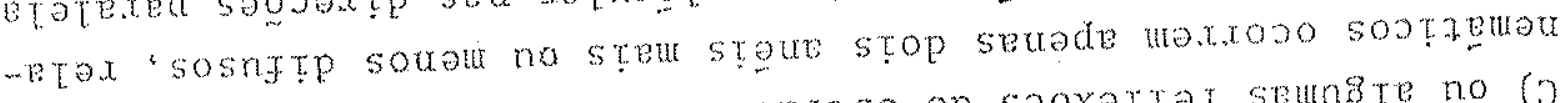

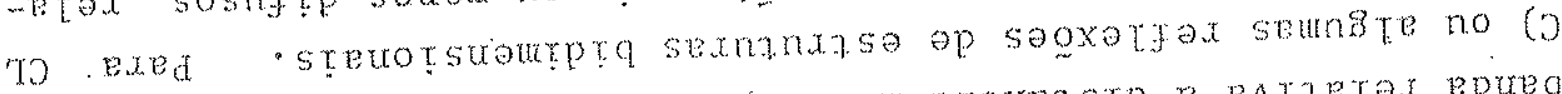

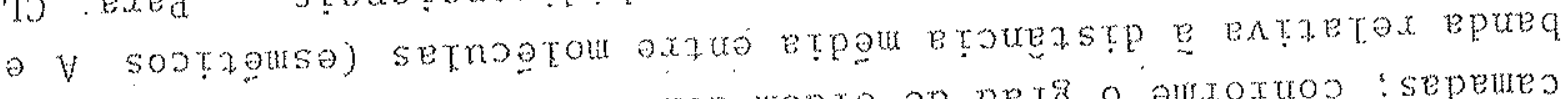

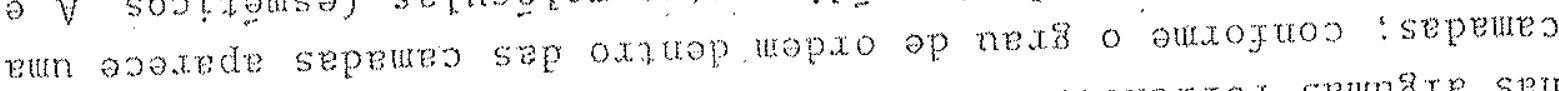

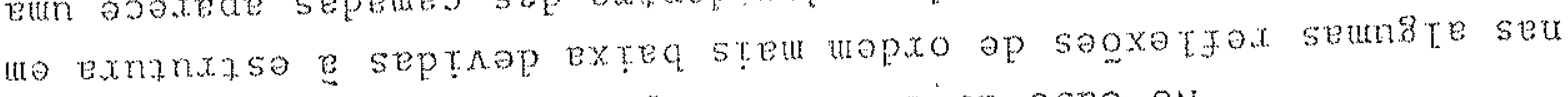

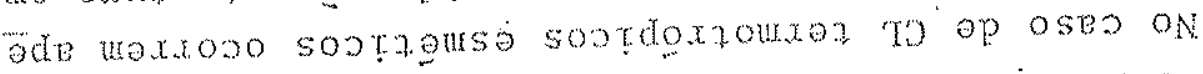

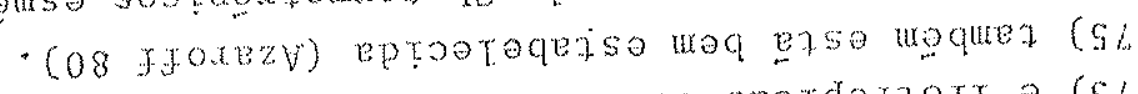

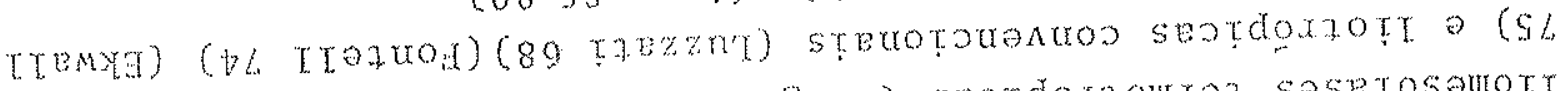

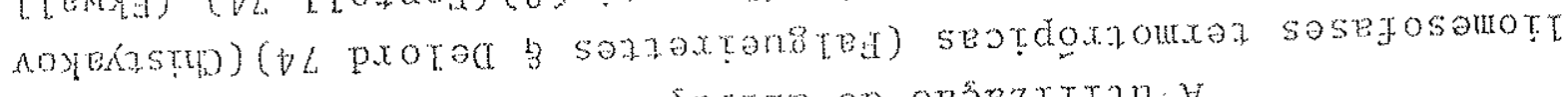
op opnas ox $x$ sotex ap opsextre op opseztran $y$

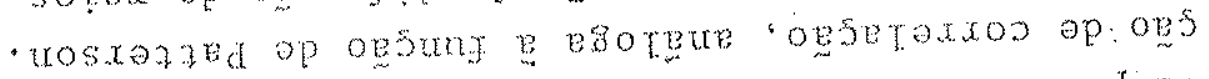

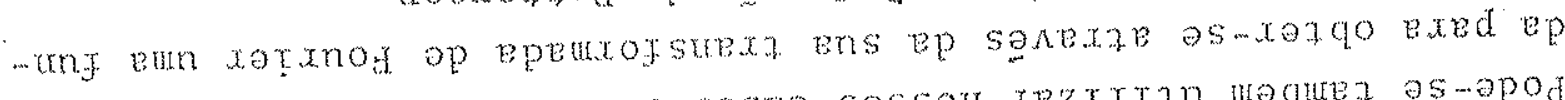

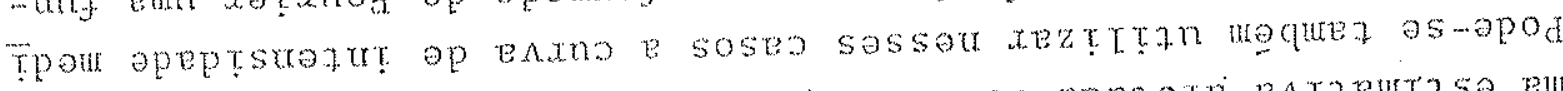

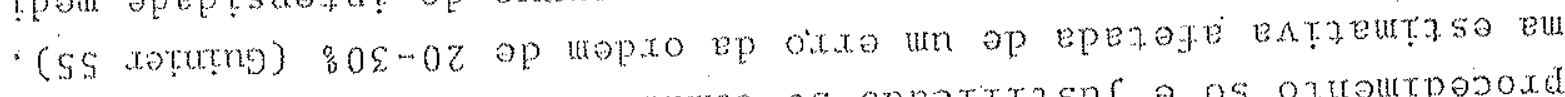

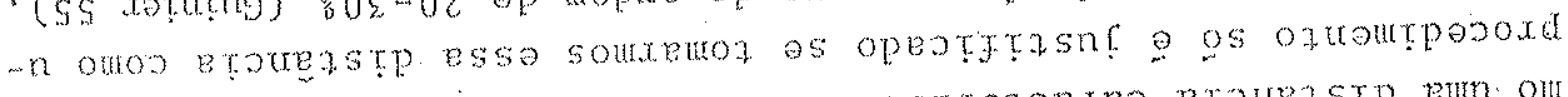

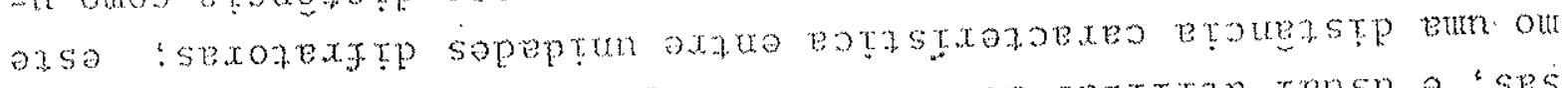

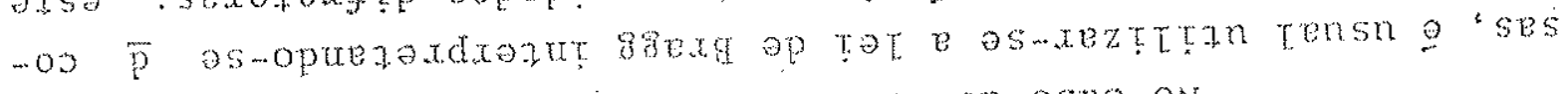

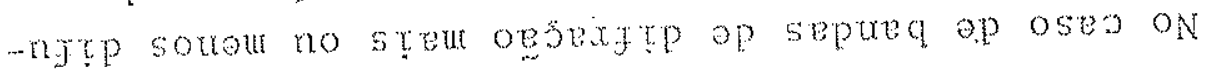

- sexodexte sep

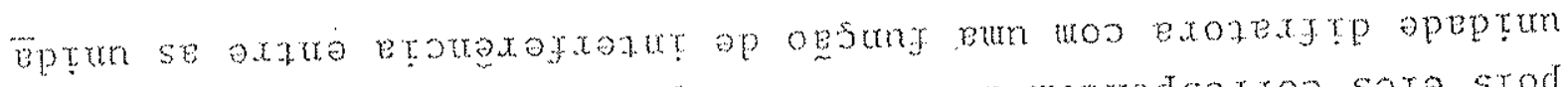

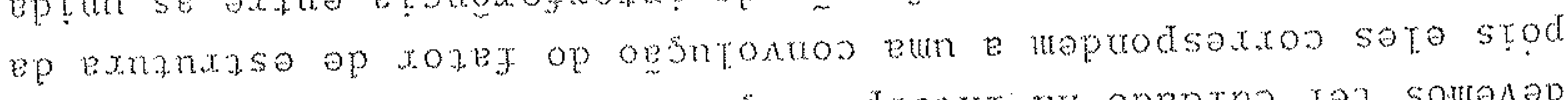

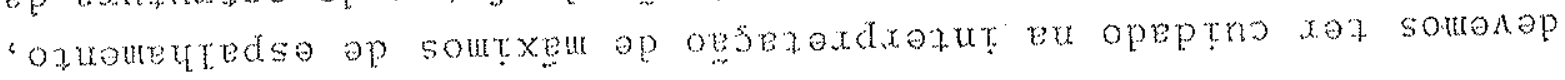

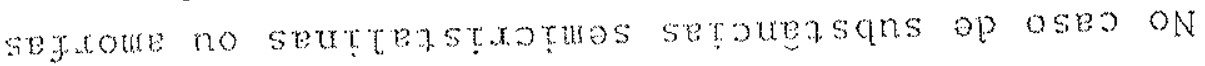

- sozoxp sopozgut 'oztrout

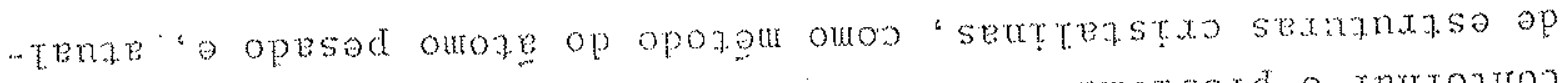

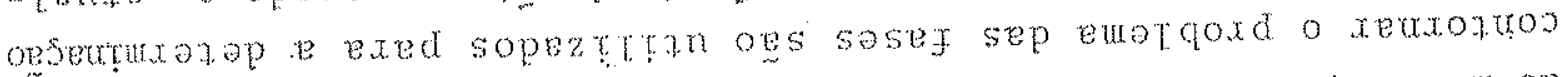

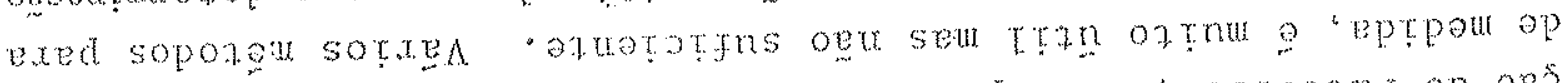

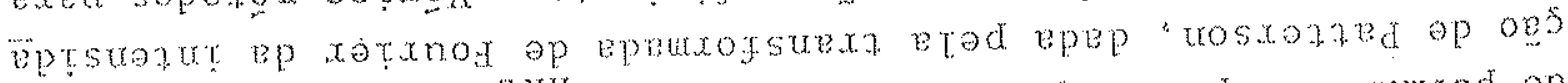

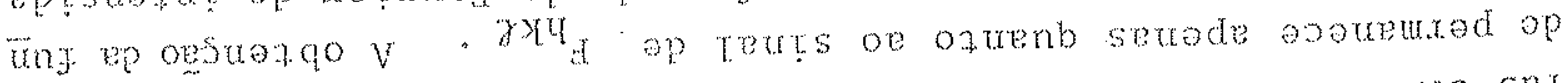

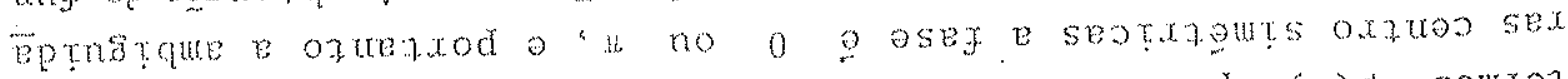

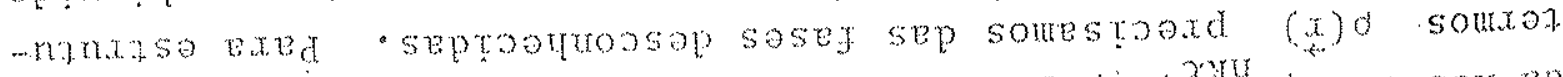

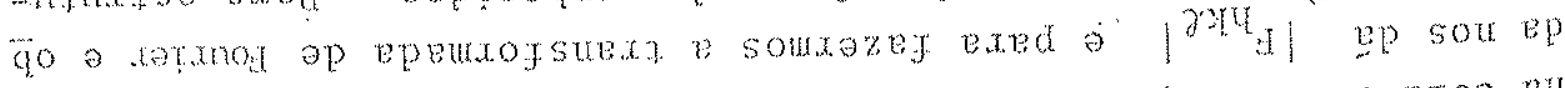

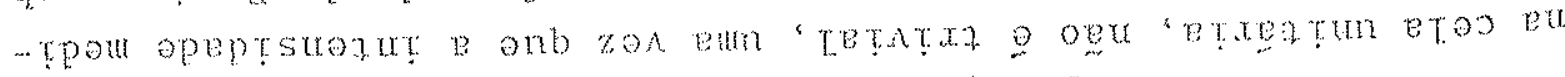

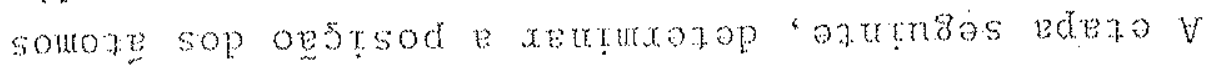

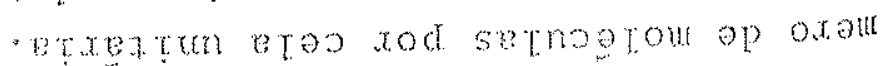

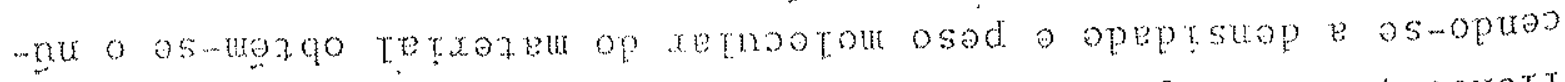

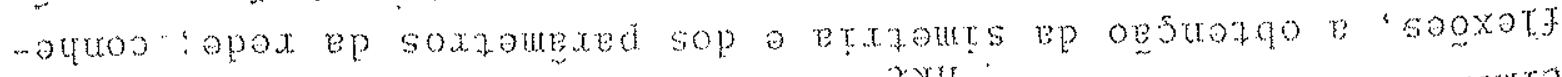

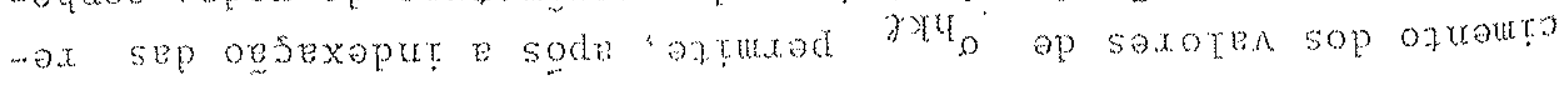

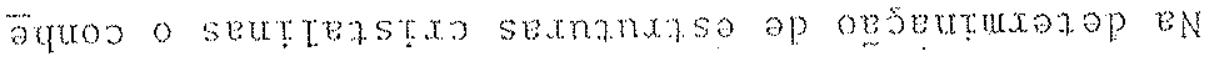




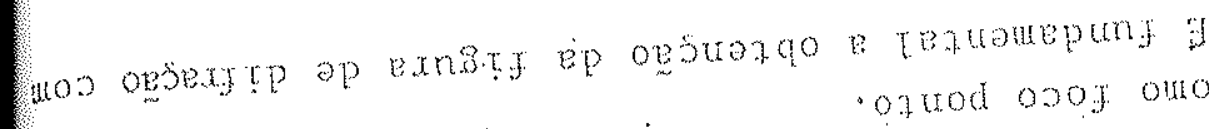

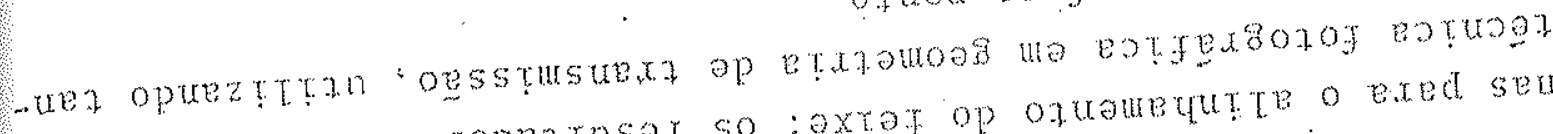

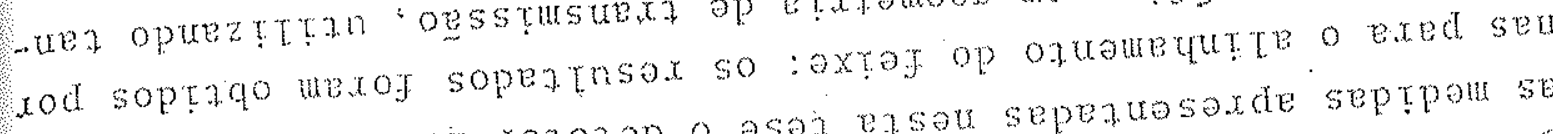

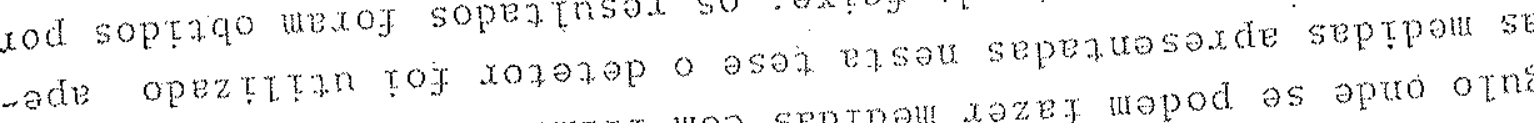

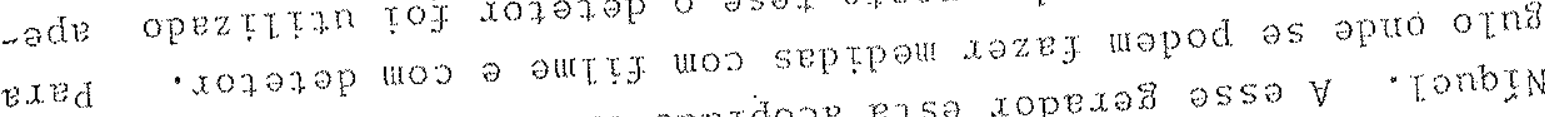

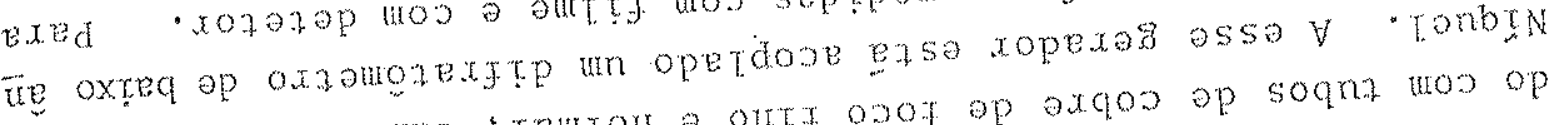

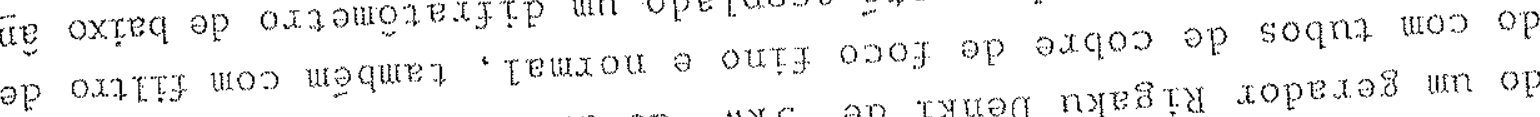

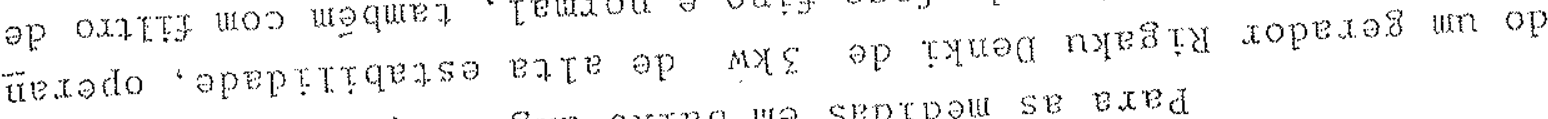
eztran tog (XVs) otrige axteq wo seppou se bxed

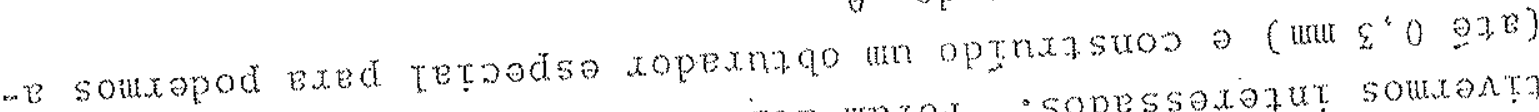

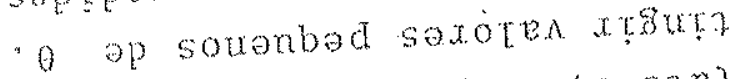

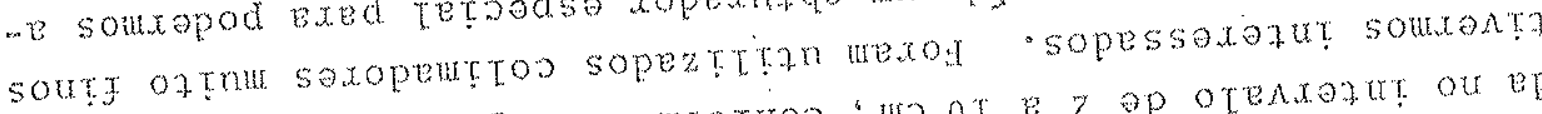

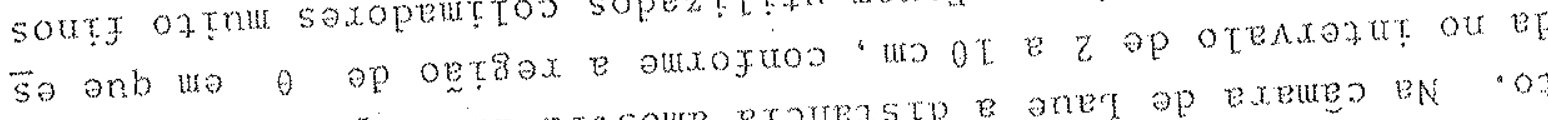

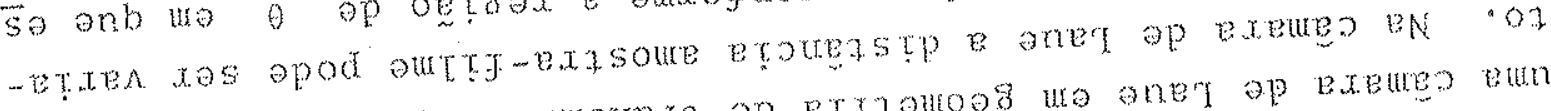

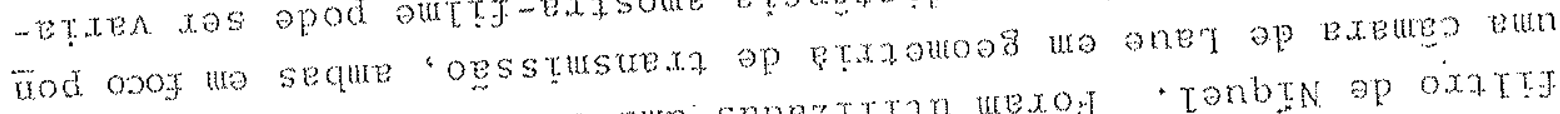

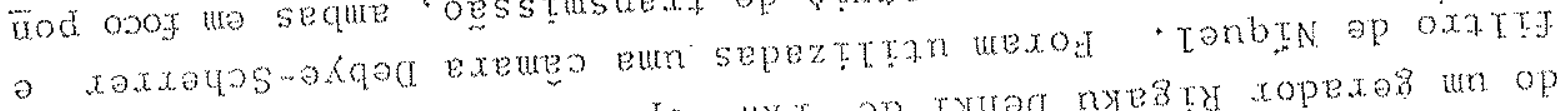

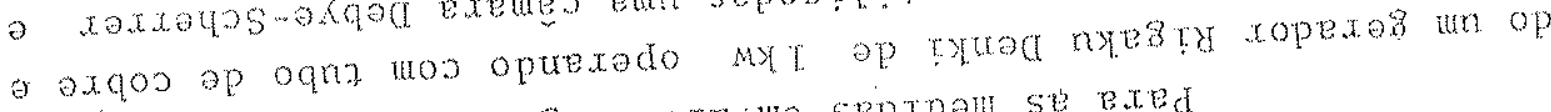

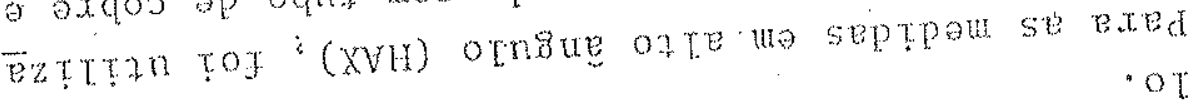

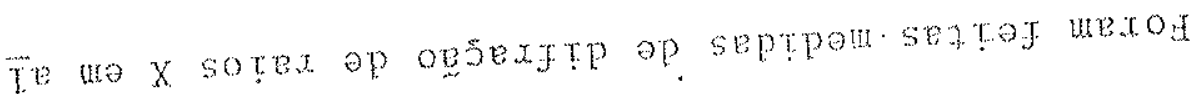

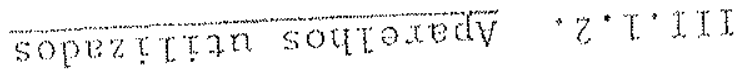

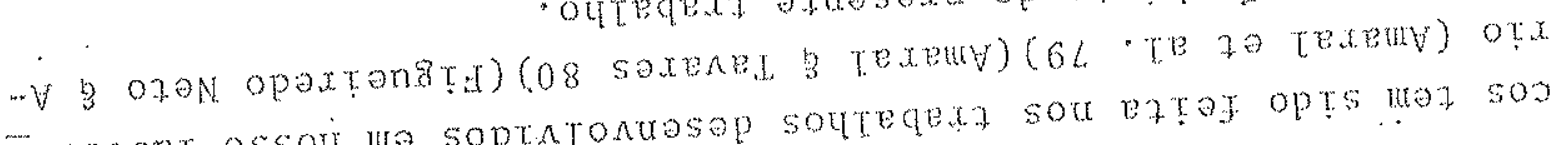

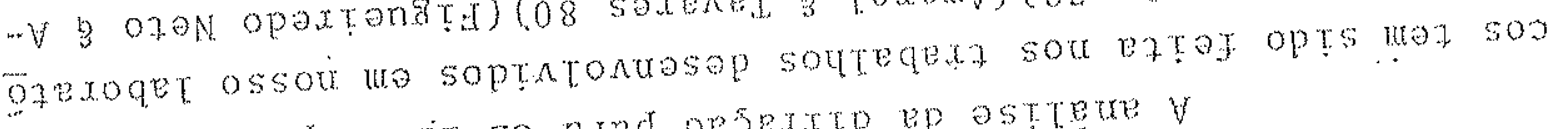

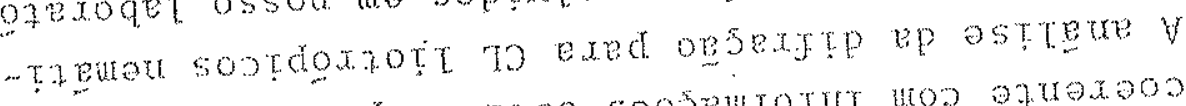

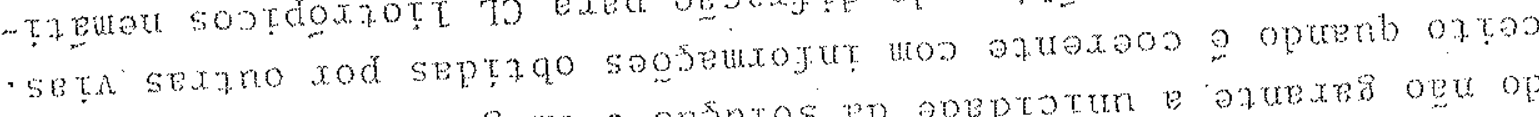

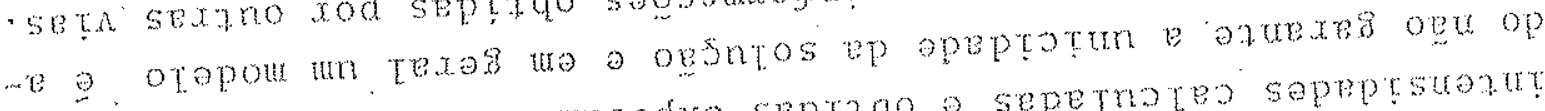

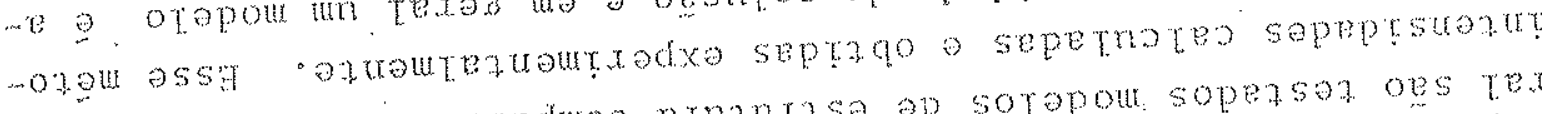

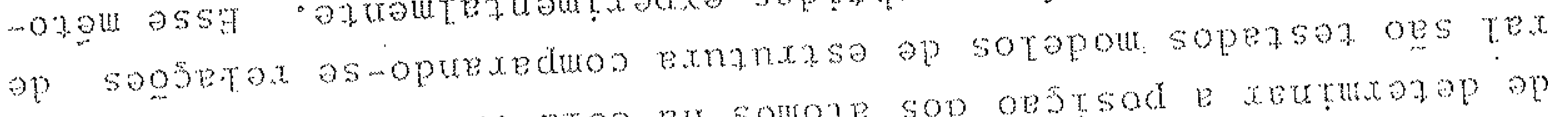

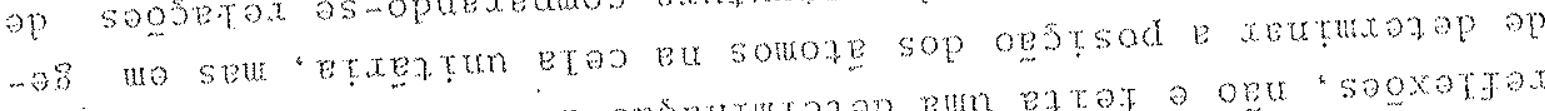

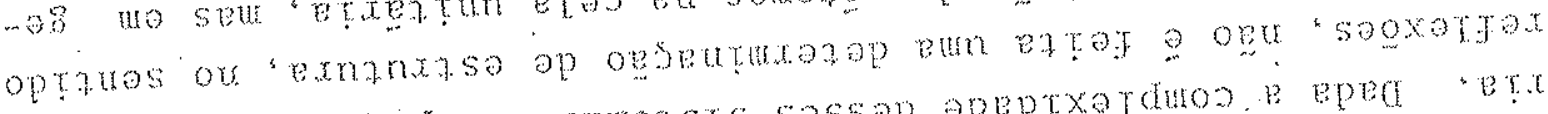

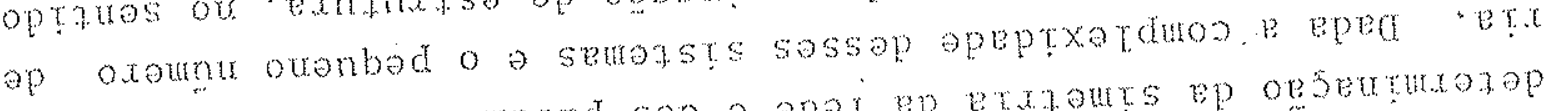

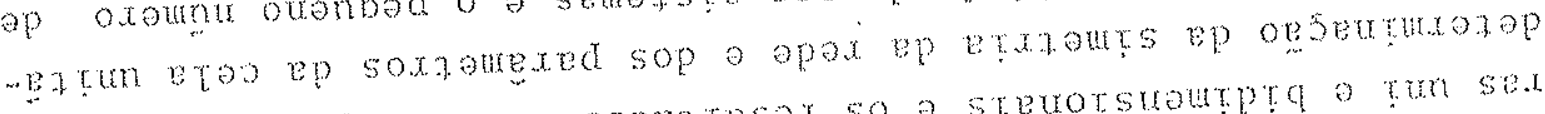

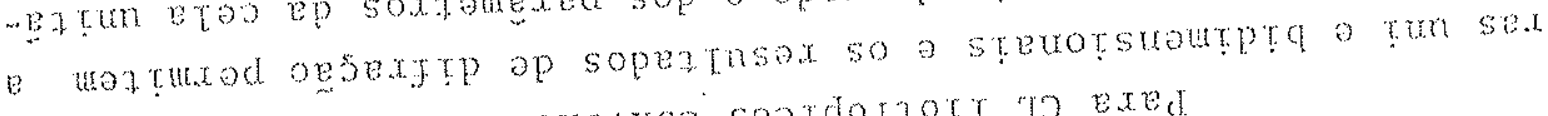

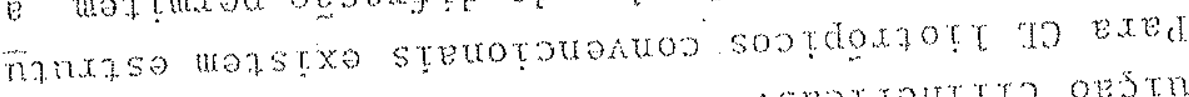

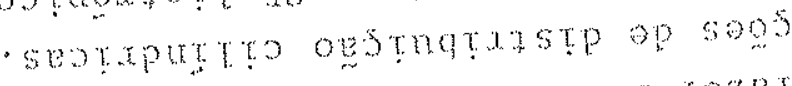

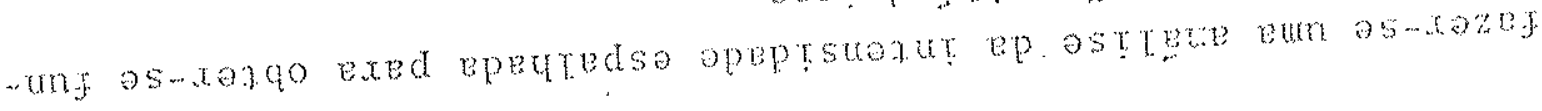




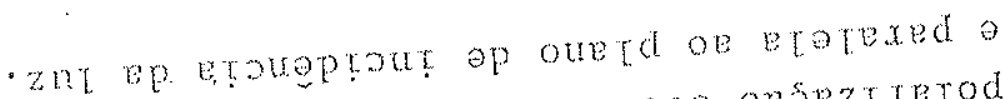

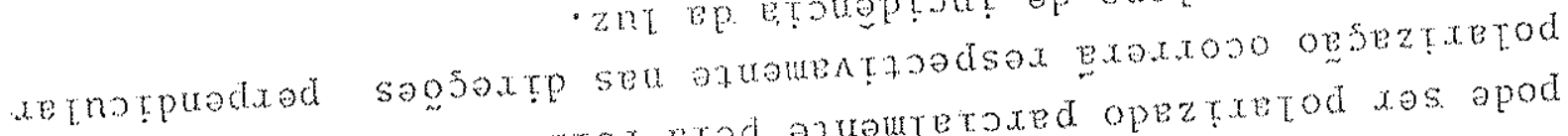

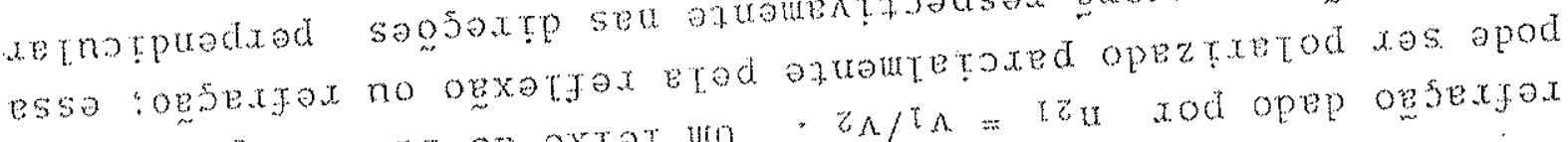

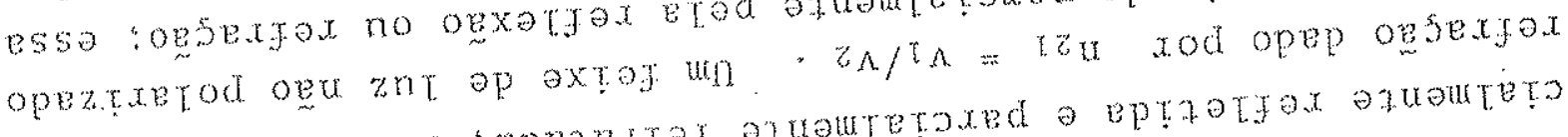

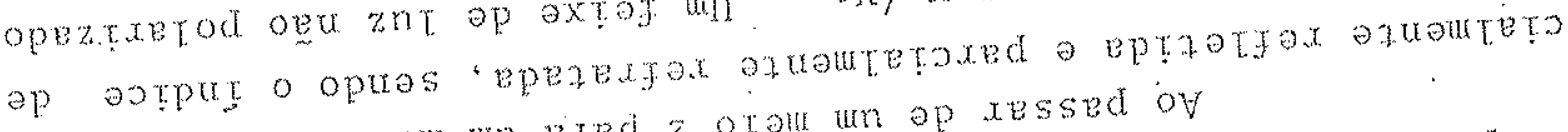

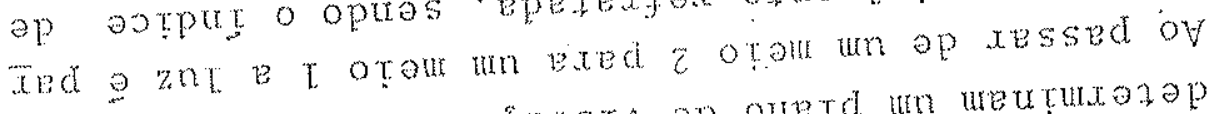

- opsedata ep onetd un ueutuxazop ozsezedoxd op

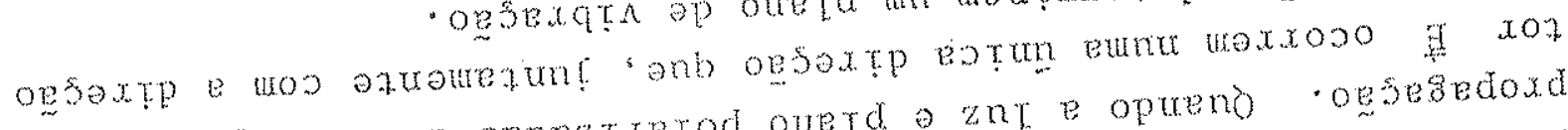
an op sogjexqu se sepezterod ouetd a znt e opteno oesesedoxd

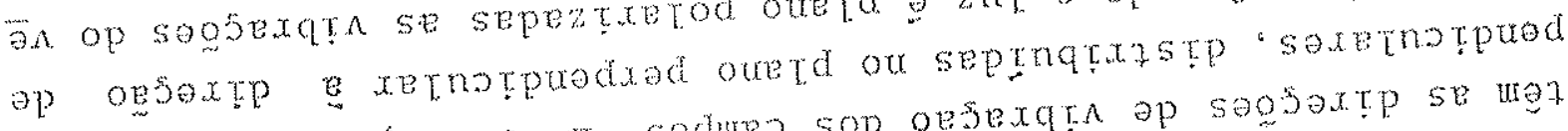

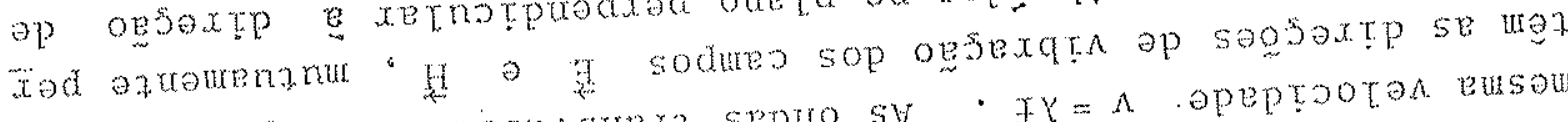

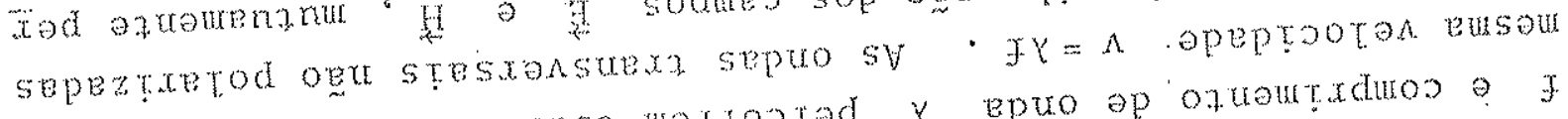

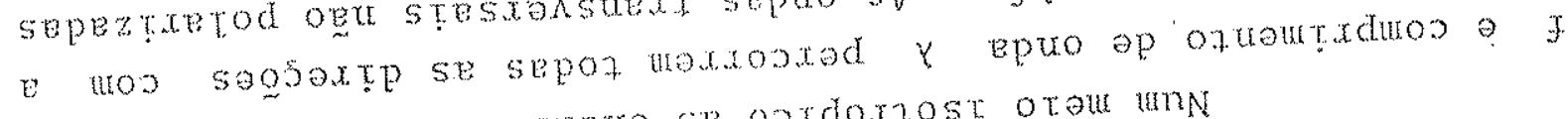

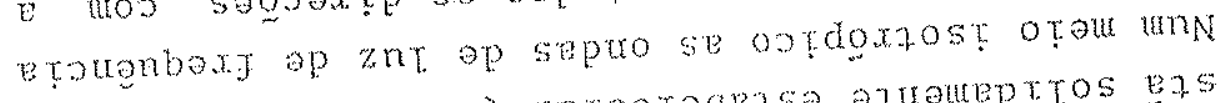

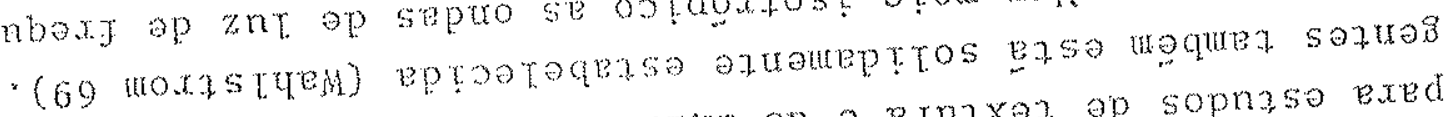

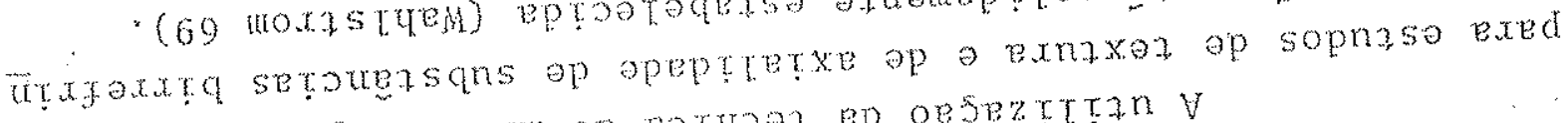
Cow exte erdossoxom op borrog ep opsertern $\mathrm{V}$

ogseztran op otdrourd "T*II

VOILAO VIdOOSOUDTW $2 \cdot \operatorname{TI}$

- ontzetox epeptrajt ex etouents

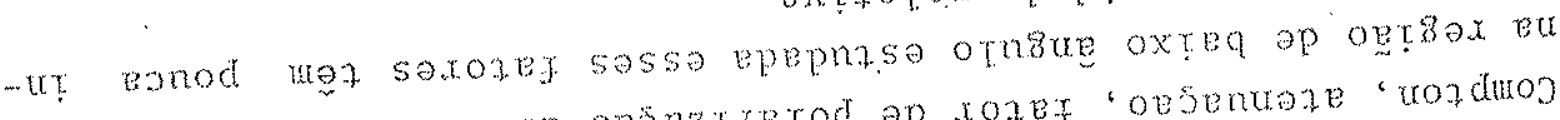

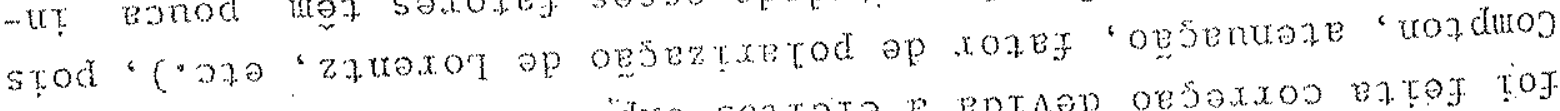

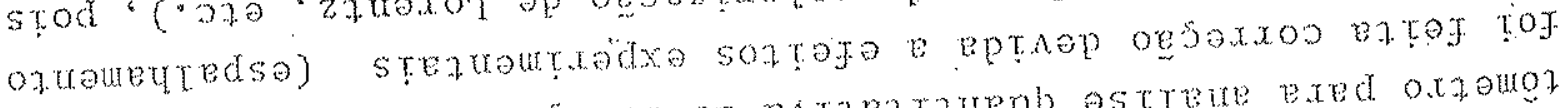

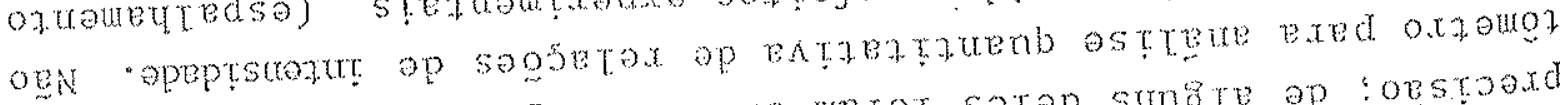

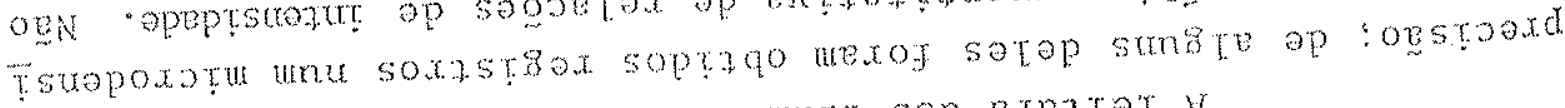

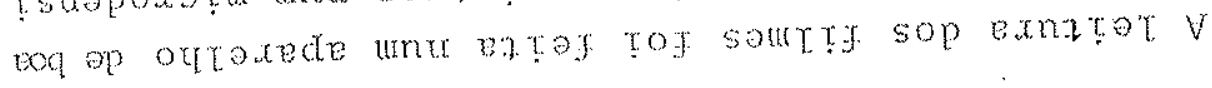

- wo 02

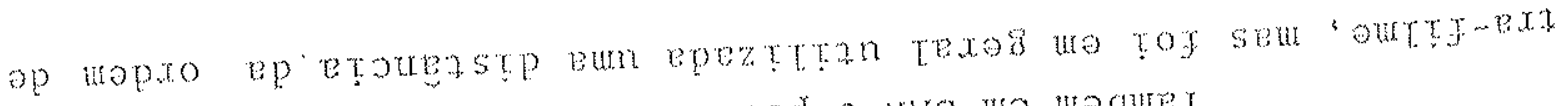

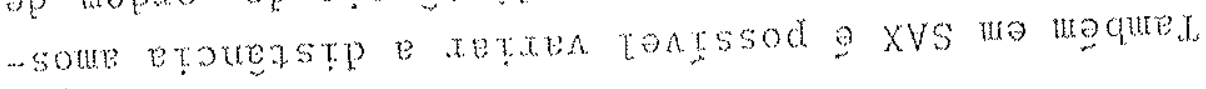

- opeptsugat

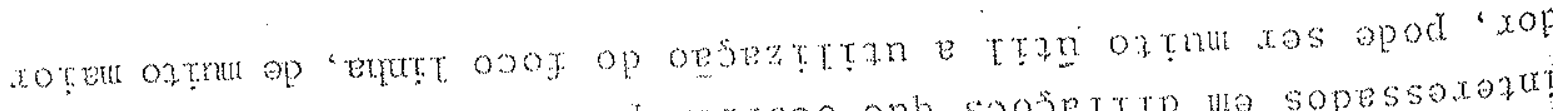

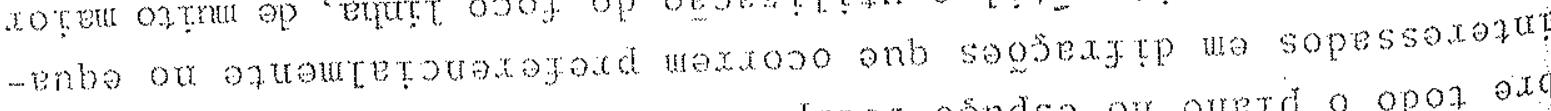

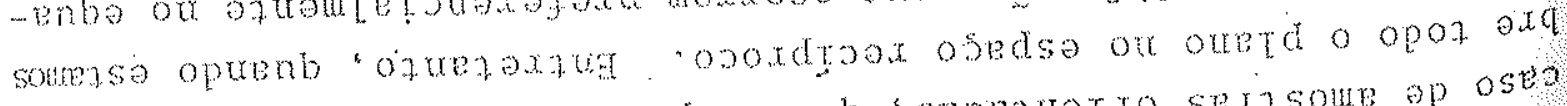

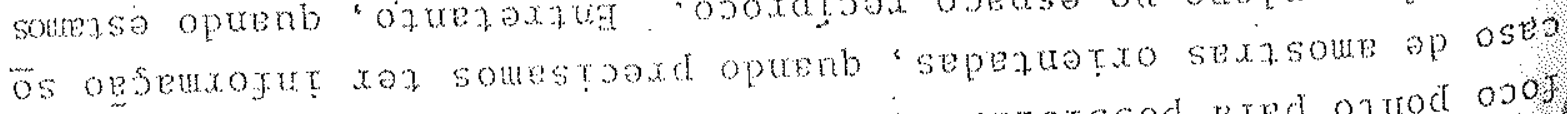

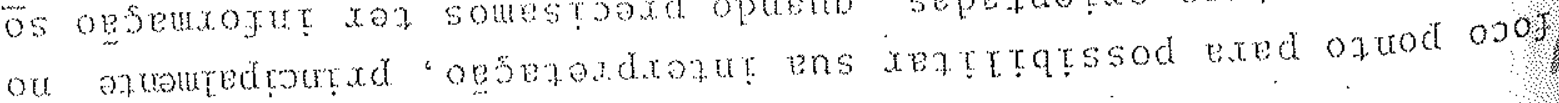




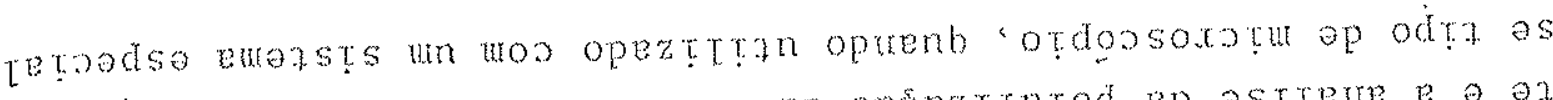

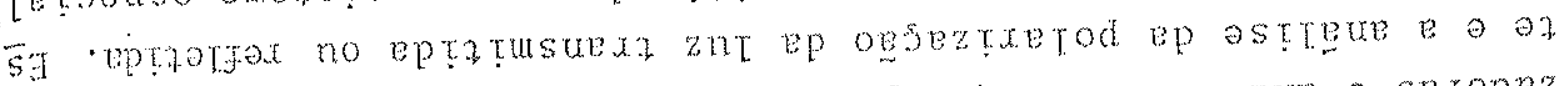

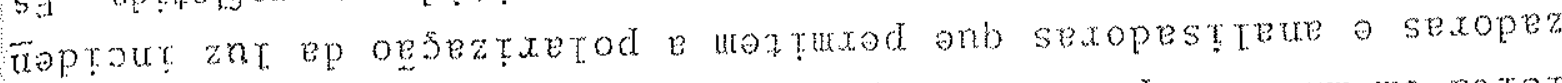

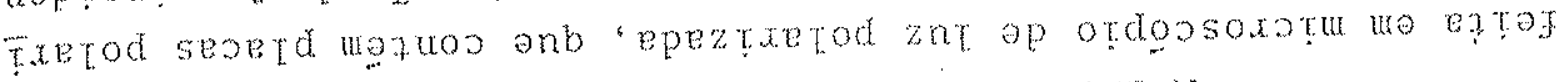
a ootdonostue otou un op apeptrexe ep astrext $V$

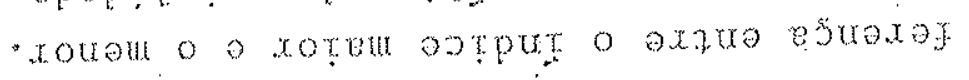

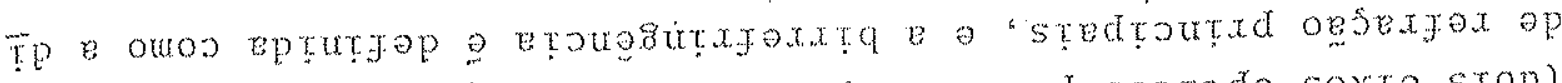

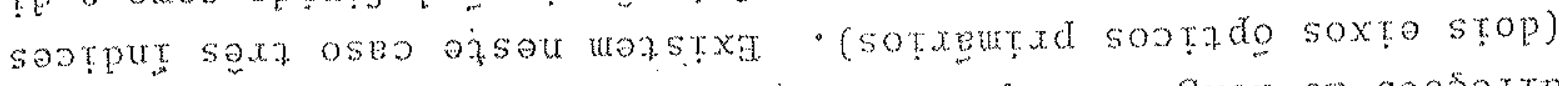
eotur o oesesedoxd ap opeprootan e stenb sep o8uot oe soojaxtp

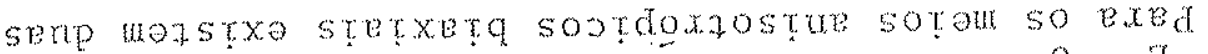

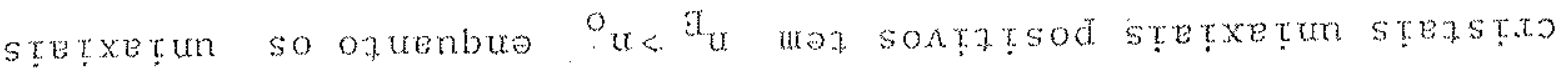

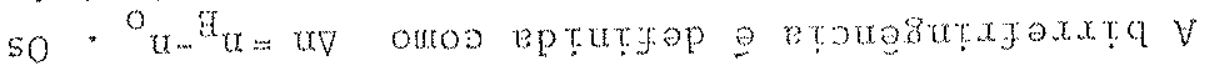

- ogsexqua op ogsextp ep ogstung emm ozexa toten

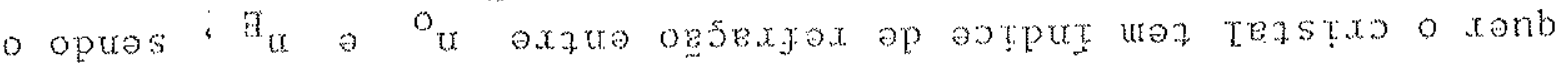

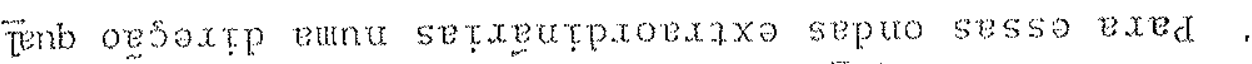

- ar operstrep o oprado oxto oe

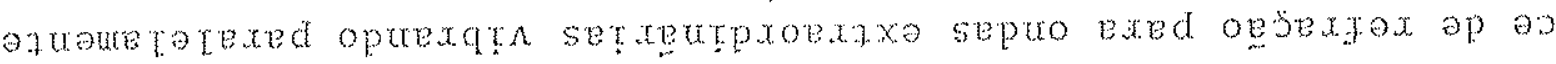

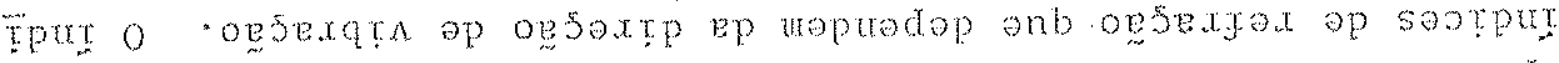

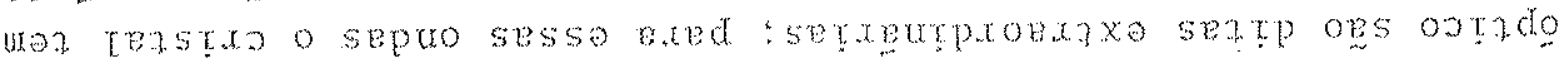

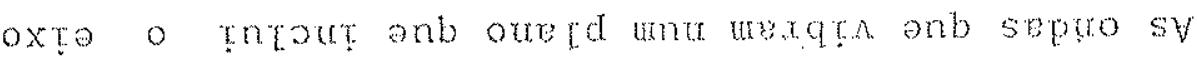

- epeztrated

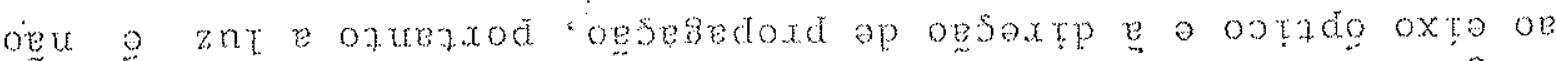

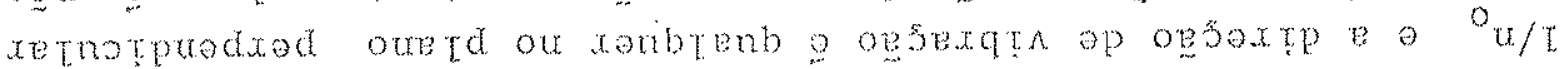

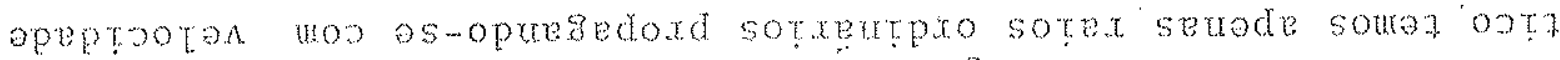

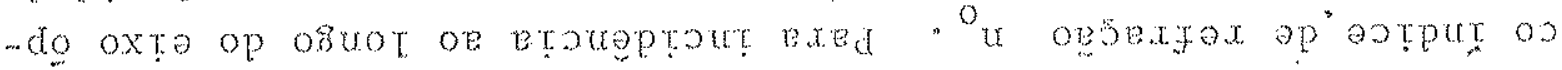

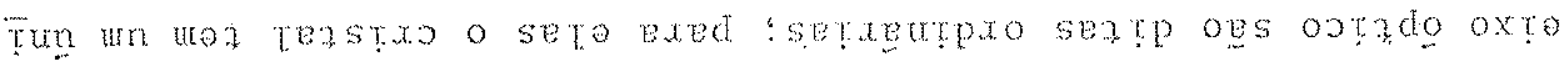

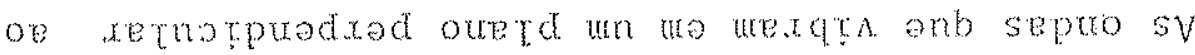

- topuestexy

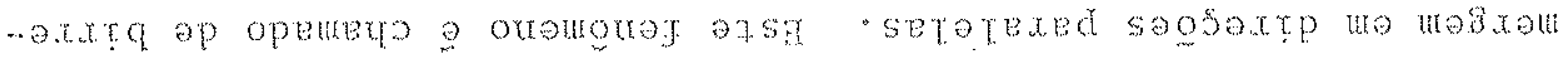

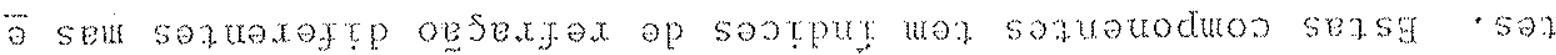

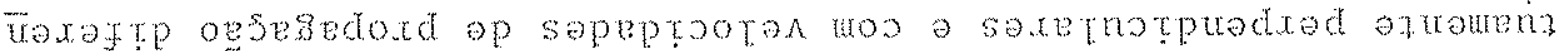

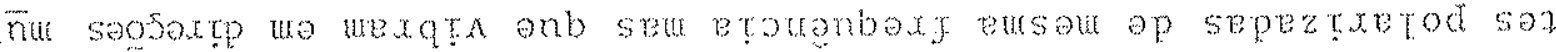

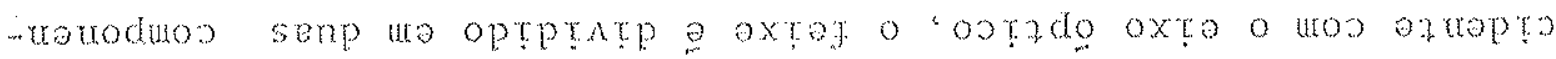

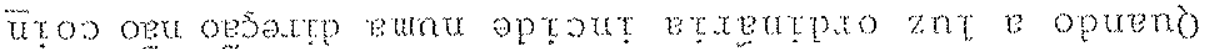

- optroxast assor 07

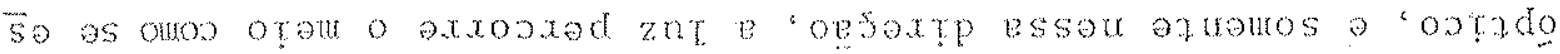

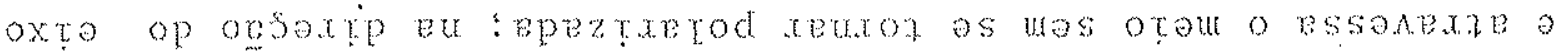

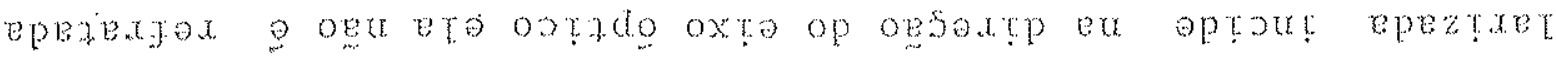

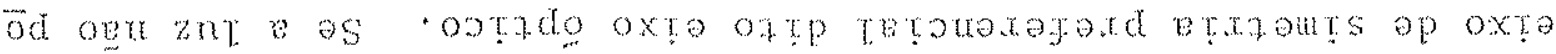
un orstxo retxetur optextostue a opou o opueno 


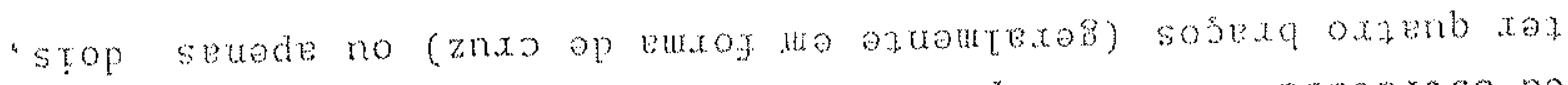

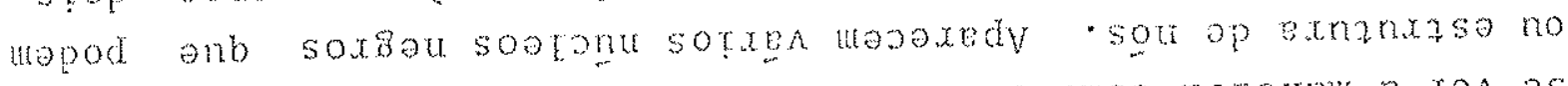

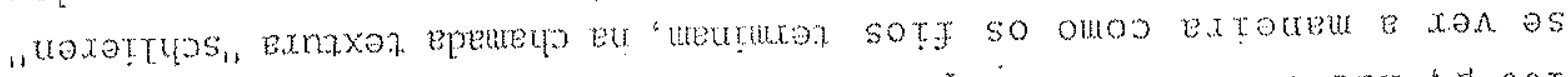

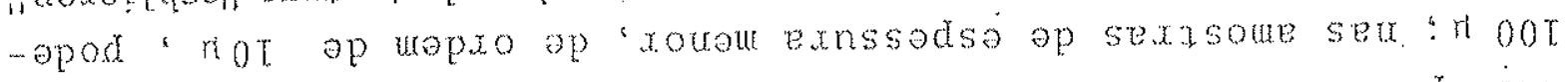
op mopxo ep exnssodse op seutd sexzsome we exsta xos opod soty

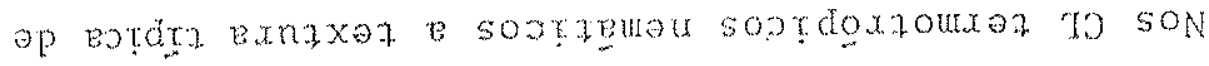

"To op exasoure eum ap opsearetro op opezse o xeztensta ap ep

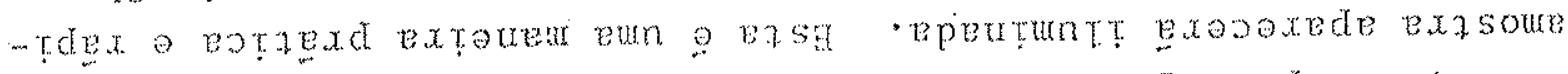

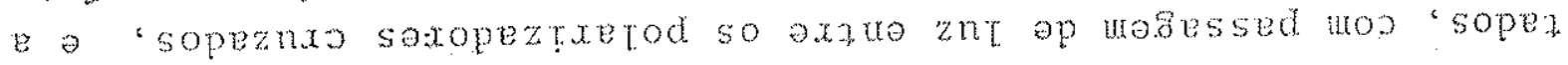

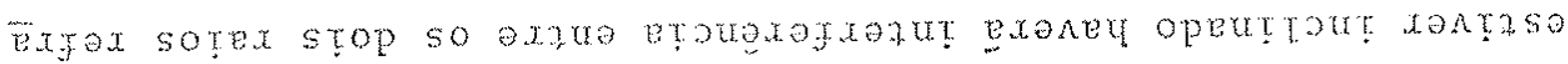

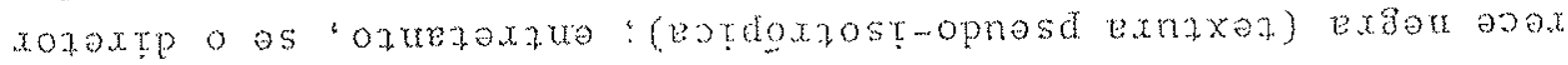

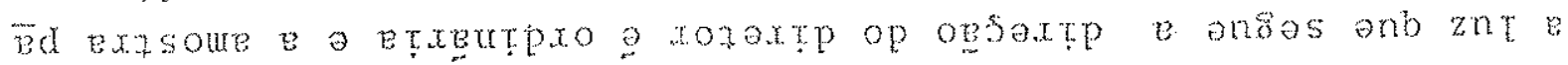
opotiodns e tetnotptedxad xontzsa xoyaxp o as sopezndo soxopez - Txerod axalu TD op exasour eun enxosqo as opteno

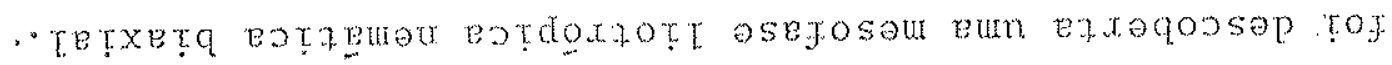

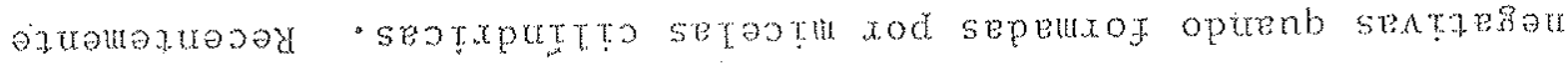

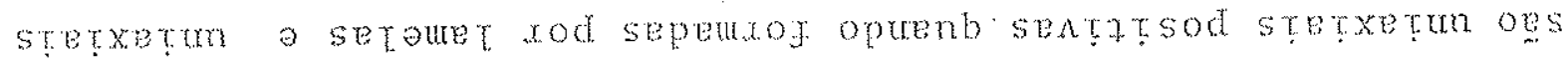

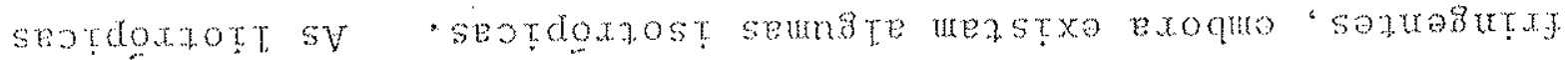

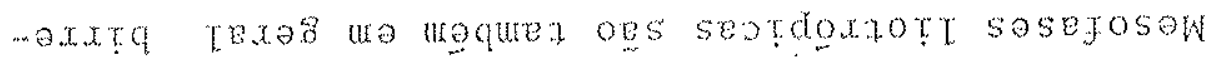

stetreta

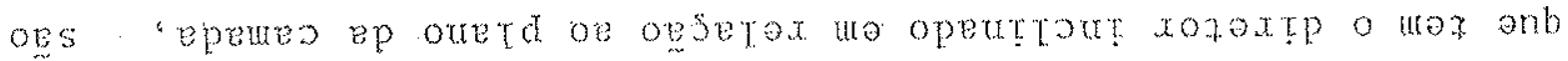

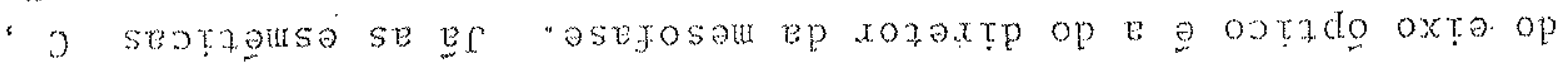

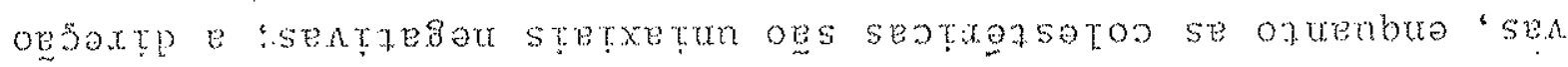

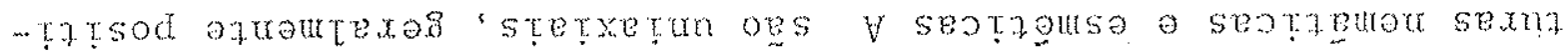

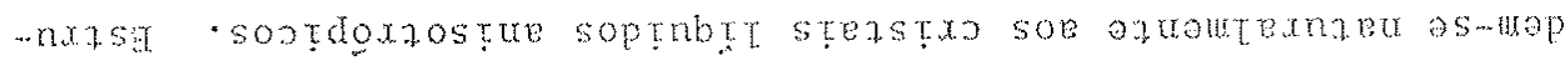

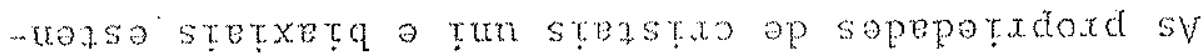

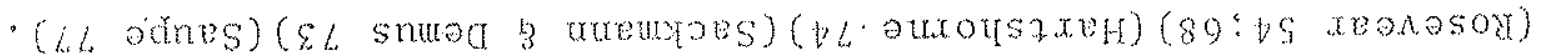

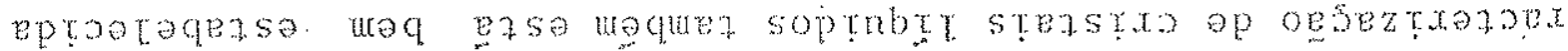

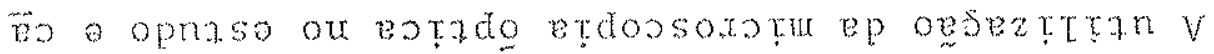

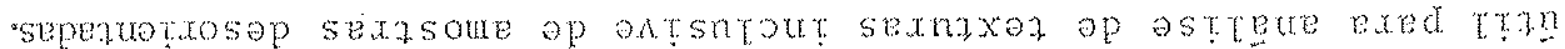

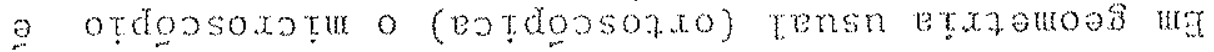

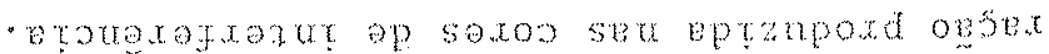

bate asmoprestgran o ofdossoxotu ou etrossooe eard am as-op ưxosut opertuxazop xas spod ppepterxe op teuts o otdossoxoxu

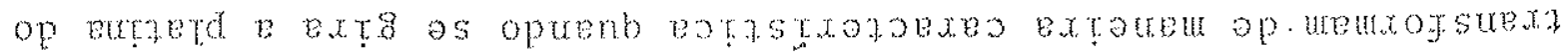

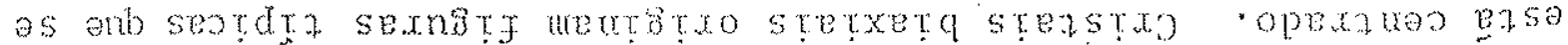

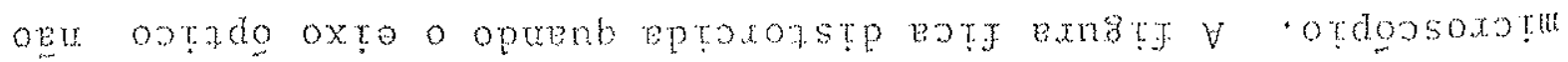

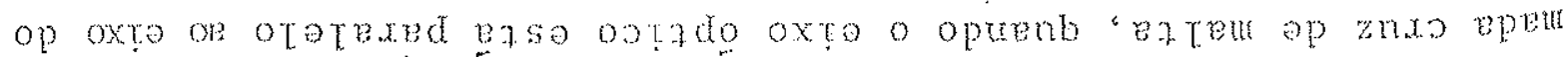

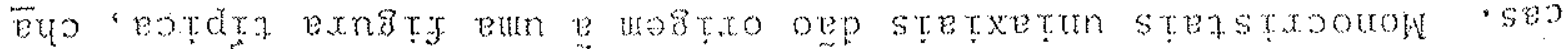

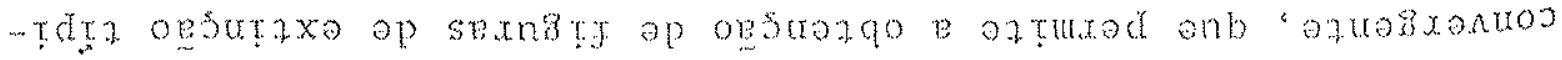

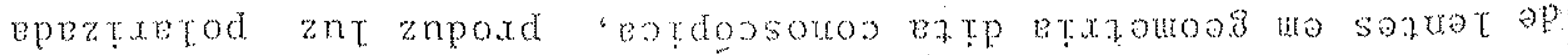




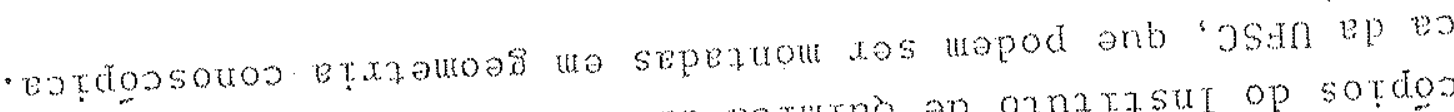

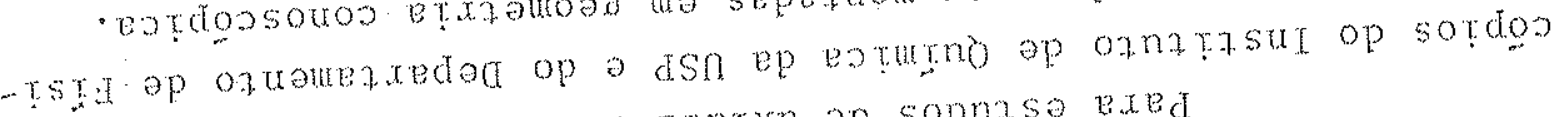

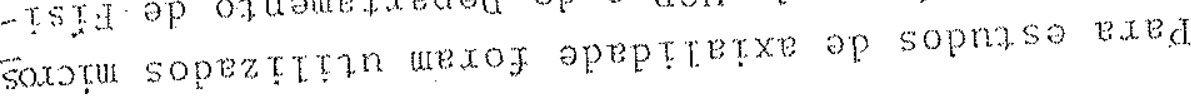

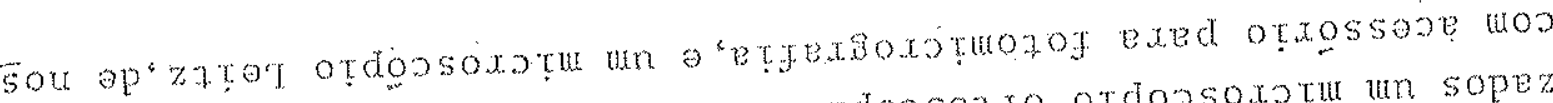
operdoos spertatod znt op pten otossoxo otdossoxotu un sopez

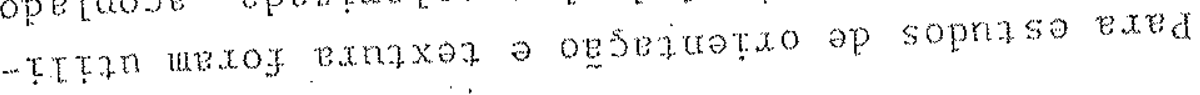

sopezttan soytaxedy $z^{\circ} z^{*}$ iti

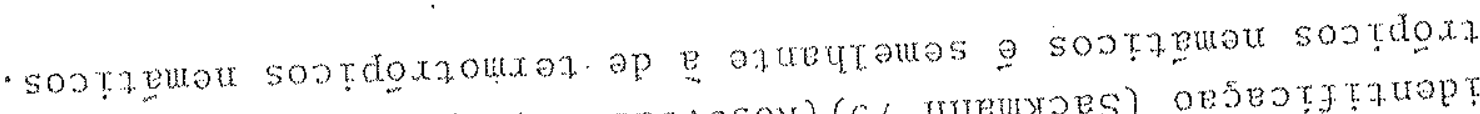

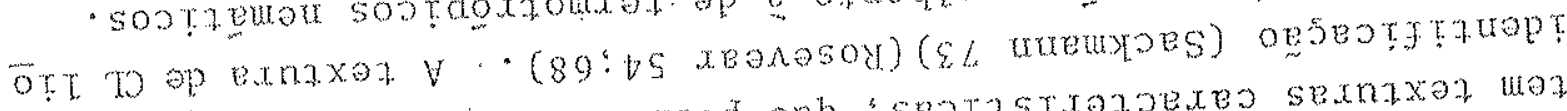

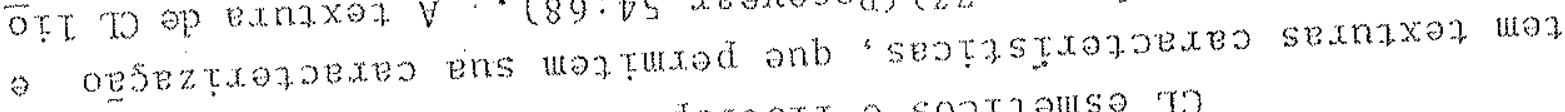

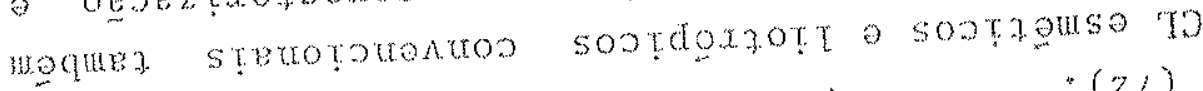

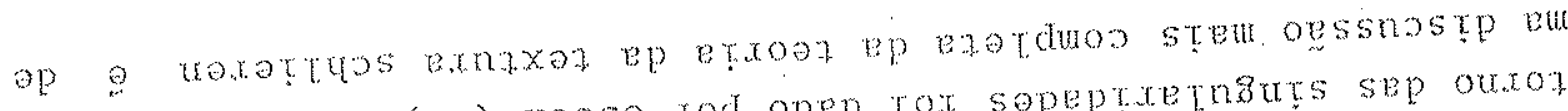

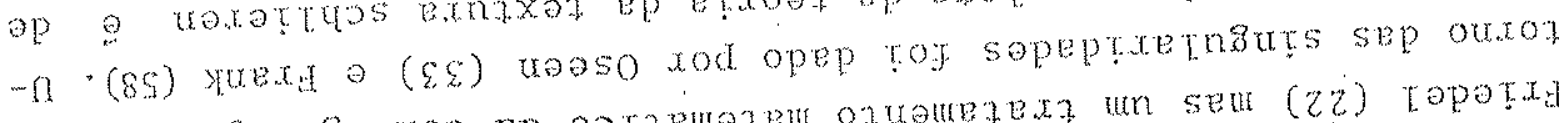

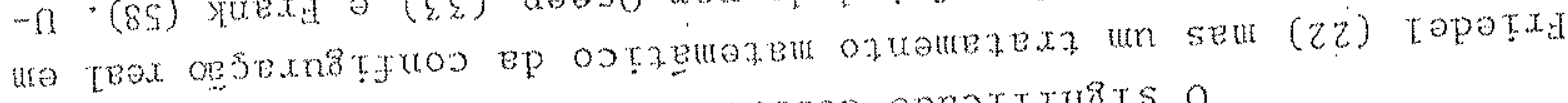

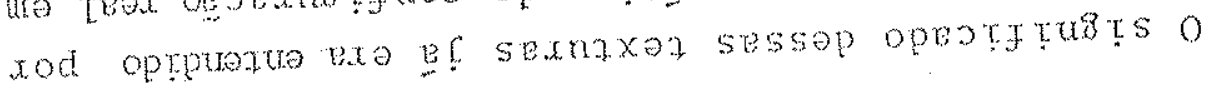

- oxer ras

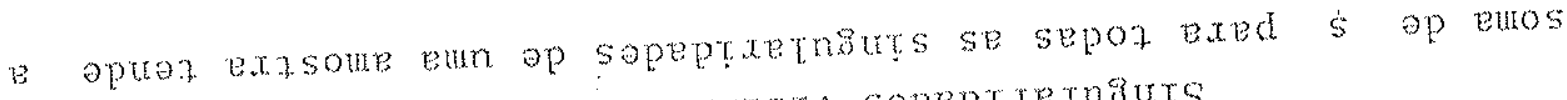

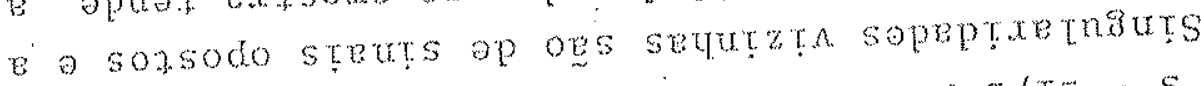

$$
2 / \mathrm{t}=\mathrm{s} \text {. pxed oxqop o } 2
$$

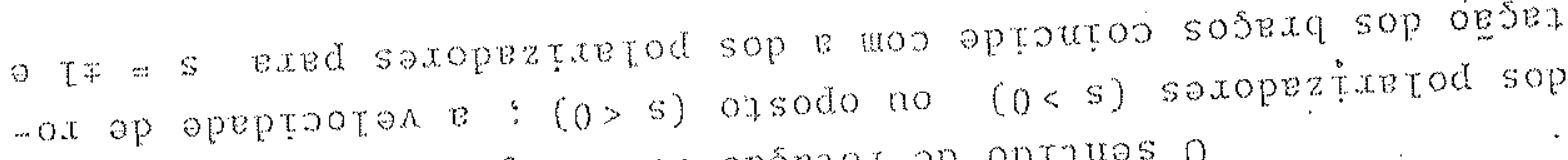
oused o hos apod sosert sop opsezox op optras o

$$
\text { - } 5050 x q \text { op oxourne } \frac{b}{T}=s
$$

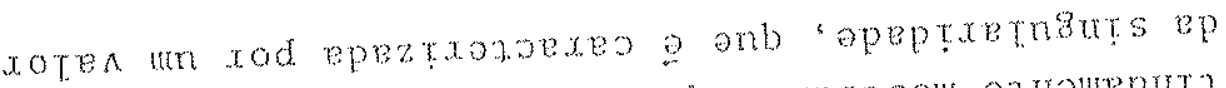

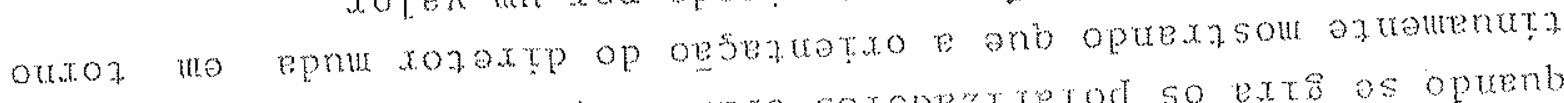

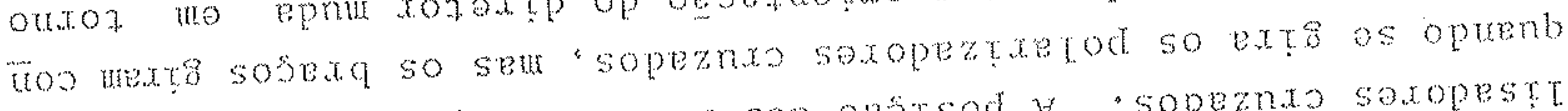

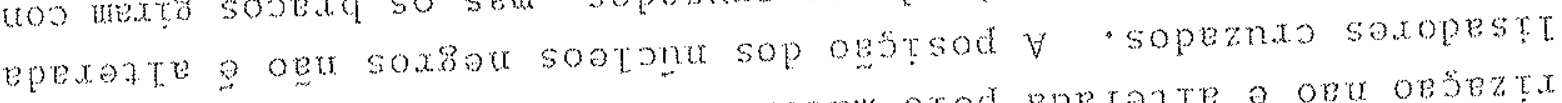

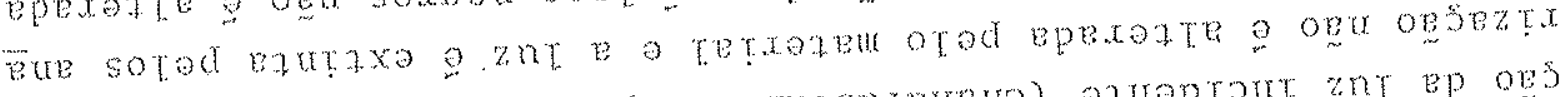

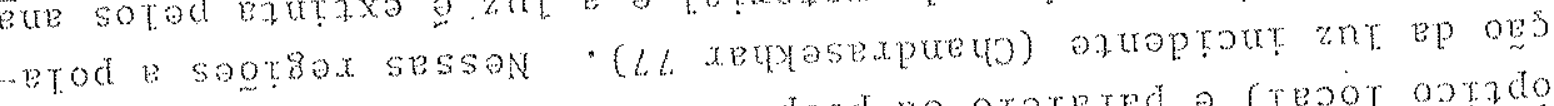

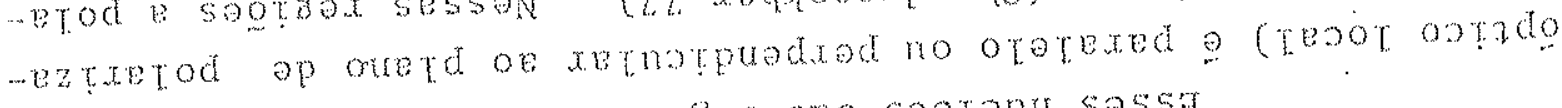

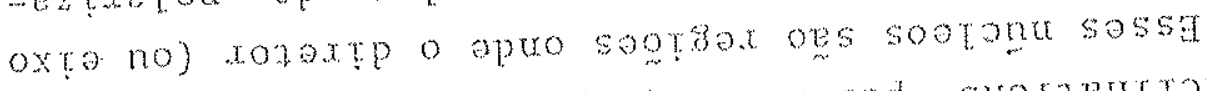

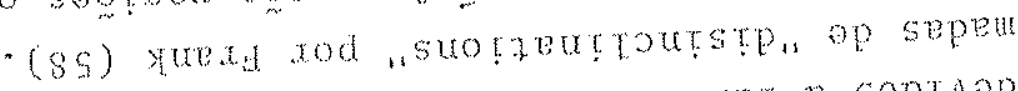

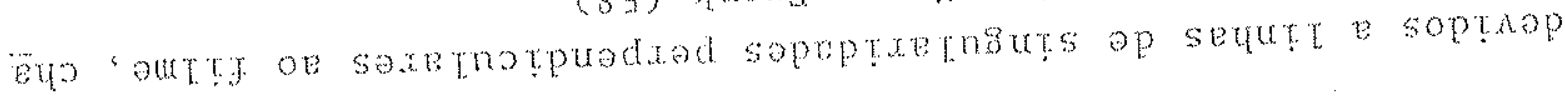




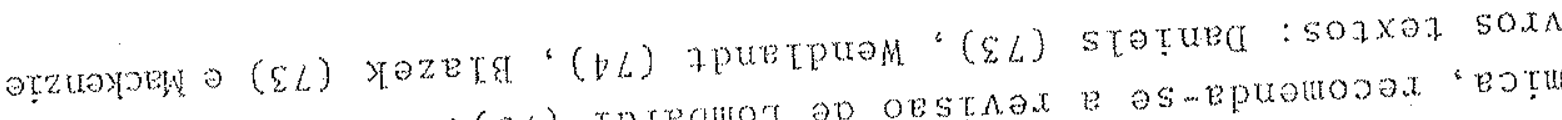

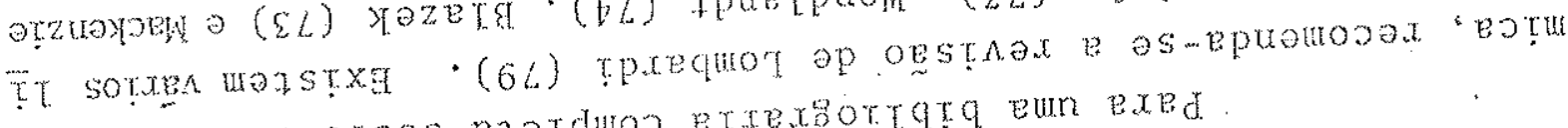

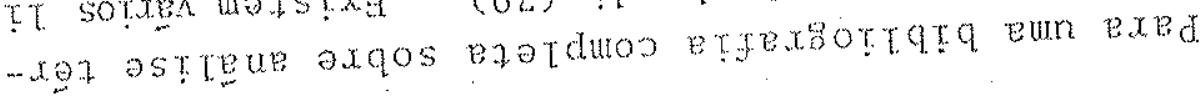

- soprdex exter

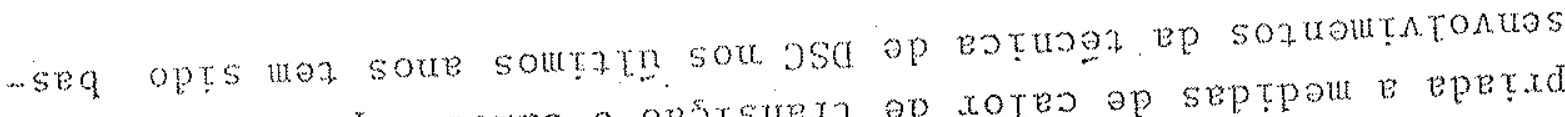

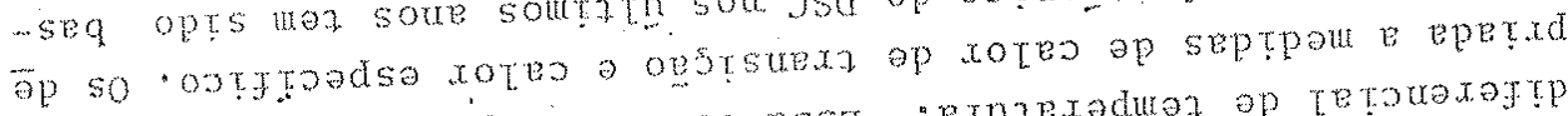

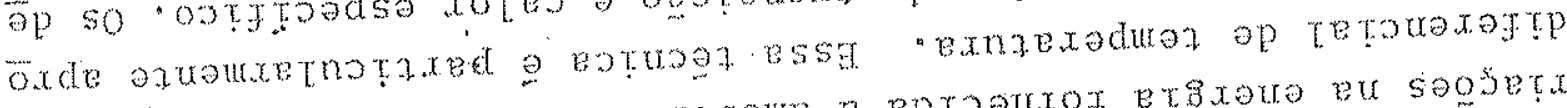

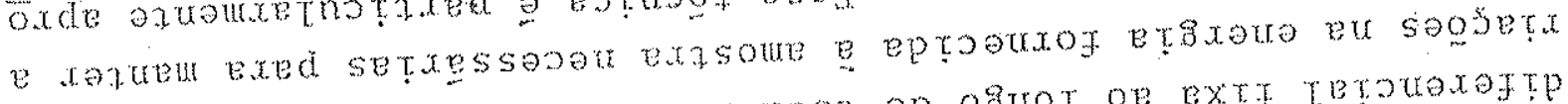

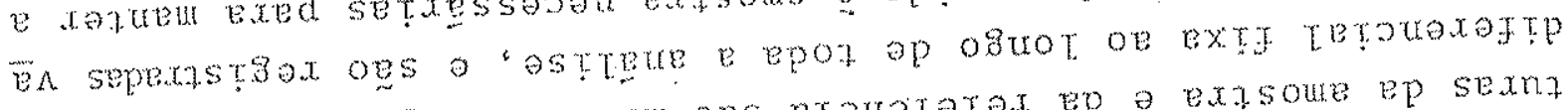

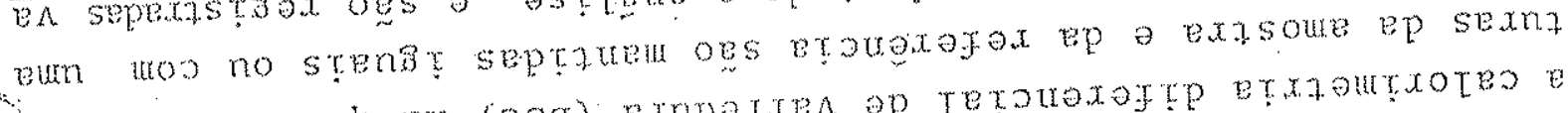

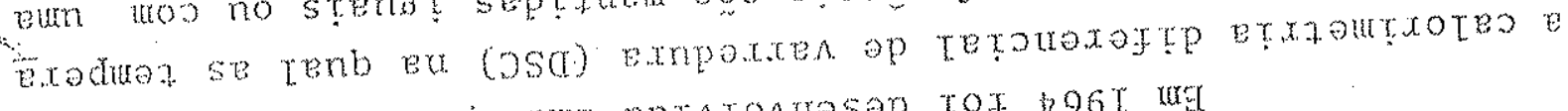

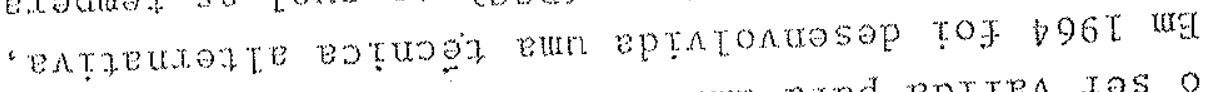

- exmaxodura epep eum exed epter xos os opsexqteo ep

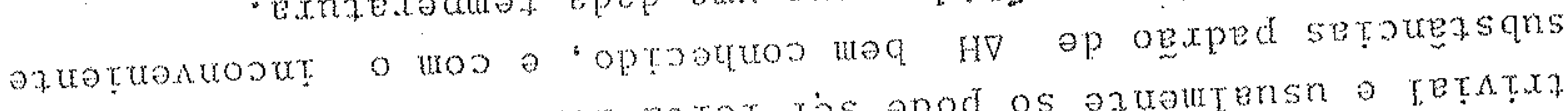

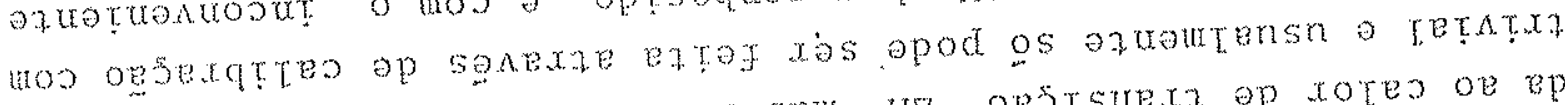

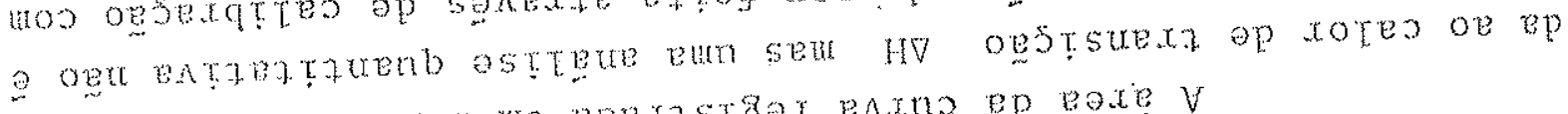

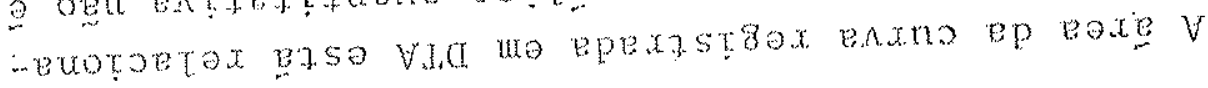

- tetx

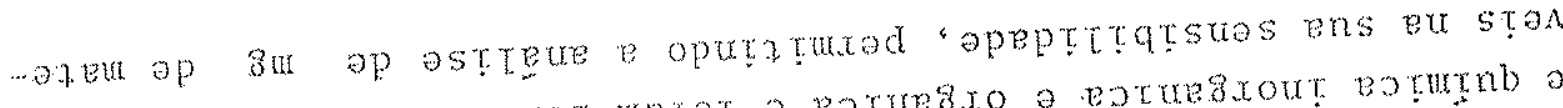

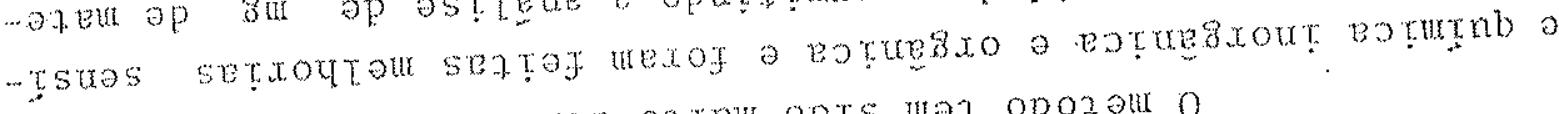

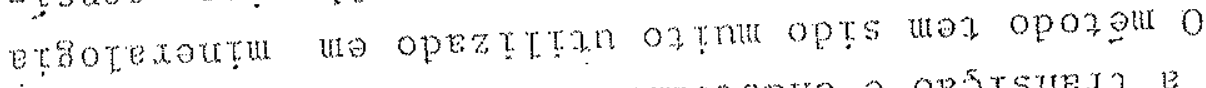

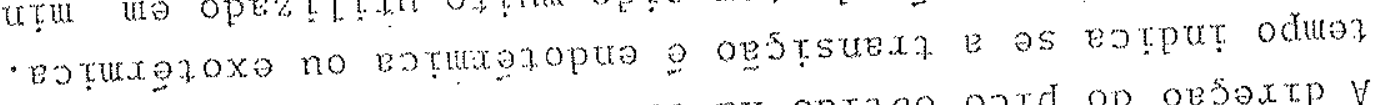

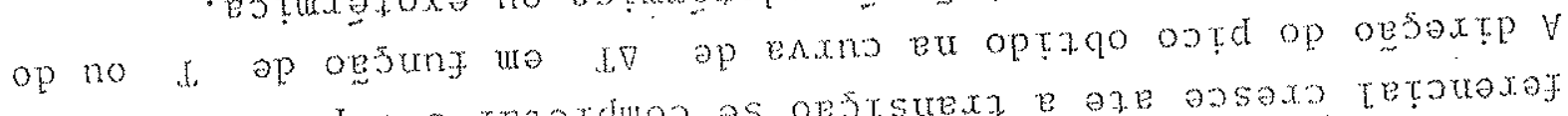

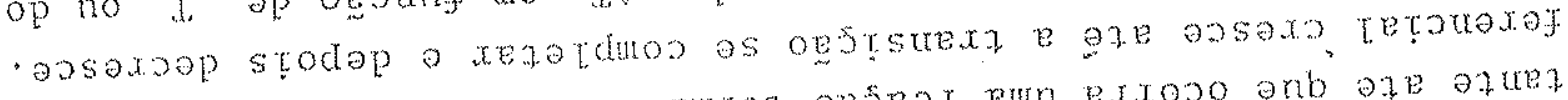

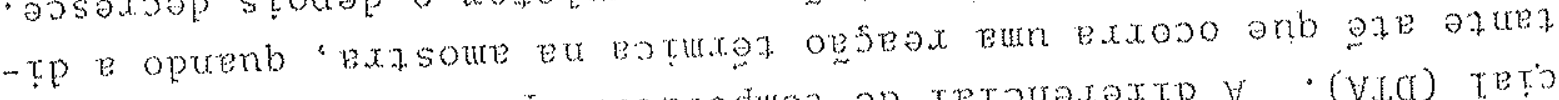

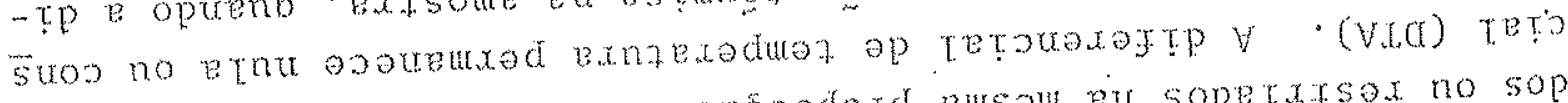

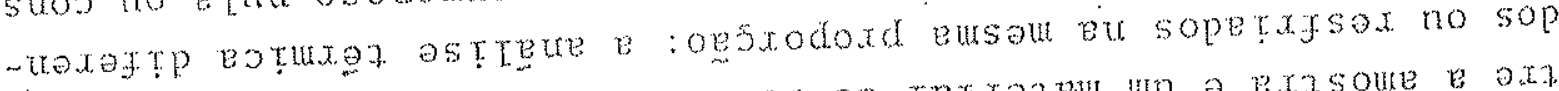

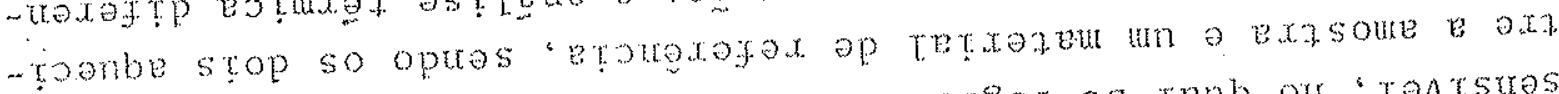

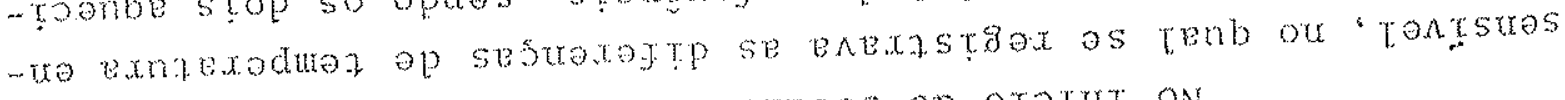
sterll opolon un optatonuesop tof otrogs op otoptr on

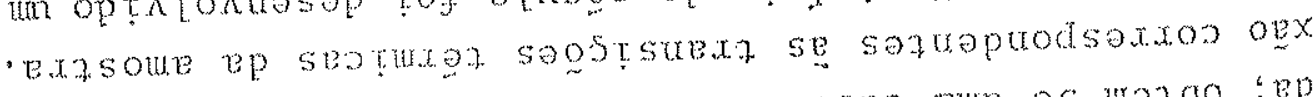

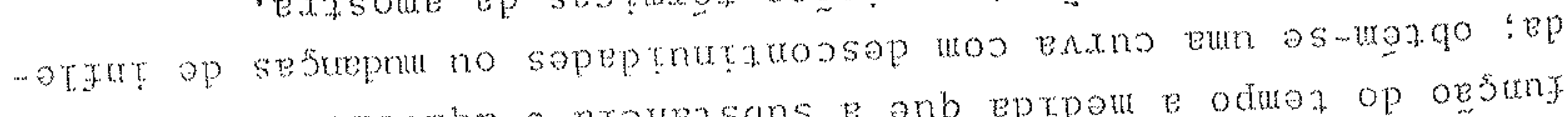

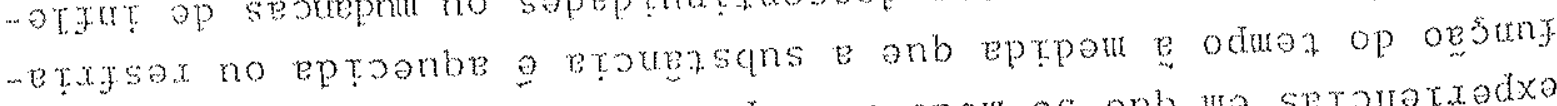

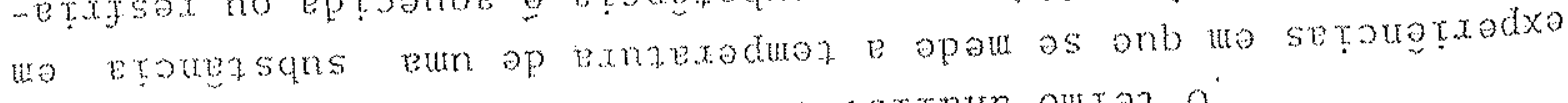

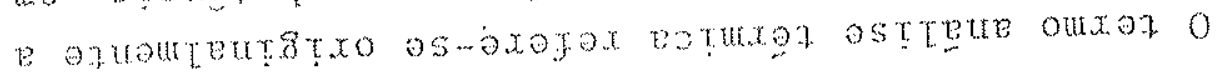

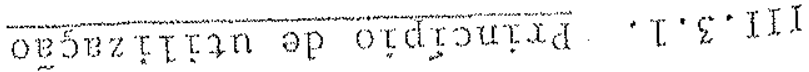




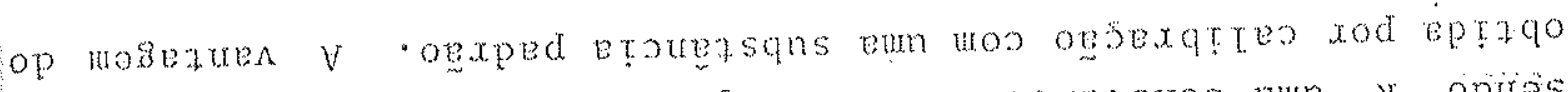

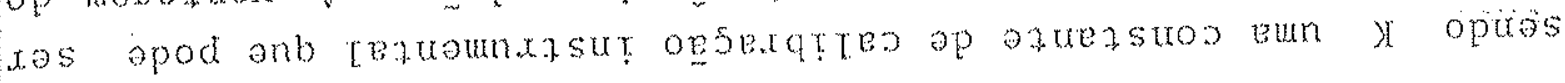

$$
\text { HV } u=V X
$$

op sontare oxd op $\mathrm{V}$ eoxe ep optrqo xas ap od u esseu op extsoure eum op hy axtreat xotes o

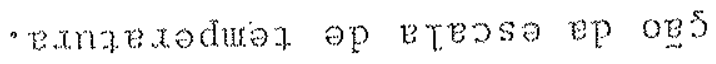

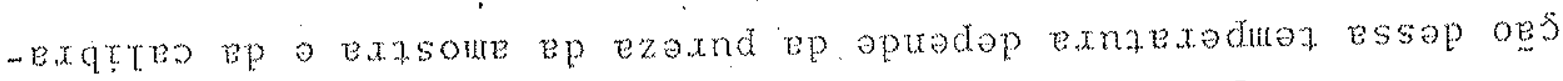

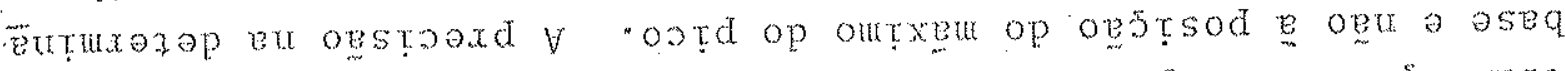

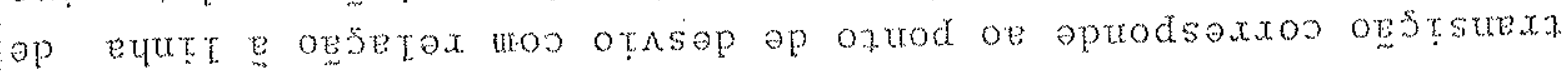

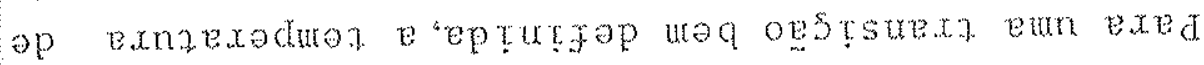

$$
\text { - oduez op opsunx ure ozTaj } 9 \text { oxa }
$$

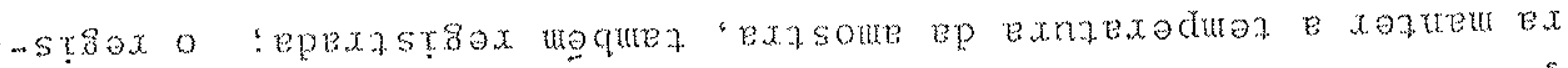

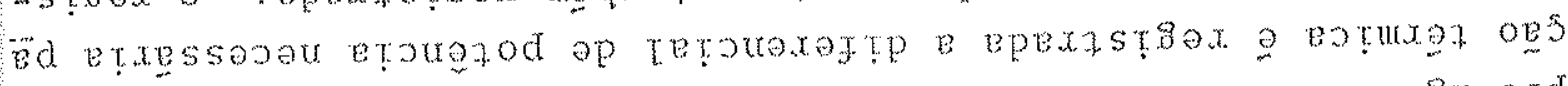

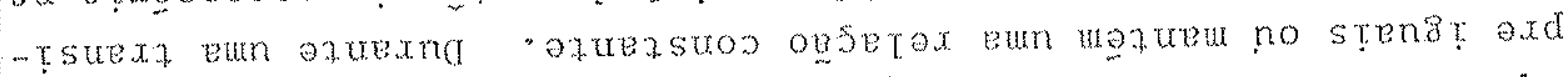

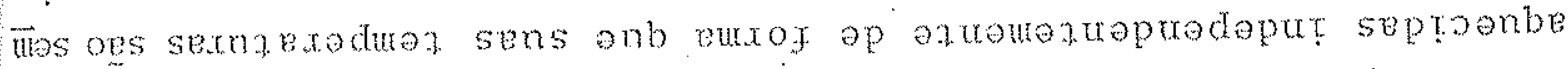

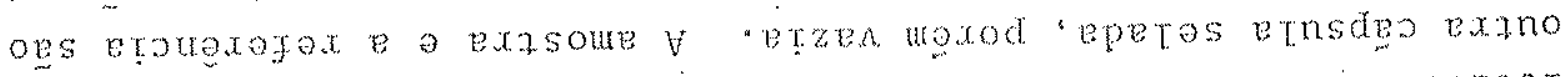

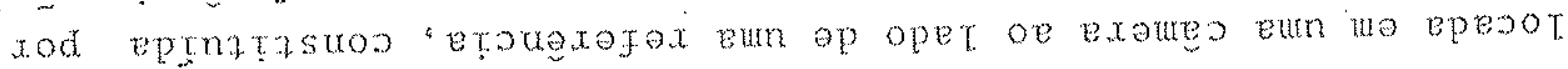

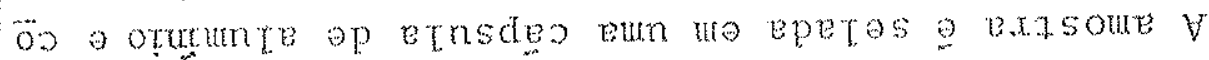

$$
\text { - optrote orwegoxitu op otrogea }
$$

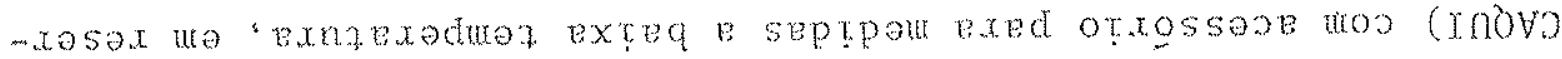

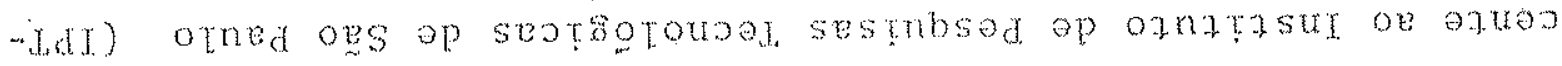

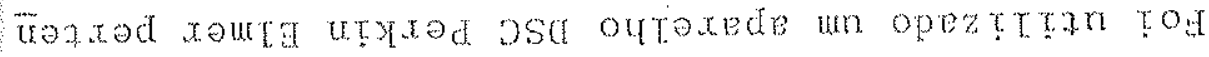

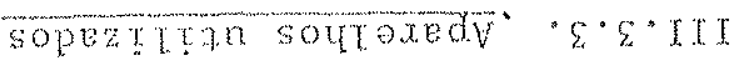

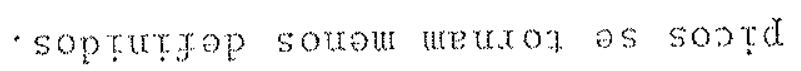

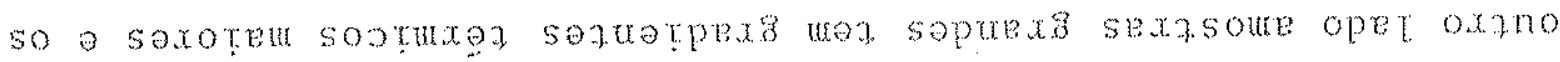

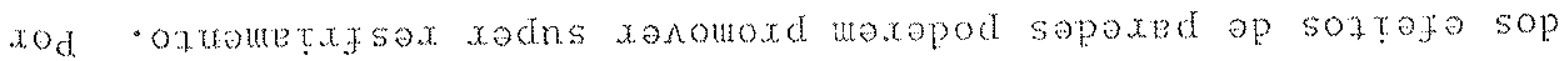

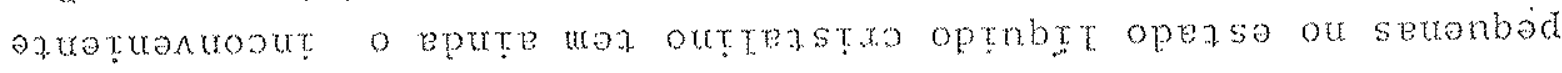

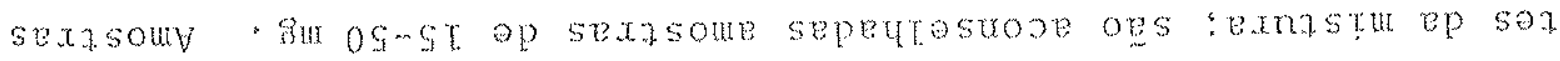

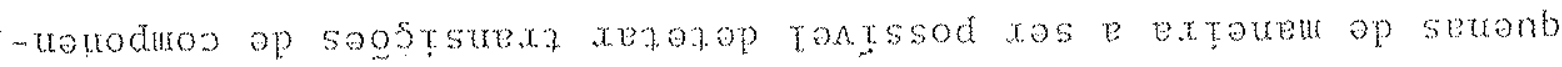

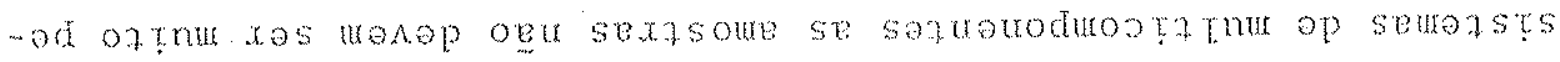
exed anb (69 weudeyp ayooxqpen) opertassax tod

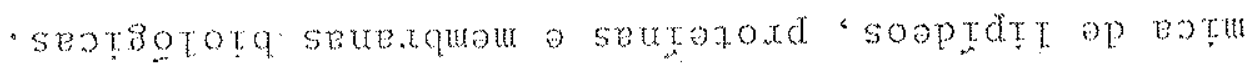

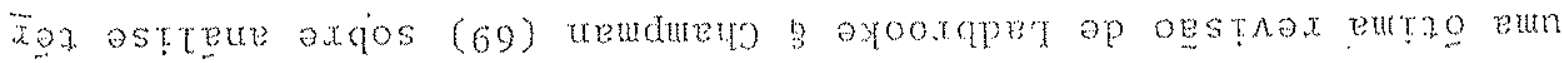

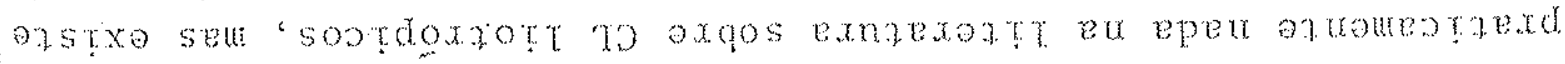

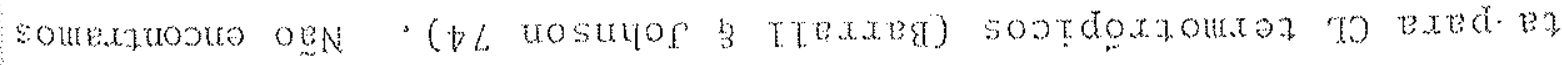

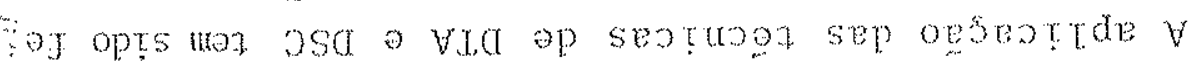




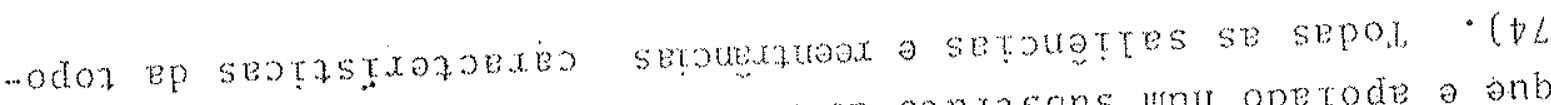

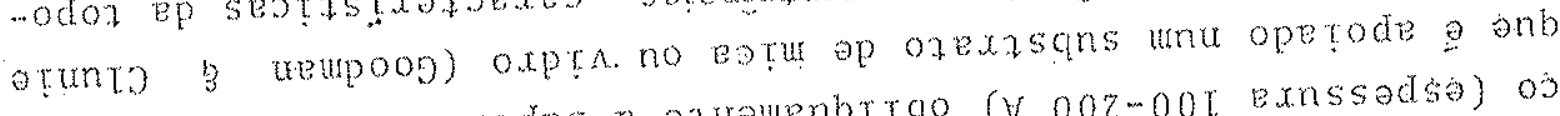

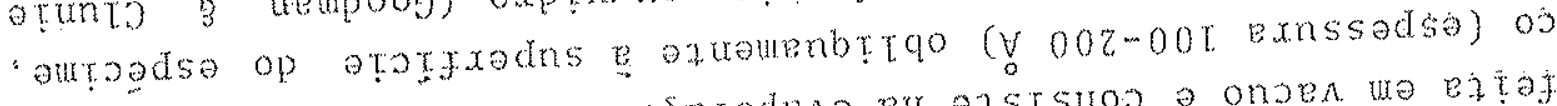

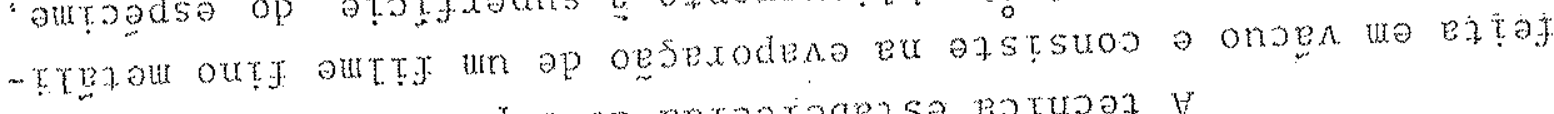

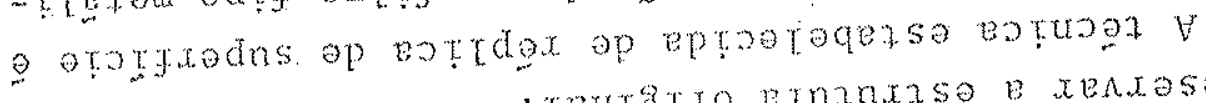

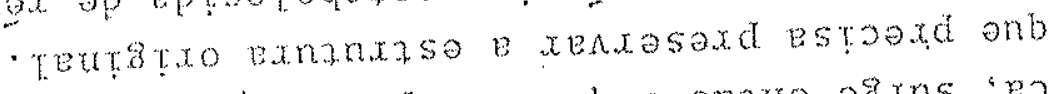

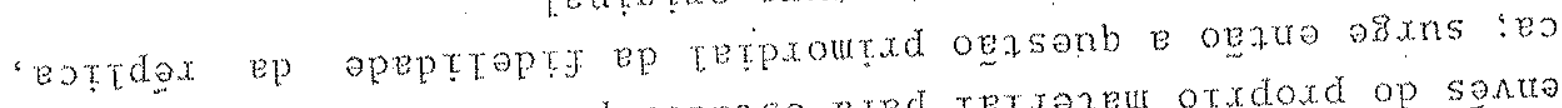
-troxato erdossoxotu xod sopnsa exed tefrazeur otxdoxd op sante

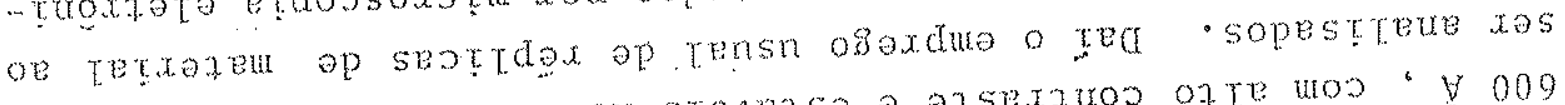

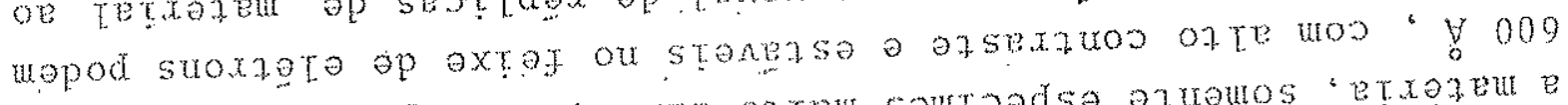

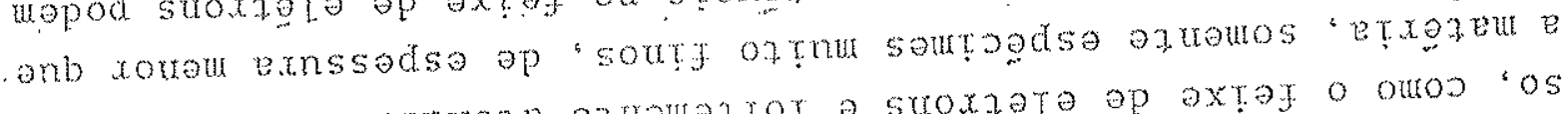

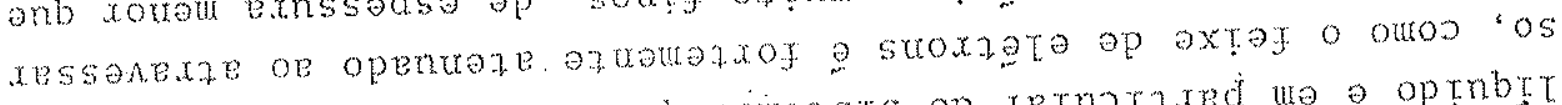

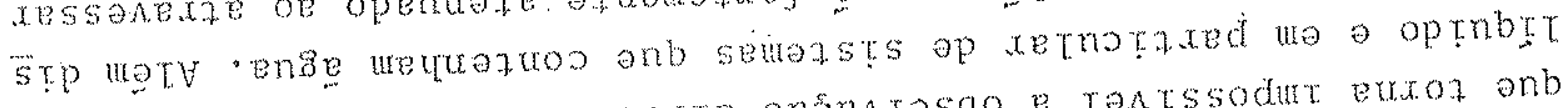
opezse ox suxasome ap baxtp ofsenxesqo v ranssodut errot onb o conoer oje me exodo oogroxigte ogdopsoxotu o

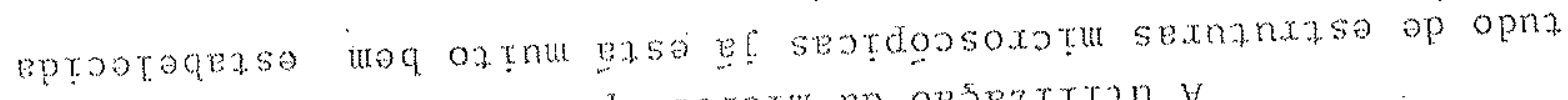

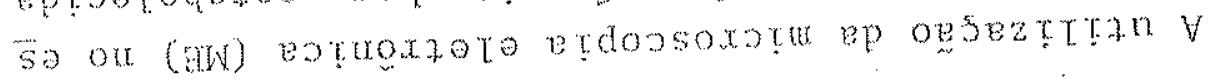

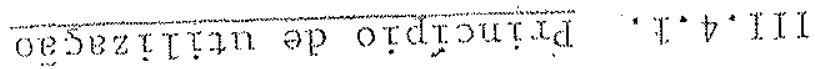

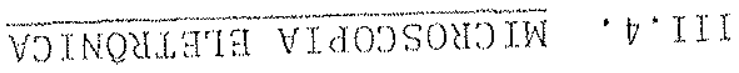

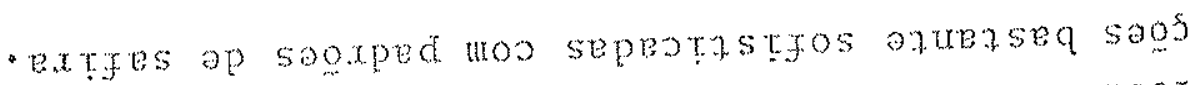

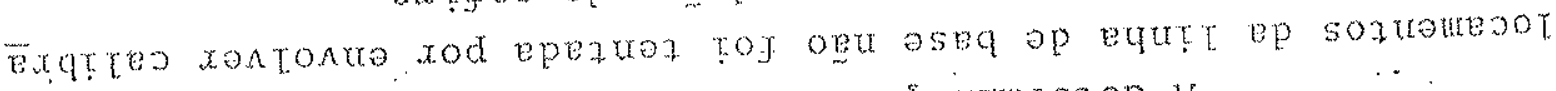

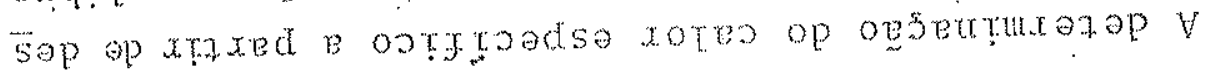

- seprrrosso

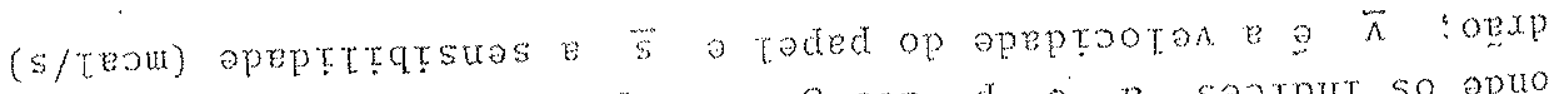

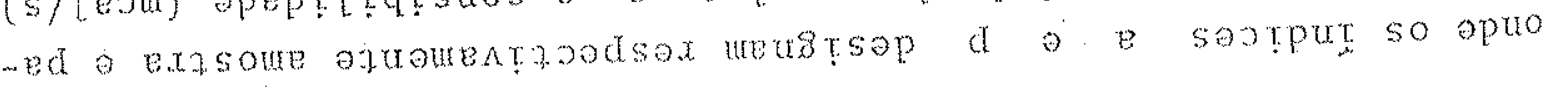

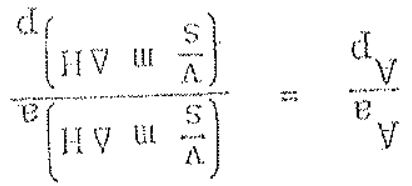

:obstar e esmopresn tepetrasop enge ap ext

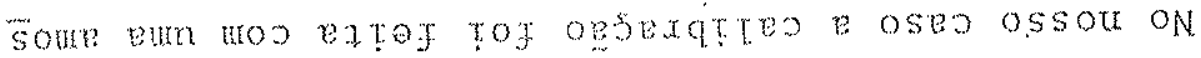

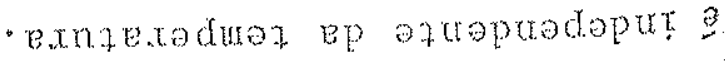

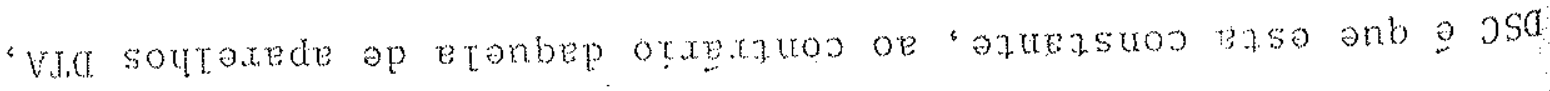




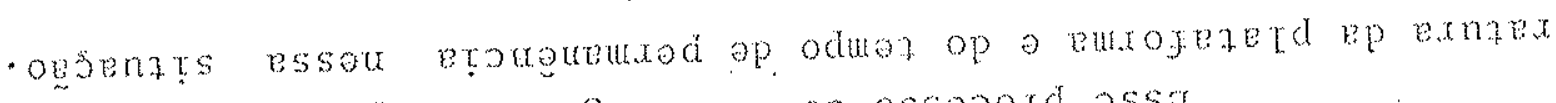
- adnor ep xapuadop ten "Butgozan op ossasond assa

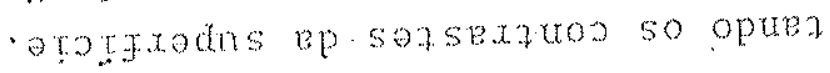

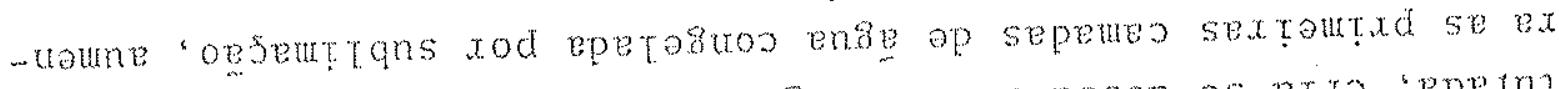

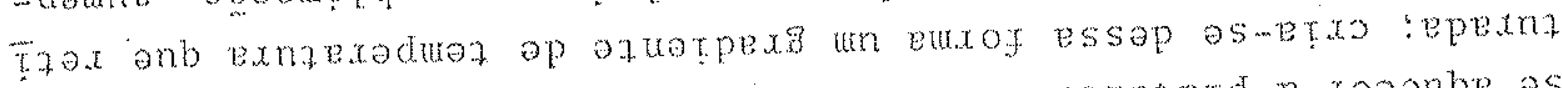

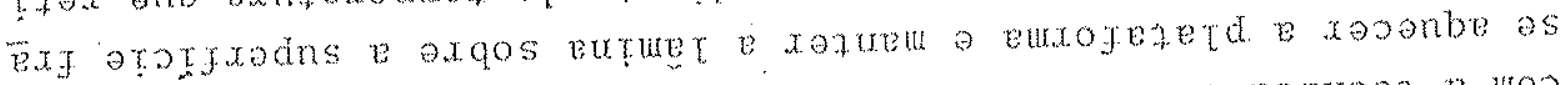

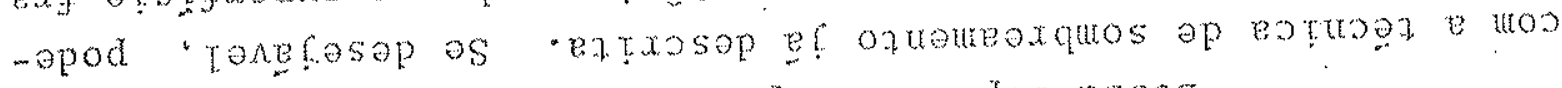

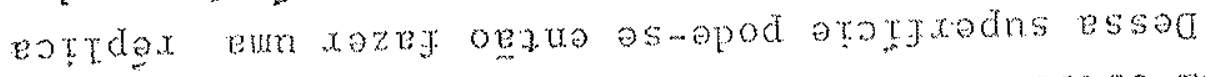

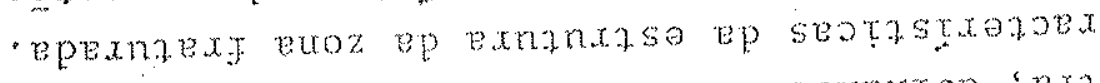

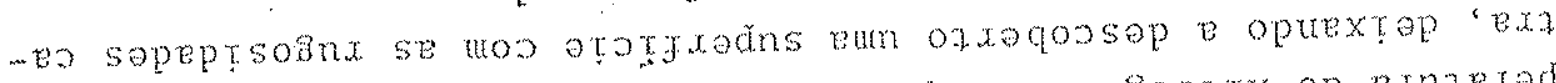

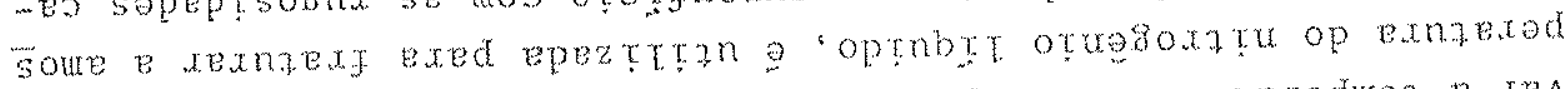

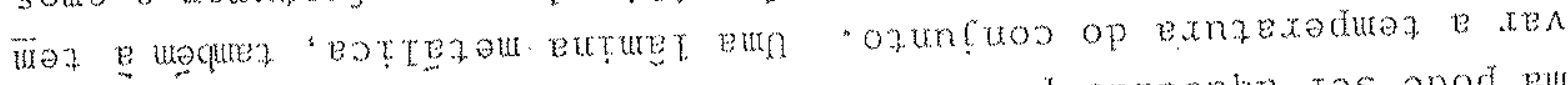

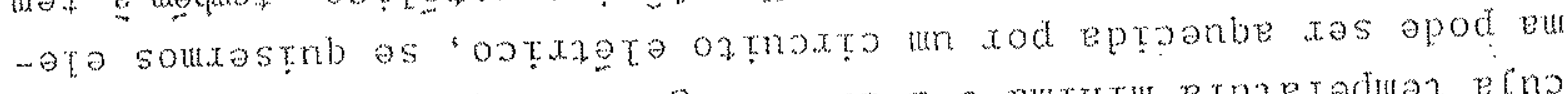

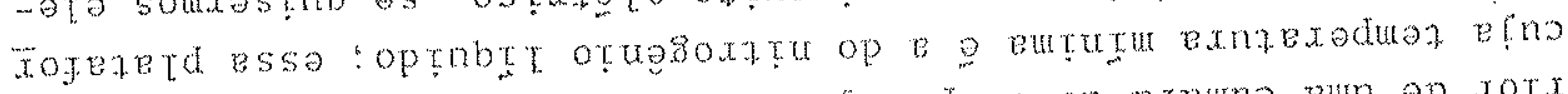

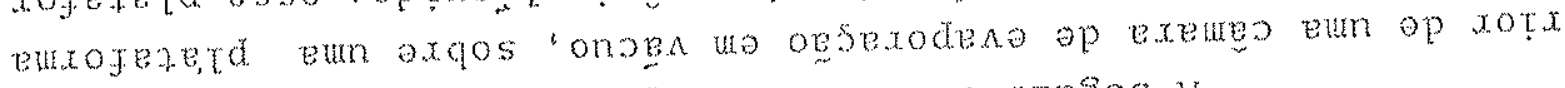

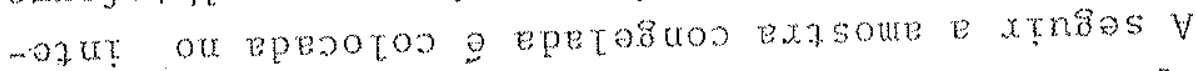

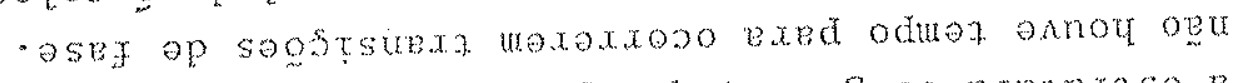

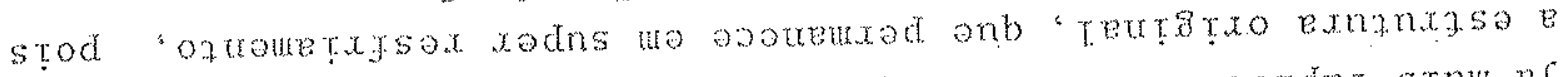

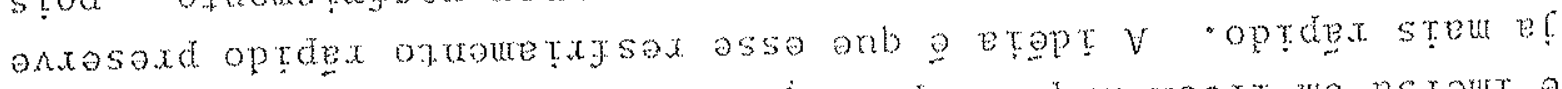

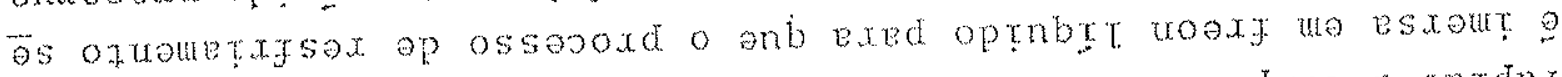

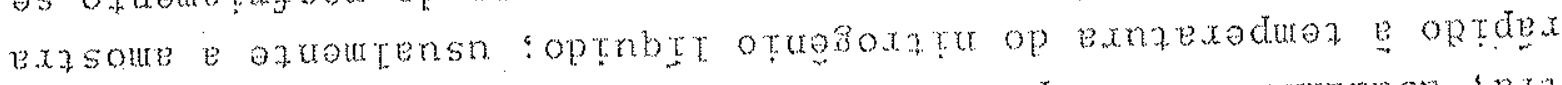

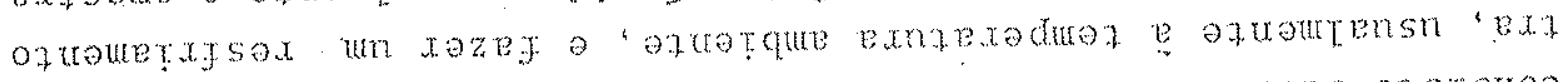

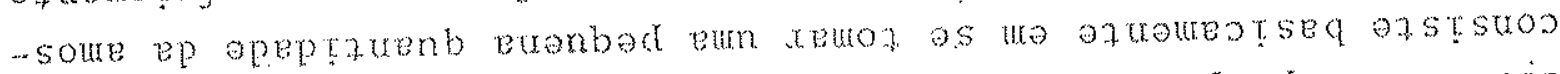

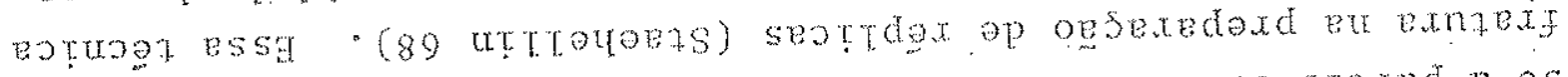

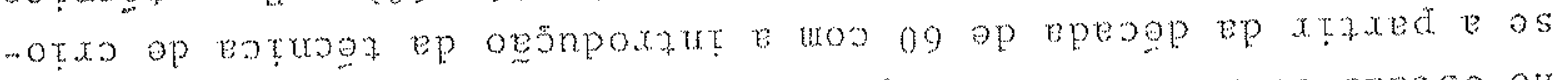

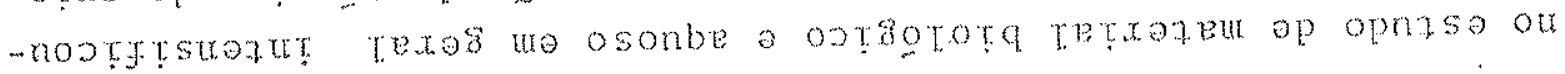

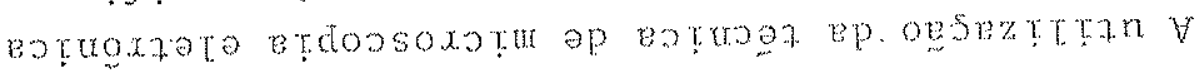

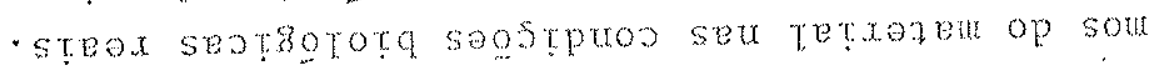

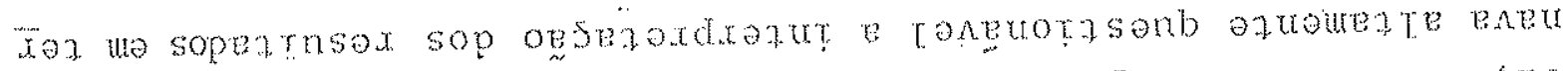

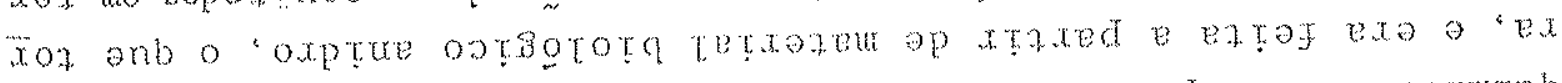

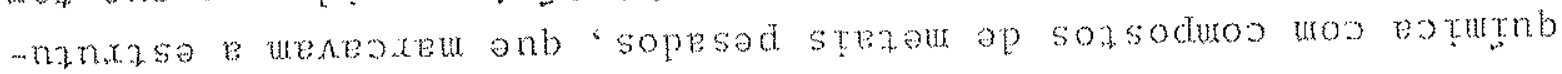

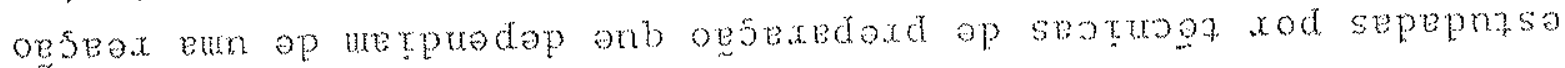

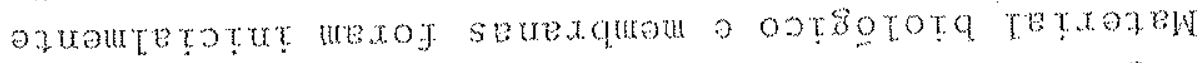

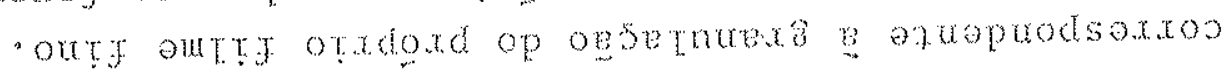

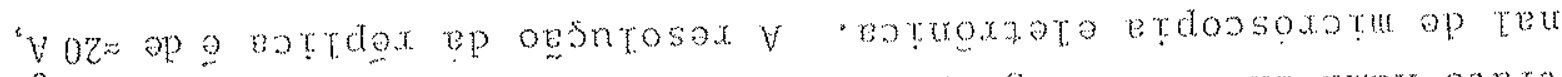

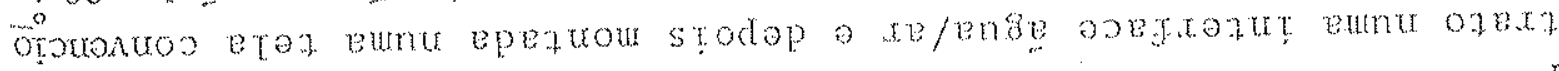

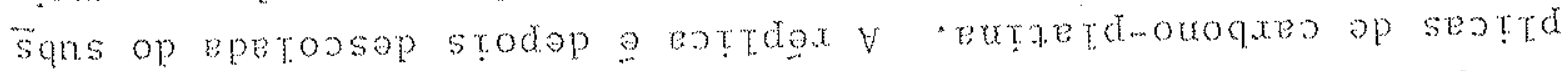

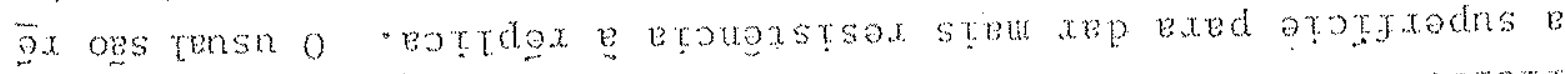

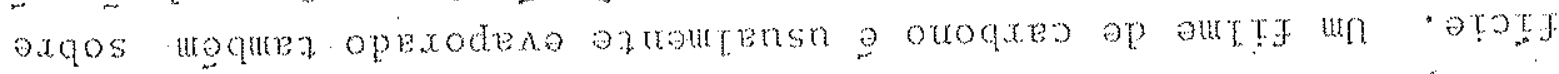

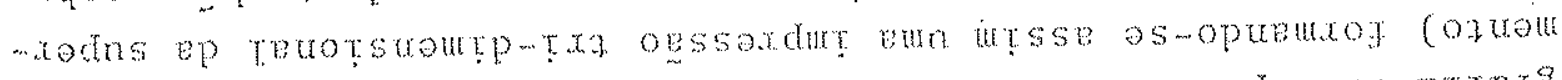

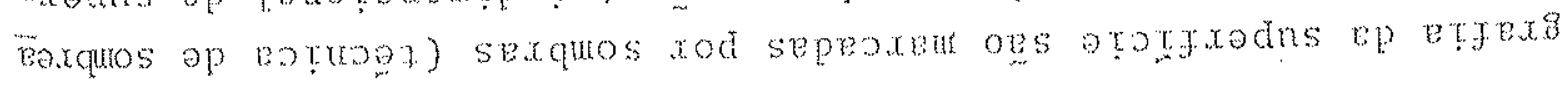




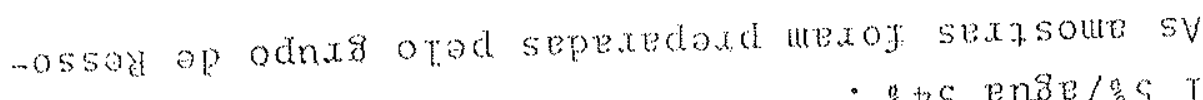

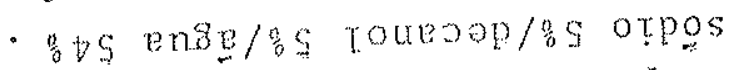

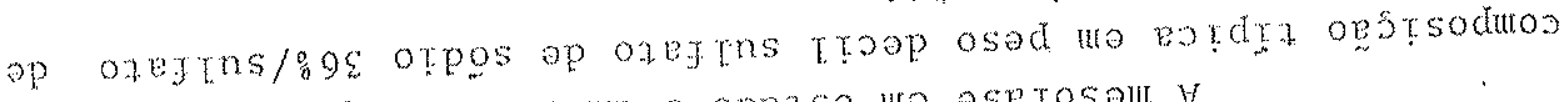

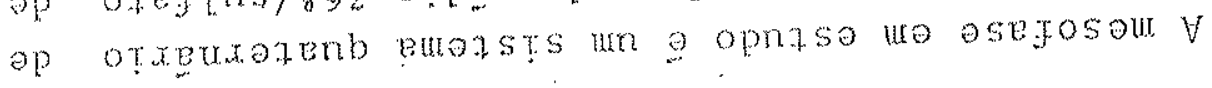

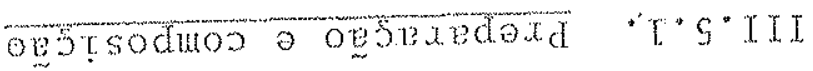

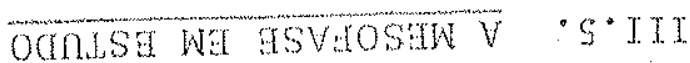

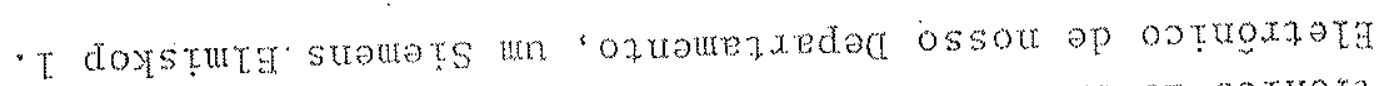

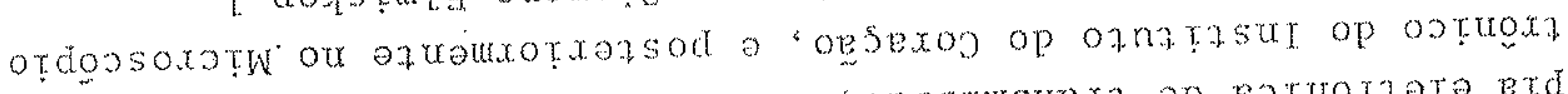

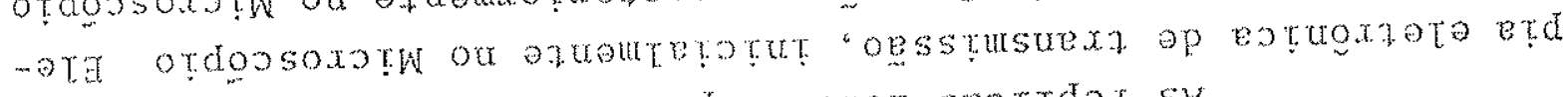
-ossoxtu xod sepestretre stodop uexog sertdex sy.

- otrozexoger op

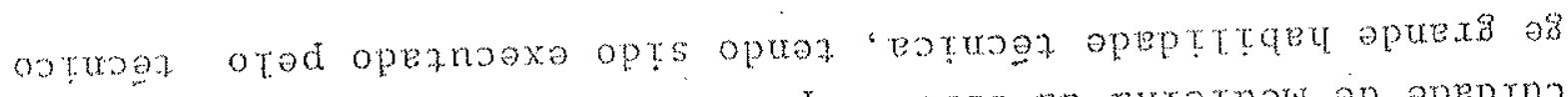

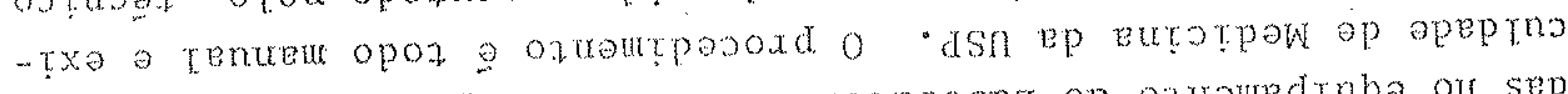

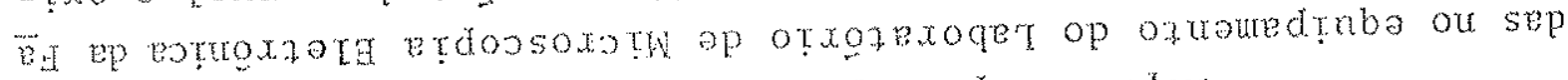

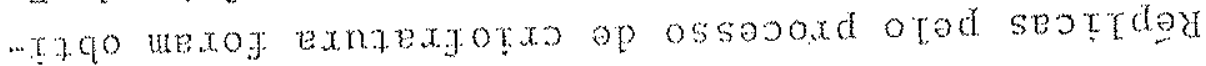

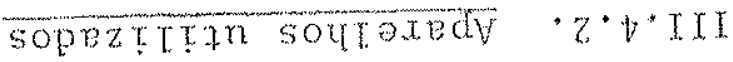

- ossasoxd op sedrze sep eungte we soleraze op ogonpora ap opep

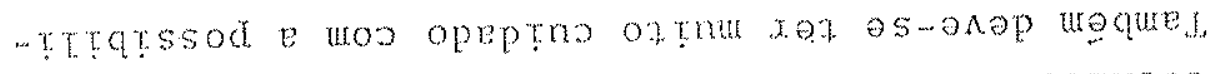

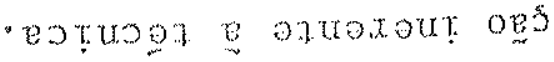

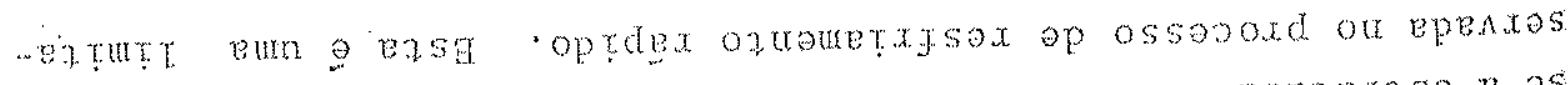

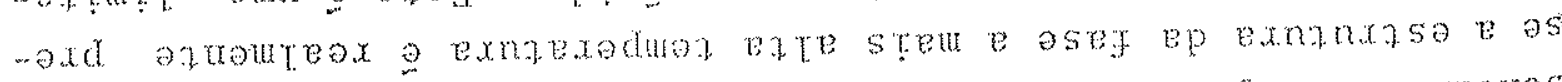

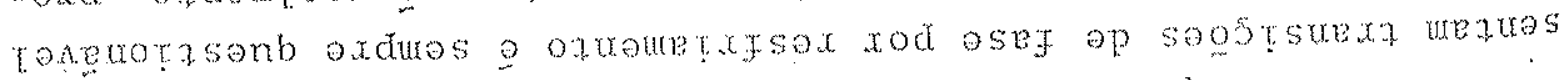
-oxde onb seurasts uos opueqrequa soureaso oprenó

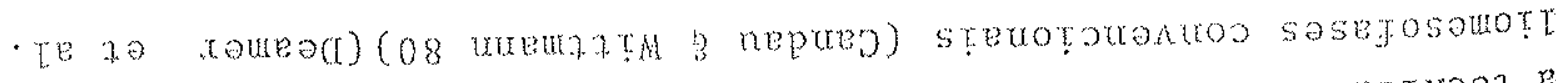

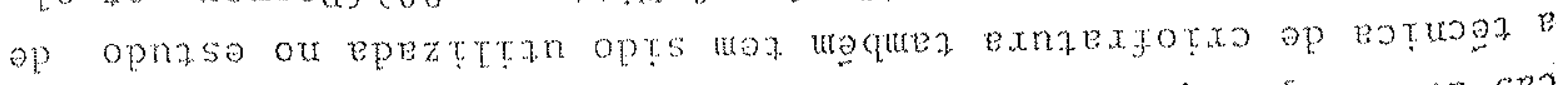

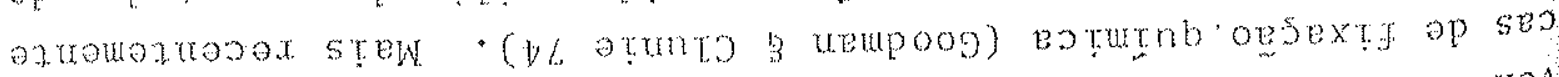

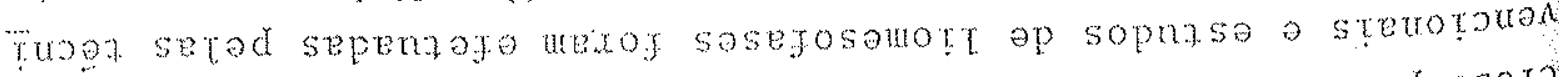

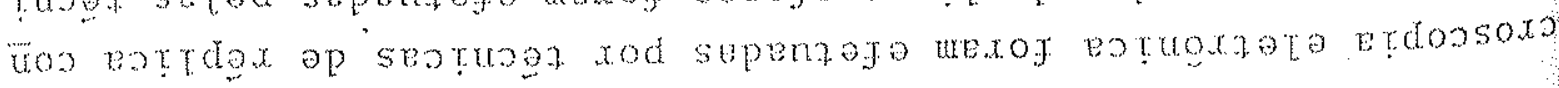

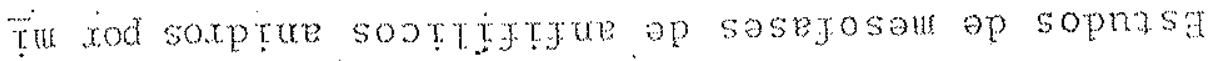

- votrdex e es-wergo stodap a somutu

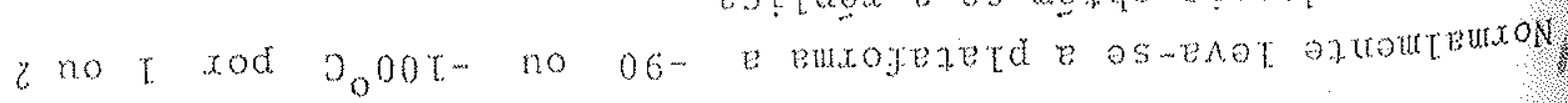


- (wot extros o rezuos was)

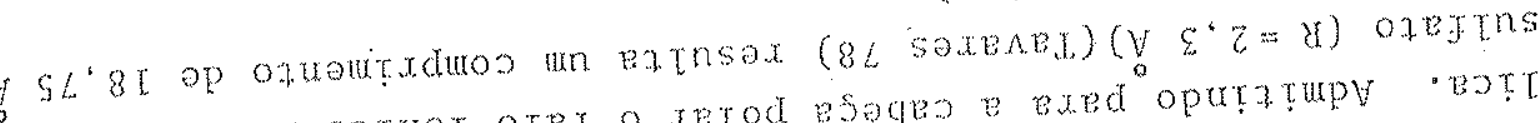

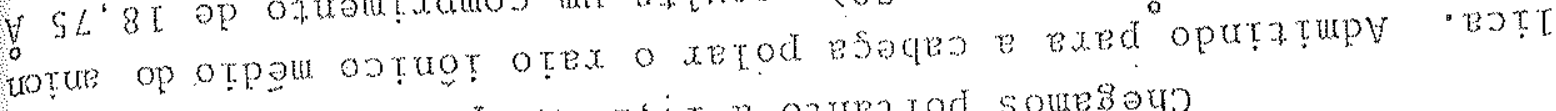

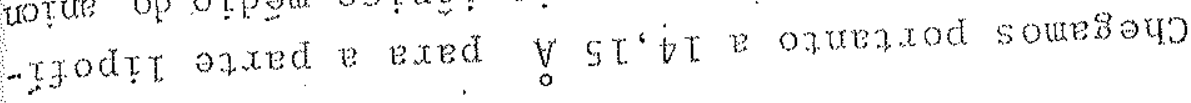

$$
x \operatorname{sgz} t+s^{*} t=x \operatorname{tg} z
$$

: (z, proxuej) xod epet

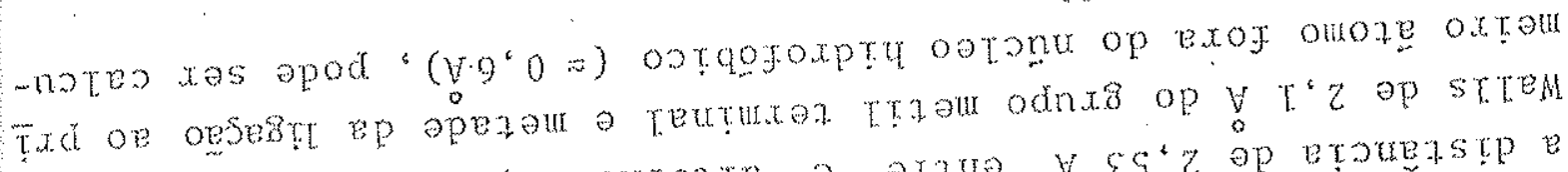

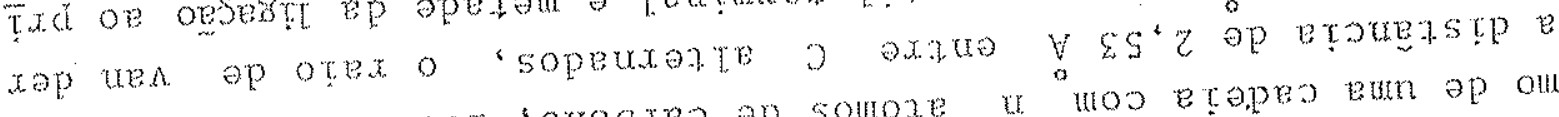

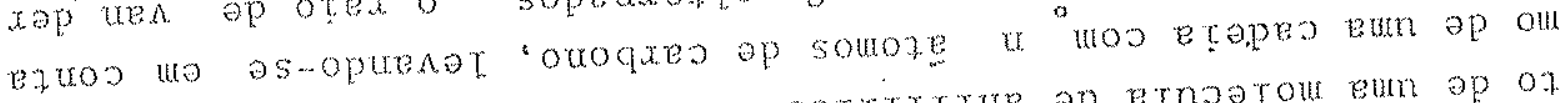

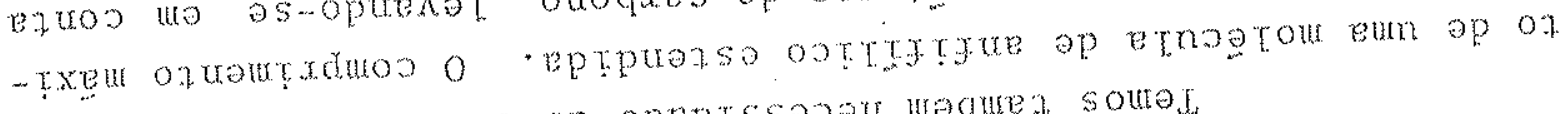

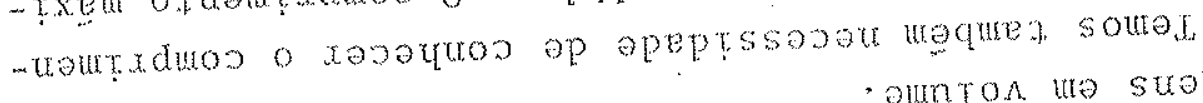

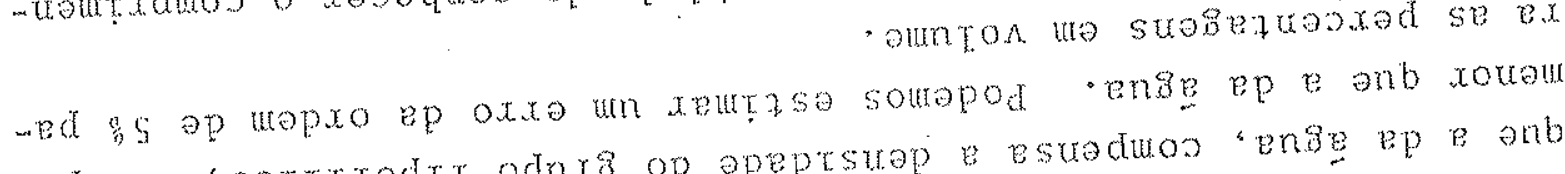

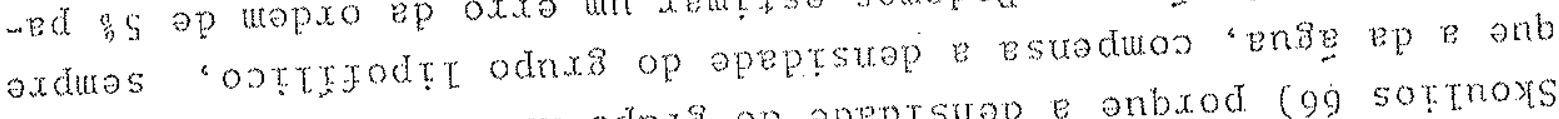

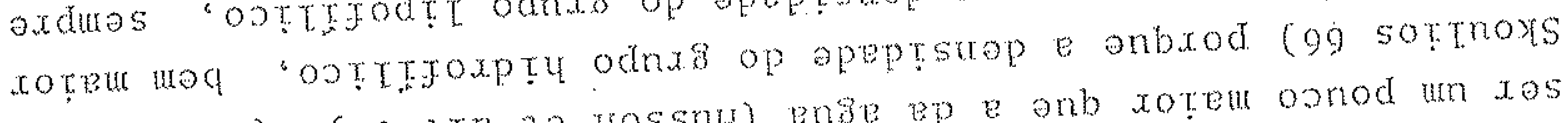

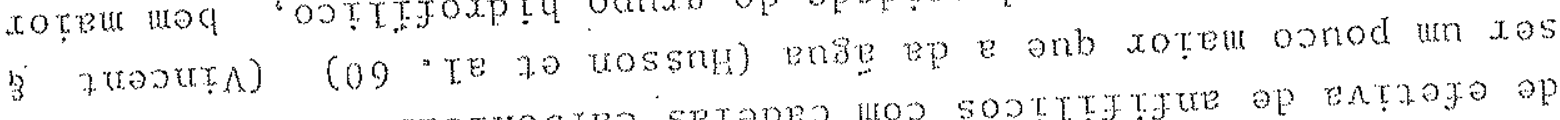

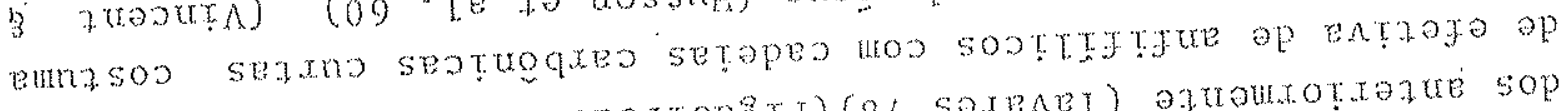

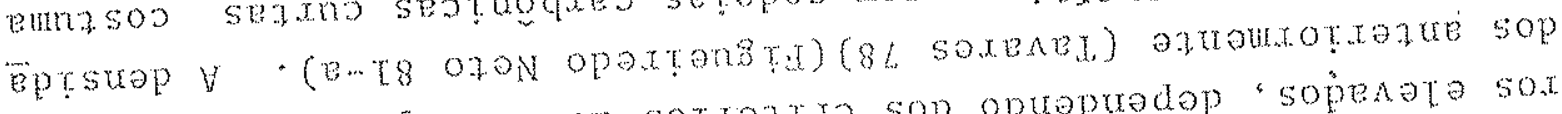

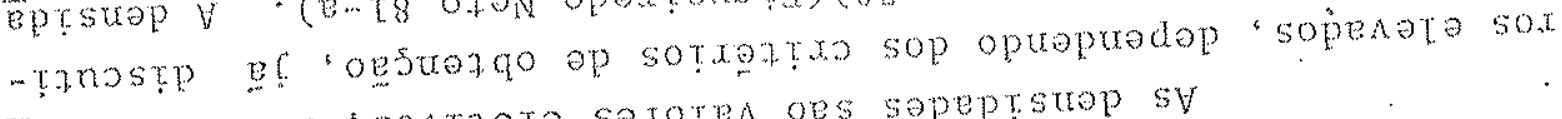
xo op soperage "sontrojo sototen oes sepeptstop sy

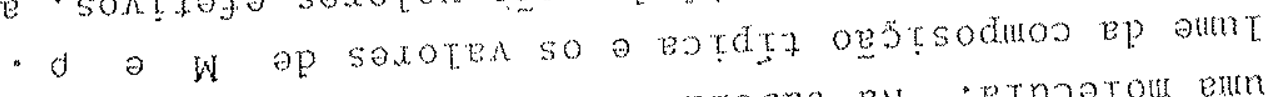

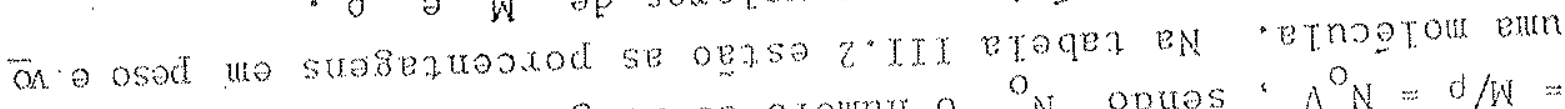

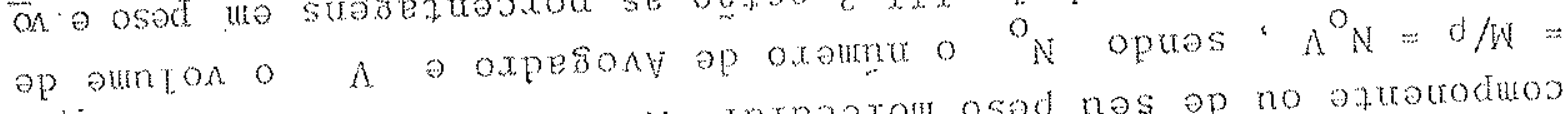

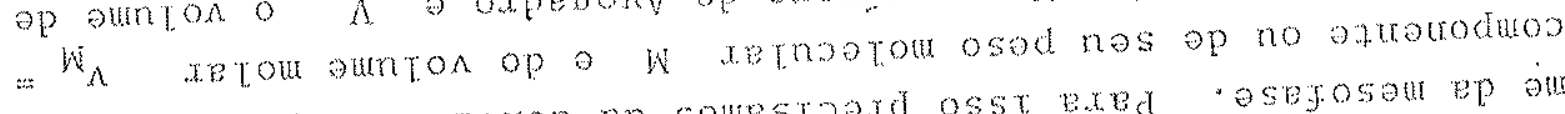

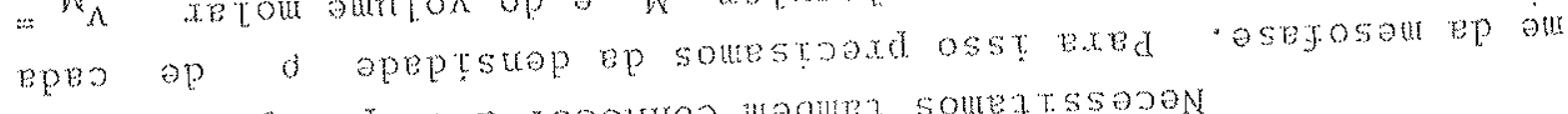

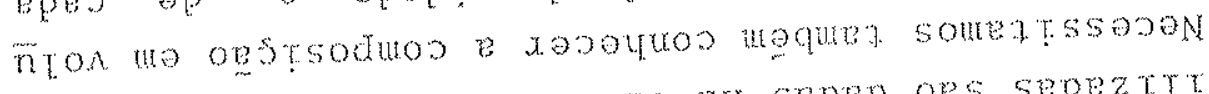

- T'Tt eroqea a sepep oes sepeztrizn sexasour sep

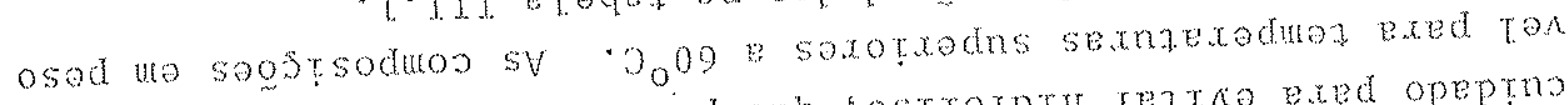

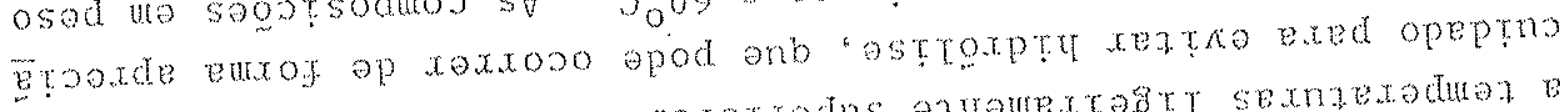

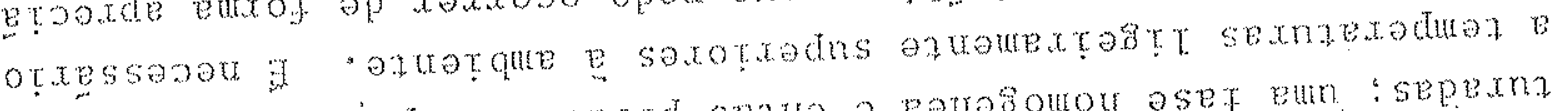

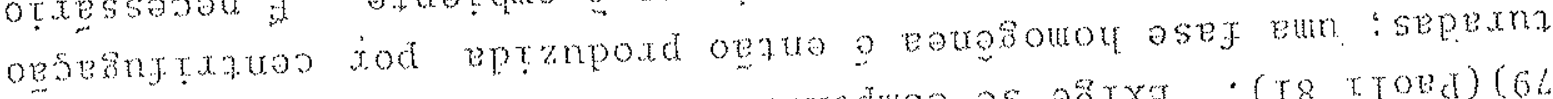

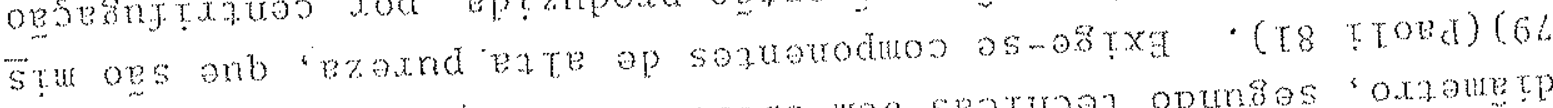

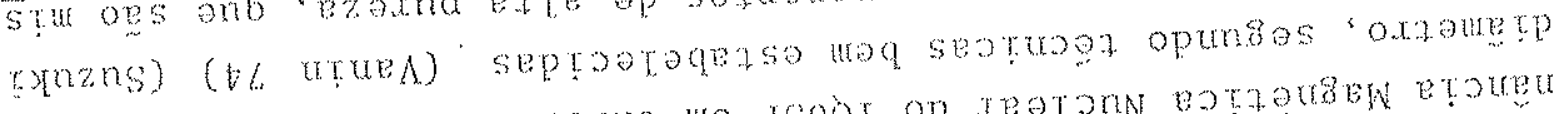

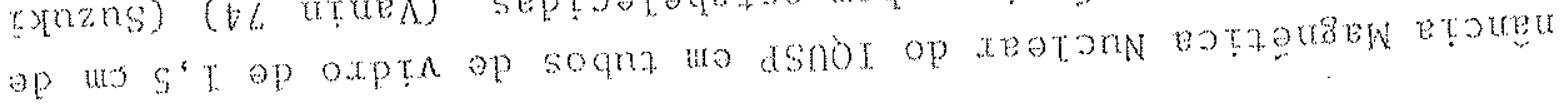




\begin{tabular}{|c|c|c|c|c|}
\hline 85 & $\forall s^{\prime}$ & $00^{\circ} \mathrm{T}$ & $00^{\circ} 8 \pi$ & $\mathrm{O}^{2} \mathrm{H}$ \\
\hline 9 & 9 & $28 \times 0$ & $6 z^{\prime} 89 \mathrm{~T}$ &.$\quad \mathrm{HO}^{6}\left(\mathrm{z}_{\mathrm{HO}}\right)^{\varepsilon} \mathrm{H}_{\mathrm{H}}$ \\
\hline$s$ & 乌 & $\operatorname{tec}^{6} \mathrm{~T}$ & bo' & $\cos ^{2} \mathrm{eN}$ \\
\hline TE & 98 & $S Z^{\prime} I$ & $0 v^{\circ} 09 z$ & $\left.\mathrm{PN}^{\varepsilon} \mathrm{OSO} \mathrm{C}^{6}\left(\mathrm{C}^{2} \mathrm{H}\right)^{\varepsilon} \mathrm{H}_{2}\right)$ \\
\hline gurtos: & $050 d y$ & d & $W$ & . \\
\hline
\end{tabular}

potatr oestsoduros ep eunton a osed ue

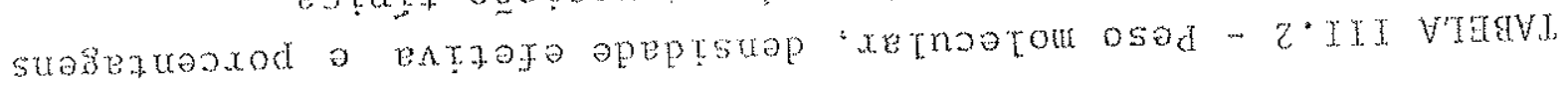

\begin{tabular}{|c|c|c|c|}
\hline $98 \mathrm{sg}$ & $6 T^{6}$ ys & $06^{\circ} 25$ & $\operatorname{eng} g$ \\
\hline $09^{\prime} \mathrm{s}$ & $18^{\circ} \mathrm{V}$ & $08 \div 5$ & rouesop \\
\hline $90^{\circ} \mathrm{S}$ & $86^{\circ} \mathrm{y}$ & $50^{\circ} \mathrm{g}$ & oN op ogejtrs \\
\hline $09^{\circ} \mathrm{S} \mathrm{C}$ & $20^{\circ} 98$ & $5 z^{\circ} 9 \%$ & $\begin{array}{r}\text { en op } \\
.07 e t \operatorname{tas} \text { Ttoop }\end{array}$ \\
\hline It exasoury & II 8xasony & t Bassour & . \\
\hline
\end{tabular}

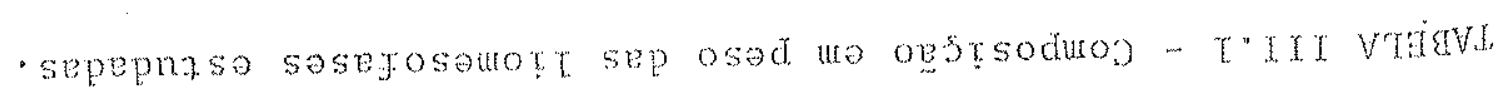




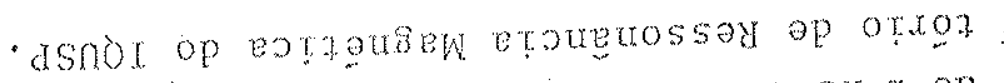

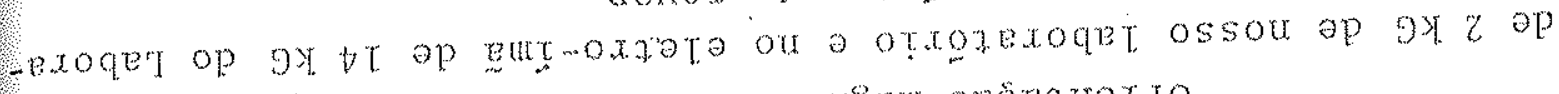

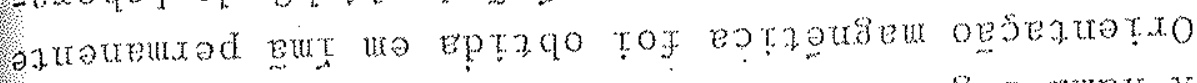

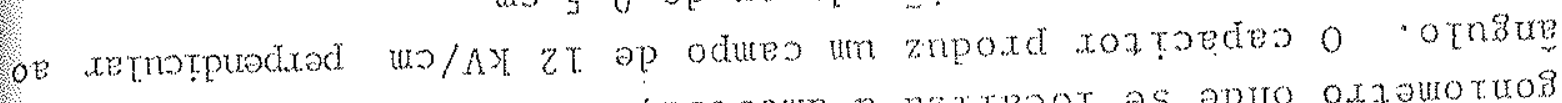
- uo $s^{2} 0$ op re ap ovtsax eume $x$ sotex op oxtex

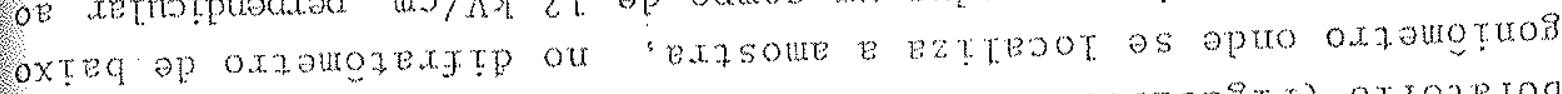

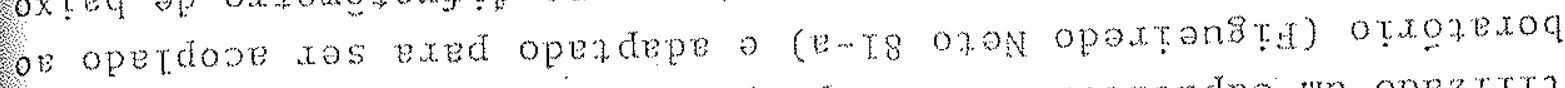
at ossou wo oprnxasuos o opeatoxd operost xortoedes un opeztt? un roj soptrate sodues ap oztaye op sopnase exed

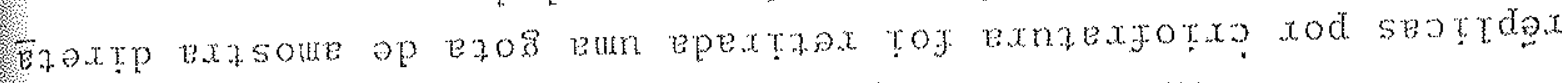

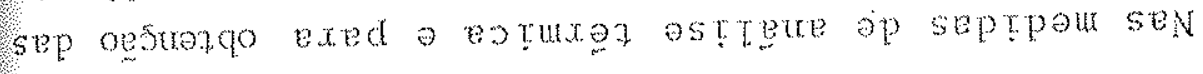

- sapeptura

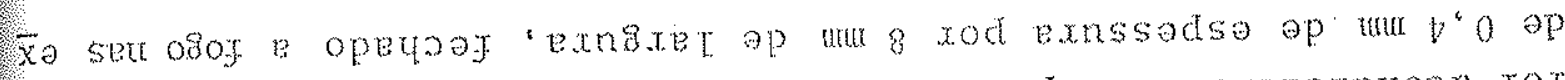

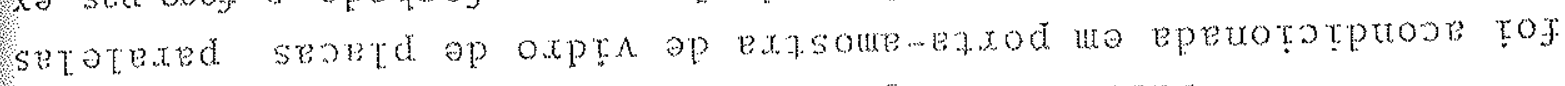

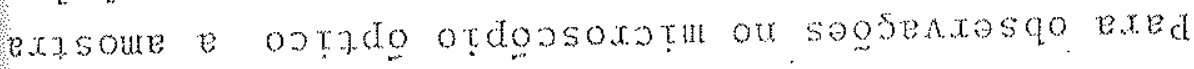

- optrater

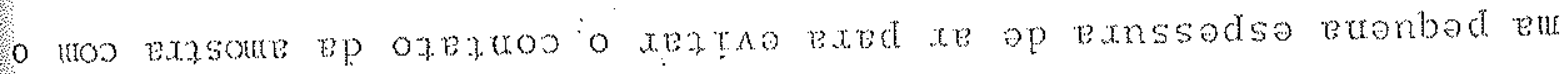

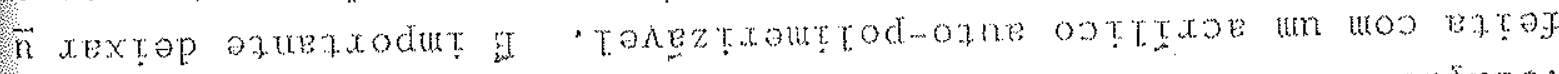

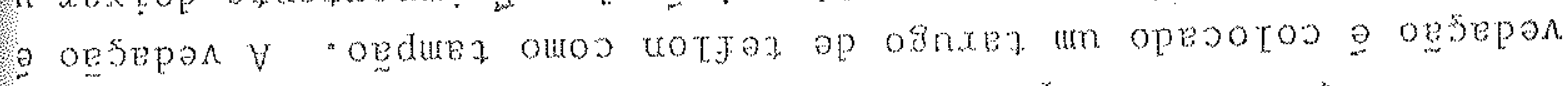
y eqtaj xas op soztre sossoxo stru soxptrdeo son

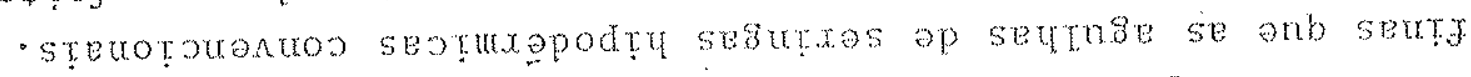

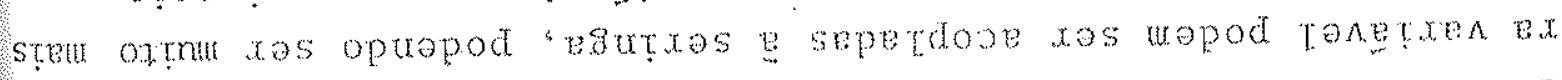

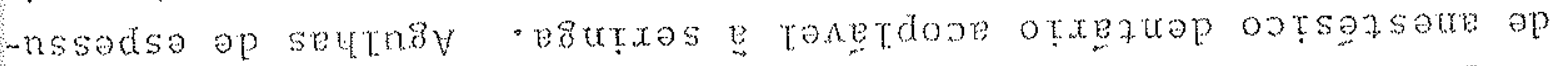

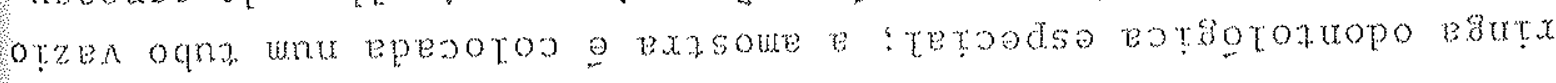

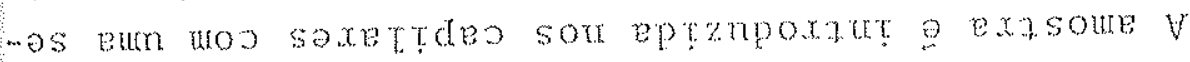

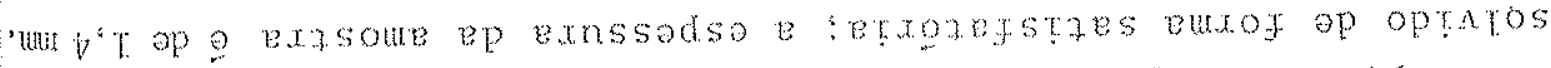

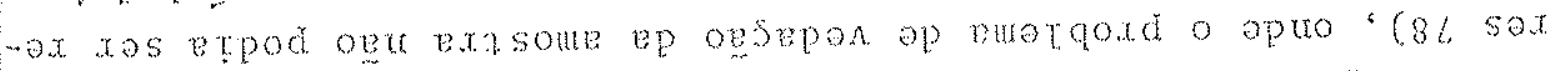

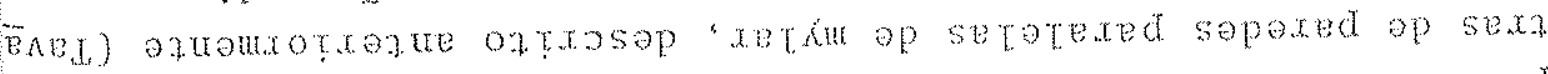

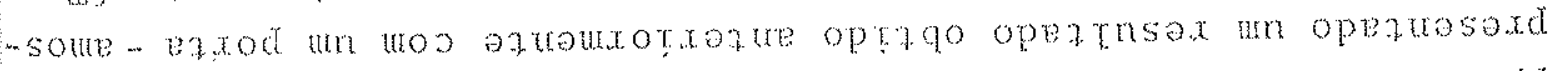

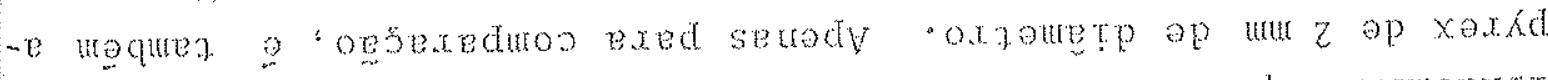

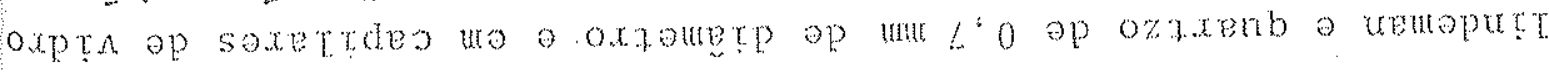

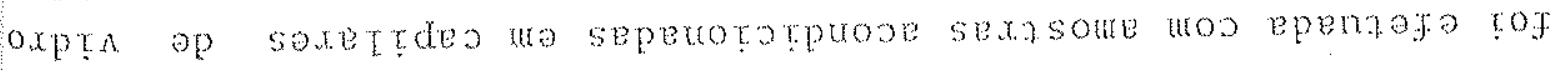

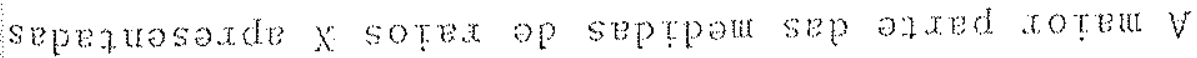




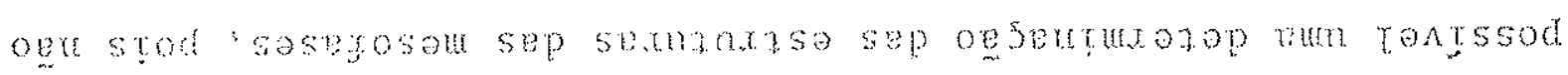

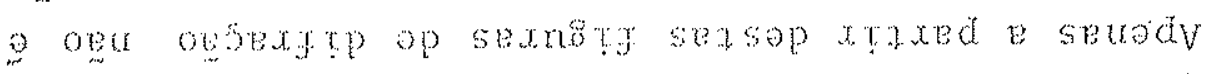

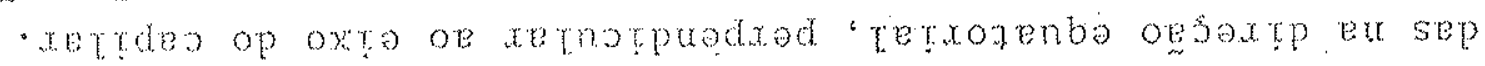

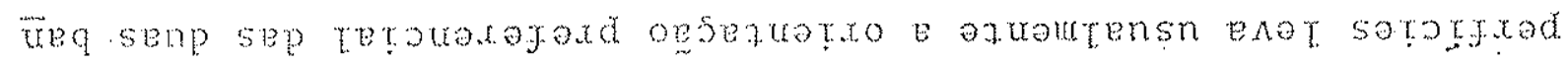

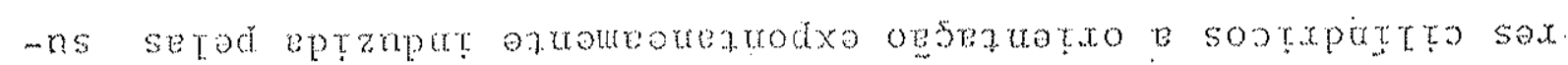

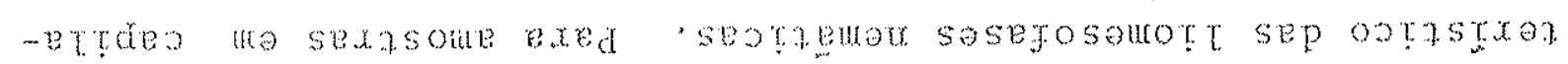

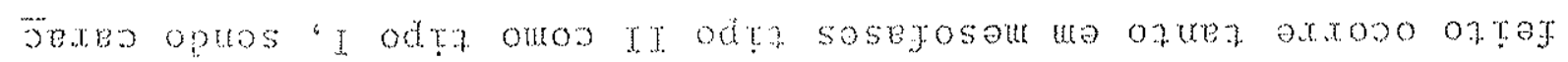

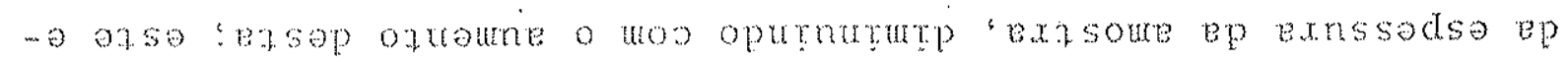

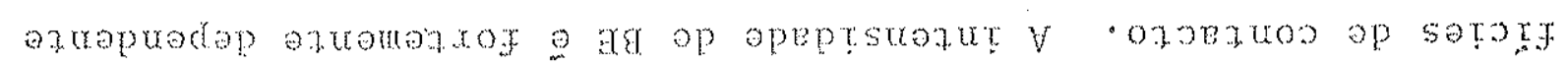

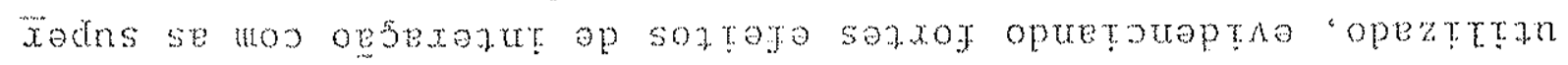

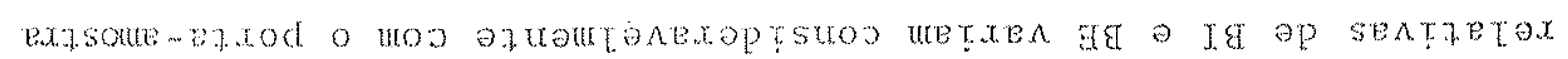

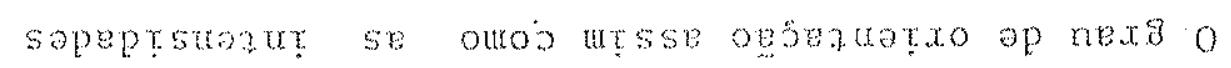

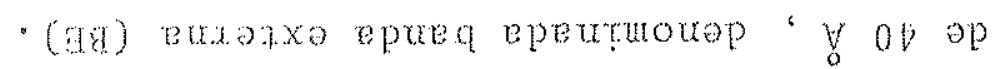

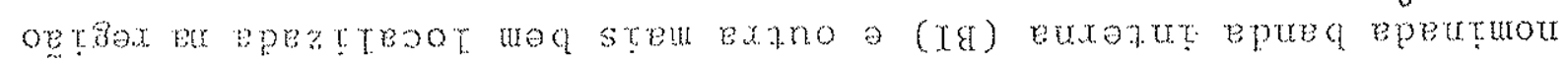

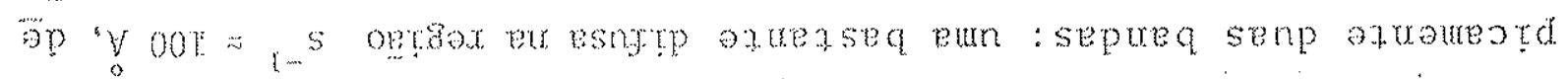

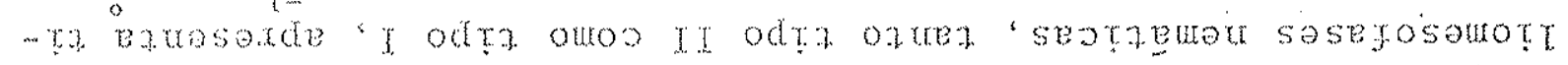

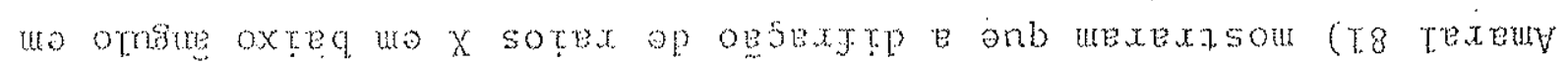

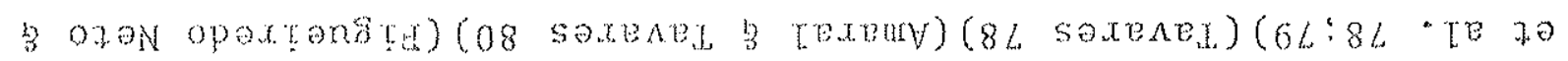
texeur) opxoraxoget ossou us sopeztrex sopnase so

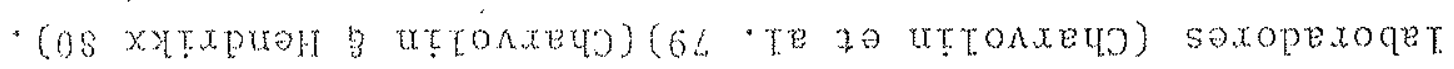

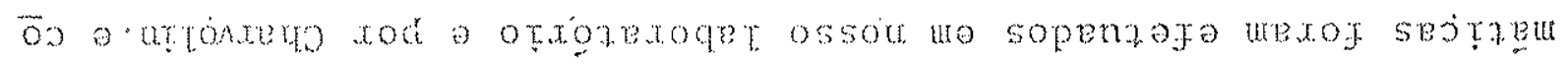
au sosasosert we $x$ sotex op oesextrp ap sopnasa

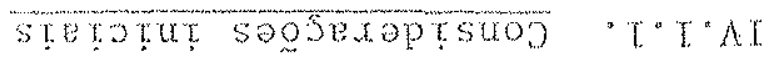

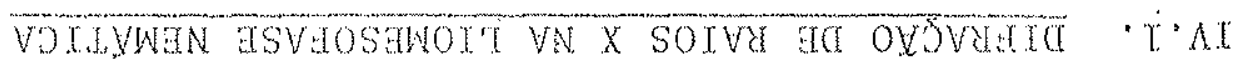

OXssnosro it sodvernsin

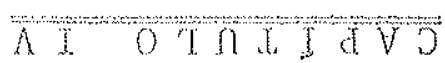




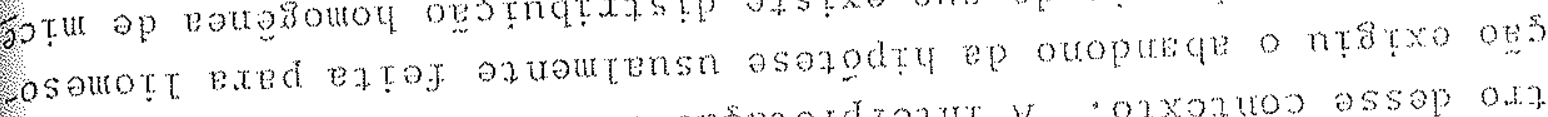

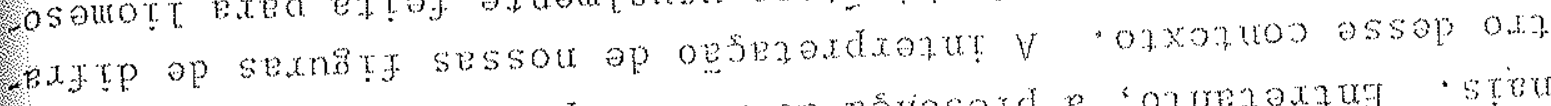

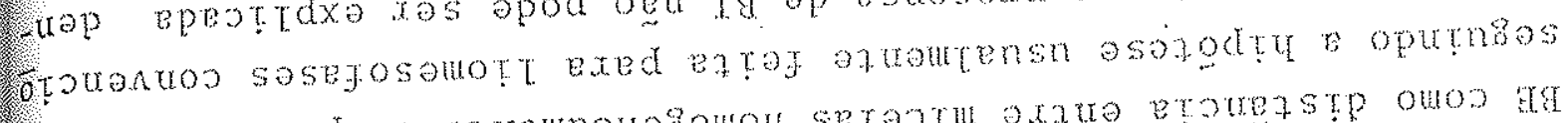

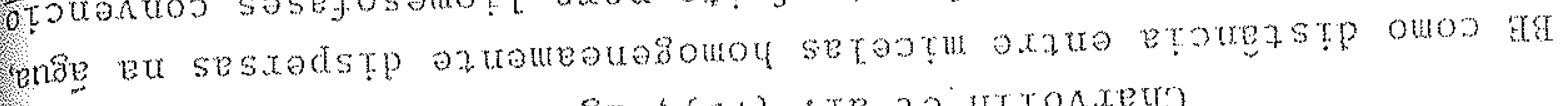

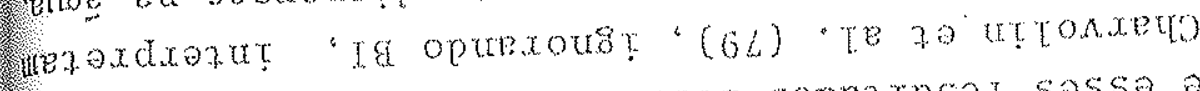

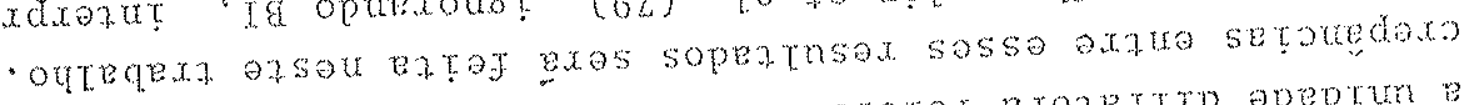

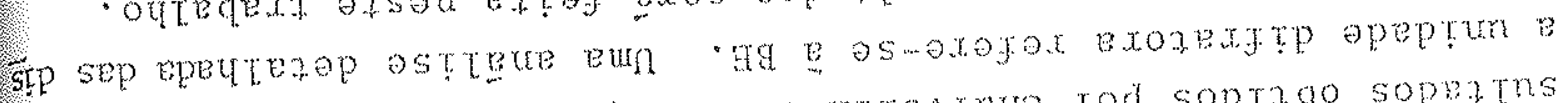

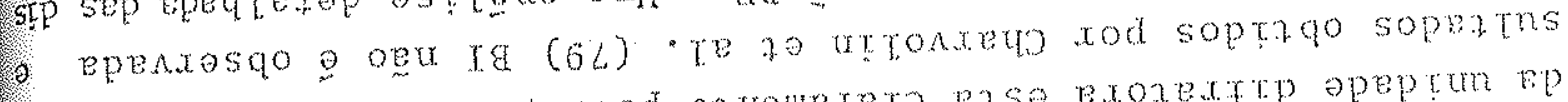

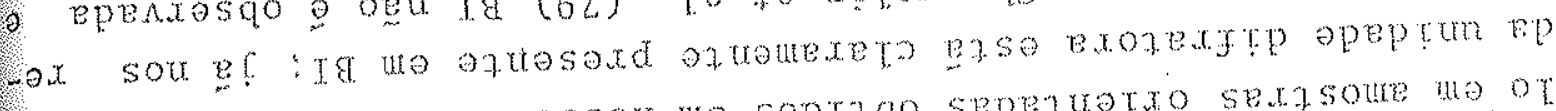

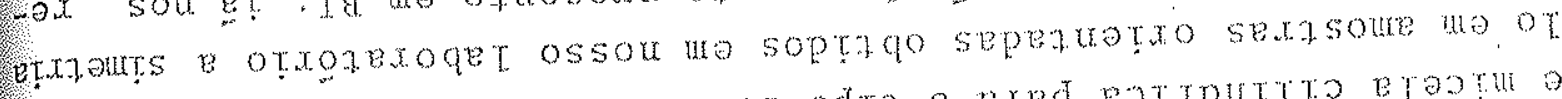

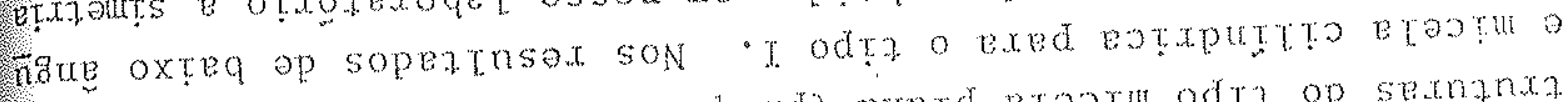

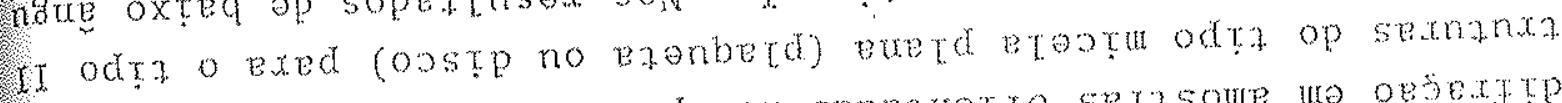

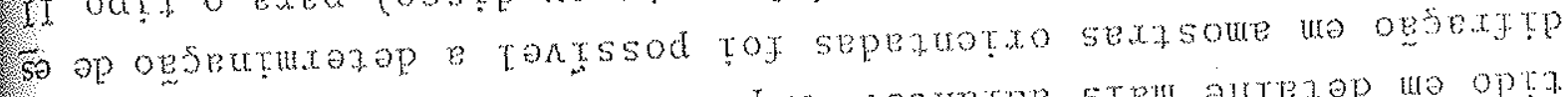

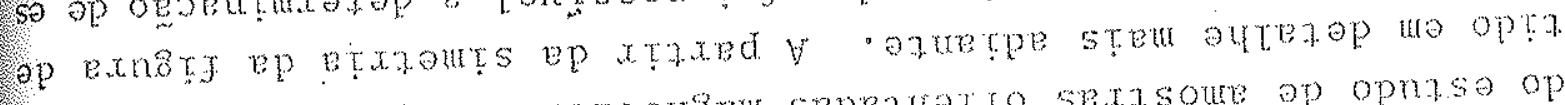

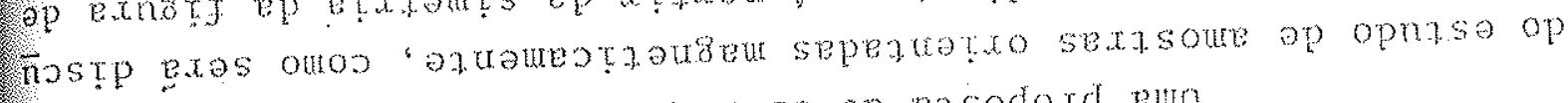

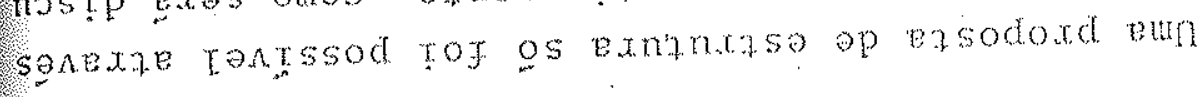

seoteratou

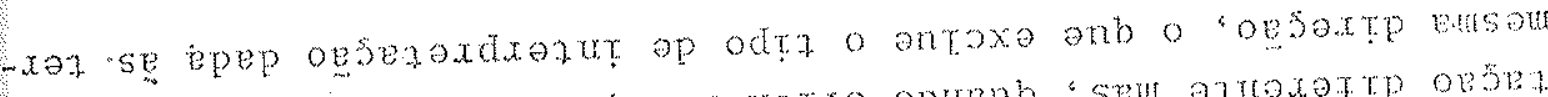

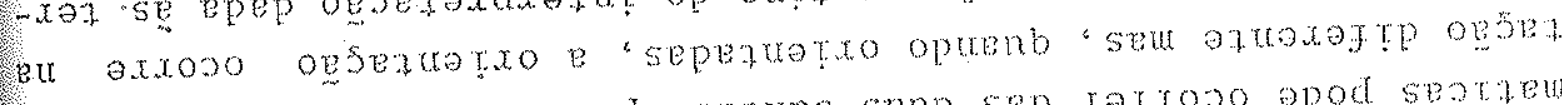

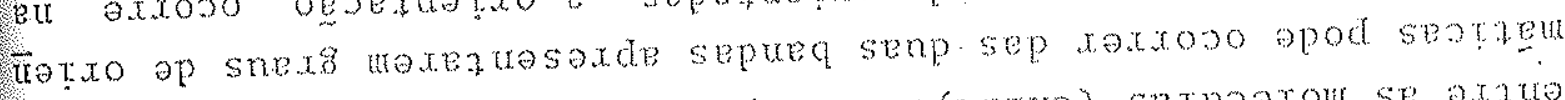

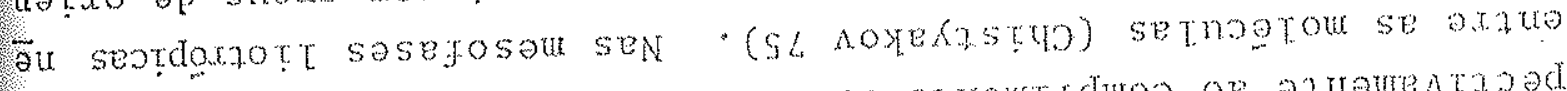

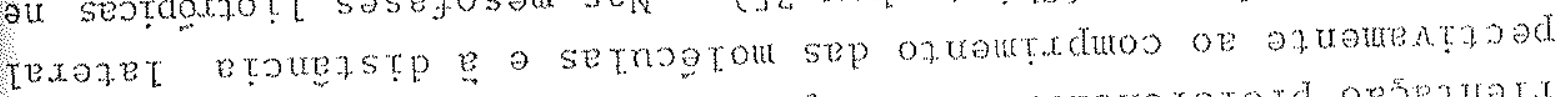

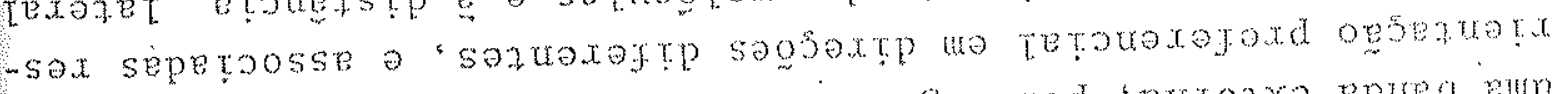

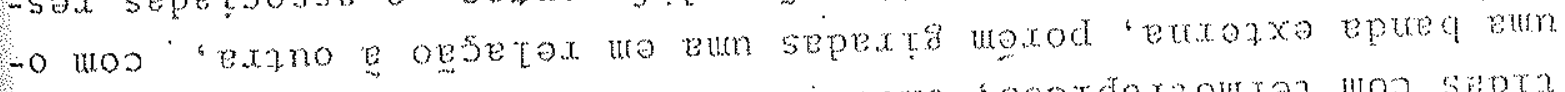

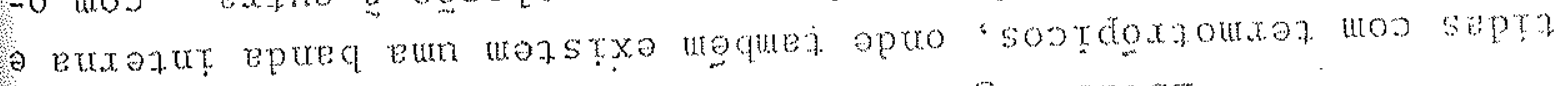

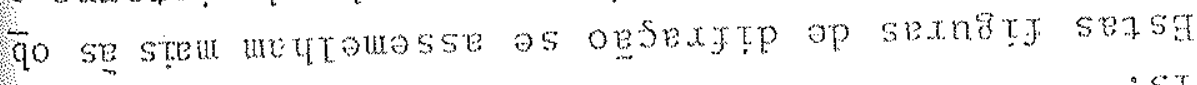

- stensn steresexot

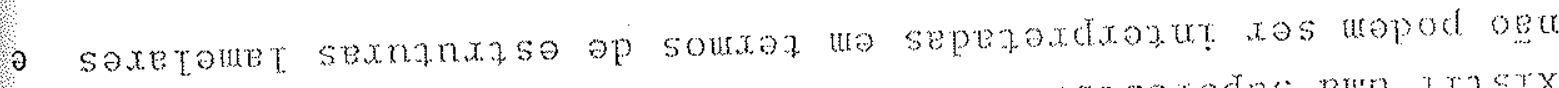

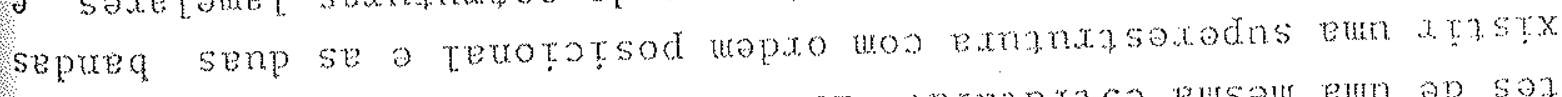

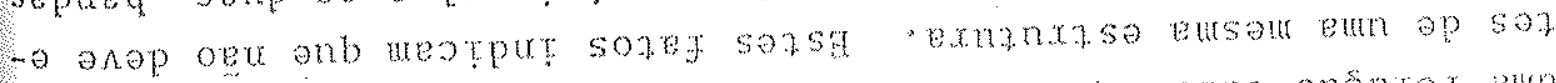

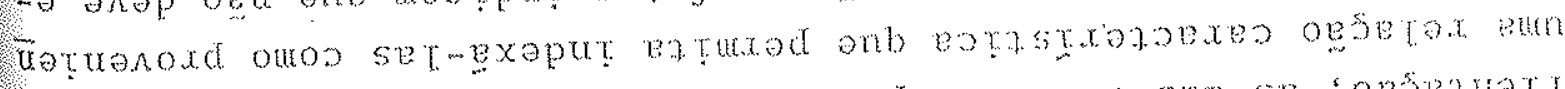

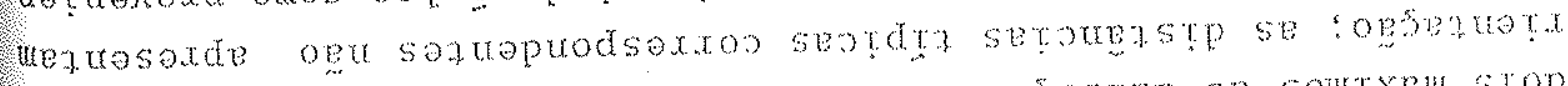

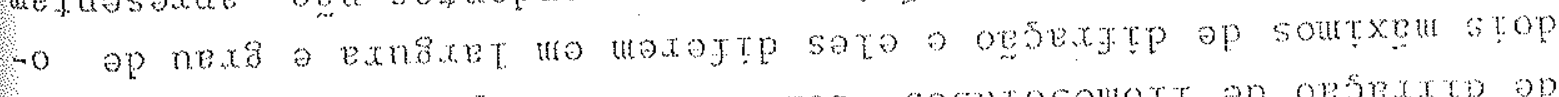

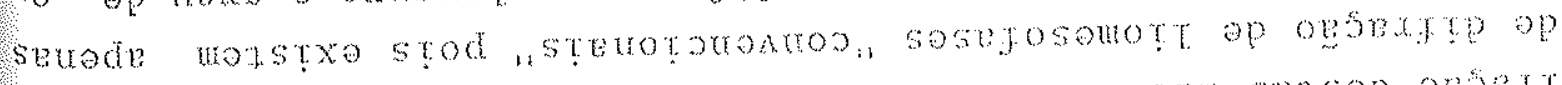

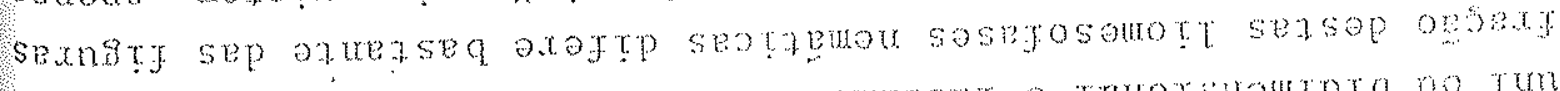

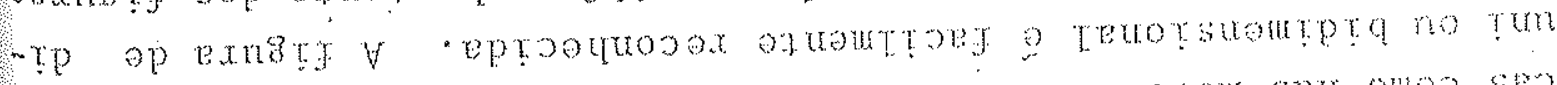

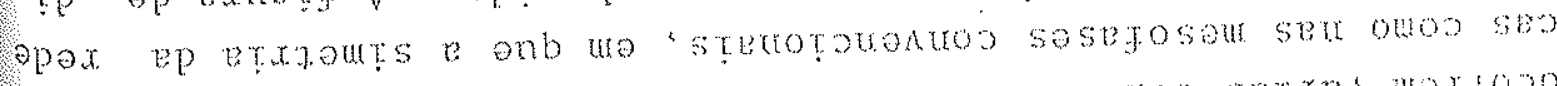

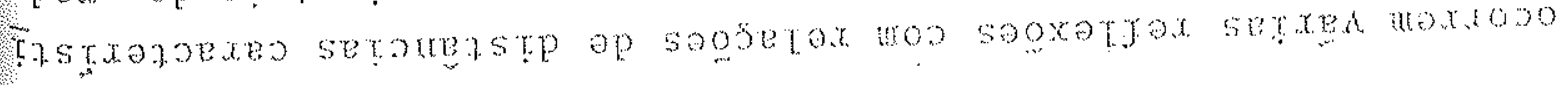




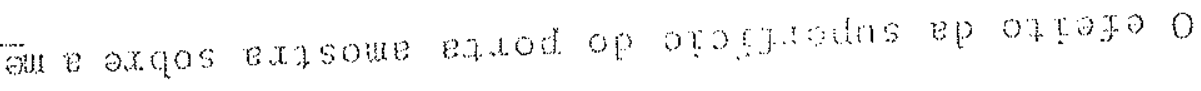

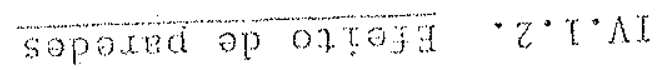

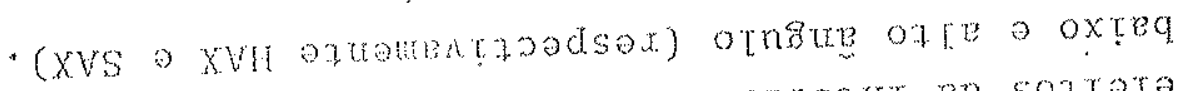

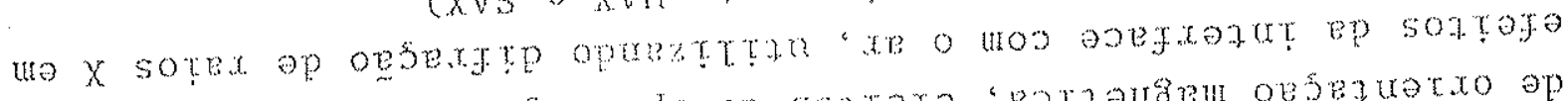

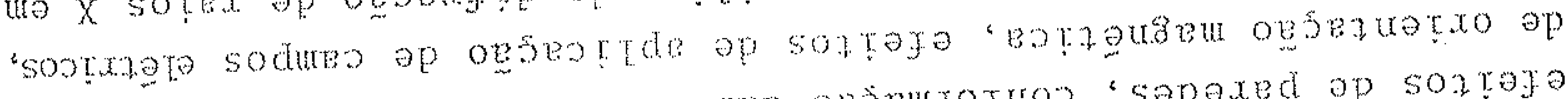

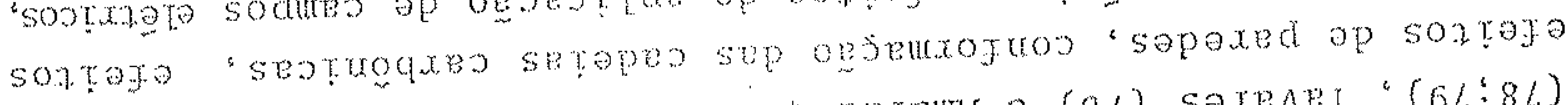

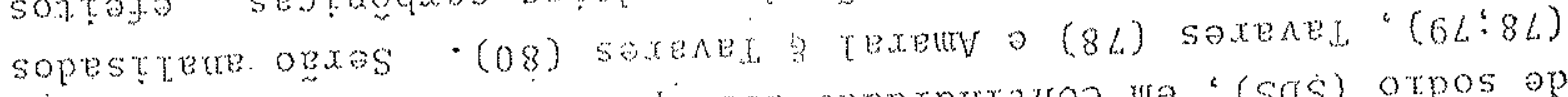

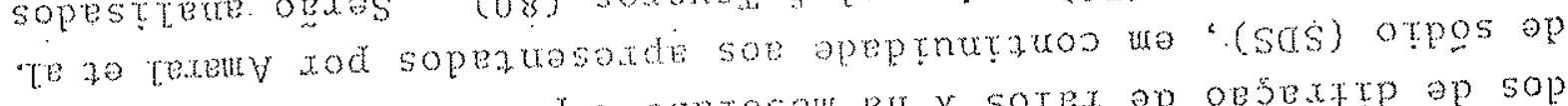

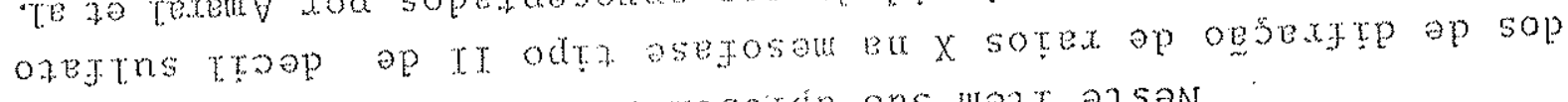

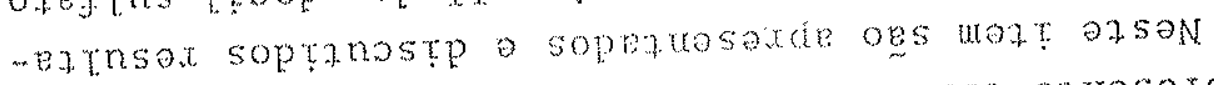

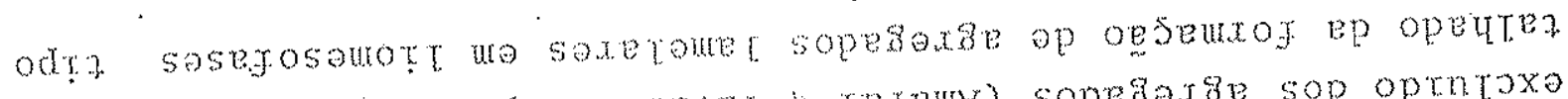

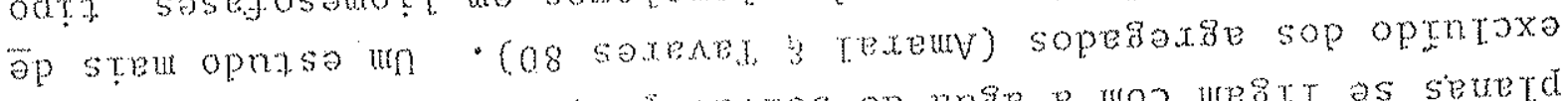

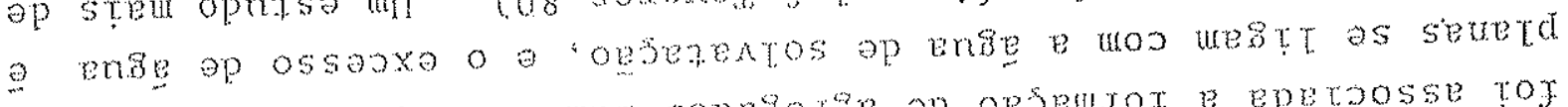

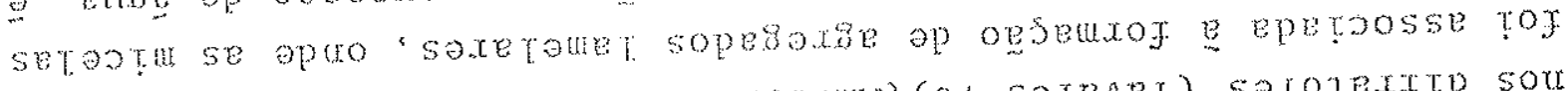

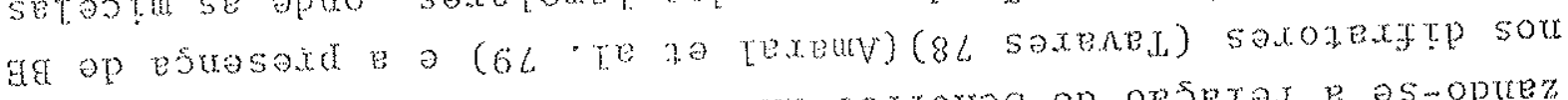

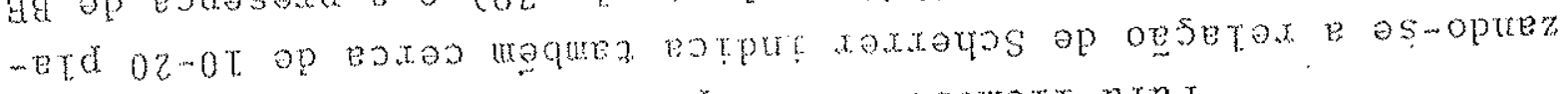

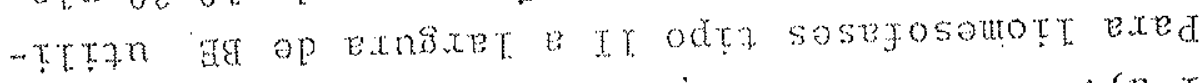

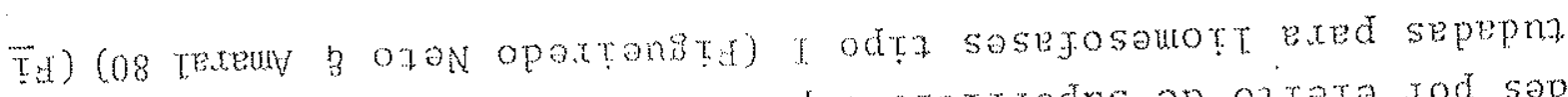

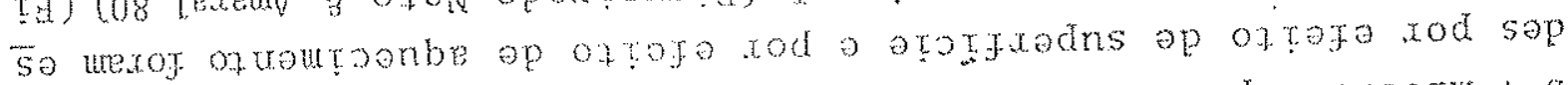

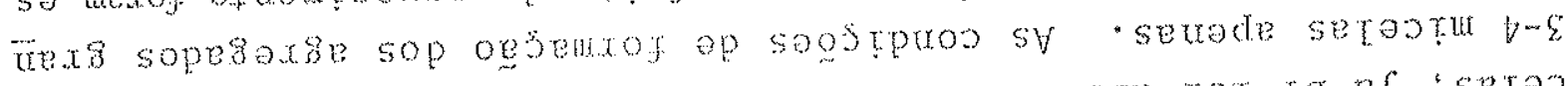

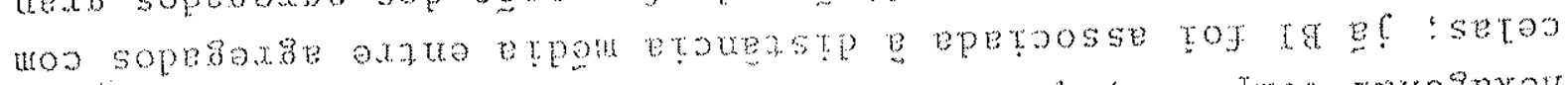

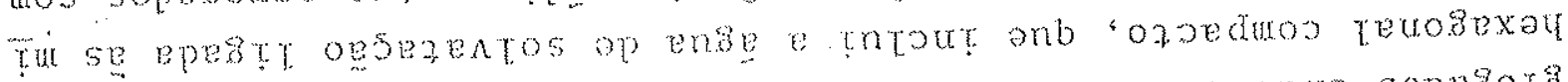

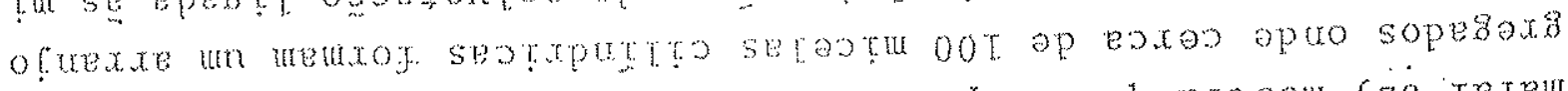

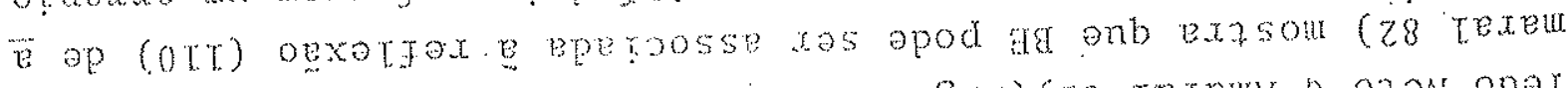

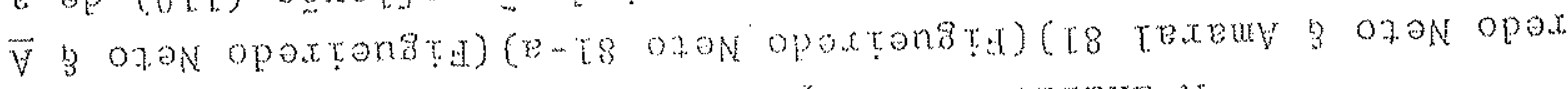

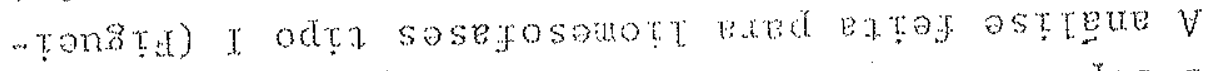

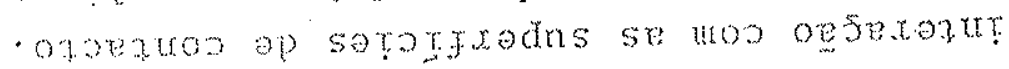

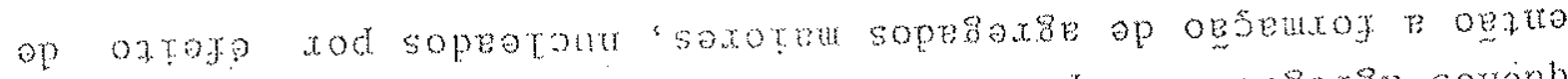

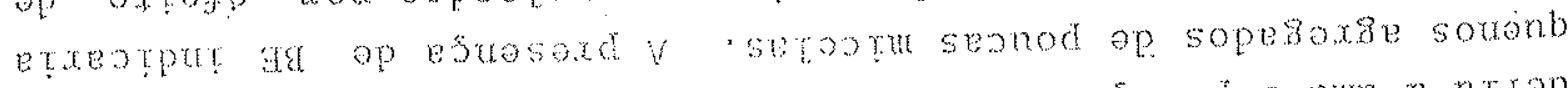

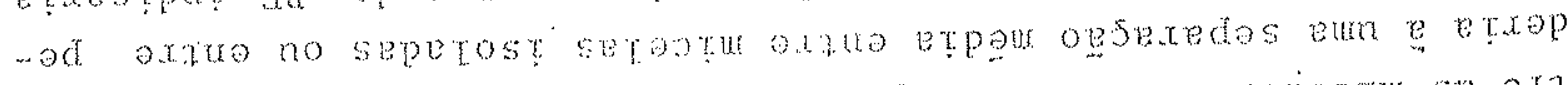

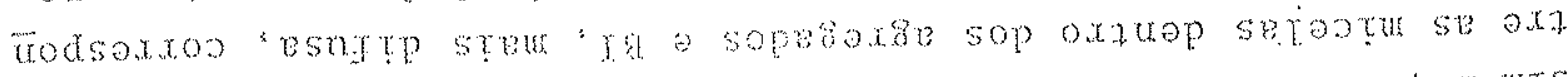

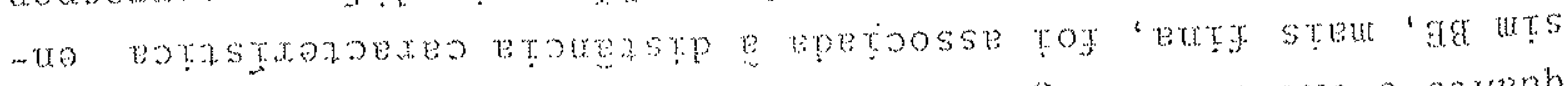

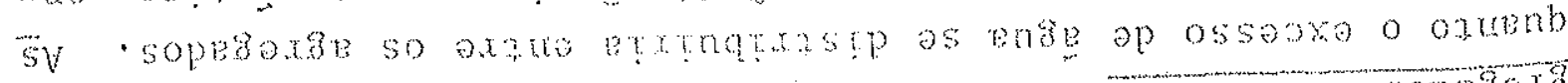

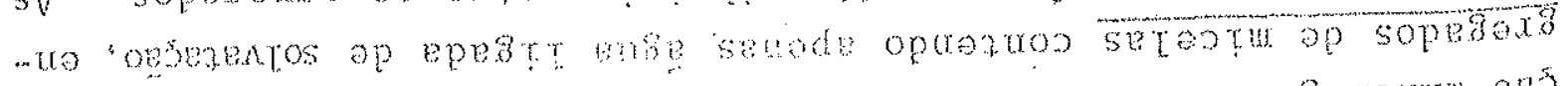

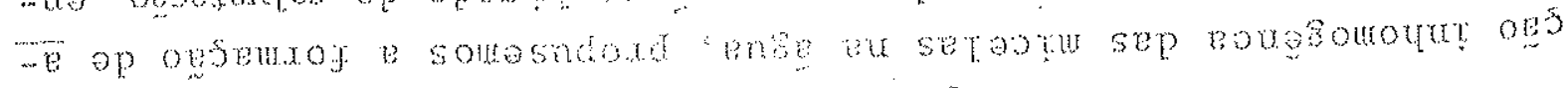

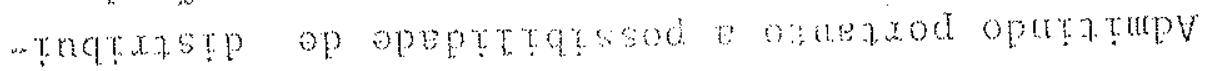




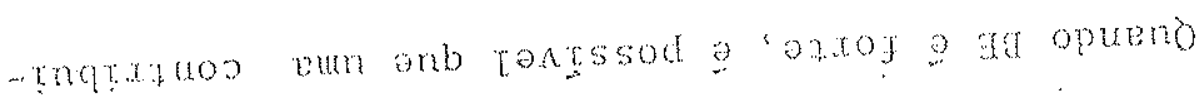

- xopenbo orr

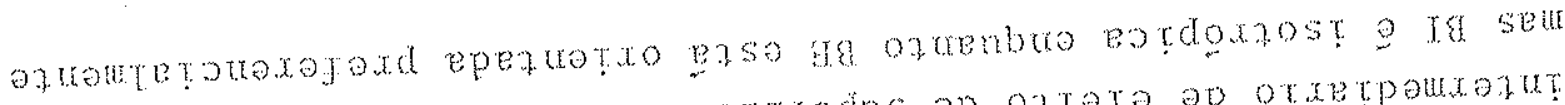

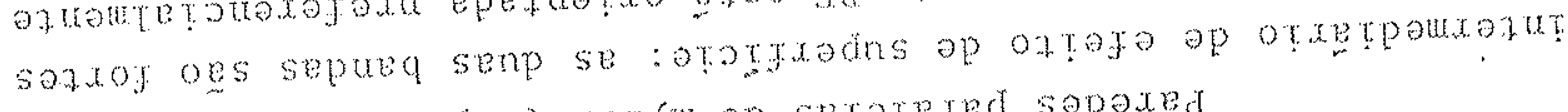
nexs un wexasom (zd) xethu op stetexed sopexed

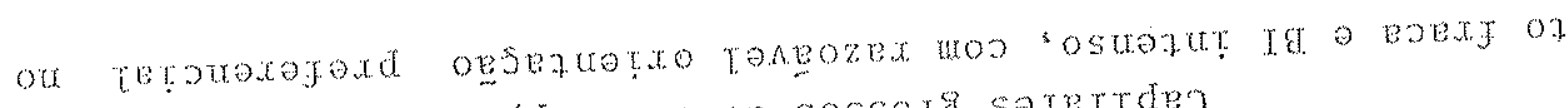

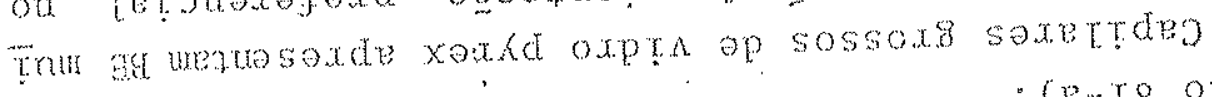

- (B.r.t8 ozan oportonsto

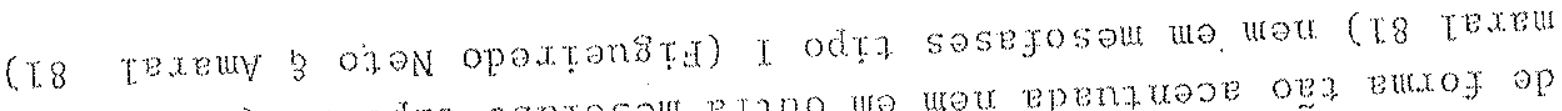

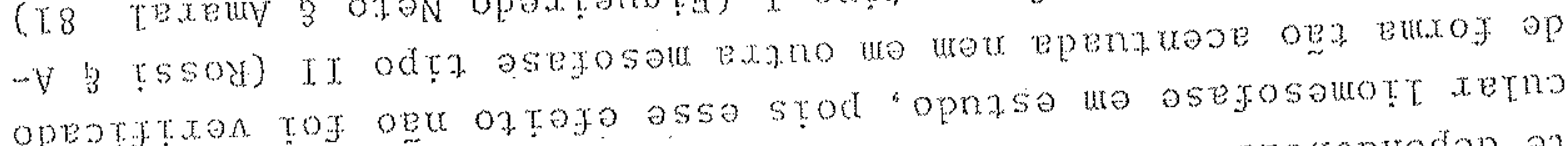

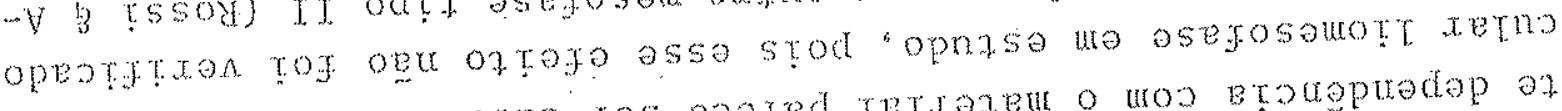

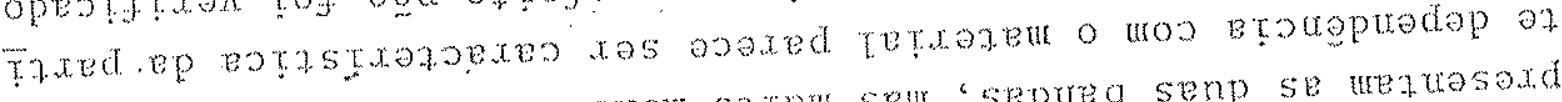

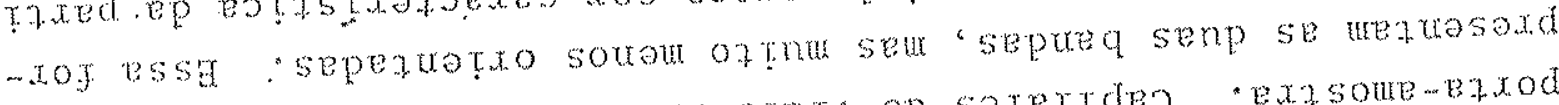

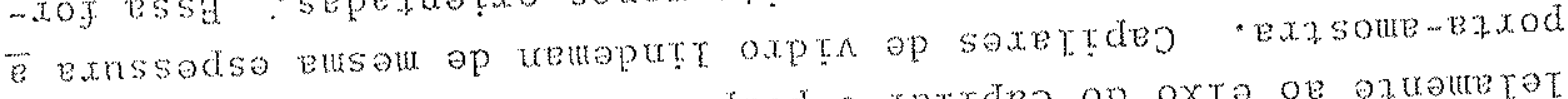

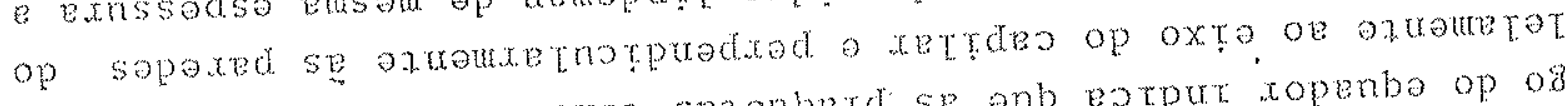

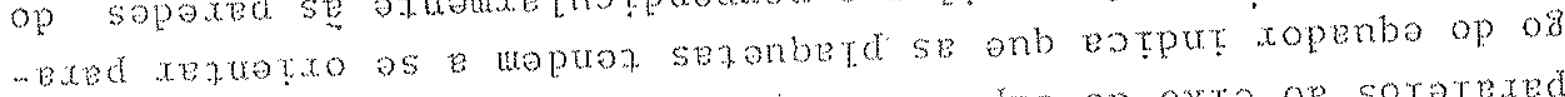

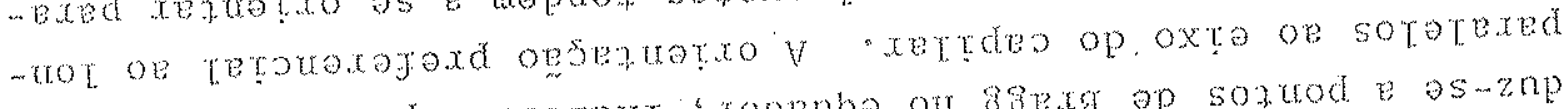

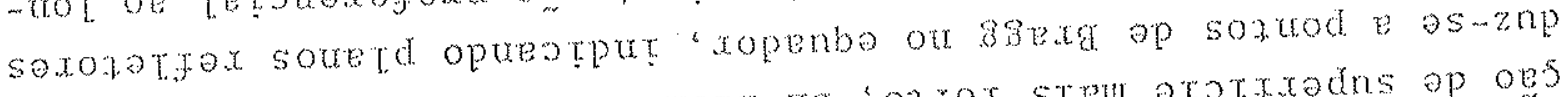

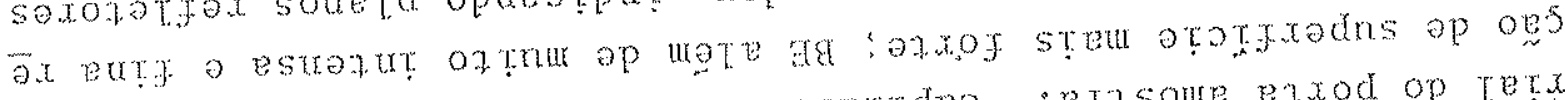

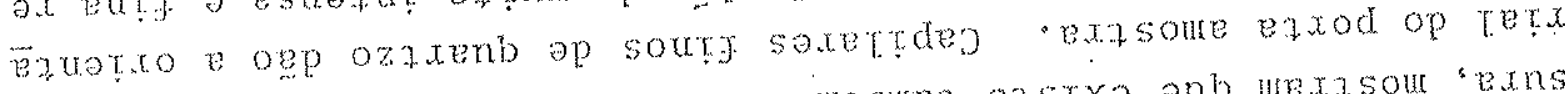

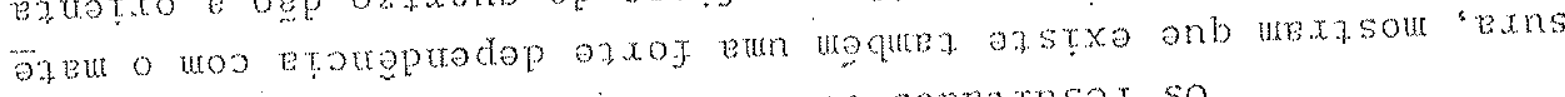

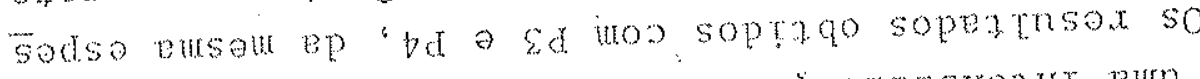

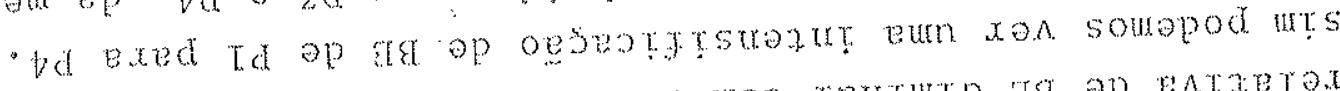

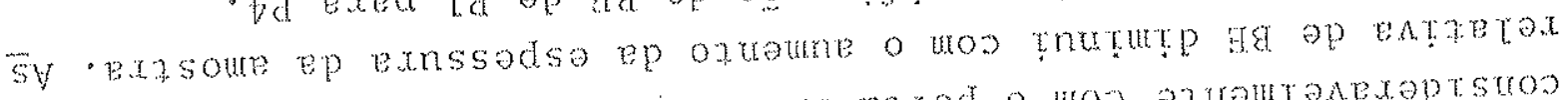

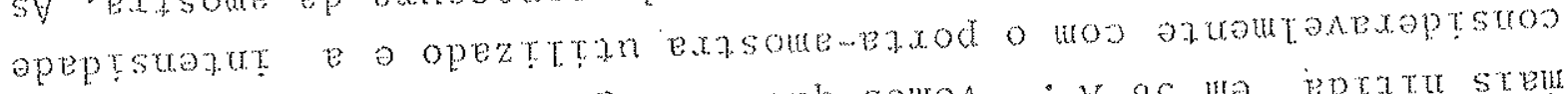

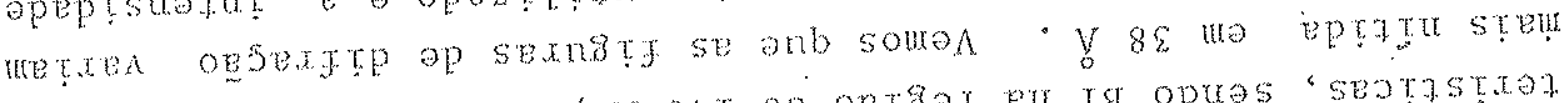

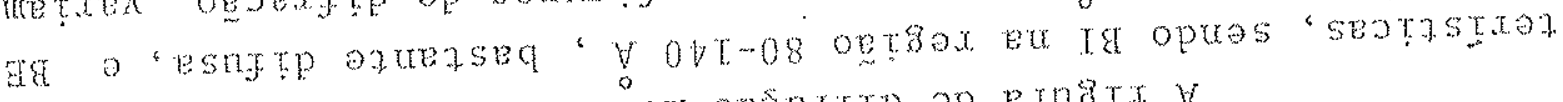

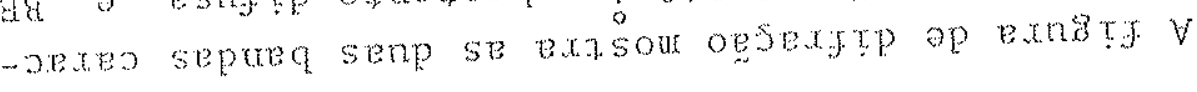

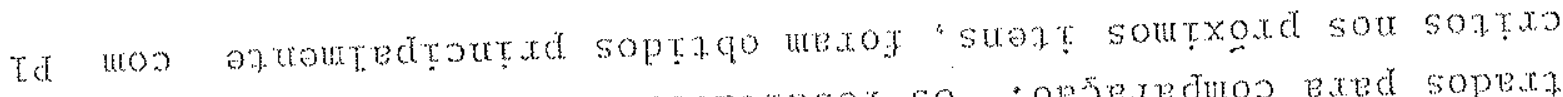
sap "oufequat atsep sonox sopeztnsox so . obsaxeduos exed sopext

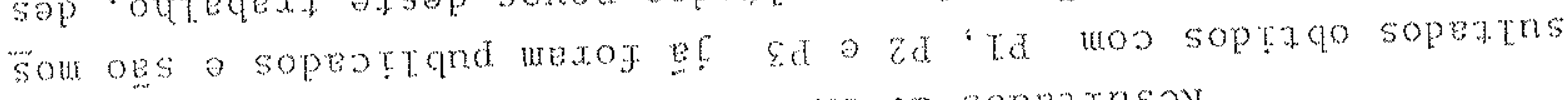

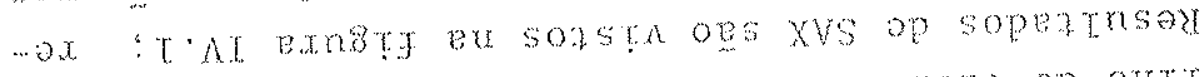

$$
\begin{aligned}
& \text { - (um l"0) wumepurt oxpra op otrif xetrdeo - ta }
\end{aligned}
$$

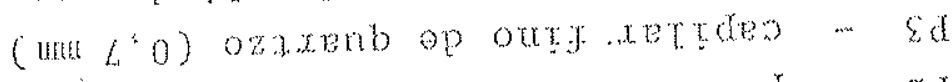

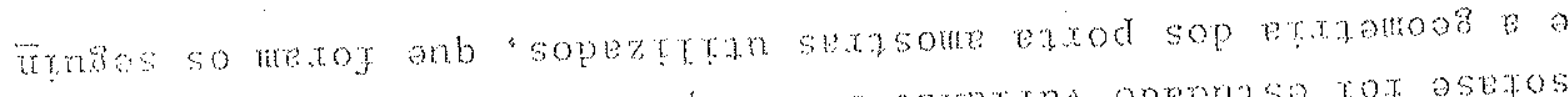

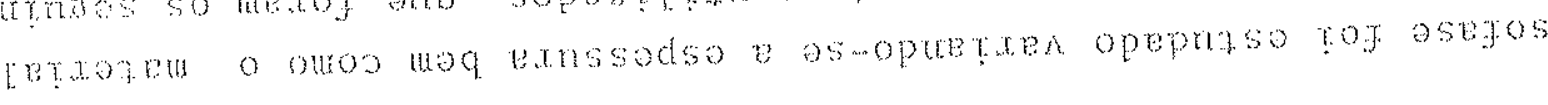




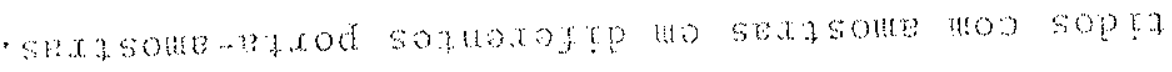

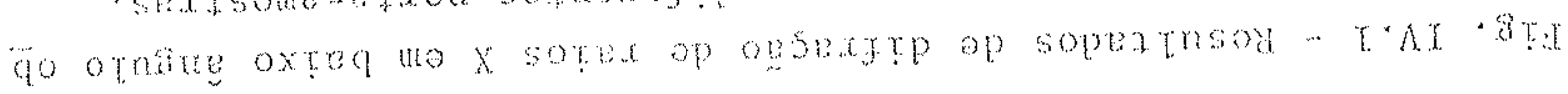

$\forall d$

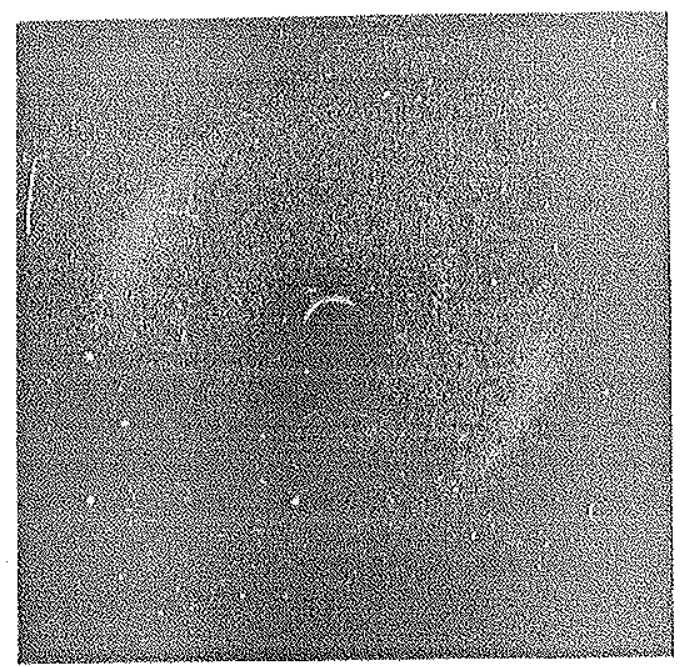

$z d$

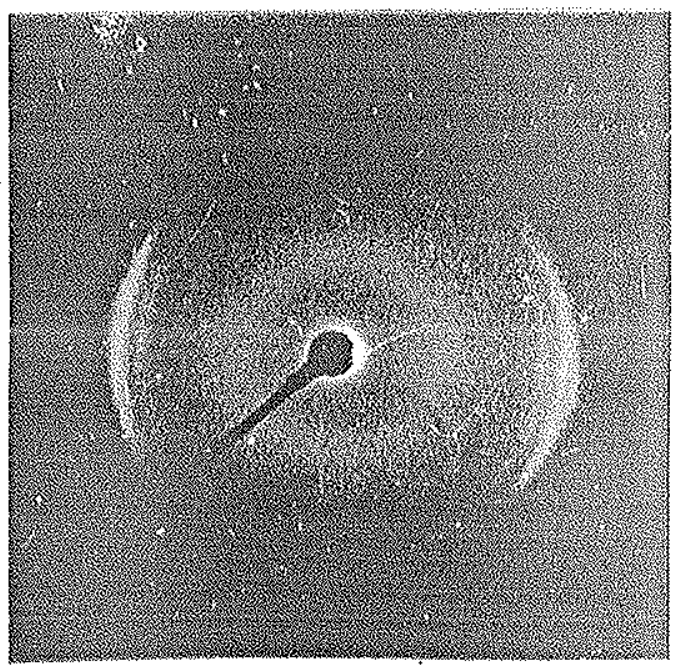

sd

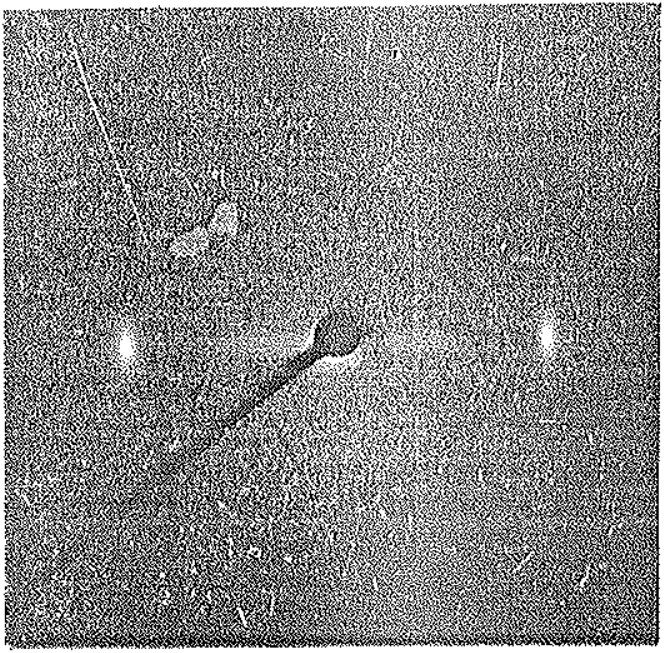

T.

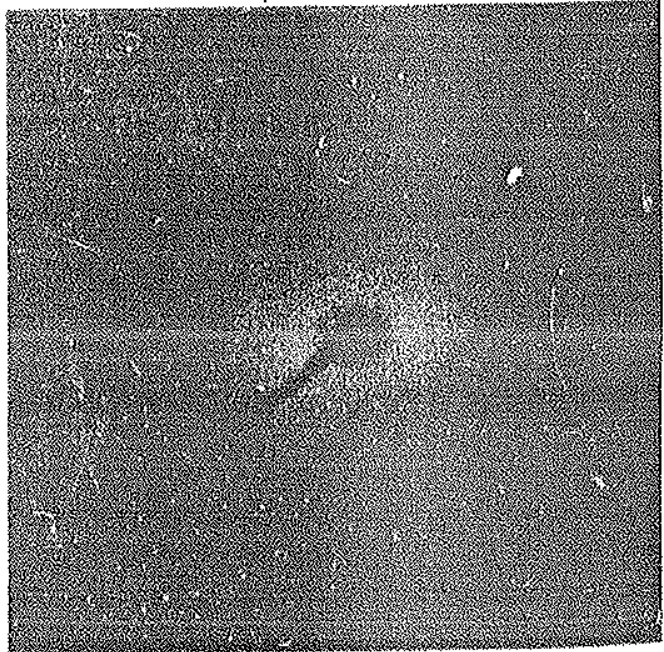




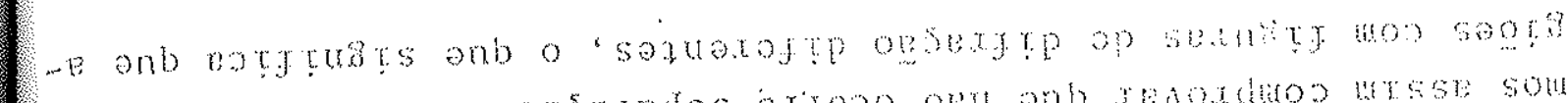

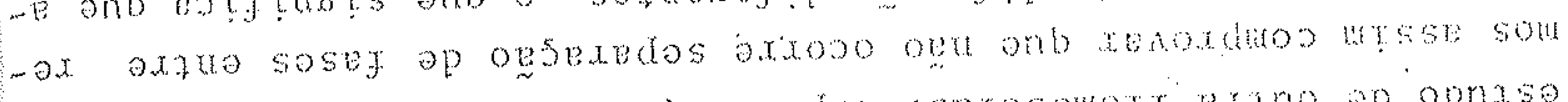

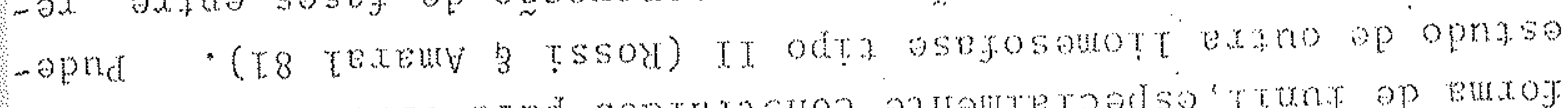

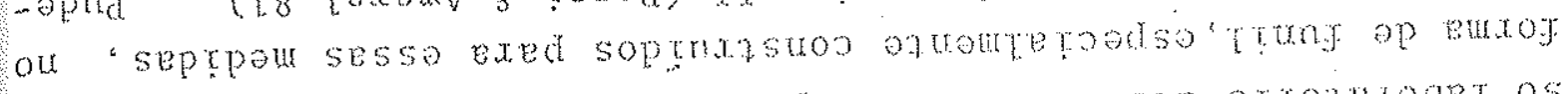

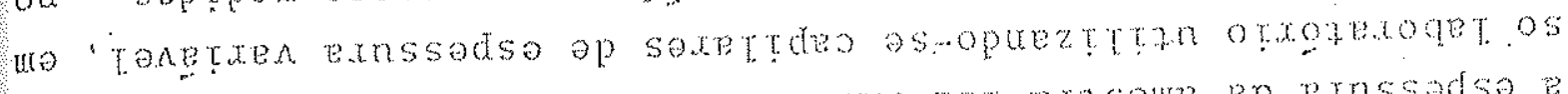

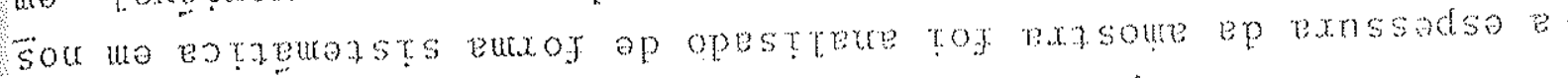
woo oesextep op exnsty ep opsetan ep pustaxd

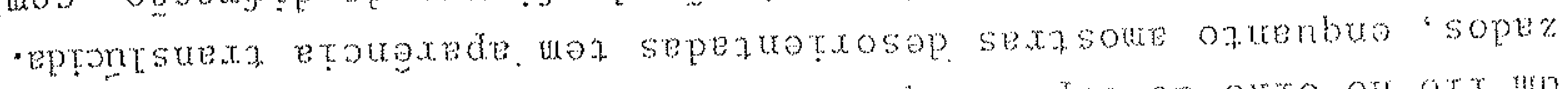

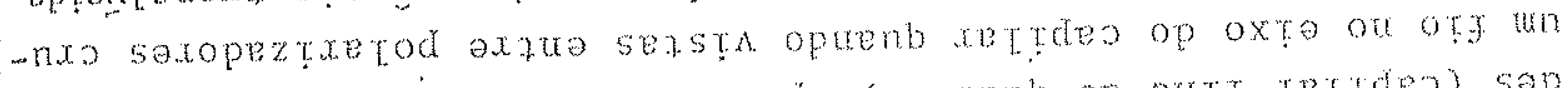

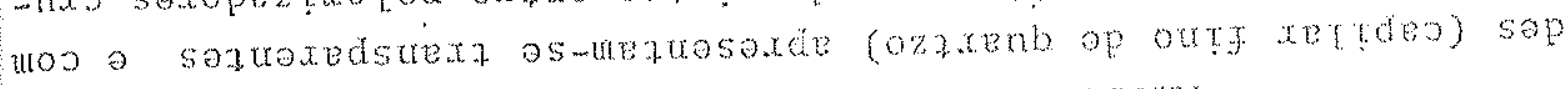

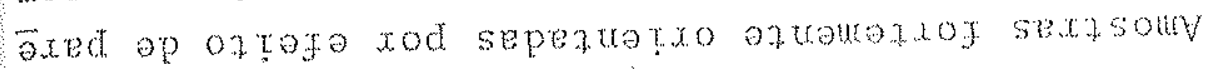

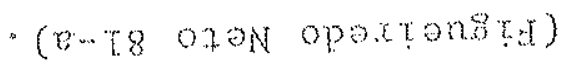

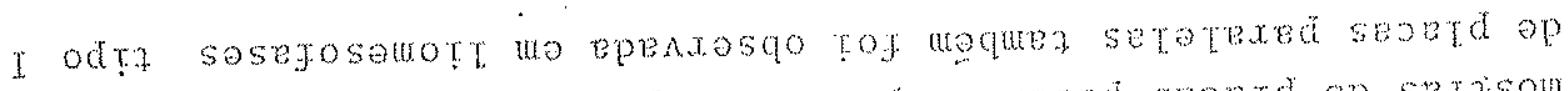

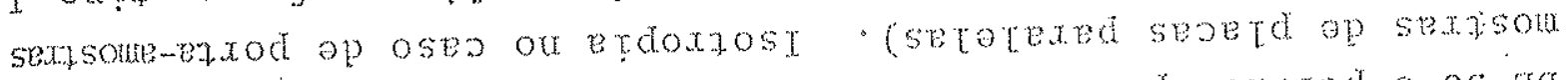

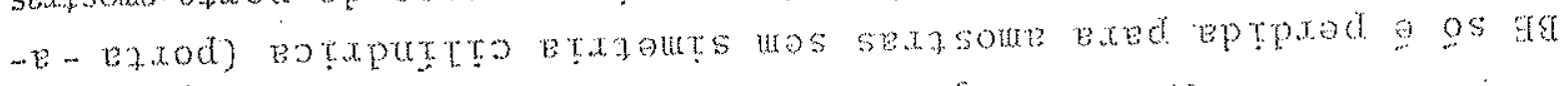

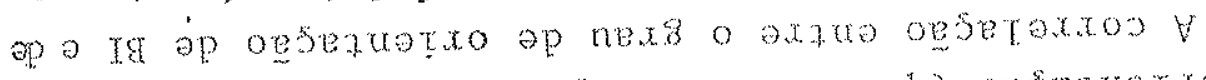

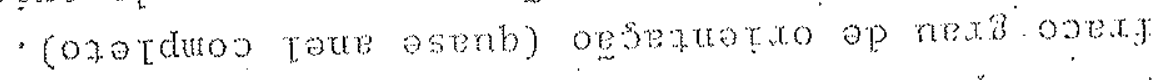

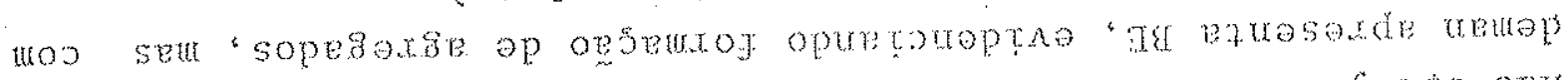

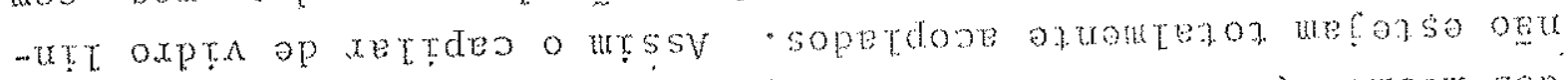

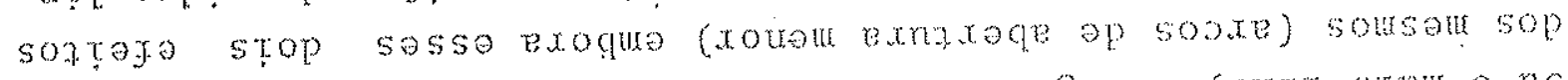

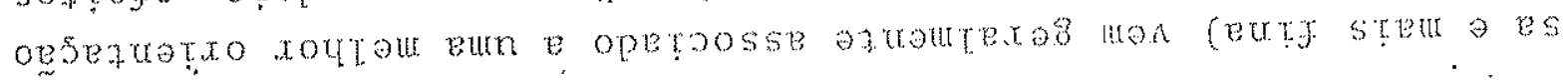

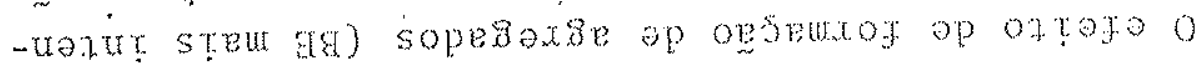

- opprationed

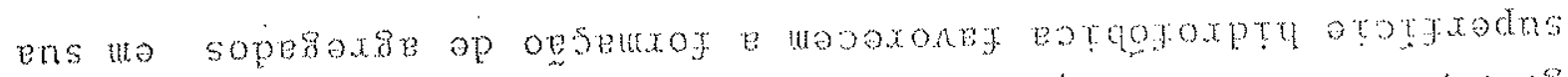

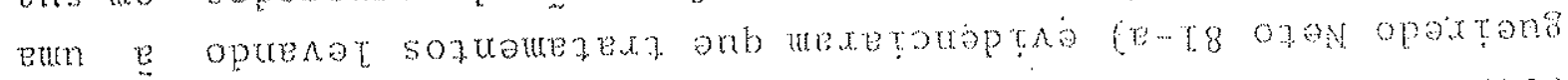

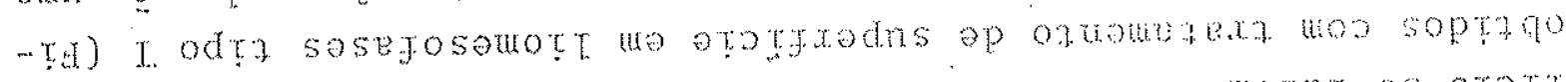

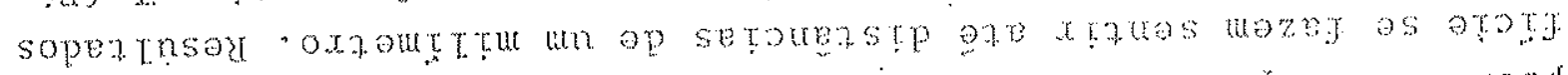

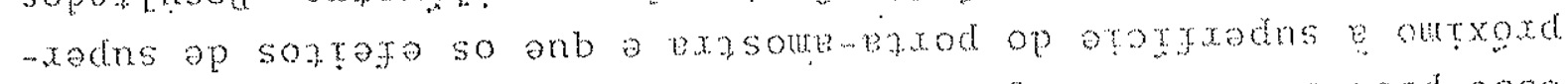

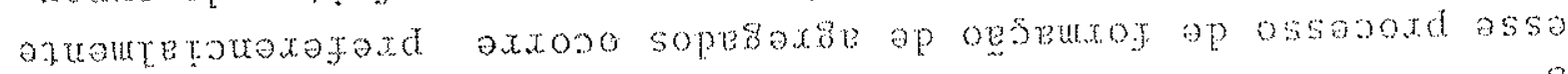

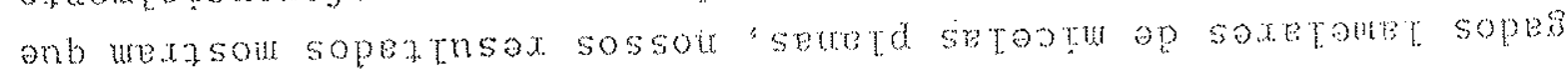

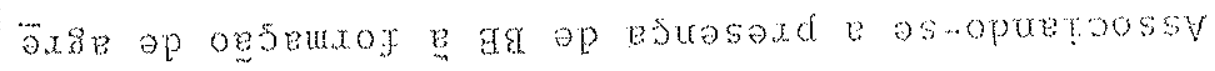

- ermara oes

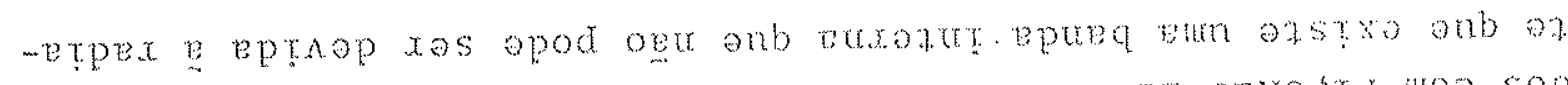

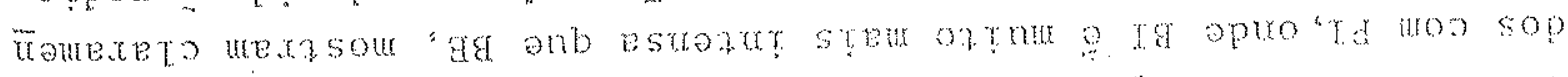

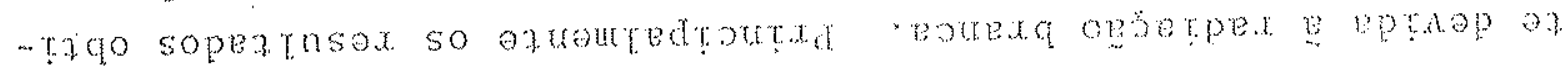

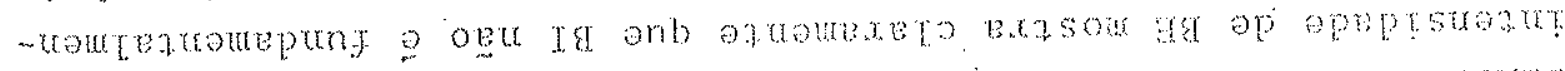

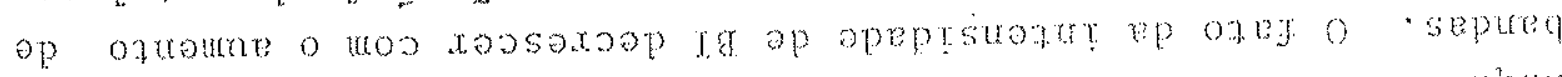

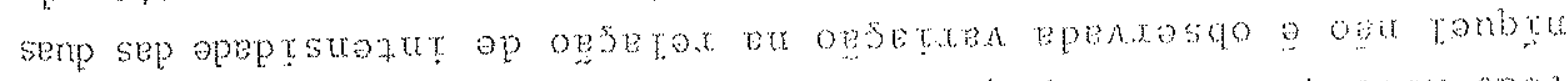

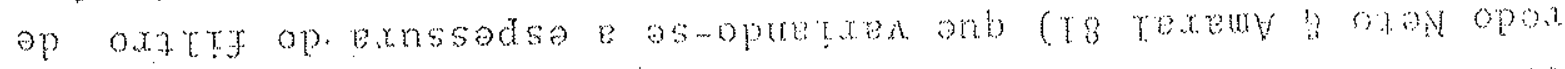

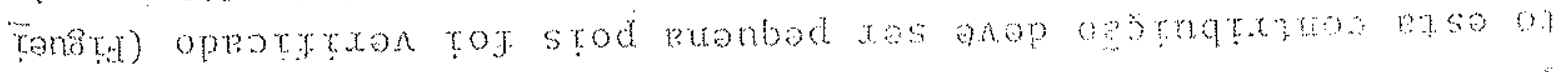

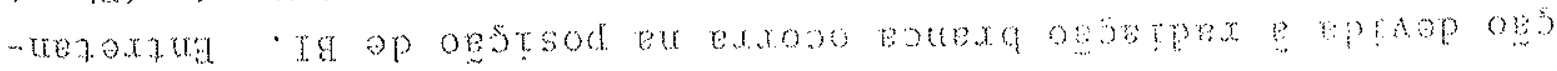




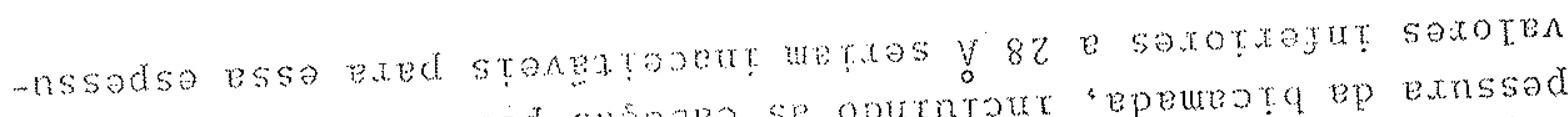

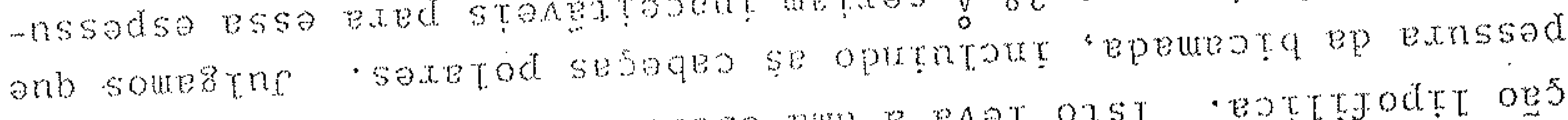

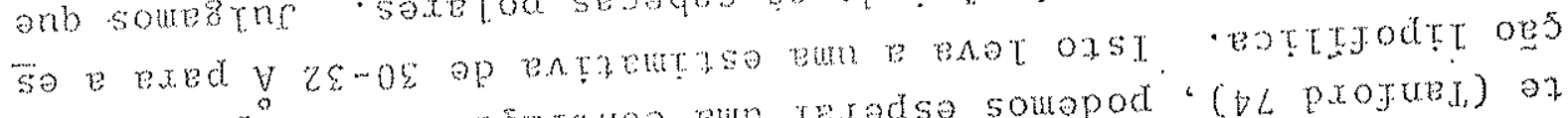

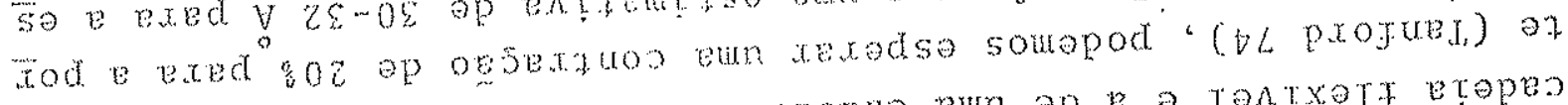

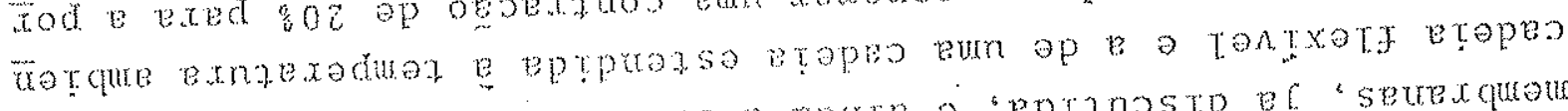

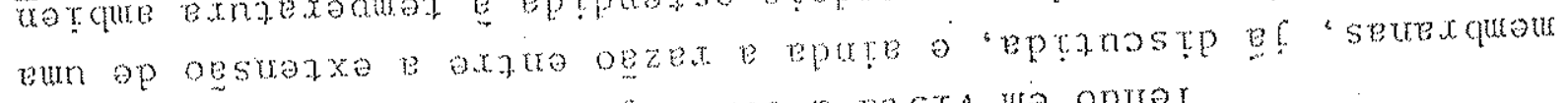

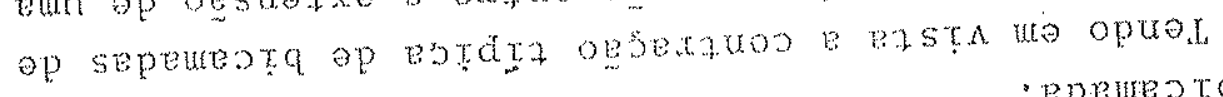

- epenesta pe texzuos ax

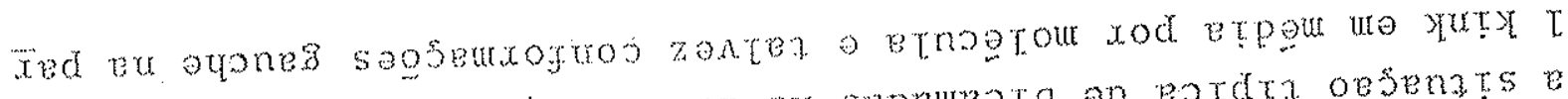

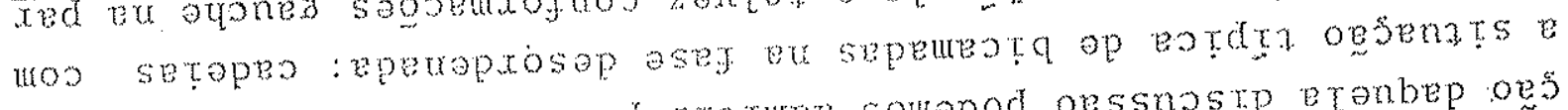

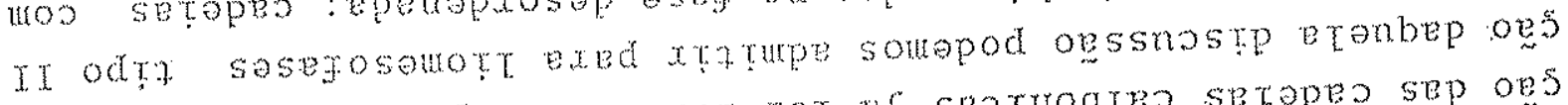

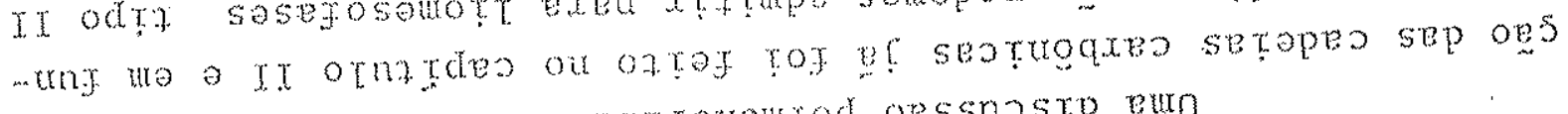

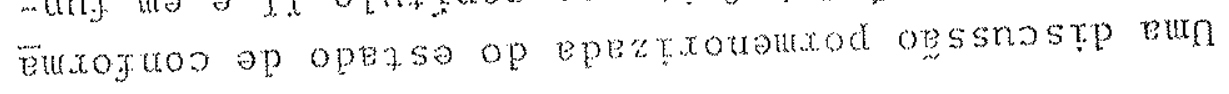

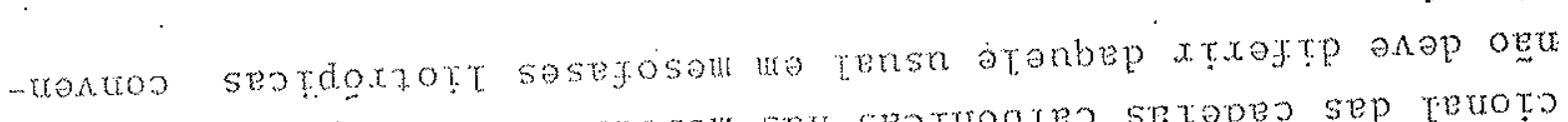

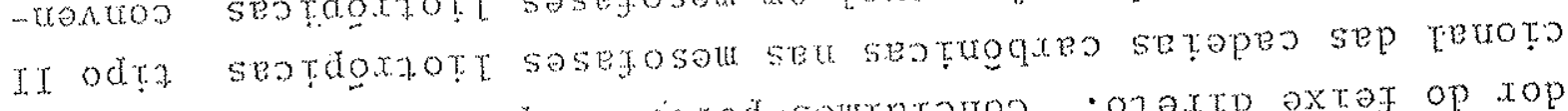

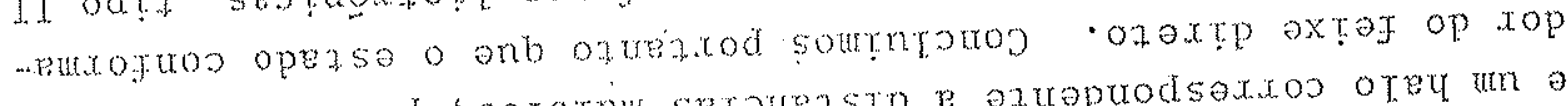

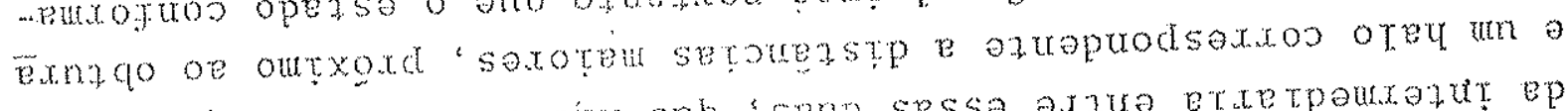

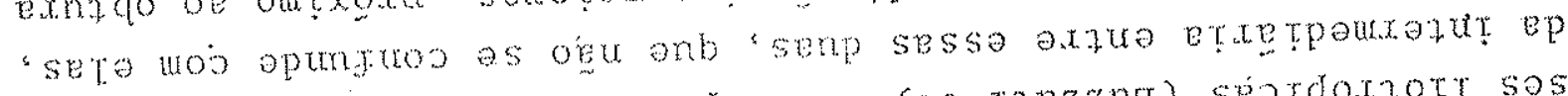

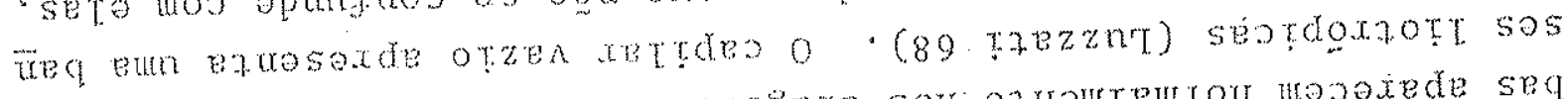

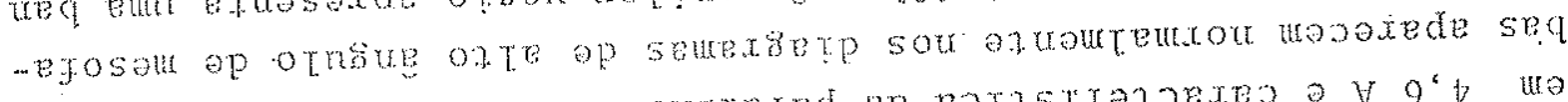

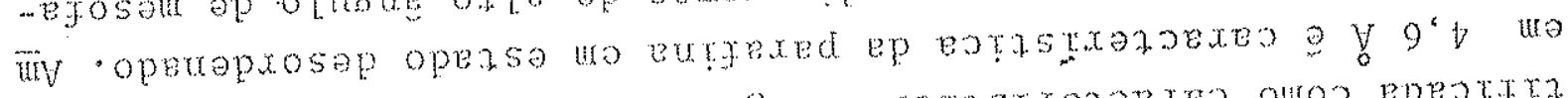

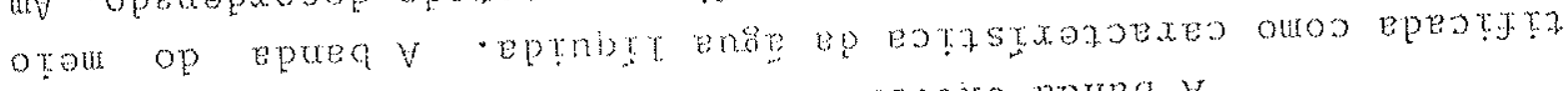

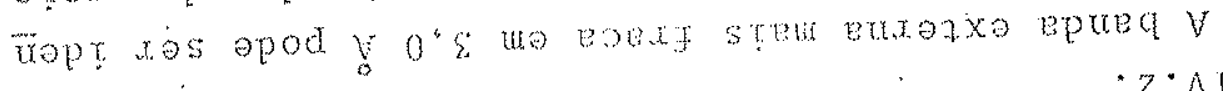

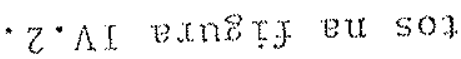

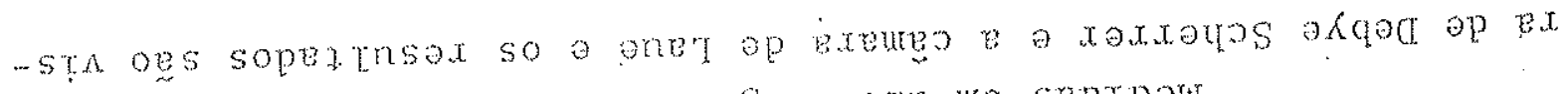

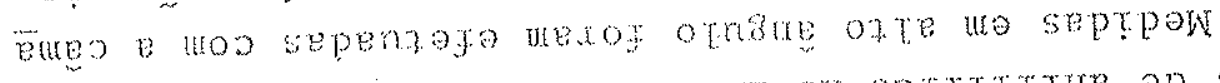

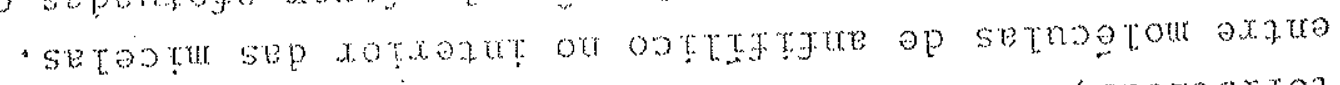

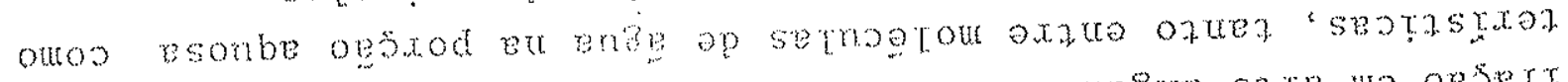

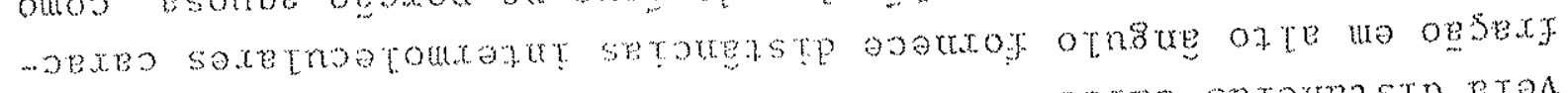

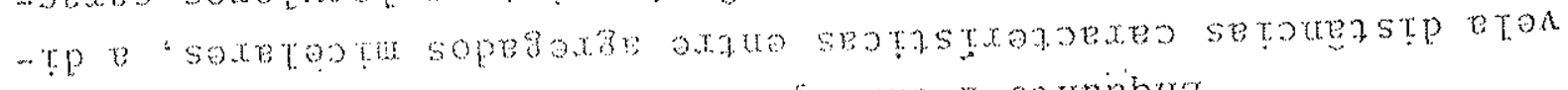

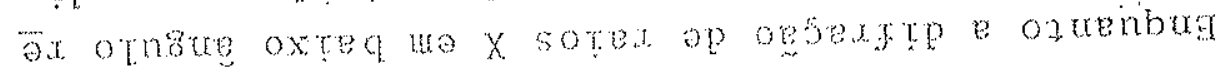

\section{spor}

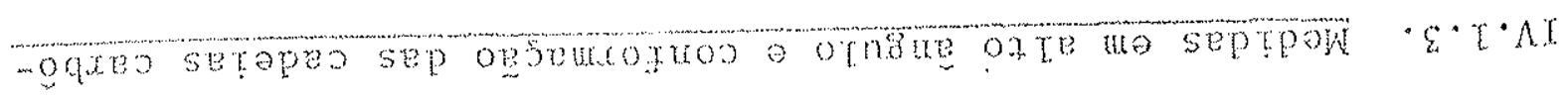

- oseg potm entur ostugute

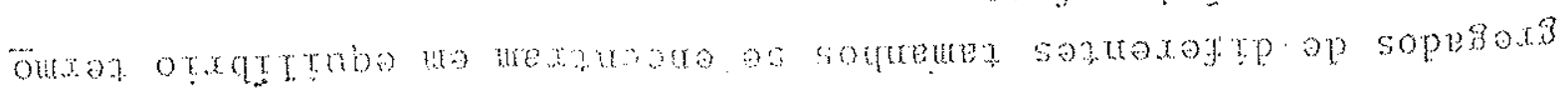




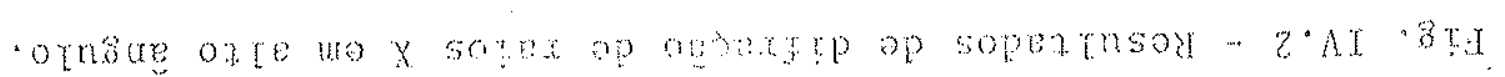

ex.2soure woo tetedes

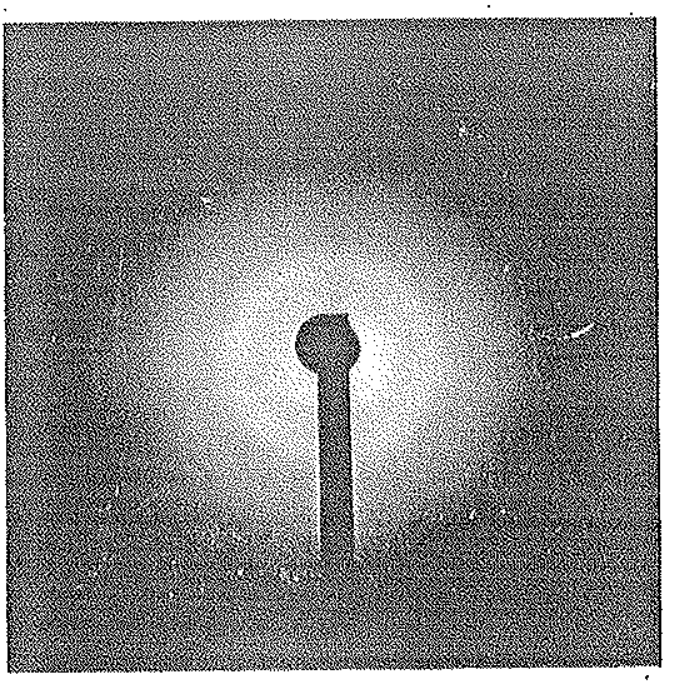

orgen xetren

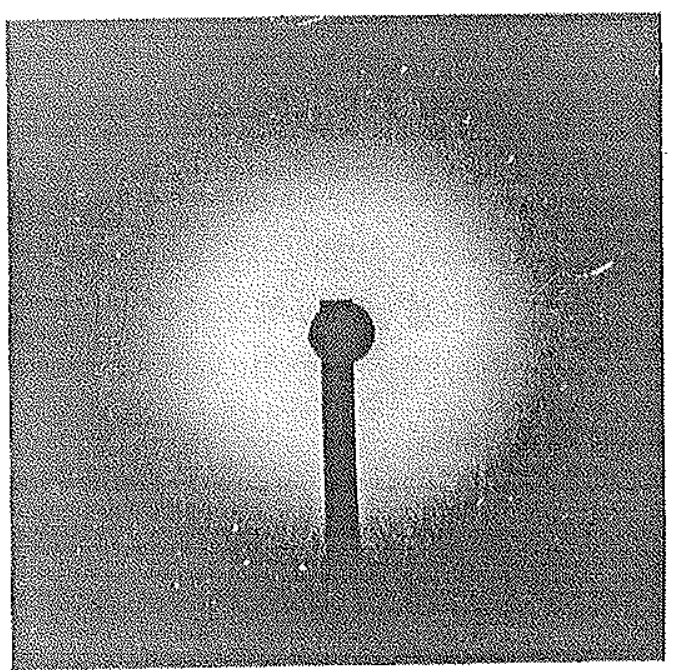

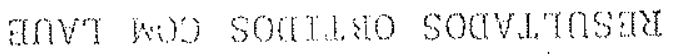

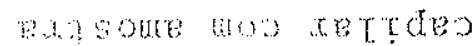

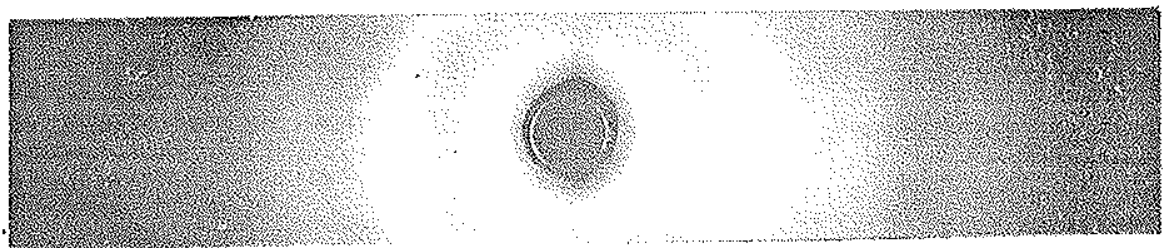

a)

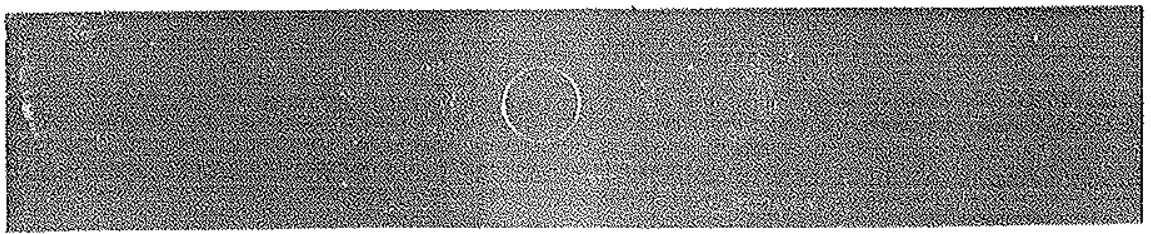

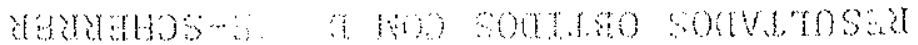




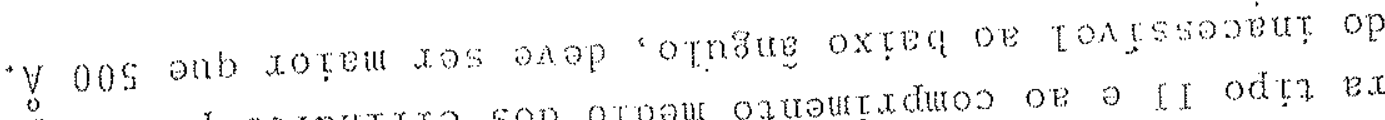

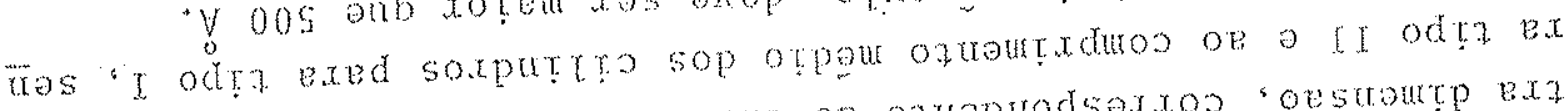

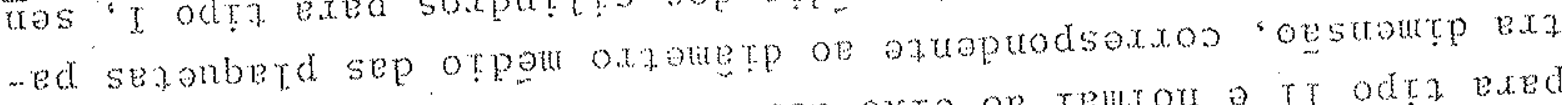

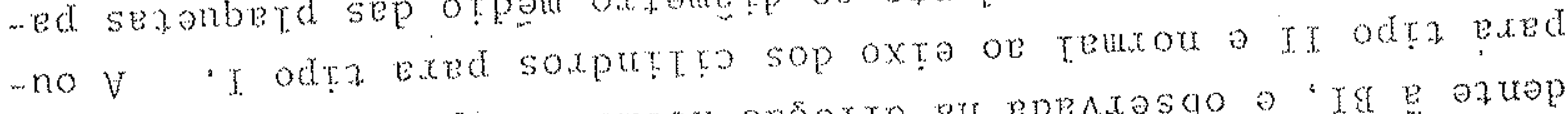

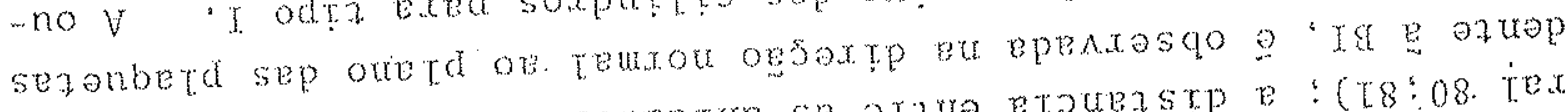

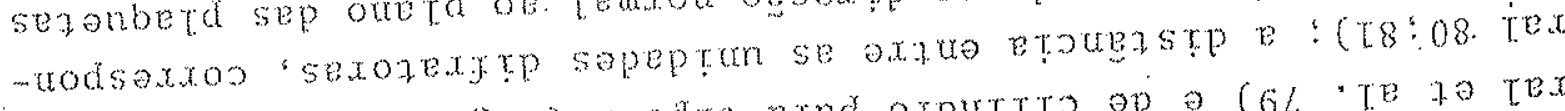

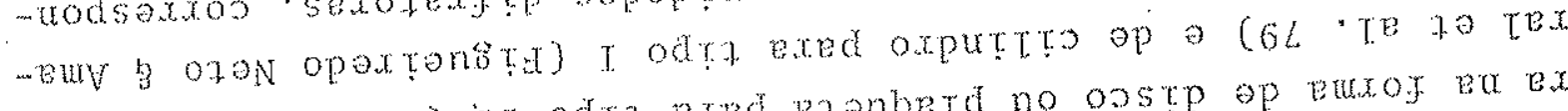

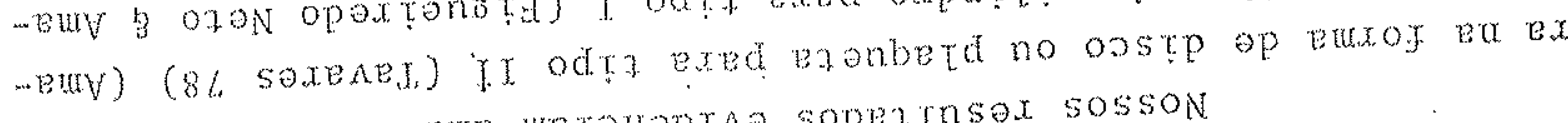

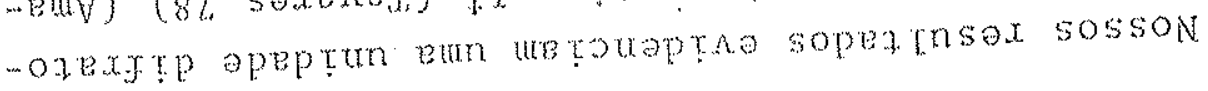

soxotxonte sopertnsox sossop oessnosta $\cdot \mathrm{V}$

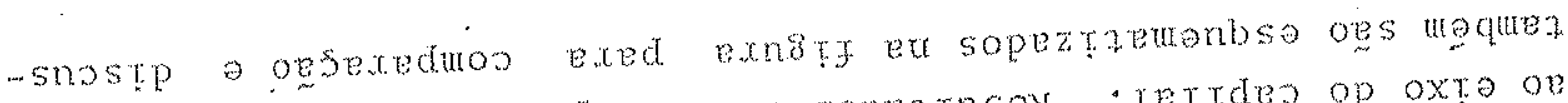

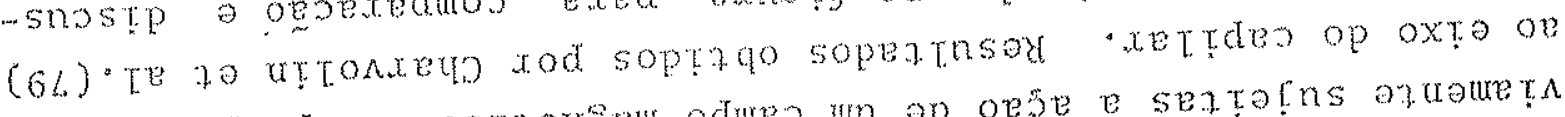

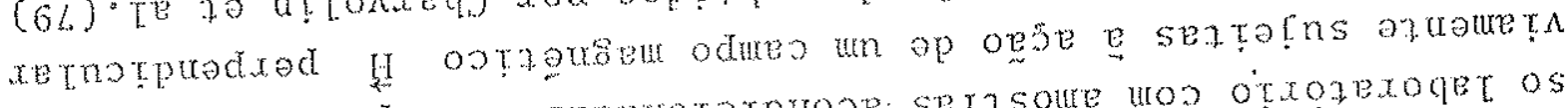

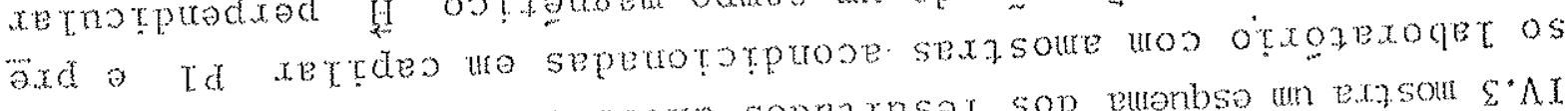

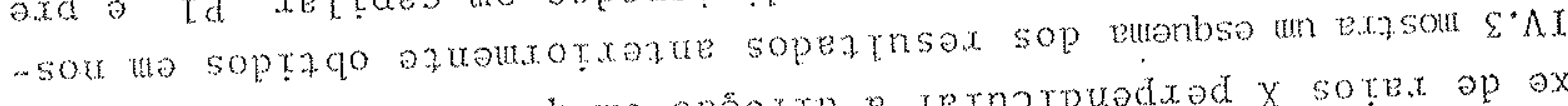
exnsty "

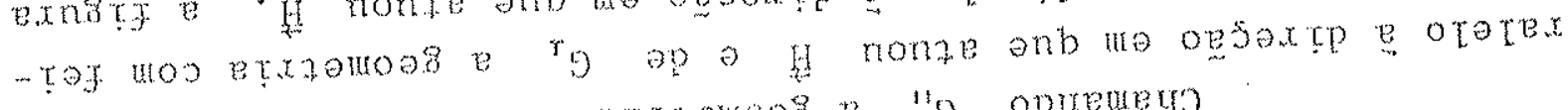

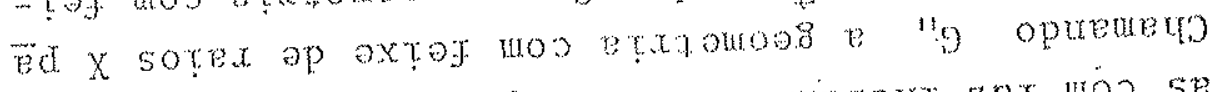

- HT oesatep ex optrptout znt wos seasta opuenb xet

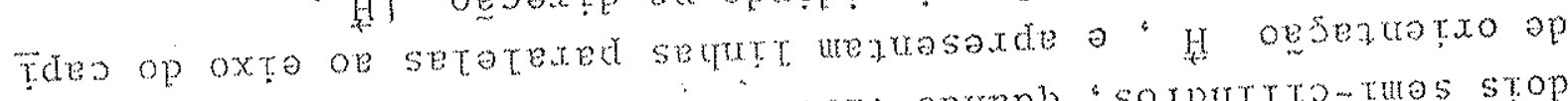

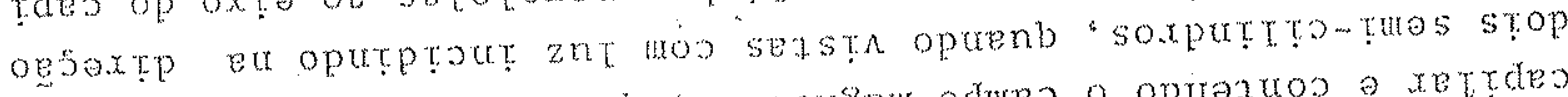

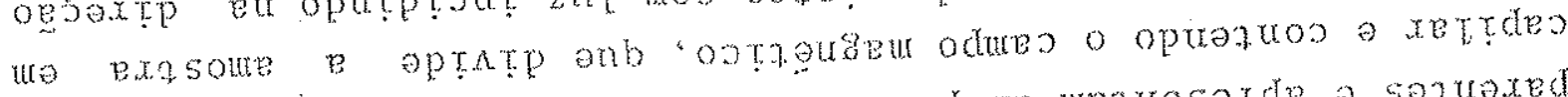

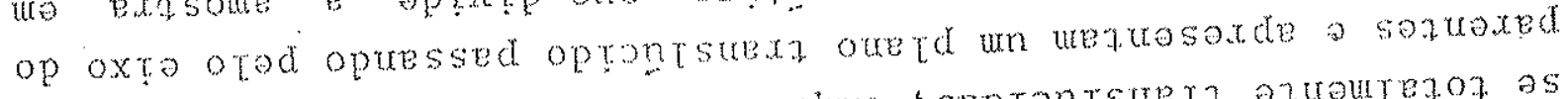

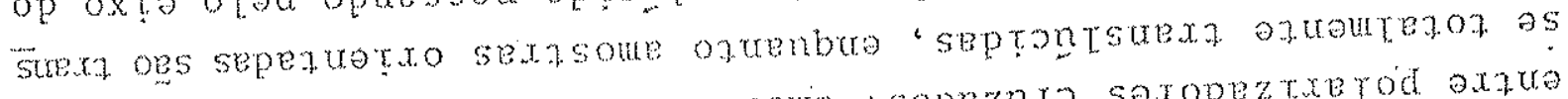

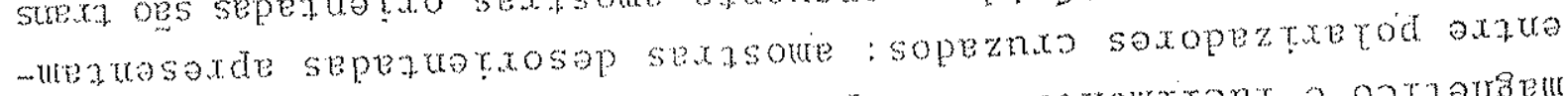

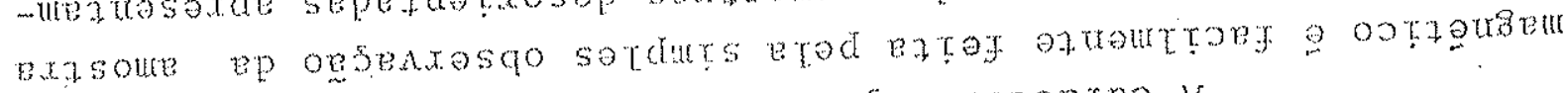

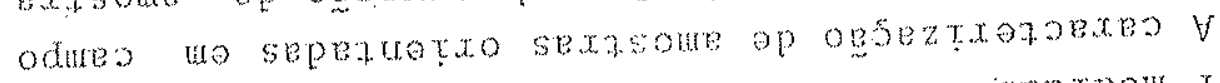

- XVS op septpom xtepurad axed o8

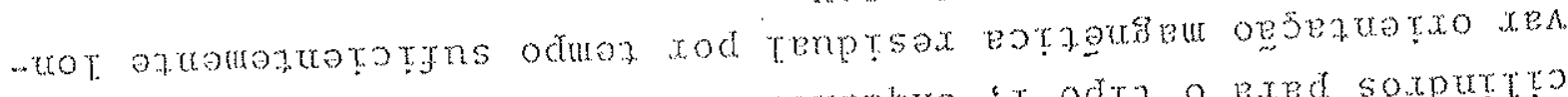

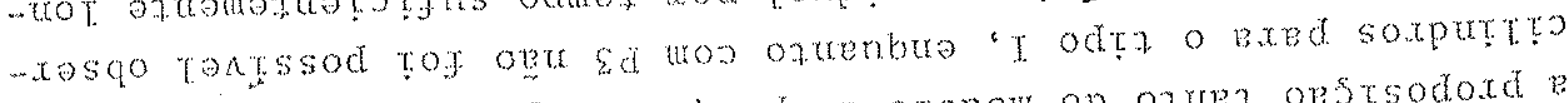

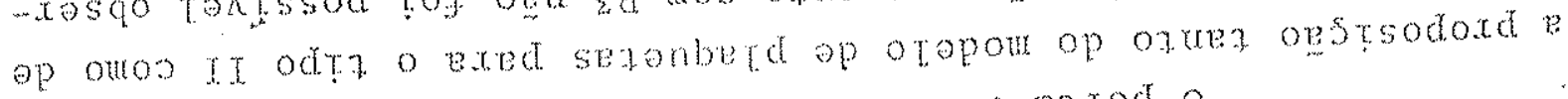

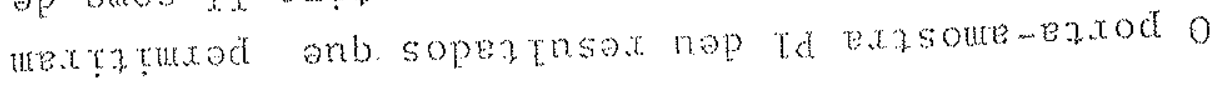

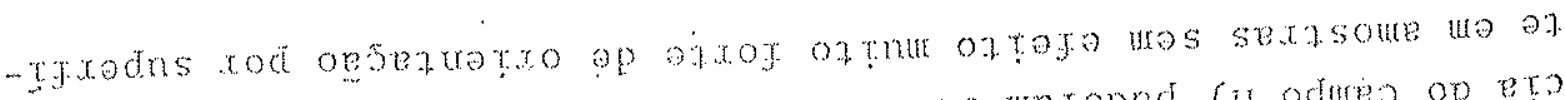

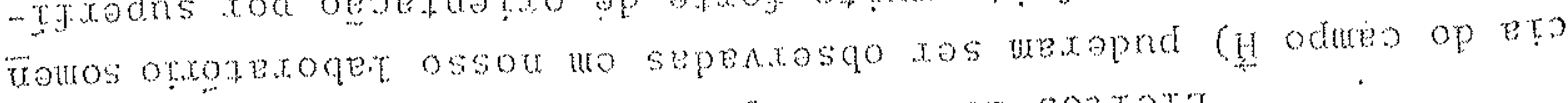

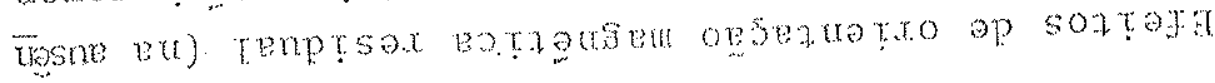

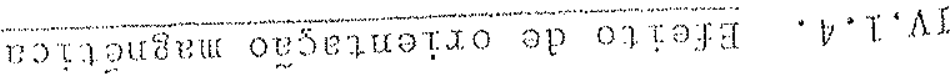




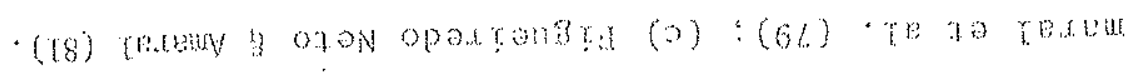

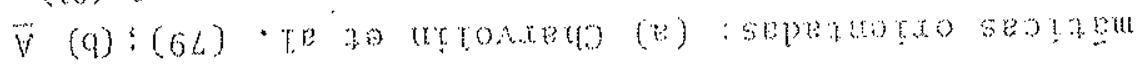

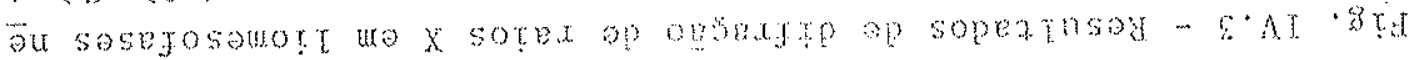

(D)

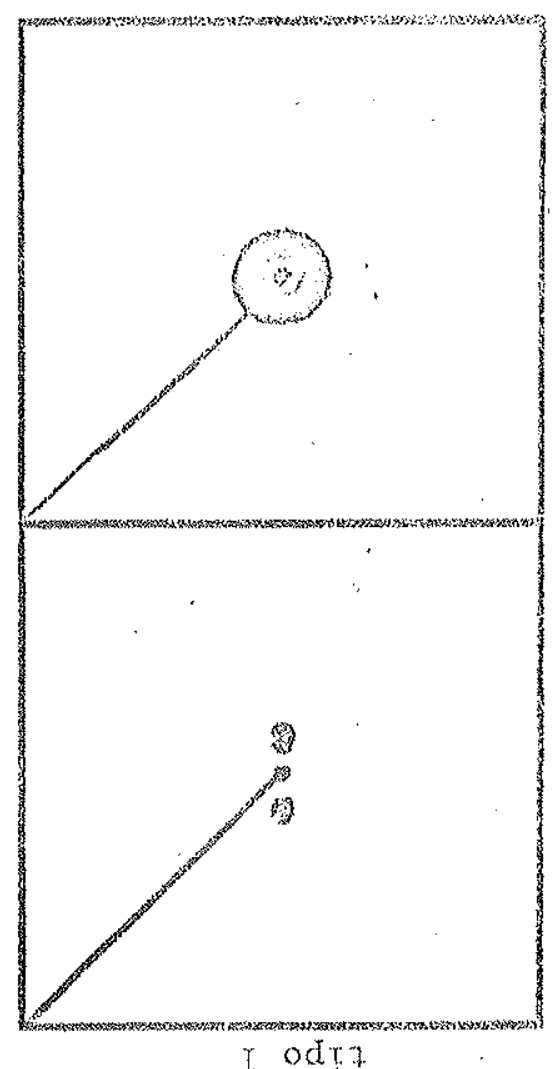

odr (a)

("o)

H

xu

(ro)

HT

$x$

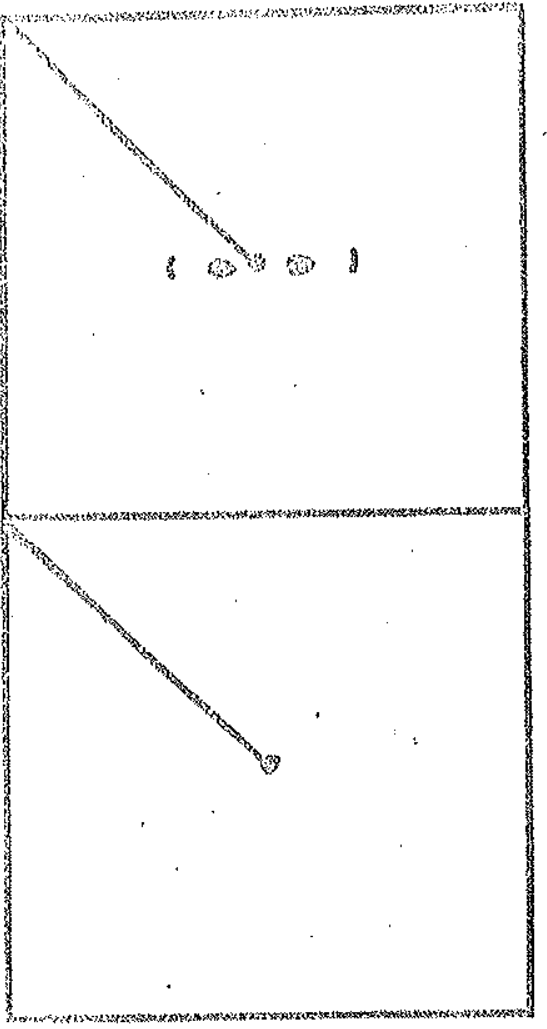

I. odta

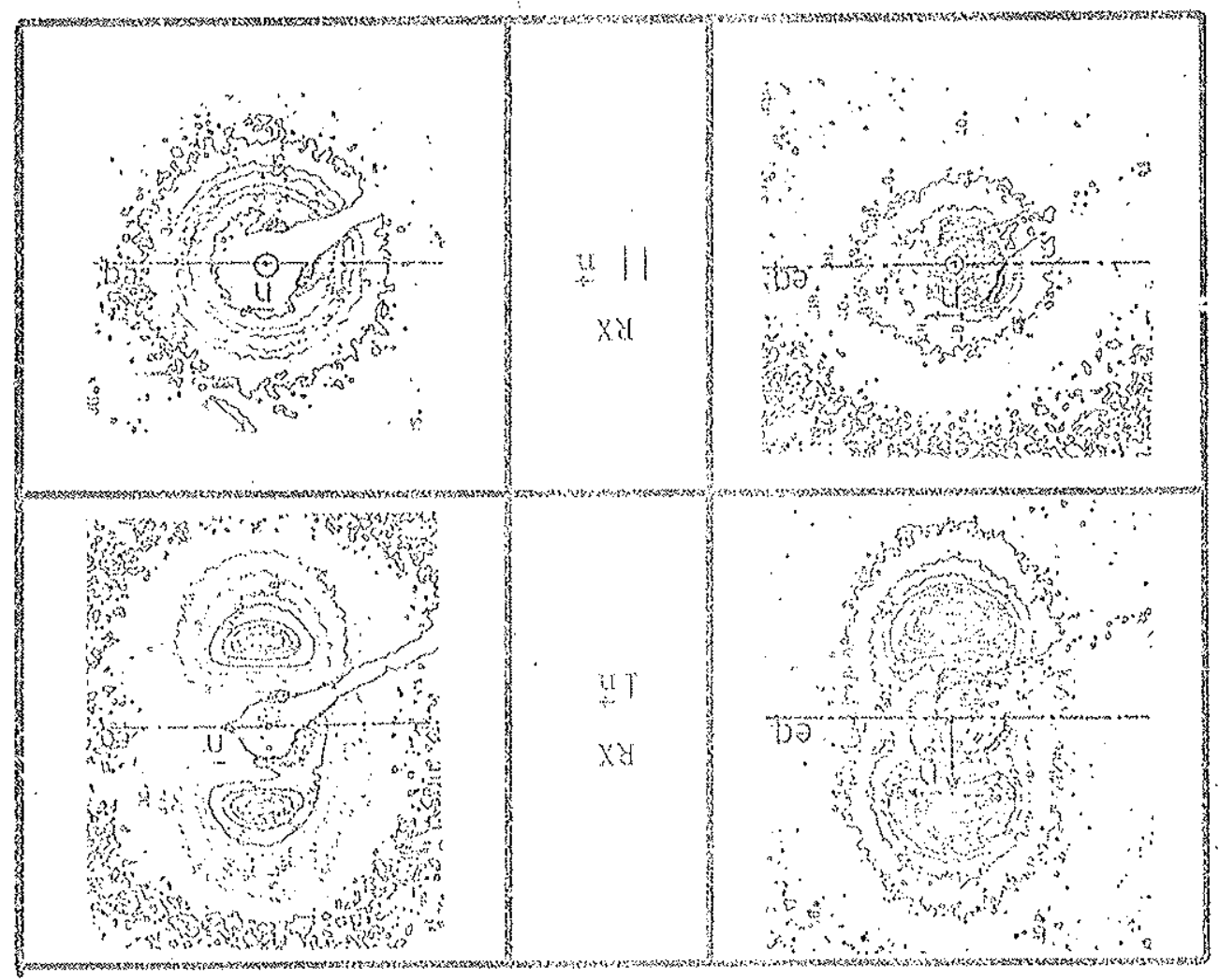

(b) 


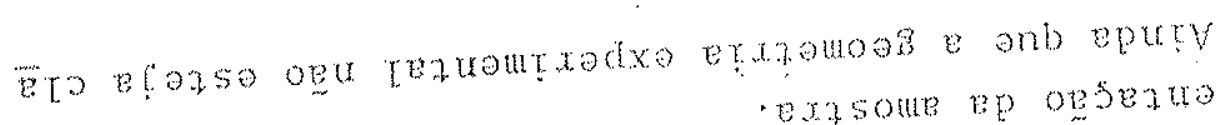

- exasour be opseanor.tosep b. optap

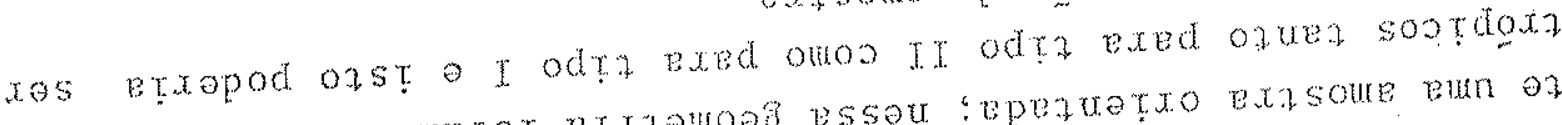

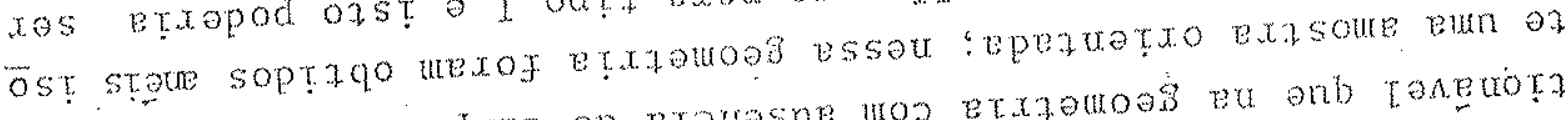

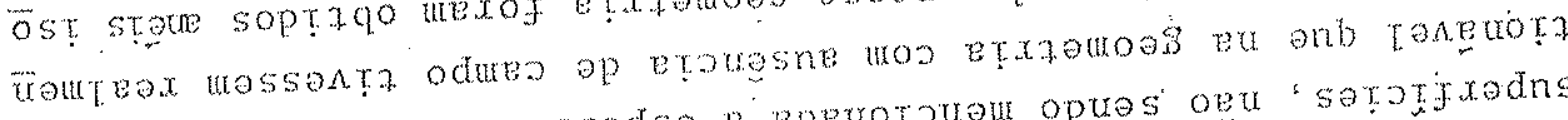

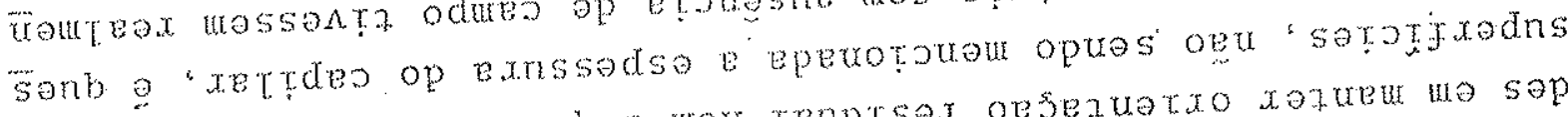

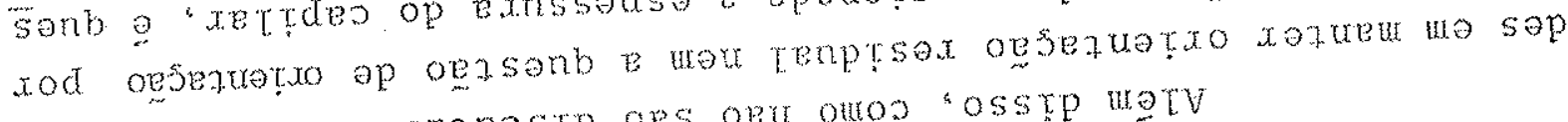

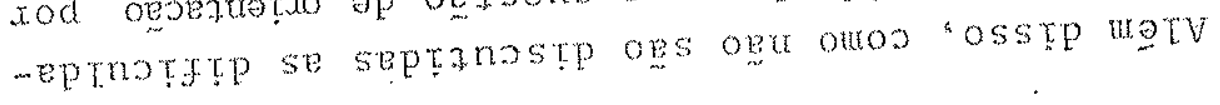

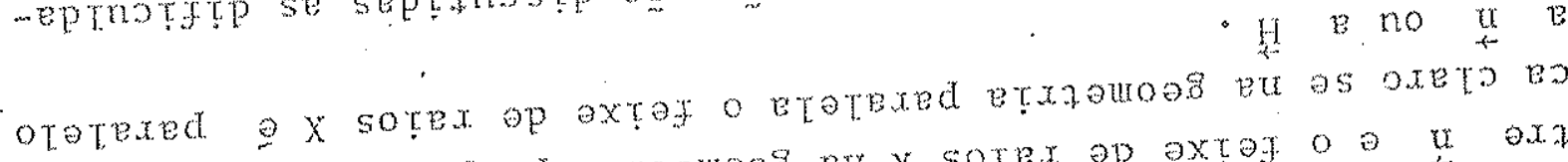

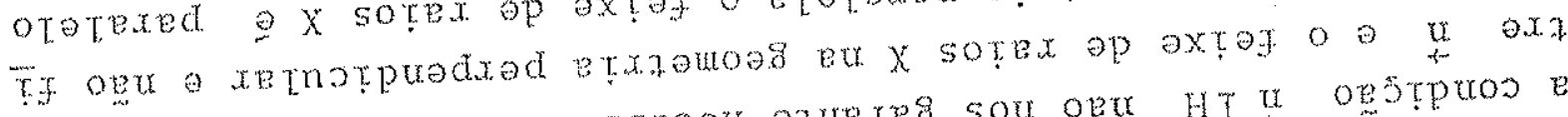

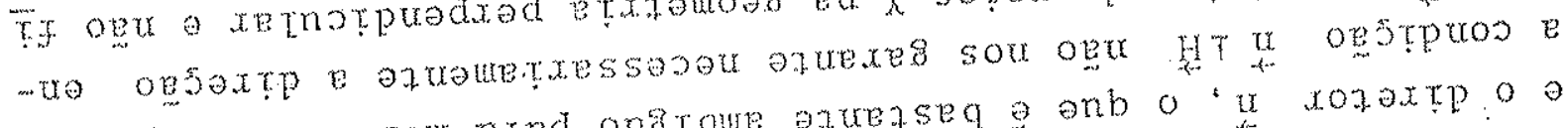

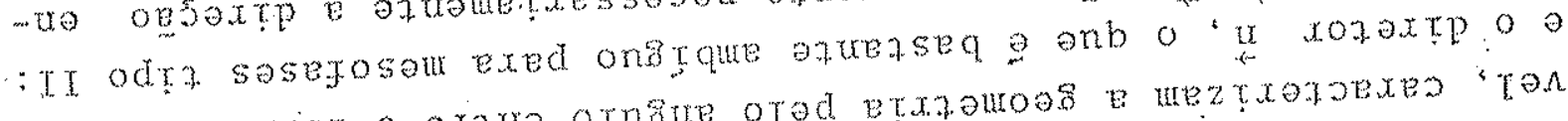

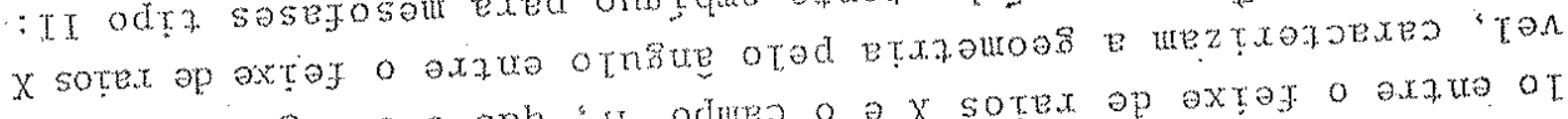

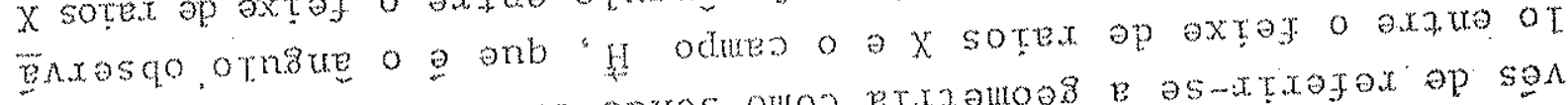

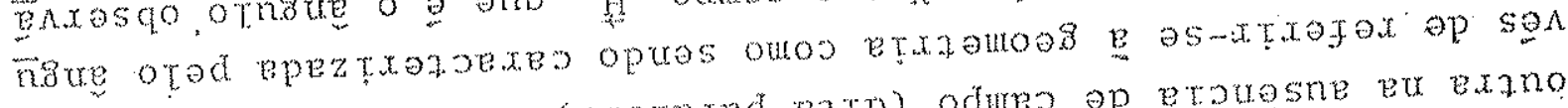

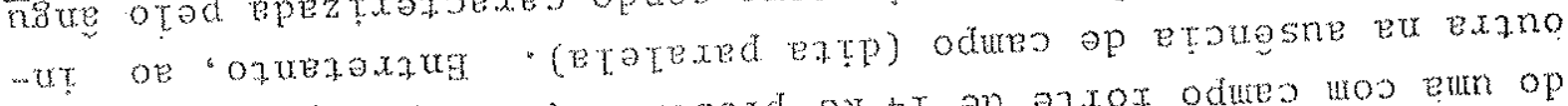

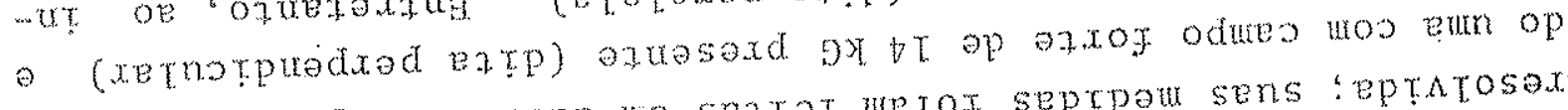

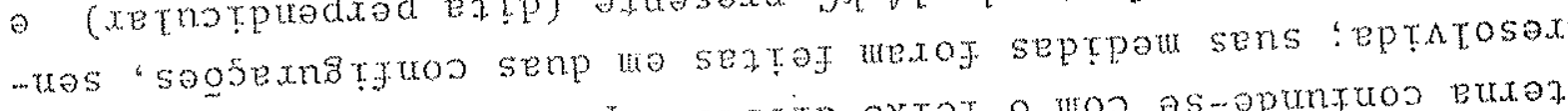
xas epod ogu Jg ofuezxod a ofaxp axtag o wos as-opunfuos buxaz -rt epreq ep oetsex g opro "onet op expues aun uros ppezteax rof sotrod smigte wa essot ep axate erouetadxa ens

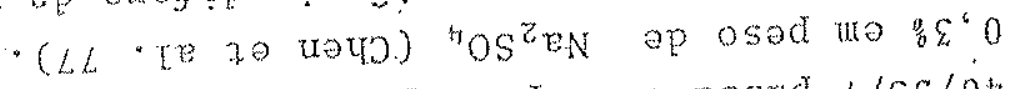

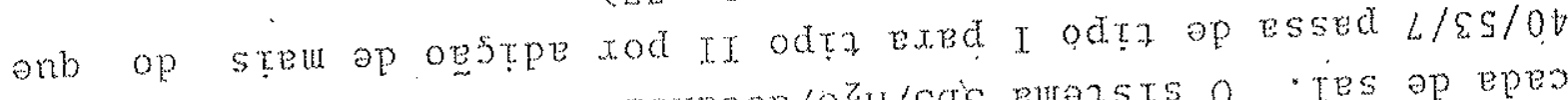

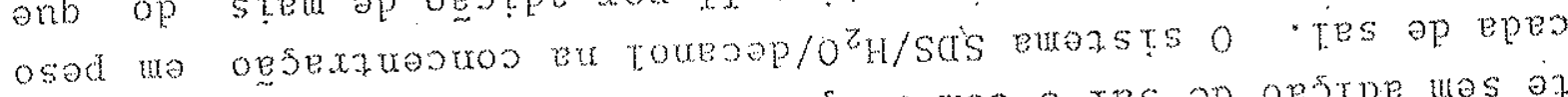

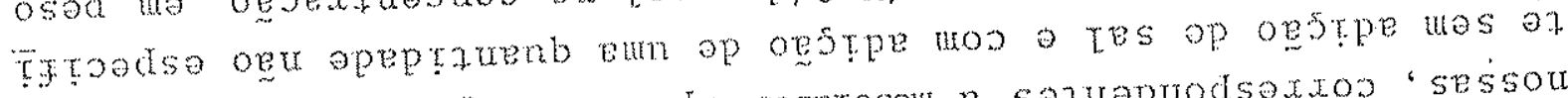

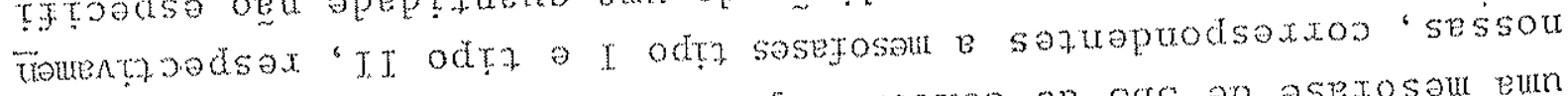

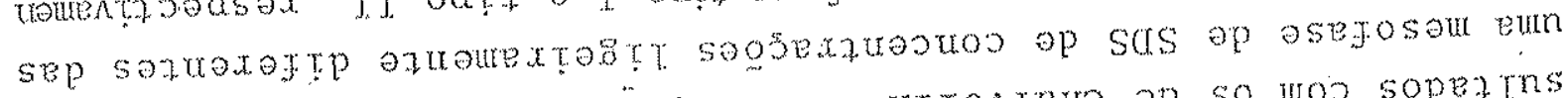

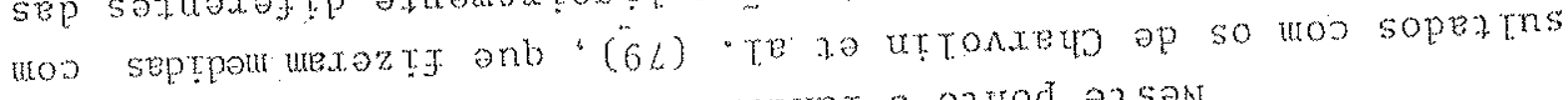

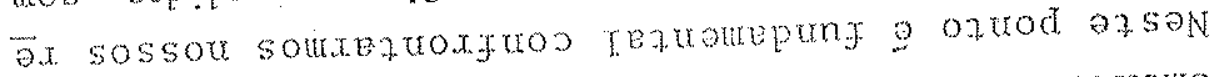

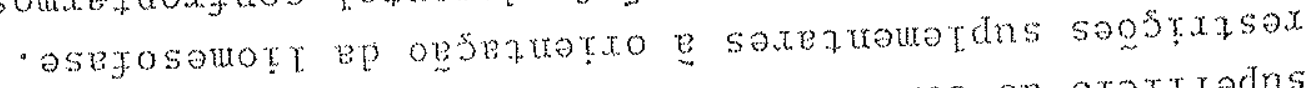

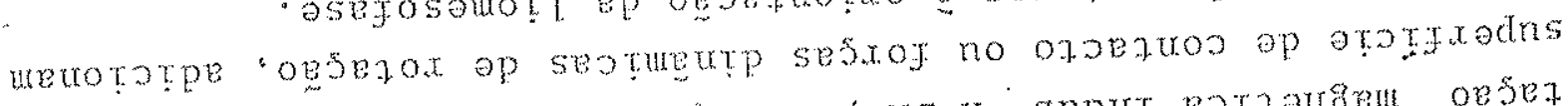

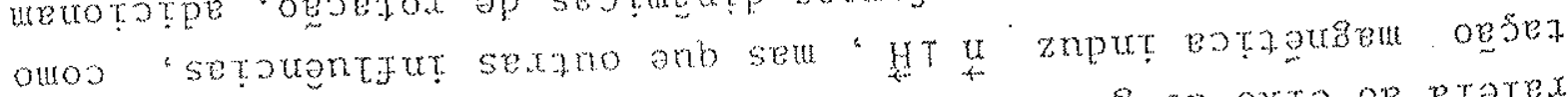

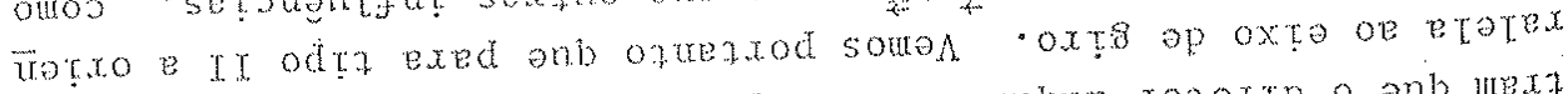

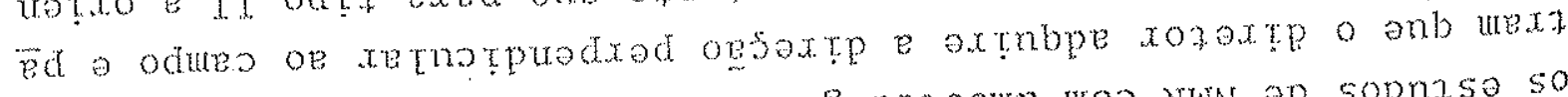

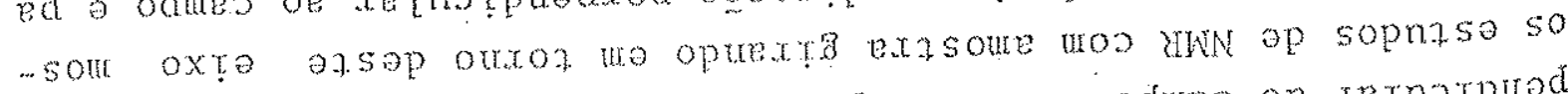

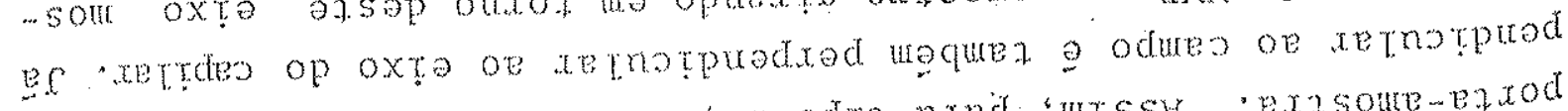

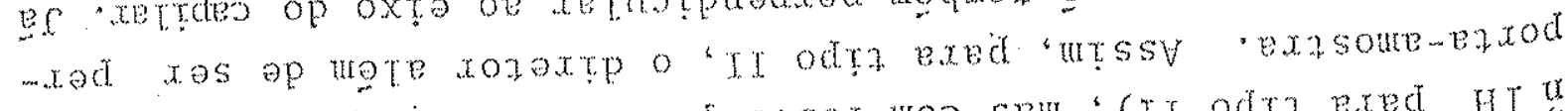

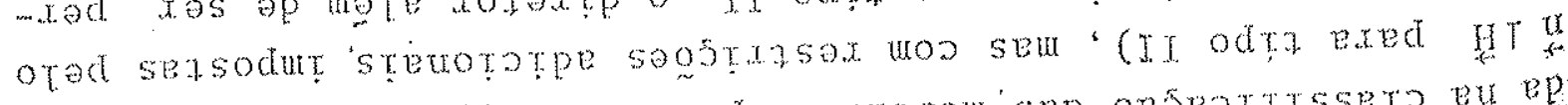

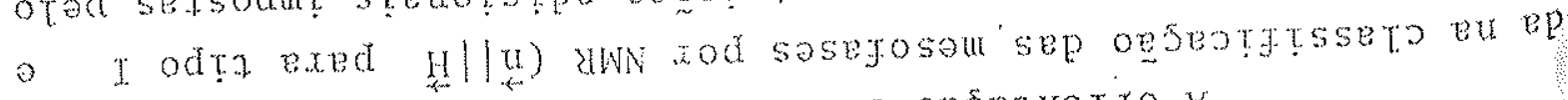

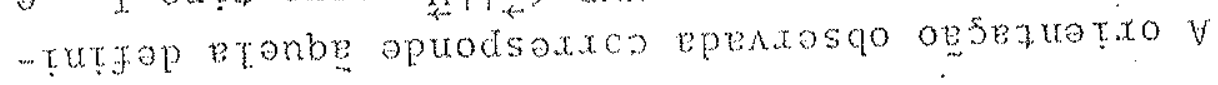


- opsoxp estun eumi u op

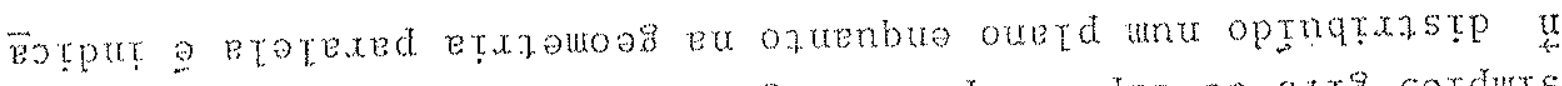

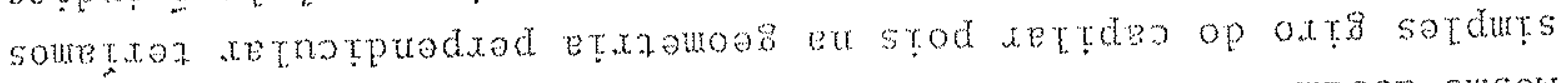
un rod septrqo opts xoz uretapod oex septpau senp se utsse ousan

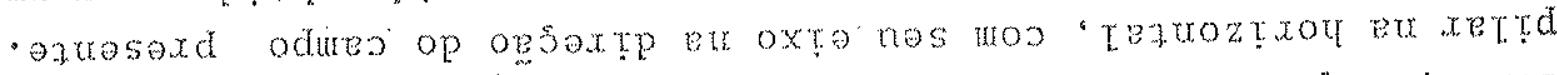

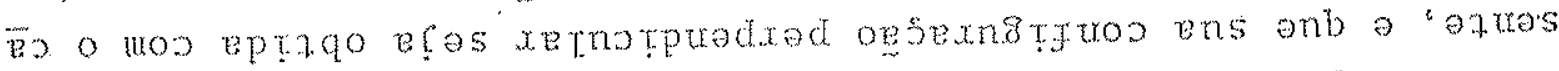

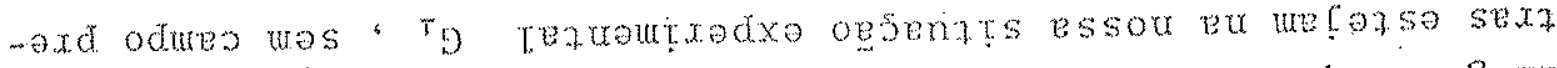

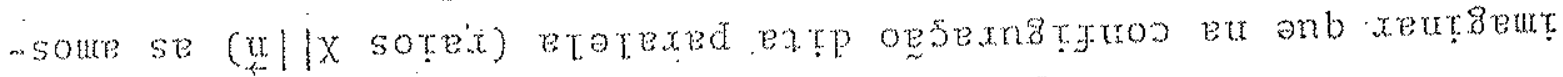
a sopeatrsox snes xetrdxe op opeptrqtssod extro

- OxTO

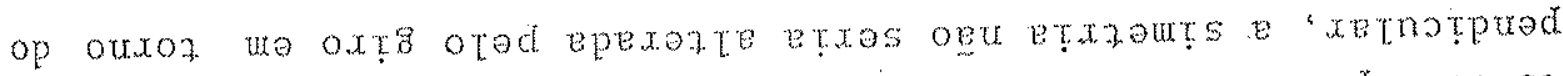

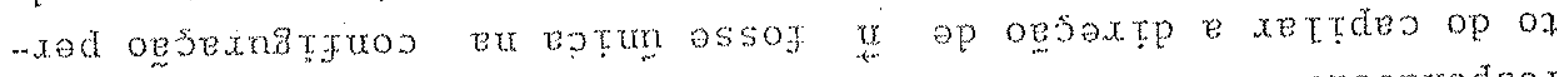

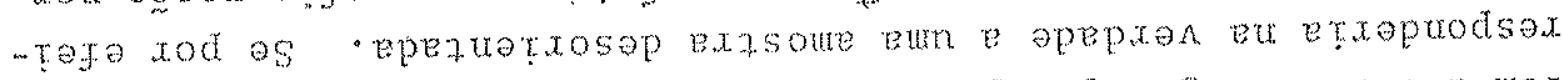

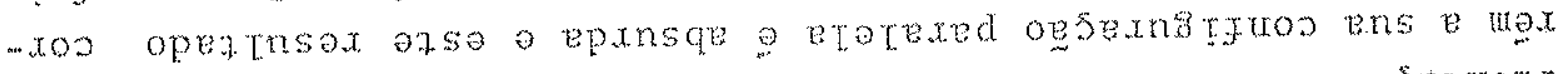

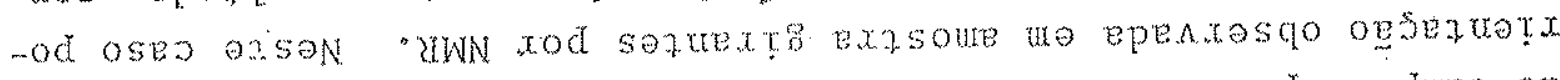

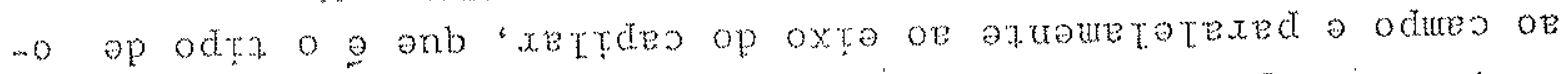

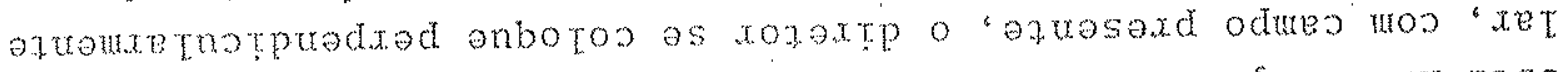

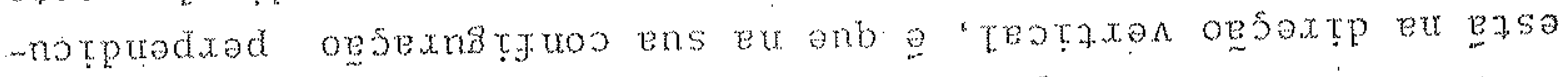

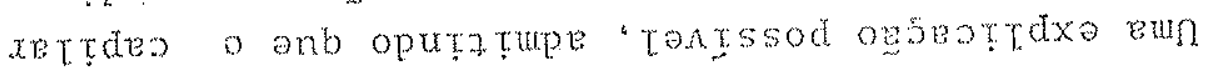

"Testr

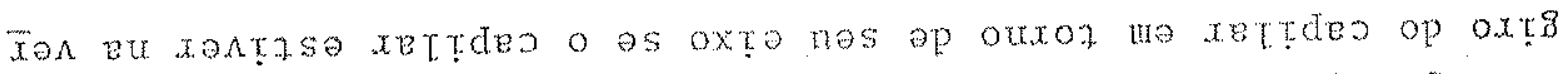

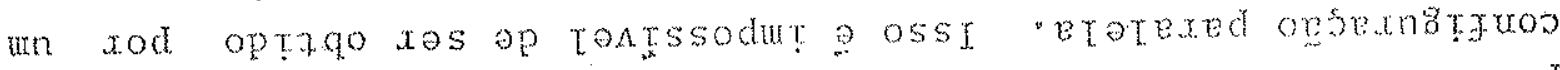

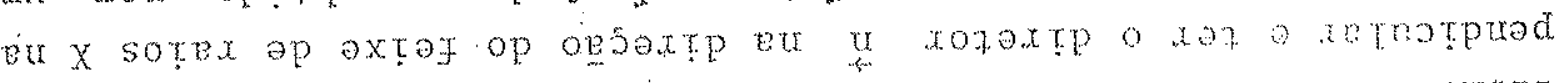

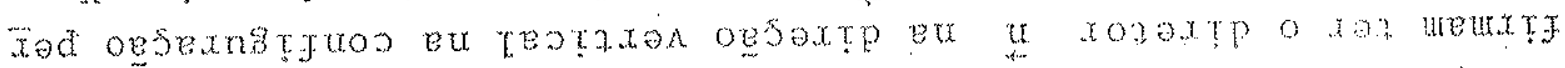

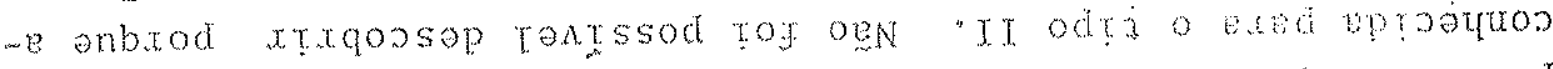

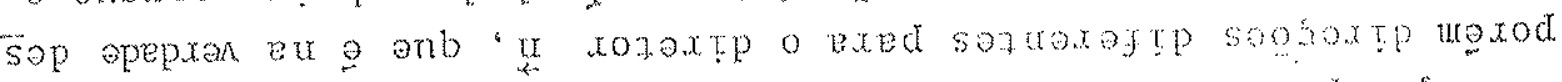

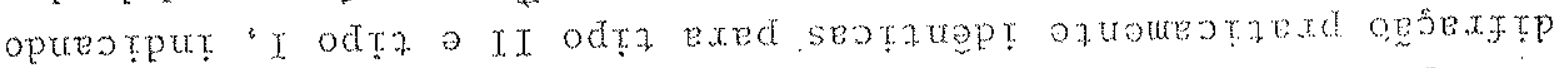

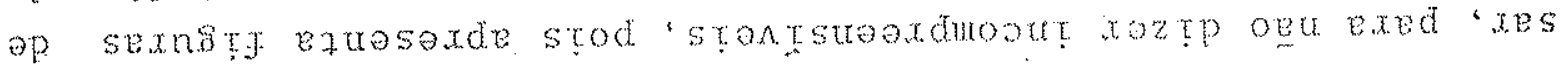

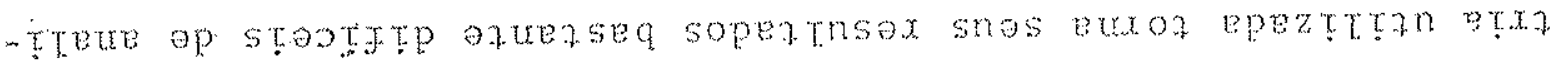

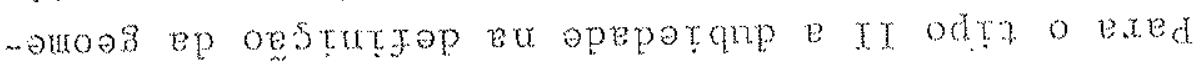

" (t8 tox

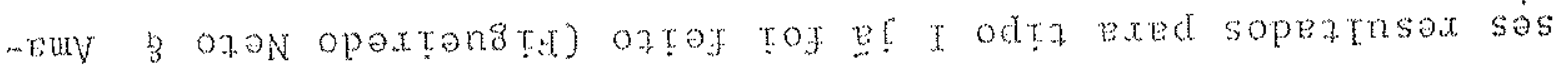

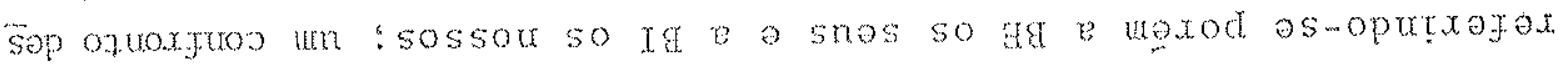

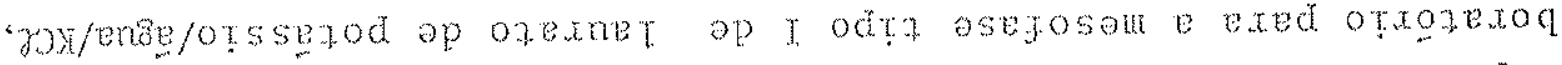

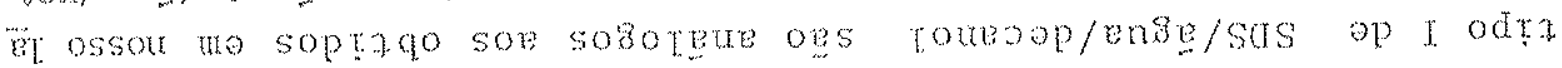

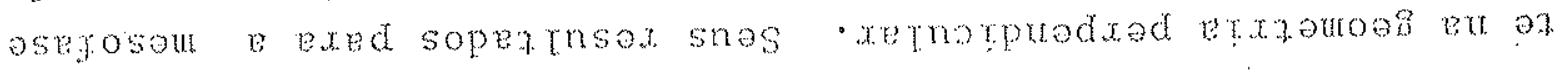

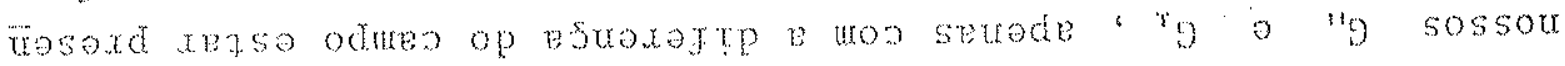

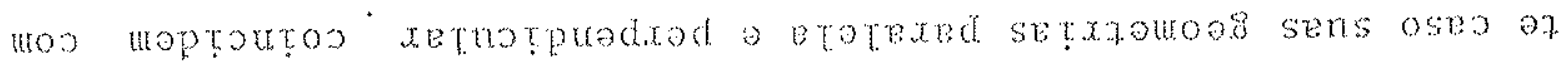

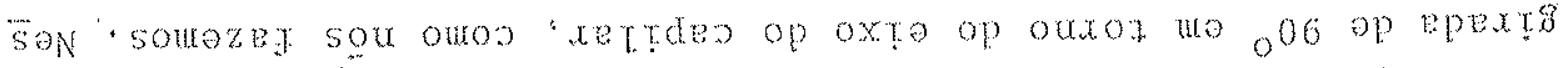

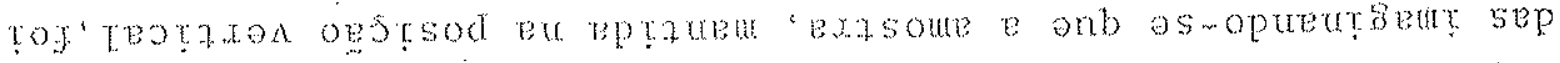

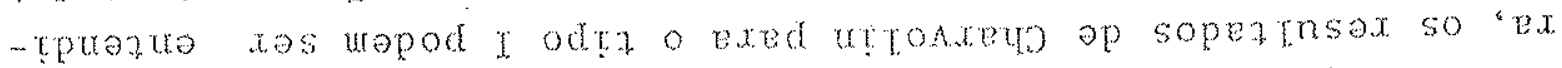




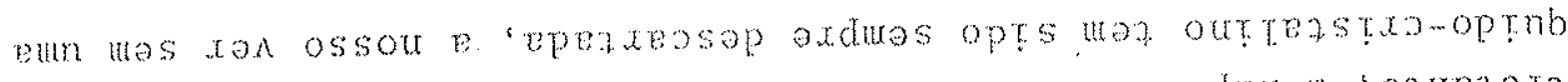

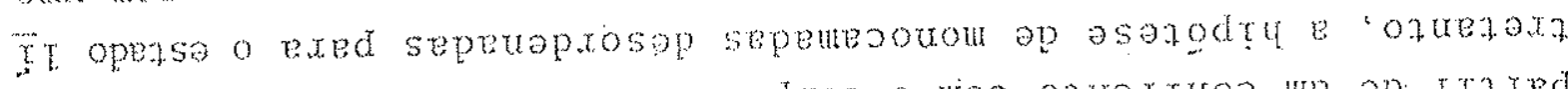

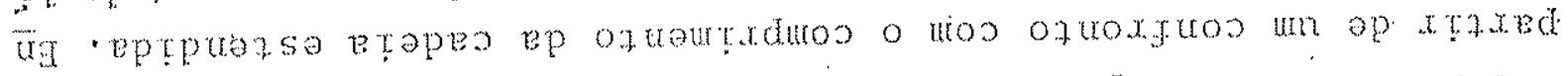

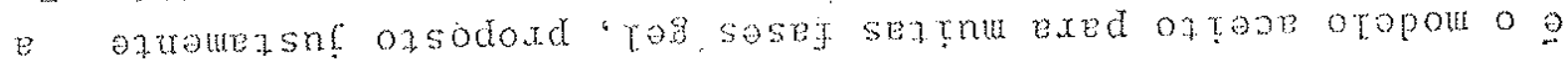

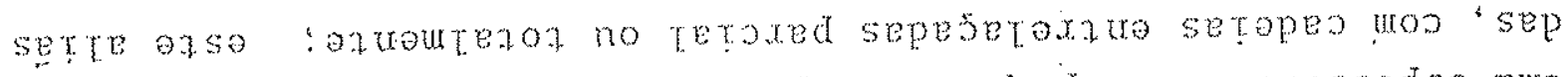

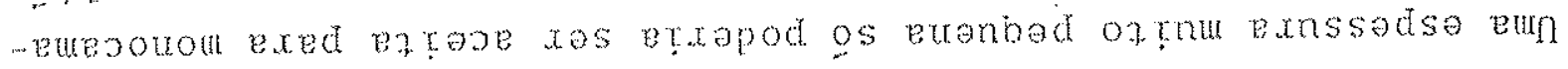

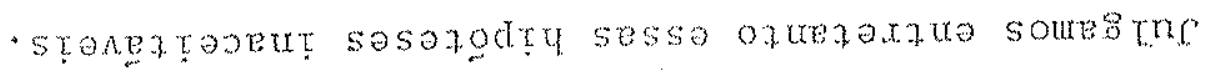

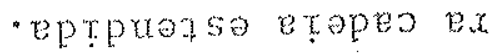

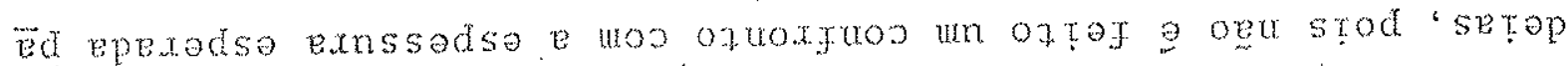

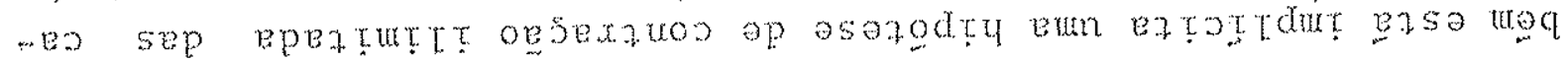

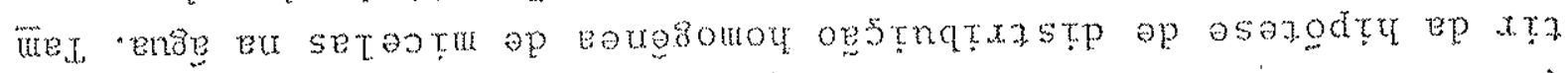

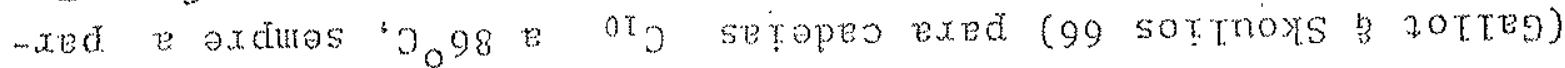
exnaxezt ex sepexaroste oes y oz op mepxo ep sepruterg op ex

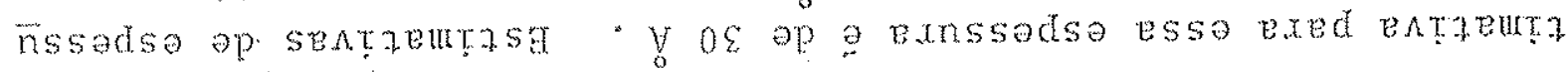

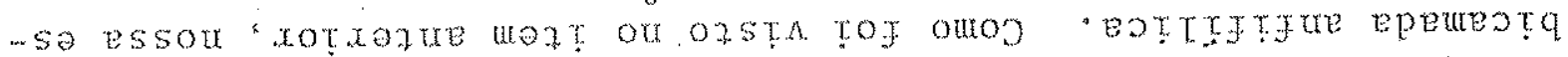
g y oz op exnssadse pun opirnquate "ense us seprngrastp ozum

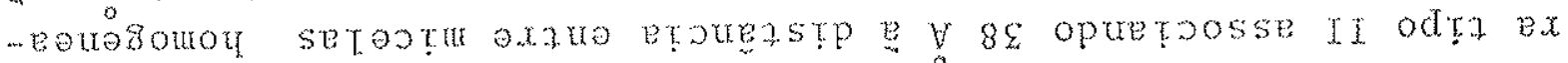

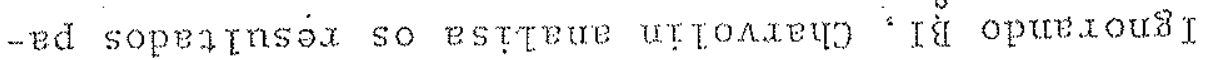

- Ha e strade waxojax as sopertnsar

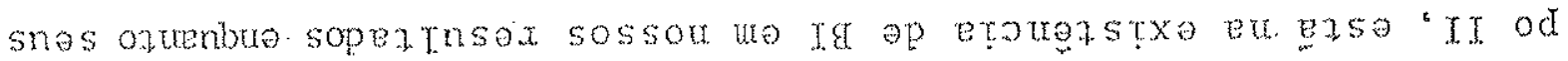

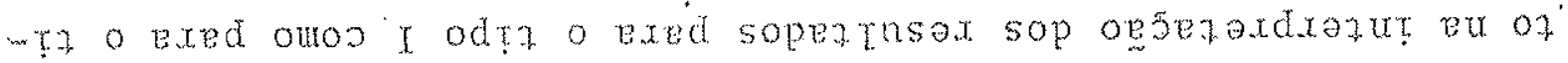

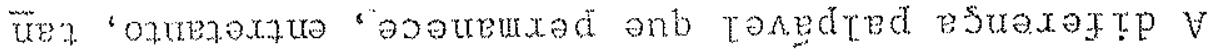

- oyteqexz ou seperotoneu opu seque "xetrdeo op tetrazeur

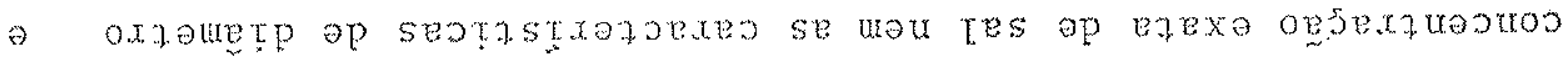

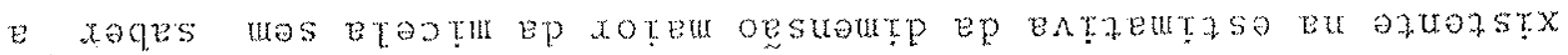

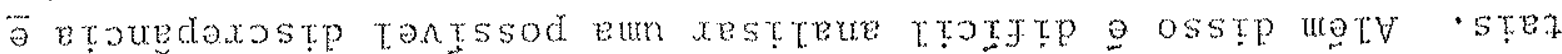

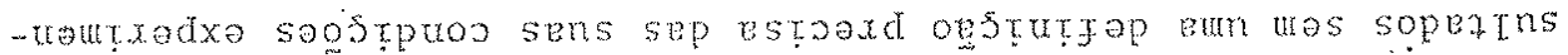

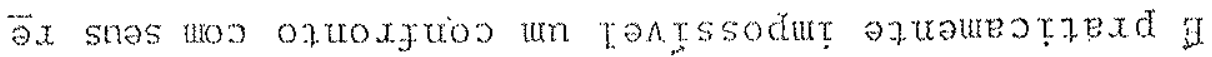

- retexed opsexnstyros op opeatnsax

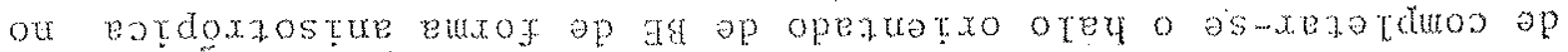

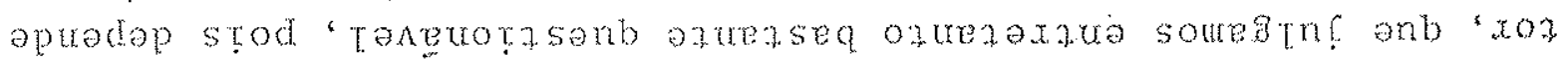

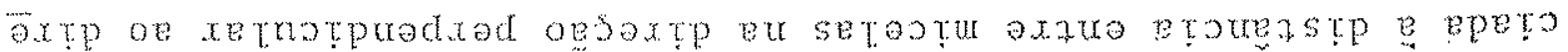

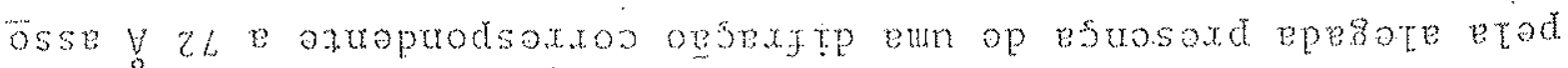

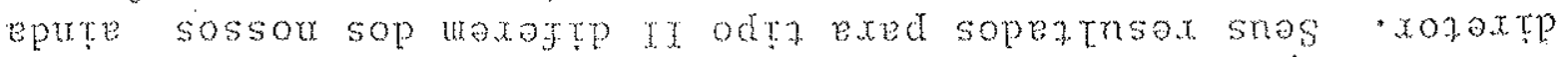

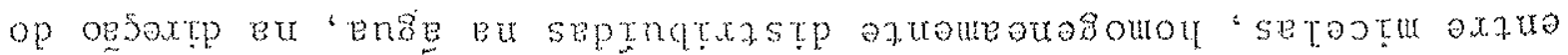

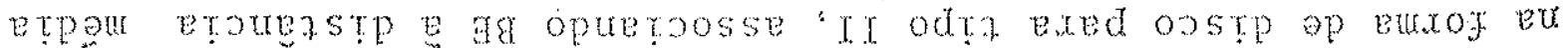

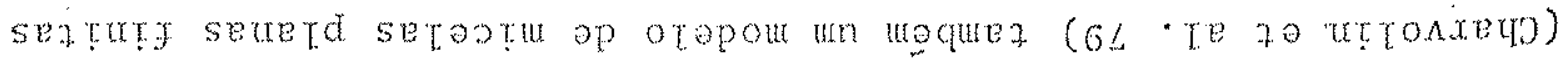

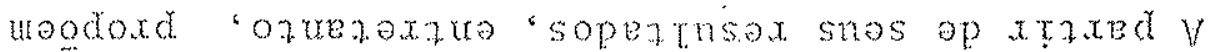

- $x$ sopex rod septpat sut r odta sesejosoll

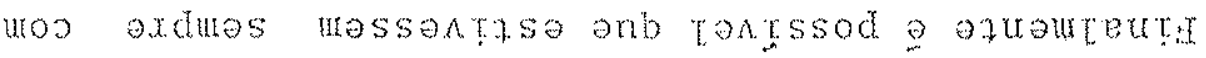




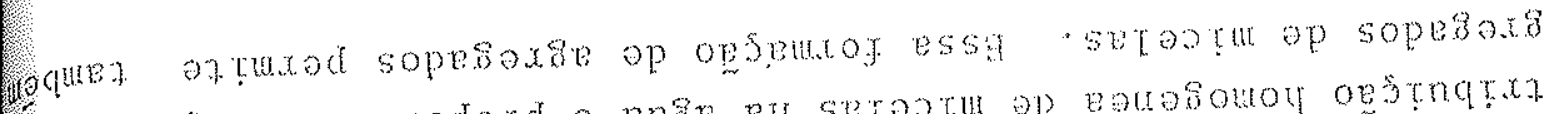

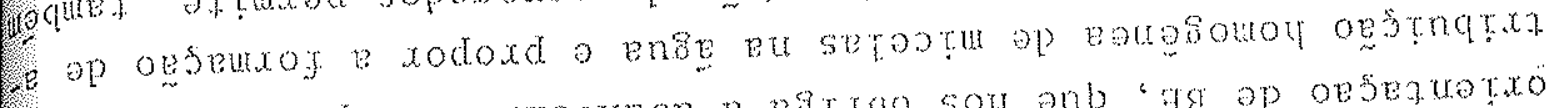

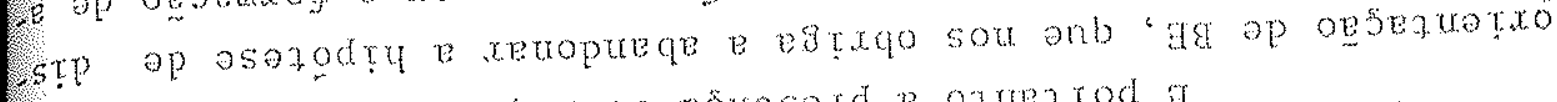

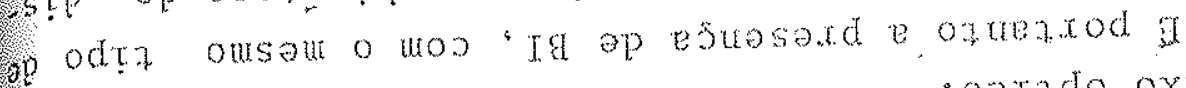

- ostrde oxte op remxor ou

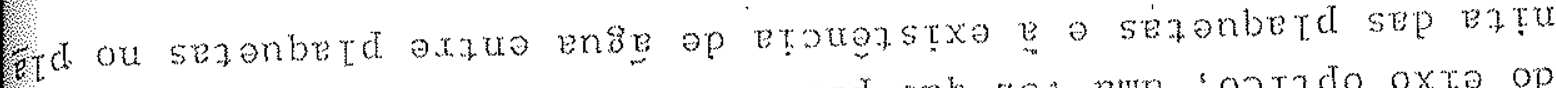

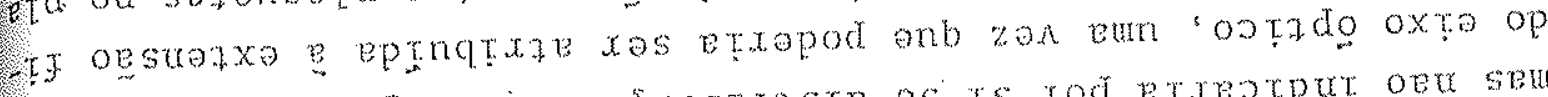

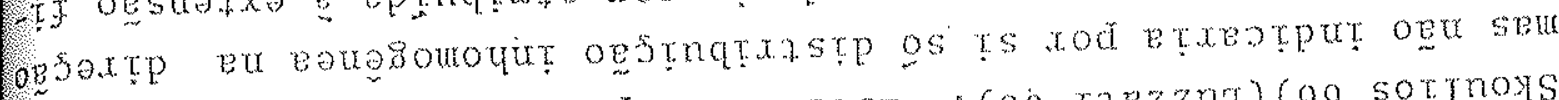

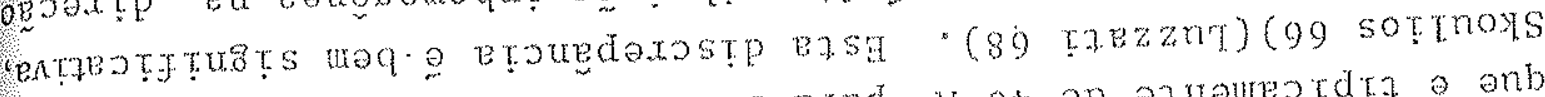

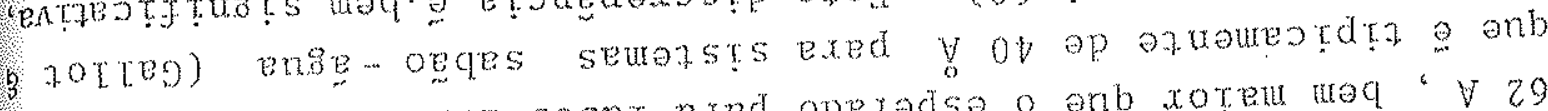

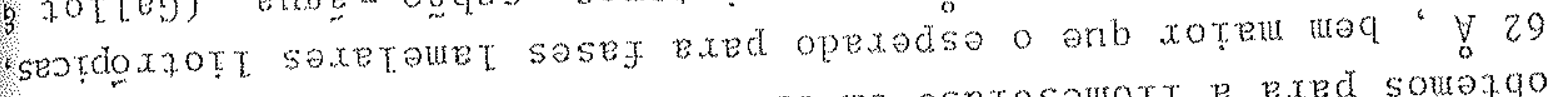

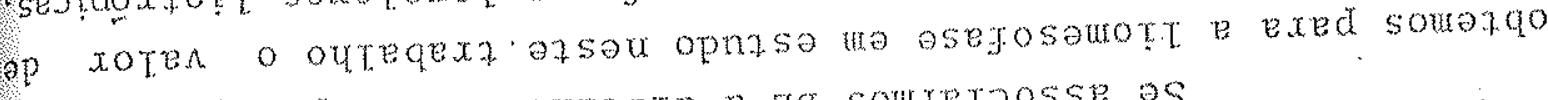

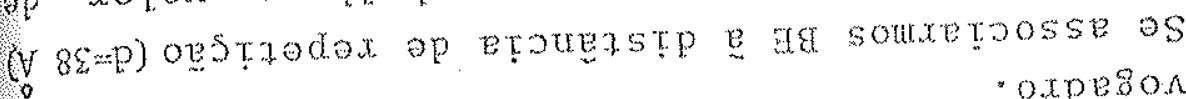

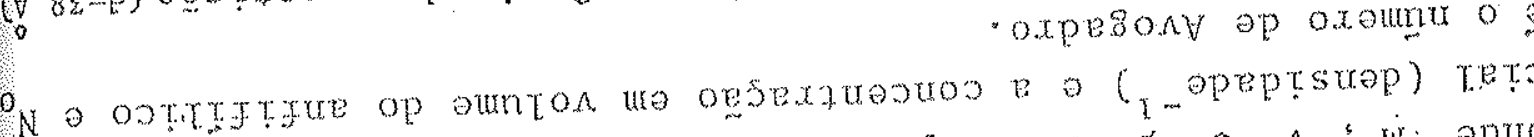

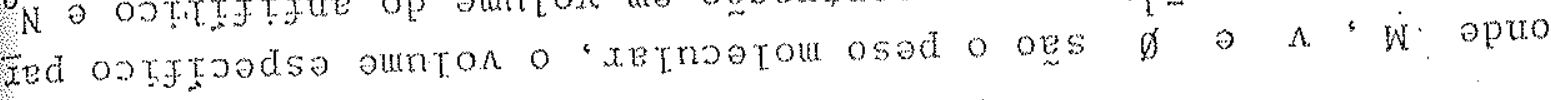

$$
\frac{0 P^{O} N}{\Lambda ?}=Y
$$

: (89 trazner) (99 sottroys

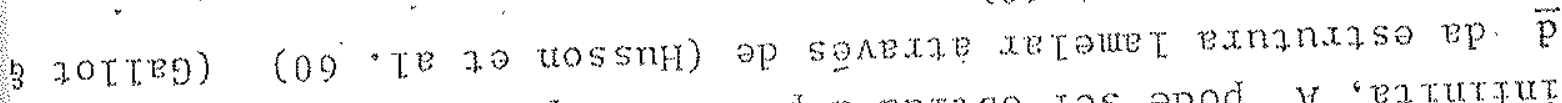

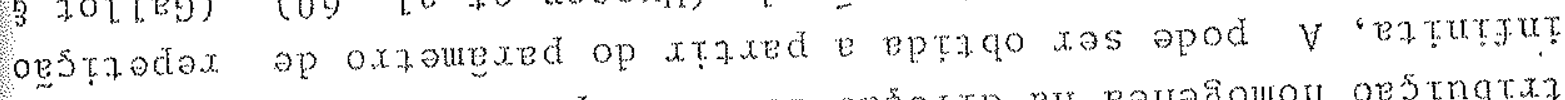

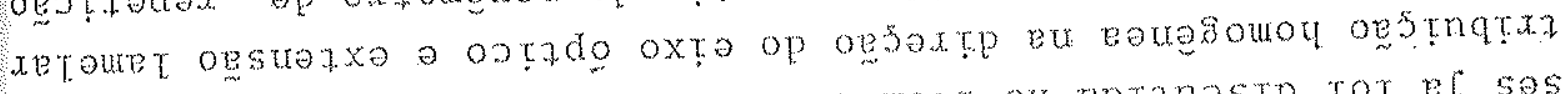

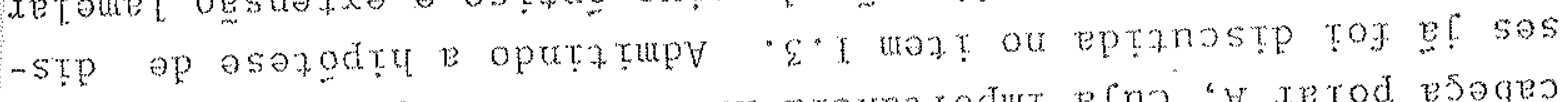

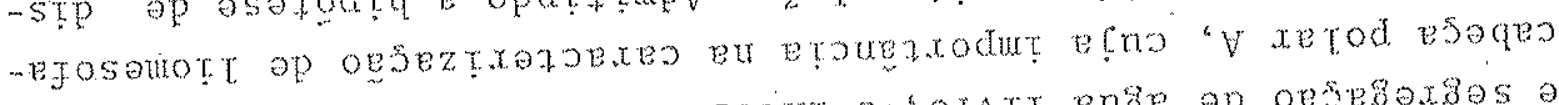

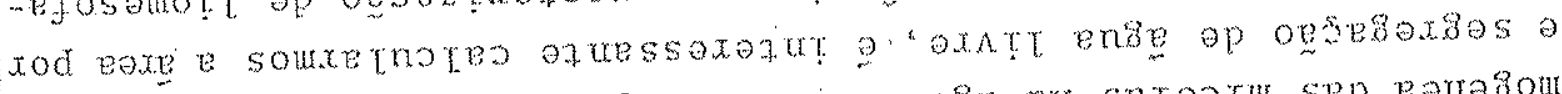

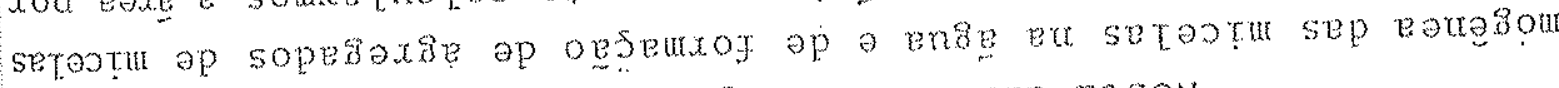
oy opu opongtaste ap azsodoxd ep opssnostp eason

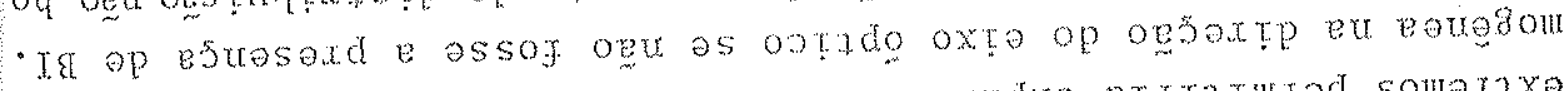

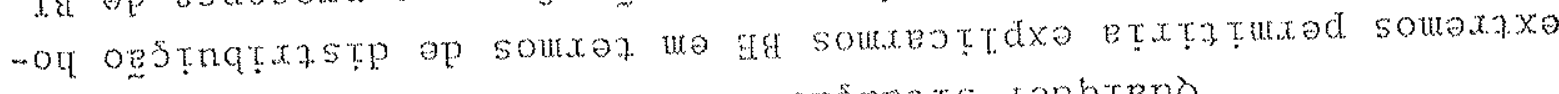

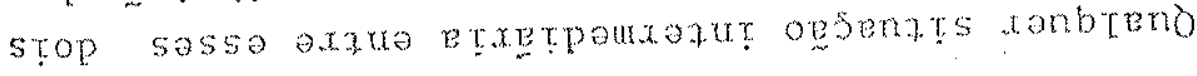

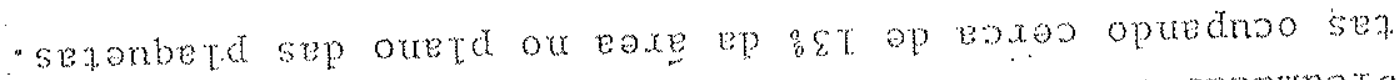

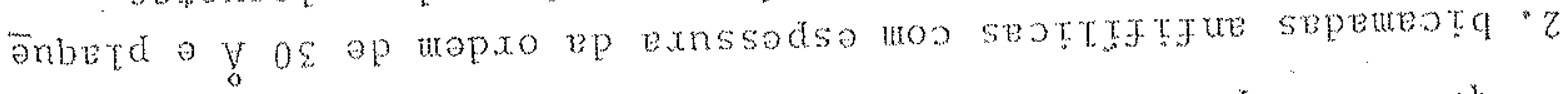
stanbetd sep oreta ou baxe ap at ap exas optrednoo sezonb

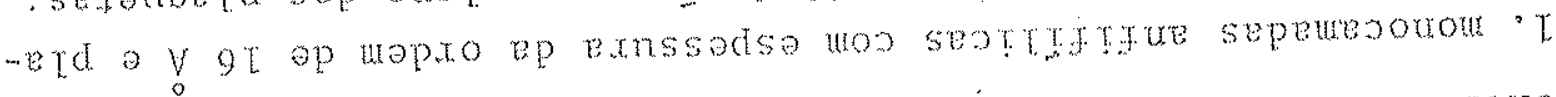

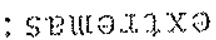

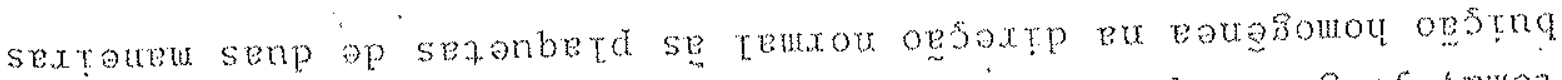

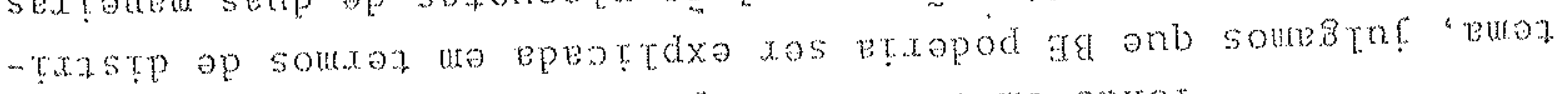
sts ossou op ounton we opstsoduos e bast me optad 


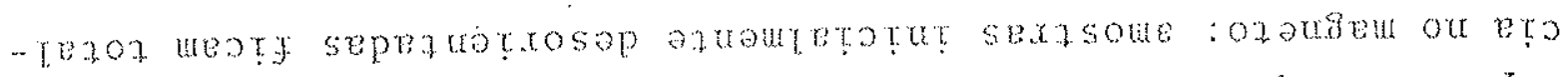

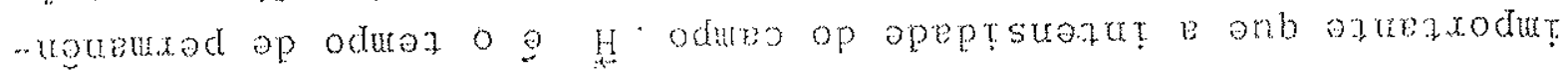

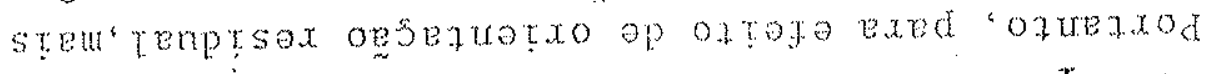

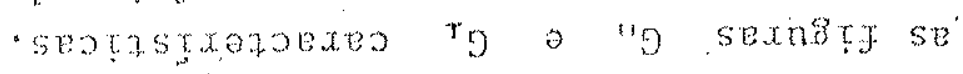

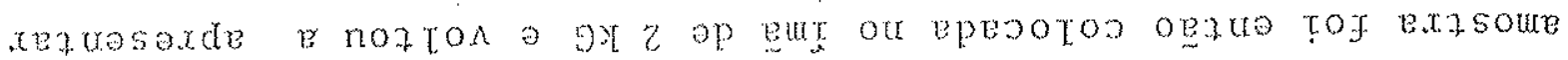

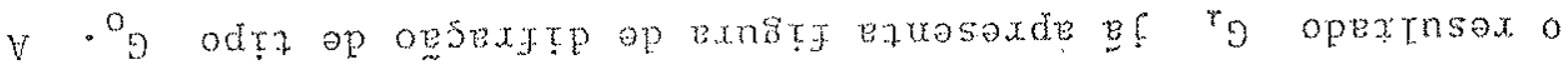
a eptprad roj tenptsox opsenuatro e em op exof setp sunge sod

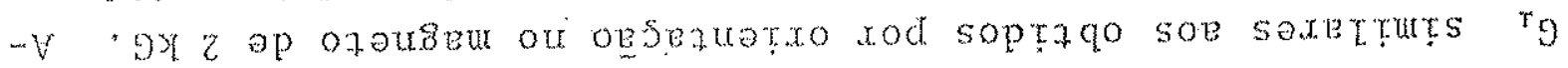

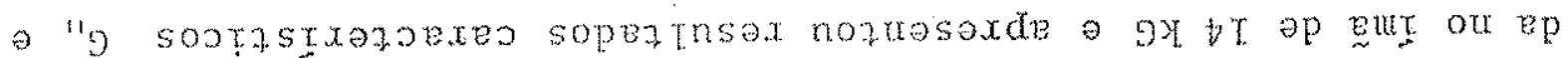

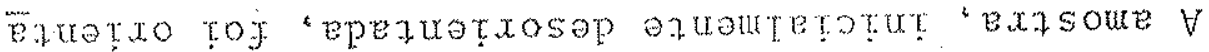

$S \cdot \Lambda J$ exngtg

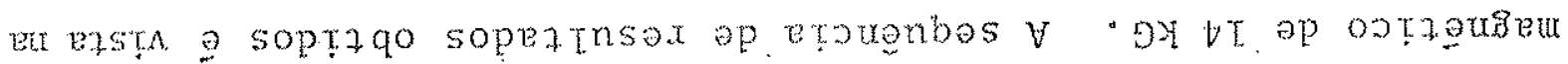
odues un asmopteztran eptadax rof ta xertes ou epezratxo ext.

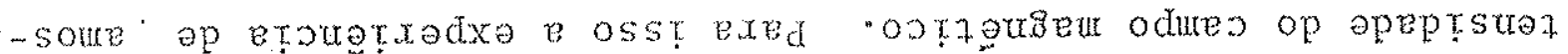

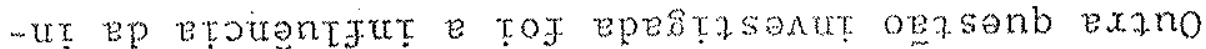

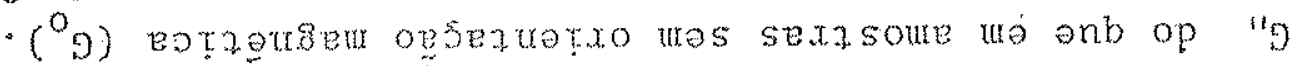

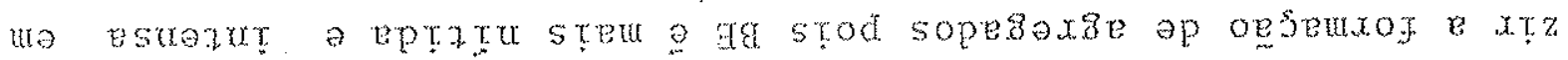
mpur essod odues op esuesexd b anb ap epure sototpur ex ostat

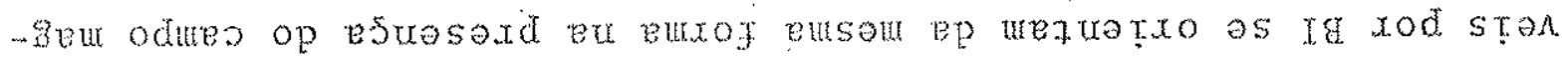

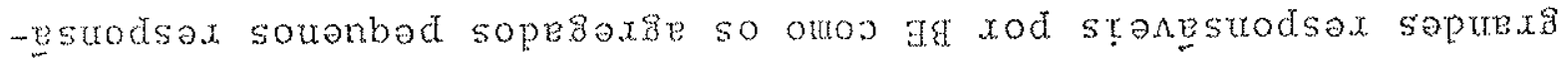
sopesenge so oztrez enb ozuzuod amtanos sourapod

- ozaxp axtaj o eav esayo onb

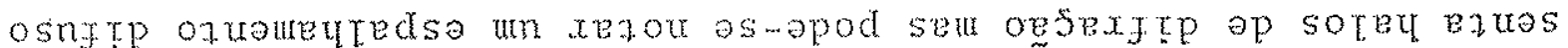

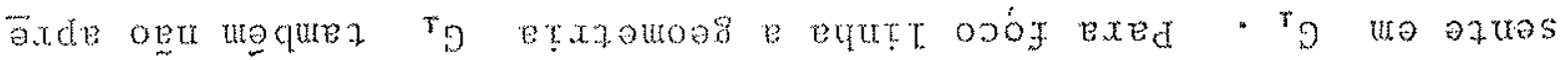

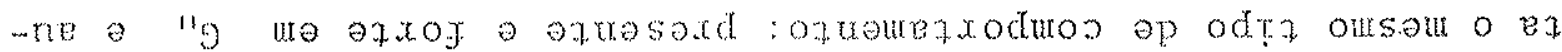

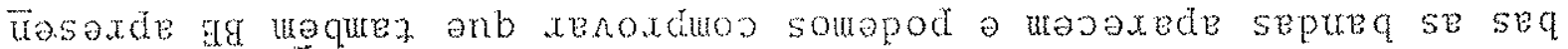

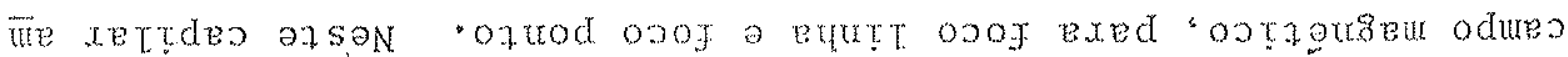

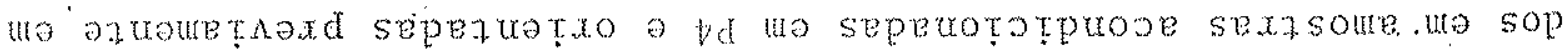

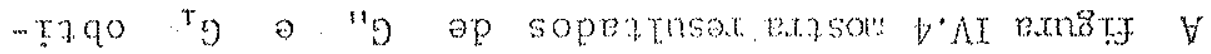

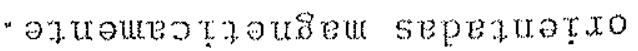

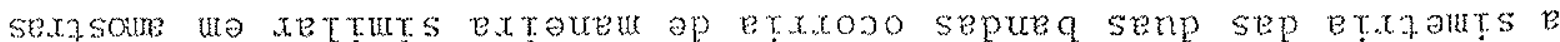

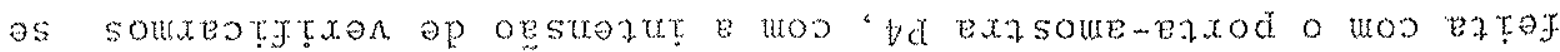

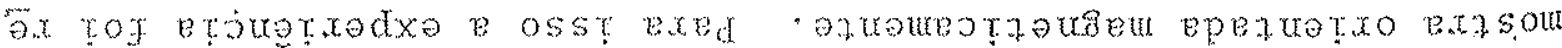

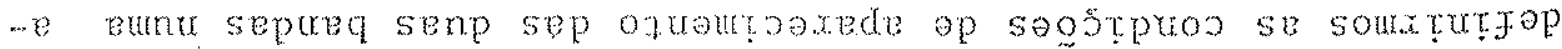

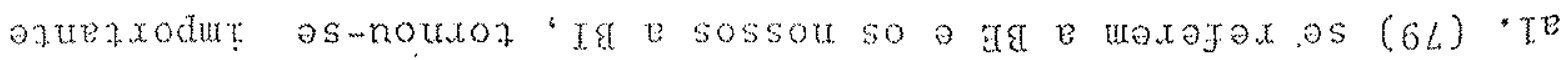

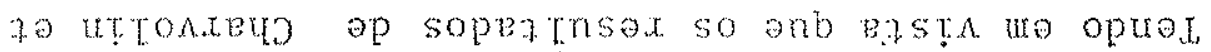

SEPTPOU SB.RON $\cdot \mathcal{C}$

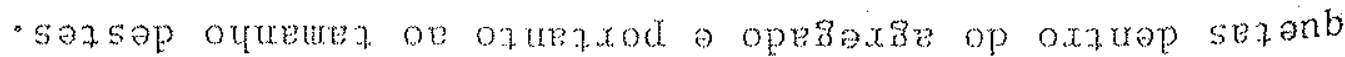

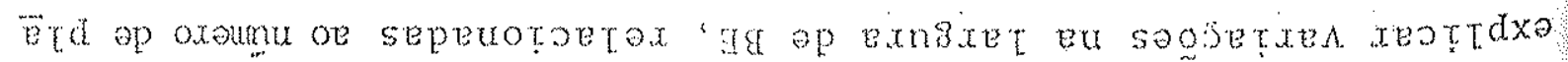




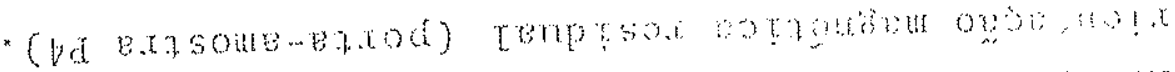

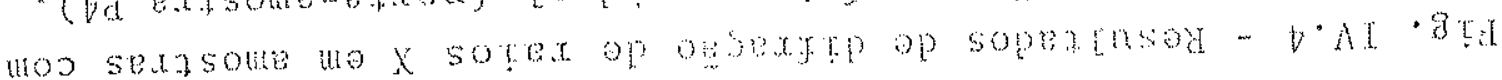
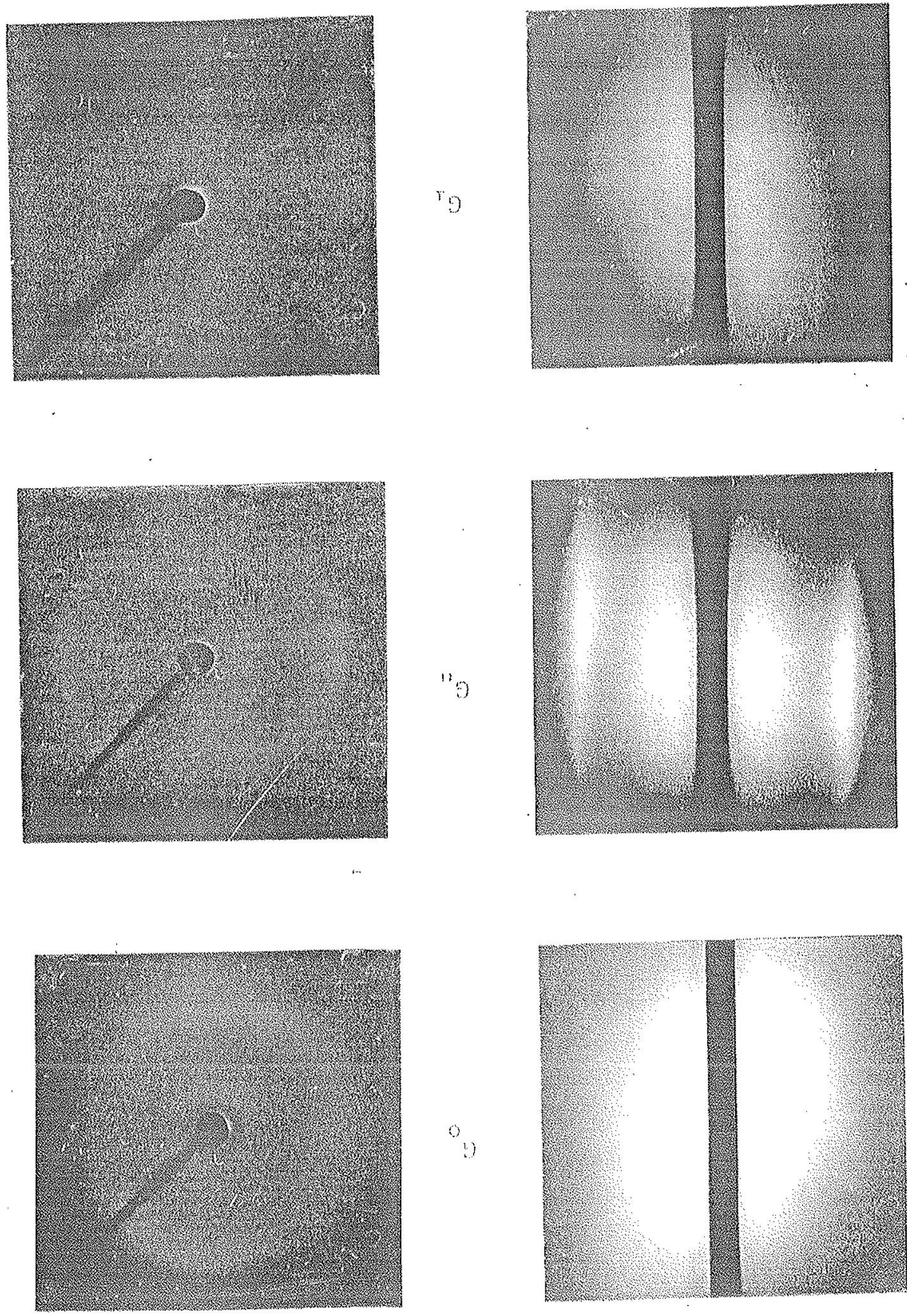

()

$0, x \operatorname{rod} 00, \mathrm{r}$

But? opor 


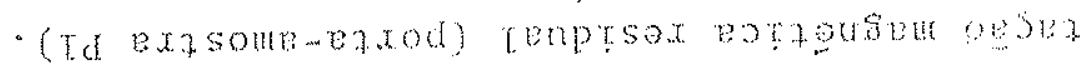

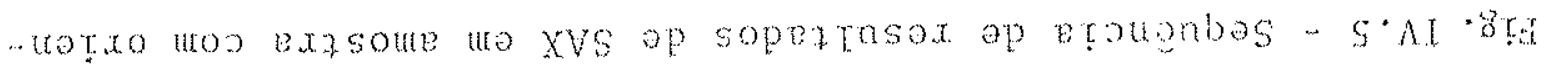

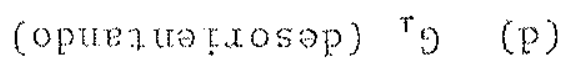

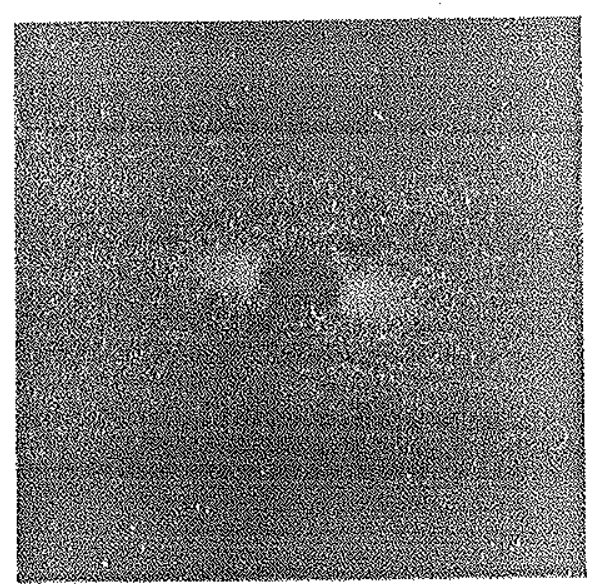

$(5 x z)^{\top} 0(0)$

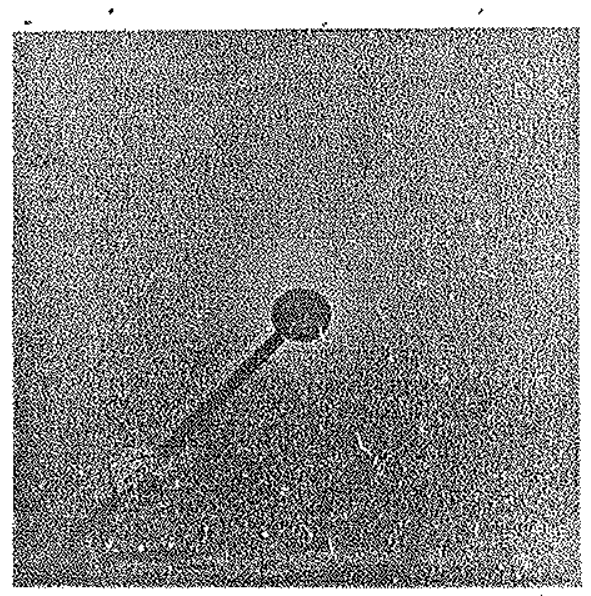

$(9 \times 2) \operatorname{Bg}(x)$

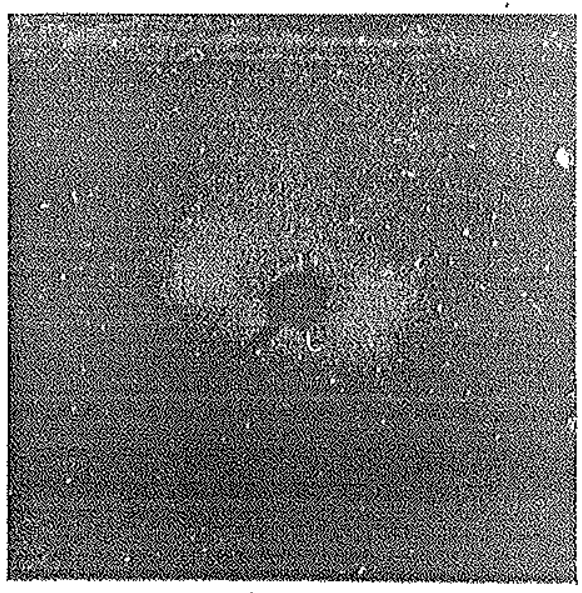

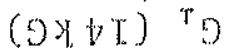

(o)

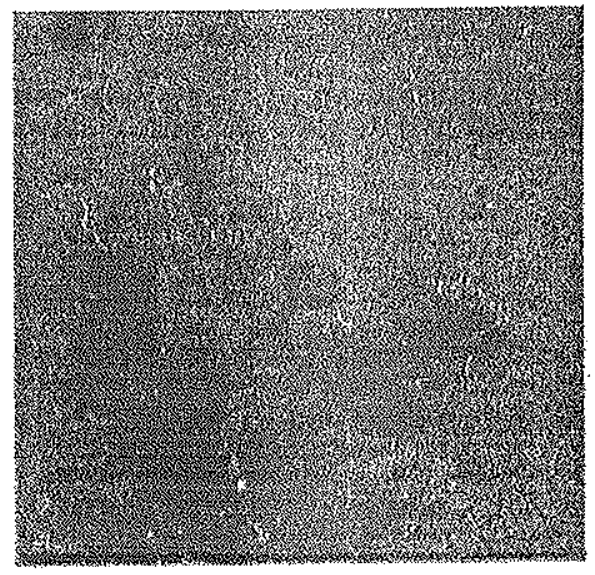

$(5 \times 1) " 5 \quad(9)$

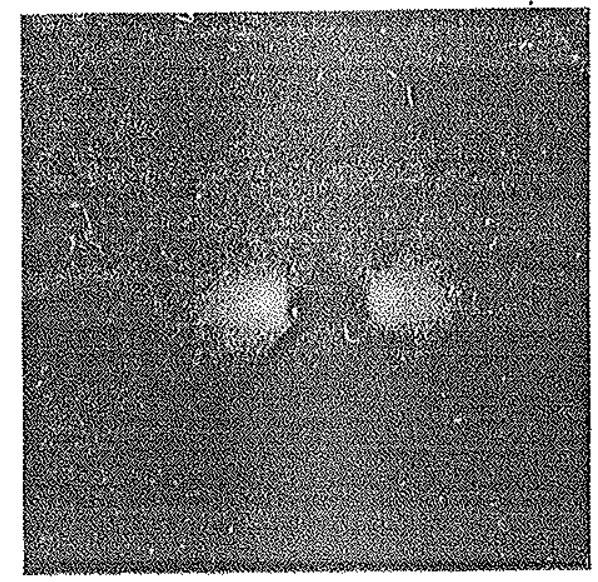

O $(\mathfrak{Q})$

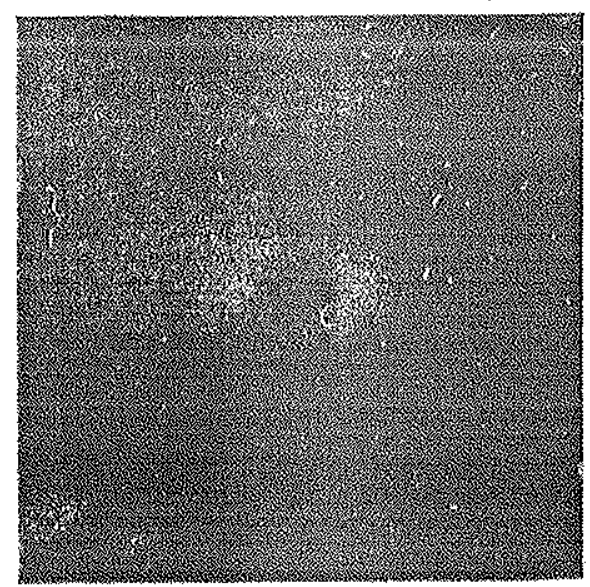




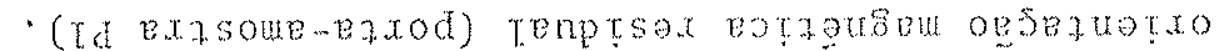

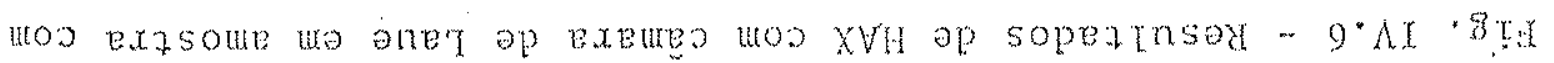

${ }^{11} \mathrm{~g}$

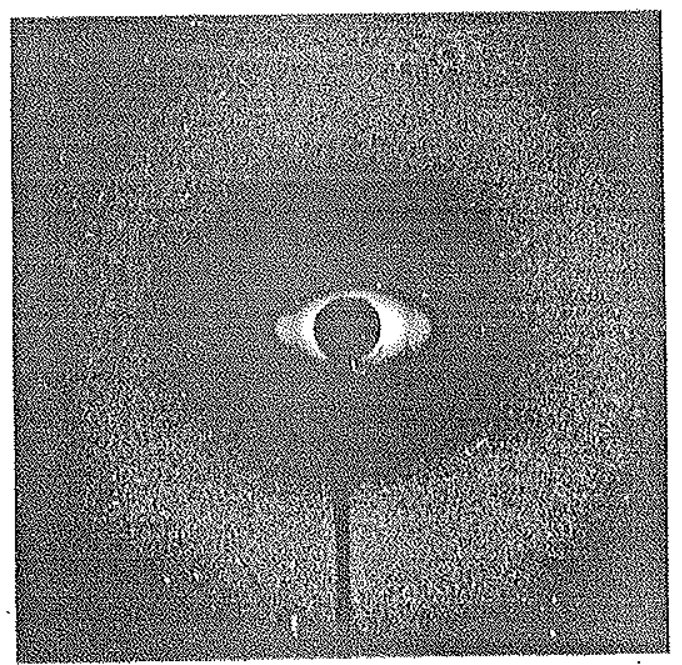

$T_{0}$

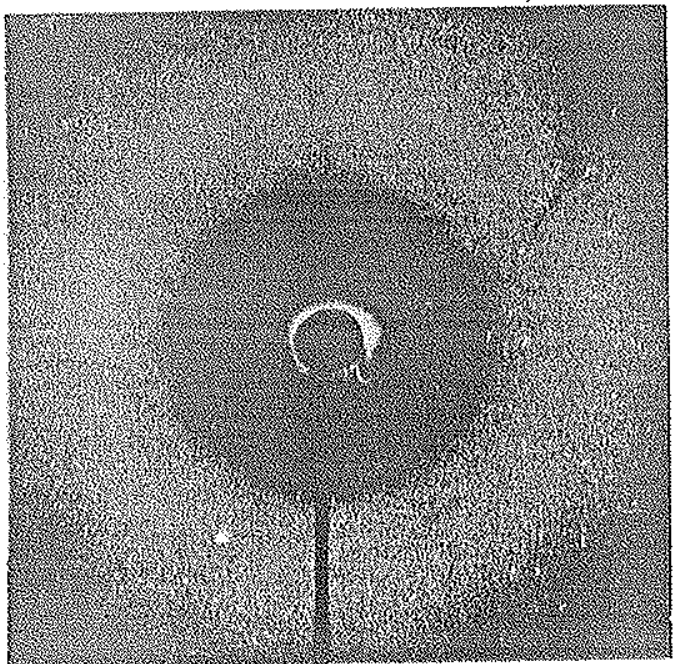

otzin aregdes

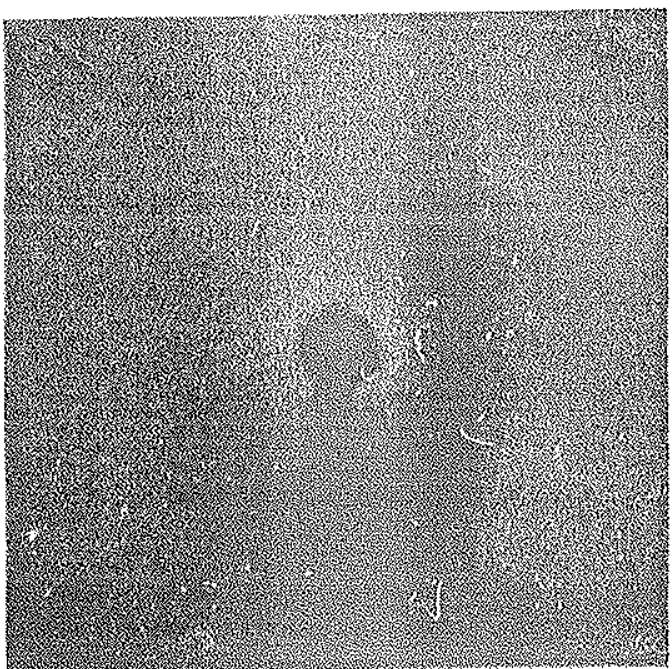




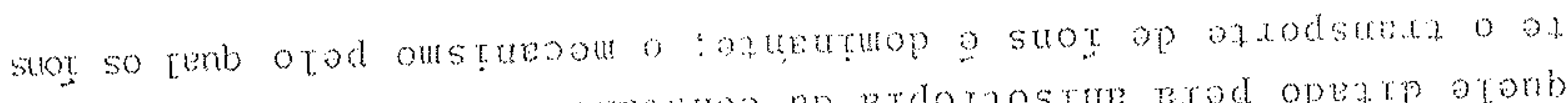

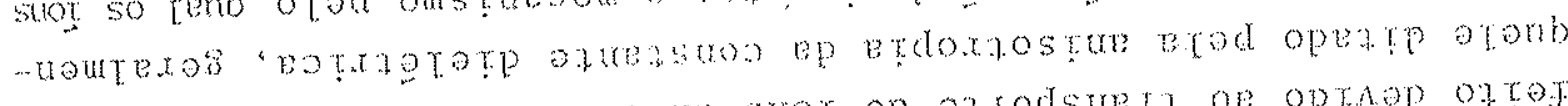

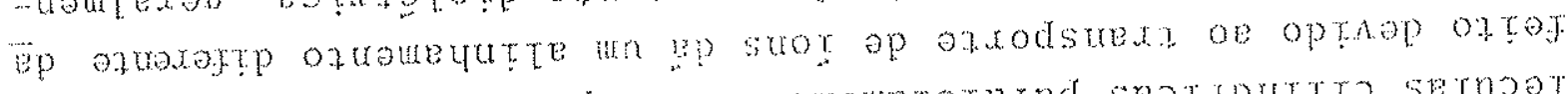

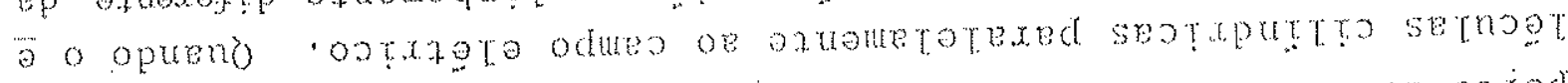

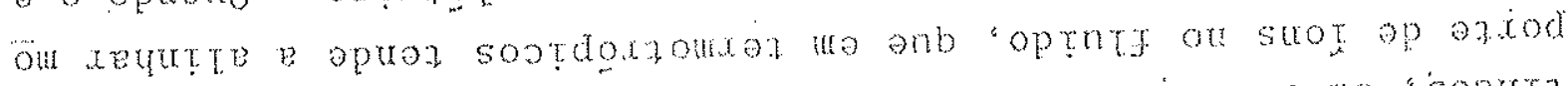

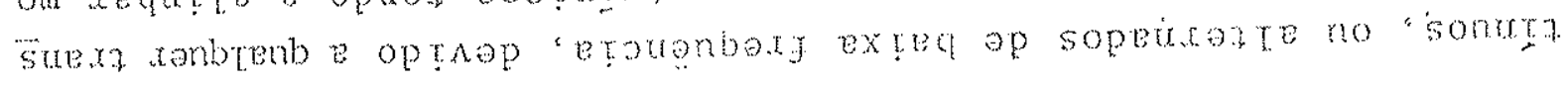

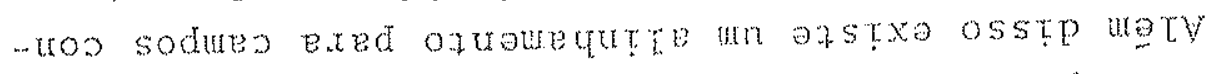

- (ge atepstot) sootut

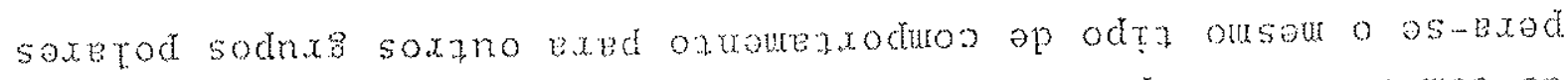

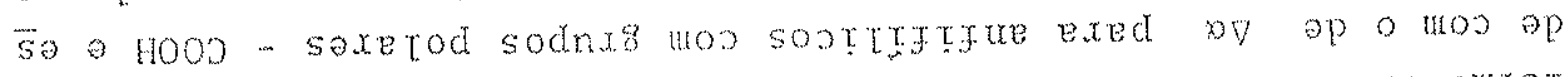

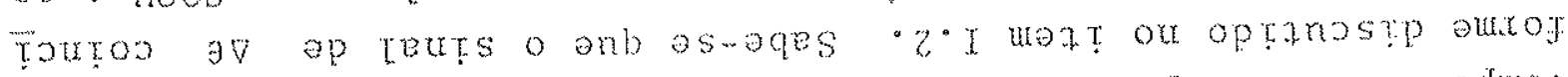

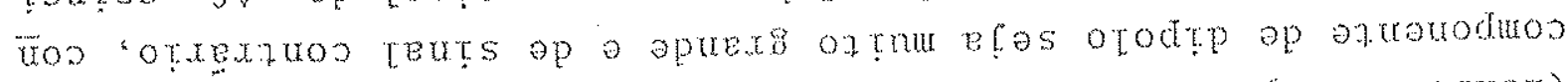

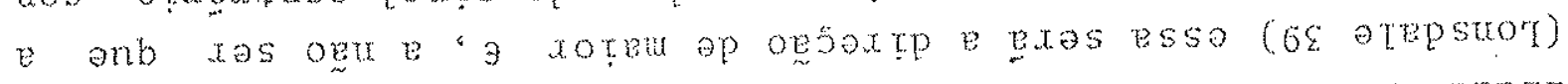

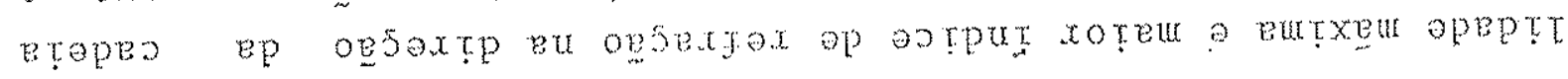
Tqeztrerod lanssod sopexnes somenoquoxper ouros

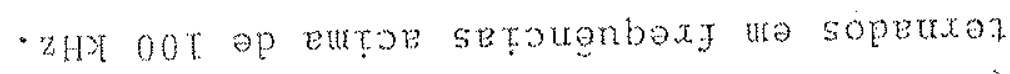

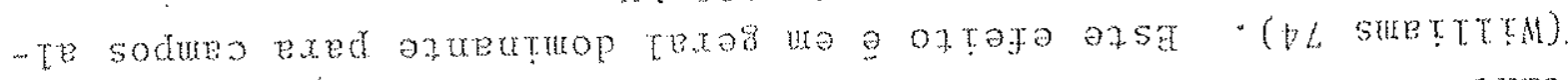

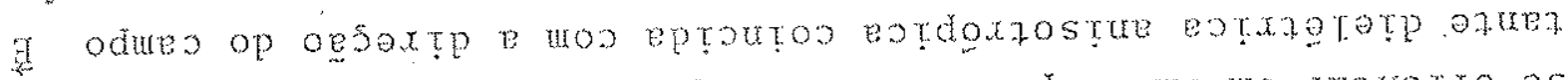

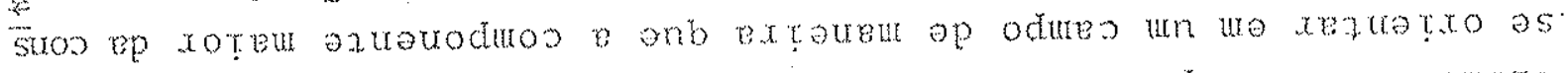

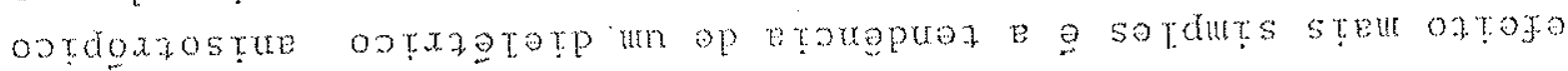

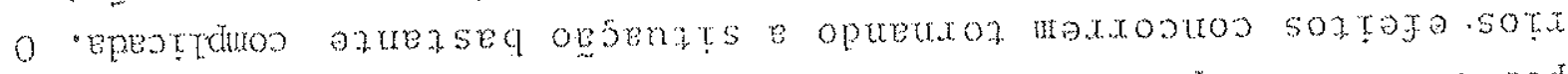

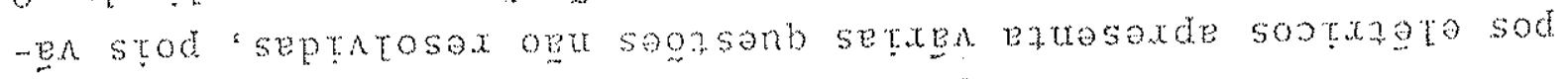

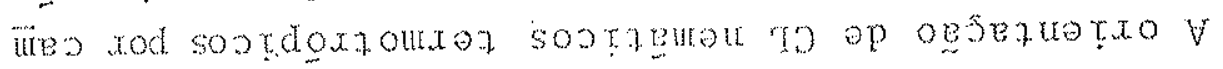

sopexadso som?Jit $v$

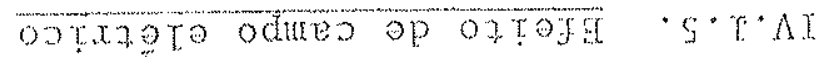

- ppex

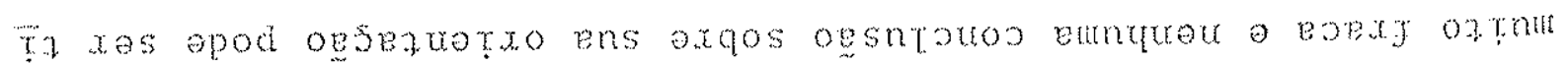

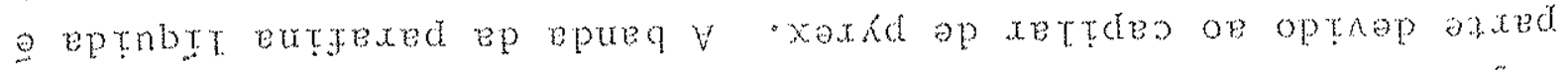

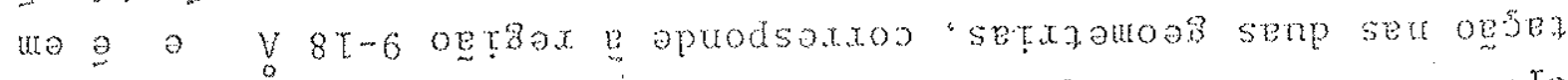

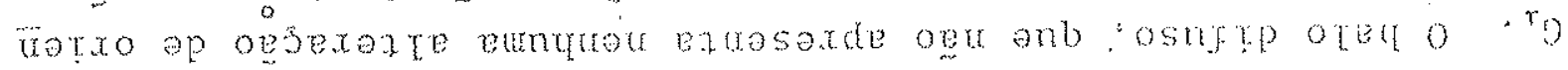

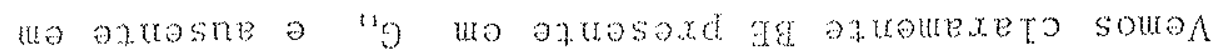

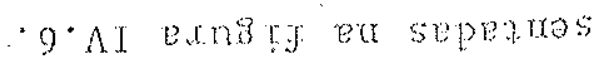

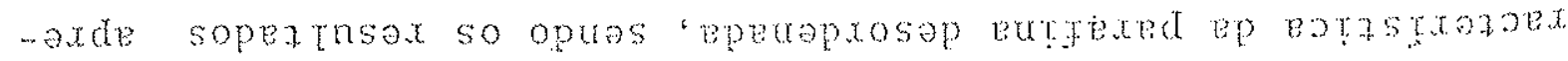

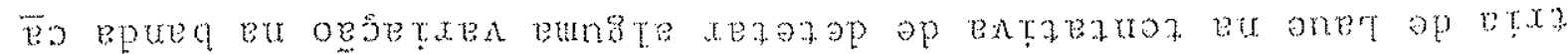

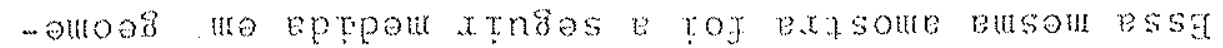

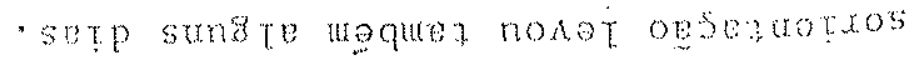

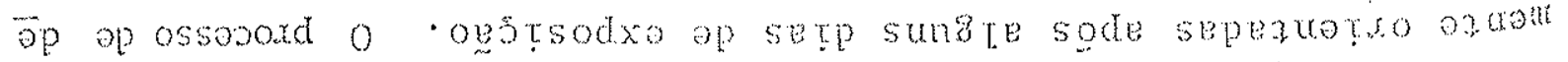




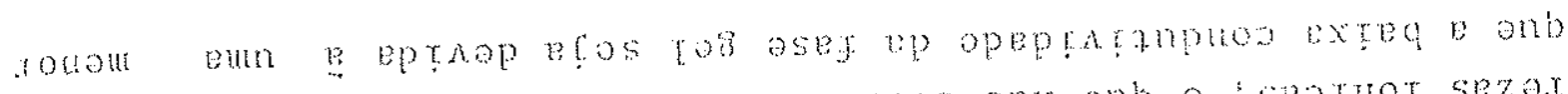

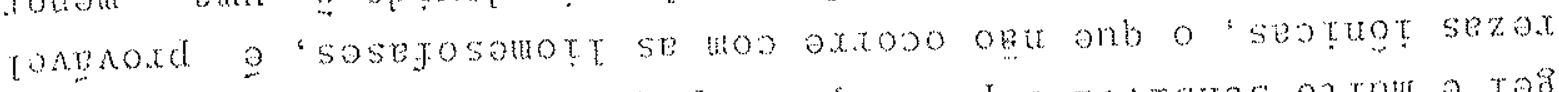

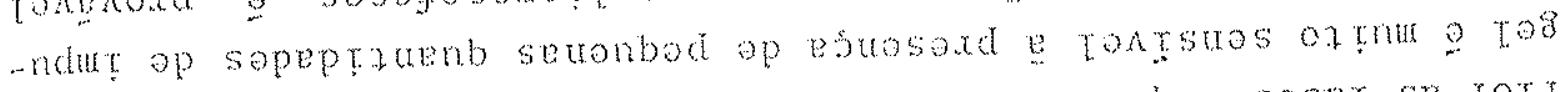

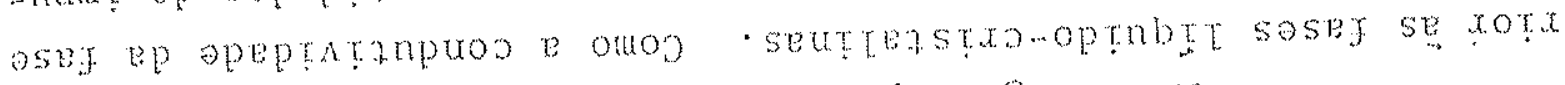

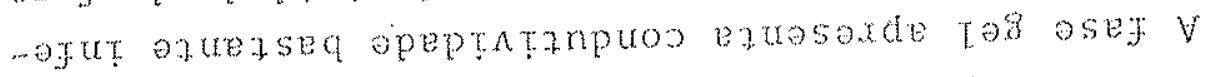

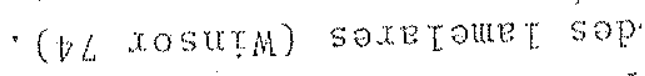

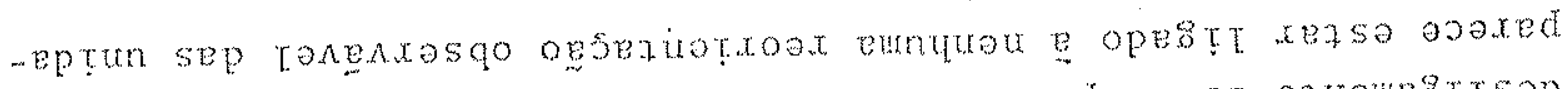

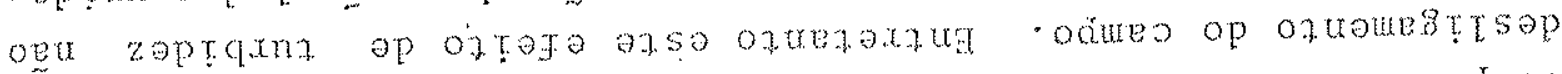

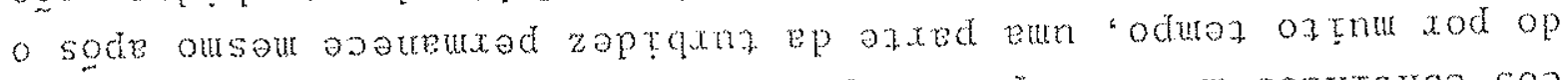

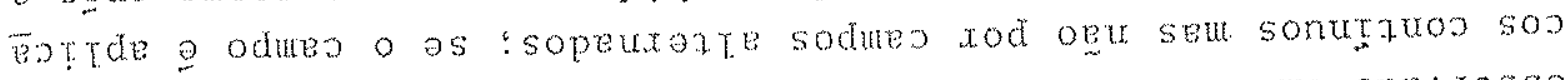

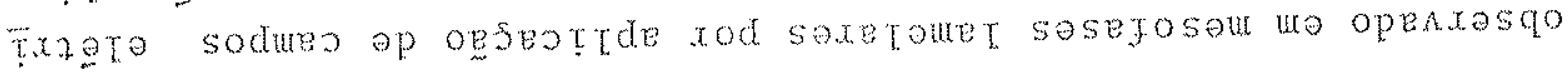

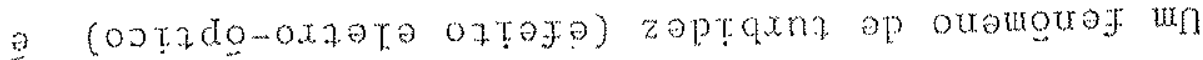

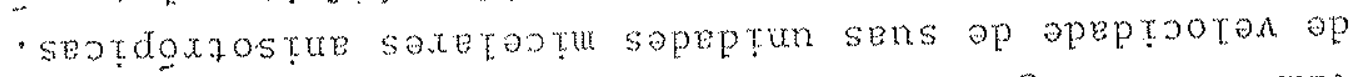

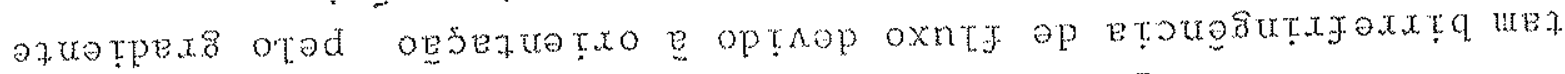

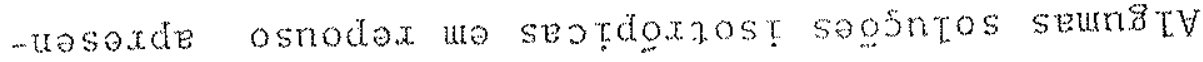

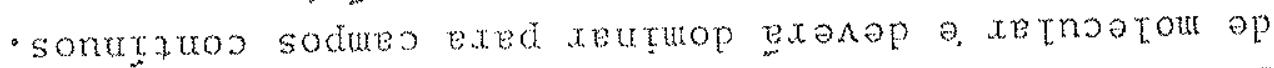

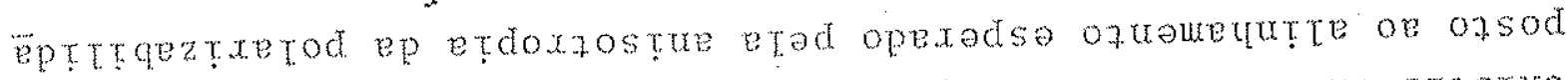

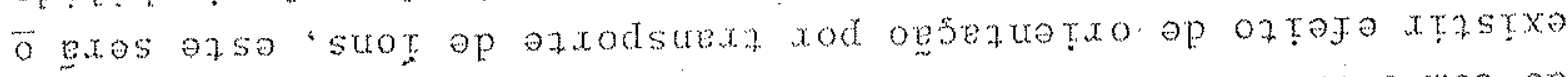

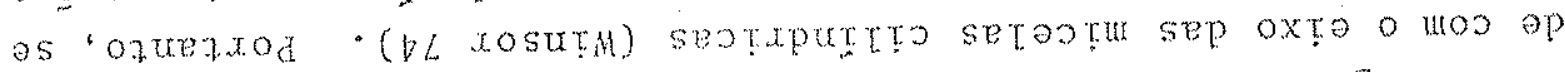

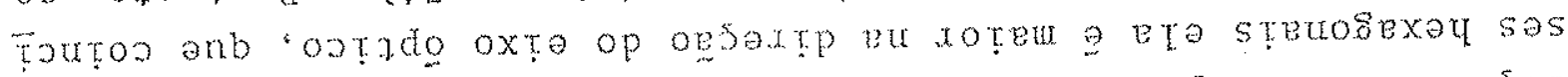

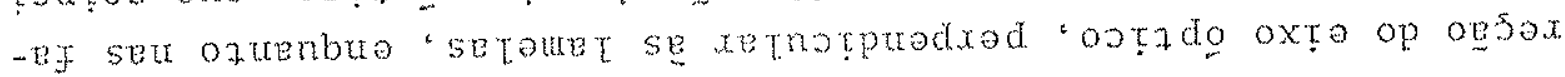

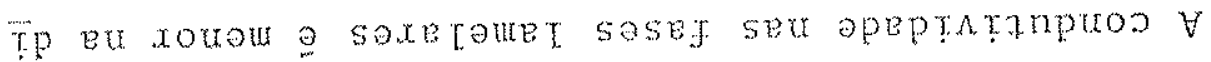

- opeptatantrom ep etroxastue ep seprpou b oxusu

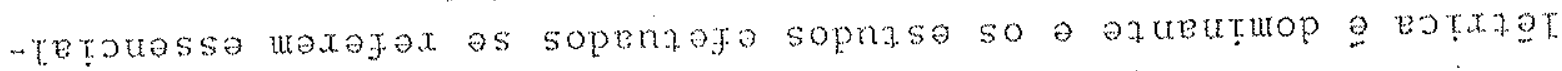

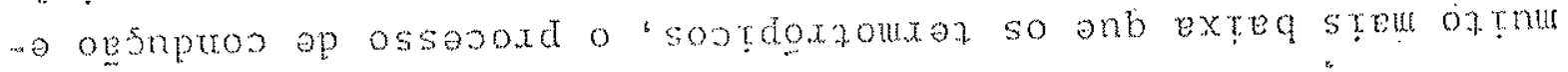

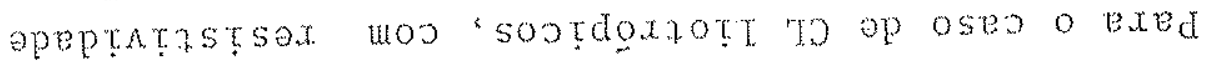

s steztoutradxes sop

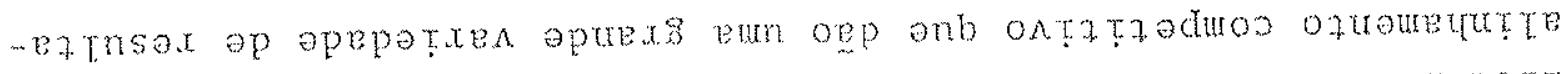

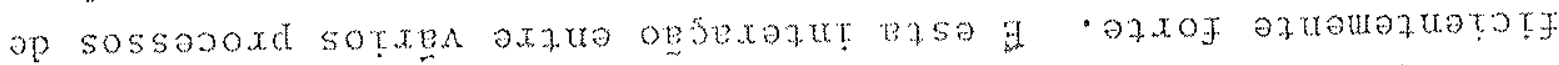

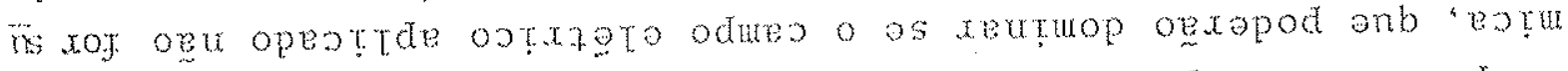

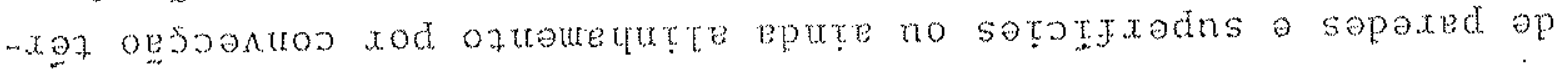

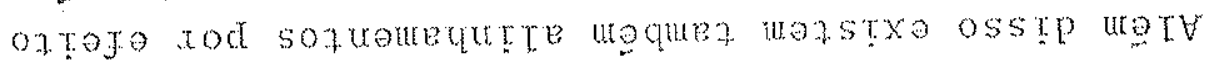

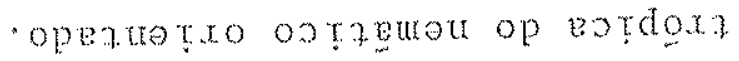
. osgue ppetsoost.

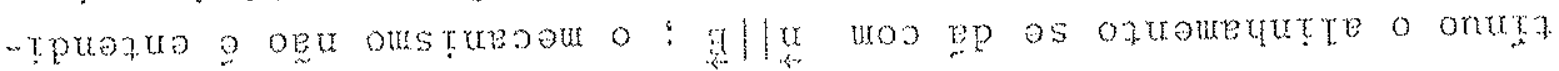

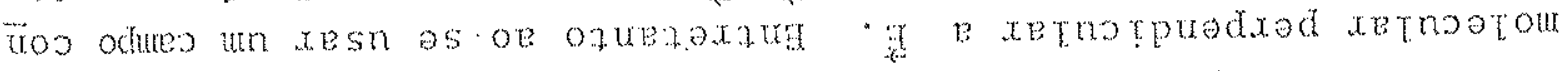

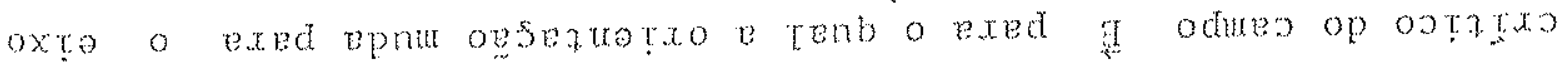

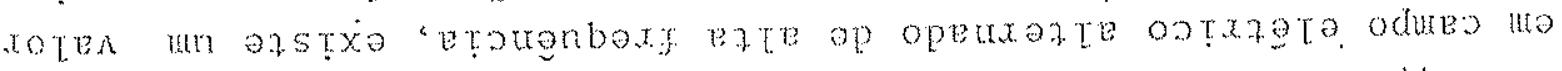

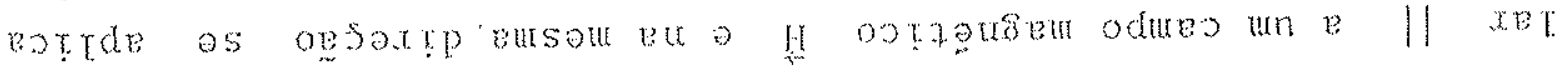

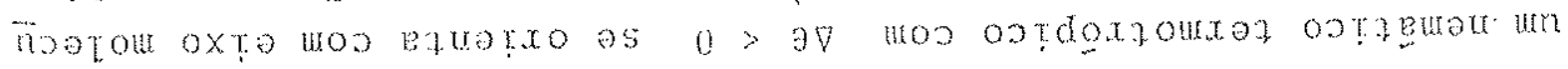

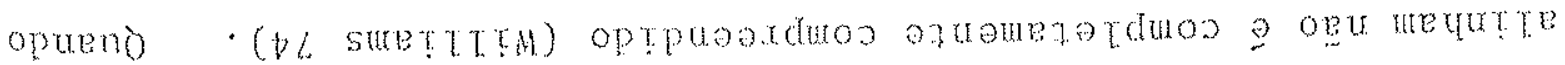




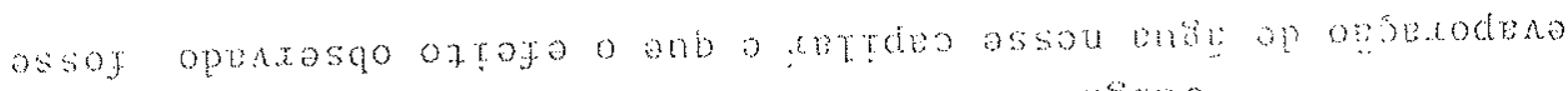

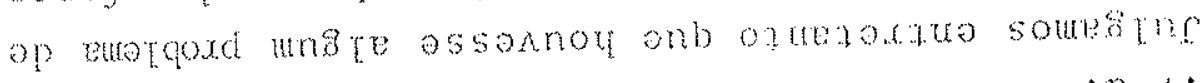

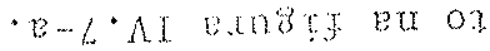

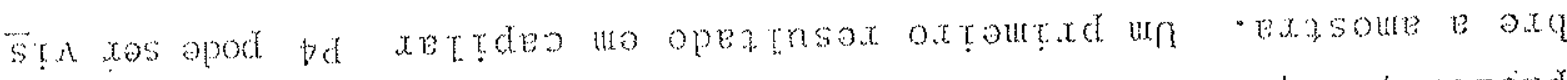

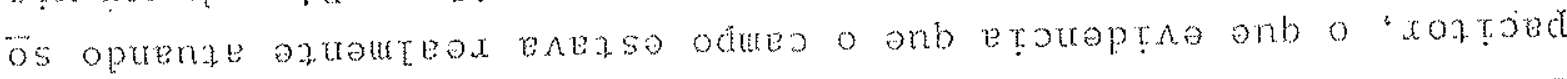

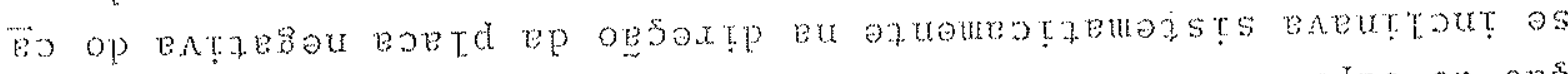

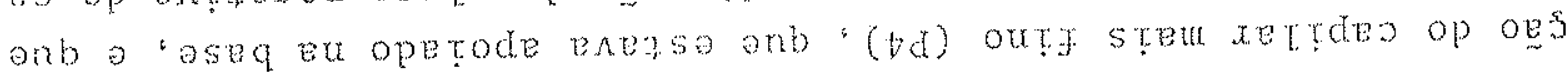

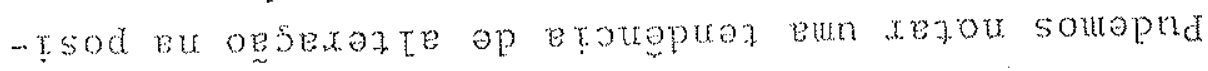

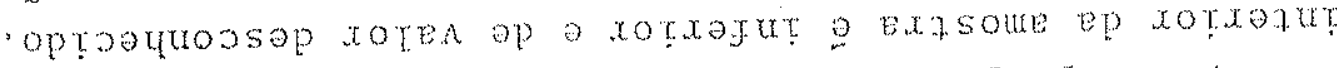

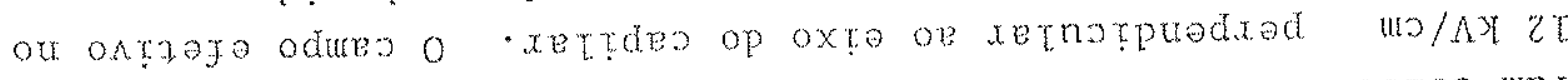

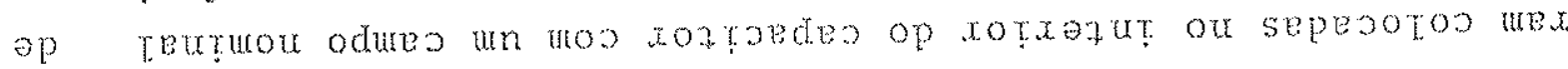
-of bd a td soxetrdeo sou seperorotpuose sexzsoury

- (T8) *te ź soutzoud op opeptatastsex op septpor

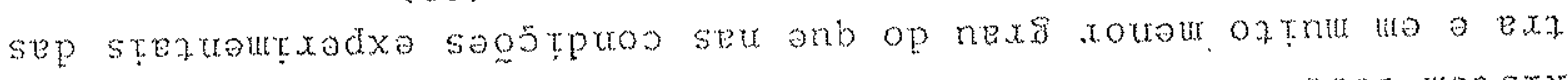

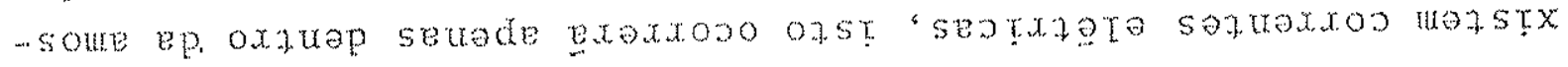
-o os anb ur oztrosep ef ofmexe o operttan tor

\section{soptago sopetirsoy $\cdot \mathrm{d}$}

- opsezaxdxazti torte ap

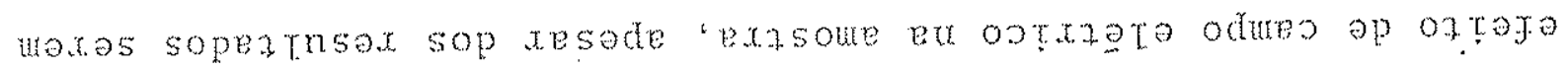

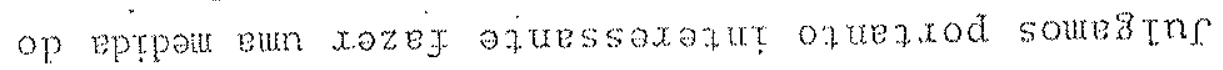

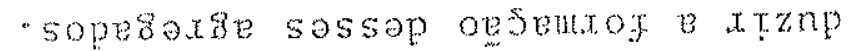

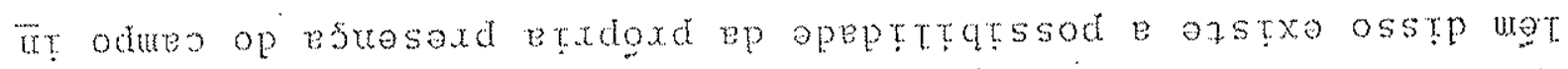

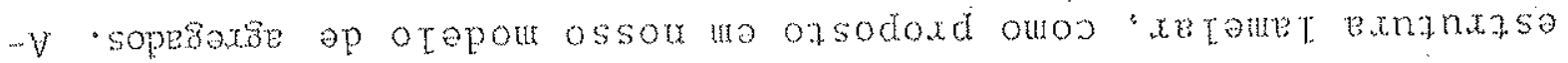

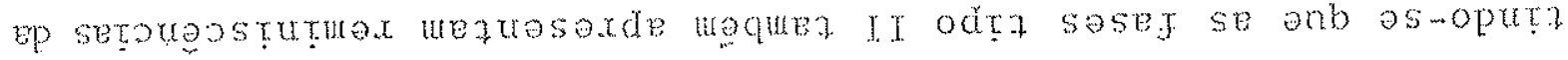

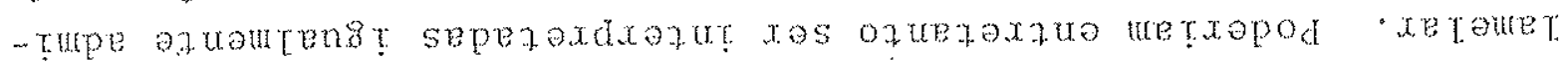

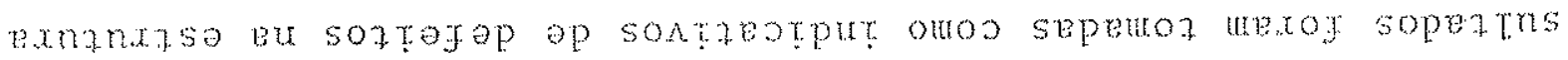

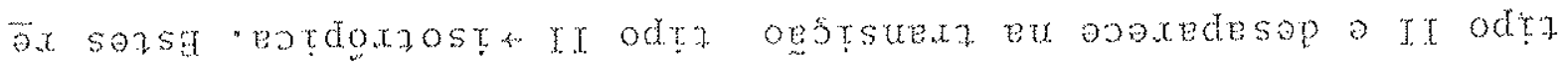

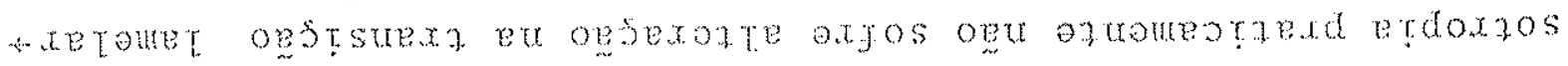

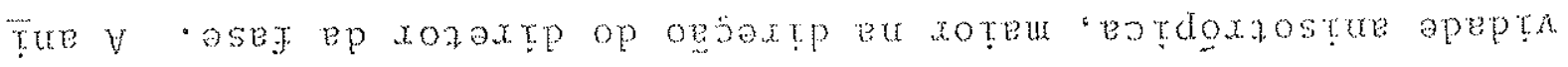

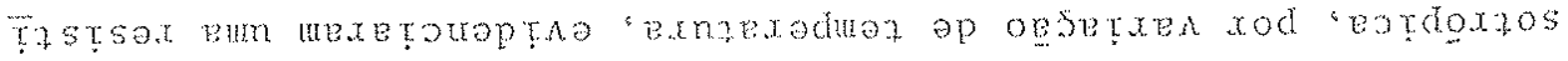

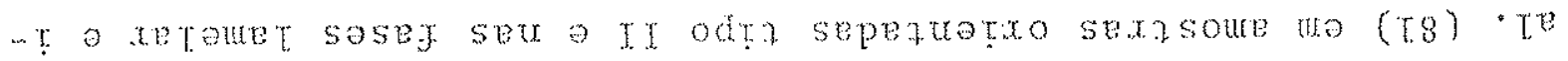

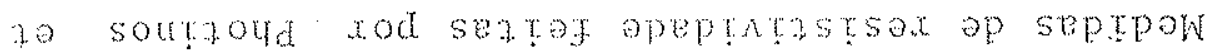

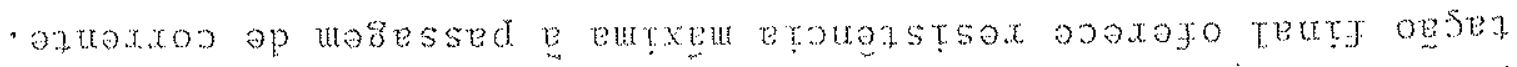

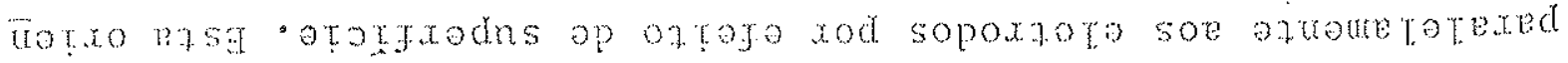

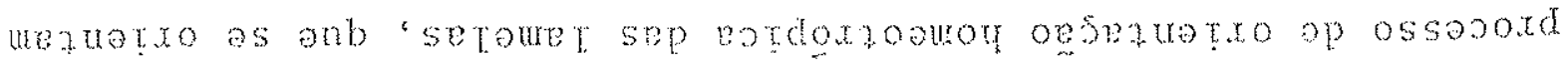

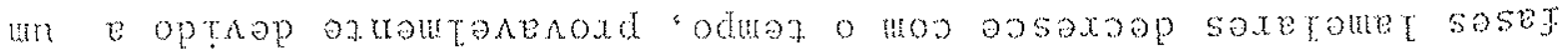

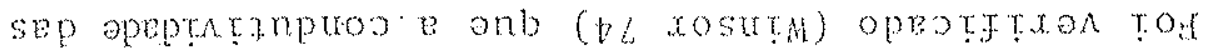

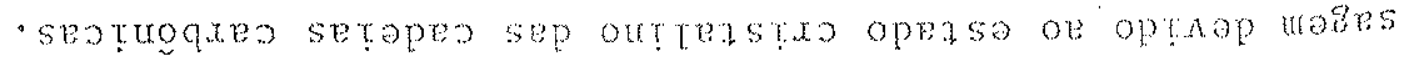

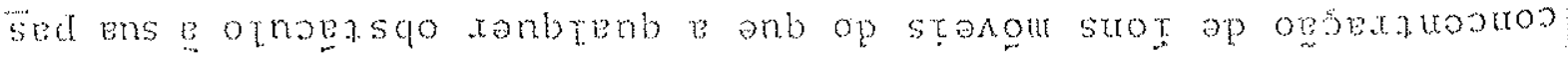




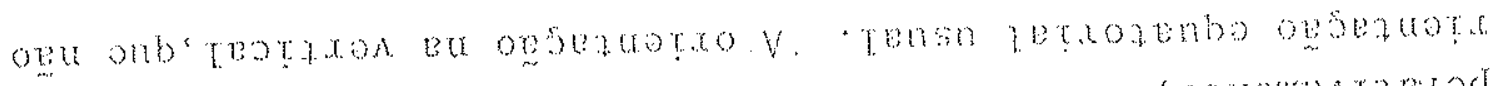

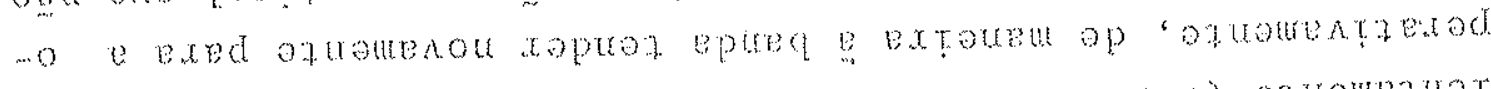

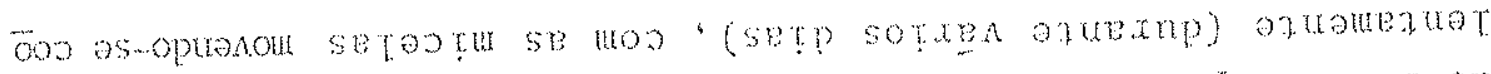

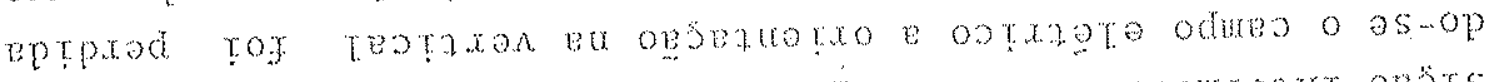

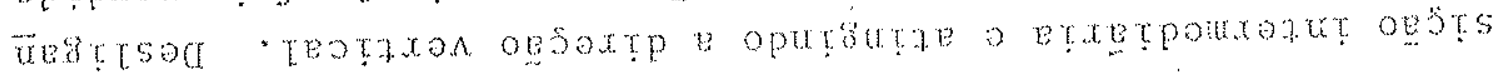

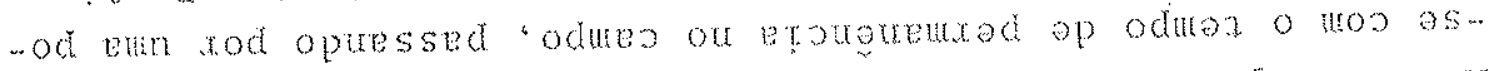

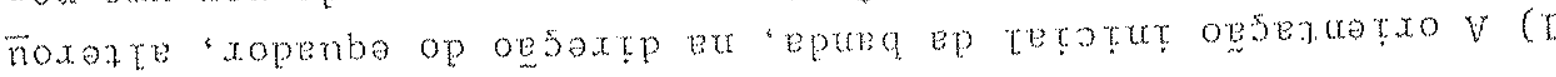

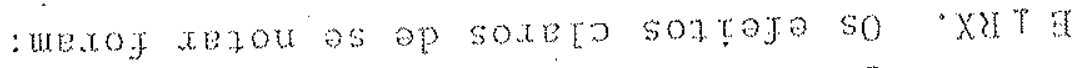

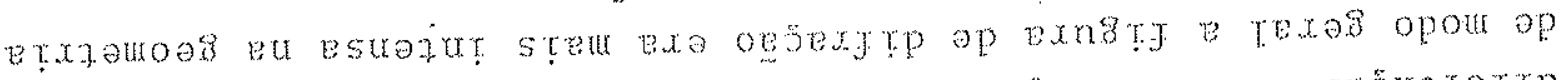

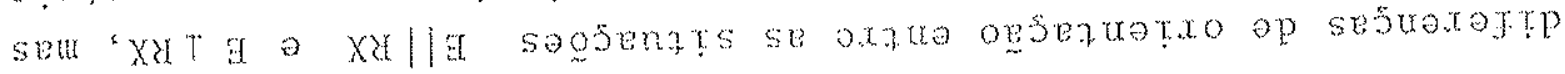

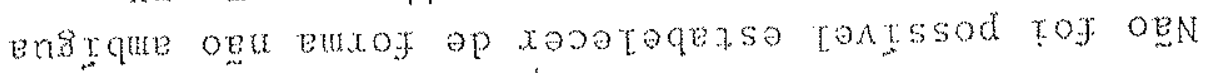

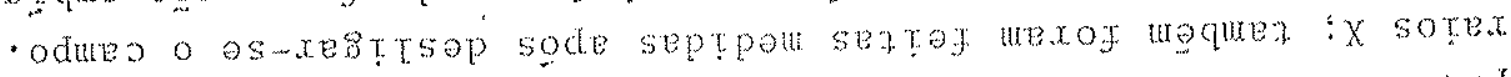

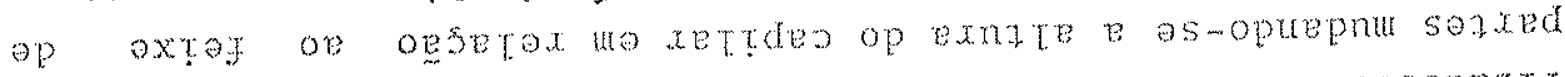

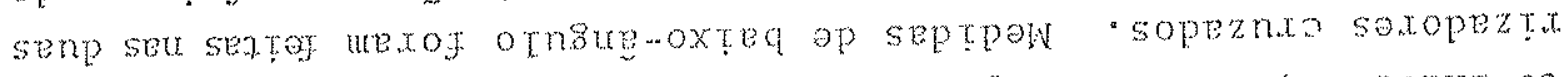

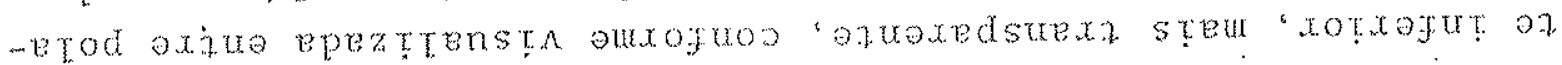

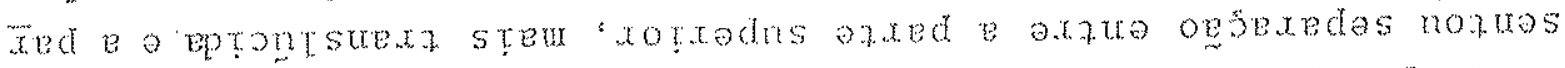

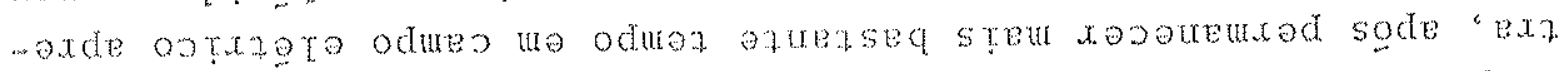

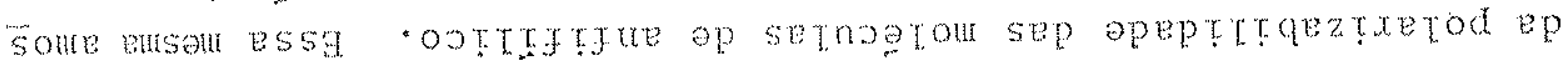

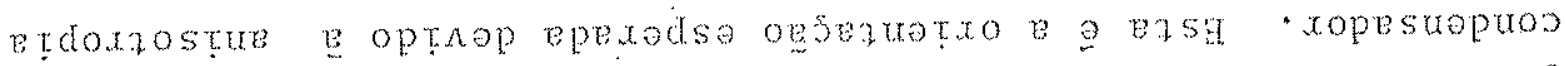

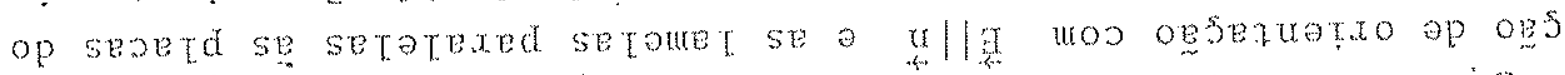

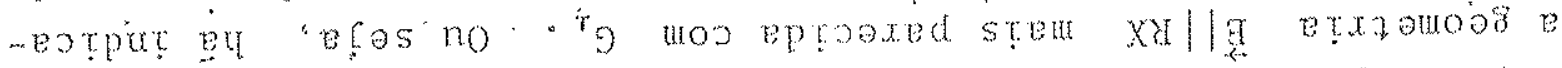

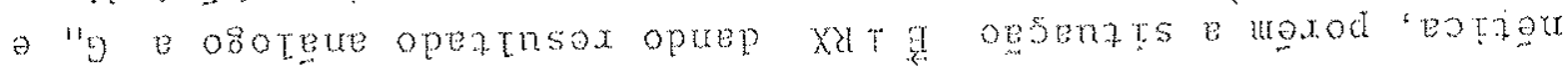

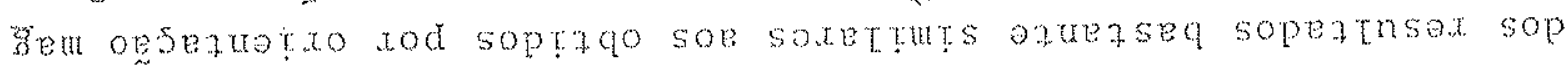

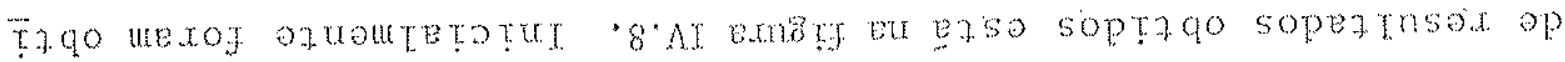

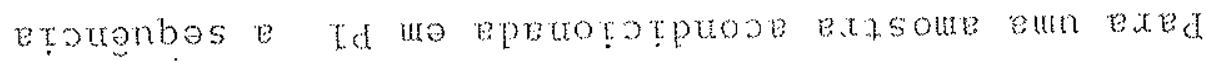

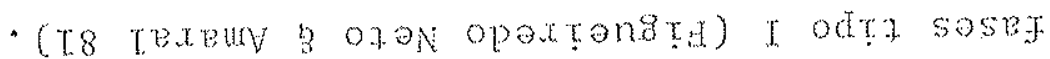

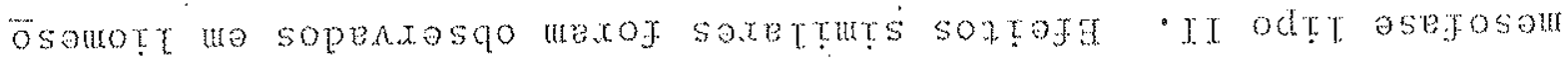

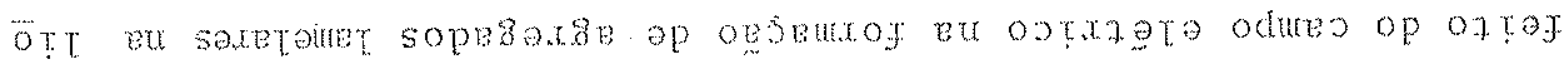
-a o poontrbart euxog ap mexerasom septpou sessa

- ozarab ap xpt

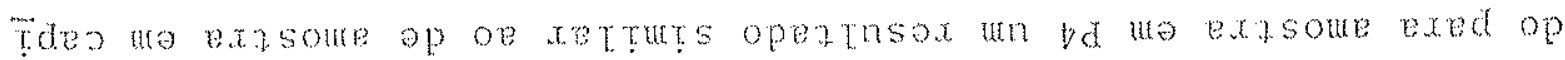

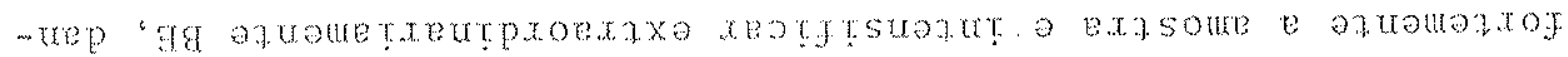

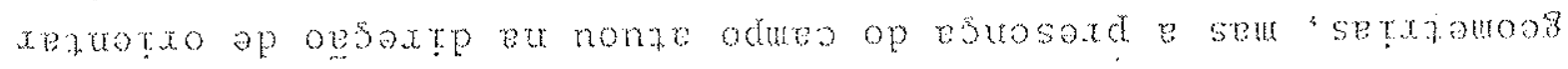
senp se axtro bsuoxogep anou ogu opeatnsox azson

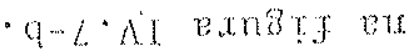

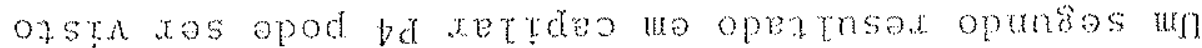

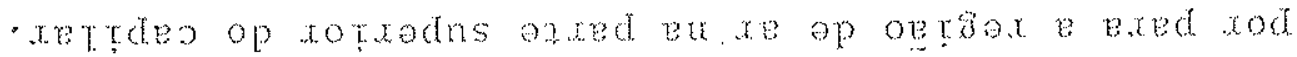

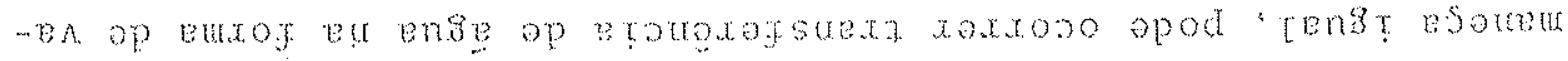

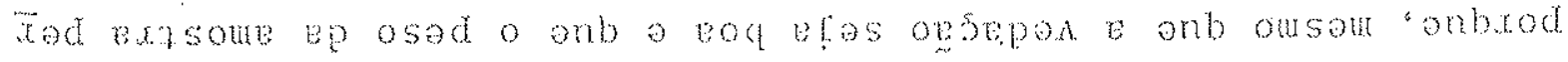

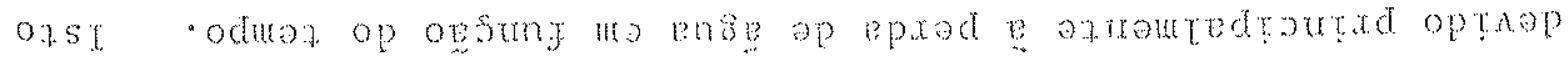




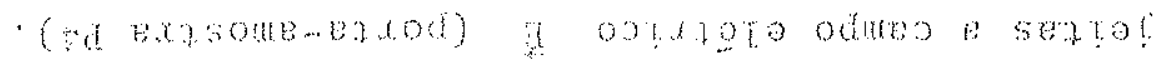

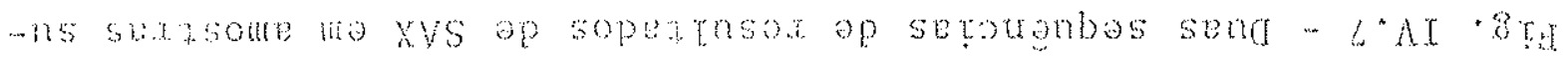
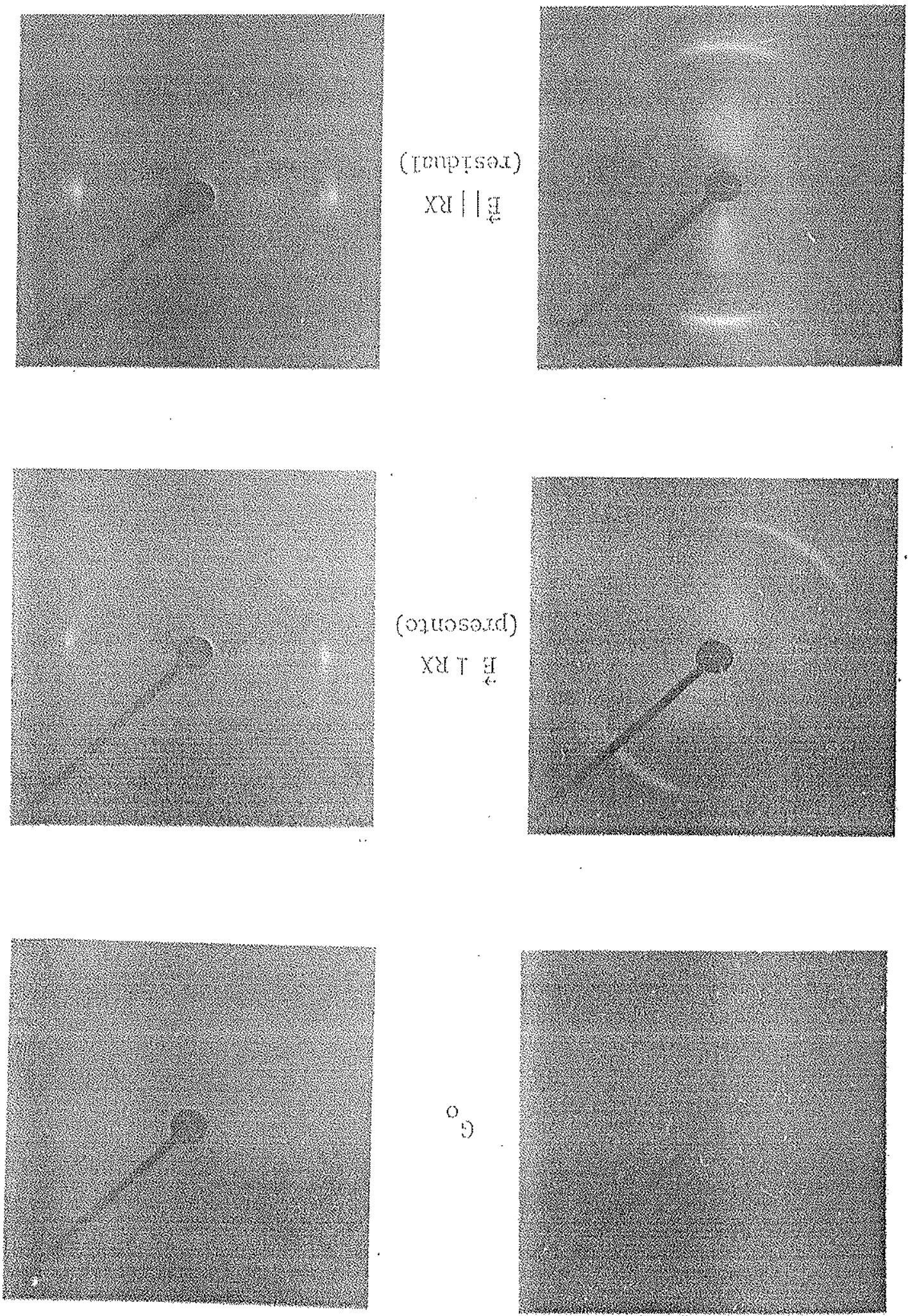

(c)

$(\varepsilon)$ 


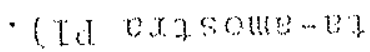

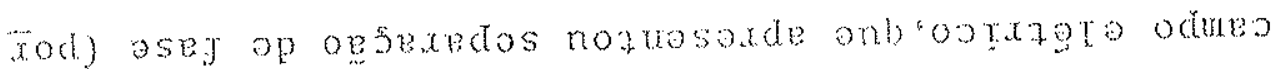

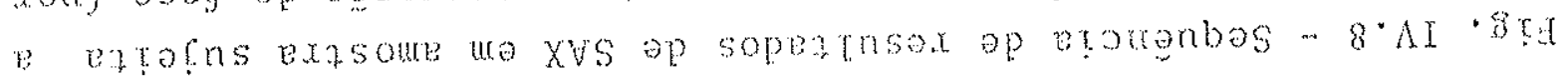

\section{$X \geq T$ i $(p)$}

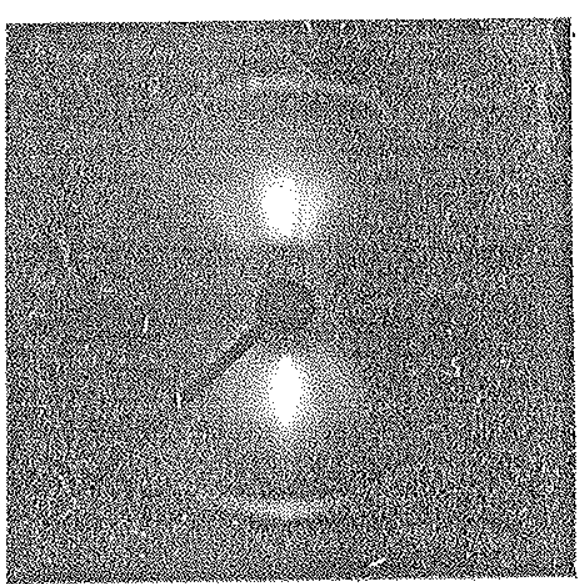

$x y \|$ (o)

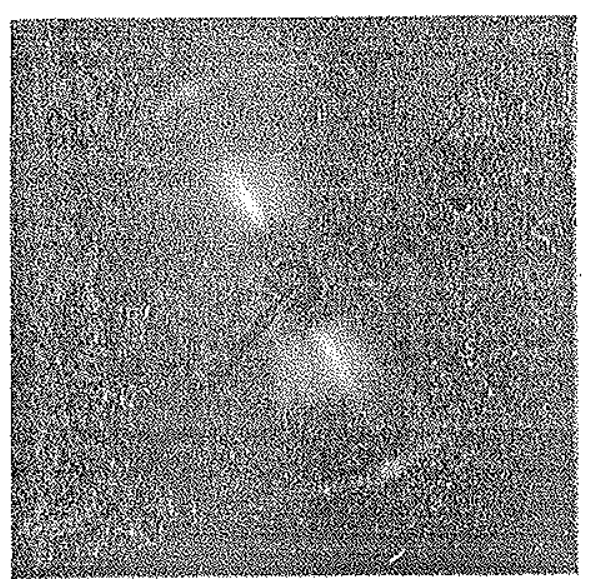

$x \geq 1 \mid$ a $(x)$

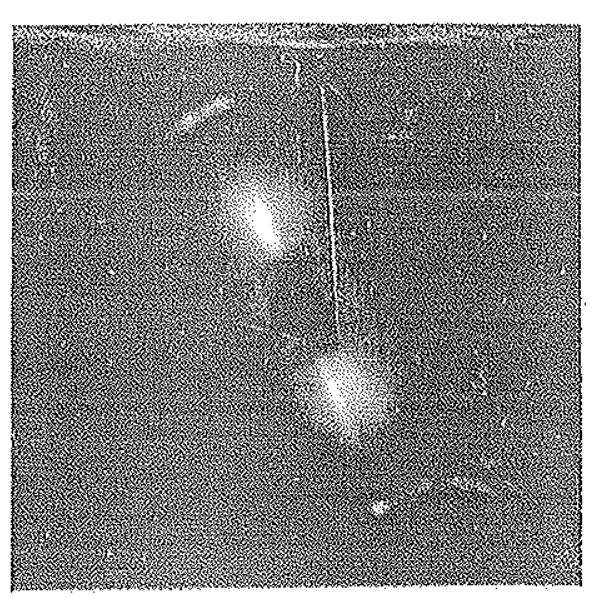

rotradns orat $x \times \Gamma(0)$

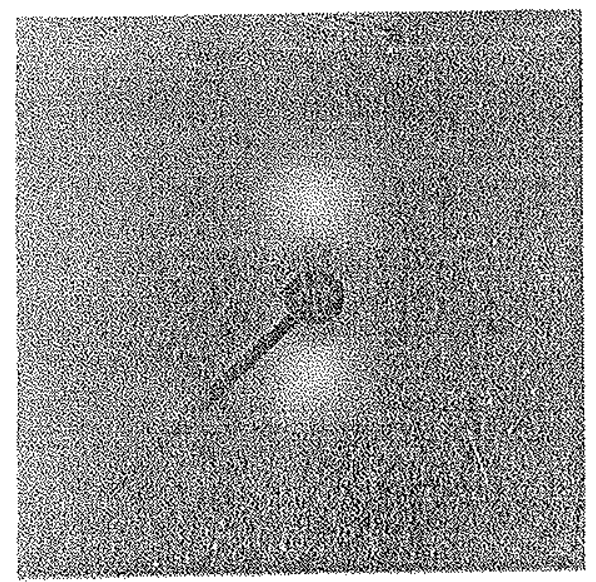

$x d r a(q)$

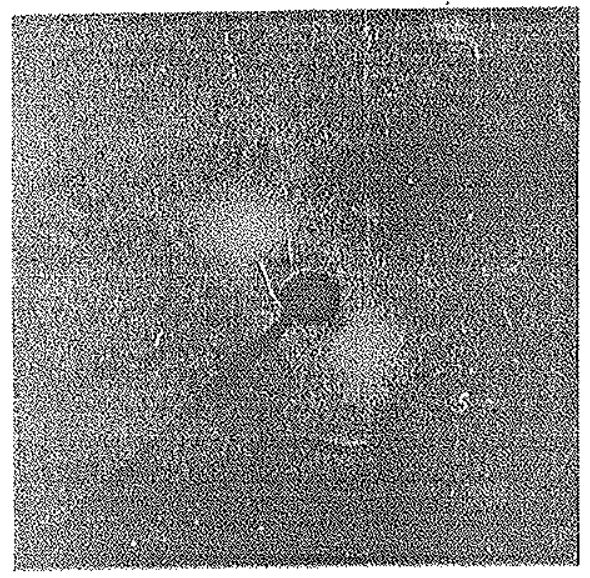

$x y+4(e)$

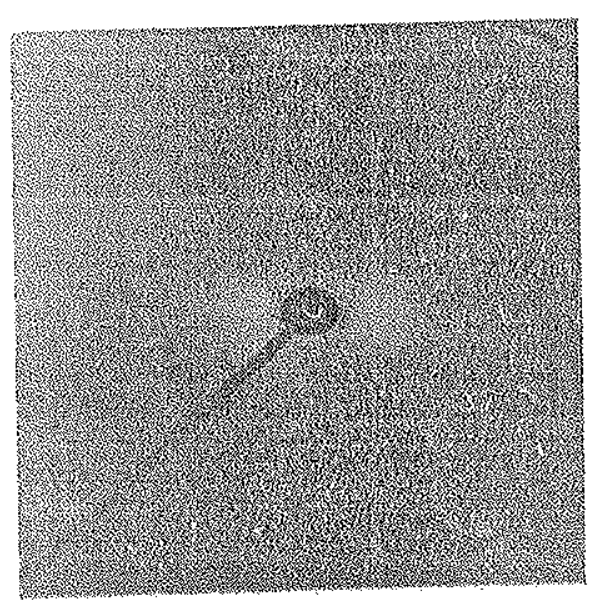

rotater orxed 


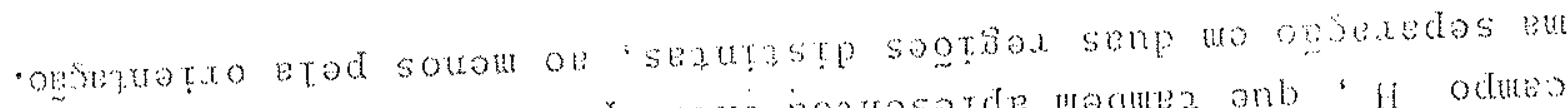

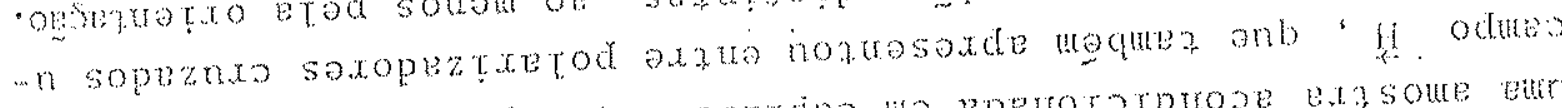

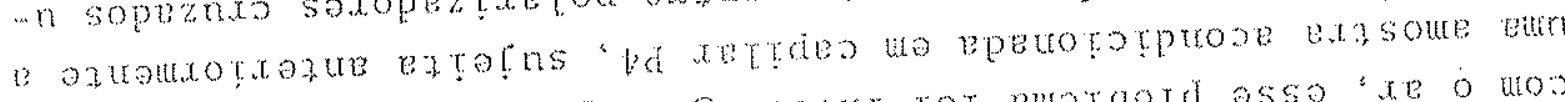

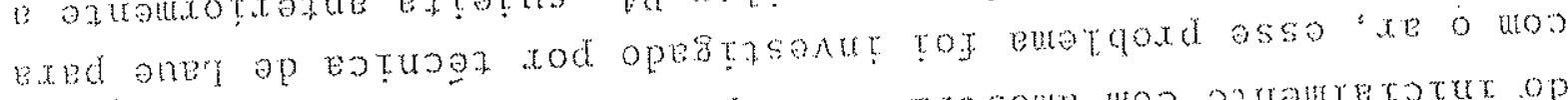

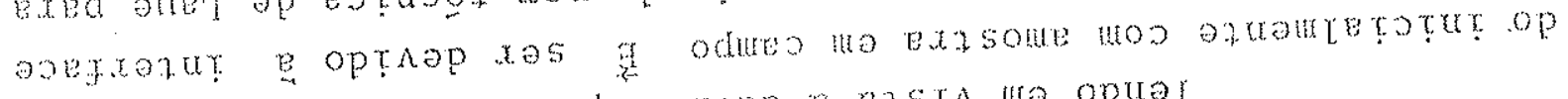

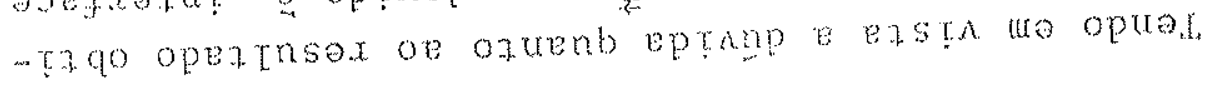

$x \in$ o

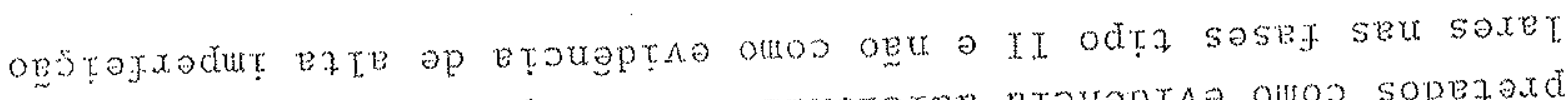

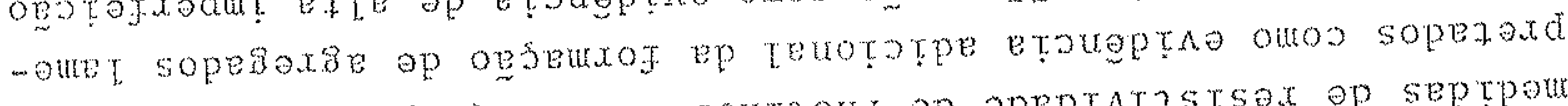

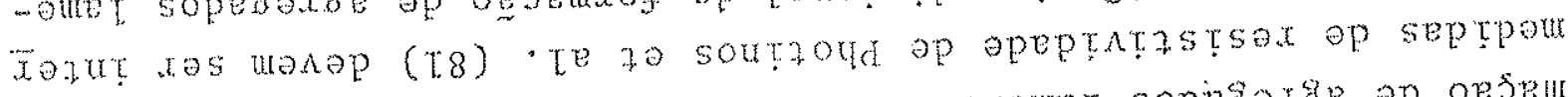

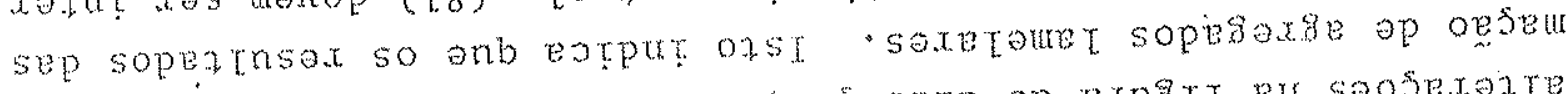

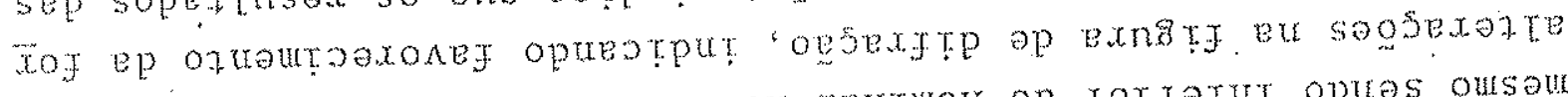

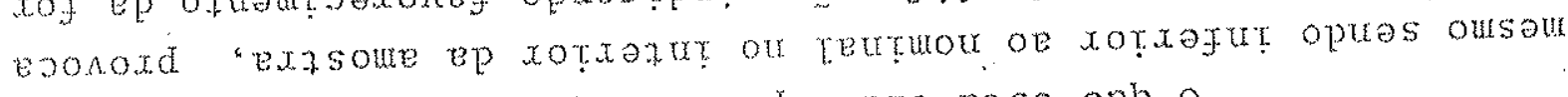
codurs op estrosaxd e anb a woxod oxeto pasa anb o

- odusa op xessed o uos azurtcut

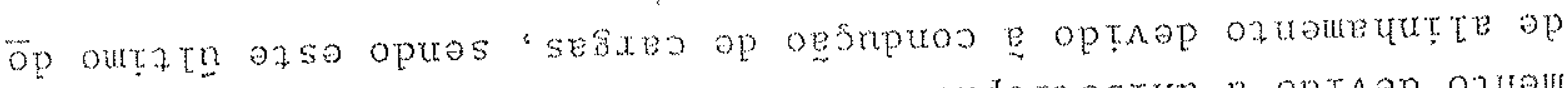

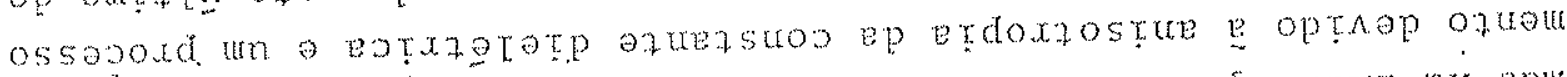

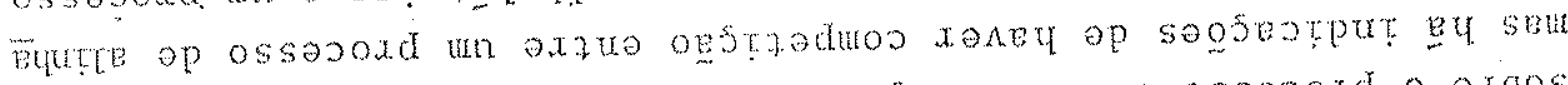

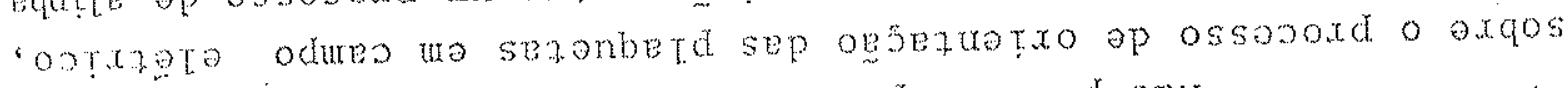

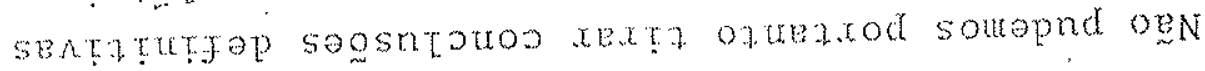

- ostrate odues un ep estresgud

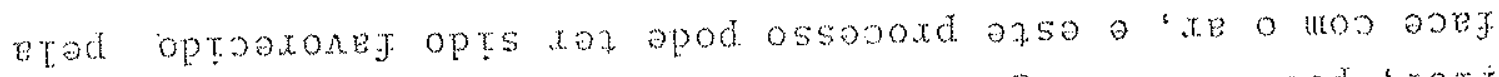

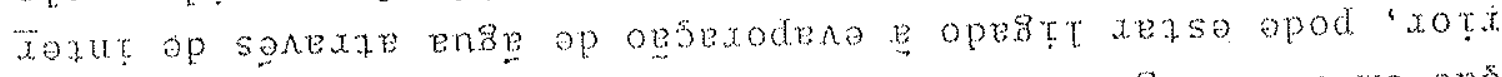

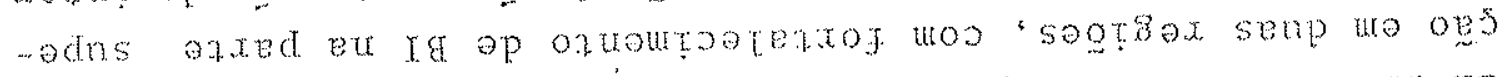

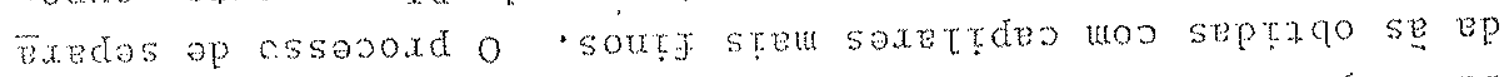

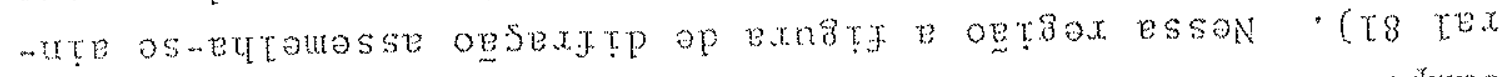

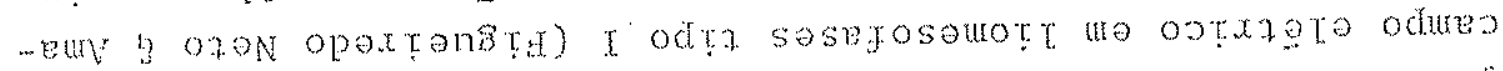

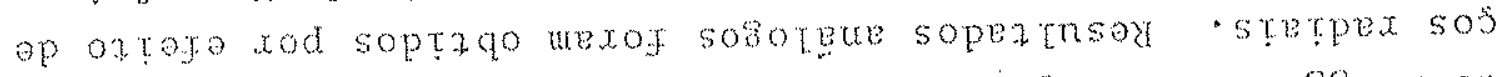

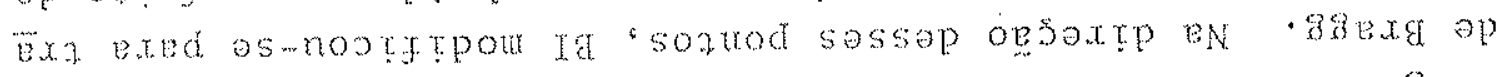

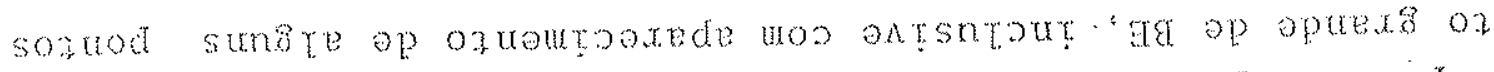

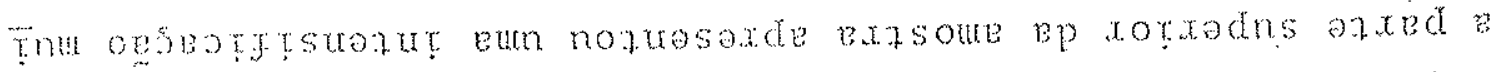

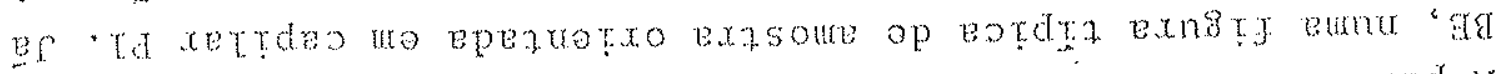

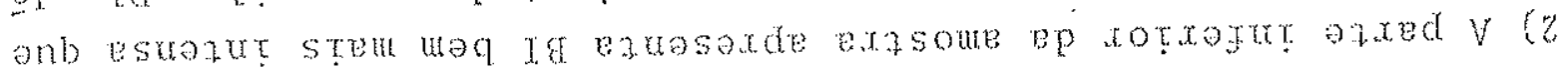

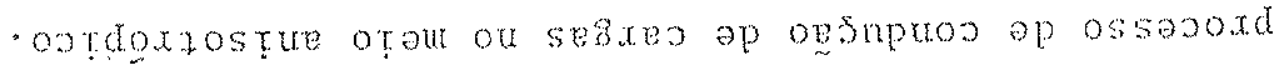

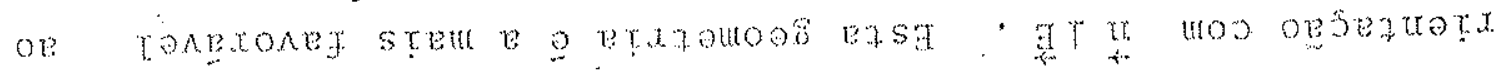

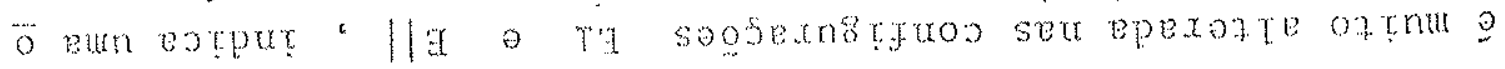




\section{- (val}

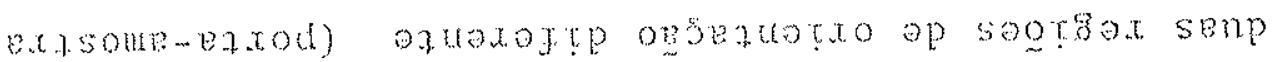

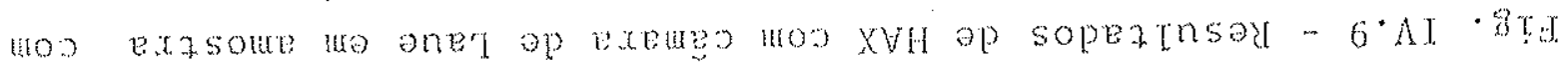

$\left.{ }^{2}\right)$

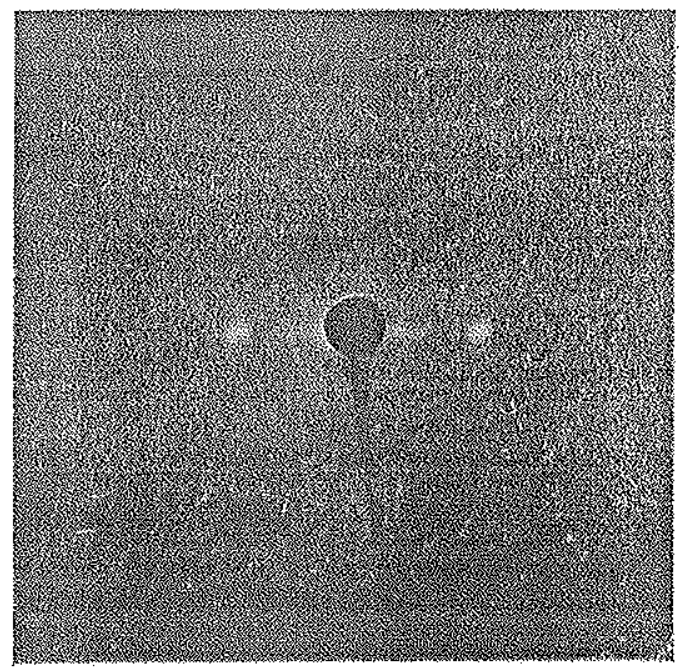

19

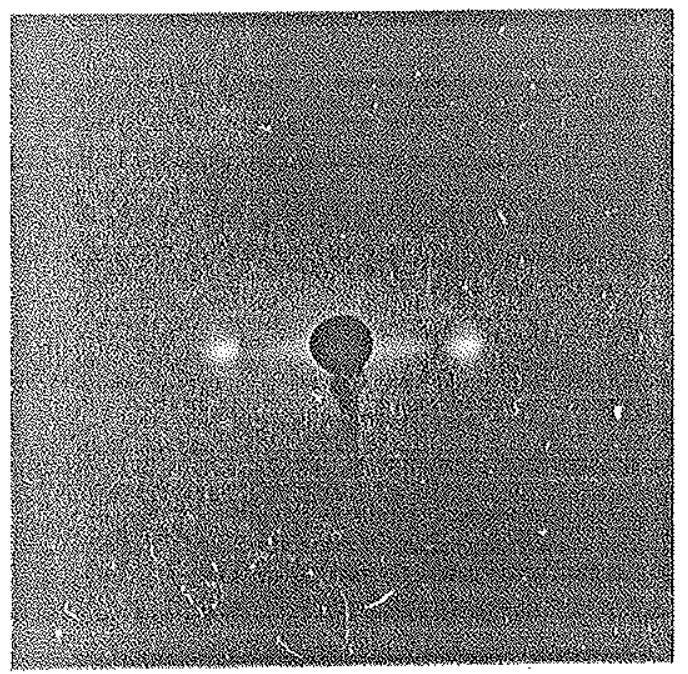

xotxaytr oxwed ep ogsezurto

"b

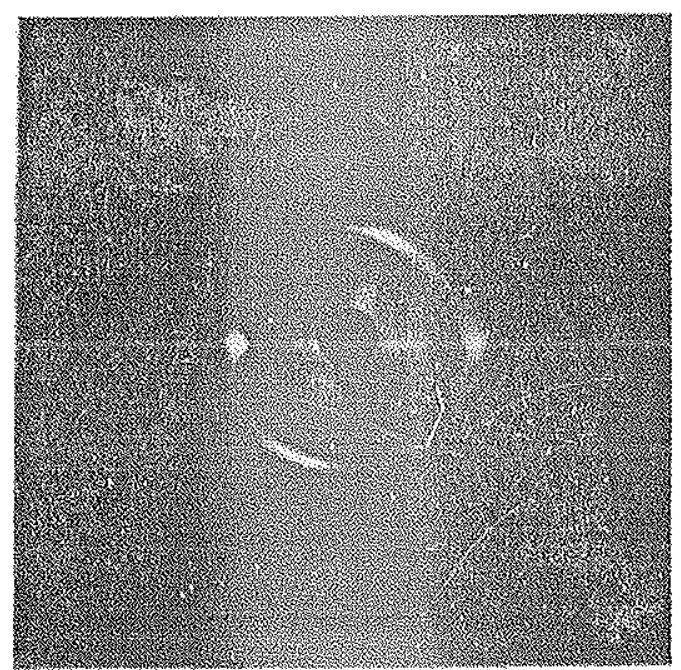

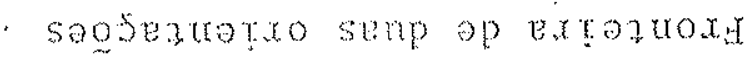




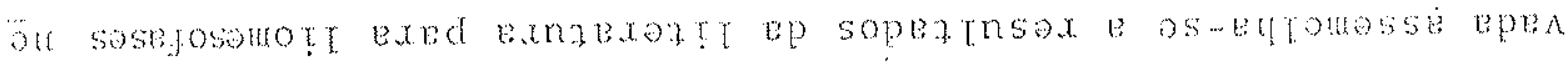

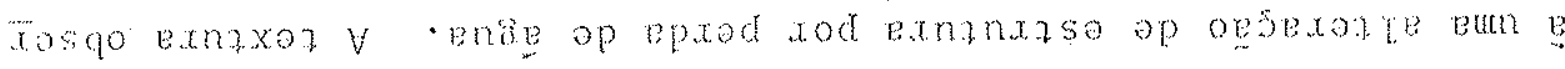

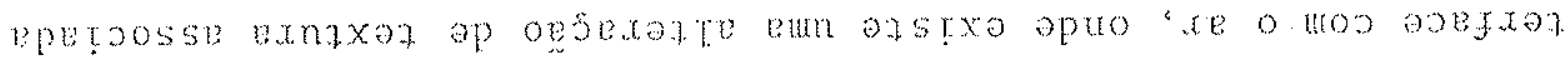

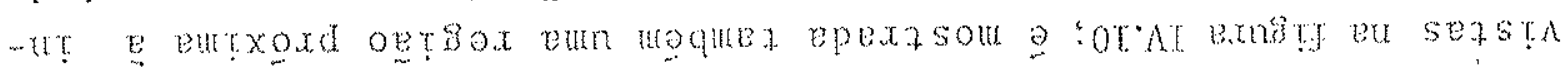

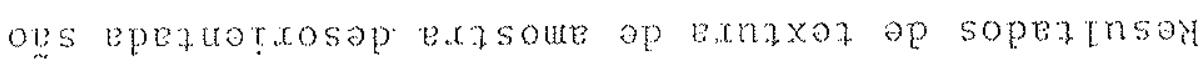

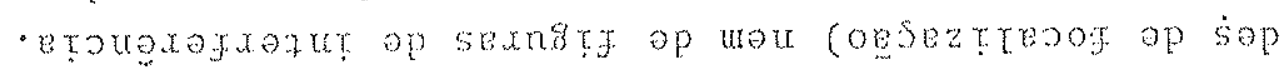

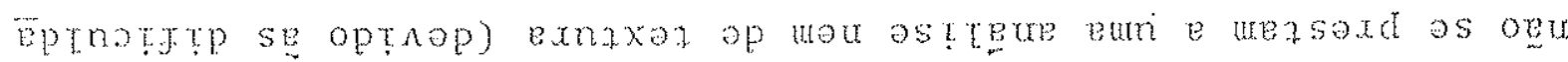

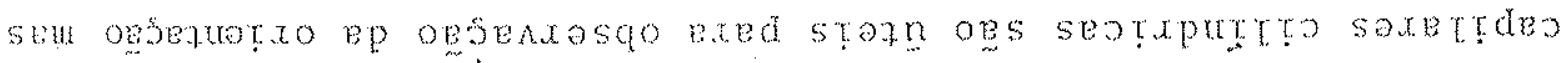

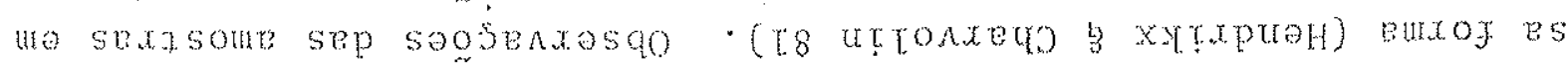

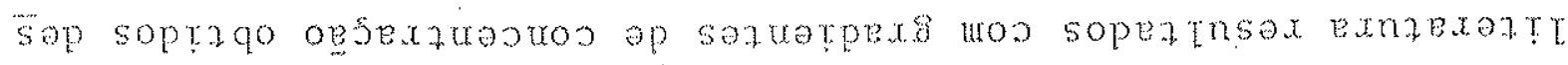

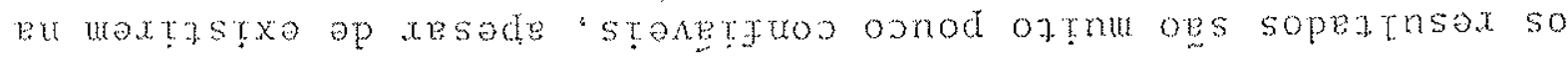

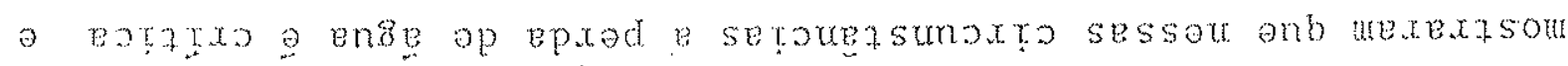
ordoosoxotu op stensn sernuruet sut sagberasqo

(e.mstet wa 8

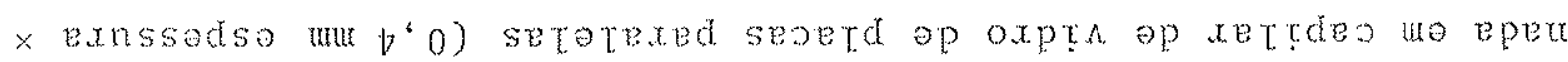

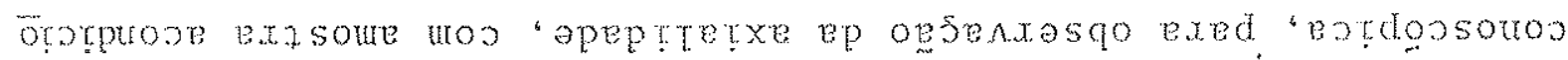

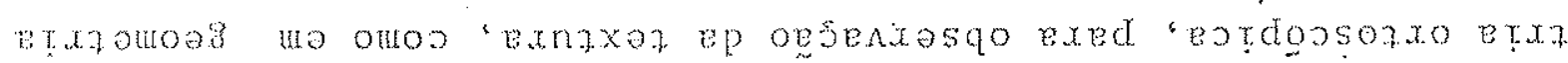

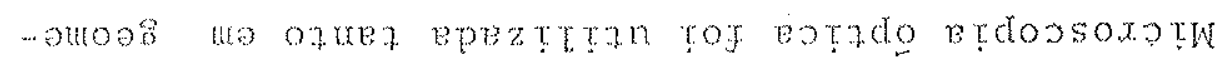

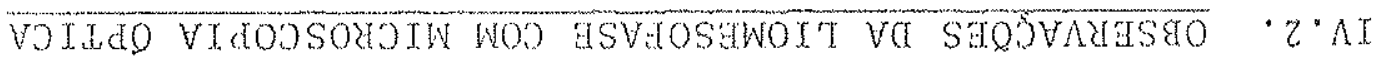

- sopesaxse sop

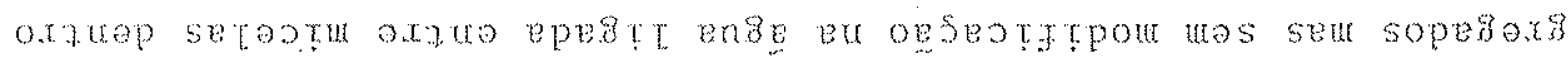

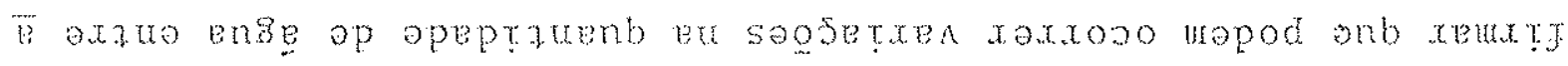

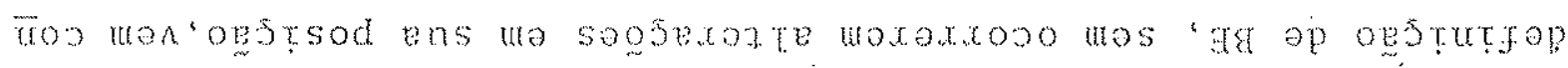

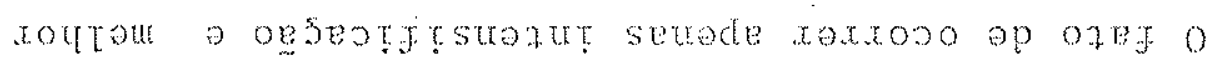

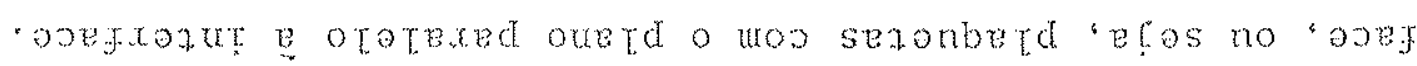

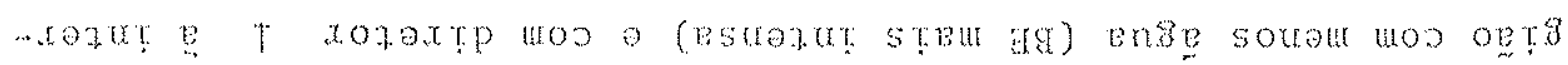

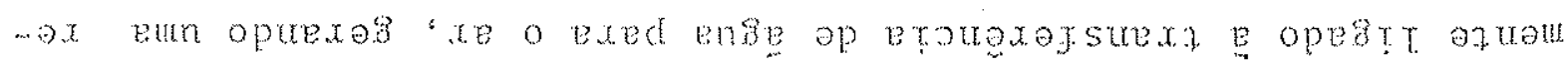

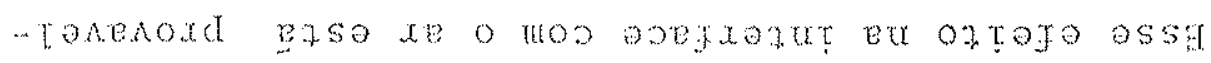

- ro ab osuatu strut wod "o epure xezou as-opod

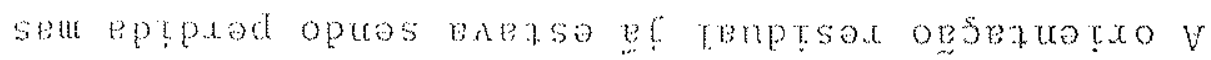

- teotaxan oxetpexale

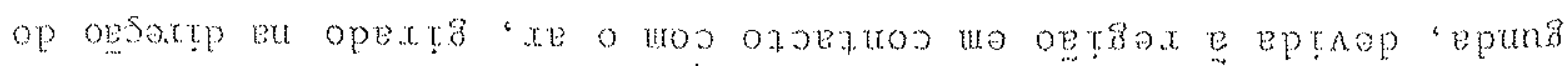

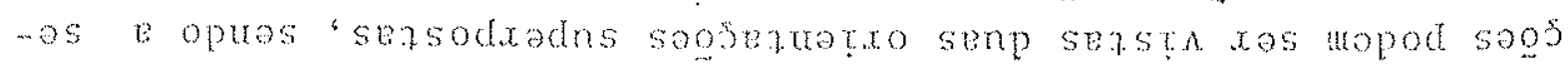

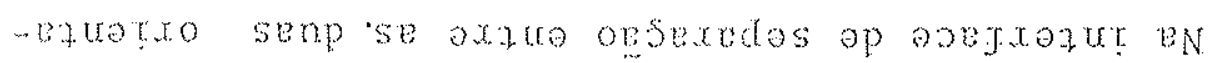

- epenosgo roj tensn terrolenba ogsezuraro

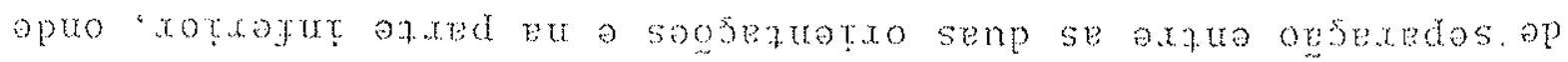

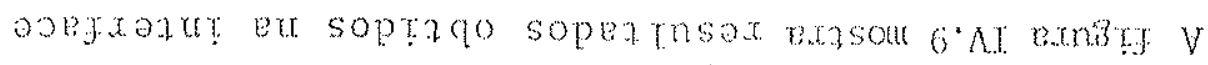




\section{- xe o luop opegarat (q)}

a.tasome ep texzuos ezxed (в)

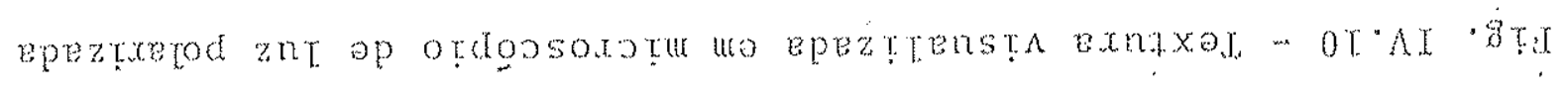

(c)

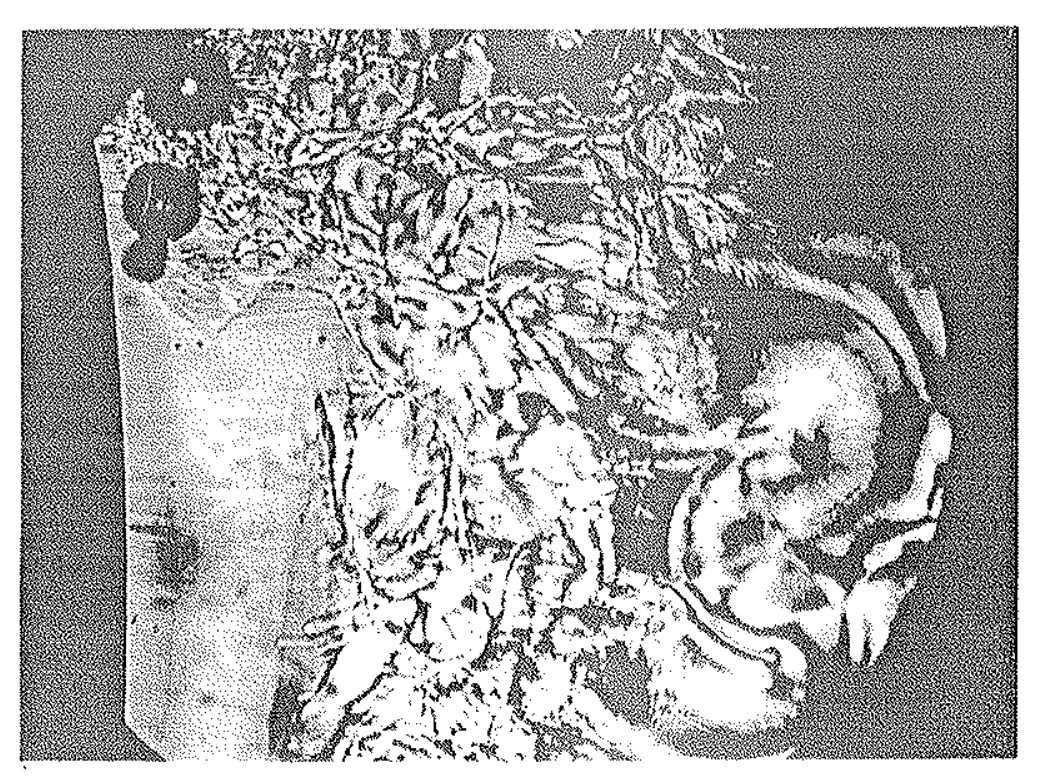

(e)

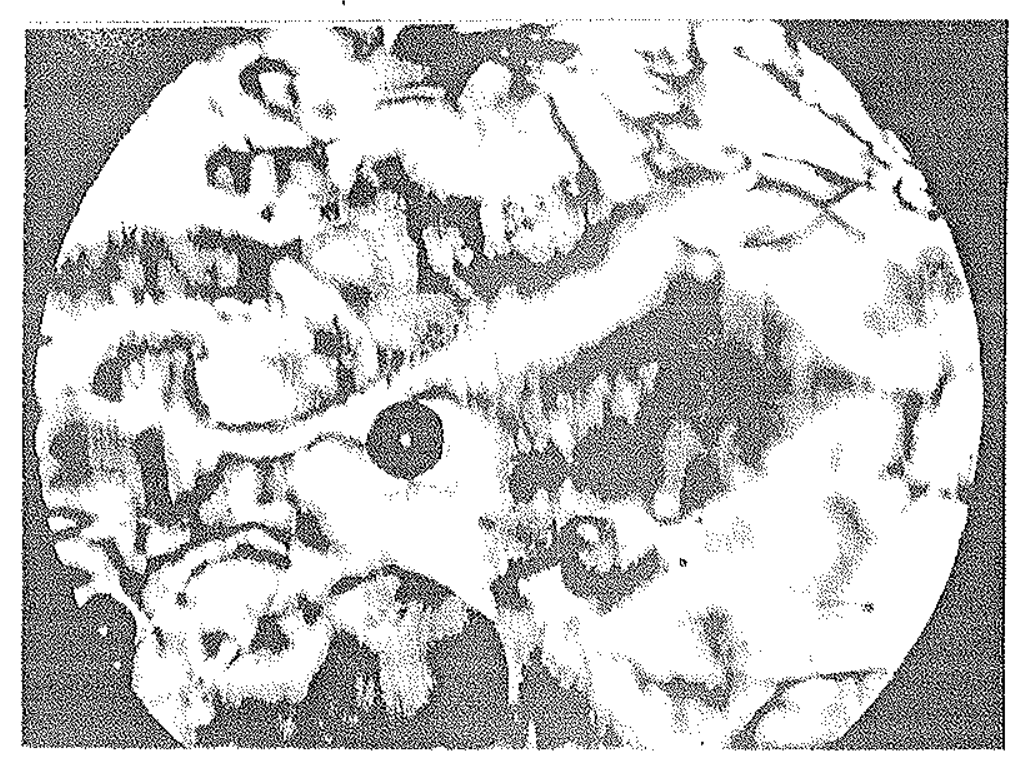


seu '

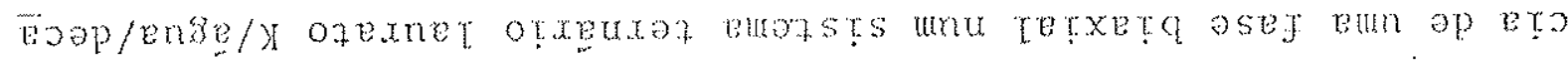

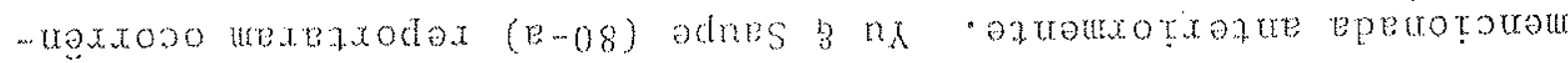

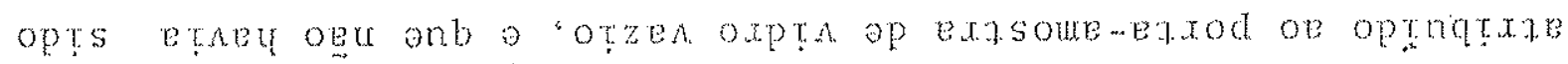

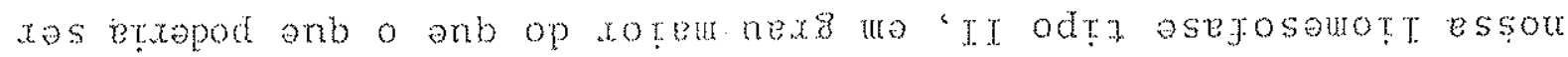

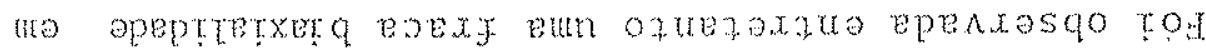

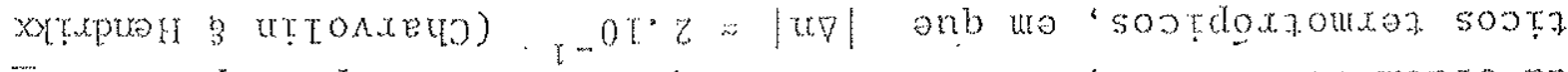

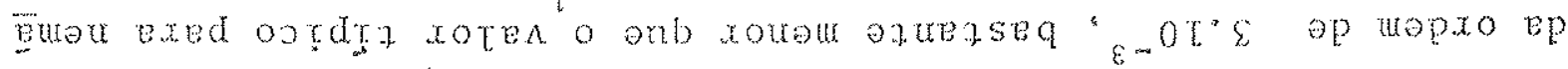

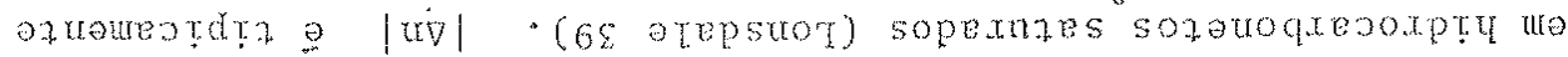
tropes ep opsaxp eu xaxoso opsexax op asput xotem op oxex o

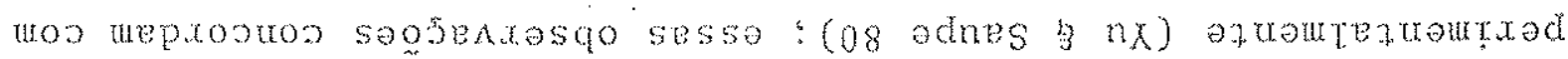

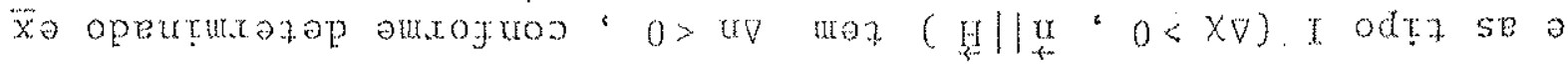

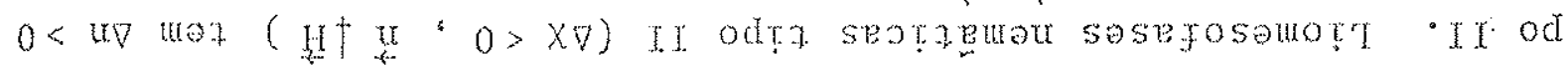

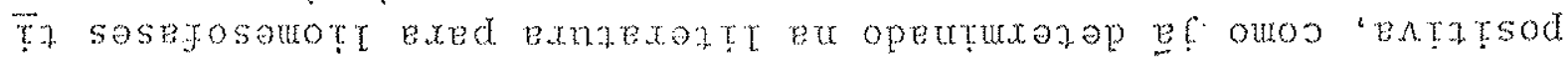

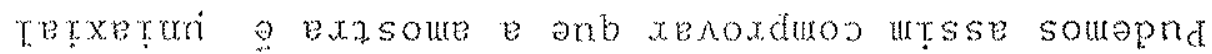

setopexed sopoxed sep oes

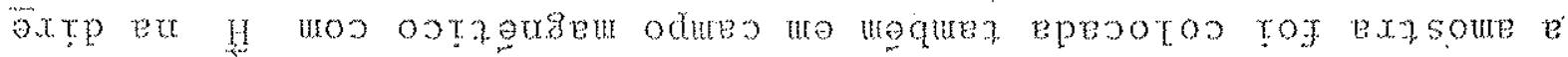

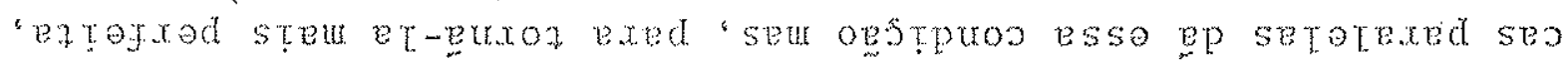

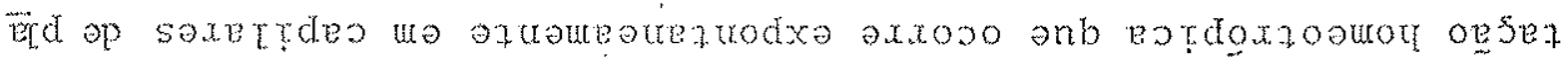

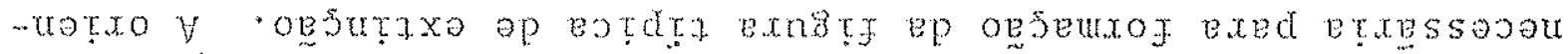

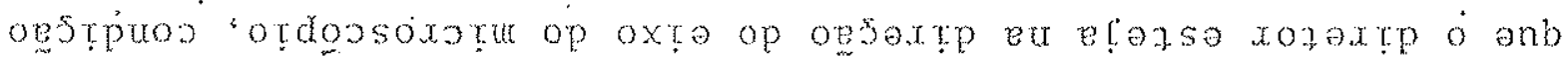
me8txe eordossonos etra

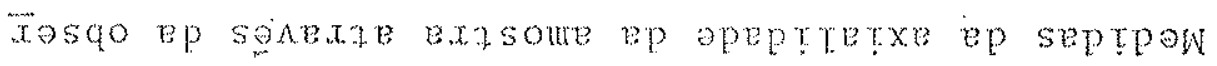

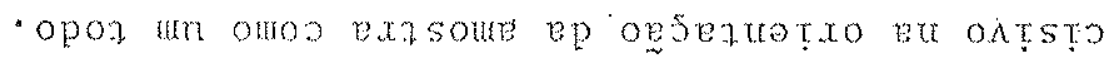

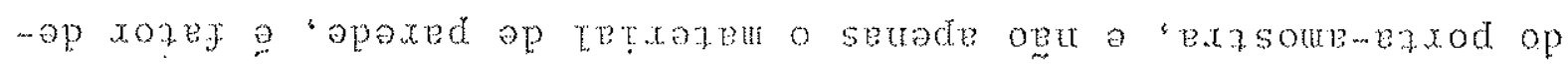

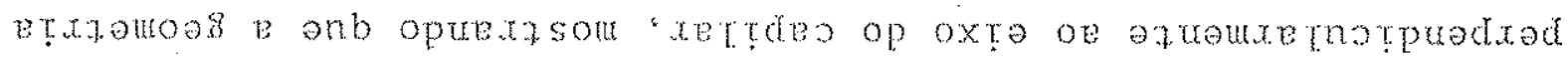

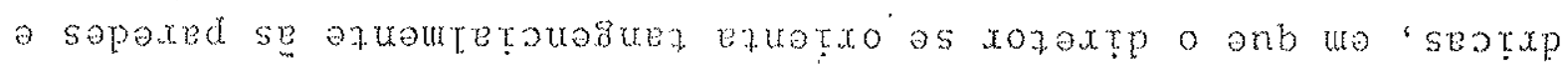

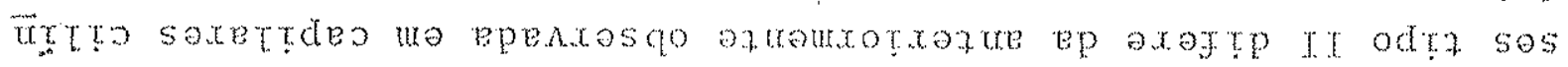

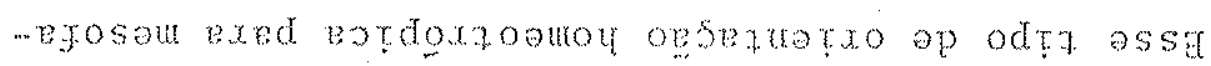

- (os adnes $3 x)$

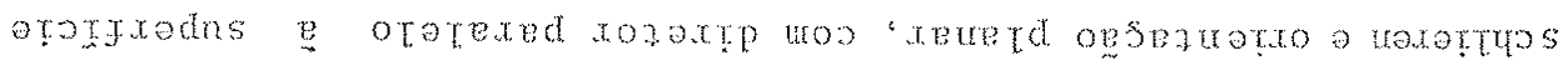

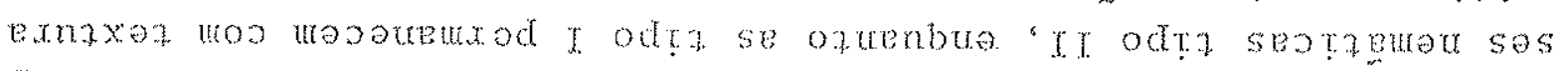

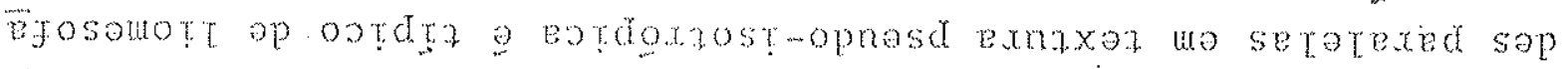

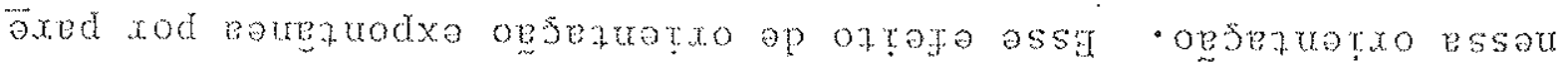

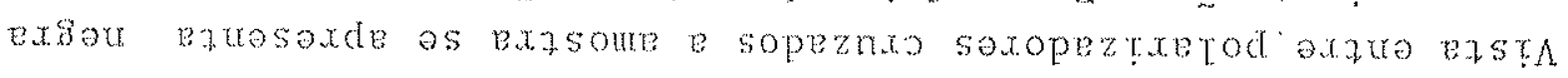

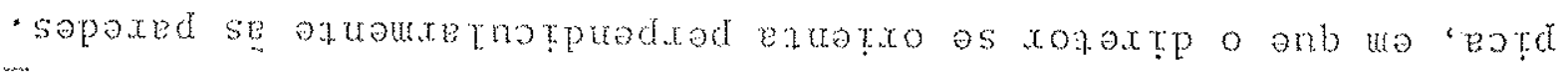

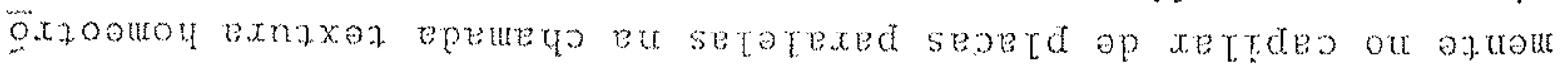

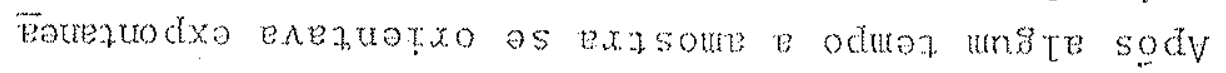

- 08 adnes $n x)$

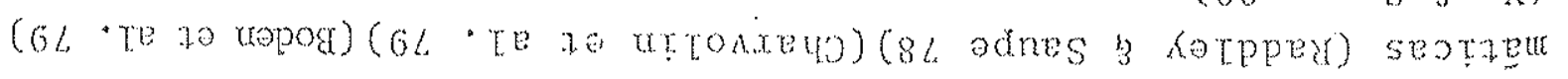




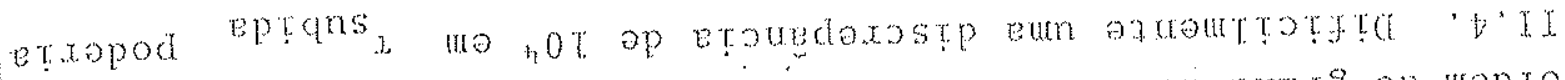

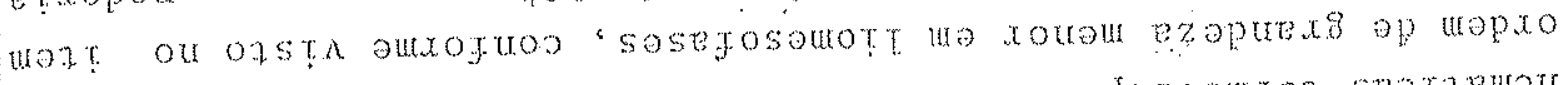

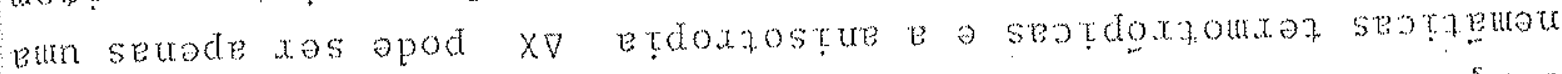

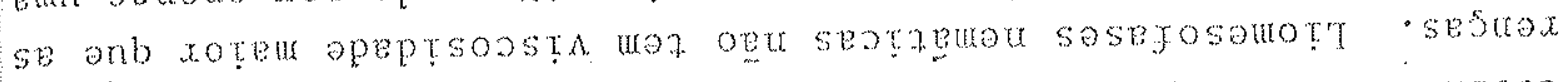
- axte sessep ezmoo oep ogu (T. rrod sepraqo eptosap a eptans ared seossordxe sV

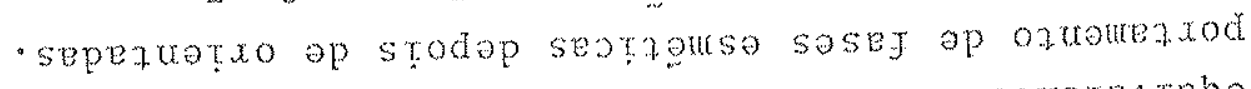

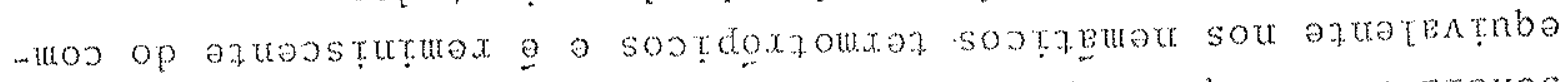

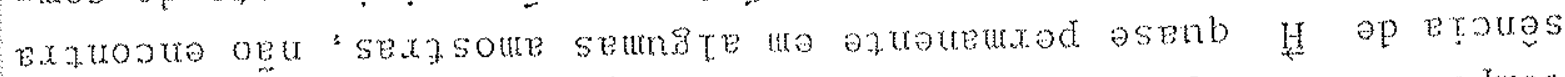

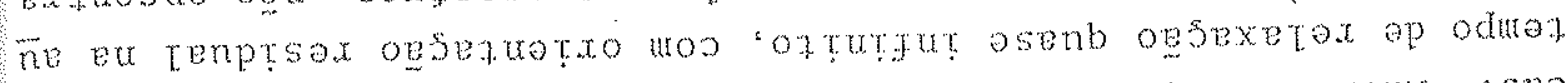

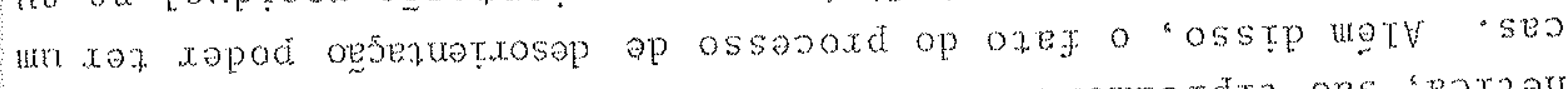

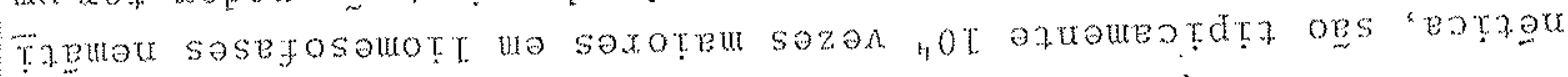

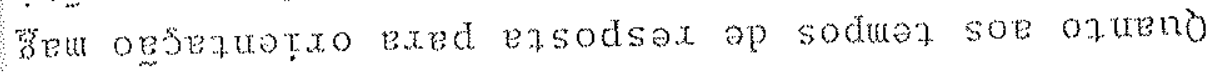

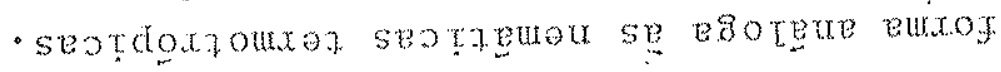

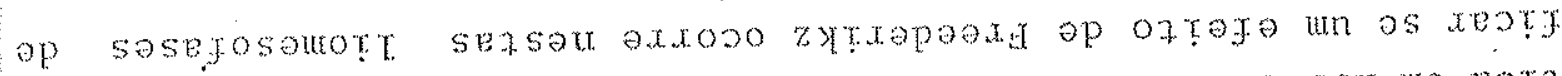

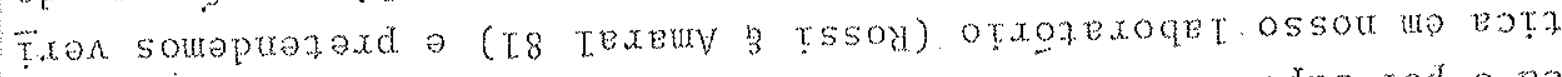

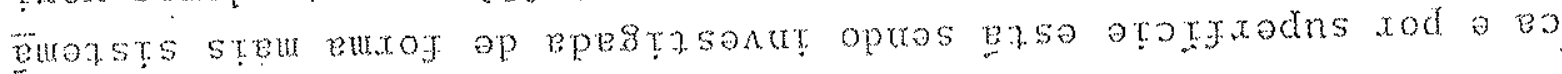

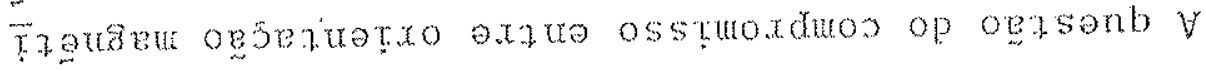

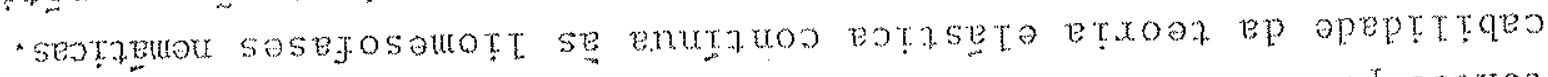

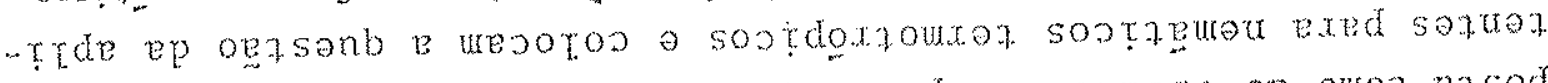

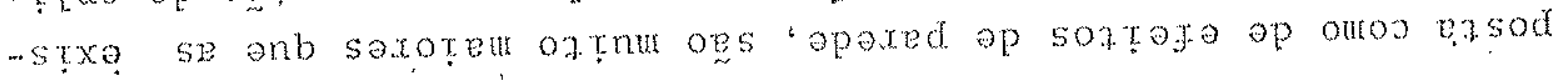
-. sar ap sodur ap oztrz "ezaptrexs op suepro sezsa

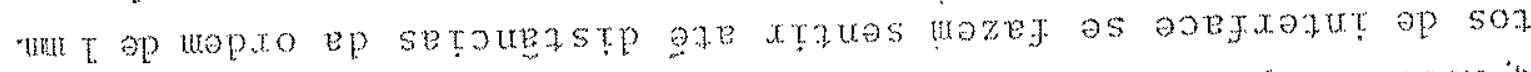

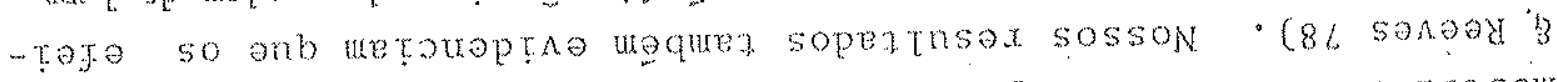

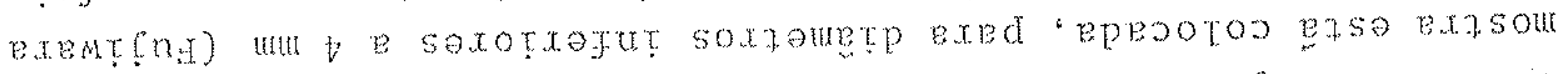
- e epro oqna op otex op estanut opsung eum dos opseanotroox op epeptoron rp ozej o merotouou wWN op sopejtnsoy

- msodsex ap soduez sossop ootzemezsts opnt.

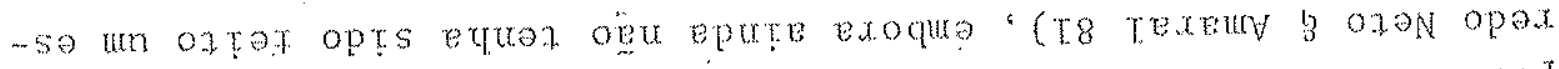

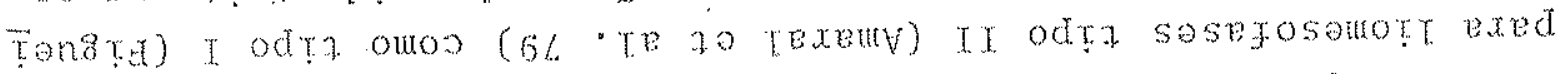

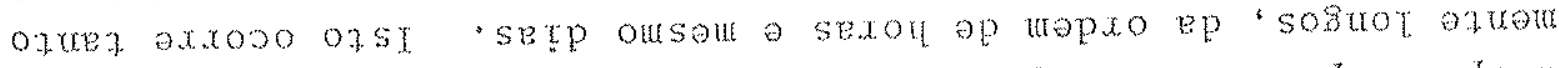

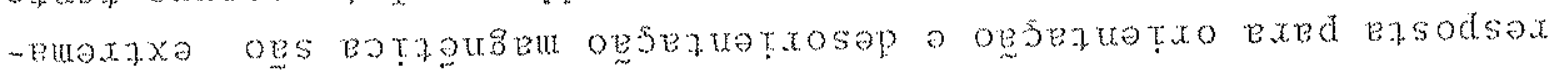

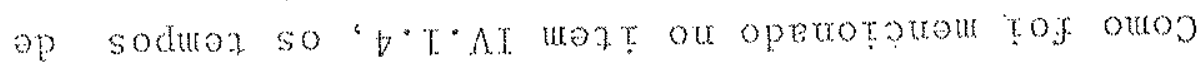

SVOWWWGN SaSVAOSAWOTT SVI VLSOASAd GA SOdWAI "s"AT

- Tr odra osegos

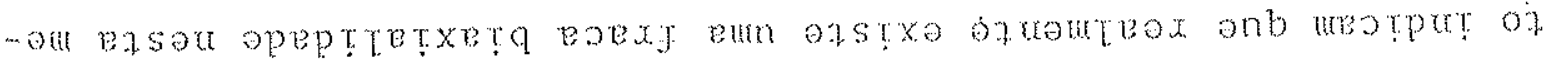

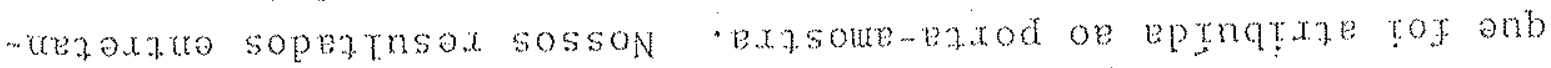




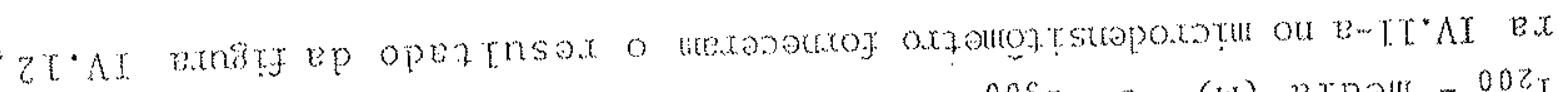

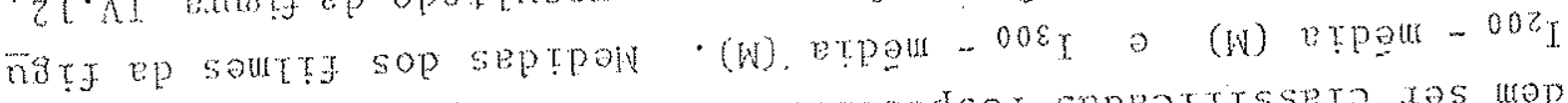

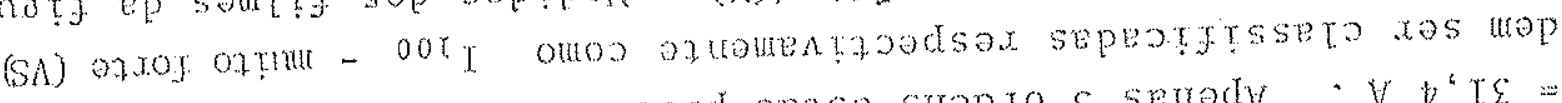

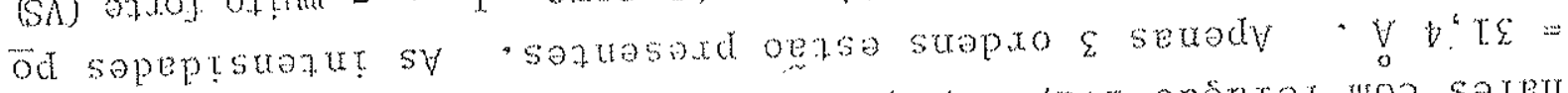

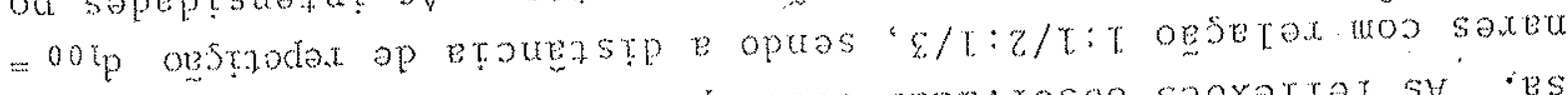

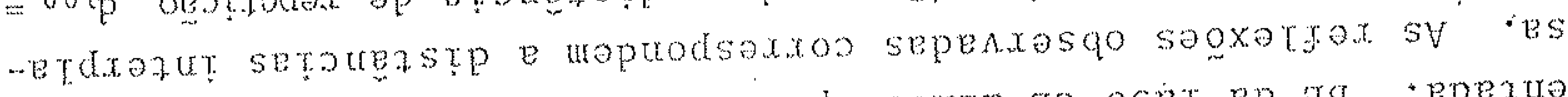

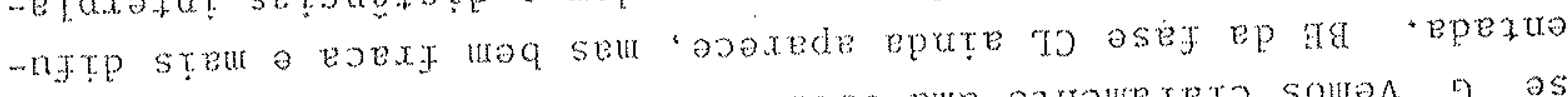

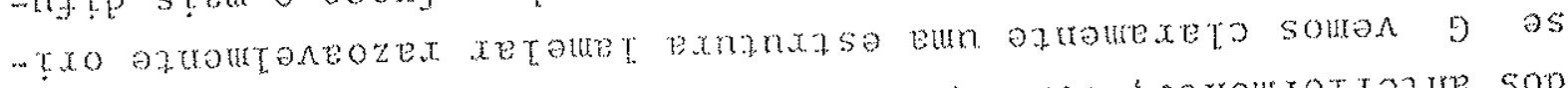

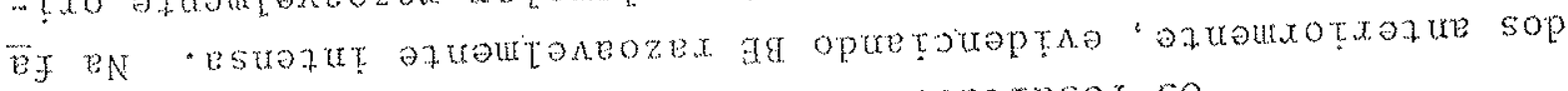

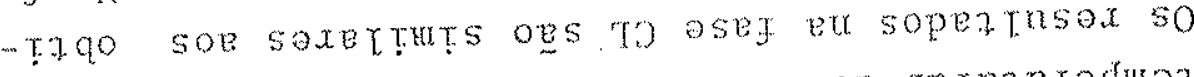

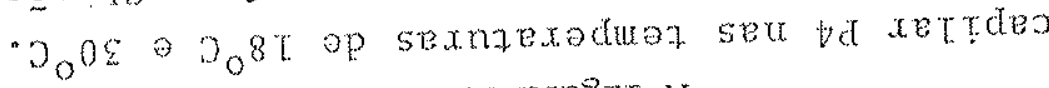

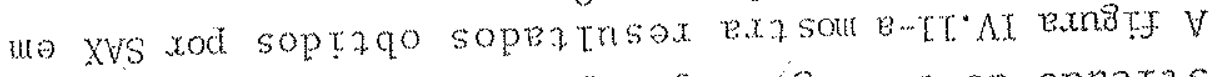

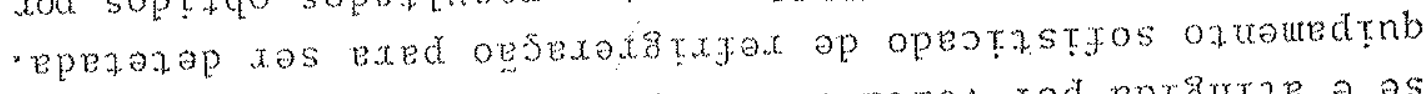

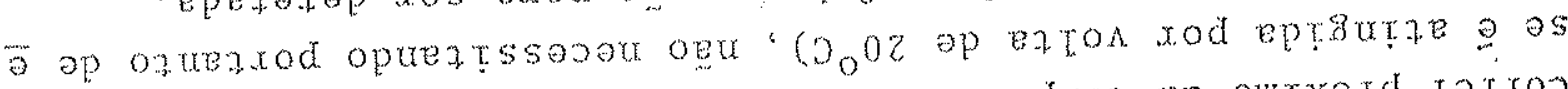

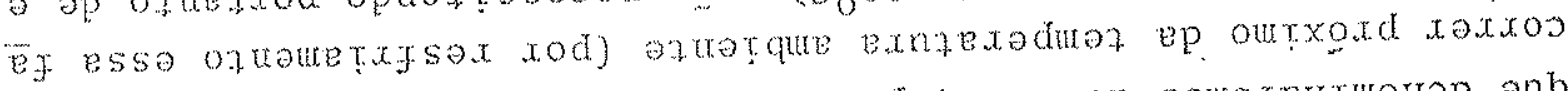

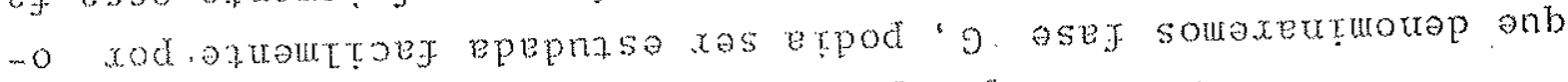

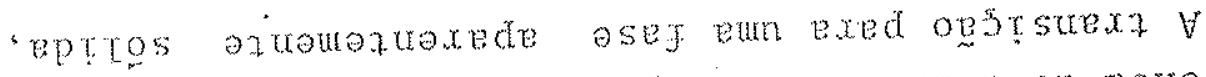

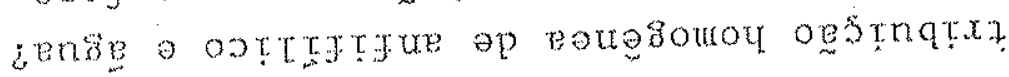

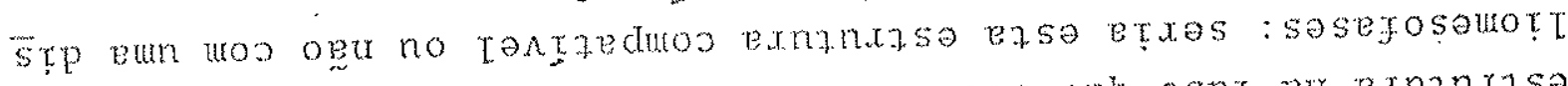

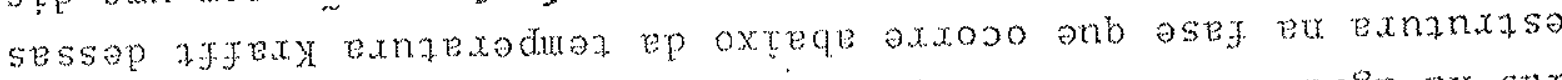

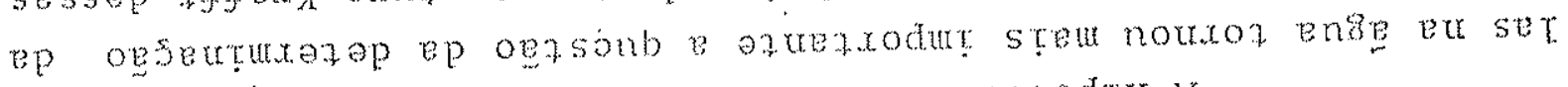
- oxtu ap boueboury ogu ogsmataste ap osorodtr $\forall$

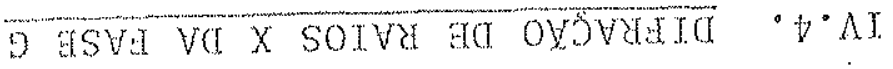

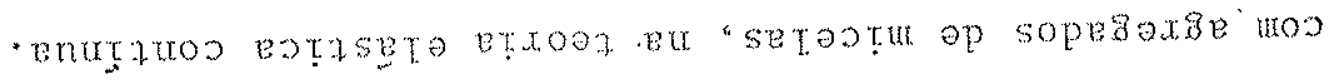

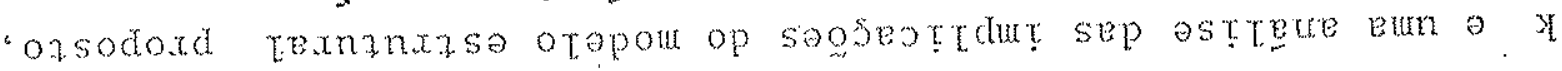

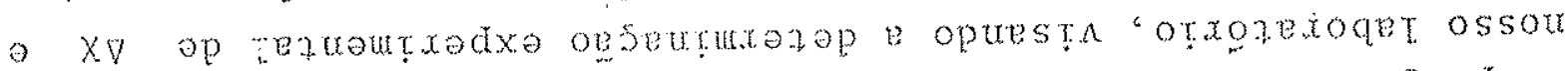

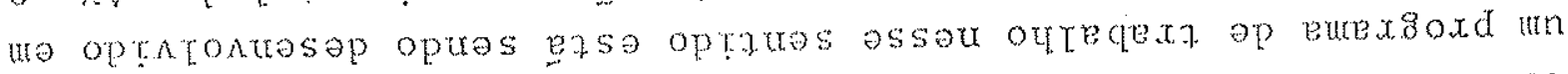

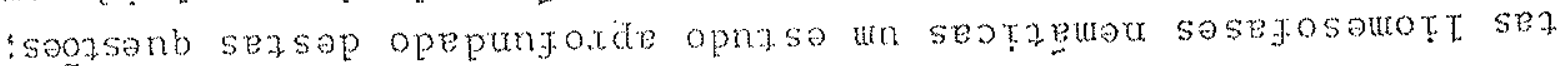

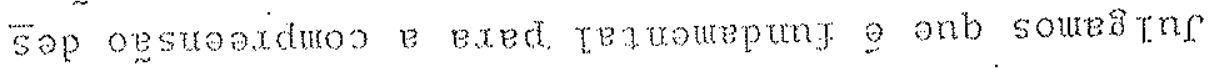

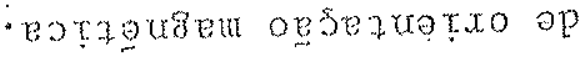

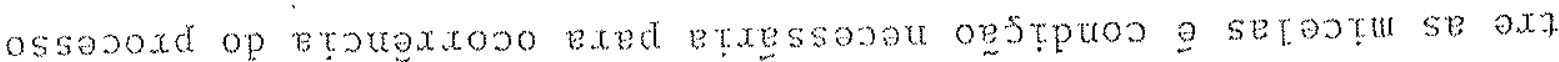

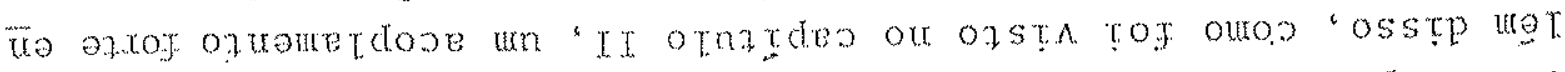

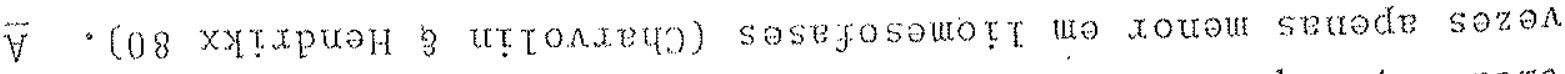

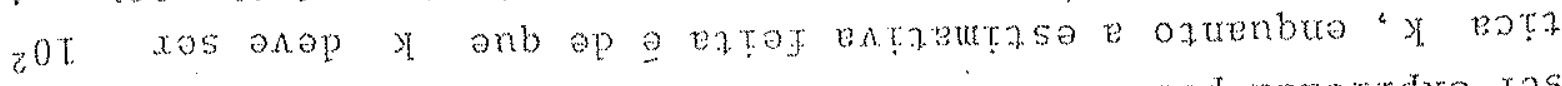

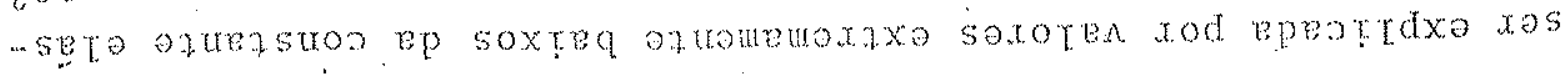

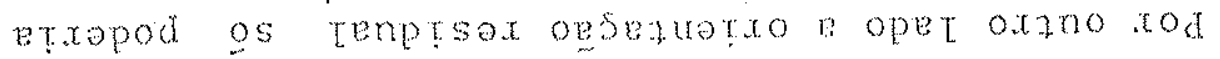

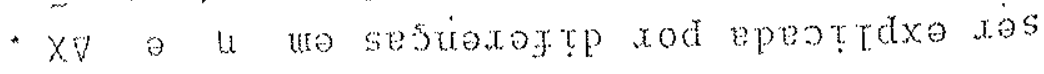




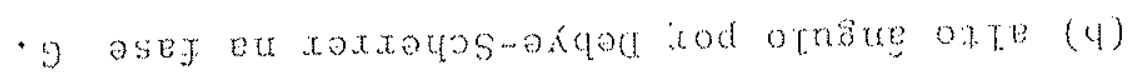

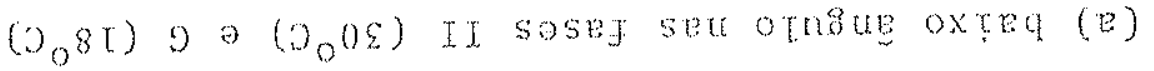

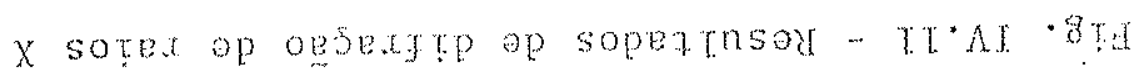
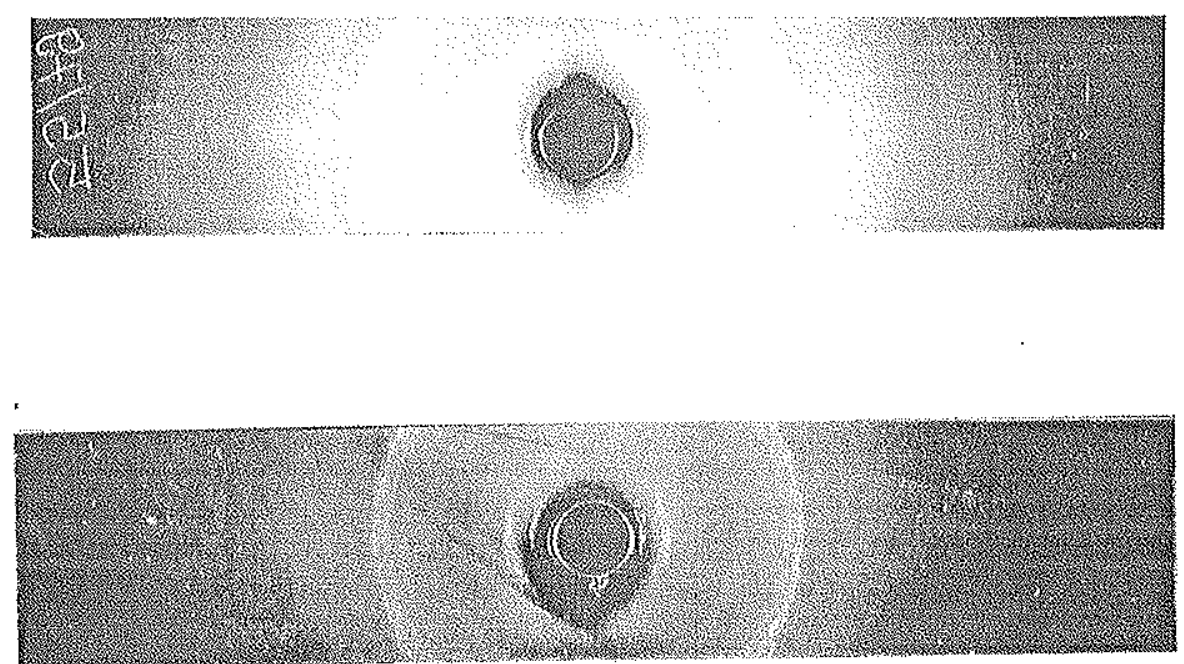

(a)

$$
200=1
$$

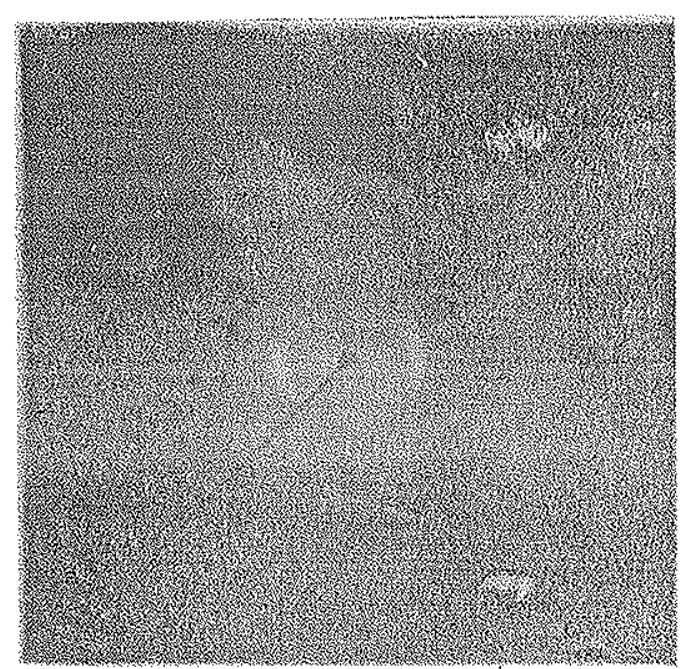

$$
08 \mathrm{O}=\mathrm{d}
$$

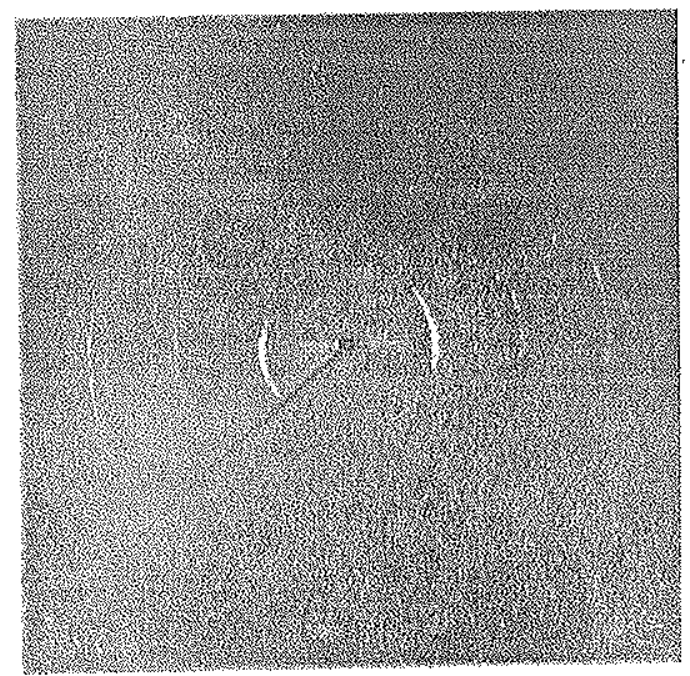

(e) 


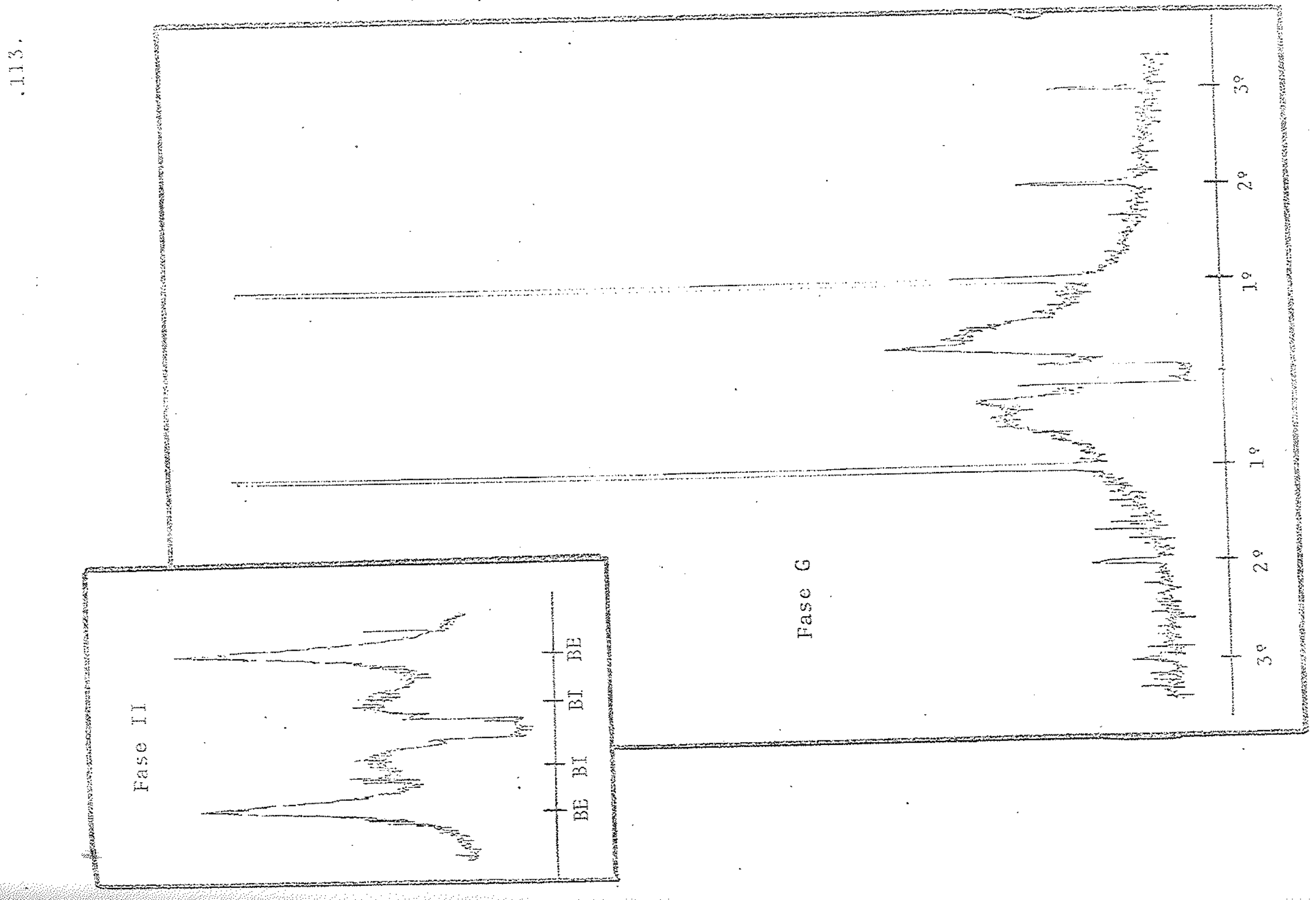




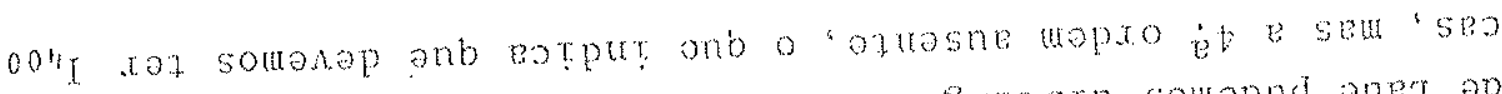

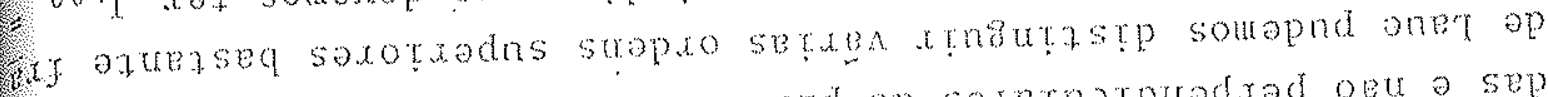

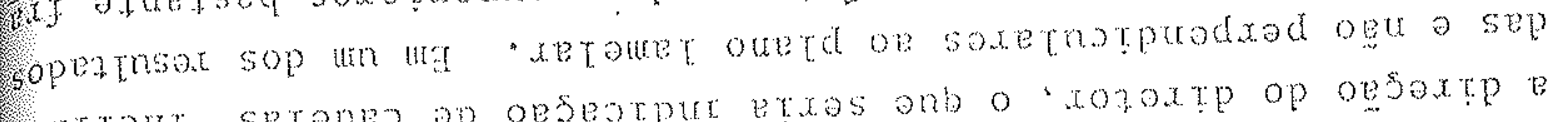

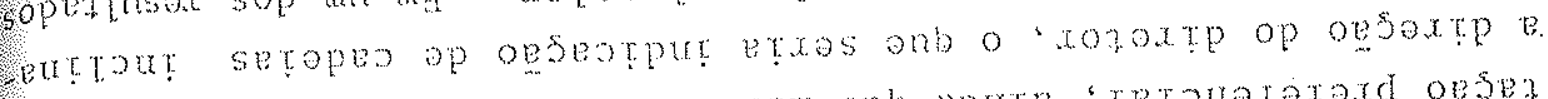

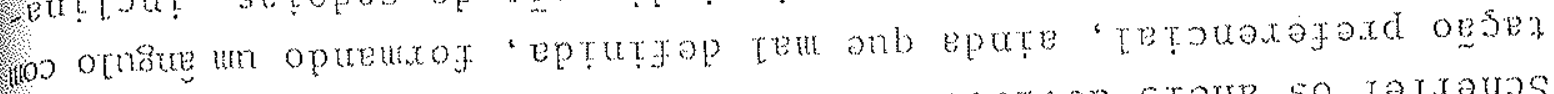

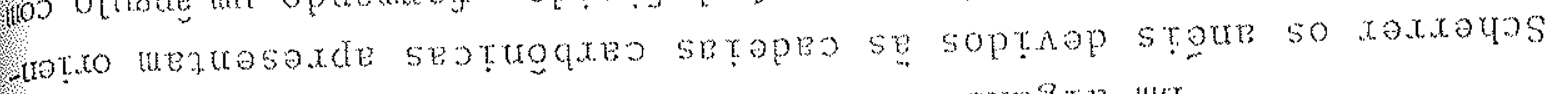

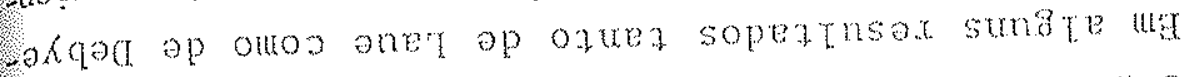

$$
\text { - (y \& z ap opentaso otpen otex) ootrtytre }
$$

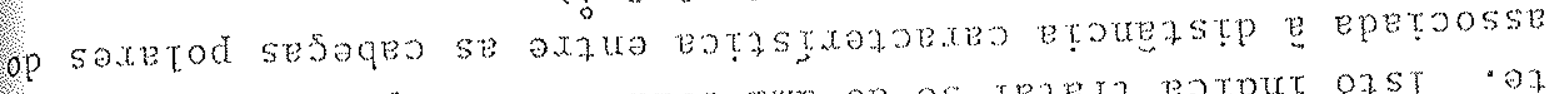

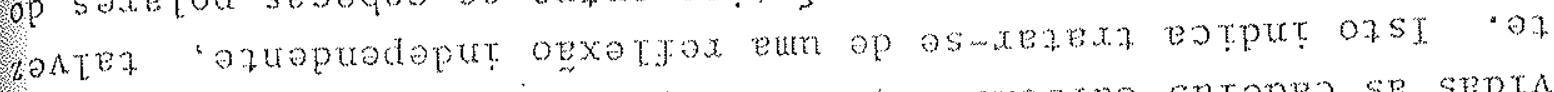

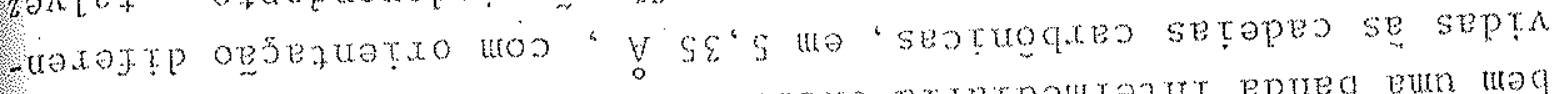

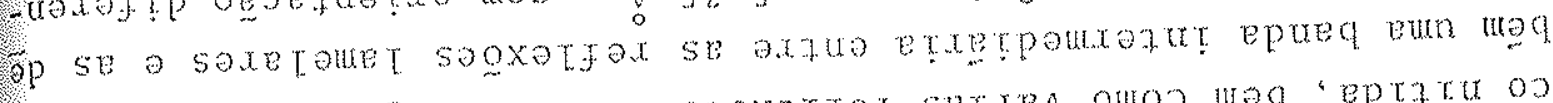

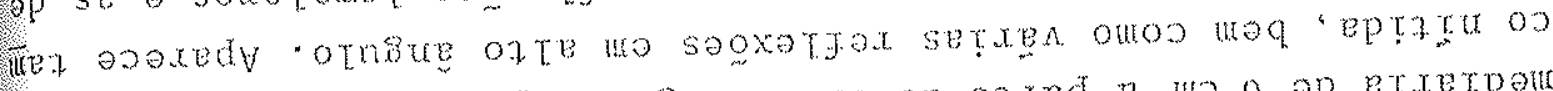

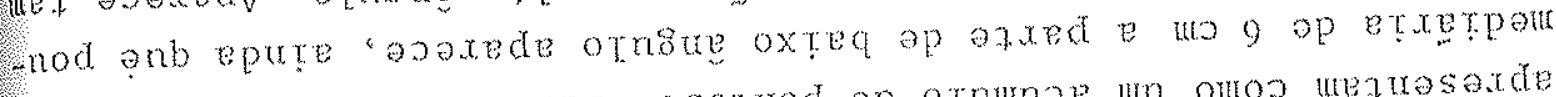

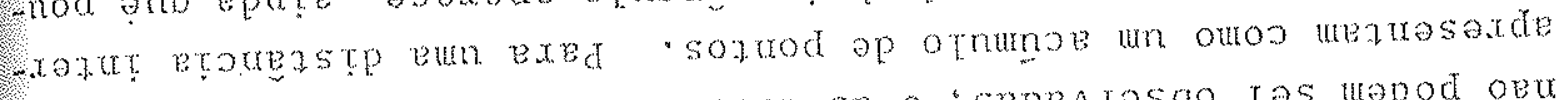

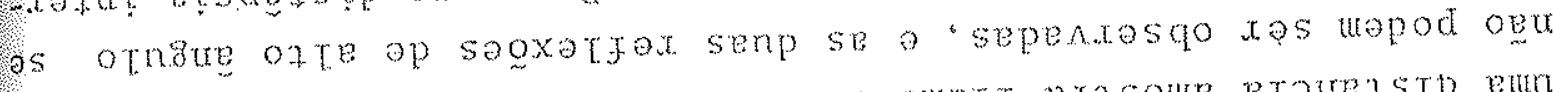

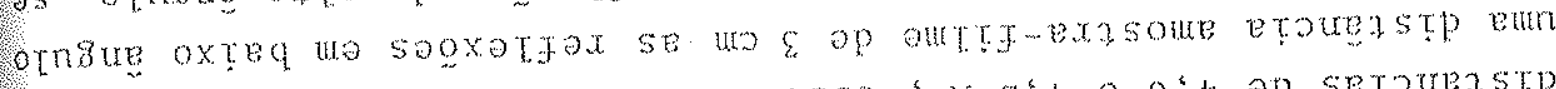

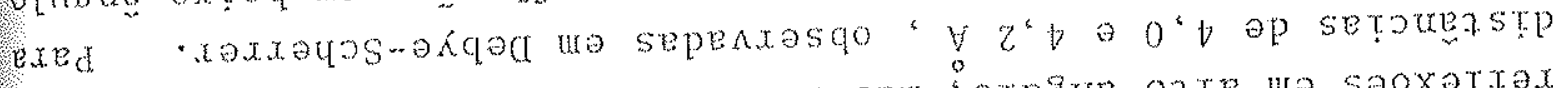

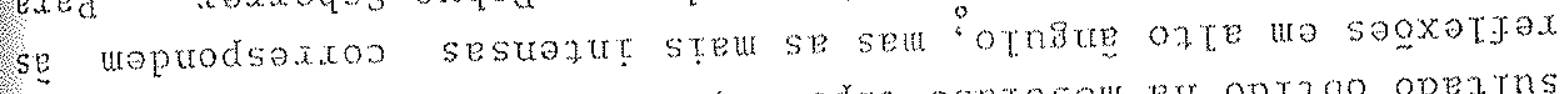

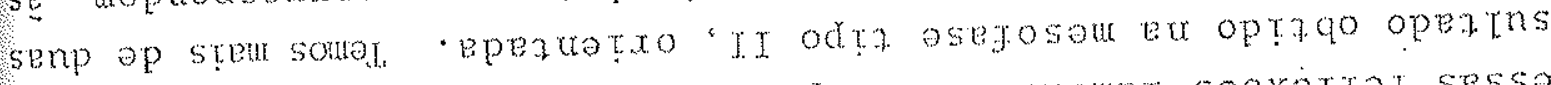

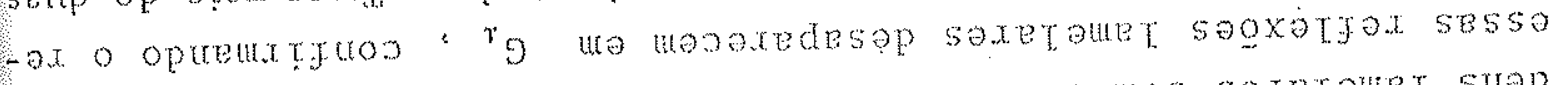

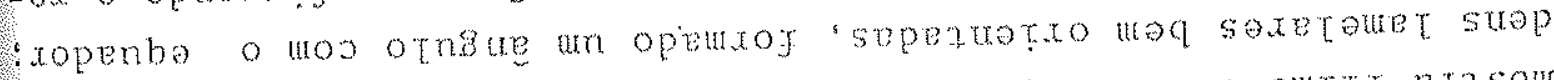

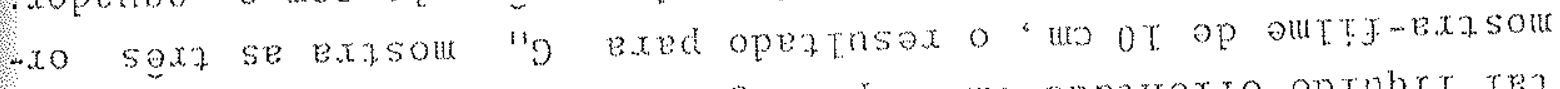

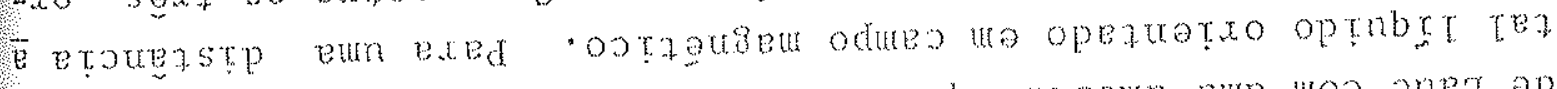

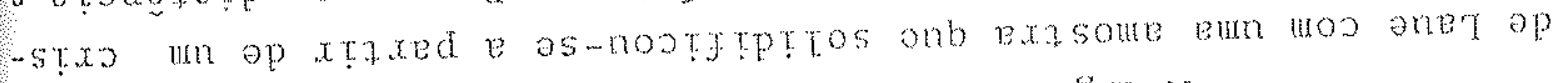

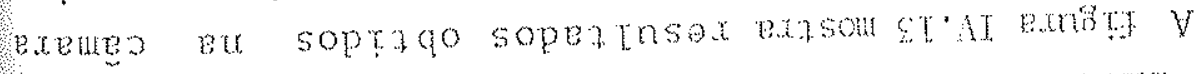

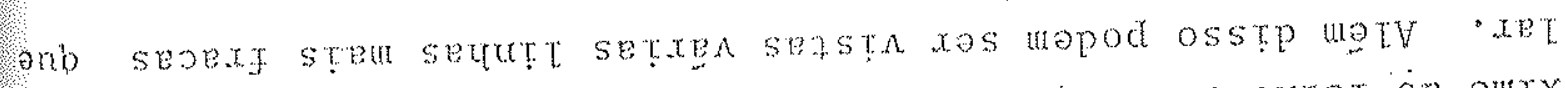

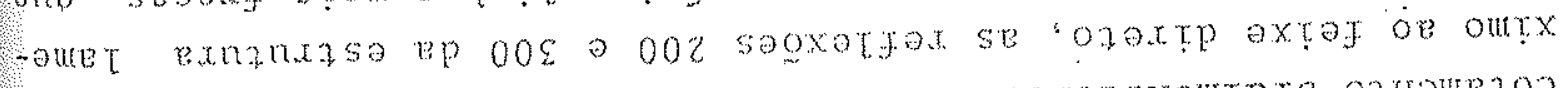

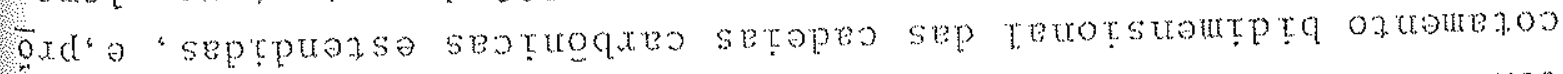

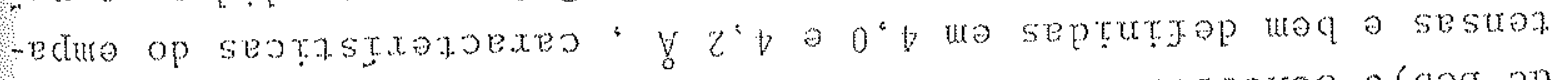

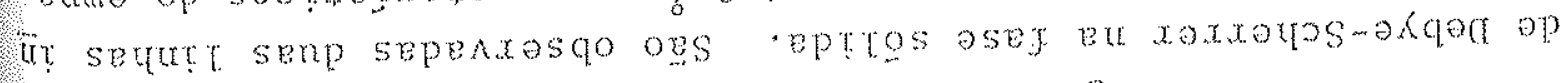

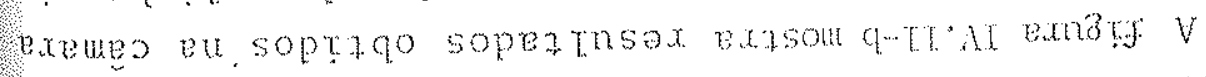

- setomet sep xotarar ou sestroqua setop

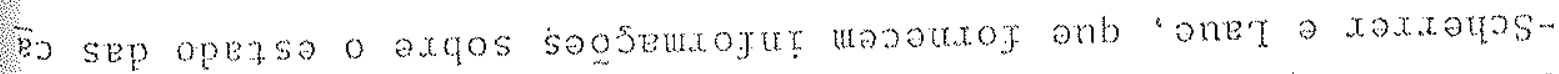

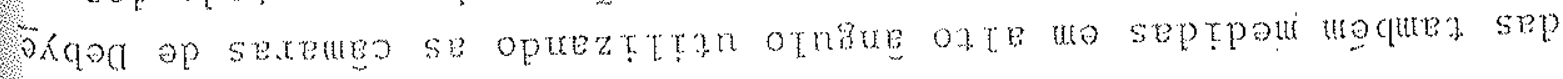

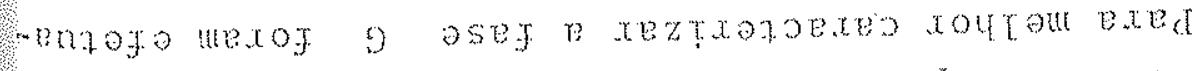

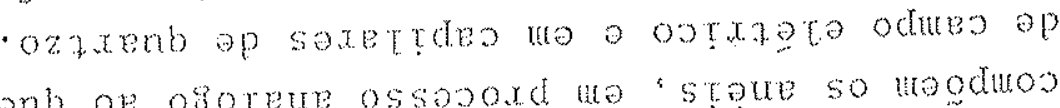

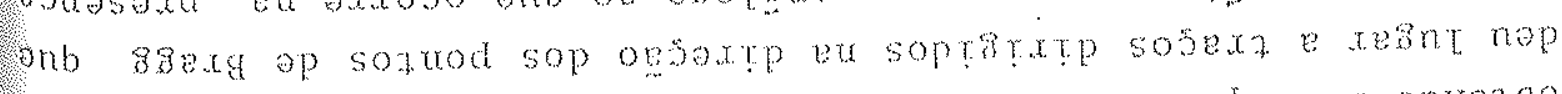

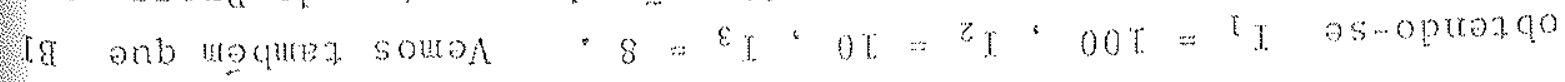




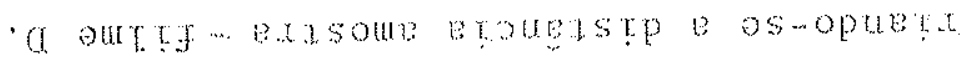

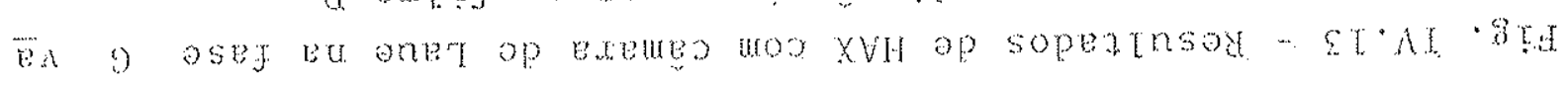

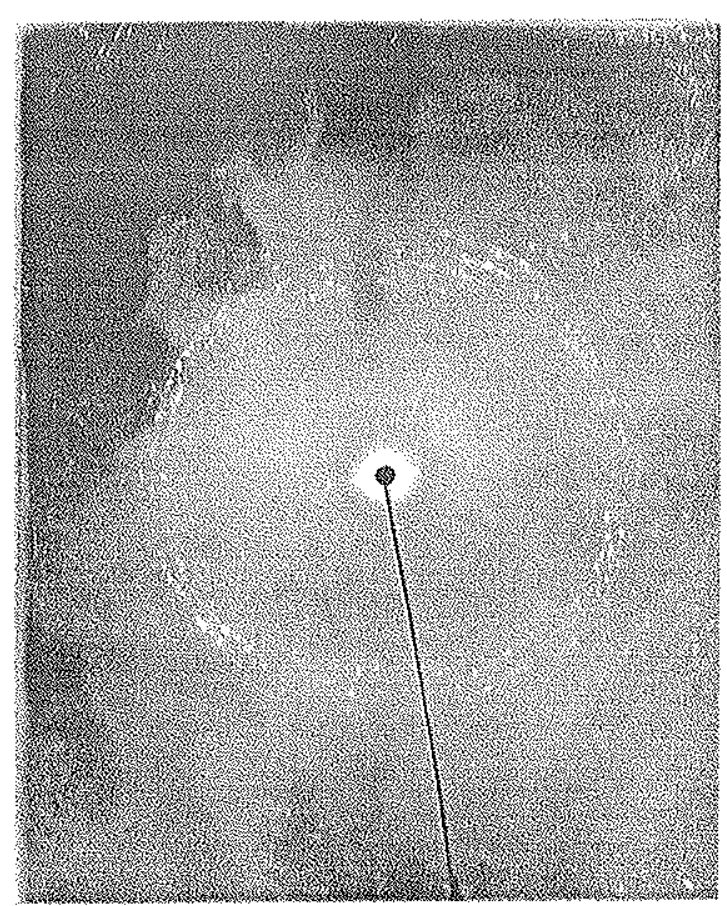

uso $9=0$

$r$

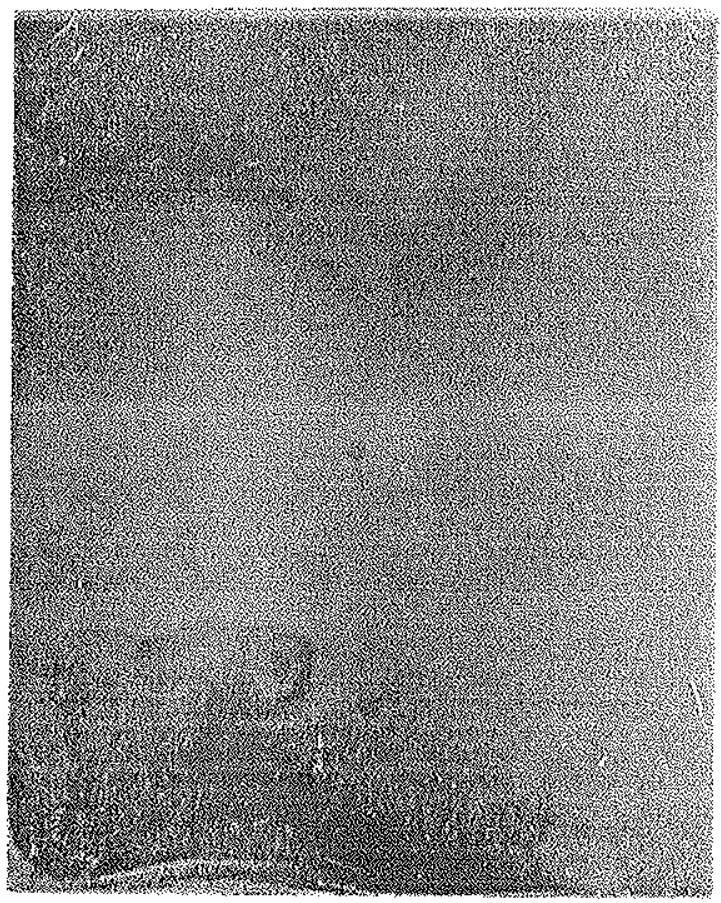

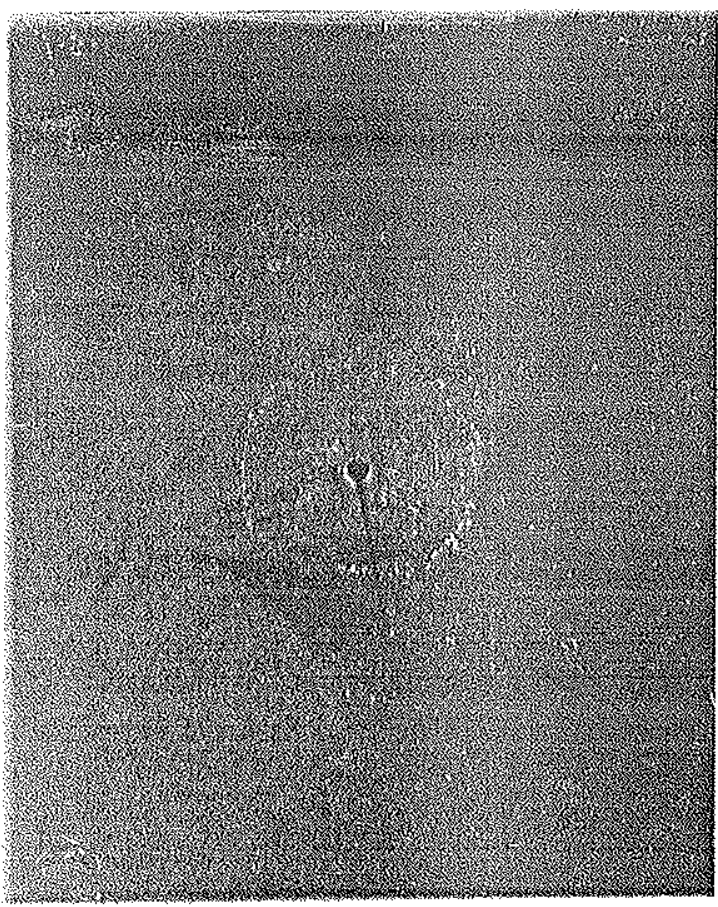

IIID $\mathrm{S} \approx \mathrm{a}$
"D

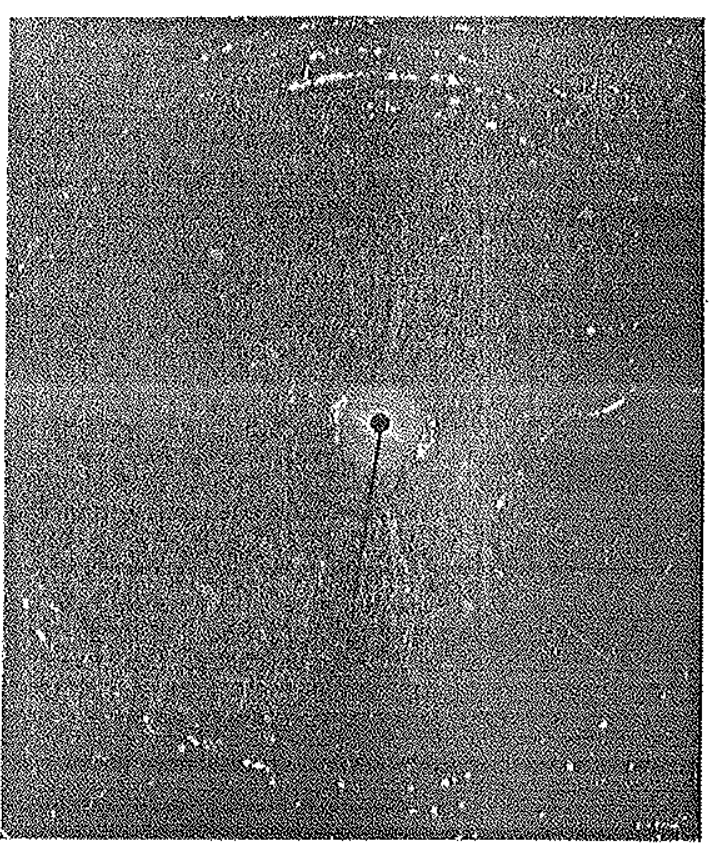

II) ot $=a$ 


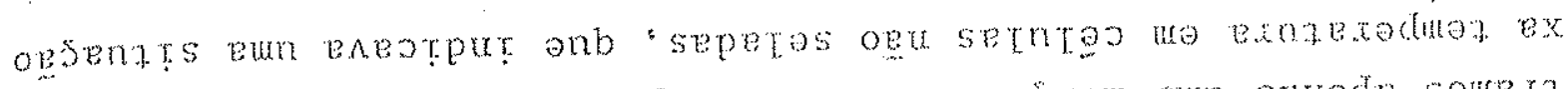

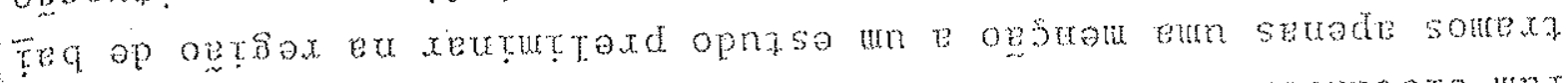

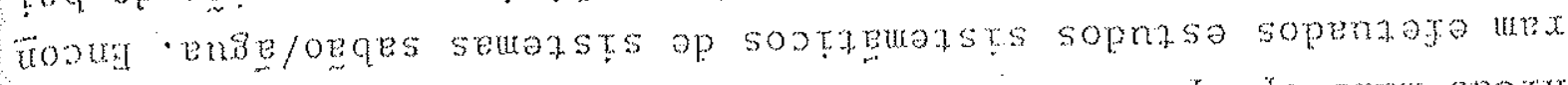
-ox opu osez op saostsuax sep opraso oe seperadoxde steu seotr 5ox sep xas retruaxte eoturea astere ep xesady

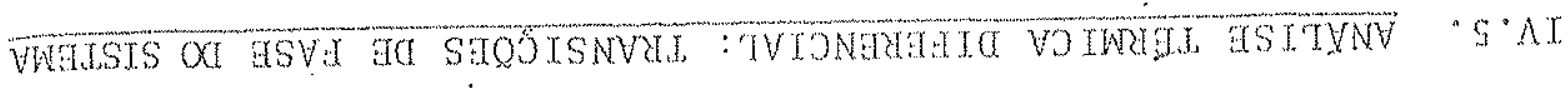

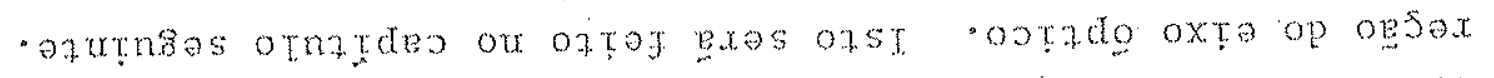

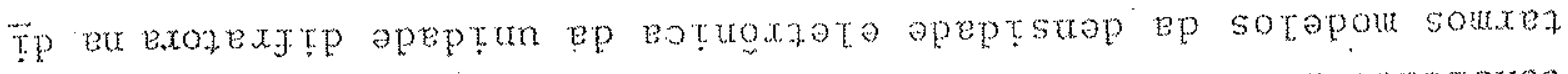

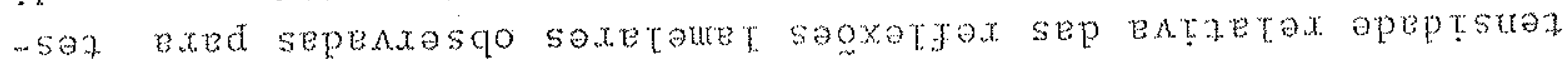

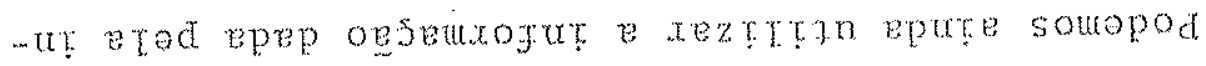

- xetauer exnanaso ep exos xezso anep ense ep azxd

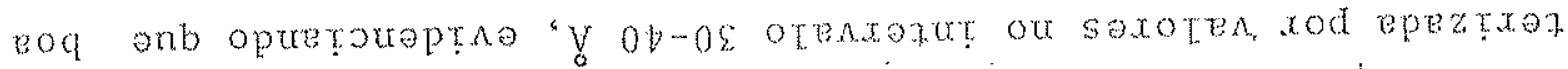

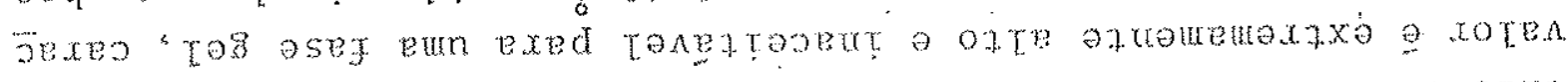

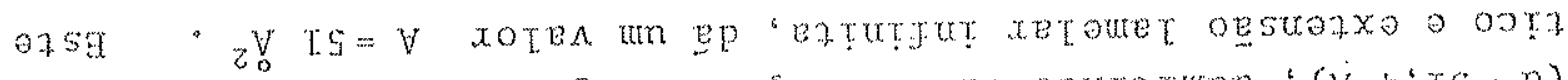

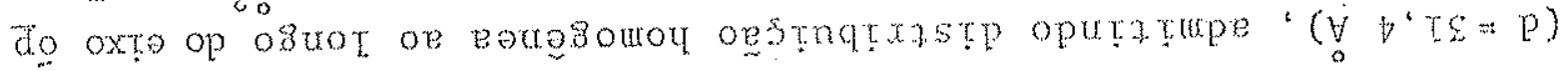

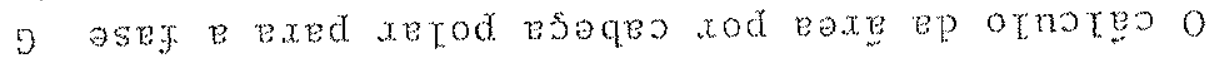

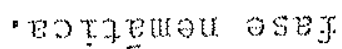

.osemot p exed ozsodoxd saxetomet sopezax8m op otopou o xaroqox

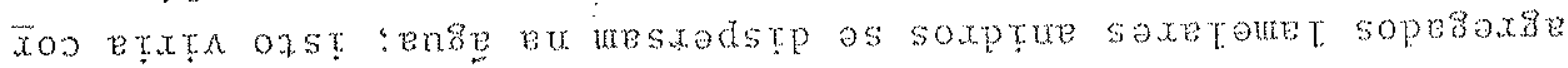

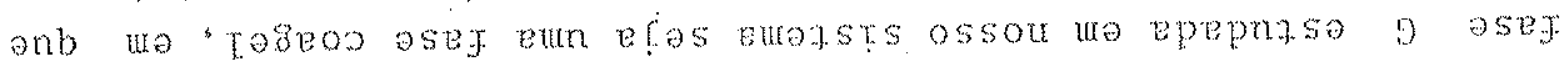

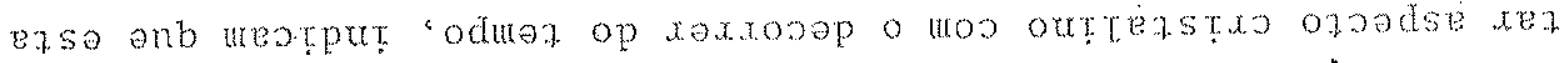

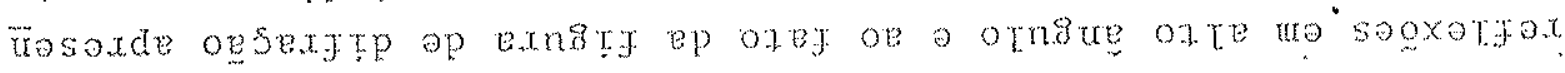
seruen op oesenxasqo ? sepette? seosmuogrt sesst

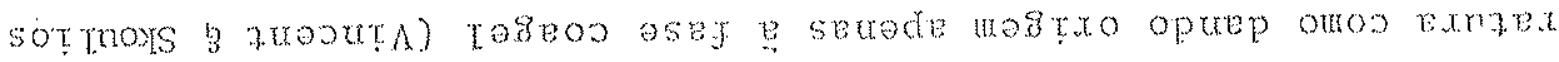

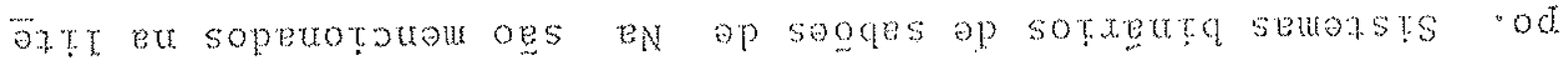

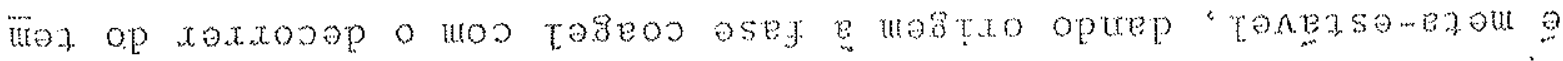

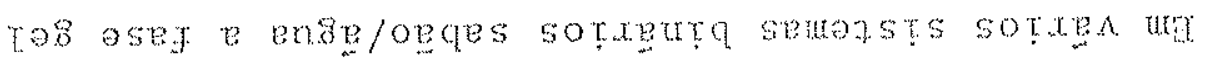

v.t mat ou septa

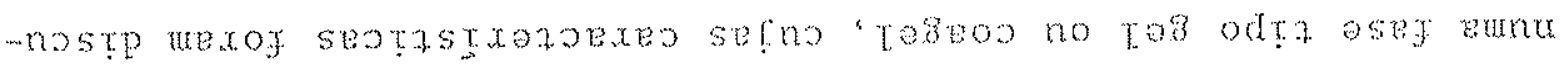

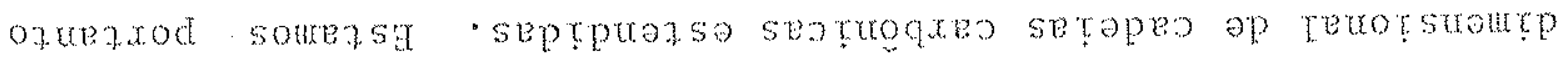

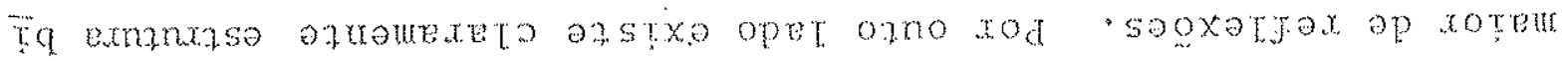

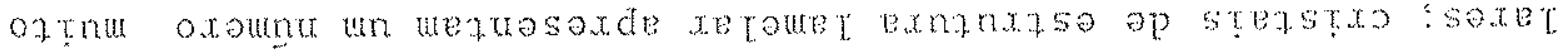

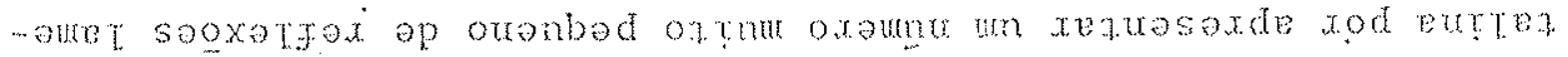

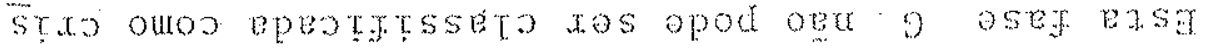

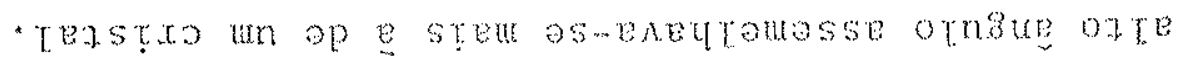

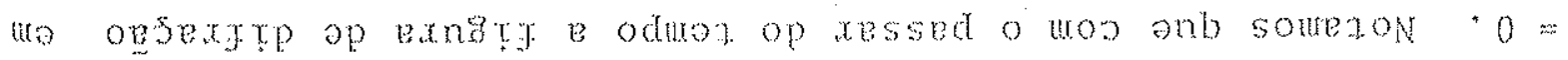




\begin{tabular}{|c|c|}
\hline$\varepsilon^{\circ} 0 \pi z^{*}$ & $L^{3} 0 \# 8^{\prime} \mathrm{EZ} \cdots$ \\
\hline $9 \times 0 * 9$ & $v^{\prime} 0$ 䎡 L"TT \\
\hline z. 再 69 & $9 \times 0 p^{\circ} 0$ \\
\hline$(3 / \mathrm{Te}) \mathrm{HV}$ & $\left(o_{0}\right) x$ \\
\hline
\end{tabular}

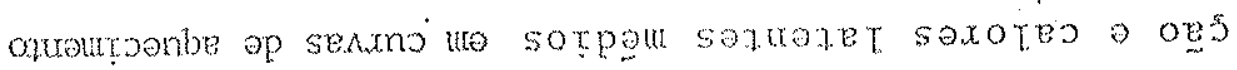

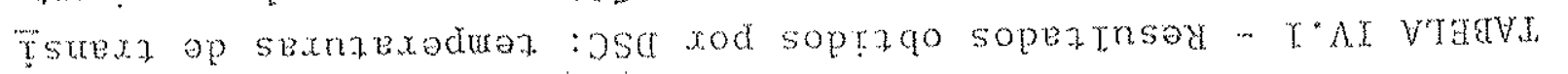

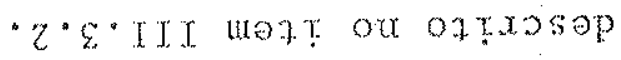

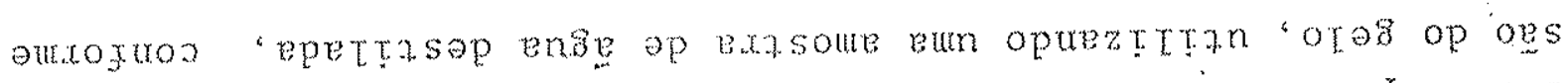

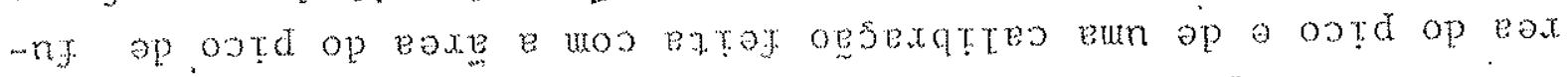

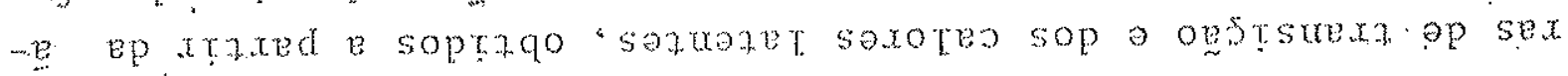
-nareduaz sep sotpeu sexoten so pp r"AT eteqea V

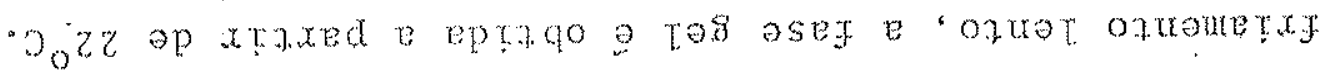

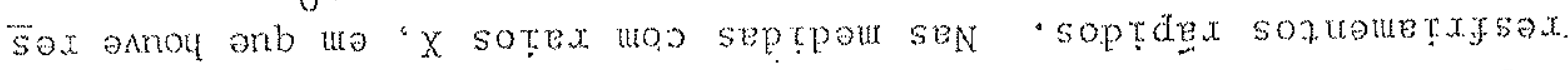

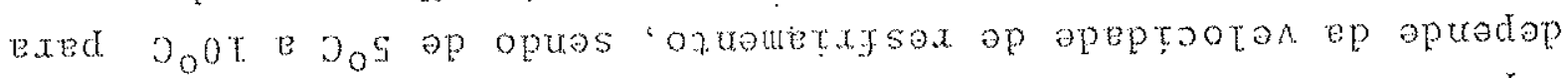

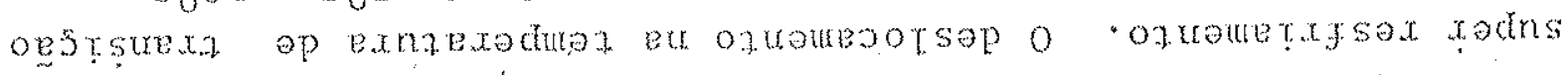

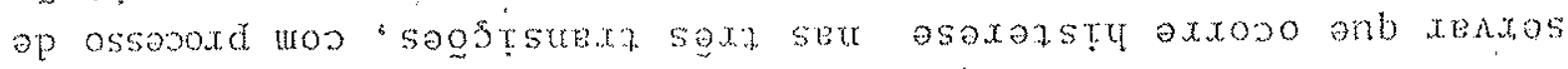

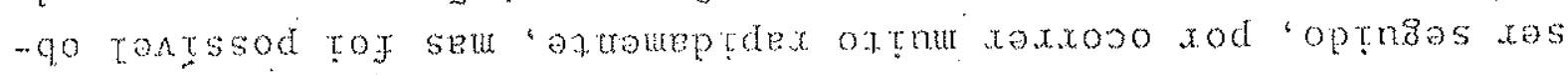

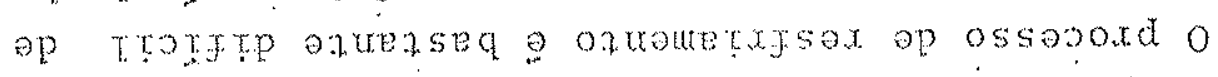

- sestisoop

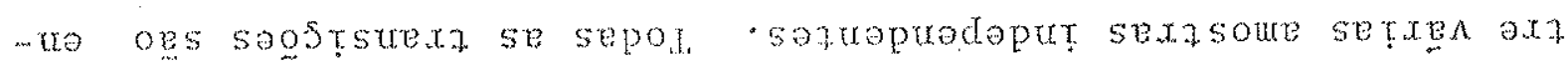

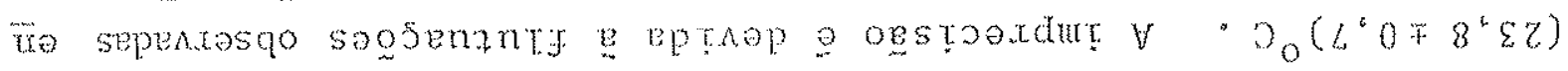

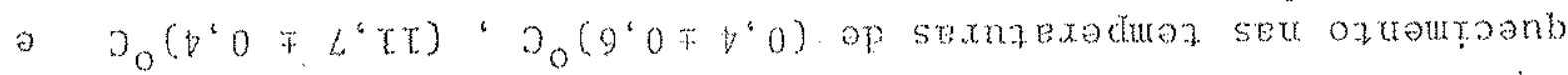

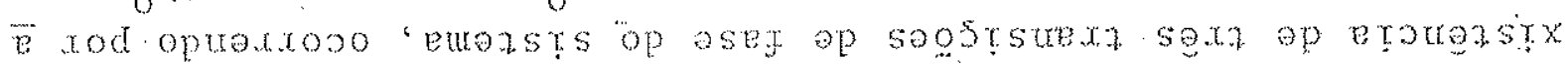

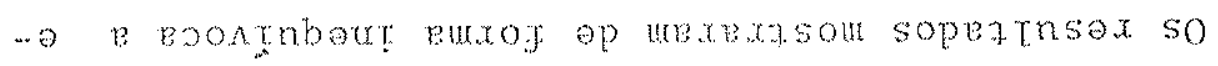

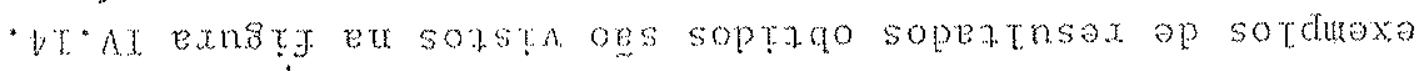

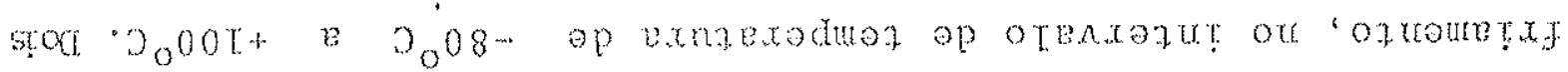

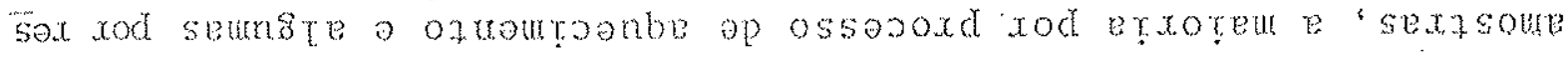

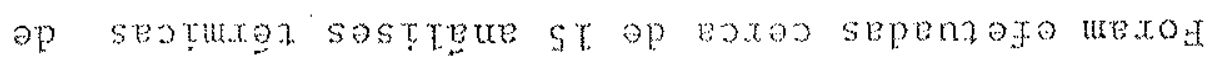

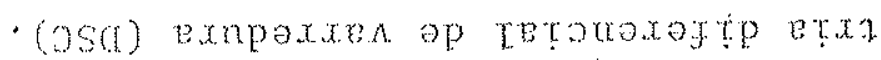

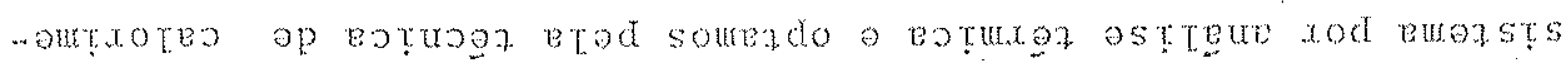
ossou op asej op sagstsuexa se xepnaso sourptood

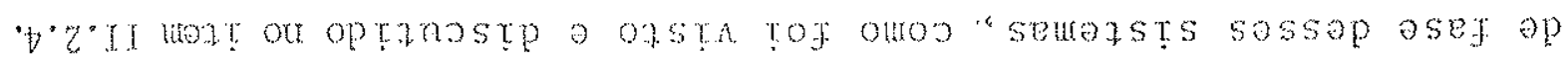

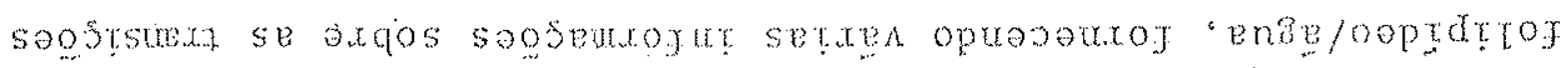

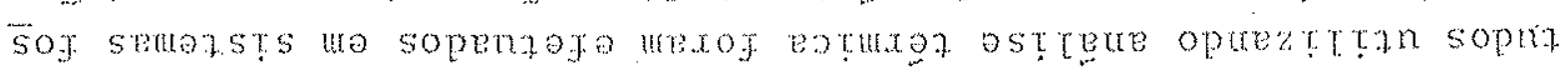

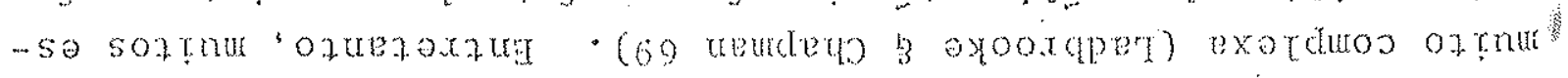



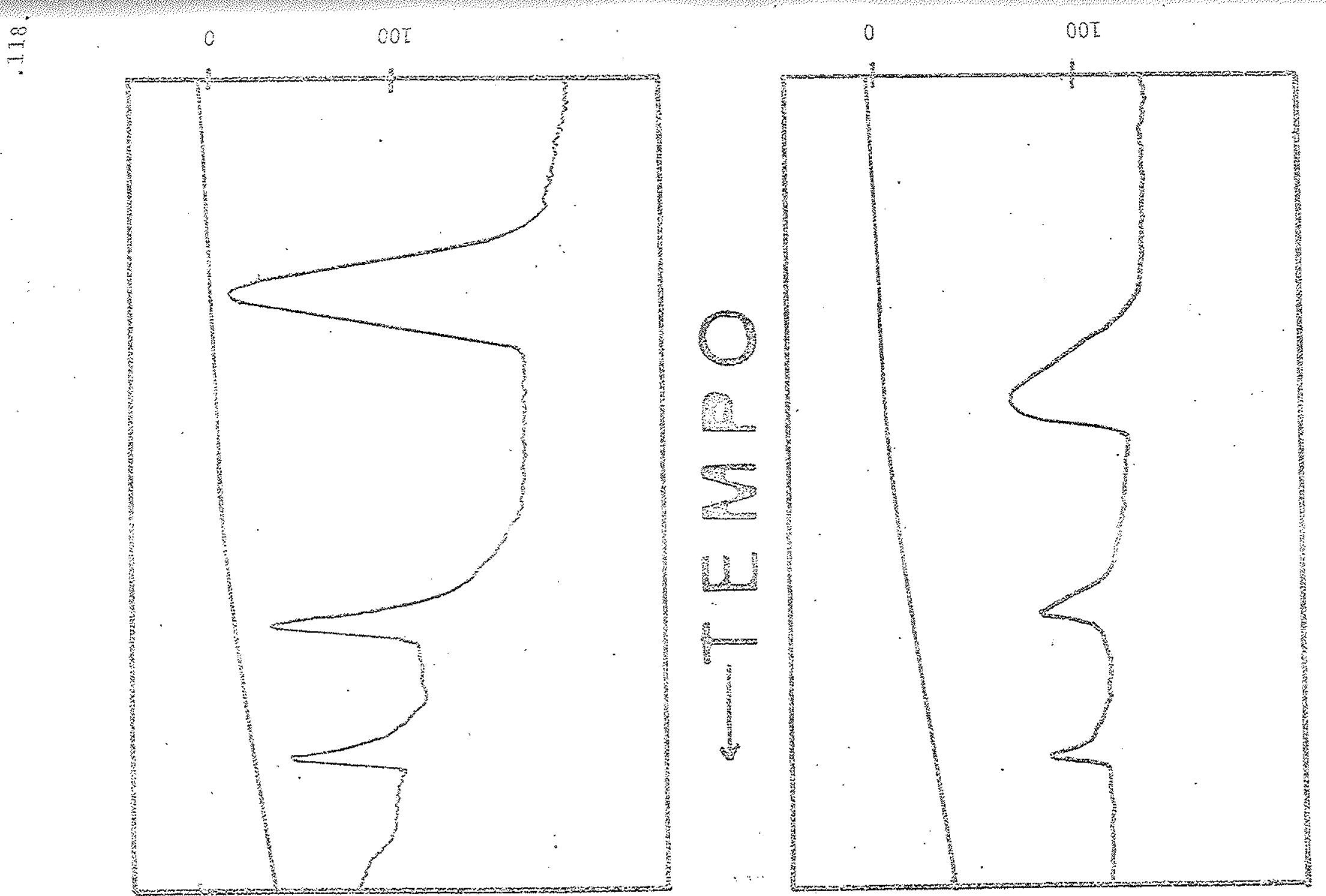

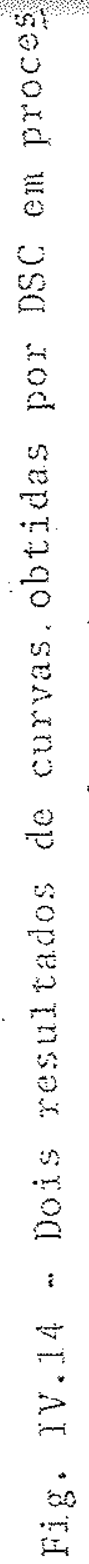

WDNESOA JU TYIONEAGIA

1. 
"oestruex easou ax

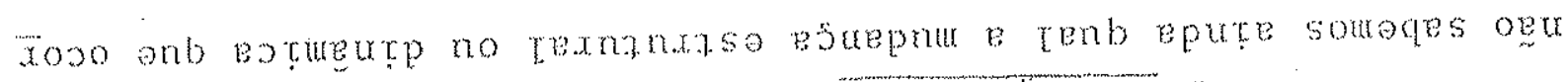

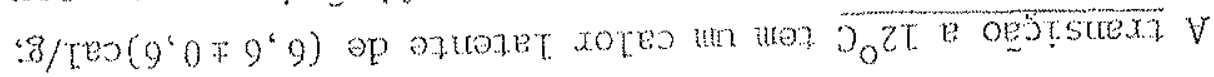

$\cdot \operatorname{sentato}$

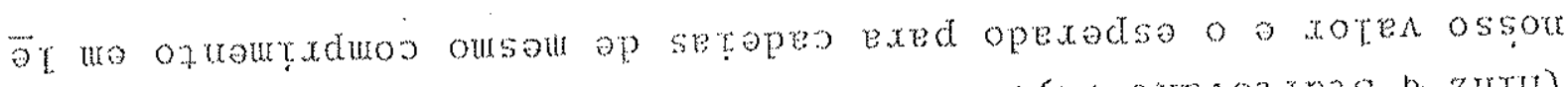

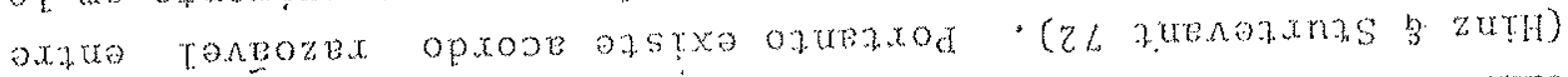

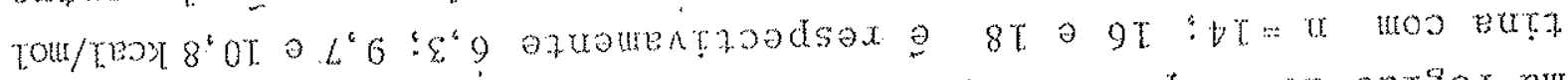

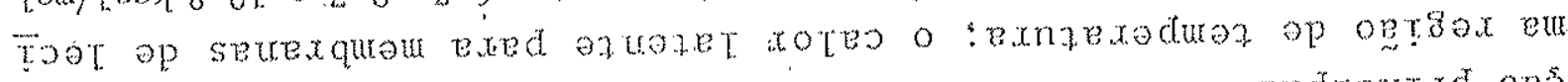

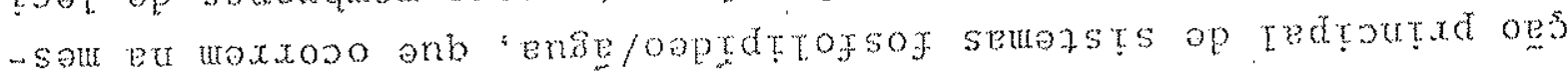

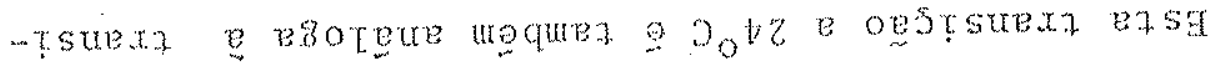

- azroxeftr xerod e5oq

एo wos onb epure "'

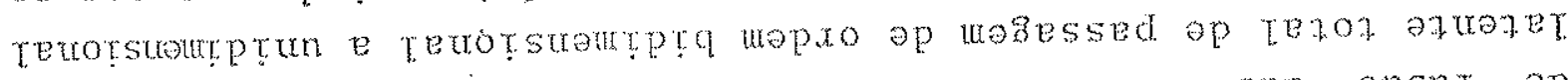

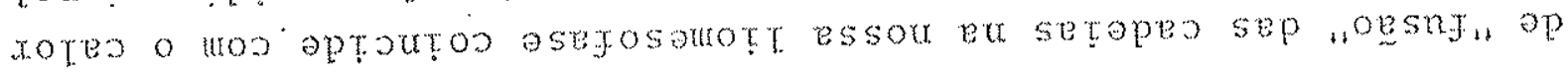

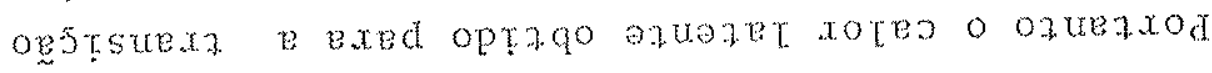

- ordoraost op

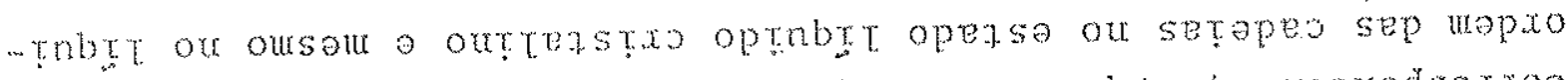

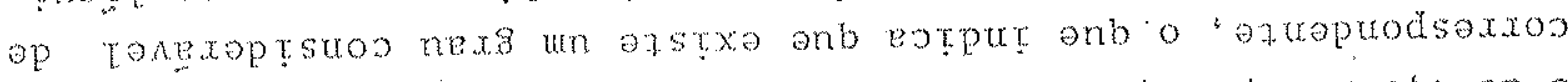

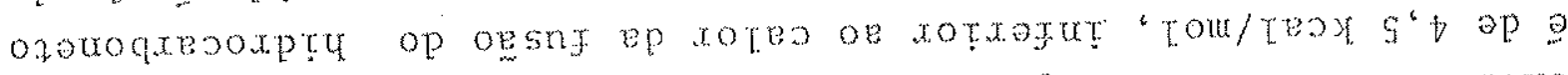

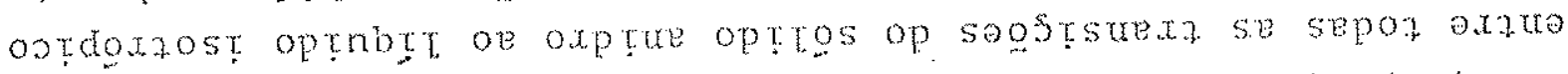

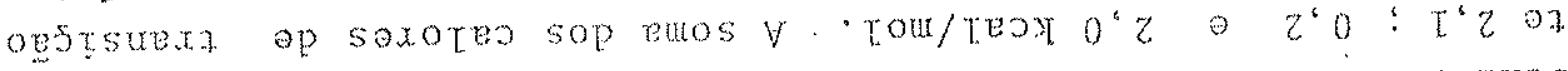

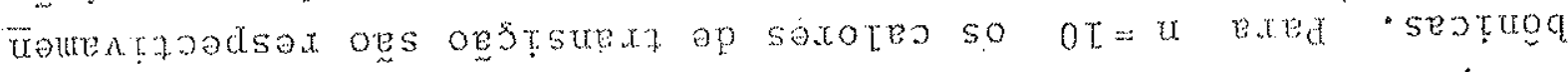

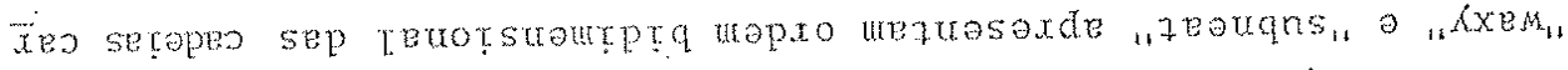

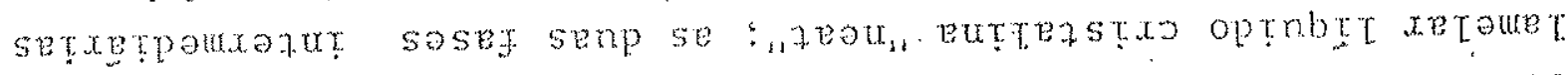

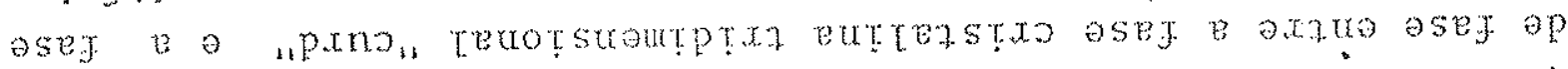

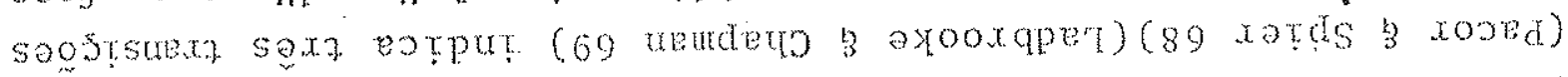

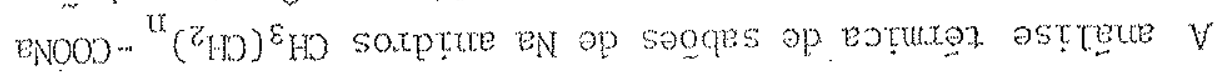

- $\operatorname{ange} 0$

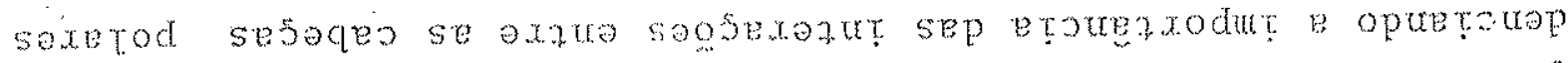

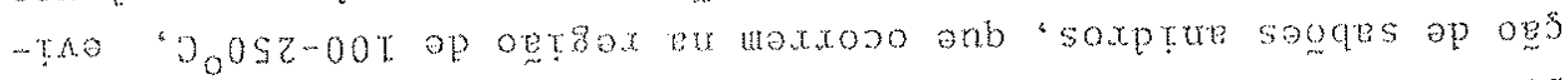

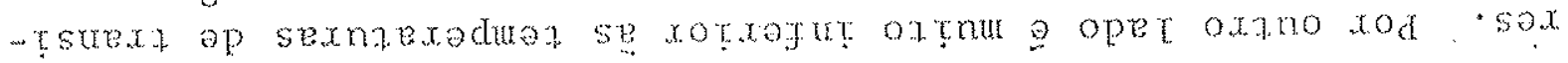

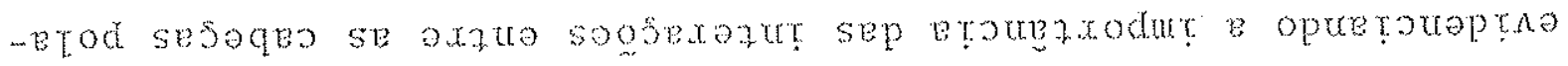
"Dos-g onb" ot $=$ u

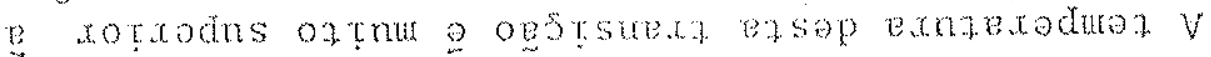

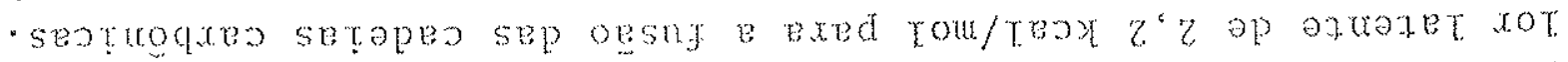

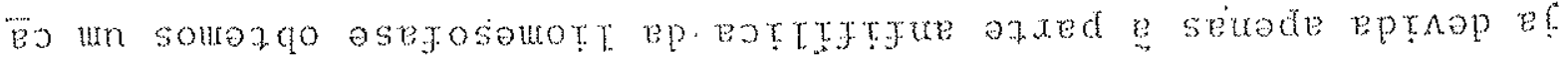

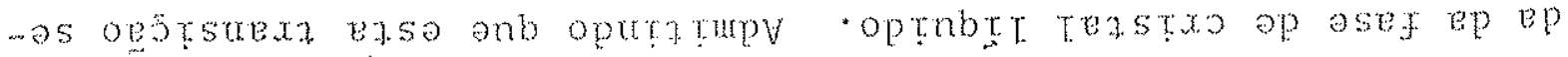

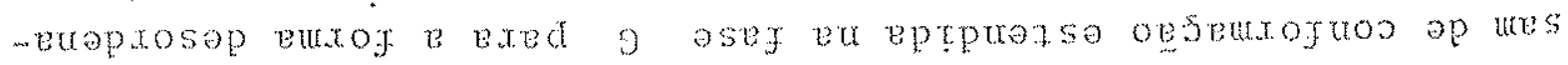

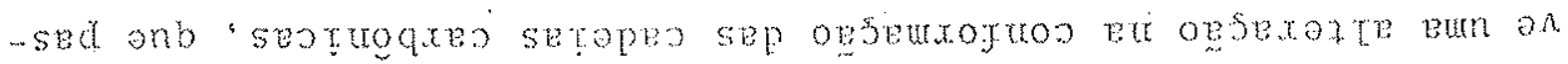

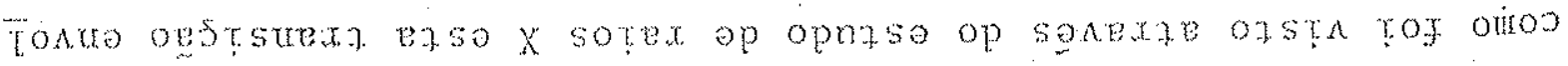

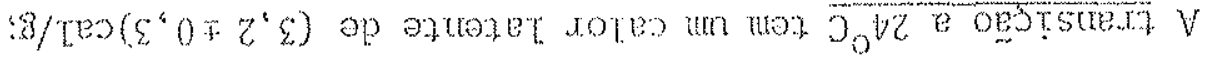




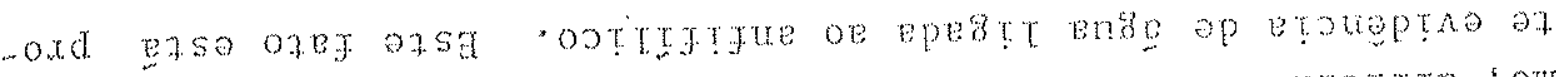

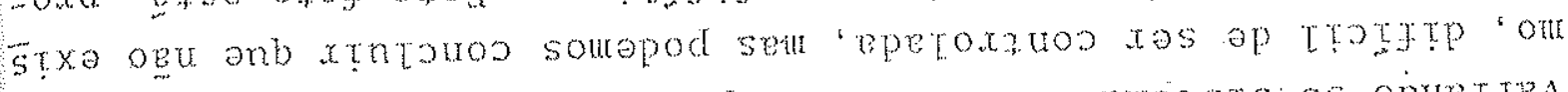

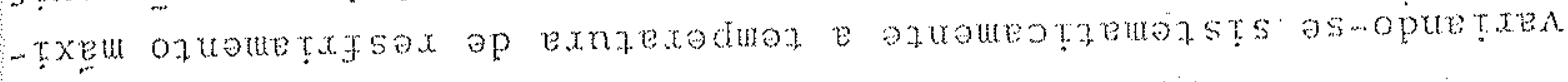
arotduos astere bun pates roj ogd ospo ossow on

"Dos un eoteretros os anb otexpourazur od

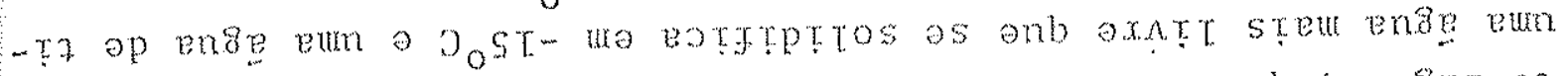

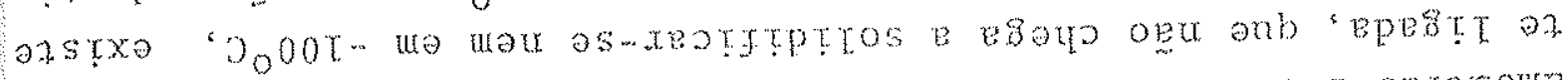

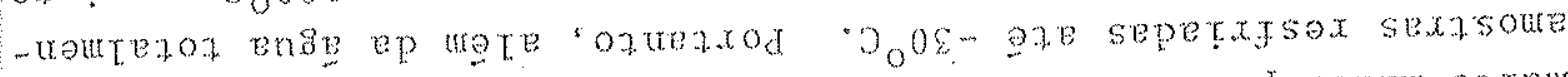
axd anb op oos- op oxtequ sepetasex sextsour exed xorpu of tru

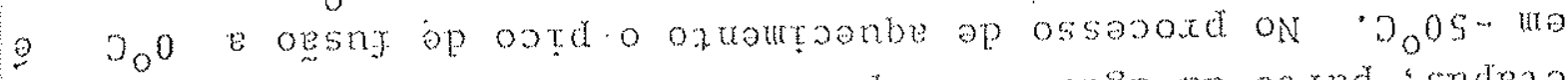

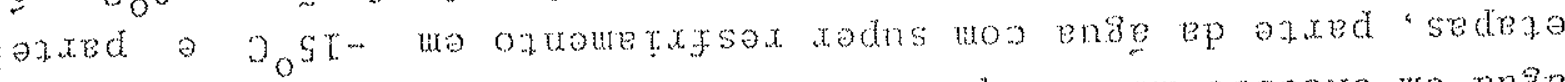

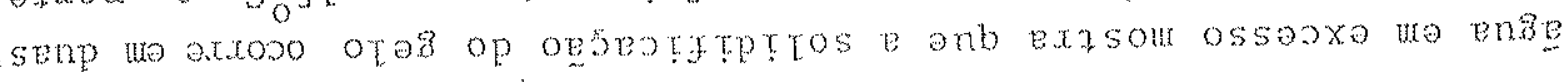

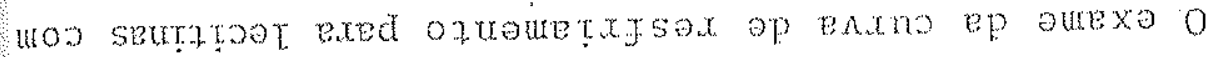

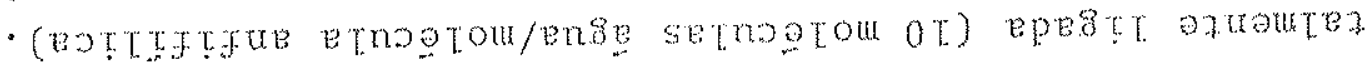

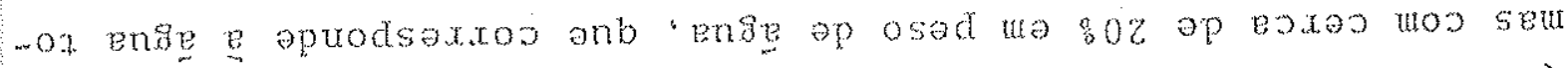

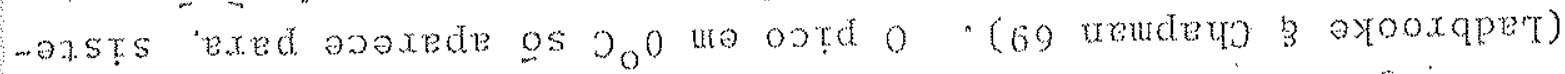

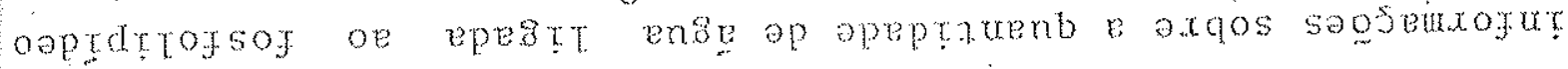

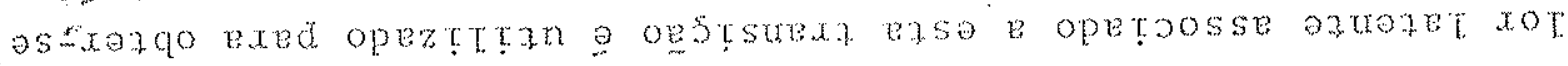

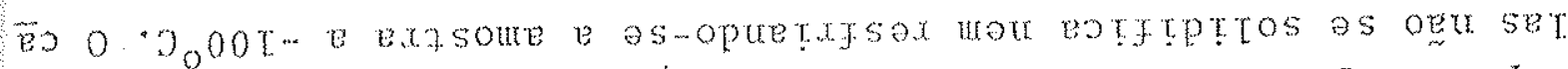
- astu se epest ense e stod 'esonbe axed ep oesne exed opexodse

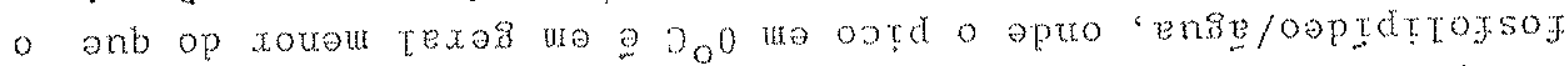

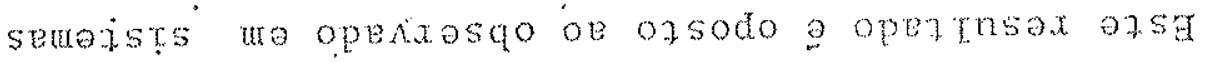

$$
\text { - opeaxasqo } 3 / \text { tro }(z ; 69) \text { roten op rotrartr }
$$

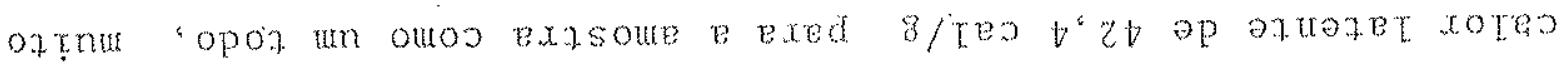

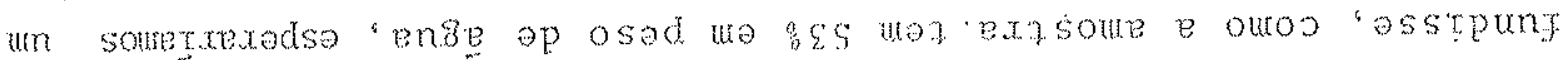
as esonbe oesxod e epoz anb opartupy oesny op ossosoxd otad az

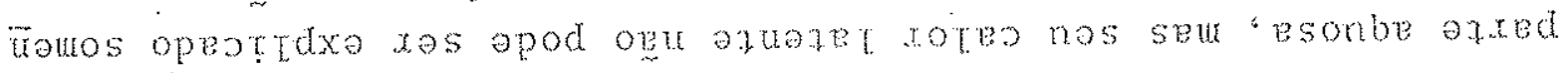

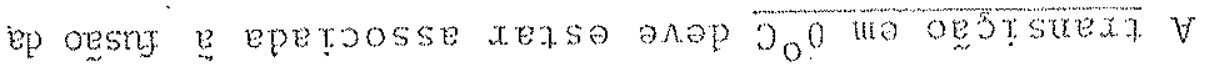

- serootu sep seotuetep no

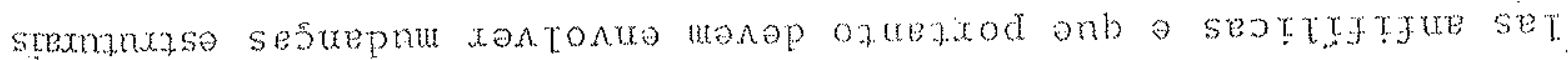
mogtoul sep opsexepxosep ep ossezoxd xod septadse xos urexopod

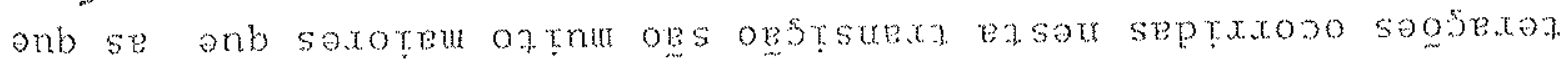

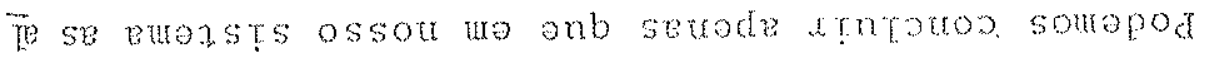

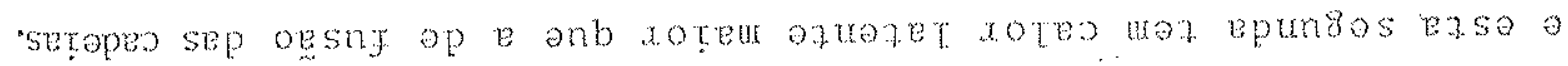

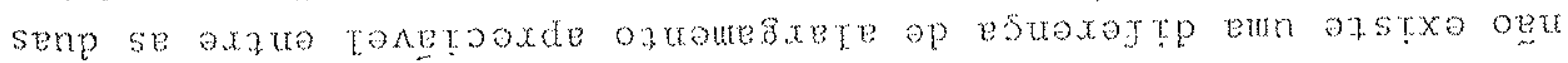

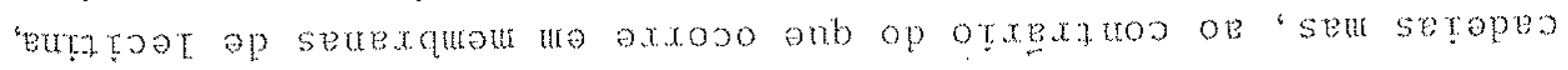

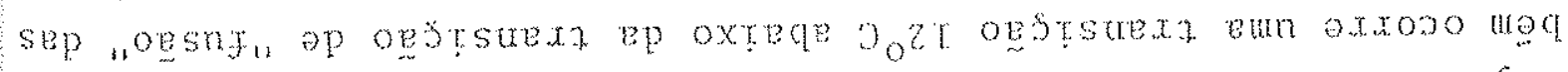

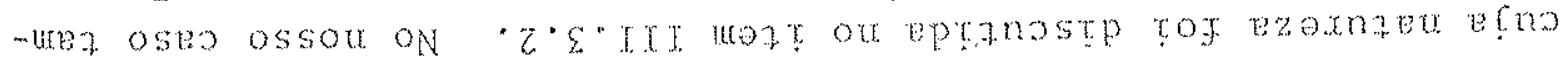

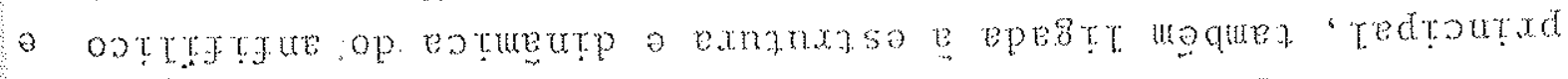

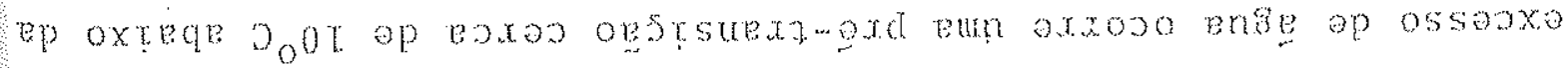
a ounbod a moo mag/opprtrojsos semotsts son 


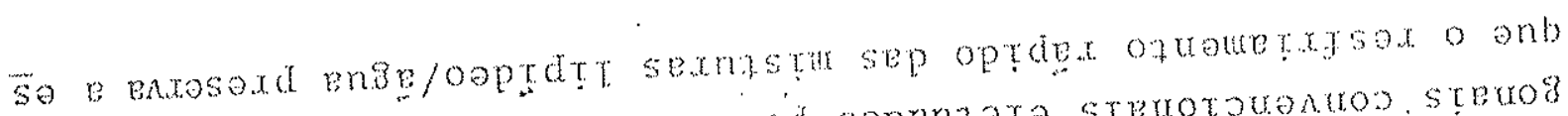

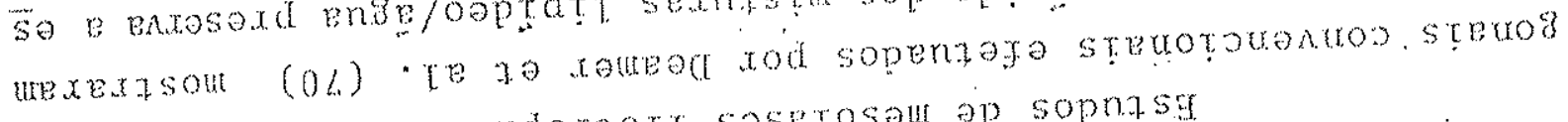

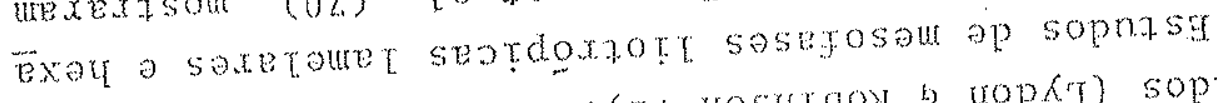

- (zh rosurgol 3 ropkt) sopenzejo mexor e? maquez aw rod sentoxzomat sasegosem op sopns

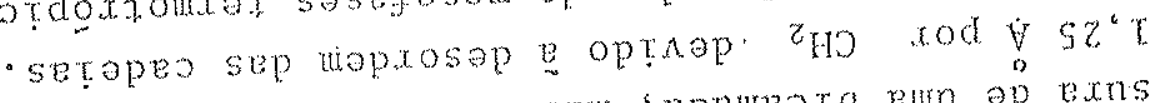

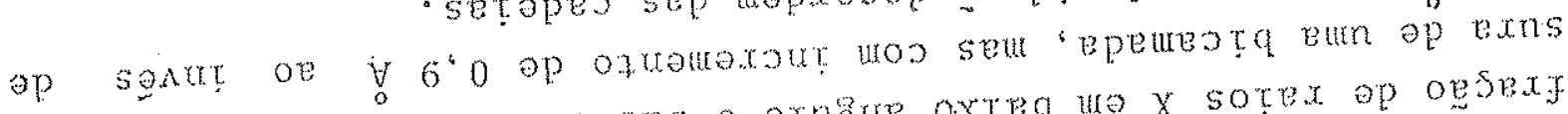

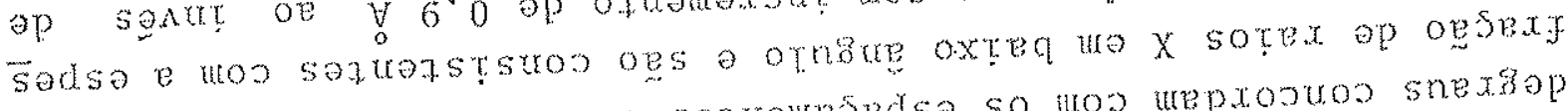

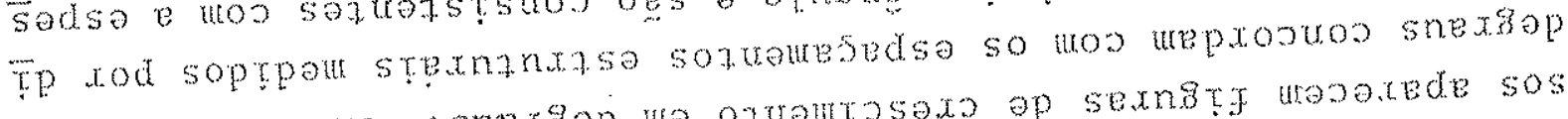

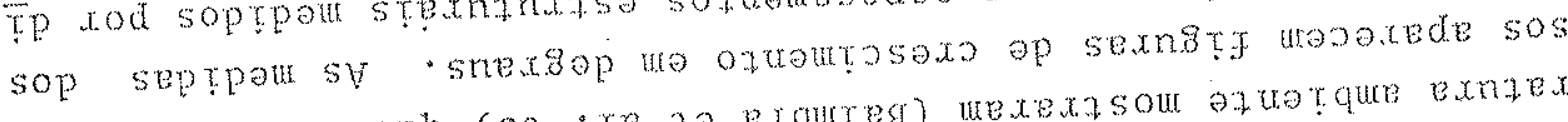

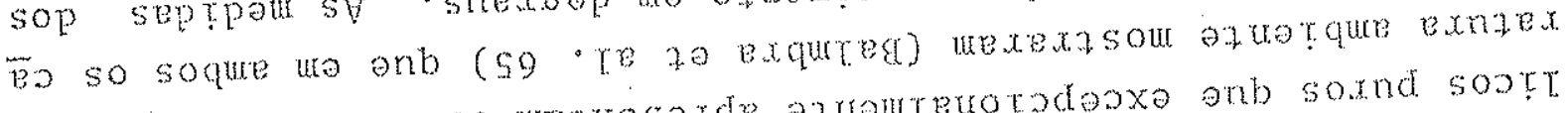

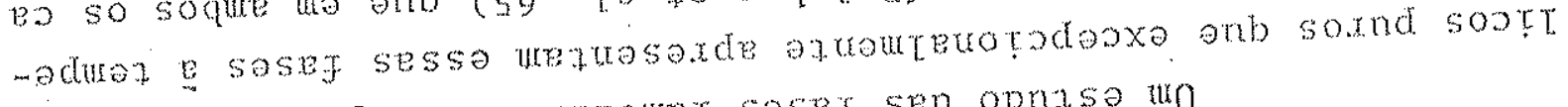

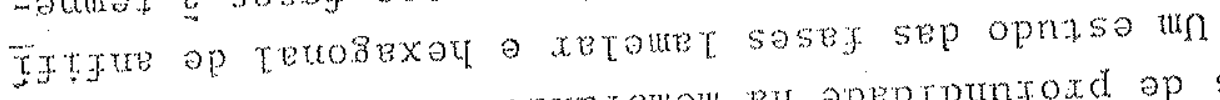

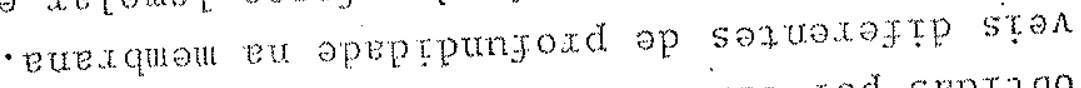

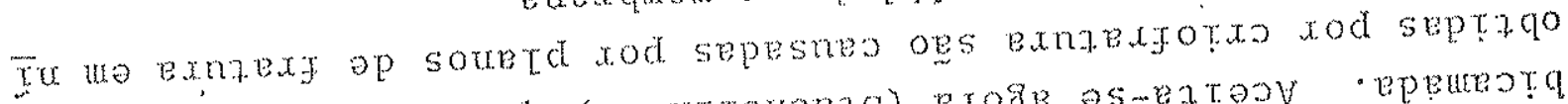

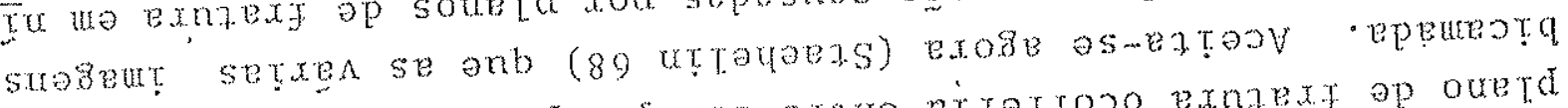

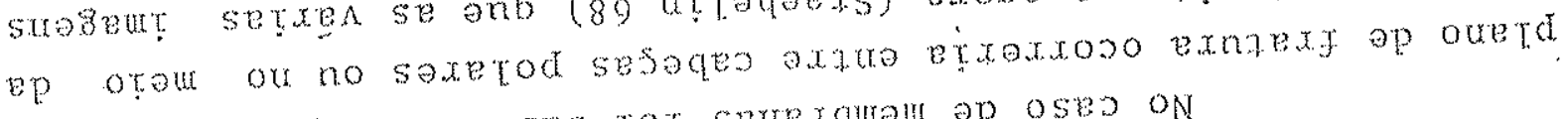
o as optanste aruezseq toj serexqua op oseo on

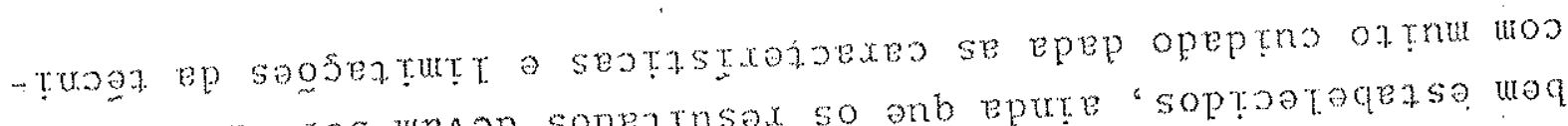

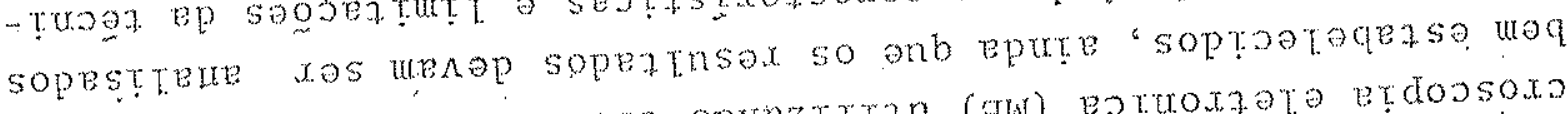

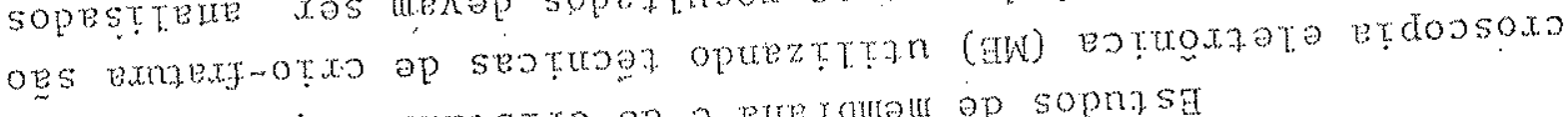

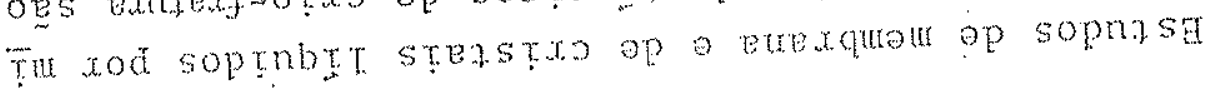

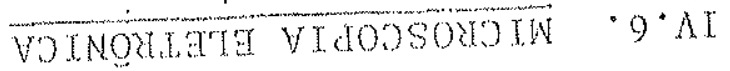

- otxozeroqet ossou he opronusap opues ezso oesox

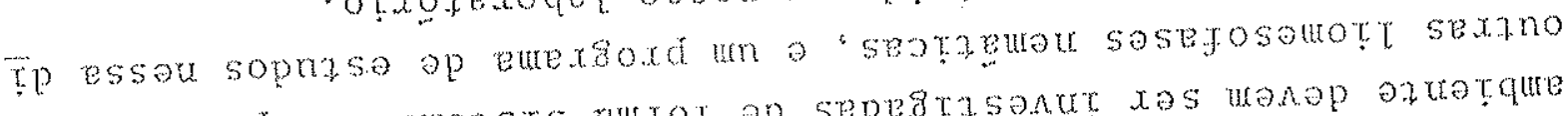

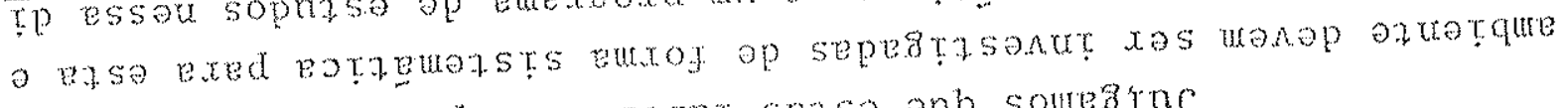

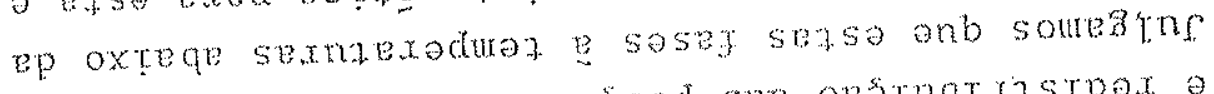

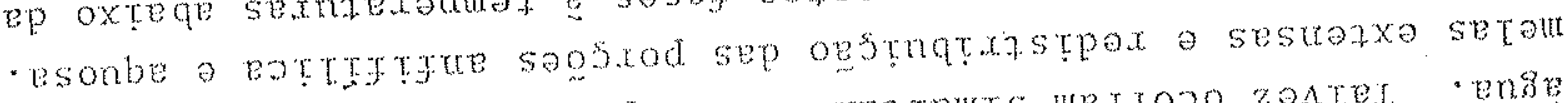

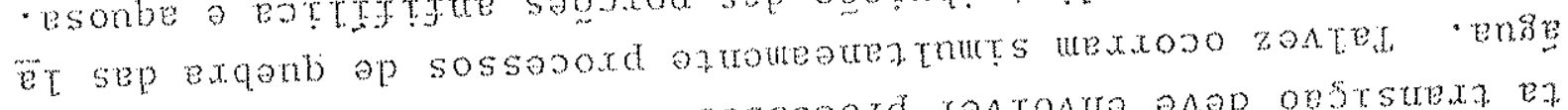

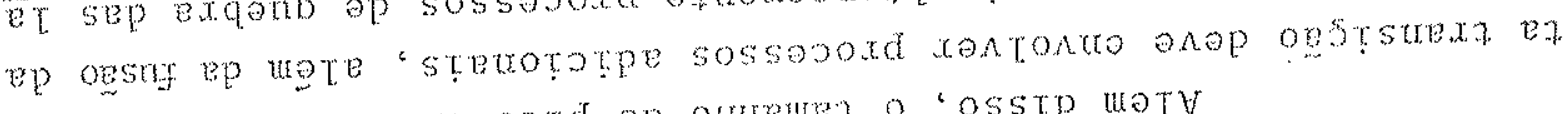
so onb atput Do ura ord op oqueury o 'osstp wetV

soseg op eursetp op enge sotrom woo sooto

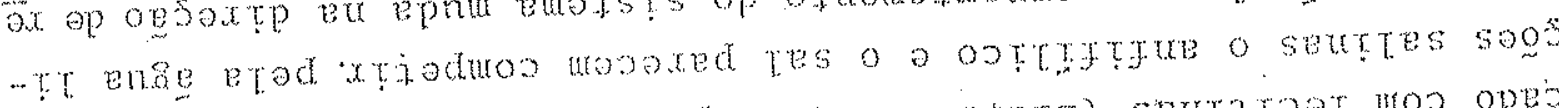

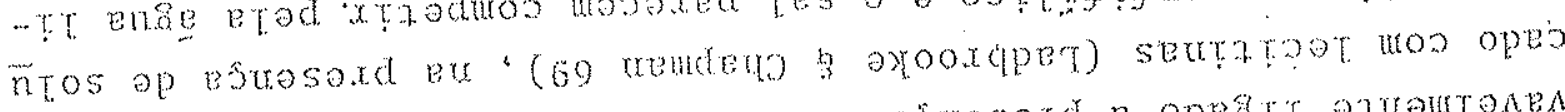

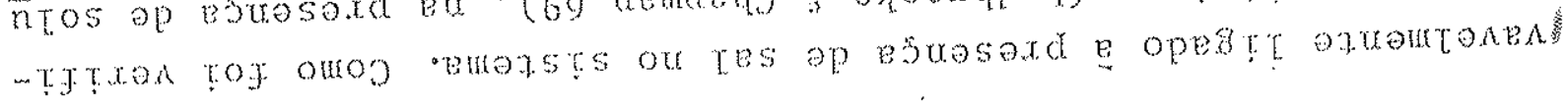




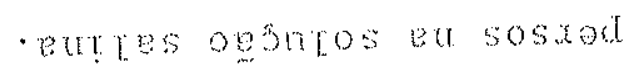

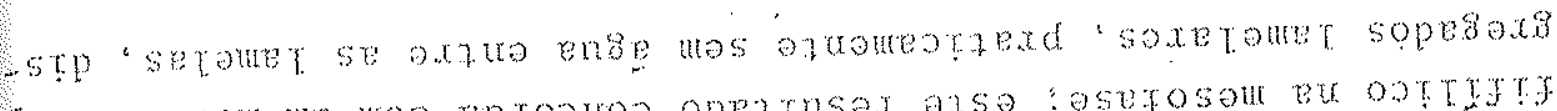

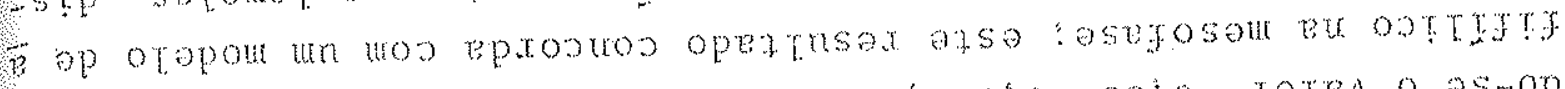

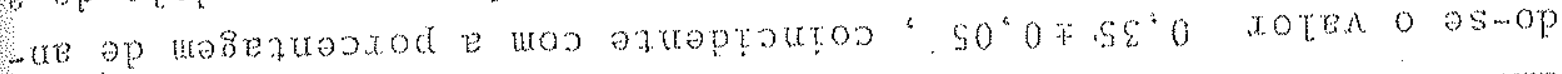

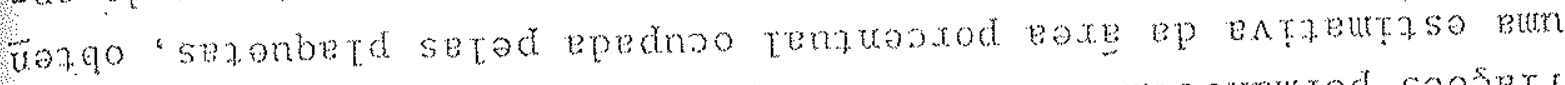

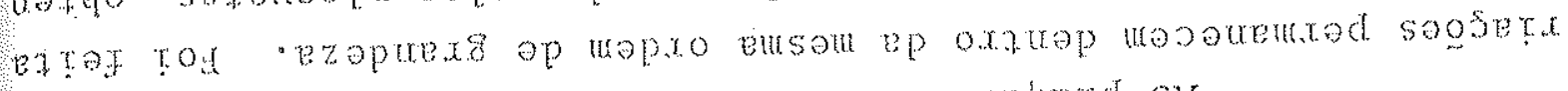

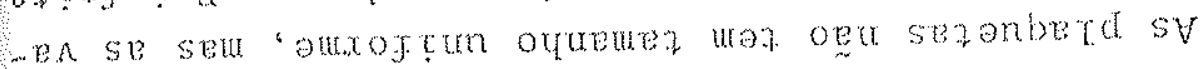

- setouret oxja ense op epeureo botr

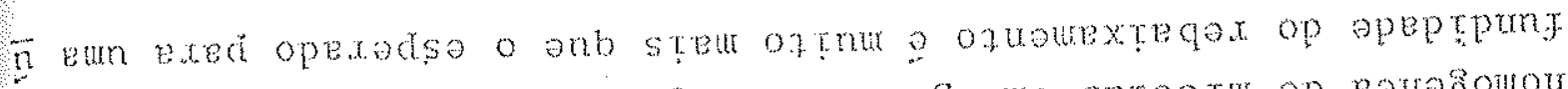

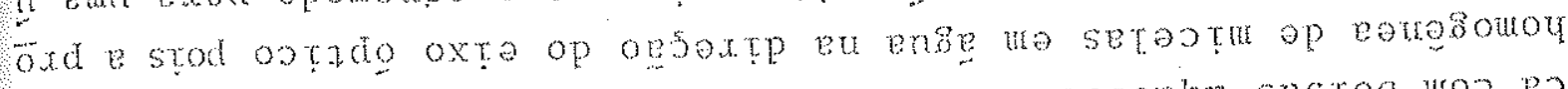

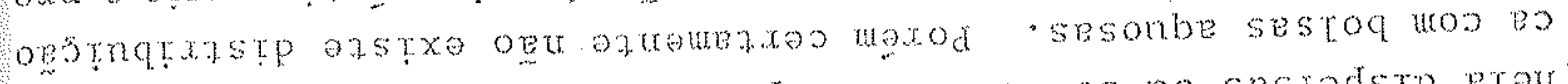

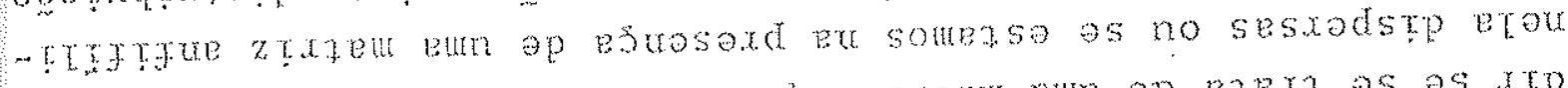

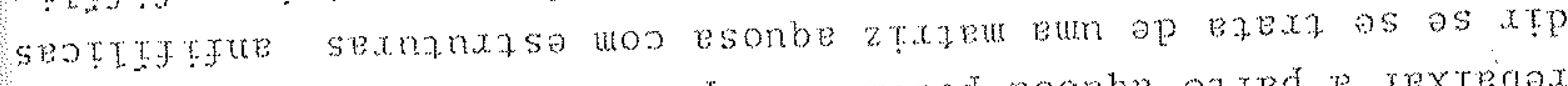

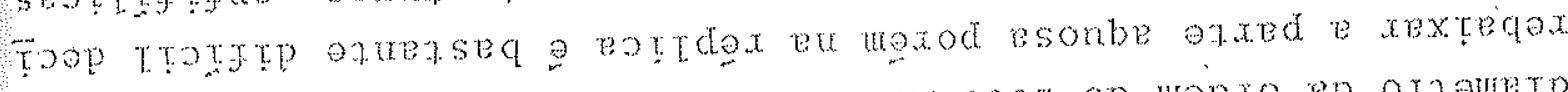

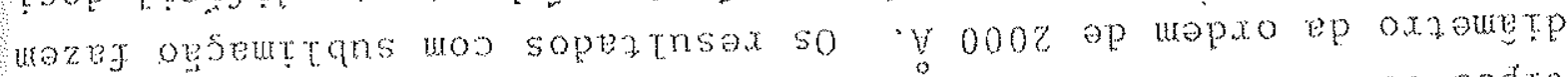

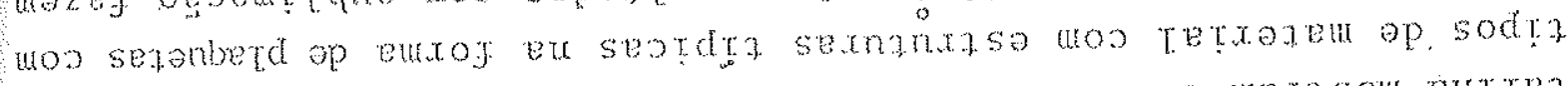

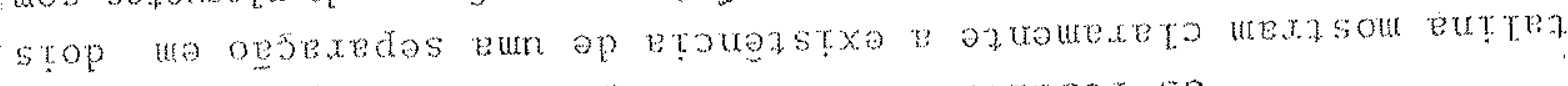
stro optrote asea ep xtaxd e soptaqo sopertusox so

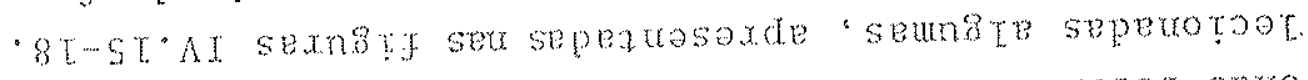

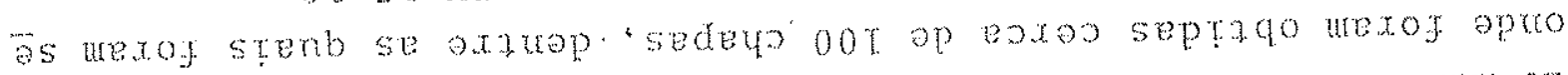

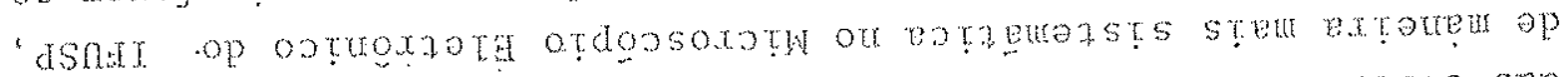

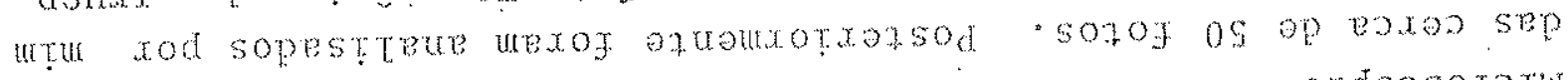

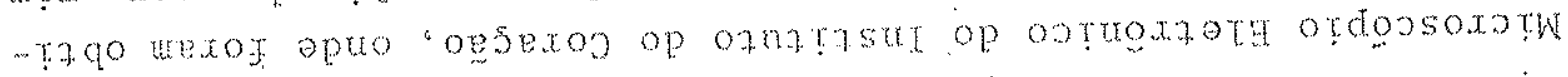

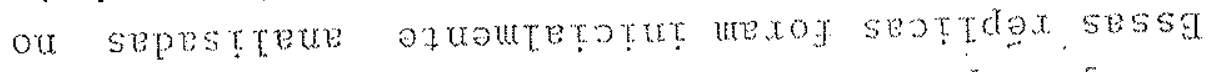

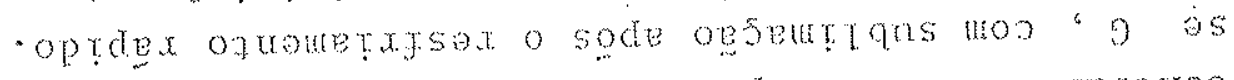

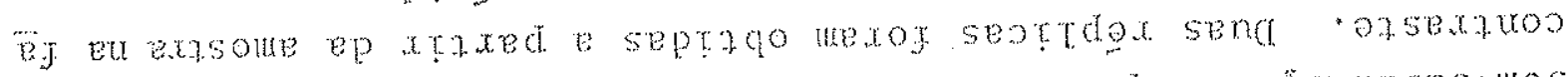

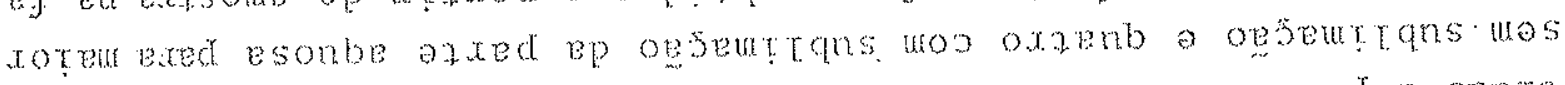

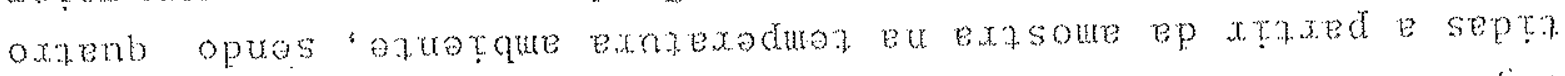

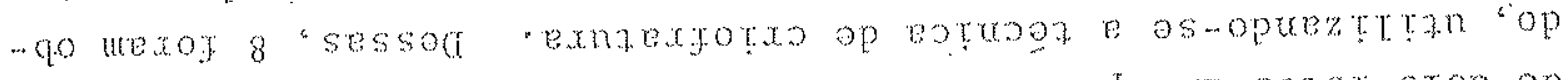

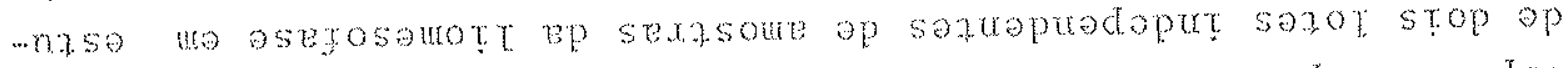

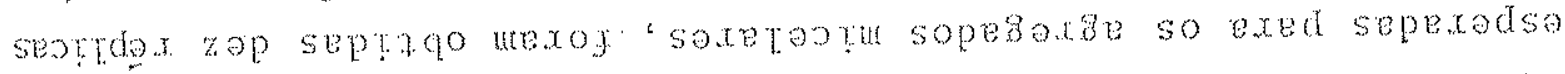

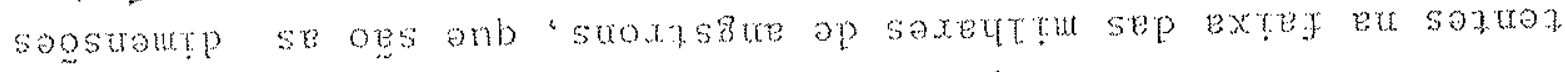

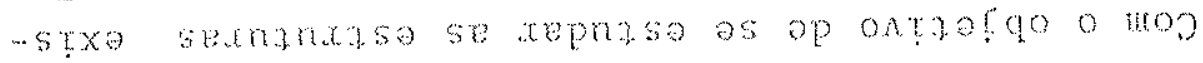

- orgaroger ossour

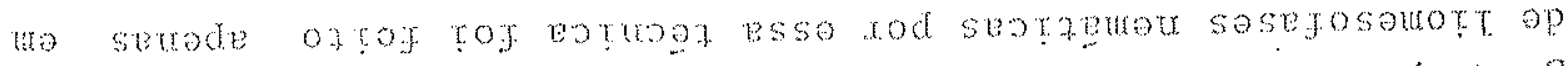

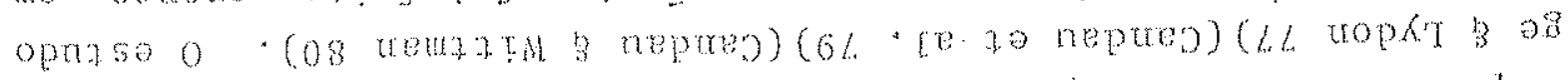

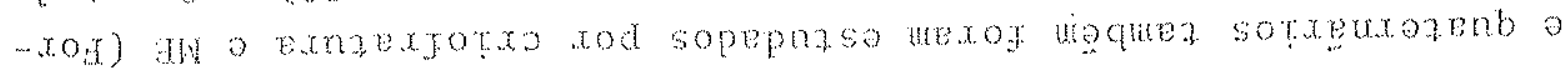

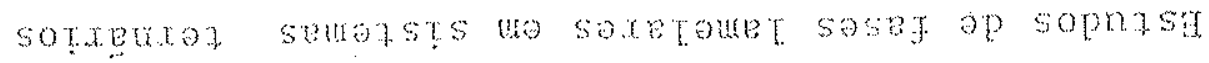

axtroxoos buxof op $x$ sotex op opsexs

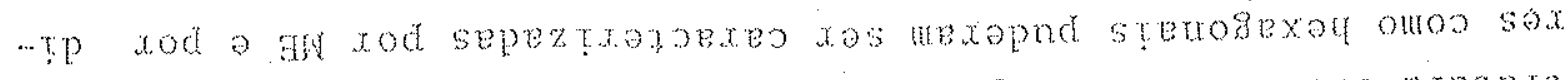

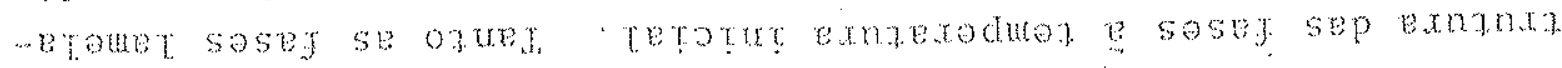




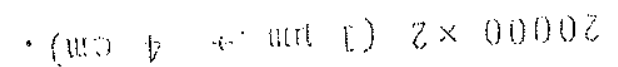

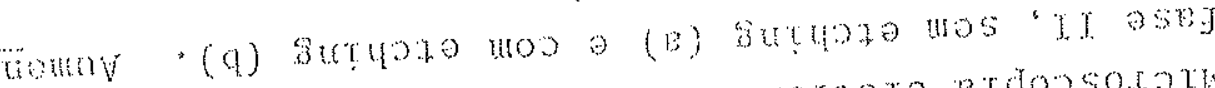

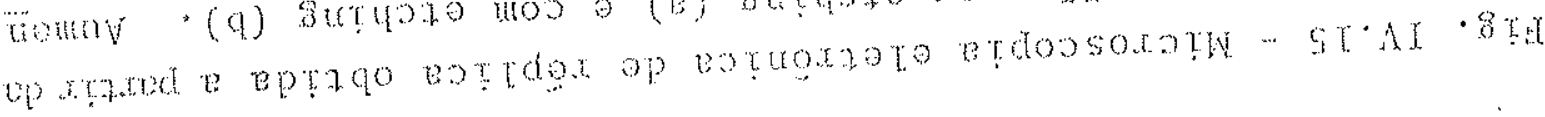

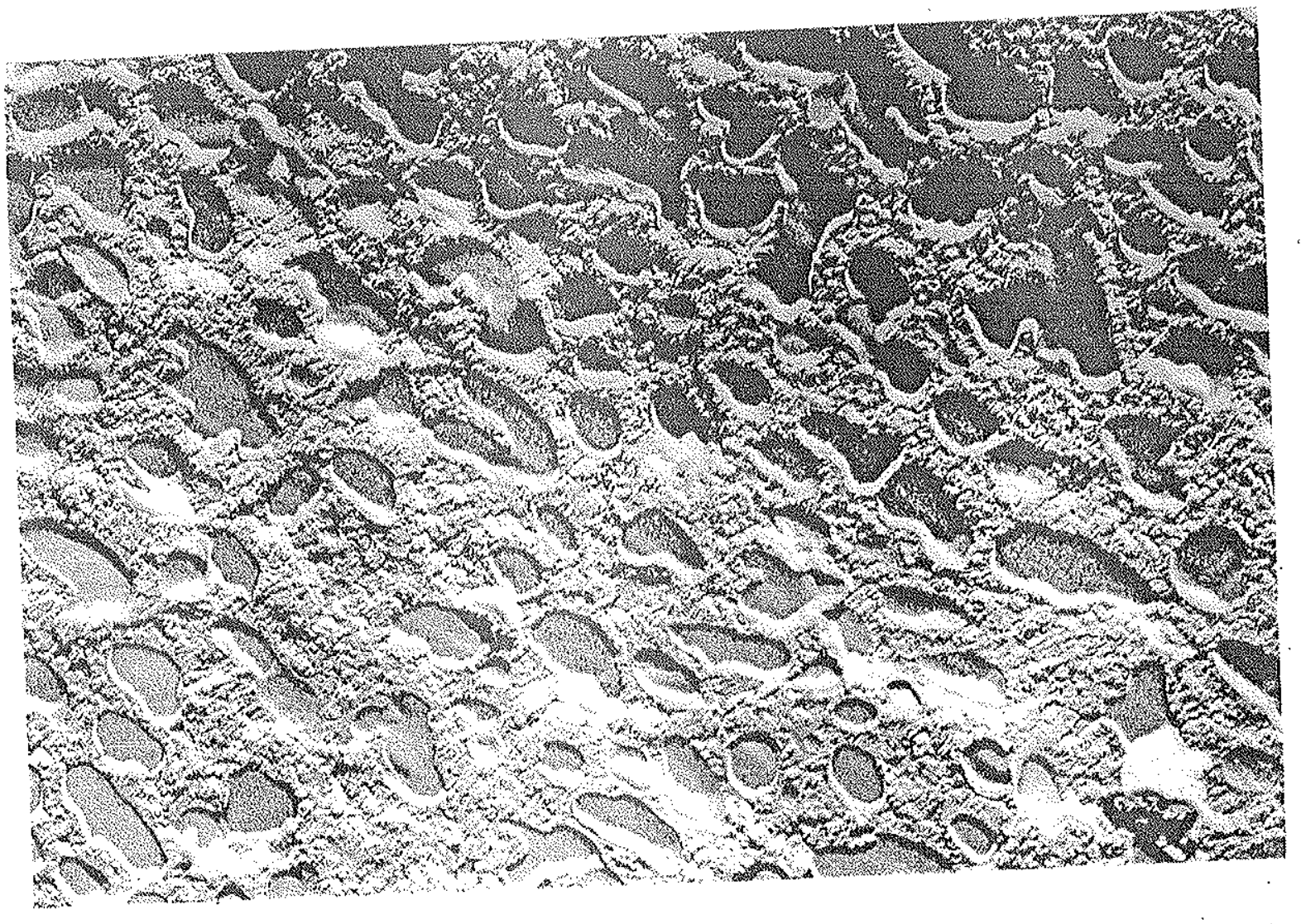

(a)

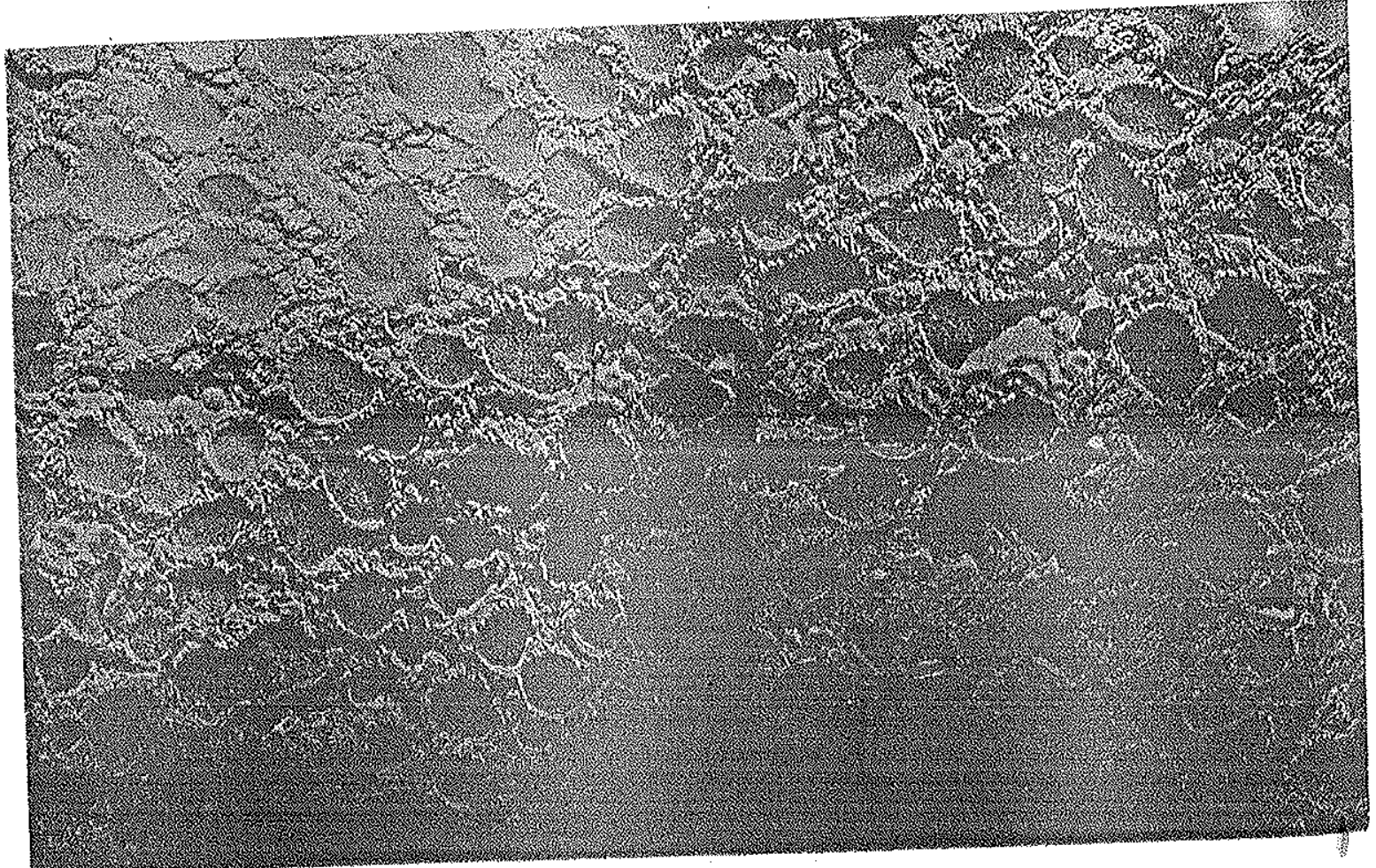

(b) 


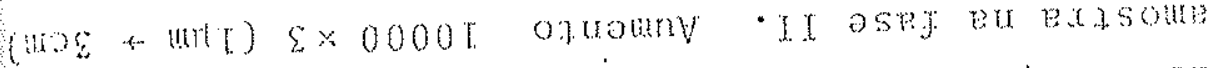

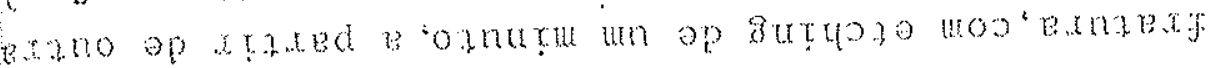

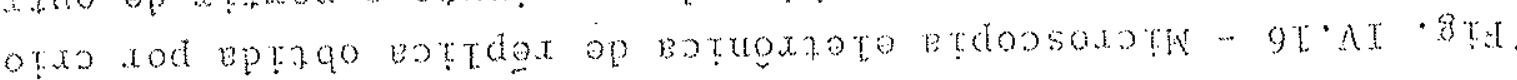

\begin{tabular}{|lll}
\hline & \\
\hline
\end{tabular}

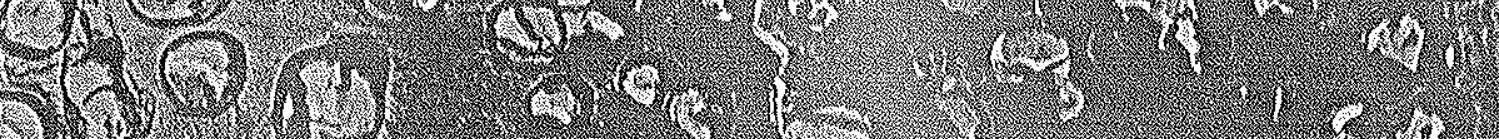

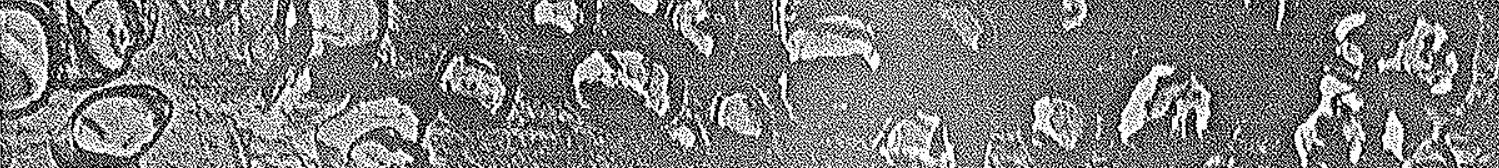

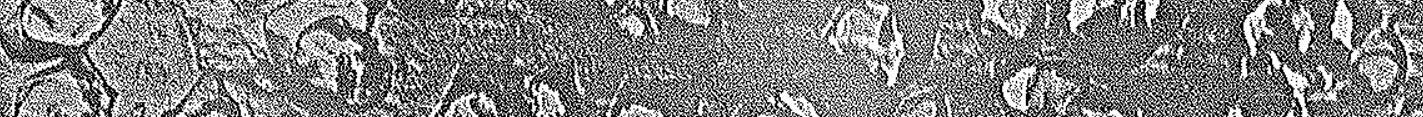
I

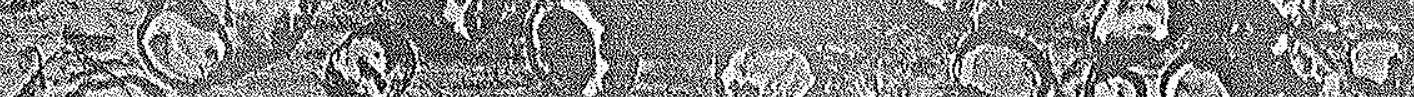

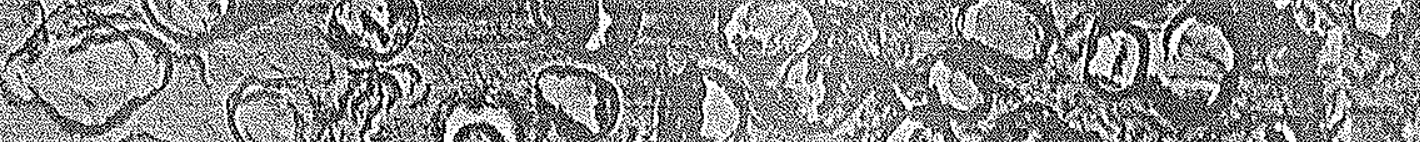
m.

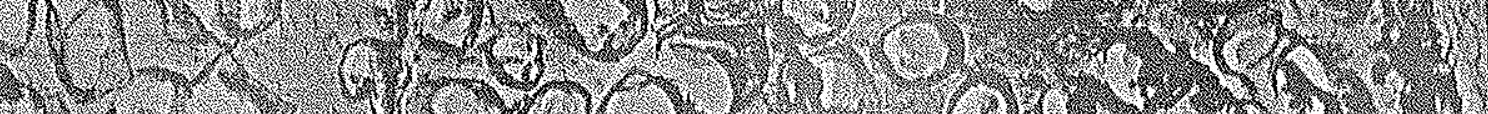
-

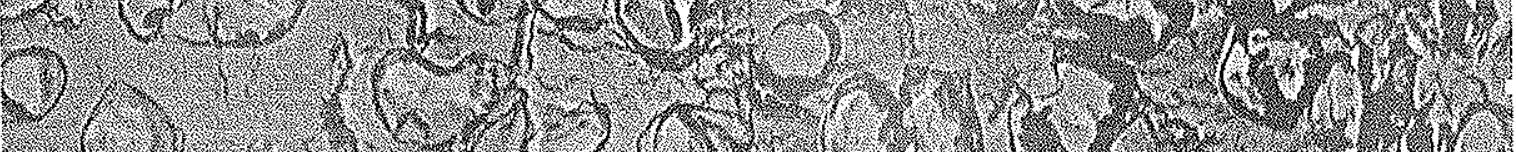

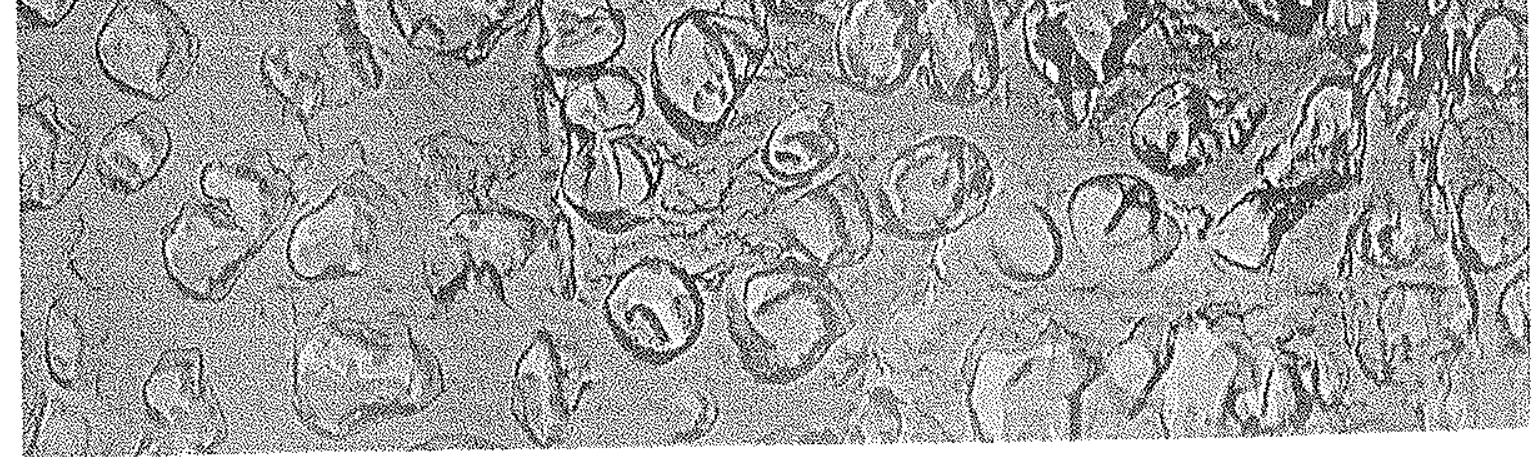




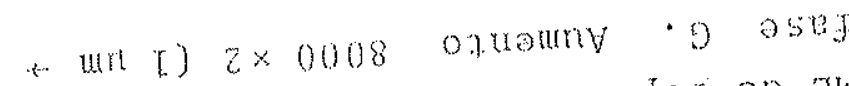

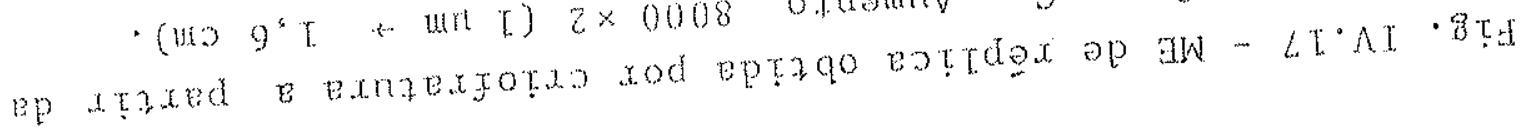
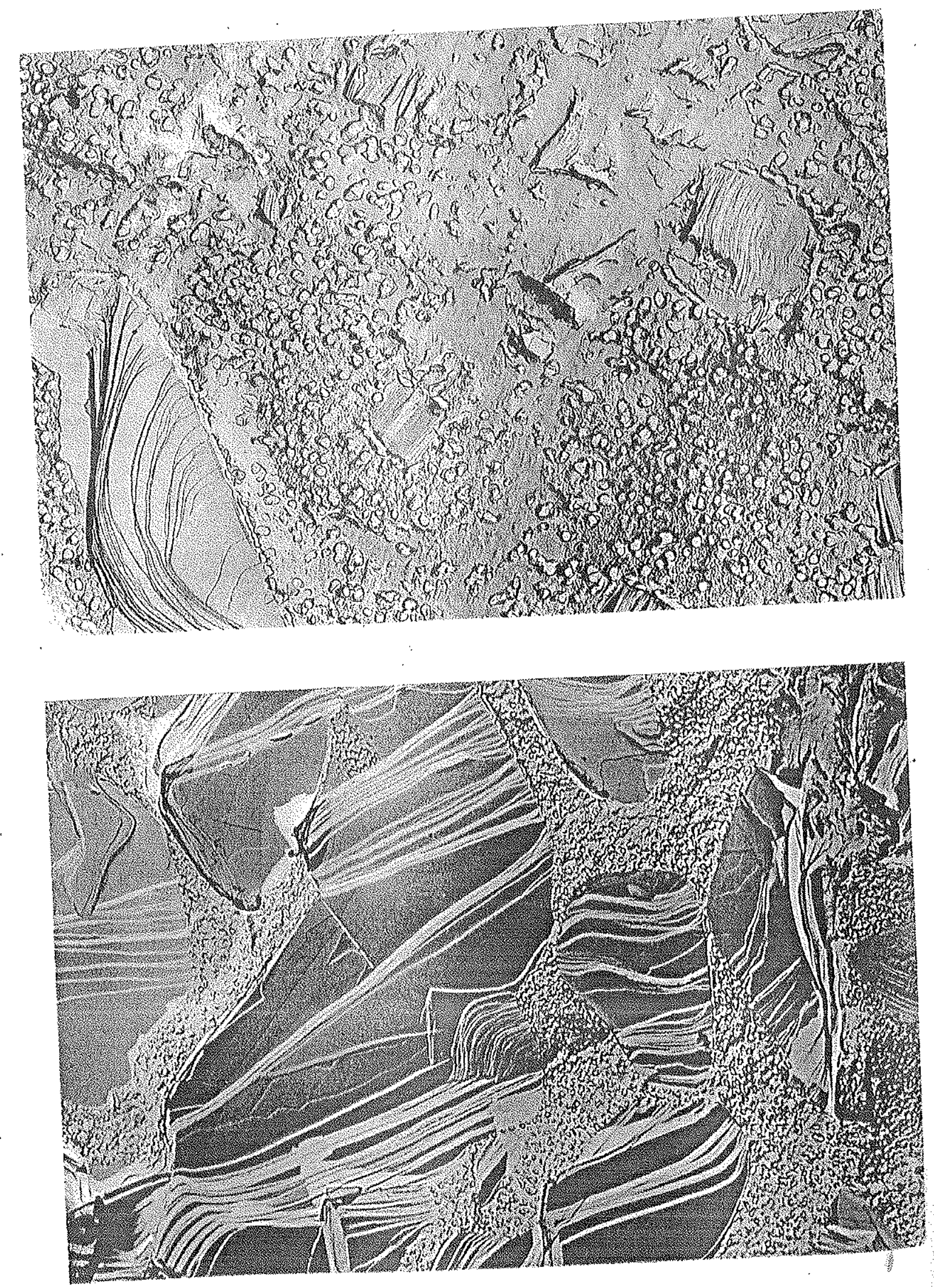


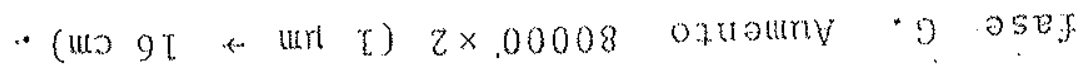

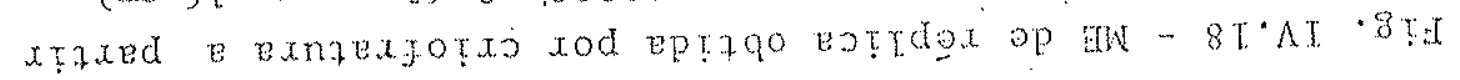
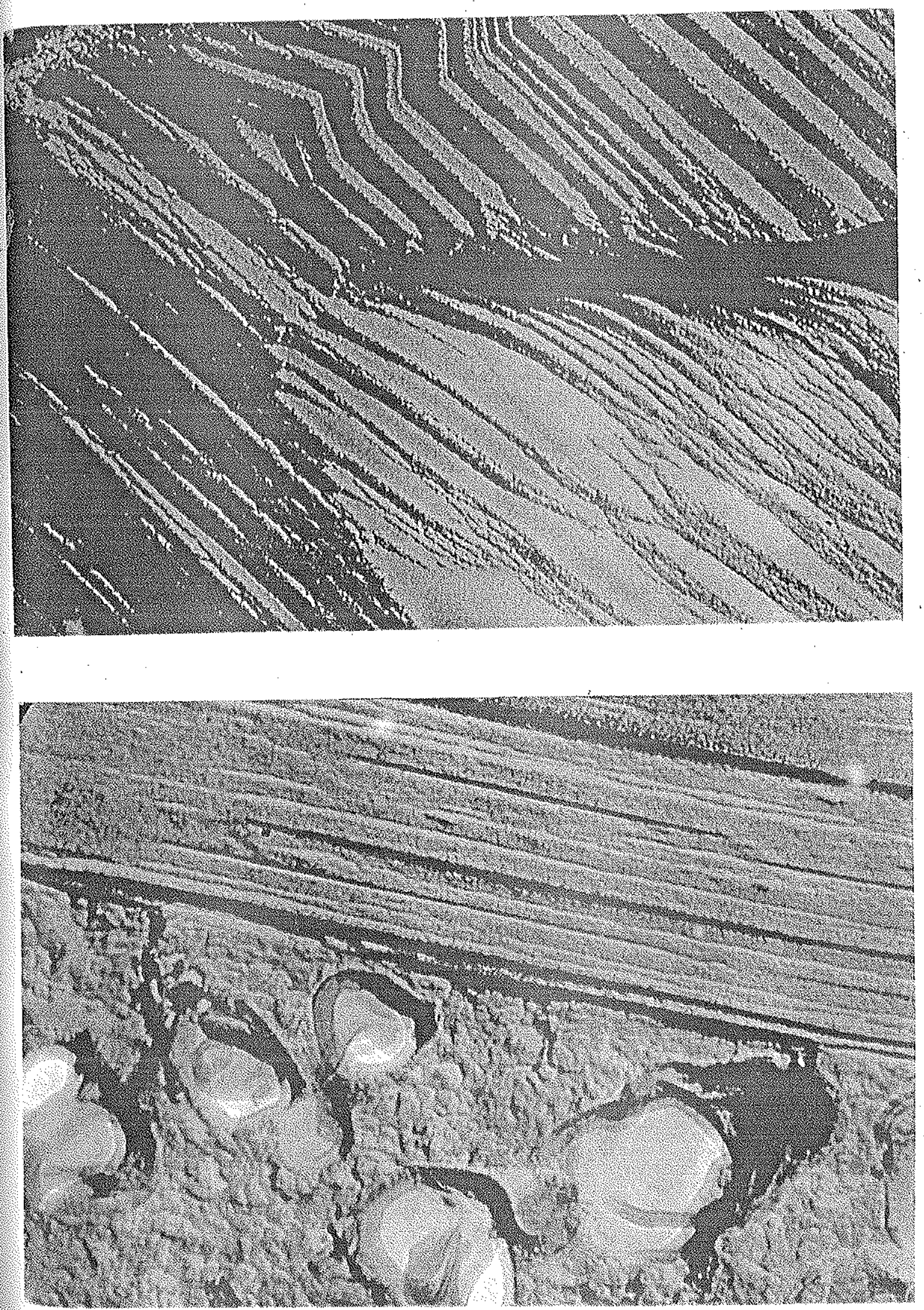
- Wh xod sepeztensta sexmanzso seu optedsax

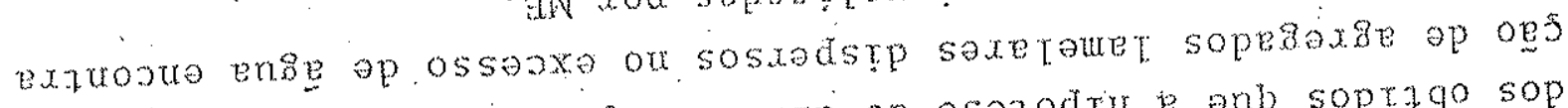

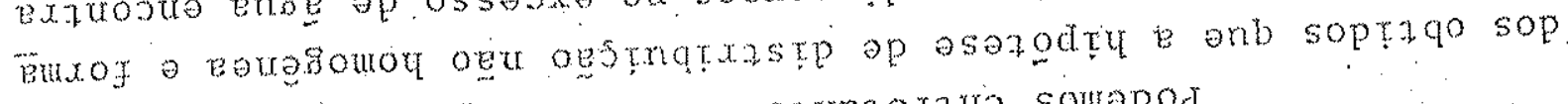

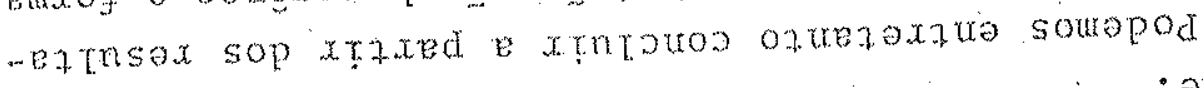

- opeptratssod es

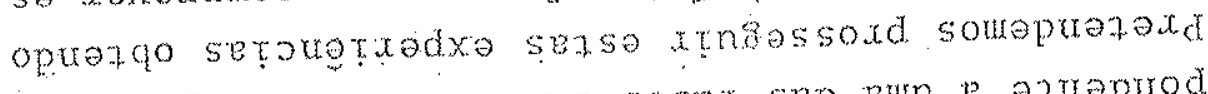

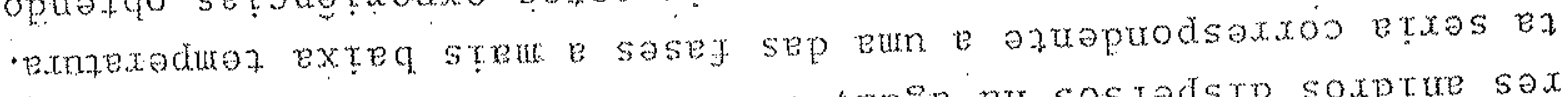

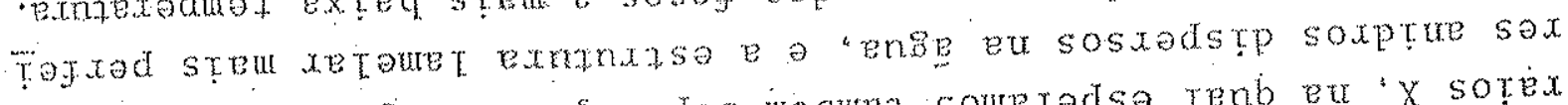

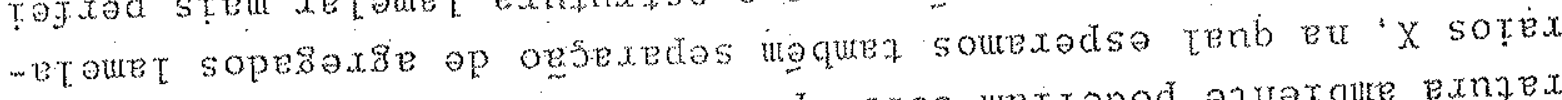

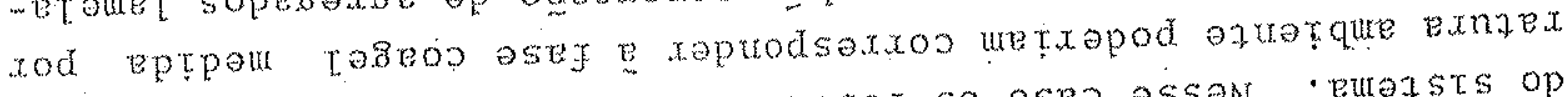

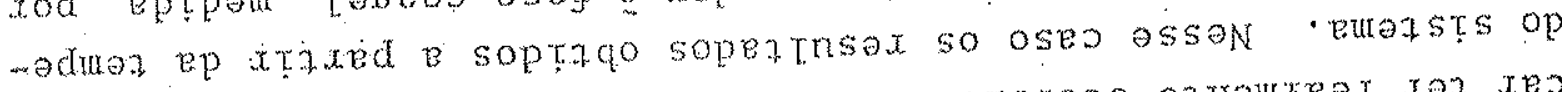

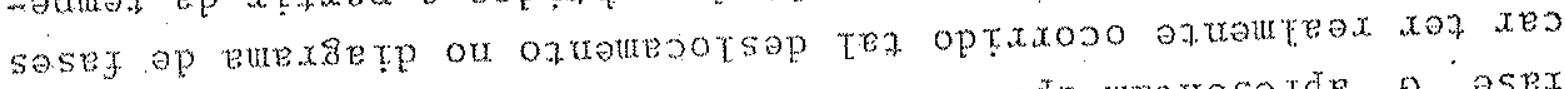

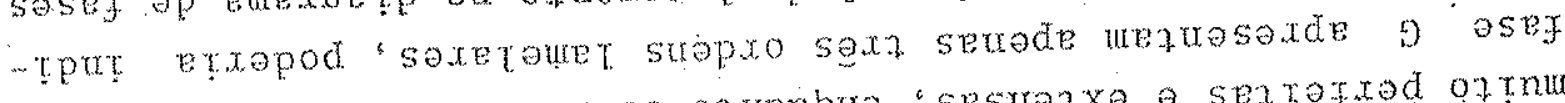

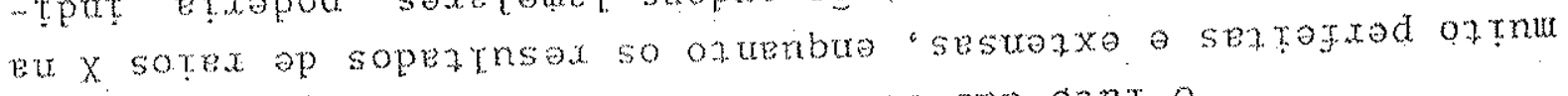

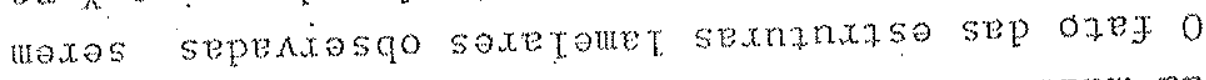

- asta extno exed opepnu equaz 9 asej ep eputa exasoure e onb

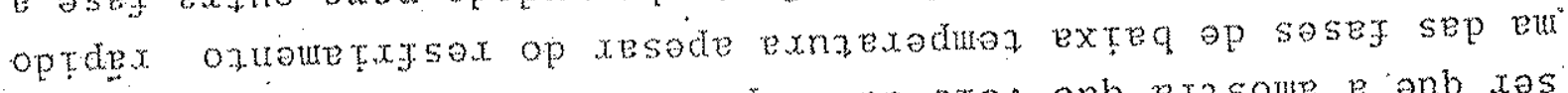

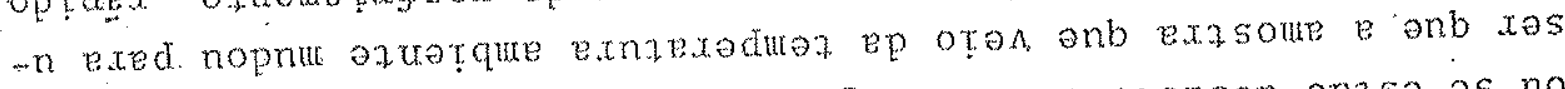

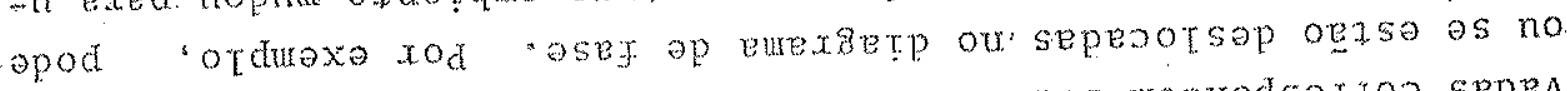

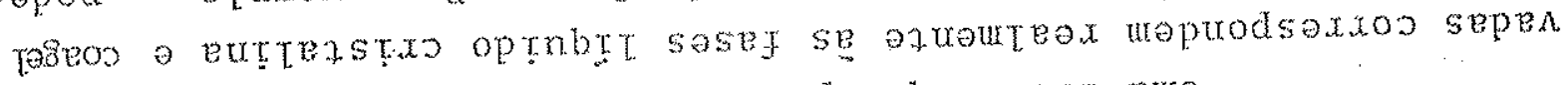

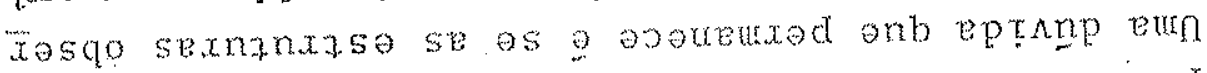

- otroue oxteq ue $x$ sotex xod eptrqo opstad

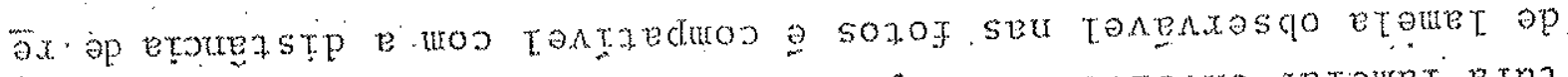

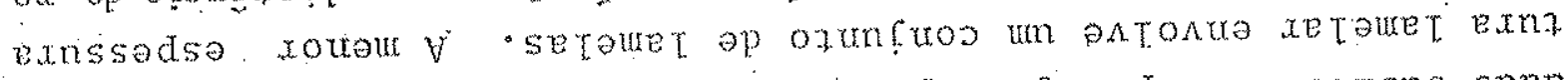

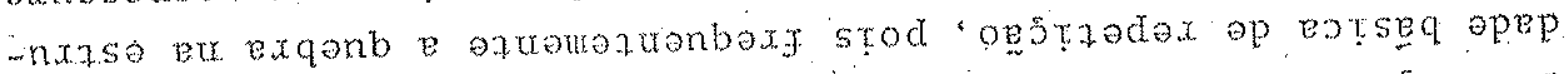

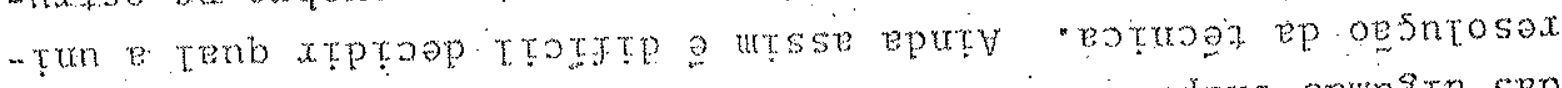
op ofturt or "seprexs oxtraseq solmoune woo sedeyo seunite sep

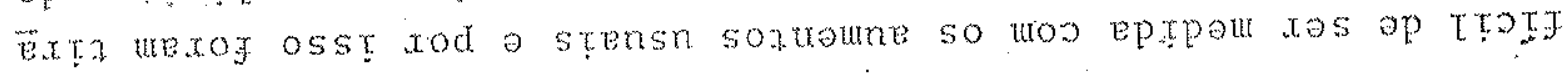

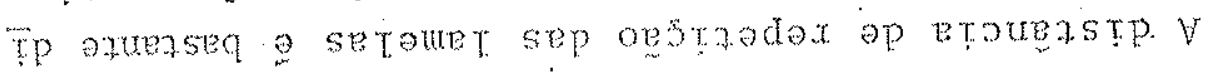

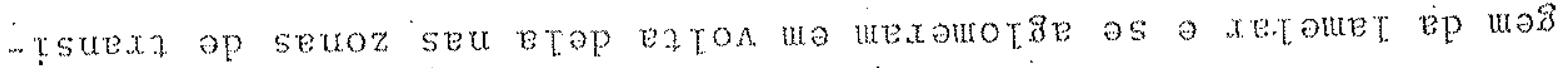

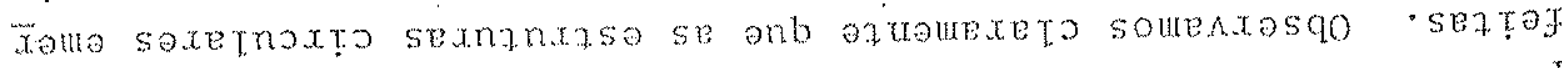

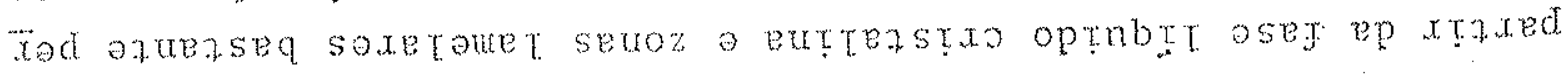

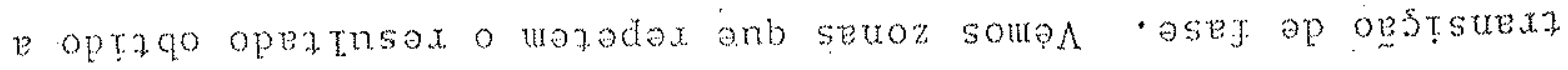

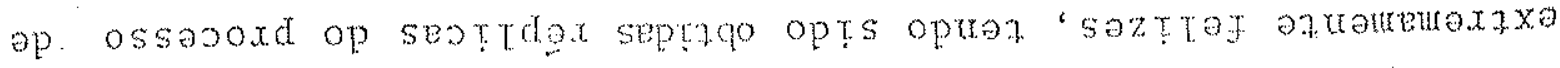

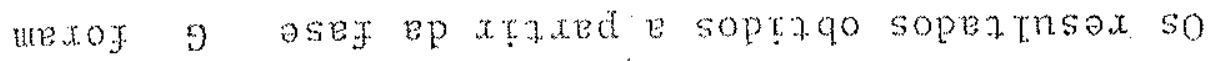




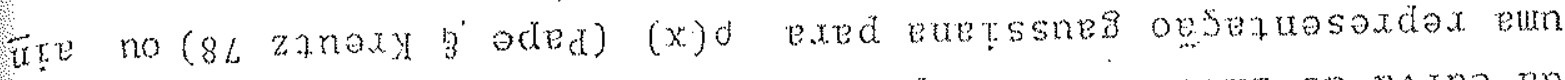
weztran asmpod (

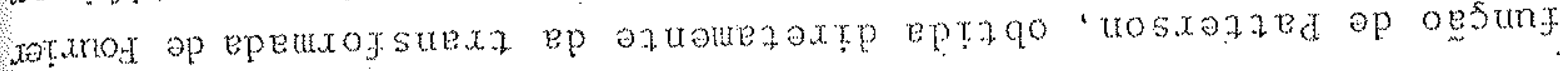
ep opsntontosep op opozou un e epeptrtatssod exzno

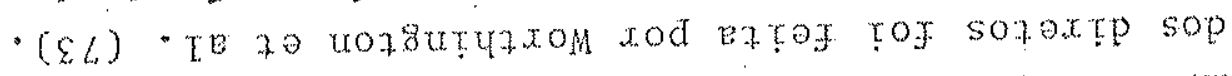

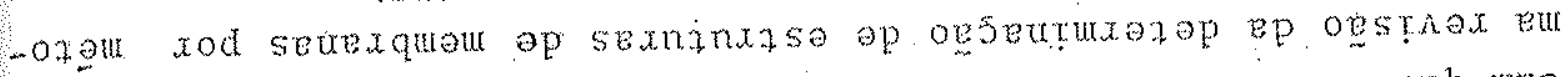

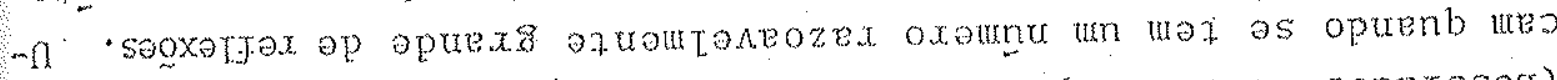

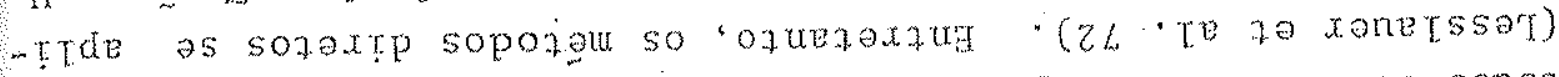

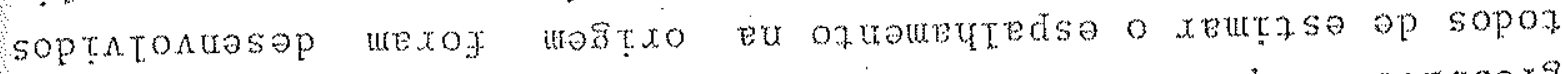

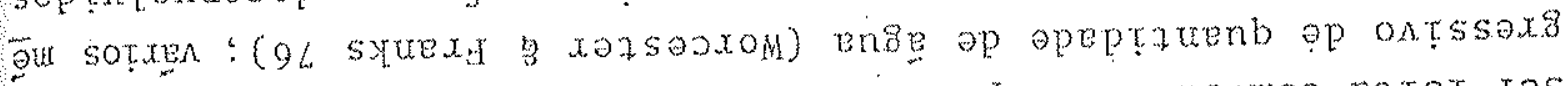

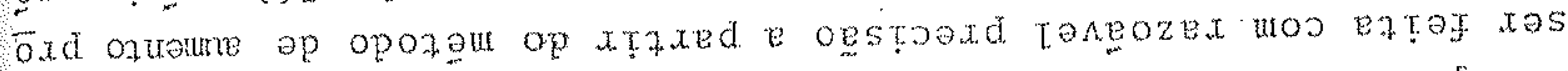

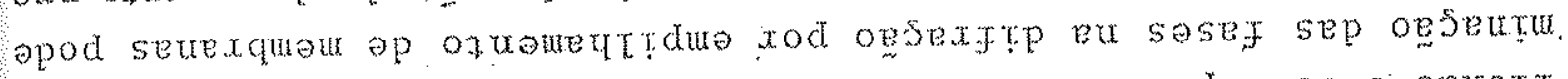

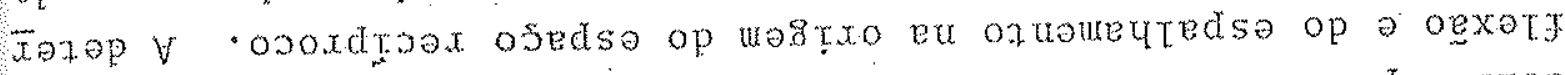

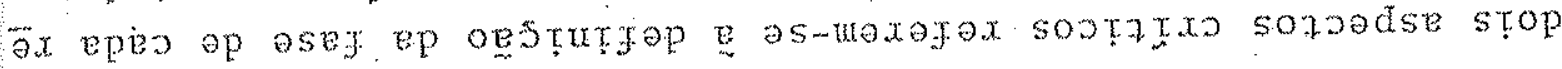

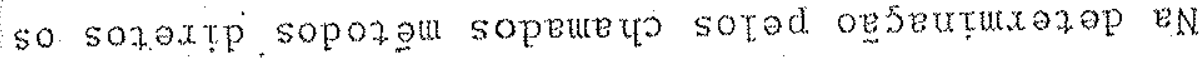

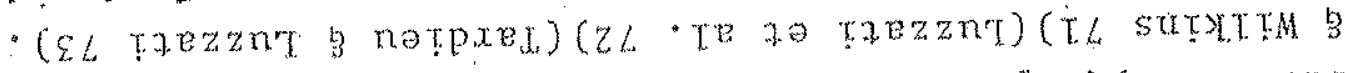

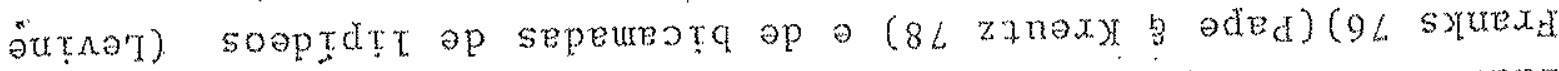

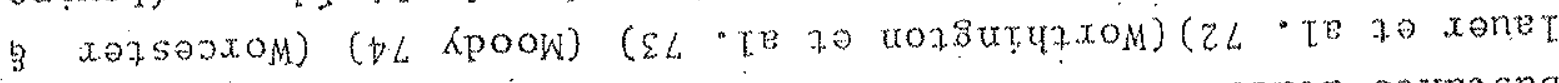

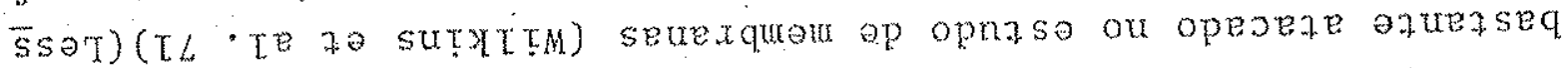
opts wat saxeteriet seoxerjox se exed sepenxasqo sapeptsugit sep

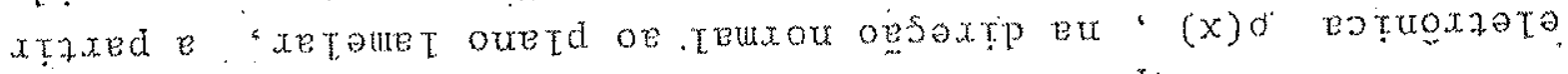

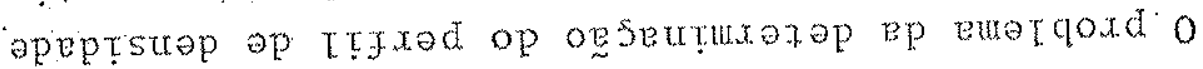

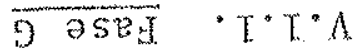

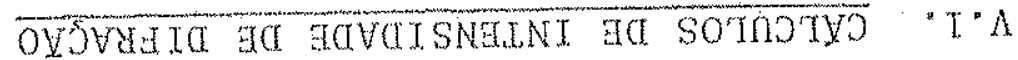

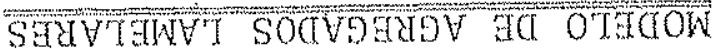

$\Lambda \quad 0 T \Omega I I d V J$ 


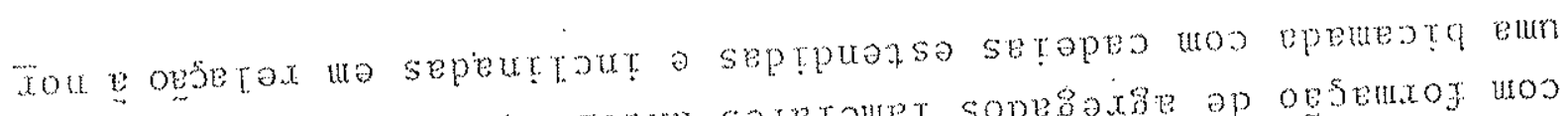

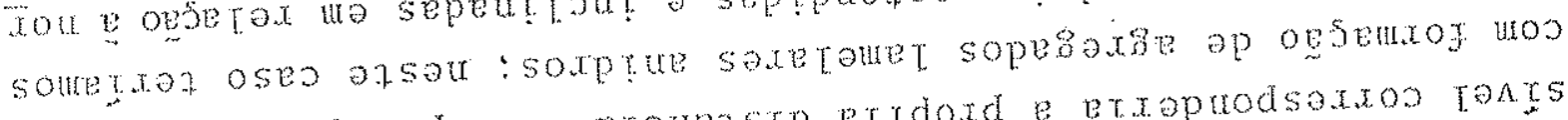

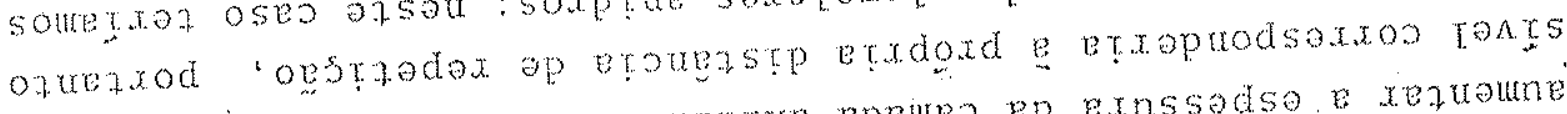

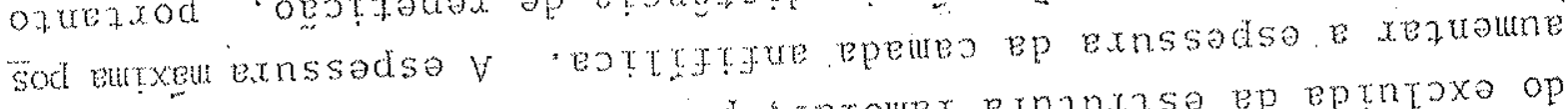

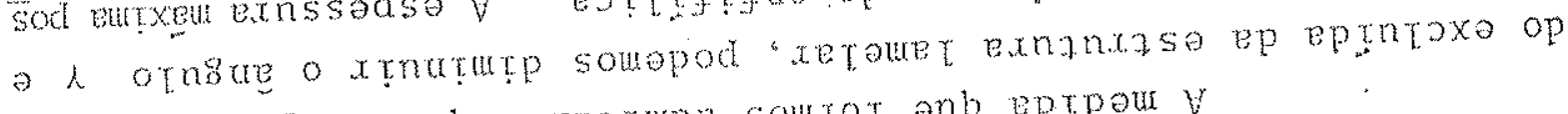
Tos pasa ense e anb opurature souxoz anb eptpou

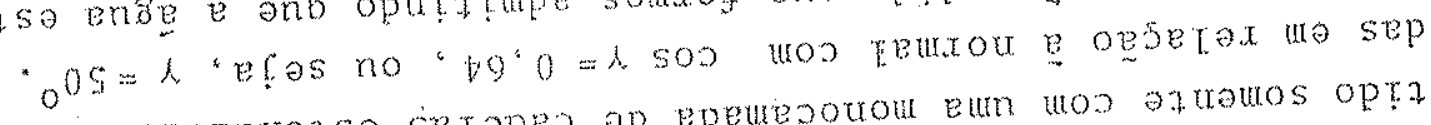

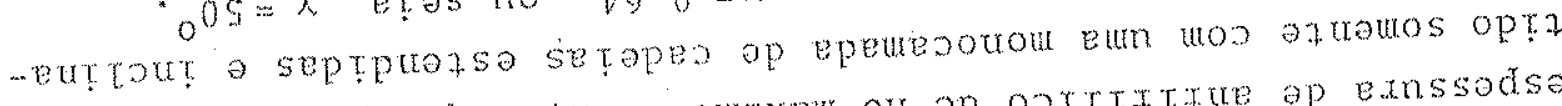

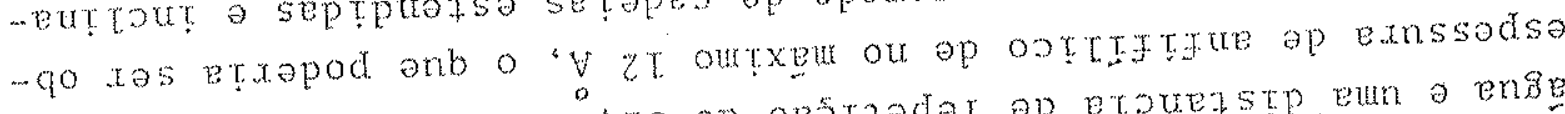

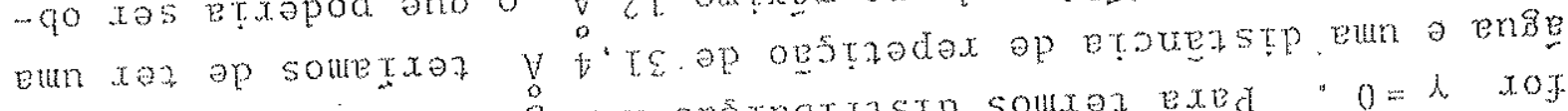

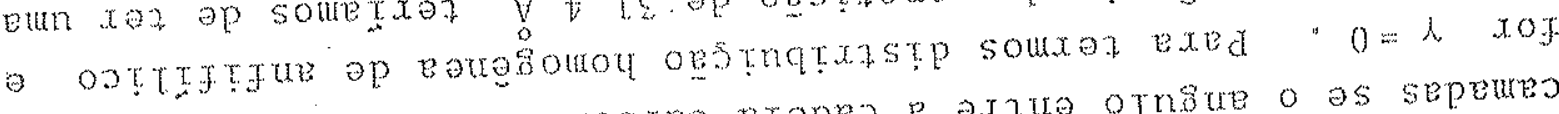

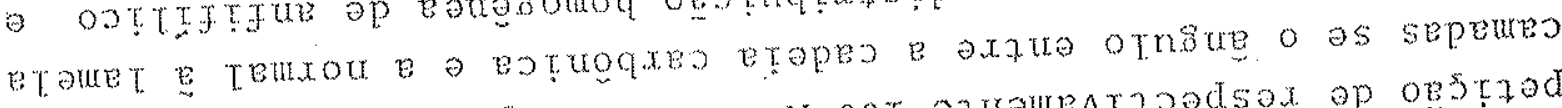

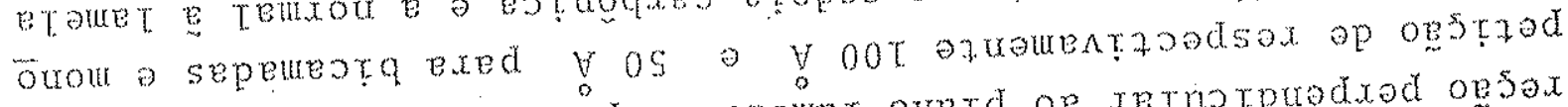

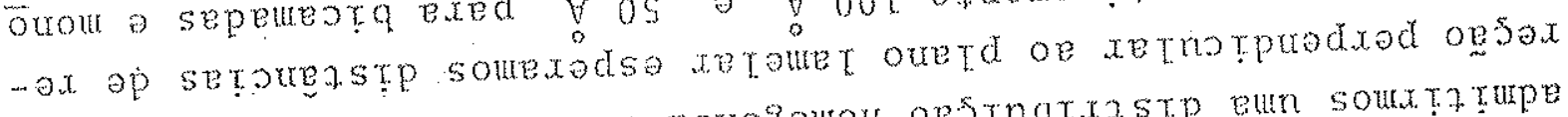

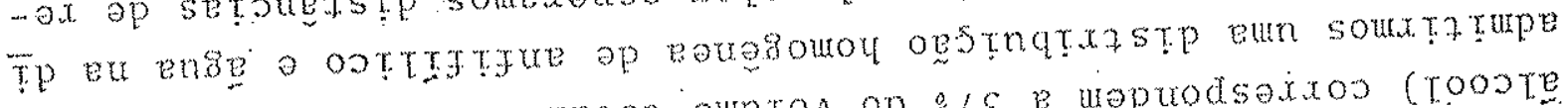

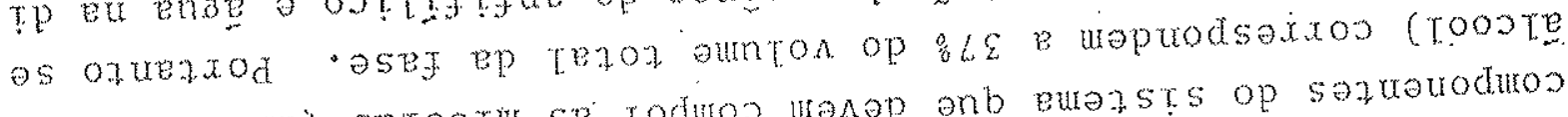

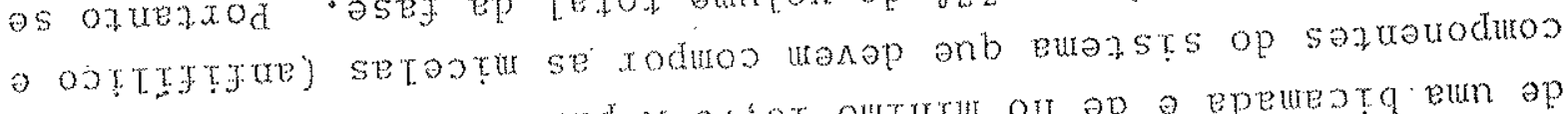

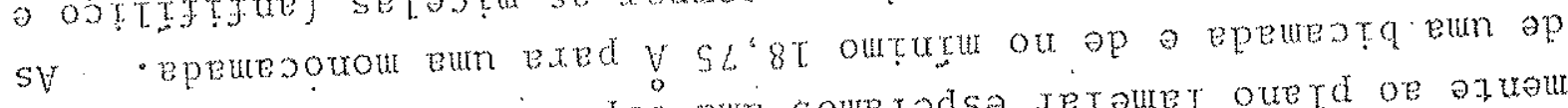

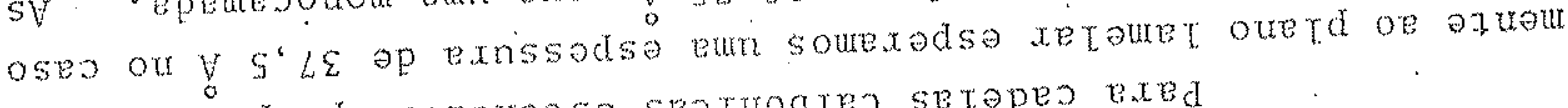

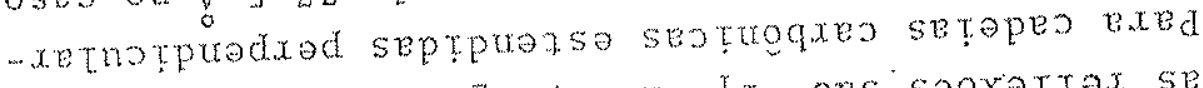

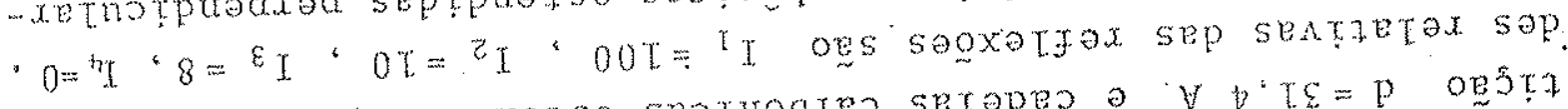

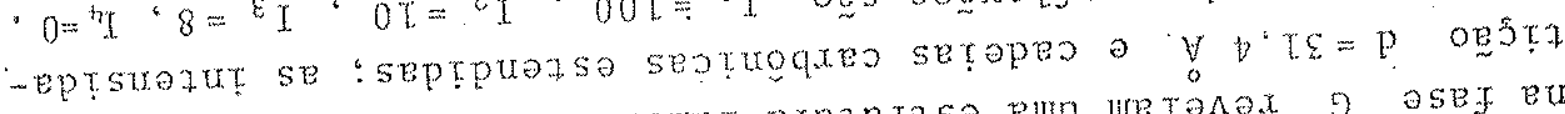

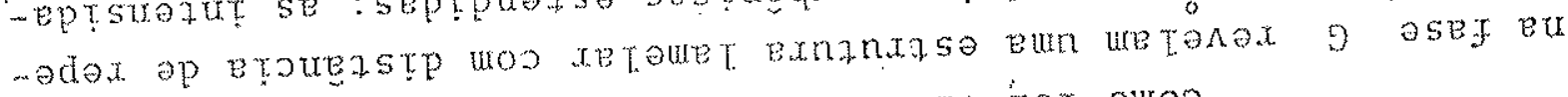

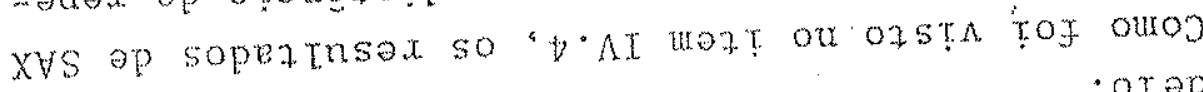
- otepon assop xizued

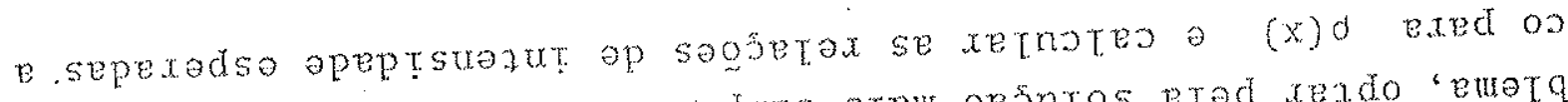

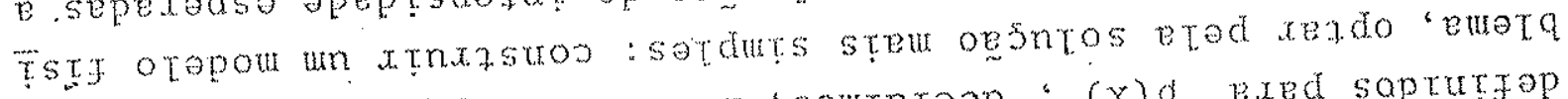

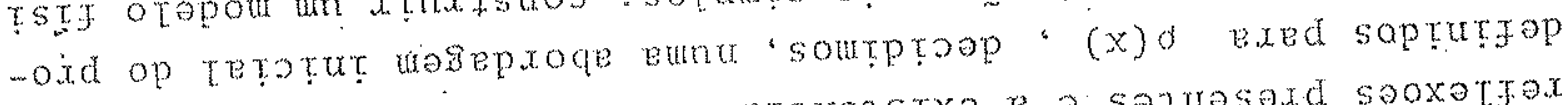

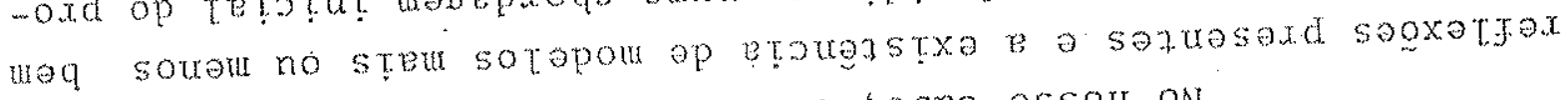
of oxamu ounbed o easta ma oproz. "Oseo ossou on

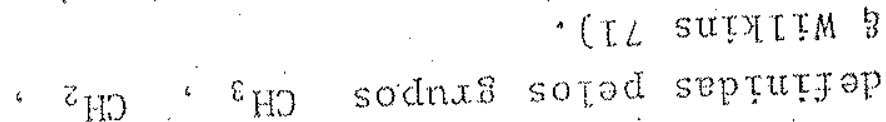

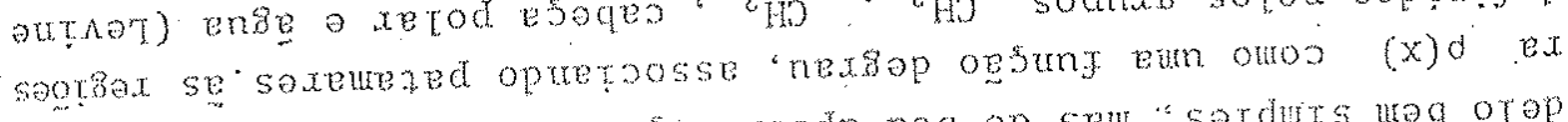

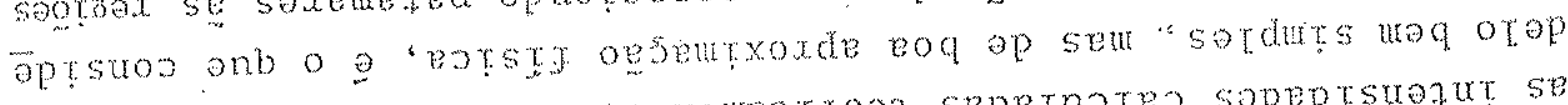

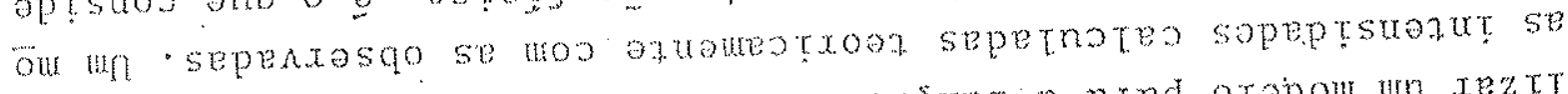

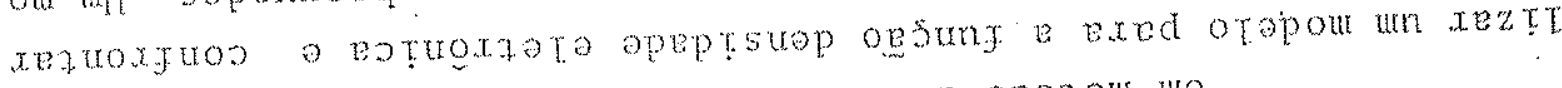

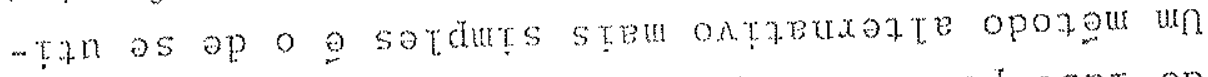

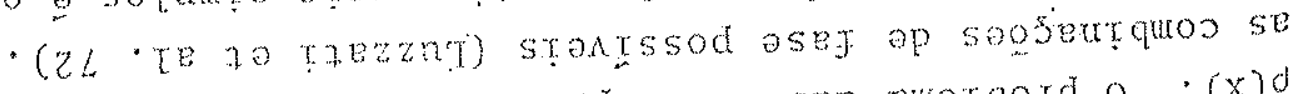

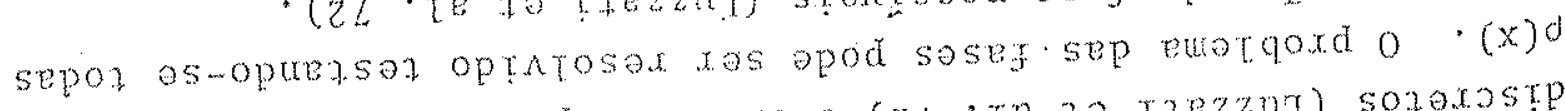

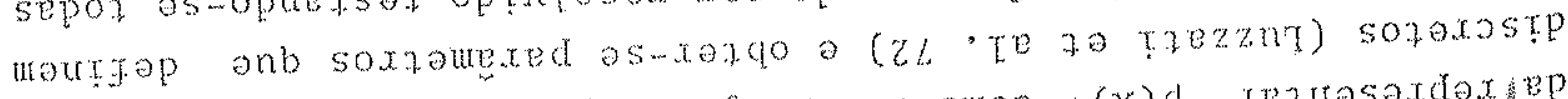

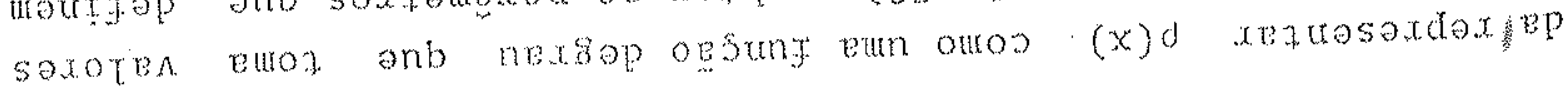




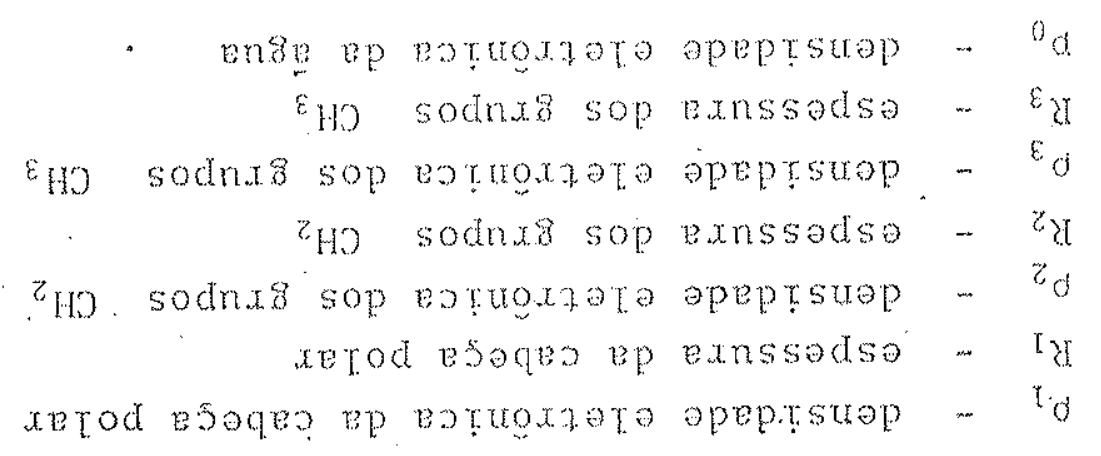

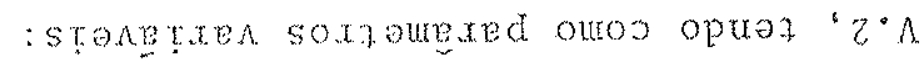

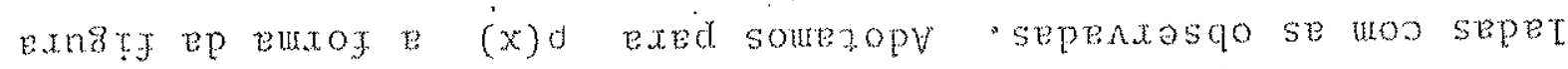

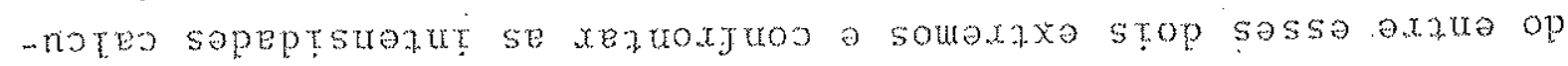
Torxa $(x)$ o exed sotopour xeasoz oefuo somantosey

- otopour ostrum un

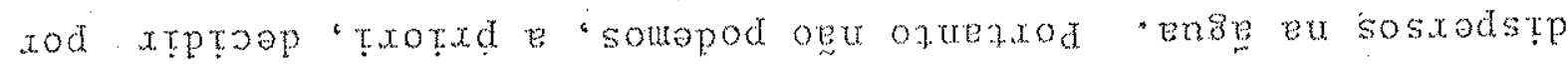

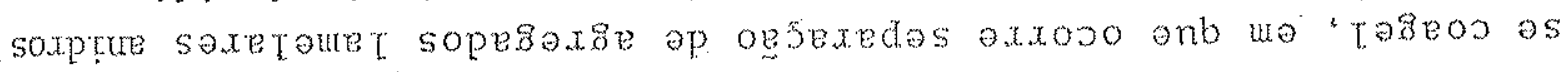

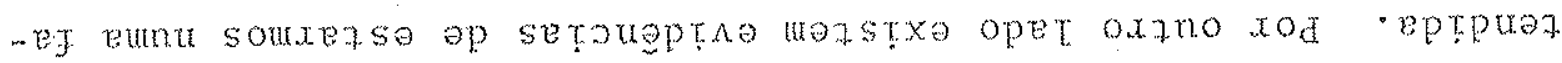

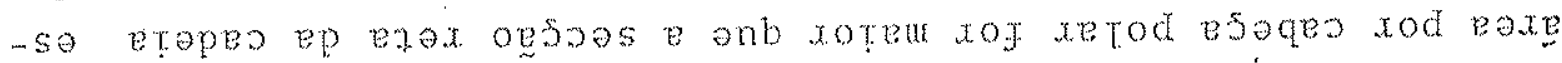

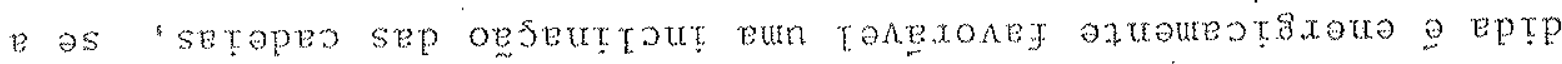

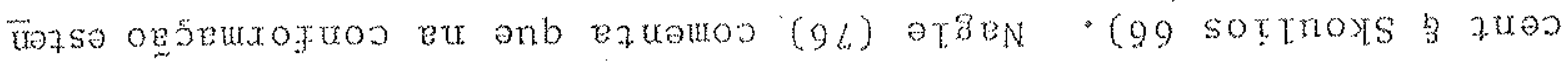

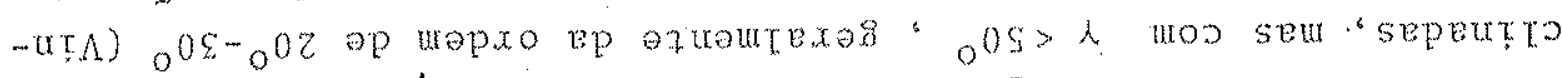

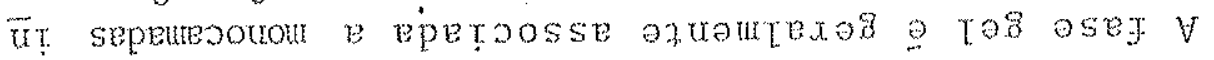

- xetoute t exmanzse ep ense ep teto

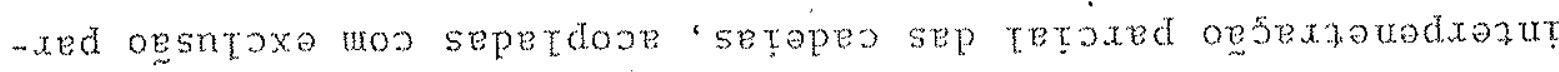

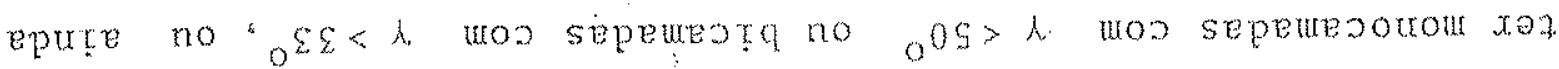

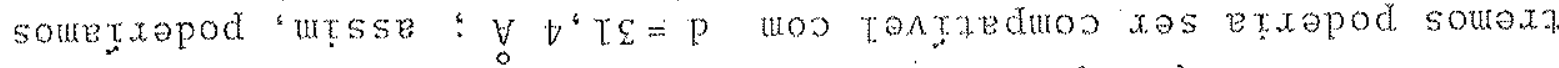
$\bar{x}_{3}$ stop sosse oxare orxetpouxarut otopou xenbteno

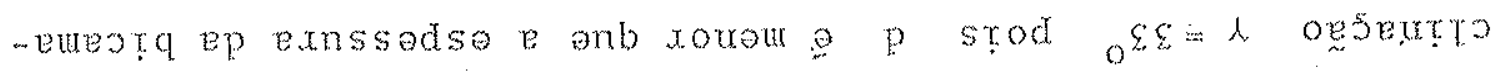

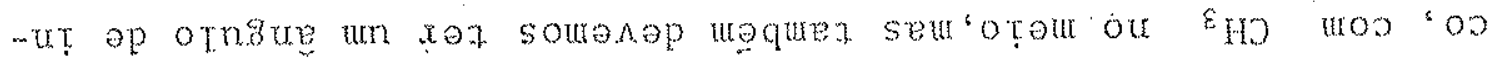

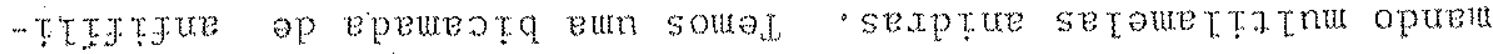

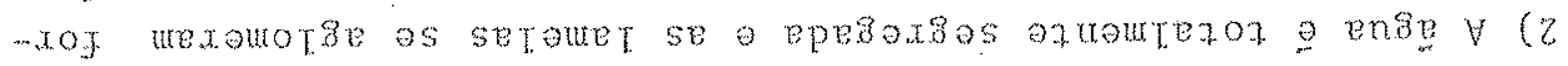
- EHe sodnx a saxerod sesoges ap opssodxadns ext -no $z_{H}$ sodnx soe axupuodsaxion un 'snexsep srop sedede

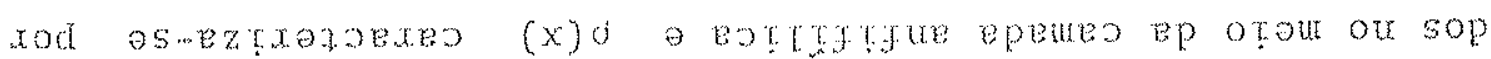

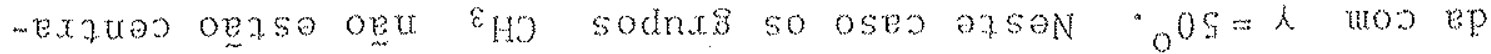

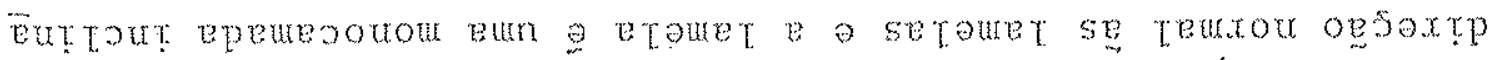

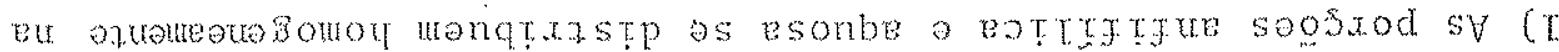

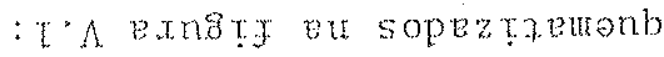

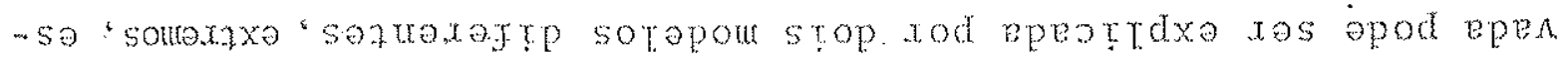

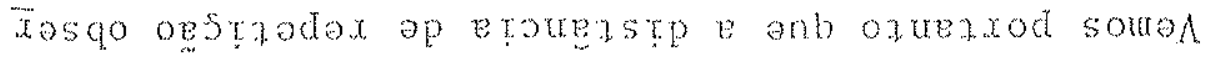

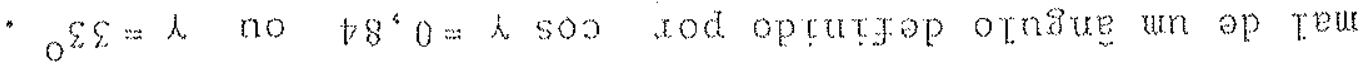


- setour sep ouet oe femcou oesarte e $x$ optos

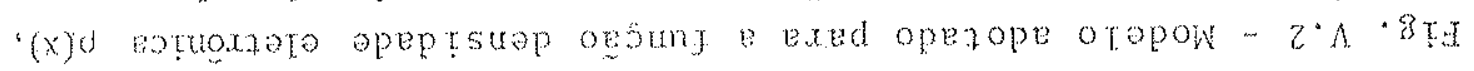

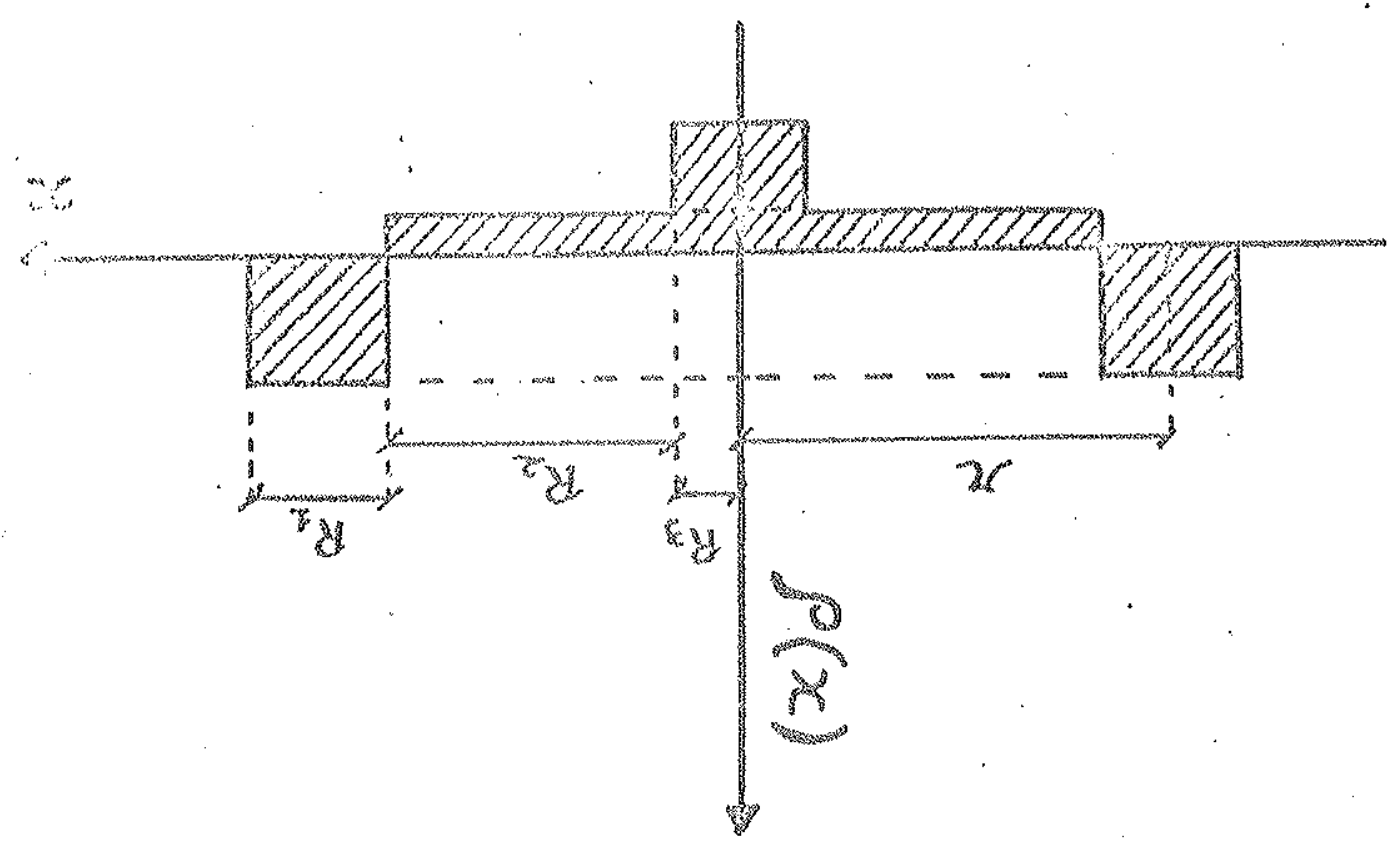

- se rouer se exa

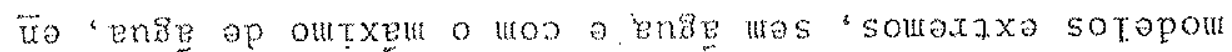

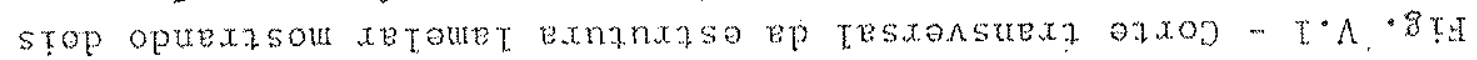

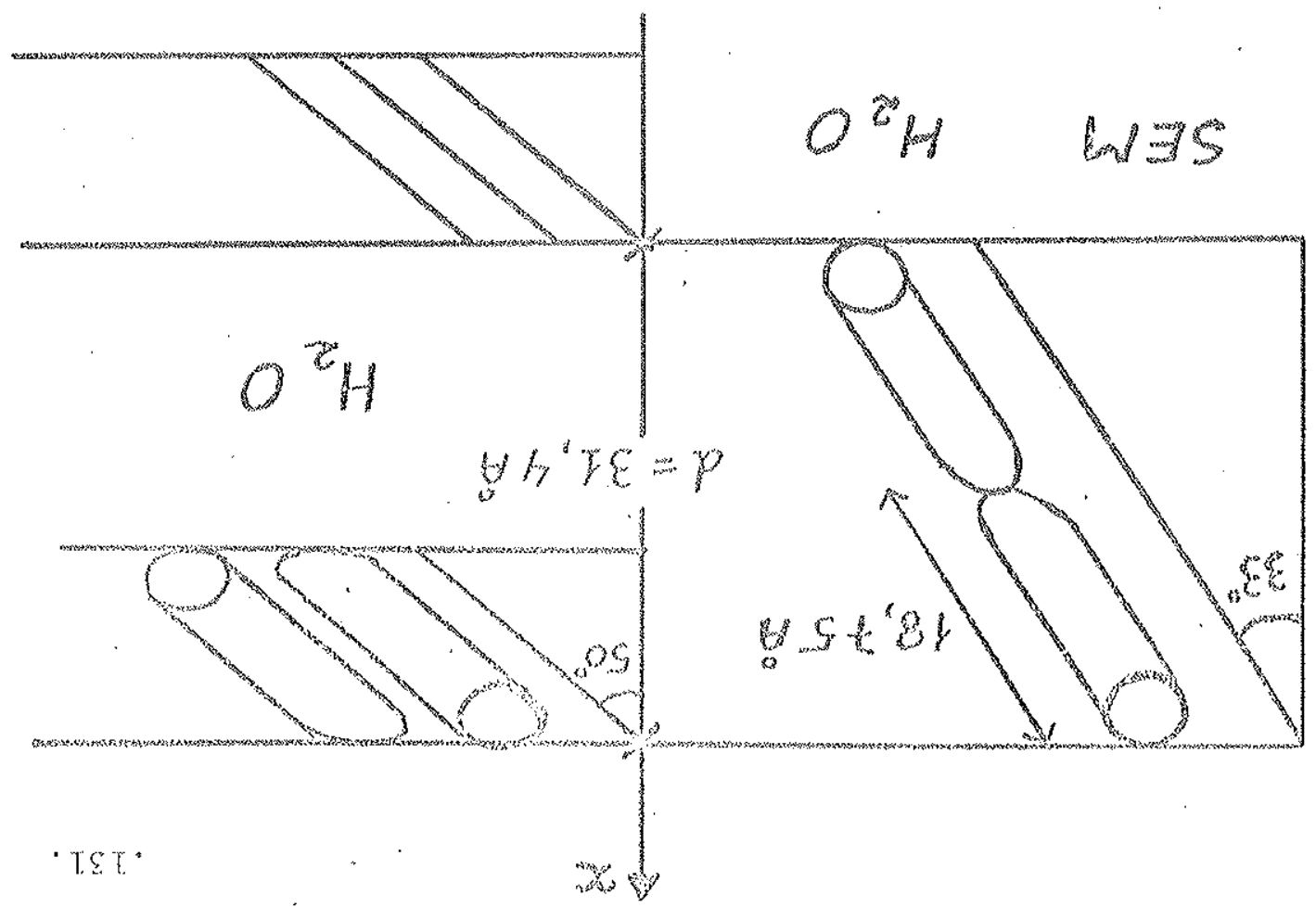




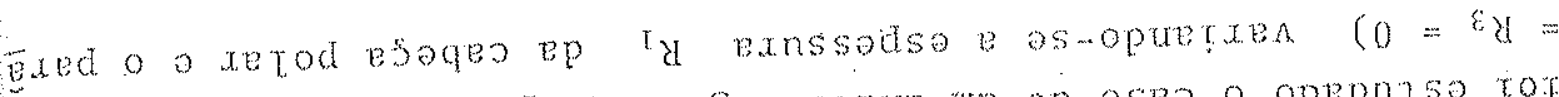

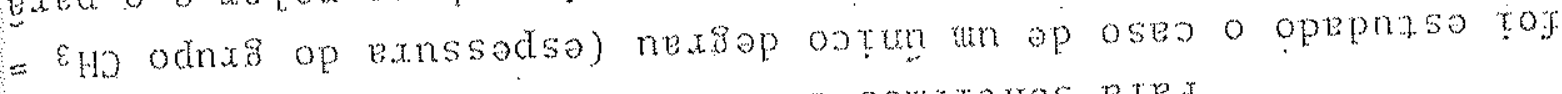
satetod sesegeo sep etomentut e soutras bxed

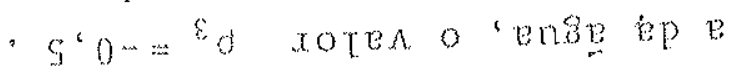

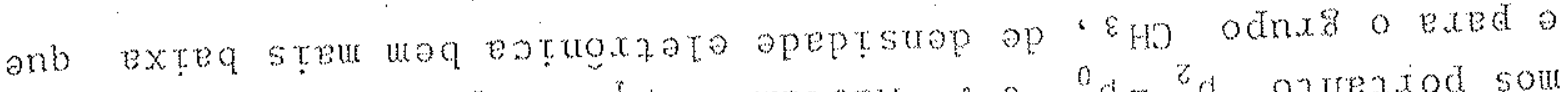

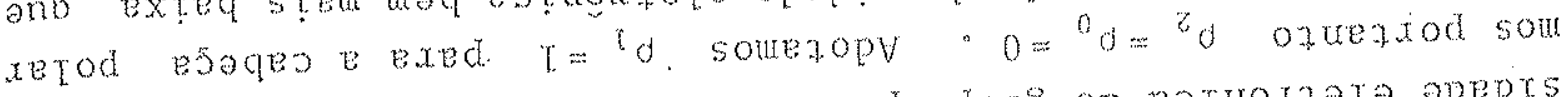

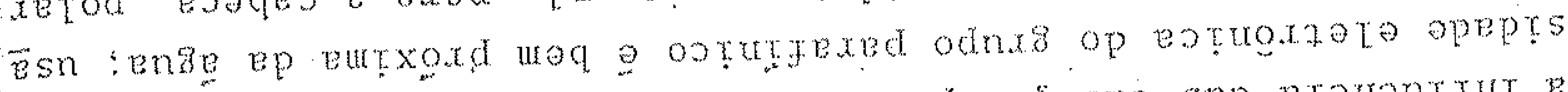

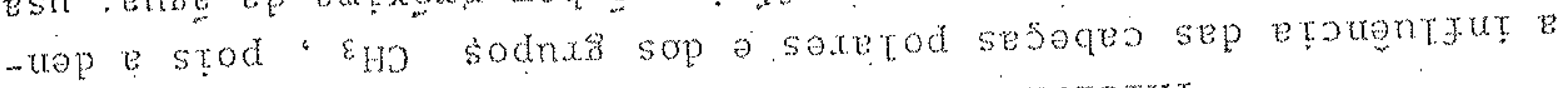

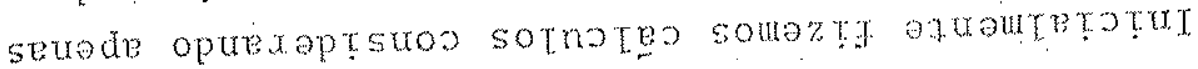

- sezred setren sep eotugrato opeptsuop

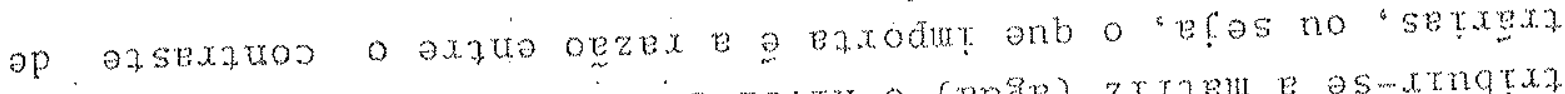

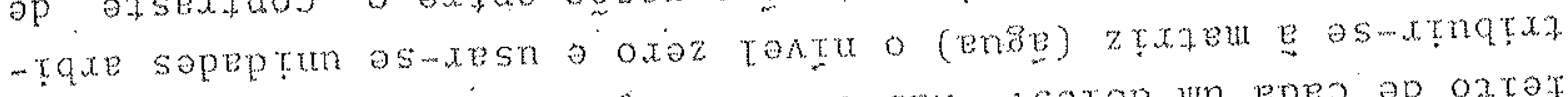
- te Tensn o (x)d ap soosentuxarop sen setop un epes op oxtor

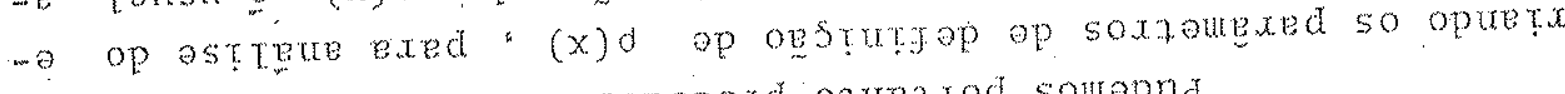

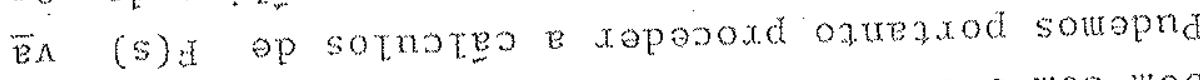

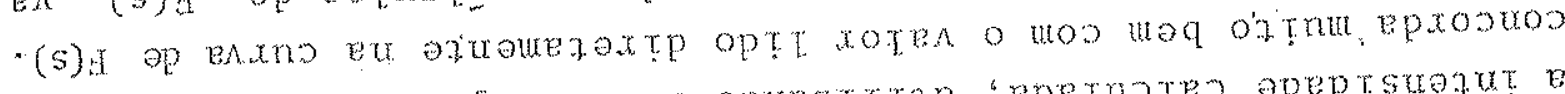

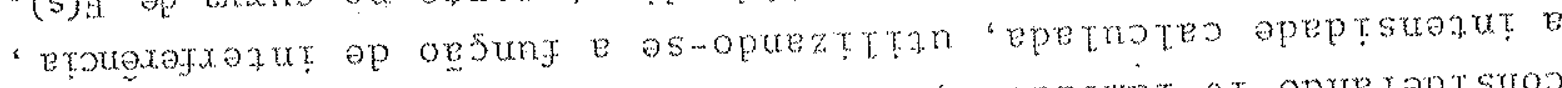

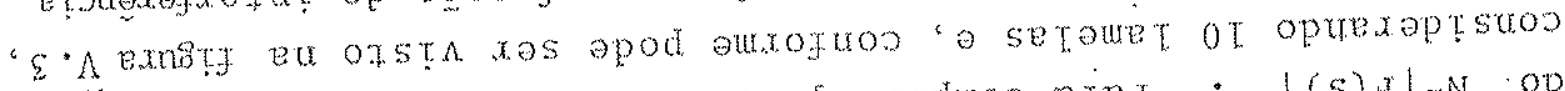

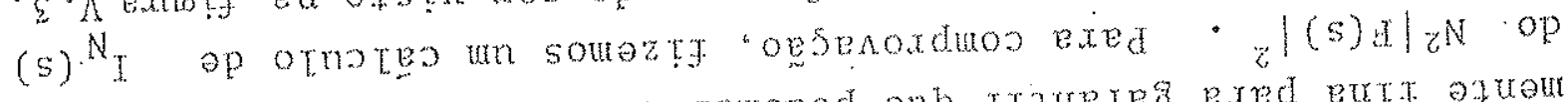

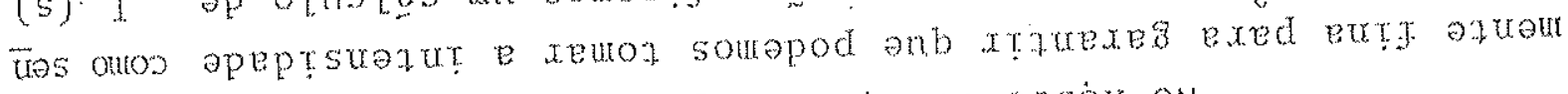
worketorins a soord sop exnixer p osto ossou on

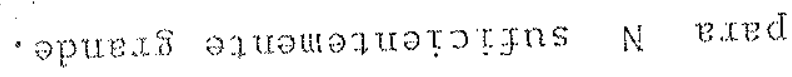

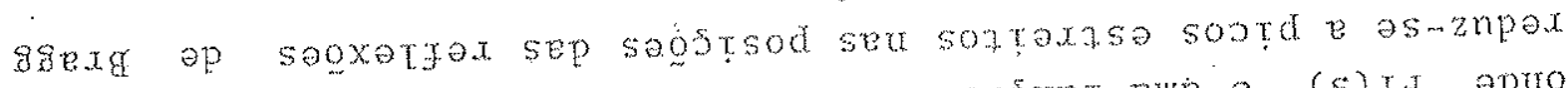

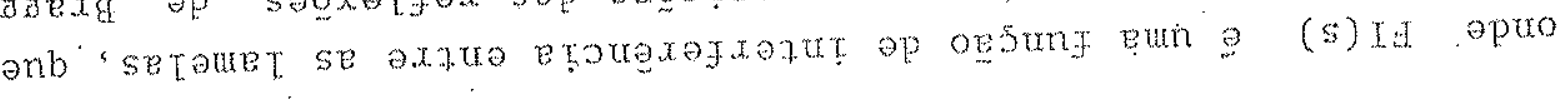

$$
\text { (s) } \mathrm{Id} z|(s) \mathrm{d}| z^{\mathrm{N}}=(s)^{\mathrm{N}} \mathrm{I}
$$

owoo batrose $x a s$ apod s?tou

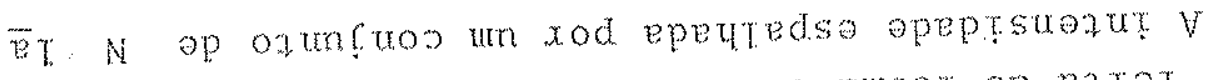

- Tetara eurof op exteg xas apod ogsexs

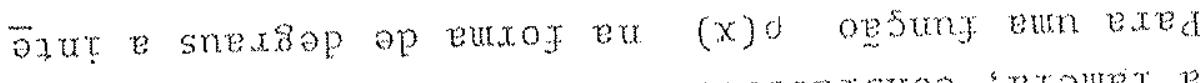

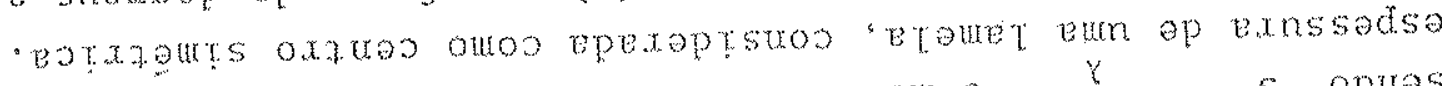

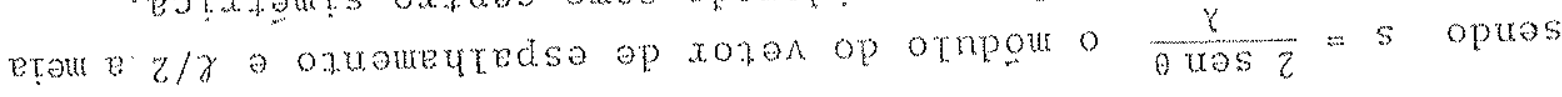

$$
x p(s x u z) \operatorname{son}(x) d \int_{z / \gamma}^{0} z=(s) d
$$

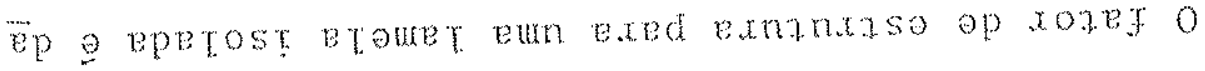




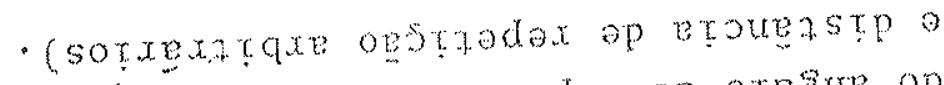
$(x)$ op soxzougxd) $a z=3$ oxuareutedso ap otnsue op

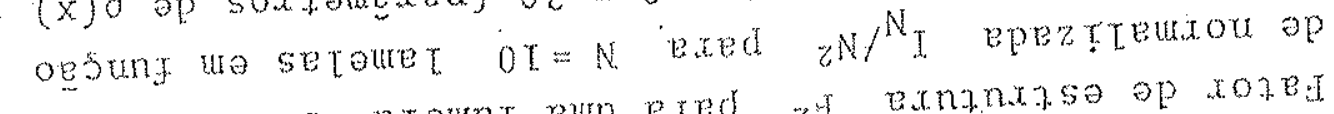

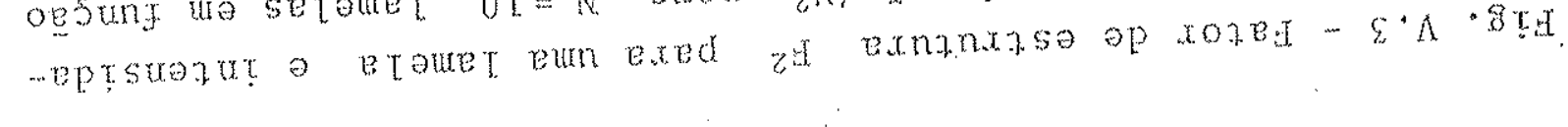

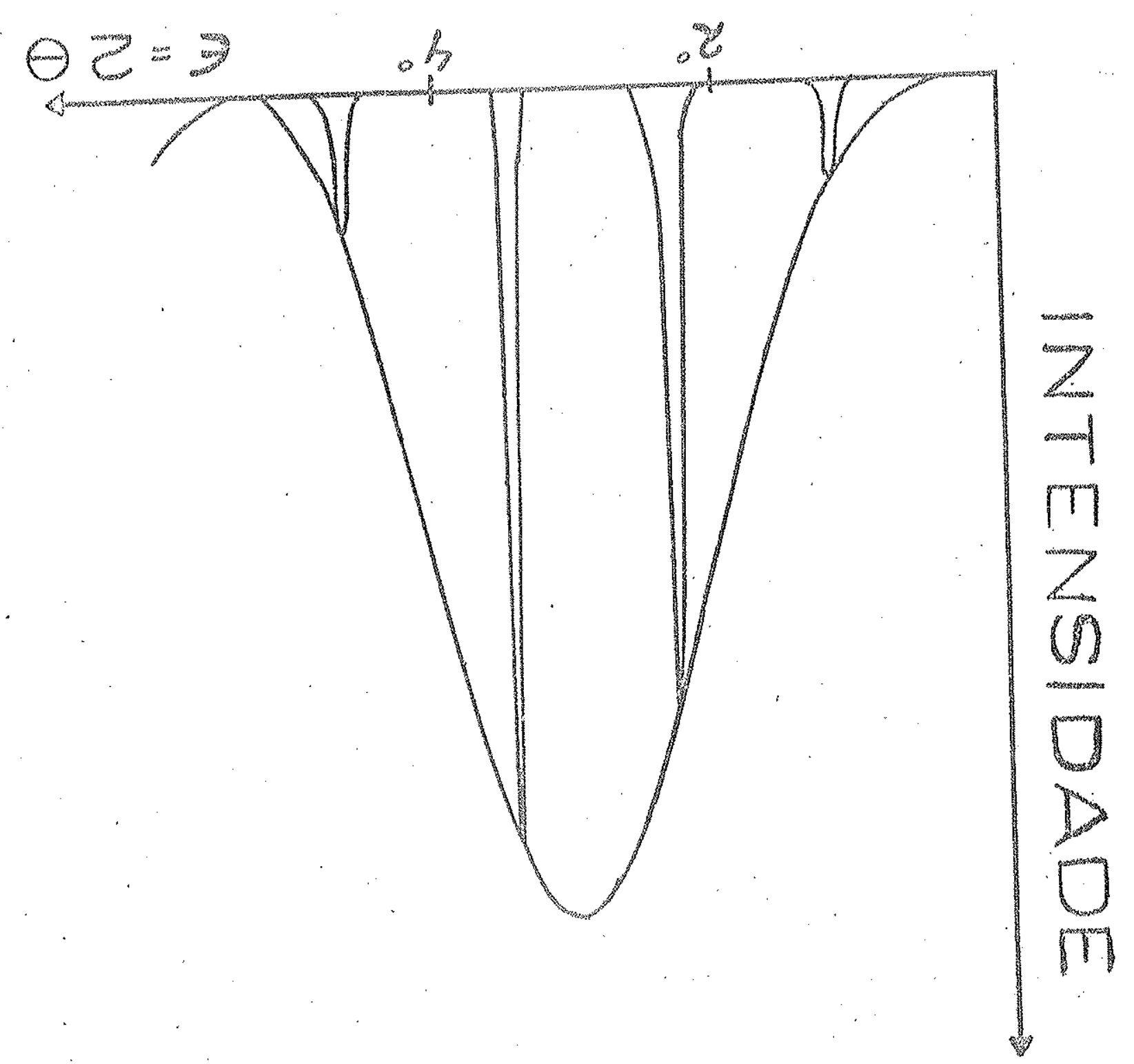




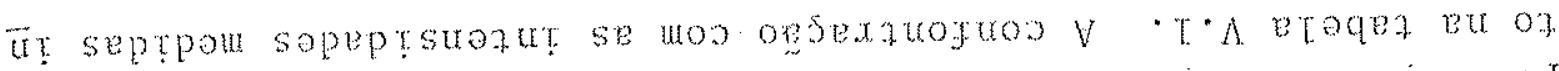
sta xes apod opertrsax o a sazupuodsoxidods op soroten so exed

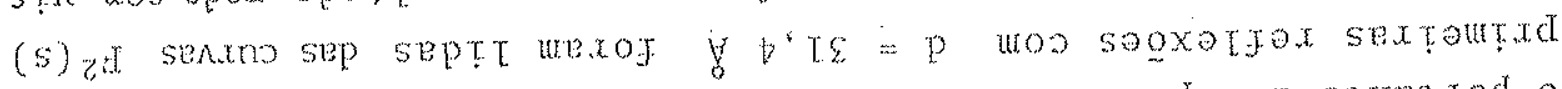

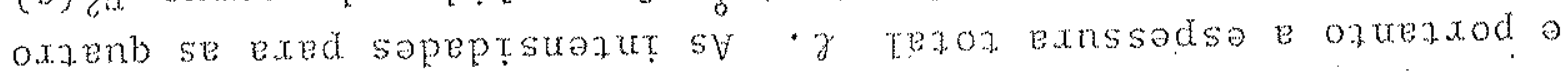

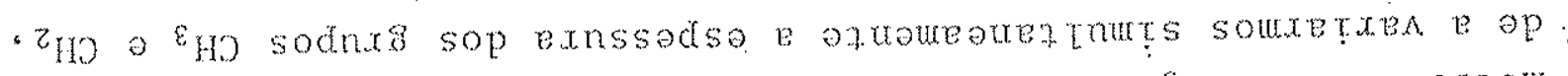

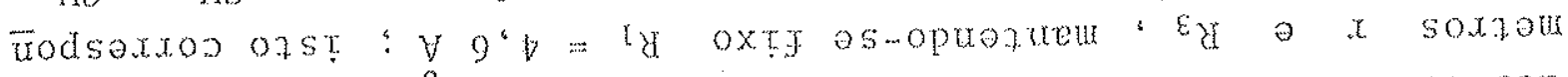

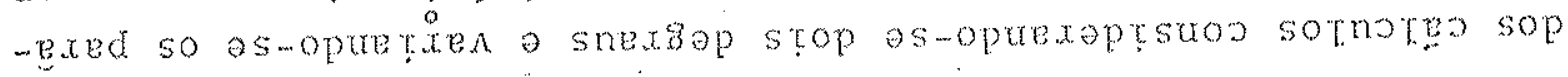
-emaga mexof sepeuejta op otopou o xetruts exed

ostratrue ap septuejourou a sepeupota op stontssod sotopou te tnuts xtruses b somexnod

- setouret arjue ense a sep - bureotrou xat opod tos asex p anb ossed oe "sexptue sepeneata op

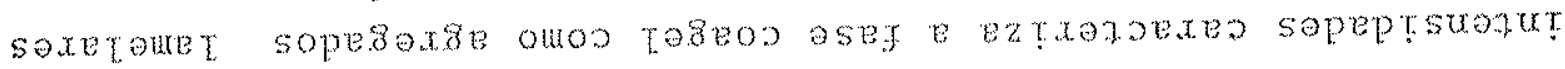

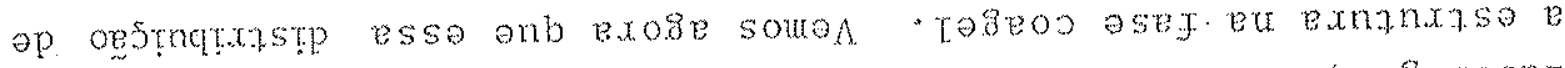

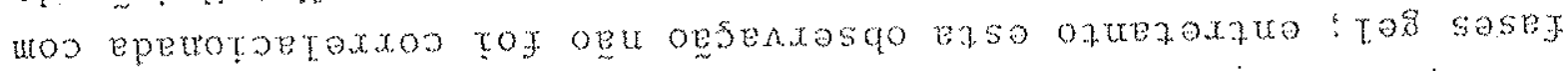

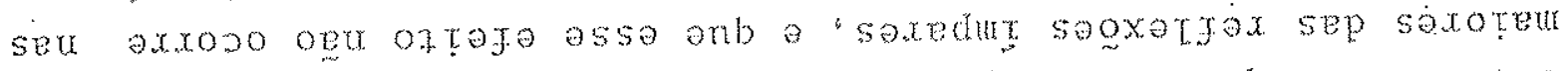

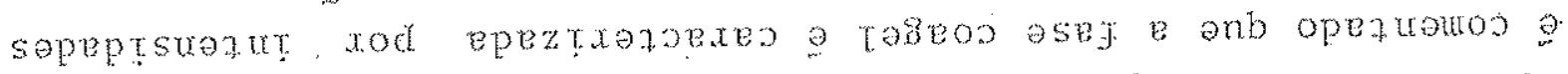

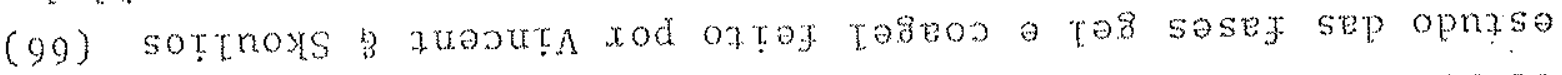

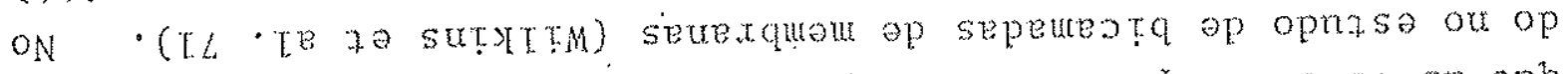

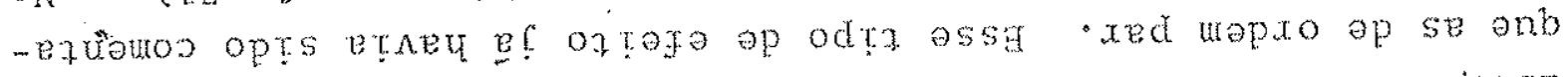

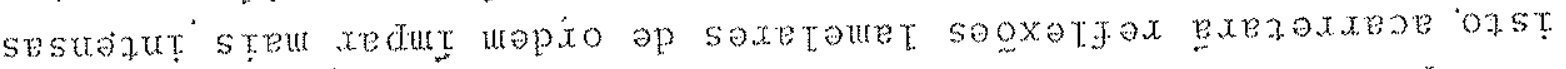

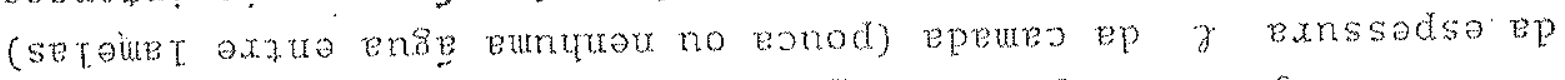

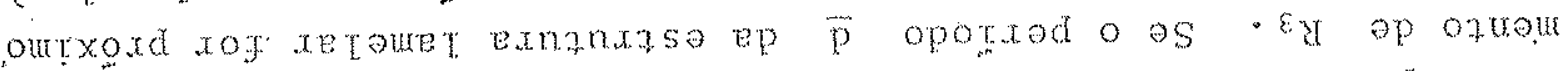

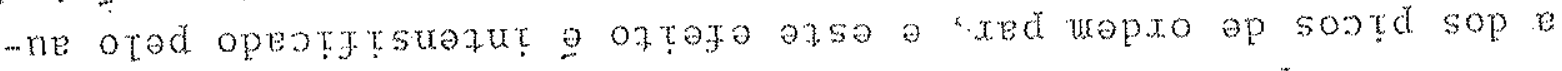

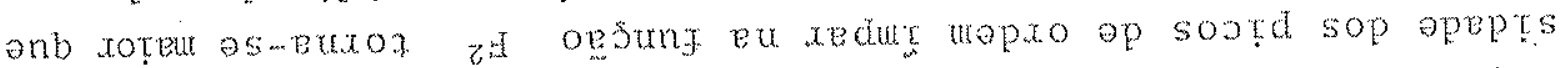

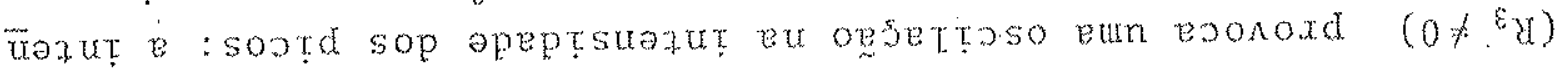

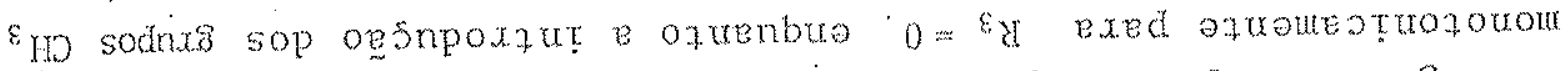

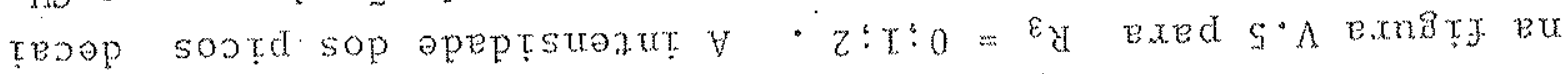

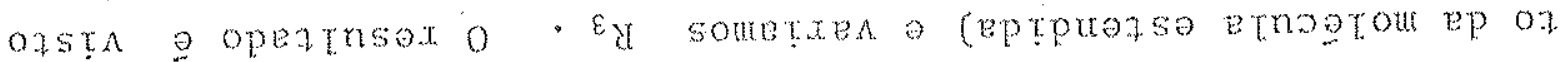

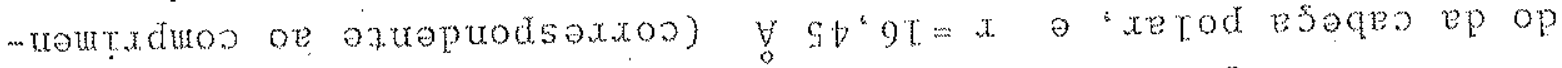

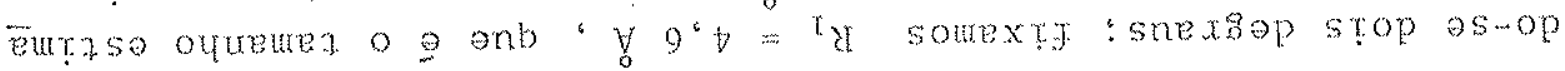
üexaptroo opernuts tog $\varepsilon_{\mathrm{H}}$ sodnx sop ofreje 0

- eate stem map

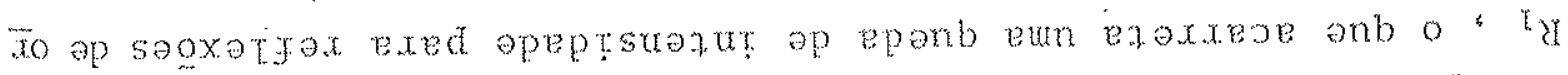

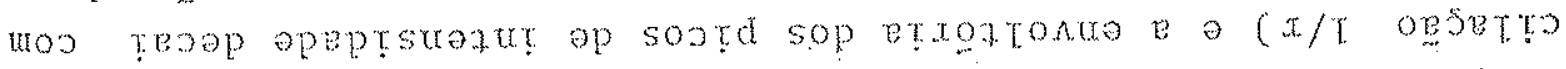
so op opogxad wos (s) d) $\bar{x}$ oxfomexed otod epermanop o oesmy

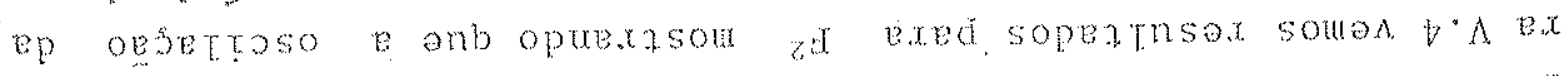

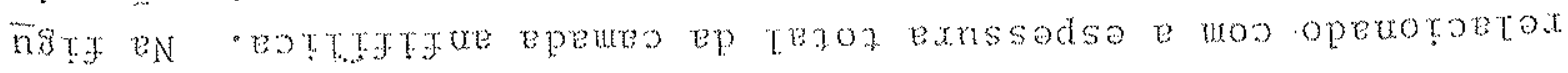

$$
z / x+z y+\varepsilon z=x
$$

$\operatorname{toc} \%$ 


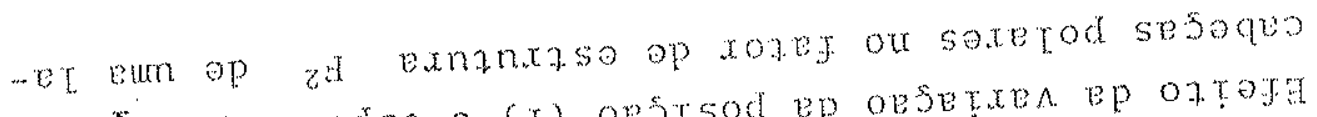

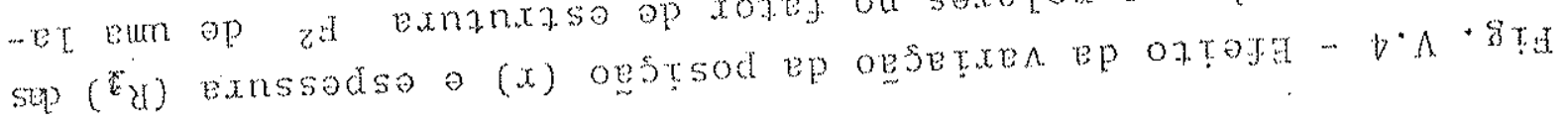

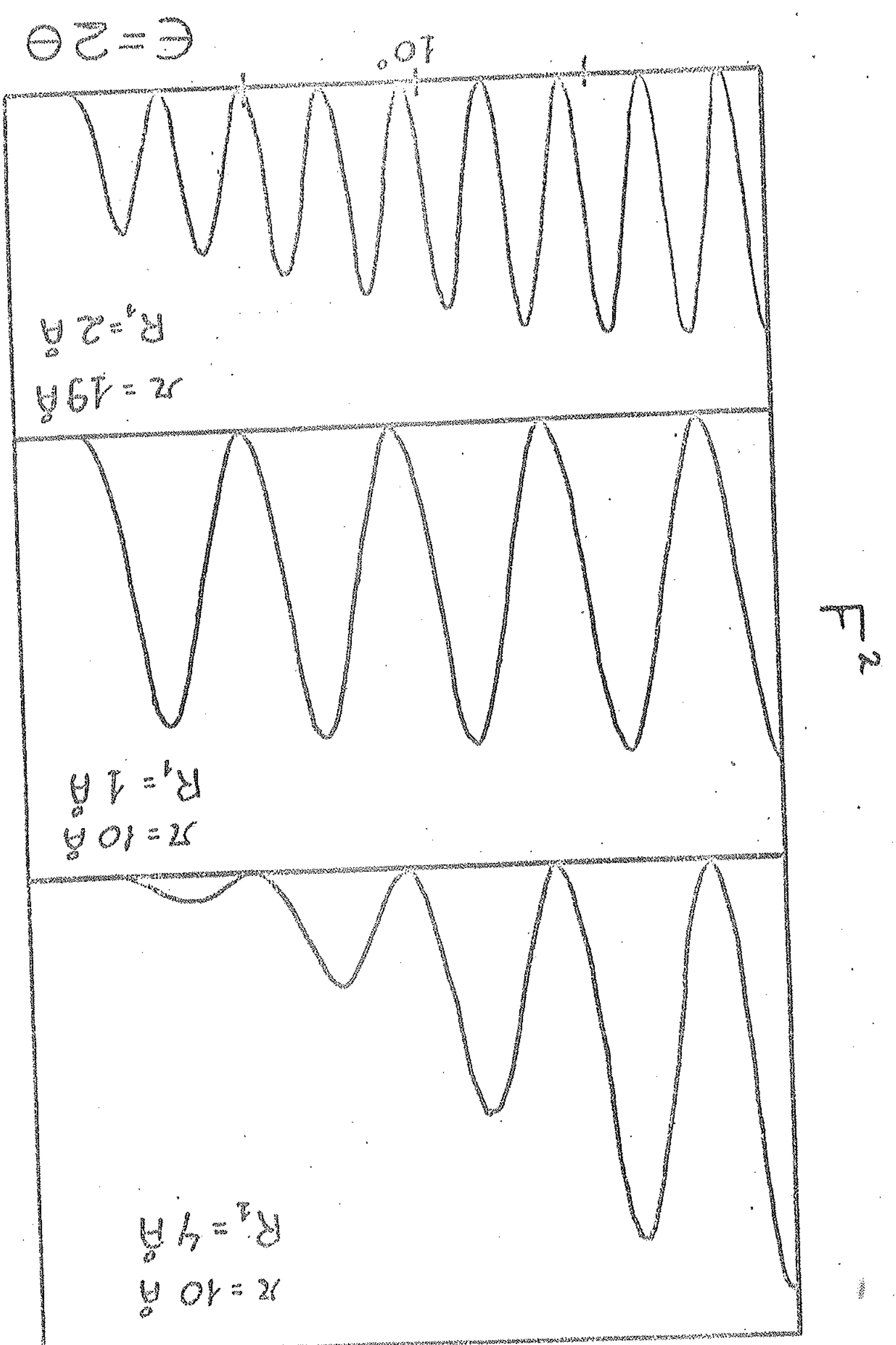


"erout eun op zi exnatise op xoats ou

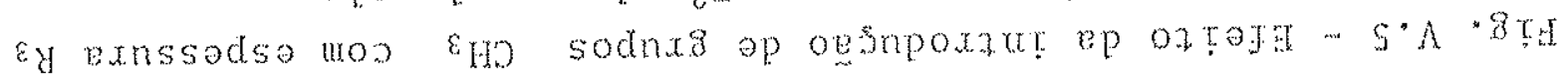

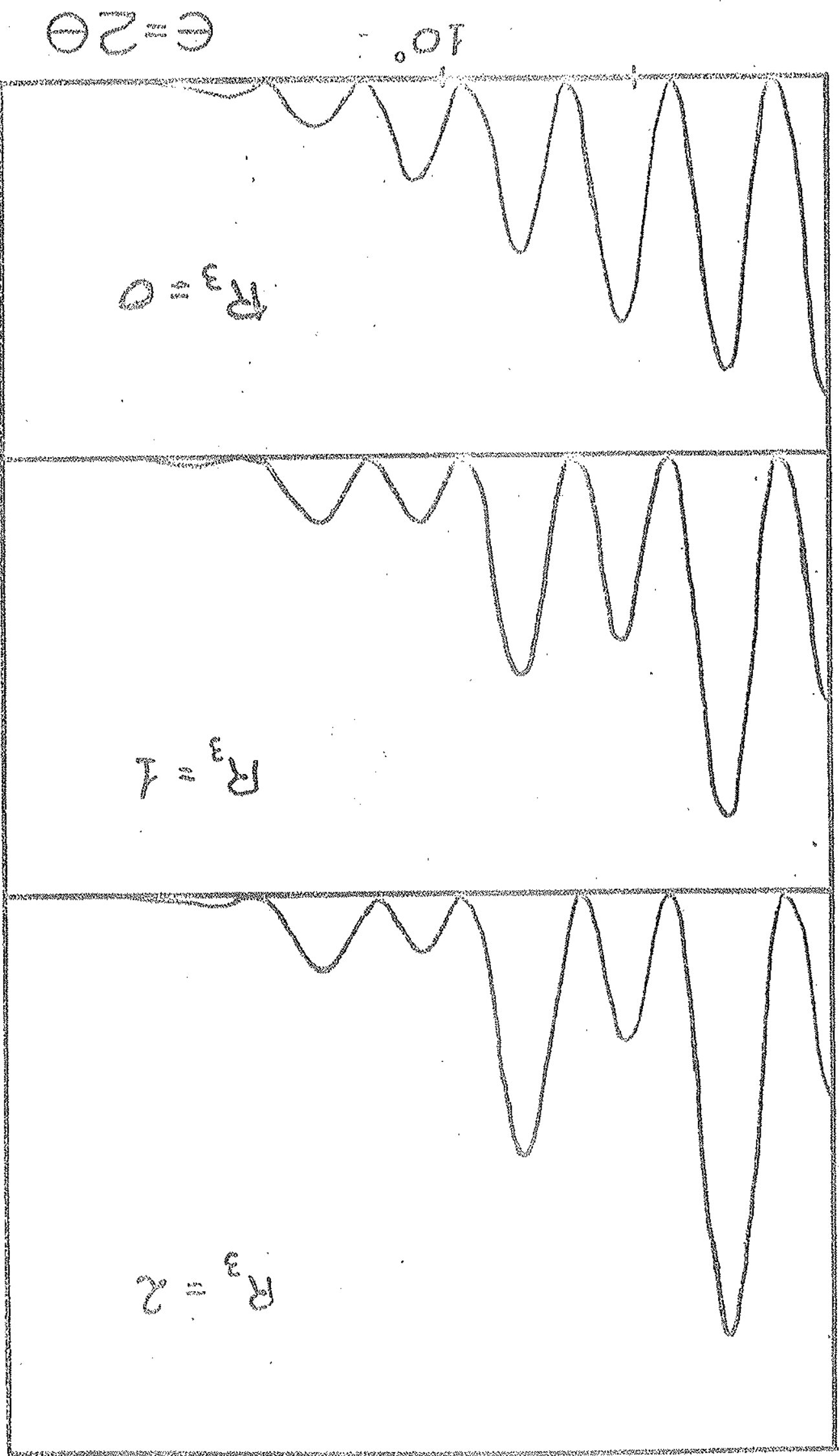




\begin{tabular}{|c|c|c|c|c|c|c|c|c|c|c|c|c|c|}
\hline 0 & 7 & $z$ & 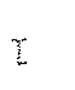 & $\angle S$ & $7 \varepsilon$ & $2 z$ & 0 & 0 & 0 & $\Sigma I$ & $9 I$ & $\Sigma Z$ & $\because$ \\
\hline 8 & $z z$ & $6 z$ & 01 & 8 & $O L$ & $0 z$ & 02 & 02 & or & 59 & $9 S$ & 97 & $\varepsilon_{i}$ \\
\hline$O T$ & ti & $9 \mathcal{Q}$ & $0 \subseteq$ & 0 & 0 & 0 & $0 z$ & 07 & 05 & 85 & 67 & 99 & $z_{I}$ \\
\hline 001 & $2 S$ & $\forall z$ & $9 T$ & 08 & 09 & v & $O I I$ & 06 & 22 & $\angle I I$ & 86 & $6 L$ & $\bar{I}$ \\
\hline \multirow[t]{2}{*}{$\hat{\mathrm{s}}$} & $z$ & $I$ & 0 & $z$ & $I$ & 0 & $z$ & I & 0 & $z$ & $T$ & 0 & $\varepsilon_{\text {兑 }}$ \\
\hline & or & $O E$ & $0 T$ & $Z I$ & $2 T$ & $2 T$ & $t T$ & $V I$ & $\nabla I$ & $\angle S^{6} T$ & $L^{*} S I$ & $L^{\circ} S T$ & $\tilde{x}$ \\
\hline
\end{tabular}

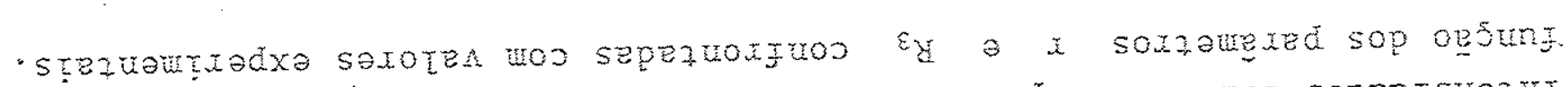

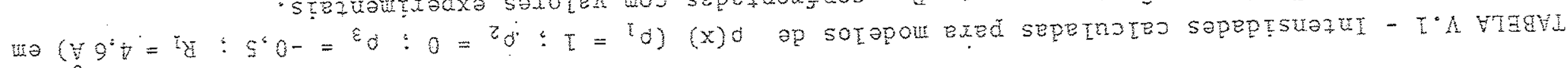




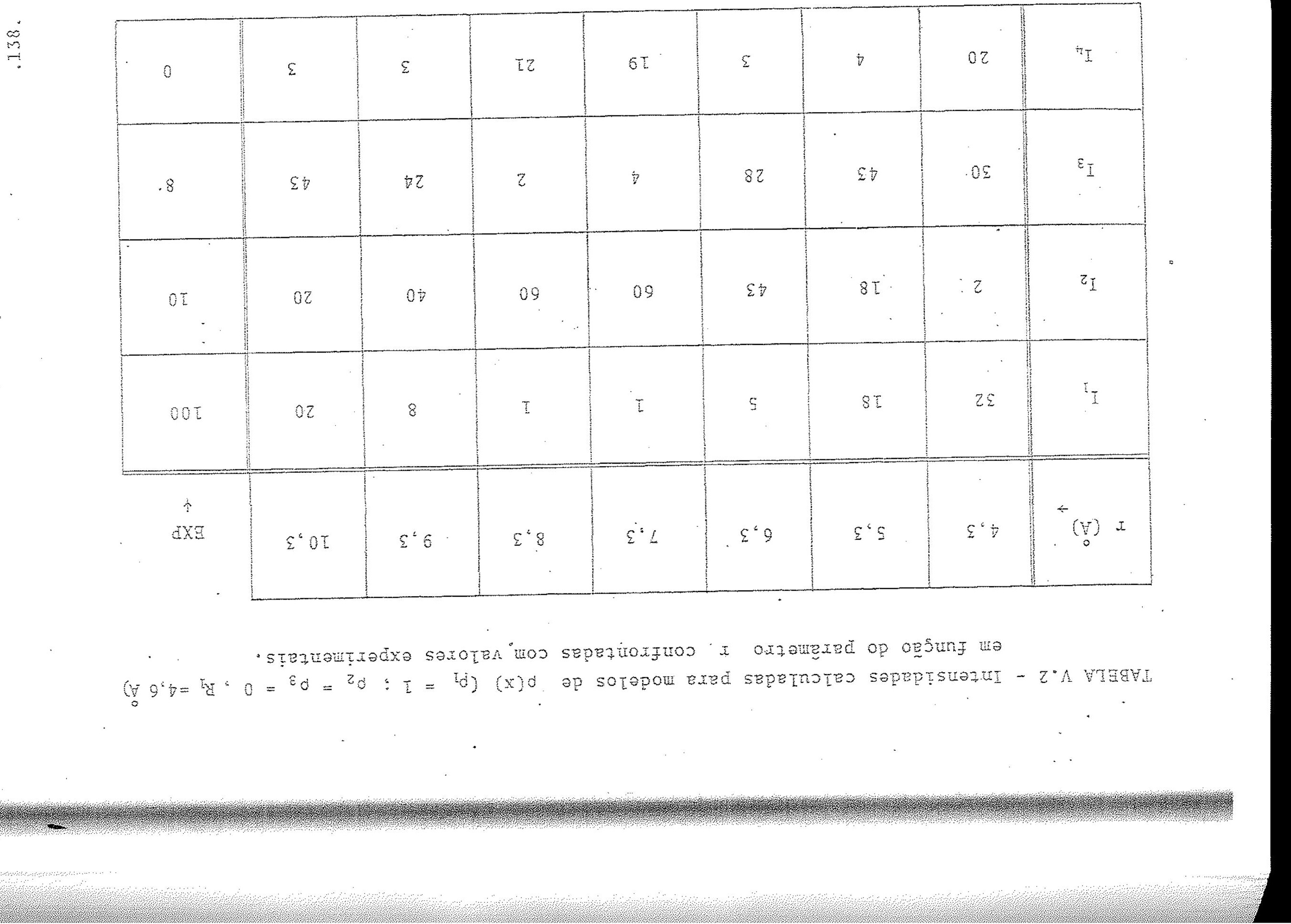




\section{- sepenasgo sexetout}

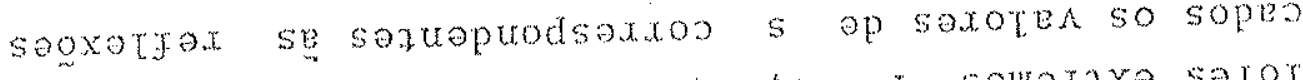

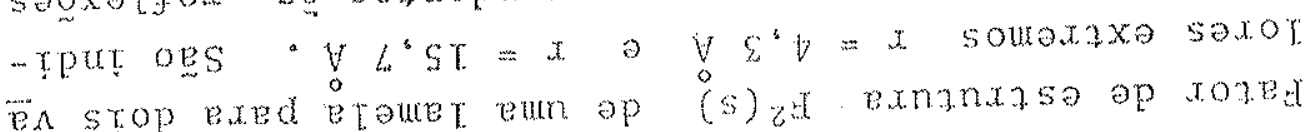

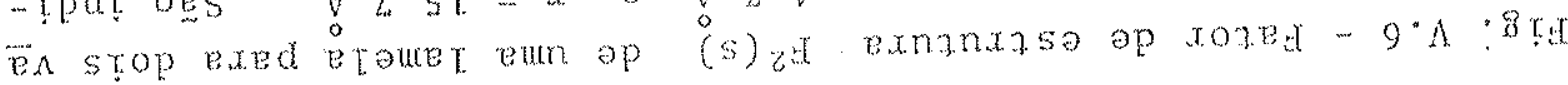

$(r-4) \&:$ Pro

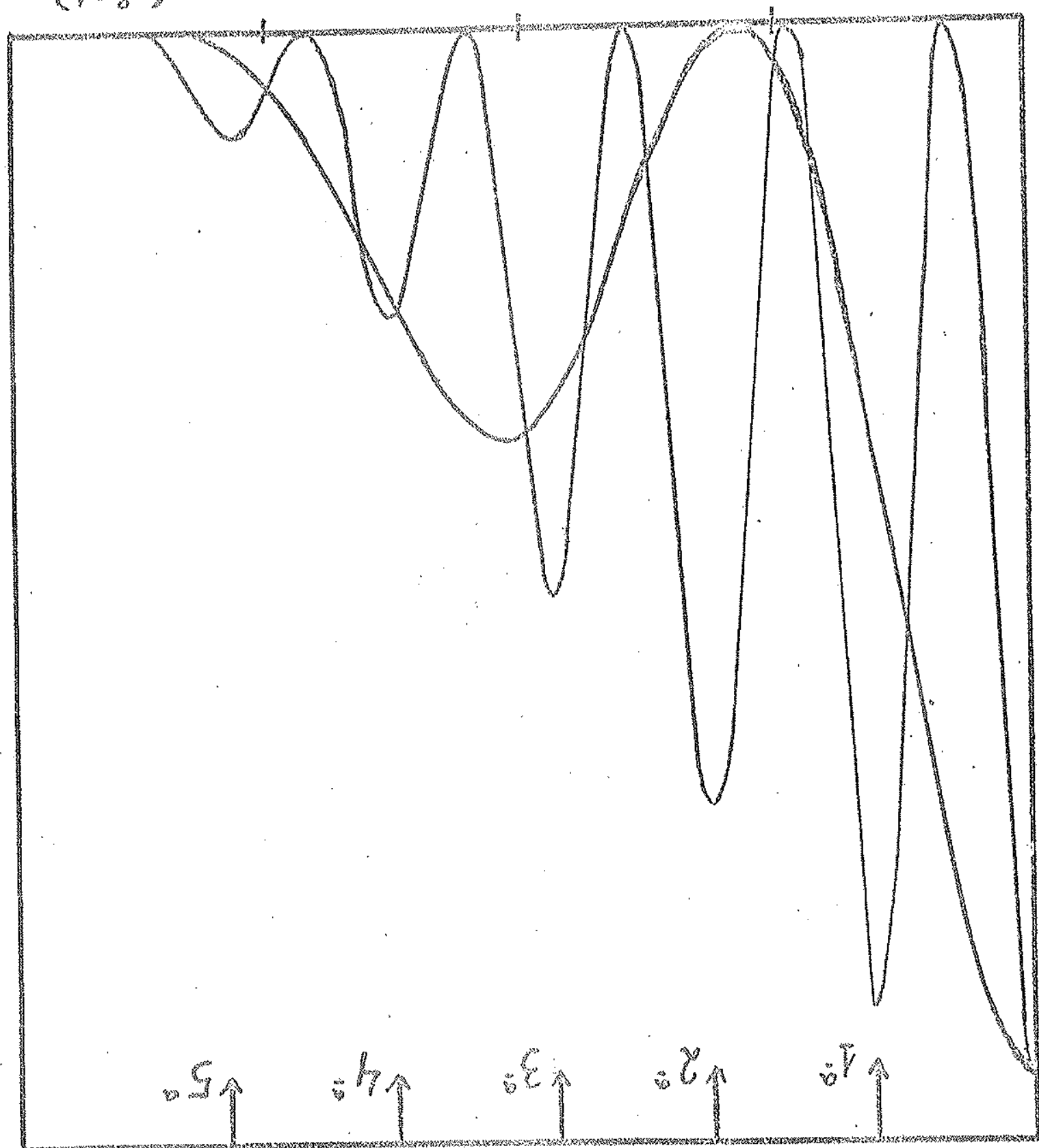




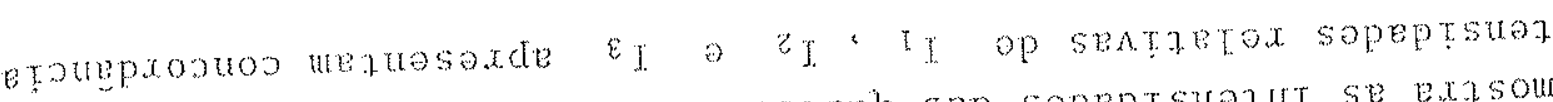

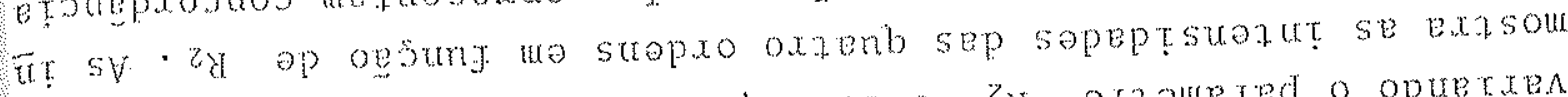

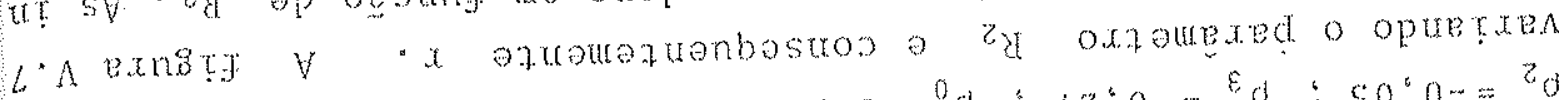

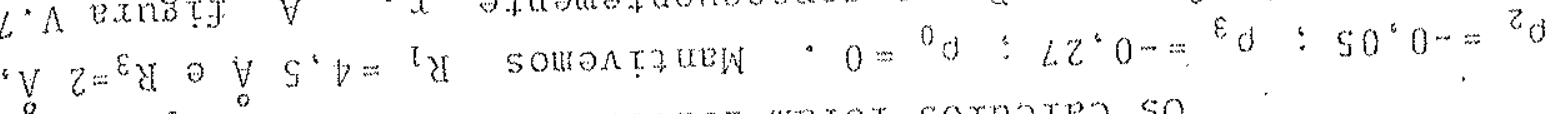

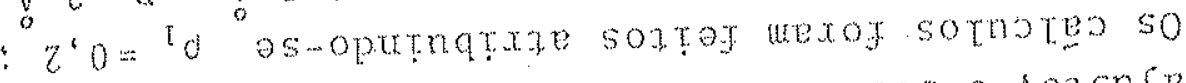

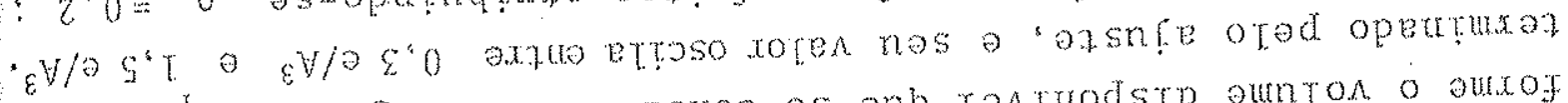

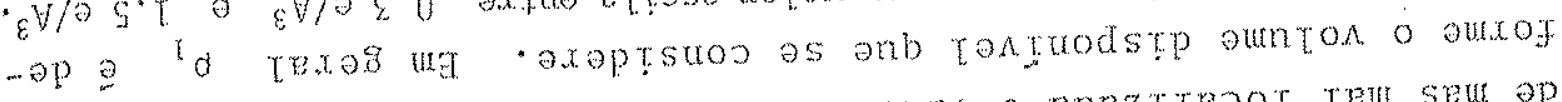

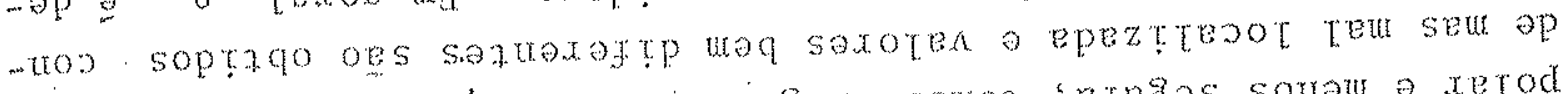

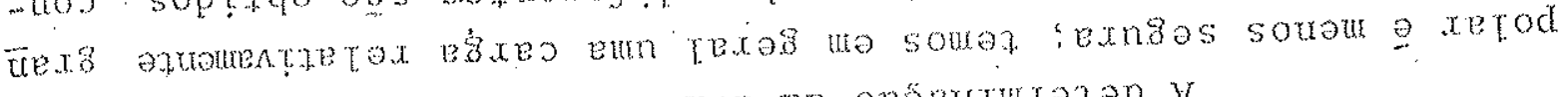

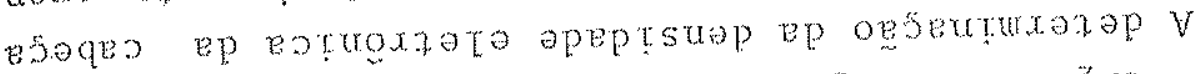

- enge a wos "Ho odnus op ajsexz

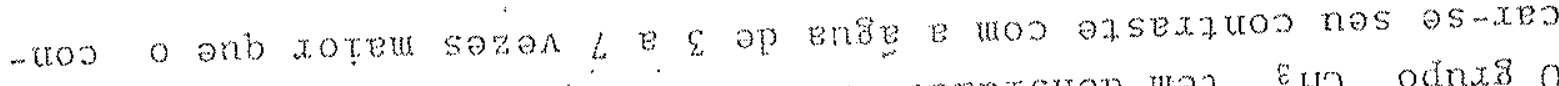

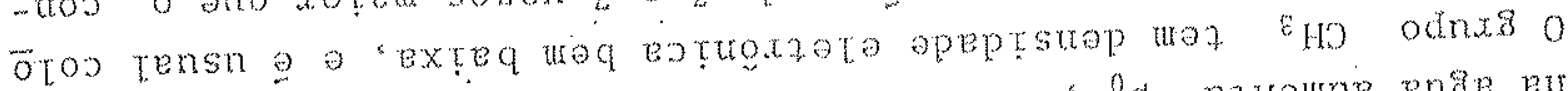

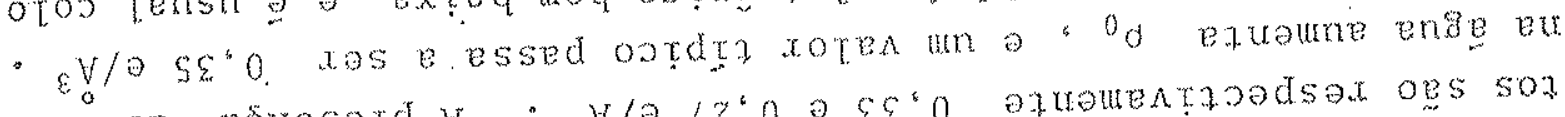

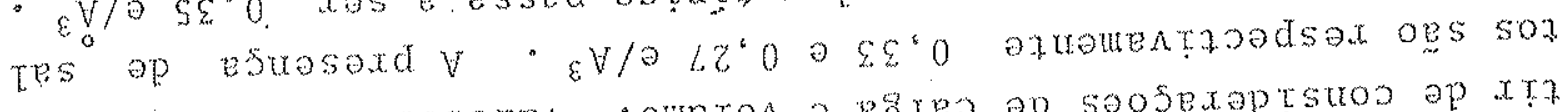

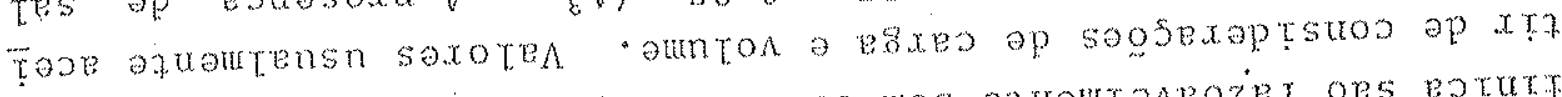

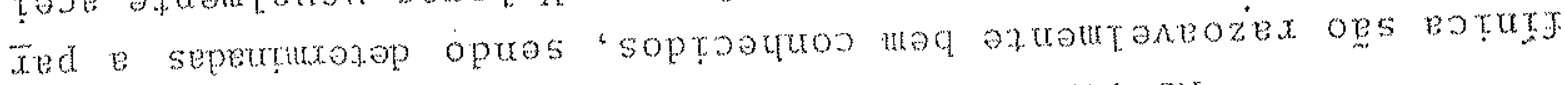

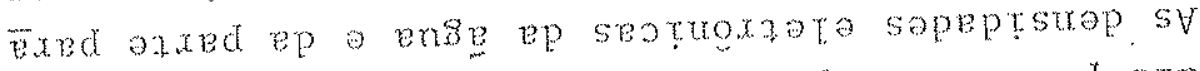

- $x$ oxaluexed op ospoxd steux assneg un xaz - qo opmexnoxd ' $(x)$ o ap sarbuezed so ext steox saxoten a nexi

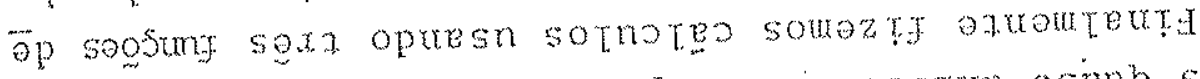

- ense eu uresadsp as soxptue asmb sepeurepta ap sax

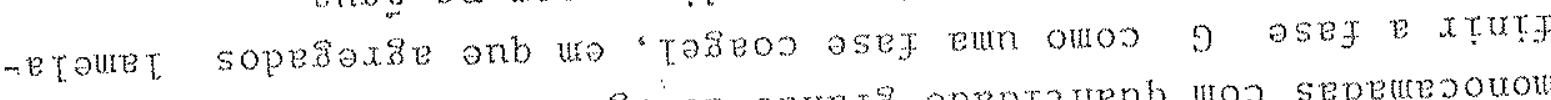
-

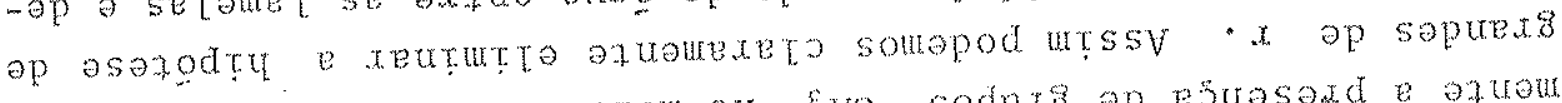

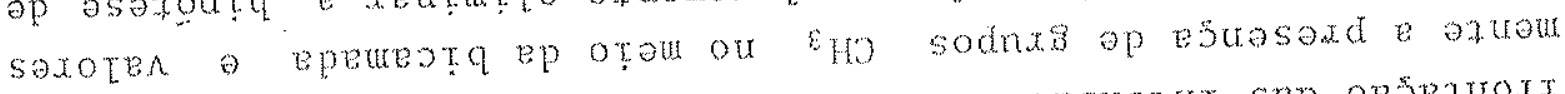

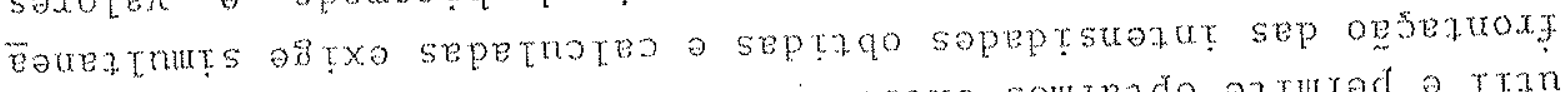

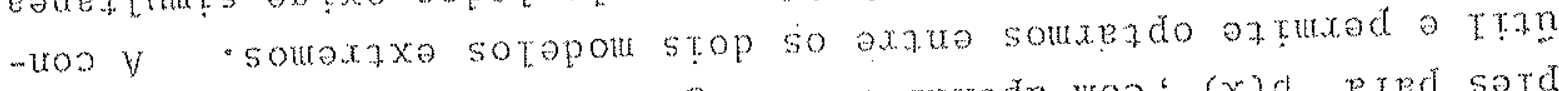

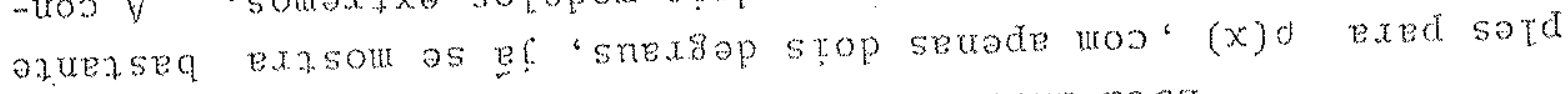
urs ontrut otopou un op xtaxd e eares astreue ejsg

sexetouet sooxetzox sep soost sod seu sopeptsuant sex Hexatyax as $x$ ap seosexate owoo xeotytxan somapod $x$ ap

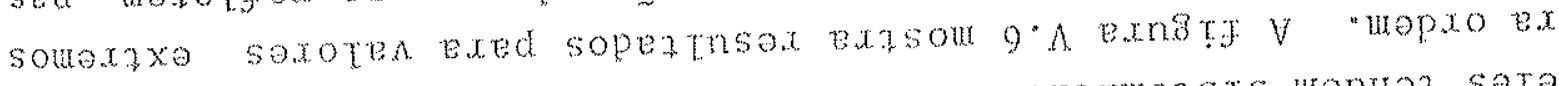

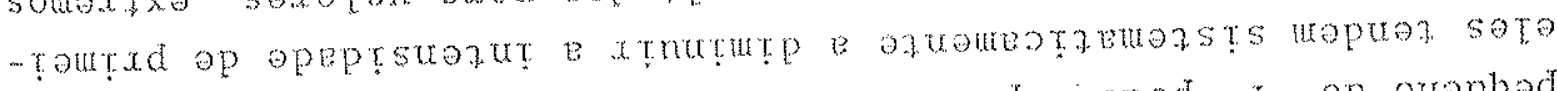

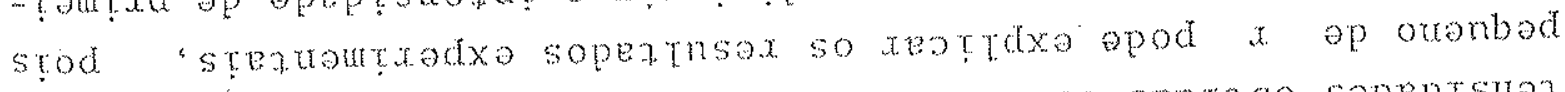

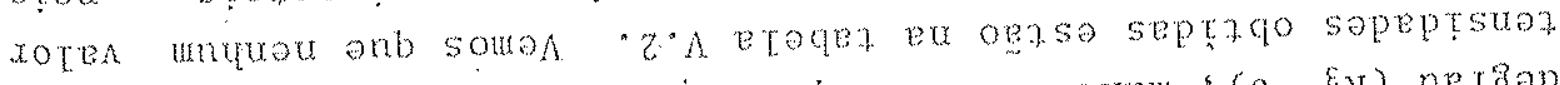

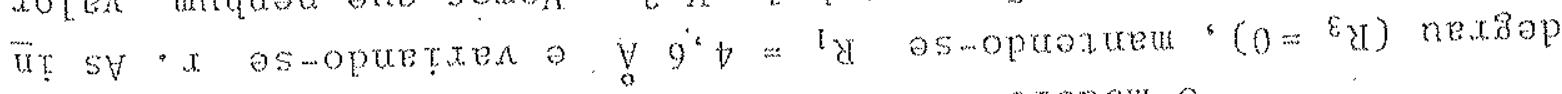
ostun un xod opetrutes por sepenesouou ep oropou 0

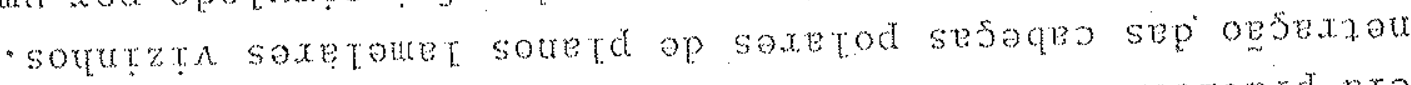

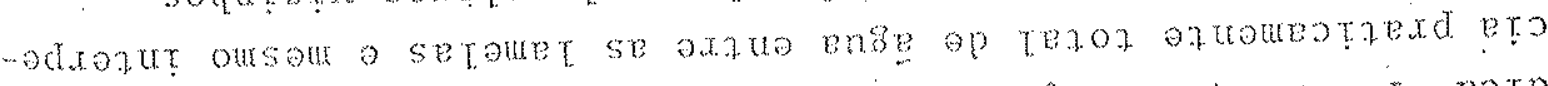

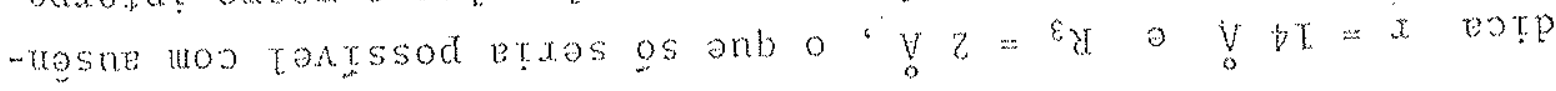




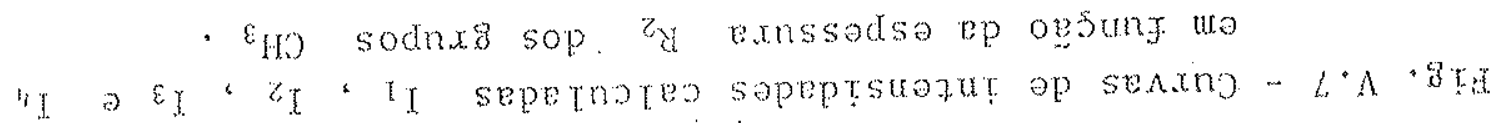

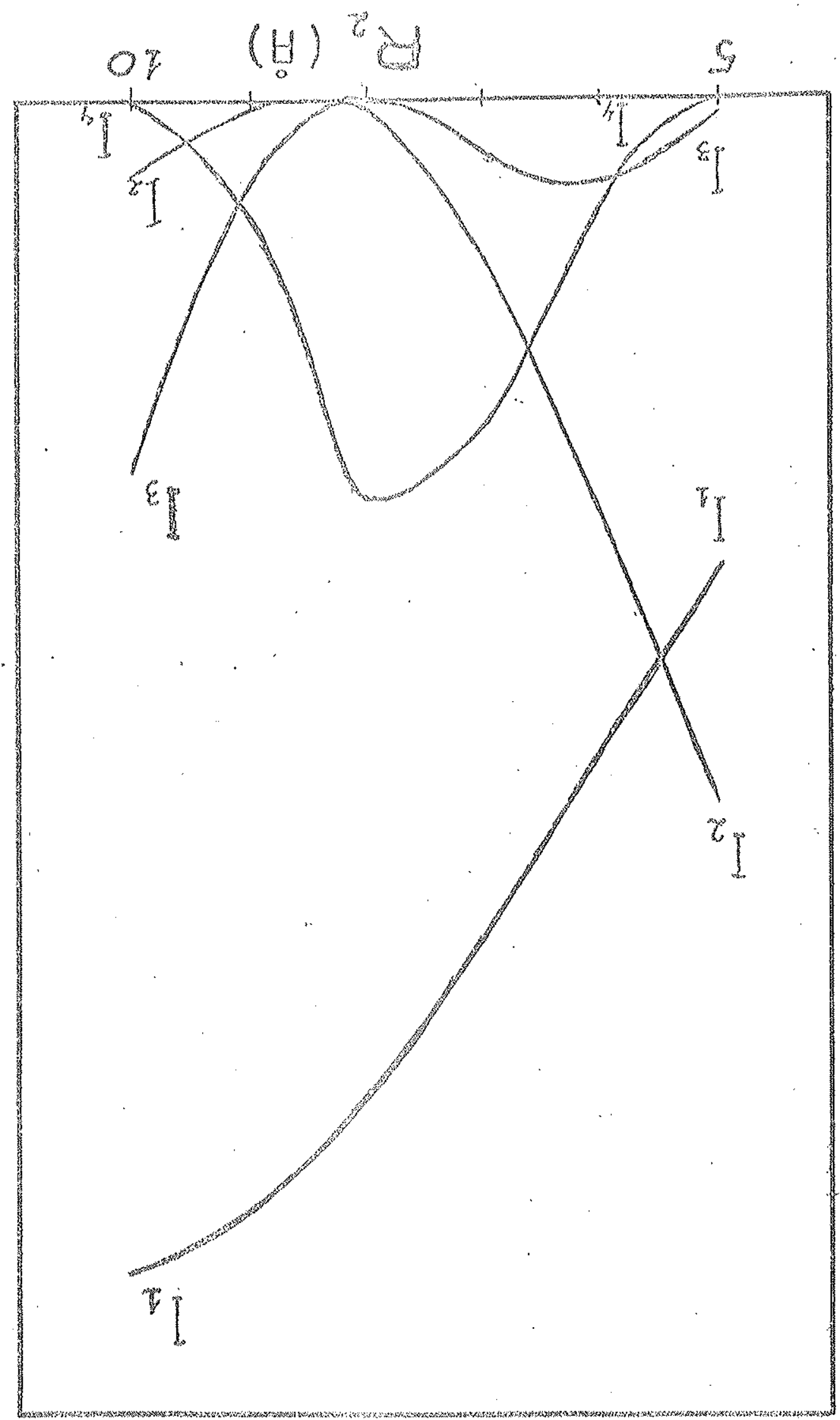

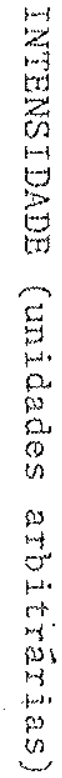




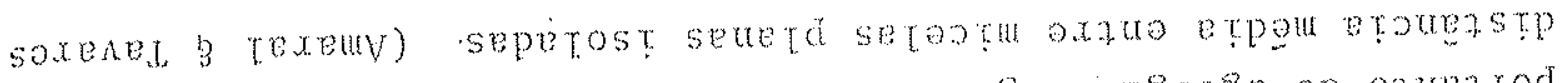

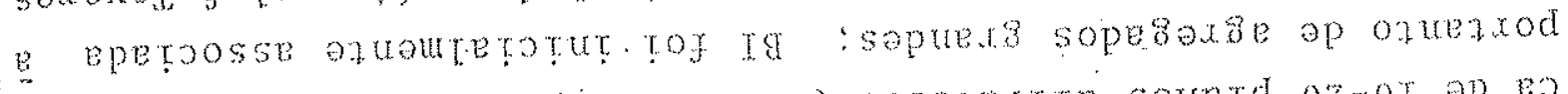

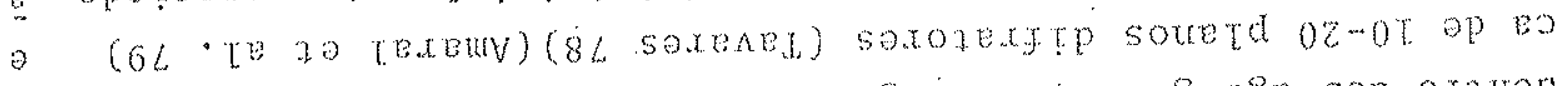

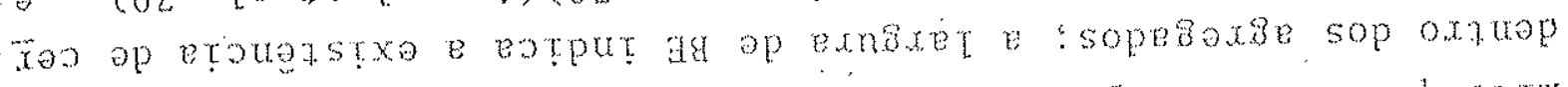

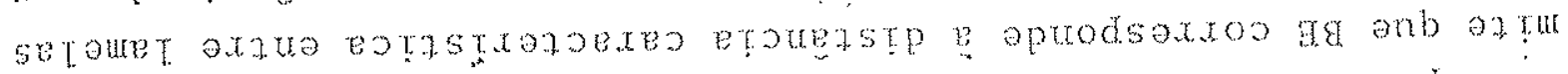

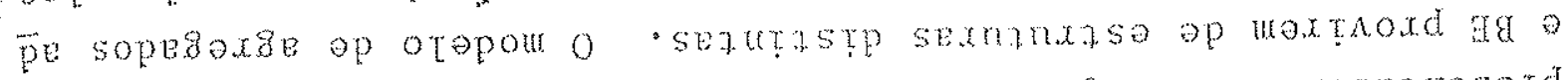

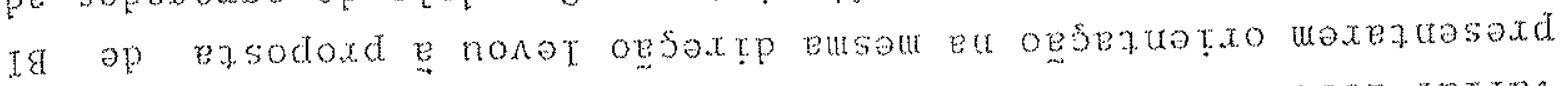

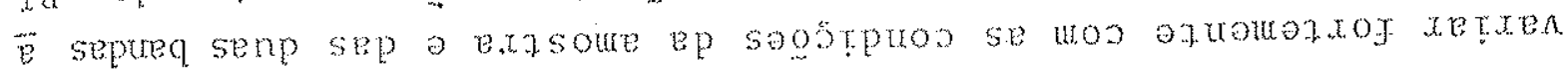

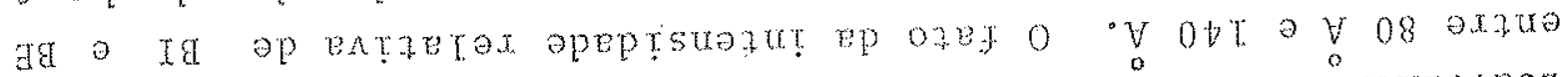

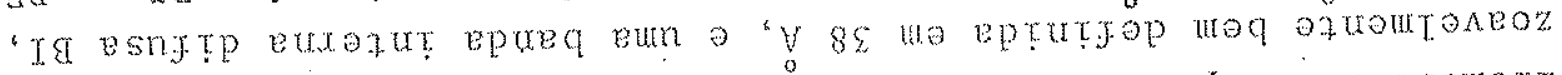

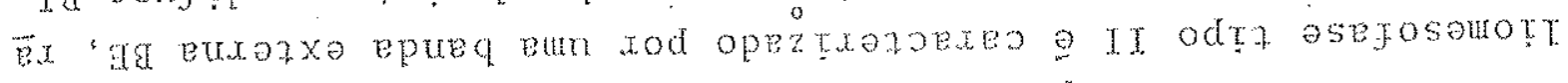

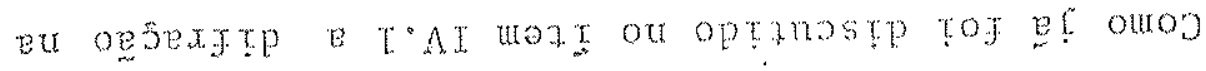

TI odth อsejosourot " 2 "T"A

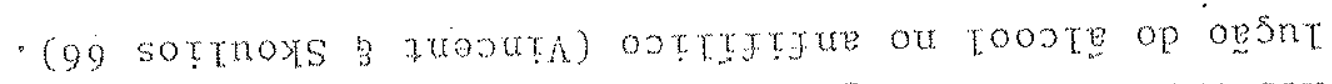
osstp e xanomoxd exed $(y$ b) ogseanatos ap enge op ppemes eum sou

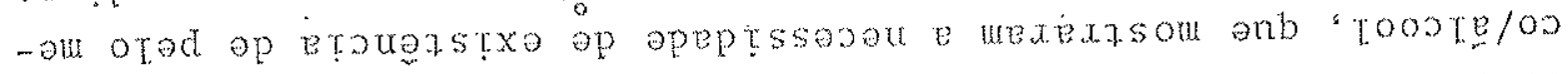

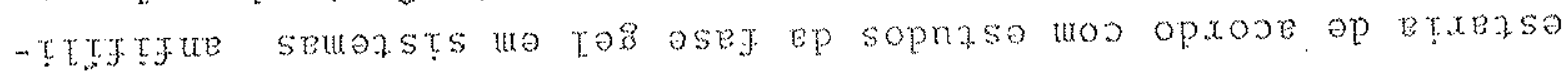

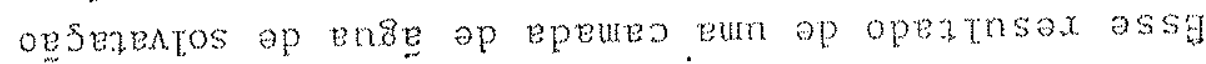

- oovar ornzue un ap telixou e opsetax ue sepruteut a sep

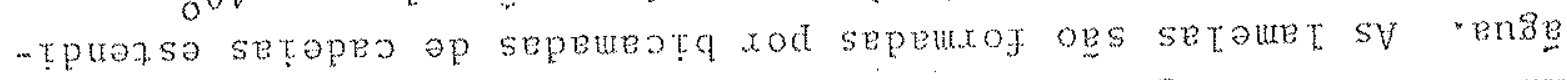

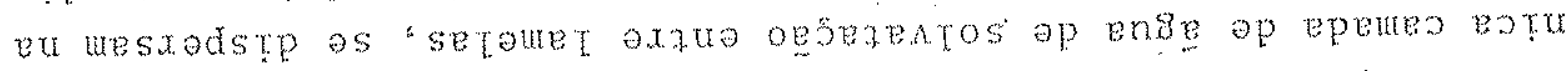

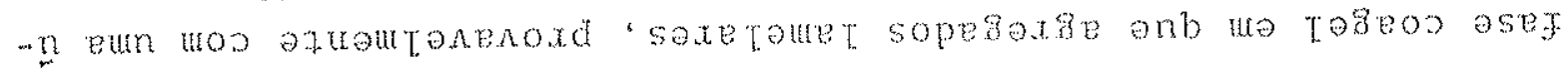

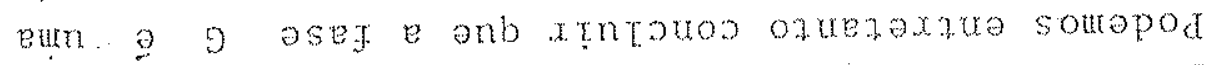

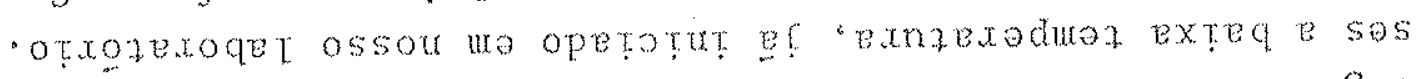

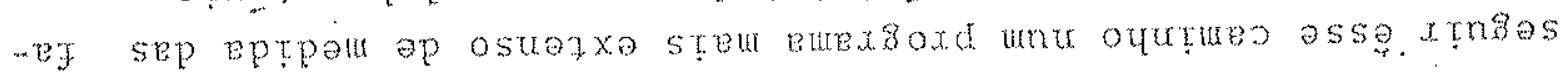

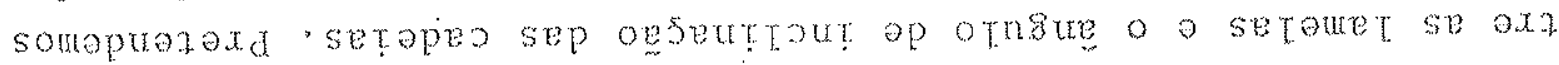

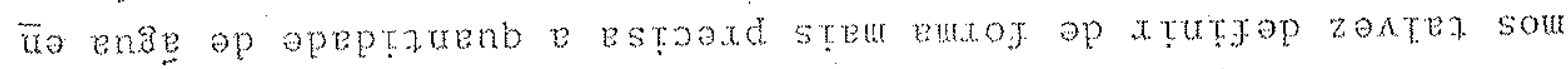

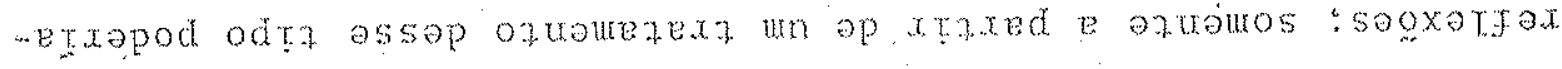

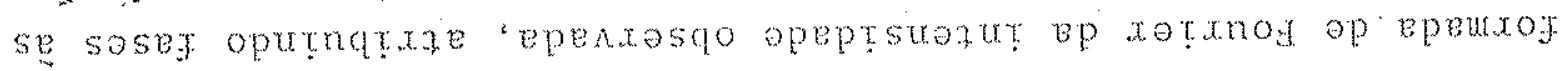

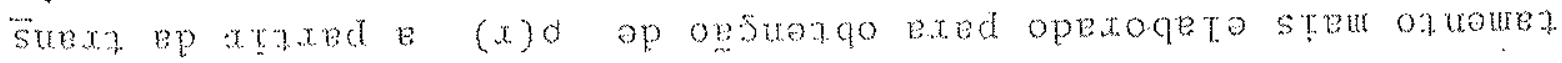

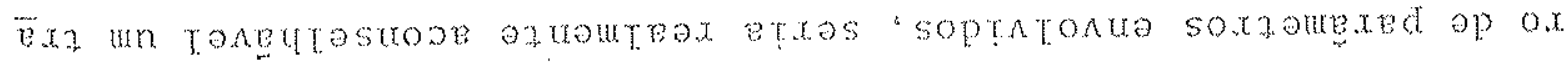

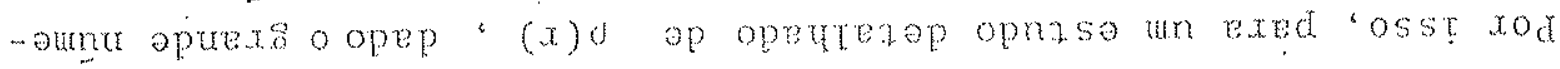

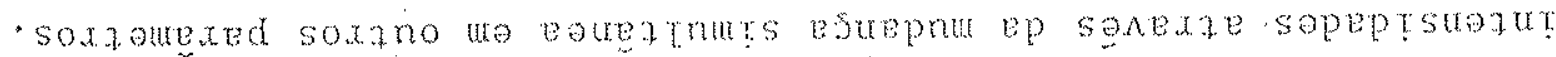

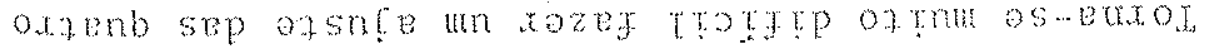

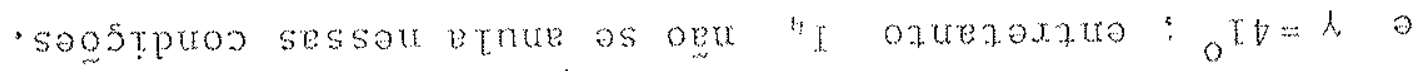

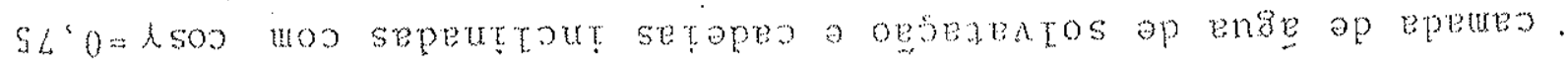

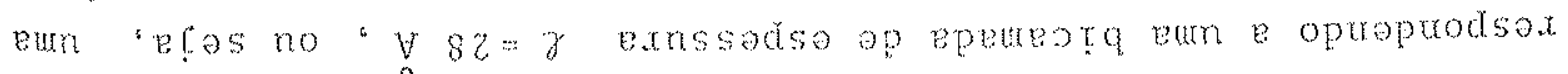

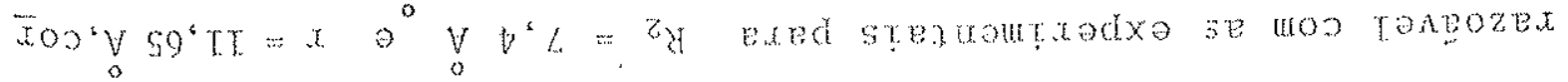




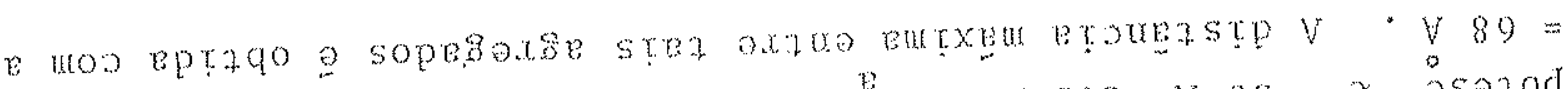

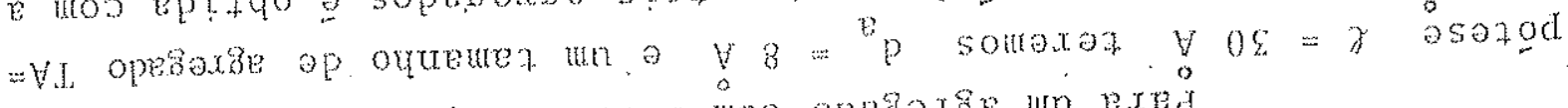

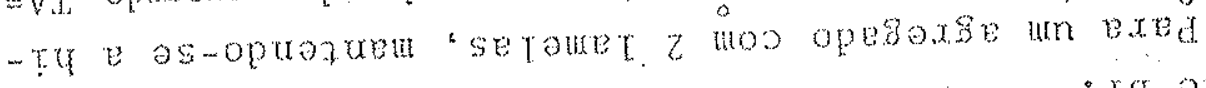

- re ep xotuater artu

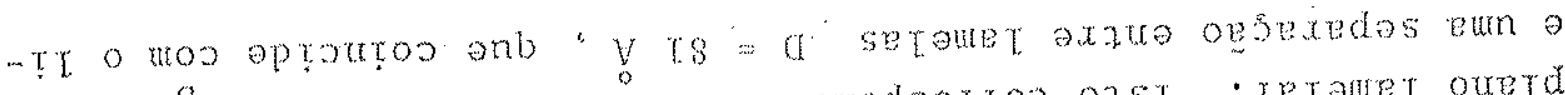

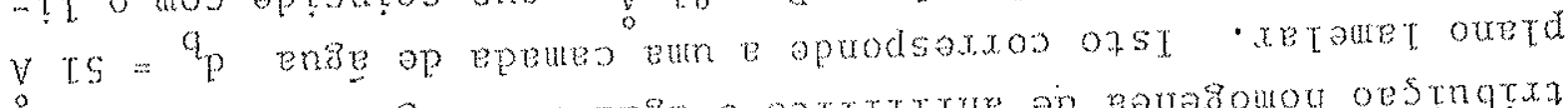

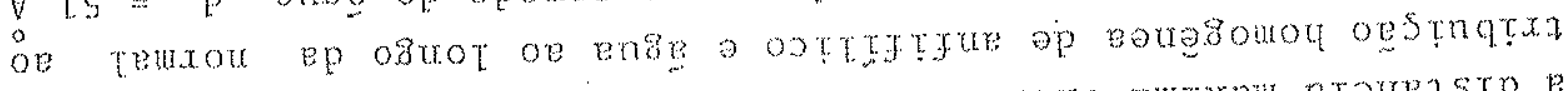

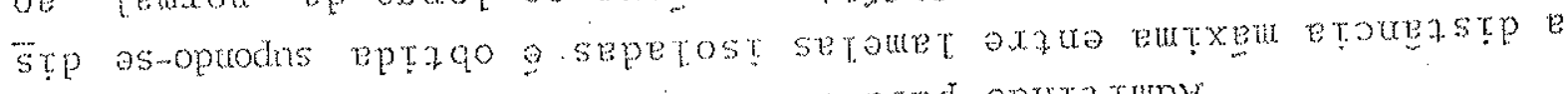

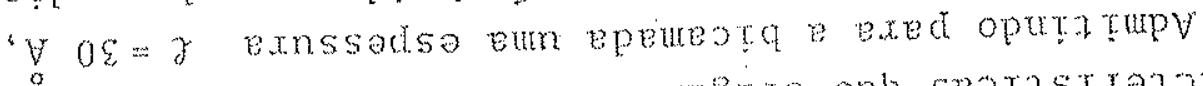

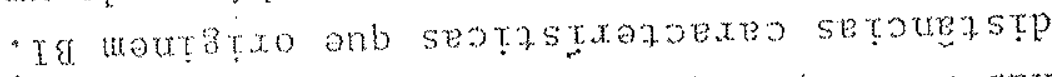

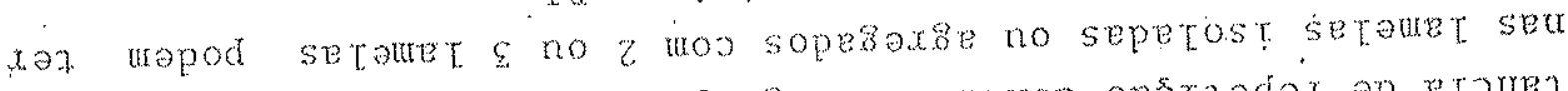

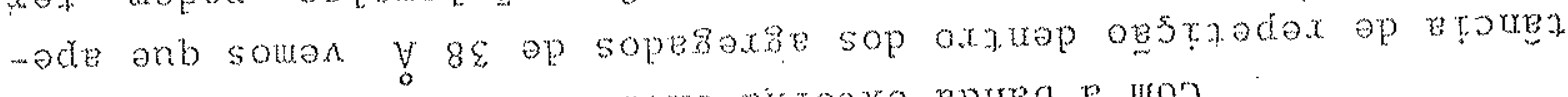

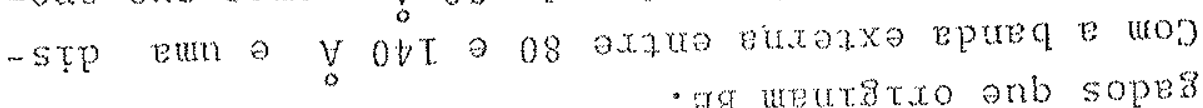

- aq wertstro onb sopeserise sousou soe

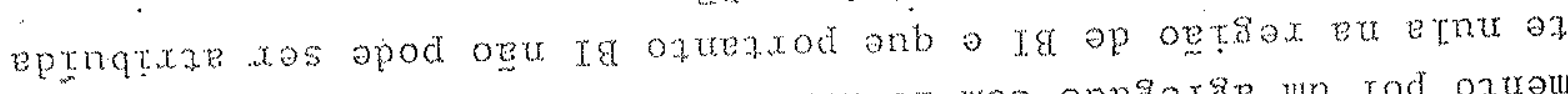

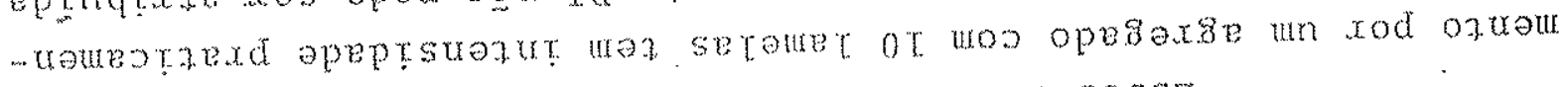
Eutedsa o onb woquea uexasou sou sopeatrsax soss

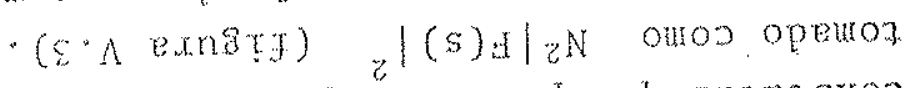

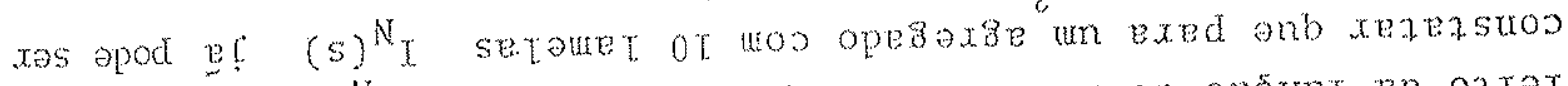

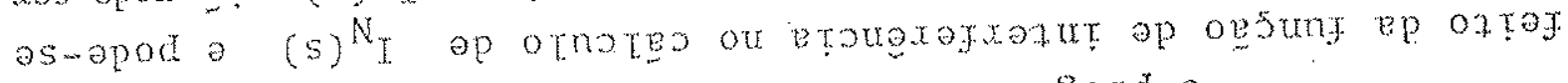
o o xessa exed azuaterotut opesn tog rutexsoxd 0 $\cdot\left(0=0^{0}\right.$ o) snexsop sext op opsury eun ouros essexdxe $\rho(x)$ d etoure epes wa ense op ${ }^{2} \mathrm{p}$ exrssadsa eum xod sepexedos seromet $N$ xod onsoduos o opesox

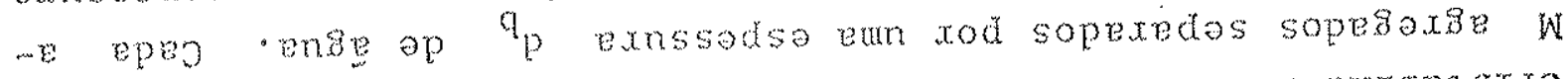

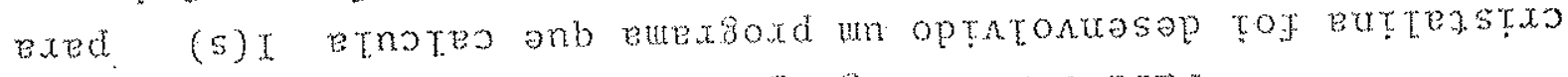

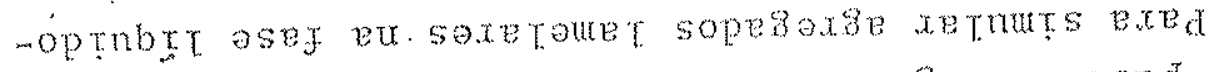

- serauter se ardue ense pe azxd op oesnpoxat a

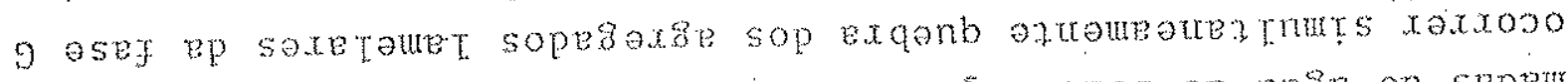

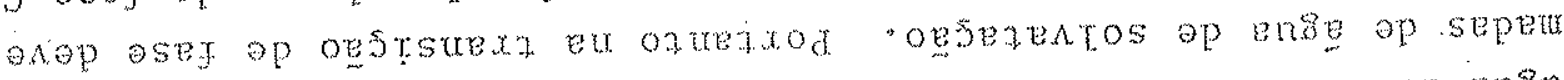

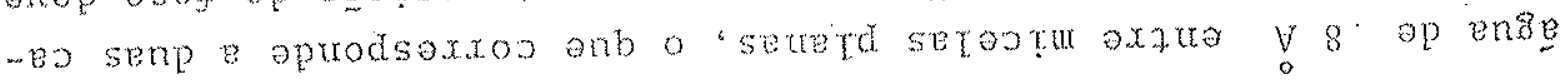

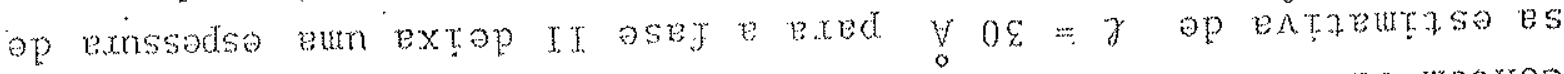

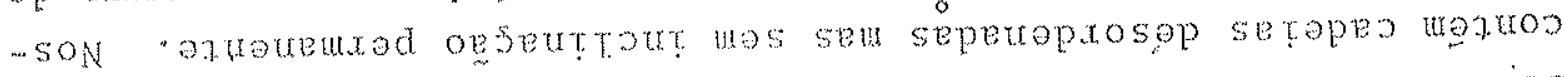

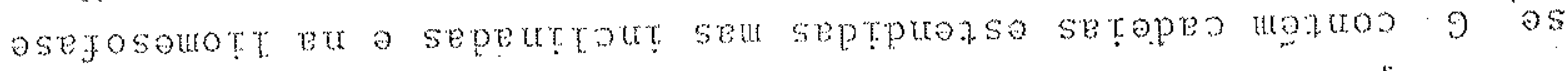

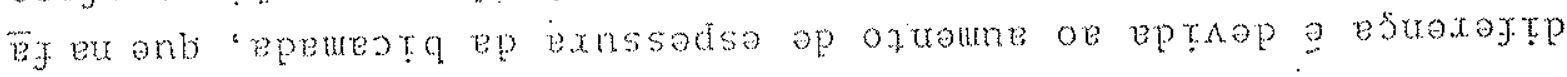

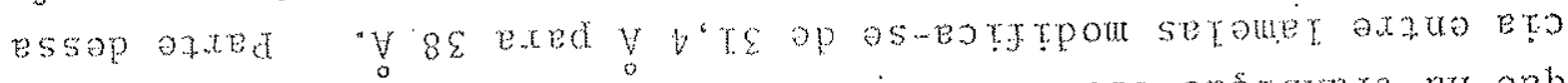

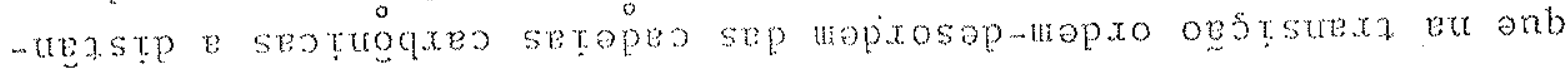
someotrtar a ased eu soptaqo sopetrasex so mon

- sopegoxse op otopon asso menx T.

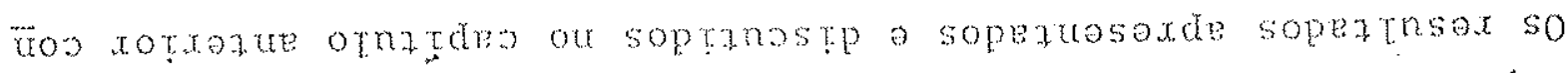

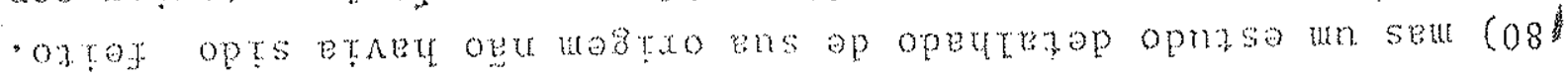




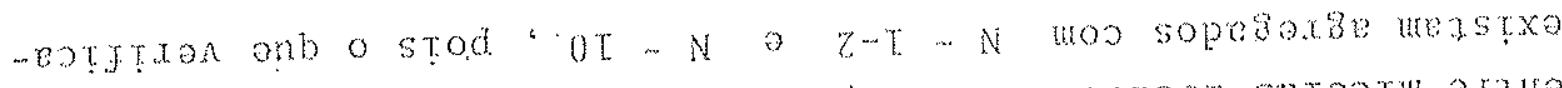

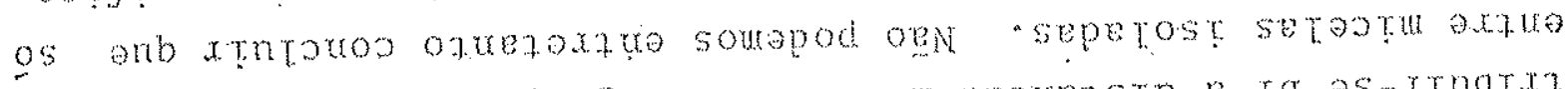

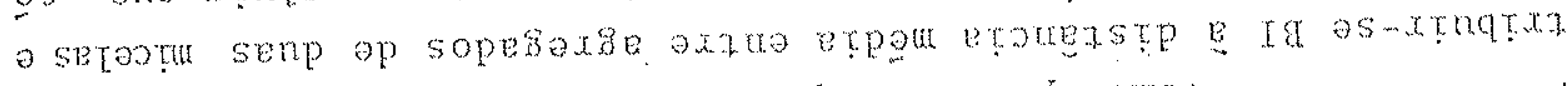

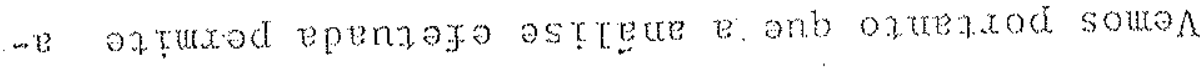

- epere

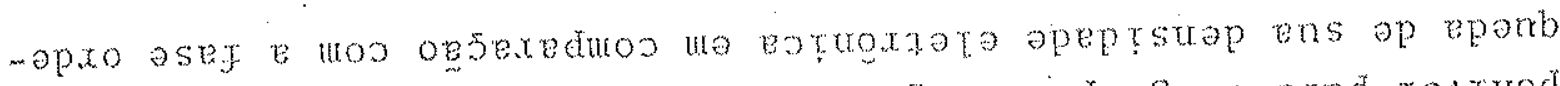

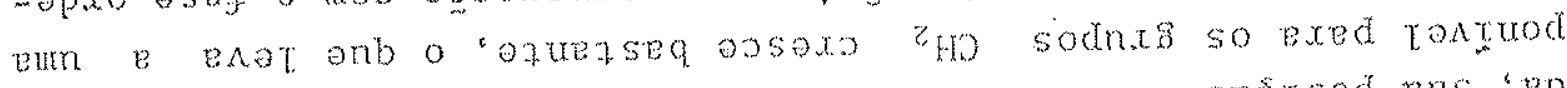

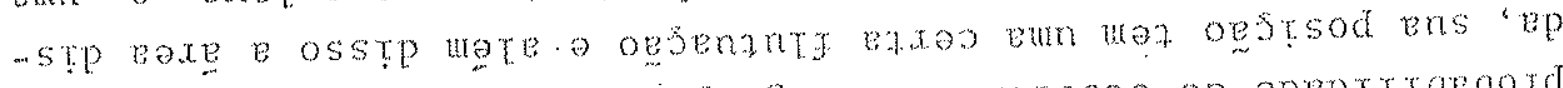

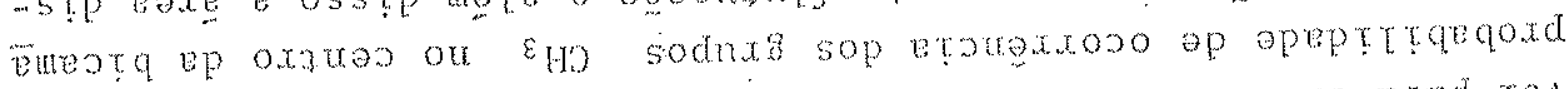
toteu rum soluxadso op xesode "stod seperepxosap setopes pxed ron

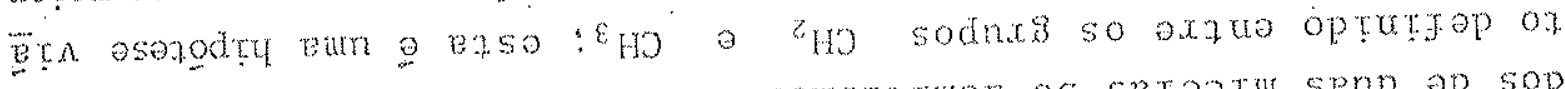

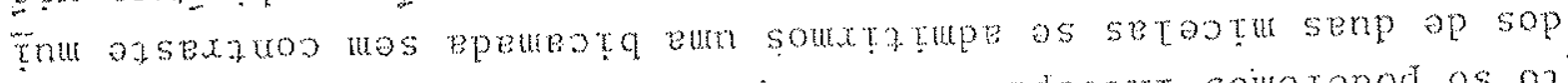

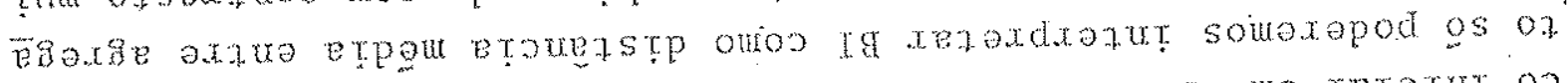

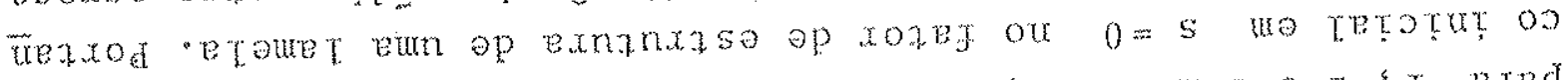

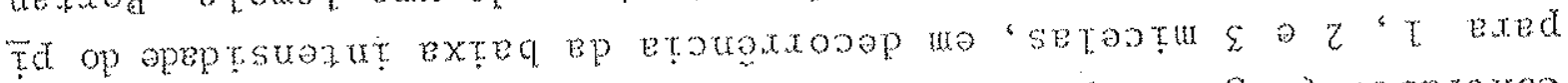

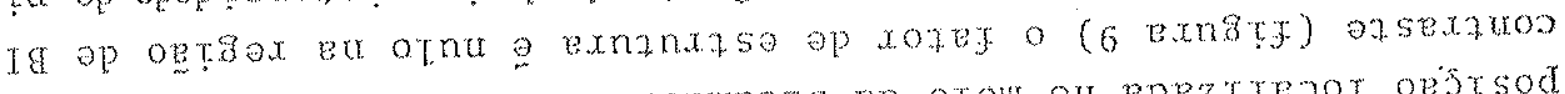

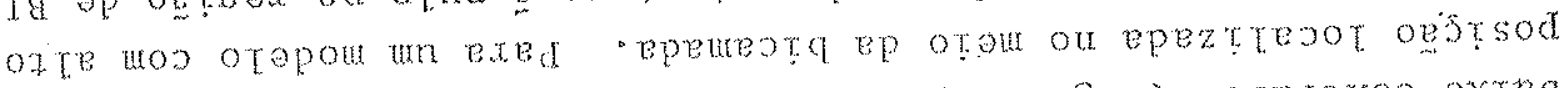

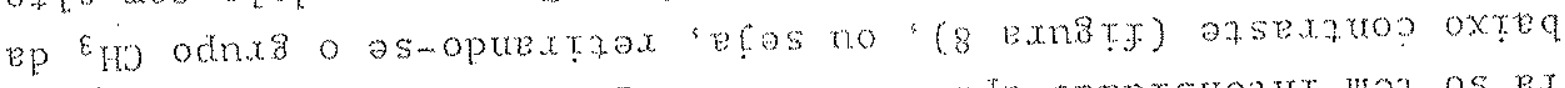

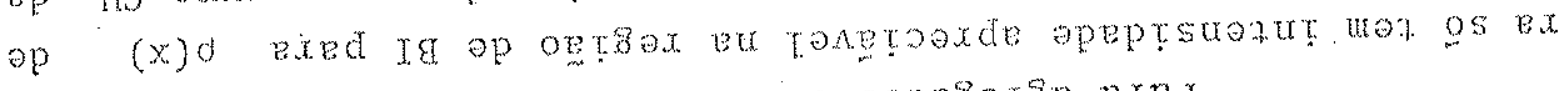
nanxss op dores o setootu senp op sopesaxie exed

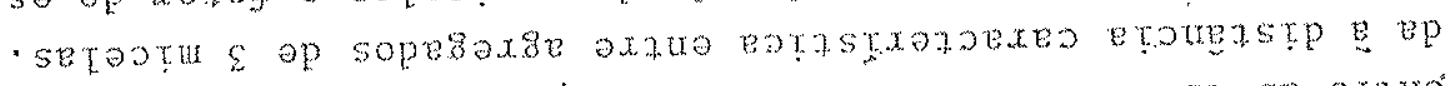

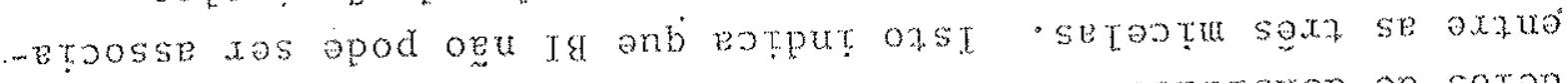

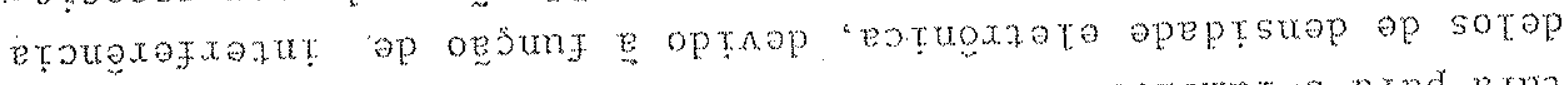

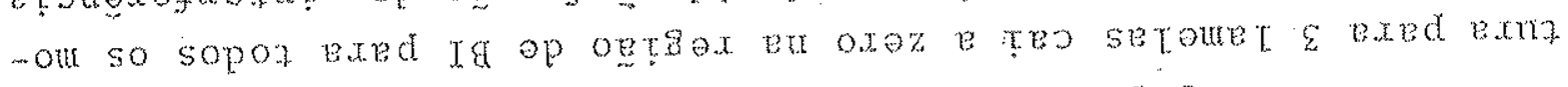

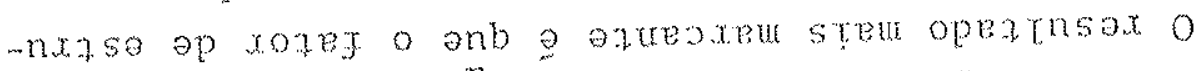

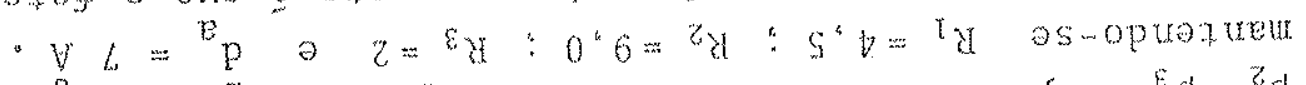

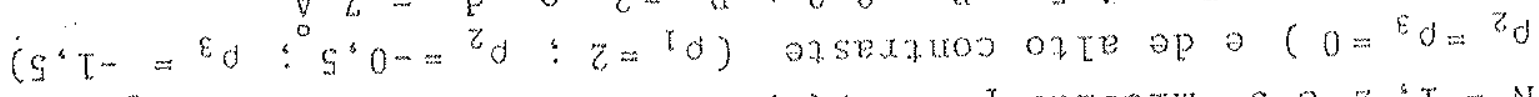

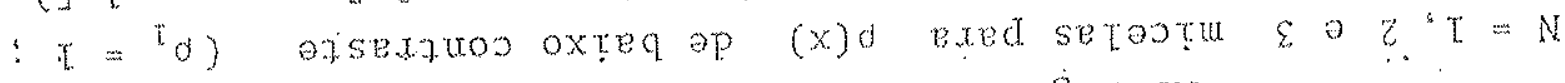

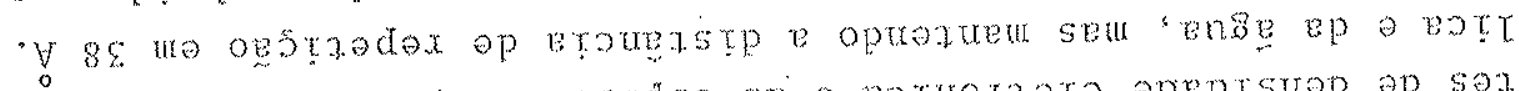

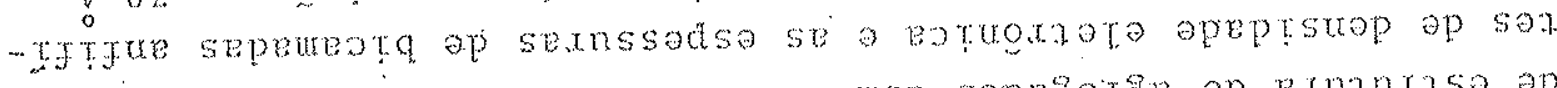

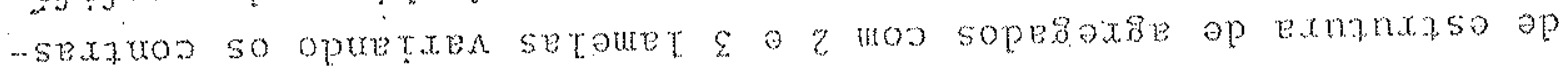

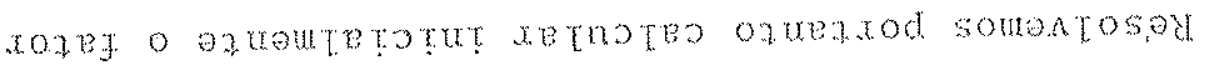

- setaut sep outed od gos ap opsadno ap eare

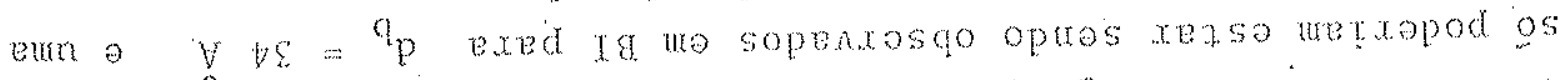

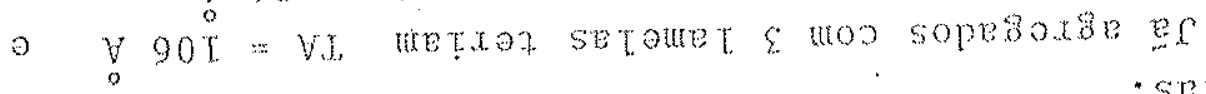

strouter sep omete

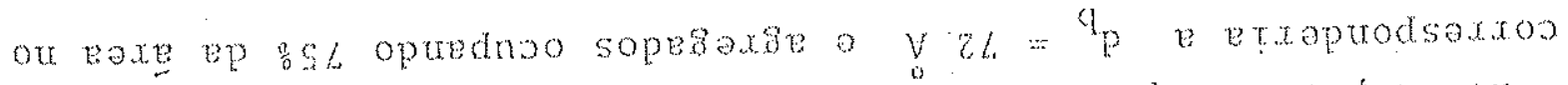

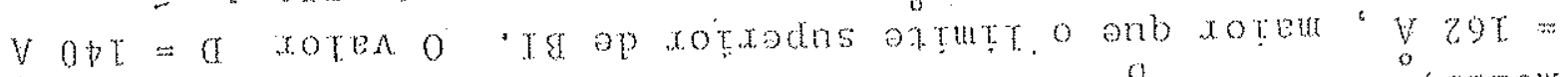

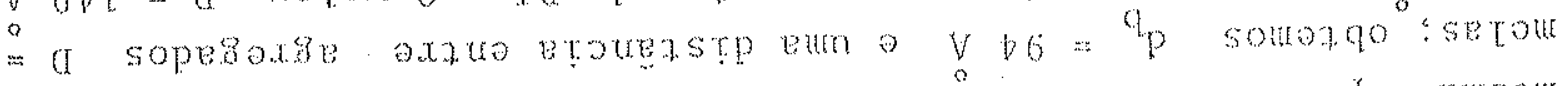

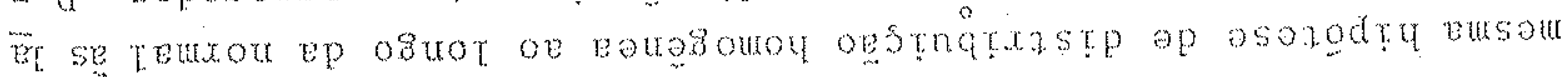




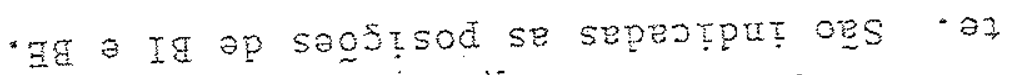

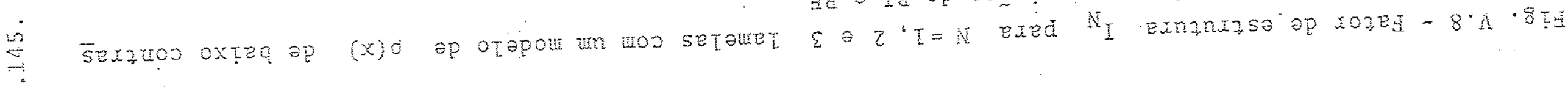

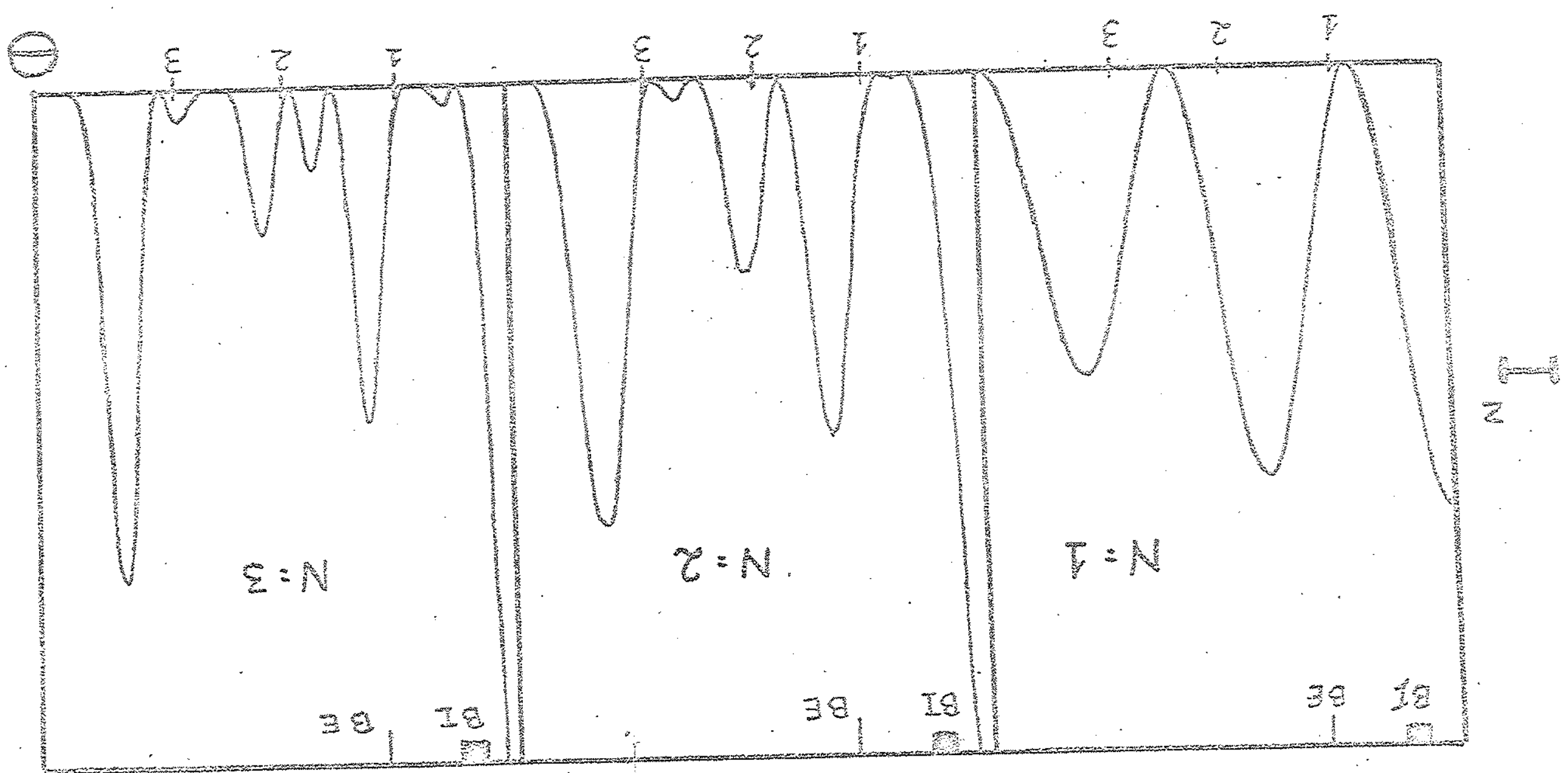


- gg $\theta$ Ig op saojtsod se sepertput oes

-27

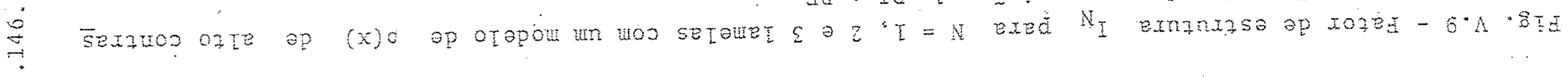

$\ominus$

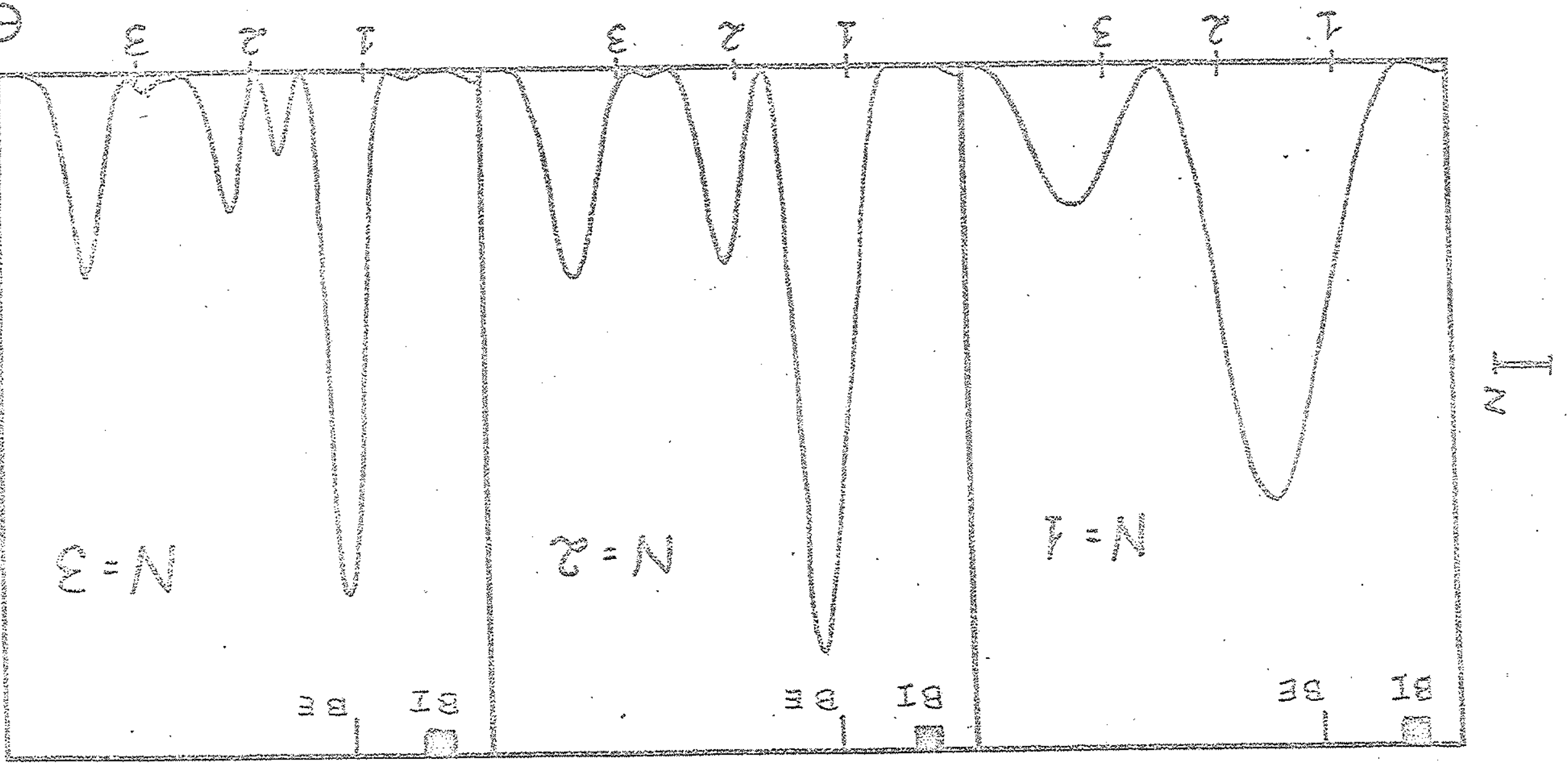


ense op sepenes senp secade wos seronberd oz-or ap soprexs sopes

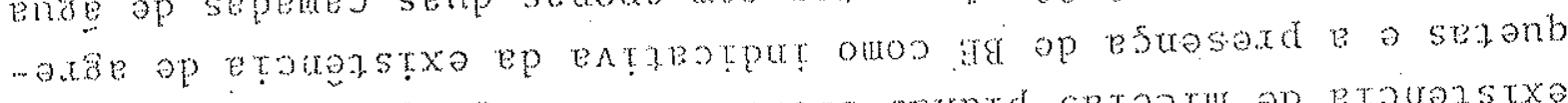

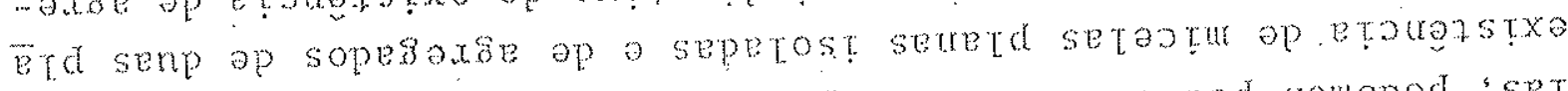

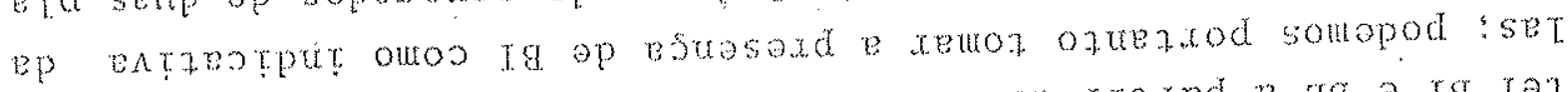

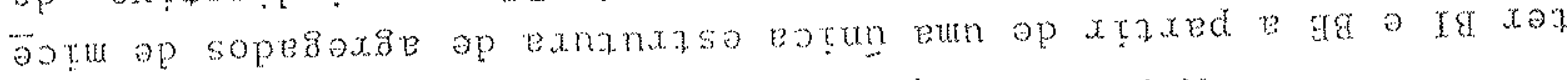
qo sompod ogu anb noxisom epenzoge trobe osteter $\mathrm{V}$

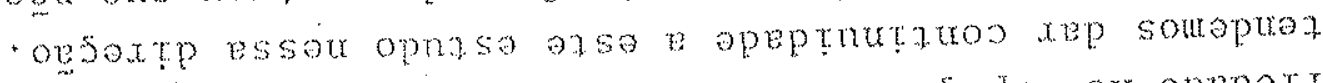

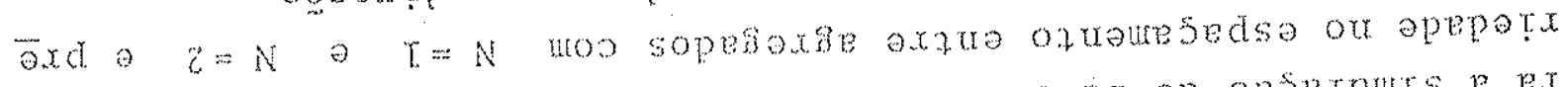

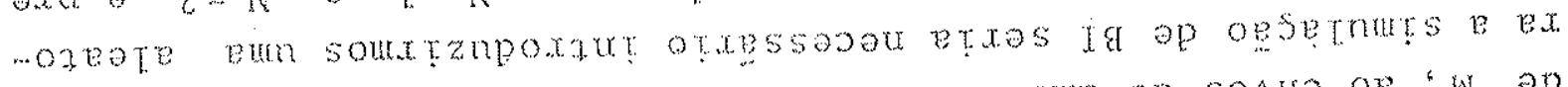

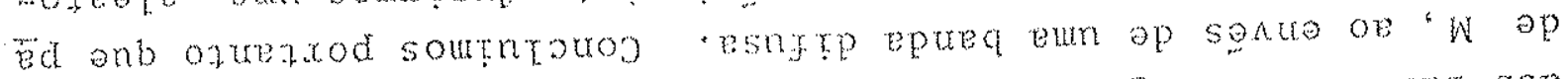

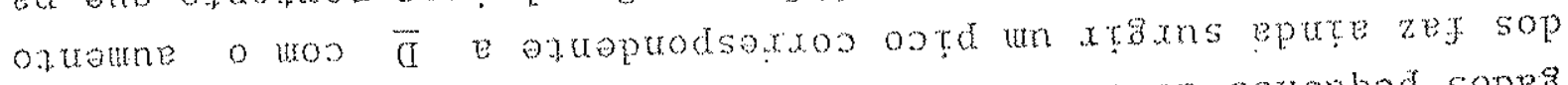

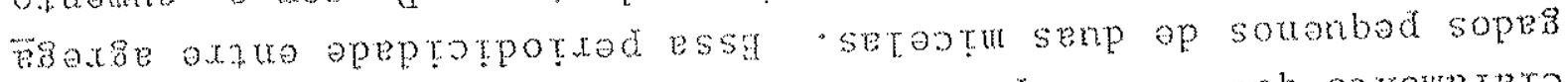

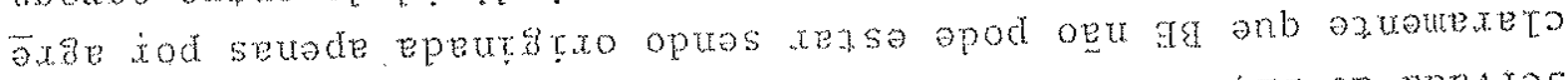

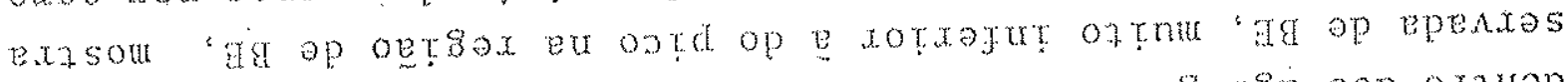

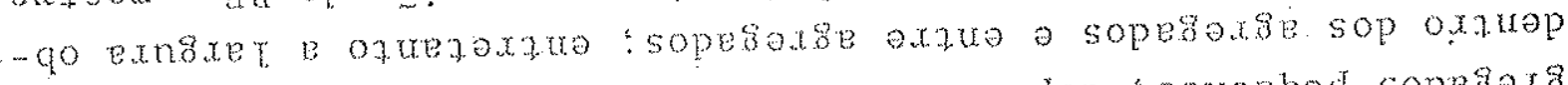
sopeptopotxad se axzue ogsetax ep opuopudap sounbad sopesexs

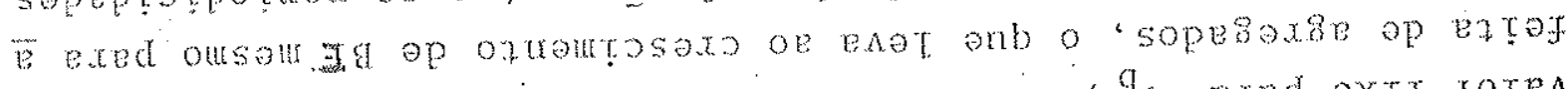

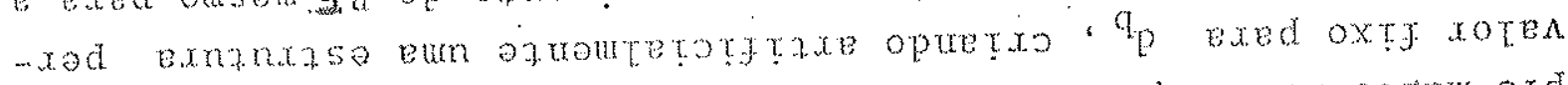

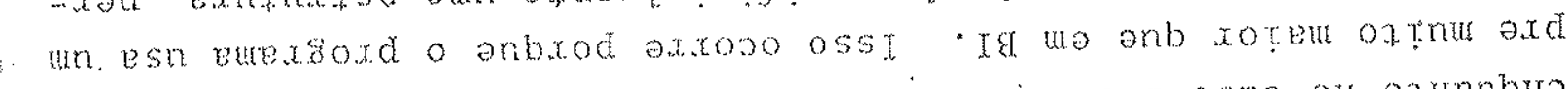

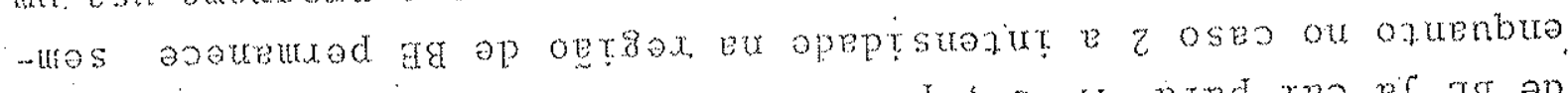
"Id ap eperost oespaxasqo opuraturad"

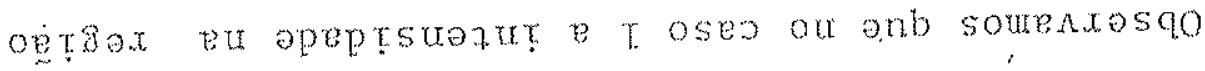

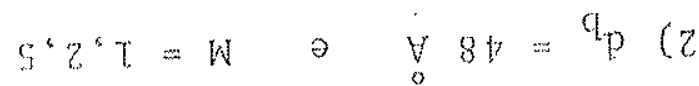

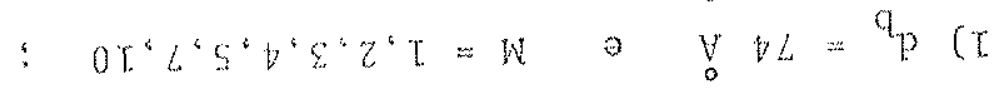

:soostproo serp ure $(y 99=V L$ osor $) \quad \forall .0 T={ }^{\circ} p$

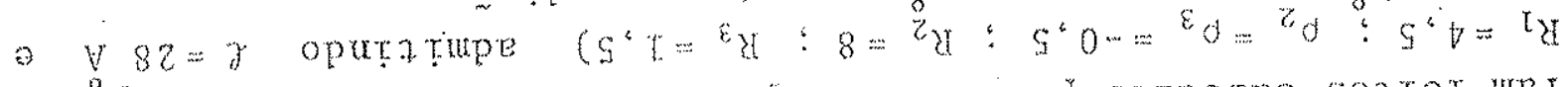

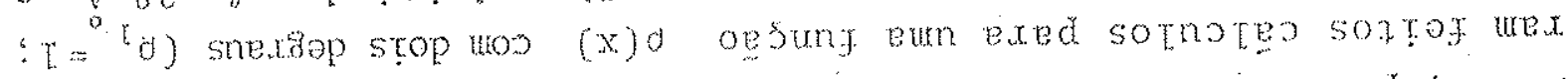

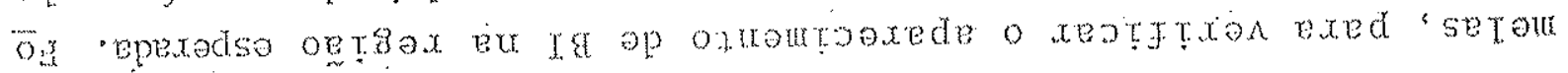

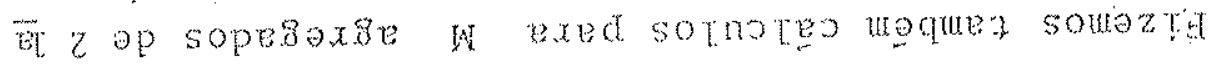

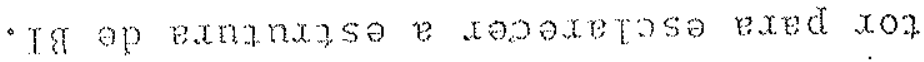

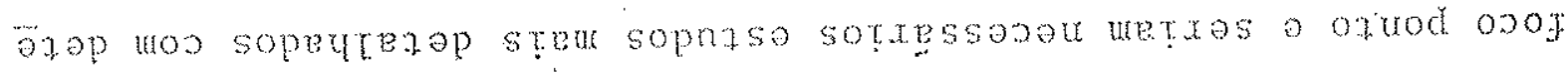

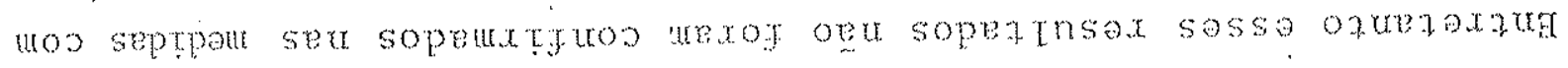

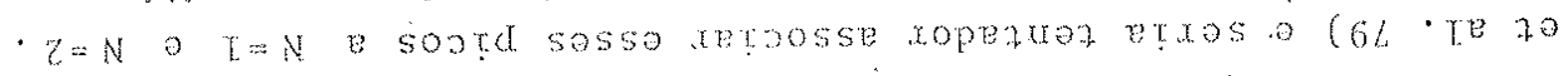

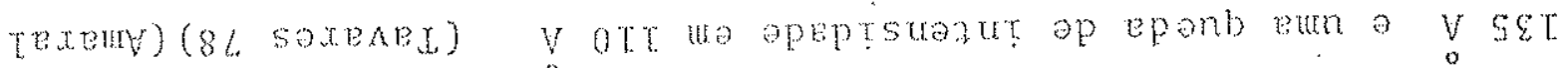

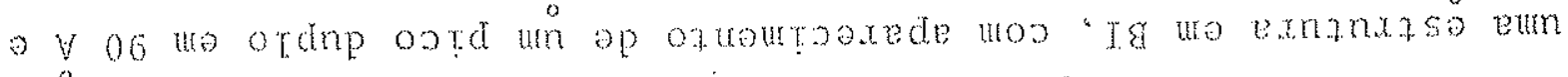

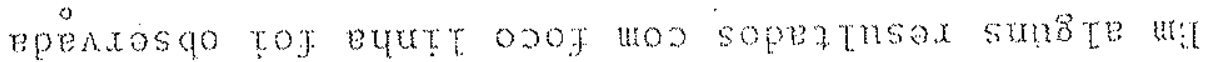

- te op ogtsox eu oxaz reo exnaraso ap xomet o $z<N$ saxoten bxed anb a sou 


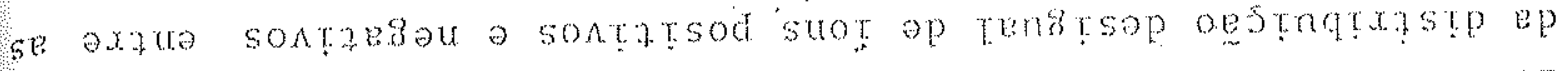

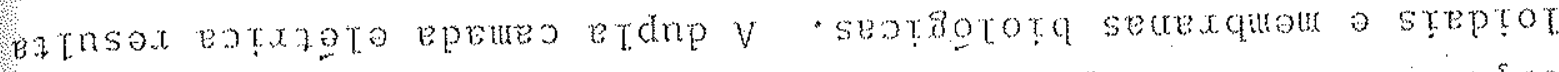

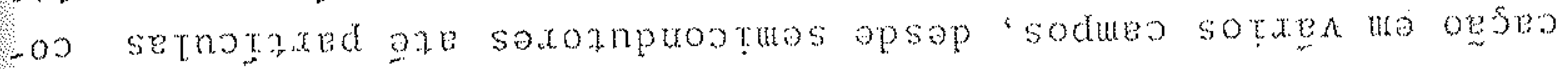

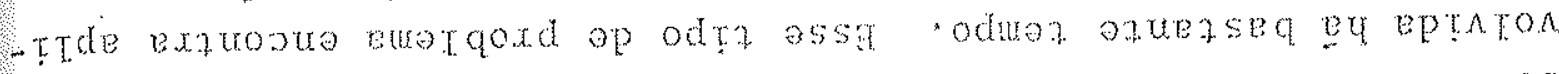

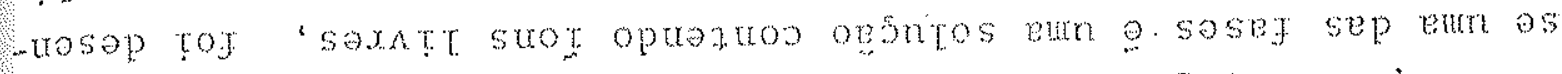

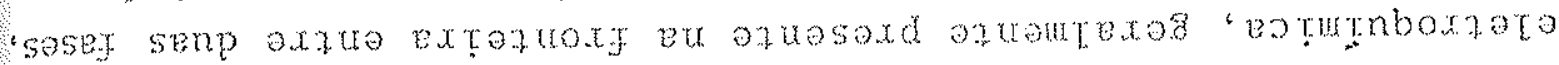

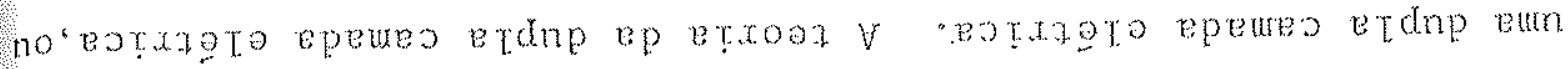
20

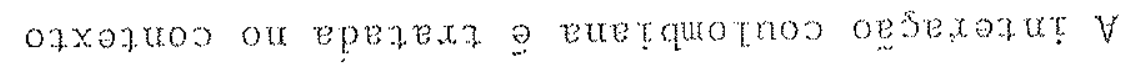

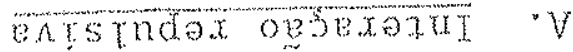

- osonbe andentos o

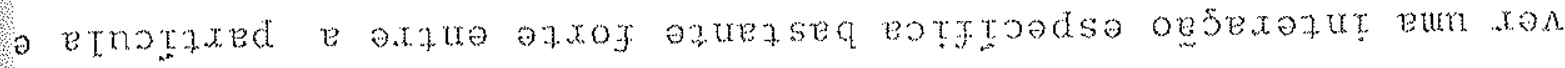

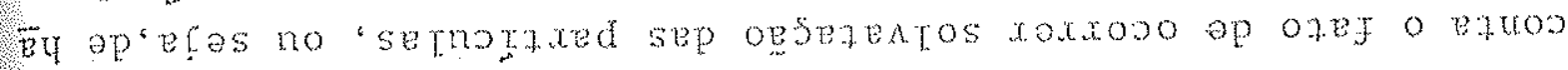

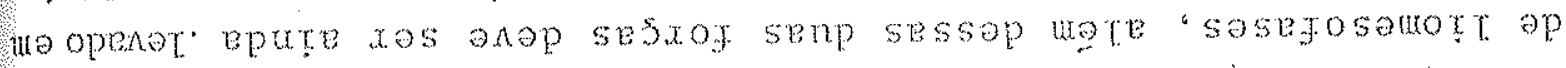
oses ox moquea a "soptritot saptotos op oses on

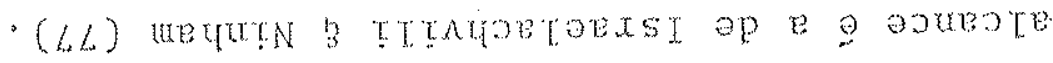

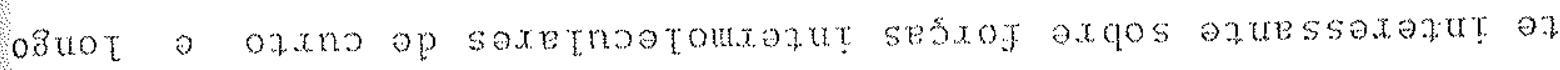

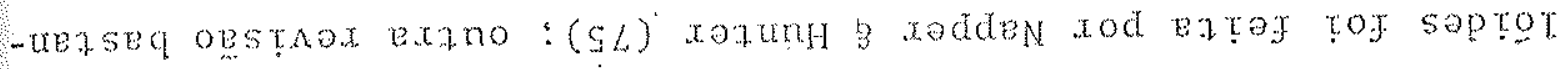

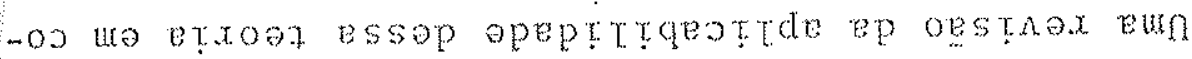

- (e-T8 oron opaxtetista)

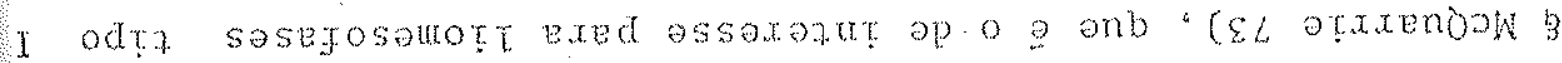

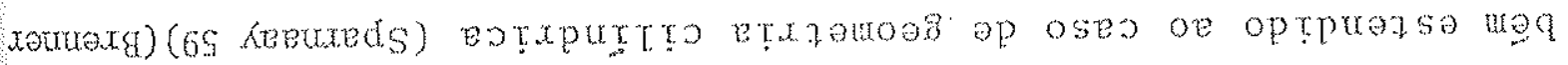

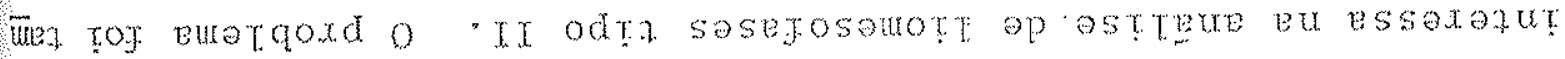

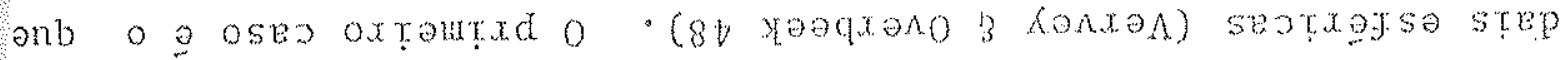

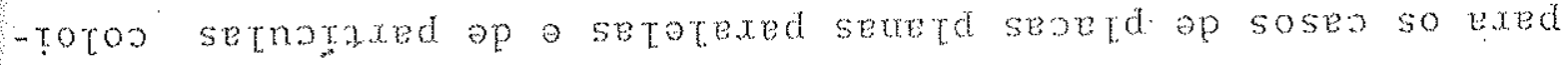

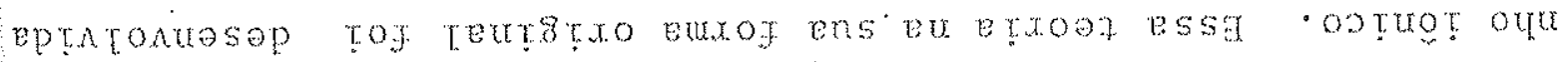

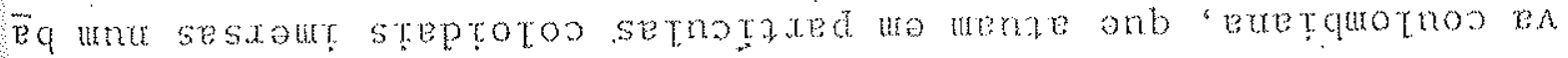

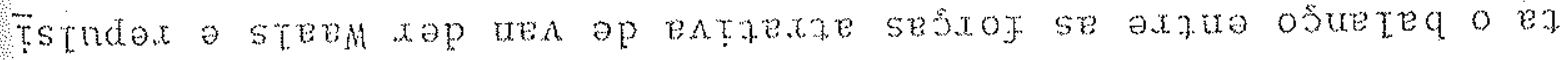

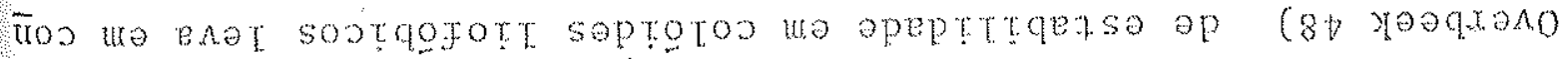

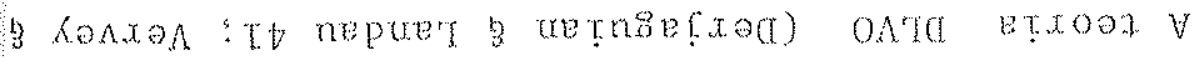

- unarastos e onb seatrytrue sernogrou sep sax -erod seseqe? se ueztroot as te stod atortadns ens wa seperax

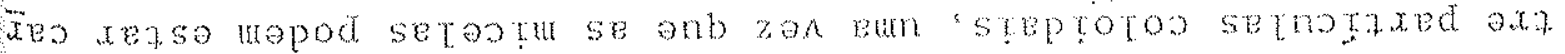

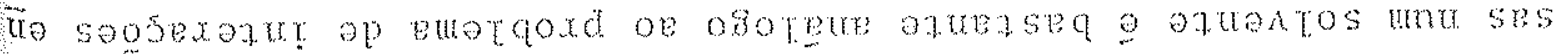

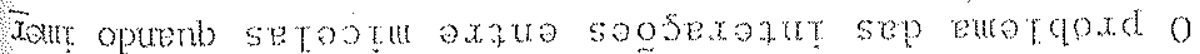

opsnporat 'T:Z'A

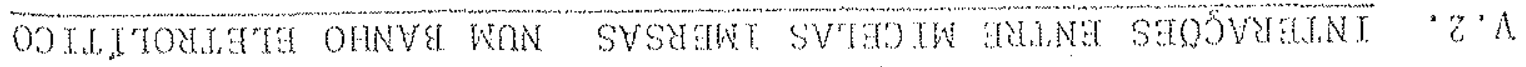




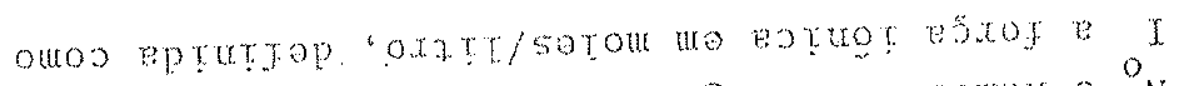

$$
\begin{aligned}
& \text { oxpesone ap oxomnu o } \mathrm{N} \\
& \text { votragre esxes op opeptra } 8
\end{aligned}
$$

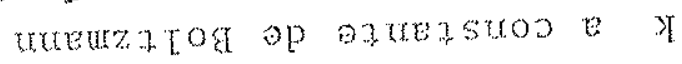

$$
\begin{aligned}
& \text { eantosqe extapexdura d d } \\
& \text { osonbe oten op extagtetp oxterstros a } 3
\end{aligned}
$$

opues

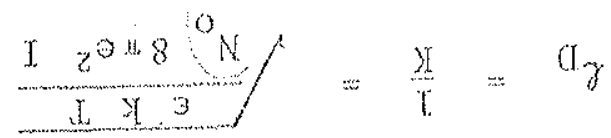

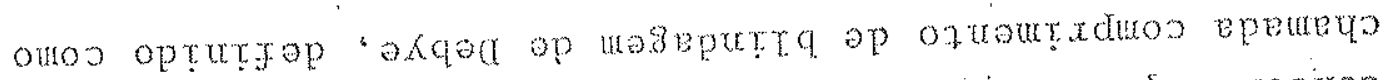

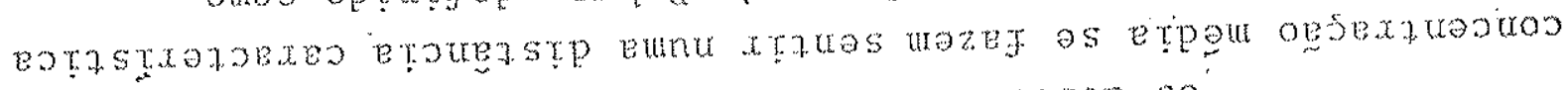

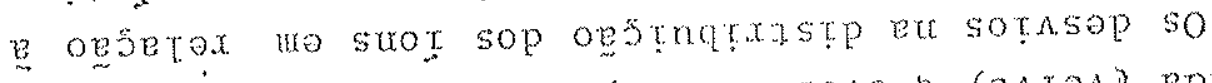

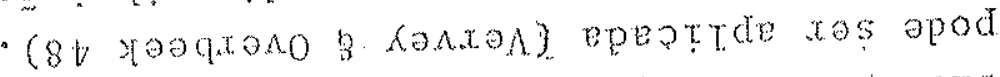

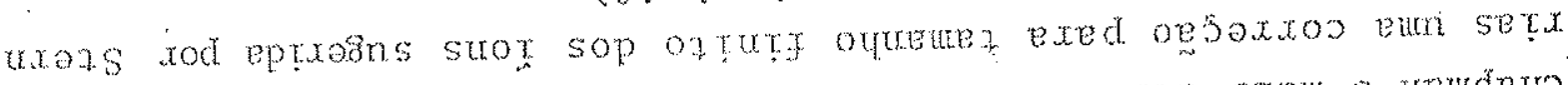

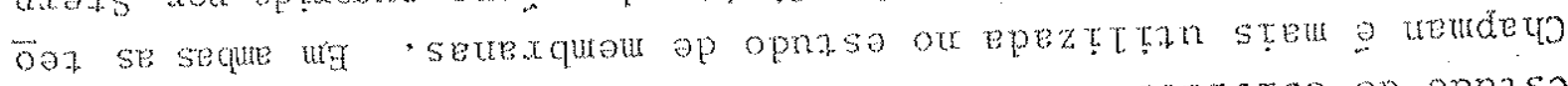

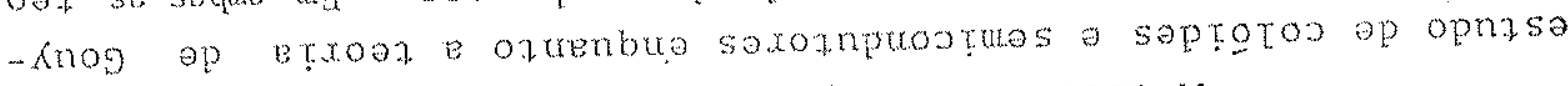

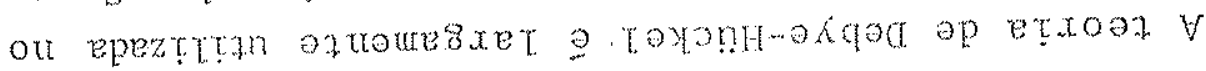

- ostseg oponow om

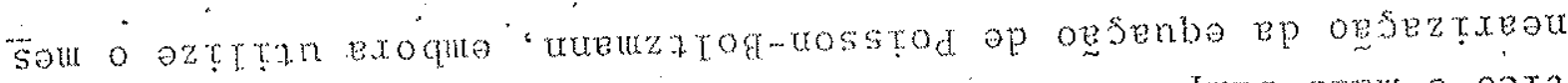

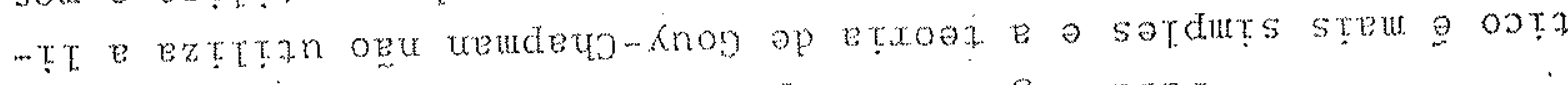

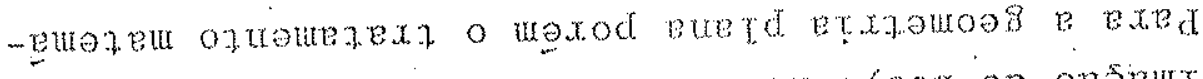

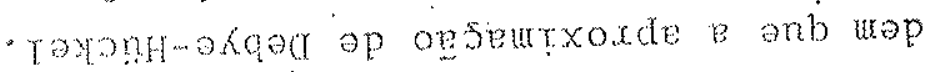

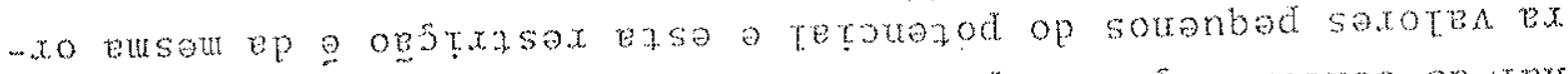

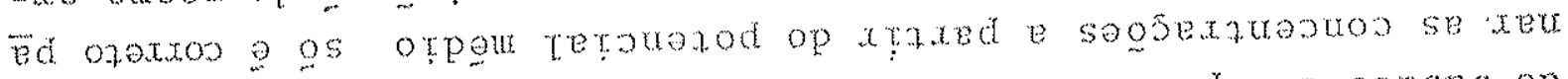

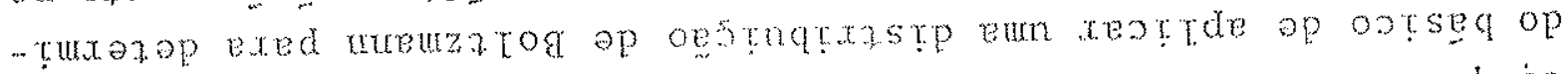

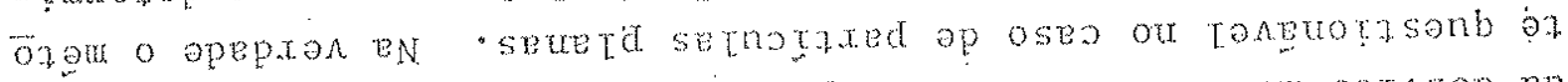

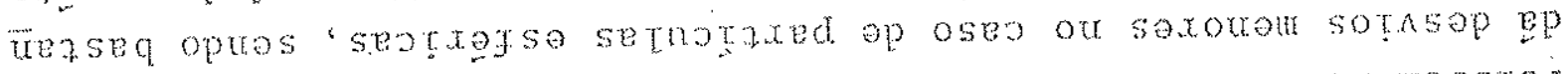

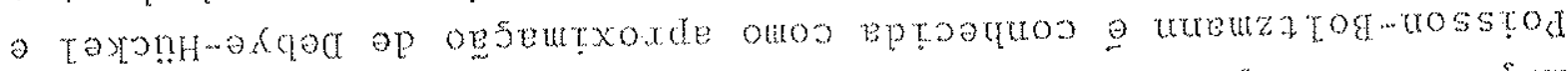

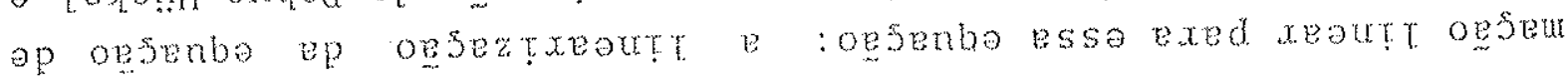

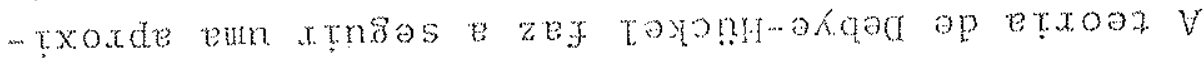

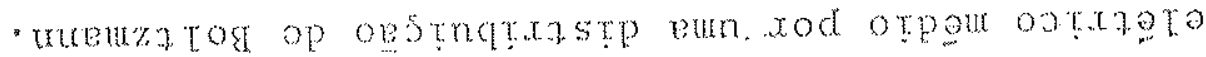

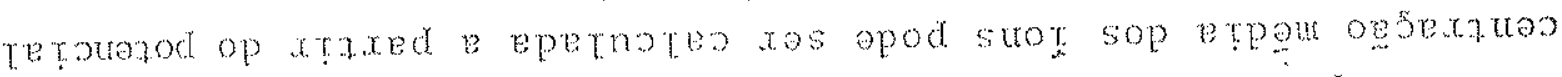
- noo a anb as-opuratupe o uosstod op opsenba ep raxed e eptaqo

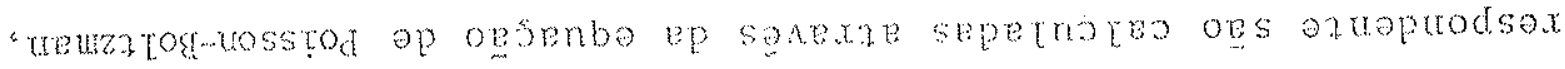

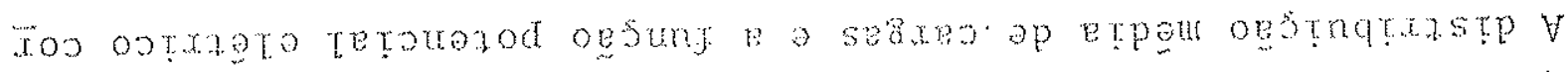

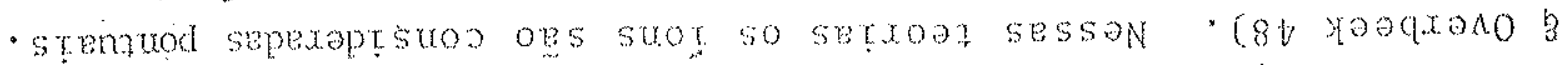

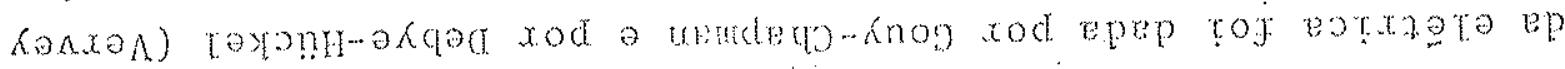

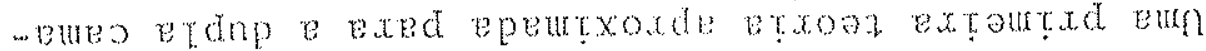




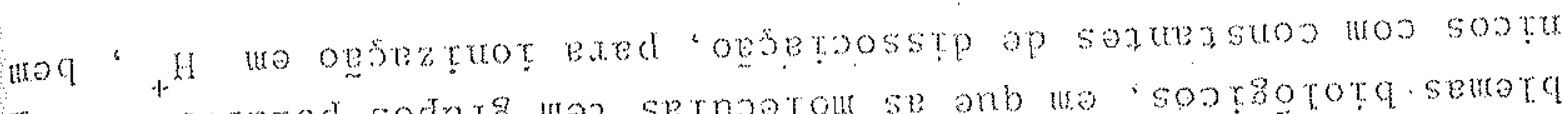

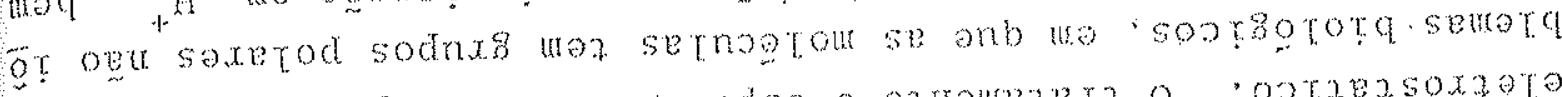

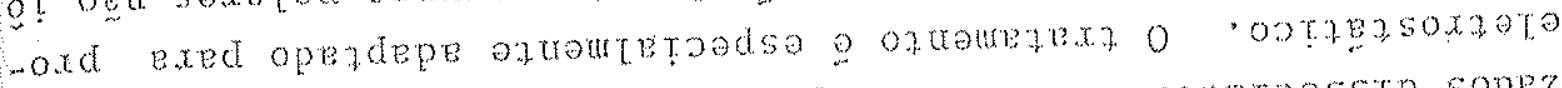

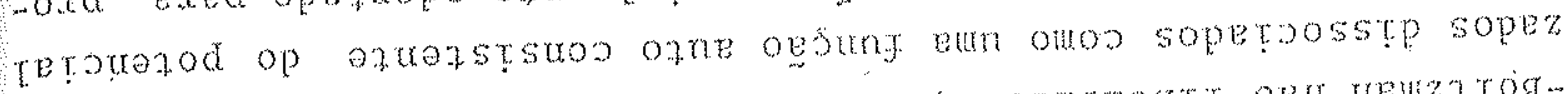

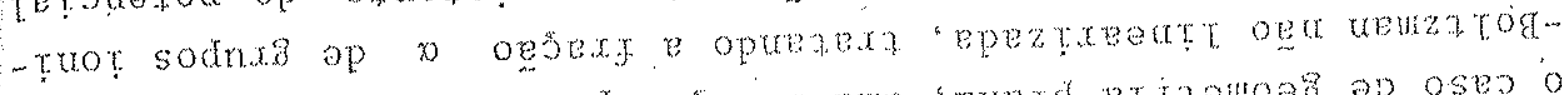

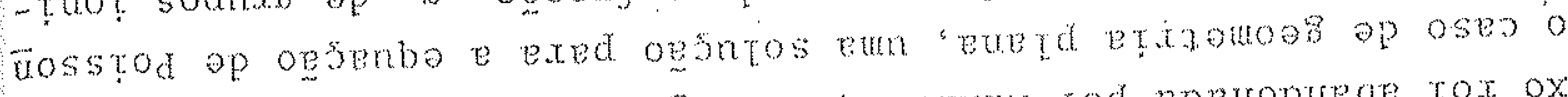

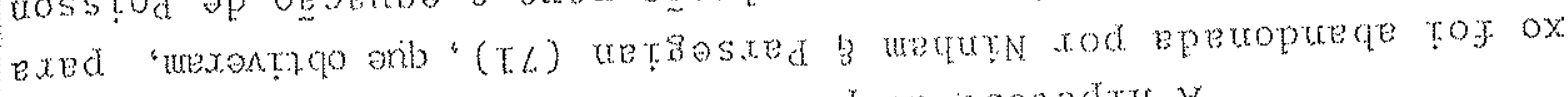

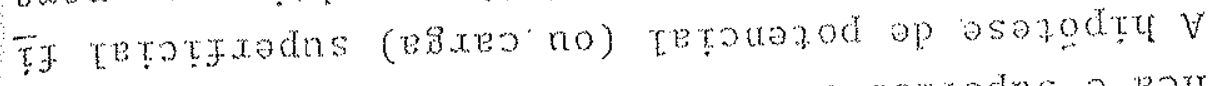

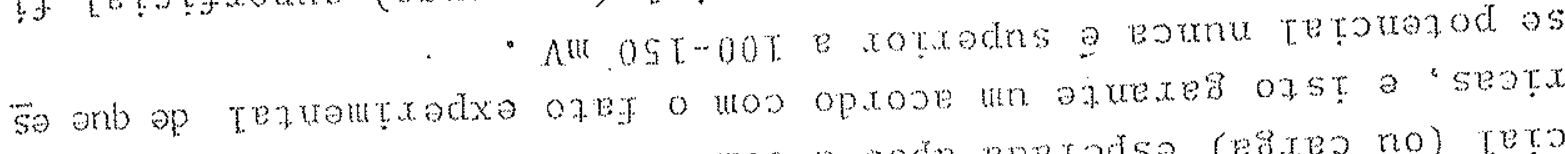

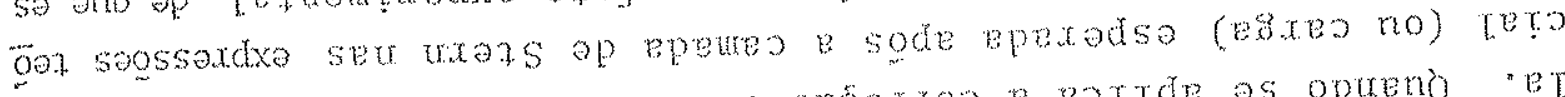

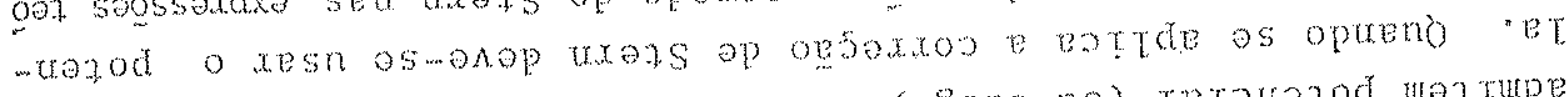

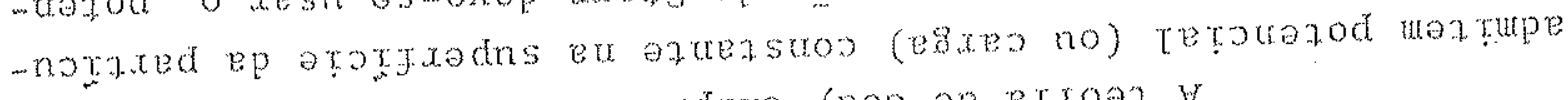

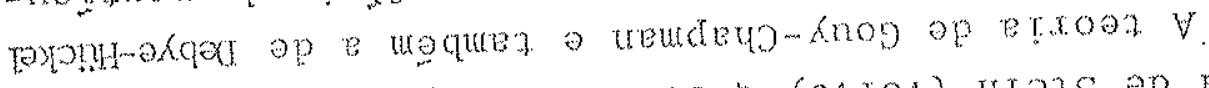

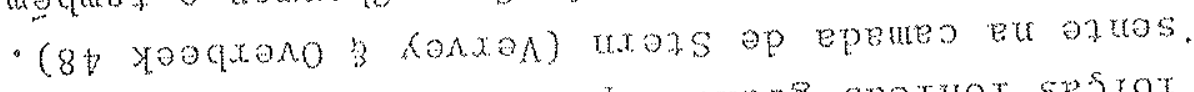

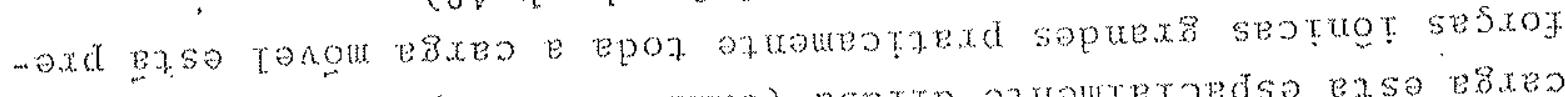

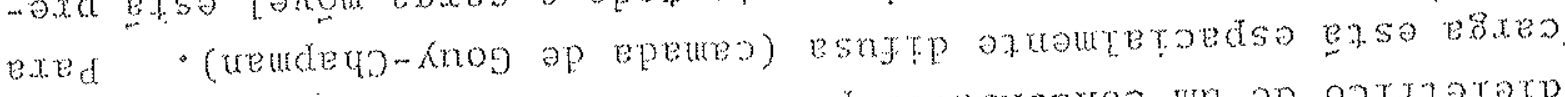

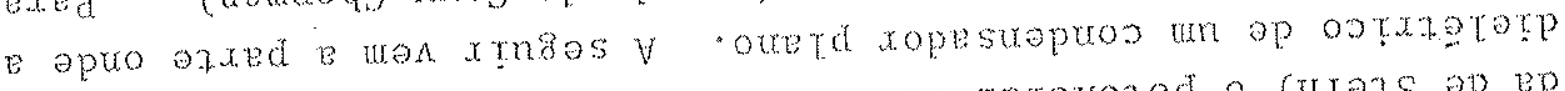

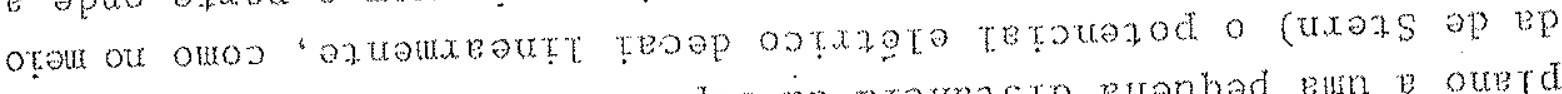

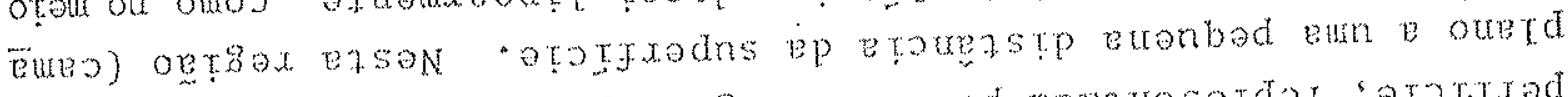

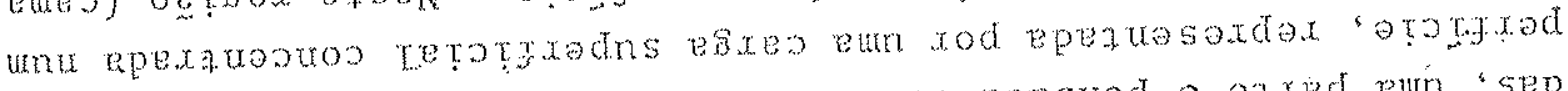

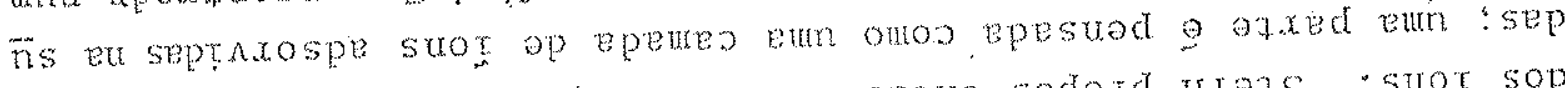

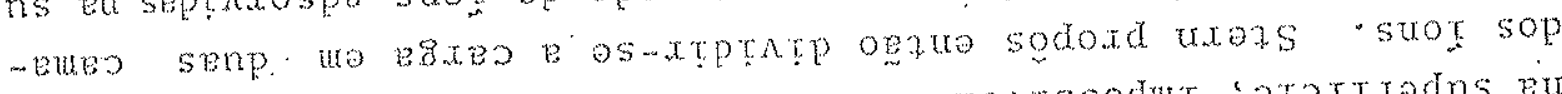
seatuta soosteute se optrep xax

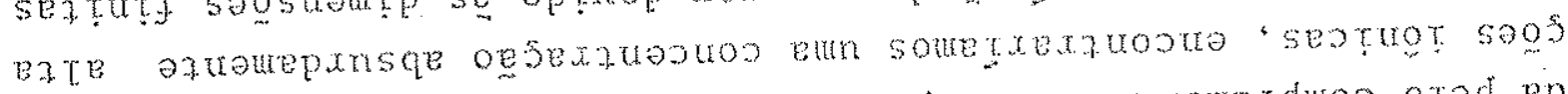

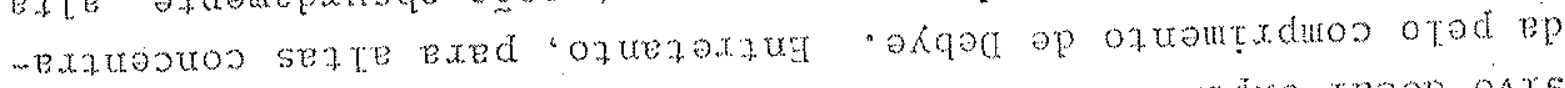

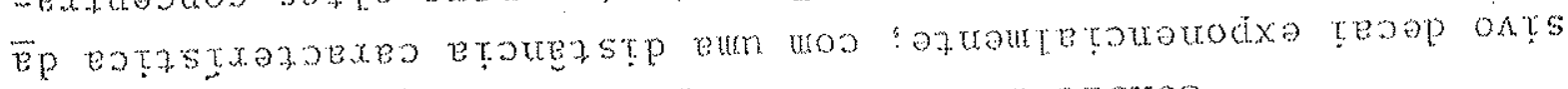

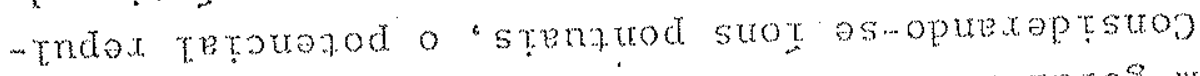

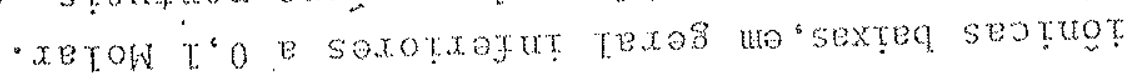

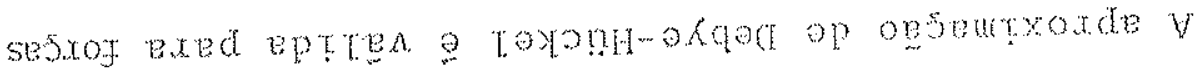

$$
\text { (V) } \quad \frac{I f}{60^{3} \zeta}=\sigma_{\gamma}
$$

rod opep a

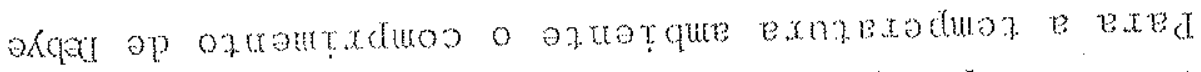

- ortat op wot op etometra

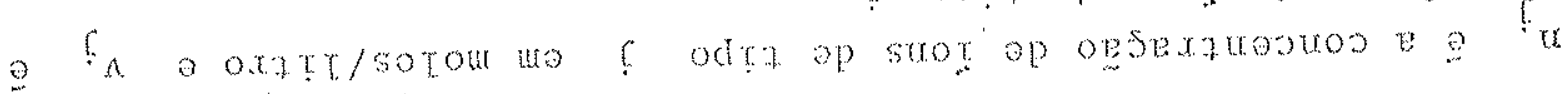
spro

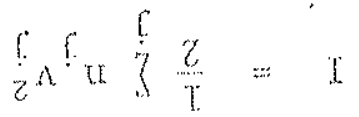




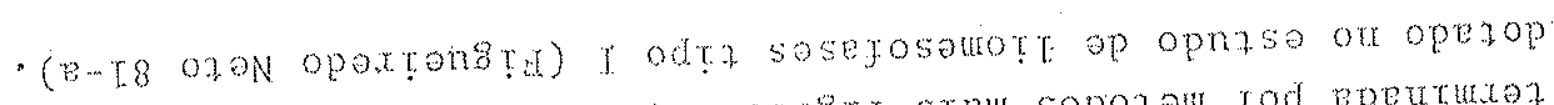

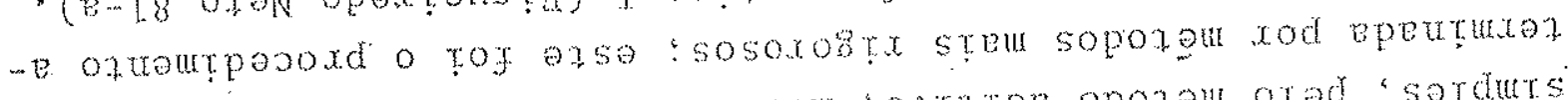

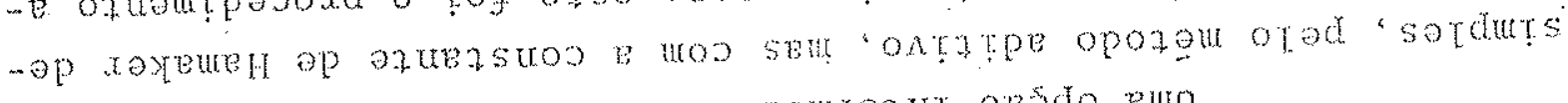

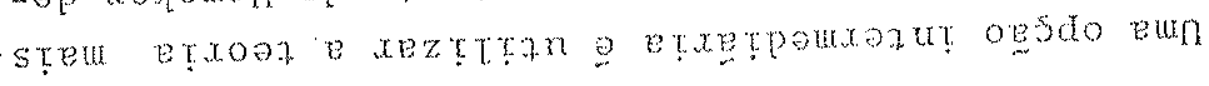

- CLe meteren 3

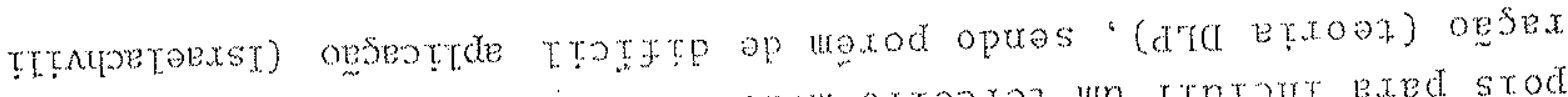

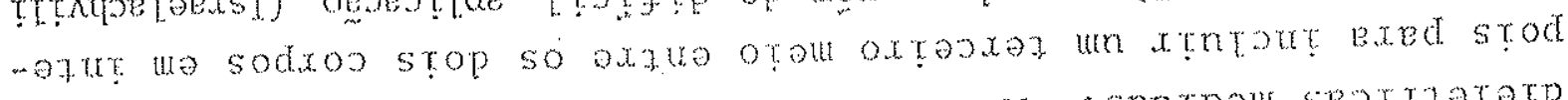

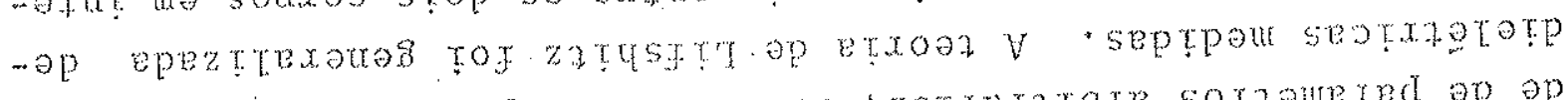

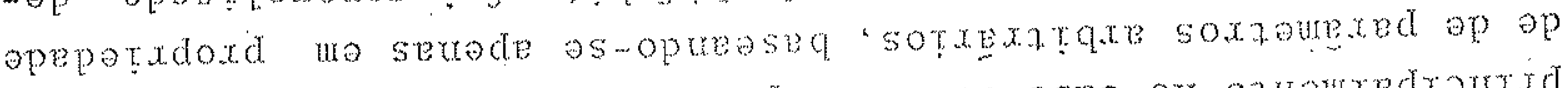

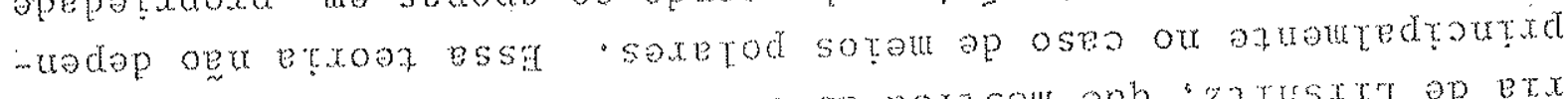

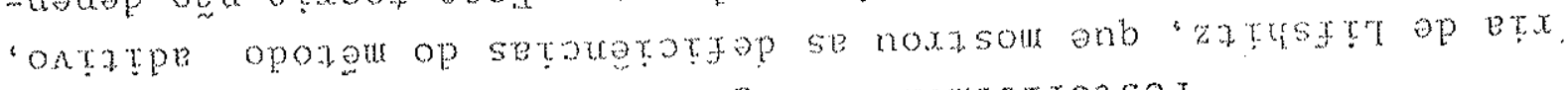

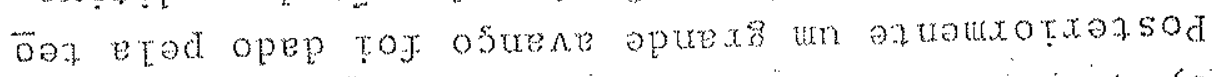

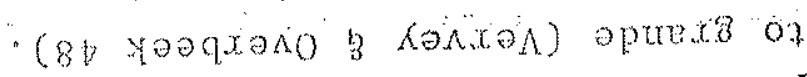

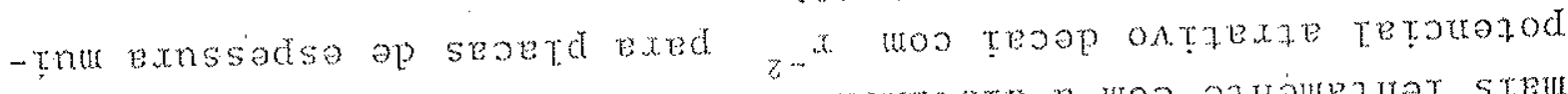

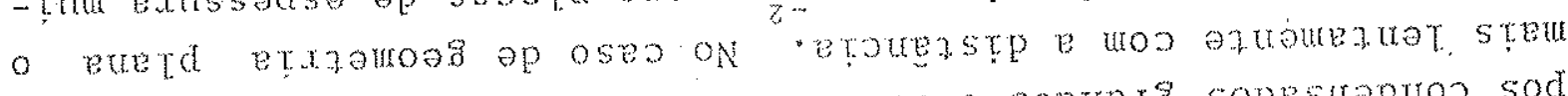
ontru oputesep "asteste xoteut ontru op 9 sopuexs sopestroptoo sod

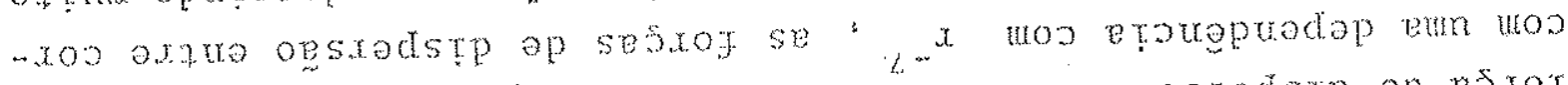

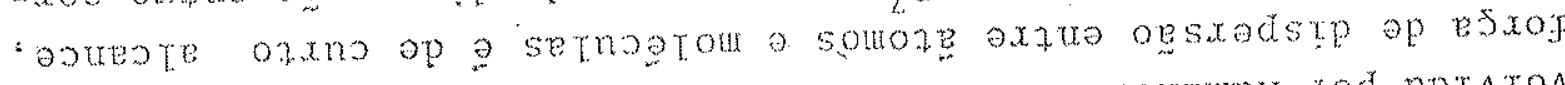

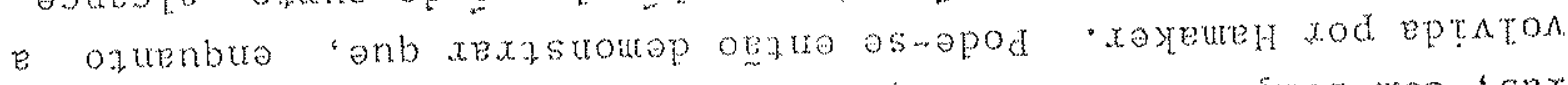

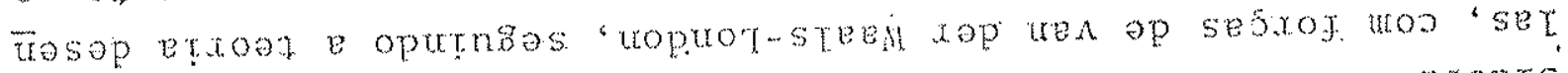

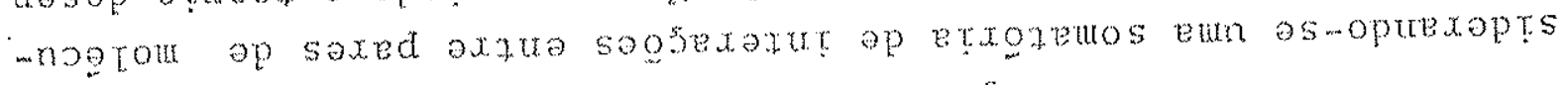

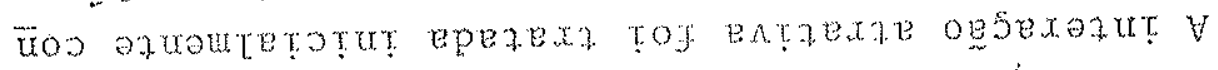

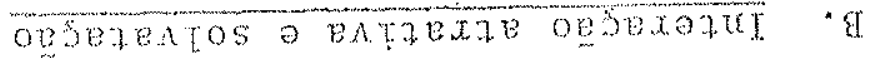

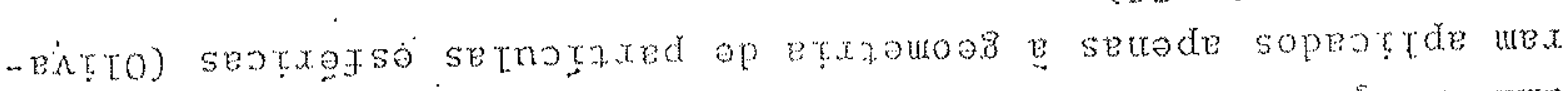

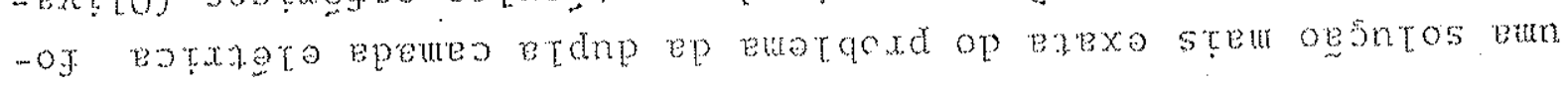

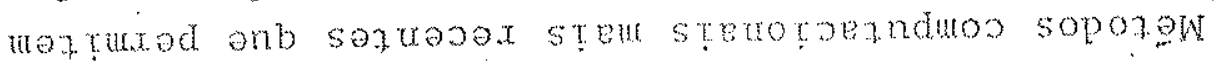

- opeptere ens

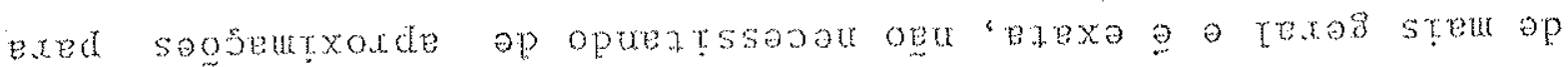

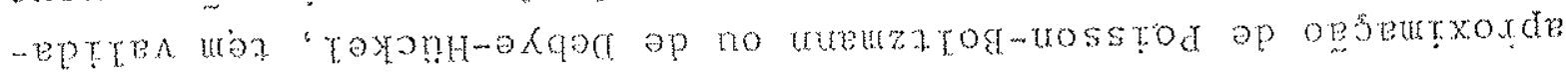

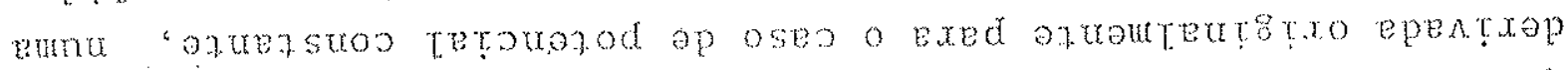

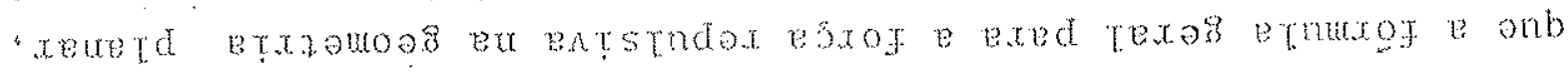

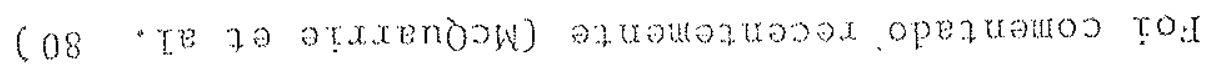

- wots op eq

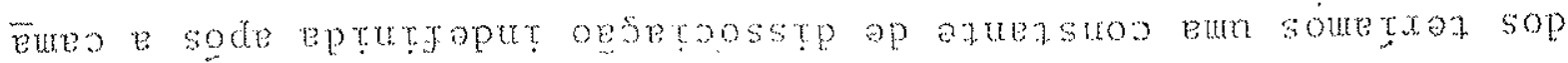

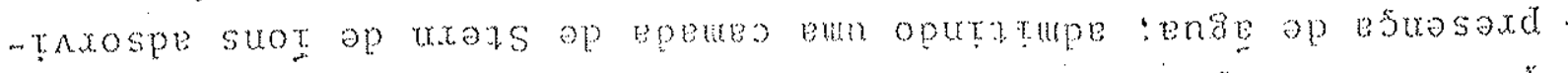

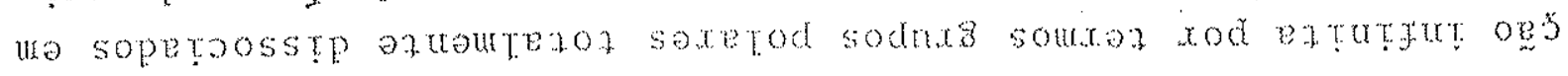

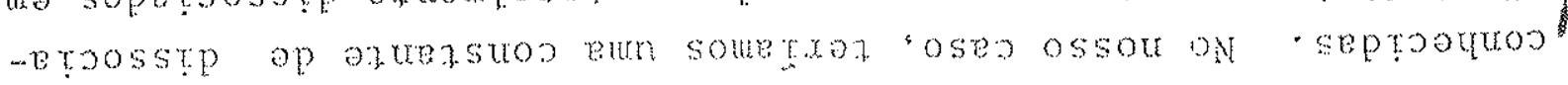




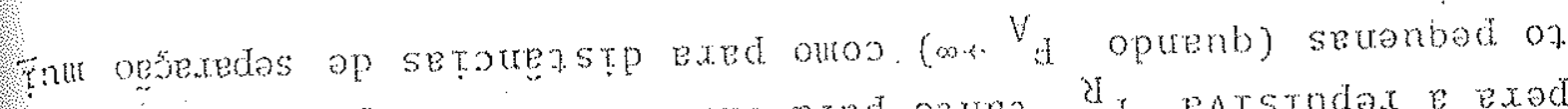

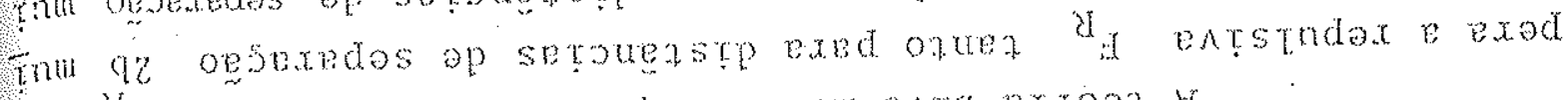

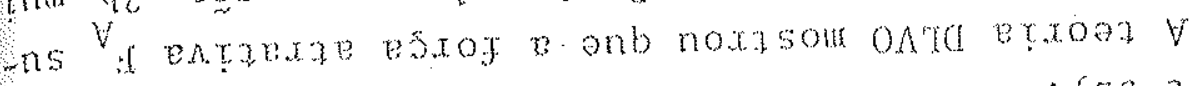

- $(25+\sin x)$ soxprtit?

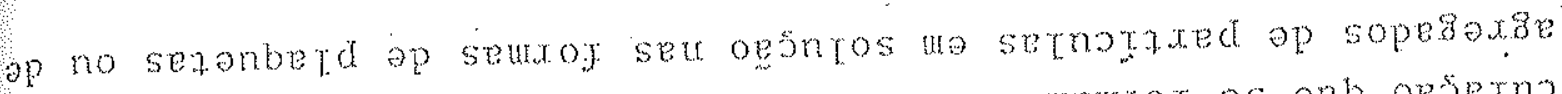

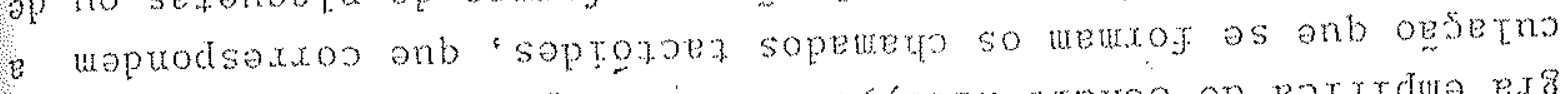

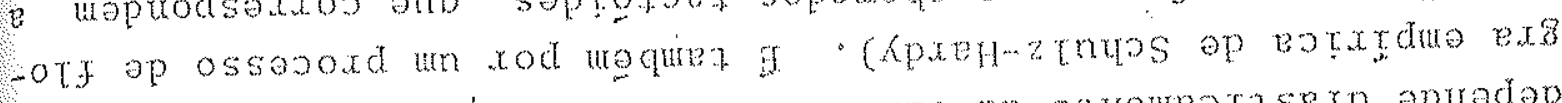

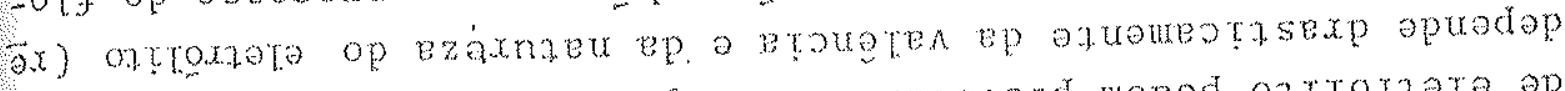

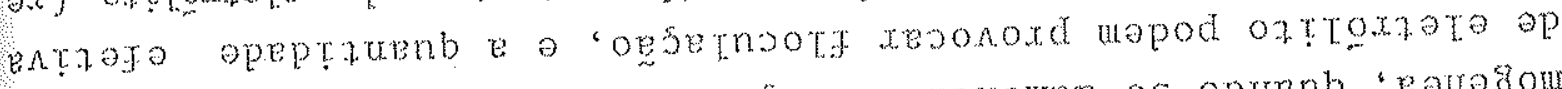

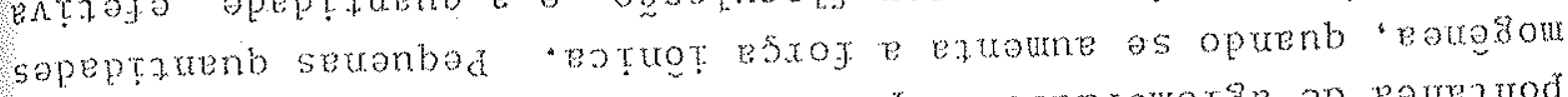

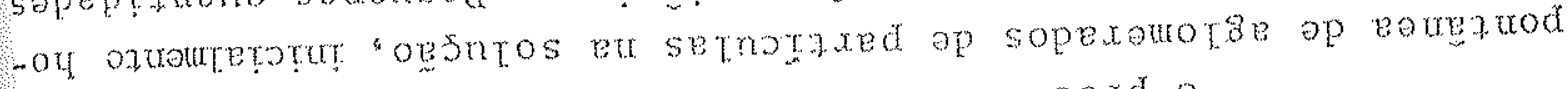

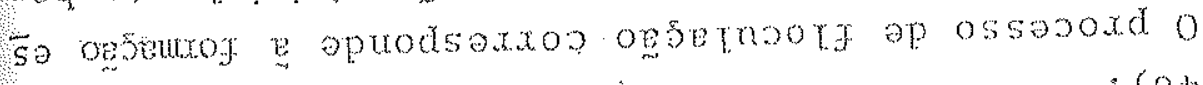

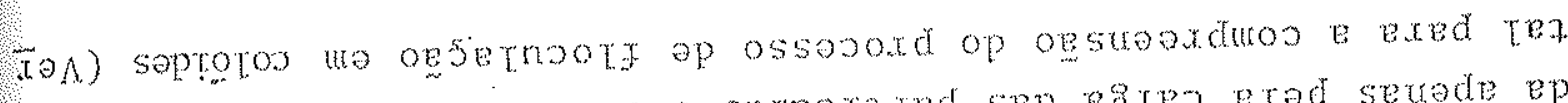

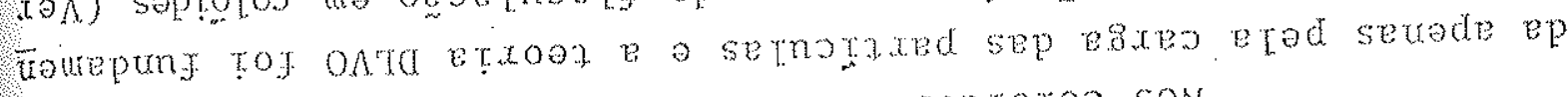

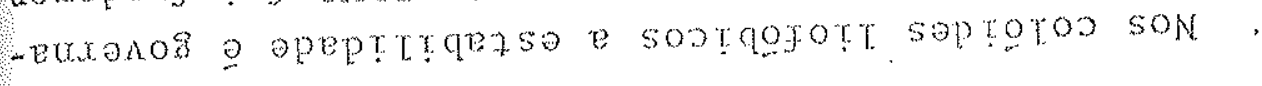

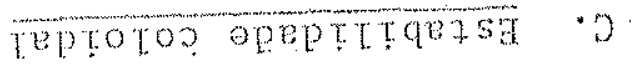

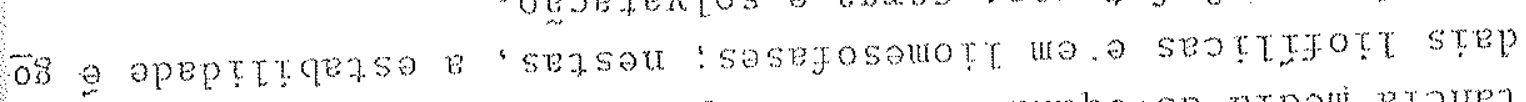

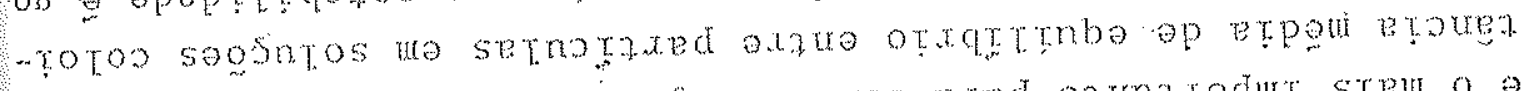

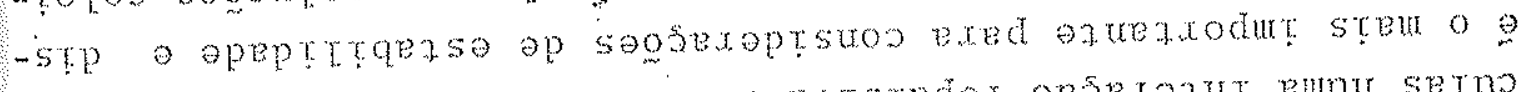

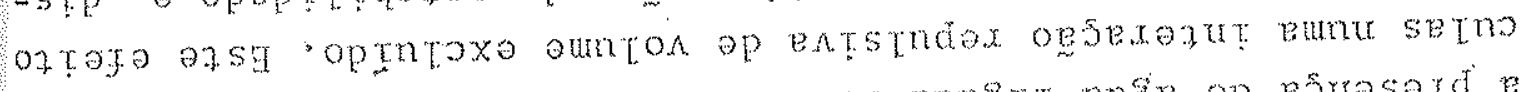

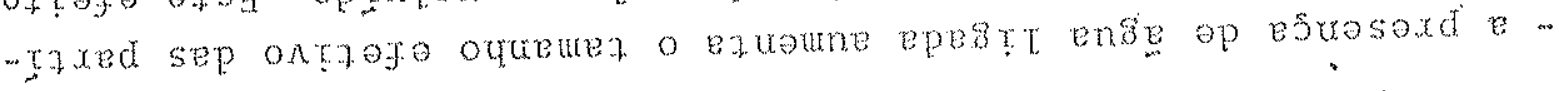
: (T8 wazkeyoty

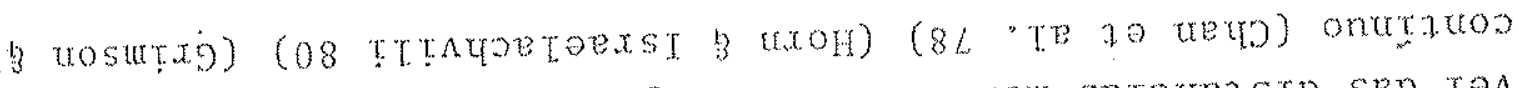

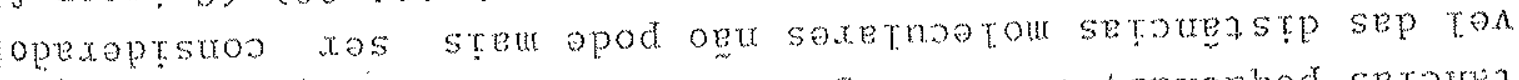

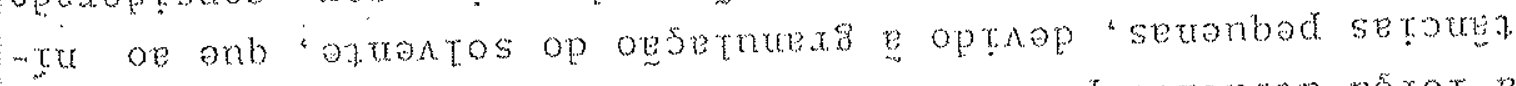

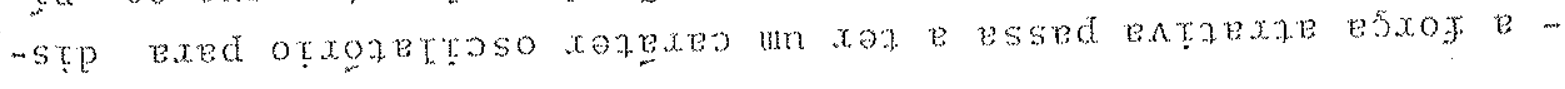

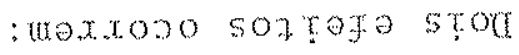

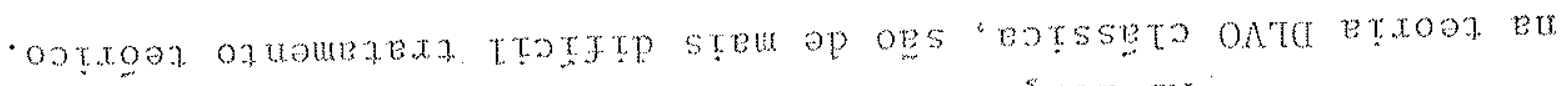
seprntour oraso opu anb oesearatos ap sesxof sy

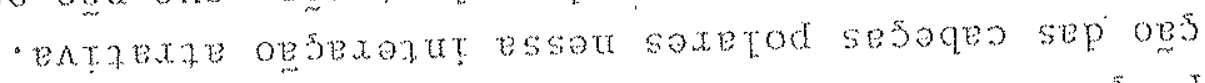

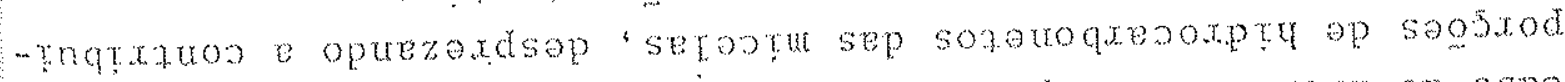

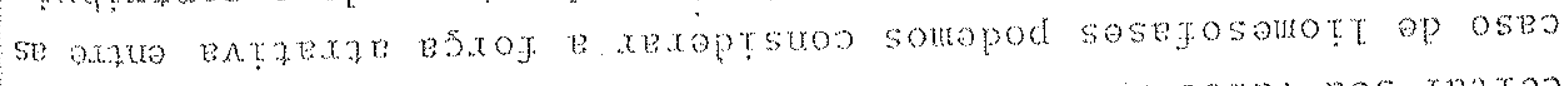

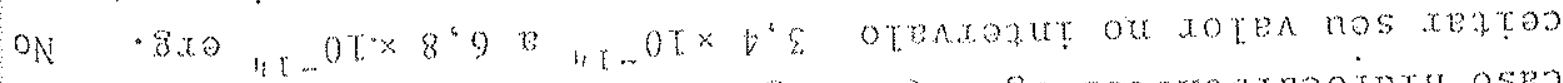

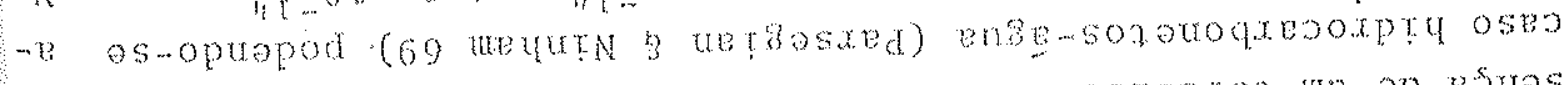

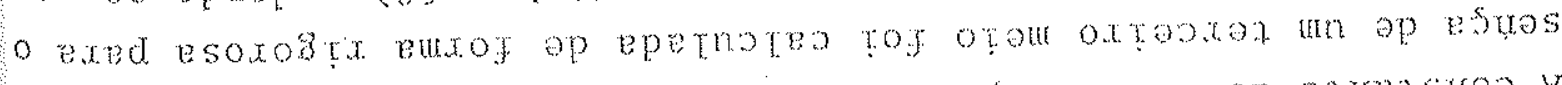

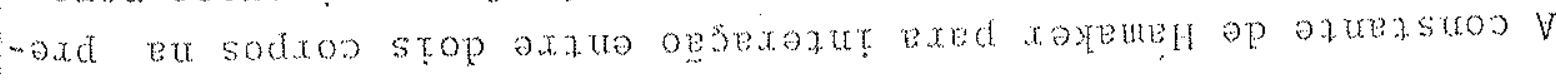




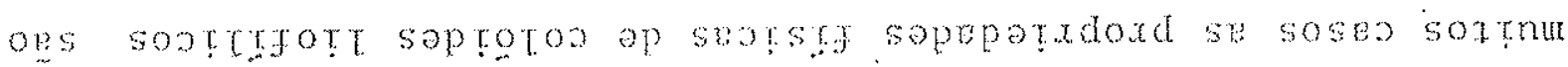

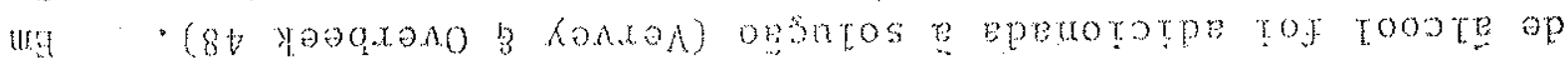

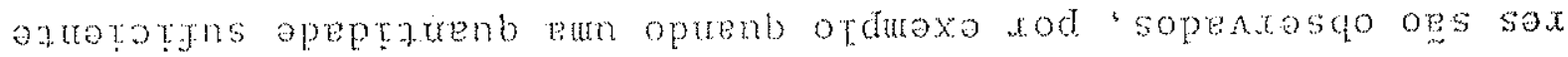

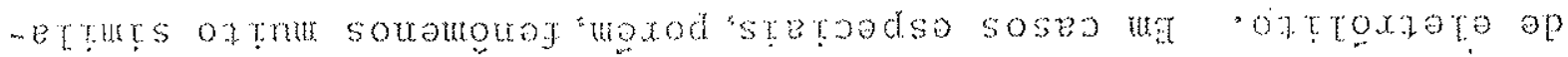

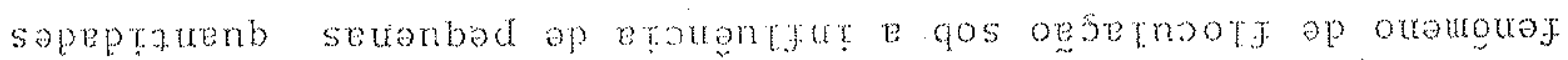

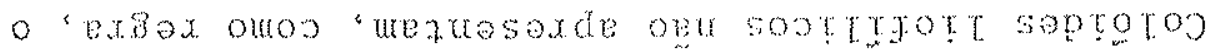

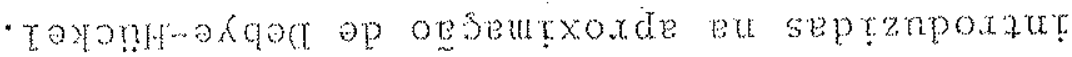

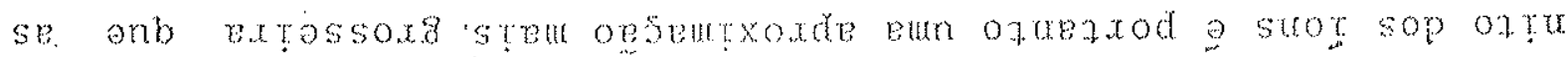

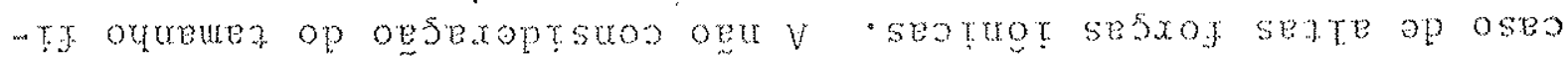

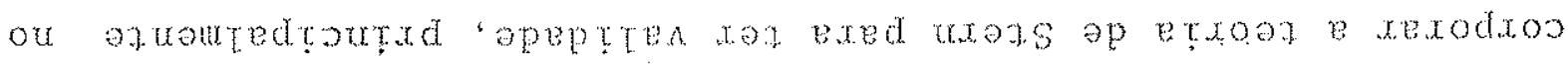

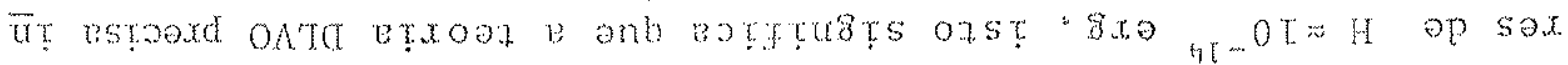
oten opp sourgpou steu sosoxosta sotnoted so omo

- (8v yeaqua 3 konxon) xorant on

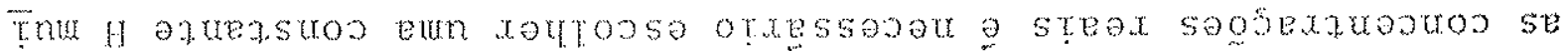

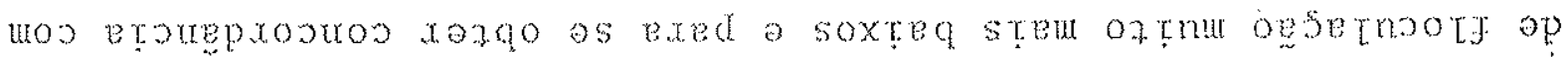

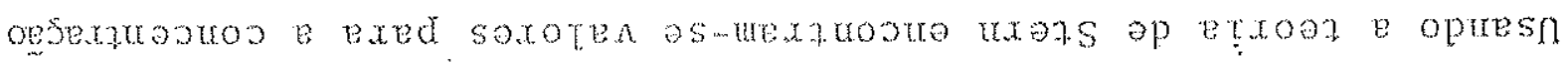

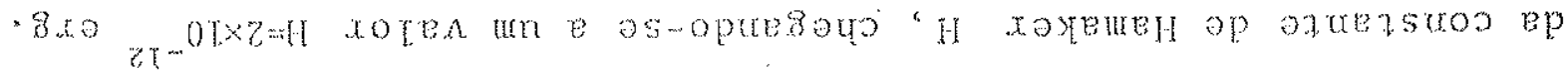

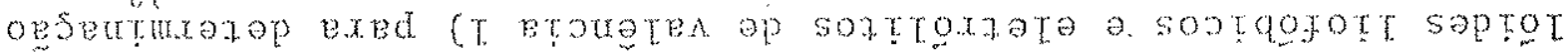

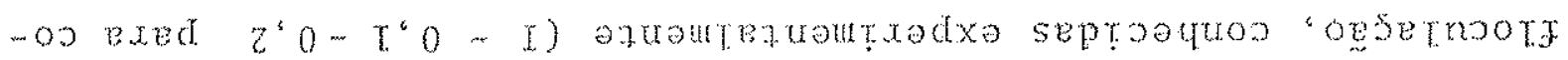

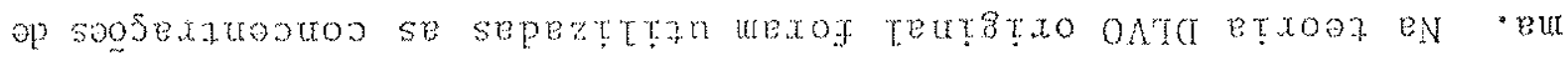

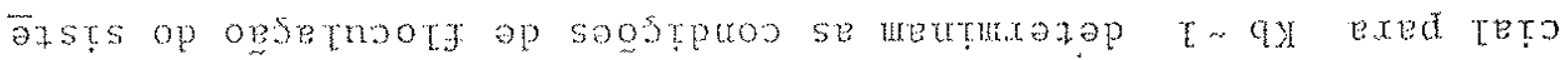

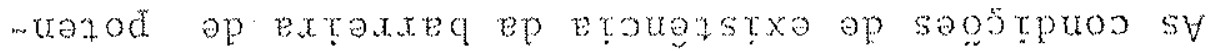

-onuetpe sten somortanostp anb'seotr

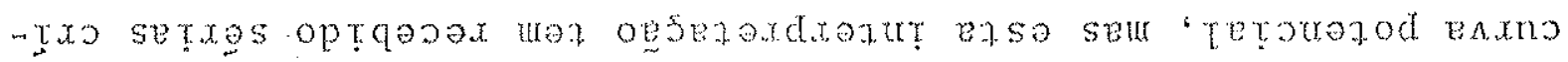

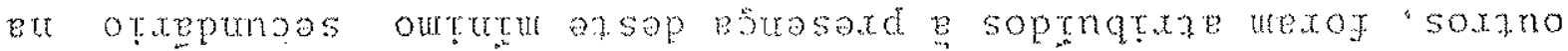
- opeqea op snxta op soosntos ue botoxastre asej em ap opjex

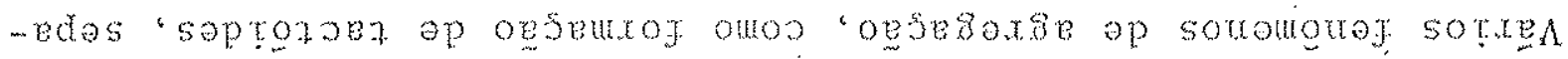

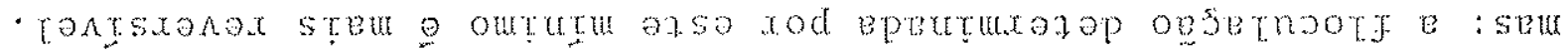

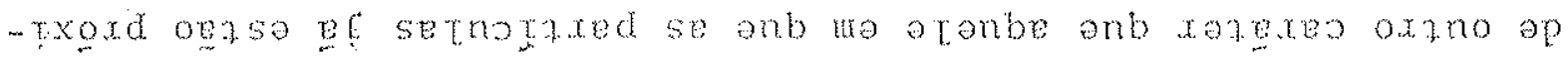

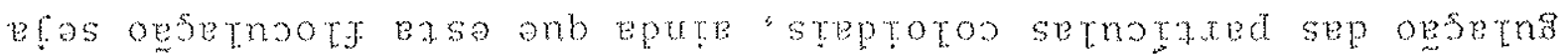

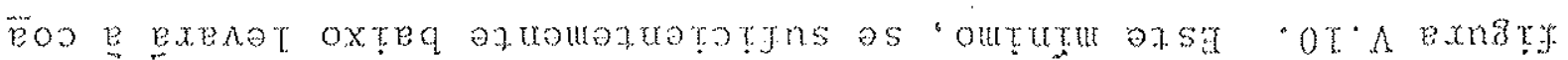

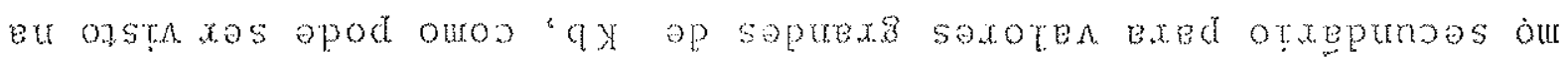

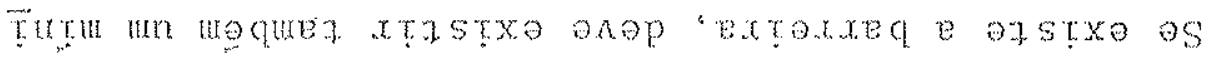

- onsetrooter ox

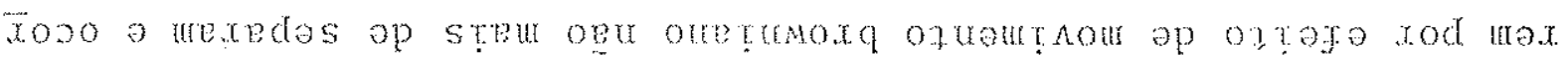

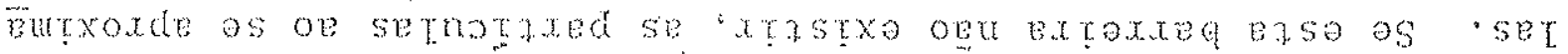

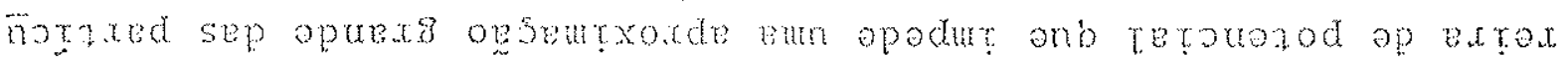

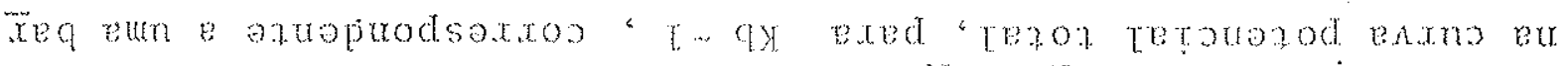

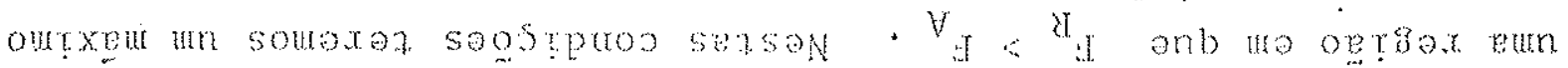

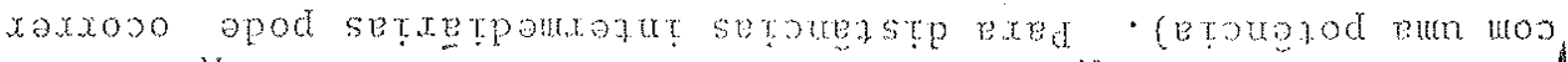

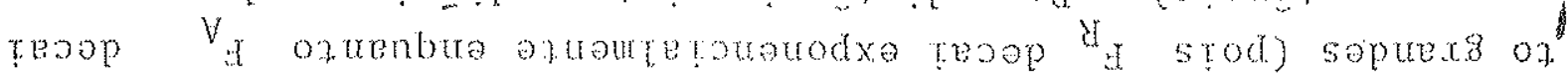




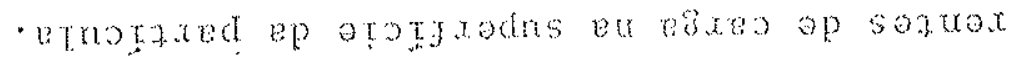

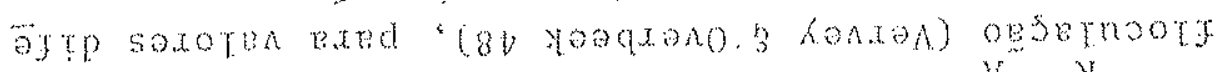

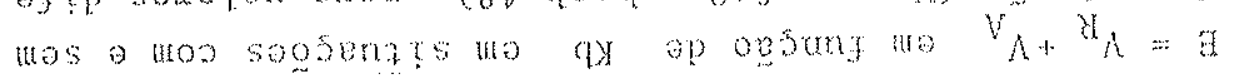

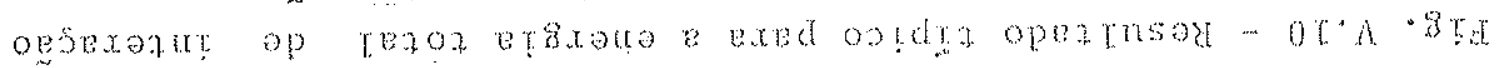

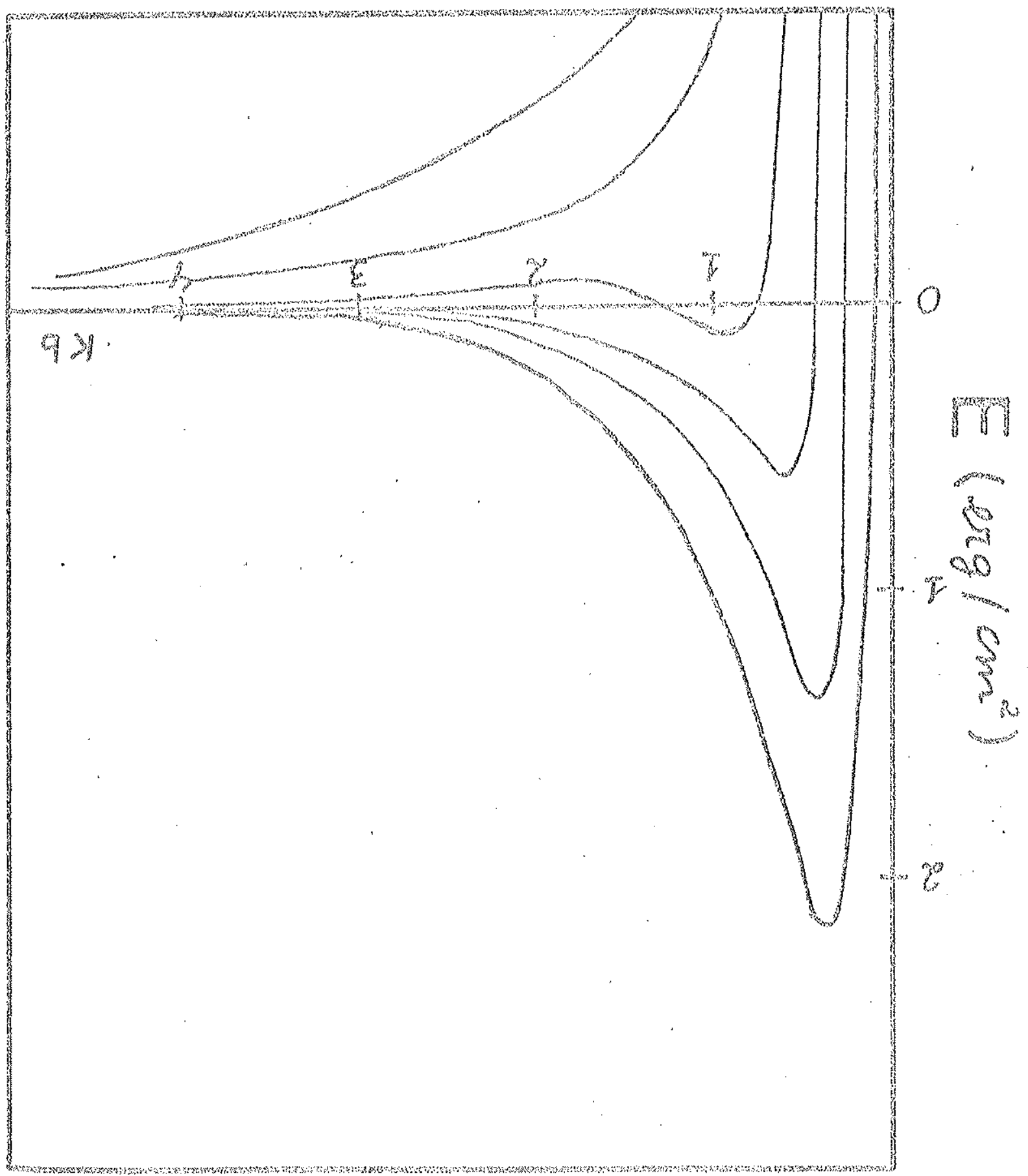




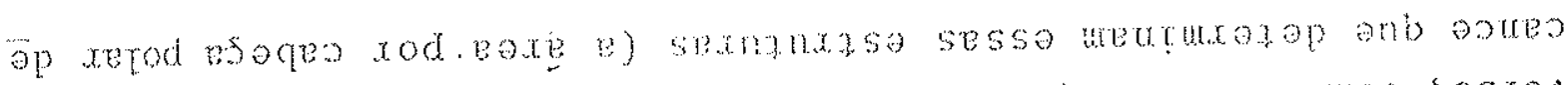

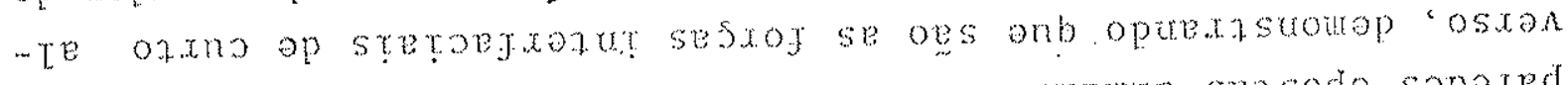

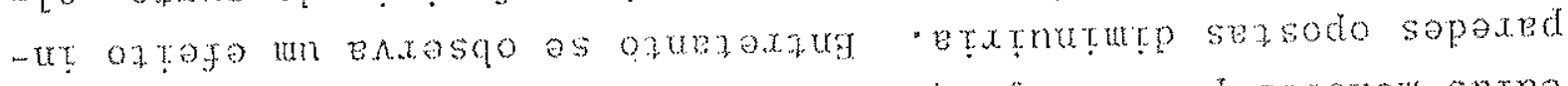

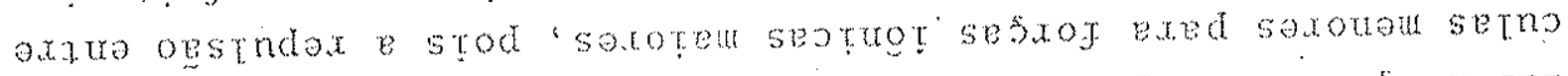

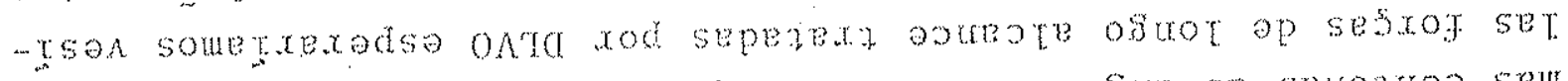

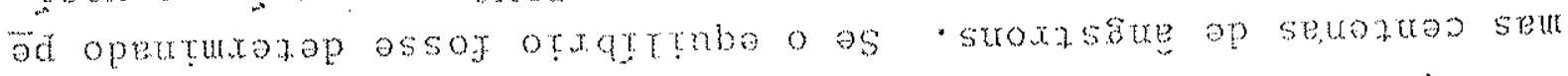

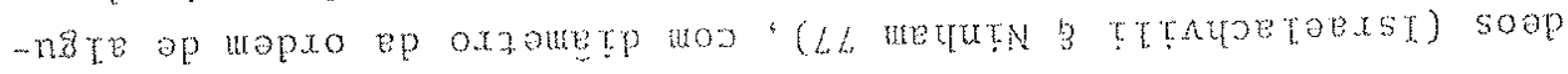

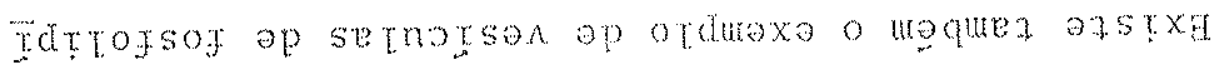

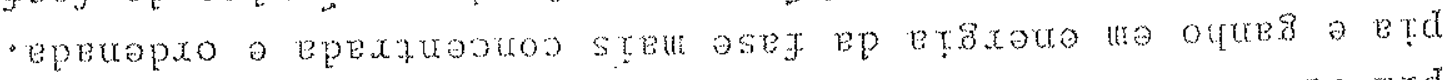

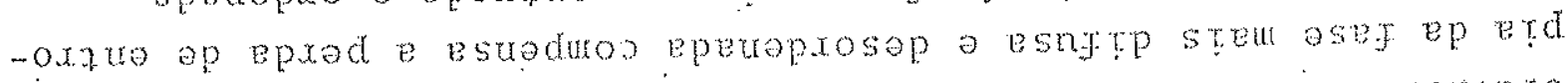
-oxzu ure oyura o apuo saseg senp no eun op semasts ue revopto

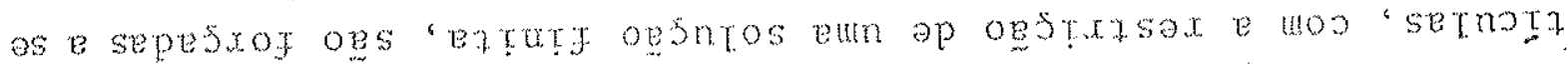

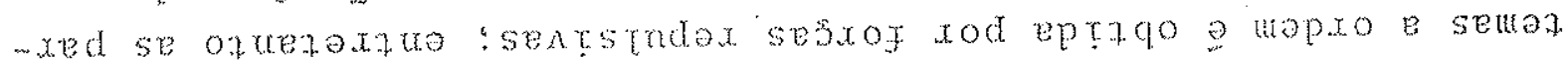

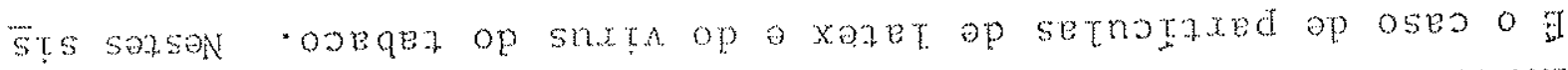

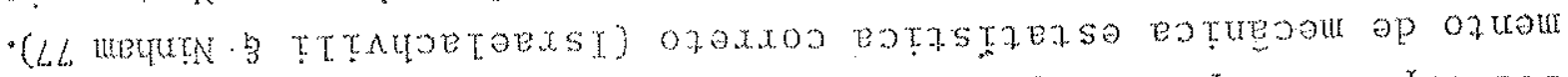

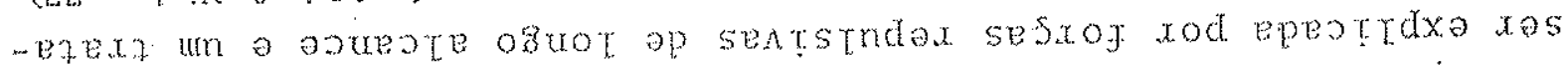

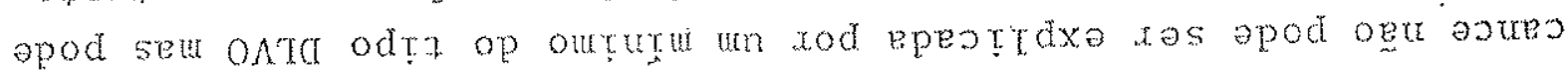

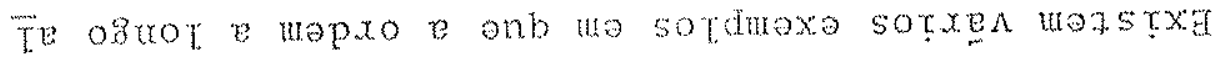

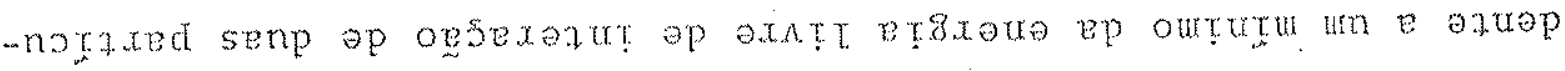

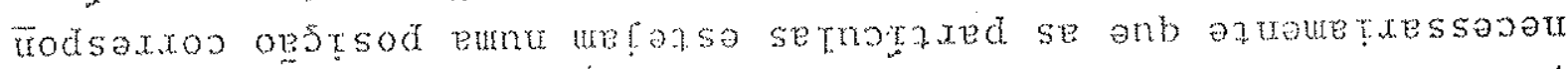

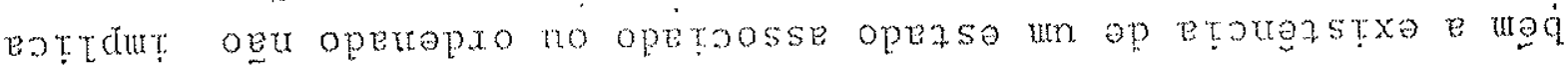

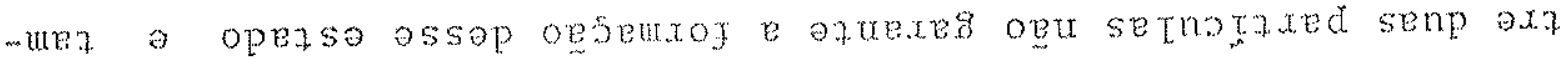

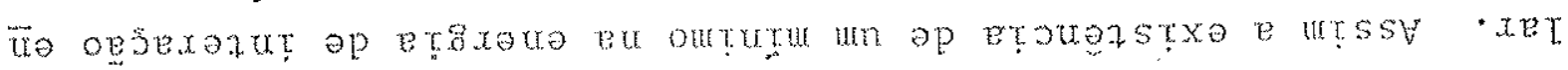

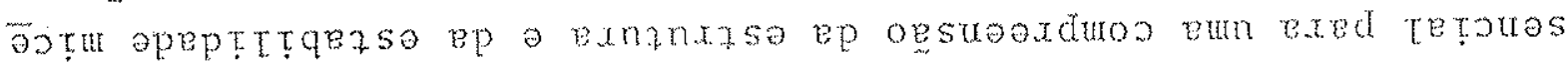
so 9 ozorduos eorasta

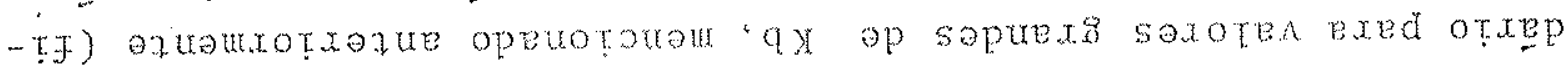

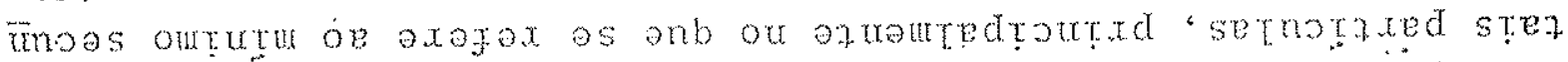
op oxmfroo un op sepepordoxd axgos oonod oxthu xate sou op

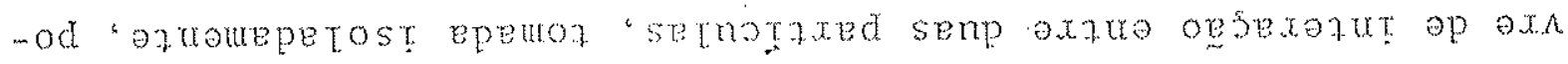

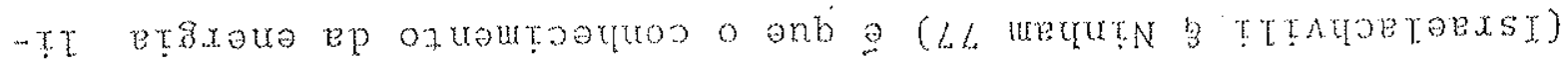

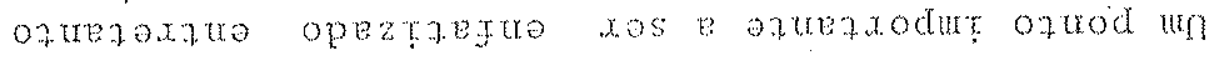

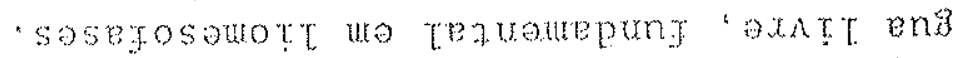

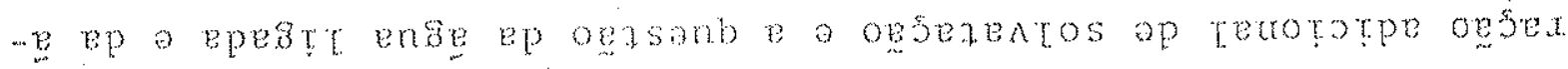

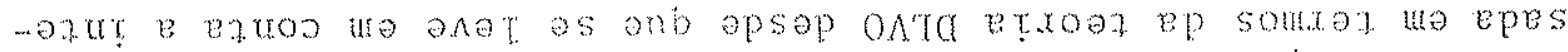

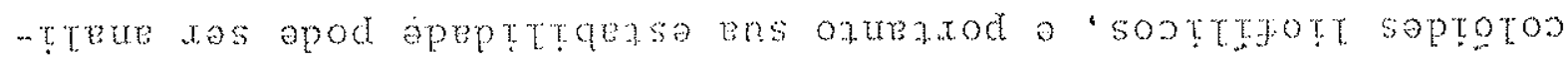

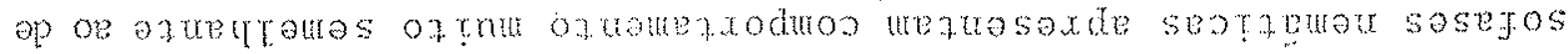
- ourt se onb op seromera ogs soley sosse sopol

- oxtroxato op sopeptanb serombed op opspe xod opep.

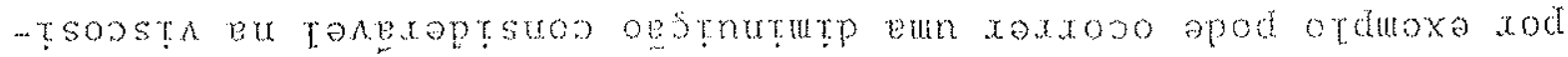

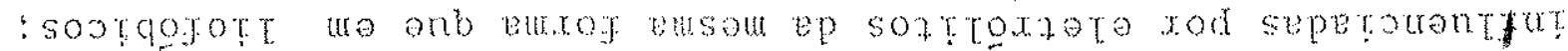


aqua ep soperthsax so somargo somoted so opuazed

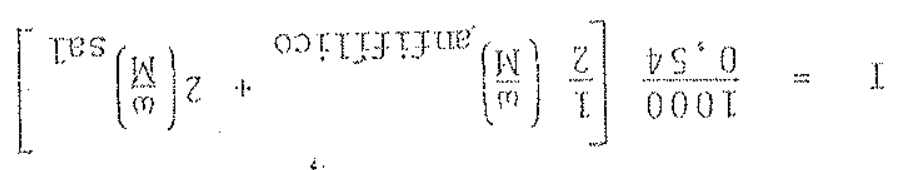

$\operatorname{rod} 0 \mathrm{e}$ ?

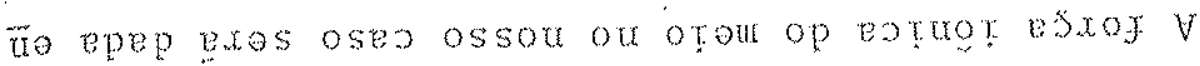

$$
\frac{W}{O_{N}^{n}} \frac{\operatorname{ts} 0}{T}=u
$$

$x o d \operatorname{pp}$

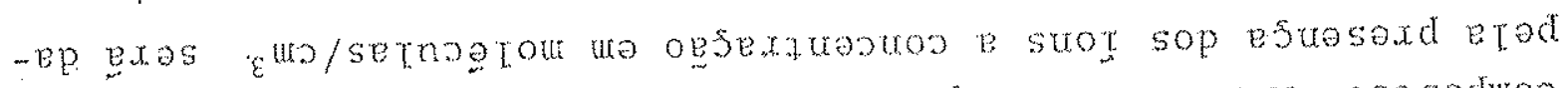
ppexate o oex enge ep epeptstop e onb as-oputarupy -ozsoduros

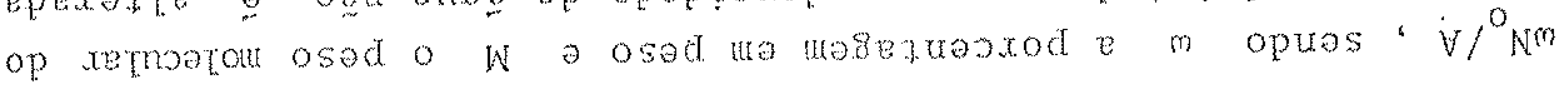

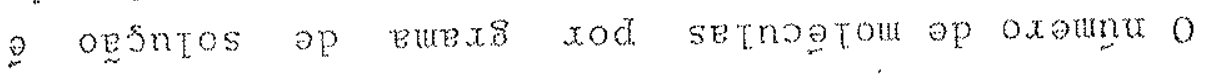

- enge eu srog ex?

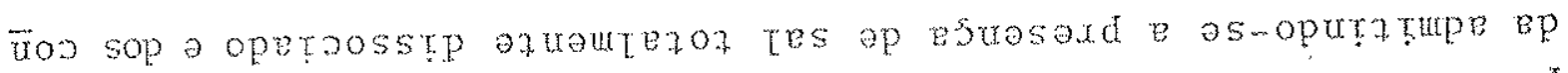
Btnoter xas anep otau op extrot esrog V asejosenot asson axed

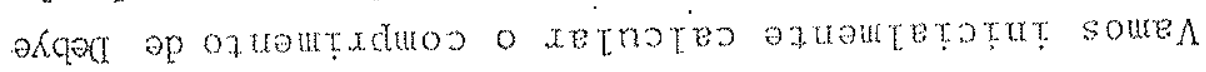

OATa xod sotmoteo $V$

II odt aseasomot BP OSTTEUV " $2 " 2 \cdot A$

- saurasts sossop opeptraezse ep a exinat

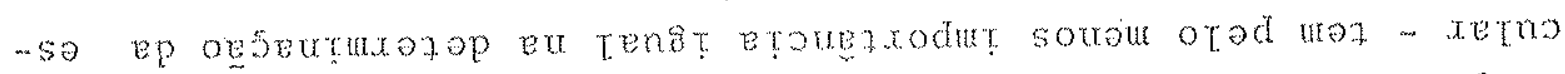

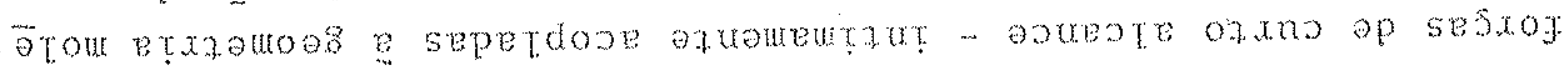

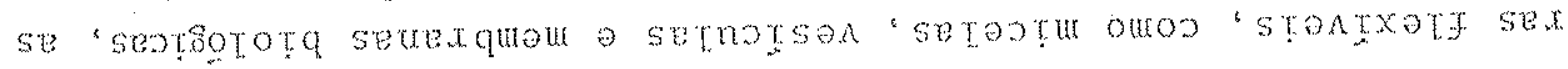

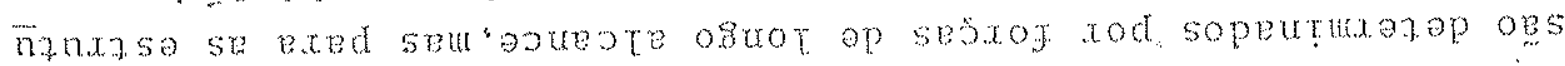

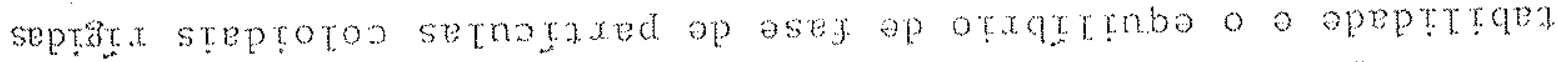

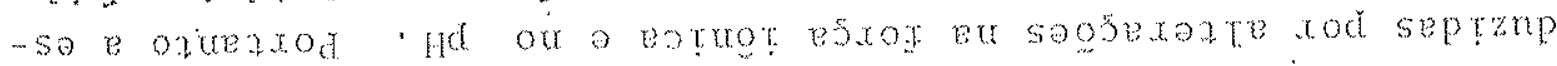

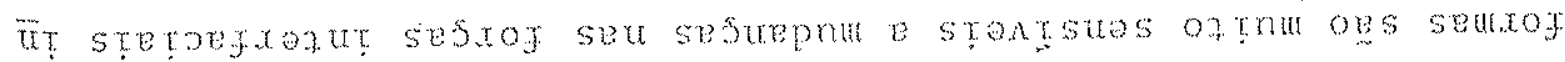

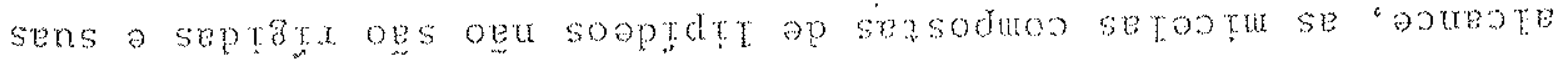

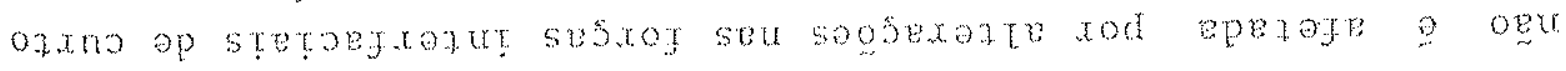
seprgtr stpptotoo sernotaxd ap euxos e ofuenbug

- (samerod sesager axtre oes

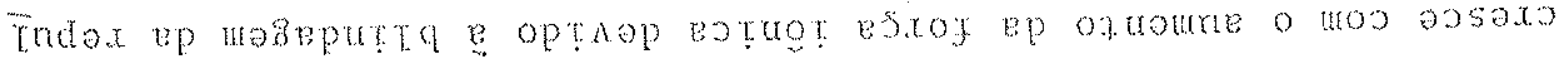




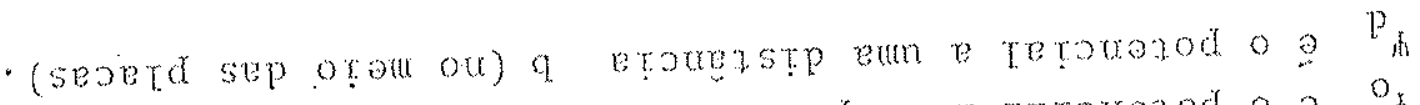

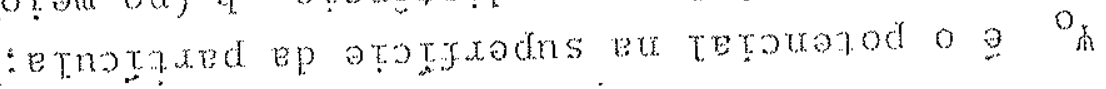

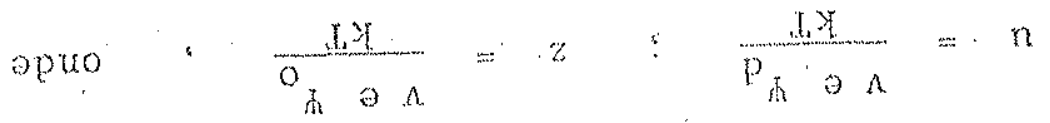
opues $\quad\left(z^{4} n\right) y\left(\frac{z^{\Lambda}}{x}\right)={ }^{x} A$

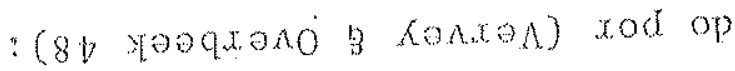

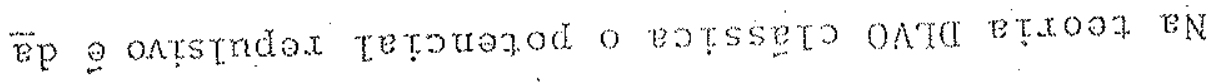
- sopertrsox

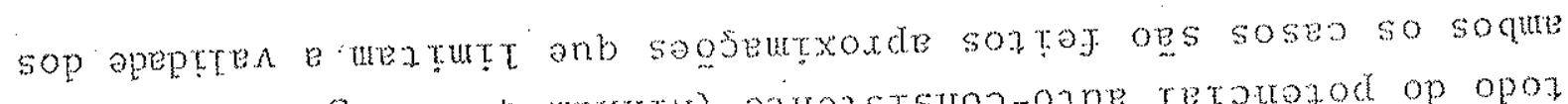

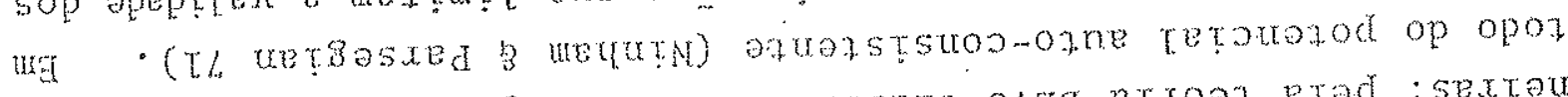

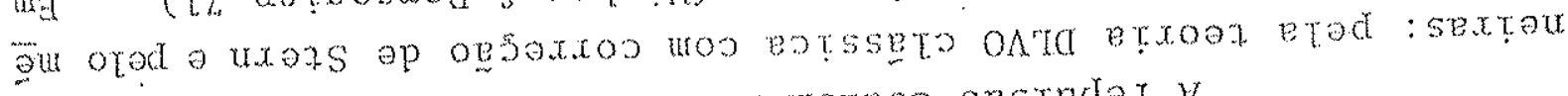
- eu senp ap epetroted rog auequotroo ogstridex $V$

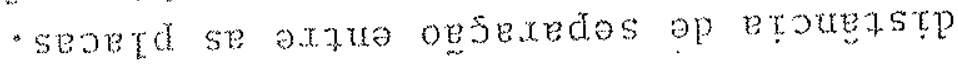

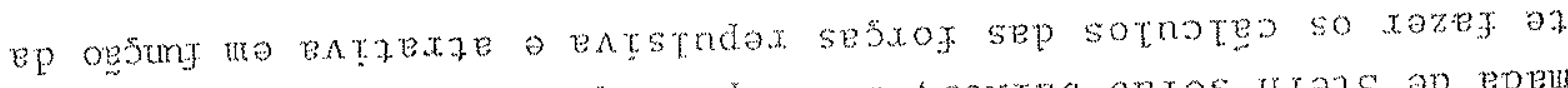

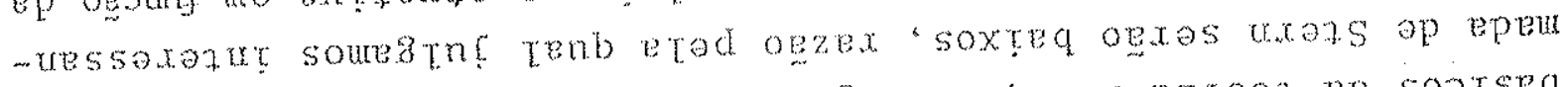

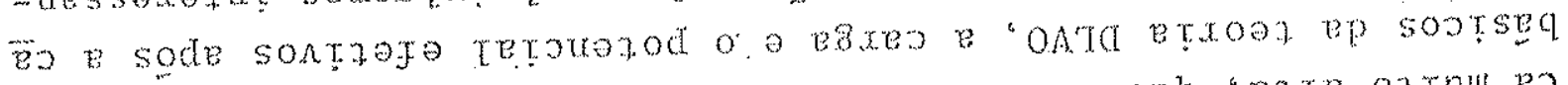
sozsodnssexd so reneurotsonb euroz antsntout anb "Bate oztrul es tron estox eun souxaz op xesade segstpuos sezsan

- soperaspe suot.

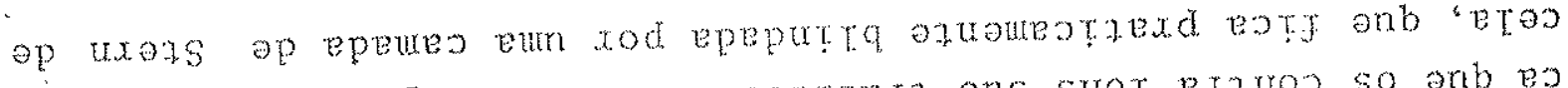
Tru ep sapepturxoxd se exron op soptzext oes swot exzuod so arb eo

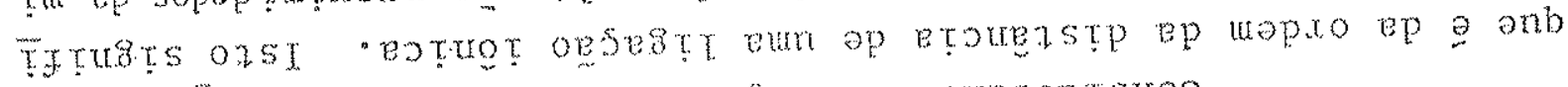

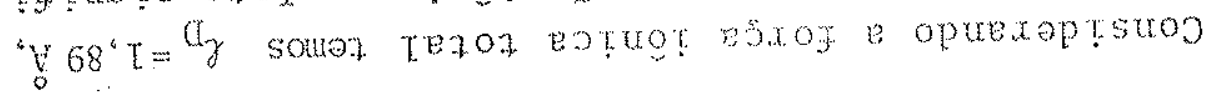

\begin{tabular}{|c|c|c|c|c|c|}
\hline $825^{\circ} 0$ & $68^{\circ} \mathrm{T}$ & $85^{\circ} 2$ & & & T:toL \\
\hline$S L S^{\circ} 0$ & $19^{\circ} 2$ & $O E^{\circ} \mathrm{T}$ & $2 v \pi$ & $90^{\circ} 0$ & tes \\
\hline $\operatorname{tsc} c^{2} 0$ & $69^{\circ} \mathrm{Z}$ & $82^{\circ} \mathrm{L}$ & $v^{2} 09 z$ & $22^{\prime} 0$ & wot exauos \\
\hline$\lambda$ & $a_{y}$ & I. & $W$ & on & trot: \\
\hline
\end{tabular}

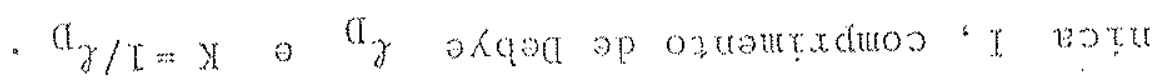

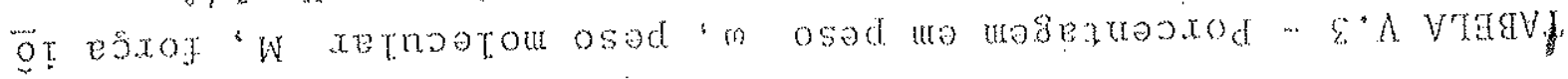




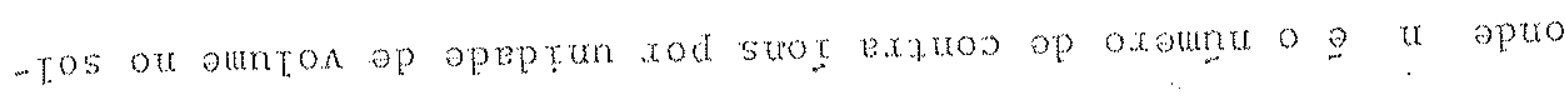

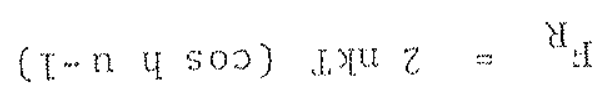

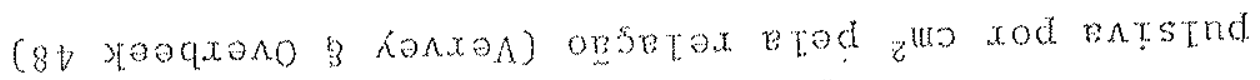

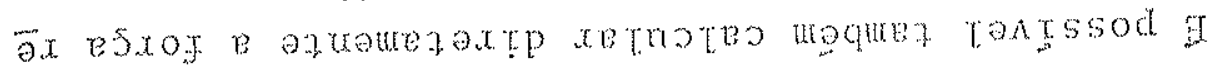

"T.t" A Bngtg ed

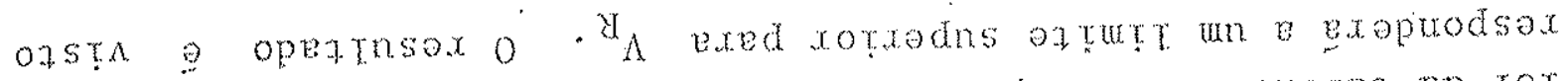

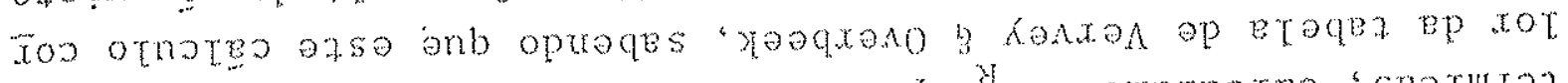

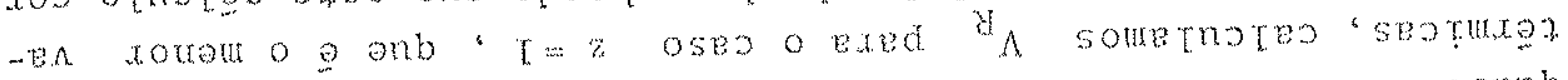

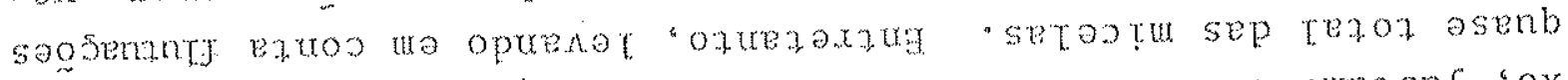

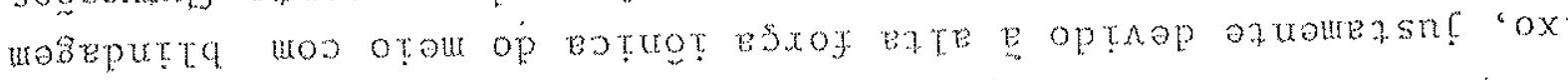

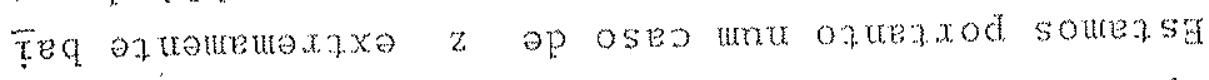

$$
6 \mathrm{~T}^{\circ} 0=z \quad \text { optrop }
$$

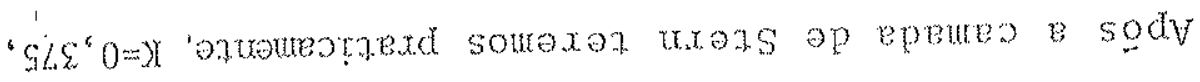

$$
\frac{x}{00 \angle 0^{\circ} 0}=z
$$

อx rotque ex

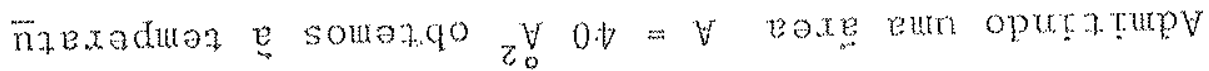

$$
\frac{L P Y Y X}{6^{6}} \frac{3}{4, y} \Rightarrow 2
$$

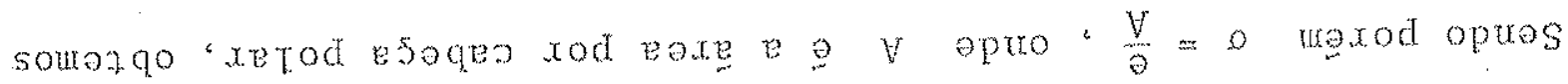

$$
\cdot 0^{2} \frac{46}{x 3}=0
$$

(8) yoog

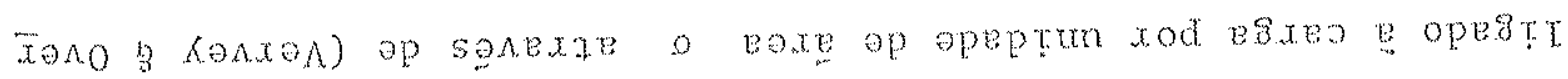

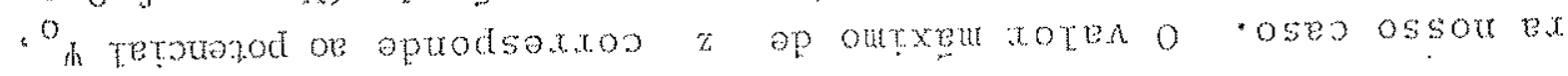
ed \% op entagutaso eun terej oxwexod sourstpaxd

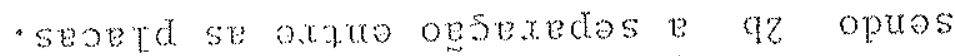

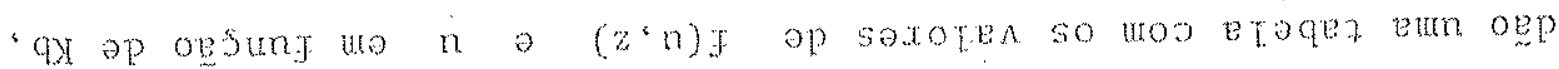

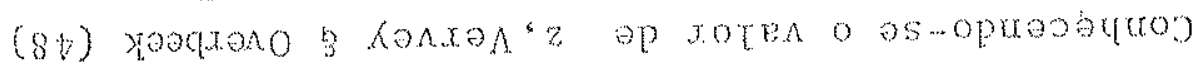

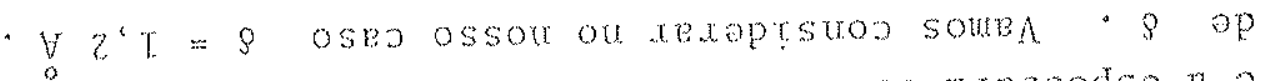

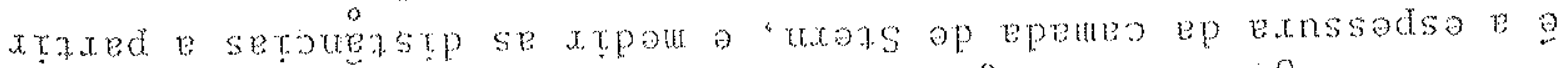

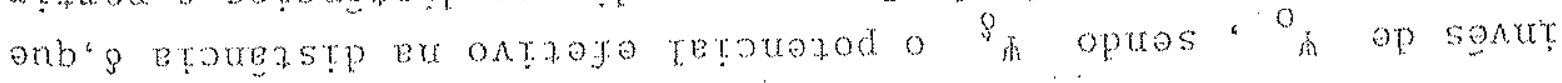

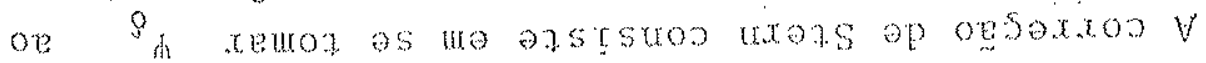




$$
\begin{aligned}
& \left\{\frac{\varepsilon(z / \gamma+q)}{z}-\frac{\varepsilon(\gamma+q)}{T}+\frac{\varepsilon q}{T}\right\} \frac{\mu \nu z}{H}-V=V \\
& \left\{\frac{z^{(z / y+q)}}{z}+\frac{z^{(\gamma+q)}}{r}+\frac{z^{q}}{\tau}\right\} \cdot \frac{\mu \beta v}{H}=V_{\Lambda}
\end{aligned}
$$

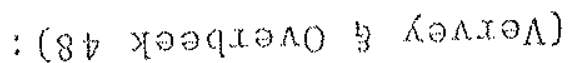

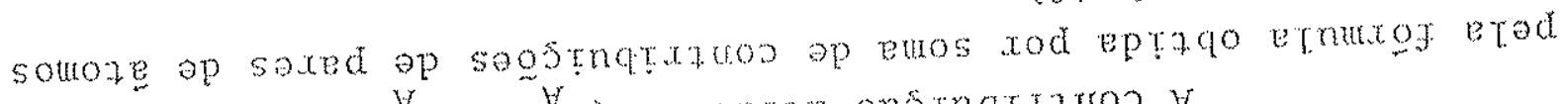

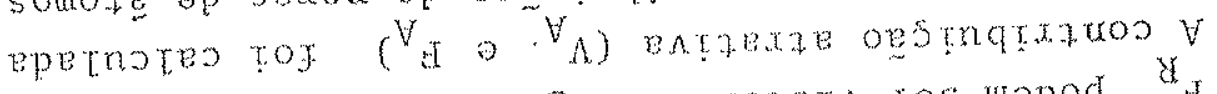

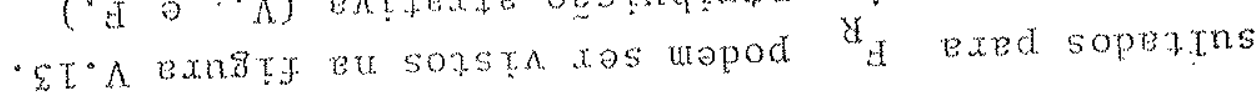

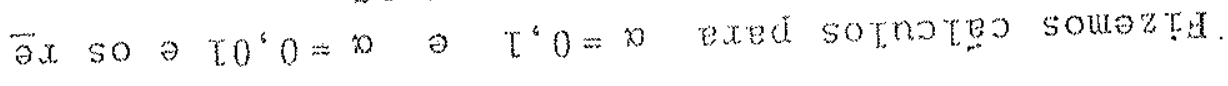

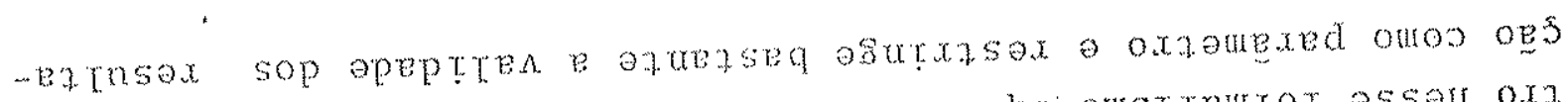

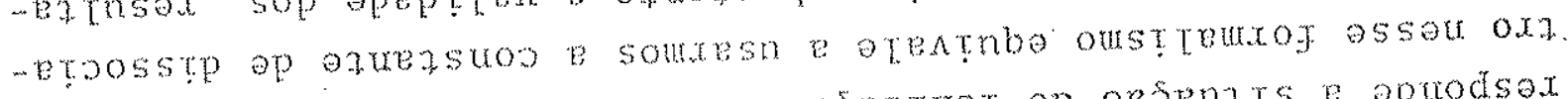

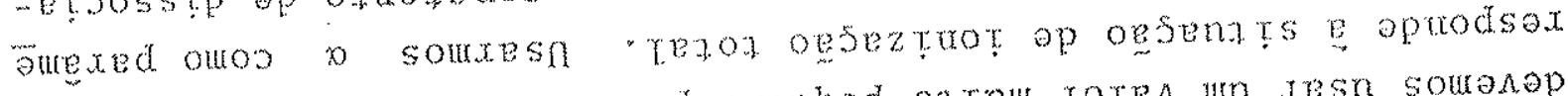

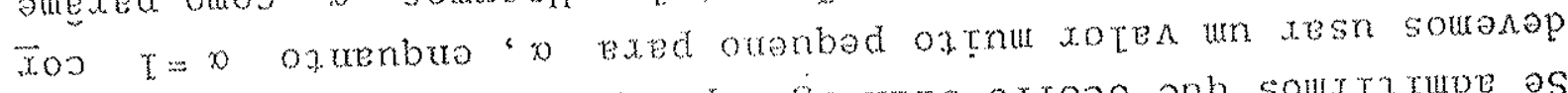

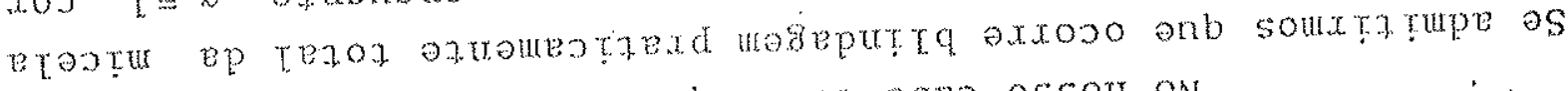
- oxzanexed olros, 0 , xesn onb somot osed ossod on

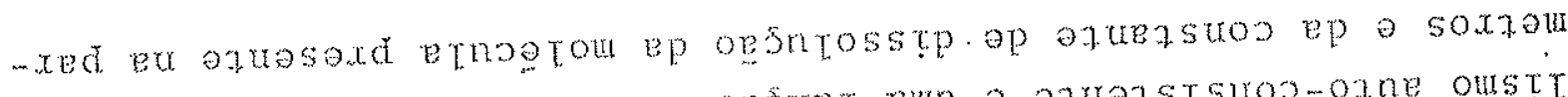

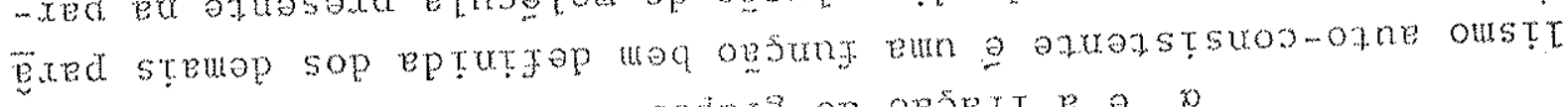
Buxof ou on 'sopetooste sodns op ogsex 2

$$
\begin{aligned}
& q x \cdot \frac{V \cdot u}{x+x}-\tau=3
\end{aligned}
$$

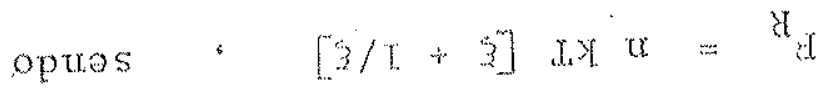

xod epep o t equeran o $\varpi_{y}$ onb satorew

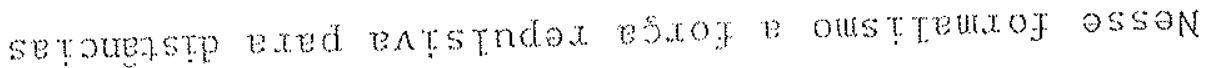
- opsentronde es

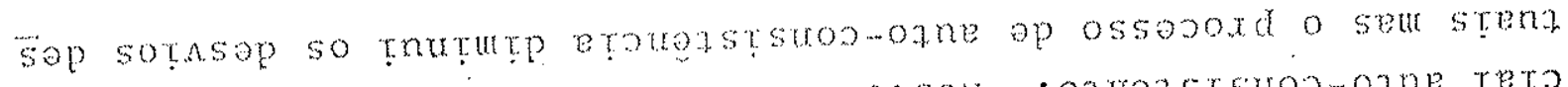

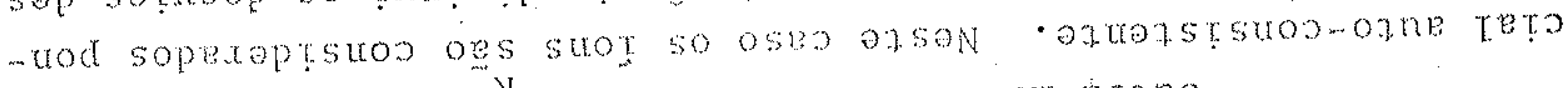

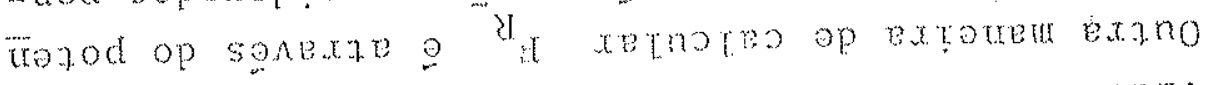

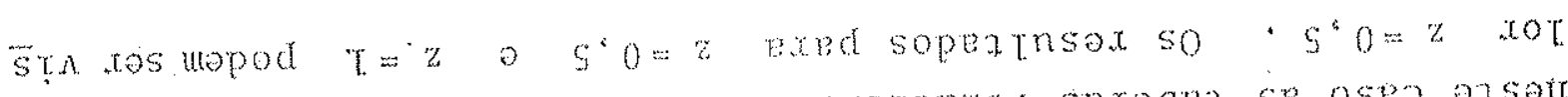

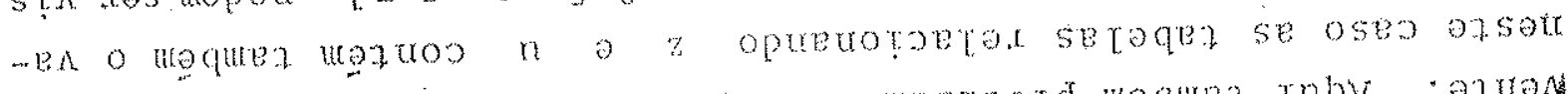

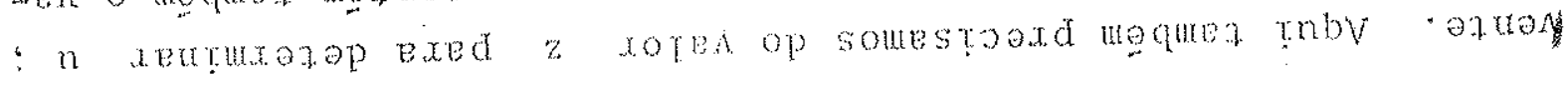


- $T=$ r racd oper

moted ${ }_{\Lambda}$ "seoerd se axzme opsexedes erou ep oesmy

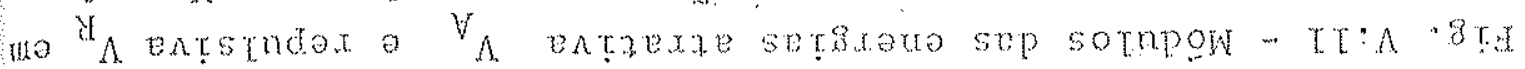

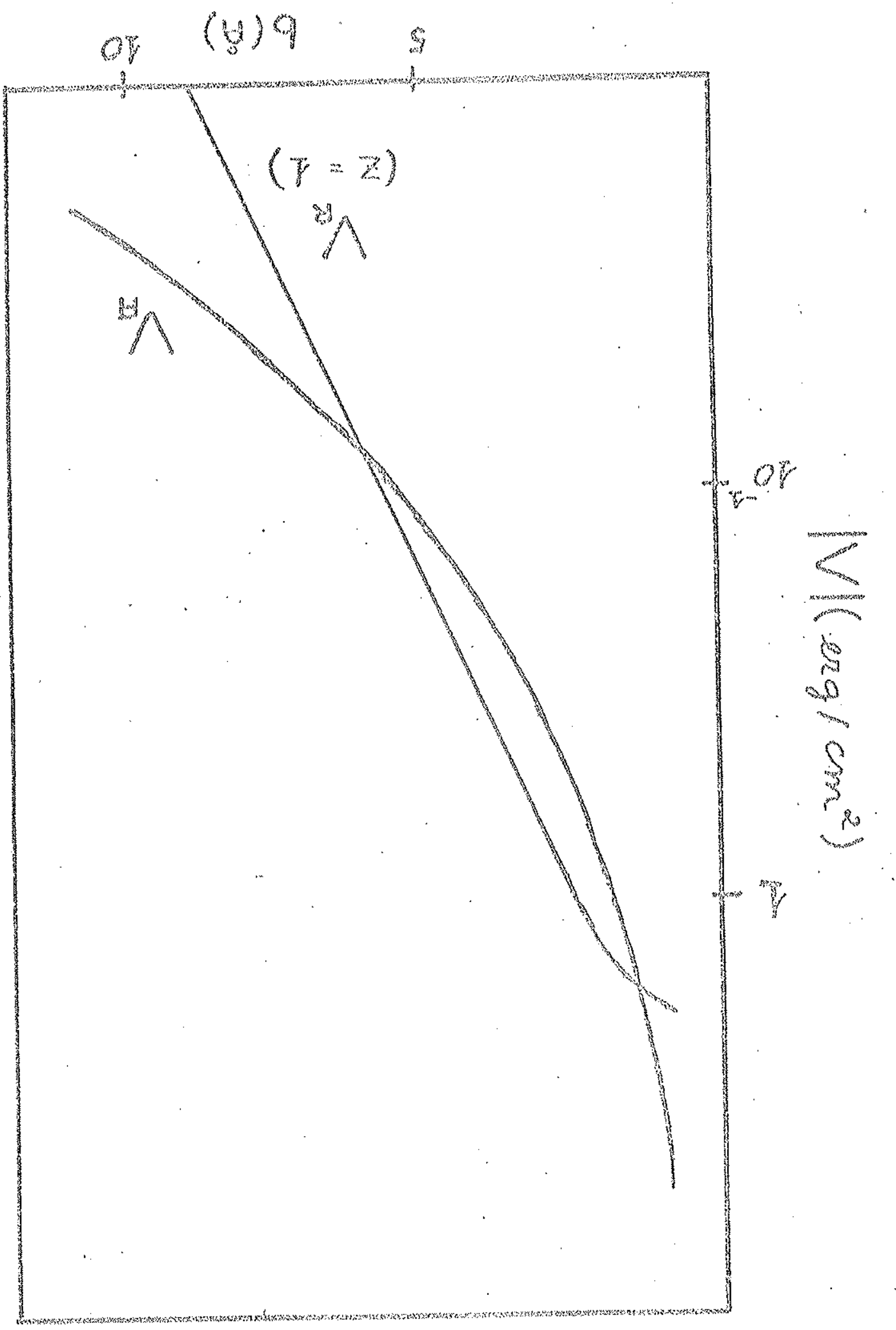


- 1 \% o $9^{\circ} 0=\%$ exad epet

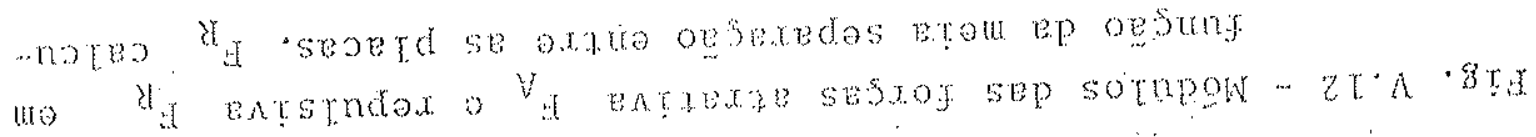

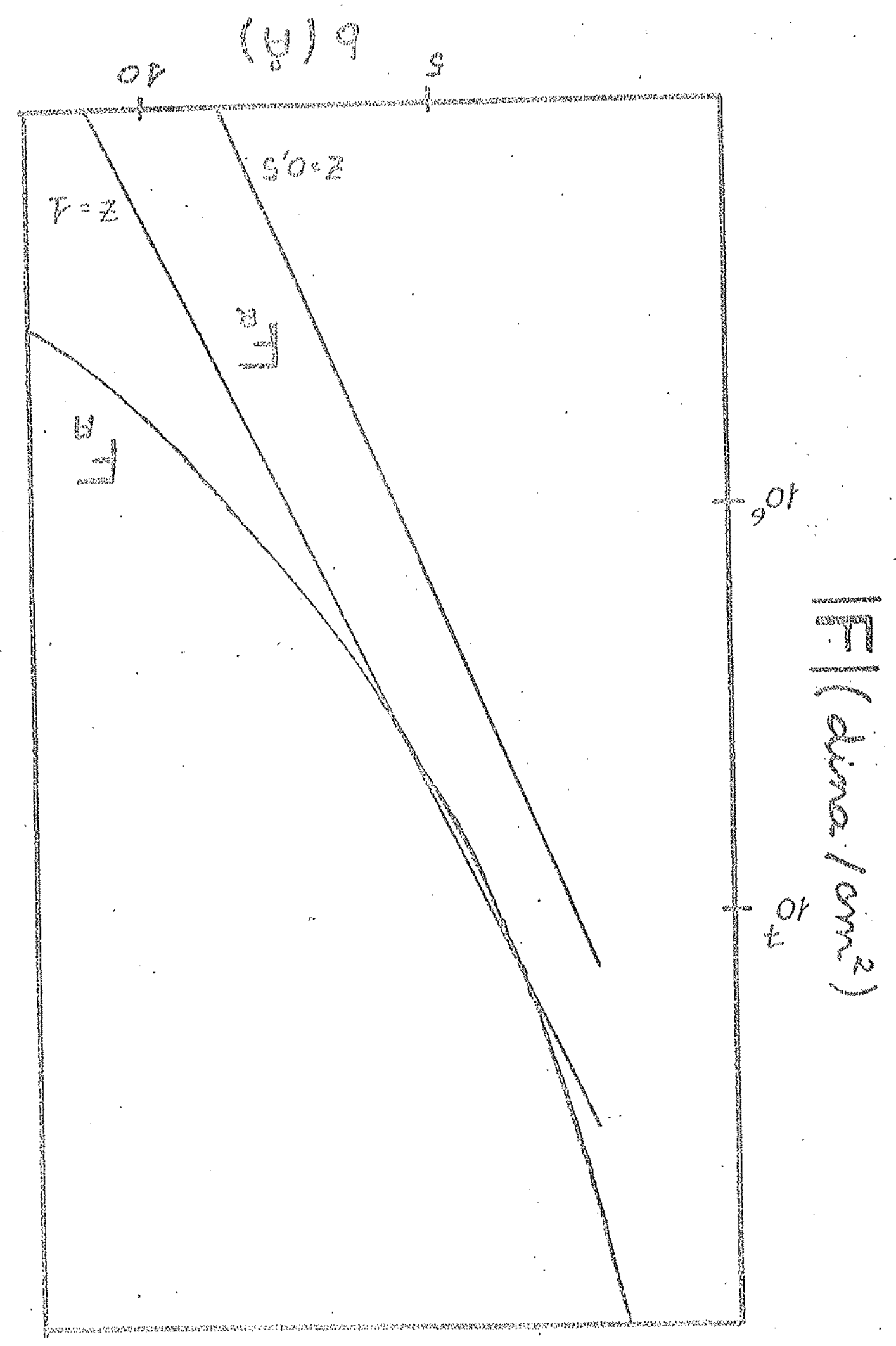




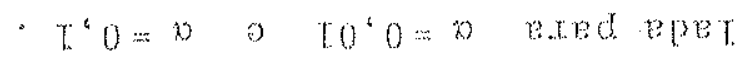

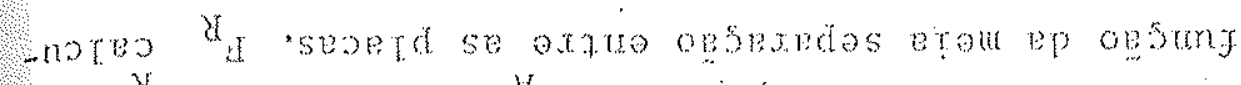

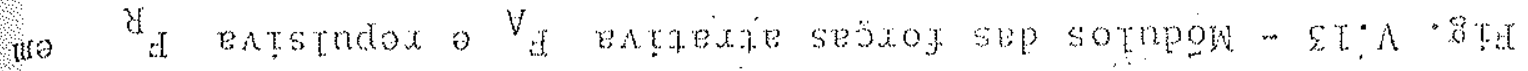

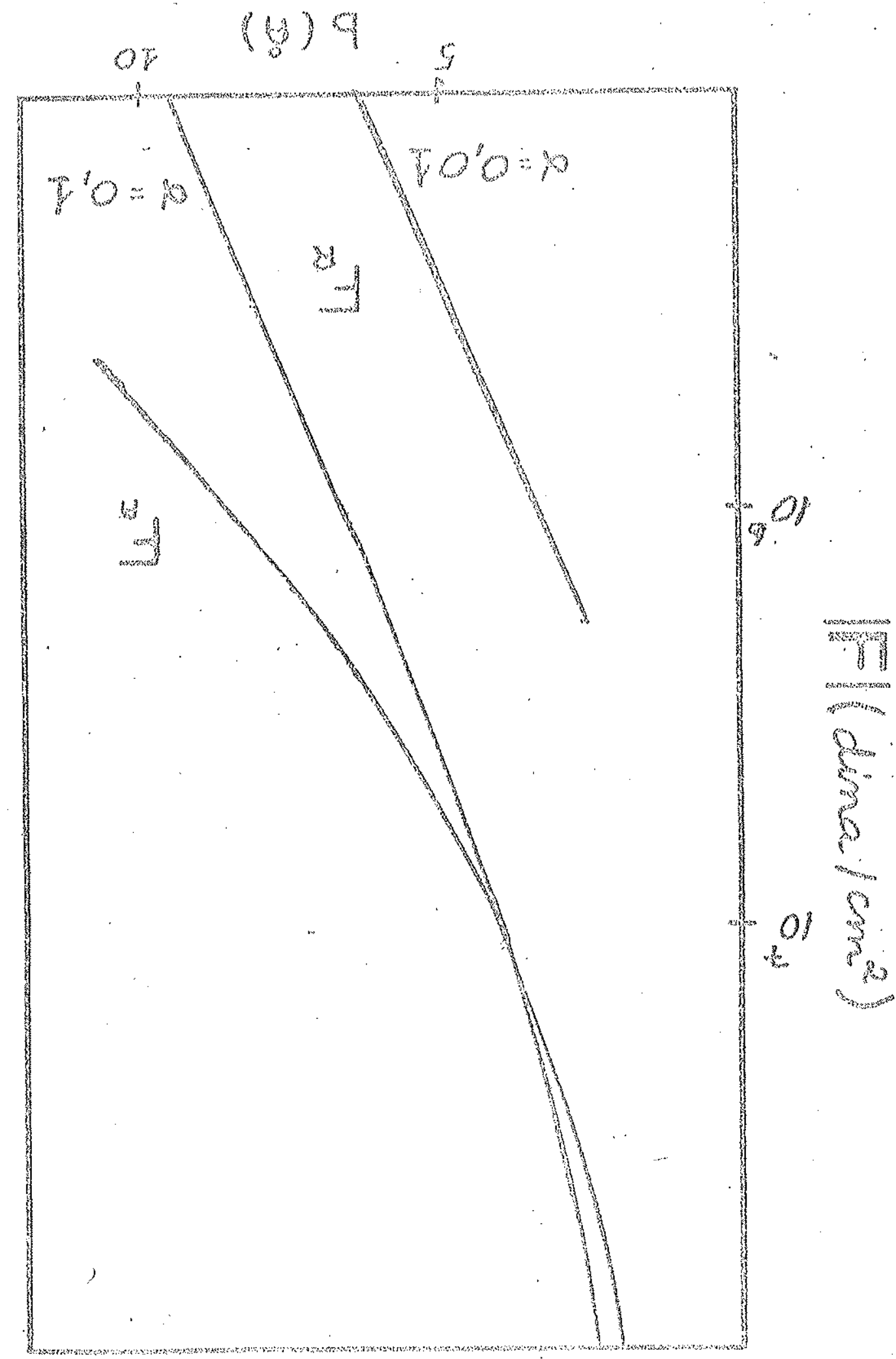


"Tetorozod op ex

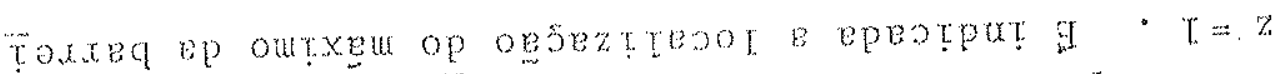

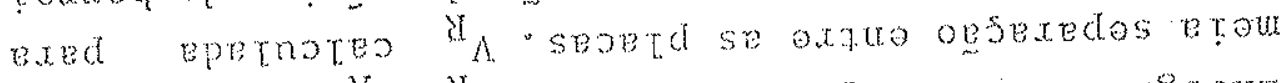

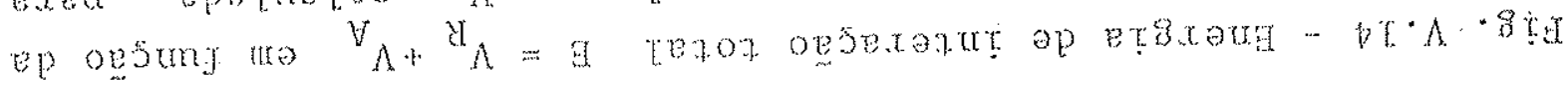

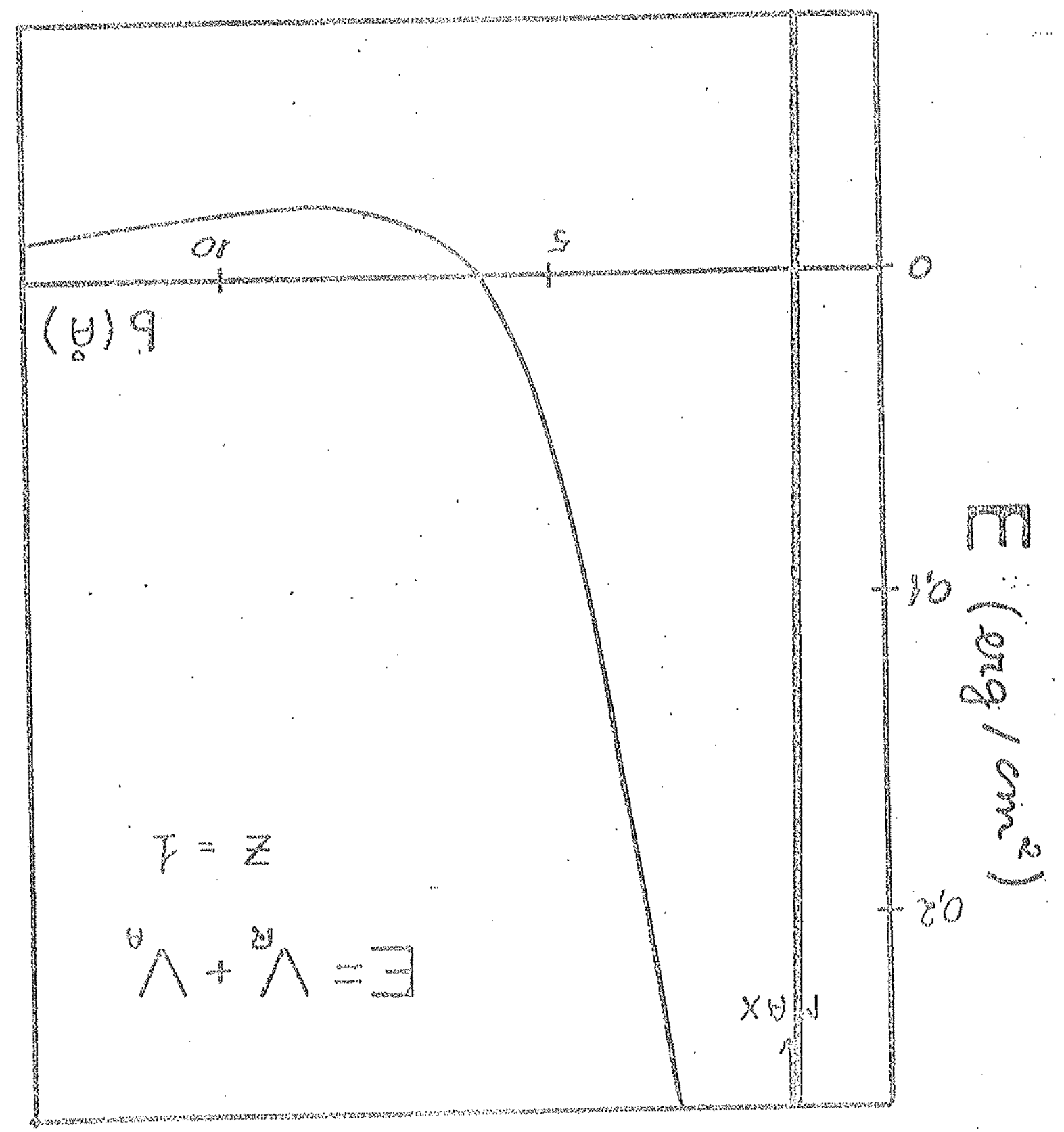




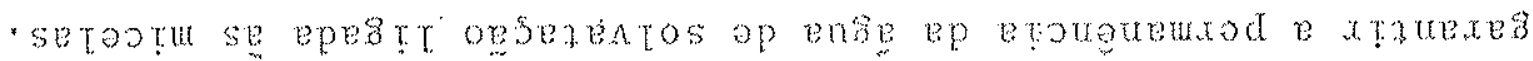

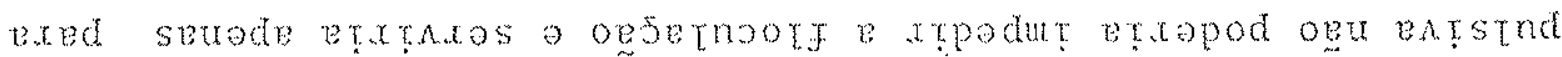

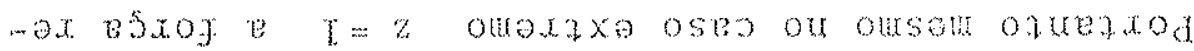

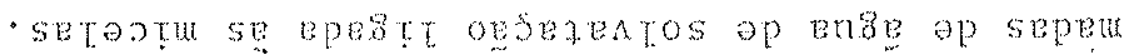

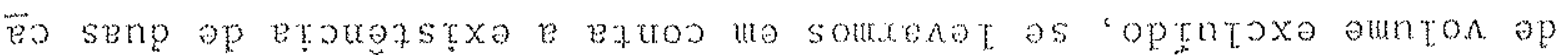

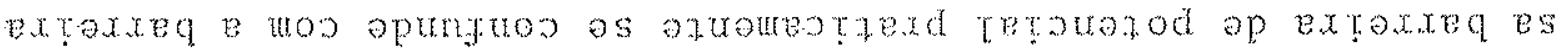
50 onb eurog op "(

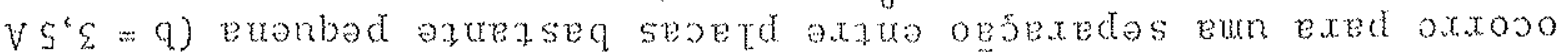

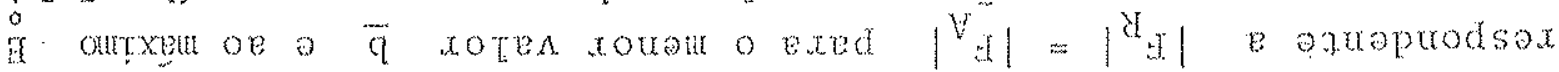

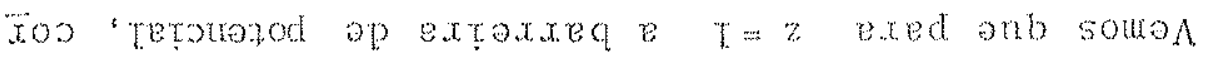

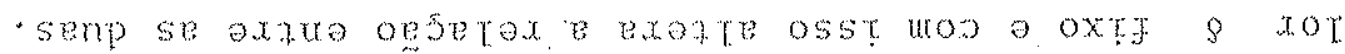

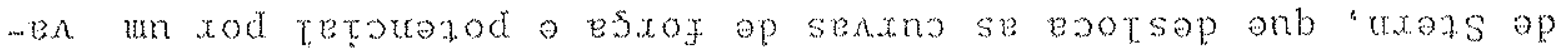

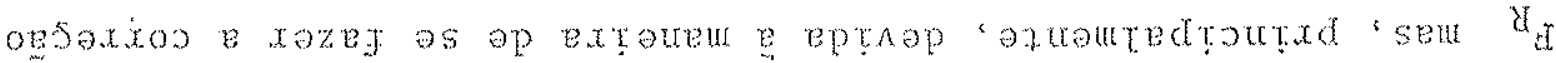

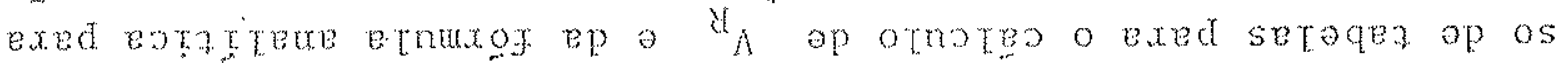

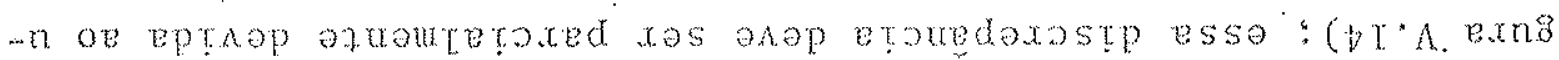

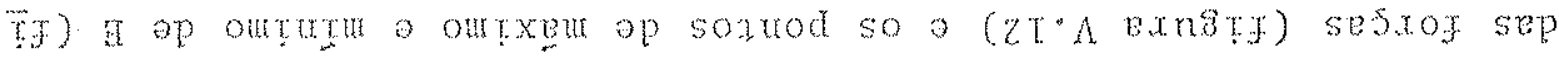

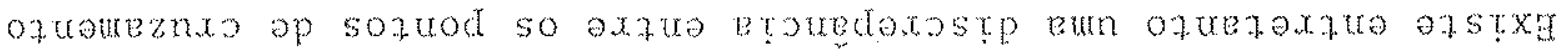

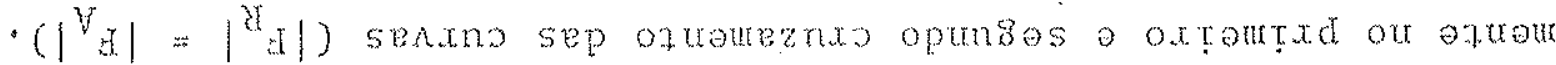

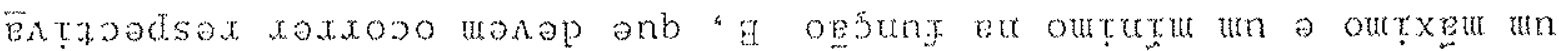

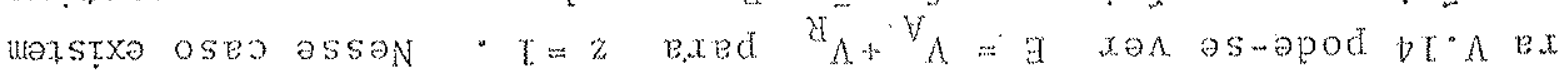

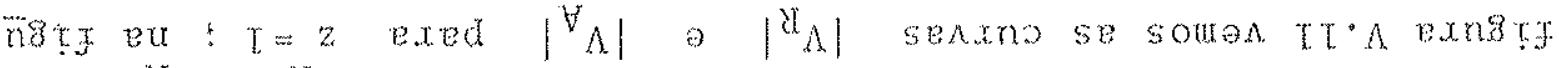

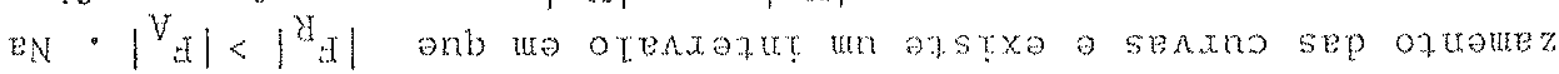

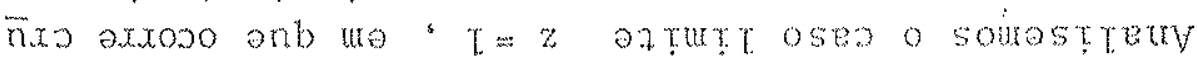

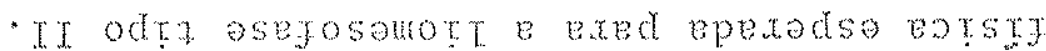

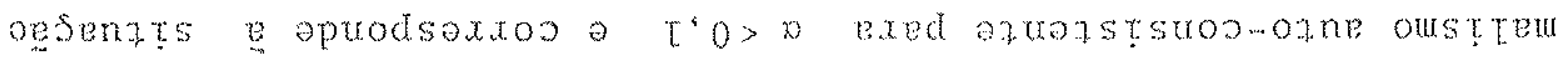

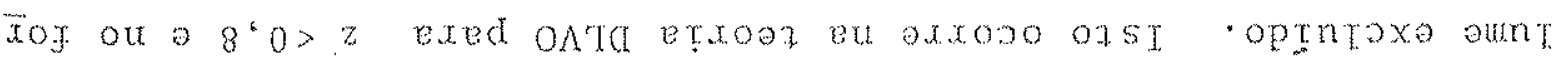

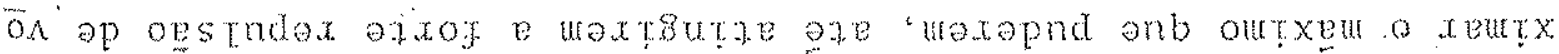

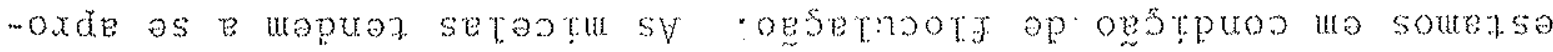

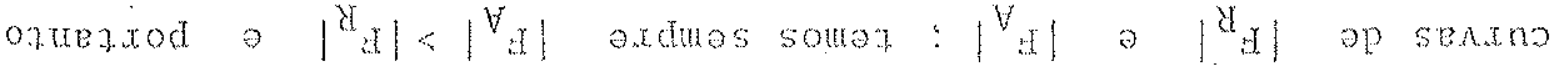

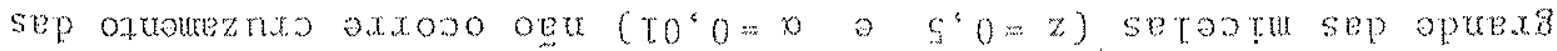

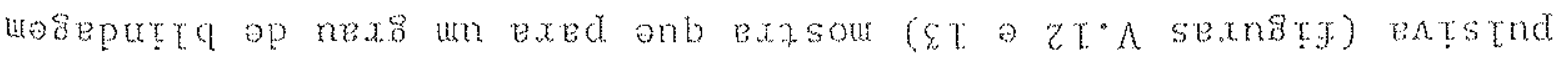

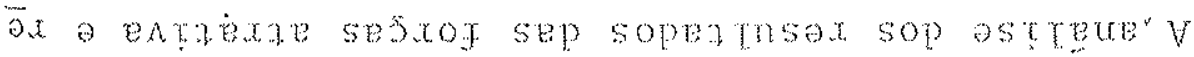

\section{Oessnosta $* 4$}

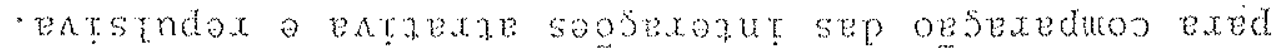

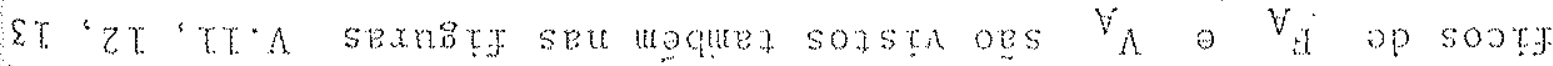

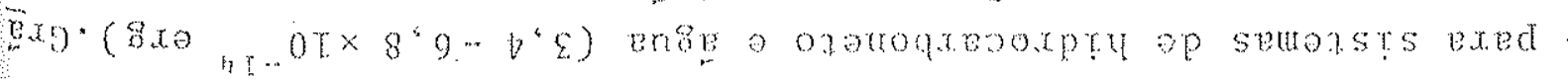

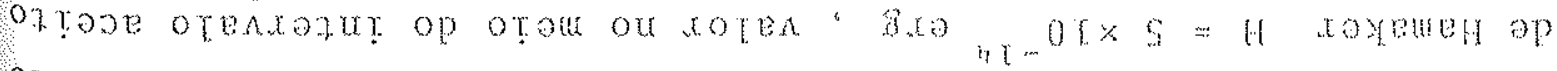

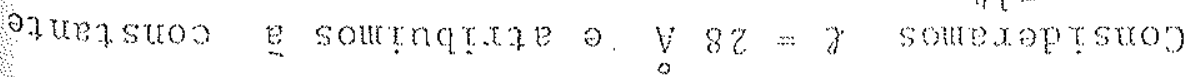




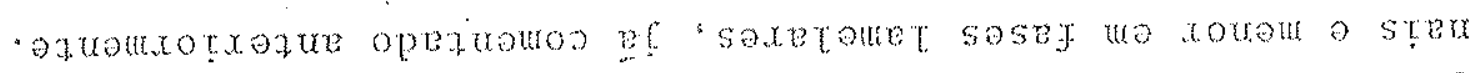

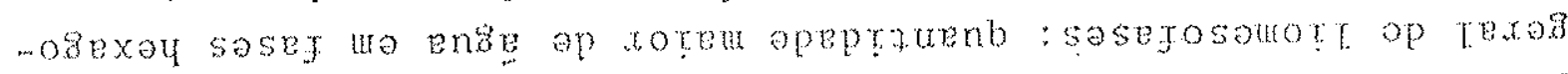

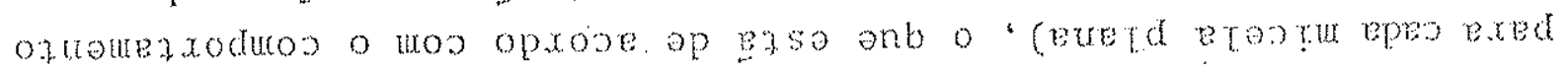

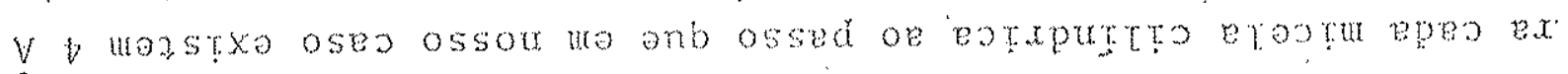

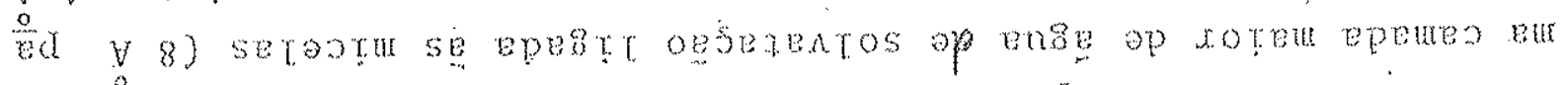

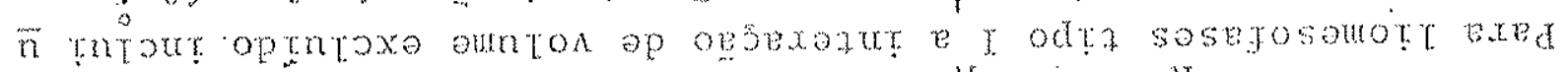

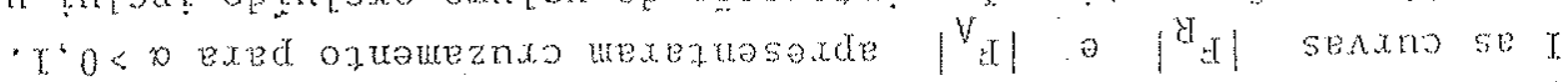

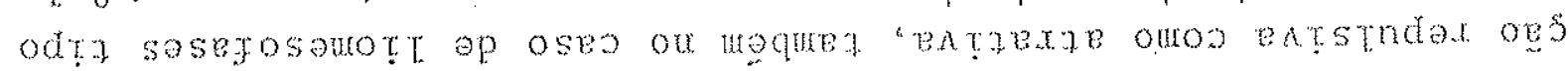

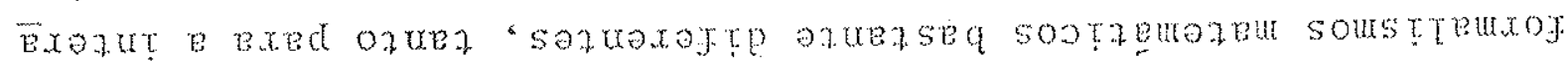

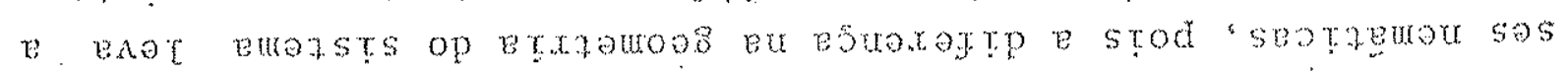

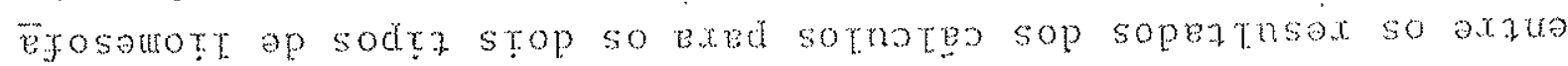

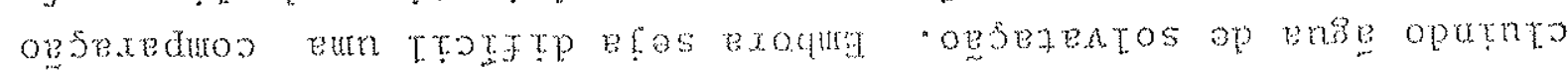

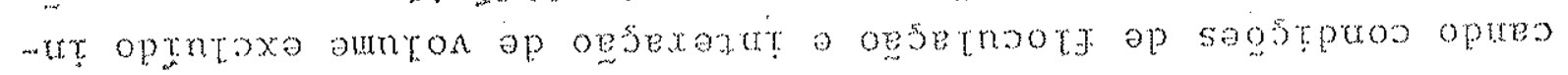

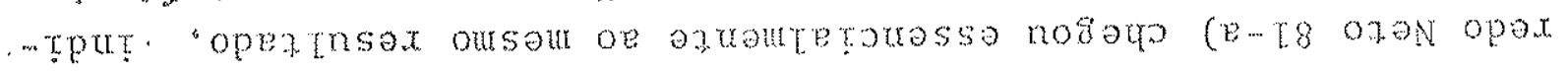

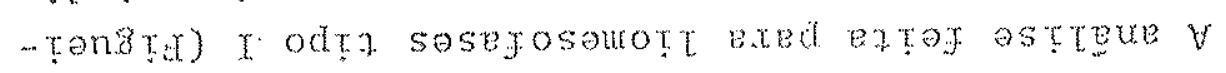

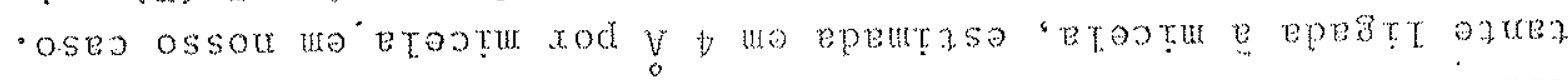

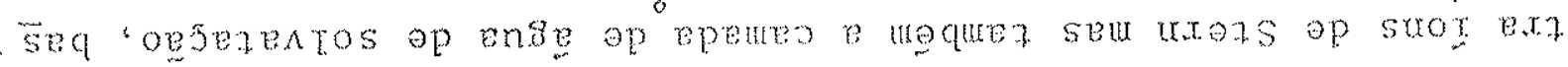

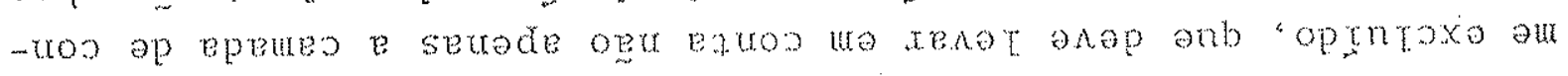

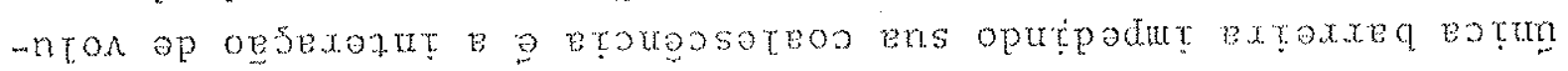

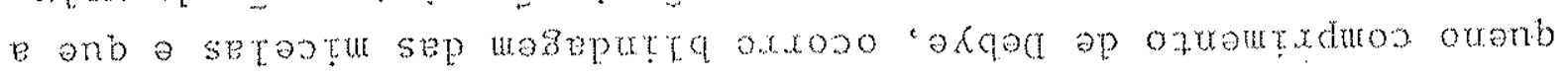

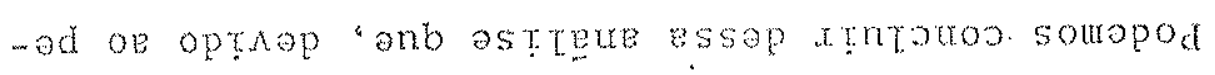

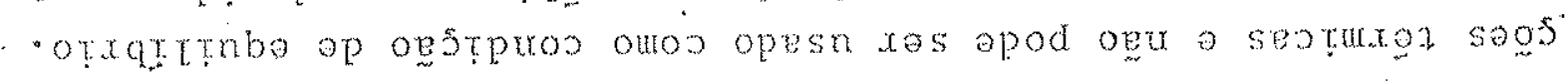

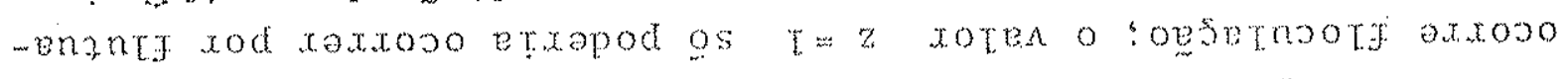

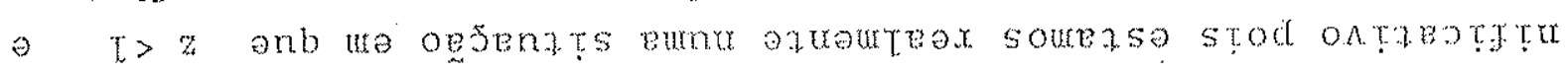

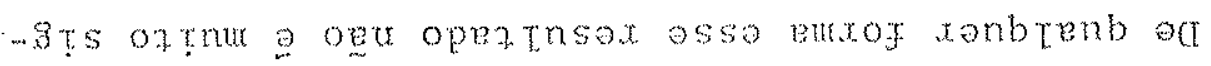

setno

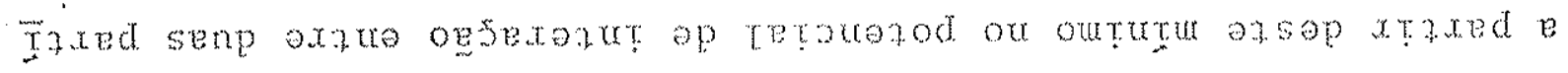

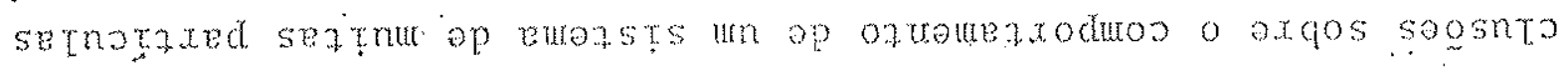

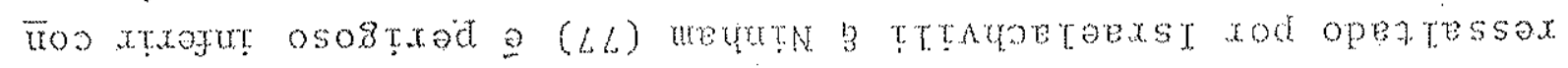

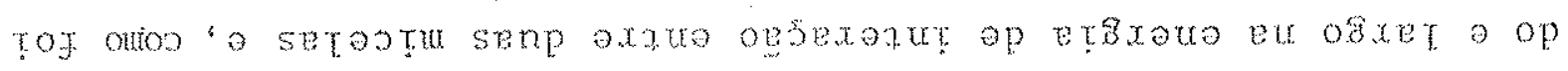

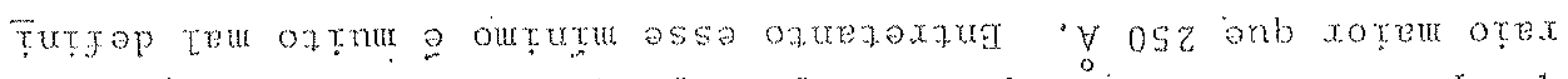

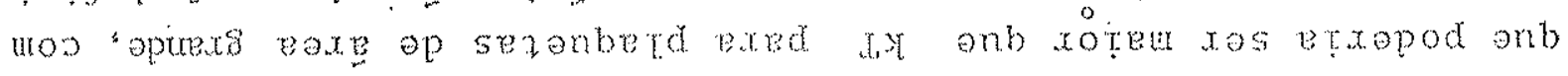

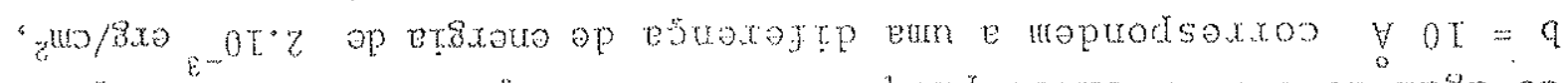

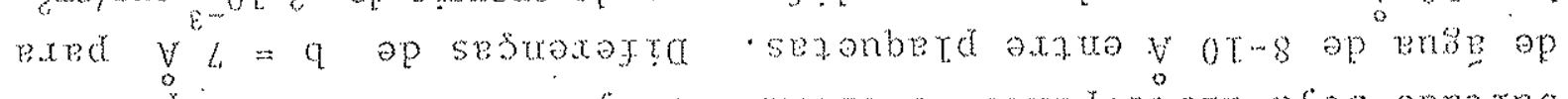

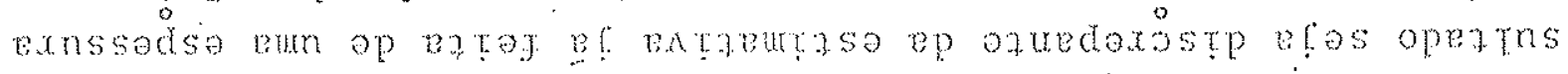

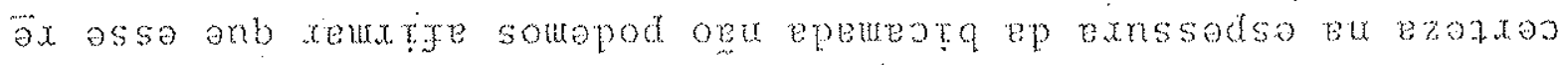

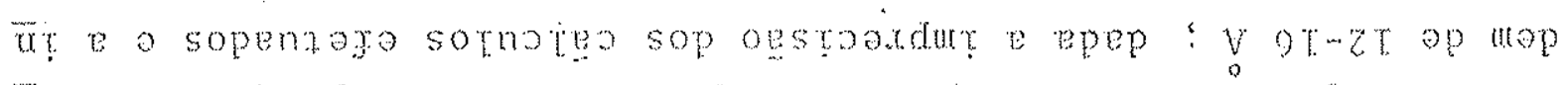

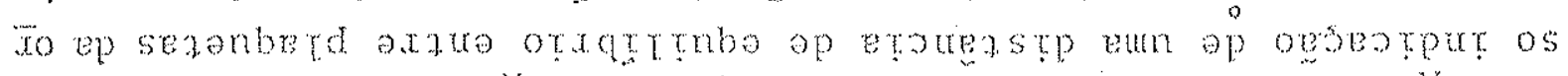

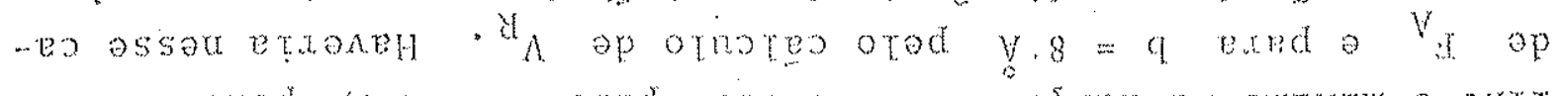

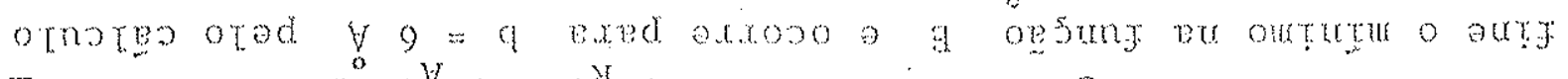

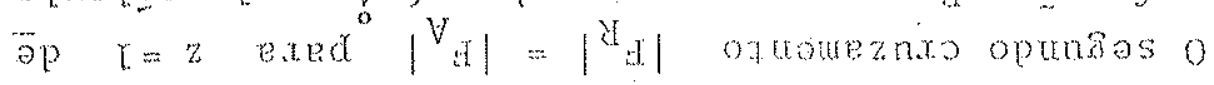




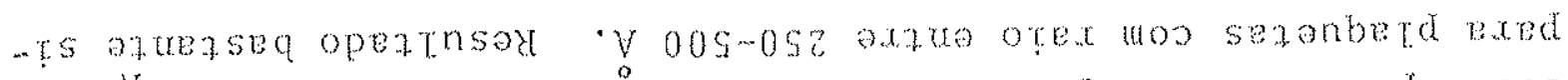

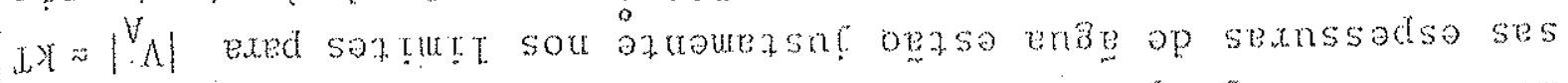

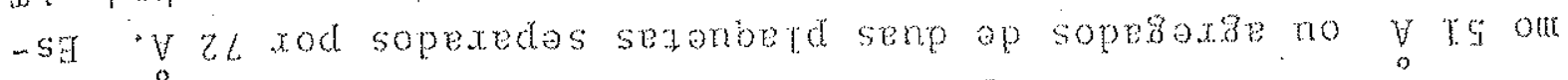

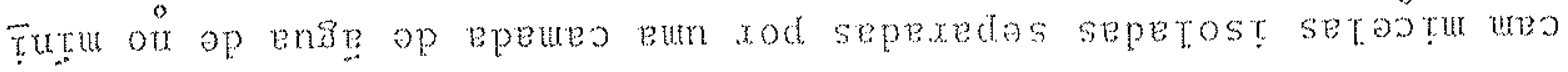

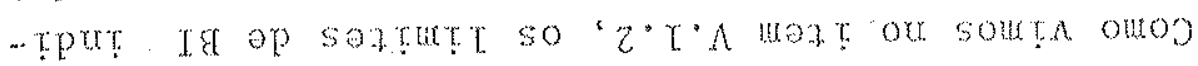

- opxazdos as steur ogu seto a dy onb roten ontru asmerroz

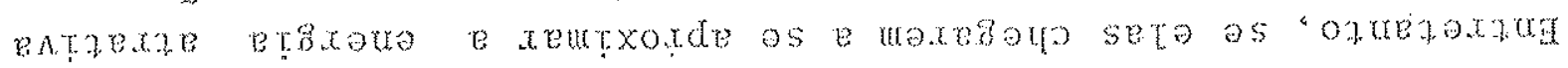

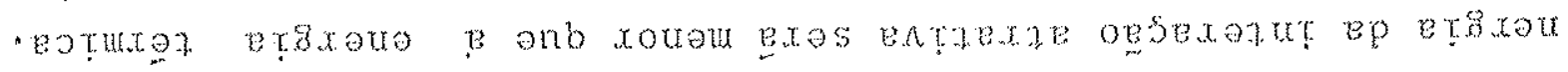

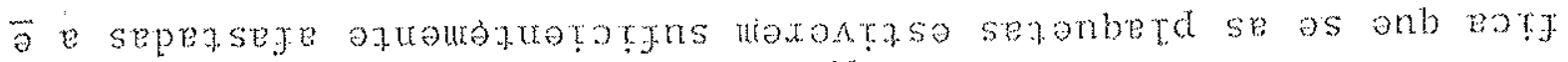

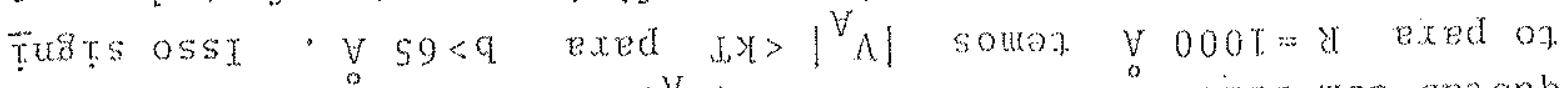

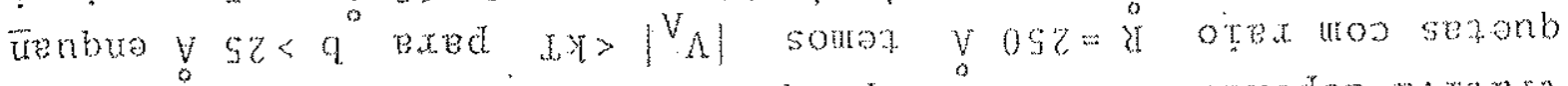

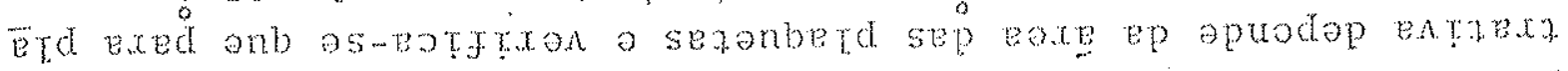

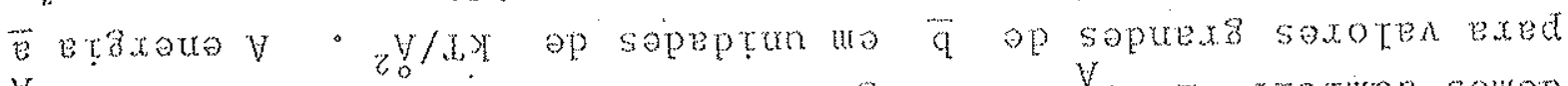

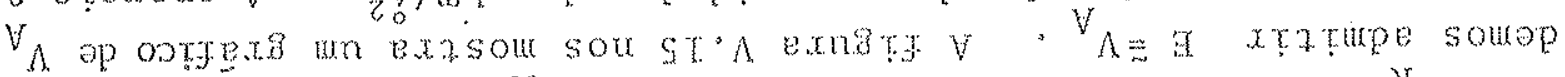

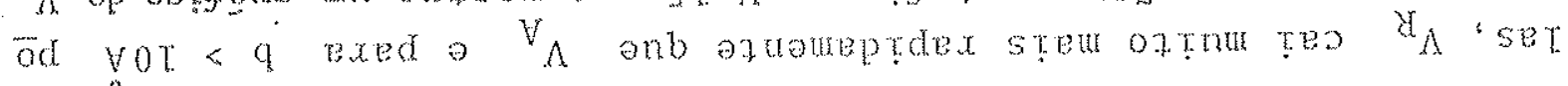

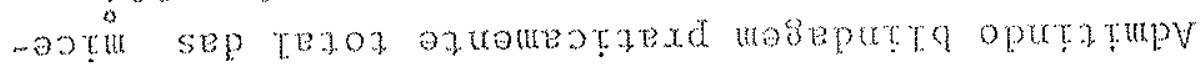

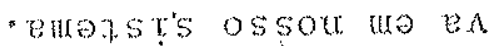

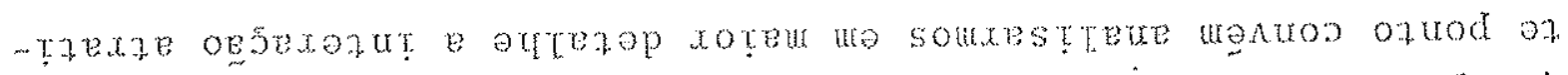

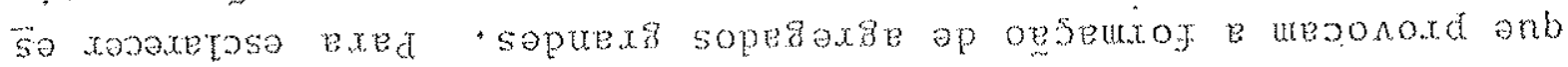

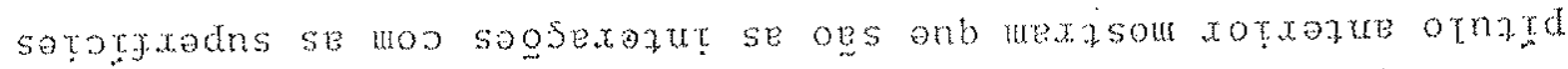

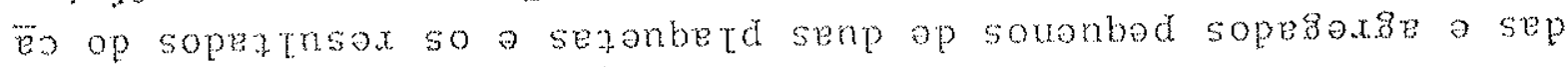

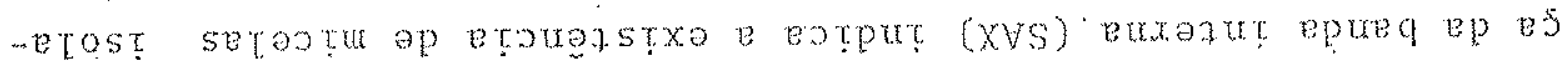

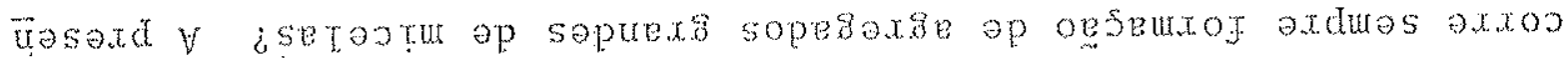

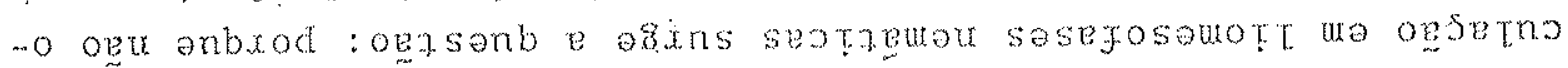

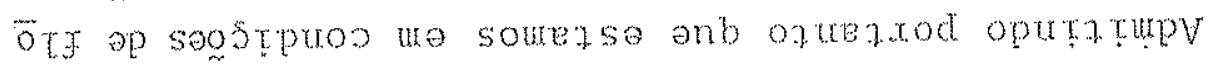

- semexts sassep oppettrqeaso e exturazop onb

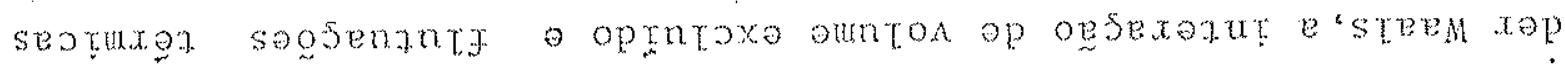

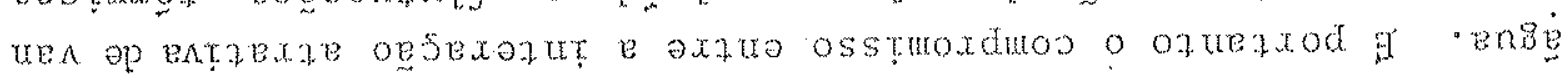

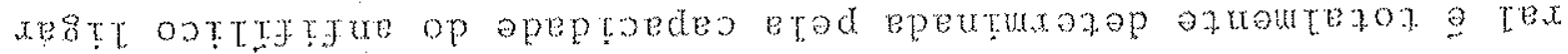

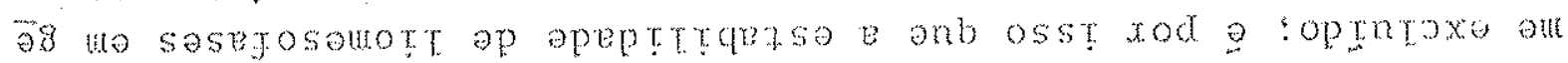

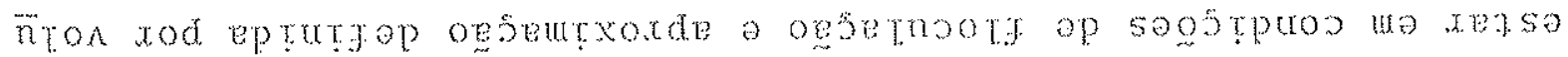
monep ugquez stemotatrantion sesegosenot "ozmatod

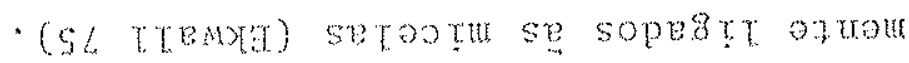

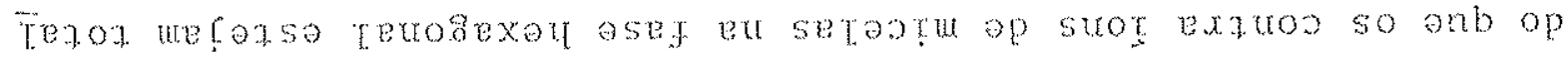

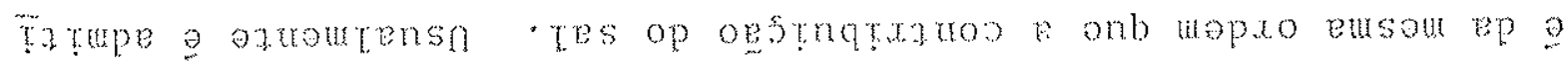

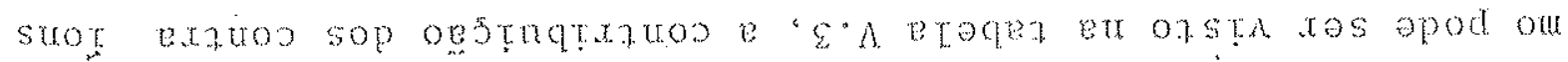

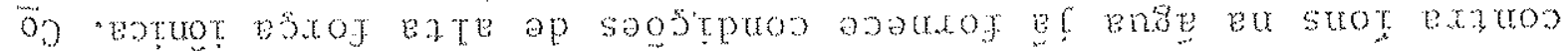

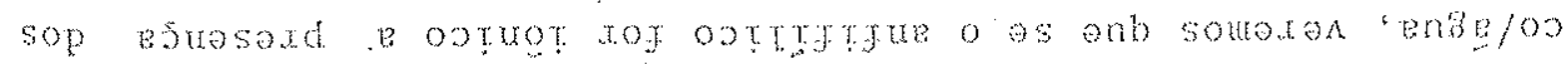

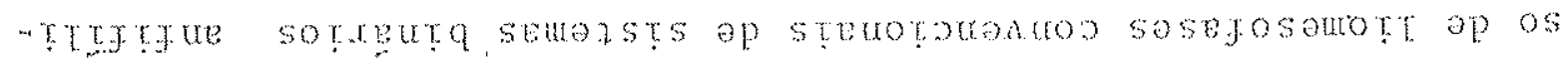

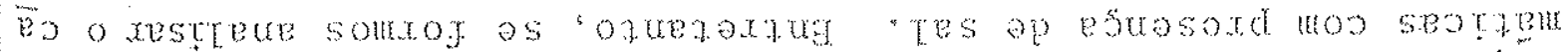

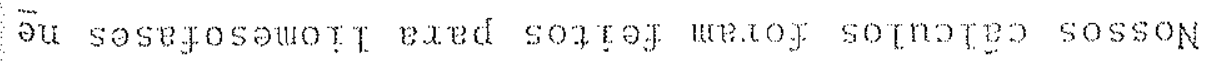




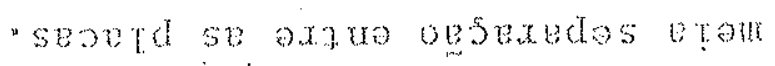

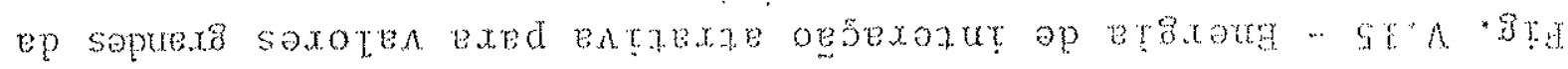

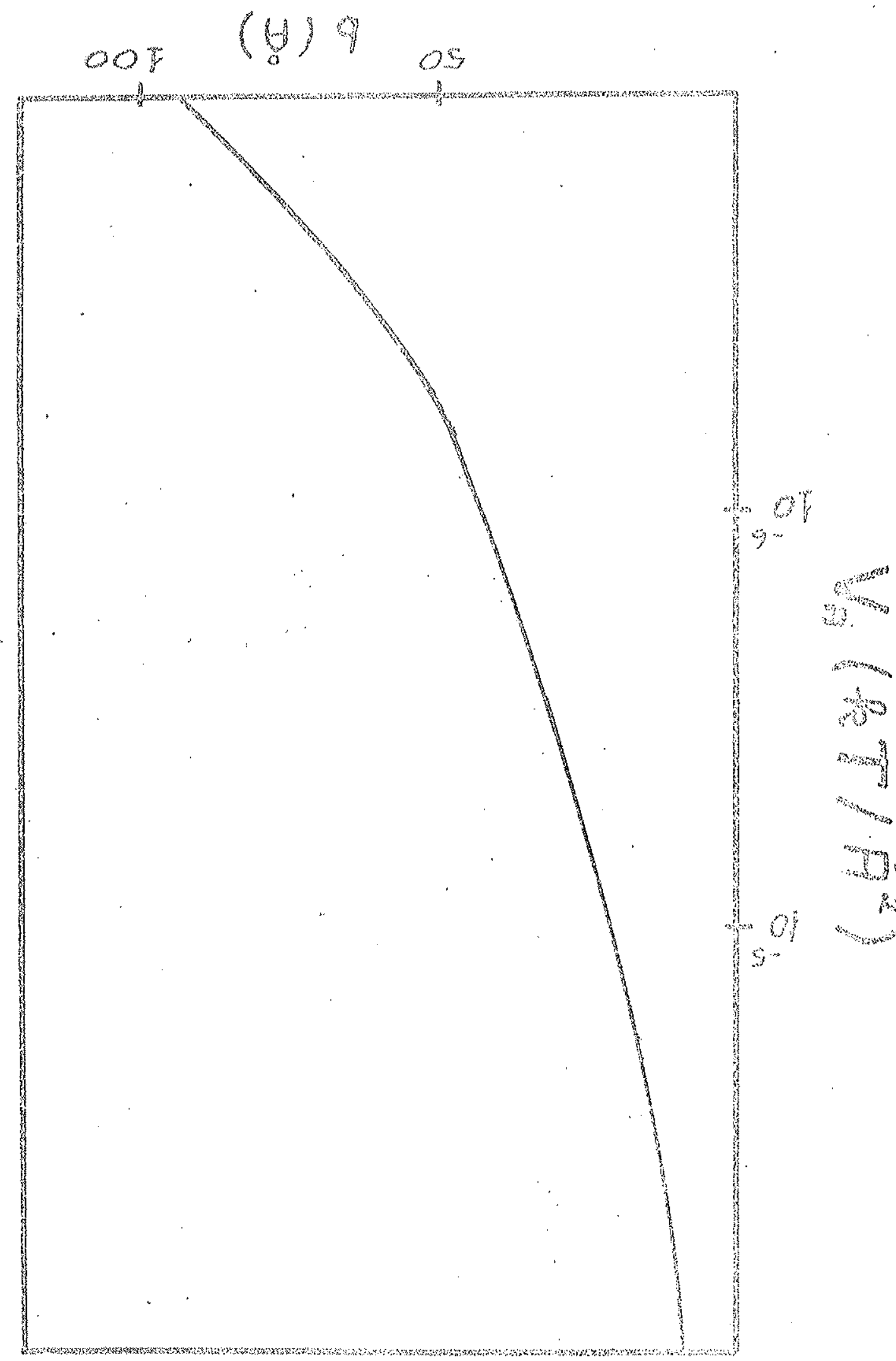




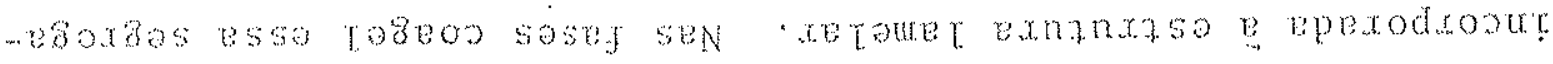

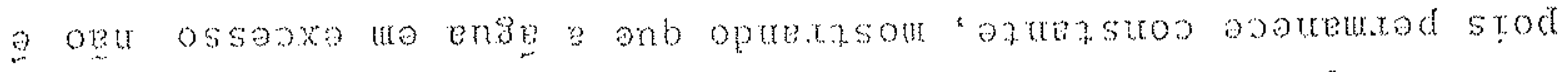

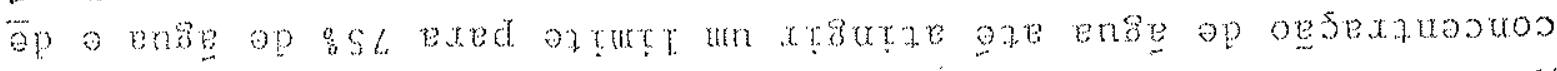

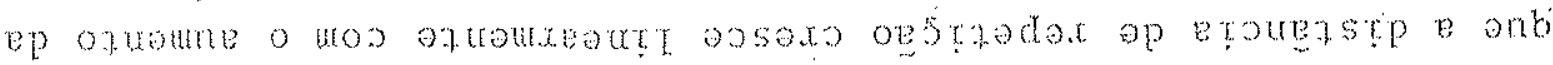

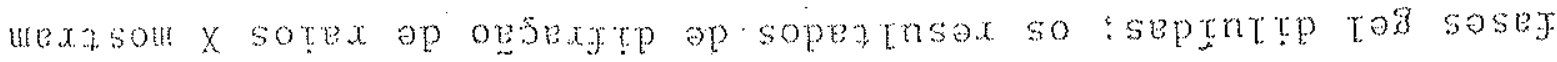

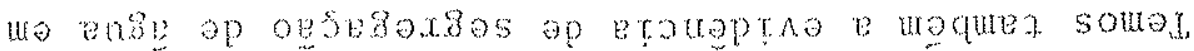

- ense/sages sotatu

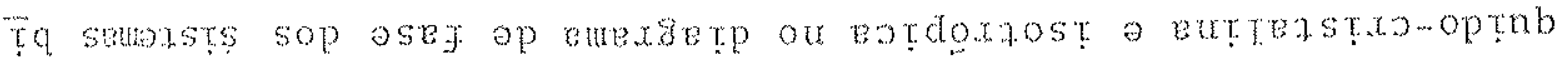

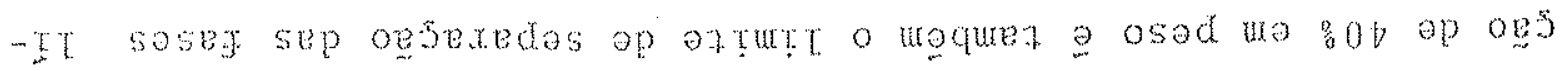

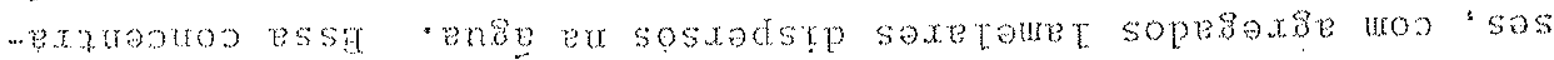

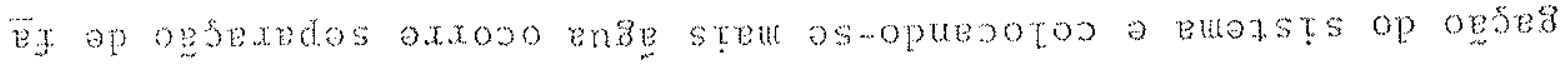

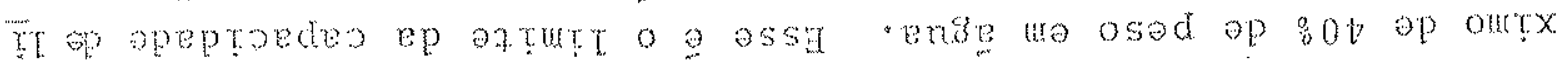

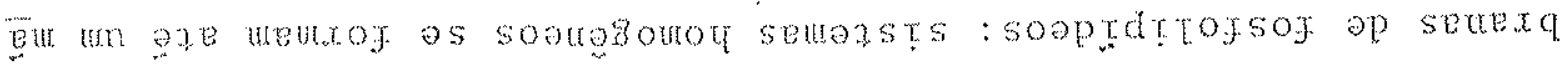

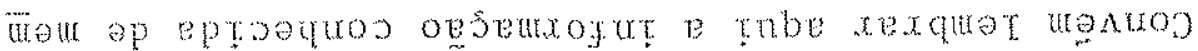

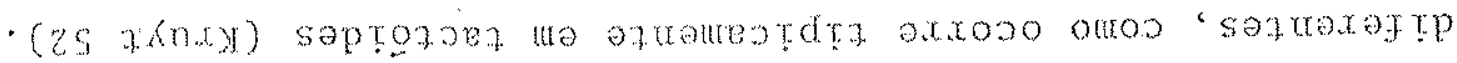

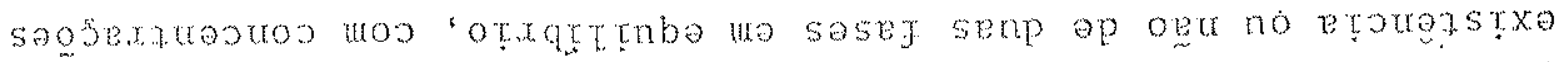

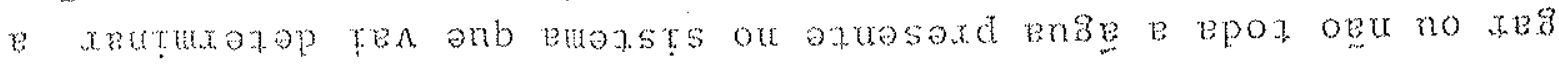

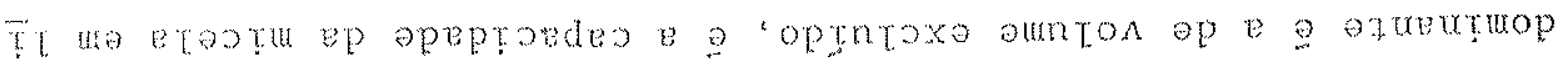

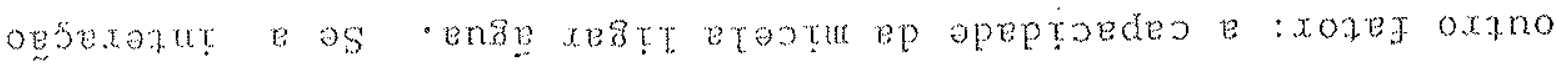

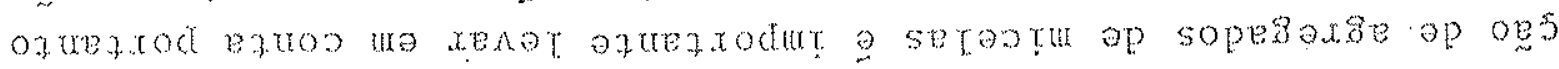
Eurog ap ogasonb easop ogseptonte xodreu bur ext

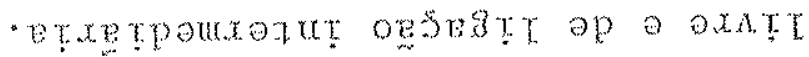

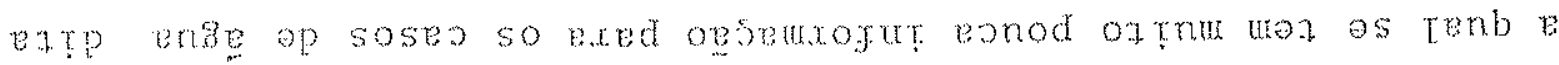

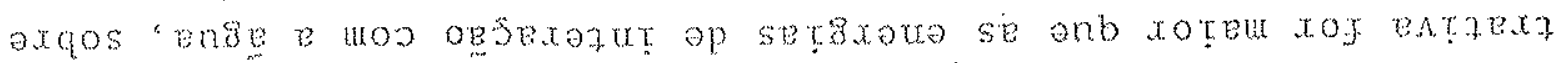

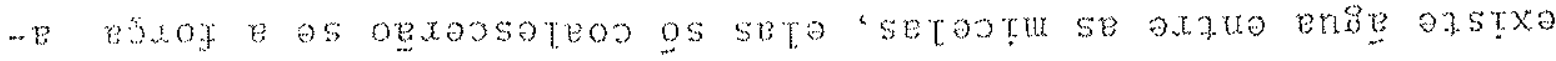

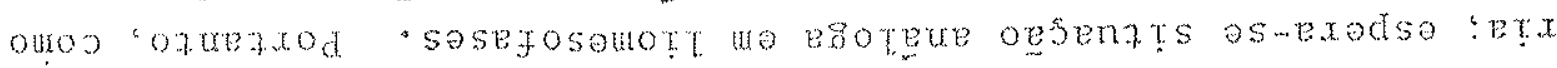

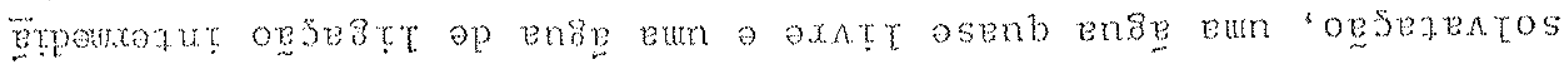

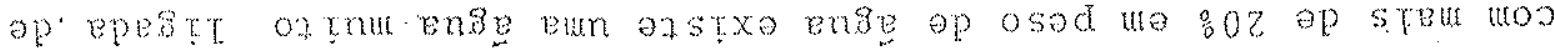

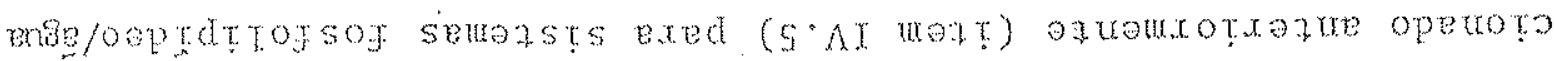

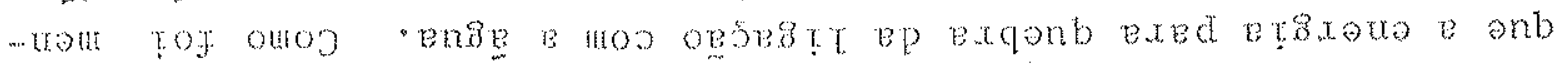

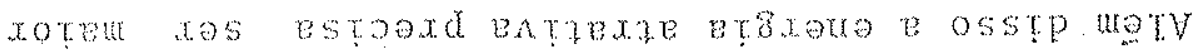

$\cdot \operatorname{sop}$

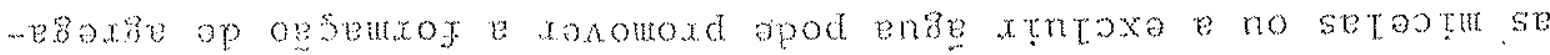

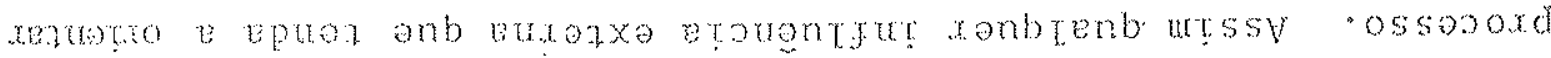

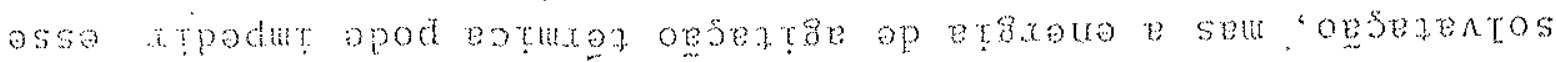

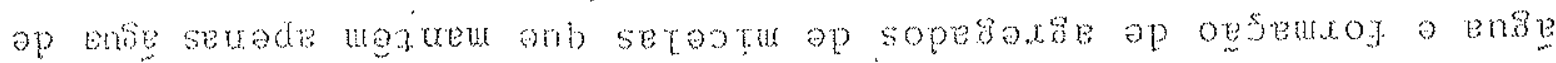

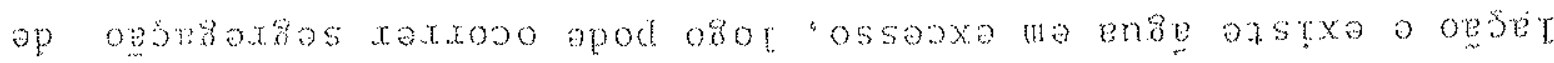

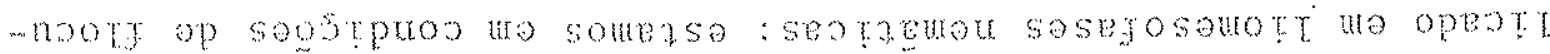

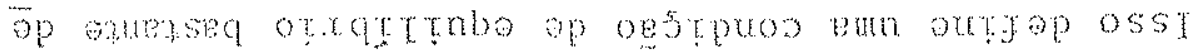

- Otrograget

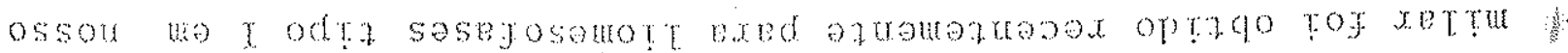




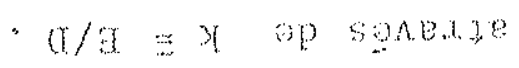

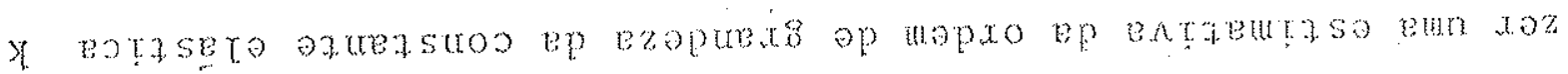

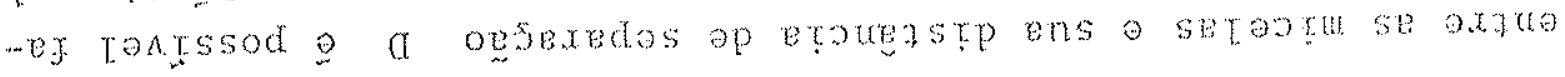

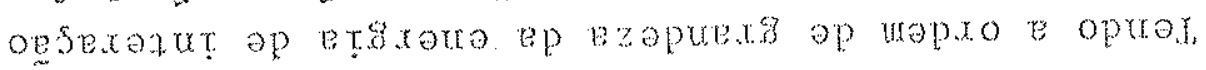

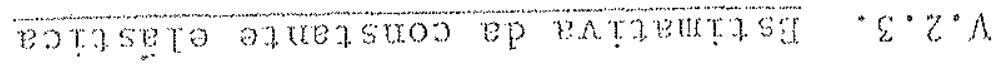

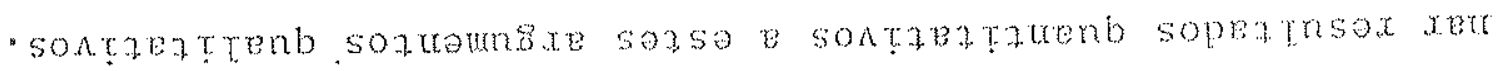

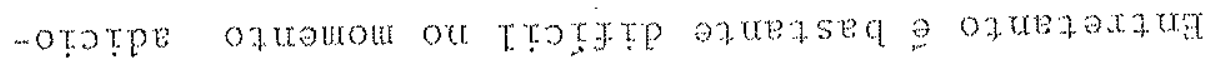

- ogsntos a toode manosax.

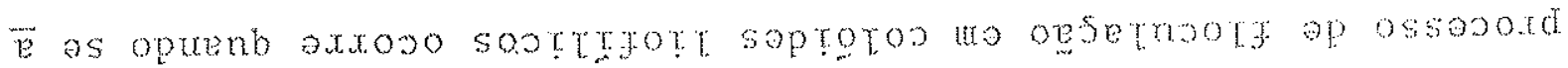

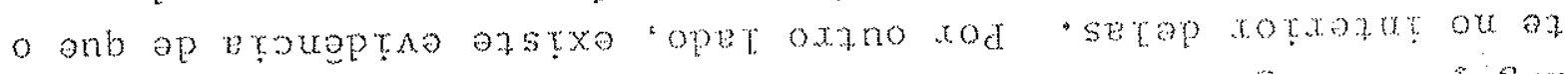

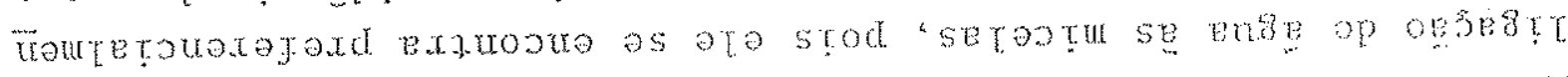

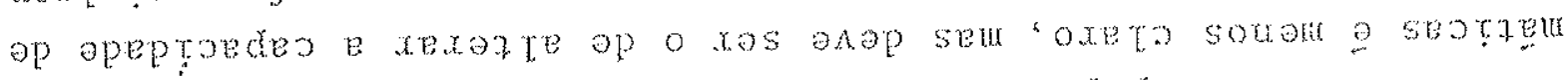

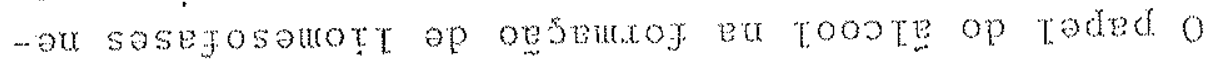

\%.

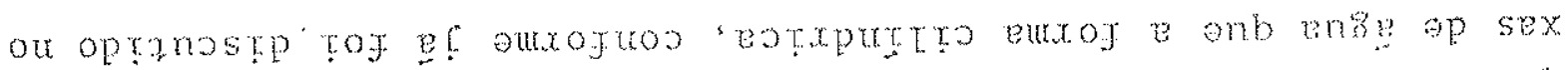

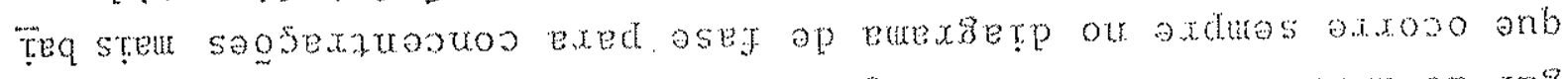

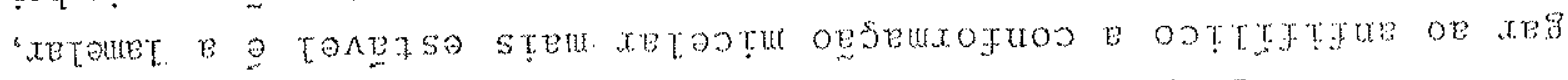

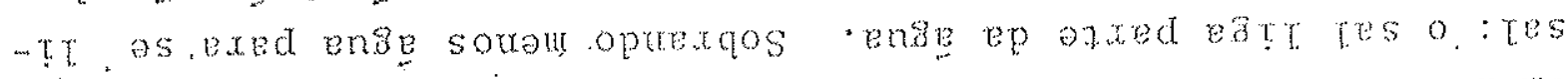

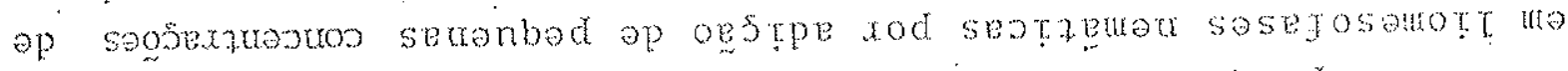

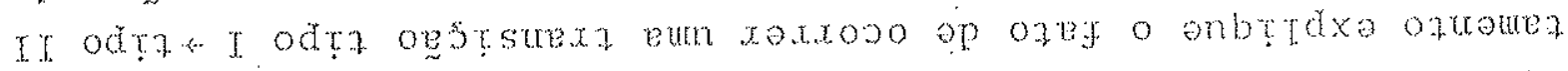
woduos op odtz assa anb ozuezrod xtrupe somepod

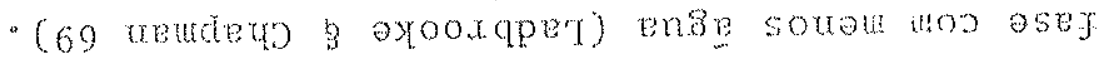

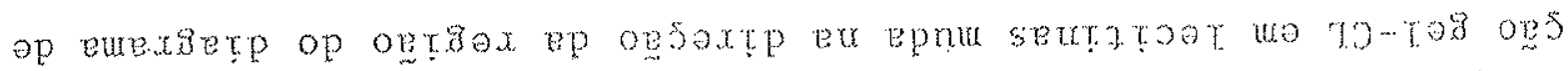

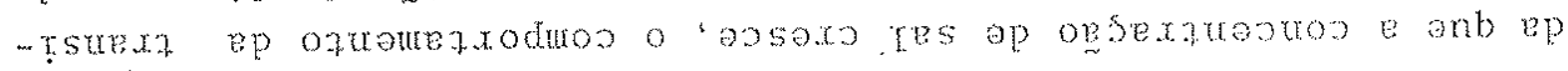

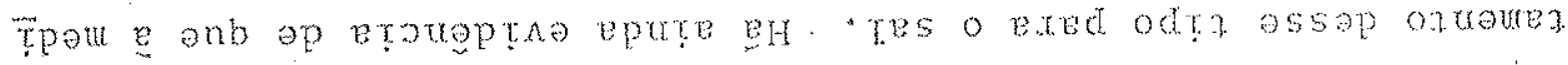

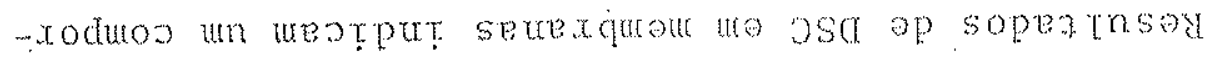

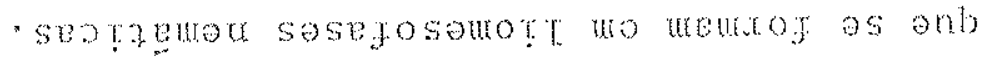

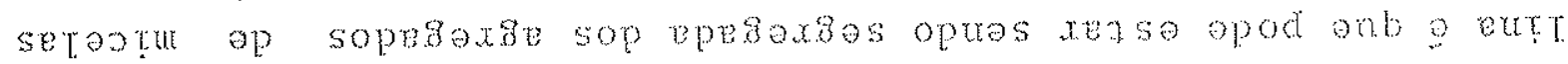

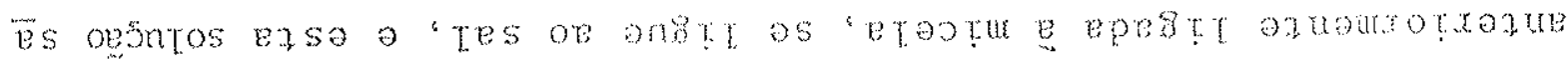

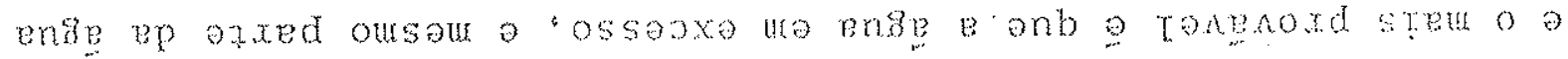

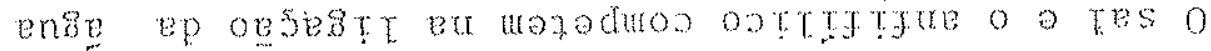

sontrpe stop soseop atotonty

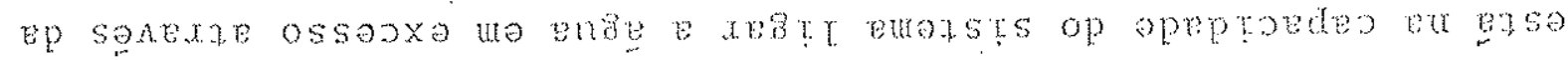

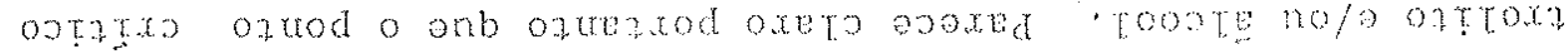

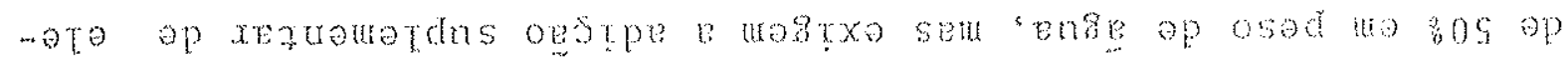

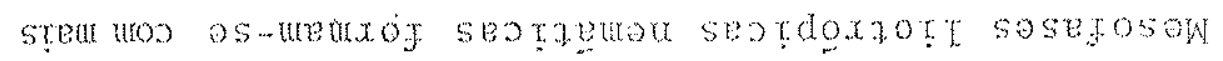

- (og sotraogs 3 anopran son

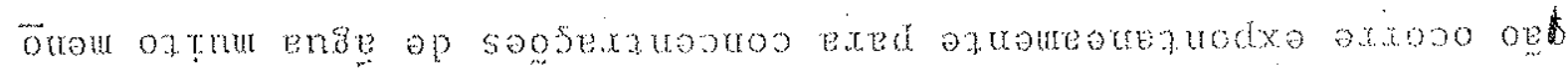




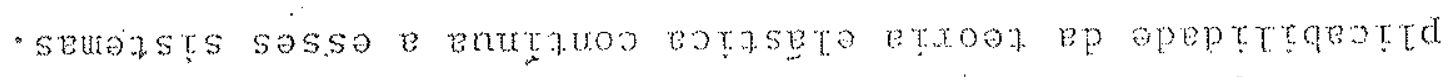

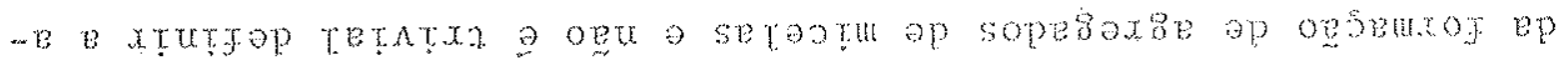

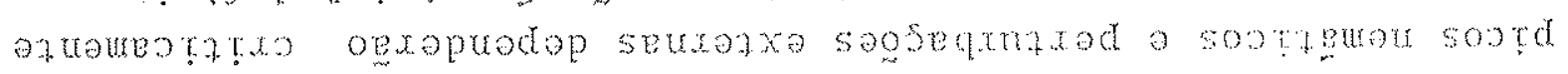

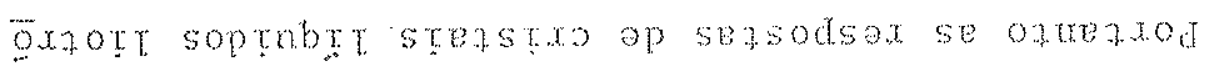

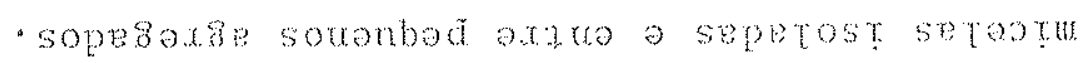

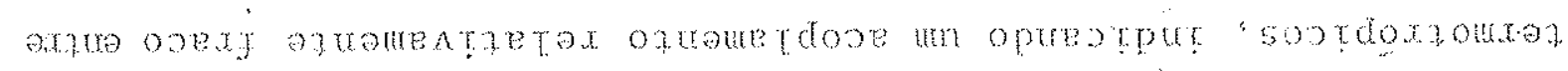

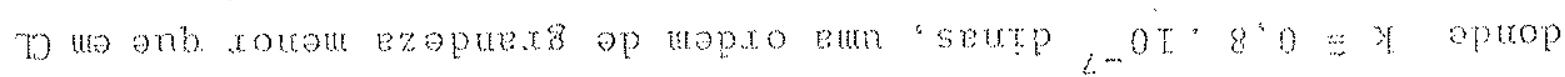

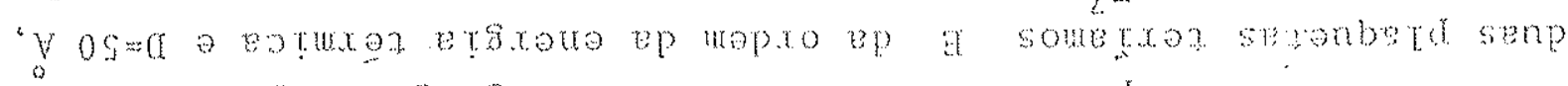
ap sounbod soperoxie no seperost seroorit exed er

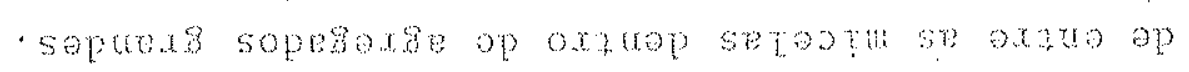

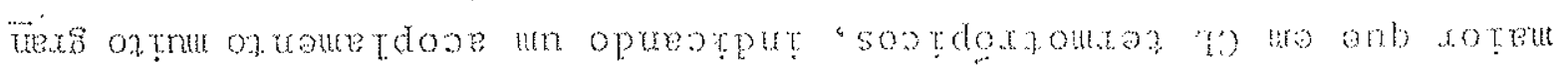

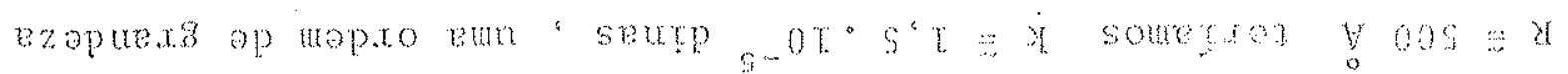

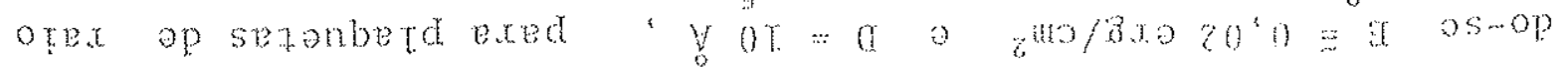

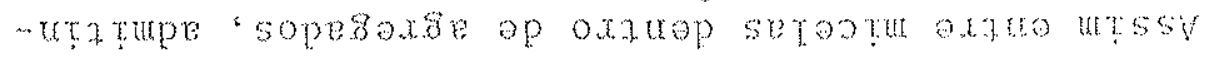




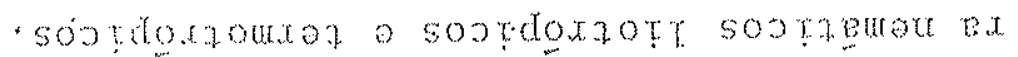

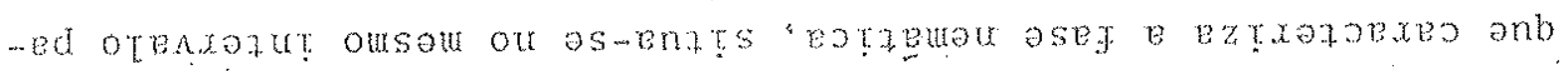

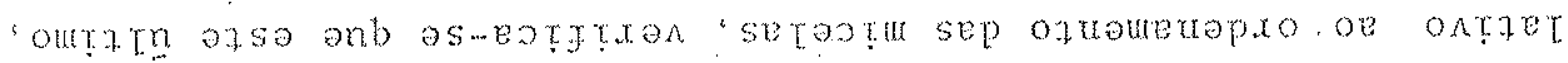

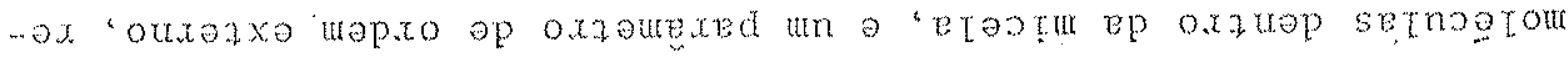

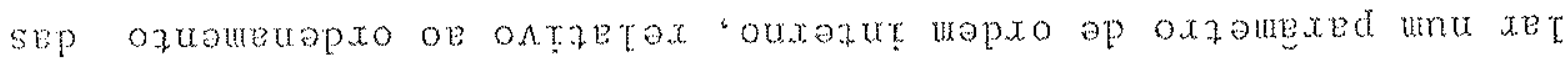

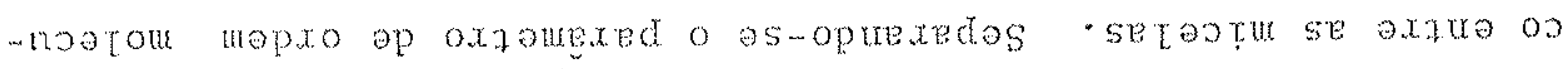

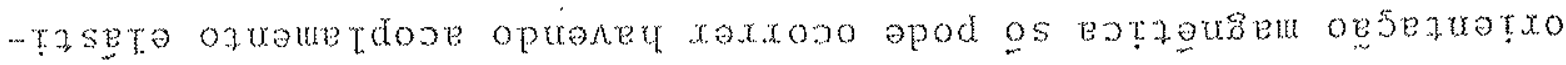

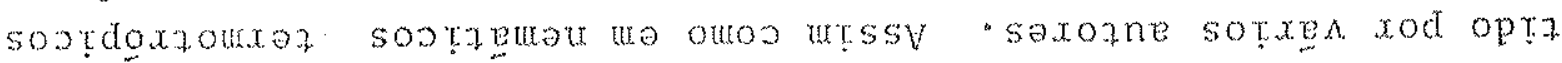

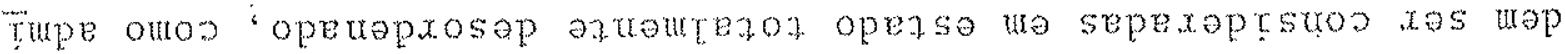

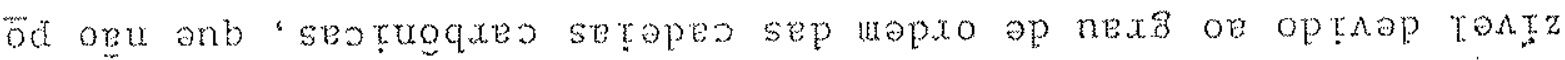

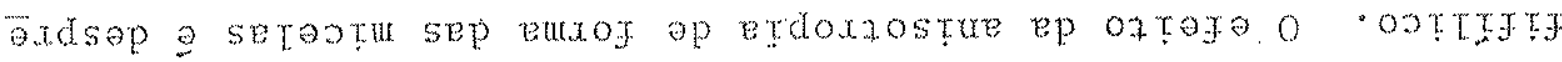

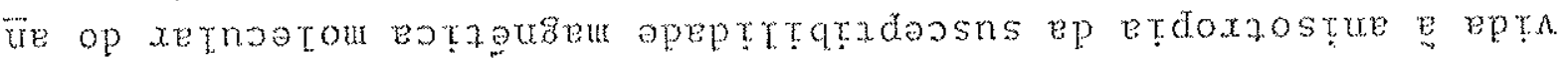

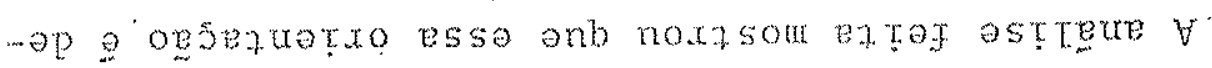

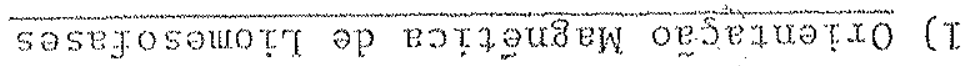

: sugr sezurnsos

sou oqteqexi ansop saosnimos $5 \%$ xtunsed souropod

- xeprase e soliasndoxd sou arib. sozunse

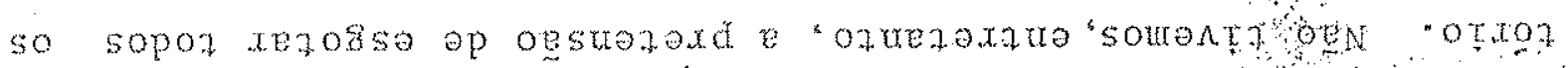

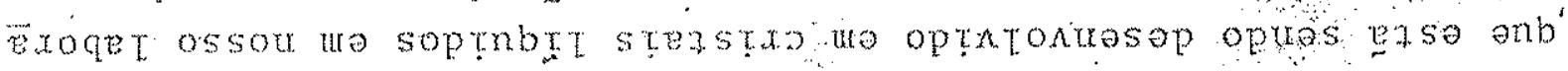

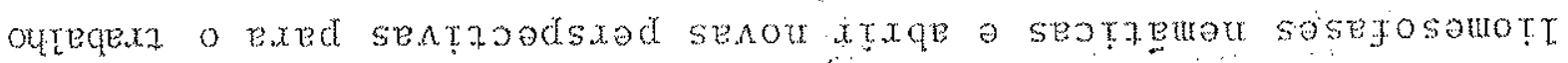

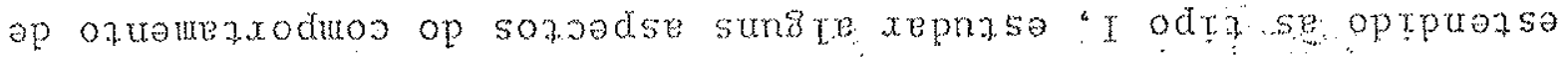

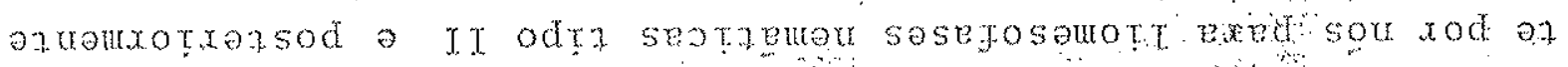

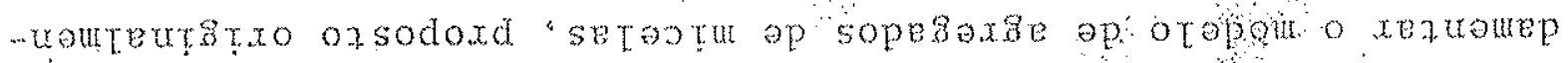

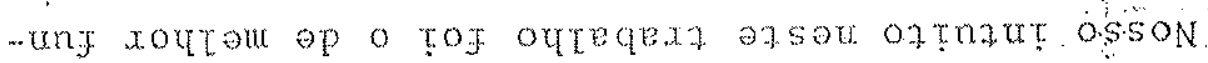

S90SATONOO

$I A O T \cap L I d V J$ 
- Teuxore ogsetex we oot ap seper

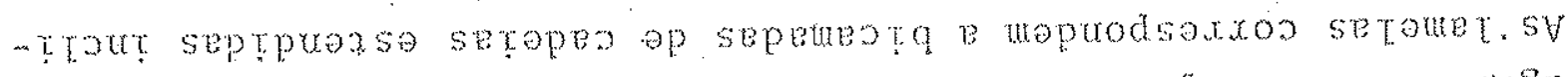

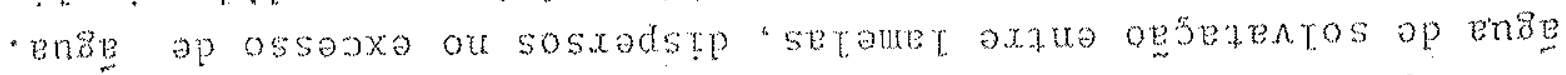

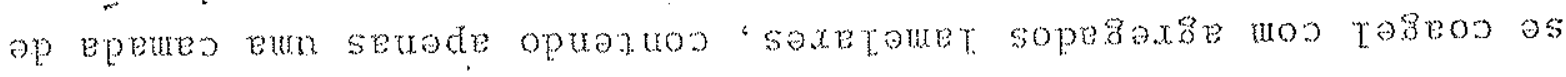

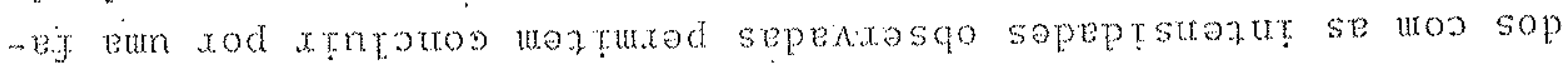

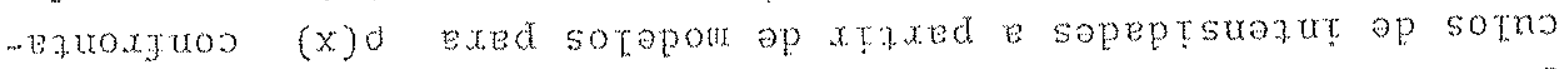

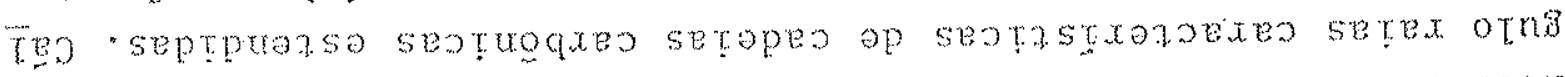

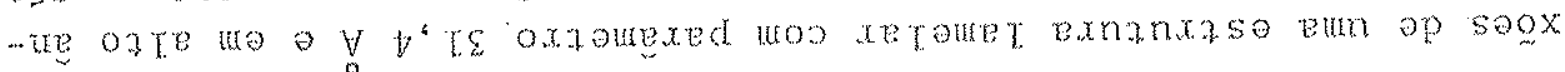
-atax \& ornsug oxegq we mextasou sopernsex so

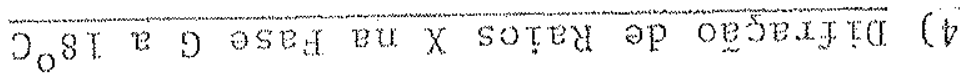

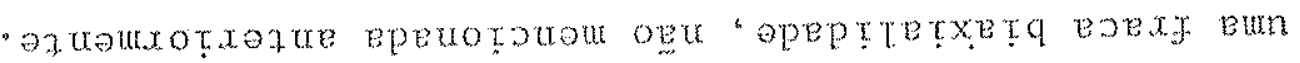

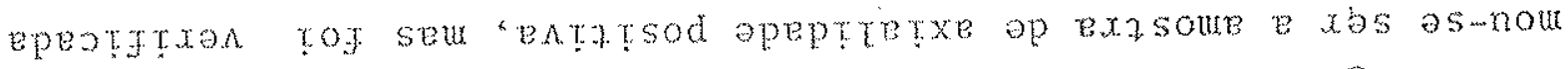

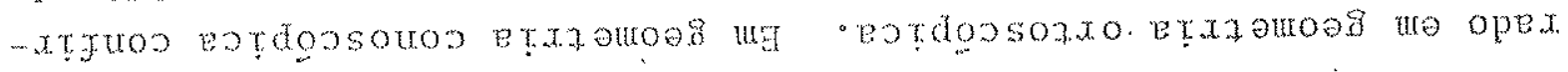

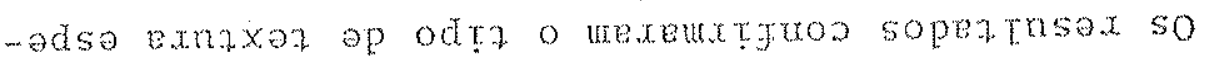

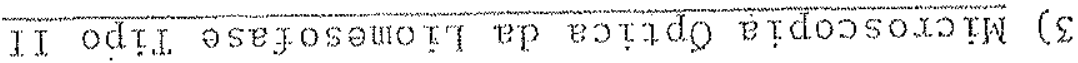

- setp ousour a sex

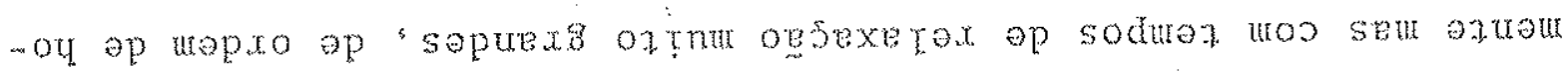

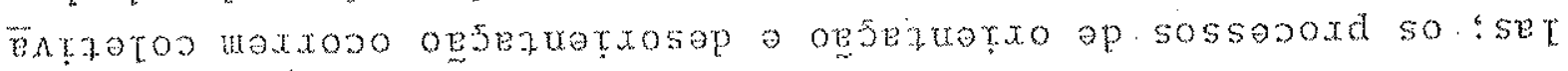

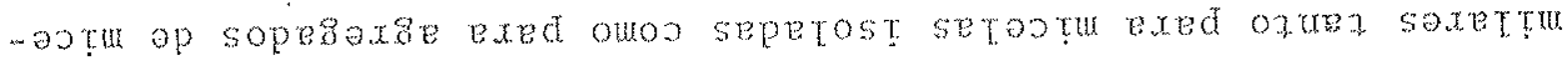

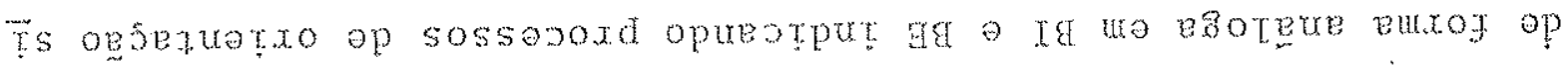

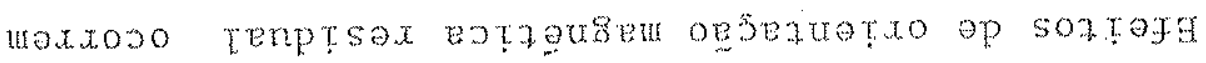

- sezanber senp ap sopesaxe ap a seper

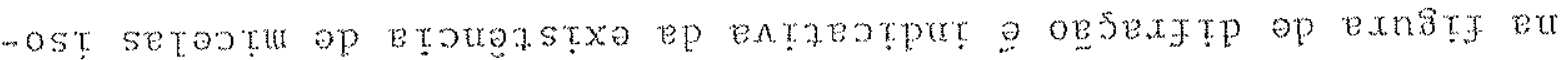

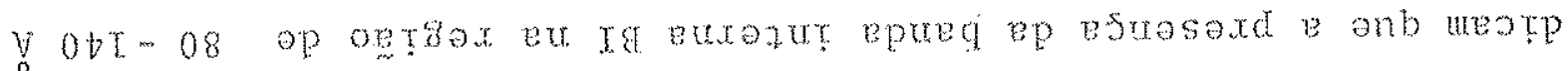

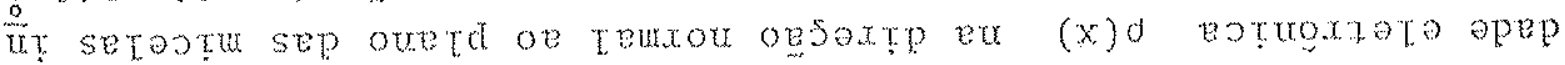

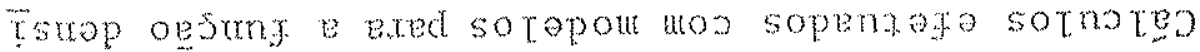

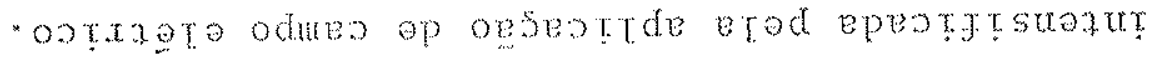

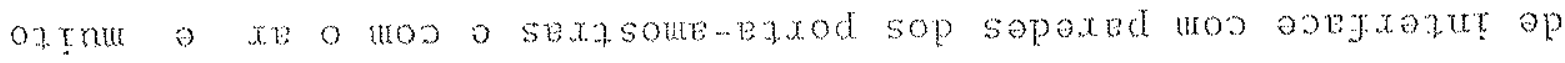

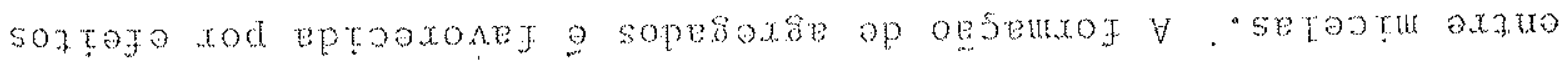

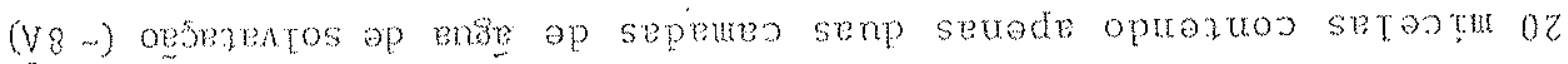

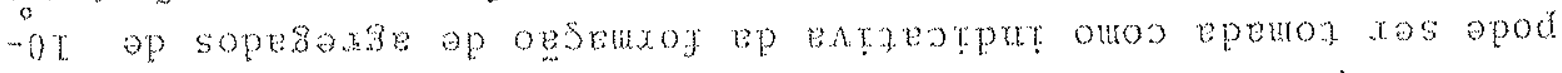

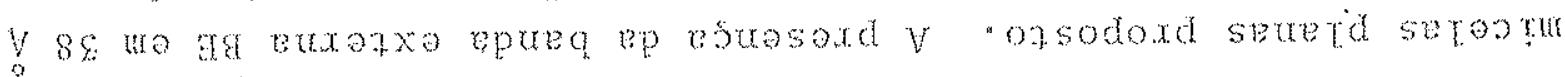

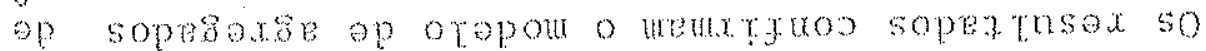

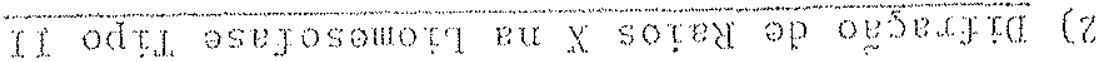




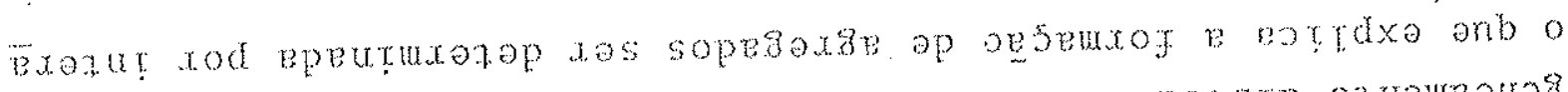

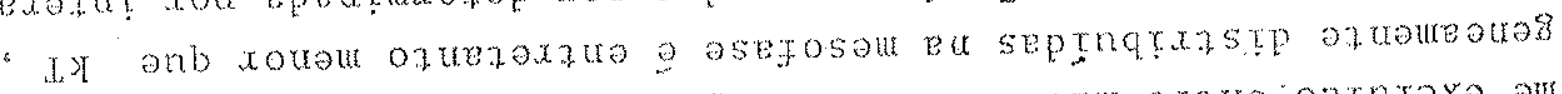

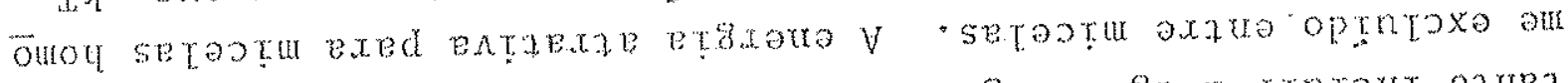

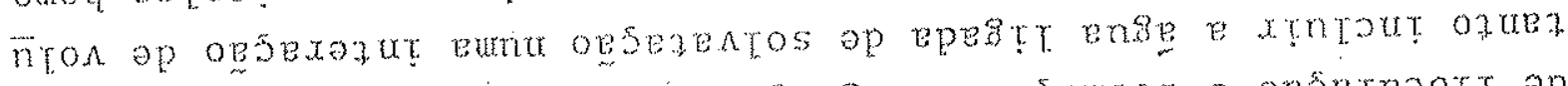

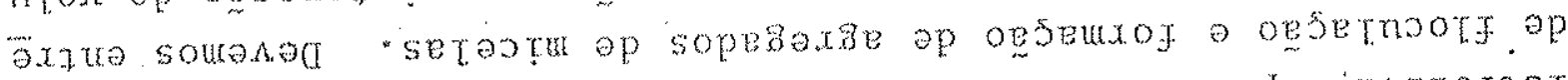

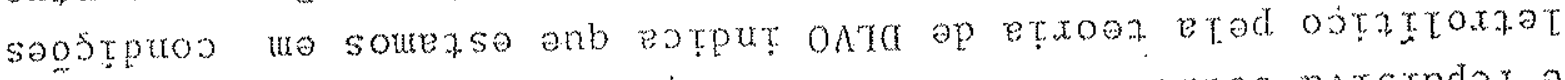

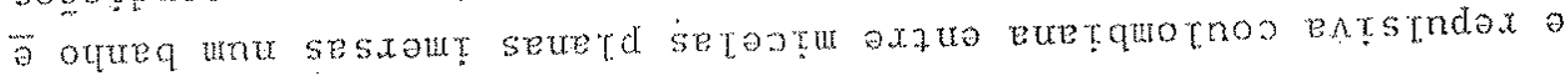

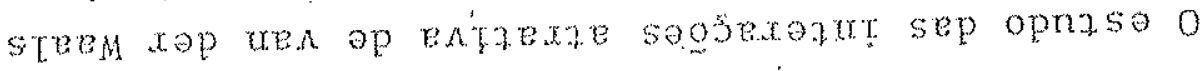

setostw axame oesexanul ep opnst (L

- eusasts op sosez op emexsetp oe ogserax

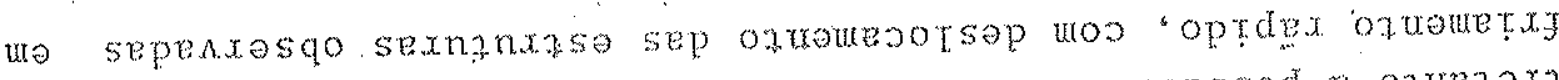

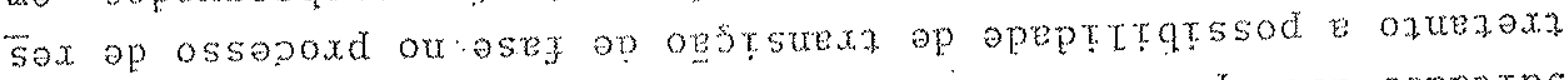
-re eptntoxe ezsa ogx sopesaxe ap otepou op otode oep sopertns

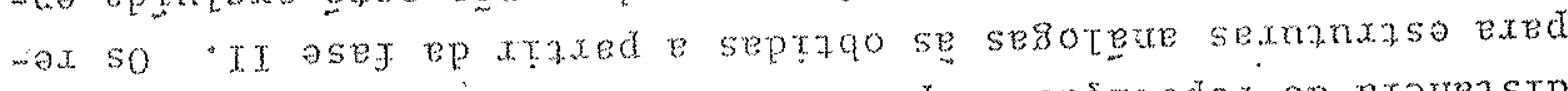

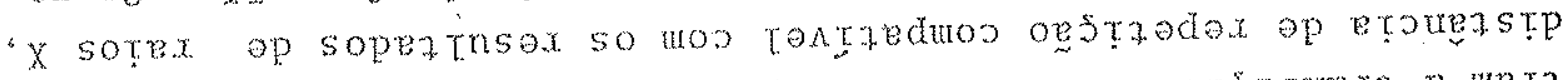

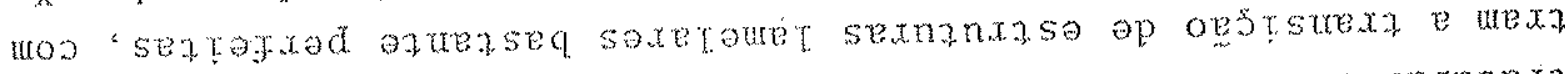

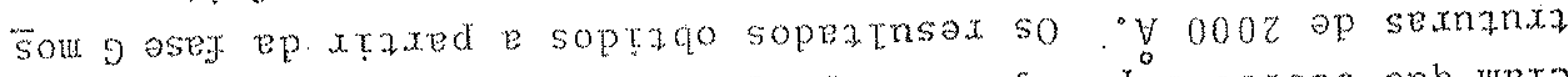

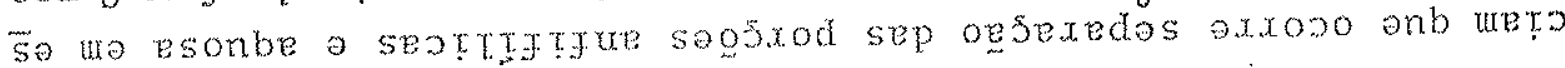
-ropta II esex ep xtaxed sopraqo sopeatros so

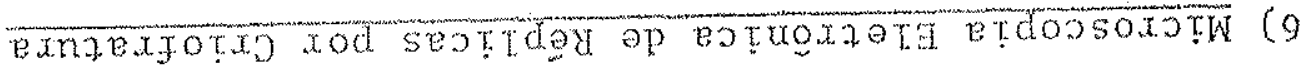

- orsng

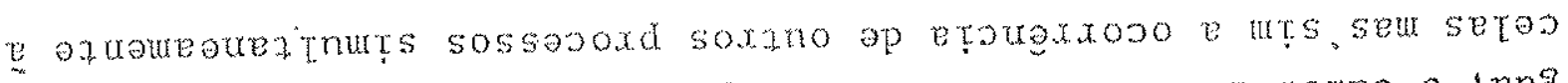

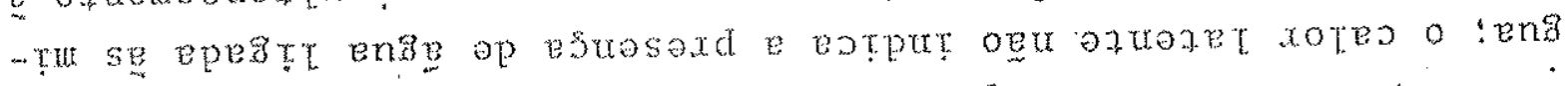
E ep oesny e moo epetoosse exse go e ogstisuext $\mathrm{V}$ sosseroxd soxano xentonue onop ozt e ossturat $\mathrm{V}$

- seteuret 5e axt

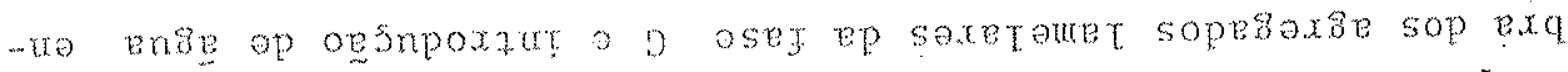

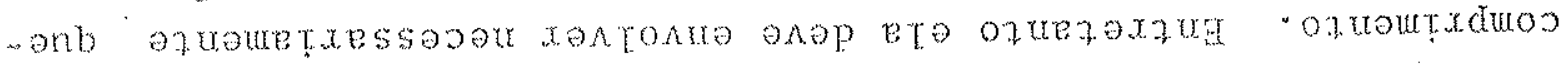

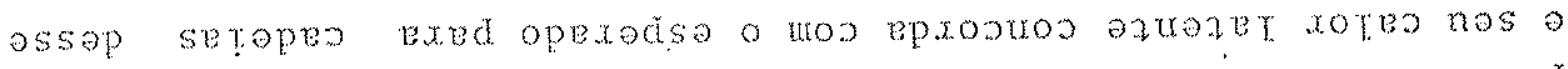

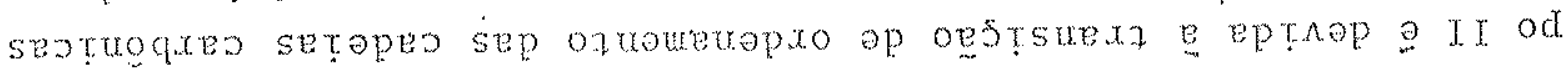

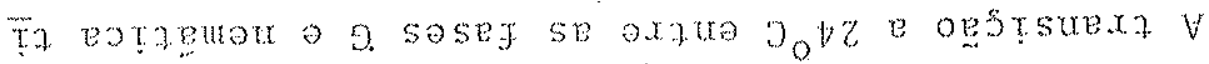

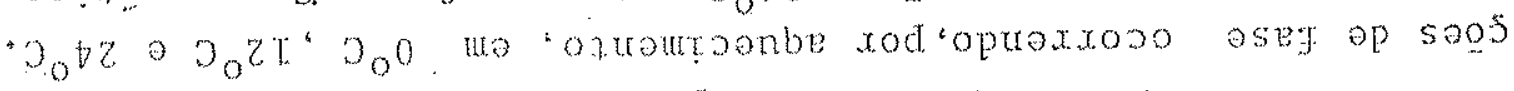

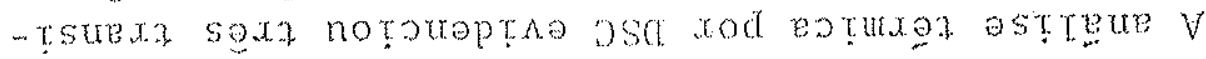


-exoqet ossor me opexasou op un a ozuatexoznop op soutequa stop we eperotu tof et sostor sext sossop megepxoqe $\mathrm{V}$

$$
\text { - ausasts op asez op soostsuex }
$$

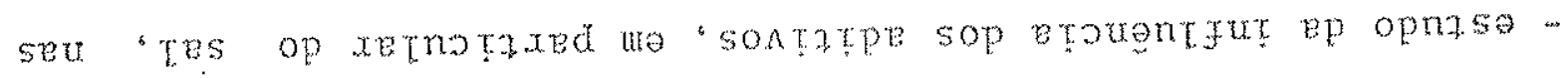
- eotrouseur

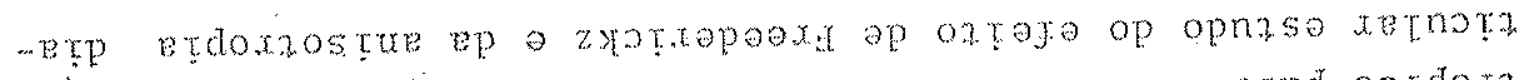

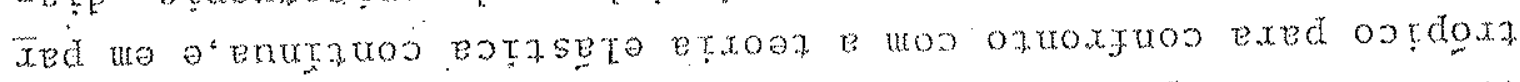

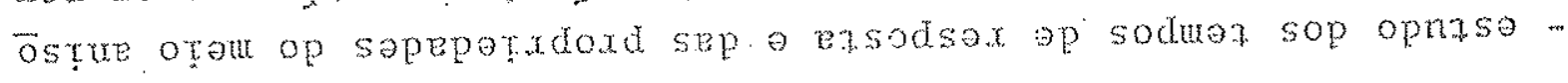

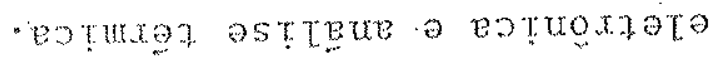

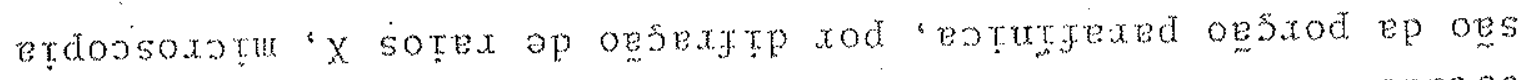

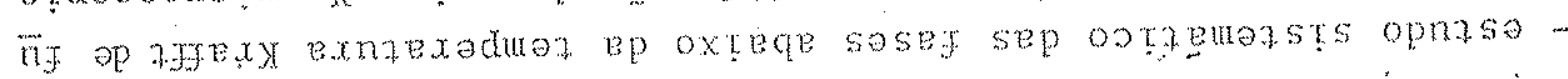
: ozrentour

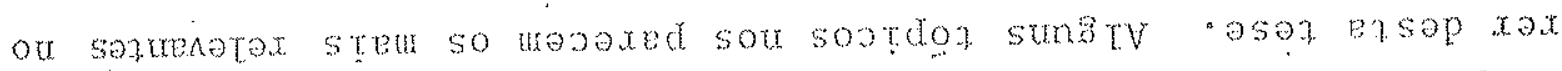

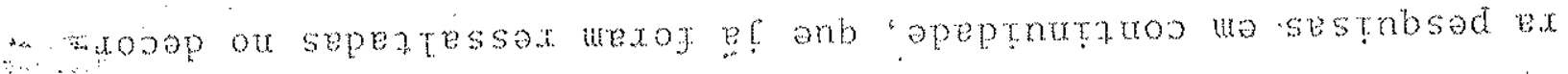
ed sentroodsad setren axge optatonusop opnaso

- sopesexse ap opsuncos op.

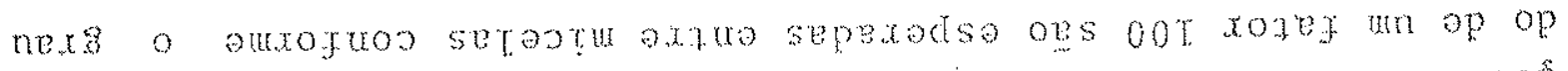

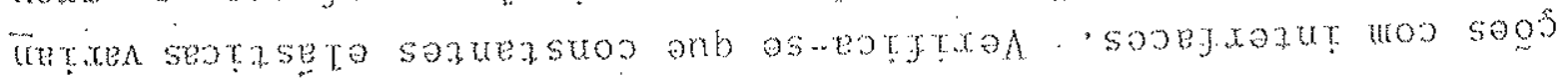




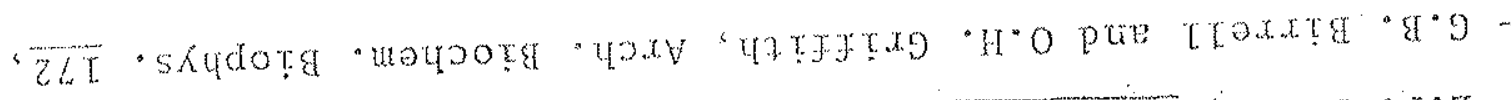

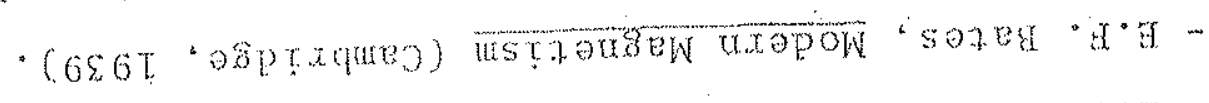

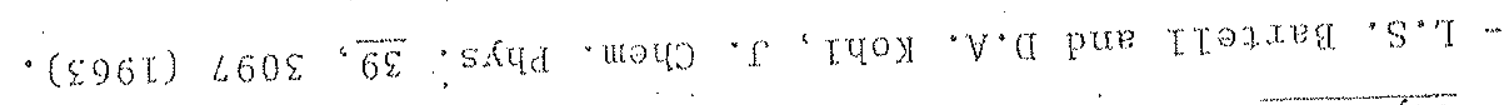

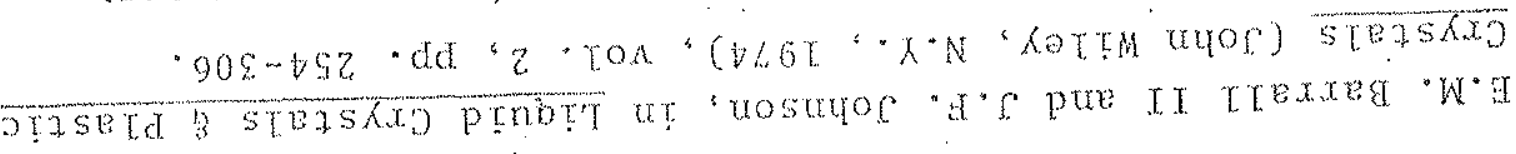
$\cdot 696 \pi \cdot x \cdot \mathrm{N} \cdot \operatorname{sen} x$

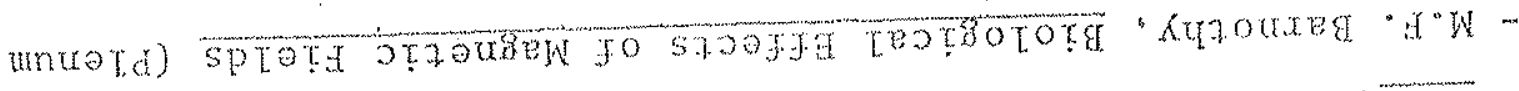

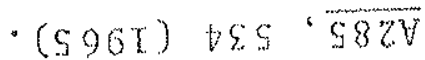

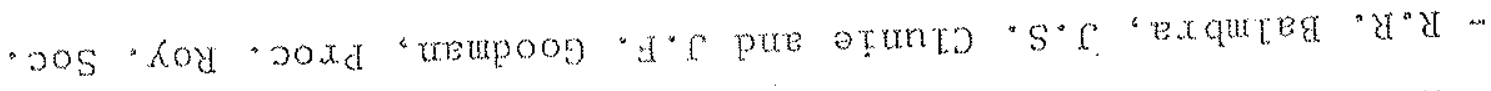

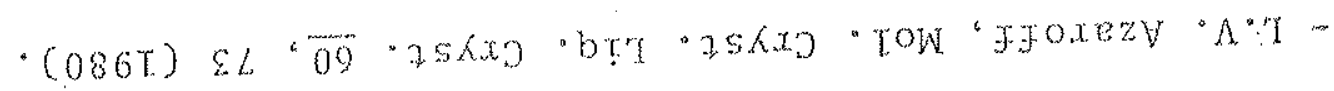

- (896: "VSR

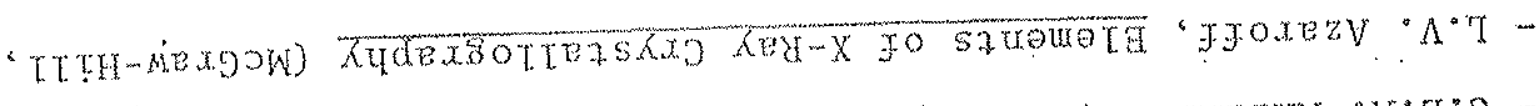

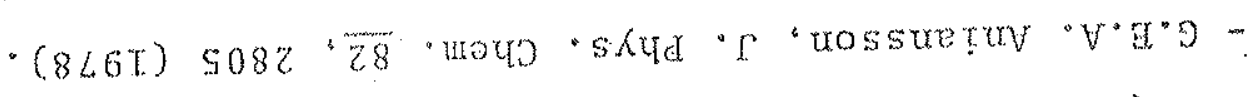

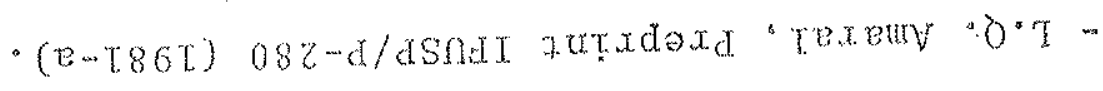

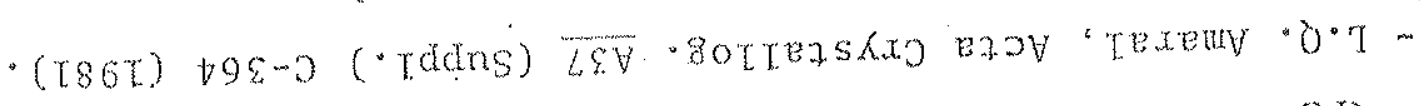
$(100+9)$

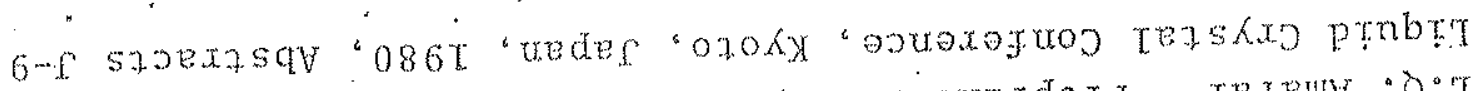

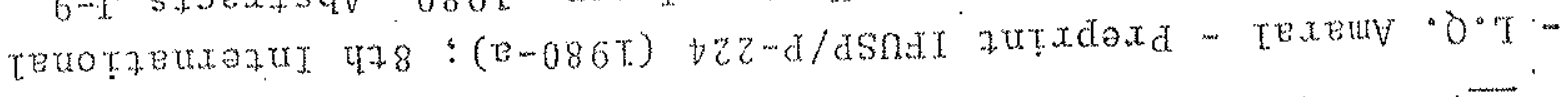
$\cdot(086 \mathrm{t}) \mathrm{s} 0 \mathrm{z} \cdot \overline{9 \mathrm{~s}}$

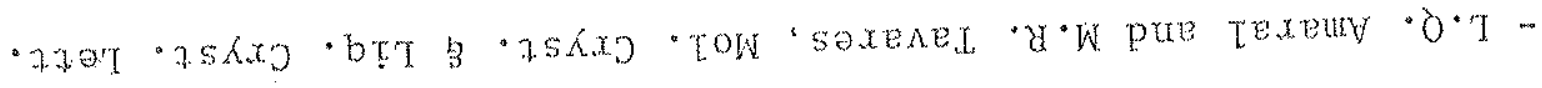

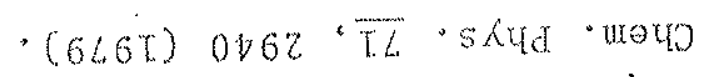

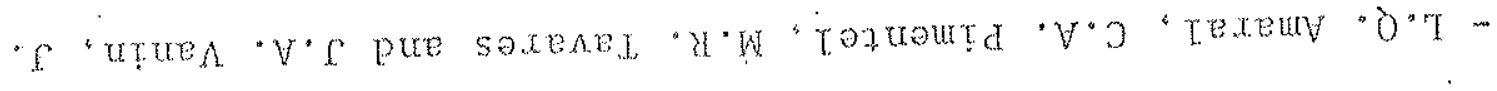

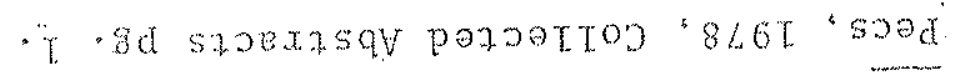

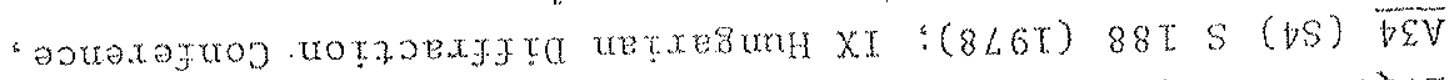

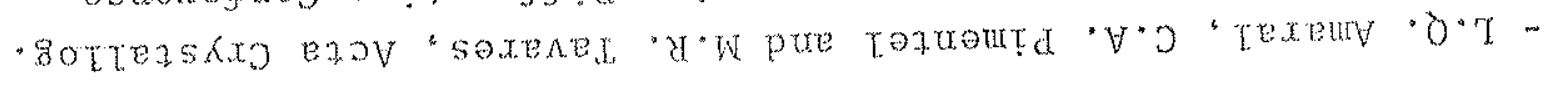

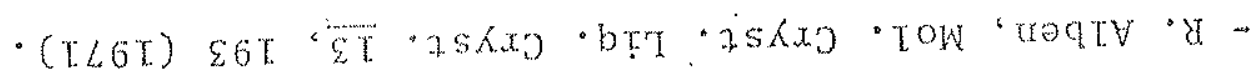




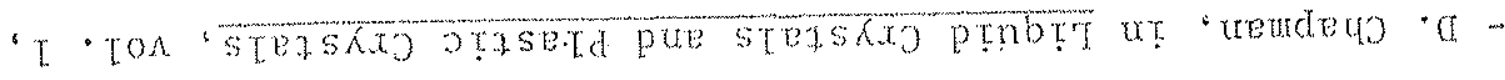
- (l96t) sot:t sptdt

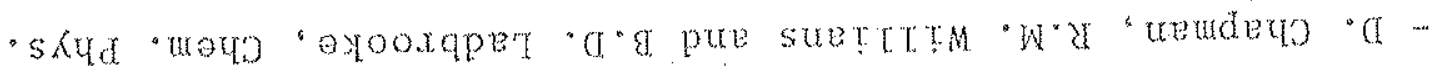

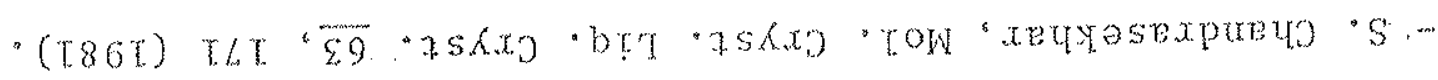
- (LLGT

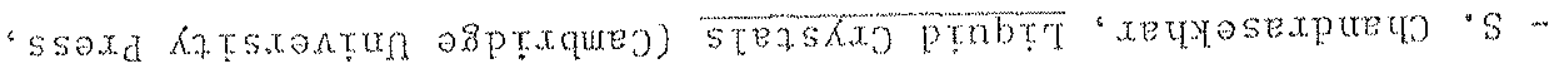

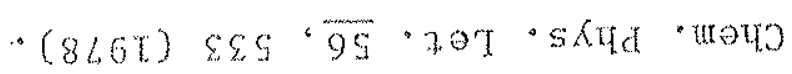

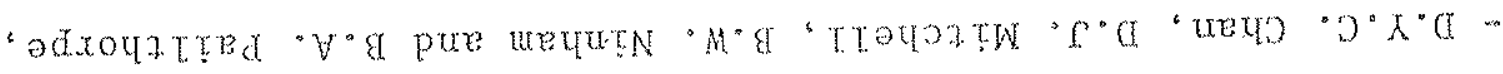
- (086T) TLT

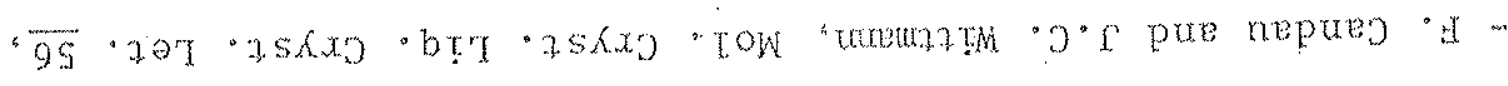

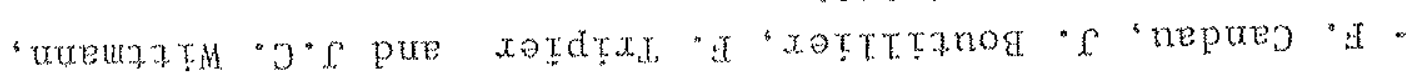

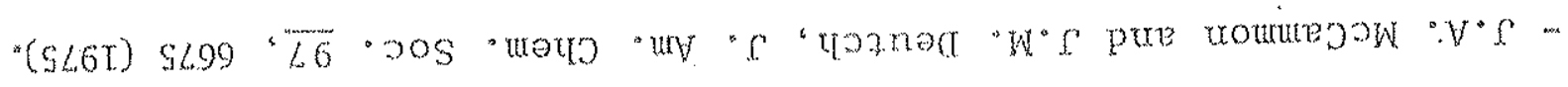

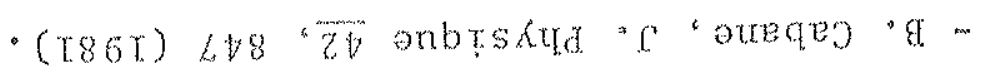

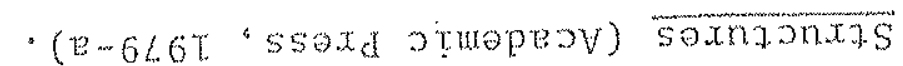

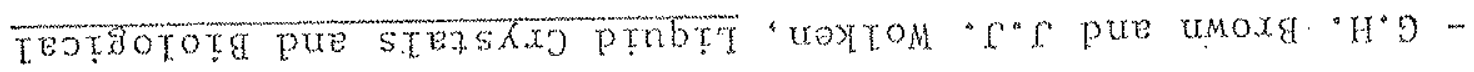

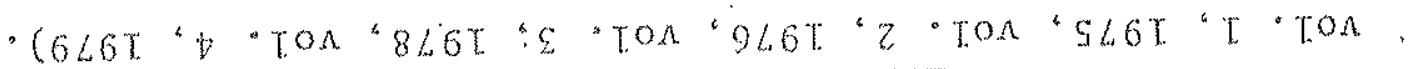

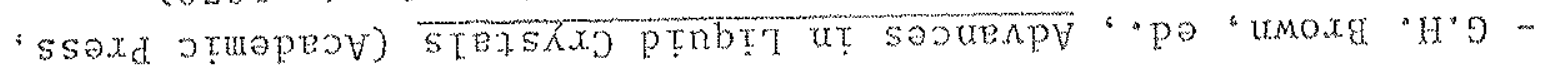
- (vL6T) T " - ct.6t "puetanato" "or xaquna tes

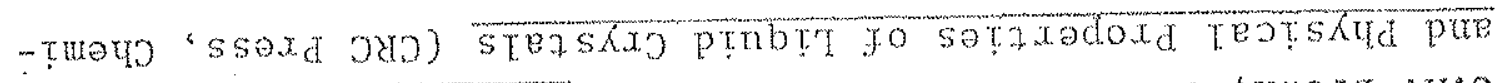

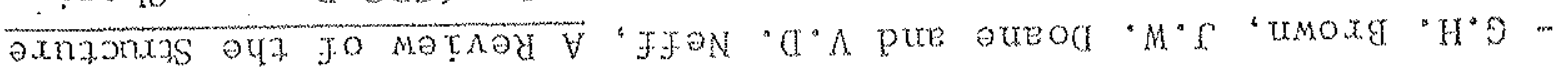
- (sLGT) TOS " $\overline{\mathrm{CT}} \cdot \mathrm{C} \cdot \mathrm{shudotg}$

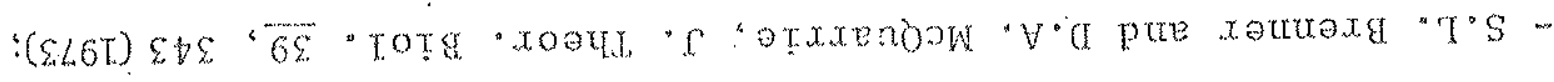

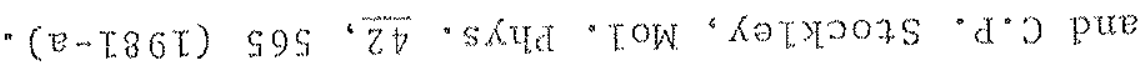

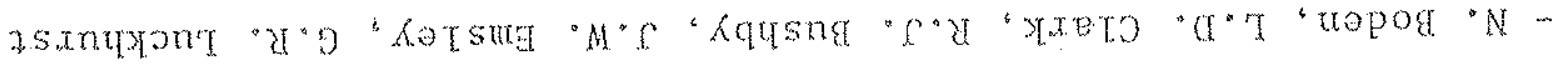

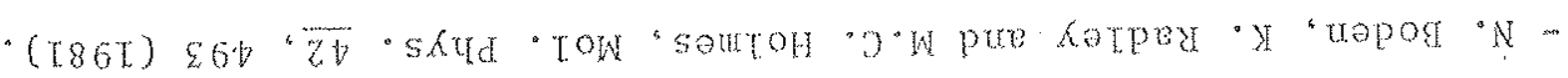
$\cdot(6460) 96 \% \cdot 59 \cdot 729 \cdot 5 \times 4 d$

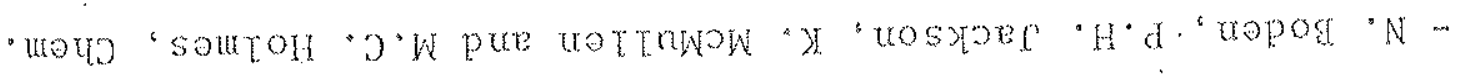
- (cL6t) zL"

" (cL6T)

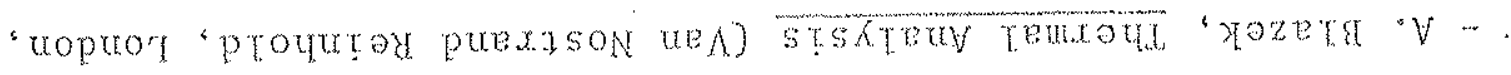
- (6960) ZVS

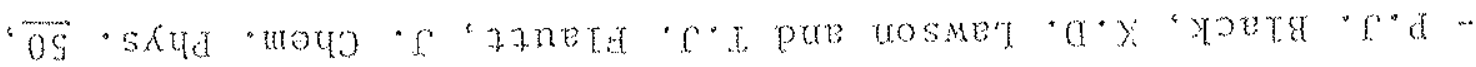




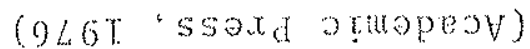

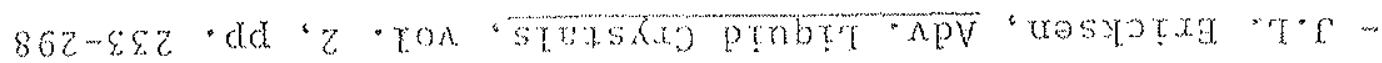

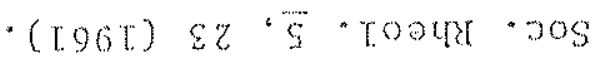

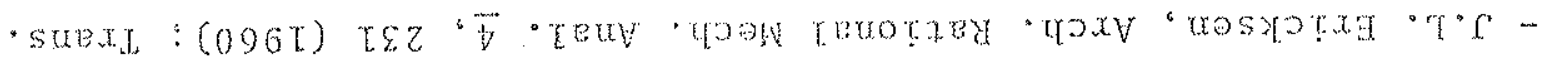

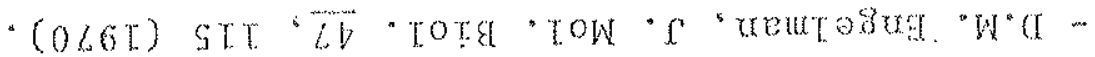

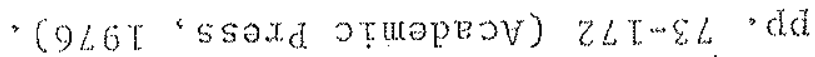

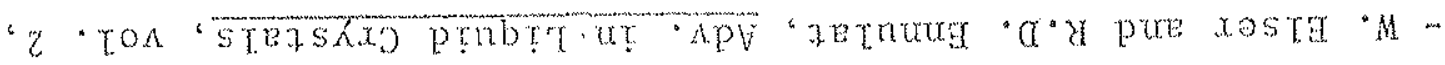
$\cdot \eta, D T-T \cdot d d \cdot T$

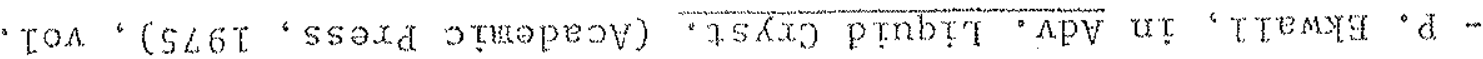

- $(696 \mathrm{~T}) 80 \mathrm{~s} \cdot \overline{\mathrm{TE}}$ oगtotos

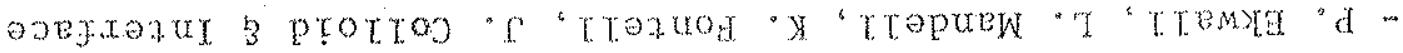

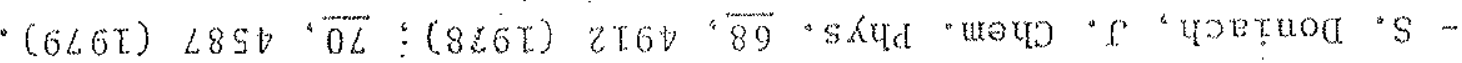

- (606t "uttueg "xosutads) ptogsox

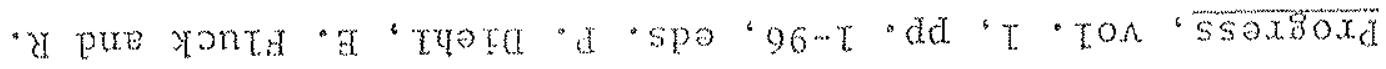

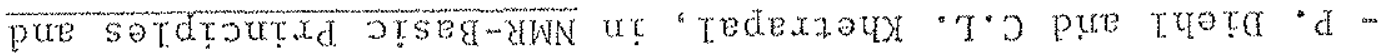

$\cdot(T+6 \mathrm{t})$

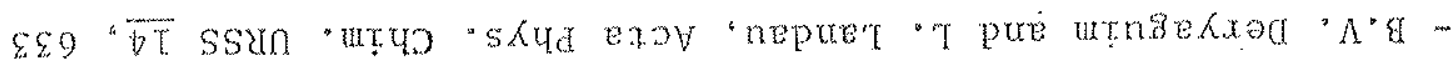

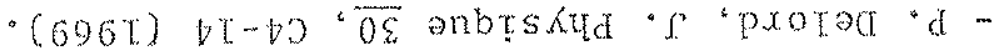

- $(996 \mathrm{~T})$

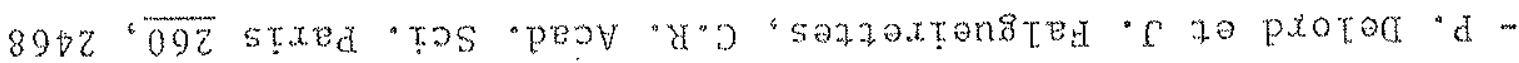

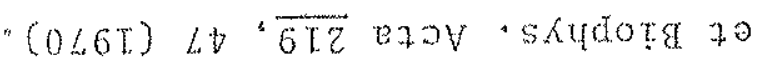

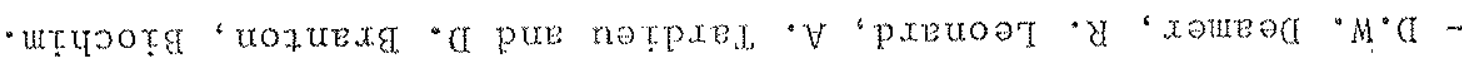

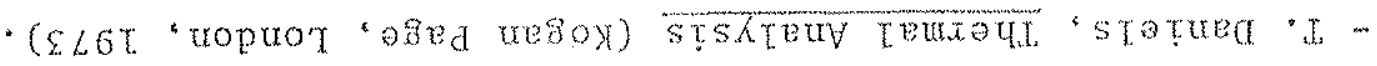

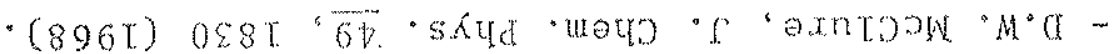
-39-str ad $\mathrm{t} \cdot \mathrm{ton}$

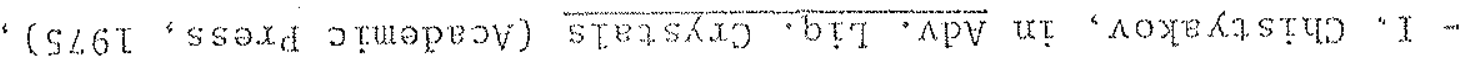
- (LL6T) $96 \%$

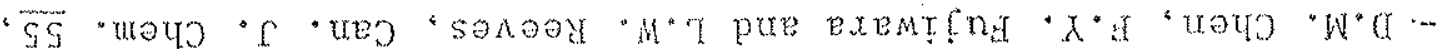
$\cdot(8-0860)$

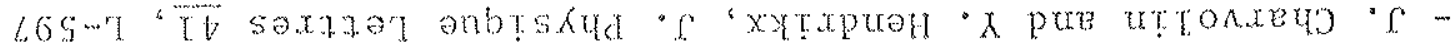
$\cdot \cos 6 \mathrm{t} \cdot \mathrm{art} x \mathrm{xg}$

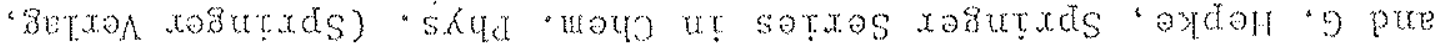

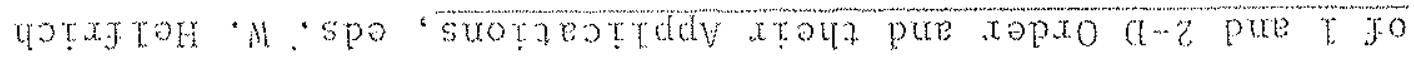

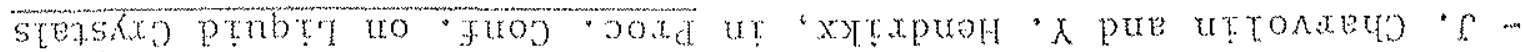

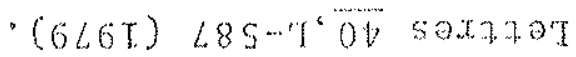

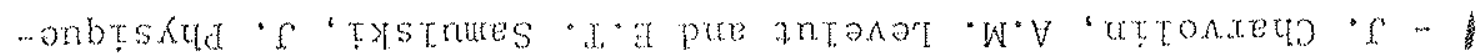




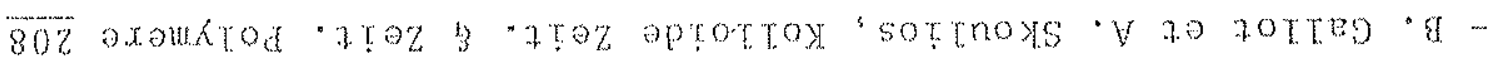

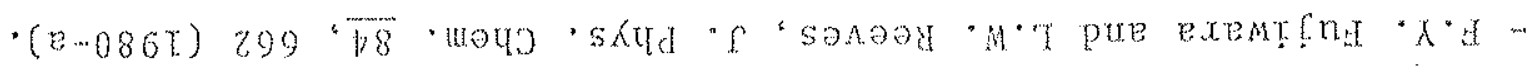

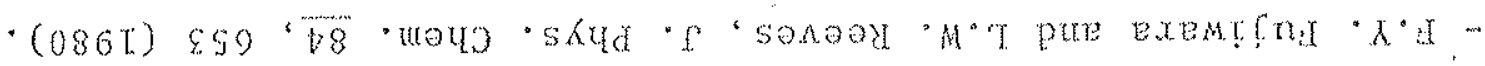
\& $9 \cdot d$ " $T$

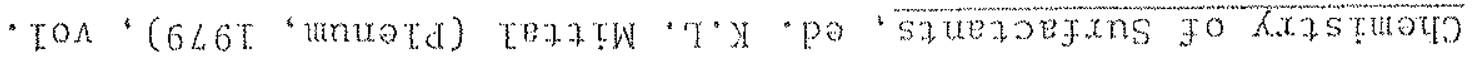

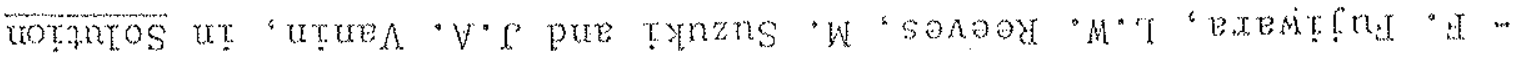

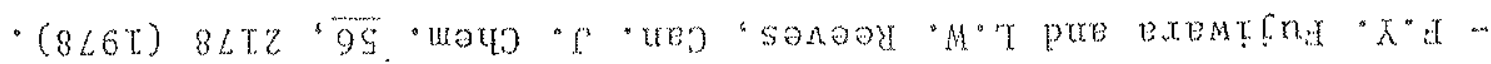

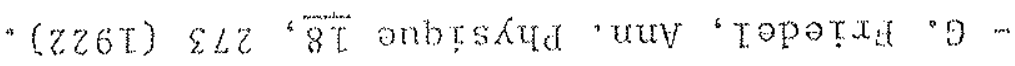

家

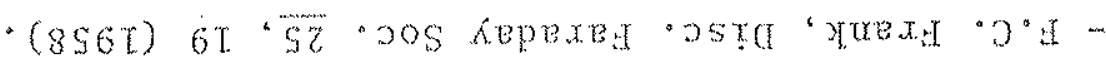
- (B.-T.86T)

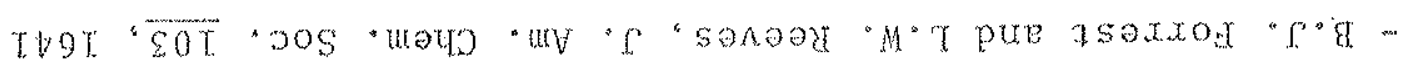

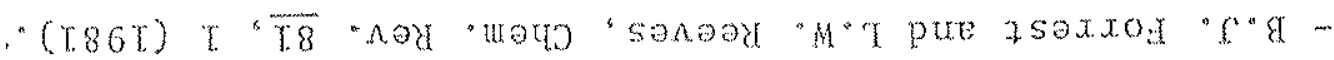
$\cdot(086 \mathrm{~T})$

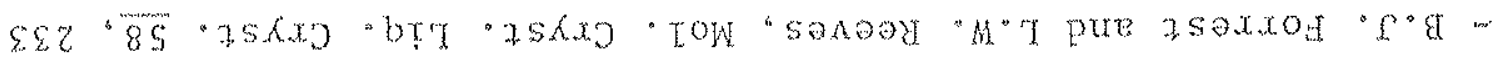
( $(L \angle 6 \mathrm{~T})$

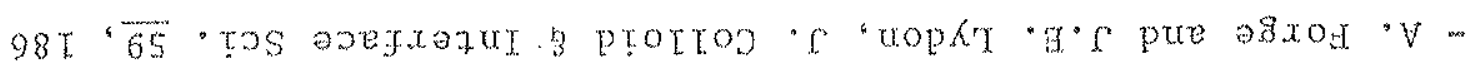

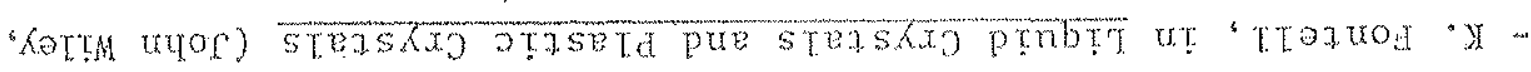

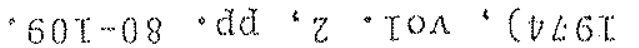

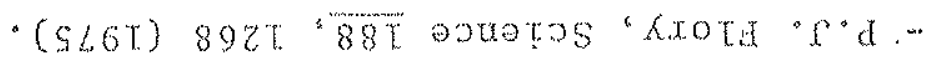

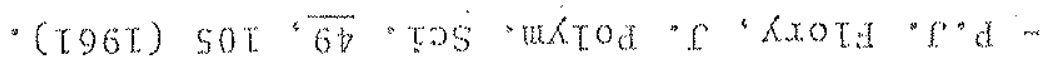

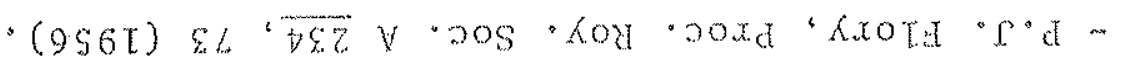

$$
\begin{aligned}
& \text { "(z86t) 0zs-d/dsnd autadoxd :(286t) }
\end{aligned}
$$

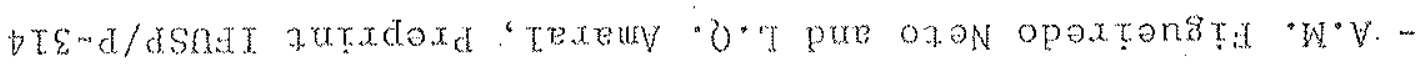

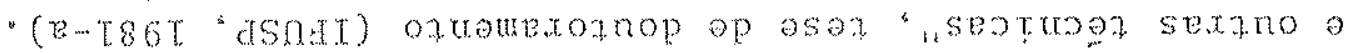

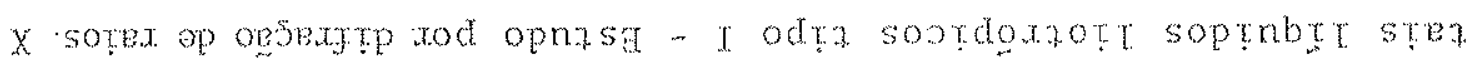

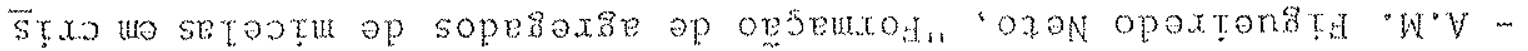
- (T.86T) $60 \mathrm{~T} \cdot \mathrm{Vh}$

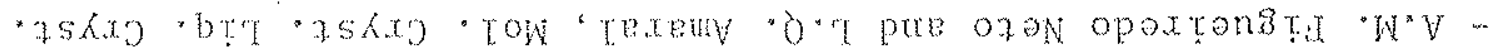

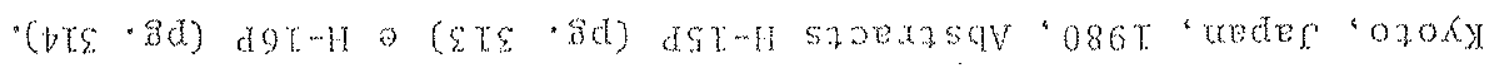

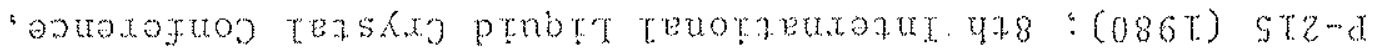

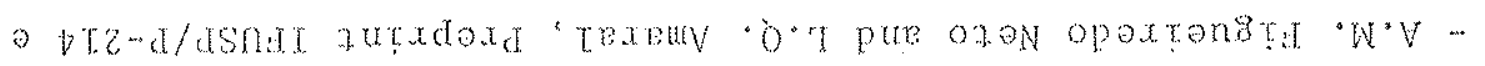

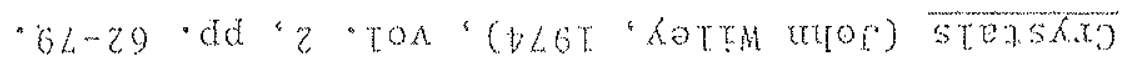

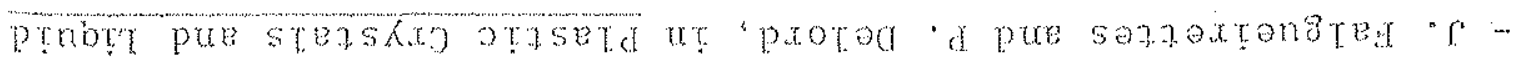

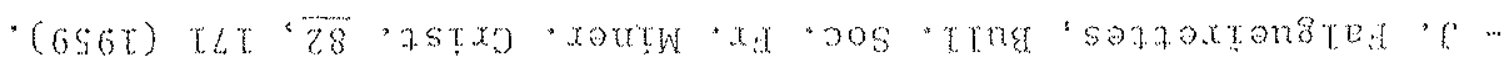




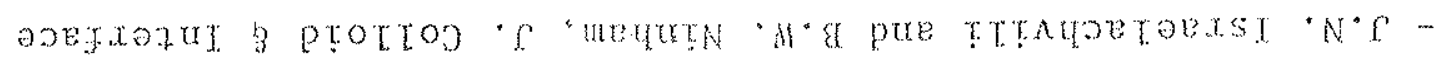
(6.6. $80 \mathrm{~T})$ T.

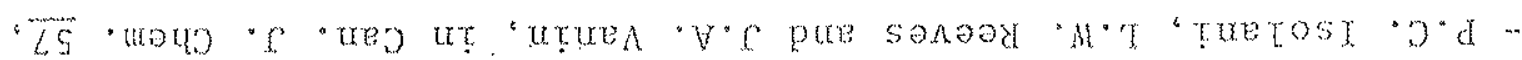

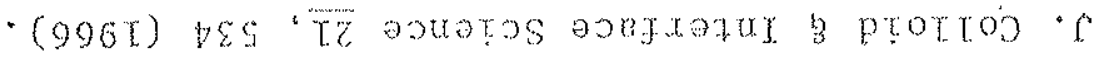

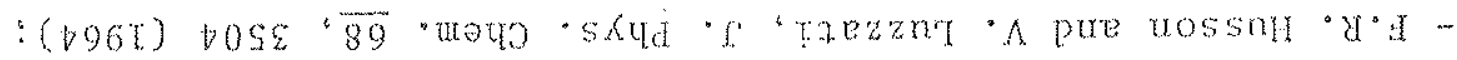
$\cdot(096 \mathrm{r})$

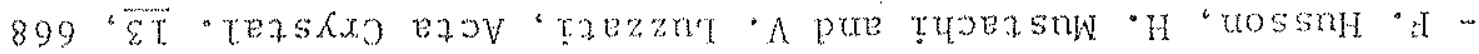
$\cdot(0860)$

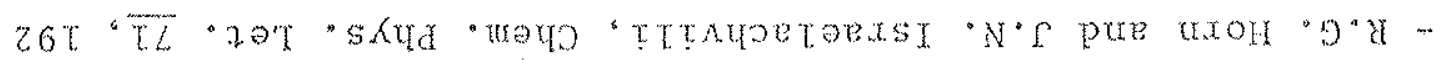

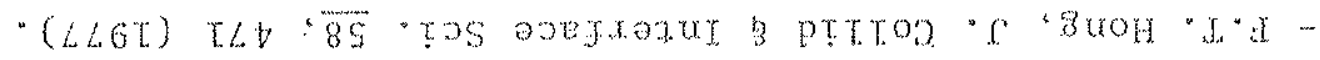

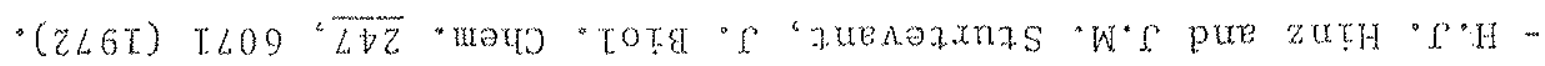

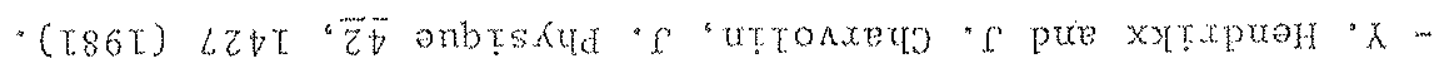

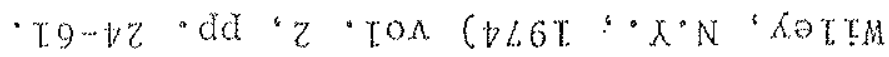

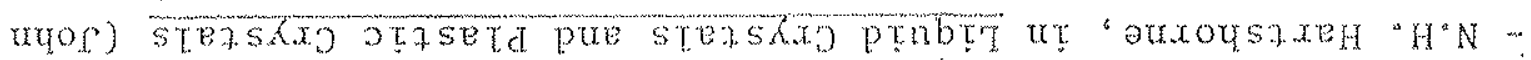
$\cdot(996 \mathrm{~T}$

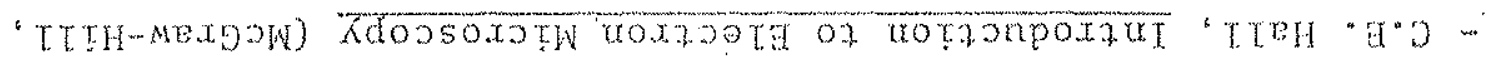
- $(956 \mathrm{t}$ "sțed "poung)

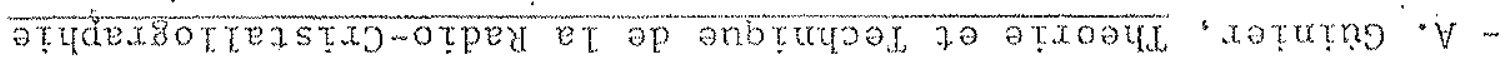

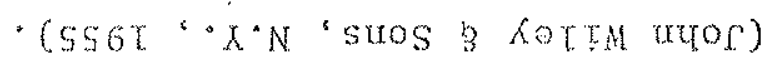

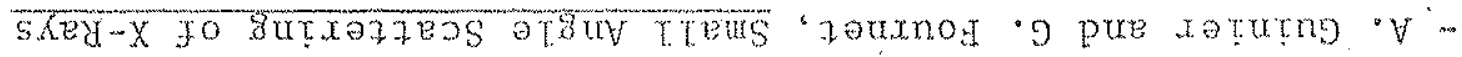

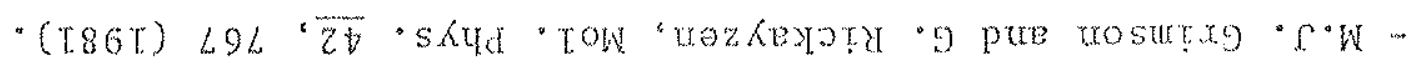

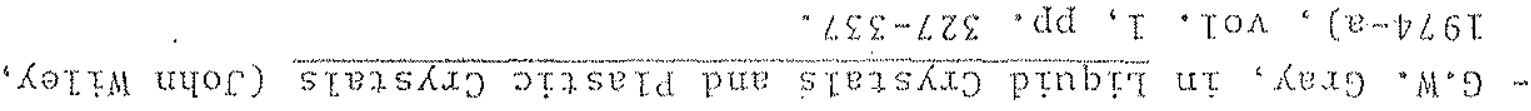

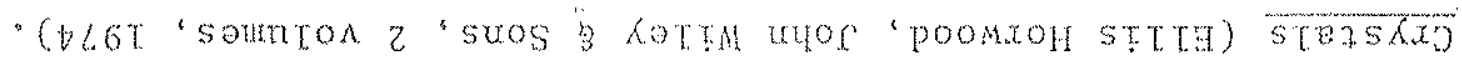

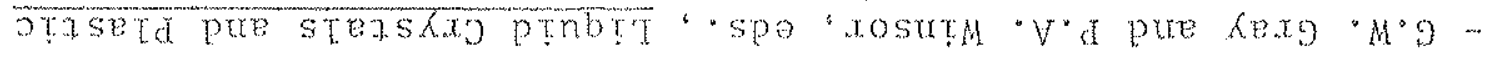

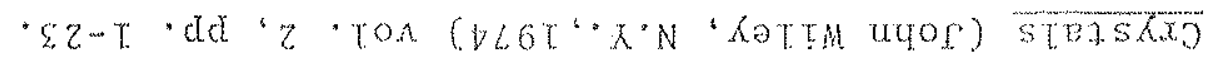

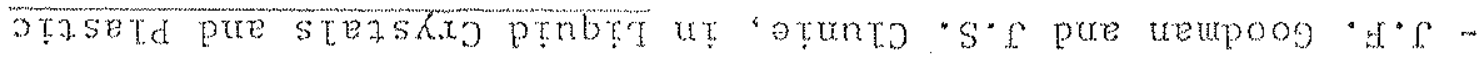
- blet 'pxojxo'ssoxd

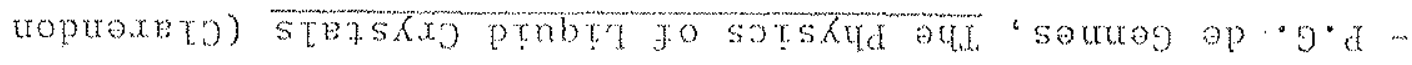

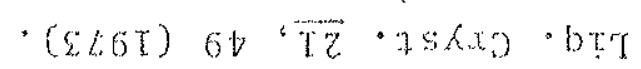

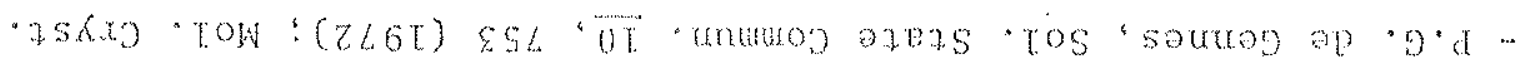

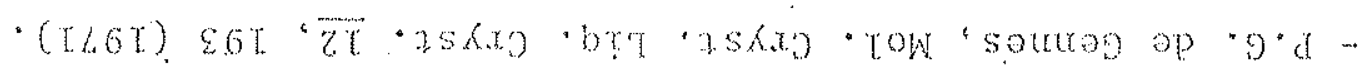

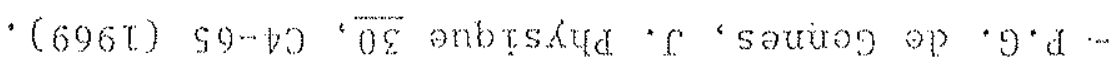

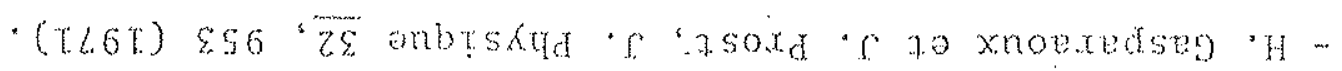




$$
\cdot(266 \mathrm{~T}) 66 \mathrm{t} \cdot \overline{69} \cdot \mathrm{T} \cdot \mathrm{S}
$$

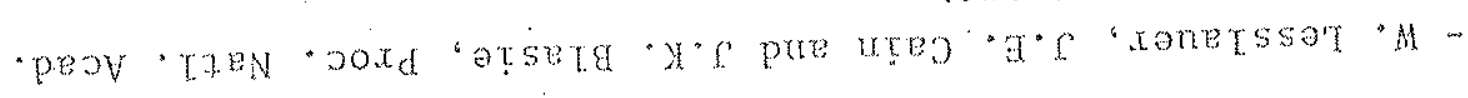
$\cdot(666 \mathrm{x} \cdot \operatorname{ss} 2 x \mathrm{~d}$

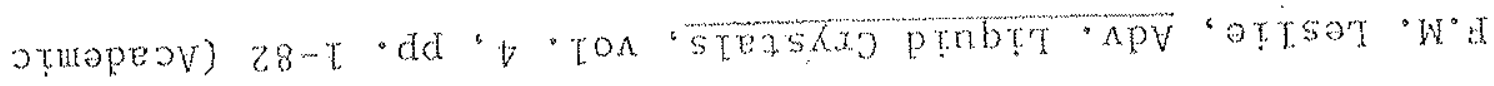

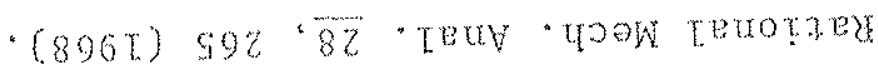

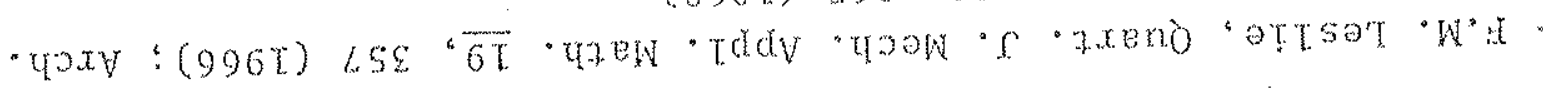
$\cdot(686 \mathrm{~T}) \mathrm{vg} \cdot \overline{0 L T V}:(686 \mathrm{~T})$ LTE

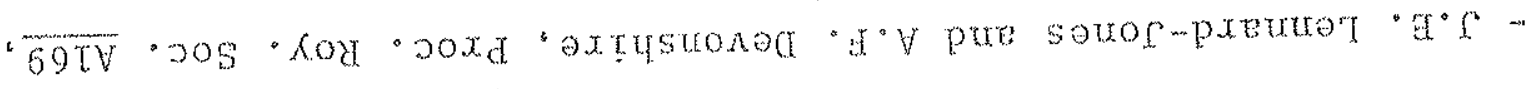

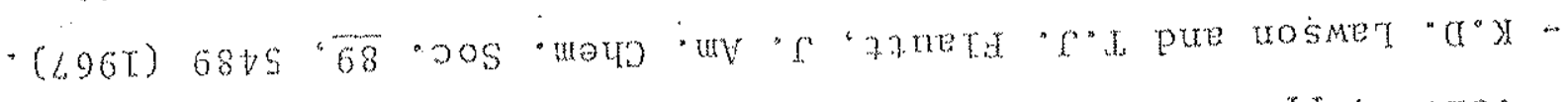
$\cdot 8 v T-z y \cdot d d \cdot c \cdot \cos$

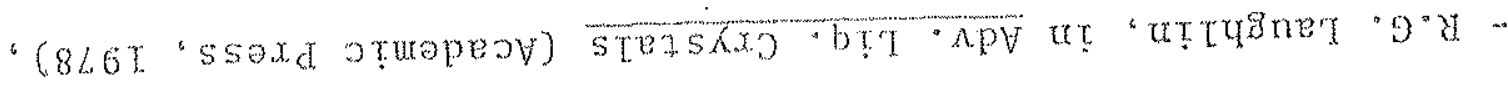

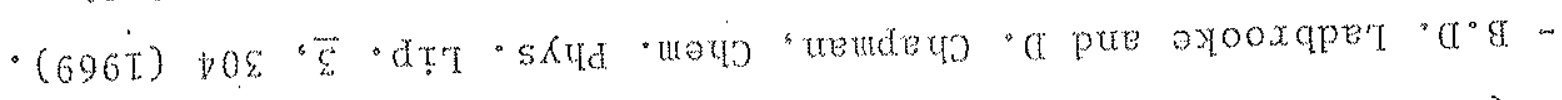

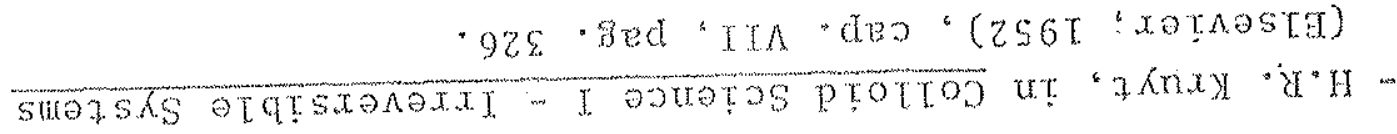

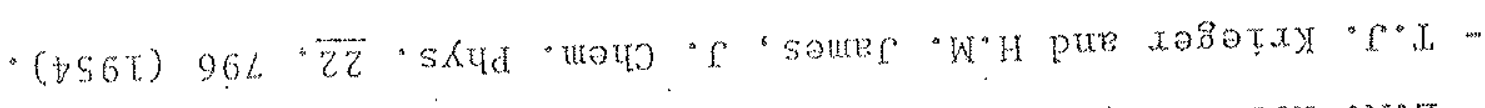

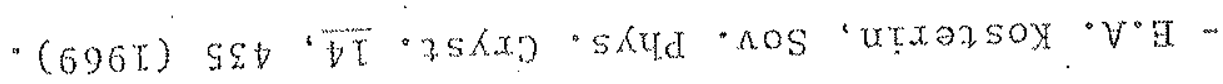

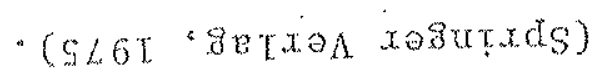

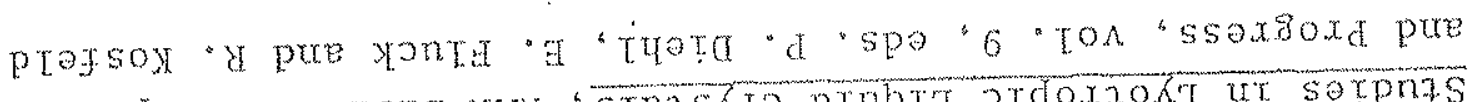

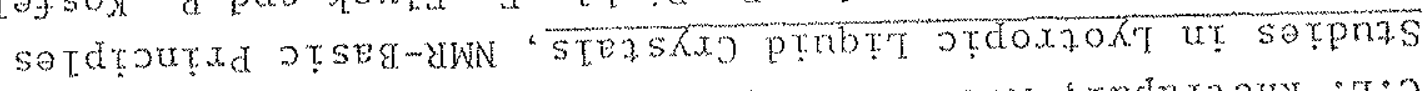

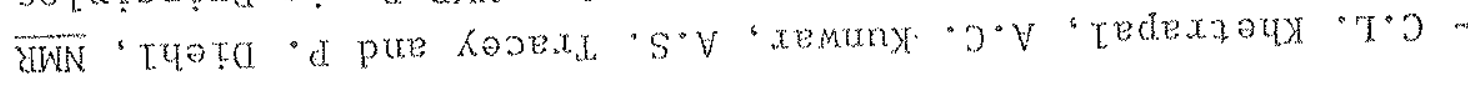

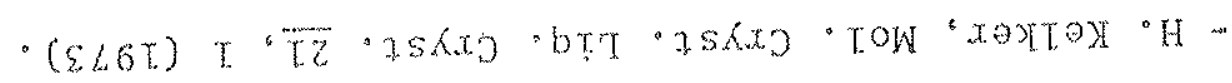

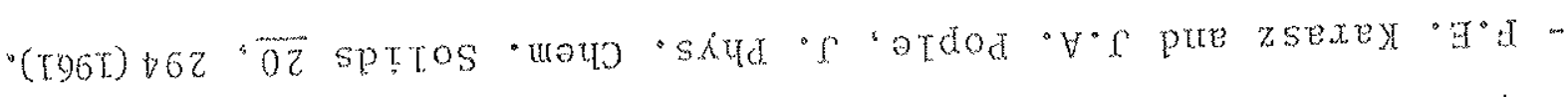
- $(96 \mathrm{G})$

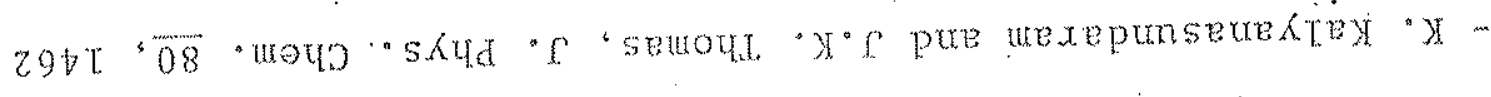

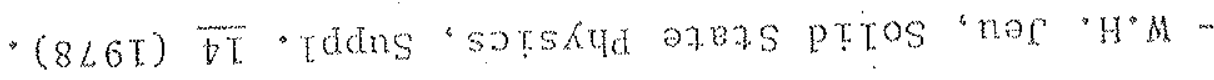

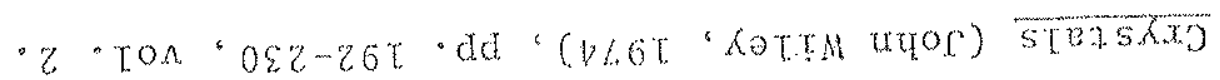

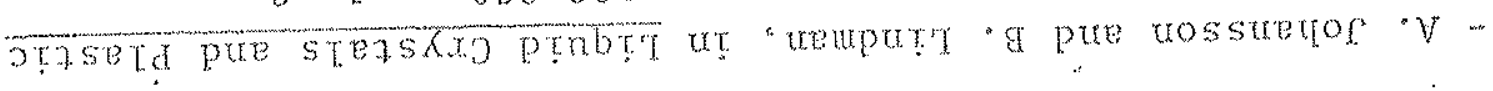
- (1.6\%) $8 \mathrm{z}$

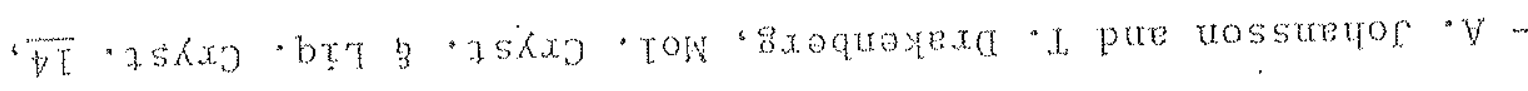
- (966t)

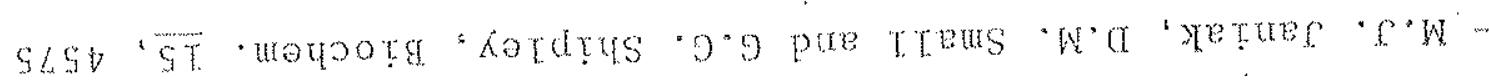
$\cdot(966 \mathrm{~T}) \& 668 \cdot 2 \mathrm{Z}$ VSn $\cdot \mathrm{TOS}$

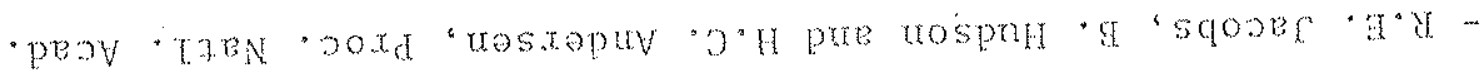

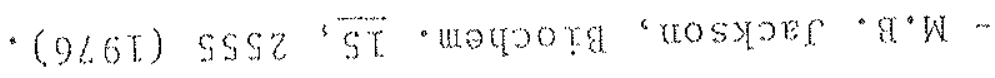




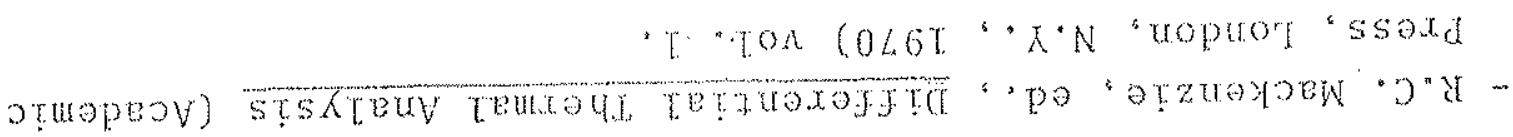
$\cdot(9660) 2985$

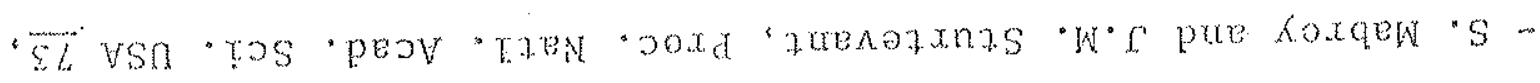

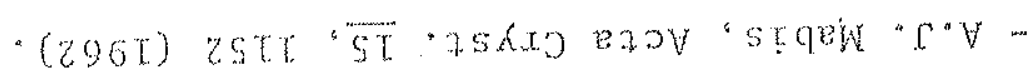
(2461) 862

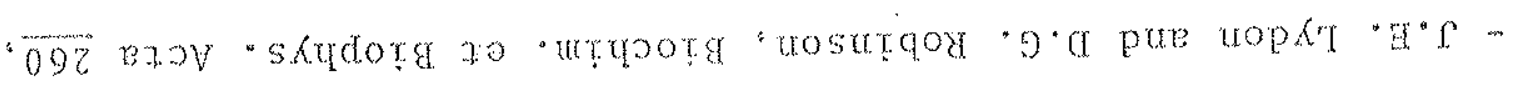

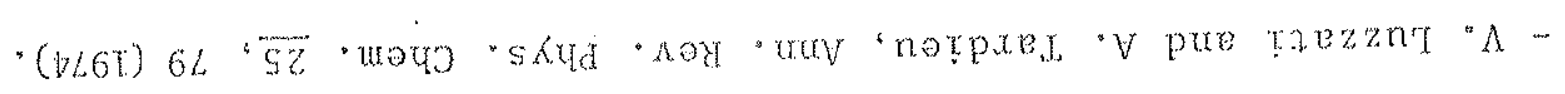
$(z<60)$

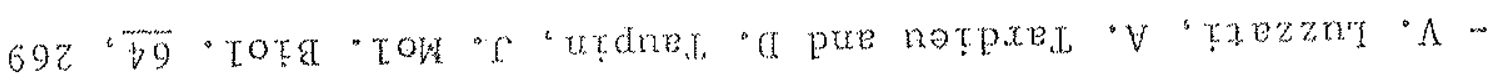

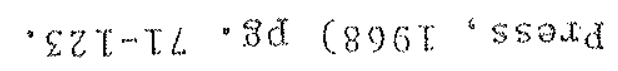

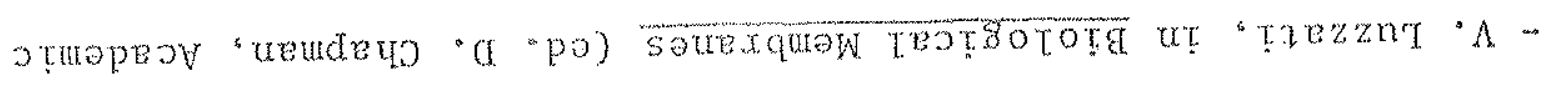

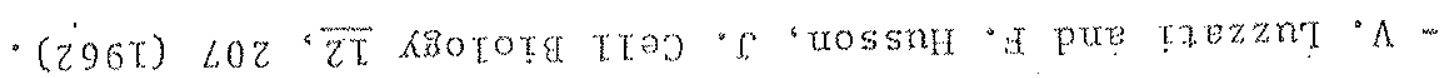
$\cdot(096 T) 099^{\circ \mathrm{CT}}$

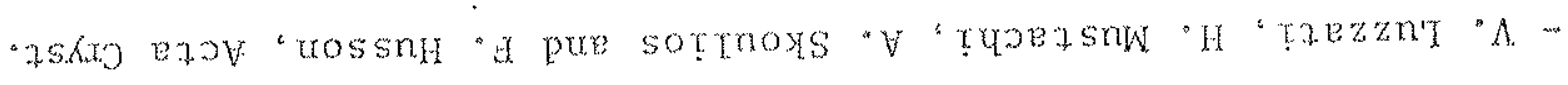
$\cdot(896 \tau) 05-80 \cdot 9 z$

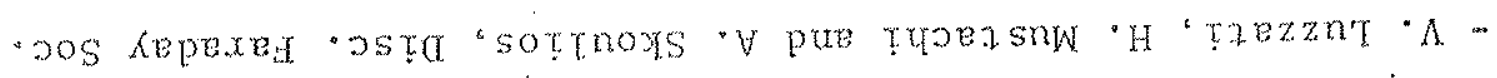

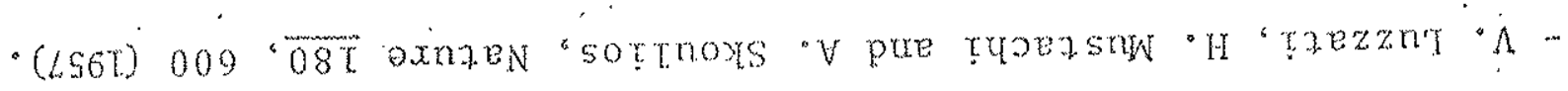

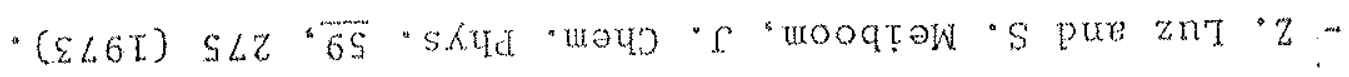

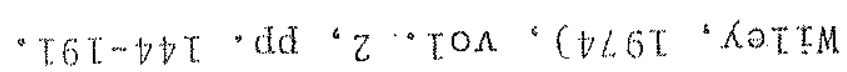

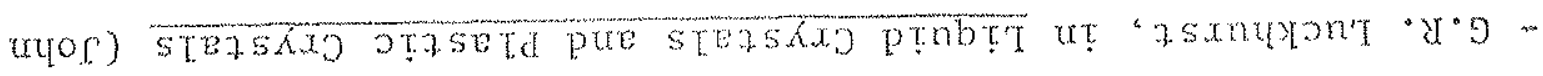

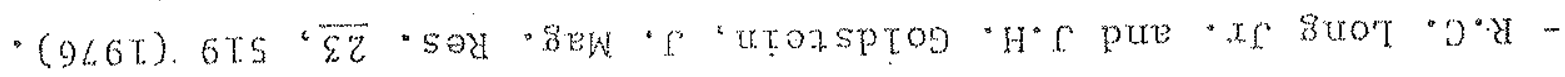

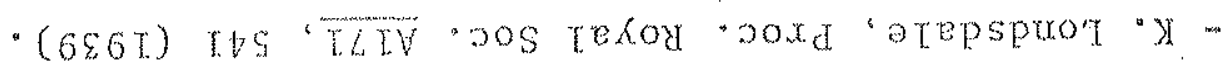

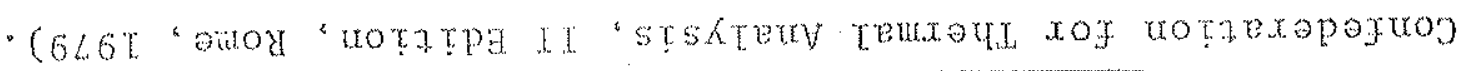

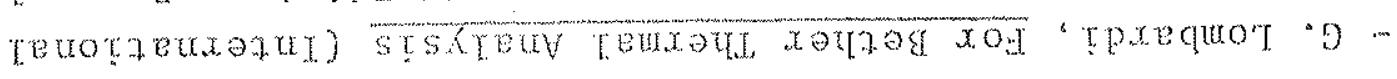

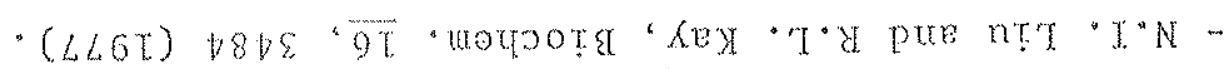
$\cdot(6461)$

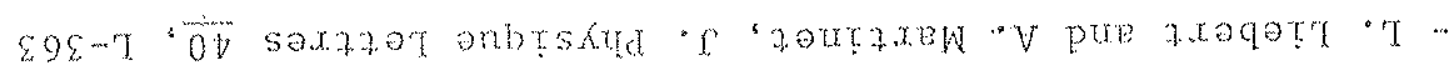
$\cdot(84.61$

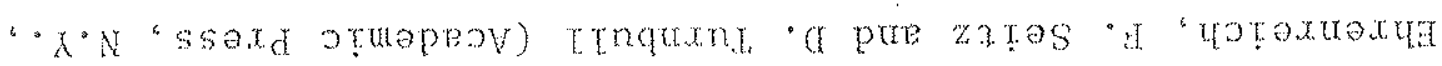

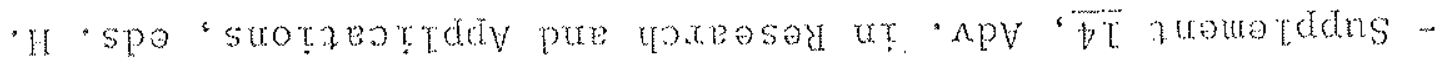

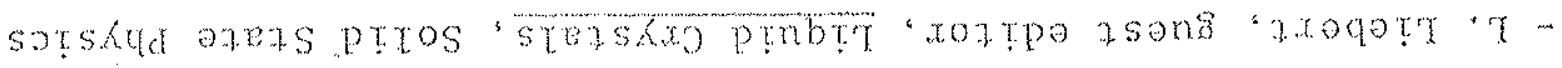

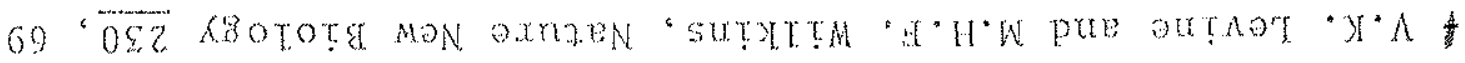


- (sL6t) st " - $(9 L 6 t) \operatorname{vgzt} " \overline{80}$ weder

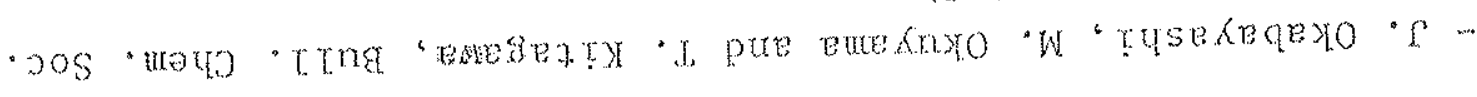

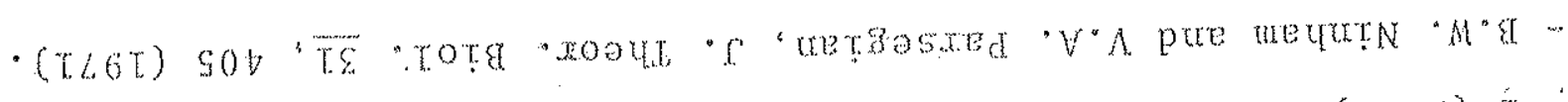
( $(266 \mathrm{~T}) \mathrm{T}$

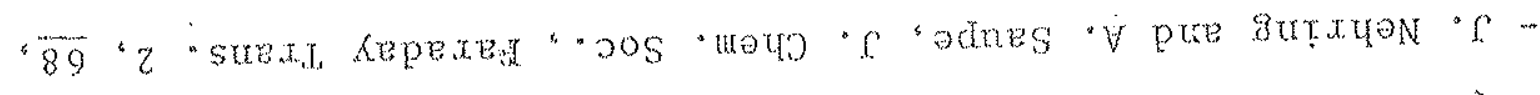

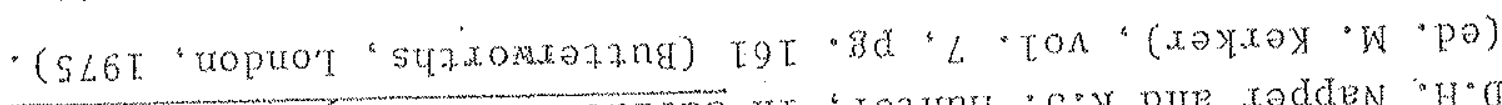

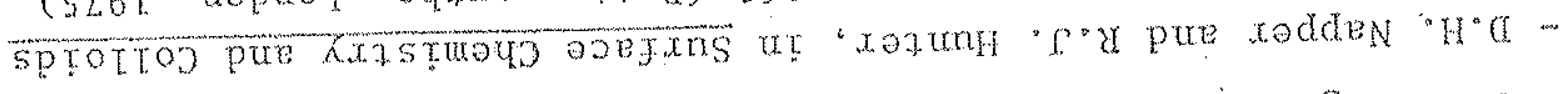

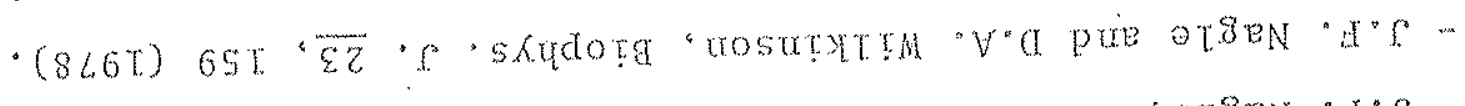

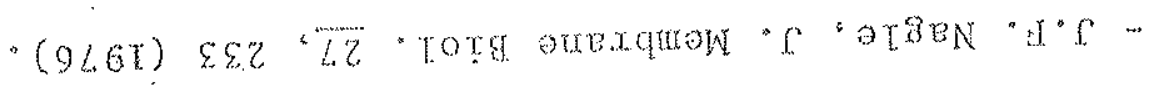

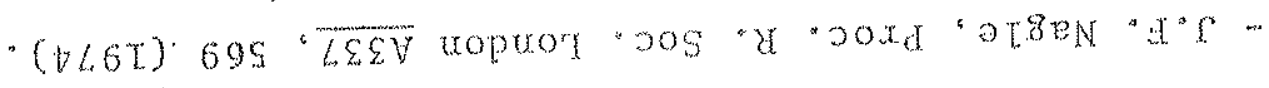

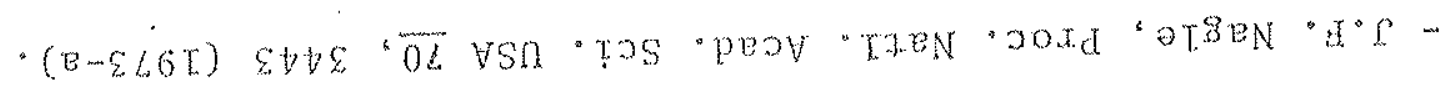

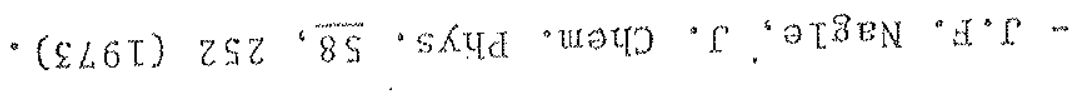

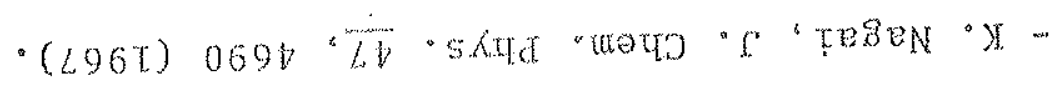

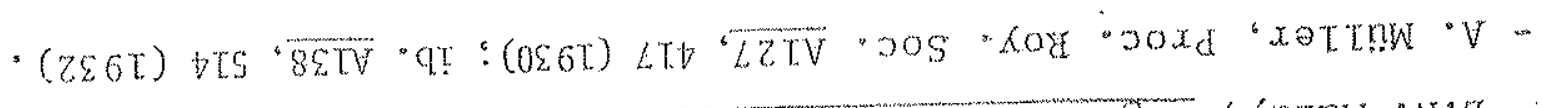

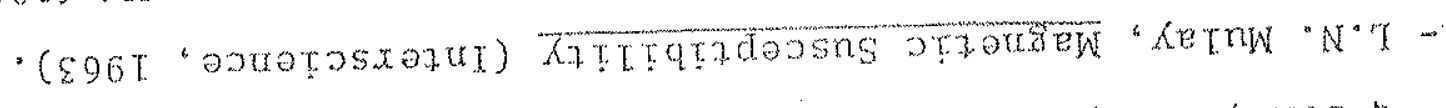
- (996t" "stros s

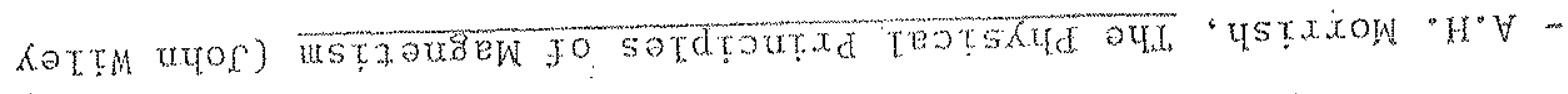

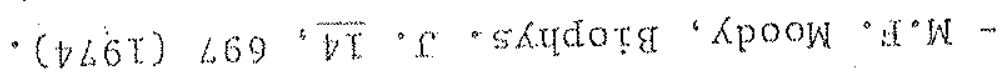

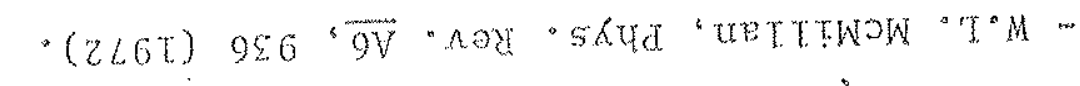

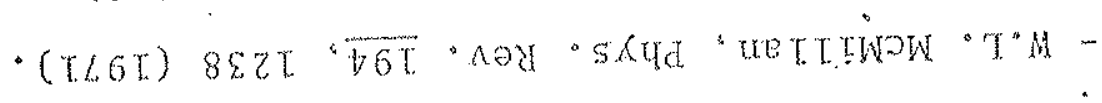

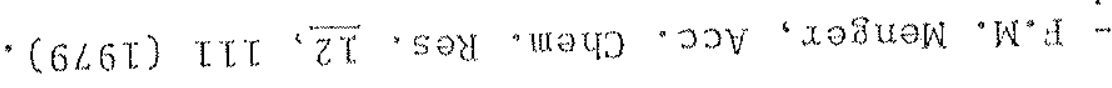
- (5L6T) T9T

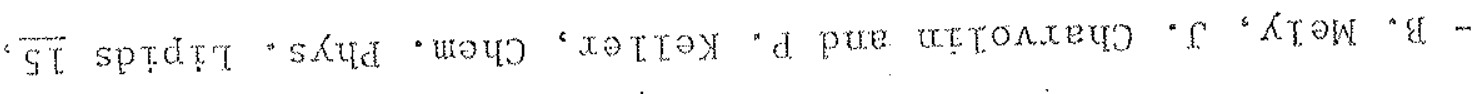

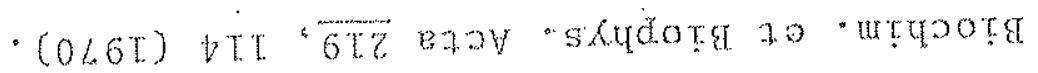

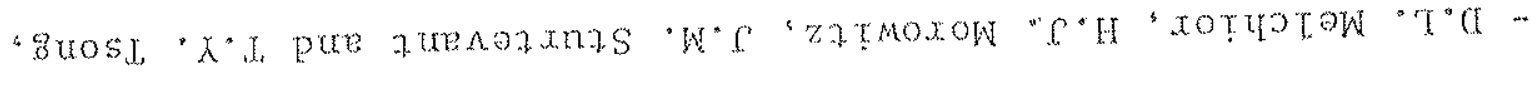

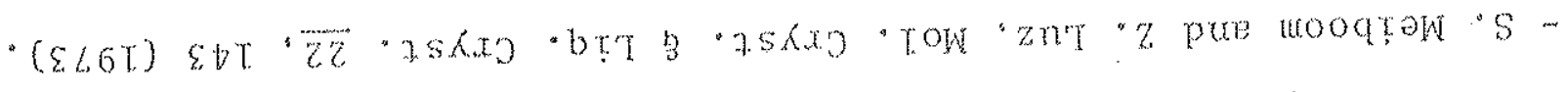

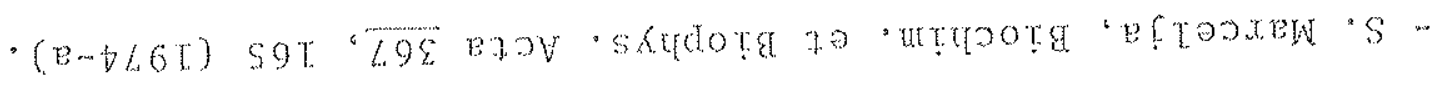

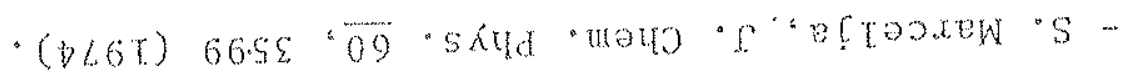

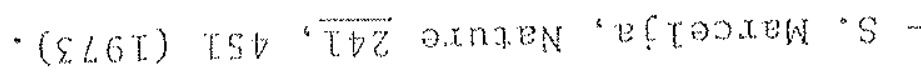
$\cdot(1.96 \mathrm{t}) \vee 2 \mathrm{~T}-68 \cdot 59 \operatorname{sot} 20 \mathrm{~s}$

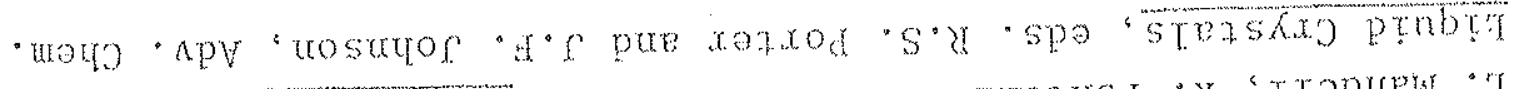

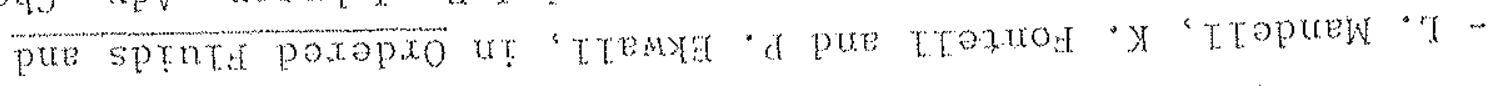




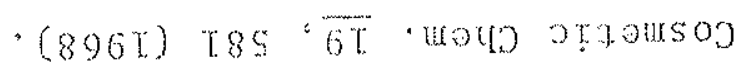

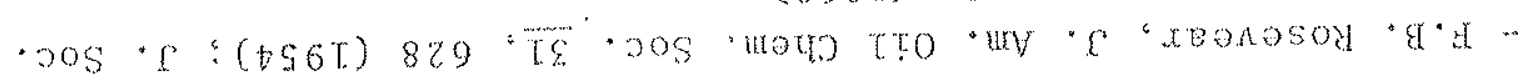

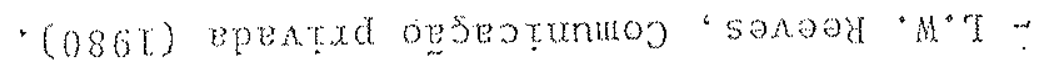

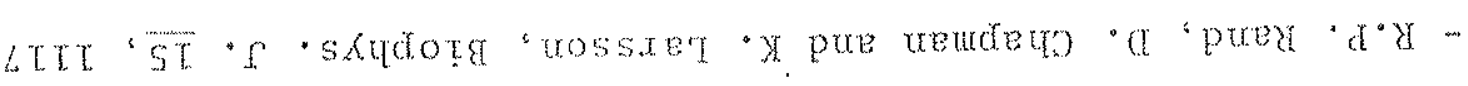

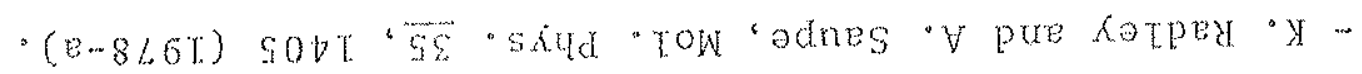

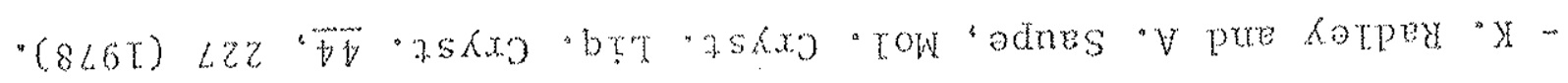
- $(966 \mathrm{~T})$ bL $\mathrm{T}$

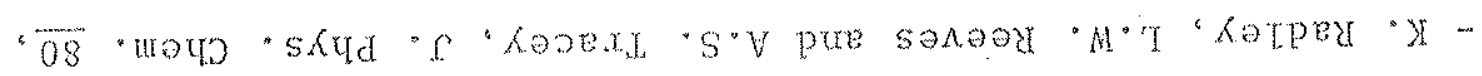

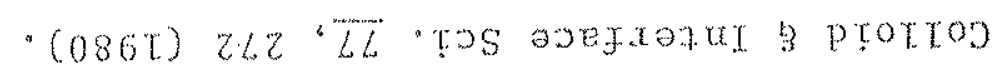

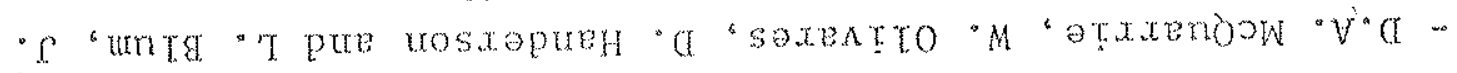

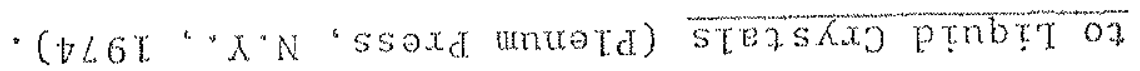

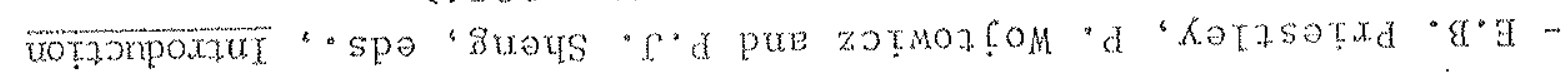

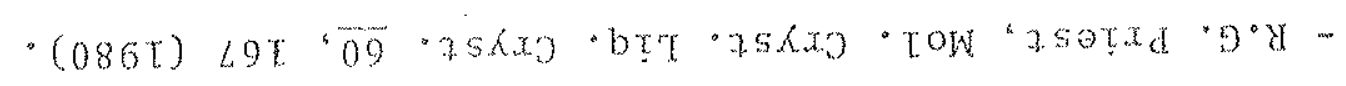

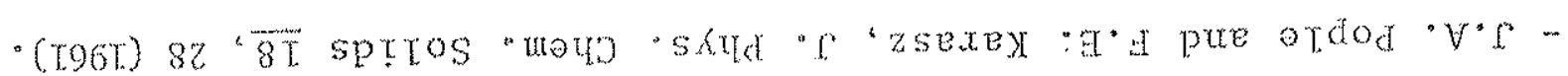
"(T.86T) L.L. "L9

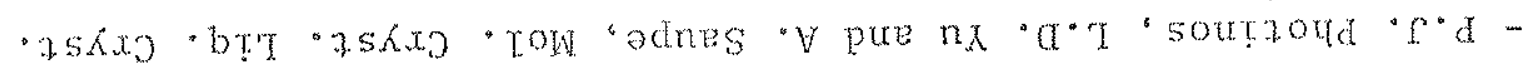

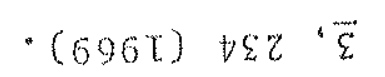

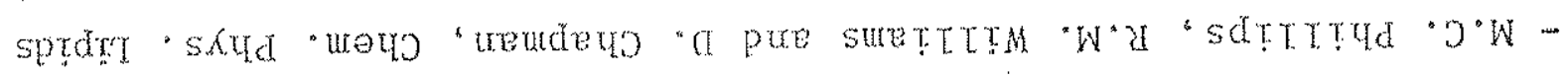

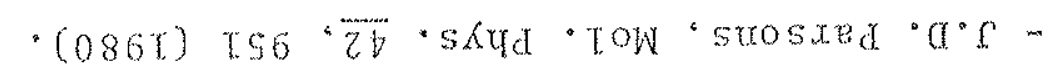

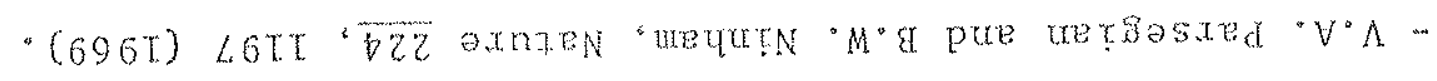

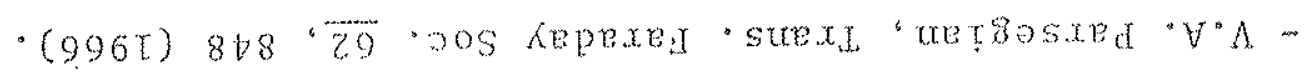

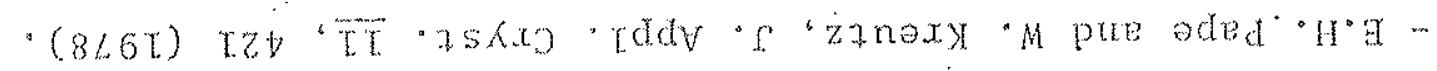

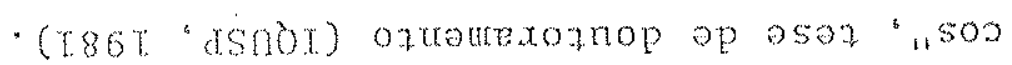

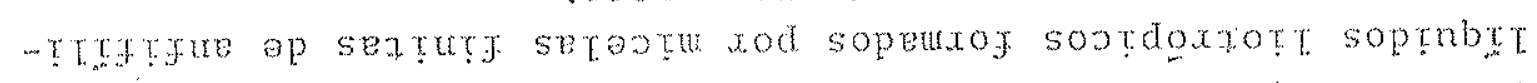

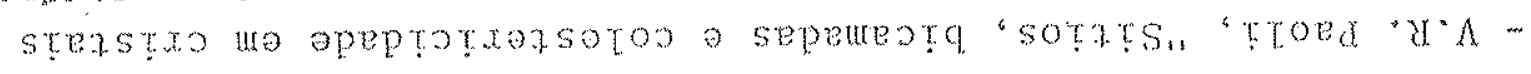

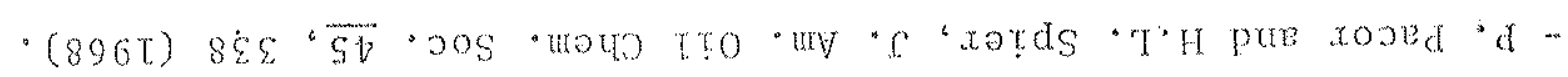

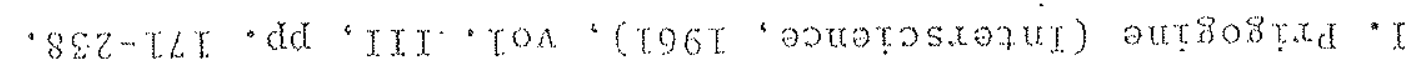

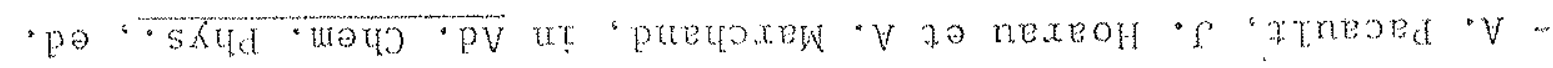

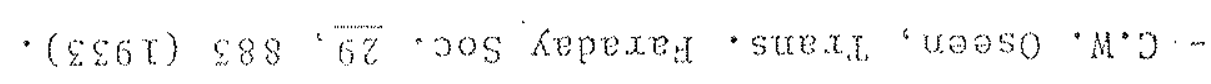

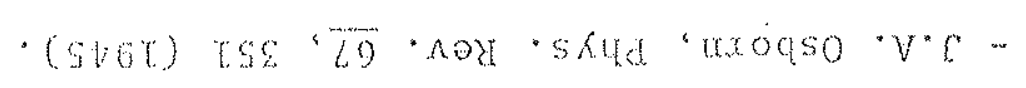

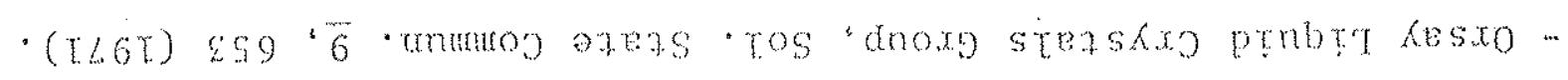

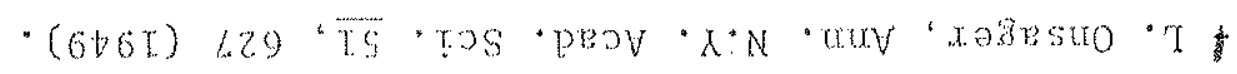


- (bL6t "

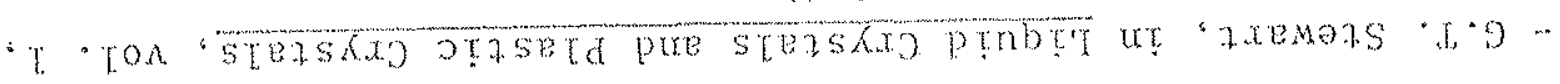
( $(6<6 \mathrm{~T})$

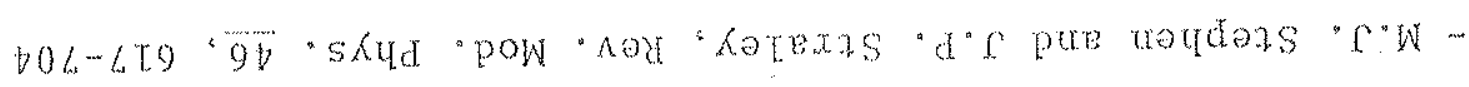

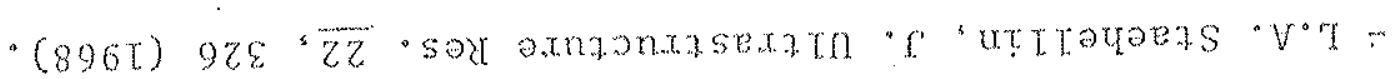
- (196T)

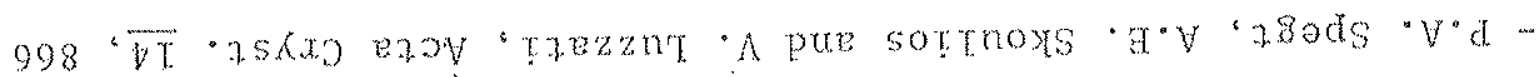
- (696T) 089

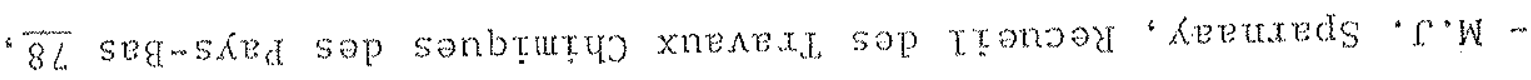

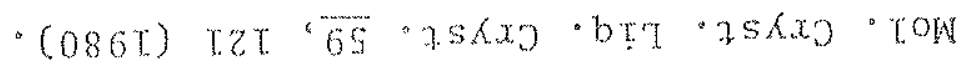

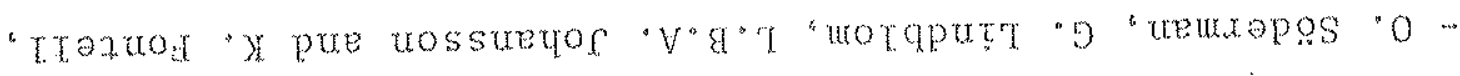
$992-68 \mathrm{~T} \cdot \cdot \mathrm{dd}$

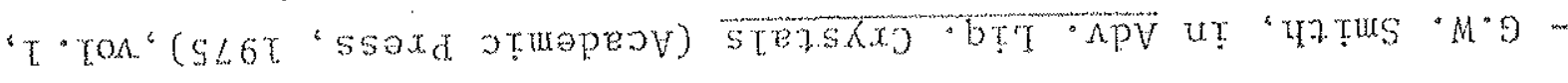

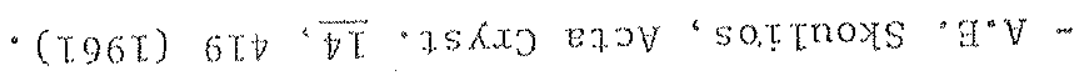

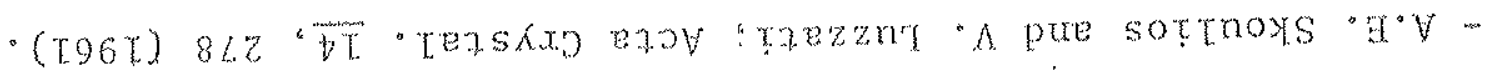
$\cdot(\theta-\angle 66 \mathrm{~T}) 60 \mathrm{~T} \cdot \overline{200} \mathrm{BT} \mathrm{OV}$

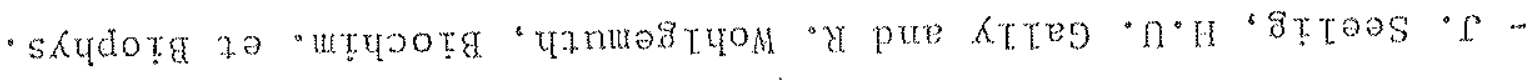

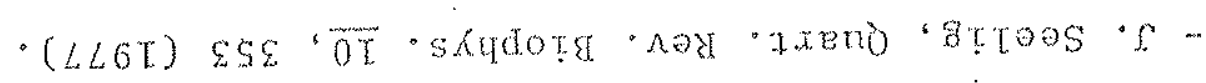

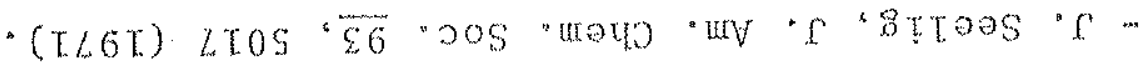

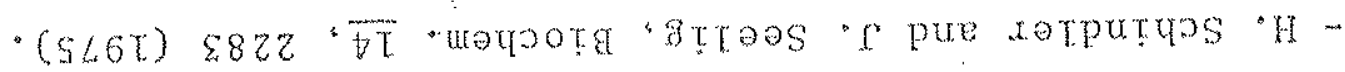

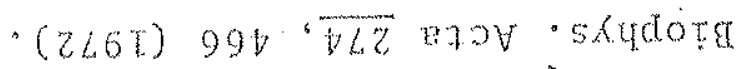

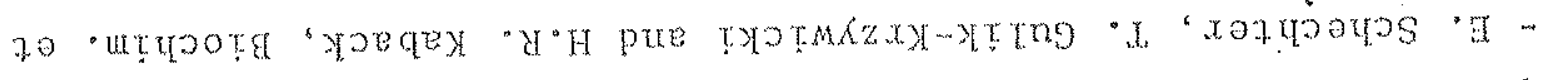

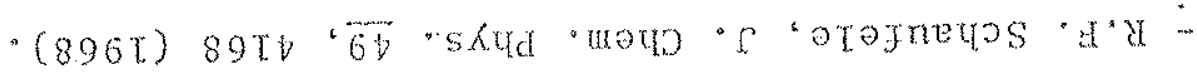

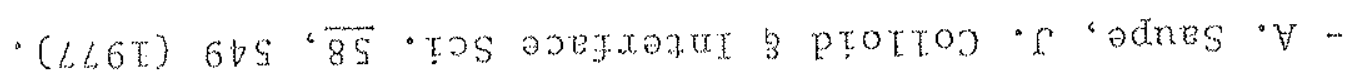

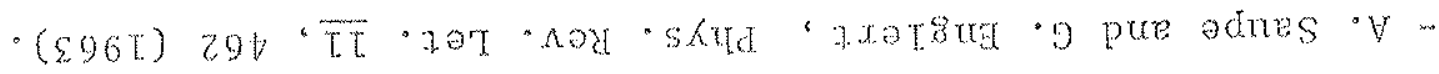

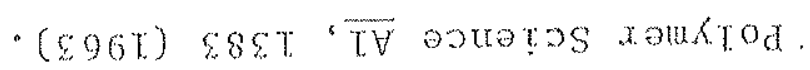

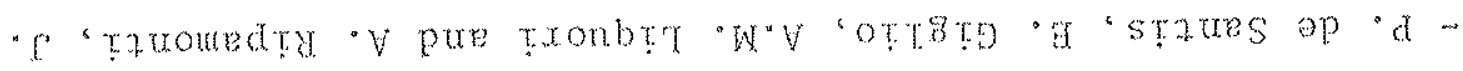

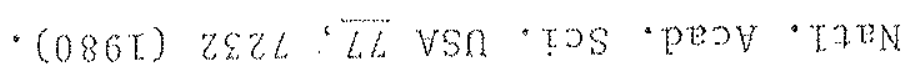

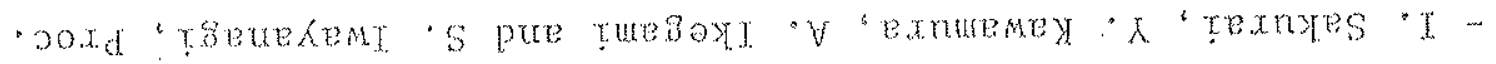

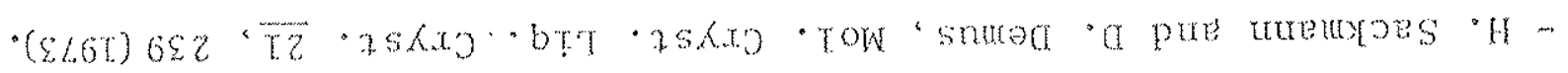

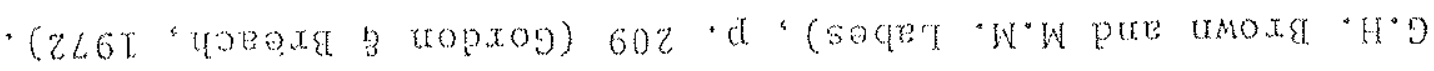

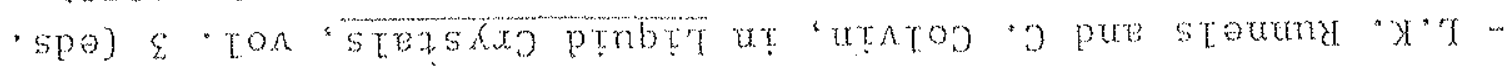

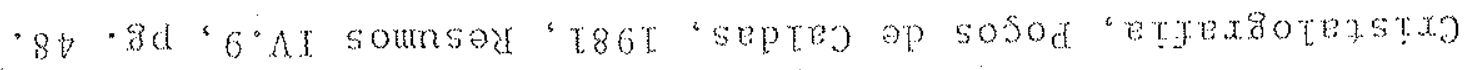

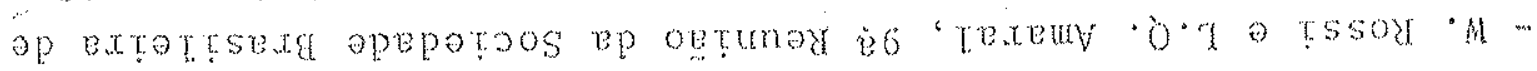




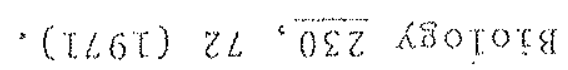

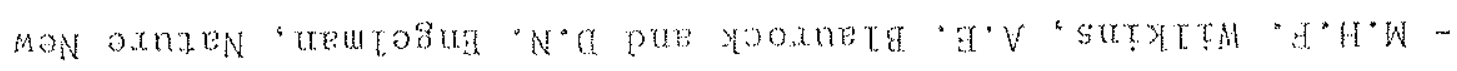

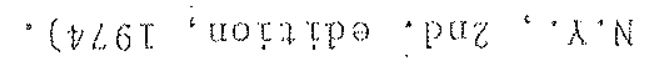

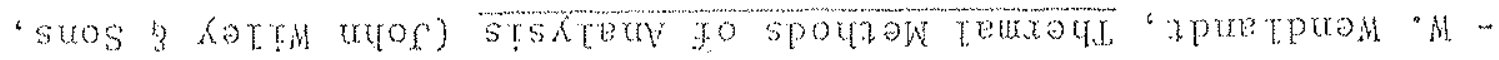

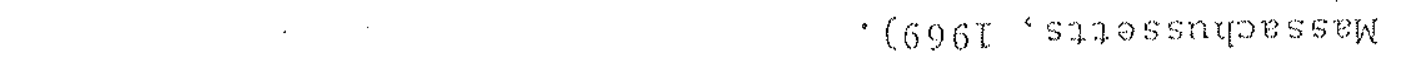

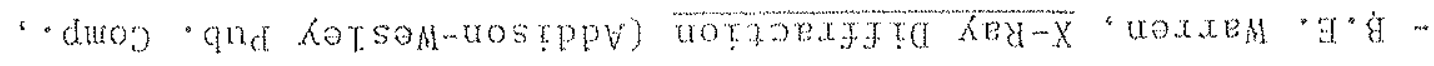

- (696t

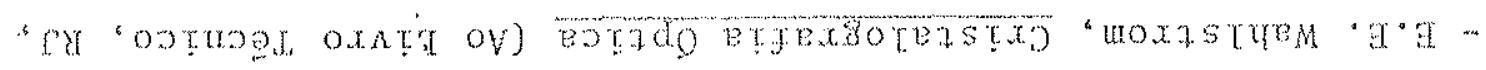

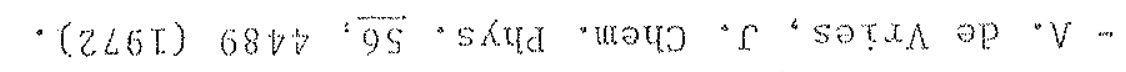

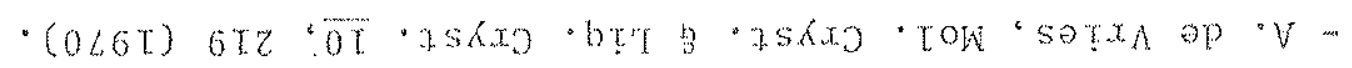
$\cdot(996 \mathrm{l})$

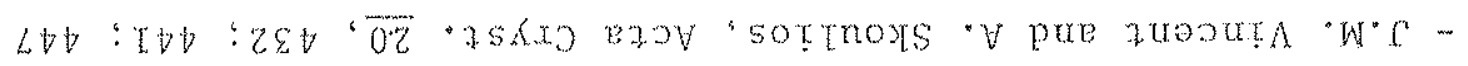

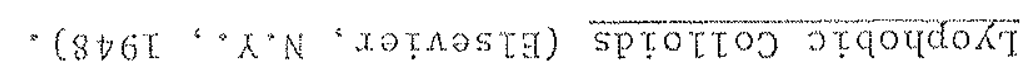

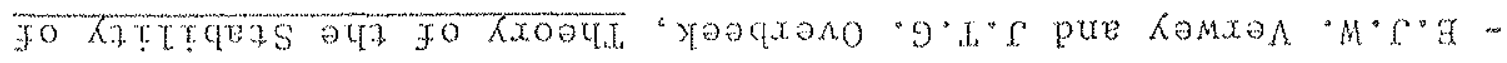
" C $646 \mathrm{~T}$

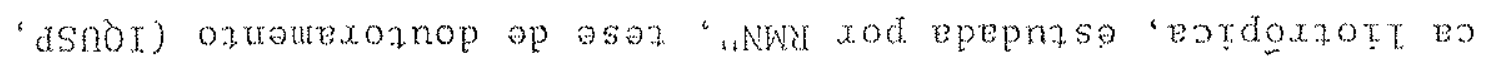

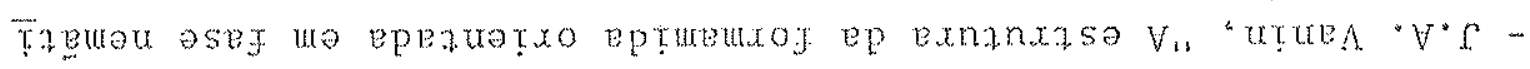

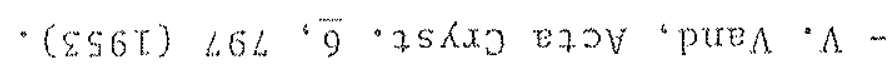

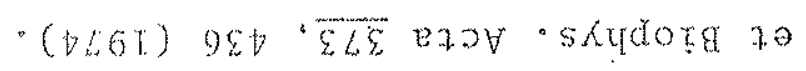

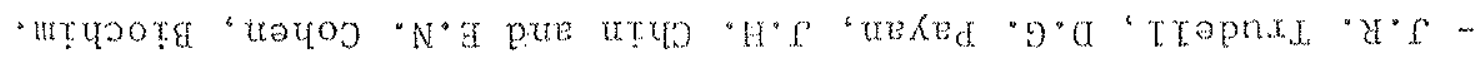
( $(1<6 \mathrm{~T})$

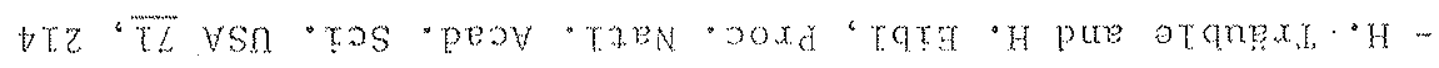

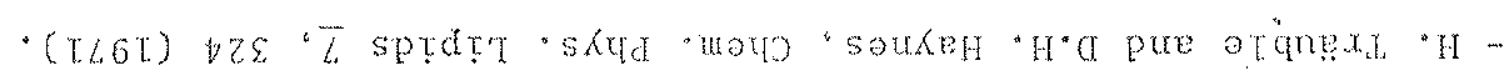

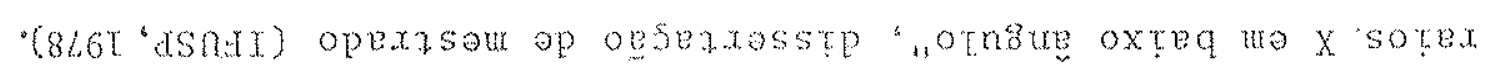

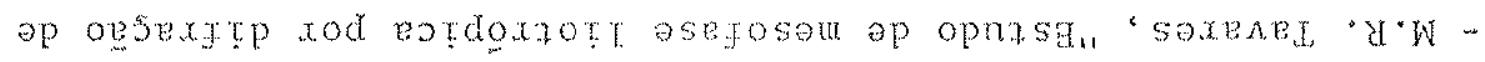

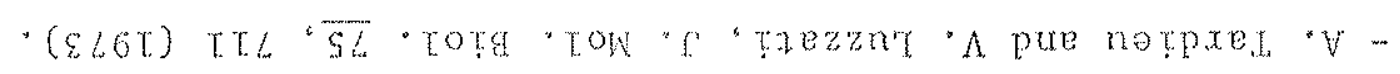

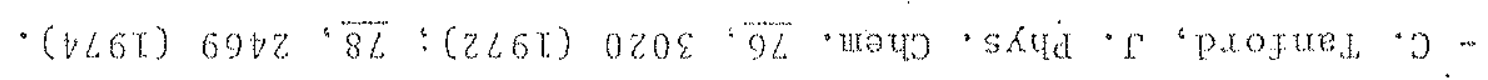

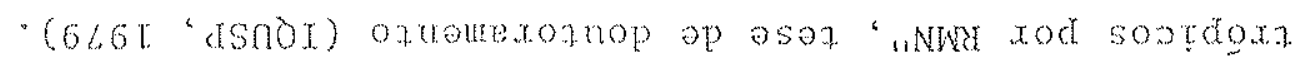

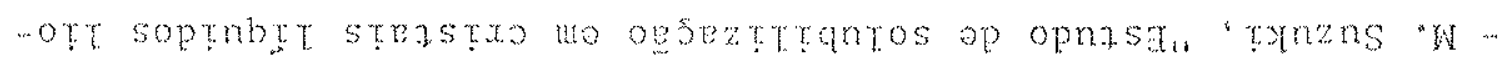

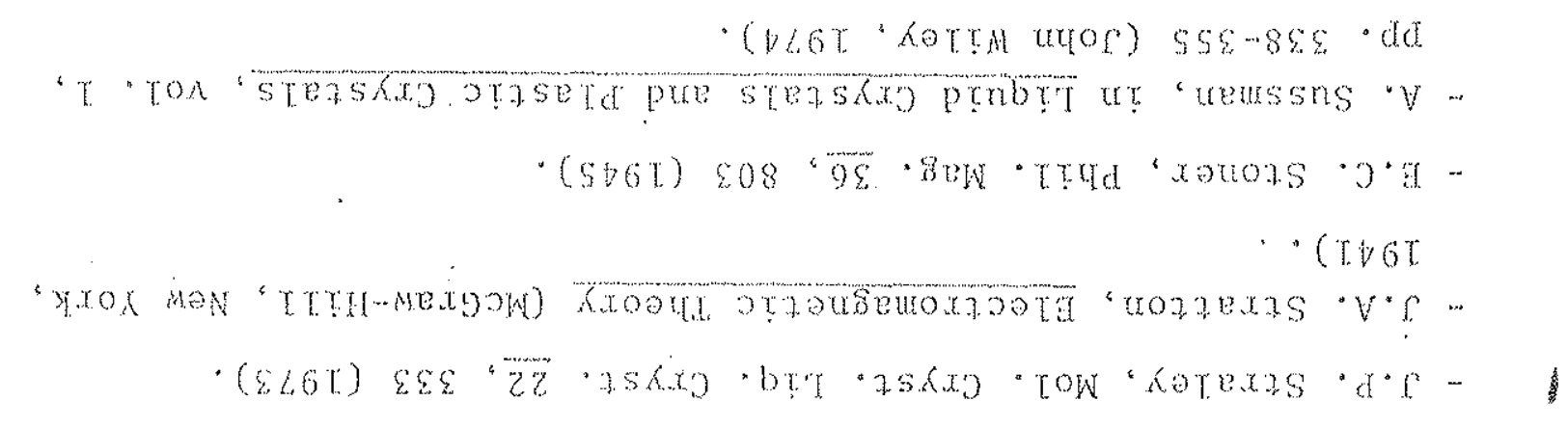




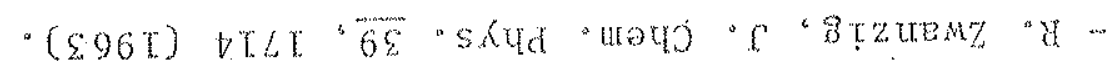

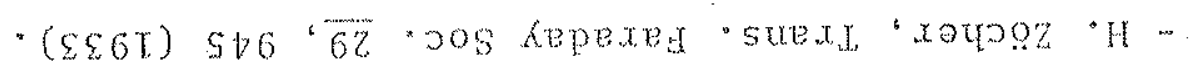

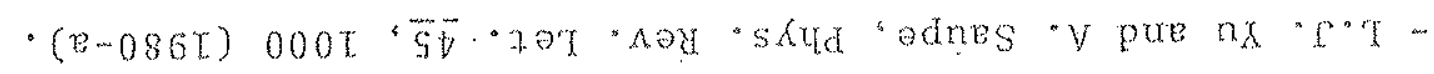

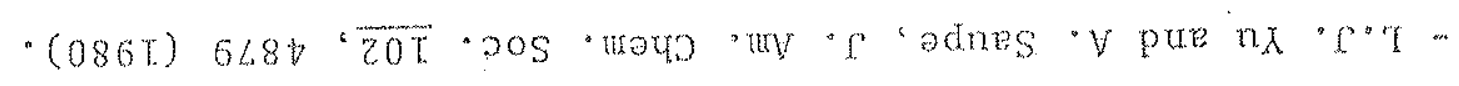

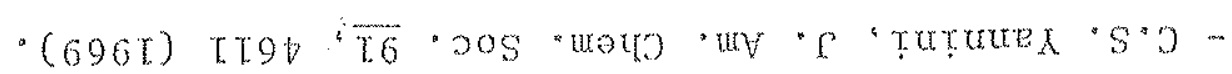

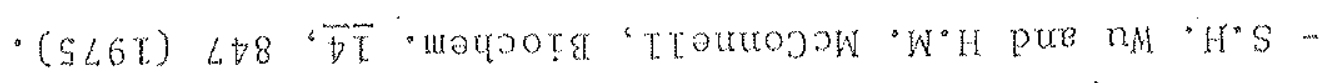
$\cdot(S 66 \mathrm{~T}) 08 \mathrm{~b}$

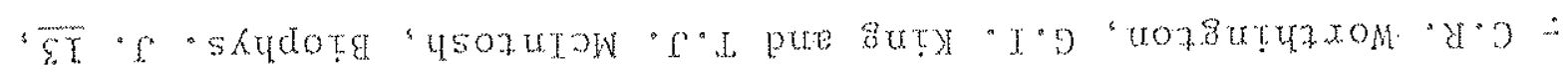

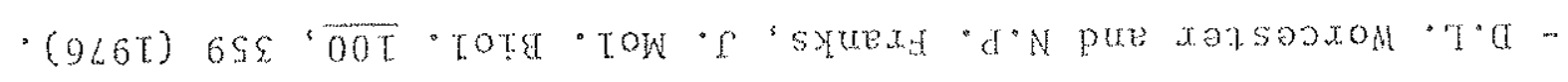
- (0t6t "aopwor 'osptxquar)

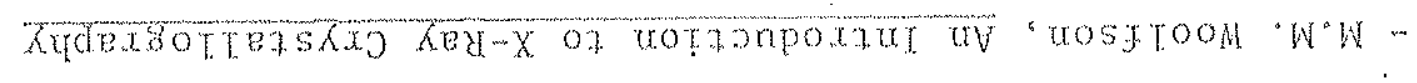

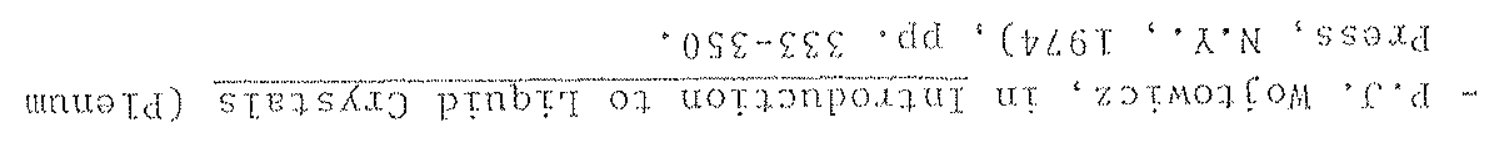

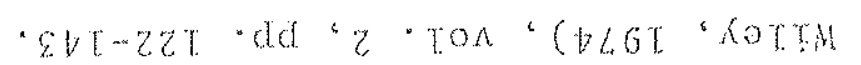

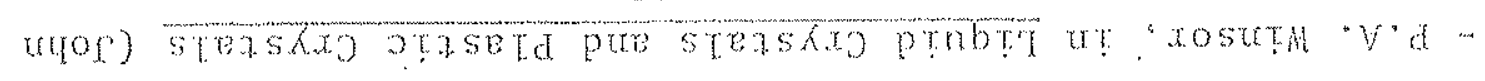

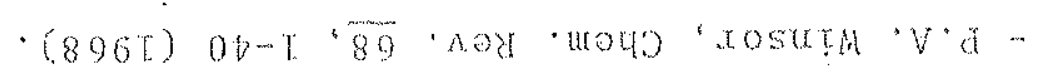

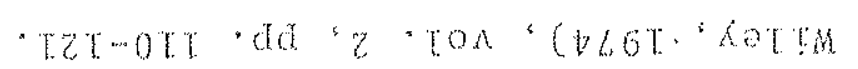

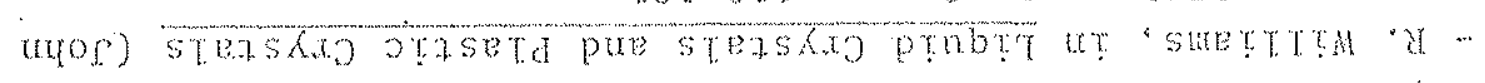

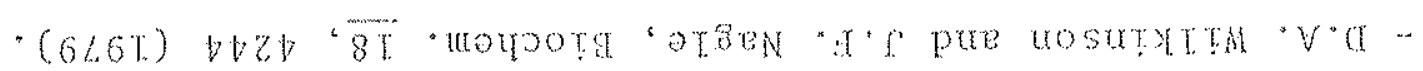

